ORGANIZED BY:

NATIONAL RESEARCH \& DEVELOPMENT

INSTITUTE FOR TEXTILES

AND LEATHER (INCDTP) - DIVISION

LEATHER AND FOOTWEAR

RESEARCH INSTITUTE (ICPI)
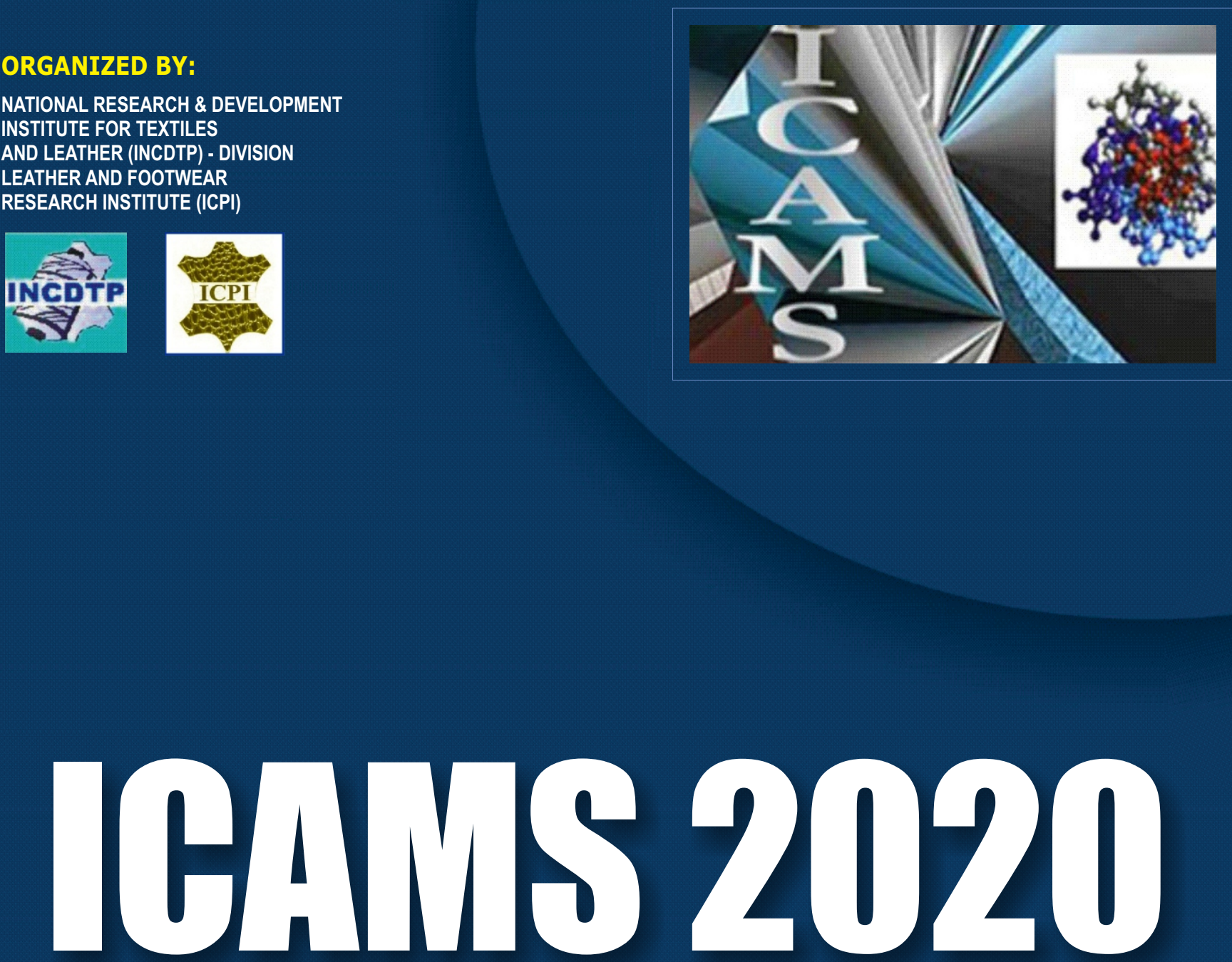

\title{
ADVANCED MATERIALS AND SYSTEMS
}

Proceedings of the 8th International Bonference

Oetober 1st - 3ri, 2020 


\section{Gheorghe COARĂ \\ Laurenția ALEXANDRESCU}

\section{EDITORS}

Proceedings of

\section{THE $8^{\text {th }}$ INTERNATIONAL CONFERENCE ON ADVANCED MATERIALS AND SYSTEMS}

1-3 October, 2020

INCDTP-ICPI

ROMANIA 


\section{Disclaimer}

This book contains full papers approved by the Scientific Committee. Authors are responsible for the content and accuracy. Opinions expressed may not necessarily reflect the position of the International Scientific Committee of ICAMS. Information in the ICAMS 2020 Conference Proceedings is subject to change without notice. No part of this book may be reproduced or transmitted in any form or by any means, electronic or mechanical, for any purpose, without the express written permission of the International Scientific Committee of ICAMS.

\section{Editura CERTEX}

certex@ns.certex.ro

București, str. Lucrețiu Patrascanu nr. 16, sector 3

Tel/ fax: 0213405515

\section{Descrierea CIP a Bibliotecii Naţionale a României}

Gheorghe COARĂ

Laurenția ALEXANDRESCU

The $8^{\text {th }}$ International Conference on Advanced Materials and Systems

Gheorghe COARĂ, Laurenția ALEXANDRESCU

Bucureşti: CERTEX, 2020

ISSN: $2068-0783$

Şef redacție:

Emilia Visileanu

Coordonator \& Coperta: Dana Gurău

Procesare text: Dana Gurău, Mihai Georgescu, Elena Ninciuleanu, Ciprian Chelaru

\section{Copyright (C) 2020}

Toate drepturile asupra acestei ediţii sunt rezervate editorilor. 


\section{SCIENTIFIC COMMITTEE}

Arife Candas ADIGUZEL ZENGIN, Ege University, Izmir, TR

Mădălina Georgiana ALBU KAYA, INCDTPICPI, RO

Laurenția ALEXANDRESCU, INCDTP-ICPI, $\mathrm{RO}$

Carmen ARIAS CASTELLANO, General

Secretary, CEC, BE

Anna BACARDIT, University of Lleida, ES

Elena BADEA, University of Craiova and

INCDTP-ICPI, RO

Bahri BASARAN, Ege University, Izmir, TR

Behzat Oral BITLISLI, Ege University, Izmir, TR

Cristina CARȘOTE, National Museum of

Romanian History, RO

Gheorghe COARĂ. INCDTP-ICPI, RO

Nizami DURAN, Mustafa Kemal University,

Antakya, TR

Maria José FERREIRA, CTCP, PT

Anton FICAI, "Politehnica" University

Bucharest, RO

Margareta FLORESCU, Bucharest Academy

of Economic Studies, RO

Carmen GAIDĂU, INCDTP-ICPI, RO

Mihaela GHICA, "Carol Davila" Univ. of

Medicine\&Pharmacy, RO

Carmen GHIȚULEASA, INCDTP, RO

Gustavo GONZALEZ-QUIJANO, Secretary

General, COTANCE, BE

Gurbuz GULUMSER, Ege University, Izmir,

Huseyin Ata KARAVANA, Ege University, Izmir, TR

Alpaslan KAYA, Mustafa Kemal University, Antakya, TR

Karel KOLOMAZNIK, Tomas Bata

University, Zlin, CZ

Jeannette LUCEJKO, University of Pisa, IT

Jianzhong MA, Shaanxi University of Science and Technology, CN

Stelian MAIER, "Gheorghe Asachi" Technical

Univ. of Iasi, RO

Minodora MARIN, INCDTP-ICPI, RO

Alcino MARTINHO, CTIC, PT

Aurelia MEGHEA, "Politehnica" University

Bucharest, RO
Aura MIHAI, “Gheorghe Asachi” Technical

Univ. of Iasi, RO

Lucreţia MIU, INCDTP-ICPI, RO

Olena MOKROUSOVA, Kyiv National

University of Technologies and Design, UA

Zenovia MOLDOVAN, University of

Bucharest, RO

Enrique MONTIEL PARREÑO, Vesica Piscis Footwear, ES

Mehmet Mete MUTLU, Ege University, Izmir, TR

Georgios PANAGIARIS, Technological

Education Institute, GR

Mirela PANTAZI, INCDTP-ICPI, RO

Dimosthenis PAPAKONSTANTINOU,

CRE.THI.DEV, GR

Vera PINTO, CTCP, PT

Victoriya PLAVAN, Kiev Nat. Univ. of

Techn\&Design, UA

Alina POPESCU, INCDTP, RO

Dmitriy SHALBUEV, East Siberia State Univ.

of Techn.\&Manag., RU

Maria SÖNMEZ, INCDTP-ICPI, RO

Paul STĂNESCU, "Politehnica” University

Bucharest, RO

Keyong TANG, Zhengzhou University, CN

Irina TITORENCU, "N. Simionescu" I.C.B.P., $\mathrm{RO}$

Jin ZHOU, Sichuan University, CN

\section{ORGANIZING COMMITTEE}

Laurentia ALEXANDRESCU, Chair,

Scientific Secretary, INCDTP - ICPI, RO Gheorghe COARA, Co-Chair, Director INCDTP - Leather and Footwear Research Institute Division (ICPI), RO

Carmen GHITULEASA, Co-Chair, General Director, National Research and Development Institute for Textile and Leather, RO

Dana GURAU, INCDTP - ICPI, RO

Ioana PIVNICERU, INCDTP - ICPI, RO

Ciprian CHELARU, INCDTP - ICPI, RO

Mihai GEORGESCU, INCDTP - ICPI, RO

Elena NINCIULEANU, INCDTP - ICPI, RO

Dan VICOVAN, INCDTP - ICPI, RO 



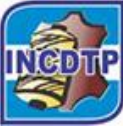

NATIONAL RESEARCH \& DEVELOPMENT INSTITUTE FOR TEXTILE AND LEATHER (INCDTP), BUCHAREST, ROMANIA
DIVISION LEATHER \& FOOTWEAR RESEARCH INSTITUTE (ICPI) BUCHAREST, ROMANIA

\section{PARTNERS}

MINISTERUL EDUCAȚIEI ȘI CERCETǍRII

MINISTRY OF EDUCATION AND RESEARCH

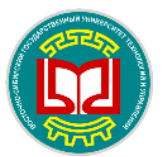

EAST SIBERIA STATE

UNIVERSITY OF

TECHNOLOGY \&

MANAGEMENT, ULANUDE, RUSSIA

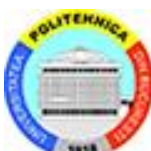

"POLITEHNICA"

UNIVERSITY BUCHAREST, ROMANIA

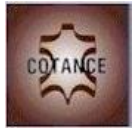

CONFEDERATION OF NATIONAL ASSOCIATIONS OF TANNERS AND DRESSERS OF THE EUROPEAN COMMUNITY

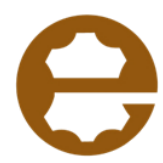

LEATHER ENGINEERING DEPARTMENT EGE UNIVERSITY, TURKEY

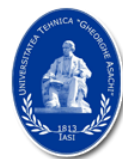

"GH. ASACHI" TECHNICAL UNIVERSITY OF IASI, ROMANIA

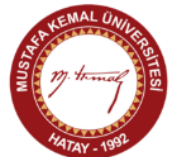

MUSTAFA KEMAL UNIVERSITY, ANTAKYAHATAY, TURKEY

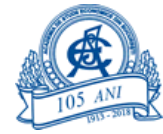

BUCHAREST ACADEMY OF ECONOMIC STUDIES, ROMANIA

sferafactor

SFERA FACTOR

THE ROMANIAN LEATHER MANUFACTURERS

ORGANIZATION
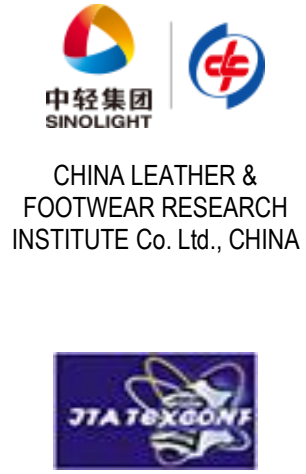

"ITA TEXCONF " ROMANIAN ENTITY WITHIN INNOVATION \& LEATHER TECHNOLOGICAL TRANSFER

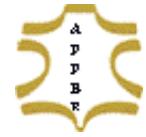

ROMANIAN LEATHER \& FUR PRODUCERS ASSOCIATION 



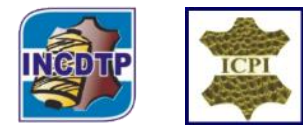

\section{FOREWORD}

ICAMS 2020 is offering the framework for presenting the latest results in research, focusing on the field of Materials Science and Innovative Technologies, which records an impressive dynamic and is recognized as a current national and European priority.

The ICAMS 2020 international event, organised by the National Institute for Research and Development for Textiles and Leather - Division Leather and Footwear Institute (INCDTP-ICPI), took place online on 0103 October 2020. ICAMS 2020 brought together different stakeholders and provides a platform for a better understanding of the European innovation ecosystem while raising awareness of the actions needed to enable synergies and drawing lessons for future actions.

The conference provides the opportunity for exchanging ideas and experience with researchers, scientists and experts at international level, and for developing new scientific contributions.

Participants virtually attended the event from several academic and research institutions, public and private sectors. Participants presented their experience on research, innovation, policies and the creation of synergies. All these inputs offered insightful elements for discussion in the different participatory sessions throughout the event.

The conference topics include, but are not limited to:

1. Advanced Materials and Nanomaterials

2. Biomaterials and Biotechnologies

3. Innovative Systems, Technologies and Quality Management

4. Ecological Processes for Circular and Neutral Economy

5. Creative Industries and Cultural Heritage

6. Education and Digitalization

We would like to thank all the participants, the International Scientific Committee, partners and all the sponsors that made this scientific event possible. ICAMS Conference has already become a tradition, contributing to the advancement of Materials Science in research, academic, social and business environments worldwide.

\section{EDITORS}

Dr. Gheorghe COARĂ

Director, INCDTP - Division: Leather and Footwear Research Institute (ICPI), RO

Dr. Laurenția ALEXANDRESCU

Scientific Secretary, INCDTP - Division: Leather and Footwear Research Institute (ICPI), RO 



\section{CONTENTS}

\section{PLENARY LECTURE}

Gustavo Gonzalez-Quijano, Dorel Acsinte, Gheorghe Bostaca

SER 2020 - A comparative approach proving the EU tanning industry's continuous striving towards sustainable development...........

SESSION 1 - ADVANCED MATERIALS AND NANOMATERIALS

Raluca Maria Aileni, Laura Chiriac, Irina Săndulache

Perspective in using chitosan films for sensors.

Suresh Aluvihara, C.S. Kalpage, P.W.S.K. Bandaranayake

The elementary characterization of anthill clay for composite materials..

Anna Bondaryeva, Olena Mokrousova

The acrylic/montmorillonite nanocomposites for leather finishing.......

Ciprian Chelaru, Mădălina-Camelia Ignat, Rodica Roxana Constantinescu Comparative study of the surface properties for some different types of leather finishes.

Laura Chirilă, Alina Popescu, Sabina Olaru, Angela Dănilă, Rodica

Roxana Constantinescu, Andreea Țigău

Bioactive textiles obtained by applying cinnamon essential oil-based emulsions.

Laura Chirilă, Marcela Corina Roșu, Sabina Olaru, Cristian Tudoran, Dragoș-Viorel Cosma, Alexandra Urda, Alice-Ortansa Mateescu, Gheorghe Mateescu, Georgiana Vasile

Cotton fabrics coated with $\mathrm{Ag}^{-\mathrm{TiO}_{2}}$ and $\mathrm{Ag}^{-\mathrm{TiO}_{2}} /$ reduced graphene oxide nanocomposites.

Durairaj Dhanapal, Bahiru Melese, Liya Shawel, Kumaravel Sanmugam Research comparison of foot plantar pressure on polyurethane visco elastic foam insole and EVA insole materials.

Ion Durbacă, Radu Iatan, Elena Surdu, Dana-Claudia Farcaş-Flamaropol Approaches to the evaluation of the mechanical properties of single-layer composite plates made of recyclable polymeric and protein materials.

Ovidiu Iordache, Elena Cornelia Mitran, Irina Săndulache, Maria Memecică, Lucia Oana Secăreanu, Cristina Lite, Elena Perdum

An overview on far-infrared functional textile materials.

Maryna Leshchyshyn Mykolaivna, Svitlana Stepanivna Garkavenko, Antonina Ivanivna Babich

Studying the similarities of deformation properties of leather materials in the process of creating a model of shoes. 
Maryna Leshchyshyn Mykolaivna, Svitlana Garkavenko, Victoria

Kernesh, Borys Zlotenko, Tetiana Kulik, Anastasia Glionik

Transformation of art objects in the 3D design process of shoe parts...

Carmen Mihai, Mădălina Alice Rus, Alexandra Gabriela Ene, Răzvan Scarlat

Digital construction of the signaling/ rescue system located in coastal aquatic areas.

Maria Sönmez, Mircea Jugănaru, Anton Ficai, Denisa Ficai, Ovidiu Oprea, Dana Gurău, Laurenția Alexandrescu, Maria Daniela Stelescu, Mihai Georgescu, Mihaela Niţuică, Alina Melinescu, Minodora Marin Dolomite surface modification with titanium and silicon precursors and its morphostructural and thermal characterisation.

Maria Sönmez, Mircea Jugănaru, Anton Ficai, Ovidiu Oprea, Roxana Trușcă, Mihai Georgescu, Dana Gurău, Denisa Ficai, Laurenția Alexandrescu, Doina Constantinescu, Maria Daniela Stelescu, Mihaela Nițuică, Minodora Marin

Improvement of mechanical, thermal and morphostructural properties of SBS thermoplastic elastomer using kaolin and dolomite microparticles with modified surface

\section{SESSION 2 - BIOMATERIALS AND BIOTECHNOLOGIES}

Maria-Cristina Anicescu, Cristina-Elena Dinu-Pîrvu, Mihaela Violeta Ghica, Valentina Anuţa, Răzvan Mihai Prisada, Marina-Theodora Talianu, Lăcrămioara Popa

Preliminary analysis of emulsion-based formulations containing pumpkin seed oil and hemp seed oil for internal use.

Mariana Daniela Berechet, Demetra Simion, Maria Stanca, Cosmin Andrei Alexe, Rodica Roxana Constantinescu, Maria Râpă, Andreea Țurcanu Antibacterial and antioxidant activities of lemon balm (Melissa officinalis L.) essential oil.

Mariana Daniela Berechet, Demetra Simion, Maria Stanca, Ciprian Chelaru, Cosmin-Andrei Alexe, Maria Râpă

The influence of alkaline extraction on some keratin hydrolysates properties.

Rodica Roxana Constantinescu, Gabriel Zăinescu, Mariana Ferdeș, Iulia Caniola

Pelt waste degradation using active microbial consortia.

Elena Dănilă, Mădălina Georgiana Albu Kaya, Mihaela Violeta Ghica, AnaMaria Bunea, Lăcrămioara Popa, Durmuș Alpaslan Kaya, Şevket Öztürk, Maria-Minodora Marin, Cristina-Elena Dinu-Pîrvu, Valentina Anuța Formulation and characterization of anti-aging cosmetic emulsions based on collagen hydrolysate and caffeine. 
Yilmaz Eren, Durmuş Alpaslan Kaya

Determination of yield and yield components in different sowing

times of black seed (Nigella sativa L.) in Hatay ecological conditions....

Mariana Ferdes, Rodica Roxana Constantinescu

Biological pretreatment of waste from the leather industry to obtain

biogas through anaerobic co-digestion processes - A review

Mariana Ferdeș, Rodica Roxana Constantinescu

Isolation and characterization of fungal and bacterial proteolytic

strains from chrome shavings.

Carmen Gaidău, Maria Stanca, Demetra Simion, Olga Niculescu,

Cosmin-Andrei Alexe, Concepcio Casas, Anna Bacardit, Stoica Tonea,

Gabriela Păun

Olipo-wet olive pomace, a new renewable source for leather retanning..

Gresy Griyanitasari, Emiliana Kasmudjiastuti, Ragil Yuliatmo,

Mohammad Zainal Abidin

Evaluation of combination tanning and natural finishing on sheep

leather with Uncaria Gambir Roxb extract.

Ovidiu Iordache, Ioana Corina Moga, Elena Cornelia Mitran,

Irina Săndulache, Maria Memecică, Lucia Oana Secăreanu, Cristina Lite,

Elena Perdum

BOD \& COD reduction from textile wastewater using bio-augmented

HDPE carriers

Meryem Karaçam, Durmuş Alpaslan Kaya

The effect of some essential oils on pathogenes that cause eczema........

Durmuş Alpaslan Kaya, Filiz Ayanoğlu

Oil glands number and oil glands diameters of Thymbra spicata var.

Spicata L. Leaves.

Lesia Maistrenko, Olga Iungin, Oleksii Savchuk, Olena Okhmat

Collagen matrices from leather industry wastes for biomedical application

Maria-Minodora Marin, Mădălina Georgiana Albu Kaya, Mihaela Violeta Ghica, Elena Dănilă, Gheorghe Coară, Lăcrămioara Popa, Ciprian Chelaru, Durmuș Alpaslan Kaya, Valentina Anuța, Cristina-Elena Dinu-Pîrvu, Ioan Cristescu

Design and evaluation of doxycycline/collagen/chondroitin sulfate delivery systems used for cartilage regeneration.

Maria-Roxana Marinescu, Elena David, Liviu-Daniel Ghiculescu

Obtaining of high density carbon materials by coke sintering resulting

from heat treatment of tar for applications in sensors manufacture.....

Kazim Mavi, Durmuş Alpaslan Kaya, Musa Türkmen, Filiz Ayanoğlu The variation of essential oil and carvacrol contents of native grown Thymbra Spicata var. Spicata $\mathrm{I}$ 
Mihaela-Doina Niculescu, Cristina Emanuela Enăscuță, Maria Stanca, Carmen Cornelia Gaidău, Cosmin Alexe, Mihai Gîdea, Marius Becheritu

Complexes based on collagen and keratin for applications in agriculture

Olga Niculescu, Carmen Gaidău, Elena Badea, Lucreția Miu, Dana Gurău, Mariana Daniela Berechet

Ecological approaches for protecting and perfuming natural sheepskin fur..

Olga Niculescu, Carmen Gaidău, Elena Badea, Lucreția Miu, Dana

Gurău, Demetra Simion

Special effect finish for bookbinding leather.

Demetra Simion, Carmen Gaidău, Jianzhong Ma, Zhang Wenbo

New nanostructured composite obtained by innovative technologies.....

Darina Zheleva, Margarita Koleva

Influence of type of pre-hair treatment from different types of animal sources on the degree of hydrolysis of keratin.

\section{SESSION 3 - INNOVATIVE SYSTEMS, TECHNOLOGIES AND QUALITY MANAGEMENT}

Anna Bacardit, Sílvia Sorolla, Concepció Casas, Lluís Ollé, Mireia Conde Synthesis of polyurethanes with low volatile organic compounds content for upholstery and automotive articles.

Mahmoud Barakat, Ahmed Ali, Islam Abdelbary, Mai Haroun

The impact of supply chain integration on operational performance through resilience under COVID-19 pandemic

Engy Bayoumi, Sara Elgazzar, Matevž Obrecht

Investigating the impact of Internet of Things on Egyptian ports sustainability

Dana Corina Deselnicu, Mihaela-Ramona Bulboacă, Dan Dumitriu,

Laurenția Alexandrescu

Analysis of the bakery industry strategic groups in Romania.

Ion Durbacă, Nicoleta Sporea, Dana-Claudia Farcaș-Flamaropol, Elena Surdu Application of the "SIX SIGMA" method for the analysis of the improvement of the environmental air quality parameters at the municipality of Bucharest, by monitoring the pollutances of NOX pollutants

Nermin Gohar, Hany Hanna, Mai H. Haroun

Factors affecting startups survival in the MENA region in the presence of COVID19.

Sandra Haddad

Developing an E-Business system to improve the downstream pharmaceutical supply chain (A study on the Egyptian market) 
Islam Saleh Hassanin

The role of Internet of Things on intelligent transport system: A

traffic optimization model.

Keyu Hou, Shunxun Li, Jingjing Li, Wei Xu, Rui Wang, Jin Zhou

Research on consumer portraits of offline fast fashion shoe stores based on IoT smart hardware.

Jilan Keshta, Hebatallah Elmesmary, Matevž Obrecht

Investigating the impact of COVID-19 on maritime supply chain sustainability and technology: a review.

Jingjing Li, Jie Zeng, Keyu Hou, Jin Zhou, Rui Wang

Application of Openpose algorithm to detect consumer behavior in store...

Alexandra Luca, Alina Iovan Dragomir

Quality management and footwear value chain. A case study of Fibalco

Alaa Othman, Ahmed Dabees, Baher Rahma, Matjaz Knez

Factors influencing the purchasing decisions of low emission cars:

comparing study between Egypt and Slovenia.

Vera Radnaeva, Dmitry Shalbuev, Nikolay Sovetkin, Khurelsukh Gaanbaatar, Solongo Khosbayar, Suvda Dashdorj, Victoriy Alekhina

Horse paws as raw material for fur industry.

Sengoda Gounder Rajamani

Innovative ecological processes with recovery of chemicals and water for reuse in leather sector - an economical and sustainable approach...

Adrian Săliștean, Carmen Mihai, Doina Toma, Sabina Olaru

Theoretical and practical aspects of the design phase for a single skin textile wing.

\section{SESSION 4 - ECOLOGICAL PROCESSES FOR CIRCULAR AND NEUTRAL ECONOMY}

Laurenția Alexandrescu, Mihai Georgescu, Maria Sönmez, Mihaela Nițuică, Maria Daniela Stelescu

Development and characterization of biodegradable polymeric composites based on butadiene-co-acrylonitrile rubber and functionalized postconsumer wood waste.

Olga Ballus, Anna Bacardit

Sustainable products in the leather industry

Rodica Roxana Constantinescu, Gabriel Zăinescu, Iulia Caniola

Smart biopolymers from protein wastes used in agriculture.

Dana Corina Deselnicu, Beatrice Alexandra Alexandrescu

Comparative analysis of sustainable development indicators at E.U. and Romanian level. 
Maria José Ferreira, Vera V. Pinto, Patrícia Costa

LIFE GREENSHOES4ALL - Footwear environmental footprint..........

Mihai Georgescu, Maria Sönmez, Laurenția Alexandrescu, Mihaela Nițuică, Maria Daniela Stelescu, Dana Gurău

Designing and obtaining wood waste and chloroprene rubber-based composites...................................................................................................

Hany Hanna, Paul Xirouchakis, Athanasios Rentizelas

Developing Integrated Green Supply Chain Drivers and Barriers Framework for Green Supply Chain Adoption, MENA Region.............

Karolis Kubilius, Violeta Valeikiene, Virgilijus Valeika

Collagen hydrolysate from chromed shavings for leather finish.

Gheorghe Lăzăroiu, Dana-Alexandra Ciupăgeanu, Lucian Mihăescu, Rodica-Manuela Grigoriu

Comparative analysis of carbon dioxide methanation technologies for low carbon society development.

Gheorghe Lăzăroiu, Lucian Mihaescu, Rodica-Manuela Grigoriu, DanaAlexandra Ciupăgeanu, Iulia Simion

Efficient poultry industry waste management approach in the bioeconomy framework

Mihaela (Vîlsan) Nițuică, Laurenția Alexandrescu, Mihai Georgescu, Maria Sönmez, Maria Daniela Stelescu, Dana Gurău, Carmen Curuțiu, Ștefania Stoleriu

Biodegradable polymeric composite based on EPDM rubber and functionalized elastomeric waste.

Mihaela (Vîlsan) Niţuică, Laurenția Alexandrescu, Mihai Georgescu, Maria Sönmez, Maria Daniela Stelescu, Dana Gurău, Carmen Curuțiu, Ştefania Stoleriu

Development and characterization of biodegradable compound based on EPDM and wood waste.

Sabina Olaru, Ionela Badea

Circular product design assessment applied to clothing products.

Mircea Constantin Opriș, Dana Corina Deselnicu Wool processing outcomes and opportunities

Mirela Pantazi-Băjenaru, Enrique Montiel Parreno, Traian Foiași,

Dana Gurău

REWEART - A $100 \%$ real circular economy manufacturing process for vegan-organic-recycled footwear 
Ion Răzvan Rădulescu, Emilia Visileanu, Răzvan Scarlat, Cătălin

Constantin, Bogdana Mitu

Comparative life cycle assessment study for fabric based

electromagnetic shielding

Carlos Ruzafa-Silvestre, Pilar Carbonell-Blasco, Elena Orgilés-Calpena,

Francisca Arán Aís

Low-pressure plasma treatment applied to polymeric materials for a

sustainable footwear industry.

Teodor Sârbu, Angela Dorogan, Cristina Grosu, Cristina Elena Stroe Innovative tool for the circular design of technical textiles.

Auksè Šliburytè, Virgilijus Valeika

Treatment of hide liming wastewater by carbon dioxide

Maria Daniela Stelescu, Daniela Ioana Constantin, Maria Sönmez, Laurenția Alexandrescu, Mihaela Niţuică, Mihai Georgescu

Development of elasto-plastic eco-nano-materials for the footwear industry..

Maria Daniela Stelescu, Mihai Georgescu, Maria Sönmez, Mihaela

Niţuică, Adriana Ștefan

Elastomeric nanomaterials based on natural rubber for the food industry..

SESSION 5 - CREATIVE INDUSTRIES AND CULTURAL HERITAGE

Traian Foiași, Mirela Pantazi-Băjenaru

Innovative and sustainable models in the ecodesign of green-vegan

footwear

Mahmoud A. Hammad, Sonja Mlaker Kač, Sandra S. G. Haddad, Habiba

S. El Rouby

Investigation of energy resources and gas discoveries in the Eastern

Mediterranean region: the case of people's expectations and social

impacts in Egypt

Mădălina Ignat, Lucreția Miu, Emanuel Hadîmbu, Claudiu Șendrea, Maria-Cristina Micu, Simona Maria Păunescu, Iulia Maria Caniola,

Elena Badea

The influence of $\mathrm{TiO}_{2}$ and $\mathrm{N}-\mathrm{TiO}_{2}$ nanopowders in natural leather

finishing for heritage or modern binding.

Alina Iovan Dragomir, Alexandra Luca

Product development of the leather good using Walton's matrix........... Lucreția Miu, Rodica Roxana Constantinescu, Alpaslan Durmuş Kaya, Emanuel Hadîmbu, Claudiu Șendrea, Maria-Cristina Micu, Simona Maria Păunescu, Iulia Maria Caniola, Elena Badea

Aspects regarding the effectiveness of volatile oils of the Tanacetum vulgare kind in the conservation of heritage objects. 
Lucreția Miu, Mariana Giurgincă, Iulia-Maria Caniola, Maria-Cristina Micu, Simona-Maria Păunescu, Claudiu Șendrea, Elena Badea Chromatic assessment of newly manufactured leather and parchment for museum purposes.

Lucreția Miu, Simona-Maria Păunescu, Maria-Cristina Micu, Iulia-Maria Caniola, Madalina Ignat, Claudiu Șendrea, Elena Badea

Chemical and physico-mechanical characterizations of leather for restoration.

Mircea Constantin Opriș, Dana Corina Deselnicu

Start-up investment for a sheep wool processing line.

Mirela Pantazi-Băjenaru, Traian Foiași

Design and manufacture of products with Romanian cultural identity

Marlena Pop

Design thinking in product development - case study: leather library..

Irina Săndulache, Mihaela-Cristina Lite, Lucia-Oana Secăreanu, ElenaCornelia Mitran, Ovidiu Iordache, Elena Perdum

Effects of UV, humidity, and high temperature exposure on linen fibers..

Lucia-Oana Secăreanu, Irina-Mariana Săndulache, Elena-Cornelia Mitran, Mihaela-Cristina Lite, Adrian Alexandru Apostol, Ovidiu Iordache, Elena Perdum

Protocol for identification and assessment of natural and synthetic textile fibers

Claudiu Șendrea, Maria-Cristina Micu, Emanuel Hadîmbu, Simona Maria Păunescu, Iulia Maria Caniola, Mădălina Ignat, Lucreția Miu, Elena Badea Micro DSC and NMR mouse studies of collagen-vegetable tannin interaction mechanism during leather making.

\section{SESSION 6 - EDUCATION AND DIGITALIZATION}

Raluca Maria Aileni, Laura Chiriac, Răzvan Ion Rădulescu

Fostering advanced textile centers through e-learning in Morocco and Jordan.

Marlena Pop, Dorina Horătău

The iteration method for developing creativity in ecodesign.

INDEX OF AUTHORS. 


\section{PLENARY LECTURE}





\title{
SER 2020 - A COMPARATIVE APPROACH PROVING THE EU TANNING INDUSTRY'S CONTINUOUS STRIVING TOWARDS SUSTAINABLE DEVELOPMENT
}

\author{
GUSTAVO GONZALEZ-QUIJANO ${ }^{1}$, DOREL ACSINTE ${ }^{2,3}$, GHEORGHE BOSTACA ${ }^{2}$ \\ ${ }^{1}$ COTANCE, 40 Rue Washington, B-1050 Brussels, Belgium, cotance@euroleather.com \\ ${ }^{2}$ APPBR - Romanian Leather \& Fur Producers Association, 93 Ion Minulescu st., sector 3, \\ 031215, Bucharest, Romania, appb.ro@gmail.com \\ ${ }^{3}$ S.C. Pielorex S.A., Jilava, Bucharest
}

\begin{abstract}
The paper presents results of the new Social and Environmental Report of the European Leather Industry (SER 2020) that follows up on the exercise done in 2012. Based on an intensive survey amongst European tanneries, led by COTANCE and industriAll-European Trade Union, company data on social indicators and environmental parameters that reflect the performance of the tanning sector were collected. Companies' data, anonymised and aggregated at national level and centrally computed at European level are presented and analysed, versus 2012 data, where appropriate (in terms of average values). Social Footprint of the EU Tanning Industry (employment contracts, age distribution in the EU force, staff retention, education, citizenship, gender balance) and Environmental Footprint of the EU Tanning Industry (chemical consumption, energy consumption, breakdown of energy sources, water consumption, removal of water pollution, waste generation, solvent consumption, costs and investments) are thoroughly discussed. Finally, Sustainability priorities / Ethical issues for the value chain and Objectives and challenges for the future are communicated in order to demonstrate the continuous striving of Europe's leather sector towards excellence in social and environmental performance.
\end{abstract}

Keywords: social report; environmental footprint; sustainable development

\section{INTRODUCTION}

The peerless quality of European leather is internationally recognized. Technological innovation, process performance, environmental protection, social accountability, design and style, are the assets that make the success story of European tanners.

The European Tanning Industry is composed of nearly 1,600 companies and 33,000 workers and 7.4 billion Euro turnover, albeit there has been a gradual concentration over the last decade. The sector has traditionally been composed of family-owned small and medium enterprises but also includes large, listed multinational companies. The average size of a European tannery is currently 21 employees; in 2000, it was 24 employees. Today, the tanning industry in Europe represents a strategic segment of the manufacturing sector, thanks to the combination of tradition and continuous innovation. These characteristics have led the European tanning sector to become a global leader in terms of both value and of quality. The EU share of global turnover is the largest, at $30 \%$, before China, Brazil, India and the other producers.

European tanners process all the main species (bovine - over $80 \%$ of production -, ovine and caprine) and supply for all the end uses for leather. The main market destination of leather has traditionally been the footwear sector. It is still the largest destination, accounting for $38 \%$ of European production. However, in recent years use in other products has increased, such as leather goods $(22 \%)$ and car interiors (13\%). 
The Social and Environmental Report of the European Leather Industry (SER 2020) is the second publication of its kind. The first, published in 2013, was a follow-up action from a previous initiative of the Social Dialogue of the European Leather sector, that adopted a protocol for the reporting of social and environmental indicators. Indeed, as early as 2009 , business and workers representatives drew up a list of parameters against which to measure the sector's performance with regard to key social and environmental criteria. This was to help companies position themselves against a European benchmark, providing the sector with a common instrument for measuring progress over time, serving as a communication tool in the leather value chain, and as a model for other regions of the world.

This SER 2020 allows you to judge for yourself the commitment of the men and women of Europe's tanneries, to sustainability.

\section{WORKING METHOD APPROACHED}

Based on an intensive survey amongst European tanneries, led by COTANCE and industriAll-European Trade Union, company data on social indicators and environmental parameters that reflect the performance of the tanning sector were collected. Companies' data, anonymised and aggregated at national level and centrally computed at European level are presented and analysed, versus 2012 data, where appropriate (in terms of average values).

The sample comprised 79 companies (5\% of the EU total) from Italy, Germany, Austria, Denmark, Sweden, UK, Spain, France, Portugal, Hungary and Romania. Although the distribution of the respondents in the EU countries does not replicate the structure of the European tanning sector, the representativeness in terms of production volume is quite high at $43 \%$ of total EU production. Company data have been collected for each year of the reference period $(2016$ - 2017 - 2018).

To enable an assessment of trends between the first and second SER, the same Key Performance Indicators (KPIs) have been considered. They constitute the most significant parameters to assess sustainability. The comparison between SERs has been made on the basis of the average results of the first SER and the current one.

For the comparison of the results, it is worth noting that the samples of the two editions of the SER differ in terms of representativeness of company size, production cycle, leather typologies and countries of reference.

In particular, the 2019 survey is characterised by a higher percentage of full cycle companies (from hides/skins to finished leather). There were also differences between the reports, in terms of production specialization of the responding companies and their wastewater treatment options. Therefore, comparison of certain KPI was not done, due to the inconsistency of reporting between the two reports, e.g. waste production and removal of pollutants. Moreover, the different sample composition led to a slight increase of some environmental indicators (chemicals consumption, waste) due to inherent differences in the processes described, skewing the final result. For example, the second report included a higher proportion of full-cycle, high volume bovine leather manufacturers for the automotive sector which as previously noted, will report more process steps and consequently, greater use of chemicals and energy, artificially distorting any comparison with the first report. 


\section{RESULTS AND DISCUSSIONS}

\section{Social Footprint of the EU Tanning Industry}

One of the pillars on which the social responsibility of the European tanning industry is based is the respect and valorisation of human resources. This is essential for an industry that combines technological innovation and craftsmanship.

\section{Employment Contracts}

More than $90 \%$ of workers in European tanneries have a permanent employment contract. Breakdown of workers in the sample is presented in Figure 1.

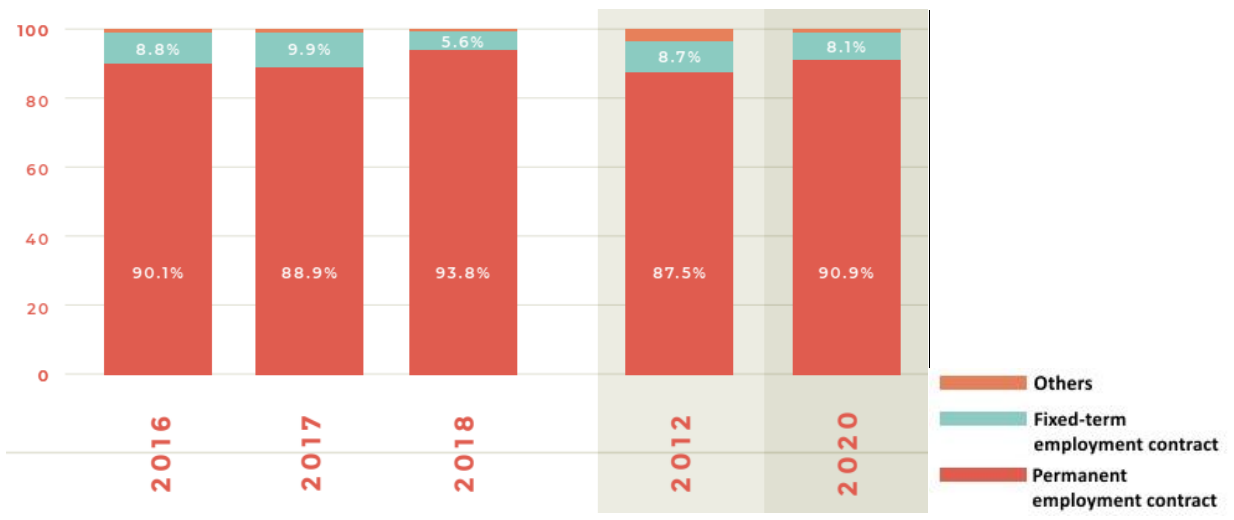

Figure 1. Breakdown of workers in the sample by employment contract, comparison SER 2012 vs. SER 2020

\section{Age Distribution in the EU Workforce}

The age distribution data revealed an increase in the over 55 age group and a decrease in the 36-45 age group. Age brackets are presented in Figure 2.

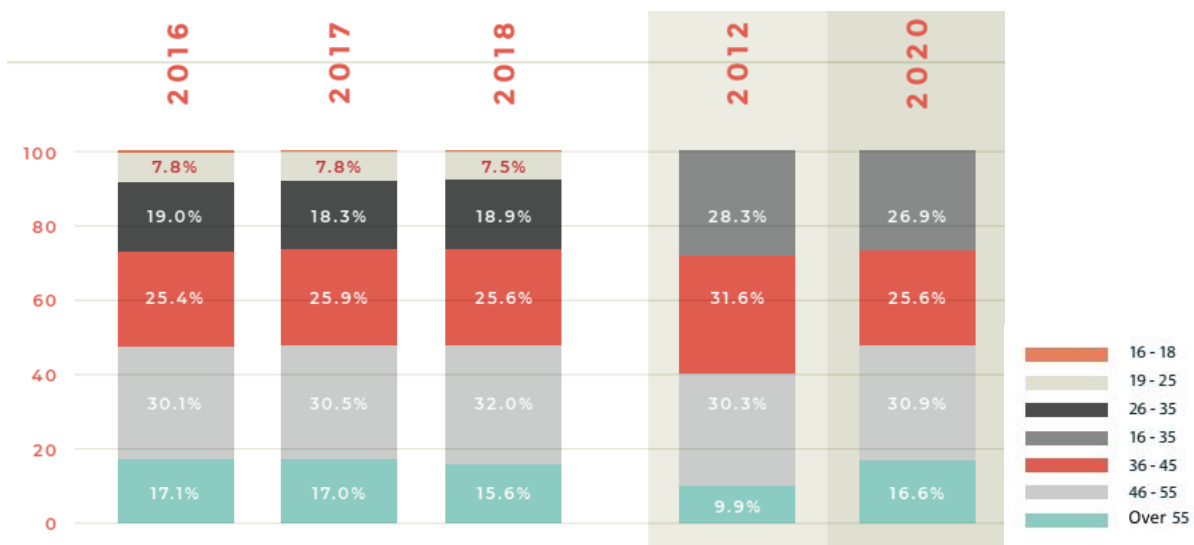

Figure 2. Age distribution of workers in the tanneries composing the sample, comparison SER 2012 vs. SER 2020 


\section{Staff Retention}

The data confirm that almost $50 \%$ of the workforce has been employed in the tanning sector for more than 10 years, 26\% from 10 to 20 years and $12 \%$ between 20 and 30 years. This confirms that employees recognise and value work in the tannery, which, despite its unwarranted negative image, is characterised by a safe and stimulating working environment.

\section{Education}

The education data reveals a more educated workforce than seen in 2012. The number of employees with qualifications of EQF $5 \& 6$ has doubled and those with EQF $3 \& 4$ has also increased, compared to 2012.

\section{Citizenship}

The number of migrant workers has doubled since the previous survey. This is linked to the increased mobility of workers in the sector in EU countries, especially in Central and Northern Europe, in the last year.

\section{Gender Balance}

The number of females in the workforce has increased slightly compared to 2012. The trend is certainly positive, although with the physical nature of tannery work, it is unlikely that gender balance will be achieved. The slight increase in female personnel could be linked to the ongoing process of transformation and technological innovation of manufacturing processes, which has reduced the very physical nature of some activities.

\section{Environmental Footprint of the EU Leather Industry}

Tanning is fundamentally an activity corresponding to the circular economy. The raw materials, hides and skins, are residues of the food industry, bio-based substances synthesized from by-products or residues of other industries are used in tanning processes, and residues from the leather process can be recovered and used by other industrial sectors, including agriculture, food, pharmaceutics and others. Finally, leather is a durable material. Leather articles will last a lifetime and can be repaired or remanufactured, going well beyond resource efficiency and recycling.

\section{Chemical Consumption}

Data collected for this survey show that between 2016 and 2018, European tanneries consumed an average $2.15 \mathrm{~kg}$ of chemicals per square metre of finished leather. Chemical products are normally applied in aqueous solution during the 'wet processes' of leather manufacture (liming, tanning, dyeing and fatliquoring) and sprayed or layered on the surface of the leather during the finishing phase. Average consumption of chemicals $\left(\mathrm{kg} / \mathrm{m}^{2}\right)$ in the tanneries composing the sample, 2016-2018, are presented in Figure 3. 


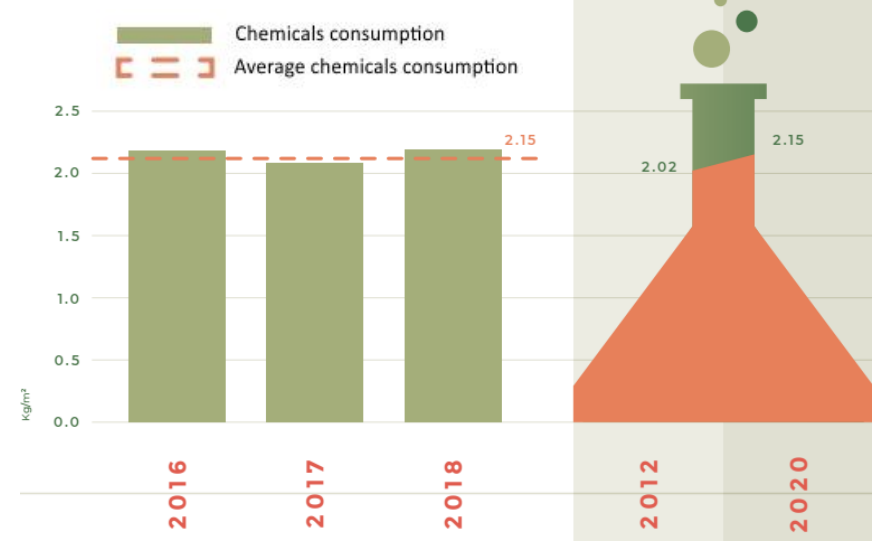

Figure 3. Average consumption of chemicals $\left(\mathrm{kg} / \mathrm{m}^{2}\right)$ in the tanneries composing the sample, comparison SER 2012 vs. SER 2020

Note: Differences in the sample composition in 2012 and 2020 do not allow a meaningful direct comparison. In 2012 there was a significantly higher proportion of tanneries starting from wet-blue which results in an average lower chemicals consumption. The closeness to the 2020 figure suggests that there has been a further reduction in chemical consumption.

\section{Energy Consumption}

Over the last three years, European tanners used an average of 1.76 Tonne of Oil Equivalent (TOE) per 1000 square metres of leather. This unit represents the amount of energy released by burning one tonne of crude oil, about 42 gigajoules or 11630 megawatt-hours. Average consumption of energy by tanneries composing the sample expressed in terms of Tonnes of Oil Equivalent (TOE) per $\mathrm{m}^{2}, 2016-2018$, is presented in Figure 4.

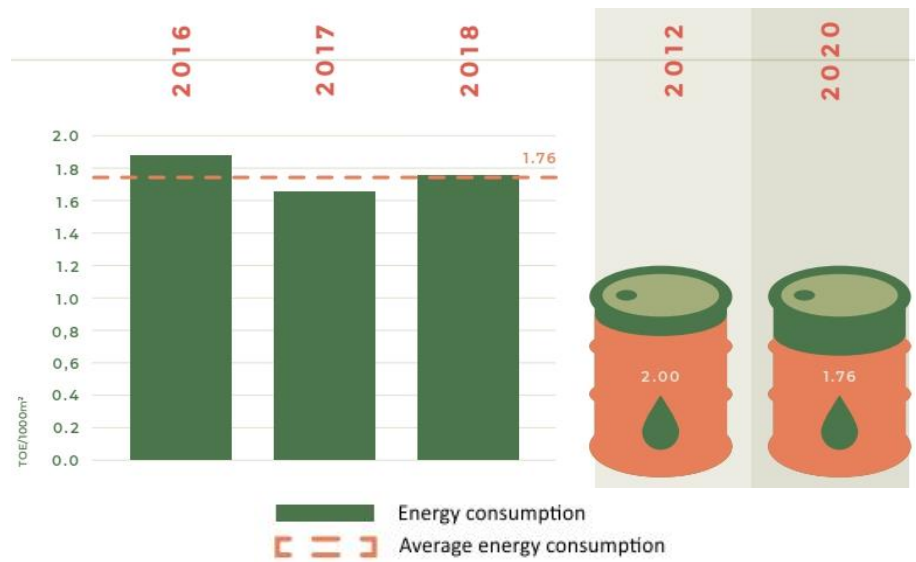

Figure 4. Comparison of the average consumption of energy expressed in terms of Tonnes of Oil Equivalent (TOE) per $\mathrm{m}^{2}$ by tanneries composing the sample of SER 2012 and SER 2020 
SER 2020 - A Comparative Approach Proving the EU Tanning Industry's Continuous Striving towards Sustainable Development

\section{Breakdown of Energy Sources}

Natural gas is the main source of energy for European tanneries, accounting for more than $2 / 3$ of total energy consumption. However, tanneries are investing more and more in renewable energy resources.

\section{Water Consumption}

In 2016-2018, European tanneries consumed an average of 0.121 cubic metres of water to produce one square metre of finished leather, about $7 \%$ less than the amount reported for years 2010-2011.

\section{Removal of Water Pollution}

Efficiency of wastewater treatments with regard to certain pollutants for the tanneries composing the sample, 2016-2018, is presented in Figure 5.

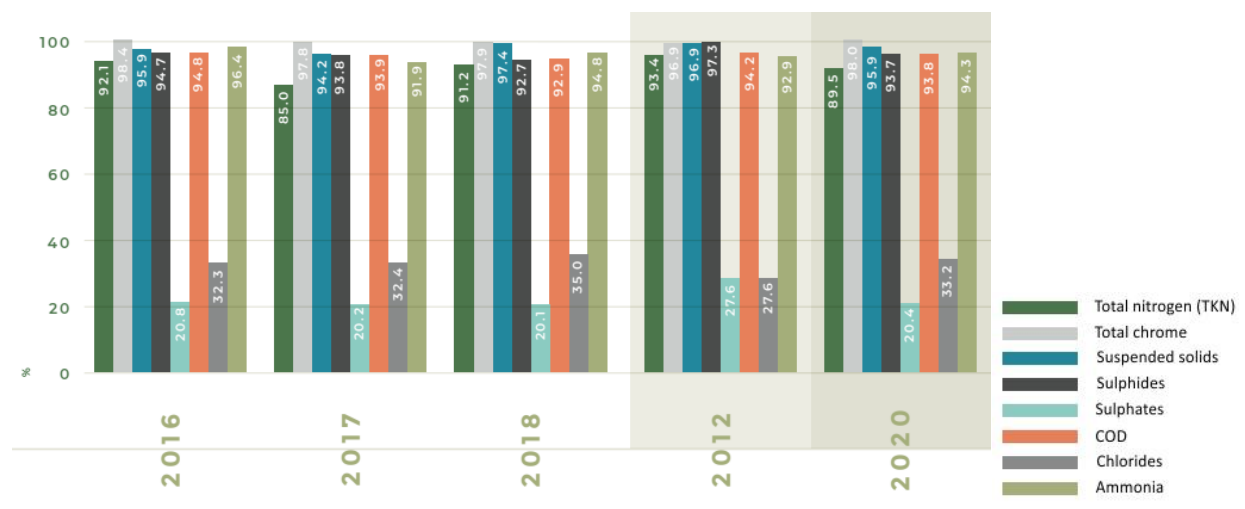

Figure 5. Efficiency of wastewater treatments with regard to certain pollutants for the tanneries composing the sample, comparison SER 2012 vs. SER 2020

\section{Waste Generation}

After valorisation of by-products, European tanneries generate an average of $2.63 \mathrm{~kg}$ of wastes per square metre of finished leather produced.

\section{Solvent Consumption}

In order to further reduce emissions to air, the European tanning sector is constantly working to reduce the use of solvents. As such, solvent consumption is a good measure for monitoring the quality of tannery air emissions. The 3-year analysis shows an average solvent consumption of $29.5 \mathrm{~g}$ per square metre of finished leather. This represents a reduction of $32 \%$ compared to the previous report.

\section{Environmental Costs and Investments}

Environmental costs in 2020 are similar to those reported in 2010, averaging around $4 \%$ of turnover. The value represents the "equilibrium level" between increased 
investments/costs and efficiency in processing (from both an environmental and economical perspective). The main directions are the following:

- Wastewater treatment

- Waste management

- Air pollution abatement

- $\quad$ Energy saving/renewable energy

\section{Sustainability Priorities / Ethical Issues for the Value Chain}

Mainly refer to:

- Fair trade (banning of export restrictions/ taxes on raw materials)

- Product quality and innovation

- Social accountability and environmental performance

- Due diligence: proactive and reactive process through which enterprises can prevent and mitigate adverse impacts related to human rights, labour rights, or the environment

- Product safety: refers to origin marking, requirements applicable to leather, process chemicals and auxiliaries for leather production

- Traceability and transparency in the supply chain

- Animal welfare

\section{Objectives and Challenges for the Future}

Important tasks for the future in our opinion seem to be:

- Protection of the term leather and guarantee the authenticity of leather in advertisements or labels and descriptions of articles and products, so that consumers can make informed purchasing choices.

- Quality of raw materials, that requires the alignment of all the links of the supply chain, from livestock breeding and transport, to slaughterhouses and hides \& skins collection and storage centres.

- Sectoral education \& training services: with an ageing population, it is important to ensure the renewal of the workforce and the transmission of knowledge as well as the provision of new skills.

- Strengthen the good governance of the sector at the international level.

\section{CONCLUSIONS}

Leather is a fascinating material in many ways. Who doesn't react to the distinctive scent of leather or the soft, warm touch of its surface? But leather invites our interest for other reasons. It is probably the oldest example of the circular economy.

Leather-making is also sensible from an ethical and environmental point of view. It is now widely understood that livestock are not slaughtered for hides or skins, as they represent only a small part of the value of an animal. The use of these raw materials is significantly better than wasting them, creating a global environmental and sanitary disaster.

The use of leather avoids the waste of a renewable resource. Using leather reduces the need for plastics or other synthetics derived from non-renewable sources, that end up in our oceans and whose micro-particles can now even be found in the food chain. 
Leather is also natural and biodegradable. However, to be deserving of the qualification of sustainable, leather must also comply with strict social and environmental standards. It is nonsensical for leather to have these extraordinary intrinsic credentials, if during its production it creates more environmental damage than it avoids, or if workers are exposed to dangerous chemicals. Just as leather must fulfil strict criteria for protection of consumers, emissions to water, land or air, risks during production must be also managed and reduced.

Europe's environmental ambitions are described in its Green Deal, the Circular Economy Action Plan and the Farm to Fork Strategy. The European Leather industry expects much from these. After the adoption of the PEFCR for leather, the industry is now advocating for zero-allocation of the environmental impact of livestock rearing to hides and skins, and participating in the development of a PEFCR for Apparel \& Footwear. In this context, $R \& D$ for cleaner production technologies continues to improve the sector's environmental performance while improving the quality of products and processes.

The Social and Environmental Report (SER 2020) illustrates all of these and the progress achieved by the European Leather Industry, since 2012, towards sustainable development.

\section{Acknowledgements}

COTANCE and IndustriAll - European Trade Union, the Social partners of the project VS/2019/0009, express their gratitude in particular to all European Leather Associations, companies and worker representatives that have offered their support and contribution to the development of all activities.

COTANCE and IndustriAll-Europe dedicate the Social \& Environmental Report (SER 2020) of the European Leather Industry to the sector's COVID-19 victims, their families and their communities.

\section{REFERENCES}

COTANCE (2020), Social and Environmental Report of the European Leather Industry, Social Dialogue Project VS/2019/0009, www.euroleather.com.

The European Commission support for the production of this publication The content is the sole responsibility of the authors and the Commission cannot be held responsible for any use which may be made of the information contained herein.

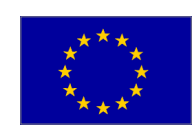

https://doi.org/10.24264/icams-2020.I.0 


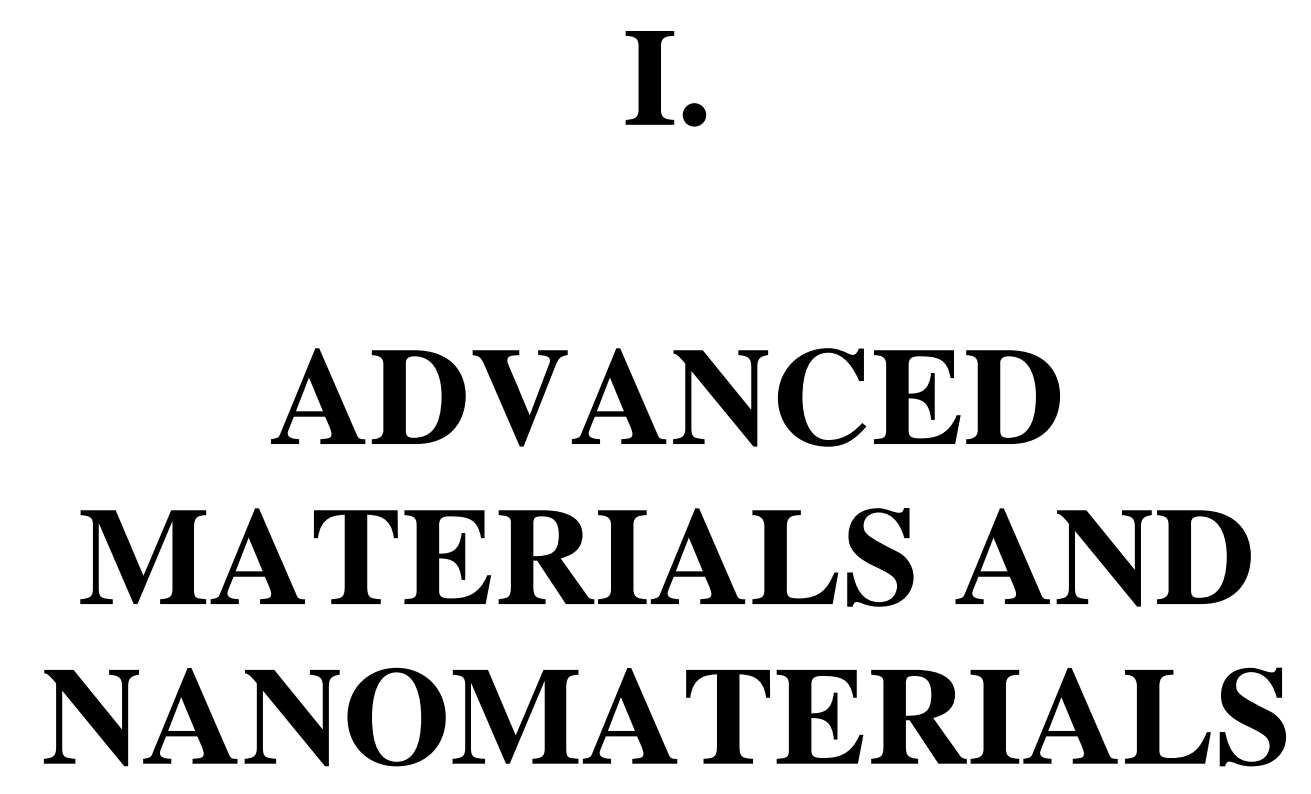




\title{
PERSPECTIVE IN USING CHITOSAN FILMS FOR SENSORS
}

\author{
RALUCA MARIA AILENI, LAURA CHIRIAC, IRINA SANDULACHE \\ INCDTP, Lucretiu Patrascanu 16, Bucharest, Romania, e-mail: raluca.aileni@incdtp.ro
}

\begin{abstract}
This paper presents some aspects concerning the possibilities of using chitosan films for sensor development. The proposed approaches consist of the development of the experimental samples using $100 \%$ cotton fabrics (plain weave) coated with a conductive paste based on chitosan (low, medium, and high molecular weight) and copper microparticles. Our samples were obtained using the scraping method and free drying $(24 \mathrm{~h})$, followed by crosslinking $2-3$ minutes at $150-160^{\circ} \mathrm{C}$. Surface resistance was investigated using the resistance tester based on two parallel electrodes. The results showed that textile coated with chitosan paste with metallic particle content has a poorly conductive character. Based on the surface resistivity, was calculated the surface conductivity. Using the VCA Optima device was investigated the surface wettability by contact angle to conclude if the samples present a hydrophilic or hydrophobic character. After these tests, we concluded that almost all samples have a hydrophilic character due in large part to the fact that this polymer (chitosan) has hydrophilic nature.
\end{abstract}

Keywords: textile, chitosan, sensors

\section{INTRODUCTION}

The scientific interest in developing chitosan films for medicine due to the antimicrobial properties, sensors, food packaging, or heavy metals collecting, was increased because chitosan is a natural mucopolysaccharide of marine origin and is considered a friendly, biodegradable and biocompatible polymer. It is already known the antibacterial effect, heavy metal adsorption effect, antioxidation effect, and film development of the chitosan. Several studies on the mechanical properties of chitosan films reinforced with the cellulose of coconut fibers found that the tensile strength of the film was increased (Bhuvaneshwari et al., 2011).

Some scientific researches show the interest in using chitosan film for the acetonebased gas sensor in order to detect acetone concentrations in human breath in case of the diagnosis of diabetes mellitus in patients (Nasution et al., 2013) or to detect heavy metals from water (Sugunan et al., 2005; Ahmed and Fekry, 2013).

However, chitosan blended with polyethylene oxide (PEO) showed lower water vapor permeability values than chitosan/poly (N-vinyl-2-pyrrolidone) (PVP) films (Li, 2008). In the case of the chitosan, films were prepared by casting method, and neutralization treatment with sodium hydroxide $(10 \% \mathrm{NaOH})$ solution was observed that the increasing in mechanical property and a reduction in swelling property, water vapor permeability, and oxygen permeability is a directly proportional relationship with the $\mathrm{NaOH}$ concentration increasing (Chang et al., 2019). A flexible chitosan film developed using lactic acid solutions as solvent was reported with an increased wettability directly depending on the lactic acid ratio used (Niamsa and Baimark, 2009).

Also, some scientific papers show the concerns in the development of the multifunctional nanocomposites of chitosan with silver nanoparticles, copper nanoparticles, and carbon nanotubes because of increased antimicrobial activity, in approximatively 10 minutes, against bacteria such as Gram-negative and Gram-positive bacteria, E. coli, Staphylococcus aureus (Morsi et al., 2017; Haldorai and Shim, 2013). Chitosan (CS) based copper oxide $(\mathrm{CuO})$ hybrid material has reported a material with high photocatalytic activity and antibacterial activity against Escherichia coli (Haldorai and Shim, 2013). 


\section{EXPERIMENTAL PART}

In our experimental part, 12 samples from $100 \%$ cotton (plain weave) were coated with thin films based 1-2\% chitosan with low (chitosan 1), medium (chitosan 2) and high molecular (chitosan 3) weight and copper microparticles (size dimension 14-25 $\mu \mathrm{m})$, being followed by drying for 24 hours at room temperature and crosslinking at $150-160^{\circ} \mathrm{C}$ for $3-5$ minutes (Table 1). In order to obtain the paste, the chitosan powder was dissolved in a $0.5-2 \%$ acetic acid solution. The chitosan film was deposited by the scraping method. In Table 1, the surface resistance was measured before $\left(\mathrm{Rs}_{1}\right)$ and after $\left(\mathrm{Rs}_{2}\right)$ crosslinking. Besides, the electroconductive behavior was appreciated before (electroconductive effect ${ }_{1}$ ) and after (electroconductive effect ${ }_{2}$ ) crosslinking. The wettability of fabric coated was investigated through contact angles for the 12 samples developed were measured by using the device VCA Optima (AST - Figure 1) and is presented in Table 2. In Figure 2 are presented images showing the canvas - surface morphology before and after chitosan-copper pasta deposition for sample no. 2.

Table 1. Electrical characterization of the experimental samples functionalized by chitosan and $\mathrm{Cu}$ microparticles

\begin{tabular}{|c|c|c|c|c|c|c|c|c|c|c|}
\hline$\dot{0}$ & 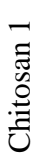 & 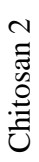 & 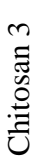 & 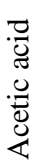 & $\begin{array}{l}\underset{N}{I} \\
\underset{I}{1}\end{array}$ & ت & $\frac{\overrightarrow{\mathfrak{C}_{1}}}{\overline{\tilde{n}}}$ & 율 & $\begin{array}{l}\vec{a} \\
\tilde{a} \\
\tilde{a}\end{array}$ & 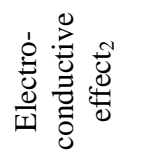 \\
\hline 1 & $\mathrm{X}$ & & & $\mathrm{X}$ & X & $\mathrm{X}$ & $10^{9}$ & antistatic & $10^{12}$ & antistatic \\
\hline 2 & $\mathrm{x}$ & & & $\mathrm{x}$ & $\mathrm{x}$ & $\mathrm{x}$ & $10^{3}$ & conductive & $10^{4}$ & conductive \\
\hline 3 & $\mathrm{x}$ & & & $\mathrm{x}$ & $\mathrm{x}$ & $\mathrm{x}$ & $10^{4}$ & conductive & $10^{4}$ & conductive \\
\hline 4 & & & $\mathrm{X}$ & $\mathrm{X}$ & $\mathrm{X}$ & $\mathrm{X}$ & $10^{7}$ & semiconductor & $10^{9}$ & antistatic \\
\hline 5 & & $\mathrm{X}$ & & $\mathrm{x}$ & $\mathrm{x}$ & $\mathrm{x}$ & $10^{4}$ & conductive & $10^{10}$ & antistatic \\
\hline 6 & $\mathrm{X}$ & & & $\mathrm{X}$ & $\mathrm{X}$ & $\mathrm{X}$ & $10^{7}$ & semiconductor & $10^{9}$ & antistatic \\
\hline 7 & & & $X$ & $\mathrm{x}$ & $\mathrm{x}$ & $\mathrm{x}$ & $10^{8}$ & antistatic & $10^{11}$ & antistatic \\
\hline 8 & & & $\mathrm{x}$ & $\mathrm{x}$ & $\mathrm{x}$ & $\mathrm{X}$ & $10^{4}$ & conductive & $10^{11}$ & antistatic \\
\hline 9 & & & $\mathrm{x}$ & $\mathrm{x}$ & $\mathrm{x}$ & $\mathrm{x}$ & $10^{10}$ & antistatic & $10^{11}$ & antistatic \\
\hline 10 & & $\mathrm{X}$ & & $\mathrm{x}$ & $\mathrm{x}$ & $\mathrm{x}$ & $10^{11}$ & antistatic & $10^{12}$ & antistatic \\
\hline 11 & & $\mathrm{x}$ & & $\mathrm{X}$ & $\mathrm{x}$ & $\mathrm{x}$ & $10^{7}$ & semiconductor & $10^{12}$ & antistatic \\
\hline 12 & & $\mathrm{x}$ & & $\mathrm{x}$ & $\mathrm{x}$ & $\mathrm{x}$ & $10^{6}$ & semiconductor & $10^{12}$ & antistatic \\
\hline
\end{tabular}

Table 2. Contact angles - VCA Optima

\begin{tabular}{|c|c|c|c|}
\hline No. & $\begin{array}{l}\text { View - Textile material after Chitosan - } \\
\text { Cu film deposition }\end{array}$ & $\begin{array}{l}\text { Contact } \\
\text { angle }\left[{ }^{0}\right]\end{array}$ & $\begin{array}{l}\text { Hydrophobic/hydrophilic } \\
\text { character }\end{array}$ \\
\hline 1 & Wifiz & 0 & Hydrophilic \\
\hline
\end{tabular}

https://doi.org/10.24264/icams-2020.I.1 


\begin{tabular}{|c|c|c|c|}
\hline No. & $\begin{array}{l}\text { View - Textile material after Chitosan - } \\
\mathrm{Cu} \text { film deposition }\end{array}$ & $\begin{array}{c}\text { Contact } \\
\text { angle }\left[{ }^{0}\right]\end{array}$ & $\begin{array}{c}\text { Hydrophobic/hydrophilic } \\
\text { character }\end{array}$ \\
\hline 2 & 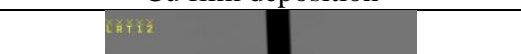 & 0 & Hydrophilic \\
\hline 3 & & 46.90 & Hydrophilic \\
\hline 4 & & 43.50 & Hydrophilic \\
\hline 5 & & 107.40 & Hydrophobic \\
\hline 6 & 12 & 0 & Hydrophilic \\
\hline 7 & 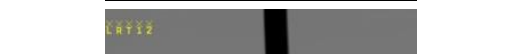 & 0 & Hydrophilic \\
\hline 8 & 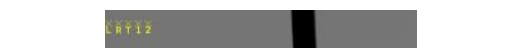 & 0 & Hydrophilic \\
\hline 9 & 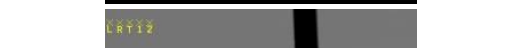 & 0 & Hydrophilic \\
\hline
\end{tabular}

https://doi.org/10.24264/icams-2020.I.1 
Perspective in Using Chitosan Films for Sensors

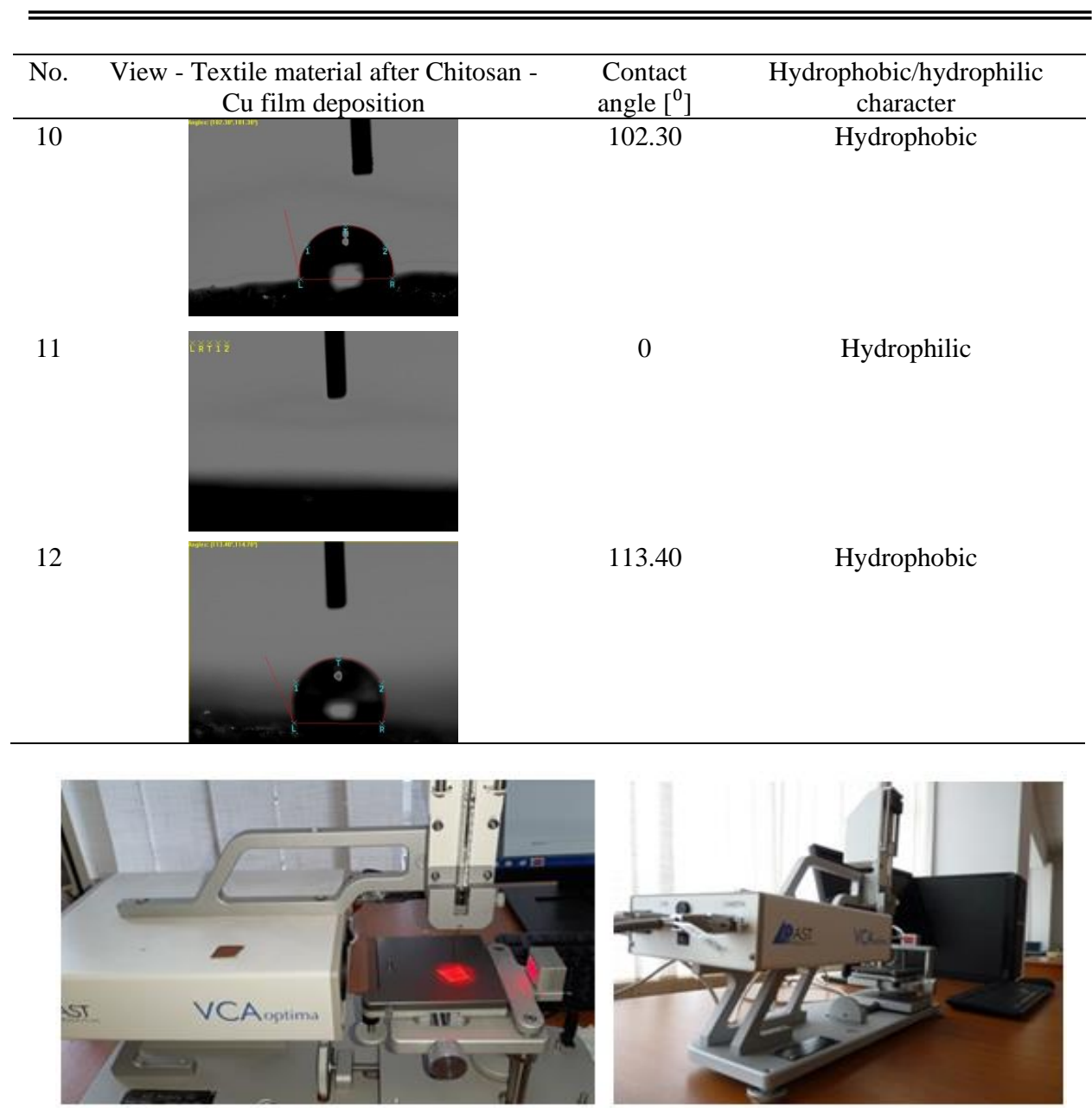

Figure 1. VCA Optima device

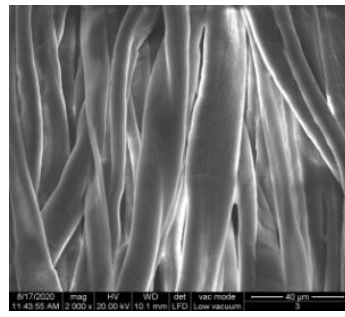

a. Sample 2 - raw fabric (magnitude 2000X)

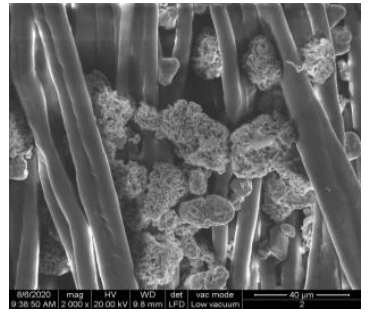

b. Sample 2 coated (magnitude 2000X)

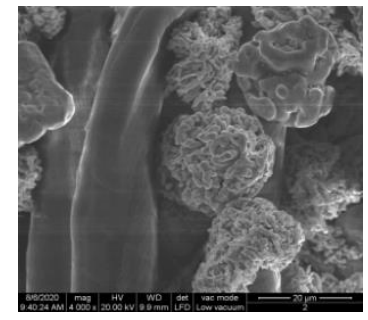

c. Sample 2 coated (magnitude 4000X)

Figure 2. SEM images showing the canvas - surface morphology before and after chitosan-copper paste deposition 


\section{DISCUSSIONS}

Following the wettability test using the VCA Optima device, we can appreciate that for samples 5, 9, and 12, the values of the contact angles are in the range [102.3113.40], but after 60 seconds, the distilled water drop $(4 \mu \mathrm{l})$ is absorbed, and the textile material sample is restored to initial condition.

From Table 1, it is evident that from the initial conductive samples $(2,3,5$, and 8$)$, after the crosslinking only samples 2 and 3 are still conductive. In the case of the samples, 2 and 3 were observed minor changes in the surface resistance, such as: for sample 2 , the surface resistance was increased with $1 \%$, respective for sample 3 , the surface resistance was not changed. After crosslinking, samples 5 and 8 become antistatic, and the surface resistances were increased with $150 \%$ for sample 5, respective with $175 \%$ for sample 8 .

Besides, it was investigated the correlation between contact angle and values of the surface resistance and because of the correlation coefficient $R_{\text {Resistance, Contact angle }}$ is 0.2756 . This means that between the contact angle values and the surface resistance (1), it is a low correlation.

$R_{\text {Resistance, Contact angle }}=\left|\begin{array}{ll}1.0000 & 0.2756 \\ 0.2756 & 1.0000\end{array}\right|$

Overall, the other samples after free drying or crosslinking become antistatic by increasing the surface resistance with $1 \%-100 \%$.

\section{CONCLUSIONS}

We can conclude that samples 2 and 3 obtained by chitosan low molecular weight and copper microparticles have the potential to be used in sensors application because they are conductive before and after crosslinking.

The surface resistance is not dependent on the wetting capacity of the fabric, and crosslinking involving a supplementary drying generates moisture loss and leads to increasing the surface resistance and decreasing the conductivity.

\section{REFERENCES}

Ahmed, R.A. and Fekry, A.M. (2013), "Preparation and characterization of a nanoparticles modified chitosan sensor and its application for the determination of heavy metals from different aqueous media", Int $J$ Electrochem Sci, 8(3), 6692-6708.

Bhuvaneshwari, S., Sruthi, D., Sivasubramanian, V., Niranjana, K. and Sugunabai, J. (2011), "Development and characterization of chitosan films", International Journal of Engineering Research and Applications, 1(2), 292-299.

Chang, W., Liu, F., Sharif, H.R., Huang, Z., Goff, H.D. and Zhong, F. (2019), "Preparation of chitosan films by neutralization for improving their preservation effects on chilled meat", Food Hydrocolloids, 90, 5061, https://doi.org/10.1016/j.foodhyd.2018.09.026.

Haldorai, Y. and Shim, J.J. (2013), "Multifunctional chitosan-copper oxide hybrid material: photocatalytic and antibacterial activities", International Journal of Photoenergy, https://doi.org/10.1155/2013/245646.

$\mathrm{Li}, \mathrm{J}$. (2008), "Characterization and performance improvement of chitosan films as affected by preparation method, synthetic polymers, and blend ratios", $\mathrm{PhD}$ diss., University of Tennessee, https://trace.tennessee.edu/utk_graddiss/464.

Morsi, R.E., Alsabagh, A.M., Nasr, S.A. and Zaki, M.M. (2017), "Multifunctional nanocomposites of chitosan, silver nanoparticles, copper nanoparticles and carbon nanotubes for water treatment: antimicrobial characteristics", International Journal of Biological Macromolecules, 97, 264-269, https://doi.org/10.1016/j.ijbiomac.2017.01.032.

https://doi.org/10.24264/icams-2020.I.1 


\section{Perspective in Using Chitosan Films for Sensors}

Nasution, T.I., Nainggolan, I., Hutagalung, S.D., Ahmad, K.R. and Ahmad, Z.A. (2013), "The sensing mechanism and detection of low concentration acetone using chitosan-based sensors", Sensors and Actuators B: Chemical, 177, 522-528, https://doi.org/10.1016/j.snb.2012.11.063.

Niamsa, N. and Baimark, Y. (2009), "Preparation and characterization of highly flexible chitosan films for use as food packaging", American Journal of Food Technology, 4(4), 162-169, https://doi.org/10.3923/ajft.2009.162.169.

Sugunan, A., Thanachayanont, C., Dutta, J. and Hilborn, J.G. (2005), "Heavy-metal ion sensors using chitosan-capped gold nanoparticles", Science and Technology of Advanced Materials, 6(3-4), 335, https://doi.org/10.1016/j.stam.2005.03.007. 


\title{
THE ELEMENTARY CHARACTERIZATION OF ANTHILL CLAY FOR COMPOSITE MATERIALS AND ADVANCED INDUSTRIAL APPLICATIONS
}

\author{
SURESH ALUVIHARA ${ }^{1 *}$, C.S. KALPAGE ${ }^{1}$, P.W.S.K. BANDARANAYAKE ${ }^{2}$ \\ ${ }^{1}$ Department of Chemical and Process Engineering, University of Peradeniya, Peradeniya, \\ 20400, Sri Lanka, sureshaluvihare@gmail.com \\ ${ }^{2}$ Department of Physics, University of Peradeniya, Peradeniya, 20400, Sri Lanka
}

\begin{abstract}
Anthill clay is a distinct soil/clay genre among common soil types because of the extraordinary stockpiling method. The small particles are carried in and erected an anthill by a small creature that it is called as termite. In generally, clay is a conspicuous raw material for industrial applications greatly and the assay of expediencies of anthill clay for advanced material applications were the prospects of the existing research. Carefully collected anthill clay samples were characterized under the physically and chemically using standard procedures and instruments. The mechanical characteristics of prepared bricks from anthill clays under $8000 \mathrm{C}$ were investigated. As the major outcomes of the existing investigation of raw clays, there were looked to 5.56 of $\mathrm{PH}$ value, $15 \%$ of natural moisture content, gap graded and symmetrically distributed arrangement of grains, $60 \%$ finer particle percentage $(<0.075 \mathrm{~mm})$ according to the weight, composition of $\mathrm{Fe}$, $\mathrm{Ti}$, $\mathrm{Ba}$ and $\mathrm{K}$ based compounds including Fe minerals with large sorption capacity for other metals. In addition that $25 \%$ of water absorption, 2.62 of bulk specific gravity, $65 \%$ of apparent porosity, 21 Mpa compressive strength and 0.4 Mpa splitting tensile strength were observed with respect to the bricks which were prepared from the anthill clay. Based on the behaviors of such anthill clay it should be an influential material in the advanced material manufacturing in the industrial purposes such as the water treatments, rigid materials, catalysts and refractors.
\end{abstract}

Keywords: anthill clay, physico-chemical characteristics, advanced industrial applications

\section{INTRODUCTION}

Anthill clay is some sort of different clay variety among well known clay species because of the availability of anthill clay. When considering the origin and formation of anthill clay, the initial factors would be similar with the origins of other clay types such as the transportation and accumulation of sediments due to the rain, wind or gravitation force. The pattern of availability is exactly significant because the anthill is prepared by termites using some of available clay type at around the location. Therefore, the characteristics of the anthill clay would be differing based upon the location. As the literature review of the existing industrial uses of other different clay varieties the following uses could be highlighted as the dominant examples (Maina et al., 2015): pottery industries; ceramic and porcelain industry; manufacturing of building materials.

Since the ancient applications were limited for mechanical applications, currently most of new innovations are being processed regarding most of different clay varieties beyond the primary uses of such clays. Among the well-known modern investigations of clays, applications of the industry of water treatment gained high benefits because the water pollution has been detected as a huge matter in the current world (Adamu et al., 2010). According to the literature reviews on the recent researches of the developments of following important approaches were emphasized as the important outcomes (Maurya et al., 2018): removal of heavy metals from the contaminated/polluted water; removal of some pathogens from contaminated water; removals of unnecessary or hazardous ions from waste water.

According to the working explanations of the most of above tasks, the main working process is adsorption, a chemical process which occurs due to the electrostatic forces of both attracting compound and attaching compound (Mahandrimanana and Joseph, 2013).

https://doi.org/10.24264/icams-2020.I.2 
Therefore, the adsorption process is a surface-based chemical process and does not involve penetration of the attached material.

Clay is usually known as a group of minerals with the silicate minerals and some of ferrous minerals (Adamu et al., 2010). Due to the diversities in the chemical compositions, clays have obtained a series of distinguished properties including some advanced characteristics such as the refractory properties, adsorption capacities and ion exchanging properties (de Oliveira et al., 2016).

Anthill clay is a specific clay type which is not enclosed as an industrially applicable material since it may have some of extraordinary characteristics. In the existing research, there were expected to investigate the primary physic-chemical characteristics of a selected unknown type of anthill clay and also to investigate some mechanical properties of prepared brick from such anthill clays.

\section{MATERIALS AND METHODOLOGY}

The raw anthill clay samples were collected from Matale region by following important precautions to maintain the accuracy of the results of further analysis - to be collected in a dry climatic occasion; usage of well cleaned non-metallic tools; storage in polythene bags. The important physical-chemical characteristics of raw anthill clays were investigated using standard methods and instruments as discussed in Table 1.

Table 1. Investigations of the physical-chemical characterizations of the clays

\begin{tabular}{cc}
\hline Physical-Chemical Characteristic & Instruments and Methodology \\
\hline Acidity $(\mathrm{pH})$ & $\begin{array}{c}\text { The } \mathrm{pH} \text { value of a prepared clay solution (distilled water) was } \\
\text { measured using a digital } \mathrm{pH} \text { meter }\end{array}$ \\
The weight loss of some selected natural clay portion and \\
the dry weight of the sample clay portion \\
Natural Moisture Content & $\begin{array}{c}\text { Mechanical sieve analysis (dry sieve analysis) of anthill clay } \\
\text { using the size range } 2 \mathrm{~mm}-0.037 \mathrm{~mm} \text { and }<0.037 \mathrm{~mm}\end{array}$ \\
Particle Size Distribution & The representative clay sample was dissolved out in distilled \\
Clay Portion & water on 0.075mm sieve (wet sieve analysis) \\
(Finer Portion $<0.075 \mathrm{~mm})$ & X-ray fluorescence (XRF) spectrometer \\
Elemental Chemical Composition &
\end{tabular}

The following definitions and equations were used in the computation of the important properties of raw clays (Verma et al., 2017).

Moisture content $=\left\{\left(\mathrm{W}_{\mathrm{I}}-\mathrm{W}_{\mathrm{F}}\right) / \mathrm{W}_{\mathrm{I}}\right\} * 100 \%$

$\mathrm{W}_{\mathrm{I}}=$ Initial weight of raw clay sample/ $\mathrm{g}$

$\mathrm{W}_{\mathrm{F}}=$ Final weight of dried clay sample/ $\mathrm{g}$

Coarse portion $=\left(\mathrm{W}_{\mathrm{C}} / \mathrm{W}_{\mathrm{D}}\right) * 100 \%$

Finer portion $=\left\{\left(\mathrm{W}_{\mathrm{D}}-\mathrm{W}_{\mathrm{C}}\right) / \mathrm{W}_{\mathrm{D}}\right\} * 100 \%$

$\mathrm{Wc}=$ Dry weight of the coarse portion $/ \mathrm{g}$

$\mathrm{W}_{\mathrm{D}}=$ Weight of dried initial clay sample/g

According to the dry sieve analysis results of clays, the particle size distribution curve was plotted on a semi-logarithm sheet, recording readings in accordance with literature (Umoru et al., 2015):

- $\quad \mathrm{D}_{10} \rightarrow \mathrm{D}_{75}=$ Diameter corresponding to $10 \% \rightarrow 75 \%$ finer percent in the particle size distribution curve/mm 
Based upon above results, the important grain size parameters of the clay were computed using the given equations and definitions (Dewangan et al., 2015).

Effective size $=\mathrm{D}_{10}$

$\mathrm{C}_{\mathrm{u}}=\mathrm{D}_{60} / \mathrm{D}_{10}$

$\mathrm{C}_{\mathrm{u}}=$ Uniformity coefficient

$\mathrm{C}_{\mathrm{c}}=\left(\mathrm{D}_{30}\right)^{2} /\left(\mathrm{D}_{60} * \mathrm{D}_{10}\right)$

$\mathrm{C}_{\mathrm{u}}=$ Coefficient of gradation

$\mathrm{S}_{0}=\left(\mathrm{D}_{75} / \mathrm{D}_{25}\right)^{1 / 2}$

$\mathrm{S}_{0}=$ Sorting coefficient

Average grain size $=\mathrm{D}_{50}$

$\mathrm{S}_{\mathrm{K}}=\left(\mathrm{D}_{25} * \mathrm{D}_{75}\right) / \mathrm{D}_{50}$

$\mathrm{S}_{\mathrm{K}}=$ Skewness

The representative clay portion sample was dried for 24 hours at the temperature of $110^{\circ} \mathrm{C}$ and analyzed using an X-ray fluorescence (XRF) spectrometer (Cultrone et al., 2004). A set of bricks were manufactured from this anthill clay under following conditions:

- Sizes were $1.5 \mathrm{~cm}$ x $6 \mathrm{~cm} \times 10 \mathrm{~cm}$ in moulds;

- The firing temperature was $800^{\circ} \mathrm{C}$ in muffle furnace;

- The firing time was about 12 hours.

The compressive strengths and splitting tensile strengths of manufactured bricks were tested using the universal tensile strength testing machine.
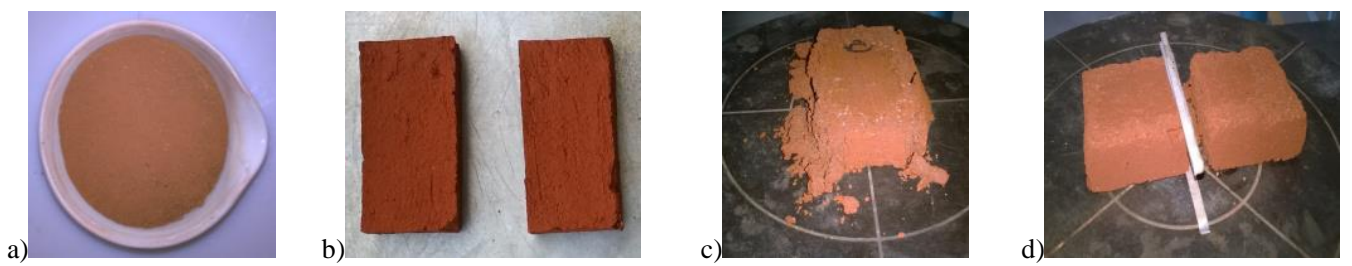

Figure 1. a) Anthill clay sample; b) Manufactured anthill clay bricks; c) Breaking the structure of bricks under compressive load and (d) under splitting tensile load

In the computation of the compressive strengths and splitting tensile strengths of bricks, following equations and definitions were used (Abu Bakar et al., 2018).

Compressive Strength $=\mathrm{P}_{\mathrm{C}} / \mathrm{A}$

$\mathrm{P}_{\mathrm{C}}=$ Applied compressive load in the failure of the structure $(\mathrm{N})$

$A=$ Surface area of the bed surface of the brick $\left(\mathrm{m}^{2}\right)$

Splitting Tensile Strength $=2 \mathrm{P} /\left(\pi^{*} \mathrm{H}^{*} \mathrm{~L}\right)$

$\mathrm{P}=$ Maximum applied load at the failure of the structure $(\mathrm{N})$

$\mathrm{H}=$ Distance between two edges (bridges) (m)

$\pi=$ Constant $(3.142)$

$\mathrm{L}=$ Splitting length $(\mathrm{m})$

Bulk densities and porosities of manufactured bricks were tested as discussed in Table 2 (Umoru et al., 2015). 
Table 2. Investigation of the mechanical properties of manufactured bricks from anthill clay

\begin{tabular}{cc}
\hline Mechanical Property of Bricks & Methodology \\
\hline Water Absorption & The weight difference between the weight of dry brick and the \\
weight of wetted brick & (24 hours immersion period in water) \\
Bulk Density & $\begin{array}{c}\text { The ratio between the dry mass of the brick and occupied } \\
\text { volume }\end{array}$ \\
Porosity & The ratio between the volume of absorbed water and the \\
volume of the brick
\end{tabular}

The above-mentioned characteristics of clay were computed using following equations and definitions based upon the obtained results.

Water Absorption $=\left\{\left(\mathrm{W}_{\mathrm{W}}-\mathrm{W}_{\mathrm{D}}\right) / \mathrm{W}_{\mathrm{D}}\right\} * 100 \%$

$\mathrm{W}_{\mathrm{D}}=$ Weight of the dried brick /g

$\mathrm{W}_{\mathrm{W}}=$ Weight of the wetted brick/g

Bulk Density $=\mathrm{W}_{\mathrm{D}} / \mathrm{V}$

$\mathrm{W}_{\mathrm{D}}=$ Dried weight of the brick $/ \mathrm{g}$

$\mathrm{V}=$ Volume of the brick $/ \mathrm{cm}^{3}$

Porosity $=\left\{\left(\mathrm{W}_{\mathrm{W}^{-}} \mathrm{W}_{\mathrm{D}}\right) / \rho * \mathrm{~V}\right\} * 100 \%$

$\mathrm{W}_{\mathrm{W}}=$ Wetted weight of the brick $/ \mathrm{g}$

$\mathrm{W}_{\mathrm{D}}=$ Dried weight of the brick /g

$\rho=$ Density of the absorbed fluid by the brick (absorbate) $/ \mathrm{gcm}^{-3}$ (For water, $\rho=1 \mathrm{gcm}^{-3}$ )

$\mathrm{V}=$ Volume of the brick $/ \mathrm{cm}^{3}$

\section{RESULTS AND DISCUSSION}

The obtained results for the physic-chemical properties of anthill clays have been shortlisted in the Table 3.

Table 3. Physical-chemical characteristics of anthill clay

\begin{tabular}{cc}
\hline Physical-Chemical Characteristic & Result \\
\hline Acidity / $(\mathrm{pH})$ & 5.56 \\
Natural Moisture Content / (\%) & $\sim 15$ \\
Weight Percentage of Coarse Particles (Sand) / (\%) & $\sim 40$ \\
Weight Percentage of Finer Particles (Clay and Silt) / (\%) & $\sim 60$ \\
\hline
\end{tabular}

According to the acidity of such clay, it is categorized as weak acidic clay because the $\mathrm{pH}$ value is between 5.5-7.0. The acidity of soil will be much considered in the agricultural and plantation purposes. The natural moisture content is a primary indicator for some sort of physic-chemical characteristics such as the porosity although it is impossible to use that one as a factor for such characteristics because it is not depending only on the characteristics of clay/soil and the natural moisture content is also depended on the environmental conditions such as the climatic conditions of the location (Mahandrimanana and Joseph, 2013). 
The finer portion was obtained as $\sim 60 \%$ according to the weight procentage which is indicated the characteristics of plasticity and cohesiveness of such soil/clay and would be a combination of clay, silt and ultrafine clay.

The elemental chemical composition of anthill clay is given in the Table 4 .

Table 4. Elemental composition of anthill clay

\begin{tabular}{ccc}
\hline Atomic Number & Element & Content $(\%)$ \\
\hline 26 & Ferrous & 82.08 \\
22 & Titanium & 4.84 \\
56 & Barium & 0.79 \\
19 & Potassium & 12.28 \\
\hline
\end{tabular}

According to the above results, it seems that the major composed metallic element is $\mathrm{Fe}$ with other trace metallic elements such as $\mathrm{Ti}$, $\mathrm{Ba}$ and $\mathrm{K}$ may be in the form of their oxides namely as $\mathrm{Fe}_{2} \mathrm{O}_{3}, \mathrm{TiO}_{2}, \mathrm{BaO}$ and $\mathrm{K}_{2} \mathrm{O}$. In the discussion of the common behaviors of such elements with their different forms, the Fe could be identified as a non-hazardous element which is also becoming a part of most of minerals. Usually the Fe minerals were identified as strong sorption or adsorption materials for some other metals such as the heavy metals in the recent researches with the applicability of clays for multi-purposes (Saat et al., 2009). Therefore, this anthill clay could be further developed or used in the waste water treatments especially for the waste water with some higher concentrations of heavy metals and also higher concentration of pathogens. The element $\mathrm{Ba}$ is also a non-toxic element although the $\mathrm{Ba}^{2+}$ is highly toxic for human body, if it is inserted into the digest system as a containable material in aqueous solutions because $\mathrm{Ba}^{2+}$ solutions may not be dissolved in hydrochloric acid and ultimately it is possible to cause some stomach problems (Baranowski et al., 2002). Therefore, it is most important the investigation of the leaching of $\mathrm{Ba}^{2+}$ into water, if this clay is selected for the water purification material. Also the element $\mathrm{K}$ is identified as a non-toxic element. But it is possible to find some effect on the alkalinity of the water, if this clay type is using in the water purifications because $\mathrm{K}$ is an alkaline metal. Alternatively, the $\mathrm{K}^{+}$has been identified as an exchangeable ion that able to replace for another cation. Therefore, it is possible to find some advanced ion exchanging applications in chemical water treatment processes. Ion exchanging is an advanced chemical treatment method for waste water to remove some undesirable ions from the waste water and to replace with some desirable ions (Maurya et al., 2018). In addition that there was not found any heavy metal nor toxic element in anthill clay under this investigation. As a further recommendation, it is possible to suggest some advanced chemical compositional analysis method which is namely as Neutron Activation Analysis (NAA) for better descriptive results. The retained clay weights on each sieve and percent finer with respect to each sieve are shortlisted in the Table 5.

The majority was obtained by the particles in the size ranges of $0.25 \mathrm{~mm}-0.5 \mathrm{~mm}$ according to the weight of the clay sample. The retained weight percentages on sieves are some better readings for the investigation of the particle size distribution. The majority of weights in large sieves indicate the coarse grained soils and the majority of weights in finer sieves indicate the fine grained soil (Verma et al., 2017).

Table 5. Particle size distribution of anthill clay (grain sizes)

\begin{tabular}{ccccc}
\hline $\begin{array}{c}\text { Sieve Size } \\
(\mathrm{mm})\end{array}$ & $\begin{array}{c}\text { Weight retained } \\
\text { on each sieve }(\mathrm{g})\end{array}$ & $\begin{array}{c}\text { Percentage of } \\
\text { weight retained }(\%)\end{array}$ & $\begin{array}{c}\text { Cumulative percentage } \\
\text { of weight retained }(\%)\end{array}$ & $\begin{array}{c}\text { Percent } \\
\text { Finer }(\%)\end{array}$ \\
\hline 2 & 0.02 & 0.04 & 0.04 & 99.96 \\
0.5 & 10.34 & 20.32 & 20.36 & 79.64 \\
\hline
\end{tabular}

https://doi.org/10.24264/icams-2020.I.2 
The Elementary Characterization of Anthill Clay for Composite Materials and Advanced Industrial Applications

\begin{tabular}{ccccc}
\hline \hline \multicolumn{1}{c}{$\begin{array}{c}\text { Pieve Size } \\
(\mathrm{mm})\end{array}$} & $\begin{array}{c}\text { Weight retained } \\
\text { on each sieve }(\mathrm{g})\end{array}$ & $\begin{array}{c}\text { Percentage of } \\
\text { weight retained }(\%)\end{array}$ & $\begin{array}{c}\text { Cumulative percentage } \\
\text { of weight retained }(\%)\end{array}$ & $\begin{array}{c}\text { Percent } \\
\text { Finer }(\%)\end{array}$ \\
\hline 0.25 & 15.61 & 30.68 & 51.04 & 48.96 \\
0.149 & 12.39 & 24.35 & 75.39 & 24.61 \\
0.074 & 3.91 & 7.68 & 83.08 & 16.92 \\
0.037 & 7.86 & 15.45 & 98.53 & 1.47 \\
$<0.037$ (pan) & 0.75 & 1.47 & 100 & 0.00 \\
\hline
\end{tabular}

The plotted particle size distribution curve of anthill clay is shown in the Figure 9.

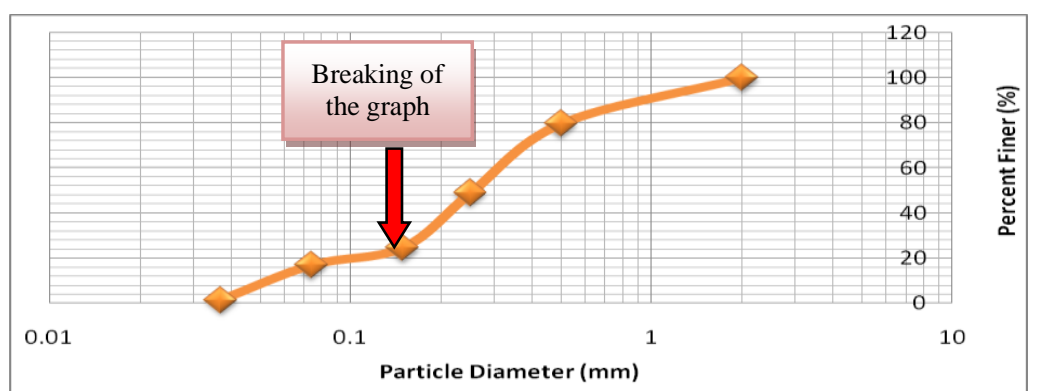

Figure 9. Particle size distribution curve of anthill clay

When considering the shape of the graph (curve), it seems the appearance of the particle size distribution curve of a gap graded clay/soil because the slope (flattened slope) of the graph was deviated from the continuous pattern and a concave shape was observed at the middle part of the graph. Those observations indicate the presence of particles in different sizes since lack of any sequence - Table 6 (Dewangan et al., 2015).

Table 6. Important readings from the particle size distribution curve

\begin{tabular}{ccccccc}
\hline Reading & $\mathrm{D}_{10}(\mathrm{~mm})$ & $\mathrm{D}_{25}(\mathrm{~mm})$ & $\mathrm{D}_{30}(\mathrm{~mm})$ & $\mathrm{D}_{50}(\mathrm{~mm})$ & $\mathrm{D}_{60}(\mathrm{~mm})$ & $\mathrm{D}_{75}(\mathrm{~mm})$ \\
\hline Value & 0.051 & 0.146 & 0.175 & 0.25 & 0.295 & 0.425 \\
\hline
\end{tabular}

Based upon the above important readings, the following important parameters were computed and interpreted as the Table 7.

Table 7. Important parameters regarding the grain sizes of clay

\begin{tabular}{ccccccc}
\hline $\begin{array}{c}\text { Type } \\
\text { of } \\
\text { Clay }\end{array}$ & $\begin{array}{c}\text { Effective } \\
\text { Size } \\
\mathrm{D}_{10}(\mathrm{~mm})\end{array}$ & $\begin{array}{c}\text { Uniformity } \\
\text { Coefficient } \\
\mathrm{C}_{\mathrm{u}}\end{array}$ & $\begin{array}{c}\text { Coefficient } \\
\text { of Gradation } \\
\mathrm{C}_{\mathrm{c}}\end{array}$ & $\begin{array}{c}\text { Sorting } \\
\text { Coefficient } \\
\mathrm{S}_{0}\end{array}$ & $\begin{array}{c}\text { Average } \\
\text { Grain Size } \\
\mathrm{D}_{50}(\mathrm{~mm})\end{array}$ & $\begin{array}{c}\text { Skewness } \\
\mathrm{S}_{\mathrm{K}}(\mathrm{mm})\end{array}$ \\
\hline $\begin{array}{c}\text { Anthill } \\
\text { Clay }\end{array}$ & 0.051 & 5.78 & 2.04 & 1.71 & 0.25 & 0.248 \\
\hline
\end{tabular}

The effective size $\left(\mathrm{D}_{10}\right)$ of a clay or soil is much useful indicator for the drainage and hydraulic conductivity of such clay or soil. If the effective size $\left(D_{10}\right)$ is too diminutive, the hydraulic conductivity will also be reduced. Therefore, the effective size $\left(D_{10}\right)$ of a clay or soil could be considered as the primary characteristic which is related with the permeability of such clay or soil. When the selecting this clay type for some water filtration applications the hydraulic conductivity would be an important factor because the filtration time is depending on the hydraulic conductivity. Apart from the 
effective size $\left(D_{10}\right)$, the shapes of the clay particles play a dominant role on the hydraulic conductivity. If the clay/soil is consisted with large amount of irregular shaped particles, the hydraulic conductivity may be increased. Usually the effective size $\left(D_{10}\right)$ of a soil or clay is using as a comparable parameter when it is available a few of different clay species to select one more uses (Verma et al., 2017).

When considering $g$ the uniformity coefficient $\left(\mathrm{C}_{\mathrm{u}}\right)$ of anthill clay, it is more close to 6. That result indicates the well graded clay type because for well graded clays/soils the uniformity coefficient $\left(\mathrm{C}_{\mathrm{u}}\right)$ is at least 6 . Besides, parameter coefficient of gradation $\left(\mathrm{C}_{\mathrm{c}}\right)$ also indicates the well grading soil/clay type because the obtained value is between 1 and 3. The sorting coefficient $\left(\mathrm{S}_{0}\right)$ is an indicator regarding the sorting of particles (grains) in the soil/clay and the higher sorting coefficient $\left(\mathrm{S}_{0}\right)$ values indicate the well sorted soils/clay (Dewangan et al., 2015).

The average grain size $\left(\mathrm{D}_{50}\right)$ and skewness $\left(\mathrm{S}_{\mathrm{K}}\right)$ are the statistical parameters regarding the particle size distribution of a soil/clay. The average grain size $\left(\mathrm{D}_{50}\right)$ could be used as a single representative value for the sizes of grains in some of a soil/clay. In some coarse grained soils, the average grain size $\left(D_{50}\right)$ values would be higher. According to the obtained value for the skewness $\left(\mathrm{S}_{\mathrm{K}}\right)$, it is found more symmetrical distribution of grains because the result was appeared in the range of 0.10-0.30 (Verma et al., 2017). The obtained results for the mechanical characterizations of anthill clay bricks are shown in Table 8.

Table 8. Mechanical characteristics of anthill clay bricks

\begin{tabular}{cc}
\hline Mechanical Characteristic & Result \\
\hline Water Absorption (\%) & 25 \\
Bulk Density $\left(\mathrm{gcm}^{-3}\right)$ & 2.62 \\
Porosity $(\%)$ & $\sim 65$ \\
Compressive Strength (MPa) & 21 \\
Splitting Tensile Strength (MPa) & 0.4 \\
\hline
\end{tabular}

According to the obtained results for the mechanical characterization of bricks, the average water absorption was detected as $\sim 25 \%$ with respect to the weight. The water absorption is an indicator about the porosity, permeability and the mechanical strengths of the structure (Vodounon et al., 2019).

The bulk density interprets some concepts regarding the density of particles whether they are heavier particles or lighter particles.

The porosity is an important characteristic regarding the applications of water treatment because the high porosity provides a large contact surface area for both water and clay which is an essential factor for the advanced chemical process "adsorption", by means of which some unnecessary components are recovered or removed from some liquid or gas compound onto the surface of some other solid compound, a process is frequently applicable in the industry of waste water treatment especially to remove some heavy metals and pathogens.

The adsorption capacity of some solid material would be varied with the properties of materials (Baranowski et al., 2002).

When considering the mechanical strengths of bricks, there were found the strengthen structure against relatively higher splitting tensile loads and higher compressive loads. The strengths of the brick structure are also depended on the porosity of structure. Therefore, the anthill clay could be further developed for some hard uses in the pure form or as a composite material (Kipsanai et al., 2017).

https://doi.org/10.24264/icams-2020.I.2 


\section{CONCLUSION AND RECOMMENDATIONS FOR FUTURE WORKS}

According to the obtained results, there were observed the gap graded clay with $\sim 60 \%$ of finer weight percentage with majority of Fe compounds without having non toxic elements and the strengthen structure against huge loads. Based on the behaviors of such anthill clay it should be an influential material in the advanced material manufacturing in the industrial purposes such as the water treatments based on adsorption and ion exchanging, rigid materials, catalysts and refractory materials. Meanwhile the entire compositional analysis of the anthill clays using some advanced analytical method such as Neutron Activation Analysis (NAA) will be recommended as a future research activity.

\section{REFERENCES}

Abu Bakar, B.H., Saari, S. and Surip, N.A. (2018), "Split tensile strength of interlocking compressed earth brick units", Journal of Built Environment, Technology and Engineering, 4, 53-58, https://doi.org/10.1063/1.5005649.

Adamu, M.B. (2010), "Fourier Transform Infrared Spectroscopic Determination of Shale Minerals in Reservoir Rocks", Nigerian Journal of Basic and Applied Science, 18(1), 6-18, https://doi.org/10.4314/njbas.v18i1.56836.

Baranowski, R., Rybak, A. and Baranowska, I. (2002), "Speciation Analysis of Elements in Soil Samples by XRF”, Polish Journal of Environmental Studies, 11(5), 473-482.

Bhattarai, J., Ghale, D.B., Chapagain, Y.P., Narendra, B.B. and Duwal, N. (2018), "Study on the physical and mechanical properties of ancient clay brick samples of Kathmandu valley, Nepal", Tribhuvan University Journal, 32(2), 1-18, https://doi.org/10.3126/tuj.v32i2.24699.

Cultrone, G., Sebastian, E., Elert, K., de la Torre, M.J., Cazalla, O. and Rodriguez-Navarro, C. (2004), "Influence of mineralogy and firing temperature on the porosity of bricks", Journal of the European Ceramic Society, 24, 547-564, https://doi.org/10.1016/S0955-2219(03)00249-8.

de Oliveira, C.I.R., Rocha, M.C.G., da Silva, A.L.N. and Bertolino, L.C. (2016), "Characterization of bentonite clays from Cubati, Paraíba (Northeast of Brazil)", Cerâmica, 62, 272-277, https://doi.org/10.1590/0366-69132016623631970.

Dewangan, P.K., Pradhan, M., and Ramtekkar, G.D. (2015), "Effect of Fragment Size, Uniformity Coefficient and Moisture Content on Compaction and Shear Strength Behavior of Coal Mine Overburden Dump Material", European Journal of Advances in Engineering and Technology, 2(12), 1-10.

Kipsanai, J.J., Namango, S.S., Muumbo, A.M. (2017), “A Study of Selected Kenyan Anthill Clays for Production of Refractory Materials", International Journal of Scientific and Research Publications, 7(9), 169-179.

Mahandrimanana, A. and Joseph, R. (2013), "Physico-Chemical Analysis for Differents Types of Clays Soils in the Areas of Analamanga, Itasy and Vakinankaratra", International Journal of Materials and Chemistry, 3(5), 99-105, https://doi.org/10.5923/j.ijmc.20130305.03.

Maina, E.W., Wanyika, H.J. and Gacanja, A.N. (2015), "Instrumental Characterization of Montmorillonite Clay by FT-IR and XRD from J.K.U.A.T Farm, in the Republic of Kenya", Chemistry and Materials Research, 7(10), 43-49.

Maurya, A., Kesharwani, L. and Mishra M.K. (2018), “Analysis of Heavy Metal in Soil through Atomic Absorption Spectroscopy for Forensic Consideration”, International Journal for Research in Applied Science \& Engineering Technology, 6(6), 1188-1192, https://doi.org/10.22214/ijraset.2018.6173.

Saat, A., Hamzah, Z. and Abu Bakar, Z. (2009), "XRF determination of major elemental contents of clay samples from North-West peninsular Malaysia", Journal of Nuclear and Related Technologies, 6(1), 230-236.

Umoru, C.I., Shuaibu, A.M., Abdullahi, I.N. and Umar, M.U. (2015), "Geotechnical Assessment of Gully Erosion at Ankpa Area, North Central Nigeria", IOSR Journal of Applied Chemistry, 8(12), 36-48.

Verma, S.K, Akhtar, S. and Shrivastava, S. (2017), "Assessment of Particles of Varied Soil by Grain Size Analysis - A Case Study in Jabalpur M.P.”, International Journal of Engineering Research and Application, 7(7), 32-37, https://doi.org/10.9790/9622-0707093237.

Vodounon, N.A., Kanali, C., Mwero, J. and Djima, M.O.A. (2019), "Splitting Tensile Strength, Physical and Durability Properties of Cement Stabilized Earth Block Reinforced with Treated and Untreated Pineapple Leaf Fibre", Journal of Materials Science Research, 8(2), 49-57. 
THE ACRYLIC/MONTMORILLONITE NANOCOMPOSITES FOR LEATHER FINISHING

\author{
ANNA BONDARYEVA ${ }^{1}$, OLENA MOKROUSOVA ${ }^{1,2}$ \\ ${ }^{1}$ Kyiv National University of Trade and Economics, Ukraine, Aa-aa@i.ua \\ ${ }^{2}$ Kyiv National University of Technologies and Design, Ukraine, olenamokrousova@ gmail.com
}

\begin{abstract}
Leather finishing is carried out by application of coatings that include polymer, pigment, solvents and any auxiliary products. The acrylic resins are employed in leather finishing to produce polymer film to create a uniform protective coating on the leather surface. To increase the operational properties of the polymer coating for leather finishing, it is proposed to use nanocomposites based on acrylic resin and modified dispersions of montmorillonite (AMC). The introduction of montmorillonite allows the polymer to be structured and provides improved physical and mechanical indexes of the leather coating. Acrylic polymers and colored modified dispersions of montmorillonite (CMDM) were used for the study. The colored montmorillonite was obtained by treating water dispersions of montmorillonite by sodium carbonate, basic chromium sulfate and anionic dyes. The AMC contained 1.5-2.0\% montmorillonite of the dry polymer residues. The use of AMC enhances the physical and mechanical properties of the leather coating. It is shown that the use of temperature at $60{ }^{\circ} \mathrm{C}$ for the formation of finishing coating enhances the structuring of the polymer matrix, which is confirmed by the $40 \%$ increase in the tensile strength of films and the $10 \%$ reduction in relative elongation at break.
\end{abstract}

Keywords: montmorillonite, coating, leather finishing

\title{
INTRODUCTION
}

The coating, which is applied to finish the surface of the leather, contains a polymer for forming a cover film; pigment for desired color; wax emulsion for coating shine and hydrophobicity; plasticizer to reduce the stiffness of the coating film or increase frost resistance; a dispersant or emulsifier to stabilize the coating composition (Zhuravsky et al., 1996; Kasyan, 2019). The largest mass fraction in the coating composition is represented by polymer (50-60 parts by weight) and pigment (10-15 parts by weight), which determines their crucial importance for the formation of a high-quality finishing coating on the leather.

The polymer is used in coating compositions as a film former to create a uniform protective coating on the leather surface. However, significant physical and mechanical loadings, multiple bending, blurring in dry and wet conditions, stretching etc. take place during leather use. This issue is especially relevant for multi-layer finishing of leather with a buffing or sanding surface. That is why the required level of the leather coating properties depends on the physico-mechanical and physico-chemical parameters of the coating films (Kasyan, 2019). Therefore, one of the ways to improve the quality of the leather coatings is the use of new effective composite materials - components of the coating compositions, which would adjust and purposefully form the necessary set of physical and mechanical properties of the polymer coating on the leather.

The use of a clay minerals has a positive structuring effect on acrylic polymers (Y1lmaz et al., 2011; Zhang et al., 2006; Ma et al., 2006).

It is known that the use of montmorillonite dispersions in sodium form helps the formation of additional chemical bonds between the surface hydroxyl groups of the mineral and the carboxyl groups of acrylate (Ma et al., 2006). The established interactions provide a change in the functional properties of the polymer (Yllmaz et al., 2011; Zhang et al., 2006) and cause the creation of nanocomposites for the leather.

https://doi.org/10.24264/icams-2020.I.3 
However, these composites do not paint the surface of the leather, which still requires the addition of pigment concentrate.

In order to increase the operational properties of the polymer coating for leather finishing and to make intensive-coloured surface it is proposed to use nanocomposites (AMC) based on acrylic resin and modified dispersions of montmorillonite. The use of montmorillonite makes the polymer structured and provides improved physical and mechanical indexes of the leather finishing coating.

\section{EXPERIMENTAL}

\section{Materials}

In order to get AMC nanocomposite acrylic resin (AR) and colored modified dispersions of montmorillonite (CMDM) were used for the study.

As an acrylic polymer (film former) it was used copolymer acrylic emulsion MBM 3 (TU 6-01-196-89), which is an aqueous dispersion of a copolymer of methacrylate, butyl acrylate and methacrylic acid in the amount of $3.0 \%$ by weight of monomers (Danylkovych et al., 2009). The molecular structure of the copolymer determined sufficient elasticity and strength of the polymer in the temperature range required for coating on the leather. The emulsion was characterized by a high molecular weight, which determines the film-forming ability required for the finishing coating. The dry residue was $38.5 \%$, the $\mathrm{pH}-4.35$.

In order to obtain colored modified dispersions of montmorillonite, bentonite clay $\mathrm{Al}_{2} \mathrm{O}_{3} \times 4 \mathrm{SiO}_{2} \times 2 \mathrm{H}_{2} \mathrm{O} \times \mathrm{nH}_{2} \mathrm{O}$ (Dashukivske deposit, Cherkasy region, Ukraine) was used. The main mineral was montmorillonite, the content was $85 \pm 3 \%$. The value of the exchange capacity was $72 \mathrm{mg}-\mathrm{eq} / 100 \mathrm{~g}$ of clay. Humidity $-27 \pm 3 \%$.

\section{Methods}

The CMDM was obtained by treating water dispersions of montmorillonite by sodium carbonate, basic chromium sulfate and anionic dyes. Namely, it was used sequential treatment of aqueous montmorillonite dispersions $(100 \mathrm{~g} / \mathrm{l})$ with sodium carbonate, basic chromium sulfate and anionic dyes. The consumption of sodium carbonate was $6 \%$ by weight of montmorillonite. Consumption of basic chromium sulfate $-10 \% \mathrm{Cr}_{2} \mathrm{O}_{3}$ by weight of the mineral, the consumption of anionic dyes in a ratio of $1: 1$ according to the mineral component. Colored modified dispersions of black (CMDM-B) and green (CMDM-G) montmorillonite were obtained.

AMC nanocomposites were prepared by sequentially adding to the colored modified montmorillonite, acrylic emulsion $20 \%$ of its concentration and water to a density of $1.050-1.060 \mathrm{~g} / \mathrm{cm}^{3}$.

Polymer films with different content of colored modified montmorillonite in the nanocomposite were formed in teflon cuvettes according to standard methods (Balberova, 1987). The polymer films were obtained by drying at $20^{\circ} \mathrm{C}, 40^{\circ} \mathrm{C}$ and $60^{\circ} \mathrm{C}$ for 48, 24 and 10 hours, respectively.

Physico-mechanical studies of polymer films based on nanocomposite AMC were performed on a rupture machine RMU-5 at a lower clamp speed of $50 \mathrm{~mm} / \mathrm{min}$ according to the standard procedure (Danylkovych et al., 2006). The conditional modulus of elasticity at $100 \%$ and $300 \%$ elongation at a temperature of $20^{\circ} \mathrm{C}$, tensile strength, elongation at break were evaluated (Danylkovych et al., 2006).

https://doi.org/10.24264/icams-2020.I.3 


\section{RESULTS AND DISCUSSION}

Physico-mechanical properties of polymer films based on AMC were determined by the content of montmorillonite in the CMDM. The results of the research indicated (Fig. 1) that the conduction of CMDM dispersions into the polymer matrix AR increased the strength $(\sigma)$ of the polymer films. The maximum level of strength of the films was achieved when the consumption of montmorillonite in the CMDM was more than $1.5 \%$ of the dry polymer residue (Fig. 1a, 1b). The use of montmorillonite increased in 3.5 times the modulus of elasticity $(100 \%)$ of acrylic films with an elongation of $100 \%$ (Fig. 1, curves).

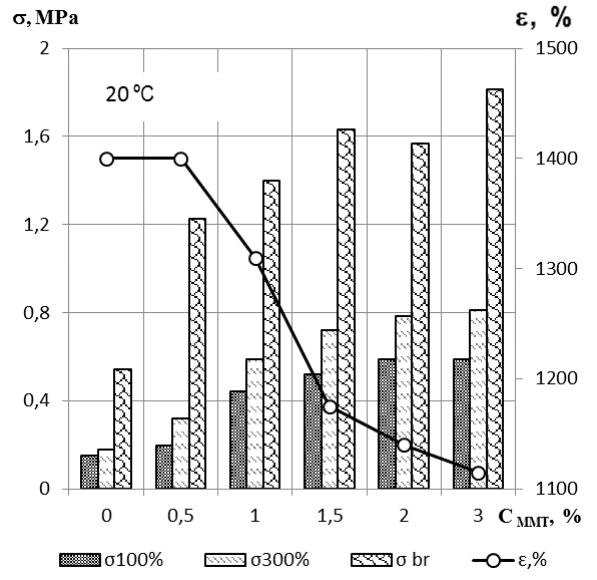

a)

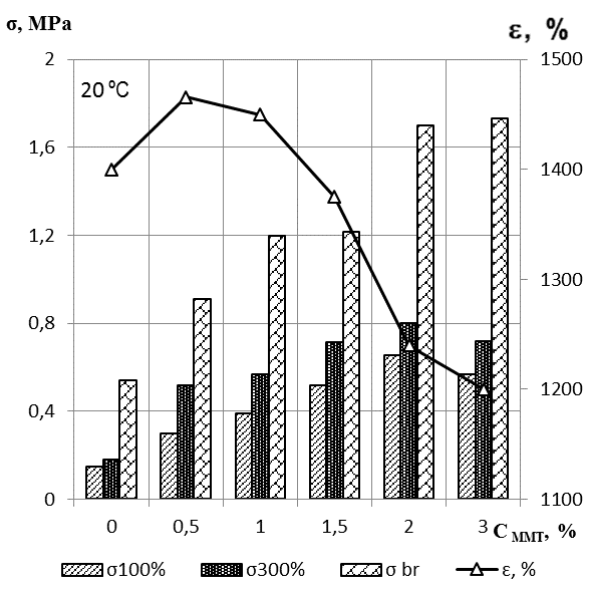

b)

Figure 1. Physico-mechanical properties of polymer films based on AMC using CMDM-B and (a) and CMDM-G (b)

The tensile strength of films ( $\sigma$ br) based on AMC increased to $1.81 \mathrm{MPa}$ in case of using CMDM-B (Fig. 1a) and to $1.73 \mathrm{MPa}$ with CMDM-G (Fig. 1b). This increase indicated that the strength of the native polymer film increased almost threefold after the using of CMDM with a consumption of 1.5-2.0\% of the dry polymer residue.

A significant increase in tensile strength (Fig. 1) of polymer films was associated with conformational strengthening of the polymer structure due to the formation of strong compact crosslinks with the involvement of active functional groups of polymer and azo dyes in colored dispersions of montmorillonite (Kovtunenko et al., 2016). Also, the use of montmorillonite nanoparticles (Mokrousova, 2010) in CMDM with a typical highly developed sorption surface of mineral particles promoted physical adsorption of the polymer and the corresponding stabilization of its structure (Kovtunenko et al., 2016; Zhang et al., 2006; Ma et al., 2006).

A further increase in the amount of montmorillonite above $2.5 \%$ in case of using CMDM-G (Fig. 1b) reduced the strength of the polymer films. This effect could be observed especially when the films were stretched by $100 \%$ and $300 \%$. This could be explained by the idea that the use of a significant amount of adsorption centers of montmorillonite affects the greater structuring of the polymer and leads to a decrease in its film-forming ability due to the high content of mineral particles between the polymer chains.

https://doi.org/10.24264/icams-2020.I.3 
The presence of CMDM mineral particles in the AMC helped to adjust the relative elongation $(\varepsilon)$ of polymer films (Fig. 1a, 1b, curves). The structuring of the polymer matrix with montmorillonite in the amount of 2.0-3.0 \% helped to reduce the relative elongation to the level of 1100-1200\%. In the case of using CMDM-G (Fig. 1b, curve) with a montmorillonite amount of $0.25-1.5 \%$ by weight of the polymer, there was a slight increase (3.0-3.5\%) in elasticity and the corresponding elongation of the polymer films. However, a further increase in the amount of CMDM-G to the level of $2.0 \%$ of montmorillonite led to a decrease of $11.5 \%$ in the relative elongation of films based on AMC (Fig. 1b, curve).

In the case of using CMDM-B (Fig. 1a, curve), a gradual decrease in relative elongation started at the montmorillonite consumption of $0.5 \%$ from the dry polymer residue. The maximum reduction of relative elongation to the level of 1180-1200 \% was typical at the montmorillonite consumption of CMDM-B more than $1.5 \%$.

Such changes in the physical and mechanical properties of films based on AMC were positive in the formation of a finishing coating for leather with the necessary resistance to operating loads, abrasion and repeated bending (Bondaryeva et al., 2020). Excessively high level of elongation and significant viscosity of polymer films can adversely affect the quality of leather finishing due to differences in physical and mechanical loads of the polymer matrix and collagen structure (Kasyan, 2019).

Further research has shown that heat treatment of polymer films based on AMC enhanced the efficiency of their structuring and changes in physical and mechanical properties (Fig. 2).

The results of research (Fig. 2) indicated the increase in the strength of films based on $\mathrm{AMC}$ after thermostating at $40^{\circ} \mathrm{C}$ and $60^{\circ} \mathrm{C}$.

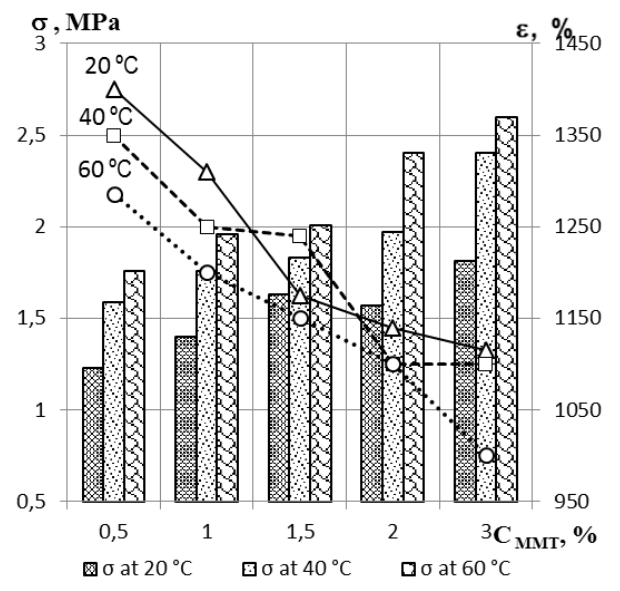

a)

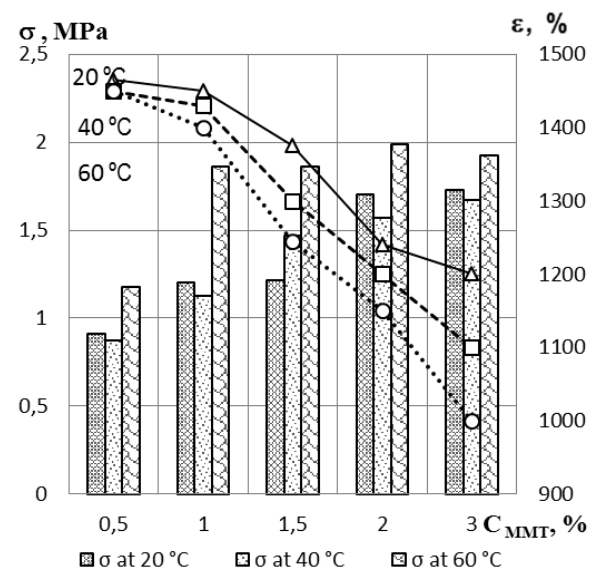

b)

Figure 2. Physico-mechanical properties of polymer films based on AMC using

CMDM-B (a) and CMDM-G (b) after thermostating

The presence of CMDM-B dispersion in the composition of the AMC (Fig. 2a) increased the strength of polymer films $\left(\sigma\right.$ at $\left.40^{\circ} \mathrm{C}\right)$ by $24 \%$ after termostating at $40^{\circ} \mathrm{C}$. In the same time, applying $60^{\circ} \mathrm{C}$ increased the strength of films $\left(\sigma\right.$ at $\left.60^{\circ} \mathrm{C}\right)$ by $40 \%$ compared to native polymer films. For the polymer films based on AMC using CMDM-

https://doi.org/10.24264/icams-2020.I.3 
$\mathrm{G}$ and consumption of montmorillonite above $1.0 \%$ by weight of the polymer (Fig. 2b) strength values $\left(\sigma\right.$ at $\left.60^{\circ} \mathrm{C}\right)$ reached the level of almost $2.0 \mathrm{MPa}$ after termostating at $60^{\circ} \mathrm{C}$ and there was no further change regardless of changes in mineral consumption in the AMC. The maximum effect of thermostating on the structuring of films based on AMC (Fig. 2b) was observed in the case of montmorillonite consumption in the range of $1.0-2.0 \%$ of the mineral by weight of the polymer residue.

According to the results of elongation studies ( $\varepsilon$ ) of films based on AMC (Fig. 2, curves), it was found that formation of such kind of films at $40^{\circ} \mathrm{C}$ and $60^{\circ} \mathrm{C}$ helped to reduce the tensile strength as well as the elongation of polymer films. The presence of CMDM dispersions in AMC reduced the elongation of polymer films to the level of $1000 \%$, which was $10 \%$ lower compared to the same parameter at $20^{\circ} \mathrm{C}$.

Our studies indicated a positive effect of thermostating at $60^{\circ} \mathrm{C}$ for effective structuring of $\mathrm{AMC}$ and improving the physical and mechanical properties of the finishing films and, subsequently, the leather coating.

\section{CONCLUSIONS}

In general, the use of mineral dispersions CMDM in the acrylic emulsion increased the physical and mechanical properties of polymer films due to their structuring, which was the result of physical adsorption and probable chemical interactions between active mineral centers and functional groups of azo dyes and polymer. The consumption of CMDM at the level of 1.5-2.0\% of montmorillonite by weight of the polymer was optimal for creating a high-quality finishing coating for leather based on AMC.

\section{REFERENCES}

Balberova, N.A. (1987), Directory of tanner: finishing. production control, Moscow: Legprombytizdat, 256 p. (in Russian).

Bondaryeva, A. and Mokrousova, O. (2020), "Formation of physico-mechanical properties of polymermineral coating for leather finishing", International scientific-practical journal "Commodities and markets”, № 2 (34), 97-107, https://doi.org/10.31617/tr.knute.2020(34)08.

Danylkovych, A.G. (2006), Workshop on chemistry and technology of leather and fur. 2nd ed., Kyiv: Phoenix, 340 p. (in Ukrainian).

Danylkovych, A.G., Mokrousova, O.R. and Okhmat, O.A. (2009), Technology and materials of leather production, Kyiv: Phoenix, 580 p. (in Ukrainian).

Kasyan, E.E. (2019), Physico-chemistry of polymer film formers for leather finishing. Kyiv: Education of Ukraine, 178 p. (in Ukrainian).

Kovtunenko, O., Travinskaya, T. and Mokrousova, O. (2016), "Thermal properties of anionic polyurethane composition for leather finishing", Materials science, 22(3), 397-402, https://doi.org/10.5755/j01.ms.22.3.10043

Ma, J., Zhang, Z., Liu, L. et al. (2006), "Application of Acrylic Coating Agent Modified by $\mathrm{Nano} \mathrm{SiO}_{2}$ ", Journal of the Society of Leather Technologists and Chemists, № 5, 188-193.

Mokrousova, O.R. (2010), "Mineral fillers for leather. Rheological properties and dispersion of their aqueous suspensions”, Bulletin of KNUTD, № 4, 256-264 (in Ukrainian).

Y1lmaz, O., Cheaburu, C.N., Gülümser, G. and Vasile, C. (2011), "Rheological behaviour of acrylate/montmorillonite nanocomposite latexes and their application in leather finishing as binders", Progress in Organic Coatings, 70(1), 52-58, https://doi.org/10.1016/j.porgcoat.2010.10.001.

Zhang, X., Liu, Q. and Zhang, W. (2006), "Nanocomposites of Acrylate-Organosilicon Resin/Layered Silicate for Leather Finishing”, Journal of the Society of Leather Technologists and Chemists, № 6, 250-254.

Zhuravsky, V.A., Kasyan, E.E. and Danylkovych, A.G. (1996), Technology of leather and fur, Kyiv: KDUTD, 744 p. (in Ukrainian). 
The Acrylic/Montmorillonite Nanocomposites for Leather Finishing

https://doi.org/10.24264/icams-2020.I.3 
COMPARATIVE STUDY OF THE SURFACE PROPERTIES FOR SOME DIFFERENT TYPES OF LEATHER FINISHES

\author{
CIPRIAN CHELARU ${ }^{1}$, MĂDĂLINA-CAMELIA IGNAT ${ }^{1}$, RODICA ROXANA \\ CONSTANTINESCU ${ }^{1}$, MINODORA MARIN ${ }^{1,2}$ \\ ${ }^{1}$ INCDTP - Division: Leather and Footwear Research Institute, 93 Ion Minulescu, Bucharest, \\ Romania, ciprian.chelaru@icpi.ro \\ ${ }^{2}$ University Politehnica of Bucharest, Faculty of Applied Chemistry and Materials Science, 1-7 \\ Gheorghe Polizu Str., 011061, Bucharest, Romania
}

\begin{abstract}
A surface characteristic of leather is an important parameter in shoe industry. During the usage, the surface of shoes is the main barrier against the environment (mostly water). The macroscopic and microscopic evaluation is useful to see the surface aspect (surface defects, continuity of finish, cracks). Test for hydrophilic/hydrophobic activity is important for the leather. In this way we can estimate if the finishing touches absorb or repel the water. Microbiological test is also important, because during an intense usage, inside the shoes are released a lot of chemicals through foot perspiration that can provide a perfect environment for development of mold and bacteria in the main structure of the shoe. The samples for this study will be five bovine leathers with different finishes.
\end{abstract}

Keywords: leather, hydrophilic/hydrophobic activity, microbiologic activity

\title{
INTRODUCTION
}

Leather industry produces a material that can be used in different other fields. One of these fields is footwear manufacturing. As we know, shoes are products with intense usage and if we take this in mind, we understand that all the components of a shoe must have some specific characteristics. Because leather is the main component that stands between the foot and the exterior environment, surface characteristic of leather is an important parameter (Serenko et al., 2014). From the start, we must assess the surface status, in order to be sure that it does not have any structural or/and mechanical defects such as holes, uneven continuity of finish, cracks so in this way, the microscopic evaluation of the samples will be performed. Permanent usage of shoes exposes them to water, that can, in time, infiltrate into the layers of leather and after a while deteriorate the inner structure of leather (Serenko et al., 2014) and finally destroy it. The hydrophilic/hydrophobic activity is an important test for the leather. It is possible to estimate if the finishing touches absorb or repel the water (Leroux and Leising, 2014). The deterioration of a shoe does not come only from the exterior. Our feet secrete perspiration (Orlita, 2004), the perfect liquid, full of chemicals, perspiration that can provide a perfect environment for development of mold and bacteria in the main structure of thr shoe (Oruko et al., 2019). The samples for this study will be five bovine leathers with different finishes.

\section{MATERIALS}

Nutrient agar and nutrient broth were purchased from Novachim (Bucharest, Romania). Staphylococcus aureus (S. aureus, ATCC 6538), Escherichia coli (E. coli, ATCC 10536), Klebsiella pneumoniae (ATCC Klebsiella pneumoniae ATCC 4352) were purchased from Novachim (Bucharest, Romania). Leather samples were prepared from cow hide leather tanned in our institute's pilot station.

https://doi.org/10.24264/icams-2020.I.4 


\section{METHODS}

Microscopic determination - S8AP0 stereomicroscope (LEICA) - it was used for surface evaluation of samples.

The dynamic contact angle modification for water was measured using a contact angle analyzer - VCA Optima XE.

Microbiological test was performed using ISO 16187:2013 standard - Footwear and footwear components. Test method to assess antibacterial activity.

\section{RESULTS}

Macroscopic test of samples - Figure 1 - provides information regarding the leather aspect and due to the different surface pattern, it is possible to estimate a potential destination for the final product. The samples P1, P2 and P3 have uniform pattern colors that recommend these leathers to be used in a small area of product. Still, P3 possesses a shiny aspect, that can be exploited on a much larger area. P4 and P5 have a graphical motif, lending itself to application on a larger surface.

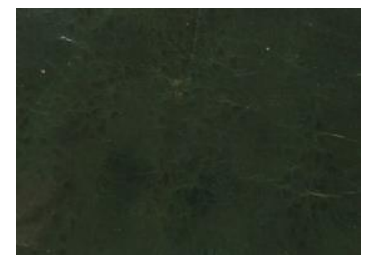

P1

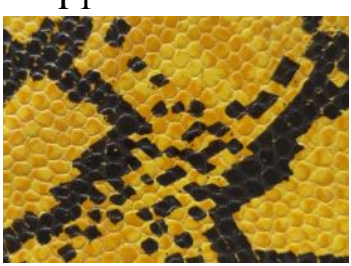

$\mathrm{P} 4$

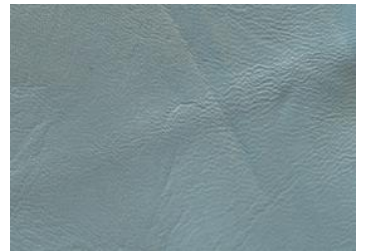

P2

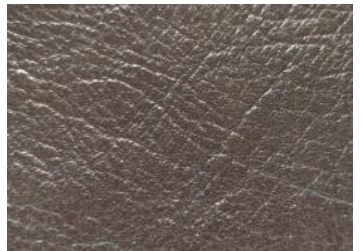

P3

Figure 1. Macroscopic aspect of leather samples

Microscopic images - Figure 2 - have been made using $20 \mathrm{x}$ magnifications for all analyzed samples. In this way we can see that the surface is smooth, no cracks or defects are present on the surface. Because of this observation, all leathers can be used to obtain footwear uppers. 


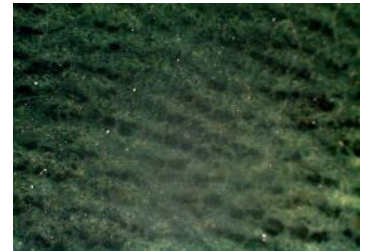

$\mathrm{P} 1$

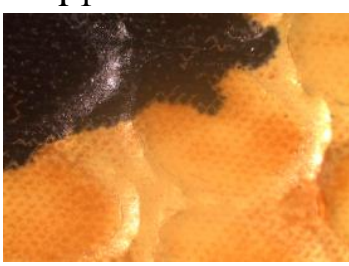

P4

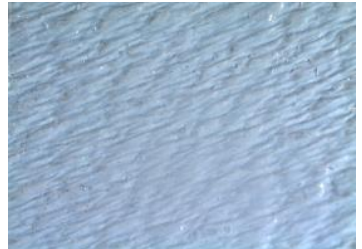

P2

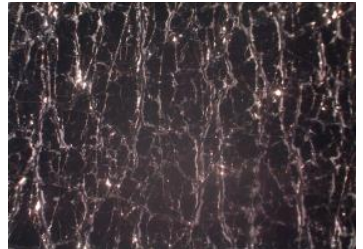

P3

Figure 2. Microscopic aspect of leather samples

Water contact angle test provides the information regarding the ability of water to wet the leather sample - Figure 3. Depending on the angle of water droplet, we have tree aspects:

- $\quad 0^{\circ}-90^{\circ}$ - surface is wettable, hydrophilic surface;

- $90^{\circ}-180^{\circ}$ - surface is not wettable, hydrophobic surface;

- $\quad$ close to $180^{\circ}$ - ultrahydrophobic surface - completely liquid-repellent, lotus effect.

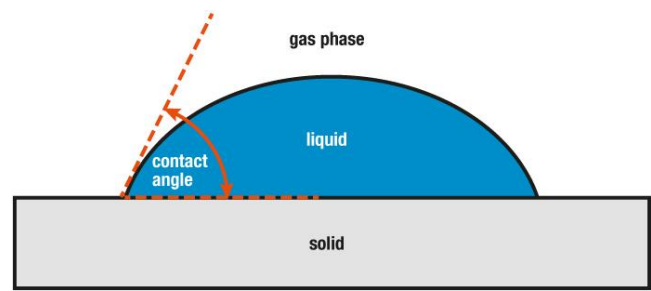

Figure 3. Contact angle example

In order to see the water behavior on leather samples the VCA Optima XE analyzer was used. Results of the individual tests are displayed in Table 1.

Table 1. Contact angle of leather samples

\begin{tabular}{ccc}
\hline No. & Sample & ca, $^{\circ}$ \\
\hline 1 & P1 & 92.74 \\
2 & P2 & 103.17 \\
3 & P3 & 99.59 \\
4 & P4 & 116.63 \\
5 & P5 & 102.14 \\
\hline
\end{tabular}

A graphical representation of contact angle is seen in Figure 4. Based on information provided regarding the contact angle, all the samples are hydrophilic. We can see that the samples P1, P3, P5 and P2 have values close to $100^{\circ}\left(92.74^{\circ}, 99.59^{\circ}, 102.14^{\circ}\right.$, 
$103.17^{\circ}$ ) and this indicates that they can provide some protection against water. On the other hand, for sample P4 an angle of $116.63^{\circ}$ was recorded, which tells us that the leather finish will provide very good protection against water.

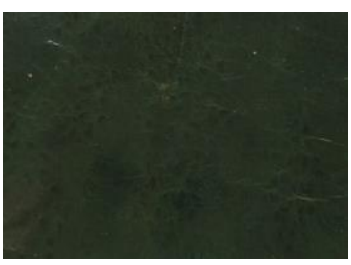

P1

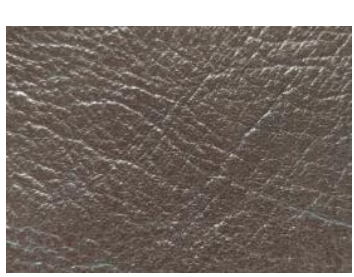

P3
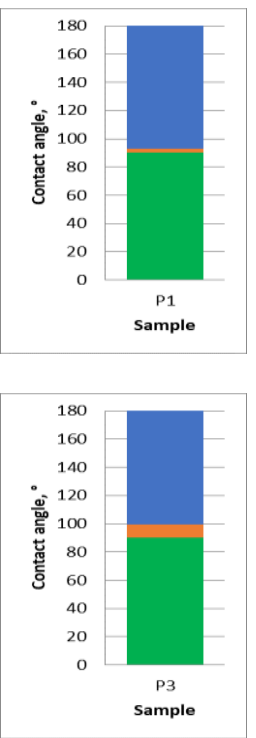

sample

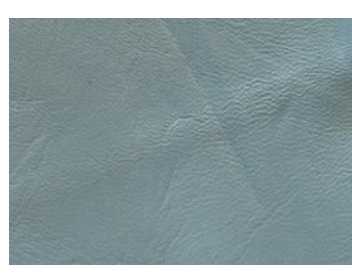

$\mathrm{P} 2$

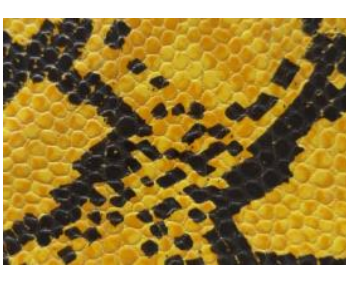

P4

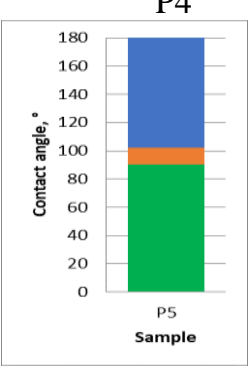

P5

Figure 4. Contact angle of leather samples

Antibacterial activity was determined for all five samples - Figure 5. Results show that all the samples have very good antibacterial activity; just after $24 \mathrm{~h}$, the percentage of bacteria that was annihilated was between $99.68 \%$ and $100 \%$. 


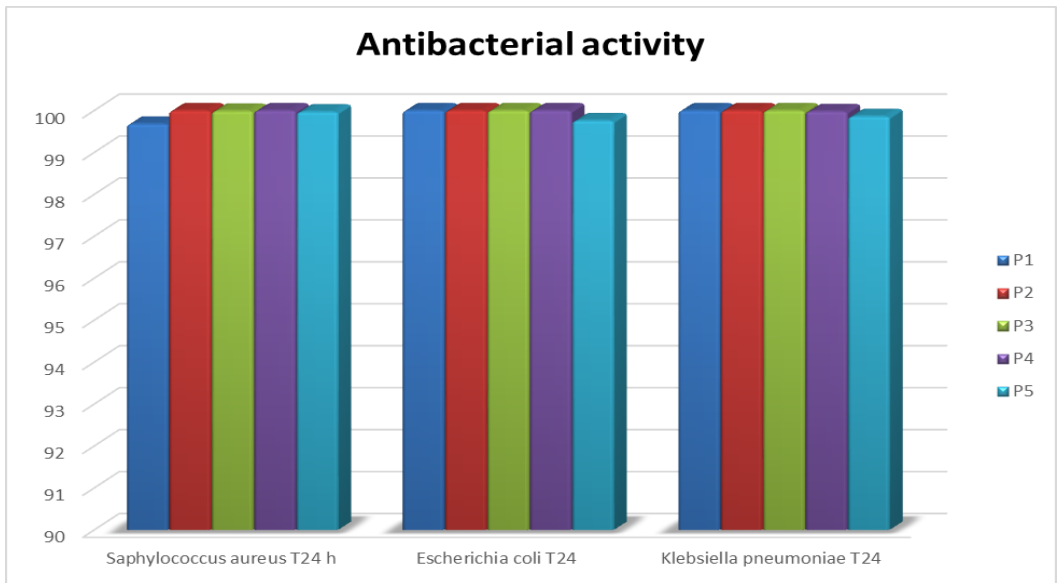

Figure 5. Antibacterial activity

\section{CONCLUSIONS}

Based on microscopic results, all the samples show a smooth surface, without cracks. The contact angle test reveal values over $90^{\circ}$ for all the samples, which indicates that leathers are hydrophobic.

All the leathers have an interesting design that can be useful to manufacture handbags, purses and/or shoes.

The antibacterial activity is very high, the Staphylococcus aureus (S. aureus, ATCC 6538), Escherichia coli (E. coli, ATCC 10536), Klebsiella pneumoniae (ATCC Klebsiella pneumoniae ATCC 4352) are eliminated in proportion of minimum $99.68 \%$ after $24 \mathrm{~h}$, therefore these types of leathers provide you a clean and safe micro environment.

\section{Acknowledgements}

The work has been funded by the Operational Programme Human Capital of the Ministry of European Funds through the Financial Agreement 51668/09.07.2019, SMIS code 124705 . The study was also financially supported by the Ministry of Research and Innovation, Program 1: Development of National System of Research-Development, Subprogram 1.2: institutional performance projects for funding the excellence in RDI, contract no 6PFE/16.10.2018 and contract no PN 19170302.

\section{REFERENCES}

Leroux, P. and Leising, C. (2014), "Processed Leather Surface Finish Using 3D Profilometry", Technica Report, Nanovea, https://doi.org/10.13140/RG.2.1.4737.9368.

Orlita, Alois (2004), "Microbial biodeterioration of leather and its control: a review", International Biodeterioration \& Biodegradation, 53(3), 157-163, https://doi.org/10.1016/S0964-8305(03)00089-1.

Oruko, R.O., Odiyo, J.O. and Edokpayi, J.N. (2019), "The Role of Leather Microbes in Human Health", in: Nar Singh Chauhan (ed.), Role of Microbes in Human Health and Diseases, https://doi.org/10.5772/intechopen.81125.

Serenko, O., Nizamova, Z., Kalinin, M., Ostrovsky, Y., Polukhina, L., Muzafarov, A. (2014), "Effect of the Morphology of Leather Surface on the Hydrophobic-Hydrophilic Properties", Advances in Materials Physics and Chemistry, 4(2), 13-19, https://doi.org/10.4236/ampc.2014.42003. 
Comparative Study of the Surface Properties for Some Different Types of Leather Finishes

https://doi.org/10.24264/icams-2020.I.4 
BIOACTIVE TEXTILES OBTAINED BY APPLYING CINNAMON ESSENTIAL OIL-BASED EMULSIONS

\author{
LAURA CHIRILA ${ }^{1}$, ALINA POPESCU $^{1}$, SABINA OLARU $^{1}$, ANGELA DANILA $^{2}$, \\ RODICA ROXANA CONSTANTINESCU ${ }^{3}$, ANDREEA TIGAU ${ }^{1}$ \\ ${ }^{1}$ National Research \& Development Institute for Textiles and Leather, 16 Lucretiu Patrascanu \\ Street, 030508, Sector 3, Bucharest, Romania, laura.chirila@incdtp.ro \\ 2 "Gheorghe Asachi" Technical University of Iasi, Faculty of Industrial Design and Business \\ Management, 29 Prof. Dr. Doc. Dimitrie Mangeron Blvd., 700050, Iasi, Romania \\ ${ }^{3}$ INCDTP - Division: Leather and Footwear Research Institute (ICPI) Division, 93 Ion Minulescu \\ Street, 031215, Bucharest, Romania
}

\begin{abstract}
In order to obtain biomaterials with potential use in the treatment of inflammatory skin conditions, this experimental study approached the immobilization on knitted fabric, made of $100 \%$ organic cotton, of oil-in-water emulsions type, based on xanthan-propolis-cinnamon essential oil, in certain formulations. For this purpose, seven experimental variants of emulsions were prepared and characterized, by specific methods, from physical-chemical and stability point of view. The functionalized textile materials were also characterized from morphological and antibacterial activity view point. The stability index, determined after 10 days, indicated that the emulsions are stable, without showing the presence of one of the flocculation, creaming / sedimentation, coalescence or Ostwald ripening phenomena. The lowest value of the turbidity was obtained for the experimental variant containing the lowest amount of essential oil and propolis. For the same variant, the highest value of viscosity was obtained, in which $0,363 \mathrm{~mL}$ water $/ \mathrm{mL}$ emulsion and $0,5 \mathrm{~mL}$ xanthan $/ \mathrm{mL}$ emulsion volume fraction was used. The textile materials treated with the synthesized emulsions based on xanthan-propolis-cinnamon essential oil shows antibacterial effect against $S$. aureus and $E$. coli test strains.
\end{abstract}

Keywords: bioactive textiles, xanthan gum, cinnamon essential oil.

\title{
INTRODUCTION
}

Textiles are an excellent carrier of different bioactive compounds through contact with the skin and have found applications for medical, hygienic, and health purposes (Alonso et al., 2013). Medical textiles are the products and constructions used for medical and biological applications such as first aid, clinical and hygienic purposes. With the growing of public awareness of potential health risks, antimicrobial properties have attracted increasing attention among practitioners and have been successfully imparted to textiles to improve the resilience against microorganisms (Dastjerdi and Montazer, 2010; Yuan and Cranston, 2008; Windler et al., 2013). Currently, antimicrobials have been playing an increasingly important role in addressing textile hygiene in clinical and sensitive environments. The development of antibacterial textiles has received extensive attention and applications in several skin disorders. This proposal was developed on the basis of the fact that a majority of skin disorders patients suffer from recurrent bacterial infections, therefore, antimicrobial textiles can be desirable alternative therapy to treat several skin inflammations by killing bacterial colonization. In order to obtain the textile materials with potential for use in the treatment of inflammatory skin conditions, this study approached the immobilization on knitted fabric, made of $100 \%$ organic cotton, of bioactive polymeric systems in certain formulations, based on xanthan-propolis-cinnamon essential oil. 


\section{EXPERIMENTAL PART}

\section{Materials}

Xanthan gum (Mayam, Romania) was used as an embedding agent of bioactive agents. Tween 80 (Sigma Aldrich, Germany) was used as a surfactant with emulsifying role and glycerol (Honeywell, USA) it has been used as a solubilizing agent with wetting role. Cinnamon leaf essential oil (Mayam, Romania) and propolis (tincture of $70 \%$ ethanol solution) (Larix SA, Romania) were used as bioactive agents. For preparation of bioactive systems the distilled water has been used. Bleached $100 \%$ knitted organic cotton was used for the functionalization processes.

\section{Emulsion Preparation Methodology}

For the achievement of the bioactive systems, initially stock solutions of $1 \%$ xanthan gum and 30\% Tween 80 solution were prepared. Further, over the previously prepared xanthan solution, glycerol was added dropwise and the system was maintained under magnetic stirring for 10 minutes. After that, Tween 80 emulsifier and distilled water separately were added under vigorous magnetic stirring. Further, after complete homogenization of the solution, the propolis and cinnamon essential oil were separately added, maintaining the magnetic stirring for 10 minutes each stage. Thus, 7 variants of emulsions have been developed that differ by: i) the nature and concentration of the embedding agent, ii) the concentration of the selected bioactive compounds and iii) the concentration of the dispersion medium (water). The selected experimental variants are presented in Table 1 .

Table 1. The concentration of constituents of xanthan gum-propolis-cinnamon essential oil polymeric system

\begin{tabular}{ccccccc}
\hline Code & $\begin{array}{c}\text { Xanthan } \\
(\mathrm{mL})\end{array}$ & $\begin{array}{c}\text { Composition of the emulsions (reported of 30 mL emulsion) } \\
\text { Tween } 80\end{array}$ & $\begin{array}{c}\text { Glycerol } \\
(\mathrm{mL})\end{array}$ & $\begin{array}{c}\text { Water } \\
(\mathrm{mL})\end{array}$ & $\begin{array}{c}\text { Essential oil } \\
(\mathrm{mL})\end{array}$ & $\begin{array}{c}\text { Propolis } \\
(\mathrm{mL})\end{array}$ \\
\hline R1 & 9 & 0.67 & 3 & 16 & 0.67 & 0.67 \\
R2 & 12 & 0.67 & 3 & 13 & 0.67 & 0.67 \\
R3 & 15 & 0.67 & 3 & 10 & 0.67 & 0.67 \\
R4 & 18 & 0.67 & 3 & 7 & 0.67 & 0.67 \\
R5 & 15 & 0.67 & 3 & 10.9 & 0.22 & 0.22 \\
R6 & 15 & 0.67 & 3 & 10.45 & 0.44 & 0.44 \\
R7 & 15 & 0.67 & 3 & 9.57 & 0.88 & 0.88 \\
\hline
\end{tabular}

\section{Immobilization of Bioactive Polymeric Systems on the Textile Materials}

The 7 experimental variants were immobilized on the textile materials by the padding method on the laboratory padder (ROACHES, UK). The treated textile materials were then subjected to the drying operation at $50^{\circ} \mathrm{C}$ for 3 minutes.

\section{Methods}

Optical Microscopy

The prepared emulsions were analyzed microscopically using an OLYMPUS BX51 optical microscope (Philippines) equipped with the OLYMPUS UC30 photodigital camera.

https://doi.org/10.24264/icams-2020.I.5 


\section{Determination of the Emulsion Stability Index}

Immediately after preparation, the emulsions were introduced into test tubes and the evolution was followed for 10 days after their preparation. The stability of the emulsions was evaluated by determining the stability index (ESI) using the relation:

$\operatorname{ESI}(\%)=\mathrm{H}-(\mathrm{Hs}+\mathrm{Hc})) / \mathrm{H}$

where: $\mathrm{H}$ - the initial height of the emulsion [cm], HS - the height of the serous layer $[\mathrm{cm}] ; \mathrm{HC}-$ the height of the creamy layer $[\mathrm{cm}]$

\section{Turbidity Analysis}

In order to determine the turbidity, the transmittance was determined, using a Camspec M501 UV-VIS Spectrophotometer. Turbidity was calculated with the relationship:

$\tau=2,303 \mathrm{~A} / \mathrm{L}$

where: $\tau_{\text {- turbidity }}\left[\mathrm{cm}^{-1}\right]$; A - absorbance; L - path length of cuvette $[\mathrm{cm}]$.

Viscosity Determination

The viscosity measurements of the obtained bioactive systems were performed with the DV2T Brookfield AMETEK viscometer. Measurements were performed in triplicate.

\section{Electron Microscopy}

Visualization of the morphology of the cotton fiber surfaces treated with the bioactive systems was performed by scanning electron microscopy using the Quanta 200 electron microscope (FEI, The Netherlands).

\section{Assessment of Antibacterial Activity}

The antibacterial activity of the treated samples in different variants was qualitatively assessed by Agar diffusion method according with the SR EN ISO 20645:2005 standard Determination of antibacterial activity-agar diffusion plate test, by using of cultures in liquid medium replicated at 24 hours of ATCC 6538 Staphylococcus aureus and ATCC 11229 Escherichia coli test strains. The textile specimens $(2 \mathrm{~cm}$ diameter $)$ are placed on the surface of the nutrient medium and then incubated at $37^{\circ} \mathrm{C}$ for $24 \mathrm{~h}$. Inhibition zones were calculated using the following formula:

$\mathrm{H}=(\mathrm{D}-\mathrm{d}) / 2$

where: $\mathrm{H}$ is the inhibition zone [mm]; $\mathrm{D}$ - the total diameter of specimen and inhibition zone $[\mathrm{mm}]$; $\mathrm{d}-$ the diameter of specimen $[\mathrm{mm}]$.

\section{RESULTS AND DISCUSSION}

The images obtained by optical microscopy for the 7 experimental variants are shown in Figure 1. According to microscopic images, the dispersed molecule phase is presented as a compact, dense small globule mass. Normally, the destabilization of the emulsions begins with the drops flocculation of essential oil followed by the phenomenon of coalescence and formation of two distinct phases (separation).

https://doi.org/10.24264/icams-2020.I.5 


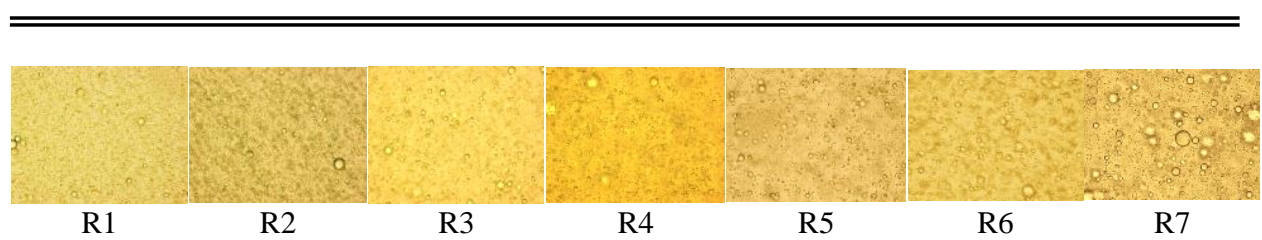

Figure 1. Optical microscopy of the resulted bioactive systems

\section{Emulsions Stability}

The calculated values for the stability index, are shown in Table 2 . The values recorded for the stability index over a period of 10 days indicate that they were stable, during this time not showing the presence of one of the flocculation, creaming / sedimentation, coalescence or Ostwald ripening phenomena. Generally, the turbidity of the bioactive polymeric systems is correlated to a great extent with their stability. Analyzing the values obtained for the 7 experimental variants it can be observed that the lowest values of the turbidity were obtained for the R5 variant (1.90) in which the smallest amount of active principle is also present (essential oil and propolis). Turbidity decreases with increasing particle size of emulsion.

Table 2. Emulsions stability

\begin{tabular}{cccccccc}
\hline Stability & R1 & R2 & R3 & R4 & R5 & R6 & R7 \\
\hline Turbidity $\left[\mathrm{cm}^{-1}\right]$ & 2.96 & 3.07 & 3.57 & 3.41 & 1.90 & 2.27 & 3.45 \\
Stability index $[\%]$ & 100 & 100 & 100 & 100 & 100 & 100 & 100 \\
\hline
\end{tabular}

\section{Viscosity}

The main viscosity indices recorded for the synthesized emulsions are shown in Table 3 .

Table 3. Viscosity indices recorded for the resulted emulsions

\begin{tabular}{cccccc}
\hline Code & $\begin{array}{c}\text { Viscosity } \\
{[\mathrm{cP}]}\end{array}$ & $\begin{array}{c}\text { SS } \\
{\left[\text { dyne/cm } / \mathrm{cm}^{2]}\right.}\end{array}$ & $\begin{array}{c}\text { SR } \\
{\left[\mathrm{s}^{-1]}\right.}\end{array}$ & $\begin{array}{c}\text { Speed } \\
{[\mathrm{RPM}]}\end{array}$ & $\begin{array}{c}\text { Temperature } \\
{\left[{ }^{\circ} \mathrm{C}\right]}\end{array}$ \\
\hline R1 & 35,41 & 65,87 & 186 & 200 & 24,9 \\
R2 & 45,91 & 85,40 & 186 & 200 & 25,2 \\
R3 & 53,08 & 98,74 & 186 & 200 & 25,3 \\
R4 & 61,30 & 114,10 & 186 & 200 & 25,3 \\
R5 & 64,08 & 119,16 & 186 & 200 & 25,4 \\
R6 & 57,75 & 107,43 & 186 & 200 & 25,3 \\
R7 & 52,25 & 97,18 & 186 & 200 & 25,3 \\
\hline
\end{tabular}

From the comparative analysis of the viscosities values it is observed that the highest value was obtained for the experimental variant R5 (64.08 cP) in which in which $0,363 \mathrm{~mL}$ water $/ \mathrm{mL}$ emulsion and $0,5 \mathrm{~mL}$ xanthan $/ \mathrm{mL}$ emulsion volume fraction were used. It can also be observed that the viscosity of the system is not dependent on the volume fraction of active principle (propolis and essential oil), the highest viscosity was obtained when using smaller volume fraction $(\varphi=0.015 \mathrm{~mL}$ oil $/ \mathrm{mL}$ emulsion $)$. The shear stress (SS) has the highest value in the case of the R5 variant, the variant obtained when using the smallest volume fraction of water. 


\section{Electron Microscopy of Textile Materials Functionalized with Bioactive Systems}

The images obtained at a magnification of $\mathrm{x} 8000$ for knitted textiles treated with bioactive systems are shown in Figure 1. The resulting micrographs reveal that the surface of the cotton fibers is covered with a thin polymeric layer deposited both on the surface of the fibers and inside the space between the fibers. However, the micrographs cannot provide information about the thickness of the deposited layer and its uniformity.

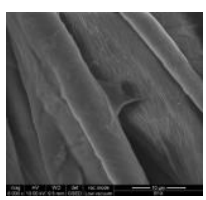

R1

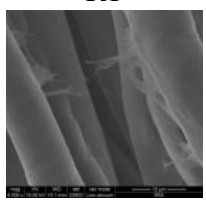

R5

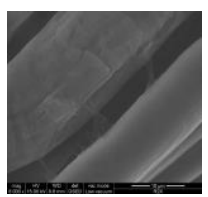

R2

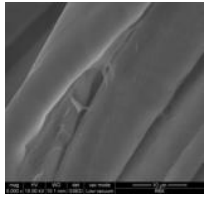

R6

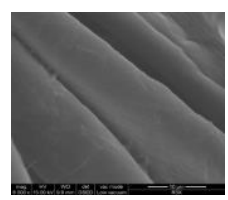

R3

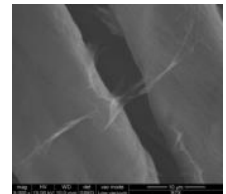

R7

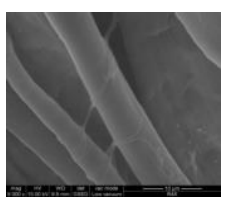

R4

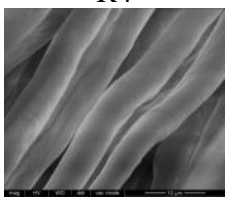

M

Figure 2. Electronic images recorded for the knitted fabrics treated with xanthan-propolis-cinnamon essential oil polymeric system

\section{Antibacterial Activity}

Images of Petri plates after 24h incubation are shown in Figure 3 and assessment of antibacterial activity is shown in Table 4.

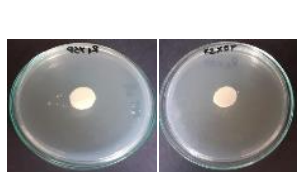

R1

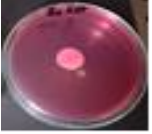

$\mathrm{R} 1$

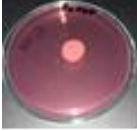

R2

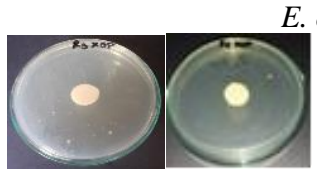

R3

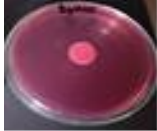

R3

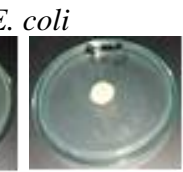

R5

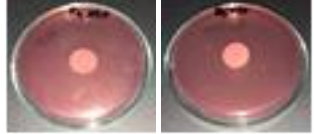

$\mathrm{R} 4$

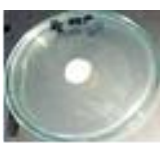

R6

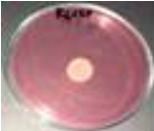

R6

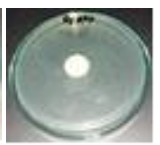

$\mathrm{R} 7$

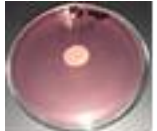

$\mathrm{R} 7$

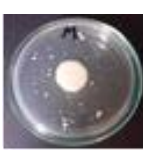

M

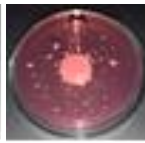

M

Figure 4. Images of Petri plates showing antibacterial effect after $24 \mathrm{~h}$ of incubation

By analyzing the obtained results, it can be concluded that the textile materials treated with synthesized emulsions based on xanthan-propolis-cinnamon essential oil have antibacterial effect against the $S$. aureus test strain, with inhibition zones between $1 \mathrm{~mm}(\mathrm{R} 5)$ and $5 \mathrm{~mm}(\mathrm{R} 2)$. In the case of E. coli strain, the presence of the inhibition zone and the growth of the test strain in the whole culture medium were not observed, being considered a satisfactory effect in terms of antibacterial activity. 
Bioactive Textiles Obtained by Applying Cinnamon Essential Oil-Based Emulsions

Table 4. Evaluation of the antibacterial activity

\begin{tabular}{ccccc}
\hline Code & \multicolumn{3}{c}{ E. coli } & \multicolumn{2}{c}{ S. aureus } \\
& $\begin{array}{c}\text { Inhibition zone } \\
{[\mathrm{mm}]}\end{array}$ & Evaluation & $\begin{array}{c}\text { Inhition zone } \\
{[\mathrm{mm}]}\end{array}$ & Evaluation \\
\hline R1 & $0(*)$ & Satisfactory effect & $0(*)$ & Satisfactory effect \\
R2 & $0(*)$ & Satisfactory effect & 5 & Satisfactory effect \\
R3 & $0(*)$ & Satisfactory effect & 3.5 & Satisfactory effect \\
R4 & $0(*)$ & Satisfactory effect & 3 & Satisfactory effect \\
R5 & $0(*)$ & Satisfactory effect & 1 & Satisfactory effect \\
R6 & $0(*)$ & Satisfactory effect & $0(*)$ & Satisfactory effect \\
R7 & $0(*)$ & Satisfactory effect & $0(*)$ & Satisfactory effect \\
M & 0 & Unsatisfactory effect & 0 & Unsatisfactory effect \\
\hline \multicolumn{5}{c}{ (*) - no inhibition zone, no multiplication }
\end{tabular}

\section{CONCLUSIONS}

Seven exprimental variants of bioactive polymer systems synthesized based on xanthan-propolis-cinamon essential oil were assessed to explore their physico-chemical, biological as well as functional property to understand the possible applications in developing of biomedical textiles. From the cumulation of the obtained data it was found that the prepared emulsions have a high degree of stability and a suitable viscosity to ensure a uniform deposition on the textile materials, on the one hand, and on the other hand to be able to be kept in a single phase until further application on textile materials. Textile materials treated with bioactive polymeric systems have shown antibacterial activity for both gram positive bacteria ( $S$ aureus) and gram negative bacteria $(E$ coli) test strains. The research on the potential developed emulsions based on xanthan-propolis-cinnamon for skin inflamations therapies by applying on textile materials is in progress, studying the potential of biocompatibility by skin irritations potential.

\section{Acknowledgments}

This work was carried out through the Nucleu Programme, with the support of MEC, project no. 4N / 08.02.2019, PN 191703 01, project title: "Multifunctional integrated systems based on nanocomposites and pharmacodynamic therapeutic agents for different skin conditions - BIOPANTEX".

\section{REFERENCES}

Alonso, C., Marti, M., Martinez, V., Rubio, L., Parra, J.L. and Coderch, L. (2013), “Antioxidant cosmeto-textiles: Skin assessment”, Eur J Pharm Biopharm, 84(1), 192-199, https://doi.org/10.1016/j.ejpb.2012.12.004.

Dastjerdi, R. and Montazer, M. (2010), "A review on the application of inorganic nano-structured materials in the modification of textiles: Focus on anti-microbial properties", Colloids Surf B, 79(1), 5-18, https://doi.org/10.1016/j.colsurfb.2010.03.029.

Yuan, G. and Cranston, R. (2008), "Recent advances in antimicrobial treatments of textiles", Text Res J, 78, 60-72, https://doi.org/10.1177/0040517507082332.

Windler, L., Height, M. and Nowack, B. (2013), "Comparative evaluation of antimicrobials for textile applications”, Environ Int, 53, 62-73, https://doi.org/10.1016/j.envint.2012.12.010. 
COTTON FABRICS COATED WITH AG-TIO2 AND AG-TIO2/REDUCED GRAPHENE OXIDE NANOCOMPOSITES

\author{
LAURA CHIRILA ${ }^{1}$, MARCELA CORINA ROSU ${ }^{2}$, SABINA OLARU ${ }^{1}$, CRISTIAN \\ TUDORAN $^{2}$, DRAGOS-VIOREL COSMA ${ }^{2}$, ALEXANDRA URDA ${ }^{2}$, ALICE-ORTANSA \\ MATEESCU $^{3}$, GHEORGHE MATEESCU $^{3}$, GEORGIANA VASILE $^{1}$ \\ ${ }^{1}$ National Research \& Development Institute for Textiles and Leather, 16 Lucretiu Patrascanu \\ Street, 030508, sector 3, Bucharest, Romania, laura.chirila@incdtp.ro \\ ${ }^{2}$ National Institute for Research and Development of Isotopic and Molecular Technologies, \\ 67-103 Donat Street, 400293, Cluj-Napoca, Romania \\ 3 "Horia Hulubei" National Institute for Research and Development in Physics and Nuclear \\ Engineering, 30 Reactorului Street, 077125, Magurele, Romania
}

\begin{abstract}
$\mathrm{Ag}-\mathrm{TiO}_{2}$ and $\mathrm{Ag}-\mathrm{TiO}_{2} /$ reduced graphene oxide nanopowders were deposited onto $100 \%$ cotton fabrics via electrostatic spraying method. The surface of cotton fabrics was pre-treated by plasma at atmospheric pressure using argon and nitrogen mixture. The as-prepared cotton fabrics were characterized in terms of structural and optical properties by X-ray diffraction (XRD) and optical reflectance measurements. The photocatalytic self-cleaning ability of $\mathrm{Ag}^{-\mathrm{TiO}_{2}}$ and $\mathrm{Ag}$ $\mathrm{TiO}_{2}$ /reduced graphene oxide coated cotton fabrics was evaluated by the photo-discoloration of methylene blue and berries juice stains, under $6 \mathrm{~h}$ simulated visible light irradiation. The combined functionalized coating on cotton fabrics demonstrated an improved photocatalytic effect compared with untreated cotton fabrics. The antimicrobial activity of $\mathrm{Ag}-\mathrm{TiO}_{2}$ and $\mathrm{Ag}-\mathrm{TiO}_{2} / \mathrm{reduced}$ graphene oxide coated cotton fabrics was tested against the Staphylococcus aureus and Candida albicans test strains as model microorganism of skin bacteria and fungi, respectively. An antimicrobial effect against the Staphylococcus aureus is observed even if the inhibition zone is not present. Untreated fabrics showed no antibacterial activity. No inhibitory effect on fungi colony growth was observed.
\end{abstract}

Keywords: $\mathrm{Ag}-\mathrm{TiO}_{2}, \mathrm{Ag}-\mathrm{TiO}_{2} /$ reduced graphene oxide, self-cleaning properties, antimicrobial activity

\title{
INTRODUCTION
}

Cotton is an important natural renewable cellulose fiber, widely used in textile industry due to its good hygroscopicity and breath ability. Cotton fabric coatings with nanoparticles exhibit antimicrobial properties, ultraviolet (UV) protection, superhydrophobicity, self-cleaning and flame retardancy (Zhang et al., 2019; Li et al., 2017). As one of the most important nanomaterial, $\mathrm{TiO}_{2}$ nanoparticles demonstrated efficient photocatalytic activity, chemical stability, biocompatibility, being used in a wide range of applications such as photocatalysis, nanomedicine, textiles, electronic devices, water sanitization (Ahmad Barudin et al., 2013).

The main goal of the presented research work was to examine the self-cleaning properties and antimicrobial activity of cotton fabric samples pre-treated with plasma and coated with $\mathrm{Ag}-\mathrm{TiO}_{2}$ and $\mathrm{Ag}-\mathrm{TiO}_{2} /$ reduced graphene oxide nanopowders.

\section{EXPERIMENTAL PART}

\section{Materials}

Bleached $100 \%$ cotton woven fabric with the weight of $168 \mathrm{~g} / \mathrm{m}^{2}$ has been used for all experiments. One percent of $\mathrm{Ag}-\mathrm{TiO}_{2}$ nanoparticles was prepared by liquid 
impregnation method using $\mathrm{TiO}_{2} \mathrm{P} 25$ powder from Degussa, silver nitrate from AlfaAesar as source of silver and distilled water, followed by a simple thermal treatment under argon atmosphere at $450^{\circ} \mathrm{C} . \mathrm{Ag}-\mathrm{TiO}_{2} /$ reduced graphene oxide $(\mathrm{Ag}-\mathrm{TiO} 2 / \mathrm{RGO})$ nanopowder was obtained by mixing $\mathrm{Ag}-\mathrm{TiO}_{2}$ nanoparticles with graphene oxide (GO) (10:1 mass ratio) followed by thermal reduction of GO under argon atmosphere at $300^{\circ} \mathrm{C}$. GO was prepared according to the previously described procedure (Pogacean $e t$ al., 2015). Methylene blue (MB) was purchased form Aldrich Sigma. Natural berries juice has been freshly prepared by freeze-thaw method of berries and then filtered.

\section{Functionalization Treatments}

A homemade model of Portable plasma generator from INCDTIM was used for pretreatment of cotton fabric samples $(25 \mathrm{~cm} \times 60 \mathrm{~cm})$, under specified experimental conditions (process gas: Ar: $\mathrm{N}_{2} /$ air, gas flow rate: 6 1/min, input power: $200 \mathrm{~W}$, plasma treatment time: $9 \mathrm{~min}$ ) for an increased adhesion of nanopowders onto cotton fabrics. The deposition of $0.1 \%$ dispersions based on $\mathrm{Ag}-\mathrm{TiO}_{2}$ and $\mathrm{Ag}-\mathrm{TiO}_{2} / \mathrm{RGO}$ nanopowders on the textile materials was performed by electrostatic spraying method using a Microbecide ${ }^{\circledR T C}-320$ Electrostatic Sprayer equipment (Singapore). The cotton coating experiments were conducted at ambient conditions on average spraying distance from samples of $80 \mathrm{~cm}$, with an flow rate adjustable of $20-240 \mathrm{ml} / \mathrm{min}$, average droplet size of 40 microns; negative electrostatic charge polarity, $1.0 \mathrm{bar}(15 \mathrm{psi})$ liquid line pressure, $2.1 \mathrm{bar}(30 \mathrm{psi})$ air line pressure and $0.6-1.2 \mathrm{kV}$ electrostatic charging voltage.

\section{Methods}

\section{X-ray Diffraction}

The structural characterization of cotton fabric pre-treated with plasma and coated with nanopowders was performed using a BRUKER D8 Advance X-ray diffractometer using $\mathrm{CuK}_{\alpha}$ radiation $(\lambda=1.54056 \AA)$ in order to identify the phase of a crystalline material.

\section{Optical Reflectance Measurements}

The optical properties of cotton fabric samples were investigated by UV-Vis spectroscopy using an UV-VIS Jasco Spectrophotometer (Japan).

\section{Assessment of Self-Cleaning Activity}

The self-cleaning properties of cotton samples pre-treated with plasma and coated with nanopowders were evaluated by photo-discoloration of methylene blue and berries juice stains on their surface. The cotton fabrics were cut into $3 \times 3 \mathrm{~cm}$ pieces. Some of them were dipped in $20 \mathrm{ppm}$ MB solution and others were stained with $10 \mu \mathrm{l}$ of berries juice, then all cotton samples were freely dried for $24 \mathrm{~h}$ in the dark. Subsequently, the stained cotton samples were exposed to visible light irradiation for $6 \mathrm{~h}$ by using the Xenotest equipment (Apolo James Heal). Evaluation of the self-cleaning ability of cotton samples pre-treated with plasma and coated with nanopowders was performed by measuring the color difference of the irradiated samples compared to non-irradiated samples (reference). Color measurements were performed according to ISO $105 \mathrm{~J} 03: 2001$, using the 
Datacolor $^{\mathrm{TM}} 650$ Spectrophotometer (Datacolor, Switzerland) and the light source was the standard illuminant D65/10. The obtained values of the lightness difference (DL*) are the average of 5 individual measurements carried out in different points on the same sample. DL* indicates a lighter color if positive and darker if negative.

\section{Antimicrobial Activity}

The antimicrobial activity of cotton samples pre-treated with plasma and coated with nanopowders was qualitatively assessed by Agar diffusion method according with the SR EN ISO 20645:2004 standard, by using of cultures in liquid medium replicated at 24 hours of ATCC 25923 Staphylococcus aureus (Gram-positive) and ATCC 10231 Candida albicans test strains. The cotton samples were cut in circular shape with a diameter of $2 \mathrm{~cm}$ and subsequently disposed on the culture medium in the middle of Petri plates, and then were incubated at $37^{\circ} \mathrm{C}(\mathrm{S}$. aureus $)$ and $30^{\circ} \mathrm{C}(\mathrm{C}$. albicans) for $48 \mathrm{~h}$. After incubation, the diameter of the clear zone $(\mathrm{mm})$ that indicates the microbial growth inhibition was measured and antimicrobial activity was evaluated.

\section{RESULTS AND DISCUSIONS}

The compared XRD patterns of pristine cotton and selected cotton sample pretreated with plasma and coated with $\mathrm{Ag}-\mathrm{TiO}_{2} / \mathrm{RGO}$ were presented in Figure 1. The diffraction peaks at $2 \theta$ values of $2 \theta=14.8^{\circ}, 16.5^{\circ}, 22.8^{\circ}$ and $34,5^{\circ}$ are assigned to the (101), (101), (002) and (040) planes of cellulose fiber (Abdelhameed et al., 2017; Ahmad et al., 2019). The reflection peak at $2 \theta=25.19$ is indexed to the (101) plane of the $\mathrm{TiO}_{2}$ anatase phase (PDF Sheet no. 21-1276). No reflection peaks related to the crystalline silver are shown, posible to its reduced amount. A slightly changed profile of broad XRD peak centered at $2 \theta \approx 15.5^{\circ}$ was observed by contribution of $(002)$ reduced graphene oxide that has a characteristic peak at $2 \theta=25^{\circ}$ (Dey et al., 2016).

The reflectance of cotton samples (Figure 2) is almost overlapped in the entire UVVis region, implying no superior UV-rays blocking properties and absorption to the visible light of the nanopowder-coated cotton samples (Chaudhari et al., 2012; Ojstršek and Fakin, 2019), probably due to the penetration of these nanoparticles into the cotton fabric material. All cotton samples show high reflectance values $(>75 \%)$ across the full visible spectrum, in accordance with their white-light grey color.

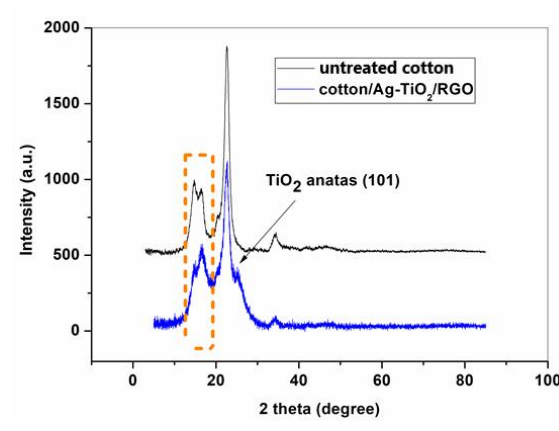

Figure 1. Comparative XRD pattern of cotton samples

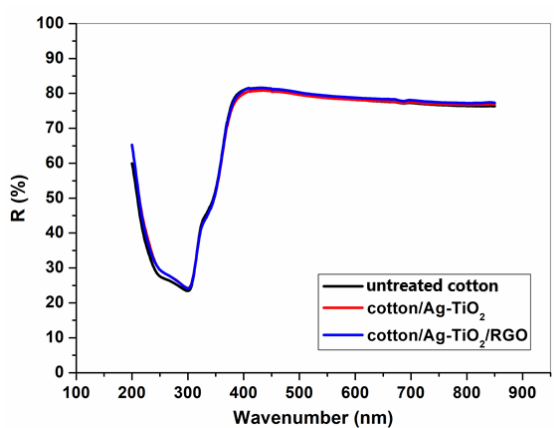

Figure 2. Comparative UV-VIS spectra of cotton samples 


\section{Self-Cleaning Activity}

The self-cleaning abilities of cotton samples pre-treated with plasma and coated with $\mathrm{Ag}-\mathrm{TiO}_{2}$ and $\mathrm{Ag}-\mathrm{TiO}_{2} / \mathrm{RGO}$ nanopowders were evaluated by monitoring the color changes due to the photocatalytic degradation of $\mathrm{MB}$ and berries juice stains. Color lightness difference $\left(\mathrm{DL}^{*}\right)$ was determined considering the non-irradiated cotton samples as reference and their values are pesented in Table 1.

Table 1. DL* values and digital photo of stained cotton fabric samples, before and after $6 \mathrm{~h}$ of simulated visible light irradiation

\begin{tabular}{|c|c|c|c|c|c|c|c|}
\hline Sample & DL* & Sample & $\mathrm{DL}^{*}$ & Sample & DL* & Sample & DL* \\
\hline $\begin{array}{l}\text { Cotton/ } \\
\text { Ag-TiO }_{2}\end{array}$ & & $\begin{array}{c}\text { Cotton/ } \\
\text { Ag- }-\mathrm{TiO}_{2} / \mathrm{RGO}\end{array}$ & & $\begin{array}{c}\text { Cotton/ } \\
\text { Ag-TiO }\end{array}$ & & $\begin{array}{c}\text { Cotton/ } \\
\mathrm{Ag}-\mathrm{TiO}_{2} / \mathrm{RGO}\end{array}$ & \\
\hline & 3.80 & & 2.29 & & 7.10 & & 3.6 \\
\hline \multicolumn{3}{|c|}{ Methylene blue } & \multicolumn{5}{|c|}{ Berries juice } \\
\hline
\end{tabular}

Note: $D^{*}=$ difference in lightness/darkness value $(+=$ lighter - = darker $)$

The obtained data revealed that the cotton samples pre-treated with plasma and coated with $\mathrm{Ag}-\mathrm{TiO}_{2}$ and $\mathrm{Ag}-\mathrm{TiO}_{2} / \mathrm{RGO}$ nanopowders showed great photodegradation efficiency of $\mathrm{MB}$ and berries juice stains. The best self-cleaning activity was obtained for cotton sample coated with $\mathrm{Ag}-\mathrm{TiO}_{2}$ nanopowder and stained with berries juice. However, the photocatalytic activity depends on the various parameters, such as nature and amount of the nanopowder as photocatalysts, light source, time exposure, type and concentration of contaminants etc.

\section{Antimicrobial Activity}

The antimicrobial and antifungal properties of textiles treated with $\mathrm{TiO}_{2}-\mathrm{Ag}$ nanoparticles (Li et al., 2017; Saraswati et al., 2019) and also $\mathrm{RGO} / \mathrm{TiO}_{2}$ nanoparticles (Karimi et al., 2014; Stan et al., 2019) are already reported.

The evaluation of the antimicrobial activity consisted in highlighting the presence or absence of the inhibition zone around the samples and the colonies formed or not on the contact surface with the environment, the results obtained being presented in Table 2.

Table 2. Antimicrobial activity of cotton fabric samples

\begin{tabular}{|c|c|c|c|c|}
\hline \multirow[b]{2}{*}{ Sample } & \multicolumn{2}{|c|}{ S. aureus } & \multicolumn{2}{|c|}{ C. albicans } \\
\hline & $\begin{array}{c}\text { Inhibition zone } \\
{[\mathrm{mm}]}\end{array}$ & Evaluation & $\begin{array}{c}\text { Inhibition zone } \\
{[\mathrm{mm}]}\end{array}$ & Evaluation \\
\hline Untreated cotton & 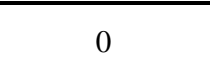 & $\begin{array}{c}\text { Satisfactory } \\
\text { effect }\end{array}$ & 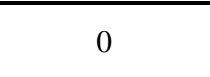 & $\begin{array}{c}\text { Unsatisfactory } \\
\text { effect }\end{array}$ \\
\hline Cotton/Ag-TiO 2 & 0 & $\begin{array}{c}\text { Satisfactory } \\
\text { effect }\end{array}$ & 1 & $\begin{array}{c}\text { Unsatisfactory } \\
\text { effect }\end{array}$ \\
\hline Cotton/Ag-TiO2/RGO & 0 & $\begin{array}{c}\text { Satisfactory } \\
\text { effect }\end{array}$ & 0 & $\begin{array}{c}\text { Unsatisfactory } \\
\text { effect }\end{array}$ \\
\hline
\end{tabular}

https://doi.org/10.24264/icams-2020.I.6 
ICAMS $2020-8^{\text {th }}$ International Conference on Advanced Materials and Systems

Table 3. Criteria for inhibition zones according to the standard SR EN ISO 20645:2005

\begin{tabular}{ccc}
\hline Inhibition zone $[\mathrm{mm}]$ & Growth & Evaluation \\
\hline$>1$ & Absence & Satisfactory effect \\
$1-0$ & & \\
0 & little & Efficiency limit \\
0 & moderate & Unsatisfactory effect \\
0 & important & \\
0
\end{tabular}

An antimicrobial effect against the Staphylococcus aureus is observed even if the inhibition zone is not present. Untreated fabrics showed no antibacterial activity. No inhibitory effect on fungi colony growth was observed.

Various factors, such as phase composition, mass loading and dispersion uniformity of nanopowders on cotton fabric, the surface modification of cotton have to be considered in order to provide antimicrobial efficacy.

\section{CONCLUSIONS}

$\mathrm{Ag}-\mathrm{TiO}_{2}$ and $\mathrm{Ag}-\mathrm{TiO}_{2} / \mathrm{RGO}$ nanopowders were successfully prepared by impregnation method. The cotton fabric samples were pre-treated with plasma and coated with $\mathrm{Ag}-\mathrm{TiO}_{2}$ and $\mathrm{Ag}-\mathrm{TiO}_{2} / \mathrm{RGO}$ nanopowders. XRD patterns confirmed the presence of nanopowders on the cotton fabric samples. UV-Vis spectral data showed a high reflectance values $(>75 \%)$ across the full visible spectrum, proving their whitelight grey appearance. The self-cleaning ability to degrade methylene blue and berries juice stains is demonstrated. An antibacterial effect against Staphylococcus aureus is observed, but no inhibitory effect on fungi colony growth of Candida albicans was present. Further strategies include the variation of different experimental parameters to increase the antimicrobial activity of cotton fabrics coated with $\mathrm{Ag}-\mathrm{TiO}_{2}$ and $\mathrm{Ag}$ $\mathrm{TiO}_{2} / \mathrm{RGO}$ nanopowders.

\section{Acknowledgments}

This work was supported by a grant of Romanian Ministry of Research and Innovation, CCCDI - UEFISCDI, Project number PN-III-P1-1.2-PCCDI-2017 0743/44PCCDI/2018, within PNCD III.

\section{REFERENCES}

Abdelhameed, R.M., Kamel, O.M.H.M., Amr, A., Rocha, J. and Silva, A.M.S. (2017), "Antimosquito activity of a titanium-organic framework supported on fabrics", ACS Appl Mater Interfaces, 9(27), 22112-22120, https://doi.org/10.1021/acsami.7b03164.

Ahmad Barudin, N.H., Sreekantan, S., Thong, O.M. and Sahgal, G. (2013), “Antibacterial activity of Ag-TiO nanoparticles with various silver contents", Materials Science Forum, 756, 238-245, https://doi.org/10.4028/www.scientific.net/MSF.756.238.

Ahmad, I., Kan, C. and Yao, Z. (2019), "Photoactive cotton fabric for UV protection and self-cleaning", RSC $A d v, 9,18106-18114$, https://doi.org/10.1039/C9RA02023C.

Chaudhari, S.B., Mandot, A.A. and Patel, B.H. (2012), "Effect of nano $\mathrm{TiO}_{2}$ pretreatment on functional properties of cotton fabric", International Journal of Engineering Research and Development, 1(9), 2429.

Dey, M.K., Reddy, A.V.R. and Satpati, A.K. (2016), "Determination of melamine by a reduced graphene oxide-gold nanoparticle composite carbon paste electrode", Anal Lett, 49(17), 2703-2715, https://doi.org/10.1080/00032719.2016.1154065. 


\section{Cotton Fabrics Coated with $\mathrm{Ag}-\mathrm{TiO}_{2}$ and $\mathrm{Ag}-\mathrm{TiO}_{2} /$ Reduced Graphene Oxide Nanocomposites}

Karimi, L., Yazdanshenas, M.E., Khajavi, R., Rashidi, A. and Mirjalili. M. (2014), "Using graphene/TiO nanocomposite as a new route for preparation of electroconductive, self-cleaning, antibacterial and antifungal cotton fabric without toxicity", Cellulose, 21(5), 3813-3827, https://doi.org/10.1007/s10570014-0385-1

Li, S., Zhu, T., Huang, J., Guo, Q., Chen, G. and Lai Y. (2017), "Durable antibacterial and UV-protective $\mathrm{Ag} / \mathrm{TiO}_{2} @$ fabrics for sustainable biomedical application", Int J Nanomedicine, 12, 2593-2606, https://doi.org/10.2147/IJN.S132035.

Ojstršek, A. and Fakin, D. (2019), "Washing durability and photo-stability of nanoTiO ${ }_{2}-\mathrm{SiO}_{2}$ coatings exhausted onto cotton and cotton/polyester fabrics", Coatings, 9, 545-557, https://doi.org/10.3390/coatings9090545.

Pogacean, F., Socaci, C., Pruneanu, S., Biris, A.R., Coros, M., Magerusan, L., Katona, G., Turcu, R. and Borodi, G. (2015), "Graphene based nanomaterials as chemical sensors for hydrogen peroxide - A comparison study of their intrinsic peroxidase catalytic behavior", Sensors Actuators, B Chem, 213, 474 483, https://doi.org/10.1016/j.snb.2015.02.124.

Saraswati, M., Permadani, R.L. and Slamet A. (2019), "The innovation of antimicrobial and self-cleaning using $\mathrm{Ag} / \mathrm{TiO} 2$ nanocomposite coated on cotton fabric for footwear application", IOP Conf. Series: Mat Sci Eng, 509, 012091, https://doi.org/10.1088/1757-899X/509/1/012091.

Stan, M.S., Nica, I.C., Popa, M., Chifiriuc, M., Iordache, O., Dumitrescu, I., Diamandescu, L. and Dinischiotu, A. (2019), "Reduced graphene oxide/ $\mathrm{TiO}_{2}$ nanocomposites coating of cotton fabrics with antibacterial and self-cleaning properties", $J$ Ind Text, 49, 277-293, https://doi.org/10.1177/1528083718779447.

Zhang, G., Wang, D., Yan, J., Xiao, Y., Gu, W. and Zang, C. (2019), "Study on the photocatalytic and antibacterial properties of $\mathrm{TiO}_{2}$ nanoparticles-coated cotton fabrics", Materials (Basel, Switzerland), 12(12), 2010-2019, https://doi.org/10.3390/ma12122010. 
RESEARCH COMPARISON OF FOOT PLANTAR PRESSURE ON POLYURETHANE VISCO ELASTIC FOAM INSOLE AND EVA INSOLE MATERIALS

\author{
DURAIRAJ DHANAPAL, BAHIRU MELESE, LIYA SHAWEL, KUMARAVEL \\ SANMUGAM \\ FTVETI, Addis Ababa,Ethiopia,dhanapal_d2k@yahoo.com
}

\begin{abstract}
The viscoelastic cushion insoles respond to the unique curves and pressures of our foot with every movement whether walking, running, playing, or exercising. Initially NASA, USA had developed viscoelastic foam insole to support astronauts during the heavy $\mathrm{G}$ force applied to the body during space flight. Nowadays $1 / 4$ inch thick layer of viscoelastic foam sandwiched between two layers of different types of foam are available commercially at a high cost. In our country, viscoelastic PU foams as cushion inserts for shoes are imported from China. But the available materials are not scientifically characterized and optimized for the composition of PU for application as an insole for treating foot abnormalities. In this project PU, the viscoelastic foam was prepared using standard polyols and dissociates used by industries to prepare memory foam mattresses. The additives which are physiologically and environmentally safe were used.
\end{abstract}

Keywords: plantar pressure, PU viscoelastic foam, EVA foam, peak values, footwear stability, cushion factor, cushion energy

\title{
INTRODUCTION
}

Footwear is one of the common commodities being used by all kinds of persons from child to aged and from poor to rich. Foot comfort on wearing footwear is the basic expectation from consumer's point of view other than protection and style (Socaciu et al., 2010). Improper footwear may lead to heel or knee or hip or back pain. Insole, one of the most important components in footwear, helps to protect the complex bony structures of the lower extremity from damage and for uniform distribution of pressure and increased contact area under the foot while standing, walking and running and thus prevent pressure spots stated that structure orthotics are custom-made shoe inserts which serve to correct or relieve misalignment and/ or pressure areas of the foot and redistributes pressure and provides shock absorption. Therefore, there is a need for new material that can be used alone as inserts in shoes in 3 to $5 \mathrm{~mm}$.

\section{Materials Used as Cushion Insole}

Polymer elastomers and foams are widely used to meet the material properties of an insole and in the sock. Among various polymers, ethylene-vinyl acetate (EVA), polyurethane (PU), and rubber-based materials are widely used in footwear. Shock absorption and dimensional stability are more important materials properties of the insole. PU foams and elastomers are proved as the most effective materials for shock absorption in footwear. Foam provides some shock attenuation and gives good pressure distribution, feels soft to the touch. An undesirable property of foams is "compression set" in which repeated loading causes the walls of the cells to collapse, leading to a loss of material thickness, elasticity, and energy absorption capacity. In contrast to PU foam, polyurethane elastomer is a soft, non-porous rubbery material. The elastomer provides good shock attenuation and pressure distribution; reasonably resistant to compression set but does not feel soft. The disadvantage of PU elastomer is the absence of pores which contribute much to the resilience of material during deformation. Therefore, 
porous viscoelastic material will fill the gap between PU elastomer and foam. In the present research work, new polyurethanes were synthesized and developed into viscoelastic foam sheets with good mechanical strength to resist.

\section{Advantages and Disadvantages of Currently Available Materials}

The foams are cellular materials, which may be either open or closed cell. Open-cell materials resemble a sponge, where the bubble walls are incomplete, allowing free exchange of air in and out of the material. In closed-cell materials, air or some other gas (usually nitrogen) is trapped within minute bubbles, which become pressurized when the material is stressed, the gas pressure aiding in the elastic recovery. In contrast to the various forms, polyurethane elastomer is a soft, non-porous rubbery material. An undesirable property of many foams is "compression set", in which repeated loading causes the walls of the cells to collapse, leading to a loss of material thickness, elasticity, and energy absorption capacity. Foam materials differ in their resistance to compression set, due to their chemical makeup and their initial stiffness. Polyurethane foams were found to be more resistant to compression set than polyethylene foams of similar density the compression set.

\section{Comparison of PU Foam and EVA Foam Materials}

The main purpose of cushion insole in shoes is to reduce the peak plantar pressure and increase the total foot contact area while standing and walking. The effectiveness of cushion insole can be determined by using high-resolution sensors in the form of the mat which can scan the plantar surface and the treks can the software can measure.
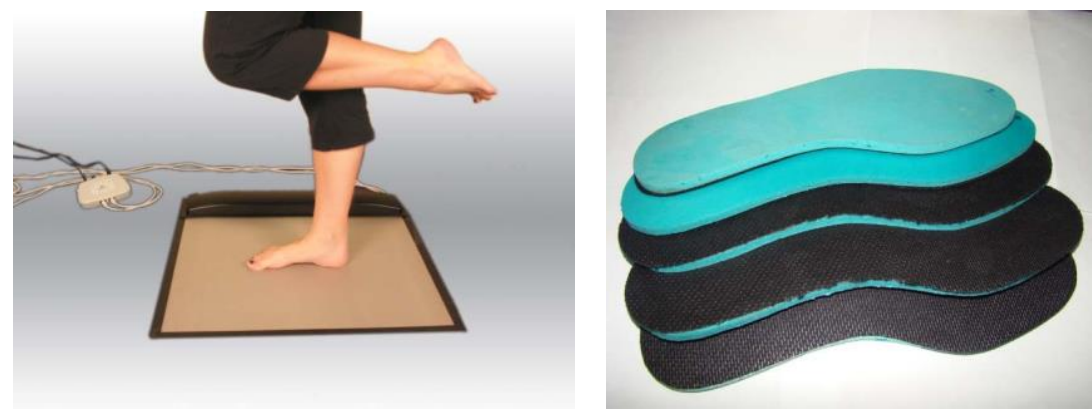

Figure 1. Foot scanner and PU Foam insole 


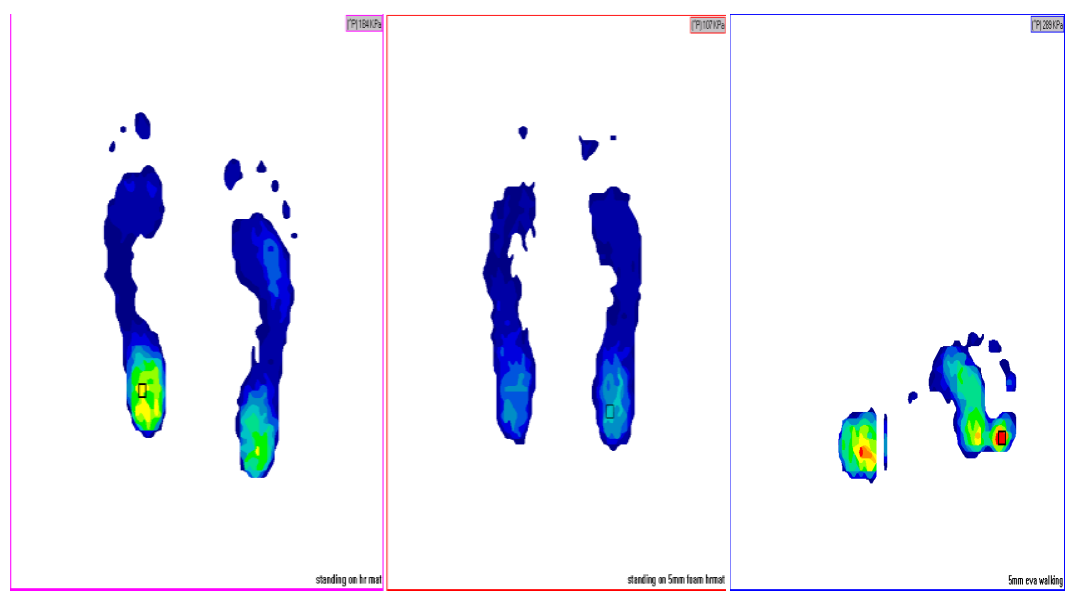

Figure 2. Foot pressure comparison along with PU Foam EVA insole

\section{DISCUSSION}

Footwear insole hardness (Shore A), density, compression set, cushion energy, cushion factor water absorption were determined by the SATRA test method. A further modification was done in the composition by changing the concentration of isocyanate and polyol and other additives such as surfactant, tin catalyst, and crosslinkers (Wood, 1982; Hock, 1998; Kaushiva, 1999). In the first trial PU, the viscoelastic foam was low resilient and very softness and water absorption was higher. Next, we modified the above chemical composition in trial second sample number 7 and 8 PU Visco elastic memory foam was higher resilience than the first trial, tensile strength was determined by SDDC lab CLRI by SATRA standard test method. Now the hardness was very higher than the first trial samples so we changing the density of the foam composition, the filler is added to increase the density for our requirements (Pratt et al., 1986). Second trial ratio 100: 80 and 100: 85 level was optimum for our requirements; the above two ratio foam was developed and now the foam was high hardness and optimum resilient that first trial samples. $10 \mathrm{~mm}$ PU Visco elastic memory foam was developed and determined by SATRA Standard test methods in SDDC Lab CLRI CHENNAI INDIA. Hardness (Shore A) compression set, density, cushion energy and cushion factor, water absorption and desorption test, tensile strength test were evaluated. These values were compared with CLRI ideal value sample ratio 100: 85 PU viscoelastic memory foam was identified and better for shoe insole. FTIR, NMR spectroscopy, and foot scan test methods were determined by standard test methods. The newly developed viscoelastic memory foam was determined to pressure studies and measurements were evaluated by GIAT Lab CLRI Chennai India. The pressure was compared with current available EVA shoe insoles, these foot scan pressure values were noted and foot pressure distribution compared with another ordinary insole. From the above results, PU viscoelastic memory foam was better than other EVA, PU, vegetable leather insole, and cork insoles (Pratt et al., 1986; Pratt, 1988). The newly developed PU viscoelastic will be mainly used for cushion insert for therapeutic footwear and it will control the foot pain, heel shock absorption and distribute the foot pressure, give the additional cushion support to the foot arches (Mihai et al., 2008; Socaciu et al., 
2010). PU viscoelastic insole gives the foot shape during walking, and running, this insole environmentally and eco-friendly shape. The project is useful for the footwear industry.

\section{CONCLUSION}

Through viscoelastic PU foams available as cushion mattress and pillow, the newly developed insole based on PU memory foam were scientifically and characterized and optimized properties for application in insole /sandals in $5 \mathrm{~mm}$ to $10 \mathrm{~mm}$ thickness. This insole may be used in footwear for flat foot, high arched foot, dropped metatarsals, planter facilities. Callus and corn due to peak plantar pressure in the diabetic foot can be treated by pressure-relieving and sock reducing effects of viscoelastic foam Physical characterization such as infrared spectroscopy, scanning electron microscopy, thermal analysis, and Fscan plantar pressure measurement were determined. This insole is better for therapeutic footwear for as cushion insole than EVA and some other leather, rubber insole.

\section{REFERENCES}

Hock, H.R. (1998), Flexible PU foam, $2^{\text {nd }}$ edition, The Dowchem Co.

Kaushiva, B.D. (1999), Structure-property relationship of flexible PU foam, Blacksburg-Virginia.

Lai, Y., Patent No. CN1730554.

Mihai, A., Dragomir, A., Berijan Gh. (2008), "Comparative study on the assessment of anthropometric parameters defining the 3D shape of the diabetic and orthotic foot", Proceedings of the $2^{\text {nd }}$ International Conference ICAMS, Bucharest.

Pratt, D.J. (1988), "Medium term comparison of shock attenuating insoles using a spectral analysis technique", J Biomed Eng, 10, 426-429, https://doi.org/10.1016/0141-5425(88)90146-X.

Pratt, D.J., Rees, P.H. and Rodgers, C. (1986), “Assessment of some shock absorbing insoles: Technical note", C Prosthet Orthot Int, 10, 43-45, https://doi.org/10.3109/03093648609103079.

Socaciu, T., Simon, M., Pop, L.D. and Freitas, M. (2010), "Research and application of Visco elastic memory foam in the field of footwear manufacturing", Scientific Bulletin of the "Petru Maior" University of Tîrgu Mureş, 7(2), 49-52.

Wood, G. (1982), Flexible Polyurethane Foams: Chemistry and Technology, Sci-Pub Ltd. Essex England. 
APPROACHES TO THE EVALUATION OF THE MECHANICAL PROPERTIES OF SINGLE-LAYER COMPOSITE PLATES MADE OF RECYCLABLE POLYMERIC AND PROTEIN MATERIALS

\author{
ION DURBACĂ, RADU IATAN, ELENA SURDU, \\ DANA-CLAUDIA FARCAȘ-FLAMAROPOL* \\ "Politehnica" University of Bucharest, Faculty of Mechanical Engineering and Mechatronics, \\ Spl.Independenței street, 313, Bucharest, Romania, e-mails: ion.durbaca@yahoo.com, \\ iatan.radu@gmail.com,elena.surdu@yahoo.com,*claudia.flamaropol@gmail.com
}

\begin{abstract}
This paper deals with the theoretical and experimental mechanical characteristics of composite plates obtained from recyclable polymer and protein matrix and fibrous reinforcement. The definition of the theoretical model of the monolayer composite material with its structural elements and the physical-mechanical evaluation of its characteristics leads to the optimal and efficient design and use of all products made of such materials. By the theoretical and experimental determination of the mechanical characteristics that define the properties of the composite material, it can be decided on its use in specific industrial technical applications.
\end{abstract}

Keywords: composite, recyclable waste, matrix, fibrous material.

\title{
INTRODUCTORY CONSIDERATIONS
}

In the context of ensuring the sustainable development of today's society, as a fundamental desideratum of humanity, the concept of integrated waste management of any kind has been developed. Thus, on the background of solving the national strategic problems of waste management and prioritizing actions to minimize waste production, recycling, composting, energy recovery and ecological storage, there was a need to use urban and / or industrial waste in order to making new materials, such as, for example, single-layer composite materials of the plate type obtained from recyclable polymer waste (PET, PVC, etc.) reinforced with fibrous waste of a protein nature (natural leather, textile fibers, etc.) (Bold, 2003; Căpăţână, 2003; Nemeș, 2013).

The theoretical model of the monolayer composite material, made by different manufacturing processes (injection, extrusion, etc.) consists of a single composite layer with the possibility of being able to demonstrate its efficiency by evaluating the mechanical characteristics of its structural components (Hadăr, 2002; Durbacă et al., 2019).

The basic unit for composite materials with fiber reinforcements is the reinforced lamella, (see Figure 1), it confers multiple possibilities of composition and correct introduction of the characteristics in the calculation of the composite.

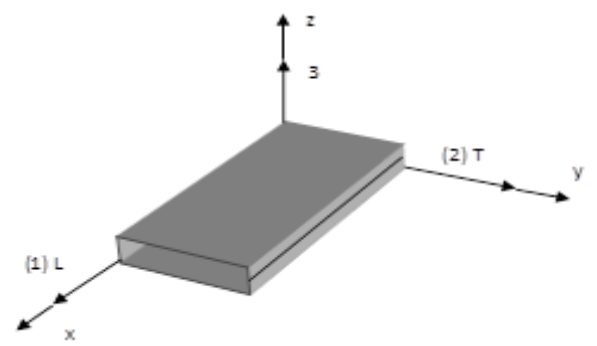

Figure 1. Theoretical model of the single-layer composite plate-type lamella (Hadăr, 2002) 
Within the lamella, the fibers that make up the reinforcement are arranged and oriented so as to lead to the achievement of the desired characteristics in the composite structural element.

The reinforced lamella is the elementary part of the composite material which consists of a sample of polymeric matrix reinforced with fibrous material in which the fibers are arranged according to the arrangement of these components in the structure of the composite assembly (random in space or plane), according to Figure 2. In this case, such structures are considered quasi-isotropic in space when the length of the fiber $L$ is much smaller than the thickness of the composite, $t_{c}$ (see Figure 2, a), and in the case of most composite structural elements, the length of the fibers it is much larger than the thickness, achieving quasi-isotropy in the plane (see Figure 2, b).

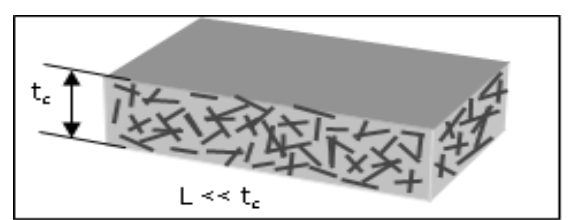

a.

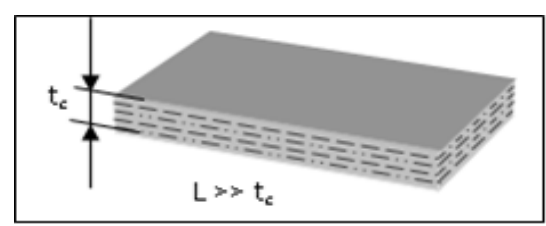

b.

Figure 2. The arrangement of the reinforced fibers in the polymeric matrix of the composite lamella: a - random orientation in space; $\mathrm{b}$ - random orientation in the plan

In structural applications where the state of tension is unpredictable, the use of composites with unidirectional reinforcement is insufficient and it is advantageous to use quasi-isotropic layers in the plane, obtained by using short fibers with random orientation.

\section{MECHANICAL CHARACTERISTICS OF SINGLE-SHEET PLATE COMPOSITES}

The physical-mechanical characteristics of composite materials reinforced with randomly oriented fibers are determined by parameters such as: fiber diameter $d f$, fiber length $L$, volumetric reinforcement fraction $w f$, fiber placement in relation to product axes and manufacturing process.

In order to establish the physical-mechanical characteristics of the composite lamella with polymeric matrix and reinforcement made of fibrous materials, a system of lamella axes is initially chosen. Therefore, in Figs. 1, above, schematically shows a lamella with unidirectional reinforcement: the direction parallel to the fibers $L$ (1) is called longitudinal, and the one perpendicular to the fibers $T(2)$ is called transverse direction and can correspond to any direction in the plane $(2,3)$. Axes $1,2,3$ are called the main axes of the material.

The main mechanical properties involved in the structural analysis of composite elements are strength and rigidity. These properties can be determined experimentally, but the tests are valid for a single fiber-matrix system, obtained with a certain manufacturing process (Hadăr, 2002; Alămoreanu and Chiriță, 1997; Durbacă et al., 2017; Păunescu, 2002; Tudorachi, 2007). Therefore, it is recommended to use theoretical and semiempirical models that allow the evaluation of mechanical characteristics based on parameters that influence the properties of the composite structure. Theoretical models are not always applicable, some direct corrections are necessary for the direct design of the 
elements, especially in the transverse direction, however for the study of mechanical characteristics in the longitudinal direction it is considered that the existing models for composites with continuous unidirectional reinforcement are sufficiently accurate (Iatan et al., 2017; Bere et al., 2012; Boboc, 2019).

Depending on the axis system adopted, for composite materials reinforced with fibers, the following mechanical characteristics necessary in the design are defined: $E_{L}$ $=E_{l}$ - longitudinal modulus of elasticity of the lamella (in direction parallel to the fibers); $E_{T}=E_{2}$ - transverse modulus of elasticity of the lamella (in a direction perpendicular to the fibers); $G_{L T}=G_{12}$ - modulus of shear elasticity of the lamella in the plane $(L, T)$ or $(1,2) ; v_{L T}=v_{12}$ and $v_{T L}=v_{21}$ - Poisson's ratios in the plane $(L, T)$ or $(1,2) ; R_{t L}$ - tensile strength of the blade in the longitudinal direction; $R_{t T}$ - tensile strength of the blade in the transverse direction; $R_{c L}$ - compressive strength of the lamella in the longitudinal direction; $R_{c T}$ - compressive strength of the transverse direction blade; $R_{f}$ ${ }_{(L T)}=R_{f(12)}$ - the shear strength of the lamella in the plane $(L, T)$ or $(1,2)$.

For theoretical analysis, it is considered a composite material with volume $v_{c}$, in which the fibers occupy the volume $v_{f}$, and the matrix the volume $v_{m}$. The same material has the weight $w_{c}$, the fibers have the weight $w_{f}$, and the matrix weight $w_{m}$. Noting with $V$ and $W$ the volumetric and gravimetric fractions, respectively, their definition is made with the help of the relations:

$v_{c}=v_{f}+v_{m} ; \quad V_{f}=v_{f} / v_{c} ; \quad V_{m}=v_{m} / v_{c}$

Expressing the masses with the help of the corresponding densities:

$\rho_{c} v_{c}=\rho_{f} v_{f}+\rho_{m} v_{m}$

where: $\rho_{c}, \rho_{f}$ and $\rho_{m}$ are the densities of composite, fiber and matrix.

The presence of gaps in the composite element significantly influences some of its mechanical properties. By increasing the gap content, the effects of properties degradation over time are generated and the results on the mechanical characteristics are scattered. A good quality composite must have less than $1 \%$ gaps, while an unsuitable one can reach a relative volume of gaps $V_{g}=5 \%$ (Hadăr, 2002).

An acceptable estimate for the longitudinal modulus of elasticity $E$ of the quasiisotropic composite in the plane, at axial stress, is obtained based on the relationships established by Gibson (Hadăr, 2002) through a series of simple relations, developed based on Cox's models (Hadăr, 2002) and allowing evaluation of the elasticity modules, distinct for the two cases:

- spatial quasi-isotropic composite:

$E=\frac{E_{f} V_{f}}{6}$

- quasi-isotropic composite in plan:

$E=\frac{E_{f} V_{f}}{3}$

The relations determined by Cox (Hadăr, 2002) for the evaluation of the transversal modulus of elasticity $G$, in the case of the composite reinforced with short fibers arranged at random are:

- spatial quasi-isotropic composite:

https://doi.org/10.24264/icams-2020.I.8 
$G=\frac{E_{f} V_{f}}{15}$

- quasi-isotropic composite in plan:

$G=\frac{E_{f} V_{f}}{8}$

For flat products made of composites reinforced with random short fibers, the following were used (Hadăr, 2002):

$G=\frac{E}{2(1+v)}$

where, the Poisson coefficient has the following values $v=1 / 4$, for spatial quasiisotropic composite and $v=1 / 3$, for plane quasi-isotropic composite.

For the calculation of the tensile strength (axial) stresses of short fiber composites randomly distributed in the plane, the following relations are used (Hadăr, 2002):

$R_{t}=\frac{2 R_{f(L T)}}{\pi}\left[1+\frac{R_{t T}}{\left(\sigma_{m}\right)_{\varepsilon_{f}^{*}}}+\ln \frac{R_{t T}\left(\sigma_{m}\right)_{\varepsilon_{f}^{*}}^{2}}{R_{f(L T)}^{2}}\right]$

where, $R_{f(L T)}, R_{t T}$ are the resistances of the unidirectional composite with continuous reinforcement calculated with the expressions (Hadăr, 2002).

For the calculation of the values of the mechanical characteristics of the composite reinforced with short fibers randomly distributed, other than those presented above, there are currently no acceptable mathematical models to allow their evaluation at values comparable to those determined experimentally.

\section{CASE STUDY}

The mechanical characteristics of a PVB / leather composite sample are analyzed, in which the matrix is represented by the PVB polymer (polyvinyl butyral), made by polycondensation of vinyl acetate with aldehydes, and the reinforcement / reinforcement material is represented by short semi-coarse crushed leather fibers; the polymer is in the form of a white-cream powder, with a density of approximately $1,2 \mathrm{~g} / \mathrm{cm}^{3}$, and the processing temperature is between $120 \div 170^{\circ} \mathrm{C}$.

Due to the presence of chromium in the skin fibers, which requires use at relatively low processing temperatures, this makes PVB a very interesting polymer matrix for the development of the case study for PVB / leather composite. Figure 3 below shows the microfractrography of some breaking surfaces of a PVB / leather composite sample, if the skin fibers are unevenly distributed in the PVB polymer matrix.

These composites were obtained by the same process used for the preparation of PVB / wood flour composite. Although there are some gaps in the polymer matrix, probably due to the defibration of the leather waste and the agglomeration of the skin fibers, a good adhesion can be given by the continuity of the agglomeration of the skin fibers in the polymer matrix PVB (de Almeida Lucas et al., 2011). As mentioned above, good adherence is usually difficult to achieve between the thermoplastic matrix and the natural fibers, due to the differences in polarity between the hydrophilic fibers 
(composed of collagen macromolecules) and the hydrophobic thermoplastic matrix. The PVB copolymer contains vinyl hydroxyl alcohol groups that can interact with the $\mathrm{OH}$ group and $-\mathrm{C}(=\mathrm{O})-\mathrm{OH}$ groups of collagen macromolecules in the skin fibers. The interaction between these groups can create an interface that manages to transfer the stress from the PVB polymer matrix on the cryogenic fracture of the skin fibers (de Almeida Lucas et al., 2011; Wehry 2002).

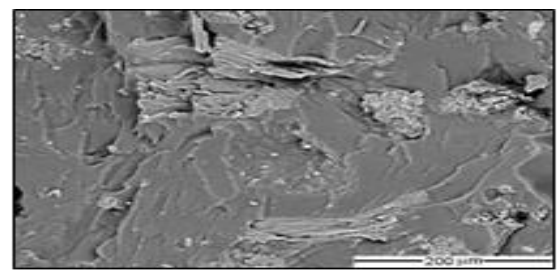

Figure 3. Microfractrography of some breaking surfaces

of a PVB / leather composite sample (de Almeida Lucas et al., 2011)

Figure 4 below shows the results of tensile tests for PVB composite with leather fibers obtained from waste recycling. The modulus of elasticity increases considerably, with the increasing content of leather fibers in the composite. The modulus of elasticity increases from $4 \mathrm{MPa}$ for PVB to $270 \mathrm{MPa}$ for composite with $70 \%$ skin fiber. Such a rapid and nonlinear growth of the modulus of elasticity with the fiber content is not frequently observed in thermoplastic composites reinforced with natural fibers. As previously presented, PVB contains plasticizer which gives it improved flexibility in composites.

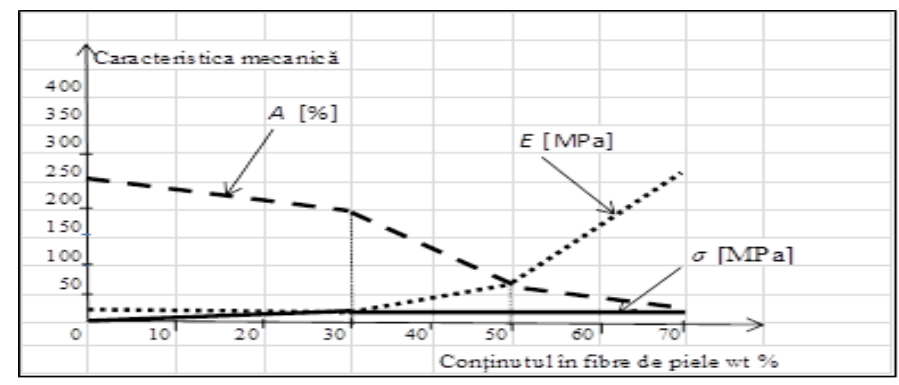

Figure 4. Variation of the mechanical properties $\left(E, \sigma_{\mathrm{r}}\right.$ and $\left.A\right)$ of the composite depending on the content of skin fibers.

In the literature there are a number of studies on thermoplastic composites containing skin fibers (de Almeida Lucas et al., 2011). Polymer matrices may include plasticized PVC, acrylonitrile-butadiene-styrene (ABS) and methyl polymethacrylate (PMMA), if the modulus of elasticity indicates different behavior. While ABS / leather and PMMA / leather composites show decreases in modulus $E$ values or a slight increase depending on the ascending content of leather fibers, PVC / leather composites show a similar behavior to PVB / leather composites, namely, the modulus elasticity is strongly influenced by the fiber content, especially in composites that exceed the skin fiber content $w_{t}=30 \%$.

It is pointed out that the polymer/leather fiber composite material, if the modulus of elasticity increases considerably with increasing amount of skin fibers, had a plasticized 
thermoplastic matrix, respectively by the existence of PVC and PVB polymeric materials used in this paper. The reduction in the tensile strength of PVB / leather composites can be attributed to a reduction in the deformation capacity of the polymer matrix, due to the concentration of leather fibers.

\section{CONCLUSIONS}

Despite the fact that the mechanical properties of the experimentally determined monolayer composite plate do not agree acceptably with those analyzed analytically, it nevertheless showed appropriate levels of use.

Therefore, the use of PVB / leather composite for industrial production, such as shoe soles and other parts of its structure (insole roof, heel, toe, etc.) it is supported by a number of mechanical properties (abrasion resistance, hardness, tear strength, adhesion to commercial adhesives, flexibility or folding capacity, resistance to repeated bending, etc.), as well as design, and have now been successfully tested in footwear companies.

By means of a simple and inexpensive processing technique (for example, an extrusion flow equipped with a single auger), PVB / leather composites have been manufactured with properties suitable for use in the footwear industry, to be returned as a raw material to the industrial chain and to facilitate the approval of recycled products by technical regulatory requirements.

\section{REFERENCES}

Alămoreanu, E. and Chiriță, R. (1997), Bars and plates from composite materials, Technical Publishing House, Bucharest.

Bere, P., Berce, P. and Nemeş O. (2012), "Phenomenological fracture model for biaxial fibre reinforced composites", Composites Part B: Engineering, 43(5), 2236-2243, https://doi.org/10.1016/j.compositesb.2012.01.073.

Boboc (Căpățână), A. (2019), "Contributions to the study of the properties of composite materials reinforced with fabrics and the effect of cyclical stresses on these properties", Doctoral thesis, "Dunărea de Jos" University of Galaţi.

Bold, O.V. (2003), Solid Waste Management, Bucharest: Matrix Rom Publishing House.

Căpățână, C. (2003), Waste, Bucharest: Matrix Rom Publishing House.

de Almeida Lucas, A., Ambrósio, J.D., Bonse, B.C. and Prado Bettini, S.H. (2011), "Natural Fiber Polymer Composites Technology Applied to the Recovery and Protection of Tropical Forests Allied to the Recycling of Industrial and Urban Residues", Advances in Composite Materials - Analysis of Natural and Man-Made Materials, Ed. Dr. Pavla Tesinova, ISBN: 978-953-307-449-8, InTech, https://doi.org/10.5772/19141.

Durbacă, A.C., Iatan, R., Durbacă, I., Diniță, A. and Vasilescu, M. (2017), "Experimental Research on the Triangular Lattice Type Polymer Based Composites Structures for Sandwich Panels Construction", Materiale Plastice, 54(4), 639-644, WOS: 00042641250000(9, IF=1,517, ISSN 0025-5289, https://doi.org/10.37358/MP.17.4.4916.

Durbacă, I., Iatan, R., Durbacă, A.C., Săcuiu, V., Mitucă Corleciuc, M. and Rusănescu, C.O. (2019), "Approaches Looking Finite Elements Analysis of a Structural Model of Lid Stratified with Cellular Polymeric Core Specific to a Pressure Vessel", Materiale Plastice, 56(1), 156-162, WOS: 000464604100031, IF=1,517, ISSN 0025-5289, https://doi.org/10.37358/MP.19.1.5142.

Hadăr, A. (2002), Structures from layered composites, Academy Publishing House and AGIR Publishing House, Bucharest.

Iatan, R., Enăchescu, G.L. and Florescu, P. (2017), Mechanical and thermal stresses in laminated composite boards, MatrixRom Publishing House, vol. 1 and 2, Bucharest.

Nemes, O. (2013), "Analytical Model Application for Adhesive Cylindrical Assemblies made by Hybrid Materials”, Materiale Plastice, 50(4), 314-318.

Păunescu, I. (2002), Urban waste management, Bucharest: Matrix Rom Publishing House.

Tudorachi, N. (2007), Thermoplastic polymer composite materials, PIM Publishing House, Iași.

Wehry, A. (2002), Ecological recycling and storage of waste, Timişoara: Orizonturi Universitare Publishing House.

https://doi.org/10.24264/icams-2020.I.8 


\author{
OVIDIU IORDACHE, ELENA CORNELIA MITRAN, IRINA SANDULACHE, \\ MARIA MEMECICA, LUCIA OANA SECAREANU, CRISTINA LITE, ELENA PERDUM \\ National R\&D Institute for Textile and Leather, Lucretiu Patrascanu 16, \\ Bucharest,Romania,office@incdtp.ro
}

\begin{abstract}
The present study was aimed at highlighting the applicability of novel generations of functional textile materials based on incorporation of safe, pyroelectric nanoparticles into fibers. The synthetic fibers with negative ions emitting properties contain semiprecious stone particles (tourmaline, monazite, opal), ceramic, charcoal, zirconium powders, aluminum titanate and mixtures of such minerals. Currently, the synthetic fibers generating pyroelectric effects are obtained by introducing minerals (e.g. superfine tourmaline powder) into melted polymers before spinning or by dispersing the minerals into the spinning solution. As polymers, polyethylene terephthalate, polyvinyl acetate, polyamide and viscose have been used. In low quantities, these minerals have almost no effect on human health. Included in large quantities, they tend to be too expensive (tourmaline, opal) and the fibers become harsh and fragile. The current generation of FIR functional textile materials faces a series of technical challenges: some of the of the used compounds are radioactive (monazite); if the particles size is too large $(0.2-0.3 \mu \mathrm{m})$, it may result in the production of highly non-uniform fibers and early wear of the mechanical parts producing installation; most of commercial pyroelectric fabrics emit a low amount of negative ions (5002600 anions/cc) and FI rays, inducing a low health effect. Clinical studies involving exposure to pyroelectric compounds have highlighted positive effects on: blood circulation, skin cell revitalizing, collagen and elastin production, sleep modulation, wounds healing and acceleration of micro-circulation, chronic pain management, improvement of vascular endothelial functions, atherosclerosis and arthritis affections etc.
\end{abstract}

Keywords: far-infrared, textiles, functional

\title{
INTRODUCTION
}

Discovered in 314 BC by Theophrastus, pyroelectricity has been used from ancient times, to heal a variety of diseases, such as anxiety, sleep disorders, cellulite, rheumatism, cardiac problems, brain dysfunction, bacterial infections, reduction of inflammation \& oxidative stress, improvement of endothelial function, increase of blood circulation and lymphatic flow, and even cancer (Lang, 2011). Such functional textiles, consist of synthetic fibers, emitting Far-Infrared-Rays (FIR), based on negative ions emitting particles, from se semiprecious stones (tourmaline, monazite, opal), ceramic powders, charcoal, zirconium, aluminum titanate and mixtures of such minerals. Currently, the synthetic fibers generating pyroelectric effects are obtained by introducing minerals (e.g. superfine tourmaline powder) into melted polymers before spinning or by dispersing the minerals into the spinning solution (Taekyung et al., 2020). As polymers, poly(ethyleneterephthalate), polyvinyl acetate, polyamide, viscose is widely used. Due to the growing health awareness and fitness activities, the global sports apparel market is estimated to generate about $\$ 184.6$ billion by 2020-2021, registering a CAGR of 4.3\% during the forecast period 2015-2020 (Allied Market Research). The work-related stress and lifestyle disorders generate a growing demand of fashionable sports apparel to maintain or improve the health state. When heat of about $37^{\circ} \mathrm{C}$ (normal body temperature) is applied to the far-infrared radiation, light energy in the range of 6 to $14 \mu \mathrm{m}$ causes resonance within constitutional molecules of the human body, by radiation, leading to molecular movement within the substrate, which is further converted into thermal energy, enhancing metabolism functions (Dyer, 2011). 


\section{PYROELECTRICITY}

Pyroelectricity is the ability of certain materials to generate an electrical potential when they are heated or cooled. As described by Lang, pyroelectricity is the manifestation of the temperature dependence on the spontaneous polarization of certain solids which may be either single crystals, or poly-crystalline aggregates (Lang, 1974). Pyroelectricity provides one of the best performing principles for the detection of temperature changes. Pyroelectric crystals, ceramics of ferroelectric materials, as well as polymers, have therefore been used since the 1960s in thermal infrared (IR) detectors, joining the earlier thermal IR detection techniques of bolometers and thermopiles (Muralt, 2005). FIR induces strong vibrational and rotational and effects at molecular level with the potential to be biologically beneficial, based on the ability of penetrating skin barrier and the underlying tissues (Figure 1), and generating heat by vibration of various structural components (fat molecules, subcutaneous proteins, water molecules).

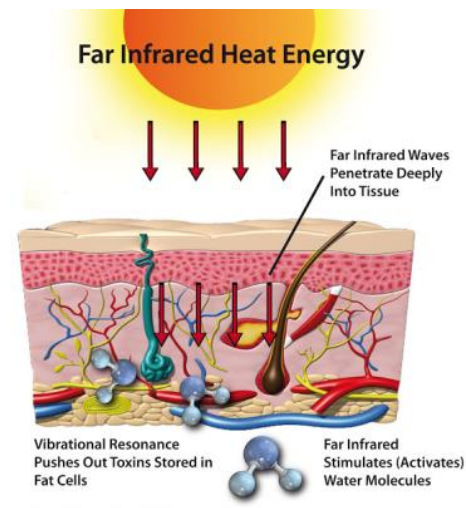

Figure 1. Representation of FIR penetration through skin (Source: www.innovationintextiles.com)

Far-infrared rays (Figure 2) increase the temperature of absorbed materials by inducing thermal energy through radiation (emission), penetration, and resonance absorption caused by the vibration of molecules among electromagnetic waves, leading to vibration of both electric and magnetic fields at the same time.

\section{ELECTROMAGNETIC SPECTRUM}
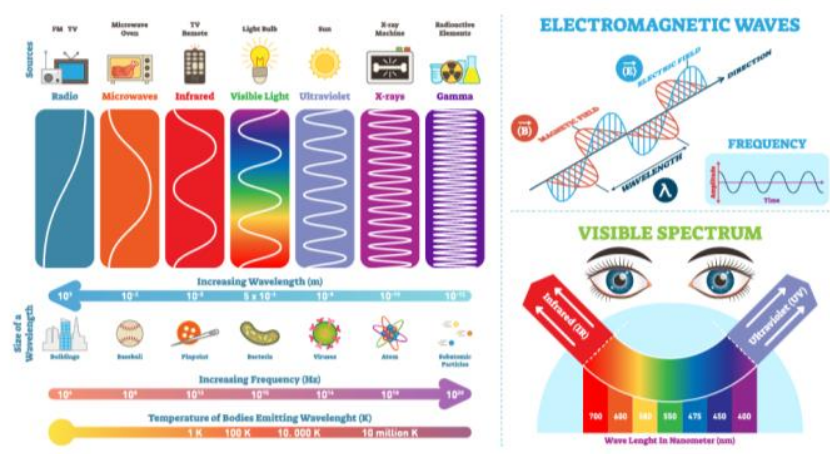

Figure 2. The electromagnetic spectrum (Source: www.muellersportsmed.com) 


\section{FAR-INRARED FUNCTIONAL MATERIALS}

Pyroelectric materials are functional materials that can generate an electrical response upon a temperature change. Modern solutions often include a combination of polypropylene and special lead-free bio-ceramics, to create special FI functional garments, which are materialized into already commercially available products, such as socks, cushion, undergarments, knee pads, trousers, bedspread, bedding and shoulder pads etc.

Functionalization materials of FIR functional products consist of a wide range of inorganic bioceramic compounds, such as: bamboo charcoal, pearl powder, tourmaline, carbide-based materials $(\mathrm{ZrC}, \mathrm{SiC})$, oxide-based materials (magnesium, zirconium, alumina, various iron oxides, germanium), photocatalytic compounds $\left(\mathrm{TiO}_{2}\right)$ (Best et al., 2008), which confer controlled infrared radiation (Figure 3).

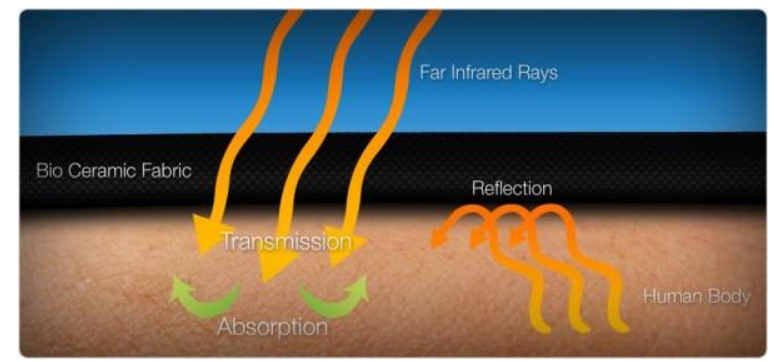

Figure 3. FIR garments functioning principle (Source: www.evidentlyhealthy.com.au)

Commercial solutions are offered by several companies, such as: Hologenix, being one of the first companies to market FIR textiles, with their Celliant products, which satisfy several criteria that regulate medical devices (Figure 4); PUMA, with its line of men's athletic apparel; French company, HT Concepts, being one of the first to create such materials, by mixing over 30 metallic oxides (from volcanic rocks) in a breathable polyurethane binder which can be coated, laminated, or printed on a fabric to reflect FIRs back to the body; titanium-mineral mix based garments, from Swiss company Schoeller; Chinese company Voll Will Enterprise, with its FIR-SKIN line of products, boasting a wide range of products, with knitted textiles infused in liquid titanium and graphite and patented bio-ceramics FI particles, such as their 3-layer edition of T+Polar product (Figure 5).
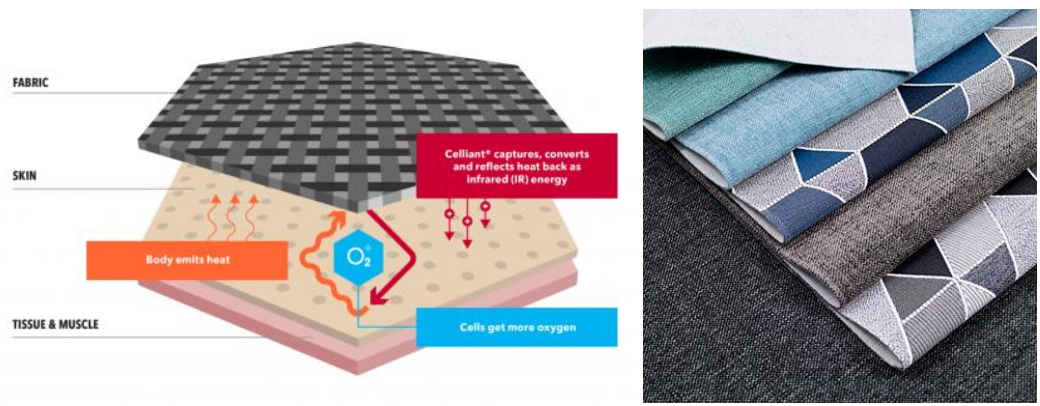

Figure 4. Celliant materials technology (by Hologenix) (Source: www.celliant.com) 
An Overview on Far-Infrared Functional Textile Materials

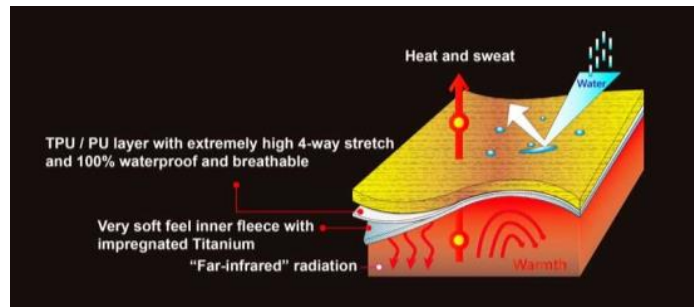

Figure 5. FIR-SKIN 3-layer edition of T+Polar (by Voll Will Enterprise) (Source: www.fir-skin.com)

Other commercially available solutions are offered by companies like: Under Armour - Athlete Recovery Sleepwear; Solvay - Emana line of products; NILIT - Nilit innergy functional materials; Toyobo - Ceram A (Figures 6-9).

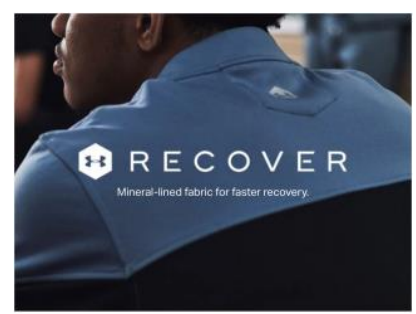

Figure 6. Athlete Recovery Sleepwear by Under Armour (Source: www.underarmour.com)

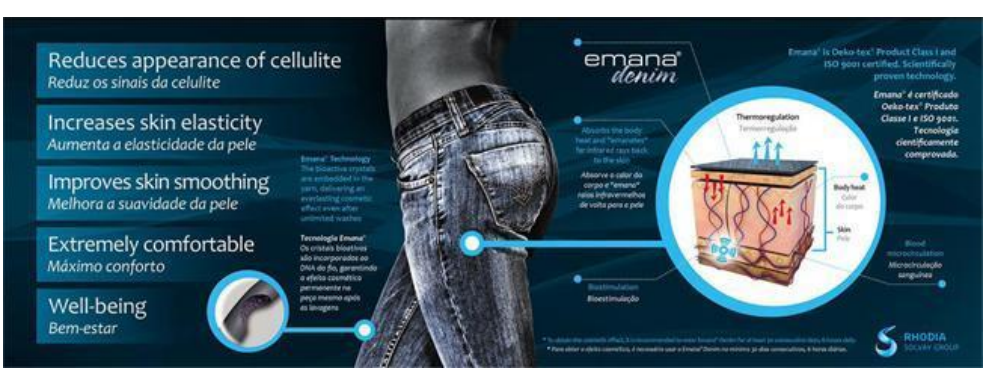

Figure 7. Emana by Solvay (Source: www.solvay.com)

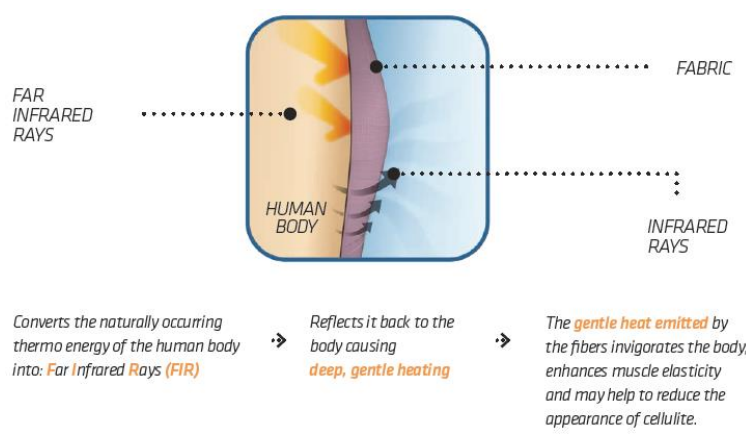

Figure 8 . Nilit innergy by Nilit (Source: www.nilit.com) 


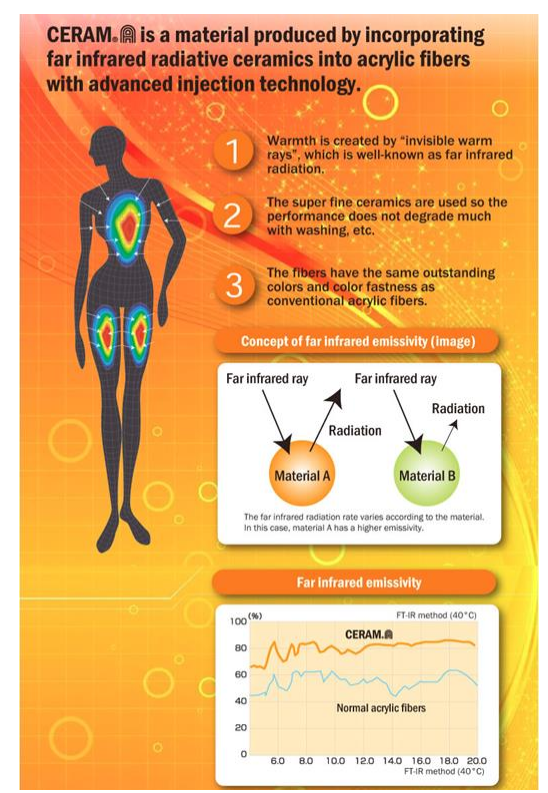

Figure 9. Ceram A by Toyobo (Source: toyobo-global.com)

The base principle for FIR functional textiles is based on two ways mechanism, once by are absorbing energy, from sunlight, and then radiate this energy back onto the body at specific wavelengths, and second, by being activated by the body's dissipated heat energy (Song et al., 2020). Thermophysiological comfort is affected by many factors, such as fiber type, yarn properties, fabric structure, finishing treatment (Sankauskaite $e t$ al., 2020). In order to obtain highly efficient functional materials, manufacturing process tends to be extremely complex, as in low quantities, the properties inducing compounds have almost no effect on wearer's health, which leads to emission of a low amount of negative ions quantity (500-2600 anion/cc), inducing a low, if any, health effect. Included in large quantities, they are too expensive (tourmaline, opal) and the fibers become harsh and fragile. Many of the used compounds have radioactive potential (monazite) (Lapidus and Doyle, 2015). Also, if the size of particles is to large (more than $0.2-0.3 \mu \mathrm{m}$ ), this will lead to obtaining of highly non-uniform fibers and early wear of mechanical parts of a textile production facility. Low cost technologies, such as polymer melt blending techniques used to incorporate minerals in nylon and poly(ethyleneterephthalate) fibers, may prove difficult to implement, due to the poor particle dispersion and weak mechanical characteristics of the fibers. Consequently, the main technical challenge is the need to efficiently blend the polymers and particles into the polymer matrices.

National R\&D Institute for Textile and Leather, INCDTP Bucharest, is running an Eureka Traditional research project, entitled "Far Infrared Rays and Anion Releasing Fabrics", acronym FairTex, consisting of six partners, from Romania and South Korea. The main objective of the project is the development of new textiles providing health and wellbeing to the users based on tailored multi-functional nanocomposites generating negative ions and far infrared rays and, protecting humans against UV-rays and microbial infections (Figure 10). 
An Overview on Far-Infrared Functional Textile Materials

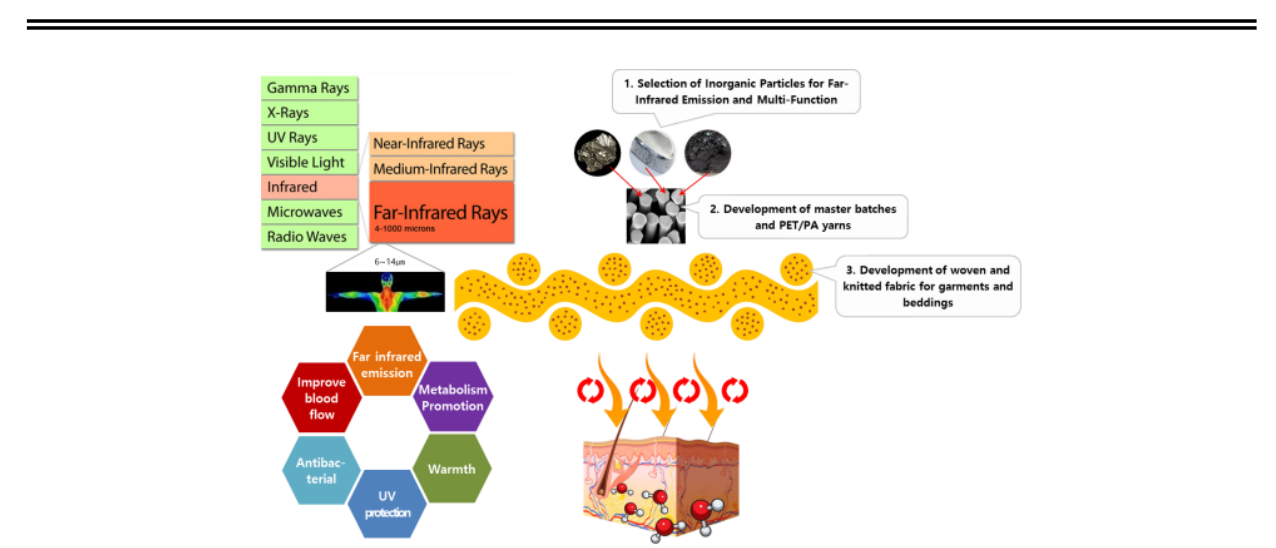

Figure 10. Overview of FairTex project

\section{CONCLUSIONS}

FIR functional textile may represent the future for alternative therapies, as they have great health benefits of FIR, ranging from regulating body heat, restoring physical function, muscle pain relieving, arthritis pain management, bronchitis etc. Even though health benefits of far infrared rays (FIR) and anion releasing fabrics are demonstrated throughout several studies, these are relatively new materials, with great potential applicability towards treatment of numerous and various health conditions.

\section{Acknowledgments}

This paper was published under Eureka Traditional program, project "Far Infrared Rays and Anion Releasing Fabrics", acronym FairTex, financed by The Executive Agency for Higher Education, Research, Development and Innovation Funding (UEFISCDI), national project ID 134E (PN-III-P3-3.5-EUK-2017-02-0044).

\section{REFERENCES}

Best, S.M., Porter, A.E., Thian, E.S. and Huang, J. (2008), "Bioceramics: Past, present and for the future", Journal of the European Ceramic Society, 28(7), 1319-1327, https://doi.org/10.1016/j.jeurceramsoc.2007.12.001.

Dyer, J. (2011), "8 - Infrared functional textiles", Functional Textiles for Improved Performance, Protection and Health, Woodhead Publishing Series in Textiles, 184-197, https://doi.org/10.1533/9780857092878.184.

Lang, S. (2011), "A 2400-year history of pyroelectricity: from Ancient Greece to exploration of the solar system”, British Ceramic Transactions, 103(2), 65-70, https://doi.org/10.1179/096797804225012765.

Lang, S.B. (1974), Ferroelectrics and Related Phenomena, Volume 2 Sourcebook of Pyroelectricity.

Lapidus, G.T. and Doyle F.M. (2015), "Selective thorium and uranium extraction from monazite: II. Approaches to enhance the removal of radioactive contaminants", Hydrometallurgy, 155, 161-167, https://doi.org/10.1016/j.hydromet.2015.03.015.

Lima, T., Jeonga, S.M., Seoa, K., Pak, J.H., Choi, Y.K. and Ju, S. (2020), "Development of fiber-based active thermal infrared camouflage textile", Applied Materials Today, 20, 100624, https://doi.org/10.1016/j.apmt.2020.100624.

Muralt, P. (2005), Encyclopedia of Condensed Matter Physics, Publisher: Amsterdam, Boston, Elsevier.

Sankauskaitė, A., Rubeziene, V., Kubilienė, D., Abraitienė, A., Baltušnikaitė-Guzaitienė, J. and Dubinskaite, K. (2020), "Investigation of Thermal Behavior of 3D PET Knits with Different Bioceramic Additives", Polymers, 12(6), 1319, https://doi.org/10.3390/polym12061319.

Song, Y.N., Li, Y., Yan, D.X., Lei, J. and Li, Z.M. (2020), "Novel passive cooling composite textile for both outdoor and indoor personal thermal management", Composites Part A: Applied Science and Manufacturing, 130, 105738, https://doi.org/10.1016/j.compositesa.2019.105738. 


\title{
STUDYING THE SIMILARITIES OF DEFORMATION PROPERTIES OF LEATHER MATERIALS IN THE PROCESS OF CREATING A MODEL OF SHOES
}

\author{
MARYNA MYKOLAIVNA LESHCHYSHYN, SVITLANA STEPANIVNA GARKAVENKO, \\ ANTONINA IVANIVNA BABICH \\ Kyiv National University of Technologies and Design, Ukraine, marfiichuk@gmail.com, \\ garkavenko.s@knutd.com.ua,basienka@ukr.net
}

\begin{abstract}
Determination of values and dependencies of deformation and physical and mechanical properties of materials of shoe models and finished products. According to the results of theoretical, analytical and marketing research, a number of experimental tests of materials have been carried out to prove the practical significance of the work, namely tests for: deformation of the vamp part of the product, uniaxial and biaxial stretching, bending, dry and wet friction, adhesion, elongation and tearing. There has been established the nature of the distribution of the total elongations of the samples of the vamps cut from different areas of the leather, as well as the ability of the leather material to be formed when improving the shape of the product or changing the shape of the shoetree. The processes of deformation of the vamp part of shoe blanks, physical and mechanical properties of different groups of modern materials and values analysis of similarity of their deformation properties have been studied. There has been created a working model-transformer for carrying out preliminary measurement of clients' feet at the individual order. The expediency of these works has been proved experimentally. A working version of a model-transformer for foot measurements has been made and as a result of the works approbation, a sample of shoes has been made. The ergonomic properties of the manufactured footwear have been improved due to the use of materials with enhanced physical and mechanical properties. The article investigates the deformation of the most vulnerable vamp part of the men's model of a typical model, as well as the physical and mechanical characteristics of leather materials for manufacturing models and shoes of this type. Providing high quality and comfort of footwear, accuracy of parameters selection of foot measurement, zones of beams and achievement of form stability of footwear with a top from genuine leathers has been predicted.
\end{abstract}

Key words: deformation, model-transformer, footwear.

\section{INTRODUCTION}

Shoe production has always been an attractive business in steady demand. This has traditionally been the work of shoemakers since ancient times, who made products by hand without the use of equipment. The production of fashion industry custom-made shoes is getting more and more popular every year and has its own segment of consumers.

In the current climate, the topical issue is the production of exclusive individual custom-made shoes that emphasize the customer status, his character and preferences.

This paper analyzes modern materials, consumer choice factors influencing the formation of the innovations range in the production of custom-made shoes, stages of improvement of technological units of foot measurement processes, stages of layout and manufacture of shoes, as well as experimental testing of materials to determine values and dependencies of deformation and physical and mechanical properties of materials of the footwear working model and the finished product, improvement of a foot measurement stage and improvement of functional and operational characteristics of a product with use of modern methods and means of production (Izovit and Naumenko, 2015).

https://doi.org/10.24264/icams-2020.I.10 


\section{SETTING OBJECTIVES}

The current task of modern small private shoe companies specializing in the production of custom-made shoes is to study the preparatory and basic processes of shoe production, namely: measuring the feet and modeling the product model, shaping the product while tightening the sock-bundle of shoes on the shoetree, etc. These studies make it possible to investigate and predict providing high quality, comfort and dimensional stability of shoes with uppers of modern classic and non-typical (python, crocodile) natural leather materials both in the design and manufacture and operation of the product, as well as after repairs and renovation or improving the product design.

Creating a comfortable and convenient shape of the product is one of the main stages of shoe production, the quality and careful implementation of which depends not only on the shape stability and comfort of shoes at the stage of operation, but also the product appearance.

At the initial stage of shoe manufacturing in the process of measuring the feet, the main length, latitude and girth parameters of the foot are selected and set. According to this the design is modeled, a model and then the product is made.

Deformation properties of materials are the most important ones, which largely determine the quality of the main technological operations of footwear production, and on which the product comfort and the preservation of its shape during operation depends.

These studies make it possible to assume and predict the possibility of using for the model manufacture as the main material of the upper shoes and a cheaper segment of materials with similar deformation and physical and mechanical properties to ensure high comfort and stability of shoes made of genuine leather materials when wearing the products.

The works of modern scientists in this direction (Kasyan, 2001; Kozar et al., 2013; Danilkovich et al., 2013; Andreeva et al., 2018; Kozar et al., 2013; Mokrousova and Okhmat, 2013; DSTU 4239-2003; EN ISO 20344: 2004; EN ISO 20345: 2004) suggest the successful use of the same basic high-value leather materials for shoe uppers in the design and manufacture of the product without losing the quality and comfort of the latter. Though there are no studies to confirm the hypothesis in using a cheap segment of materials for the manufacture of the layout, the quality and comfort of the finished product will be high. Therefore, this hypothesis must be tested and confirmed experimentally, because the change in the material type and its properties during the layout of the product and its production can significantly affect the comfort of the finished product and its appearance. These processes have been insufficiently studied, which indicates the relevance of this study.

\section{RESEARCH RESULTS}

In the process of researching and studying the customer base of enterprises working on individual orders, it has been found out that the products price is not the predominant indicator of importance for consumers, but the appearance, comfort and quality of materials are predominant. Therefore, the issues of creating comfortable shoes for manufacturing products must be addressed more deeply at the stage of measuring the feet and layout. To study the issues of improving the stages of measurement, layout and manufacturing the products, its reduction without losing quality and comfort. 
Based on the results of theoretical and analytical, marketing and experimental research, a model-transformer of shoes for preliminary foot measurement was designed (Fig. 1, a) and prototypes of the product model (Fig. 1, b) were made, which were also partially subjected to experimental tests. mechanical properties according to certain importance factors.

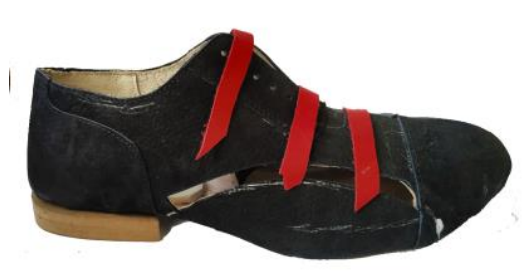

a)

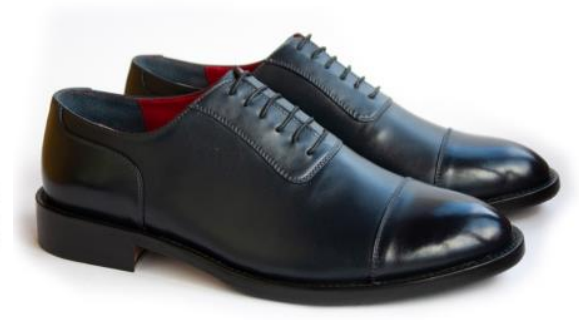

b)

Figure 1. a) Model-transformer shoes for preliminary foot measurement; b) Prototype of a model of manufactured shoes

To study the quality of leather for the shoe upper, 4 samples of genuine leather from different manufacturers have been selected. In general, the organoleptic characteristics of the leather for the shoe uppers are presented in Table 1.

Table 1. Organoleptic characteristics of leather for shoe uppers

\begin{tabular}{ccccc}
\hline Indicator & \multicolumn{4}{c}{ Leather sample } \\
& 1 & 2 & 3 & 4 \\
\hline Name & cattle & crocodile & python & ostrich \\
Code number & 350-P15- 5505 & $650-\mathrm{P}-154352$ & $100-\mathrm{P}-110601$ & 200-P-151215 \\
Colour & bordeaux & blue & white & black \\
Lace & Natural lace & Clearly defined & Natural & Natural clear \\
condition & condition & segments of lace & distinct scales & holes \\
\hline
\end{tabular}

To assess the leather quality according to physical and mechanical properties there has been determined the tensile strength of the material, the relative elongation at a strain of $10 \mathrm{MPa}$, the resistance of the coating to repeated bending, the adhesion of the coating film to wet and dry skin. Samples of the materials described above have been used to evaluate the deformation properties of the materials. According to the methods described in DSTU (DSTU 4239-2003; EN ISO 20344: 2004; EN ISO 20345: 2004) for the relevant tests three samples cut from different leather areas have been taken, they mimicked the vamp ( 3 options). One vamp is cut from the cheprak part in the longitudinal direction, the second - from the cheprak part in the transverse direction, the third is cut from the extreme part of the leather cheprak part in the longitudinal direction. There are totally 15 samples (EN ISO 20345: 2004).

Therefore, the samples selected for research have been evaluated for quality in terms of physical, mechanical and deformation properties. The results of the research are presented in Table. 2 As it can be seen from the data presented in the above tables, the studied leather samples in terms of indicators fully meet the standard requirements for leather for shoe uppers (DSTU 4239-2003; EN ISO 20344: 2004). 
Studying the Similarities of Deformation Properties of Leather Materials in the Process of Creating a Model of Shoes

Table 2. Physico-mechanical tests of skins

\begin{tabular}{lccc}
\hline Sample & $\begin{array}{c}\text { Tensile strength, } \\
\times 9.8 \mathrm{MPa}\end{array}$ & $\begin{array}{c}\text { Relative elongation, with } \\
\text { a load of 9.8 MPa, \% }\end{array}$ & $\begin{array}{c}\text { Elongation at } \\
\text { breaking, \% }\end{array}$ \\
\hline 1 & 1,7 & 54,0 & 80,0 \\
2 & 1,5 & 35,0 & 65,0 \\
3 & 1,6 & 35,0 & 58,0 \\
4 & 1,8 & 24,0 & 59,0 \\
Standard & 1,5 & $20,0-40,0$ & - \\
requirements & & & \\
\hline
\end{tabular}

Therefore, it should be noted that all samples of genuine leather for the shoe uppers according to the indicators of chemical composition, physical and mechanical properties fully meet the requirements of regulatory documents and are safe for human feet.

The second stage of the research was studying the properties of products in order to verify and confirm the similarity of the deformation properties of materials.

For the first type of research, 270-size vamps were selected, designed on a shoetree of style 9112 of medium fullness.

In the process of forming, the vamps on the shoetree are subjected to forced stretching simultaneously in several directions, i.e. there is a multi-axis deformation.

According to the method (Kozar et al., 2013), a grid of lines is applied on the flesh side of the detail imitating the vamp, this grid forms squares of size $20 \times 20 \mathrm{~mm}$. Transverse lines are drawn perpendicular to the inflection line of the vamp and numbered in Arabic numerals, longitudinal lines are numbered in Roman numerals on the inside and outside of the vamp.

In the process of forming, the vamps on the shoetree are subjected to forced stretching simultaneously in several directions, i.e. there is a multi-axis deformation.

Tightening by machine is performed manually. At manual tightening the operation is carried out on nails by means of pincers and a hammer. And it is necessary to consider the fact that the machine movements are identical both on the effort applied to preparation and on frequency of movement reproducibility.

In the process of forming, the vamps on the shoetree are subjected to forced stretching simultaneously in several directions, i.e. there is a multi-axis deformation.

At manual tightening everything is on the contrary. The master cannot reproduce his movements with the same effort and frequency physically, so each movement is different and each tightened area of the workpiece has a different amount of material stretching, but we are interested in this option.

After tightening and performing various groups of forming operations, the samples are kept for some time on the shoetree and then removed. Measurements of material deformation along the total length of lines and sides of squares have been performed during the study 3 times with a caliper under a microscope with an accuracy of $0.1 \mathrm{~mm}$.

The values of measuring the size of each square of the six samples of the first, second and third options after deformation have been averaged and taken into account changes in deviations of $\pm 2.5 \%$ or more from the original size.

The reliability of the experimental research results has been assessed by traditional methods of mathematical statistics. The standard deviation $\sigma_{\mathrm{B}}$, the coefficient of variation $v$ and the parameters reflecting the proximity of the research results is the accuracy of the test $\delta$ were determined (Danilkovich et al., 2013).

The results of tests of experimental leather and samples indicate the ability of the material to forming and repeated deformation (Fig. 2).

https://doi.org/10.24264/icams-2020.I.10 
When forming the workpiece of the shoe upper, the leather structure can change significantly as a result of tensile and flexural deformations: the bundles of fibers are able to navigate under the action of tensile loads and bend elastically.

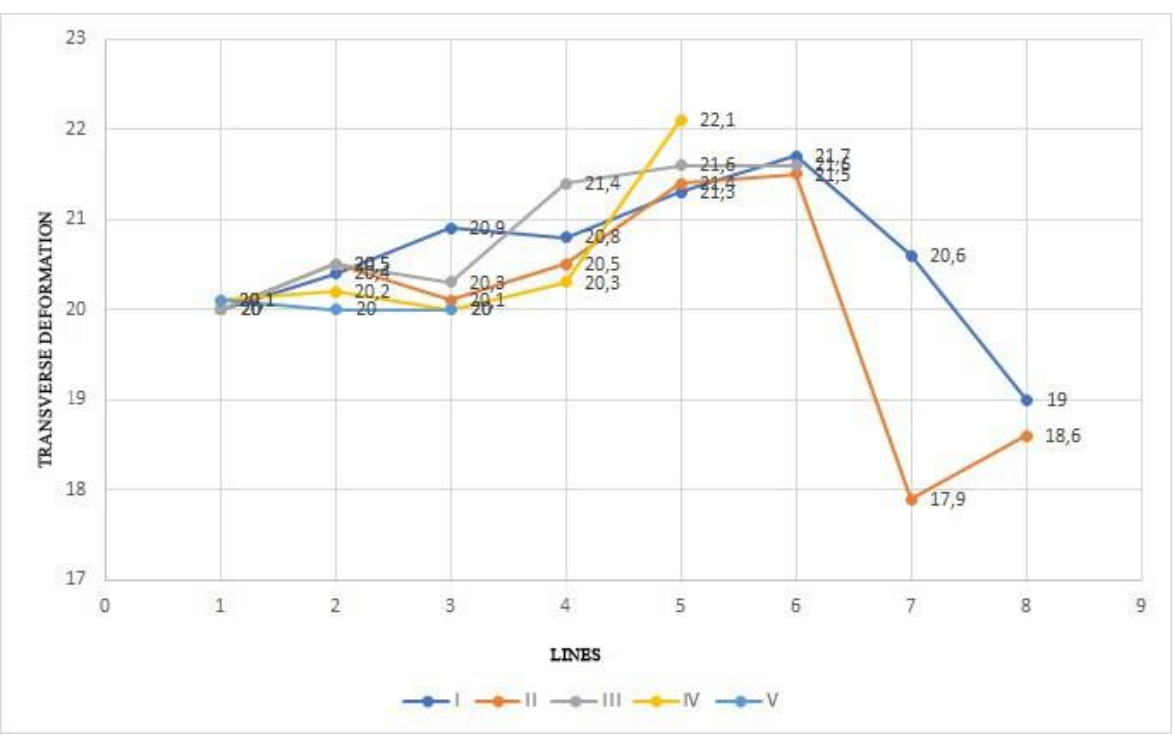

Figure 2. The results of transverse deformation

\section{CONCLUSIONS}

Genuine leather has a wide range of applications. Most of it is aimed at making leather for the shoe uppers`. Therefore, quality control of genuine leather is a very important factor. Since a person spends a long time in shoes, it is important to feel comfortable and convenient. Given the relevance and feasibility of the purpose of this study, to assess the quality, samples of selected leather of exotic animals of foreign production have been chosen.

The processes of deformation of the vamp part of shoetrees, physical and mechanical properties of different groups of modern materials and analysis of the values of similarity of their deformation properties have been investigated.

The working model-transformer for carrying out preliminary foot measurement of customers at the individual order has been created, and as a result of approbation of the works, the sample of footwear has been made.

The expediency of these works has been proved experimentally. The ergonomic properties of the manufactured footwear have been improved due to the use of materials with enhanced physical and mechanical properties.

Providing high quality and comfort of footwear, accuracy of selection of parameters of foot measurement, zones of beams and achievement of form stability of footwear with a top from genuine leather at formation on shoetrees of various styles and forms has been predicted. 
Studying the Similarities of Deformation Properties of Leather Materials in the Process of Creating a Model of Shoes

\section{REFERENCES}

Andreeva, O.A., Hryshchenko, I.M. and Zvarych, I.T. (2018), Features of leather and fur raw materials: Svit uspichy, Ukraine, p. 451.

Danilkovich, A.G., Hryshchenko, I.M. and Lishchuk, V.I. (2013), Innovative technologies for the production of leather and fur materials and products: a monograph, p. 344.

Izovit, T. and Naumenko I. (2015), “Trends in Ukrainian leather production market”, Light industry, № 4. p $2-4$

Kasyan, E.E. (2001), Fundamentals of leather and fur technology: a textbook.

Kozar, O.P., Mokrousova, O.R. and Konoval, V.P. (2013), "Evaluation of relaxation and deformation characteristics of leather for shoe uppers filled with natural minerals", Bulletin of the Kyiv National University of Technologies and Design - №4. - P. 107-115.

Kozar, O.P., Mokrousova, O.R. and Victor, M.T. (2013), "Evaluation of the stability of leather modified with organic mineral compositions", Scientific notes. - Lutsk: - Issue. 41. - P. 135-137.

Mokrousova, O.R. and Okhmat, E.A., (2013), "Formation of operational properties of leather using montmorillonite", Materials IX Int. Scientific-Practical Conference "Leather and fur in the XXI century: technology, quality, ecology, education", Ulan-Ude, Russia, Publishing house VSGUTU, - P. 83-92.

*** (2003), Derzhspozhivstandard of Ukraine (Effective from 01-10-2004), Textile and leather materials and household products. Basic hygienic requirements: DSTU 4239-2003.

*** (2009), Derzhspozhivstandard of Ukraine (Effective from 2012-01-01), Personal protective equipment. Professional shoes. Shoe test methods (EN ISO 20344: 2004 / AC: 2005 / A1: 2007, IDT): DSTU EN ISO 20344: 2009.

*** (2012), Derzhspozhivstandard of Ukraine, Personal protective equipment. Household shoes. Technical conditions (EN ISO 20345: 2004 / AC: 2007 / A1: 2007, IDT): DSTU EN ISO 20345: 2009. 


\title{
TRANSFORMATION OF ART OBJECTS IN THE 3D DESIGN PROCESS OF
} SHOE PARTS

\author{
MARYNA LESHCHYSHYN, SVITLANA GARKAVENKO, VICTORIA KERNESH, \\ BORYS ZLOTENKO, TETIANA KULIK, ANASTASIA GLIONIK \\ Kyiv National University of Technology and Design, Ukraine, marfiichuk@gmail.com, \\ garkavenko.s@knutd.com.ua,kievfoot@ukr.net, zlotenko.bm@knutd.com.ua, \\ kulik.ti@knutd.com.ua,gloynik.nastia@gmail.com
}

\begin{abstract}
The analysis and classification of shaping methods in the design of shoe bottom parts, and the investigation of research methods of automation of the design process of shaping shoe bottom parts to increase the efficiency of the design process and reduce the complexity of manufacturing products at the stages of technological preparation. Based on the use of means of transformation and harmonization, the structure of the stages of shaping of art objects to obtain modern shapes was developed, which made it possible to implement a new approach to the artistic design of footwear. On the basis of the developed approaches to the choice of shapes and shaping methods sketches were created and 3D models of shoe bottom parts with the subsequent program analysis of loading on a sole were developed. New shapes shoe bottom parts have been developed with the help of transformation principles and a model has been made by automated design methods, which can be used in technological preparation for the manufacture of light industry products. Regularities of transformation processes for improvement of artistic design of footwear on the principles of bionics and stylization are established. Given that the complexity of structures and the emergence of new spatial solutions pose designers more complex technical tasks for the implementation of design projects, a method was proposed of calculating the strength and stability of products of complex geometric shapes, taking into account the materials from which they are made. As a result of the performed work, women's shoes of the "shuttle" design with an over seam back allowance were developed. Given the fact that the shoes should be comfortable and meet ergonomic indicators, the materials, the shape of the pad, and the design of the model were selected. The purpose of this shoe is everyday wearing, focused on the youth category of consumers. The used technique allows shaping a wide range of various prototypes of footwear, using a small number of transformed products. Determining the most loaded areas made it possible to propose measures to improve the design of shoes of non-standard models. And the combination of the given techniques with modern high-tech production helps to save means of the manufacturer and to increase the service life of footwear.
\end{abstract}

Key words: footwear, shaping, transformation.

\section{INTRODUCTION}

At all times, footwear was a necessity, a means of protecting the human foot from the adverse effects of the environment. During thousands of years of its existence, shoe production has undergone significant changes: from hand-made by individual craftsmen to mass production of large batches of the same type of shoes in large factories.

One of the key factors in shaping the modern footwear market is the impact of fashion trends that are changing very fast, especially for women's shoes. The assortment of women's shoes has the tendency to constantly change and update compared to men's. The main factors for this are the change of the season, fashion trends, the emergence of new materials and improvements in production technologies, as well as the constant need of women to change the assortment depending on the psychological feeling. Therefore, women usually do more purchases in the season than men (https://buklib.net/books/28806).

The trends of modern fashion popularize footwear models of complex shapes, which requires the use of non-traditional methods and innovative design technologies. Properly organized three-dimensional structure and a pronounced transformation of the shape of the product create the preconditions for the integrity and harmony of the shape of the shoe. 


\section{SETTING OBJECTIVES}

Design today is advancing within the framework of global large-scale trends that correct everyday concepts. This is expressed in the methods of non-standard shaping, as well as the introduction of new technological materials in combination with color palettes, the result of which guarantees the achievement of artistic expressiveness of the shape of products. Shoes, as a three-dimensional structure, are a complex system of parts that are placed in a specific compositional relationship.

Despite the variety of existing solutions in the field of shaping, today there is a need for research and search for new promising developments in shoe design. Today, 3D technologies are gaining wide popularity in various industries, and their use in the design and manufacture of footwear will be promising. The latest solutions allow improving the transmission of information and design of shoe parts using CAD software, simplifying the process of models designing and reducing the complexity of production.

\section{RESEARCH RESULTS}

Among the main methods of shaping are: combinatorial shaping, transformation, stylization, the method of shaping the form based on a natural analogue (bionics), color shaping (Ustin, 2007; Zaeva-Burdonskaya and Kurasov, 2008).

The richest source of inspiration for the designer is wildlife, which is the basis for finding a decorative combinatorial element. When appealing to nature, the search for a harmonious interaction of content (biological function of the organism), form (the way of existence of the organism) and material (of which the organism itself is composed) is assumed. The shape of a unified element creates a certain image and character of an object. A more complex process of product shaping is the combination of finished objects (Detkina and Fukin, 2010).

When designing the shape, the craftsmen first started from natural forms. Later, in the process of studying the environment, man began to establish a generalized thinking, which contributed to the development of new shapes, based on the properties of materials and their purpose.

Today, any object, even a building, can be a source of inspiration for designers. The basis for the transformation and creation of sketches of shoes was chosen architectural structure, namely the medical center Enter Architecture (https://novate.ru/blogs/ 060311/17011). The Australian design studio "Louns" has completed the construction of a modern medical and expressive complex "John Curtin school of medical research", the concept of which reflects the progressive methods of work and the desire to improve and develop technology (Fig. 1). Based on the art object, a design based on the principles of visual transformations, which are important for structuring the artistic qualities of the transformative properties of shoes, was proposed (Fig. 2).

An example of an artistic search for the shaping of the bottom of shoes on the basis of various architectural structures is shown in Fig. 3.

The complex shape of shoe parts requires a responsible approach to ensuring their performance. It is well known that the shoe in the process of wearing receives various loads that cause different types of deformation of its parts. Within the specified limits, these deformations are useful and necessary for the comfortable well-being of the shoe owner. With a rigid shoe design, a feeling of discomfort and fatigue quickly occurs. And vice versa - flexible and elastic design helps to maintain comfort for a long time. However, too flexible shoes also quickly cause fatigue, and insufficient fixation of the foot can cause injury. In addition, in the event of significant deformations, stresses may occur in the materials of shoe parts that exceed their strength and cause the destruction of these parts. Therefore, at the stage of designing shoes, their strength calculation is very important. 

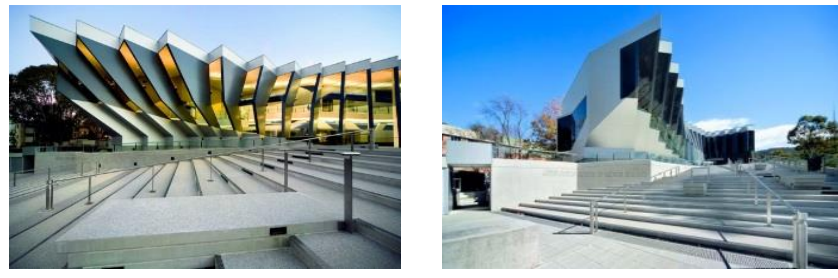

Figure 1. Art object Enter Architecture
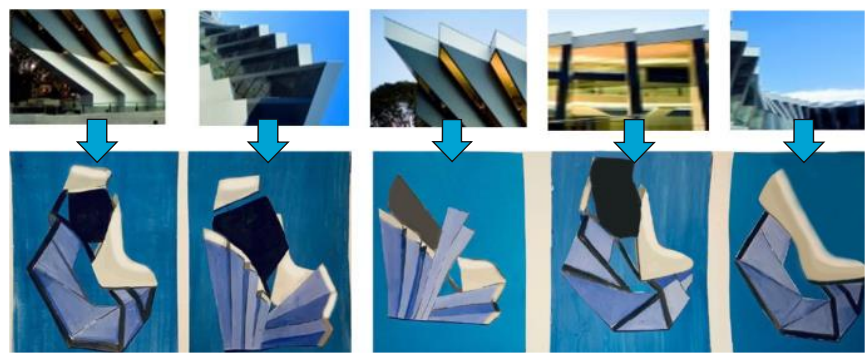

Figure 2. Elements of transformation that were used in modeling of shoes
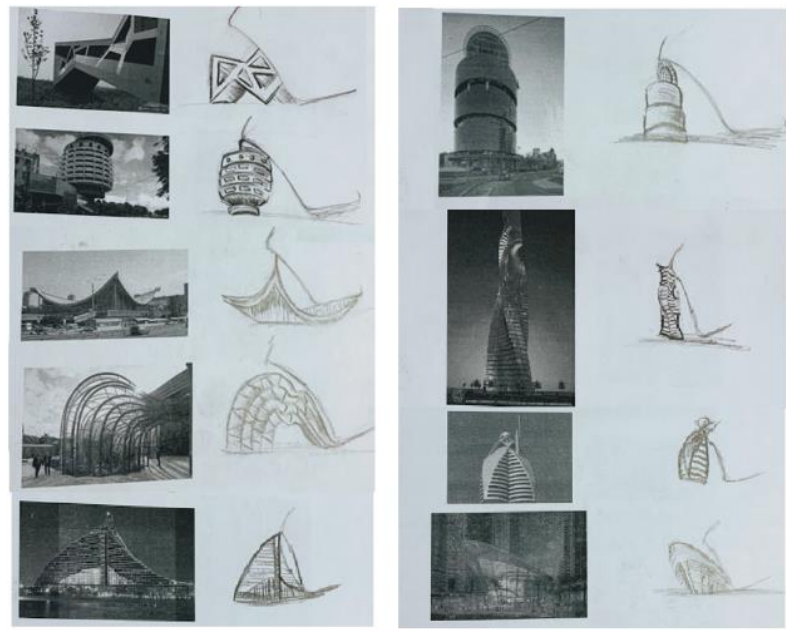

Figure 3. Transformation of art objects into shoes construction

Typical types of deformation of parts traditionally include: bending, shear, torsion, tension and compression.

The relationship between strain and normal stresses in a material is established by Hooke's law (Pisarenko et al., 2004). In the general case, this dependence has the form: $\sigma=E \varepsilon$,

where $\sigma$ - is the stress; $\varepsilon$ - is the relative deformation; $E$ - is the modulus of elasticity of the material (Young's modulus).

The calculation of polymer parts of shoes is complicated by the fact that they are not elastic bodies, and for them cannot be applied with high accuracy classical methods of resistance of materials and the theory of elasticity (Tager, 2007). For such elements, you 
can use the function of the relationship between deformation and normal stresses in polymeric materials in the form (Lebedev, 1991):

$\sigma^{m}=E \varepsilon$,

where $m$ - is the exponent, which varies from 0.6 to 1 depending on the type of material (at $m=1$, the body exhibits elastic properties, which is characteristic of metals).

In the process of designing shoe parts, you can start from the conditions of providing strength or providing the necessary rigidity. Each element can be represented as a simple part - a rod, a plate or a tube. This will make it possible to use known engineering methods of calculation taking into account the properties of the materials from which these elements of footwear are made. For example, in Kulik, 2017a, we obtained expressions for the calculation of cantilevered elements of the polymer shoe bottom under the action of a distributed load (Fig. 4, a), and Kulik, 2017b presented a method for calculating the polymer elements of the rod shape during torsion (Fig. 4, b).

The required strength and rigidity of the part can be ensured by choosing its rational geometric parameters, because the physical and mechanical properties of the already selected material cannot be influenced. Such parameters can be the thickness $h$ or radius $r$ of the part.

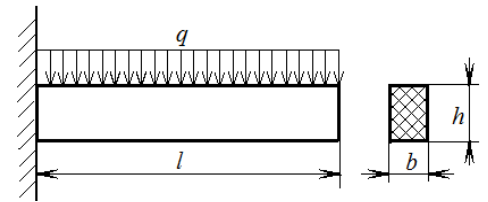

a)

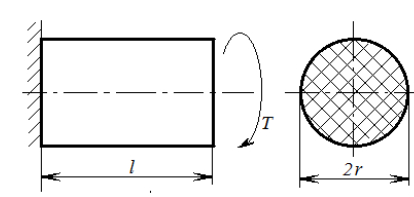

b)

Figure 4. Calculation schemes for the study of the stress-strain state of cantilevered polymer elements of footwear: $a$ - under the action of a uniform load; $b$ - when twisting

When cantilever bending of a part of rectangular cross section $b \times h$, loaded with distributed force (see Fig. 4, a), the required strength of the part is provided by the thickness determined from the expression:

$h=\sqrt{q \cdot l^{2} \cdot(1 / m+2) /(b \cdot[\sigma])} \cdot$

The condition of rigidity will be:

$$
h=2 \cdot \sqrt{\frac{\frac{1}{m}+z}{2^{m+1} \cdot m \cdot b \cdot E \cdot\left[\theta_{\max }\right]}}
$$

or

$$
h=2 \cdot \sqrt[\frac{1}{m}+2]{\frac{q^{m} \cdot l^{2 m+n} \cdot\left(\frac{1}{m}+2\right)}{2^{m+2} \cdot(m+1) \cdot b \cdot E \cdot\left[y_{\max }\right]}} .
$$

In the given expressions: $b, h, l$ - respectively width, thickness and length of the part; $q$ - is the magnitude of the load; $m$ - is the exponent that characterizes the elastic properties of the polymeric material; $[\sigma]$ - the strength of the material of the part; $[\theta]-$ angle of rotation; $[y]$ - deflection.

For the part of the rod shape shown in the diagram of Fig. 4, b, the minimum radius is determined at which the appropriate strength will be provided:

$$
r=\sqrt[3]{(T /[\tau]) \cdot(1 / m+3)}
$$

or required rigidity: 
$r=\frac{1}{m}+3 \sqrt{\frac{T \cdot l^{1 / m} \cdot(1 / m+3)}{\sqrt[m]{G \cdot[\varphi]}}}$.

In the given expressions: $r, l$ - radius and length of the rod element; $T$ - torque; $m$ - is the exponent that characterizes the elastic properties of the polymeric material; $[\tau]-$ allowable tangential stresses for the material; $[\varphi]$ - allowable angle of rotation; $G$ - is the shear modulus. To date developed a large number of different software that simplifies the process of designing shoes, to receive materials cutting schemes, to investigate manufacturing processes, to model behavior of footwear parts of various loadings.

In this study, the software product Rhinoceros 3D was used for automated design of shoe uppers and bottoms. In fig. 5 a sketch of the developed shoes is shown.

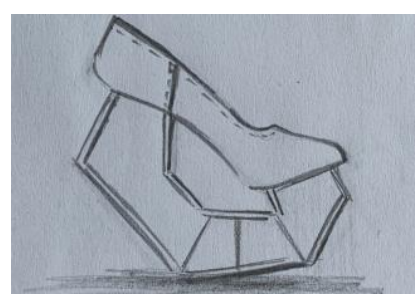

Figure 5. Sketch of shoes

SolidWorks software was used to analyze the loads that will occur in the elements of the shoe while wearing it. Fig. 6 illustrates the uneven distribution of pressure on the surface of the sole, taking into account the angle of the foot. Fig. 7 presents the analysis of stresses and strains in the sole of the shoe during operation.

As a result of modeling, it was found that the weakest part of the structure is its middle section. It is this area that receives the greatest deformation loads. To strengthen it, it was proposed to use an additional element of reinforcement, which is shown in Fig. 8. The finished product obtained by the developed project is presented in Fig. 9.

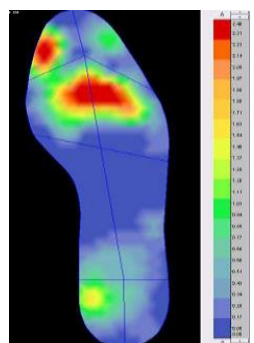

Figure 6. Weight distribution on the insole of shoes

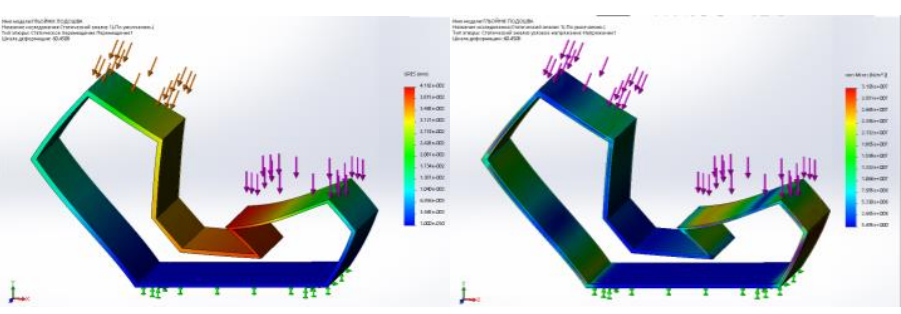

a)

Figure 7. Distribution of stresses $(a)$ and deformations $(b)$ arising in the sole of the shoe under the action of operating loads

A feature of the bottom of the developed shoes is the presence of a platform: the design of the classic platform has been transformed into a geometric object that has not only a functional purpose, but also acts as a decor. The model is based on contrasting colors that give the shoes a more aesthetic, brighter look. All details of the top are made of natural materials. The insole assembly consists of a main insole, a half insole and a metal insole; padding, which fills the space between the contours of the lasting edge of the lasted shoes. The method of forming the workpiece is external lasting and force lasting. The platform is made by a method of stamping from the rolled metal tape $2 \mathrm{~mm}$ thick (ST-3 steel). 


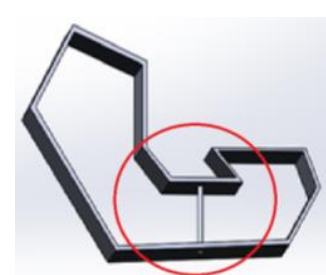

Figure 8. The element of structural reinforcement

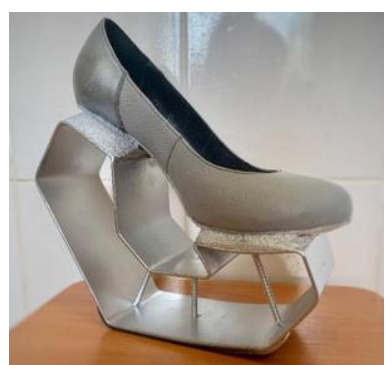

Figure 9. The made specimen

\section{CONCLUSIONS}

The study considered the methods of visual transformations that are important for structuring the artistic qualities of shoes. The used technique allows shaping a wide range of various prototypes of footwear, using a small number of transformed products. And the combination of the given techniques with modern high-tech production helps to save means of the manufacturer and to increase the service life of footwear.

Given that the complexity of structures and the emergence of new three-dimensional solutions pose more complex technical challenges for designers to implement design projects, the possibility of calculating the strength and dimensional stability of products of complex geometric shapes was analyzed taking into account the characteristics of the materials from which they are made.

Possibilities of the modern software for designing of footwear and modeling of its behavior in the course of operation are considered. Determining the most loaded areas made it possible to propose measures to improve the design without the manufacture and study of an experimental sample, which is of great importance in the manufacture of shoes of non-standard models.

As a result of the performed work, women's shoes of the "shuttle" design with an over seam back allowance were developed. Given the fact that the shoes should be comfortable and meet ergonomic indicators, the materials, the shape of the pad, and the design of the model were selected. The purpose of this shoe is everyday wearing, focused on the youth category of consumers.

\section{REFERENCES}

Detkina, D.N. and Fukin, V.A. (2010), The use of illusions of visual perception in the artistic modeling of footwear, IIC MGUDT, Moscow.

Kulik, T.I. (2017a), "Investigation of the stress-strain state of cantilevered elements of the polymer bottom of shoes under the action of distributed load", Bulletin of the Kyiv National University of Technology and Design, Technical Sciences Series, № 6 (116), pp. 66-73.

Kulik, T.I. (2017b), "Method of calculating the rod elements of the bottom of the shoe during torsion", Bulletin of Kherson National Technical University, № 1, pp. 130-135.

Lebedev, V.S. (1991), Technological processes of machines and apparatus in the production of consumer services, Legprombytizdat, Moscow.

Pisarenko, G.S., Kvitka, O.L. and Umansky, E.S. (2004), Resistance of Materials, Higher School, Kyiv.

Tager, A.A. (2007), Physical chemistry of polymers, Scientific world, Moscow.

Ustin, V.B. (2007), Composition in design, AST: Astrel, Moscow.

Zaeva-Burdonskaya, E.A. and Kurasov, S.V. (2008), Shaping in environmental design. Stylization method, MGHPU: SG Stroganov, Moscow.

*** Assortment of leather shoes, https://buklib.net/books/28806/.

*** Expressive architecture of the Australian Medical Center, https://novate.ru/blogs/060311/17011/. 
DIGITAL CONSTRUCTION OF THE SIGNALING/ RESCUE SYSTEM LOCATED IN COASTAL AQUATIC AREAS

\author{
CARMEN MIHAI ${ }^{1}$, MADALINA ALICE RUS ${ }^{2}$, ALEXANDRA GABRIELA ENE ${ }^{1}$, \\ RAZVAN SCARLAT ${ }^{1}$ \\ ${ }^{1}$ The R\&D National Institute for Textiles and Leather, 16, Patrascanu Str, sector 3, Bucharest, \\ Romania,carmen.mihai@incdtp.ro \\ 2 "Dunarea de Jos" University of Galati, Faculty of Engineering, 47, Domneasca Str, Galati, \\ Romania,mrus@ugal.ro
}

\begin{abstract}
For signaling and rescue, at international level, modular systems made of composite structures are used, which ensure the maintaining at the water surface of them, in a fixed point, in any meteorological conditions etc. According to the diversity of activities carried out in the marine and/ or river sector and depending on them, worldwide signaling systems are applied: for the safety of navigation, for data recording and processing activities and for the marking of ecological protection areas (National Strategy for Sustainable Development of Romania 2030, 2017; La Depeche, 2014). For this last category, the signaling of the navigable channel is made in accordance with the "Basic Regulations for Navigation on the Danube" edited by the Danube Commission, 1991 edition, Budapest, while for the maritime buoyage system the IALA agreement is used, concluded in Paris, on the 15th April 1982 and involves the use of floats and buoys made of composite materials. The work aims to create a digital signaling/ rescue system applied in the coastal aquatic area (Council Regulation (EC) No. 509/2006; Decision No. 1600/2002/EC). In this respect, using FEM modelling, the geometric domain was defined, the composite structure of which the system is made was modeled, the structure with finite elements was generated (discretization, properties modelling, specific finite elements obtaining), the constraints and loads were modeled and finally, in the post-processing stage, the results (deformation, Von Mises stress, displacements) were visualized and studied.
\end{abstract}

Keywords: modelling, FEM, numerical analysis, digital construction.

\title{
INTRODUCTION
}

The worldwide signaling systems are applied as follows:

i) For the safety of navigation, in order to carry out the vessel traffic in safe conditions, by marking the passage corridors to avoid the areas with reefs or rocks (Multiannual National Strategic Plan on Aquaculture 2014-2020).

ii) For data recording and processing activities, in order to record the parameters that characterize the marine environment, namely: data on speed and direction of surface and depth currents, wind speed and direction, water and air temperature etc. (EC Communication, 2018).

iii) For the marking of ecological protection areas, in order to protect the areas considered ecological reserves; tourist and leisure activities, within the beaches and marinas; marking the risk areas: (deep waters with rocky bottom and strong currents) and for marking the passage corridors of the boats based on the beaches (EC Communication, 2006).

For this last category, the signaling of the navigable channel is made in accordance with the "Basic Regulations for Navigation on the Danube" edited by the Danube Commission, 1991 edition, Budapest, while for the maritime buoyage system the IALA agreement is used, concluded in Paris, on the 15th April 1982 and involves the use of floats and buoys made of composite materials (EEA Report No 8/2012). 


\section{MATERIALS AND METHODS}

For modelling the geometric domain of the problem, the sketcher module was used.

The dimensional constraints for the Part Design module used in order to obtain the 3D image were: height: $600 \mathrm{~mm}$; base diameter: $1000 \mathrm{~mm}$. Figure 1 sequentially visualizes the dimensioning of the ME, made using Part Design (Shaft).
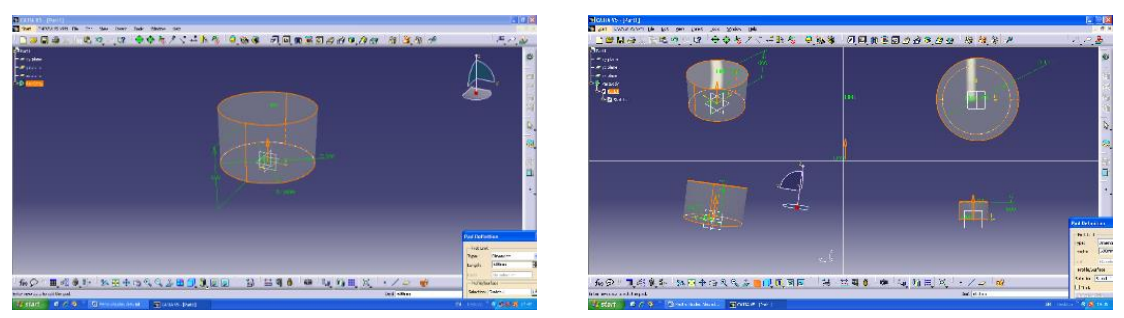

Figure 1. Sequential configuration made with Part Design

After checking the model and discretizing the created geometry, the calculation conditions were identified, the values corresponding to for 4 bf and 8 bf were identified, respectively (Table 1 ).

It should be noted that, in the situation where the system will be located in running water, hydrometeorological conditions (necessary for calculation) cannot be more difficult than those in the open sea, so the stress to which the system was subjected were the most difficult.

Table 1 . The values corresponding for 4 bf and $8 \mathrm{bf}$

\begin{tabular}{lll}
\hline Beaufort Scale & \multicolumn{1}{c}{$4 \mathrm{bf}$} & \multicolumn{1}{c}{$8 \mathrm{bf}$} \\
\hline Wind speed & $11-15 \mathrm{kt}(20-88 \mathrm{~km} / \mathrm{h})$ & $34-40 \mathrm{kt}(62-74 \mathrm{~km} / \mathrm{h})$ \\
Sea conditions & Height wave, max. $1.5 \mathrm{~m} ;$ & Height wave: 6-7.5 m; \\
& Small waves with rolling crests & Big waves with arched crest \\
On the land & The dust rises; & The trees branches break; \\
& $\begin{array}{l}\text { The pennant stretches, taking a } \\
\text { horizontal position }\end{array}$ & The vehicles can go out of control \\
\end{tabular}

The constraints for both 4 bf and 8 bf were done on the entire circumference of the lower base (which is submerged in water) (Figure 2).
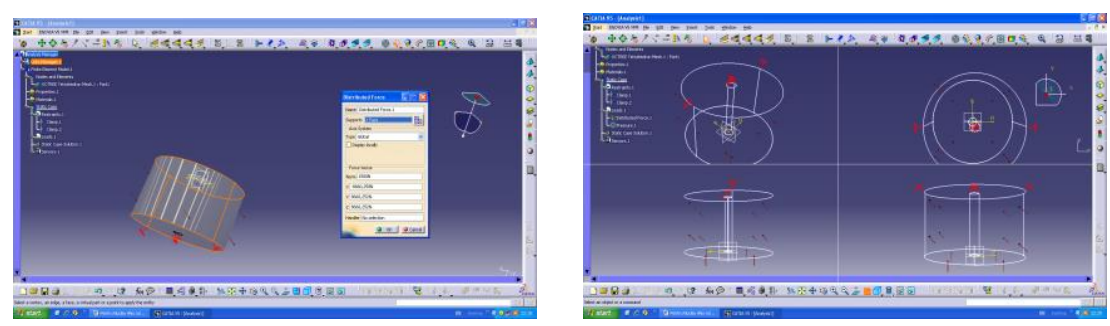

Figure 2. Preparation for simulation: $a-$ single view; $b$ - multiple view

The loads were directed to: distributed force and distributed pressure. The views of these constraints are shown in figure 3. 
The material from which the signaling/ rescue system is considered to be made has the following characteristics (for both simulation conditions, 4 bf and $8 \mathrm{bf}$ ):

- warp// weft: $100 \%$ PES// 100\% PES;

- warp// weft length density: 167dtex/ f32x2 / 250Z// 167dtex/ f32x2/ 120Z;

- breaking force, min. 300// $315 \mathrm{daN}$;

- warp// weft elongation at break, max. 20// $21 \%$.

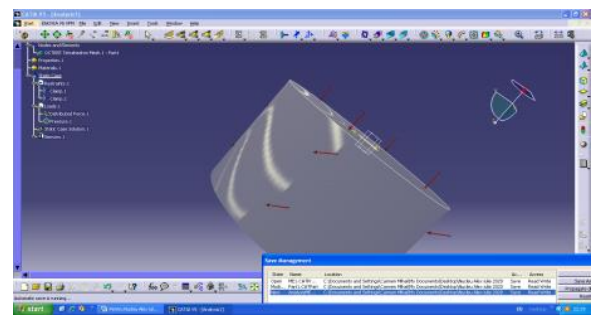

Figure 3. The views of the constraints

\section{RESULTS AND DISCUSSIONS}

Post-processing allowed the visualization of the results (for both stress conditions, at $4 \mathrm{bf}$ and at $8 \mathrm{bf}$ ): deformation of the structure under the effect of dynamic pressure, Von Mises stress fields and distribution of displacement vectors. The state of tension in the system (possible cracks) that may occur at the contact of the composite structure with the fluid in turbulent motion was predicted using the Von Mises criterion.

\section{Post-Processing Results - Stress at 4 bf}

Figure 4 shows the images corresponding to the model calculation at 4 bf. Figure $4 \mathrm{~b}$ and $4 \mathrm{c}$ show the interior deformation of the ME1 on different segments, visualization possible with the help of the section plane manipulated with the help of the compass (rotations, displacements). By dynamically changing the position of the plan, the results can be viewed in real time.

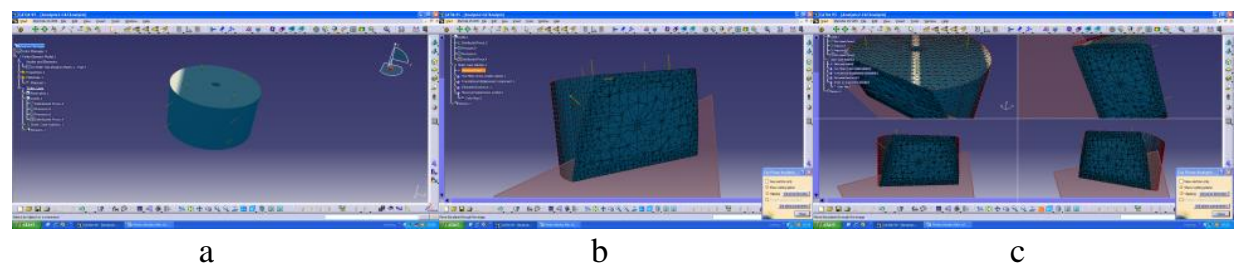

Figure 4. Deformation visualization of the signaling/ rescue system located in coastal areas; a - deformation ME1 from SC4; b - deformation SC4 and ME1 interior single view; c - SC4 deformation and ME1 interior - multiple view

The Von Mises Stress result highlights the range of values for the minimum and maximum stresses that can occur in the required system at $4 \mathrm{bf}$. According to the analysis, the resulting values fall in the range [5.26e+004; 1.78e+006] N/m². Given the value for admissible resistance of the textile material, it can be predicted that this and implicitly the composite structure will withstand the distributed force imposed for $4 \mathrm{bf}$. 
The obtained images are shown in figure 5 and successively highlights the Von Mises values of the system.

The image obtained for the main stresses in the system subjected to the action of the distributed force and pressure for 4 bf is presented in figure 6 . The main voltage tensor is represented in 3D in each node and is in the range of $[-2.81 \mathrm{e}+006 ;+2.99 \mathrm{e}+006] \mathrm{N} / \mathrm{m}^{2}$.

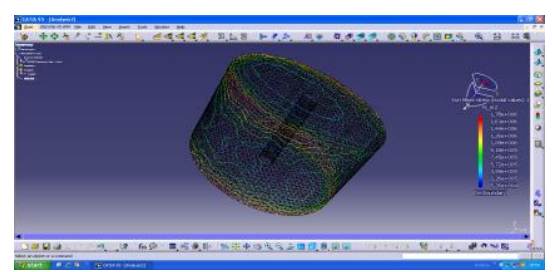

$\mathrm{a}$

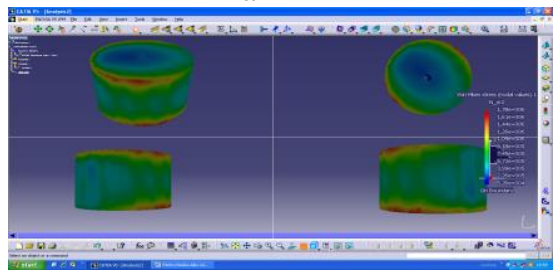

C

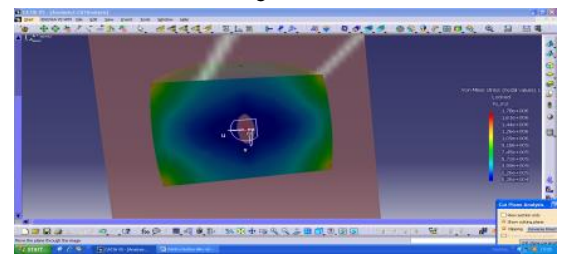

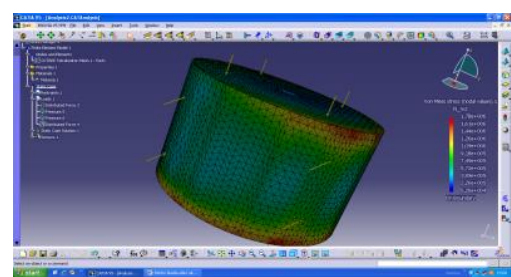

b

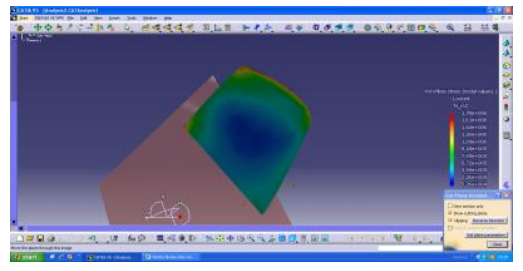

d

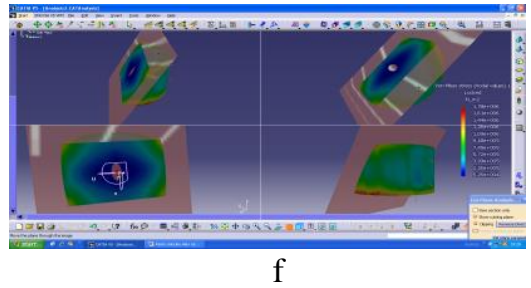

Figure 5. Nodal values visualization of Von Mises stress for the signaling/ rescue system located in coastal areas (maritime and fluvial): $a, b$ - image obtained with mesh view - single view; c - multiple view; d, e, f - cut plane analysis

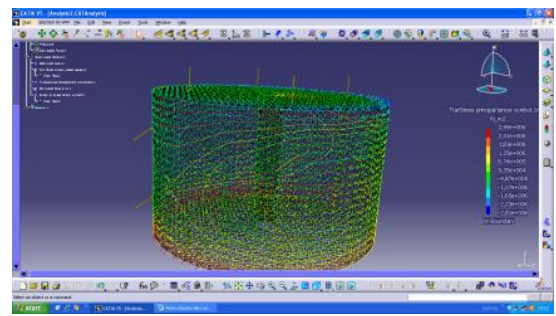

Figure 6. The main stress tensor for the signaling/ rescue system located in coastal areas (maritime and fluvial)

\section{Post-Processing Results - Stress at 8 bf}

The images corresponding to the calculation at a stress of 8 bf are shown in figure $7 \mathrm{a}$ and $7 \mathrm{~b}$ highlight the deformation of the system by means of its representation with the discretization network; in figure $8 \mathrm{c}$ is visualized the interior deformation on different segments (cut plane analysis). 
The analysis of the images highlights a large deformation of the system in the sense of implosion of the area exposed to the dynamic stresses that appear especially in the sea - shore direction.

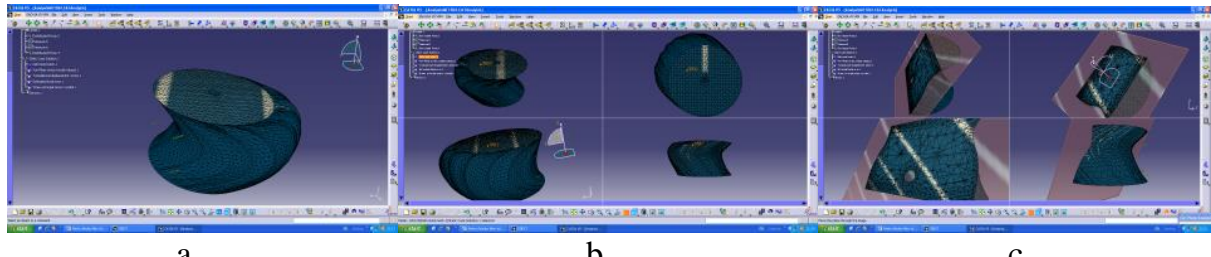

a

b

Figure 7. Deformation of the signaling/ rescue system located in coastal areas (maritime and fluvial): a - material and mesh representation - single view; b - material and mesh representation - multiple view; c - cut plane analysis - multiple view

According to the analysis performed for the stress at $8 \mathrm{bf}$, the resulting values for Von Mises Stress (on node) fall within the range [3.15e+008; $1.07 \mathrm{e}+010] \mathrm{N} / \mathrm{m}^{2}$, for certain values exceeding the admissible resistance of the material, so the system will resist to the distributed force and pressures required for $8 \mathrm{bf}$.

The images obtained for Von Mises at a stress of 8 bf are shown in figure 8 .

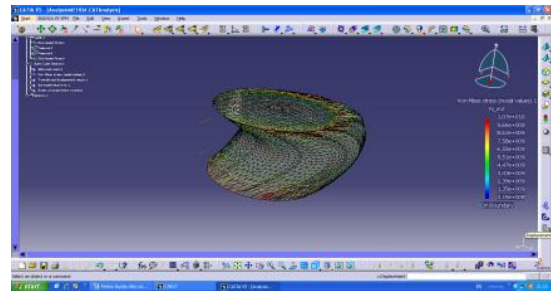

a
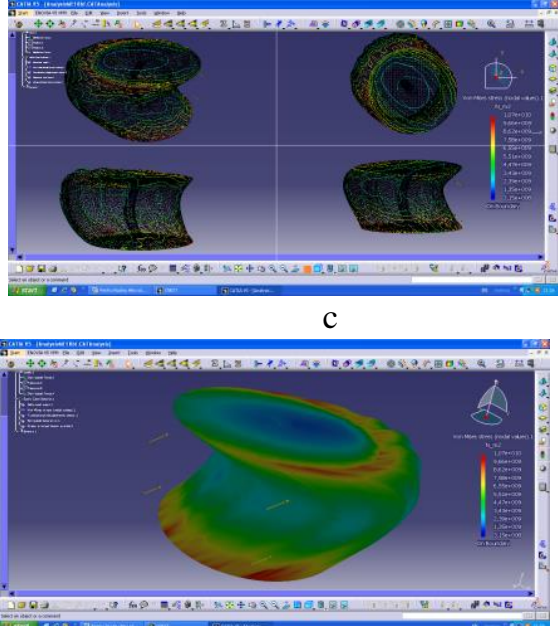

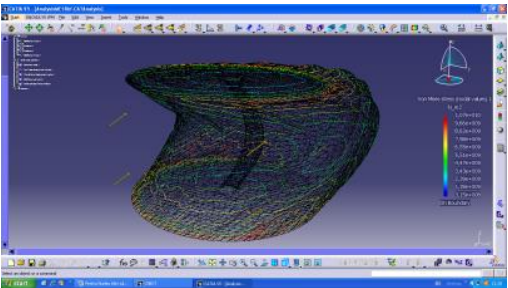

b

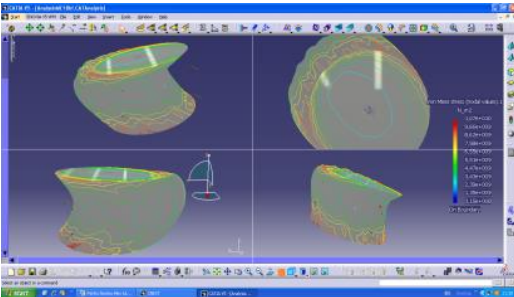

d

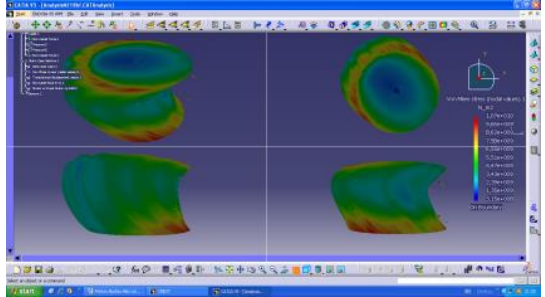

f

Figure 8. Visualization of the Von Mises system values - 8 bf: a -material and mesh; $\mathrm{b}$ - mesh; c, d - representation in multiple view; e - material representation; $\mathrm{f}$ - material representation - multiple view 
Figure 9 allows the visualization of the main stress tensor highlighting the fact that the values in each node and are in the range of $[-1.68 \mathrm{e}+010 ; 1.8 \mathrm{e}+010] \mathrm{N} / \mathrm{m}^{2}$.

\section{CONCLUSIONS}

The modelling with FEM allowed the prediction of the possible responses of the signaling/ rescue system located in the aquatic coastal area.

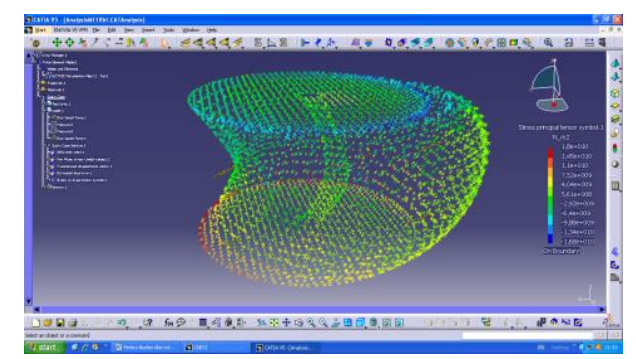

Figure 9. The main stress tensor for $8 \mathrm{bf}$

The post-processing performed for conditions of 4 bf and 8 bf showed that the designed digital system that has as material from the composite structure made by specialists from INCDTP will resist the difficult conditions imposed by the field of use.

\section{REFERENCES}

Ene, A. (2020), "High tech composite structures for sustainable development of biodiversity and aquatic ecosystems (4AquaSave)" (in Romanian) - Project no. PN 19170202.

*** (2002), Decision No. 1600/2002/EC of the European Parliament and of the Council of 22 July 2002 laying down the Sixth Community Environment Action Programme (JO L 242, 10.9.2002, p.1).

*** (2006), Communication from the Commission - Halting the loss of biodiversity by 2010 - and beyond Sustaining ecosystem services for human well-being, http://eur-lex.europa.eu/LexUriServ /site/en/com / 2016/com2016 0216en01.pdf

*** (2006), Council Regulation (EC) No. 509/2006, "Moules de bouchot", EC no.: FR-TSG-0007-004828.12.2006

*** (2012), EEA Report No 8/2012, European waters - assessment of status and pressures, https://www.eea.europa.eu/publications/european-waters-assessment-2012

*** (2013), Decision No 1386/2013/EU of the European Parliament and of the Council of 20 November 2013 on a General Union Environment Action Programme to 2020 'Living well, within the limits of our planet'

*** (2014), https://www.ladepeche.fr/article/2014/07/23/1923284

*** (2015), Multiannual National Strategic Plan on Aquaculture 2014-2020, Operational Programme for Fisheries and Maritime Affairs, available at: https://www.madr.ro/docs/fep/2015/popam-20142020/PSNMA-2014-2020-versiune-oficiala-15.04.2015.pdf.

*** (2017), National Strategy for Sustainable Development of Romania 2030, Goal 14 - Conserve and sustainably use the oceans, seas and marine resources for sustainable development, http://dezvoltaredurabila.gov.ro/web/obiective/odd14/

*** (2018), Communication from the Commission to the European Parliament, the Council, the European Economic and Social Committee and the Committee of the Regions, A European strategy for data, Brussels, 29.4.2018 COM (2018) 229. 
DOLOMITE SURFACE MODIFICATION WITH TITANIA AND SILICA PRECURSORS AND ITS MORPHOSTRUCTURAL AND THERMAL CHARACTERISATION

\author{
MARIA SÖNMEZ ${ }^{1}$, MIRCEA JUGĂNARU ${ }^{2}$, ANTON FICAI ${ }^{3}$, DENISA FICAI ${ }^{3}$, OVIDIU \\ OPREA $^{3}$, DANA GURĂU ${ }^{1}$, LAURENȚIA ALEXANDRESCU ${ }^{1}$, MARIA DANIELA \\ STELESCU $^{1}$, MIHAI GEORGESCU ${ }^{1}$, MIHAELA NIȚUICA ${ }^{1}$, ALINA MELINESCU $^{3}$, \\ MINODORA MARIN ${ }^{1}$ \\ ${ }^{1}$ INCDTP - Division: Leather and Footwear Research Institute, 93 Ion Minulescu st., Bucharest, \\ Romania,maria.sonmez@icpi.ro \\ ${ }^{2}$ Protect Styl Industry S.R.L., 30 Bulevardul Unirii, 600192, Bacau, Romania, \\ mircea.juganaru@protectstyl.ro \\ ${ }^{3}$ Politehnica University of Bucharest, Faculty of Applied Chemistry and Material Science, 1-7 \\ Polizu St., Bucharest, Romania, anton.ficai@upb.ro
}

\begin{abstract}
The aim of the paper is to modify the surface of dolomite with titania $\left(\mathrm{TiO}_{2}\right)$ and silica $\left(\mathrm{SiO}_{2}\right)$ precursors, in order to use it as a potential reinforcement material in a polymeric matrix or for environmental applications (photocatalyst for the degradation of organic pollutants based on $\mathrm{TiO}_{2}$ ). The dolomite surface modification was performed by 2 methods. The first method consisted in modifying the direct dolomite surface with $\mathrm{SiO}_{2}$ and $\mathrm{TiO}_{2}$. The second method consisted in the initial treatment of dolomite with TEOS, in order to form silanol bonds, followed by the addition of $\mathrm{SiO}_{2}$ and $\mathrm{TiO}_{2}$ precursors. The obtained powders were characterized by FTIR, SEM-EDS and DSC-TG. The FTIR spectra prove the formation of the silica network while the samples modified with PDMS exhibit the characteristic peaks of methyl groups from PDMS. In EDS, the presence of the characteristic elements of dolomite (calcium, magnesium, oxygen and carbon) can be observed. When analyzing the modified dolomite powders the presence of titanium and silicon can be observed. The characteristic morphology of the dolomite is preserved in all the samples but, the surface of the larger particles is decorated with smaller particles proving the functionalization of the dolomite, according to the two routes. The thermal analysis is characteristic for dolomite-based materials, the main difference between the samples appearing as a consequence of the burning of the organic part of PDMS, which occur between 400 and $600^{\circ} \mathrm{C}$.
\end{abstract}

Keywords: Dolomite, $\mathrm{SiO}_{2}, \mathrm{TiO}_{2}$.

\title{
INTRODUCTION
}

Dolomite is a mineral composed of layers of carbonate ions $\left(\mathrm{CO}_{3}^{-2}\right)$ separated by alternating layers of the calcium $\left(\mathrm{Ca}^{2+}\right)$ and the magnesium $\left(\mathrm{Mg}^{2+}\right)$ ions. Dolomite has properties similar to calcite $\left(\mathrm{CaCO}_{3}\right)$, but is harder and has a higher resistance to acid attack (DeArmitt, 2019). Due to its high abundance in nature, dolomite has a low price (Correia et al., 2015). Dolomite is considered a promising catalyst in obtaining biofuel, due to its composition (mainly $\mathrm{CaCO}_{3}$ and $\mathrm{Mg}$ ), which is decomposed at high temperatures (thermal activation/calcination) into $\mathrm{CaO}$ and $\mathrm{MgO}$ (Ñústez-Castaño et al., 2019). Other applications of dolomite include: adsorbent for retaining heavy metals ( $\mathrm{Zn}$, $\mathrm{Ni}$, etc.) from wastewater, photocatalyst for degradation of organic pollutants (bisphenol A, chlorophenols, etc.) in the presence of UV light (Nagase et al., 2014), filler in various polymeric matrices (polyester resins, alginates, polypropylene, etc.) (Saidi et al., 2019; Huang et al., 2019; Ozdemir et al., 2017). Although dolomite can be used in a wide range of applications, it is not exploited to its true potential, at the industrial level. This is because it requires additional surface treatments to induce specific features of interest. These functionalities are reflected in improved mechanical, chemical, flame retardant properties, etc., which makes it a viable material at the industrial level, the 
quality / price ratio being superior to carbon nanotubes, carbon fibers, etc. Therefore, this paper aims to modify the surface of dolomite powder with $\mathrm{SiO}_{2}$ and $\mathrm{TiO}_{2}$ precursors, by two simple methods, in order to use it as a potential reinforcing material in polymer matrices or for environmental applications (photocatalyst for degradation of organic pollutants based on $\mathrm{TiO}_{2}$ ).

\section{MATERIALS AND METODS}

\section{Materials}

The following raw materials were used in the experiments: Dolomite powder type DOLOFLOR, with 30-33\% $\mathrm{CaO}, 18-20 \% \mathrm{MgO}, \mathrm{pH}=9.67$, from SC CEMROM SA; Tetraethyl orthosilicate (TEOS) - reagent grade, 98\%, 208.33 wt\%; Poly(dimethylsiloxane) - viscosity 500cSt $\left(25^{\circ} \mathrm{C}\right.$ ) (lit.), mol wt $\sim 17,250$; Titanium (IV) isopropoxide, $\geq 97 \%$, molecular weight: 284.22 , density: $0.96 \mathrm{~g} / \mathrm{mL}$ at $20^{\circ} \mathrm{C}$ (lit.) from Sigma Aldrich, Ethyl Alcohol - for analysis, molecular weight: 46.07 acquired from Chimreactiv SRL.

\section{Method} (2019).

Dolomite powder was treated according to a methodology published by Sonmez et al.

Method 1: $100 \mathrm{~g}$ of dolomite was transferred to a plastic container over which excess ethanol was added (so as to cover the entire surface of the powder) and magnetically stirred at $300 \mathrm{rpm}$ and $40-50^{\circ} \mathrm{C}$ for $30 \mathrm{~min}$. Then, the functionalizing agents $(10 \mathrm{~mL}$ polydimethylsiloxane-PDMS or $10.5 \mathrm{~mL}$ titanium isopropoxide - $\mathrm{TiO}_{2}$ ) were added as fine droplets, and stirring was continued at the same parameters as above, for another $2 \mathrm{~h}$. Into the glass containing the dolomite powder $/ 10 \%$ titanium isopropoxide, $10 \mathrm{~mL}$ of distilled water was added for the hydrolysis process to occur, and stirring was continued for another $20 \mathrm{~min}$. The two types of powders (Dolomite/10\% PDMS, symbolized in the paper as Sample 7 and Dolomite $/ 10 \% \mathrm{TiO}_{2}$ - Sample 8) obtained, were filtered under vacuum, washed with ethanol in abundance (at least 3 times to eliminate unreacted agents), dried in an oven with hot air at $80^{\circ} \mathrm{C}$ for $\sim 24 \mathrm{~h}$, ground and characterized (FTIR, EDS, SEM, DSC-TG).

Method 2 (sol-gel): involves, compared to method 1, the initial treatment of dolomite with TEOS in order to form reactive $\mathrm{Si}-\mathrm{OH}$ bonds on the surface (at $300 \mathrm{rpm}, 40-50^{\circ} \mathrm{C}$ for $1 \mathrm{~h}$ ), followed by the addition of functionalizing agents (PDMS or titanium isopropoxide). The amount of TEOS $(18.6 \mathrm{~mL})$ was calculated to obtain in the end $5 \mathrm{~g} \mathrm{SiO}_{2}$ and $5 \mathrm{~g}$ of $\mathrm{TiO}_{2}$ ( $24.67 \mathrm{~mL}$ titanium isopropoxide), the rest of the stages being identical to method 1 . Thus, 2 powder variants were also obtained: Dolomite/5\%TEOS/5\%PDMS - Sample 9 and Dolomite/5\% TEOS/5\% $\mathrm{TiO}_{2}$ - Sample 10. 


\section{RESULTS AND DISCUSSION}

\section{FTIR Spectra of Raw / Modified Dolomite Powder}

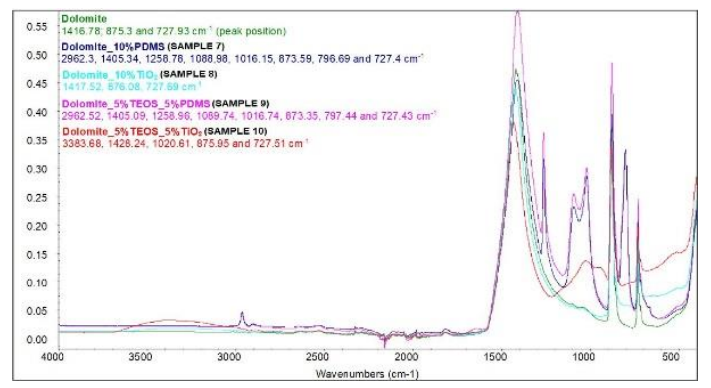

Figure 1. FTIR spectra of raw dolomite powder / modified with $\mathrm{SiO}_{2}$ and/or $\mathrm{TiO}_{2}$ precursors

The FTIR spectrum obtained on raw dolomite, highlights the bands from 1416.78, associated with the asymmetric stretching vibration $\left(v_{\mathrm{as}}\right)$ of $\left(\mathrm{CO}_{3}\right)^{2-}$ groups, the band from $873.3 \mathrm{~cm}^{-1}$ (associated $\gamma-$ out of plane bending vibration of bonds $\mathrm{O}-\mathrm{C}-\mathrm{O}$ ) and the 727.93 $\mathrm{cm}^{-1}$ band, associated with $\delta-$ in plane bending vibration of $\mathrm{O}-\mathrm{C}-\mathrm{O}$ in $\left(\mathrm{CO}_{3}\right)^{2-}$ group (Mroczkowska-Szerszeń and Orzechowski, 2018). In Sample 7, in addition to the characteristic dolomite bands, the PDMS bands can also be identified. Thus, the band from $2962.3 \mathrm{~cm}^{-1}$ (is associated with the stretching vibration of $\mathrm{CH}_{3}$ bonds) and the bands from $1088.98,1016.15 \mathrm{~cm}^{-1}$ are associated with the vibration of $\mathrm{Si}-\mathrm{O}-\mathrm{Si}$ bonds. The band from $1258.78 \mathrm{~cm}^{-1} \mathrm{~s}$ associated with symmetrical deformations of the $\mathrm{CH}_{3}$ bonds from the $\mathrm{Si}-\mathrm{CH}_{3}$ group, and the one from $796.69 \mathrm{~cm}^{-1}$ represents the stretching vibration of the Si-O bonds (Cui et al., 2018). In the spectrum obtained on Sample 9 can be identified both the characteristic bands of PDMS and silica. In the case of Sample 10, the adsorption band from $3383.68 \mathrm{~cm}^{-1}$ associated with the stretching vibration of hydrogen bonds and the $\mathrm{Si}-\mathrm{O}-\mathrm{Si}$ band from silica can be identified. The characteristic bands of the $\mathrm{TiO}_{2}$ group could be revealed by FTIR (Sample 8 and 10), only at values below $500 \mathrm{~cm}^{-1}$. However, the presence of the element Ti has been proven by EDS in the case of both powders containing $\mathrm{TiO}_{2}$ precursor.

\section{SEM-EDS Analysis of Raw and Modified Dolomite Powders}

The SEM images obtained on raw dolomite (Figure 2, image A) show a high heterogeneity of particle sizes with variations from $1,512 \mu \mathrm{m}$ to $14.08 \mu \mathrm{m}$. According to the literature, generally in raw dolomite, two types of aggregates can be observed: fine with dimensions between 1-4 $\mu \mathrm{m}$ and large with dimensions between 5-14 $\mu \mathrm{m}$ (Gruszecka-Kosowska et al., 2017; Huang et al., 2019). Moreover, the shape of the particles in dolomite is not well defined (irregular) and the aggregates have a smooth surface, without visible pores. The characteristic morphology of dolomite is preserved in all modified powders (Figure 2, B-E), with the mention that the surface of large particles is decorated with smaller particles which demonstrates the functionalization of dolomite, according to the two methods. Thus, in the case of Samples 7 and 9 (Fig. 2, B and C), the particle size from $\mathrm{SiO}_{2}$ precursors varies between 718.3 and $956.5 \mathrm{~nm}$. Smaller particle sizes could be observed in Sample 10, especially at large magnifications-20.000x, with size variations between 113.6, 145.2 and $154 \mathrm{~nm}$. The EDS spectrum obtained on raw dolomite (Figure 2, A) highlights the main elements in its composition: $\mathrm{Ca}, \mathrm{Mg}, \mathrm{C}$ and $\mathrm{O}$ 
without identifying other elements considered impurities, which demonstrates the advanced purity of the dolomite used.

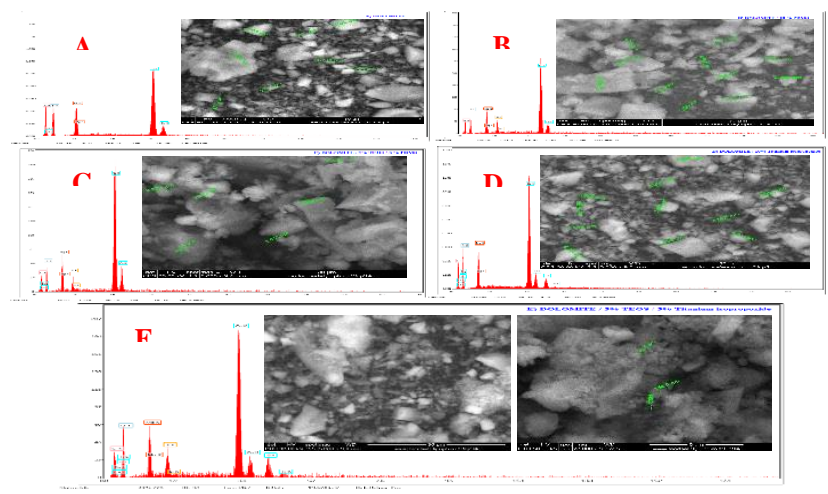

Figure 2. SEM-EDS analysis of: (A) raw dolomite, (B) Sample 7, (C) Sample 9, (D) Sample 8 and (E) Sample 10, magnification 5000 and 20000x

In Sample 7 (B) and Sample 9 (C) in addition to the dolomite elements, the presence of $\mathrm{Si}$ can also be observed, which confirms that the chosen modification route was optimal. Similar, in Sample 8 (D) the element Ti can be identified, and in Sample 10 (E) the elements Ti and Si.

\section{Thermal Analysis}

To evaluate the thermal decomposition behavior of raw dolomite and modified with $\mathrm{TiO}_{2}$ and/or $\mathrm{SiO}_{2}$ precursors, the DSC-TG analysis was performed, and the obtained curves are shown in Figure 3. In raw dolomite (Figure 3Error! Reference source not found., A), a mass loss of $1.57 \%$ is observed in the range $\mathrm{RT}-600^{\circ} \mathrm{C}$. A weak endothermic effect below $100^{\circ} \mathrm{C}$ indicates an initial water loss (water physically absorbed into the mineral). After $600^{\circ} \mathrm{C}$ the sample loses $45.81 \%$ of the mass up to $900^{\circ} \mathrm{C}$, the residual mass being 52.97\%. This is the interval in which $\mathrm{MgCO}_{3}$ and $\mathrm{CaCO}_{3}$ decompose by eliminating $\mathrm{CO}_{2}$. The endothermic effect that accompanies this decomposition process is wide, asymmetrical, with a minimum at $849.5^{\circ} \mathrm{C}$, and a shoulder at $813.8^{\circ} \mathrm{C}$. According to data from the literature (Yang et al, 2019), the first to decompose is $\mathrm{MgCO}_{3}$ (endothermic effect at $813.8^{\circ} \mathrm{C}$ ). $\mathrm{CaCO}_{3}$ decomposes at a higher temperature (endothermic effect at $849.5^{\circ} \mathrm{C}$ ). The inflection point on the DTG curve is at $829^{\circ} \mathrm{C}$ (until there the decomposition of $\mathrm{MgCO}_{3}$ dominates, and at temperatures $>829^{\circ} \mathrm{C}$ the decomposition of $\mathrm{CaCO}_{3}$ dominates). By decreasing the water and considering the total decompositions, a (mass) composition of $\mathrm{MgCO}_{3} \sim 26 \%$ and $\mathrm{CaCO}_{3} \sim 73 \%$ can be estimated. In Sample 7 (image B) a mass loss of $6.05 \%$ up to $600^{\circ} \mathrm{C}$ is observed. In addition to traces of water removed up to $150^{\circ} \mathrm{C}$ (according to the weak endothermic effect at $75^{\circ} \mathrm{C}$ ) there is a higher mass loss after $300^{\circ} \mathrm{C}$ most likely generated by oxidation of PDMS and transformation into $\mathrm{SiO}_{2}$ (weak exothermic effect at $386.5^{\circ} \mathrm{C}$ ). After $600^{\circ} \mathrm{C}$ the decomposition of the two carbonates takes place. Besides the mass loss of $13.71 \%$ between $725-793^{\circ} \mathrm{C}$ attributed to the decomposition of $\mathrm{MgCO}_{3}$ (endothermic effect at $757.9^{\circ} \mathrm{C}$ ) and the mass loss of $25.03 \%$ between $793-900^{\circ} \mathrm{C}$ attributed to the decomposition of $\mathrm{CaCO}_{3}$ (endothermic effect at $818.6^{\circ} \mathrm{C}$ ) a mass loss between $600-725^{\circ} \mathrm{C}$ of $4.66 \%$ can also be observed, with a weak endothermic effect at $690.6^{\circ} \mathrm{C}$. It can be 
attributed to the formation of a $\mathrm{Ca}$ or $\mathrm{Mg}$ silicate $\left(\mathrm{MSiO}_{3}\right.$ type). In this case there is a decrease in carbonate decomposition temperatures, more pronounced for $\mathrm{MgCO}_{3}$.

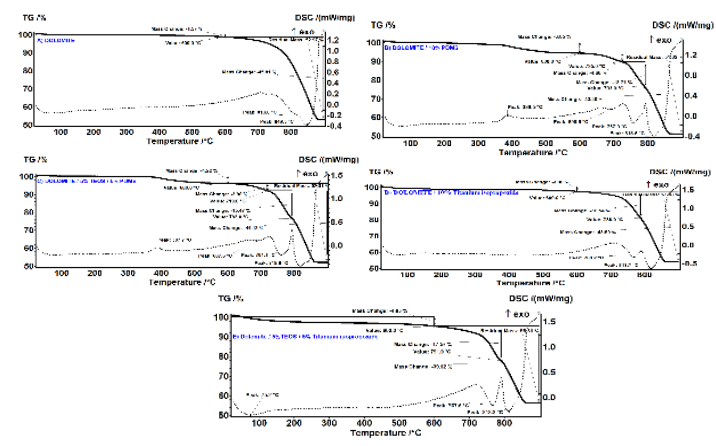

Figure 3. Thermal analysis of powders: (A) Dolomite; (B) Sample 7; (C) Sample 9; (D) Sample 8 and (E) Sample 10

In Sample 9 (image C) there is a mass loss of $4.22 \%$ up to $600^{\circ} \mathrm{C}$. In addition to traces of water removed up to $150^{\circ} \mathrm{C}$ (according to the weak endothermic effect at $80^{\circ} \mathrm{C}$ ) there is a greater loss of mass after $300^{\circ} \mathrm{C}$, most likely generated by oxidation of PDMS and transformation into $\mathrm{SiO}_{2}$ (weak exothermic effect of at $387.7^{\circ} \mathrm{C}$ ). The smaller amount of PDMS in the sample leads to a lower weight loss. Considering the thermal analysis from Sample 10 (image E), the oxidative degradation of TEOS occurs without being able to revealing sudden mass losses or thermal effects. After $600^{\circ} \mathrm{C}$ the decomposition of the two carbonates takes place. In the range of $600-719^{\circ} \mathrm{C}$ the reaction between $\mathrm{SiO}_{2}$ and carbonates takes place (obtaining $\mathrm{MSiO}_{3}$ ) with a mass loss of $4.22 \%$ and a weak endothermic effect at $687.6^{\circ} \mathrm{C}$. The decomposition of $\mathrm{MgCO}_{3}$ occurs predominantly in the range of $719-793^{\circ} \mathrm{C}$ (endothermic effect at $761.1^{\circ} \mathrm{C}$ ), the mass loss being $15.47 \%$. Between $793-900^{\circ} \mathrm{C}, \mathrm{CaCO}_{3}$ decomposes predominantly (endothermic effect at $818.6^{\circ} \mathrm{C}$ ), with a mass loss of $24.68 \%$. In this case there is a decrease in the decomposition temperatures of carbonates. In Sample 8 (image D), a mass loss of $3.08 \%$ up to $600^{\circ} \mathrm{C}$ is observed. Below $100^{\circ} \mathrm{C}$ a weak endothermic effect is observed, generated by the loss of water absorbed in the sample. After $150^{\circ} \mathrm{C}$ there are a number of weak endothermic effects, which can be attributed to the decomposition of traces of organic matter. Considering the thermal analysis from Sample 10, the oxidative degradation of titanium isopropoxide occurs without any sudden loss of mass or visible thermal effects. The main decomposition stage starts after $600^{\circ} \mathrm{C}$ and represents $43.50 \%$ up to $900^{\circ} \mathrm{C}$. At this stage, the decomposition of $\mathrm{MgCO}_{3}$ takes place, with the endothermic effect from $768.2^{\circ} \mathrm{C}$ and the decomposition of $\mathrm{CaCO}_{3}$ with the endothermic effect from $818.7^{\circ} \mathrm{C}$. The inflection point on the DTG curve occurs at $788^{\circ} \mathrm{C}$, and can be considered that below $788^{\circ} \mathrm{C}$ the decomposition of $\mathrm{MgCO}_{3}$ predominates and then the decomposition of $\mathrm{CaCO}_{3}$ predominates. It is observed that the addition of Ti isopropoxide (which is already $\mathrm{TiO}_{2}$ ) decreases the decomposition temperatures of carbonates. Sample 10 (image E) records a mass loss of $4.83 \%$ up to $600^{\circ} \mathrm{C}$. In addition to traces of water removed at low temperature (endothermic effect at $75.2^{\circ} \mathrm{C}$ ), there is an oxidative degradation of TEOS and $\mathrm{Ti}$ isopropoxide, without major thermal effects. After $600^{\circ} \mathrm{C}$ the decomposition of the two carbonates takes place in stages. $\mathrm{Up}$ to $791^{\circ} \mathrm{C}$ the decomposition of $\mathrm{MgCO}_{3}$ predominates (endothermic effect at $767.6^{\circ} \mathrm{C}$ ) with a mass loss of $17.57 \%$. After $791^{\circ} \mathrm{C}$ the decomposition of $\mathrm{CaCO}_{3}$ predominates accompanied by the endothermic effect at $813.3^{\circ} \mathrm{C}$, with a mass loss of $21.55 \%$. For this sample, the formation of $\mathrm{Mg}$ or $\mathrm{Ca}$ silicate 


\section{Dolomite Surface Modification with Titania and Silica Precursors and Its Morphostructural and Thermal Characterization}

is not observed before the decomposition of $\mathrm{MgCO}_{3}$. There are two possible explanations, either the formation of the silicate overlaps with the decomposition of the carbonate and can no longer be revealed as separate processes, or $\mathrm{SiO}_{2}$ has already combined with $\mathrm{TiO}_{2}$.

\section{CONCLUSIONS}

The dolomite powder was modified according to the two methods presented, in order to be used as a reinforcing agent in obtaining composite materials. Additionally, the presence of $\mathrm{TiO}_{2}$ can ensure the photo-catalytic activity, and the obtained powders can be used in environmental applications.

\section{Acknowledgements}

This work was supported by: 1. Contract No. 183 CI/2018, 2. Contract no. 6PFE/2018, Acronym: PERFORM-TEX-PEL, and 3. PN 19170302 / 2019.

\section{REFERENCES}

Correia, L.M., de Sousa Campelo, N., Novaes, D.S., Cavalcante Jr., C.L., Cecilia, J.A., Rodríguez-Castellón, E. and Vieira, R.S., (2015), "Characterization and application of dolomite as catalytic precursor for canola and sunflower oils for biodiesel production", Chemical Engineering Journal, 269, 35-43, https://doi.org/10.1016/j.cej.2015.01.097.

Cui, X., Zhu, G., Pan, Y., Shao, Q., Zhao, C., Dong, M., Zhang, Y. and Guo, Z. (2018), "Polydimethylsiloxanetitania nanocomposite coating: Fabrication and corrosion resistance", Polymer, 138, 203-210, https://doi.org/10.1016/j.polymer.2018.01.063

DeArmitt, C. (2019), "Fillers and Filled Plastics", Kirk-Othmer Encyclopedia of Chemical Technology, 1-27, https://doi.org/10.1002/0471238961.0609121206011203.a0.

Gruszecka-Kosowska, A., Baran, P., Wdowin, M. and Franus, W., (2017), "Waste dolomite powder as an adsorbent of $\mathrm{Cd}, \mathrm{Pb}(\mathrm{II})$, and $\mathrm{Zn}$ from aqueous solutions", Environmental Earth Sciences, 76(15), 1-12, https://doi.org/10.1007/s12665-017-6854-8.

Huang, Y.X., Liu, M.J., Chen, S., Jasmi, I.I., Tang, Y. and Lin, S., (2019), "Enhanced adsorption and slow release of phosphate by dolomite-alginate composite beads as potential fertilizer", Water Environment Research, 91(8), 797-8, https://doi.org/10.1002/wer.1122.

Mroczkowska-Szerszeń, M. and Orzechowski, M. (2018), "Infrared spectroscopy methods in reservoir rocks analysis - semiquantitative approach for carbonate rocks", Nafta-Gaz, Rok LXXIV, 11, 802-812, https://doi.org/10.18668/ng.2018.11.04.

Nagase, H., Tsujino, H., Kurihara, D., Saito, H., and Kawase, M. (2014), “A role for calcium hydroxide and dolomite in water: Acceleration of the reaction under ultraviolet light", Chemosphere, 101, 8-14, https://doi.org/10.1016/j.chemosphere.2013.11.042.

Ñústez-Castaño, S.A., Villamizar-Castro, D.O. and Vargas-Solano, E.M., (2019), "Evaluation of dolomite as catalyst in the transesterification reaction using palm oil (RBD)", DYNA, 86(209), 180-187, http://dx.doi.org/10.15446/dyna.v86n209.74126.

Özdemir, F., Ayrilmis, N., and Mengeloğlu, F. (2017), "Effect of dolomite powder on combustion and technological properties of WPC and neat polypropylene", Journal of the Chilean Chemical Society, 62(4), 3716-3720, https://doi.org/10.4067/s0717-97072017000403716.

Saidi, M.A.A., Mazlan, F.S., Hassan, A., Rasid, R.A., and Rahmat, A.R. (2019), "Flammability, thermal and mechanical properties of polybutylene terephthalate/dolomite composites", Journal of Physical Science, 30(3), 175-189, https://doi.org/10.21315/jps2019.30.3.11.

Sonmez, M., Alexandrescu, L., Georgescu, M., Gurau, D., Chelaru, C., Juganaru, M., Ficai, A. and Trusca, R (2019), "Influence of adding functionalized microparticles on the physical-mechanical, structural, and processability properties of thermoplastic rubber", Leather and Footwear Journal, 19(1), 29-40, https://doi.org/10.24264/lfj.19.1.4

Yang, H., Dong, H., Zhang, T. et al. (2019), "Calcined Dolomite: An Efficient and Recyclable Catalyst for Synthesis of $\alpha, \quad \beta$-Unsaturated Carbonyl Compounds", Catalysis Letters, 149, 778-787. https://doi.org/10.1007/s10562-018-2632-9. 


\title{
IMPROVEMENT OF MECHANICAL, THERMAL AND MORPHOSTRUCTURAL PROPERTIES OF SBS THERMOPLASTIC ELASTOMER USING KAOLIN AND DOLOMITE MICROPARTICLES WITH MODIFIED SURFACE
}

\author{
MARIA SÖNMEZ ${ }^{1}$, MIRCEA JUGANARU ${ }^{2}$, ANTON FICAI ${ }^{3}$, OVIDIU OPREA ${ }^{3}$, ROXANA \\ TRUȘCA ${ }^{3}$, MIHAI GEORGESCU ${ }^{1}$, DANA GURĂU ${ }^{1}$, DENISA FICAI ${ }^{3}$, LAURENȚIA \\ ALEXANDRESCU ${ }^{1}$, DOINA CONSTANTINESCU ${ }^{4}$, MARIA DANIELA STELESCU ${ }^{1}$, \\ MIHAELA NIȚUICA ${ }^{1}$, MINODORA MARIN ${ }^{1}$ \\ ${ }^{1}$ INCDTP - Division: Leather and Footwear Research Institute, 93 Ion Minulescu st., Bucharest, \\ Romania,maria.sonmez@icpi.ro \\ ${ }^{2}$ Protect Styl Industry S.R.L., 30 Bulevardul Unirii, 600192, Bacau, Romania, \\ mircea.juganaru@protectstyl.ro \\ ${ }^{3}$ Politehnica University of Bucharest, Faculty of Applied Chemistry and Material Science, 1-7 \\ Polizu St., Bucharest, Romania, anton.ficai@upb.ro \\ ${ }^{4}$ SC MONOFIL SRL, 5 F Gheorghe Caramfil str, Savinesti, Neamt, Romania, \\ monofil.srl@gmail.com
}

\begin{abstract}
The aim of this paper was to assess the influence of the modification of the surface of dolomite and kaolin with $\mathrm{SiO}_{2}$ and $\mathrm{TiO}_{2}$ precursors, on the block copolymer styrene-butadiene-styrene (SBS) type thermoplastic rubber properties. These composite materials were obtained by compounding SBS with various ratio of powders. Based on the SEM images it can conclude that the powders were homogenously dispersed in to the SBS matrix. The dolomite particles can be clearly identified in the SEM images as particles of 10 micrometers. The samples obtained with modified dolomite have similar morphology. The EDS elemental distribution confirming a good corroboration between the elements of the dolomite, kaolin and titanium or silicon elements. Based on the thermal analysis according to the residual mass, the presence of $20 \%$ mineral phase can be confirmed. According to the DSC curves a strong stabilization of the composite appears, because of the presence of the mineral component. According to the physical-mechanical data all the composite materials exhibit improved mechanical properties. Additionally, the modification of the kaolin and/or dolomite bring important improvements in mechanical properties. The samples 13 and 14 exhibit high tensile and tear strength. These composites can be used for various applications, such as, for instance, soles for firefighters' footwear.
\end{abstract}

Keywords: SBS thermoplastic rubber, interface, functionalization

\section{INTRODUCTION}

Styrene-butadiene block copolymers are an important class of synthetic rubber, composed of central rubber blocks and polystyrene ends. Hard styrene blocks induce mechanical and abrasion resistance, while butadiene improves flexibility and toughness. SBS rubber is widely used as an impact modifier in various plastics and adhesives, sealing materials, gaskets, rubber bands for wires and cables, shoe soles, toy parts and bitumenbased products for road paving and roofing applications, etc. (Jin et al., 2021). To improve performance (mechanical, thermal, etc.), styrene-butadiene-styrene (SBS) rubber is often compounded with other polymers (PP, PS, etc.) and/or other reinforcing agents $\left(\mathrm{TiO}_{2}\right.$, $\mathrm{SiO}_{2}$, carbon black, clay, talcum, graphene, $\mathrm{CaCO}_{3}, \mathrm{MgCO}_{3}$, multi-walled carbon nanotubes-MWCNTs, fullerene (C60), nanodiamond, etc.) (Bicy et al., 2018; Abdelsalam et al., 2019; Das et al., 2019; Sönmez et al., 2019; Costa et al., 2014; Perov, 2018). However, the interaction between the rubber matrix/filler is affected by several factors such as the chemical/physical interactions between the surface of the filler/polymer particles, the structure of the filler network, the shape, size, proportion of the filler, etc. 
(Abdelsalam et al., 2019). In the literature, there are no studies on the effect of dolomite and/or kaolin microparticles functionalized with $\mathrm{SiO}_{2}$ and $\mathrm{TiO}_{2}$ precursors on the physical-mechanical, morpho-structural and thermal properties of SBS rubber. Improving the properties of SBS rubber is of major importance in the industry, as it is used in a wide range of applications and by using low cost and high availability fillers, improved properties can be obtained.

\section{MATERIALS AND METODS}

\section{Materials}

The following raw materials were used for the processing of composites: Styrenebutadiene-styrene rubber (SBS) - type Eurruber from Tecnofil SME, Italy; Kaolin - calcined (Snowpaque), with particle size from 2 to $50 \mu \mathrm{m}$ and layer thickness from $13.2 \mathrm{~nm}$ to 19.25 $\mathrm{nm}$, purchased from Bridgexim SRL; DOLOFLOR dolomite powder, with the following composition: $\mathrm{CaO}-30-33 \%, \mathrm{MgO}-18-20 \%, \mathrm{pH}=9.67$, neutralization number $-59.55 \% \mathrm{CaO}$, particle size of several tens of $\mu \mathrm{m}$, manufacturer SC CEMROM SA.

\section{Method}

Composites based on SBS reinforced with $5 \mathrm{~g}$ kaolin powder and/or varying amounts (30-75g) of modified/unmodified dolomite relative to $100 \mathrm{~g}$ SBS were obtained according to Table 1 .

Table 1. Formulation based on SBS rubber reinforced with modified/unmodified particles, wt ratio

\begin{tabular}{|c|c|c|c|c|c|c|}
\hline Sample code / Raw materials & SBS & 11 & 12 & 13 & 14 & 15 \\
\hline SBS Rubber & 100 & 100 & 100 & 100 & 100 & 100 \\
\hline Kaolin & - & 5 & - & - & - & - \\
\hline Kaolin/10\%PDMS & - & - & 5 & - & - & - \\
\hline Kaolin/10\% $\mathrm{TiO}_{2}$ & - & - & - & 5 & - & - \\
\hline Dolomite & - & 30 & - & - & - & - \\
\hline Dolomite/5\%TEOS+5\% PDMS & - & - & 30 & - & - & 20 \\
\hline Dolomite/5\%TEOS+5\% $\mathrm{TiO}_{2}$ & - & - & - & 30 & 20 & - \\
\hline Dolomite $/ 10 \% \mathrm{TiO}_{2}$ & - & - & - & - & 55 & - \\
\hline Dolomite/10\%PDMS & - & - & - & - & - & 55 \\
\hline
\end{tabular}

The surface of kaolin and dolomite was modified by a methodology similar to the one described in the article published by Sönmez et al. (2019). Prior to the actual processing on the Brabender, the first step was assigned to drying the raw materials at $100^{\circ} \mathrm{C}$ in a hot air oven for several hours. The second stage consisted of processing the composites on a Brabender mixer, at $160^{\circ} \mathrm{C}$ and at different rotational speeds ( $30 \mathrm{rpm}, \sim 1$ minute, and at 130 $\mathrm{rpm}, \sim 3$ minutes). Depending on the amount of powder added (mixtures 14 and 15), the processing time increased by 2-3 minutes or until the total and uniform incorporation of the powders into the SBS mass took place. From the processed mixtures, plates with the size of 150x150x4 mm (length $\mathrm{x}$ width $\mathrm{x}$ thickness) were obtained in a metal mold, by the hot compression method. Press processing parameters: the temperature of the platters (upper and lower) was set at $170^{\circ} \mathrm{C}$, preheating and pressing time -4 minutes, cooling the platters -10 minutes. From the resulting composite plates, specimens with standardized dimensions 
and shapes were stamped (dumbbell specimen - tensile strength, elongation at break, modulus, and from the trouser-type specimen - tear strength was determined), in order to perform physical-mechanical tests. Following the physical-mechanical determinations, the tested specimens were used for thermal (DSC-TG) and morpho-structural analyses (SEM, EDS).

\section{RESULTS AND DISCUSSION}

\section{Energy Dispersive Spectroscopy (EDS) and SEM Analysis}
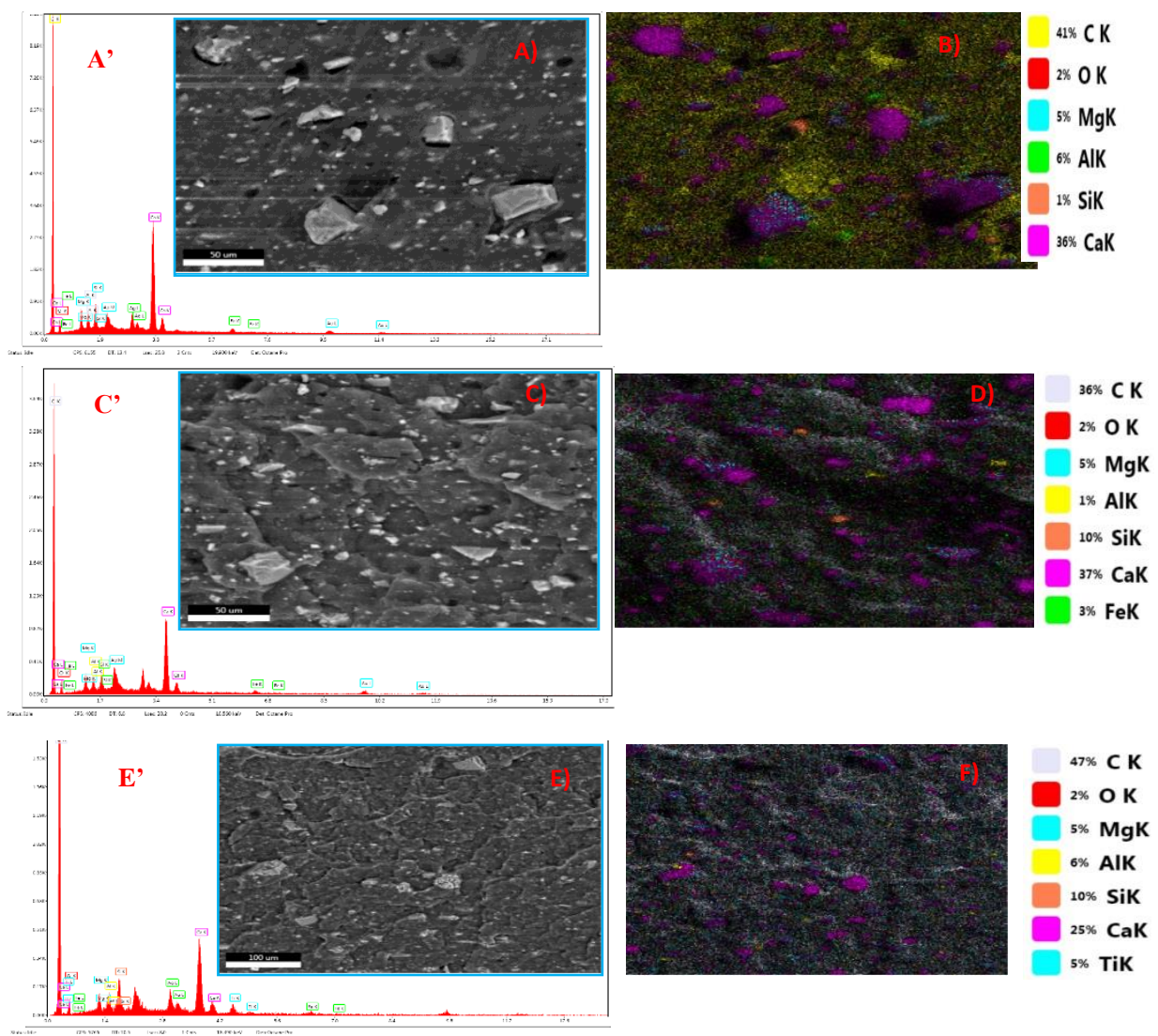

Figure 1. SEM image, EDS spectra and map of: Sample 11 (A, $\mathbf{A}^{\prime}$ and B); Sample 12 $\left(\mathbf{C}, \mathbf{C}^{\prime}\right.$ and D) and Sample 13 (E, E' and F)

Figure 1, image A for sample 11 in which the reinforcing agents are not chemically modified, kaolin and/or dolomite particles can be observed but also defects (of the order of tens of $\mu \mathrm{m}$ ) due to the detachment of the dispersed phase, which denotes a weak interaction between phases. In image $C$ of sample 12, due to surface modification, the large defects visible in sample 11 are not specific, probably due to a better matrix/ reinforcing agent interaction. In sample 13 the morphology changes considerably, the polyhedral reinforcing agent particles seem to be better embedded in the polymer matrix, 
and the rupture takes place mainly through the SBS matrix. The EDS elemental distribution (Figure 1, images B, D and F) confirming a good corroboration between the elements of the dolomite and titanium or silicon elements. The EDS spectra (images A', $\mathrm{C}^{\prime}$ and E') highlight the presence of component elements in dolomite (mainly $\mathrm{Ca}, \mathrm{Mg}$ ), kaolin (Al, Si, Fe) (Sönmez et al., 2019) and functionalizing agents (and additionally from PDMS, and/or silica from TEOS, Ti element).

\section{DSC-TG Thermal Analysis}

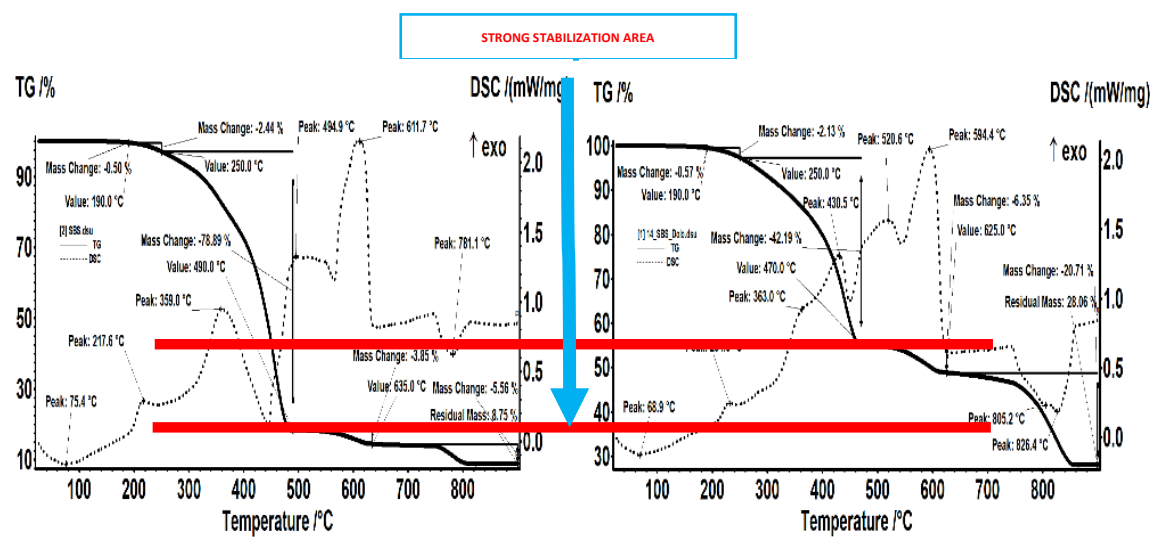

Figure 2. Thermal analysis of SBS rubber (left) and Sample 14 (right)

Thermal analysis performed on SBS rubber shows a relatively good stability up to $190^{\circ} \mathrm{C}(0.5 \%$ mass loss being caused by the elimination of traces of solvent - endothermic effect with a minimum of $75.4^{\circ} \mathrm{C}$ ). In the interval $190-250^{\circ} \mathrm{C}$ there is a mass loss of $2.44 \%$, accompanied by an exothermic effect with a maximum of $217.6^{\circ} \mathrm{C}$, which indicates an oxidation phenomenon. The main stage of mass loss (78.89\%) takes place in the range of $250-490^{\circ} \mathrm{C}$ and is accompanied in the first part by an exothermic effect with a maximum of $359^{\circ} \mathrm{C}$, then by an endothermic effect generated by breaking some bonds and eliminating fragments such as $\mathrm{C} 11 \mathrm{H} 12$ (1-cyclopentene-1-1yl-benzene). In the range 490$635^{\circ} \mathrm{C}$ there is a mass loss of $3.85 \%$, the process being accompanied by two separate effects with maximums at 494.9 and $611.7^{\circ} \mathrm{C}$ (both exothermic effects are wide and asymmetric indicating several overlapping reactions). In principle, in this interval the residual carbon mass is oxidized. The last stage, between $635-820^{\circ} \mathrm{C}$ represents an endothermic decomposition process (mass loss is $5.56 \%$ and the minimum effect is at $781.1^{\circ} \mathrm{C}$ ). The residual mass is $8.75 \%$ and can most likely be associated with the filler used in SBS stabilization.

Sample 14 is relatively stable up to $190^{\circ} \mathrm{C}(0.57 \%$ mass loss being caused by the elimination of traces of solvent - endothermic effect with a minimum of $68.9^{\circ} \mathrm{C}$ ). In the interval $190-250^{\circ} \mathrm{C}$ there is a mass loss of $2.13 \%$, accompanied by an exothermic effect with a maximum of $231.5^{\circ} \mathrm{C}$, which indicates an oxidation phenomenon. This process takes place at a slightly higher temperature than in the control SBS sample, indicating that the incorporation of dolomite has a protective role against this oxidation process. The main stage of weight loss $(42.19 \%)$ takes place in the range of $250-470^{\circ} \mathrm{C}$ and is accompanied by two exothermic effects, partially overlapping with maximums at $363^{\circ} \mathrm{C}$ 
and $430.5^{\circ} \mathrm{C}$. Basically, the addition of dolomite delays the first oxidation process and modifies the decomposition mechanism, intervening in this interval a second oxidation process. In the range $470-625^{\circ} \mathrm{C}$ there is a mass loss of $6.35 \%$, the process being accompanied by two separate effects with maximums at 520.6 and $594.4^{\circ} \mathrm{C}$ (both exothermic effects are wide and asymmetric indicating several overlapping reactions). In principle, in this interval the residual carbon mass is oxidized. Although we have less organic matter, we have more residual carbon mass burned during this time. A possible explanation is given by the fact that in the previous stage, instead of the elimination of some large organic molecules, only a partial oxidation took place. The last stage, between $625-860^{\circ} \mathrm{C}$, represents an endothermic decomposition process (mass loss is $20.71 \%$ and the minimum effect is at 805.2 and $826.4{ }^{\circ} \mathrm{C}$ plus a shoulder at a lower temperature). The endothermic effect is clearly formed by the superposition of at least 3 individual endothermic effects. The lower temperature shoulder is also associated with the existing filler in SBS. The other two effects are specific to dolomite. It is normal for the mass loss at this stage to be higher, because in addition to the decomposition that was present in SBS, we also have the decomposition of dolomite in the sample. The residual mass is $28.06 \%$, white-gray, consisting mainly of metal oxides. Considering approximately $8 \%$ the residual mass related to SBS and based on the thermal analysis of dolomite (Sönmez et al., 2020), it can be estimated that the mass of the reinforcing agent is $\sim 40 \%$ which is in accordance with the proposed working method.

\section{Physical-Mechanical Characterisation}

Table 2 shows the physical-mechanical values obtained for SBS-based composites reinforced with kaolin and/or various amounts of dolomite. In the case of mixtures containing kaolin and modified/unmodified dolomite (samples 11-13) respectively modified dolomite powder (samples 14-15) tensile strength, presents values with 130 , 141, 212, 182 and 94\% higher compared to the control sample - SBS. A similar trend was observed in the case of the module at $300 \%$, elongations at break and tear strength, these being clearly higher compared to the control test - SBS. This clearly demonstrates that the modification of the surface of kaolin and/or dolomite with $\mathrm{SiO}_{2}$ and $\mathrm{TiO}_{2}$ precursors contributes to the reduction of the surface energy between phases, which leads to a good compatibility and implicitly to improved mechanical properties.

Table 2. Values of physical-mechanical characteristics of SBS and composites

\begin{tabular}{lcccccc}
\hline Symbol /Property & SBS & 11 & 12 & 13 & 14 & 15 \\
\hline Hardness, ${ }^{\circ}$ ShA & $60 \pm 0.05$ & $80 \pm 0.05$ & $85 \pm 0.1$ & $89 \pm 1$ & $91 \pm 0$ & $96 \pm 2.1$ \\
Tensile strength, & $1.29 \pm 0.13$ & $2.97 \pm 0.26$ & $3.11 \pm 0.07$ & $4.03 \pm 0.08$ & $3.64 \pm 0.07$ & $2.5 \pm 0.13$ \\
N/mm & & & & & & \\
Modulus at 300\% & - & $2.87 \pm 0$ & $2.89 \pm 0.04$ & $4.03 \pm 0.08$ & - & $2.23 \pm 0.3$ \\
Elongation at & $120 \pm 57.73$ & $320 \pm 34.64$ & $340 \pm 20$ & $300 \pm 0$ & $300 \pm 0$ & $360 \pm 3$ \\
break, \% & & & & & & \\
Tear strength, & $8.97 \pm 1.84$ & $17.11 \pm 0.71$ & $17.79 \pm 0.17$ & $21.52 \pm 1.15$ & $23 \pm 0.01$ & $16 \pm 0.19$ \\
N/mm & & & & & & \\
\hline
\end{tabular}

These improved mechanical properties are maintained even at high amounts of dolomite, compared to the data presented in the literature, where even the addition of $5 \%$ dolomite in PC/ABS reduces tensile strength and elongation at break, and the amounts between $10-15 \%$ almost completely compromise the properties due to the high susceptibility of dolomite to the phenomenon of agglomeration and void formation 
(Ezenkwa et al., 2019). Similar observations of diminishing elongation at break were also reported in the case of $\mathrm{SBS} /$ nanoCaCO 3 composites (at a loading degree of only 1-3 phr), attributed to the restriction of polymer chain mobility (Vahidi and Azizi, 2017).

\section{CONCLUSIONS}

Composite materials based on SBS and modified or unmodified kaolin and/or dolomite were obtained. According to the mechanical properties, it can be concluded that surface modification of the fillers can bring important improvements especially for tensile and tear strength. Furthermore, the presence of the filler leads to important thermal stabilization. These composites can be used for various applications, such as, for instance, soles for firefighters' footwear.

\section{Acknowledgements}

This work was supported by: 1. Ctr. No. 183 CI/2018 - Thermoplastic polymer and nano/micro particle-based fireproof protection sole for firefighters, and 2. Contract no. 6PFE/2018, Acronym: PERFORM-TEX-PEL.

\section{REFERENCES}

Abdelsalam, A.A., Araby, S., El-Sabbagh, S.H., Abdelmoneim, A. and Hassan, M.A. (2019), "Effect of carbon black loading on mechanical and rheological properties of natural rubber/styrene-butadiene rubber/nitrile butadiene rubber blends", Journal of Thermoplastic Composite Materials, 20(10), 1-18, https://doi.org/10.1177/0892705719844556.

Bicy, K., Abraham, J, Maria, J.H., J. and Sabu, T. (2018), "Styrene-butadiene copolymers", Encyclopedia of Polymer Science and Technology, 1-23, https://doi.org/10.1002/0471440264.pst353.pub2.

Costa, P., Silvia, C., Viana, J.C. and Lanceros Mendez, S. (2014), "Extruded thermoplastic elastomers styrenebutadiene-styrene/carbon nanotubes composites for strain sensor applications", Composites: Part B, 57, 242-24, https://doi.org/10.1016/j.compositesb.2013.10.006.

Das, C., Bansod, N.D., Kapgate, B.P., Rajkumar, K. and Das, A. (2019), "Incorporation of titania nanoparticles in elastomer matrix to develop highly reinforced multifunctional solution styrene butadiene rubber composites", Polymer, 162, 1-10, https://doi.org/10.1016/j.polymer.2018.12.022.

Ezenkwa, O.E., Ismail, A.S., Saidi, A.A.M., Rashid, A.R., Hassan, A. and Arjmandi, R. (2019), "Mechanical and thermal properties of dolomite filled polycarbonate/acrylonitrile butadiene styrene composites", PERINTIS eJournal, 9(1), 1-14.

Jin, J., Gao, Y., Wu, Y., Liu, S., Liu, R., Wei, H., Qian, G. and Zheng, J. (2021), "Rheological and adhesion properties of nano-organic palygorskite and linear SBS on the composite modified asphalt", Powder Technology, 377, 212-221, https://doi.org/10.1016/j.powtec.2020.08.080.

Perov, N.S. (2018), "Relaxation properties of thermoplastic styrene-butadiene block copolymer with nanodiamond filler", Inorganic Materials: Applied Research, 9(4), 670-674, https://doi.org/10.1134/S2075113318040275.

Sönmez, M., Alexandrescu, L., Georgescu, M., Gurau, D., Chelaru, C., Juganaru, M., Ficai, A. and Trusca, R. (2019), "Influence of adding functionalized microparticles on the physical-mechanical, structural, and processability properties of thermoplastic rubber", Leather and Footwear Journal, 19(1), 29-40, https://doi.org/10.24264/1fj.19.1.4.

Sönmez, M., Juganaru, M., Ficai, A., Ficai, D., Oprea, O., Gurau, D., Alexandrescu, L., Stelescu, M.D., Georgescu, M, Nituica, M., Melinescu, A. and Marin, M. (2020), "Dolomite surface modification with titania and silica precursors and its morphostructural and thermal characterization", Proceedings of the $8^{\text {th }}$ International Conference on Advanced Materials and Systems, 1-3 October, Bucharest, Romania, ISSN 2068-0783.

Vahidi, M. and Azizi, H. (2017), "Preparation and properties of styrene butadiene styrene/nano calcium carbonate thermoplastics elastomer composite foams: Morphology and mechanical properties", Cellular Polymers, 36(5), 251-268, https://doi.org/10.1177/026248931703600502. 


\section{II.}

\section{BIOMATERIALS \\ AND BIO- \\ TECHNOLOGIES}


PRELIMINARY ANALYSIS OF EMULSION-BASED FORMULATIONS CONTAINING PUMPKIN SEED OIL AND HEMP SEED OIL FOR INTERNAL USE

\author{
MARIA-CRISTINA ANICESCU, CRISTINA-ELENA DINU-PIRVU*, MIHAELA VIOLETA \\ GHICA, VALENTINA ANUTA, RĂZVAN MIHAI PRISADA, MARINA-THEODORA \\ TALIANU, LĂCRĂMIOARA POPA \\ "Carol Davila" University of Medicine and Pharmacy, Faculty of Pharmacy, Physical and \\ Colloidal Chemistry Department, 6 Traian Vuia Str., 020956, Bucharest, Romania, \\ cristina.dinu@umfcd.ro
}

\begin{abstract}
With a long tradition in pharmaceutical design, emulsions are functional formulations that can maintain their adaptive power connected with the new formulation requirements. Hence, this study proposed preliminary assays concerning the obtaining of natural emulsions for oral administration, incorporating pumpkin seed oil and hemp seed oil as oil phases, with lecithin as emulsifying agent. Using emulsification method, $\mathrm{O} / \mathrm{W}$ and $\mathrm{W} / \mathrm{O}$ emulsions were prepared and characterized from a stability point of view considering organoleptic parameters, conductivity properties followed by an extensive superficial analysis by fitting two different goniometric approaches like contact angle and pendant drop models. The emulsions obtained were stable, homogeneous, their properties being reflected by composition. Conductivity values confirmed the type of emulsions, completing their profile. Superficial analysis revealed that lecithin can sustain a proper stability due to a variation of surface tension values around $25 \mathrm{mN} / \mathrm{m}$. The mean contact angle values ranging between $31.87 \pm 0.51^{\circ}$ and $44.01 \pm 5.48^{\circ}$ defined an adequate wettability, being correlated with the internal structure. To conclude, this preliminary study offered important data concerning the stability of some emulsions for oral delivery, accessing natural biocompatible components. On this way, it can be created multifunctional systems with nutritional value, but also special vehicles designed for drug delivery.
\end{abstract}

Keywords: emulsions, vegetable oils, superficial analysis

\title{
INTRODUCTION
}

Defined as classical coarse dispersions, with multiple applications in pharmaceutical domain, emulsions are colloidal metastable systems composed of two immiscible phases, usually an oil and an aqueous phase (Leal-Calderon et al., 2007). The internal phase will be dispersed in a continuous phase and stabilized ideally with an emulsifier which is recognized for its contribution to the diminishing of the interfacial tension at the contact of the two phases being characterized by superficial and electrical properties (Liu et al., 2020). Simple ternary systems will be resulted, being known as $\mathrm{O} / \mathrm{W}$ and W/O emulsions (Leal-Calderon et al., 2007). Thus, the emulsifier is considered the key element with a major impact for emulsion stability, by avoiding phase separation phenomena, influencing the quality profile and the biopharmaceutical properties of the final formulation (Costa et al., 2019). In this direction, soy lecithin is a natural and safe biocompatible emulsifier with a high content of phospholipidic fractions, where phosphatidylcholine is dominant, presenting amphiphilic properties due to the presence of positive and negative charges (van Hoogevest and Wendel, 2014). It is appreciated for its stabilization power in emulsion manufacturing, largely selected in the formulation of oral preparations on a concentration domain between $1-15 \%$, as a function of each phase concentration and the intended type of emulsion, combined or not with other emulsifiers (Traynor et al., 2014, Ponphaiboon et al., 2018). To create multifunctional emulsions, vegetable oils as natural by-products can be considered a good option in order to ensure the generation of a personalized system, due to their 
biocompatibility and their potential therapeutic outcomes which are studied for centuries and correlated with the relief of some imbalances of the human body normal functions (Górecki et al., 2016). Pumpkin seed oil can be a potent antioxidant due to its composition based on fatty acids and carotenoids, with physiological effects at cellular level. Particular actions are found to be appreciated in the cure of the urinary tract infections with a specific extent on the cure of benign prostatic hyperplasia (Alhakamy et al., 2019). Being rich in omega-3 and omega-6 fatty acids with a high amount of vitamin E, hemp seed oil has multiple benefits for human body, based on antiinflammatory and antioxidant actions which are implied in the prevention of cardiovascular disease, metabolic ailments or degenerative disorders (Miculková et al., 2017).

Considering these statements, this preliminary study aimed to obtain and evaluate a group of $\mathrm{O} / \mathrm{W}$ and W/O emulsions for oral delivery, using lecithin as an emulsifier and two vegetable oil phases, namely pumpkin seed oil and hemp seed oil. It was observed the impact of the emulsifier for each type of formulation with repercussions on physical appearance and stability parameters.

\section{MATERIALS AND METHODS}

\section{Materials}

Liquid soy lecithin was purchased from Amitex (Amitex Agro Product Private Ltd, India), organic certified pumpkin seed oil and hemp seed oil were supplied from NaturalniProdukti.com. Distilled water was used as aqueous phase.

\section{Preparation of $\mathrm{O} / \mathrm{W}$ and W/O Emulsions}

Four formulations, as it can be observed in Table 1, were prepared using 10-50\% oil phase, being distinguished here pumpkin seed oil and hemp seed oil. Water volume was adapted in order to obtain $\mathrm{O} / \mathrm{W}$ or $\mathrm{W} / \mathrm{O}$ emulsions, selecting lecithin $6-10 \%$ as an emulsifier. Using emulsification method, $100 \mathrm{~mL}$ of each emulsion were prepared with respect to the following preparation method: the oil phase was measured in a graduated cylinder. Lecithin was weighed at the analytical balance, then placed in a proper mortar and triturated with small amounts of oil phase until the primary emulsion will be formed. In the last step, the water gently warmed was added in small portions until 100 $\mathrm{mL}$ of emulsion were obtained. The obtained emulsions were shortly coded as: E1, E2, $\mathrm{E} 3$ and $\mathrm{E} 4$, being further remembered in the present paper.

Table 1. Composition of $\mathrm{O} / \mathrm{W}$ and $\mathrm{W} / \mathrm{O}$ emulsions

\begin{tabular}{ccccc}
\hline Sample & $\begin{array}{c}\text { Pumpkin seed oil } \\
(\% \mathrm{v} / \mathrm{v})\end{array}$ & $\begin{array}{c}\text { Hemp seed oil } \\
(\% \mathrm{v} / \mathrm{v})\end{array}$ & $\begin{array}{c}\text { Lecithin } \\
(\% \mathrm{~m} / \mathrm{v})\end{array}$ & $\begin{array}{c}\text { Distilled water } \\
(\% \mathrm{v} / \mathrm{v})\end{array}$ \\
\hline E1 & 10 & - & 10 & 80 \\
E2 & - & 10 & 10 & 80 \\
E3 & 50 & - & 6 & 44 \\
E4 & - & 50 & 6 & 44 \\
\hline
\end{tabular}

\section{Organoleptic Analysis}

The organoleptic analysis was performed after preparation and over seven days. In 
this case, for each formulation were followed characteristics like appearance, consistency, fluidity, homogeneity, color and the presence of instability clues like phase separation. The dilution test was realized to confirm structural modifications in the internal structure of emulsions considering two ratios of dilution 1:4 and 1:10 which were compared with the primary system.

\section{Conductometry Analysis}

Conductivity analysis was carried out using Consort C931 conductivity meter (De Bruyne Instruments, Belgium) designed as a multifunctional platform which was planned for various determinations, being equipped with a platinum electrode calibrated with a saline potassium chloride solution. The determinations were performed in triplicate.

\section{Evaluation of Superficial Properties}

For the evaluation of superficial properties considering the free superficial energy and contact angle measurements, CAM-101 apparatus, equipped with a Hamilton syringe and a C 209-30 needle was used (KSV Instruments Ltd., Finland). Young equation was fitted with two models of analysis: the contact angle (CA) and pedant drop methods. The measurements were performed in triplicate.

\section{RESULTS AND DISCUSSION}

The O/W emulsions formulated with pumpkin seed oil and hemp seed oil were kept their stability over a week from the preparation time. It was observed a homogeneous appearance with a semi-fluid structure which was correlated with the composition of the systems. The yellow color was specific and attributed to the components: the oil phase and the emulsifier. It can be mentioned that $\mathrm{O} / \mathrm{W}$ emulsion with pumpkin seed oil had an intense color than one with hemp seed oil and can be associated with its composition rich in carotenoids. On the other hand, the W/O systems had similar characteristics, considering the aspect and the color parameters, excepting their consistency which was highly viscous and correlated with an elevated amount of oil phase of $50 \%$ which was also responsible for some oxidative phenomena visualized in the superior portion of the vials. In the formulation process for future studies, the attention must be awarded to the addition of proper antioxidants.

After the completion of dilution test, were obtained several data about structural behaviour of $\mathrm{O} / \mathrm{W}$ and $\mathrm{W} / \mathrm{O}$ emulsions, offering preliminary information that were correlated with the future experimental results. Thus, it was observed that $\mathrm{O} / \mathrm{W}$ emulsions E1 and E2 exhibited a good stability after the addition of water fractions. The color intensity was slightly yellow without an intense appearance. No phase separation was observed and the homogeneity was proper. On the opposite, in the case of W/O emulsions, was noted an initiation of phase separation after several minutes of examination, thus, these systems are more susceptible to experience instability events.

Conductivity evaluation is essential for internal structure analysis of fluid colloidal dispersions. On this way, according to Table 2, was confirmed the emulsion type for each sample, obtaining the mean values of conductivity $(\mu \mathrm{S} / \mathrm{cm})$, after samples measuring in triplicate, at $25^{\circ} \mathrm{C}$ at 7 and 14 days after preparation, the values being between 397.66-1081.00 $\mu \mathrm{S} / \mathrm{cm}$ and 604.00-1326.66 $\mu \mathrm{S} / \mathrm{cm}$, respectively. An increase in conductivity after $800 \mu \mathrm{S} / \mathrm{cm}$ was specific for $\mathrm{O} / \mathrm{W}$ emulsions E1 and E2 which 
contained a high amount of aqueous phase of $80 \%$, while the inversed type represented by E3 and E4 had lower values being correlated with the incorporation of a large concentration of oil phase.

Table 2. Conductivity values for the $\mathrm{O} / \mathrm{W}$ and $\mathrm{W} / \mathrm{O}$ emulsions prepared with pumpkin seed oil and hemp seed oil tested at $25^{\circ} \mathrm{C}$ and mean values \pm SD at 7 days and 14 days from preparation time

\begin{tabular}{cccc}
\hline Emulsion & $\begin{array}{c}\text { Mean values of } \\
\text { conductivity } \pm \mathrm{SD}(\mu \mathrm{S} / \mathrm{cm}) \\
\text { after } 7 \text { days }\end{array}$ & $\begin{array}{c}\text { Mean values of } \\
\text { conductivity } \pm \mathrm{SD}(\mu \mathrm{S} / \mathrm{cm}) \\
\text { after } 14 \text { days }\end{array}$ & $\begin{array}{c}\text { Emulsion } \\
\text { type }\end{array}$ \\
\hline E1 & $934.66 \pm 1.59$ & $1111.00 \pm 10.00$ & $\mathrm{O} / \mathrm{W}$ \\
$\mathrm{E} 2$ & $1081.00 \pm 4.58$ & $1326.66 \pm 10.40$ & $\mathrm{O} / \mathrm{W}$ \\
$\mathrm{E} 3$ & $397.66 \pm 5.13$ & $712.33 \pm 6.42$ & $\mathrm{~W} / \mathrm{O}$ \\
$\mathrm{E} 4$ & $401.00 \pm 2.64$ & $604.00 \pm 19.15$ & $\mathrm{~W} / \mathrm{O}$ \\
\hline
\end{tabular}

It is important to note that the inclusion of lecithin as an emulsifier will influence the conductivity due to its amphiphilic structure, with cationic and anionic charges. Thus, the main components that will influence the electrical properties of the emulsions are water molecules and the emulsifier.

Considering the superficial properties, the formulated emulsions E1, E2, E3 and E4 were analysed by applying goniometric principles. The superficial tension was determined using the equation Young (Popa et al., 2013):

$\gamma_{\mathrm{SG}}=\gamma_{\mathrm{SL}}+\gamma_{\mathrm{LG}} \cdot \cos \theta$

The equation was correlated with two representative models that can offer valuable information considering the surface properties and peculiarities of the internal structure for both $\mathrm{O} / \mathrm{W}$ and $\mathrm{W} / \mathrm{O}$ emulsions: the pendant drop and contact angle models. Thus, in Table 3, are presented results specific for both types of determinations, while in Figure 1 , it can be depicted a variation of mean superficial tension values in time $(9 \mathrm{~s})$ for each emulsion and also the variation of the mean values of contact angle in time $(4 \mathrm{~s})$. The mean values of surface tension had a similarity over the analysis for both type of emulsions, being a mark of their stability. It can be considered that lecithin as a potent emulsifier will create at the oil/water interface a monomolecular layer with the aim to decrease the interfacial tension, forming an electrical barrier that keeps the particles dispersed in the continuous medium.

Table 3. Results of the mean values $\pm \mathrm{SD}$ after superficial assays for $\mathrm{O} / \mathrm{W}$ and $\mathrm{W} / \mathrm{O}$ emulsions prepared with vegetable oils and lecithin

\begin{tabular}{cccc}
\hline Emulsion & $\begin{array}{c}\text { Surface tension } \\
\text { Stm }(\mathrm{mN} / \mathrm{m}) \\
\text { Pendant drop model }\end{array}$ & $\begin{array}{c}\text { Surface tension } \\
\text { StM }(\mathrm{mN} / \mathrm{m}) \\
\text { Contact angle model }\end{array}$ & $\begin{array}{c}\text { Contact } \\
\text { angle } \\
\left({ }^{\circ}\right)\end{array}$ \\
\hline E1 & $25.14 \pm 0.97$ & $32.21 \pm 5.11$ & $40.46 \pm 4.89$ \\
E2 & $23.46 \pm 0.60$ & $24.40 \pm 2.88$ & $32.05 \pm 2.01$ \\
E3 & $23.32 \pm 0.37$ & $25.23 \pm 2.92$ & $31.87 \pm 0.51$ \\
E4 & $25.35 \pm 2.34$ & $26.69 \pm 2.53$ & $44.01 \pm 5.48$ \\
\hline
\end{tabular}




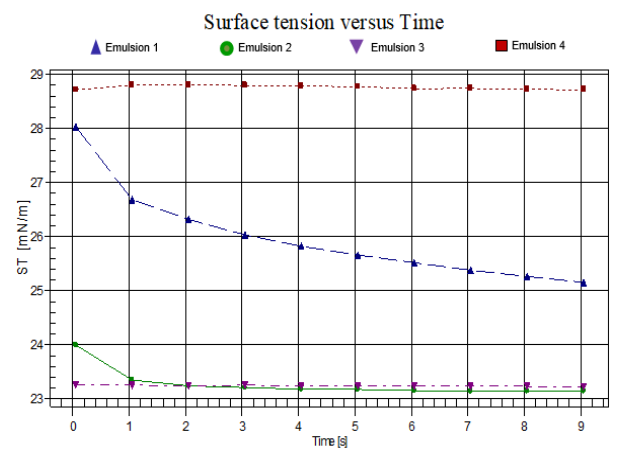

a)

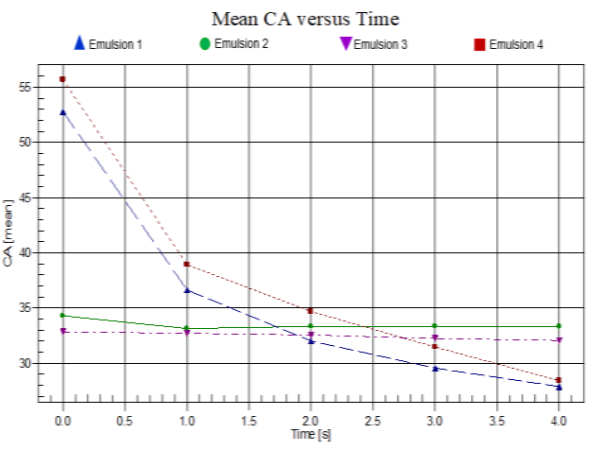

b)

Figure 1. Graphical representations for superficial parameters:

a) Cumulative profiles showing the variation of superficial tension $(\mathrm{mN} / \mathrm{m})$ versus time (s) for O/W and W/O emulsions E1, E2, E3 and E4

b) Cumulative profiles showing the variation of contact angle for $\mathrm{O} / \mathrm{W}$ and $\mathrm{W} / \mathrm{O}$ emulsions expressed as mean contact angle $\left({ }^{\circ}\right)$ as a function of time (s)

The contact angle model applied in the case of each emulsion, gave information concerning the wettability properties, exploring the hydrophilic or hydrophobic behaviour of $\mathrm{O} / \mathrm{W}$ and $\mathrm{W} / \mathrm{O}$ formulations. Using this model, in Figure 2 are proposed a series of determinations with the most representative results, where it can be observed that the $\mathrm{O} / \mathrm{W}$ emulsions $\mathrm{E} 1$ and $\mathrm{E} 2$ are characterized by a high wettability, being observed changes in the contact angle value on a period of 4 seconds. In the case of E2, where the contact angle keeps it value around $35^{\circ}$, wasn't observed major changes. In the case of W/O emulsions E3 and E4 with 50\% oil phase, a slightly hydrophobic character of the droplets was dominant for $\mathrm{E} 4$, with significant modifications of hysteresis from $55.69^{\circ}$ to $28.40^{\circ}$. Considering the placement of contact angle values in the domain of $0-90^{\circ}$ for each formulation it was experienced an incomplete wettability, being deducted that the adhesion forces at the solid surface are superior than cohesion ones, but not as powerful to generate a complete wettability to reach a $0^{\circ}$ contact angle. As a final note, the hydrophilicity will be increased for the emulsions in the following order, from the most hydrophobic through the most hydrophilic structures: $\mathrm{E} 4>\mathrm{E} 1>\mathrm{E} 2>\mathrm{E} 3$.

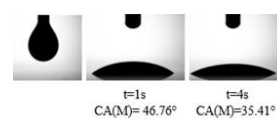

a)

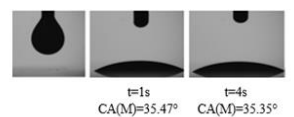

b)

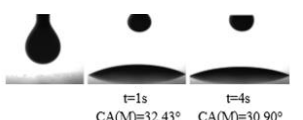

c)

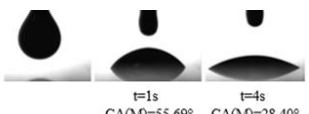

d)

Figure 2. Pendant drops and evolution of contact angle for $\mathrm{O} / \mathrm{W}$ and $\mathrm{W} / \mathrm{O}$ emulsions from $1 \mathrm{~s}$ to $4 \mathrm{~s}$ : case a) contact angle variation for emulsion E1 with pumpkin oil; b) contact angle variation for emulsion E2 with hemp seed oil; c) contact angle variation for emulsion E3 with pumpkin seed oil, and d) contact angle variation for emulsion E4 with hemp seed oil 


\section{CONCLUSIONS}

It can be appreciated that the emulsions prepared with vegetable oils are functional colloidal dispersions with a complex pattern which was analysed in the present preliminary study. In this direction, were obtained stable, homogeneous systems, with a proper appearance which was correlated with the composition. O/W emulsions had a superior stability than W/O type, due to a large amount of lecithin selected in the formulation. The conductivity evaluation confirmed the type of each emulsion. The results of superficial analysis proved the lecithin influence in the diminishing of surface tension, in order to promote stable systems, as well as the wettability behaviour which was characterized by contact angles under $90^{\circ}$, exhibiting the dynamics occurred between hydrophilic and hydrophobic forces at a solid surface. Based on their properties, the designed emulsions can be attractive systems with biocompatible components for oral administration, being a suitable option for drug formulation.

\section{Acknowledgements}

This paper was financially supported by "Carol Davila" University of Medicine and Pharmacy through Contract No. CNFIS-FDI-2020-0604 (MEDEX-III) funded by the Ministry of Education and Research, Romania, from the Institutional Development Fund for Public Universities - FDI 2020.

\section{REFERENCES}

Alhakamy, N.A., Fahmy, U.A. and Ahmed, O. (2019), “Attenuation of Benign Prostatic Hyperplasia by Optimized Tadalafil Loaded Pumpkin Seed Oil-Based Self Nanoemulsion: In Vitro and In Vivo Evaluation", Pharmaceutics, 11(12), 1-13, https://doi.org/10.3390/pharmaceutics11120640.

Costa, C., Medronho, B., Filipe, A., Mira, I., Lindman, B., Edlund, H. and Norgren, M. (2019), "Emulsion Formation and Stabilization by Biomolecules: The Leading Role of Cellulose", Polymers, 11(10), 1-18, https://doi.org/10.3390/polym11101570.

Górecki, M., Sosada, M., Pasker, B., Pająk, M. and Fraś, P. (2016), "Preparation of Ibuprofen Emulsions with Rapeseed Phospholipids and Vegetable Oils", Ind. J. Pharm. Ed. Res., 50(2), 271-276, https://doi.org/10.5530/ijper.50.2.7

Leal-Calderon, F., Schmitt, V. and Bibette, J. (2007), "Emulsion Science Basic Principles”, 2nd ed., Springer, Verlag, New York, 1-4, https://doi.org/10.1007/978-0-387-39683-5.

Liu, Y., Wei, Z.C., Deng, Y.Y., Dong, H., Zhang, Y., Tang, X.J., Li, P., Liu, G. and Zhang, M.W. (2020), "Comparison of the Effects of Different Food-Grade Emulsifiers on the Properties and Stability of a Casein-Maltodextrin-Soybean Oil Compound Emulsion”, Molecules, 25(3), 1-16, https://doi.org/10.3390/molecules25030458.

Mikulcová, V., Kašpárková, V., Humpolíček, P., Buňková, L. (2017), "Formulation, Characterization and Properties of Hemp Seed Oil and Its Emulsions", Molecules, 22(5), 1-13, https://doi.org/10.3390/molecules22050700.

Ponphaiboon, J., Limmatvapirat, S. and Limmatvapirat, C. (2018), "Influence of Emulsifiers on Physical Properties of Oil/Water Emulsions Containing Ostrich Oil", Key Engine. Mat., 777, 592-596, https://doi.org/10.4028/www.scientific.net/KEM.777.592.

Popa, L., Ghica, M.V., Albu, M.G., Ortan, A. and Dinu-Pirvu, C.E. (2013), "Hysteresis of Contact Angle. Dynamic Wettability Studies of Collagen and Doxycycline Porous Matrices Crosslinked with Tannic Acid", Dig. J. Nanomater. Biostruct., 8(3), 937-943.

Traynor, M., Burke, R., Frias, J.M. Gaston, E. and Barry-Ryan, C. (2013), "Formation and stability of an oil in water emulsion containing lecithin, xanthan gum and sunflower oil”, Int. Food. Res. J., 20(5), 2173 2181.

van Hoogevest, P. and Wendel, A. (2014), “The use of natural and synthetic phospholipids as pharmaceutical excipients", Eur. J. Lipid Sci. Technol., 116(9), 1088-1107, https://doi.org/10.1002/ejlt.201400219. 
ANTIBACTERIAL AND ANTIOXIDANT ACTIVITIES OF LEMON BALM (Melissa officinalis L.) ESSENTIAL OIL

\author{
MARIANA DANIELA BERECHET ${ }^{1}$, DEMETRA SIMION ${ }^{1}$, MARIA STANCA ${ }^{1}$, \\ COSMIN-ANDREI ALEXE ${ }^{1}$, RODICA ROXANA CONSTANTINESCU ${ }^{1}$, \\ MARIA RÂPĂ ${ }^{2}$, ANDREEA ȚURCANU ${ }^{2}$ \\ ${ }^{1}$ INCDTP - Division Leather and Footwear Research Institute, 031215 Bucharest, Romania; \\ marianadanielaberechet@yahoo.co.uk; demetra.simion@yahoo.com; maria.stanca@icpi.ro; \\ cosminandrei.alexe@yahoo.com;rodica.roxana@yahoo.com \\ ${ }^{2}$ Faculty of Materials Science and Engineering, University Politehnica of Bucharest, 313 Spl. \\ Independentei, 060042 Bucharest, Romania; rapa_m2002@yahoo.com; \\ andreea.turcanu90@yahoo.com
}

\begin{abstract}
Lemon balm (Melissa officinalis L.) belongs to the Lamiaceae family. Essential oil extracted from the aerial parts of lemon balm has been investigated for the protection of fruits during storage, as insecticidal, as well as in medicine, due to its bioactive properties. In this paper, the composition and identification of components from Melissa officinalis L. essential oil were determined by gas chromatography coupled with mass spectrophotometry (GC/MS) analysis. Total phenol content (TPC) and the scavenging activity towards 2,2-diphenyl-1-picrylhydrazyl (DPPH') and 2,2'-azinobis (3-ethylbenzthiazoline)-6-sulfonic acid $\left(\mathrm{ABTS}^{+\bullet}\right.$ ) free radicals were evaluated by UV-VIS spectrometry. Antibacterial activities were carried out against Staphylococcus aureus, Escherichia coli and Klebsiella pneumoniae. Seventeen bioactive compounds were found as constituents of Melissa officinalis L. essential oil, among which o-cymene (19.735\%), dehydro-p-cymene $(17.180 \%)$, and limonene $(11.589 \%)$ were found as the major components. Attenuated total reflectance Fourier transform infrared spectroscopy (ATR-FT-IR) provided a confirmation for the chemical components of lemon balm essential oil identified by GC/MS. The values recorded for TPC and antioxidant activity were as follows: $54.72 \mathrm{mg} \mathrm{GAE} / \mathrm{g}$ dry substance, $28.53 \%$ for DPPH, and $46.17 \%$ for ABTS assays, respectively. $100 \mu \mathrm{L}$ lemon balm essential oil proved total antibacterial activity against the tested microorganisms. The results showed that the Melissa officinalis L. may be a good candidate as plant-derived antioxidant and antibacterial agent for medical footwear, wound dressings and other medical applications.
\end{abstract}

Keywords: Melissa officinalis L., bioactive compounds, antioxidant activity, antibacterial activity

\title{
INTRODUCTION
}

Lemon balm (Melissa officinalis L.) belongs to the Lamiaceae family. Melissa officinalis essential oil is characterized by a fresh, lemon aroma, slightly sweet,color pale yellow and a watery viscosity. Lemon balm oil soothes and has excellent qualities in combating depression. It has a sedative effect, helps in cases of panic, slow down the heartbeat, lowers blood pressure and is a good tonic for the heart. The monoterpene oxygenated and sesquiterpenes were found as the major components of $M$. officinalis essential oil (Ghasemi Pirbalouti et al., 2019). Antimicrobial and antioxidant activities of M. officinalis essential oil can be attributed to its polyphenol compounds. These natural antioxidants are responsible for reactive oxygen species (ROS) scavenging capacity (Lin et al., 2012). In a study performed by Anastasaki et al. (2017) it was shown that the total phenol content (TPC) depends on the extraction solvent used in the maceration technique. Thus, in the case of plant extract with petroleum ether, the TPC was found $0.30 \pm 0.03 \mathrm{mg}$ of Gallic acid equivalent (GAE) per $\mathrm{g}$ of dry plant weight $(\mathrm{dw})$, and $64.29 \pm 5.70 \mathrm{mg} / \mathrm{g} \mathrm{GAE} / \mathrm{g}$ by using of methanol as extraction medium. Essential oil extracted from aerial parts of lemon balm has been investigated fortheprotection of fruits during storage (El Ouadi et al., 2017), as insecticidal (Ghasemi Pirbalouti et al., 2019), as well as in medicine (Lin et al., 2012), due to its bioactive 
properties. However, M. officinalis essential oil administered in doses higher than $1 \mathrm{~g} \mathrm{~kg}^{-1}$ produced acute oral toxicity in rat model (Stojanović et al., 2019). M. officinalis essential oil $(0,0.25$ and $0.5 \%(\mathrm{w} / \mathrm{v}))$ together with chitosan and zinc oxide have been used for the preparation of antimicrobial biodegradable composite film having food packaging application, due to the good antimicrobial activity against $E$. coli, antioxidant activity and mechanical properties (Sani et al., 2019).

\section{MATERIALS AND METHODS}

\section{Material}

Lemon balm (Melissa officinalis L.) essential oil was acquired from Solaris Plant SRL-Radix (Darmstadt, Germany). Cotton material was used for impregnation with different concentrations of essential oils in order to simulate the footwear lining or bandages. Other chemical reagents for analysis were analytical grade.

\section{Methods}

\section{Preparation of Tested Materials}

Discs of cotton samples (20 mm diameter cotton discs, three for each sample) were treated with different amounts of lemon balm essential oil: $30 \mu \mathrm{L}, 50 \mu \mathrm{L}$, and $100 \mu \mathrm{L}$, labeled as $\mathrm{BBCR}_{30}, \mathrm{BBCR}_{50}$, and $\mathrm{BBCR}_{100}$, respectively. Cotton samples without essential oil were used as control.

\section{GC/MS Analysis}

In order to determine the bioactive compounds from lemon balm essential oil, a Thermo Scientific gas chromatograph coupled with mass spectrometer, DSQ II MS, equipped with TR-5 MS non-polar capillary column $(60 \mathrm{~m} \times 0.25 \mu \mathrm{m} \times 0.25 \mu \mathrm{m})$ was used. The temperature was set in the range between room temperature to $350^{\circ} \mathrm{C}$, heating rate was programmable between 0.1 and $120^{\circ} \mathrm{C} / \mathrm{min}$, temperature of split/splitless injector was in the range of $50^{\circ} \mathrm{C}$ and $375^{\circ} \mathrm{C}$, and pneumatic control system of pressure/carrying helium gas flow was of $5 \%$.

\section{ATR-FTIR Spectroscopy}

ATR-FTIR analysis was performed with a FT-IR/ATR spectrometer-Jasco 4200 operating in the range of 4000 to $600 \mathrm{~cm}^{-1}$, with a spectral resolution of $0.5 \mathrm{~cm}^{-1}$.

\section{Determination of Total Phenol Content (TPC)}

The total phenol content was determined using Folin-Ciocalteu method. To $0.5 \mathrm{~mL}$ of the sample, $3.6 \mathrm{~mL} \mathrm{H}_{2} \mathrm{O}$, and $0.24 \mathrm{~mL}$ Folin-Ciocalteu reagent were added. After 5 min, $0.68 \mathrm{~mL}$ of $7.5 \% \mathrm{Na}_{2} \mathrm{CO}_{3}$ were added and the resulted solution was mixed and allowed to stand for $30 \mathrm{~min}$ at $40^{\circ} \mathrm{C}$. The absorbance at $750 \mathrm{~nm}$ was measured using a UV-Vis spectrophotometer, and calculated based on a calibration curve with Gallic acid $\left(0-500 \mathrm{mg} / \mathrm{mL}, \mathrm{R}^{2}=0.995\right)$ previously established. The total phenol content was expressed as Gallic acid equivalent (GAE) in $\mathrm{mg} / \mathrm{g}$ of dry weight. 
DPPH Radical-Scavenging Activity

The 2,2-diphenyl-1-picrylhydrazyl (DPPH) radical-scavenging activity was evaluated using the method proposed by Sharma et al. (2017). $2.5 \mathrm{~mL}$ of DPPH solution (100 $\mu \mathrm{M}$ obtained by dissolving in ethanol) was added to $500 \mu \mathrm{L}$ of sample (concentration of $60 \mathrm{mg} / \mathrm{mL}$ ). The mixture resulted was shaken vigorously and kept in the dark for $30 \mathrm{~min}$ at room temperature. The decrease in absorbance of the analyzed solution at $517 \mathrm{~nm}$ was monitored using an UV-Vis spectrophotometer. Radical scavenging antioxidant activity of sample was calculated according to the equation:

DPPH free radical scavenging activity $(\%)=\left[\left(\mathrm{A}_{\mathrm{c}}-\mathrm{A}_{\mathrm{s}}\right) / \mathrm{A}_{\mathrm{c}}\right] \times 100$

where: $A_{C}-$ absorbance of control, $A_{s}-$ absorbance of sample.

\section{ABTS Free Radical Scavenging Assay}

The experiment was carried out using an improved ABTS discoloring assay (Huang et al., 2017). The 2,2'-azino-bis-(3-ethylbenzothiazoline-6-sulfonate) radical cation $\left(\mathrm{ABTS}^{+\bullet}\right.$ ) was produced by reacting $7 \mathrm{mM}$ stock solution of ABTS with $2.45 \mathrm{mM}$ potassium persulphate and allowing the mixture to stand in the dark for at least $16 \mathrm{~h}$ at room temperature prior to use. The $\mathrm{ABTS}^{+\bullet}$ solution was diluted to an absorbance of 0.7 \pm 0.05 at $750 \mathrm{~nm}$. $140 \mu \mathrm{L}$ of essential oil $(60 \mathrm{mg} / \mathrm{mL})$ were mixed with $4 \mathrm{~mL}$ ABTS $^{+\bullet}$ solution and kept in the dark, at room temperature for 6 min. The affinity of essential oil to quench ABTS free radical was evaluated according to the following equation:

ABTS free radical scavenging activity $\left.(\%)=\left[\left(\mathrm{A}_{\mathrm{C}}-\mathrm{A}_{\mathrm{s}}\right) / \mathrm{A}_{\mathrm{C}}\right)\right] \times 100$

where: $A_{C}-$ absorbance of control, $A_{s}$-absorbance of sample.

All the experiments were carried out in triplicates $(n=3)$ for each sample and mean average values with standard deviation $( \pm \mathrm{SD})$ were reported.

\section{Microbiological Analyses}

Microbiological analyses were performed according to ISO 20743:2013, Textiles Determination of antibacterial activity of textile products, the absorption method.

\section{RESULTS AND DISCUSSIONS}

The composition and bioactive compounds from lemon balm essential oil were determined by GC-MS analysis. The major compounds found in lemon balm essential oil were (\% of area): $19.735 \%$ of $o$-cymene, $17.180 \%$ of dehydro-p-cymene and $11.589 \%$ of limonene. In this study, seventeen bioactive compounds were detected as constituents of Melissa officinalis essential oil representing more than $50 \%$ of the composition. El Ouadi et al. (2017) reported the P-mentha-1,2,3-triol (13.1\%), P-menth3 -en-8-ol (8.8\%), piperitenone oxide (8.4\%) and Z-piperitone oxide $(7.3 \%)$ as the main compounds identified in M. officinalis essential oil. A comparison of our results with the previous reports (Ghasemi Pirbalouti et al., 2019; Stojanović et al., 2019) evidenced some differences in the volatile composition of the plant assigned to the geographic origin of the plant sample, methods of extraction, and environmental factors.

The ATR-FTIR spectrum showed in Figure 1 reveals the spectral bands at: $2920 \mathrm{~cm}^{-1}$, $2857 \mathrm{~cm}^{-1}$ (aliphatic C-H), $1727 \mathrm{~cm}^{-1}, 1673 \mathrm{~cm}^{-1}, 1632 \mathrm{~cm}^{-1}, 1444 \mathrm{~cm}^{-1}$ (C=C stretching vibration of the aromatic ring from $p$-cymene), $1379 \mathrm{~cm}^{-1}$ (isopropyl methyl group symmetric bending vibration), $1232 \mathrm{~cm}^{-1}$ (C-O-C stretching), $1191 \mathrm{~cm}^{-1}, 1154 \mathrm{~cm}^{-1}$, 
$1118 \mathrm{~cm}^{-1}$ ( $\mathrm{CH}_{3}$ deformations), $1022 \mathrm{~cm}^{-1}, 985 \mathrm{~cm}^{-1}$ (para-substituted phenyl) (Valderrama and Rojas De, 2017), $885 \mathrm{~cm}^{-1}, 841 \mathrm{~cm}^{-1}$ (C-H bend pattern from dehydro$p$-cymene and limonene), $743 \mathrm{~cm}^{-1}$, (aromatic C-H out-of-plane bend from $o$-cymene).

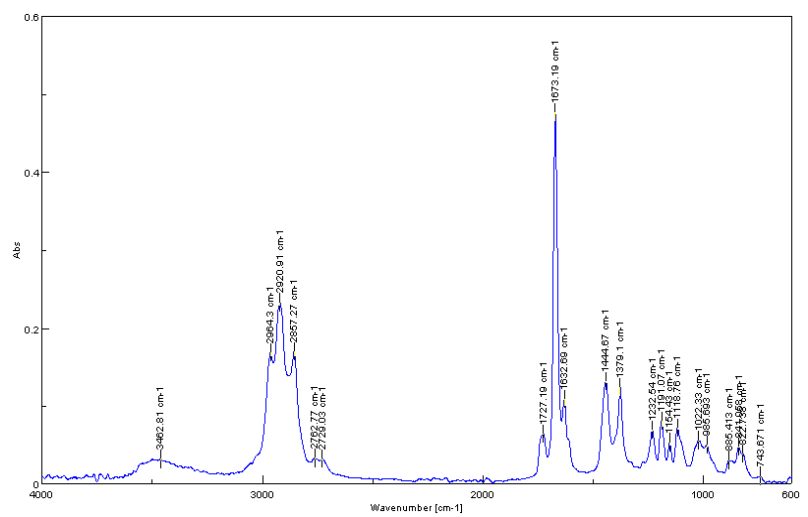

Figure 1. ATR-FTIR spectrum of Melissa officinalis essential oil

The M. officinalis essential oil was evaluated for the total phenol content and antioxidant activities by using DPPH and ABTS radical scavenging bioassays. The results are shown in Table 1.

Table 1. TPC and the radical scavenging activity results for $M$. officinalis essential oil

\begin{tabular}{ccc}
\hline $\begin{array}{c}\text { TPC } \\
\text { (mg GAE/g dry substance) }\end{array}$ & $\begin{array}{c}\text { DPPH free radical } \\
\text { scavenging activity }(\%)\end{array}$ & $\begin{array}{c}\text { ABTS free radical } \\
\text { scavenging activity }(\%)\end{array}$ \\
\hline $54.72 \pm 4.8$ & $28.53 \pm 5.4$ & $46.17 \pm 4.7$ \\
\hline
\end{tabular}

The results from Table 1 prove the potential of $M$. officinalis essential oil to be used as antioxidant agent. The total phenol content is comparable with similar reported values for extracts in petroleum ether (Lin et al., 2012; Anastasaki et al., 2017). Antibacterial properties were performed against Staphylococcus aureus ATCC 6538 (Table 2), Escherichia coli ATCC 10536 (Table 3) and Klebsiella pneumonia ATCC 4352 (Table 4).

Table 2. Resistance against Staphylococcus aureus of cotton samples treated with $M$. officinalis essential oil

\begin{tabular}{lccccc}
\hline Sample & Images & $\mathrm{T}_{0}(\mathrm{UFC} / \mathrm{mL})$ & $\mathrm{T}_{24}(\mathrm{UFC} / \mathrm{mL})$ & $\mathrm{R}(\%)$ & $\mathrm{Log}_{10}$ \\
\hline $\begin{array}{l}\text { Inoculum } \\
\text { concentration }\end{array}$ & $1 \times 10^{5}$ & - & - & - \\
Control & $1 \times 10^{5}$ & $1.115 \times 10^{3}$ & 98.88 & 1.95 \\
$\mathrm{BBCR}_{30}$ & $1 \times 10^{5}$ & $3.5 \times 10^{2}$ & 99.65 & 3.46 \\
$\mathrm{BBCR}_{50}$ & $1 \times 10^{5}$ & $1.6 \times 10^{1}$ & 99.98 & 3.79 \\
$\mathrm{BBCR}_{100}$ & $1 \times 10^{5}$ & 4 & 100 & 4.4 \\
\hline
\end{tabular}

https://doi.org/10.24264/icams-2020.II.2 
Table 3. Resistance against Escherichia coli of cotton samples treated with $M$. officinalis essential oil

\begin{tabular}{lccccc}
\hline Sample & Images & $\mathrm{T}_{0}(\mathrm{UFC} / \mathrm{mL})$ & $\mathrm{T}_{24}(\mathrm{UFC} / \mathrm{mL})$ & $\mathrm{R}(\%)$ & $\mathrm{Log}_{10}$ \\
\hline $\begin{array}{l}\text { Inoculum } \\
\text { concentration } \\
\text { Control }\end{array}$ & $1 \times 10^{5}$ & - & - & - \\
$\mathrm{BBCR}_{30}$ & $1 \times 10^{5}$ & $1.12 \times 10^{3}$ & 98.88 & 1.96 \\
$\mathrm{BBCR}_{50}$ & $1 \times 10^{5}$ & $5.44 \times 10^{2}$ & 99.46 & 2.26 \\
$\mathrm{BBCR}_{100}$ & $1 \times 10^{5}$ & $1.6 \times 10^{1}$ & 99.98 & 3.79 \\
& & $1 \times 10^{5}$ & 1 & 100 & 5 \\
\hline
\end{tabular}

Table 4. Resistance against Klebsiella pneumonia of cotton samples treated with $M$. officinalis essential oil

\begin{tabular}{lccccc}
\hline Sample & Images & $\mathrm{T}_{0}(\mathrm{UFC} / \mathrm{mL})$ & $\mathrm{T}_{24}(\mathrm{UFC} / \mathrm{mL})$ & $\mathrm{R}(\%)$ & $\mathrm{Log}_{10}$ \\
\hline $\begin{array}{l}\text { Inoculum } \\
\text { concentration }\end{array}$ & $1 \times 10^{5}$ & - & - & - \\
Control & $1 \times 10^{5}$ & $7.2 \times 10^{2}$ & 99.2 & 2.14 \\
BBCR $_{30}$ & $1 \times 10^{5}$ & $7 \times 10^{1}$ & 99.93 & 3.15 \\
BBCR50 & $1 \times 10^{5}$ & $2.4 \times 10^{1}$ & 99.98 & 3.62 \\
BBCR $_{100}$ & $1 \times 10^{5}$ & 0 & 100 & 5 \\
& & & & & \\
\hline
\end{tabular}

The results from Tables 2-4 showed that the cotton samples treated with $100 \mu \mathrm{L}$ lemon balm essential oil have $100 \%$ resistance against Staphylococcus aureus, Escherichia coli and Klebsiella pneumonia.

\section{CONCLUSION}

Melissa officinalis L. essential oil was investigated for its antioxidant by TPC, DPPH and ABTS methods and showed $54.72 \mathrm{mg}$ GAE/g dry substance, $28.53 \%$ for DPPH, and $46.17 \%$ for ABTS assays, respectively. The antibacterial activity tested against Staphylococcus aureus, Escherichia coli and Klebsiella pneumonia on cotton samples treated with different concentrations of essential oil proved $100 \%$ UFC reduction for samples treated with $100 \mu \mathrm{L}$ of essential oil. The main bioactive compounds detected by GC-MS, o-cymene, dehydro- $p$-cymene and limonene are responsible for the high antioxidant and antibacterial activities. Melissa officinalis L. essential oil could be used as potential bioactive agent for medical footwear, wound dressings and other medical applications. 


\section{Acknowledgements}

The research was carried out under project no. 19/2019,PN 1917 01 02,CREATIV_PIEL, 4PERFORM-TEX-PEL project, funded by Romanian Ministry of Education and Research.

\section{REFERENCES}

Anastasaki, E., Zoumpopoulou, G., Astraka, K., Kampoli, E., Skoumpi, G., Papadimitriou, K., Tsakalidou, E and Polissiou, M. (2017), "Phytochemical analysis and evaluation of the antioxidant and antimicrobial properties of selected herbs cultivated in Greece", Industrial Crops \& Products, 108, 616-628, https://doi.org/10.1016/j.indcrop.2017.06.066.

El Ouadi, Y., Manssouri, M., Bouyanzer, A., Majidi, L., Bendaif, H., Elmsellem, H., Shariati, M.A. Melhaoui, A. and Hammouti, B. (2017), "Essential oil composition and antifungal activity of Melissa officinalis originating from north-east Morocco, against postharvest phytopathogenic fungi in apples", Microbial Pathogenesis, 107, 321- 326, https://doi.org/10.1016/j.micpath.2017.04.004.

Ghasemi Pirbalouti, A., Nekoei, M., Rahimmalek, M. and Malekpoor, F. (2019), "Chemical composition and yield of essential oil from lemon balm (Melissa officinalis L.) under foliar applications of jasmonic and salicylic acids", Biocatalysis and Agricultural Biotechnology, 19, 101144, https://doi.org/10.1016/j.bcab.2019.101144.

Huang, X., Dai, Y., Cai, J., Zhong, N., Xiao, H., McClements, D.J. and Hu, K. (2017), "Resveratrol encapsulation in core-shell biopolymer nanoparticles: Impact on antioxidant and cancer activities", Food Hydrocolloids, 64157-165, https://doi.org/10.1016/j.foodhyd.2016.10.029.

Lin, J.T., Chen, Y.C., Lee, Y.C., Rolis Hou, C.W., Chen, F.L. and Yang, D.J. (2012), “Antioxidant, antiproliferative and cyclooxygenase-2 inhibitory activities of ethanolic extracts from lemon balm (Melissa officinalis L.) leaves", LWT, 49(1), 1-7, https://doi.org/10.1016/j.lwt.2012.04.009.

Sani, I.K., Pirsa, S. and Tağı, Ş. (2019), "Preparation of chitosan/zinc oxide/Melissa officinalis essential oil nano-composite film and evaluation of physical, mechanical and antimicrobial properties by response surface method", Polymer Testing, 79, 106004, https://doi.org/10.1016/j.polymertesting.2019.106004.

Sharma, S., Gupta, A., Chik, S.M.S.T., Kee, C.G., Mistry, B.M., Kim, D.H. and Sharma, G. (2017), "Characterization of keratin microparticles from feather biomass with potent antioxidant and anticancer activities", International Journal of Biological Macromolecules, 104, 189-196, https://doi.org/10.1016/j.ijbiomac.2017.06.015.

Stojanović, N.M., Randjelović, P.J., Mladenović, M.Z., Ilić, I.R., Petrović, V., Stojiljković, N., Ilić, S., Radulović, N.S. (2019), "Toxic essential oils, part VI: Acute oral toxicity of lemon balm (Melissa officinalis L.) essential oil in BALB/c mice", Food and Chemical Toxicology, 133, 110794, https://doi.org/10.1016/j.fct.2019.110794.

Valderrama, A. and Rojas De, G. (2017), "Traceability of Active Compounds of Essential Oils in Antimicrobial Food Packaging Using a Chemometric Method by ATR-FTIR", American Journal of Analytical Chemistry, 8, 726-741, https://doi.org/10.4236/ajac.2017.811053. 
THE INFLUENCE OF ALKALINE EXTRACTION ON SOME KERATIN HYDROLYSATES PROPERTIES

\author{
MARIANA DANIELA BERECHET ${ }^{1}$, DEMETRA SIMION ${ }^{1}$, MARIA STANCA ${ }^{1}$, \\ CIPRIAN CHELARU ${ }^{1}$, COSMIN-ANDREI ALEXE ${ }^{1}$, MARIA RÂPĂ ${ }^{2}$ \\ ${ }^{1}$ INCDTP - Division Leather and Footwear Research Institute, 031215 Bucharest, Romania; \\ marianadanielaberechet@yahoo.co.uk; demetra.simion@yahoo.com;maria.stanca@icpi.ro; \\ ciprian.chelaru@icpi.ro; cosminandrei.alexe@yahoo.com \\ ${ }^{2}$ Faculty of Materials Science and Engineering, Politehnica University of Bucharest, $313 \mathrm{Spl}$. \\ Independentei,060042 Bucharest, Romania; rapa_m2002@yahoo.com
}

\begin{abstract}
Keratin is a fibrous protein abundant in nature, being the component of wool, hair, hooves, horns, feathers, and claws. Keratin is one of the most valuable natural biopolymers due to its chemical versatility and biological performance. At the molecular level, keratin is distinguished from other biopolymers by its high concentration of cysteine-containing sulfur. Two keratin hydrolysate batches were obtained in alkaline medium, at a constant concentration of $8 \% \mathrm{NaOH}$ and $75^{\circ} \mathrm{C}$ $\left(\mathrm{KerNa}_{75}\right), 85^{\circ} \mathrm{C}\left(\mathrm{KerNa}_{85}\right)$, and $95^{\circ} \mathrm{C}\left(\mathrm{KerNa}_{95}\right)$, and at a fixed temperature of $99^{\circ} \mathrm{C}$ and different concentrations of $\mathrm{NaOH}$, i.e. $3 \%\left(\mathrm{KerNa}_{99}\right), 5 \%\left(\mathrm{KerNa}_{99}\right)$, and $8 \%\left(\mathrm{KerNa}_{99}\right)$, respectively. Physical-chemical analyses showed that the protein content ranging between $83.60 \%$ for $\mathrm{KerNa}_{75}$ and $88.88 \%$ for KerNa3 ${ }_{99}$, while the total nitrogen was found $13.83 \%$ and $14.67 \%$ in the case of $\mathrm{KerNa}_{75}$ and KerNa3 ${ }_{99}$, respectively. Dynamic light scattering analysis showed that the particle sizes decreased with the increased concentration in the reaction medium. The average particle size was between $1352 \mathrm{~nm}$ and $1771 \mathrm{~nm}$ for the samples obtained at a temperature of $99^{\circ} \mathrm{C}$ and with lower values between $463.3 \mathrm{~nm}$ and $571.6 \mathrm{~nm}$ for the samples obtained with $8 \% \mathrm{NaOH}$. The Fourier transform infrared (FT-IR) spectra evidenced the specific bands of keratin-specific proteins and sulfur compounds. Experiments were also performed to evaluate the antioxidant activity and the growth of Tamino and Mirastar wheat plants by applying the treatments with $3 \%$ and $5 \%$ concentrations of $\mathrm{KerNa}_{99}$ on wheat seeds. These experiments showed an improvement in the wheat plant growth during 10 days of observation compared to control sample. The results recommend the potential use for keratin hydrolysates in the medical, pharmaceutical, cosmetics fields, and also as fertilizers in agriculture.
\end{abstract}

Keywords: keratin hydrolysates, alkaline hydrolysis, keratin properties

\title{
INTRODUCTION
}

Keratin is one of the most valuable natural biomaterials due to its chemical versatility and biological performance (Râpă et al., 2020). Keratin is an abundant fibrous protein in nature being the component of wool, hair, horns hooves, feathers, claws and epithelial cells (Râpă et al., 2020; Aluigi et al., 2013). At the molecular level, the keratin is distinguished from other proteins (such as fibroin or collagen) by high concentration of cysteine - containing sulfur (7-20\% of the total amino acid residues) (Hearle, 2000; Seghir et al., 2020). Cysteine residues are present in oxidized form of cystine residues, characterized by covalent disulfide bonds, which gives the mechanical, thermal stability and chemistry properties (Brandelli, 2008; Feughelman, 2002). In particular, keratins extracted from wool can be classified into two groups: proteins with intermediate filaments and matrix proteins. Intermediate filament proteins have low sulfur keratins with molecular weights in the range of 40-60 $\mathrm{kDa}$ and $\alpha$-helix structure. Matrix proteins are keratin with a lot of sulfur, and molecular weights in the range of $11-26 \mathrm{kDa}$. Matrix keratins surround keratins in proteins with intermediate filaments and interact with them through intermolecular disulfide bonds (Plowman, 2003). Keratins extracted from wool are biodegradable (Yamauchi et al., 1996), support 
growth and adhesion of fibroblasts (Tachibana et al., 2002) and osteoblasts (Tachibana et al., 2005). Keratin is a structural protein with interesting properties in terms of biocompatibility and an absorbent ability for heavy metal ions and volatile organic compounds (Râpă et al., 2020). Keratin is an ideal biopolymer for wound healing due to the amino acid sequence that induces cell adhesion, mitogenic and chemotactic activity and mediates changes in gene expression (Râpă et al., 2020). The development of techniques for the efficient production of biomass from poultry farming or sheep breeding for keratin extraction should prove to be very useful for the sustainable management of huge waste. Denaturation techniques, such as methods based on reduction (Zeng and Lu, 2014), oxidation (Brown et al., 2016) and enzymatic hydrolysis and ionic liquids (Rajabinejad et al., 2018) are benchmarks for obtaining good yields and soluble keratin (Hearle, 2000).

In this paper we aimed to modify the properties of some keratin hydrolysates obtained by alkaline extraction under the influence of temperature and concentration variation of the working environment. Experiments were also performed to evaluate the antioxidant activity and bioactive properties by assessing the growth of Tamino and Mirastar wheat plants by applying the treatments with $3 \%$ and 5\% concentrations of KerNa899.

\section{MATERIALS AND METHODS}

\section{Materials}

Pearl sodium hydroxide $(\mathrm{NaOH})$ was acquired from Lachner, Neratovice, Czech Republic. Sheep wool was purchased from local sheep farmers. The other materials are analytical grade.

\section{Methods}

\section{Extraction of Keratin Hydrolysate}

Keratin hydrolysate was obtained by alkaline hydrolysis with $\mathrm{NaOH}$. Two series of keratins were obtained by varying the temperature or concentration during the production process. Alkaline hydrolysis was made with mechanical stirring for $3 \mathrm{~h}$. The first batch of keratins was obtained at constant temperature of $99^{\circ} \mathrm{C}$ and different concentrations of $\mathrm{NaOH}$ : 3\% (KerNa3 $\left.{ }_{99}\right), 5 \%$ (KerNa599) and 8\% (KerNa899) (w/w). The second batch of keratins was obtained at a constant concentration of $\mathrm{NaOH} 8 \%$ $(\mathrm{w} / \mathrm{w})$ and modified temperatures, i.e. $75^{\circ} \mathrm{C}\left(\mathrm{KerNa}{ }_{75}\right), 85^{\circ} \mathrm{C}\left(\mathrm{KerNa} 8_{85}\right)$, and $95^{\circ} \mathrm{C}$ (KerNa895).

\section{Characterization of Keratin Hydrolysates}

The physical-chemical characteristics for keratin hydrolysates obtained were analyzed according to the standardized and in-house methods: SR EN ISO 4684:2006 (dry matter), SR EN ISO 4047:2008 (ash content), SR ISO 5397:1996 (total nitrogen content and protein). The size particles and zeta potential of keratins were measured by Dynamic light scattering (DLS) technique with Zetasizer Nano-ZS device from Malvern (Malvern Hills, UK). The results of the analyses are expressed as the average values of three determinations. ATR-FT-IR analysis was performed with a FT-IR/ATR spectrometer Jasco 4200 operating in the range of 4000 to $550 \mathrm{~cm}^{-1}$, with spectral resolution of $0.5 \mathrm{~cm}^{-1}$. 
The antioxidant activity by 2,2-diphenyl-1-picrylhydrazyl (DPPH) and 2,2'-azino-bis (3ethylbenzthiazoline)-6-sulfonic acid $\left(\mathrm{ABTS}^{+\bullet}\right.$ ) free radicals for $\mathrm{KerNa}_{99}$ was also determined by measuring the maximum absorbance recorded at $517 \mathrm{~nm}$ and $750 \mathrm{~nm}$, respectively. Experiments were also performed to evaluate the growth of Tamino and Mirastar wheat plants by applying the treatments with $\mathrm{KerNa}_{99}$ at two concentrations of $3 \%$ and $5 \%$ over a period of 10 days. Three samples were prepared for each wheat type consisted of 10 seeds: a control sample treated with water, a sample treated with 3\% $\mathrm{KerNa}_{99}$ and a sample treated with $5 \% \mathrm{KerNa}_{99}$

\section{RESULTS AND DISCUSSIONS}

The main physical-chemical characteristics of keratins hydrolysate extracts obtained with $3 \%, 5 \%$ and $8 \%$ solution of $\mathrm{NaOH}$ and constant temperature at $99^{\circ} \mathrm{C}$ are shown in table 1 .

Table 1. Physical-chemical characteristics of $\mathrm{KerNa}_{99}, \mathrm{KerNa}_{99}$ and $\mathrm{KerNa}_{99}$ hydrolysate extracts

\begin{tabular}{lccc}
\hline Characteristics & KerNa399 & KerNa599 & KerNa899 \\
\hline Dry matter $(\%)$ & 10.70 & 11.75 & 12.60 \\
Ash $(\%)^{*}$ & 8.32 & 11.40 & 11.03 \\
Total nitrogen $(\%)^{*}$ & 14.67 & 13.96 & 13.89 \\
Protein content $(\%)^{*}$ & 88.88 & 84.60 & 84.21 \\
pH (units of pH) & 10.31 & 10.34 & 10.77 \\
\hline * Values reported at dry substance & &
\end{tabular}

The keratin hydrolysates obtained have values from $13.89 \%$ to $14.67 \%$ for total nitrogen content and between $84.21 \%$ and $88.88 \%$ for the protein content and the dry matter content increased from $10.70 \%$ to $12.60 \%$ with the increasing of concentration in the reaction medium (table 1 ).

Table 2. Particle sizes and zeta potential of $\mathrm{KerNa}_{99}, \mathrm{KerNa}_{99}$ and $\mathrm{KerNa}_{99}$ hydrolysate extracts

\begin{tabular}{|c|c|c|c|c|c|c|c|c|c|}
\hline \multirow{3}{*}{$\begin{array}{c}\text { Keratin } \\
\text { hydrolysate }\end{array}$} & \multicolumn{6}{|c|}{ Particle populations $(\%)$ and size $(\mathrm{nm})$} & \multirow{3}{*}{$\begin{array}{l}\text { Average, } \\
\text { d, nm }\end{array}$} & \multirow[t]{3}{*}{ Pdl } & \multirow{3}{*}{$\begin{array}{c}\text { Zeta } \\
\text { potential, } \\
\mathrm{mV}\end{array}$} \\
\hline & \multicolumn{2}{|c|}{$\begin{array}{c}\text { Majority } \\
\text { population } 1\end{array}$} & \multicolumn{2}{|c|}{$\begin{array}{c}\text { Majority } \\
\text { population } 2\end{array}$} & \multicolumn{2}{|c|}{$\begin{array}{c}\text { Majority } \\
\text { population } 3\end{array}$} & & & \\
\hline & Size & $\%$ & Size & $\%$ & Size & $\%$ & & & \\
\hline KerNa399 & 446 & 27.1 & 2111 & 50 & 4723 & 22.9 & 1358 & 0.744 & -11 \\
\hline KerNa599 & 117.3 & 11.5 & 798.6 & 88.5 & - & - & 1697 & 0.993 & -12.1 \\
\hline KerNa899 & 142.9 & 6.7 & 614 & 73.4 & 5497 & 19.9 & 1771 & 1 & -12.7 \\
\hline
\end{tabular}

As the concentration of $\mathrm{NaOH}$ increased from $3 \%$ to $8 \%$, the size of the constituent nanoparticles decreases in the keratin hydrolysate extraction medium (Table 2) due to the increase of the degree of hydrolysis of keratin chains. DLS analysis of keratin hydrolysates shows the presence of majority populations starting from $117.3 \mathrm{~nm}$ $(11.5 \%)$ for KerNa599, $142.9 \mathrm{~nm}(6.7 \%)$ for KerNa899, and $446 \mathrm{~nm}$ (27.1\%) for $\mathrm{KerNa}_{99}$ to $5497 \mathrm{~nm}(19.9 \%)$ for KerNa899. The zeta potential was between $-11 \mathrm{mV}$ for $\mathrm{KerNa}_{99}$ and $-12.7 \mathrm{mV}$ for $\mathrm{KerNa}_{99}$ (Table 2). The main physical-chemical characteristics of keratin hydrolysate extracts obtained at $8 \% \mathrm{NaOH}$ and temperatures of $75^{\circ} \mathrm{C}, 85^{\circ} \mathrm{C}$, and $95^{\circ} \mathrm{C}$ are shown in table 3 . 
The Influence of Alkaline Extraction on Some Keratin Hydrolysates Properties

Table 3. Physical-chemical characteristics of $\mathrm{KerNa}_{75}, \mathrm{KerNa}_{85}$ and $\mathrm{KerNa}_{95}$ hydrolysate extracts

\begin{tabular}{lccc}
\hline Characteristics & KerNa875 & KerNa885 & KerNa895 \\
\hline Dry matter (\%) & 3.11 & 4.25 & 4.74 \\
Ash $(\%)^{*}$ & 15.11 & 11.53 & 10.97 \\
Total nitrogen (\%)* & 13.83 & 14.35 & 14.56 \\
Protein content (\%)* & 83.60 & 87.06 & 88.19 \\
pH (units of pH) & 11.93 & 11.42 & 10.61 \\
\hline * Values reported at dry substance & &
\end{tabular}

The keratin hydrolysates obtained with $8 \% \mathrm{NaOH}$ and different temperatures showed an increase of the total nitrogen values from $13.83 \%$ in the case of $\mathrm{KerNa}_{75}$ to $14.56 \%$ in the case of $\mathrm{KerNa}_{95}$ and of the protein substance from $83.60 \%$ in $\mathrm{KerNa}_{75}$ to $88.19 \%$ in $\mathrm{KerNa}_{95}$ (table 3).

Table 4. Particle sizes and zeta potential of $\mathrm{KerNa}_{75}, \mathrm{KerNa}_{85}$ and $\mathrm{KerNa}_{95}$ hydrolysate extracts

\begin{tabular}{|c|c|c|c|c|c|c|c|c|c|}
\hline \multirow{3}{*}{$\begin{array}{c}\text { Keratin } \\
\text { hydrolysate }\end{array}$} & \multicolumn{6}{|c|}{ Particle populations (\%) and size (nm) } & \multirow{3}{*}{$\begin{array}{c}\text { Average, } \\
\mathrm{d}, \mathrm{nm}\end{array}$} & \multirow[t]{3}{*}{ Pdl } & \multirow{3}{*}{$\begin{array}{c}\text { Zeta } \\
\text { potential, } \\
\mathrm{mV}\end{array}$} \\
\hline & \multicolumn{2}{|c|}{$\begin{array}{c}\text { Majority } \\
\text { population } 1\end{array}$} & \multicolumn{2}{|c|}{$\begin{array}{c}\text { Majority } \\
\text { population } 2\end{array}$} & \multicolumn{2}{|c|}{$\begin{array}{c}\text { Majority } \\
\text { population } 3\end{array}$} & & & \\
\hline & Size & $\%$ & Size & $\%$ & Size & $\%$ & & & \\
\hline KerNa875 & 93.51 & 9.2 & 510.3 & 79.3 & 5062 & 11.5 & 463.3 & 0.682 & -18.9 \\
\hline KerNa885 & 96.17 & 10.3 & 590.8 & 76.7 & 5146 & 13 & 563.1 & 0.760 & -18.3 \\
\hline $\mathrm{KerNa}_{95}$ & 73.66 & 8.3 & 541.8 & 81.6 & 5320 & 10 & 571.6 & 0.796 & -17.1 \\
\hline
\end{tabular}

Particle size measurement shows values from $73.66 \mathrm{~nm}(8.3 \%)$ in $\mathrm{KerNa}_{95}$ and $96.17 \mathrm{~nm}(10.3 \%)$ in $\mathrm{KerNa}_{85}$ to $510.3 \mathrm{~nm}$ (79.3) in $\mathrm{KerNa}_{75}$ and $590.8 \mathrm{~nm}(76.7 \%)$ in $\mathrm{KerNa}_{75}$ to $5062 \mathrm{~nm}(11.5 \%)$ in $\mathrm{KerNa}_{75}$ and $5320 \mathrm{~nm}(10 \%)$ in $\mathrm{KerNa}_{95}$. The potential zeta values were between $-17.1 \mathrm{mV}$ at $\mathrm{KerNa}_{95}$ to $-18.9 \mathrm{mV}$ for $\mathrm{KerNa}_{75}$ (table 4).

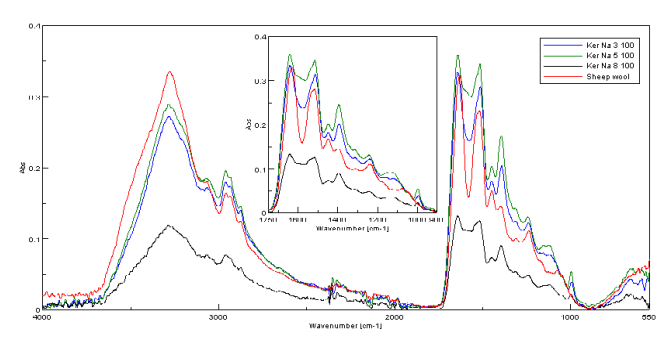

(a)

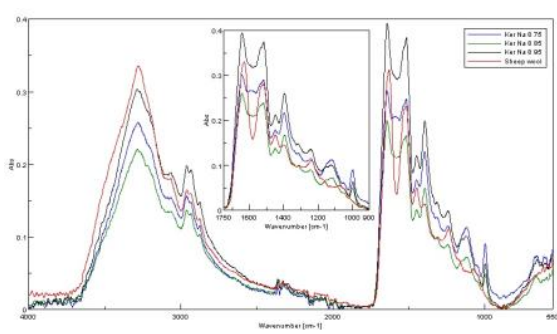

(b)

Figure 1. ATR-FTIR spectra for: KerNa3 ${ }_{99}, \mathrm{KerNa}_{99}$, and $\mathrm{KerNa}_{99}$ (a) and KerNa875, $\mathrm{KerNa}_{85}, \mathrm{KerNa}_{95}$ (b) compared with the spectrum of sheep wool

The ATR-FTIR spectra showed in Figure $1(\mathrm{a}, \mathrm{b})$ the spectral bands specific to proteins and sulfur compounds resulting from the hydrolysis process by breaking the S$\mathrm{S}$ bonds of cystine (Khosa and Ullah, 2014). Keratin is easier to break into smaller specific fragments after breaking these S-S bonds between macromolecular chains

https://doi.org/10.24264/icams-2020.II.3 
(Pavia et al., 2008). The spectral band from $3280-3274 \mathrm{~cm}^{-1}$ can be attributed to the stretching vibration of $-\mathrm{O}-\mathrm{H}$ and $-\mathrm{N}-\mathrm{H}$ (Amide A) (Mohanty et al., 2005). The absorption peak around $2960 \mathrm{~cm}^{-1}$ is attributed to the asymmetric extent of $-\mathrm{CH}_{2}$ (Amide B). The specific band from $1630-1637 \mathrm{~cm}^{-1}$ is assigned to the extension of $\mathrm{C}=\mathrm{O}$ (Amide I). The absorption peaks between $1517-1549 \mathrm{~cm}^{-1}$ and $1242-1237 \mathrm{~cm}^{-1}$ correspond to the $\mathrm{N}-\mathrm{H}$ band coupled to the $\mathrm{C}-\mathrm{H}$ (Amide II) range and the $\mathrm{C}-\mathrm{H}$ (Amide III) range, respectively. The absorption bands at $670 \mathrm{~cm}^{1}$ and $578-541 \mathrm{~cm}^{-1}$ can be attributed to the extension of the C-S bond and the S-S bond, as well as to the deformation of the $\mathrm{C}-\mathrm{C}$ bond corresponding to keratin specific sulfur compounds.

KerNa899 hydrolysate extract showed antioxidant activity measured by DPPH method of $23.65 \pm 1.4 \%$ and by ABTS method of $99.86 \pm 2.0 \%$. The values are similar with feather origin keratin hydrolysates with $0.2 \mathrm{mg} / \mathrm{mL}$ concentration (Oluba et al., 2019).

$\mathrm{KerNa}_{99}$ was selected to treat the wheat seeds because this type has a higher value for dry matter $(12.60 \%)$ and important protein content $(84.21 \%)$. Tamino wheat treated with both concentrations of $\mathrm{KerNa}_{99}$ increased more than the control sample and Mirastar wheat treated with $5 \% \mathrm{KerNa}_{99}$ increased more compared to the control sample in the 10 days of the experiment. Tamino wheat increased in length by $44.3 \%$ in the case of treatment with $3 \% \mathrm{KerNa}_{99}$ and by $19.9 \%$ when it was treated with $5 \% \mathrm{KerNa}_{99}$ compared to the control sample (figure 2). Mirastar wheat seeds grew less, with $25.49 \%$ for treatment with $3 \% \mathrm{KerNa}_{99}$ compared to the control sample, while in the case of $5 \%$ $\mathrm{KerNa}_{99}$ it increased by $50.3 \%$ compared to water treated seeds (figure 3 ).

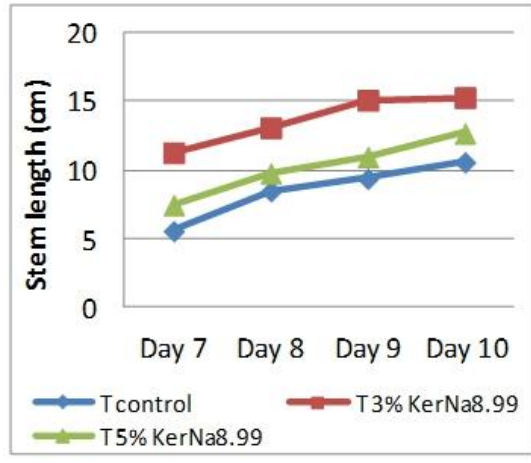

Figure 2. The increase in Tamino wheat stem length $(\mathrm{cm})$

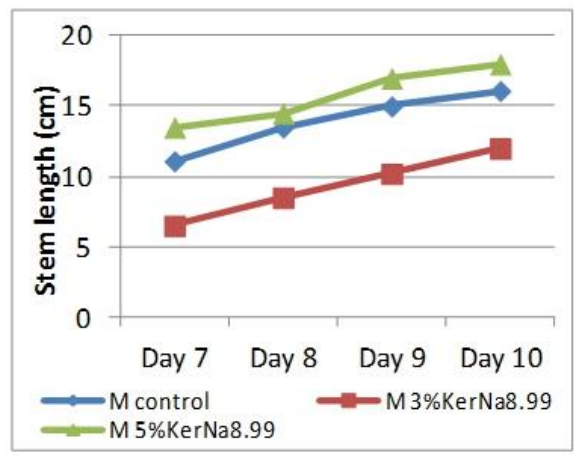

Figure 3. The increase in Mirastar wheat stem length $(\mathrm{cm})$

\section{CONCLUSION}

Two keratin hydrolysate batches were obtained from sheep wool by alkaline hydrolysis at different concentrations and temperatures of the reaction medium. The physical-chemical characteristics of the keratins showed values of the protein substance between $84.21 \%$ and $88.88 \%$ corresponding to total nitrogen content of $13.83 \%$ and $14.67 \%$. Particle size measurements showed a decrease in particle size with increasing concentration of the reaction medium. The ATR-FTIR spectra showed spectral bands specific to proteins and sulfur compounds from keratin. KerNa8 99 hydrolysate extract had the highest antioxidant activity, due to the highest level of hydrolysis. Experiments performed on growth Tamino and Mirastar wheat plants by applying 5\% KerNa899 treatments showed an improvement in the wheat plant growth in first 10 days of 
observation compared with control sample. Keratin hydrolysates can be used in the medical, pharmaceutical, cosmetics fields, and also as fertilizers in agriculture.

\section{Acknowledgements}

This work was supported by a grant of the Romanian Ministry of Education and Research, CCCDI-UEFISCDI project number PN-III-P2.1-PTE-2019-0655, contract 5PTE, BIOTEHKER, and 4PERFORM-TEX-PEL project.

\section{REFERENCES}

Aluigi, A., Corbellini, A., Rombaldoni, F., Zoccola, M. and Canetti, M. (2013), "Morphological and structural investigation of wool-derived keratin nanofibres crosslinked by thermal treatment", International Journal of Biological Macromolecules, 57, 30-37, https://doi.org/10.1016/j.ijbiomac.2013.02.013.

Brandelli, A. (2008), "Bacterial keratinases: useful enzymes for bioprocessing agroindustrial wastes and beyond", J Food Bioprocess Technology, 1, 2, 105-116, https://doi.org/10.1007/s11947-007-0025-y.

Brown, E.M., Pandya, K., Taylor, M.M. and Liu, C.-K. (2016), "Comparison of methods for extraction of keratin from waste wool", J Agricultural Sciences, 7, 10, 670, https://doi.org/10.4236/as.2016.710063.

Feughelman, M. (2002), "Natural protein fibers", Journal of Applied Polymer Science, 83, 3, 489-507, https://doi.org/10.1002/app.2255.

Hearle, J. (2000), "A critical review of the structural mechanics of wool and hair fibre", International Journal of Biological Macromolecules, 27, 2, 123-138, https://doi.org/10.1016/S0141-8130(00)00116-1.

Khosa, M.A. and Ullah A. (2014), "In-situ modification, regeneration, and application of keratin biopolymer for arsenic removal", Journal of Hazardous Materials 278, 360-371, https://doi.org/10.1016/j.jhazmat.2014.06.023.

Mohanty, A.K., Misra, M. and Drzal, L.T. (2005), Natural Fibers, Biopolymers, and Biocomposites, CRC press, https://doi.org/10.1201/9780203508206.

Oluba, O.M., Akpor, O.B., Alabi, O.O., Shoyombo, A.J., Adeyonu, A.G. and Adebiyi, F.D. (2019), "Antioxidant activity and in vitro protein digestibility of chicken feather protein hydrolysates", https://doi.org/10.21203/rs.2.14464/v1.

Pavia, D.L., Lampman, G.M., Kriz, G.S. and Vyvyan, J.A. (2008), Introduction to Spectroscopy, Cengage Learn, 4th edn, Belmont, pp. 752.

Plowman, J.E. (2003), "Analytical Technologies in the Biomedical and Life Sciences", Journal of Chromatography B, 787, 63-76, https://doi.org/10.1016/S1570-0232(02)00211-8, PMID: 12659734.

Rajabinejad, H., Zoccola, M., Patrucco, A., Montarsolo, A., Rovero, G. and Tonin, C. (2018), "Physicochemical properties of keratin extracted from wool by various methods", J Textile Research Journal, 88, 21, 2415-2424, https://doi.org/10.1177/0040517517723028.

Râpă, M., Gaidau, C., Stefan, L.M., Matei, E., Niculescu, M., Berechet, M.D., Stanca, M., Tablet, C., Tudorache, M., Gavrila, R., Predescu, C. and Vidu, R. (2020), "New Nanofibers Based on Protein ByProducts with Bioactive Potential for Tissue Engineering", Materials, 13, 3149 , https://doi.org/10.3390/ma13143149.

Seghir, B.B., Hemmami, H., Soumeia, Z., Laouini, S.E., Rebiai, A., Amor, I.B., Souici, I. and Beki, A. (2020), "Preparation methods keratin and nanoparticles keratin from wool: a review", Algerian Journal of Chemical Engineering, 01, 5-11, https://doi.org/10.5281/zenodo.3930645.

Tachibana, A., Furuta, Y., Takeshima, H., Tanabe, T. and Yamauchi, K. (2002), "Fabrication of wool keratin sponge scaffolds for long-term cell cultivation", Journal of Biotechnology, 93, 165-170, https://doi.org/10.1016/S0168-1656(01)00395-9.

Tachibana, A., Kaneko, S., Tanabe, T. and Yamauchi, K. (2005), "Rapid fabrication of keratin-hydroxyapatite hybrid sponges toward osteoblast cultivation and differentiation", Biomaterials, 26, 297-302, https://doi.org/10.1016/j.biomaterials.2004.02.032.

Yamauchi, K., Yamauchi, A., Kusunoki, T., Kohda, A. and Konishi, Y. (1996), "Preparation of stable aqueous solution of keratins, and physiochemical and biodegradational properties of films", Journal of Biomedical Materials Research 31, 439-444, https://doi.org/10.1002/(SICI)10974636(199608)31:4\%3C439::AID-JBM1\%3E3.0.CO;2-M.

Zeng, C.H. and Lu, Q. (2014), "Study on the recovery of waste wool by combining reduction and metallic salt methods", Advanced Materials Research, 881, 551-555, Trans Tech Publ, https://doi.org/10.4028/www.scientific.net/AMR.881-883.551. 
PELT WASTE DEGRADATION USING ACTIVE MICROBIAL CONSORTIA

\author{
RODICA ROXANA CONSTANTINESCU ${ }^{1}$, GABRIEL ZAINESCU ${ }^{1}$, MARIANA FERDES ${ }^{2}$, \\ IULIA CANIOLA ${ }^{1}$ \\ ${ }^{1}$ INCDTP - Division Leather and Footwear Research Institute, 93, Ion Minulescu Str., Bucharest, \\ Romania,rodica.roxana@yahoo.com \\ ${ }^{2}$ University Politehnica of Bucharest, Faculty of Biotechnical Systems Engineering, Romania
}

\begin{abstract}
In tanneries, environmental problems have special implications in terms of optimizing the consumption of used chemicals, applied technologies, the degree of recovery of useful substances from leather waste. Biodegradation is the process by which organic substances are broken down by microorganisms. From an ecological point of view, biodegradability assessments of new materials and compounds in the industry are essential to understand and quantify their impact on the environment. The sustainable development of the leather industry has focused on major environmental issues, such as clean production methods and waste management. Tanneries generate huge quantities of solid wastes as pelt waste. If these bio-waste materials are not utilized properly, they are potential source of pollution. Microbiological degradation of pelt waste is amongst the permanent concerns of leather processing units. The process may have the purpose of decomposing waste to exploit by-products as biocompost or to obtain proteases through a biotechnological process. These enzymes can be used after purification in various processes that have animal protein as a substrate. They can also be used in raw state for enzymatic hydrolysis. The paper aims at development of an experimental model on the bioenzymatic degradation process of protein waste from tanneries.
\end{abstract}

Keywords: tanneries, pelt waste, enzymes.

\title{
INTRODUCTION
}

Tanneries generate huge quantities of solid wastes as fleshings. If these bio-waste materials are not utilized properly, they are potential source of pollution. Leather has a complex composition comprising collagen, keratin, elastin, albumins and globulins (Sundar et al., 2017). Each of these compounds can be degraded under certain environmental conditions (temperature, humidity, $\mathrm{pH}, \mathrm{O}_{2}$ concentration) under the action of enzyme complexes synthesized by a variety of microorganisms (bacteria and fungi). Leather waste degradation occurs by means of proteolytic enzymes (Tang et al., 2021).

Worldwide research for leather waste recycling is aimed at obtaining protein composites, through biochemical treatments with microorganisms/enzymes and obtaining hydrolyzed proteins and biocomposites for various uses in forestry. Currently, the entire amount of solid waste resulting from leather processing is deposited in the landfill, leading to the accumulation of chemicals in the soil, with possible negative effects on the ecosystems (Thankaswamy et al., 2018).

Microbiological degradation of pelt waste is amongst the permanent concerns of leather processing units. Microorganisms (fungi and bacteria) play an important role in solving these problems (Constantinescu et al., 2015).

Molds or filamentous fungi are eukaryotic spore-forming microorganisms, feeding by absorption, easily adaptable, because they have the ability to form induced enzymes depending on the nature of the substrate they are found, causing degradation (Rangel Serrano et al., 2003).

Enzymes are organic substances, generally proteins, known like biocatalyst to multiples chemicals reactions and are commercially exploited in detergent, food, 
pharmaceutical, diagnostics and fine chemicals industry. They are between the more notable biomolecules due their extraordinary specificity and catalytic power, which are higher that the catalytic power of catalyst produced by man (Gupta et al., 2019).

The main enzymes that are of interest to the leather industry are as follows:

- Proteases because they hydrolyze the protein fraction of dermatan sulfate, making the collagen more accessible to water and reducing the attachment of the basal layer. In addition, they act in the removal of globular proteins;

- Lipases, which hydrolyze fats, oils and greases present in the hypoderm;

- Keratinases, which hydrolyze the keratin of hair and epidermis and break down the disulfide bonds of this molecule (Hussain et al., 2020).

Most degradations found on leather waste are due to the action of fungi, a microorganism group very rich in enzymes. The following strains have been identified: Penicillium chrysogenum, $P$. rugulosum, $P$. brevicompactum, $P$. luteum, $P$. decubens, $P$. aculeatum, $P$. funiculosum, Aspergillus niger, A. fumigatus, A. ochraceus, A. ventii, A. flavus, A. oryzae, Mucor mucedo, Rhizopus nigricans, Paecylomyces varioti, Scopulariopsis brevicaulis, Verticillium glaucum, Trichoderma species (Zhang et al., 2017; Wosten, 2019).

Microorganisms (bacteria and fungi) are an excellent source of these enzymes and can be used as such for various purposes, including in the leather industry .

In recent years there have been numerous attempts to degrade hide waste by treatment with alkaline proteases in order to obtain protein products. Enzymatic methods use commercially available alkaline proteases, at moderate temperatures and for short periods of time. This relatively simple method can be a solution for the removal of potentially harmful waste. A variant of the method is based on the proteolytic activity of microbial cells, especially of microorganisms isolated from this waste.

The potential of these enzymes, used in the pure state or as extracellular enzymes elaborated by cultures of microorganisms, is much higher and it is considered that their application in other processes in the near future creates a major field of research advance in the field (Calin et al., 2017).

The paper aims at development of an experimental model on the bioenzymatic degradation process of protein waste from tanneries.

\section{EXPERIMENTAL}

Samples of ground protein waste were used in the experiments. They are grayyellowish, with hard, slightly gelatinous, wet consistency.

\section{Materials and Methods}

The samples taken in the study were protein waste resulting from different hide processing stages from tanneries. Different-looking hide samples were selected, which were numbered as follows:

P 1 - hide fleshings from Pielorex (white - gray), with smooth outer surface,

P 2 - hide fleshings from Pielorex, with rough surface,

P 3 - hide fleshings from ICPI, with smooth surface,

P 4 - hide fleshings from ICPI, with rough surface.

In order to determine the $\mathrm{pH}$ value of the samples, from each protein waste were taken $1 \mathrm{~cm}^{2}$ fragments, which were suspended in Erlenmeyer vials with $25 \mathrm{ml}$ physiological serum. 
Also, samples of the same surface were suspended in Erlenmeyer vials with $25 \mathrm{ml}$ of nutrient broth and incubated at $28^{\circ} \mathrm{C}$ for 72 hours. The resulting culture liquids were used for decimal dilutions. These were seeded by incorporation into Petri dishes with nutrient agar, in three repetitions, in order to obtain isolated colonies and to determine the number of viable cells.

The plates were incubated for 24 hours at $28^{\circ} \mathrm{C}$ and then the number of isolated colonies was estimated by calculating the average of the values obtained in the three repetitions performed for each dilution.

The study of the morphological characters of isolated microorganisms was performed by optical microscopy observations on Gram stained smears, which were examined under a microscope.

The ability of isolated strains to hydrolyze proteins was determined by gelatin and casein hydrolysis assay.

Also, in all the gelatin and casein hydrolysis testing experiments, controls were used, represented by Petri dishes or tubes with non-inoculated media.

\section{RESULTS AND DISCUSSION}

Determination of $\mathrm{pH}$ value revealed the $\mathrm{pH}$ change of the physiological serum after immersion of hide samples, from $\mathrm{pH} 6.4$ to values between 6.6-8.6.

The calculation of the number of viable cells obtained on plates with nutrient agar medium resulted in values between $4 \cdot 107$ for hide samples P 1 and P 2, and 5 107 for sample P 3, while sample P 4 showed a relatively lower number of bacterial cells -2 . $106 / \mathrm{cm}^{2}$ (Table 1).

Table 1. Determining the number of cells obtained on plates with nutrient agar medium

\begin{tabular}{ccccc}
\hline No. & Sample & $\mathrm{pH}$ & Number of bacteria $/ \mathrm{cm}^{2}$ sample & Number of isolated strains \\
\hline 1. & P 1 & 6.88 & $4 \cdot 10^{7}$ & 6 \\
2. & P 2 & 6.85 & $4 \cdot 10^{7}$ & 4 \\
3. & P 3 & 6.65 & $5 \cdot 10^{7}$ & 5 \\
4. & P 4 & 8.67 & $2 \cdot 10^{6}$ & 5 \\
\hline
\end{tabular}

From the four samples analyzed, a total number of 20 bacterial strains were isolated using the serial decimal dilution method, which was seeded on nutrient agar. Most strains showed colonies of white-beige color, with matte, flat, rough surface and irregular edges. In the case of sample P 3, the strains forming spherical colonies on the nutrient agar predominated, of white color, with smooth surface and even edges.

The P 4/4 and P 4/5 strains differed in color and appearance of the formed colonies, these being dark beige, semi-glossy, flat, with smooth surface and even edges.

The microscopic examination of the Gram stained smears enabled verification of the purity of cultures and showed that most of the strains were represented by Grampositive bacilli, with rounded heads, arranged in short chains of 2 to 4 cells or in irregular agglomerations. 
Pelt Waste Degradation Using Active Microbial Consortia

Table 2. Morphological culture and biochemical characters of isolated bacteria strains

\begin{tabular}{|c|c|c|c|c|c|c|}
\hline No. & Strain & Colony morphology & Cell morphology & $\begin{array}{l}\text { Gram } \\
\text { reaction }\end{array}$ & $\begin{array}{r}\text { Protein } \\
\text { (after } 96 \\
\text { gelatin }\end{array}$ & $\begin{array}{l}\text { olysis } \\
\text { bation) } \\
\text { casein }\end{array}$ \\
\hline 1. & $\mathrm{P} 1 / 1$ & $\begin{array}{l}\text { White, matte colony } \\
\text { with rough surface } \\
\text { and irregular edges }\end{array}$ & $\begin{array}{l}\text { Bacilli with } \\
\text { rounded heads, in } \\
\text { short chains, with } \\
\text { central spore }\end{array}$ & + & +++ & +++ \\
\hline 2. & $\mathrm{P} 1 / 2$ & $\begin{array}{l}\text { Small white, matte, } \\
\text { flat colony with } \\
\text { rough surface and } \\
\text { irregular edges }\end{array}$ & $\begin{array}{l}\text { Bacilli with } \\
\text { rounded heads, in } \\
\text { short chains, with } \\
\text { central spore }\end{array}$ & + & +++ & ++ \\
\hline 3. & $\mathrm{P} 1 / 3$ & $\begin{array}{l}\text { White-beige, matte, } \\
\text { flat colony with } \\
\text { rough surface and } \\
\text { irregular edges }\end{array}$ & $\begin{array}{l}\text { Bacilli with } \\
\text { rounded heads, in } \\
\text { short chains, with } \\
\text { central spore }\end{array}$ & + & +++ & ++ \\
\hline 4 & $\mathrm{P} 1 / 4$ & $\begin{array}{l}\text { White, matte, flat } \\
\text { colony with rough } \\
\text { surface and irregular } \\
\text { edges }\end{array}$ & $\begin{array}{l}\text { Bacilli with } \\
\text { rounded heads, in } \\
\text { short chains, with } \\
\text { central spore }\end{array}$ & + & +++ & ++ \\
\hline 5 & $\mathrm{P} 1 / 5$ & $\begin{array}{l}\text { White-beige, matte, } \\
\text { flat colony with } \\
\text { rough surface and } \\
\text { irregular edges }\end{array}$ & $\begin{array}{l}\text { Bacilli with } \\
\text { rounded heads, in } \\
\text { short chains, with } \\
\text { central spore }\end{array}$ & + & +++ & ++ \\
\hline 6 & $\mathrm{P} 1 / 6$ & $\begin{array}{l}\text { White-beige, matte, } \\
\text { flat colony with } \\
\text { rough surface and } \\
\text { irregular edges }\end{array}$ & $\begin{array}{l}\text { Bacilli with } \\
\text { rounded heads, in } \\
\text { short chains, with } \\
\text { central spore }\end{array}$ & + & +++ & ++ \\
\hline 7 & $\mathrm{P} 2 / 1$ & $\begin{array}{l}\text { White, flat colony } \\
\text { with rough surface } \\
\text { and irregular edges }\end{array}$ & $\begin{array}{l}\text { Bacilli with } \\
\text { rounded heads, in } \\
\text { short chains, with } \\
\text { central spore }\end{array}$ & + & +++ & ++ \\
\hline 8 & $\mathrm{P} 2 / 2$ & $\begin{array}{l}\text { White, flat colony } \\
\text { with rough surface } \\
\text { and irregular edges }\end{array}$ & $\begin{array}{l}\text { Bacilli with } \\
\text { rounded heads, in } \\
\text { short chains, with } \\
\text { central spore }\end{array}$ & + & +++ & ++ \\
\hline 9 & $\mathrm{P} 2 / 3$ & $\begin{array}{l}\text { White, flat colony } \\
\text { with rough surface } \\
\text { and irregular edges }\end{array}$ & $\begin{array}{l}\text { Bacilli with } \\
\text { rounded heads, in } \\
\text { short chains, with } \\
\text { central spore }\end{array}$ & + & +++ & ++ \\
\hline 10 & P $2 / 4$ & $\begin{array}{l}\text { White, flat colony } \\
\text { with rough surface } \\
\text { and irregular edges }\end{array}$ & $\begin{array}{l}\text { Bacilli with } \\
\text { rounded heads, in } \\
\text { short chains, with } \\
\text { central spore }\end{array}$ & + & +++ & ++ \\
\hline 11. & P $3 / 1$ & $\begin{array}{l}\text { Large white, round, } \\
\text { matte, flat colony } \\
\text { with smooth surface } \\
\text { and even edges }\end{array}$ & $\begin{array}{l}\text { Bacilli with } \\
\text { rounded heads, in } \\
\text { short chains or } \\
\text { agglomerations, } \\
\text { with central spore }\end{array}$ & + & +++ & +++ \\
\hline 12 & $\mathrm{P} 3 / 2$ & $\begin{array}{l}\text { Large white, round, } \\
\text { matte, flat colony } \\
\text { with smooth surface } \\
\text { and even edges }\end{array}$ & $\begin{array}{l}\text { Bacilli with } \\
\text { rounded heads, in } \\
\text { short chains or } \\
\text { agglomerations, }\end{array}$ & + & +++ & +++ \\
\hline
\end{tabular}

https://doi.org/10.24264/icams-2020.II.5 


\begin{tabular}{|c|c|c|c|c|c|c|}
\hline \multirow[t]{2}{*}{ No. } & \multirow[t]{2}{*}{ Strain } & \multirow[t]{2}{*}{ Colony morphology } & \multirow[t]{2}{*}{ Cell morphology } & \multirow[t]{2}{*}{$\begin{array}{l}\text { Gram } \\
\text { reaction }\end{array}$} & \multicolumn{2}{|c|}{$\begin{array}{l}\text { Protein hydrolysis } \\
\text { (after } 96 \mathrm{~h} \text { incubation) }\end{array}$} \\
\hline & & & & & gelatin & casein \\
\hline & & & with central spore & & & \\
\hline 13 & $\mathrm{P} 3 / 3$ & $\begin{array}{l}\text { Large white, round, } \\
\text { matte, flat colony } \\
\text { with smooth surface } \\
\text { and even edges }\end{array}$ & $\begin{array}{l}\text { Bacilli with } \\
\text { rounded heads, in } \\
\text { short chains or } \\
\text { agglomerations, } \\
\text { with central spore }\end{array}$ & + & +++ & +++ \\
\hline 14 & P $3 / 4$ & $\begin{array}{l}\text { Large white, round, } \\
\text { matte, flat colony } \\
\text { with smooth surface } \\
\text { and even edges }\end{array}$ & $\begin{array}{l}\text { Bacilli with } \\
\text { rounded heads, in } \\
\text { short chains or } \\
\text { agglomerations, } \\
\text { with central spore }\end{array}$ & + & +++ & +++ \\
\hline 15 & P $3 / 5$ & $\begin{array}{l}\text { Large white, round, } \\
\text { matte, flat colony } \\
\text { with smooth surface } \\
\text { and even edges }\end{array}$ & $\begin{array}{l}\text { Bacilli with } \\
\text { rounded heads, in } \\
\text { short chains or } \\
\text { agglomerations, } \\
\text { with central spore }\end{array}$ & + & +++ & +++ \\
\hline 16 & $\mathrm{P} 4 / 1$ & $\begin{array}{l}\text { Cream-white, matte } \\
\text { colony with smooth } \\
\text { surface and even } \\
\text { edges }\end{array}$ & $\begin{array}{l}\text { Bacilli with } \\
\text { rounded heads, in } \\
\text { short chains or } \\
\text { agglomerations, } \\
\text { with central spore }\end{array}$ & + & +++ & +++ \\
\hline 17 & $\mathrm{P} 4 / 2$ & $\begin{array}{l}\text { White-beige, matte, } \\
\text { flat colony with } \\
\text { rough surface and } \\
\text { irregular edges }\end{array}$ & $\begin{array}{l}\text { Bacilli with } \\
\text { rounded heads, in } \\
\text { short chains or } \\
\text { agglomerations, } \\
\text { with central spore }\end{array}$ & + & +++ & +++ \\
\hline 18 & $\mathrm{P} 4 / 3$ & $\begin{array}{l}\text { Beige, matte, flat } \\
\text { colony with rough } \\
\text { surface and irregular } \\
\text { edges }\end{array}$ & $\begin{array}{l}\text { Bacilli with } \\
\text { rounded heads, in } \\
\text { short chains or } \\
\text { agglomerations, } \\
\text { with central spore }\end{array}$ & + & +++ & +++ \\
\hline 19 & P $4 / 4$ & $\begin{array}{l}\text { Dark beige, semi- } \\
\text { glossy, flat colony } \\
\text { with smooth surface } \\
\text { and even edges }\end{array}$ & $\begin{array}{l}\text { Bacilli with } \\
\text { rounded heads, in } \\
\text { short chains, } \\
\text { arranged in pairs } \\
\text { or isolated, with } \\
\text { central spore }\end{array}$ & + & - & +++ \\
\hline 20 & $\mathrm{P} 4 / 5$ & $\begin{array}{l}\text { Dark beige, semi- } \\
\text { glossy, flat colony } \\
\text { with smooth surface } \\
\text { and even edges }\end{array}$ & $\begin{array}{l}\text { Bacilli with } \\
\text { rounded heads, in } \\
\text { short chains, } \\
\text { arranged in pairs } \\
\text { or isolated, with } \\
\text { central spore }\end{array}$ & + & - & +++ \\
\hline
\end{tabular}

Gelatin hydrolysis testing revealed that of the 20 isolated strains 18 resulted in complete liquefaction of the culture medium whereas, two strains, P 4/11 and P 4/12 (isolated from sample P 4), did not show the ability to hydrolyze gelatin.

Testing the capacity of casein hydrolysis led to similar results for both experimental variants used. Thus, all the strains tested determined the hydrolysis of the substrate and clarification of the culture medium around the bacterial growth areas.

https://doi.org/10.24264/icams-2020.II.5 


\section{CONCLUSION}

Microbiological analysis of 4 samples of hide waste revealed the presence of bacteria and fungi. The density of bacteria developed on hide samples was estimated at 4-5 107 cells $/ \mathrm{cm}^{2}$ in the case of samples P $1-\mathrm{P} 3$ and $2 \cdot 106$ cells $/ \mathrm{cm}^{2}$ in the case of $\mathrm{P} 4$.

The vast majority of isolated colonies have a morphology characteristic of the species of the genus Bacillus, with irregular shapes. Microscopic observations revealed the presence of bacillary, sporulated, Gram-positive cell forms. The morphology of the colonies and cells, as well as the response to Gram staining, confirms that the bacteria belong to the genus Bacillus.

As a result of the isolation in pure cultures, 20 bacteria strains have been obtained, representing a first collection of bacteria that contaminate hide samples.

The proteolytic capacity of the tested strains was highlighted by the gelatin and casein hydrolysis test.

\section{Acknowledgements}

This project is funded by the Ministry of Research and Innovation within Program 1 - Development of the national R\&D system, Subprogram 1.2 - Institutional Performance - RDI excellence funding projects, Contract no 6 PFE/16.10.2018.

\section{REFERENCES}

Călin, M., Constantinescu-Aruxandei, D., Alexandrescu, E., Răut, I. and Lazăr, V. (2017), "Degradation of keratin substrates by keratinolytic fungi", Electronic Journal of Biotechnology, 28, 101-112, https://doi.org/10.1016/j.ejbt.2017.05.007.

Constantinescu, R.R., Stefan, D.S., Meghea, A. and Zainescu, G. (2015), "Pelt waste degradation using fungi strains", Buletinul Stiintific UPB, Seria B, 77(4), 123-132.

Gupta, S., Ponsubbiah, S., Gupta, S.K. and Mandal, S. (2019), "Sustainable Value Creation From Leather Solid Wastes: Preparation Of Shoe Soling Material Using Nano Fillers", IULTCS Congress Dresden, June 25-28, pp.87-92.

Hussain, F.S., Memon, N., Khatri, Z. and Memon, S. (2020), "Solid waste-derived biodegradable keratin sponges for removal of chromium: A circular approach for waste management in leather industry", Environmental Technology \& Innovation, 20, https://doi.org/10.1016/j.eti.2020.101120.

Rangel Serrano, A., Maldonado, V.M. and Kösters, K. (2003), "Characterization of Waste Materials in Tanneries for Better Ecological Uses”, Journal of the American Leather Chemists Association, 98, P43484.

Silveira, A., Moreno, R.J., Correia, M.J. and Ferro, V. (2019), “A method for the rapid evaluation of leather biodegradability during the production phase", Waste Management, 87(15), 661-671 https://doi.org/10.1016/j.wasman.2019.03.003.

Sundar, V.J., Ramamurthy, G. and Sastry, T.P. (2017), "Study on plant growth promoter from proteineous wastes from leather industry", Leather and Footwear Journal, 17(2), 87-90, https://doi.org/10.24264/lfj.17.2.2.

Tang, Y., Zhao, J., Zhang, Y., Zhou, J. and Shi, B (2021), "Conversion of tannery solid waste to an adsorbent for high-efficiency dye removal from tannery wastewater: A road to circular utilization, Chemosphere, 263, https://doi.org/10.1016/j.chemosphere.2020.127987.

Thankaswamy, S.R., Sundaramoorthy, S., Palanivel, S. and Ramudu, K.N. (2018), "Improved microbia degradation of animal hair waste from leather industry using Brevibacterium luteolum (MTCC 5982)", Journal of Cleaner Production, 189, 701-708, https://doi.org/10.1016/j.jclepro.2018.04.095.

Wösten, H.A.B. (2019), "Filamentous fungi for the production of enzymes, chemicals and materials", Current Opinion in Biotechnology, 59, 65-70, https://doi.org/10.1016/j.copbio.2019.02.010.

Zhang, J., Han, Z., Teng, B. and Chen, W. (2017), "Biodeterioration process of chromium tanned leather with Penicillium sp.", International Biodeterioration \& Biodegradation, 116, 104-111, https://doi.org/10.1016/j.ibiod.2016.10.019.

https://doi.org/10.24264/icams-2020.II.5 


\title{
FORMULATION AND CHARACTERIZATION OF ANTI-AGING COSMETIC
} EMULSIONS BASED ON COLLAGEN HYDROLYSATE AND CAFFEINE

\author{
ELENA DĂNILĂ ${ }^{1}$, MĂDĂLINA GEORGIANA ALBU KAYA², MIHAELA VIOLETA \\ GHICA $^{3 *}$, ANA-MARIA BUNEA ${ }^{4}$, LĂCRĂMIOARA POPA ${ }^{3}$, DURMUS ALPASLAN KAYA ${ }^{5}$, \\ ŞEVKET ÖZTÜRK ${ }^{6}$, MARIA-MINODORA MARIN ${ }^{1,2}$, CRISTINA-ELENA DINU-PÎRVU ${ }^{3}$, \\ VALENTINA ANUȚA ${ }^{3}$ \\ ${ }^{1}$ University Politehnica of Bucharest, Faculty of Applied Chemistry and Materials Science, 1-7 \\ Gheorghe Polizu Str., 011061, Bucharest, Romania \\ ${ }^{2}$ National Research and Development Institute for Textile and Leather, Division Leather and \\ Footwear Research Institute, Collagen Department, 93 Ion Minulescu Str., 031215, Bucharest, \\ Romania \\ 3 "Carol Davila" University of Medicine and Pharmacy, Faculty of Pharmacy, Physical and \\ Colloidal Chemistry Department, 6 Traian Vuia Str., 020956, Bucharest, Romania, \\ mihaelaghica@yahoo.com \\ ${ }^{4}$ University of Bucharest, Faculty of Biology, 91-95 Splaiul Independenței Str., 050095 Bucharest, \\ Romania \\ ${ }^{5}$ Mustafa Kemal University, Faculty of Agriculture, Department of Field Crops, 31034, Antakya- \\ Hatay, Turkey \\ ${ }^{6}$ Hatay Metropolitan Municipality, 31034, Antakya-Hatay, Turkey
}

\begin{abstract}
The goal of this work was to formulate and characterize some O/W emulsions, designed as skin anti-aging creams. The cosmetic formulations based on collagen hydrolysate, caffeine and natural ingredients (essential and vegetable oils) were evaluated organoleptically, in terms of $\mathrm{pH}$ morphological, superficial and rheological properties. The rheological measurements were carried out at 23 and $32^{\circ} \mathrm{C}$, and the shear stress versus shear rate ascending and descending rheograms were built, as well as the flow patterns viscosity versus shear rate. All emulsions obtained are stable at different temperatures and the $\mathrm{pH}$ values correspond to the skin physiological one, indicating that cosmeceuticals can be safely applied to the skin. Results from the optical microscopy analysis show that all emulsions presented a creamy and foamy appearance. The superficial profiles, quantified through contact angle at solid/liquid interface, indicated a high emulsions hydrophilicity degree. The emulsions showed a pseudoplastic and thixotropic behaviour, facilitating the flow formulations and the topical application. The flow properties were quantified according to the Power law model, and the thixotropic analysis was performed using specific descriptors as thixotropic area and thixotropic index. The designed emulsions presented physico-chemical properties adequate for cosmetic skin care product formulations.
\end{abstract}

Keywords: collagen hydrolysate, anti-aging cosmetic cream, physico-chemical characteristics

\section{INTRODUCTION}

Skin aging is characterized by a progressive deterioration of the skin functional properties, linked to alterations of dermal connective tissue due to the changes at the cell, gene and protein levels. Skin aging can be divided into two basic processes: intrinsic aging and photoaging (Zhou et al., 2020; Jung-Won et al., 2019).

Collagen, being an important protein for the skin, is essential in the structure and functioning of the extracellular matrix in the dermis (Albu, 2011). Visibly thin skin, fine lines and wrinkles are the first signs of collagen reduction in the skin due to the aging process. In addition to the anti-wrinkle effect, collagen is used in cosmetics also for the hydration it offers by forming a film on the skin surface that prevents the loss of transepidermal water and protects skin by external factors (Aguirre-Cruz et al., 2020).

The most popular systems employed in the manufacture of cosmetics are emulsions (Arrieta-Escobar et al., 2019). To incorporate protein (like collagen) into cosmetic 
products (e.g. emulsions), it is appropriate that the proteins are water-soluble and reaggregation of peptides is avoided. Hydrolysates of proteins are often added into cosmetic products (Dănilă et al., 2019b; Li et al., 2008).

The aim of this study was to formulate and evaluate some O/W emulsions based on collagen hydrolysate and other natural ingredients such as vegetable and essential oils, and caffeine, that can be used as anti-age creams.

\section{MATERIALS AND METHODS}

\section{Materials}

Vegetable oils and butter (mango butter, almond oil, rice oil, coconut oil), emulsifiers (glyceryl stearate; cetearylolivate and sorbitanolivate), floral waters (linden, neroli, melissa, hammamelis), xanthan gum, caffeine, allantoin, essential oils were purchased from a local pharmacy. Type I collagen hydrolysate was obtained by acidic hydrolysis as we previously described (Dănilă et al., 2019b).

\section{Preparation of the O/W Emulsions}

According to Table 1, the ingredients of phase A (oils and emulsifier) and phase B (floral water, distilled water, collagen hydrolysate, caffeine) were heated in a water bath in two heat-resistant Berzelius beakers, periodically homogenizing the composition. When both phases reach a temperature of about $70-75^{\circ} \mathrm{C}$, they were removed from the water bath; and phase B was slowly added over phase A under continuous stirring. The mixing continued for 10 minutes, avoiding as much as possible the aeration of the emulsion. The beaker was placed in a cold-water bath under continuous stirring for 3 minutes. In the cooled composition, the ingredients of phase $\mathrm{C}$ (allantoin, xanthan gum, essential oils) were added and mixed slowly. The obtained emulsion was transferred to a sterile container.

Table 1. Composition of the dermatocosmetic O/W emulsions

\begin{tabular}{clcccc}
\hline Phase & Ingredients, \% & Emulsion 1 & Emulsion 2 & Emulsion 3 & Emulsion 4 \\
\hline A & almond oil (mL) & 10 & 20 & - & - \\
A & rice oil (mL) & - & - & 10 & 15 \\
A & mango butter $(\mathrm{g})$ & 12.6 & 11.1 & - & - \\
A & coconut oil $(\mathrm{g})$ & - & - & 12.6 & 16.1 \\
A & Bella emulsifier $(\mathrm{g}) *$ & 5 & 5 & - & - \\
A & Olliva emulsifier $(\mathrm{g}) * *$ & - & - & 5 & 5 \\
B & floral water (mL)*** & 10 & 10 & 10 & 10 \\
B & distilled water $(\mathrm{mL})$ & 60 & 50 & 60 & 50 \\
B & collagen hydrolysate $(\mathrm{g})$ & 1 & 2 & 0.5 & 2 \\
B & caffeine (g) & 0.5 & 1 & 0.4 & 1 \\
C & allantoin (g) & 0.4 & 0.2 & - & 0.2 \\
C & xanthan gum $(\mathrm{g})$ & - & 0.2 & 0.5 & 0.2 \\
C & essential oil (mL)**** & 0.5 & 0.5 & 0.5 \\
\hline
\end{tabular}

* Bella emulsifier: glyceryl stearate; $* *$ Olliva emulsifier: cetearyl olivate, sorbitan olivate

*** Linden, neroli, melissa, hammamelis; **** Eucalyptus, pine, rosemary, oregano

\section{pH Determination}

The $\mathrm{pH}$ of dermatocosmetic emulsions $\mathrm{pH}$ was evaluated using an inoLab $\mathrm{pH}$ meter. 
Optical Microscopy Analysis

The morphology of the designed emulsions was carried out using a LEICA optical microscope model S8AP0, with increase power of 20-160x.

\section{Goniometric Evaluation}

The superficial properties were performed with CAM 101 (KSV Instruments), using the contact angle routine: liquid drop (water) was spread on a solid surface (emulsioncoated slide test), analyzing the modifications of liquid drop shape on this surface.

\section{Rheological Analysis}

The flow properties of the emulsions were conducted with a rotational viscometer Multi-Visc Rheometer (Fungilab), equipped with TR 8 and TR 10 standard spindles, and a ThermoHaake P5 Ultrathermostat to ensure a constant temperature $\left(23 \pm 0.1^{\circ} \mathrm{C}\right.$ and $32 \pm 0.1^{\circ} \mathrm{C}$ ). The operational conditions were previously reported (Ghica et al., 2016).

\section{RESULTS AND DISCUSSION}

All emulsions are homogeneous, stable, with no phase separation and with a smell specific to the ingredients. The W/O emulsions presented a $\mathrm{pH}$ between 5.5 and 6 , compatible with the skin $\mathrm{pH}$. The morphology of the emulsions was performed by optical microscopy analysis and the images obtained are presented in Figure 1.

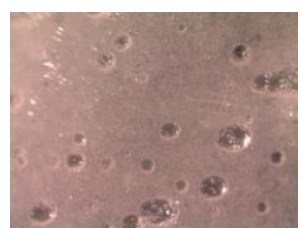

Emulsion 1

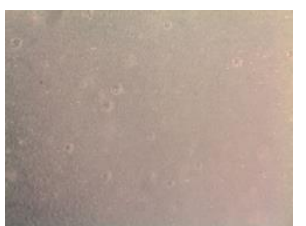

Emulsion 2

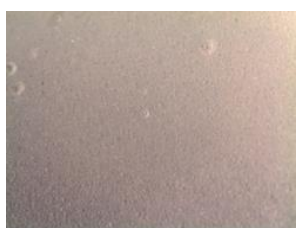

Emulsion 3

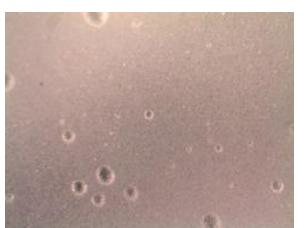

Emulsion 4

Figure 1. Optical microscopy images of W/O dermatocosmetic emulsions

From Figure 1 it can be noticed that all emulsions present a "foam-like" aspect. The differences between the microscopic images are due to various vegetable oils incorporated in the oil phase of the emulsions, with a different content of fatty acids.

The superficial properties of the emulsions were quantified through contact angle $\left(\mathrm{CA}^{\circ}\right)$ values. The drop shape (Figure 2) was monitored with a digital camera and mathematically described by the Young equation:

$\gamma_{\mathrm{SG}}=\gamma_{\mathrm{SL}}+\gamma_{\mathrm{LG}} \cdot \cos \theta$

where $\gamma_{S G}$ is the interfacial tension $S / G, \gamma_{S L}-$ the interfacial tension $S / L, \gamma_{L G}-$ the superficial tension $\mathrm{L} / \mathrm{G}$ and $\theta-$ the contact angle.

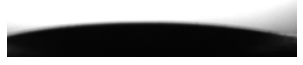

a) $\mathrm{CA}=14.21^{\circ} \pm 1.46$

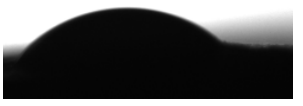

b) $\mathrm{CA}=33.03^{\circ} \pm 2.63$

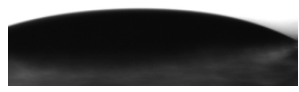

c) $\mathrm{CA}=20.71^{\circ} \pm 1.91$

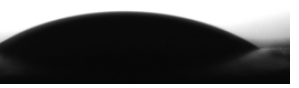

d) $\mathrm{CA}=27.66^{\circ} \pm 1.57$

Figure 2. Images of the drop shape for the W/O dermatocosmetic emulsions:

a) Emulsion 1; b) Emulsion 2; c) Emulsion 3; d) Emulsion 4 
It can be remarked that contact angle values are about 1.3-2.3 times higher for the Emulsions 2 and 4 compared to the Emulsions 1 and 3. The CA recorded values indicated a marked hydrophilicity of the emulsions, a quality requirement important in their design, with direct implications in skin hydration capacity.

The rheograms - viscosity as a function of shear rate - were built (Figure 3) and the Power law model was used to quantify the flow behaviour of the formulations:

$\eta=\mathrm{m} \cdot \dot{\gamma}^{-\mathrm{n}}$

where $\mathrm{m}$ parameter represents the viscosity obtained for the shear rate value of $1 \cdot \mathrm{s}^{-1}$, and $\mathrm{n}$ is the flow behaviour index (Dănilă et al., 2019a).

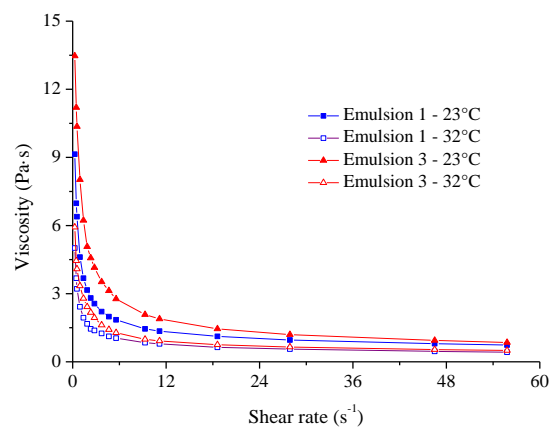

a)

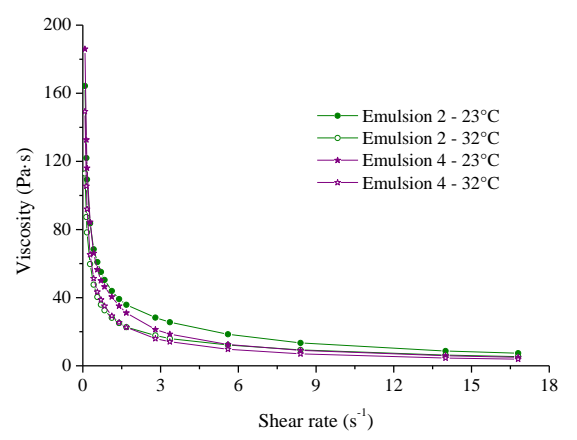

b)

Figure 3. Plots of viscosity versus shear rate, evaluated at $23^{\circ} \mathrm{C}$ and $32^{\circ} \mathrm{C}$, for:

a) Emulsion 1 and Emulsion 3; b) Emulsion 2 and Emulsion 4

As can be seen from Figure $3 a-b$, the emulsion viscosity decreased at shear rate increase showing a pseudoplastic behaviour at both working temperatures. The pseudoplastic behaviour is a requirement needed for the dermatocosmetic emulsions, both conditioning aspect and spreading as a continuous film at skin level.

The parameters $\mathrm{n}$ and $\mathrm{m}$, specific to the Power law model, are listed in Tables 2 and 3 for both working temperatures.

Table 2. Values for the determination coefficient and parameter specific to the Power law model, and for the thixotropic descriptors determined at $23^{\circ} \mathrm{C}$

\begin{tabular}{ccccccc}
\hline Formulation & $\mathrm{R}^{2}$ & $\mathrm{~m}$ & $\mathrm{n}$ & $\mathrm{S}_{\text {asc }}\left(\mathrm{Pa} \cdot \mathrm{s}^{-1}\right)$ & $\mathrm{S}_{\text {thix }}\left(\mathrm{Pa} \cdot \mathrm{s}^{-1}\right)$ & $\mathrm{T}_{\text {hyst } \%}$ \\
\hline Emulsion 1 & 0.9964 & 4.414 & 0.523 & 1423.334 & 91.303 & 6.41 \\
Emulsion 2 & 0.9976 & 44.794 & 0.508 & 1706.082 & 163.295 & 9.57 \\
Emulsion 3 & 0.9936 & 7.211 & 0.517 & 1763.773 & 123.175 & 6.98 \\
Emulsion 4 & 0.9981 & 39.813 & 0.606 & 1189.556 & 132.985 & 11.47 \\
\hline
\end{tabular}

Table 3. Values for the determination coefficient and parameter specific to the Power law model, and for the thixotropic descriptors determined at $32^{\circ} \mathrm{C}$

\begin{tabular}{ccccccc}
\hline Formulation & $\mathrm{R}^{2}$ & $\mathrm{~m}$ & $\mathrm{n}$ & $\mathrm{S}_{\text {asc }}\left(\mathrm{Pa} \cdot \mathrm{s}^{-1}\right)$ & $\mathrm{S}_{\text {thix }}\left(\mathrm{Pa} \cdot \mathrm{s}^{-1}\right)$ & $\mathrm{T}_{\text {hyst } \%}$ \\
\hline Emulsion 1 & 0.9931 & 2.435 & 0.525 & 819.094 & 43.105 & 5.26 \\
Emulsion 2 & 0.9991 & 29.991 & 0.529 & 1179.270 & 121.677 & 10.32 \\
Emulsion 3 & 0.9976 & 3.135 & 0.481 & 963.843 & 61.379 & 6.37 \\
Emulsion 4 & 0.9987 & 30.061 & 0.631 & 916.505 & 96.107 & 10.48 \\
\hline
\end{tabular}

https://doi.org/10.24264/icams-2020.II.6 
The highest values obtained for the determination coefficients $\mathrm{R}^{2}$ (between 0.9936 and 0.9981 at $23^{\circ} \mathrm{C}, 0.9931$ and 0.9991 at $32^{\circ} \mathrm{C}$ respectively) indicated that the experimental data well fitted the Power law rheological model. The most fluid formulation is Emulsion 1, the $\mathrm{m}$ parameter having the lowest value. The maximum values for $\mathrm{m}$ parameter are recorded for the Emulsions 2 and 4, about 6-10 times higher comparing to emulsions 1 and 3 at $23^{\circ} \mathrm{C}$ and about $10-15$ times at $32^{\circ} \mathrm{C}$.

The forward and backward rheograms - shear stress as a function of shear rate were also recorded (Figure 4) and the thixotropic behaviour was quantified through thixotropy area $\left(\mathrm{S}_{\text {thix }}\right)$ and thixotropy index $\left(\mathrm{T}_{\text {hyst } \%}\right)$.

$\mathrm{T}_{\text {hyst } \%}=\left(\mathrm{S}_{\text {thix }} / \mathrm{S}_{\text {asc }}\right) \cdot 100$

where $\mathrm{S}_{\text {asc }}$ is the area enclosed under the forward rheogram. Thixotropic systems are

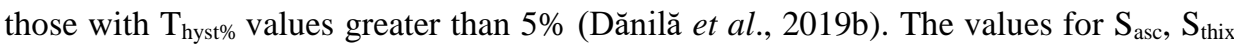
and $\mathrm{T}_{\text {hyst } \%}$ are given in Table 2 and Table 3 for both working temperatures.
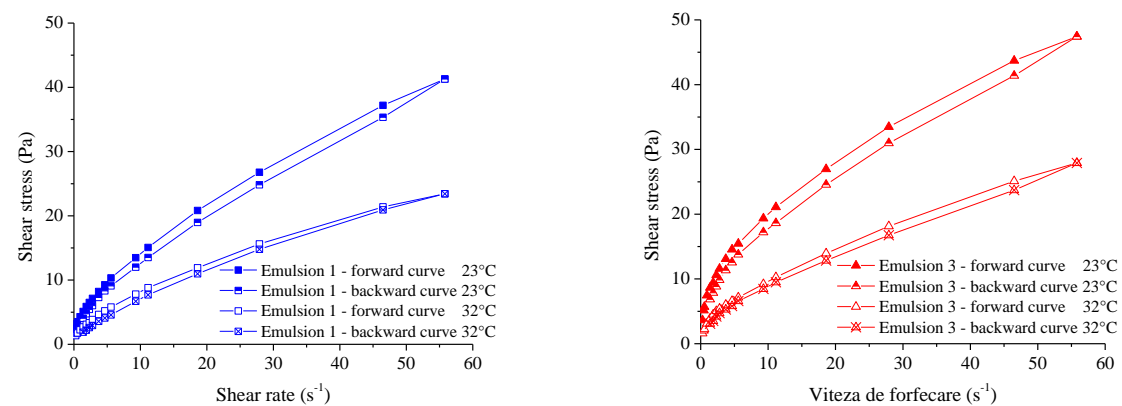

a)
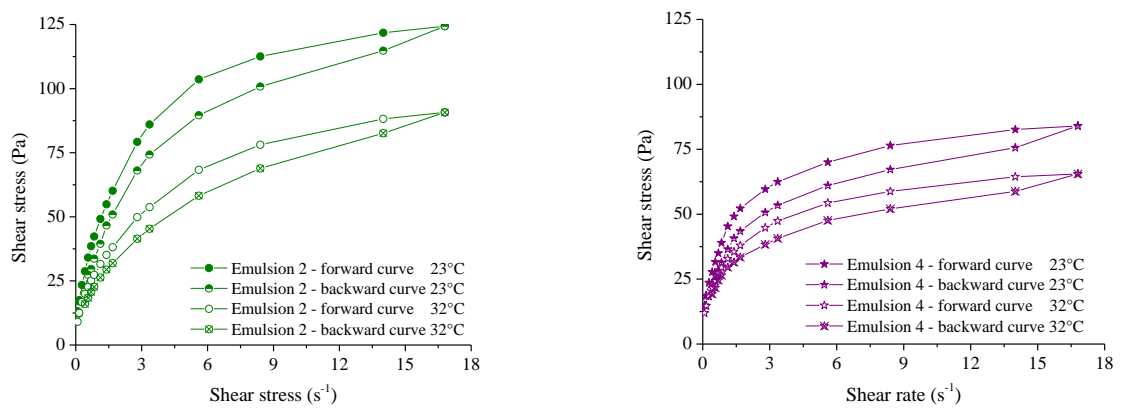

b)

Figure 4. Forward and backward rheograms, shear stress versus shear rate, evaluated at

$23^{\circ} \mathrm{C}$ and $32^{\circ} \mathrm{C}$, for: a) Emulsion 1 and Emulsion 3; b) Emulsion 2 and Emulsion 4

Figure 4a-b indicates that backward curve is placed under forward curve, indicating that for the same shear rate, the shear stress for the return curve is smaller. The thixotropic character is highlighted by the thixotropy index higher than $5 \%$ for both temperatures. The thixotropic behaviour is also a quality requirement targeted in emulsions design, allowing the transformation of a viscous product in a more fluid one, easy to spread.

Aside, the emulsions composition that influenced both their viscosity and thixotropic descriptors, another influence factor is related to the temperature. Thus, for 
temperature increase it can be noticed a m parameter decrease for all emulsions, about 1.3- 2.3 times, and a decrease of thixotropic parameters about 1.3-2.1 times.

\section{CONCLUSIONS}

All emulsions obtained are stable and the $\mathrm{pH}$ values correspond to the skin physiological one, indicating that cosmeceuticals can be safely applied to the skin. The optical microscopy analysis shows that all emulsions presented a creamy and foamy appearance. The superficial profiles, quantified through contact angle, indicated a high emulsions hydrophilicity degree. The emulsions showed a pseudoplastic and thixotropic behaviour, facilitating the flow formulations and the topical application. It can be concluded that the designed emulsions presented physico-chemical properties adequate for cosmetic skin care product formulations.

\section{Acknowledgements}

The work has been funded by the Operational Programme Human Capital of the Ministry of European Funds through the Financial Agreement 51668/09.07.2019, SMIS code 124705. This paper was financially supported by "Carol Davila" University of Medicine and Pharmacy through Contract No. CNFIS-FDI-2020-0604 (MEDEX-III) funded by the Ministry of Education and Research, Romania, from the Institutional Development Fund for Public Universities - FDI 2020.

\section{REFERENCES}

Albu, M.G. (2011), Collagen Gels and Matrices for Biomedical Applications, Lambert Academic Publishing, Saarbrücken.

Aguirre-Cruz, G., León-López, A., Cruz-Gómez, V., Jiménez-Alvarado, R. and Aguirre-Álvarez, G. (2020), "Collagen Hydrolysates for Skin Protection: Oral Administration and Topical Formulation", Antioxidants, 9(2), 181, https://doi.org/10.3390/antiox9020181.

Arrieta-Escobar, J.A., Bernardo, F.P., Orjuela, A., Camargo, M. and Morel, L. (2019), "Incorporation of Heuristic Knowledge in the Optimal Design of Formulated Products: Application to a Cosmetic Emulsion", Computers and Chemical Engineering, 122, 265-274, https://doi.org/10.1016/j.compchemeng.2018.08.032.

Dănilă, E., Kaya, D.A., Ghica, M.V., Albu Kaya, M.G., Negrea, C., Popa, L. and Nitipir, C. (2019a), "Rheological Properties and Stability of Dermatocosmetic Emulsions with Collagen and Natural Ingredients Used as Color Correcting Cream and Cream Foundation”, Revista de Chimie, 70 (6), 19281933, https://doi.org/10.37358/RC.19.6.7249.

Dănilă, E., Moldovan Z., Albu Kaya, M.G. and Ghica, M.V. (2019b), "Formulation and Characterization of some Oil in Water Cosmetic Emulsions Based on Collagen Hydrolysate and Vegetable Oils Mixtures", Pure and Applied Chemistry, 91(9), 1493-1507, https://doi.org/10.1515/pac-2018-0911.

Ghica, M.V., Hîrjău, M., Lupuleasa, D. and Dinu-Pîrvu, C.E. (2016), "Flow and Thixotropic Parameters for Rheological Characterization of Hydrogels", Molecules, 21(6), E 786 , https://doi.org/10.3390/molecules21060786.

Jung-Won, S., Soon-Hyo, K., Ji-Young, C., Jung-Im, N., Chang-Hun, H., Hye-Ryung, C. and Kyung-Chan Park. (2019), "Molecular Mechanisms of Dermal Aging and Antiaging Approaches", International Journal of Molecular Sciences, 20 (9), 2126, https://doi.org/10.3390/ijms20092126.

Li, G.Y., Fukunaga, S., Takenouchi, K. and Nakamura, F. (2008), "Comparative Study of the Physiological Properties of Collagen, Gelatin and Collagen Hydrolysate as Cosmetic Materials", International Journal of Cosmetic Science, 27(2), 101-106, https://doi.org/10.1111/j.1467-2494.2004.00251.x.

Zhou, Z., Liu, C., Wan, X. and Fang, L. (2020), "Development of a W/O Emulsion Using Ionic Liquid Strategy for Transdermal Delivery of Anti-Aging Component $\alpha$-Lipoic Acid: Mechanism of Different Ionic Liquids on Skin Retention and Efficacy Evaluation", European Journal of Pharmaceutical Sciences, 141, 105042, https://doi.org/10.1016/j.ejps.2019.105042. 


\title{
DETERMINATION OF YIELD AND YIELD COMPONENTS IN DIFFERENT SOWING TIMES OF BLACK SEED (Nigella sativa L.) IN HATAY ECOLOGICAL CONDITIONS
}

\author{
YILMAZ EREN, DURMUS ALPASLAN KAYA* \\ Mustafa Kemal University, Faculty of Agriculture, Department of Field Crops, \\ dak1976@msn.com
}

\begin{abstract}
This study was carried out in order to determine the yield and yield components of Nigella sativa L. grown in different cultivation periods in Hatay ecological conditions at Mustafa Kemal University, Faculty of Agriculture, Medical Plants trial area in 2018-2019. The experiment was laid out at spilt plot in randomized blocks with three replications. Trial; The parcels split in random blocks were carried out with 3 repetitions according to the trial pattern. In the trial, sowing was carried out on 15 November, 1 January, 15 February, 1 March, 15 March, 1 April, 15 April, 1 May and 15 May. In the study, some properties related to phenological, herbal properties, yield and yield components were examined during field trial and after harvest. As a result of the measurements taken, the plant height is $19.03-54.00 \mathrm{~cm}$, the number of branches is $8.80-3.78$ number/plant the number of plant capsules 3.73-20.67 number/plant, thousand grain weight is $3.04-2.10 \mathrm{~g}$, seed yield is $3.65-51.81 \mathrm{~kg} \cdot \mathrm{da}^{-1}$, constant oil rate \% was found between 22.47-32.07 values. Due to the increase of summer temperatures early in Hatay ecological conditions, the yield and yield factors of summer planting decreased in this period compared to winter planting. For this reason, November 1-15 is recommended as the most suitable sowing time for black seed in Hatay ecological conditions.
\end{abstract}

Keywords: Nigella sativa L., planting time, Hatay.

\section{INTRODUCTION}

Black seed (Nigella sativa L.), whose homeland is Southern Europe and West Asia, is an annual plant from the Ranunculaceae family. There are 20 species of Nigella genus in the world. Black seed is a pile rooted herbaceous plant that is cultivated in many countries, especially in the Eastern Mediterranean. There are 12 species of Nigella in Turkey. Among these species, Nigella sativa L., Nigella damascena, Nigella arvensis L. seeds are used in the treatment of snake and scorpion stings, old tumors, abscess treatment and skin rash, inflammation of the head area and the treatment of colds. It is also a milk enhancer, appetizer, spice used on bread and buns (Baytop 1984; Gün, 2012). Since the seeds and oils of black seed have a rich potential for synthetic and new drugs, it is seen that the importance given to black seed cultivation is increasing day by day (Karaman, 1999).

The yields in the provinces where the black seed culture in Turkey; Kahramanmaraş $200 \mathrm{~kg} \cdot \mathrm{da}^{-1}$, Denizli $174 \mathrm{~kg} \cdot \mathrm{da}^{-1}$, Kilis $161 \mathrm{~kg} \mathrm{da}^{-1}$, Afyonkarahisar $159 \mathrm{~kg} \mathrm{da}^{-1}$, Antalya $151 \mathrm{~kg} \mathrm{da}^{-1}$, Kars $141 \mathrm{~kg} \mathrm{da}^{-1}$, Ankara $127 \mathrm{~kg} \mathrm{da}^{-1}$, Kayseri $127 \mathrm{~kg} \mathrm{da}^{-1}$, Konya $117 \mathrm{~kg}$ $\mathrm{da}^{-1}$, Nevşehir $111 \mathrm{~kg} \mathrm{da}^{-1}$, Samsun $111 \mathrm{~kg} \mathrm{da}^{-1}$, Isparta $108 \mathrm{~kg} \mathrm{da}^{-1}$, Kütahya $102 \mathrm{~kg} \mathrm{da}$ 1, Sivas $101 \mathrm{~kg} \mathrm{da}^{-1}$, Eskişehir $100 \mathrm{~kg} \mathrm{da}^{-1}$, Kırşehir $100 \mathrm{~kg} \mathrm{da}^{-1}$, Çorum kg da ${ }^{-1}$, Malatya $94 \mathrm{~kg} \mathrm{da}^{-1}$, Uşak $89 \mathrm{~kg} \mathrm{da}^{-1}$, Balıkesir $86 \mathrm{~kg} \mathrm{da}^{-1}$, Burdur $82 \mathrm{~kg} \mathrm{da}^{-1}$, Yozgat $70 \mathrm{~kg} \mathrm{da}^{-1}$, Bursa $76 \mathrm{~kg} \mathrm{da}^{-1}$, ve Karabük $20 \mathrm{~kg} \mathrm{da}^{-1}$ (Anonymous, 2019).

Climatic conditions, environment and growing factors, planting time and similar factors affect the cultivation of black seed. For this reason, it is important to determine the breeding characteristics specific to different regions related to black seed. This study was carried out with the aim of determining the yield and yield components of black seed (Nigella sativa L.) at different planting times in Hatay ecological conditions. 


\section{MATERIALS AND METHODS}

In the experiment carried out, seed of the black cumin (Nigella sativa L.) plant obtained from Eskişehir Agricultural Research Institute in Hatay ecological conditions was used as a material. In the district of Antakya in the province of Hatay where the trial was conducted, the Mediterranean climate prevails with hot and dry summers and warm and rainy winters, and it snows only a few days of the year. The temperature ranges between -6.3 and $+43^{\circ} \mathrm{C}$ and the climatic characteristics differ significantly depending on the altitude. Annual rainfall is $877-1174 \mathrm{~mm}$. In Hatay, the wind is light and medium strong from north and northeast directions in winter months, and moderately strong and strongly from south and southwest directions in summer. Climate data for the months of the study are given in table 1 .

Table 1. Climate data for the months of the study

\begin{tabular}{lccccc}
\hline & $\begin{array}{c}\text { Min. } \\
\left({ }^{\circ} \mathrm{C}\right)\end{array}$ & $\begin{array}{c}\text { Max. } \\
\left({ }^{\circ} \mathrm{C}\right)\end{array}$ & $\begin{array}{c}\text { Average } \\
\left({ }^{\circ} \mathrm{C}\right)\end{array}$ & $\begin{array}{c}\text { Relative } \\
\text { humidity } \\
(\%)\end{array}$ & $\begin{array}{c}\text { Total } \\
\text { precipitation } \\
\left(\mathrm{mm}=\mathrm{kg} / \mathrm{m}^{2}\right)\end{array}$ \\
\hline November 2018 & 4.6 & 26.4 & 14.3 & 76.1 & 200.7 \\
December 2018 & 0.6 & 19.4 & 10.3 & 85.9 & 361.9 \\
January 2019 & -0.7 & 15.8 & 8.1 & 76.5 & 312.1 \\
February 2019 & 3.8 & 17.5 & 10.0 & 79.7 & 235.7 \\
March 2019 & 2.0 & 24.3 & 12.6 & 74.7 & 222.4 \\
April 2019 & 5.6 & 29.5 & 15.2 & 73.3 & 93.7 \\
May 2019 & 10.1 & 37.9 & 22.3 & 59.2 & 0.3 \\
June 2019 & 15.7 & 38.0 & 25.6 & 69.3 & 5.0 \\
July 2019 & 20.7 & 36.4 & 27.3 & 66.7 & 15.6 \\
August 2019 & 22.5 & 35.7 & 28.0 & 70.6 & 4.4 \\
\hline
\end{tabular}

In the experiment, black seed seeds were sown in the trial area with 3 replications at 9 different sowing times (15 November, 15 January, 15 February, 1 March, 15 March, 1 April, 15 April, 1 May and 15 May). It was not planted in December due to adverse weather conditions. In the experiment, the plots were formed in 5 rows of $4 \mathrm{~m}$ in length and the planting density was applied as $30 \times 30 \mathrm{~cm}$. In the experiment, although it was planted at 9 sowing times, yields were obtained from the plants only in 5 periods. Since there was no germination in the plants planted in the periods of April 1, April 15, May 1 and May 15, data could not be determined from the sowing on these dates. In the experiment, weed control and irrigation were performed as required during the growing period of the plants, and the plants were harvested between July and August. In the experiment, $6 \mathrm{~kg} \mathrm{da}^{-1}$ ammonium sulphate $\left(\mathrm{NH}_{4}\right) 2 \mathrm{SO}_{4}$ fertilizer was applied during the stalking period of the plants.

In this study, germination period, vegetation period, beginning of flowering, blooming times, plant height, number of branches, number of capsules, thousand kernel weight, seed yield per plant, seed yield, fixed oil ratio, oil yield, fixed and essential oil components were examined. Some of the properties examined were subjected to Duncan test for statistical comparison. 


\section{RESULTS AND DISCUSSION}

\section{Germination Periods}

In the study carried out in different plantings in Hatay climatic conditions, the germination periods were reached as 28 days in 15 November sowing, 20 days in 15 January sowing, 23 days in 15 February sowing, 20 days in 1 March sowing, and 15 March 24 days. It was also understood that different sowing periods do not matter on the germination time of the black cumin plant (Table 2).

\section{Vegetation Periods}

The vegetation periods obtained in different plantings under the ecological conditions of Hatay are given in Table 2. According to the results, the longest vegetation period was reached in 15 November sowing and the shortest vegetation period was reached in 15 March sowing. It is thought that the heavy rainfall, which is effective in the long vegetation period in October 15, is due to the fact that the weather is not hot enough and the day time is short. In other sowing dates, it was observed that the weather not too cold and the length of the day were effective in shortening the vegetation period.

\section{Beginning of Flowering}

Beginning of flowering times obtained in the experiment conducted in different sowing dates in the ecological conditions of Hatay are given in Table 2. Considering the results obtained, the longest flowering period was obtained from 15 November (189 days) and the shortest flowering period from 15 March (92 days). It was concluded that different sowing days were obtained in different sowing dates and the weather temperature affects the beginning of flowering.

\section{Flowering Days}

In this study carried out in different sowing dates under the ecological conditions of Hatay, the flowering period was obtained as 14 days in 15 November sowing, 7 days in 15 January sowing, 6 days in 15 February sowing, 8 days in 1 March sowing and 7 days in 15 March sowing (Table 2). It was concluded that the longest flowering day was 14 days in 15 November sowing and 6 days in 15 February sowing as the shortest time in sowing on different dates. It has been understood that low or high air temperature during flowering is an important factor in different flowering times.

Table 2. Phenological observations of black cumin grown at different sowing times

\begin{tabular}{cccccc}
\hline Sowing dates & Harvest dates & $\begin{array}{c}\text { Germination } \\
\text { periods } \\
\text { (days) }\end{array}$ & $\begin{array}{c}\text { Beginning } \\
\text { of flowering } \\
\text { (days) }\end{array}$ & $\begin{array}{c}\text { Flowering } \\
\text { (days) }\end{array}$ & $\begin{array}{c}\text { Vegetation } \\
\text { periods } \\
\text { (days) }\end{array}$ \\
\hline $15^{\text {th }}$ Nov. 2018 & $10^{\text {th }}$ June 2019 & 28 & 189 & 14 & 207 \\
$15^{\text {th }}$ Jan. 2019 & $17^{\text {th }}$ June 2019 & 20 & 143 & 7 & 147 \\
$15^{\text {th }}$ Feb. 2019 & $19^{\text {th }}$ June 2019 & 23 & 107 & 6 & 126 \\
$01^{\text {st }}$ Mar. 2019 & $28^{\text {th }}$ June 2019 & 20 & 95 & 8 & 120 \\
$15^{\text {th }}$ Mar. 2019 & $08^{\text {th }}$ July 2019 & 24 & 92 & 7 & 109 \\
\hline
\end{tabular}




\section{Plant Height}

The plant height of black cumin sown in different periods in Hatay ecological conditions varies between $19.03-54 \mathrm{~cm}$, while the highest plant height is $54 \mathrm{~cm}$ in 15 November sowing and the lowest plant height is in sowing with 19.03 and 15 February. (Table 3).

\section{Number of Branches}

It is seen that the number of branches of black cumin planted in different sowing times in the ecological conditions of Hatay is in the range of 3.78-8.80 pieces/plant. (Table 3). It is seen that the highest value is in 15 November sowing with 8.80 pieces/plant and the lowest value is in 15 February sowing with 3.78 pieces/plant.

\section{Number of Capsules}

The number of capsules in the black cumin plant grown in different sowing periods in the ecological conditions of Hatay was between 3.73-20.67 units/plant, and the highest value was obtained from 15 November sowing with 20.67 units/plant and the lowest value 3.73 from 15 February sowing. (Table 3).

\section{Thousand Kernel Weight}

One thousand kernel weight of black cumin grown in different sowing periods in Hatay ecological conditions was between 2.10-3.04 g and the highest value was $3.04 \mathrm{~g}$ in 15 March sowing and the lowest value was obtained in 2.10 and 15 February sowing. (Table 3).

\section{Seed Yield per Plant}

It was determined that the plant yield of black cumin grown in different sowing periods in Hatay ecological conditions varies between 0.21-3.10 g, the highest seed yield per plant was $3.10 \mathrm{~g}$ in 15 November sowing and the lowest seed yield per plant was in planting on February 15 with $0.21 \mathrm{~g}$ (Table 3).

Table 3. Morphological observations and Duncan groups of black cumin grown in different sowing periods

\begin{tabular}{lcccccc}
\hline Sowing dates & $\begin{array}{c}\text { Plant } \\
\text { height } \\
(\mathrm{cm})\end{array}$ & $\begin{array}{c}\text { Number of } \\
\text { branches } \\
\text { (pieces/plant) }\end{array}$ & $\begin{array}{c}\text { Number of } \\
\text { capsules } \\
\text { (pieces/plant) }\end{array}$ & $\begin{array}{c}\text { Thousand } \\
\text { kernel } \\
\text { weight }(\mathrm{g})\end{array}$ & $\begin{array}{c}\text { Seed } \\
\text { yield per } \\
\text { plant }(\mathrm{g})\end{array}$ & $\begin{array}{c}\text { Seed } \\
\text { yield } \\
\left(\mathrm{kg} \mathrm{da}^{-1}\right)\end{array}$ \\
\hline $15^{\text {th }}$ Nov. 2018 & $54.00 \mathrm{a}$ & $8.80 \mathrm{a}$ & $20.67 \mathrm{a}$ & $2.20 \mathrm{~b}$ & $3.10 \mathrm{a}$ & $51.81 \mathrm{a}$ \\
$15^{\text {th }}$ Jan. 2019 & $21.43 \mathrm{c}$ & $4.12 \mathrm{c}$ & $5.88 \mathrm{~d}$ & $2.37 \mathrm{~b}$ & $0.55 \mathrm{~d}$ & $9.27 \mathrm{~d}$ \\
$15^{\text {th }}$ Feb. 2019 & $19.03 \mathrm{~d}$ & $3.78 \mathrm{c}$ & $3.73 \mathrm{e}$ & $2.10 \mathrm{~b}$ & $0.21 \mathrm{e}$ & $3.65 \mathrm{e}$ \\
01 ${ }^{\text {st }}$ Mar. 2019 & $28.87 \mathrm{~b}$ & $5.33 \mathrm{~b}$ & $8.52 \mathrm{~b}$ & $2.73 \mathrm{ab}$ & $2.43 \mathrm{~b}$ & $40.27 \mathrm{~b}$ \\
$15^{\text {th }}$ Mar. 2019 & $21.33 \mathrm{c}$ & $4.18 \mathrm{c}$ & $6.90 \mathrm{c}$ & $3.04 \mathrm{a}$ & $1.25 \mathrm{c}$ & $21.65 \mathrm{c}$ \\
\hline
\end{tabular}

\section{Seed Yield}

The seed yield of black seed grown in different sowing periods in Hatay ecological conditions was between $3.65-51.81 \mathrm{~kg} \mathrm{da}^{-1}$, and the highest seed yield was obtained in 
$51.81 \mathrm{~kg} \mathrm{da}^{-1}$ in 15 November sowing and the lowest yield was obtained in $3.65 \mathrm{~kg} \mathrm{da}^{-1}$ in 15 February sowing. (Table 3).

\section{Fixed Oil Ratio}

In the ecological conditions of Hatay, the oil content in black cumin was observed between 22.47-32.07\% in different planting periods. The highest oil content was obtained with $32.07 \%$ in 15 March sowing and the lowest with $22.47 \%$ in 15 January sowing. (Table 4).

\section{Oil Yield}

The fixed oil yield of the black cumin grown in different sowing periods in Hatay varied between 2.03-23.05 $1 \mathrm{da}^{-1}$ and the highest yield was obtained from $23.05 \mathrm{l} \mathrm{da}^{-1}$ and 15 November sowing. (Table 4).

Table 4. Fixed oil ratio, oil yield and Duncan groups of black cumin grown in different sowing periods

\begin{tabular}{lcc}
\hline Sowing dates & $\begin{array}{c}\text { Fixed oil ratio } \\
(\%)\end{array}$ & $\begin{array}{c}\text { Oil yield } \\
\left(1 \mathrm{da}^{-1}\right)\end{array}$ \\
\hline $15^{\text {th }}$ November 2018 & 24.73 & $23.05 \mathrm{a}$ \\
$15^{\text {th }}$ January 2019 & 22.47 & $3.74 \mathrm{c}$ \\
$15^{\text {th }}$ February 2019 & 30.87 & $2.03 \mathrm{c}$ \\
$01^{\text {st }}$ March 2019 & 26.80 & $19.41 \mathrm{ab}$ \\
$15^{\text {th }}$ March 2019 & 32.07 & $12.60 \mathrm{~b}$ \\
\hline
\end{tabular}

\section{Fixed Oil Composition}

When the main components of fixed oil are examined; It was determined that linoleic acid methyl ester, oleic acid methyl ester, palmitic acid, stearic acid and linoleic acid ethyl ester. It was observed that the fixed oil components of black seed did not show a significant change according to different cultivation periods (Table 5).

Table 5. Fixed oil components of black seed grown in different sowing periods

\begin{tabular}{|c|c|c|c|c|c|c|c|}
\hline RT & Components & Cas \# & $\begin{array}{l}15^{\text {th }} \\
\text { Nov }\end{array}$ & $\begin{array}{c}15^{\text {th }} \\
\text { Jan }\end{array}$ & $\begin{array}{l}15^{\text {th }} \\
\mathrm{Feb}\end{array}$ & $\begin{array}{l}01^{\mathrm{st}} \\
\text { Mar }\end{array}$ & $\begin{array}{l}15^{\text {th }} \\
\text { Mar }\end{array}$ \\
\hline 5.23 & Stearic acid & $112-61-8$ & 0.03 & 1.43 & 1.35 & 1.91 & 1.97 \\
\hline 12.94 & Palmitic acid & $112-39-0$ & 11.56 & 12.23 & 10.55 & 15.27 & 16.74 \\
\hline 18.63 & Oleic acid, methyl ester & $2345-29-1$ & 21.62 & 18.75 & 19.69- & 20.73 & 21.62 \\
\hline 20.14 & Linoleic acid, methyl ester & $56599-58-7$ & 52.24 & 57.42 & 58.78 & 52.61 & 49.32 \\
\hline 24.25 & Linoleic acid ethyl ester & $544-35-4$ & 2.13 & 2.68 & 2.16 & 2.04 & 2.03 \\
\hline
\end{tabular}

\section{Essential Oil Composition}

When the main components of essential oil are examined; It was determined that linoleic acid methyl ester, o-Cymene, ethyl oleate, $\alpha$-Thujene and palmitic acid ethyl ester. It has been observed that the essential oil components of black seed oil show significant changes according to different planting periods. (Table 6). 
Determination of Yield and Yield Components in Different Sowing Times of Black Seed (Nigella sativa L.) in Hatay Ecological Conditions

Table 6. Essential oil components of black seed grown in different sowing periods

\begin{tabular}{|c|c|c|c|c|c|c|c|}
\hline RT & Components & Cas \# & $\begin{array}{l}15^{\text {th }} \\
\text { Nov }\end{array}$ & $\begin{array}{l}15^{\text {th }} \\
\text { Jan }\end{array}$ & $\begin{array}{l}15^{\text {th }} \\
\mathrm{Feb}\end{array}$ & $\begin{array}{l}01^{\text {st }} \\
\text { Mar }\end{array}$ & $\begin{array}{l}15^{\text {th }} \\
\text { Mar }\end{array}$ \\
\hline 6.51 & $\alpha$-Thujene & $2867-05-2$ & 8.34 & 9.60 & 6.74 & 6.06 & 3.14 \\
\hline 11.11 & $\gamma$-Terpinene & $99-85-4$ & 2.03 & 3.19 & 2.14 & 1.57 & 0.26 \\
\hline 12.65 & o-Cymene & $527-84-4$ & 20.29 & 28.19 & 21.55 & 16.41 & 11.32 \\
\hline 39.96 & Thymoquinone & $490-91-5$ & 2.91 & 2.51 & 1.88 & 1.21 & 0.26 \\
\hline 42.37 & Palmitic acid ethyl ester & $628-97-7$ & 6.65 & 3.78 & 4.44 & 7.99 & 10.25 \\
\hline 46.97 & Stearic acid ethyl ester & $111-61-5$ & 0.98 & 0.48 & 0.56 & 1.15 & 1.93 \\
\hline 47.75 & Ethyl oleate & $111-62-6$ & 14.94 & 9.33 & 10.59 & 16.73 & 23.39 \\
\hline 49.19 & Linoleic acid ethyl ester & $544-35-4$ & 28.36 & 26.03 & 39.82 & 36.26 & 40.03 \\
\hline
\end{tabular}

As a result, it has been determined that different sowing periods are quite effective on the yield and yield elements of black cumin grown in Hatay ecological conditions. Low or high air temperature, long or short day duration caused different results on yield and efficiency factors. If the black seed cultivation is to be done for oil and seeds in Hatay, it has been determined that the most suitable sowing date is 15 November. In the sowings made on this date, it is better for the plants to branch and form capsules as they complete their development. As a result, it is thought to result in a better result than other sowing dates in terms of seed and oil yields.

\section{Acknowledgements}

This study was funded by the Scientific Research Projects Commission of Hatay Mustafa Kemal University. (HMKU-BAP-Project No:19YL013).

\section{REFERENCES}

Baytop, T. (1984), “Türkiye'de Bitkiler ile Tedavi (Geçmişte ve Bugün)”, İstanbul Üniversitesi Eczacılık Fakültesi Yayınlarl, no: 3255, page 211-212.

Gün, M. (2012), "Kutsal Tohum (Nigella sativa): Çörek Otunun İyileştirici Etkisine İlişkin Bazı Bilgiler", Lokman Hekim Journal, 2(1), 43-46, https://dergipark.org.tr/tr/download/article-file/643364.

Karaman, A. (1999), "Çörek otunda (Nigella damascena) farklı ekim zamanlarının tohum verimi ve kaliteye etkisi üzerinde bir araştırma”, Çukurova Üniversitesi Fen Bilimleri Enstitüsü Yüksek Lisans Tezi, Adana.

*** (2019), Türkiye İstatistik Kurumu datas, http://www.tuik.gov.tr/ 


\title{
BIOLOGICAL PRETREATMENT OF WASTE FROM THE LEATHER INDUSTRY TO OBTAIN BIOGAS THROUGH ANAEROBIC CO-DIGESTION PROCESSES - A REVIEW
}

\author{
MARIANA FERDES ${ }^{1}$, ROXANA RODICA CONSTANTINESCU ${ }^{2}$ \\ ${ }^{1}$ University Politehnica Bucharest, Romania, marianaferdes@yahoo.com \\ ${ }^{2}$ Leather and Footwear Research Institute, Bucharest, Romania, rodica.roxana@yahoo.com
}

\begin{abstract}
The leather industry produces a relatively high amount of hard degradable waste, with a negative impact on the environment. This waste usually contains two major categories of residues, namely animal fat and residues containing mostly proteins, suitable for biogas conversion. Obtaining biogas from leather waste by co-digestion with vegetable waste can be a sustainable and ecofriendly alternative to conventional energy from fuels. Anaerobic co-digestion is now considered to reduce waste from various sources and turn it into energy, generating in addition a digestate used as fertilizer in agriculture. This waste is relatively resistant to transformation into an easily fermentable substrate for anaerobic digestion to produce biogas. Thus, the methane yield can be significantly improved by biological treatments with enzymes and enzyme complexes, with microorganisms selected and used as inoculum or by mixed methods, which include other physical or chemical treatments. This paper aims to show the main methods of biological treatment of leather industry waste, in order to increase the yield of biogas by co-digestion with plant materials.
\end{abstract}

Keywords: leather industry, waste, biogas, proteases, lipases, microorganisms

\section{INTRODUCTION}

The global reduction of conventional energy resources, as well as restrictive legislation on the level of environmental pollution, have created premises for the identification and exploitation of new energy sources, economic and non-polluting.

The anaerobic fermentation process is considered to be a key technology for the sustainable use of biomass consisting of the organic fraction of industrial waste, municipal solid waste, animal manure, plant debris, aquatic biomass, but also energy crops suitable for this process (Nallathambi Gunaseelan, 1997). Recently, the anaerobic fermentation process has gained special attention due to environmental protection by reducing greenhouse gas emissions, and generation of biogas, a promising source of renewable energy. The benefits of anaerobic fermentation technology are also reflected in the stability and agronomic quality of the fertilizer obtained. This method of treatment is in accordance with the provisions of the European Union, which involve the reduction and recovery of waste, as well as the promotion of clean technologies (Scano et al., 2014).

Anaerobic fermentation is a process of decomposition of the organic substrate in the presence of several species of bacteria, under controlled environmental conditions, in the absence of oxygen (Chen and Neibling, 2014; Zupancic and Grlic, 2012).

The composition of the biogas resulting from the anaerobic fermentation process varies depending on the raw material used, but also on the fermentation conditions. In general, biogas consists of two main components, methane $\left(\mathrm{CH}_{4}\right)$ and carbon dioxide $\left(\mathrm{CO}_{2}\right)$, along with small amounts of hydrogen $\left(\mathrm{H}_{2}\right)$, hydrogen sulfide $\left(\mathrm{H}_{2} \mathrm{~S}\right)$, nitrogen $\left(\mathrm{N}_{2}\right)$, ammonia $\left(\mathrm{NH}_{3}\right)$, oxygen $\left(\mathrm{O}_{2}\right)$ and water vapor (Valenti et al., 2016).

\section{Phases of the Anaerobic Fermentation Process}

Biogas production by anaerobic fermentation is a complex process consisting of 4 phases, which are the following: hydrolysis, acidogenesis, acetogenesis and 
methanogenesis, produced under the action of several species of bacteria (Gould, 2014; Khalid et al., 2011).

Bacteria degrade the substrate through enzymes that are macromolecules of protein origin, acting as biocatalysts, with the help of which living cells can produce complex reactions in a short time. By catalyzing biochemical reactions, they play a key role in the biosynthesis and degradation of living matter, occurring in all animal, plant and microorganisms. Bacterial enzymes are exo or endo enzymes that greatly accelerate the rate of biochemical reactions (Gerardi, 2003).

\section{Hydrolysis}

In the first stage, the hydrolysis, fermentative bacteria transform insoluble organic matter into sugars, amino acids and fatty acids. At this stage, microorganisms such as Clostridium, Micrococcus, Bacteroides, Butyrivibrio, Fusobacterium, Selenomonas and Streptococcus act (Cirne et al., 2007) and produce hydrolysis enzymes involved in the degradation of substrate.

\section{Protein Hydrolysis}

Proteins are polymers of amino acids, joined by peptide bonds, whose hydrolysis is mediated by extracellular proteases that give rise to polypeptides and free amino acids (Karlsson et al., 2014). During the hydrolysis step of anaerobic digestion, proteins are first converted to individual amino acids or peptides with short amino acid chains. During the fermentation stage, the breakdown of amino acids takes place, and the amino groups are released as ammonia $\left(\mathrm{NH}_{3}\right)$ or ammonium $\left(\mathrm{NH}_{4}{ }^{+}\right)$(Schnürer and Jarvis, 2010). During the protein degradation period, ammonia $\left(\mathrm{NH}_{3}\right)$ is released into the liquid part of the substrate in the fermenter, and can inhibit the fermentation process.

Organic waste contains proteins and fats, which are rich in energy and produce a relatively large amount of methane in biogas. Substrates with high protein and fat content, generally come from the leather and food industries, being useful in biogas production because they have high methanogenic potential (up to 500-600 1/ kg volatile solids) (Hejnfelt and Angelidaki, 2009).

The main materials from the leather industry are characterized by a high content of proteins and lipids, derived from leather processing.

The leather-making process generates substantial quantities of solid waste before and after the tanning stage, which are a potential source in the anaerobic digestion of biogas process (Agustini et al., 2018). The main solid residues are hide and leather shavings (Piccin et al., 2016) and sludge from wastewater treatment plants (Mella et al., 2016). Some important characteristics of these residues include the high organic load and the difficulty of degradation of recalcitrant and poorly biodegradable compounds (Mannucci et al., 2010).

The inhibition of the bioprocess of wastewaters treatment in the wastewater treatment plants, due to the presence of chromium has been demonstrated for concentrations of $140 \mathrm{mg} / \mathrm{L}$, which cause a $50 \%$ reduction of bacterial activity. The other tanning agent class, which is popularly used, is based on vegetable tannins, which are extracted from plants. (Kalyanaraman et al., 2015). The toxicity of tannins can be associated with several mechanisms such as enzyme inhibition, substrate deprivation and loss of metal ions (Bhoite and Murthy, 2015). 
Hydrolysis of Fats

The lipids represented by fats, oils and / or greases are mainly glycerol esters with three long-chain fatty acids, which form triglycerides. Lipids are part of various waste streams: leather and food industry, domestic sewage, industrial effluents, food processing, wool washing, food oil manufacturing (Karlsson et al., 2014).

In general, fats consist of glycerol and various fatty acids released by biodegradation. The enzymes that break down fats are the lipases, which are produced by aerobic or facultative aerobic microorganisms.

\section{Acidogenesis}

Acidogenesis is the fastest stage in the process of anaerobic conversion of complex organic matter, being also called the stage of acid fermentation. The monomers resulting from the hydrolysis phase are degraded by various anaerobic and optionally anaerobic bacteria into organic acids (acetic, propionic and butyric acid), volatile fatty acids, alcohols, hydrogen $\left(\mathrm{H}_{2}\right)$, carbon dioxide $\left(\mathrm{CO}_{2}\right)$ and ammonia (Kalyuzhnyi et al., 2000).

\section{Acetogenesis}

In the third phase, acetogenic bacteria convert volatile fatty acids and alcohols into hydrogen $\left(\mathrm{H}_{2}\right)$, carbon dioxide $\left(\mathrm{CO}_{2}\right)$ and acetic acid, which is the substrate for the last stage of the process, methanogenesis (Yi et al., 2014).

\section{Methanogenesis}

In the last phase of the anaerobic fermentation process, methanogenesis, are involved the methanogenic bacteria, which are very sensitive to changes in environmental factors, such as $\mathrm{pH}$ and temperature. Under these conditions, methanogenic bacteria are considered to be the factor that limits the rate of the anaerobic fermentation process (Chen et al., 2008). During this stage, the microorganisms convert the hydrogen and acetic acid previously formed into methane $\left(\mathrm{CH}_{4}\right)$ and carbon dioxide $\left(\mathrm{CO}_{2}\right)$.

Obtaining biogas from leather waste by co-digestion with vegetable waste can be a sustainable and eco-friendly alternative to conventional energy from fuels. Anaerobic co-digestion is now considered to reduce waste from various sources and turn it into energy, generating in addition a digestate used as fertilizer in agriculture.

\section{Microbial Proteases}

Microbial proteases are synthesized from the exponential growth phase and are primary metabolites of major importance for the metabolic functions of the cell. Both bacteria and fungi can produce proteases, depending on the species and environmental conditions.

Keratinolytic protease is a new generation of proteolytic enzymes with the ability to degrade recalcitrant keratin proteins like leather, feathers, horn, hooves, nails, hairs etc as well as potentially de- grade normal proteins (Brandelli, 2008). These keratinous wastes are difficult to degrade due to the dense polypeptide tightly packed by several hydrogen bonds and hydrophobic interactions. In addition,s trong disulfide cross-linking of protein chains confers high mechanical stability and resistance to proteolytic degradation of keratins by common proteases (figure 1) (Parry and North, 1998; Kreplak et al., 2004). Their unique property to degrade tough rigid protein structure has 


\section{Biological Pretreatment of Waste from the Leather Industry to Obtain Biogas through Anaerobic Co-Digestion Processes - A Review}

made them attractive biocatalysts in industrial areas dealing with those substrates like dehairing of skins and hides, detergent additives (Gupta and Ramnani, 2006). However, many researchers found that not only is keratinase a superior enzyme among those of the protease world but also the fermented feathers hydrolysate produced by keratinase gives a rich low cost substrate for biogas production and has an important potentiality to economic important plants (Gurav and Jadhav, 2013; Paul et al., 2013). A recent study found that fermented chicken feathers hydrolysate by Paenibacillus woosongensis TKB2 is a potential anti Staphylococcus agent (Paul et al., 2015).

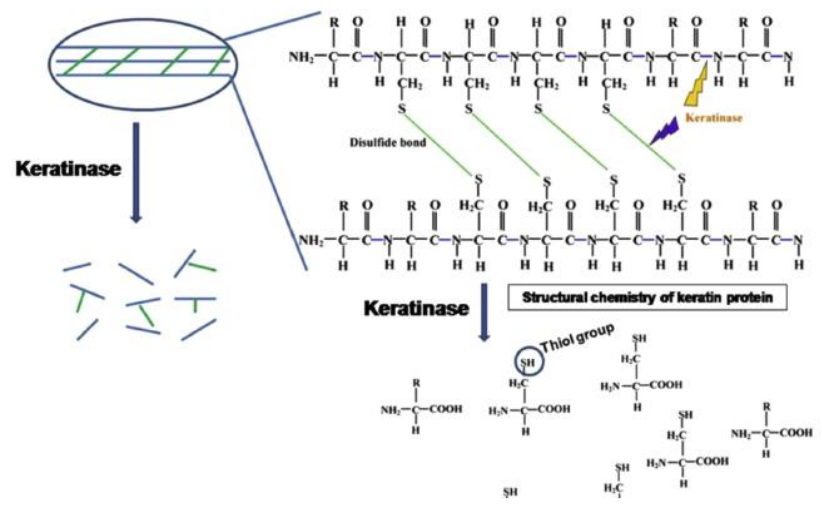

Figure 1. Mode of action of keratinolytic protease enzyme (Paul et al., 2016)

The characteristics of some bacterial proteases are shown in Table 1.

Table 1. Bacterial proteases (Paul et al., 2016)

\begin{tabular}{llll}
\hline \multicolumn{1}{c}{ Microorganism } & $\begin{array}{c}\text { Optimum } \\
\mathrm{pH}\end{array}$ & $\begin{array}{c}\text { Optimum } \\
\text { temperature, }{ }^{\circ} \mathrm{C}\end{array}$ & Type \\
\hline Bacillus sp. strain 221 & - & - & - \\
Bacillus sp. strain DZ100 & 12.5 & 85 & Serine Protease \\
Serratia marcescens P3 & 6.5 & $40-45$ & Metalloprotease \\
Chryseobacterium gleum & 8 & 30 & Metalloprotease \\
Bacillus sp. P7 & 9 & 55 & Serine Protease \\
Streptomyces sp. strain AB1 & 11.5 & 75 & Serine Protease \\
Bacillus subtilis strain RM-01 & 9 & 45 & Serine Protease \\
Bacillus licheniformis ER-15 & 11 & 70 & Serine Protease \\
Bacillus pumilus ZED17 & 8 & 40 & - \\
Bacillus mojavensis A21 & $8-11$ & 60 & Serine Protease \\
Pseudomonas aeruginosa C11 & 7.5 & 50 & Metalloprotease \\
\hline
\end{tabular}

An important feature of waste from the textile industry is the presence of various toxic components for microorganisms, which can inhibit both the multiplication of microbial cells and the entire process of anaerobic digestion.

The main form of solid tanned waste, constituting about $10 \%$ of the total weight of raw materials processed, is chromium and protein in the form of "chrome shavings" (CRS), which are small, thin pieces of leather generated during the hide shaving operation and represent high value protein-based waste (Pillai and Archana, 2012; John Sundar et al., 2011) Enzymatic treatment of CRS has been used to recover chromium 
and protein from CRS. Alkaline proteases like alcalase and combination of trypsin and esterase are used in the preparation of soluble collagen hydrolysate from CRS called chrome cake (Kupec et al., 2002).

Microbial growth on CRS necessitates the capacity of the strain to grow in highly concentrated chromium containing environment (Thacker et al., 2006). Some research (Katsifas et al., 2004) reported a chromium tolerant strain of Aspergillus carbonarius that degrades CRS in solid state fermentation process and they propose it as a useful tool in the tanning industries for the management of CRS and recovery of valuable Cr.

In other studies, the use of the keratinolytic isolate Bacillus subtilis P13 which is able to grow in alkali-free CRS, offers a promising prospect for the biodegradation of CRS along with recovery of chromium from the liquefied waste and additionally generates a valuable byproduct that can be used in biogas production (Pillai and Archana, 2012).

\section{CONCLUSION}

The leather-making process generates substantial quantities of solid waste before and after the tanning stage, which are a potential source of substrate for biogas production, in the co-digestion process.

The main solid residues are hide and leather shavings and sludge from wastewater treatment plants. Some important characteristics of these residues include the high organic load and the difficulty of degradation of recalcitrant and poorly biodegradable compounds.

The most important in the biological treatment are enzyme-producing microorganisms, able to synthesize proteases and lipases.

The methane yield can be significantly improved by biological treatments with enzymes and enzyme complexes, with microorganisms selected and used as inoculum or by mixed methods, which include other physical or chemical treatments.

\section{REFERENCES}

Agustini, C.B., Spier, F., da Costa, M. and Gutterres, M. (2018), "Biogas production for anaerobic codigestion of tannery solid wastes under presence and absence of the tanning agent", Resources, Conservation \& Recycling, 130, 51-59, https://doi.org/10.1016/j.resconrec.2017.11.018.

Bhoite, R.N. and Murthy, P.S. (2015), "Biodegradation of coffee pulp tannin by Penicillium verrucosum for production of tannase, statistical optimization and its application”, Food Bioprod. Process, 94, 727-735, https://doi.org/10.1016/j.fbp.2014.10.007.

Brandelli, A. (2008), "Bacterial keratinases: useful enzymes for bioprocessing agroindustrial wastes and beyond", Food. Bioproc.Technol, 1, 105-116, https://doi.org/10.1007/s11947-007-0025-y.

Chen, L. and Neibling, H. (2014), Anaerobic digestion basics, University of Idaho, 1-6.

Chen, Y., Cheng, J.J. and Creamer, K.S. (2008), "Inhibition of anaerobic digestion process: a review", Bioresource Technology, 99, 4044-4064, https://doi.org/10.1016/j.biortech.2007.01.057.

Cirne, D.G., Lehtomaki, A., Bjornsson, L. and Blackall, L.L. (2007), "Hydrolysis and microbial community analyses in two-stage anaerobic digestion of energy crops", J. Appl. Microbiol., 103, 516-527, https://doi.org/10.1111/j.1365-2672.2006.03270.x.

Gerardi, M.H. (2003), The Microbiology of Anaerobic Digesters, Published by John Wiley\&Sons, Inc., Hoboken, New Jersey, ISBN 0-471-20693-8.

Gould, M.C. (2014), Bioenergy and anaerobic digestion in Bioenergy - Biomass to biofuels, edited by A Dahiya, Ed. Academic Press, ISBN 9780124081208.

Gupta, R. and Ramnani, P. (2006), "Microbial keratinases and their prospective applications: an overview", Appl. Microbiol. Biotechnol., 70, 21-33, https://doi.org/10.1007/s00253-005-0239-8.

Gurav, R.G. and Jadhav, J.P. (2013), "A novel source of biofertilizer from feather biomass for banana cultivation", Env. Sci. Pollut. Res, 20, 4532-4539, https://doi.org/10.1007/s11356-012-1405-z.

Hejnfelt, A. and Angelidaki, I. (2009), "Anaerobic digestion of slaughterhouse by-products", Biomass Bioenerg., 33, 1046-1054, https://doi.org/10.1016/j.biombioe.2009.03.004. 


\section{Biological Pretreatment of Waste from the Leather Industry to Obtain Biogas through Anaerobic Co-Digestion Processes - A Review}

John Sundar, V., Raghavrao, J., Muralidharan, C. and Mandal, A.B. (2011), "Recovery and utilization of chromium-tanned proteinous wastes of leather making: a review", Crit Rev Environ Sci Technol, 41, 2048-75, https://doi.org/10.1080/10643389.2010.497434.

Kalyanaraman, C., Kameswari, K.S.B. and Rao, J.R. (2015), "Studies on enhancing the biodegradation of tannins by ozonation and Fenton's oxidation process", J. Ind. Eng. Chem. 25, 329-337, https://doi.org/10.1016/j.jiec.2014.11.012.

Kalyuzhnyi, S., Veeken, A. and Hamelers, B. (2000), "Two - particle model of anaerobic solid - state fermentation", Water Science and Technology, 41, 43-50, https://doi.org/10.2166/wst.2000.0054.

Karlsson, A., Björn, A., Shakeri Yekta, S. and Svensson, B.H. (2014), "Improvement of the biogas production process. Explorative project (EP1)", Biogas Research Center, Linköping University, Sweden.

Katsifas, E.A., Giannoutsou, E., Lambraki, M., Barla, M. and Karagouni, A.D. (2004), "Chromium recycling of tannery waste through microbial fermentation", $J$ Ind Microbiol Biotechnol, 31, 57-62, https://doi.org/10.1007/s10295-004-0115-z.

Khalid, A., Arshad, M., Anjum, M., Mahmood, T. and Dawson, L. (2011), "The anaerobic digestion of solid organic waste", Waste Management, 31, 1737-1744, https://doi.org/10.1016/j.wasman.2011.03.021

Kreplak, L., Doucet, J., Dumas, P. and Briki, F. (2004), "New aspects of the $\alpha$-helix to $\beta$ - sheet transition in stretched hard $\alpha$ - keratin fibers", Biophys. J., 87, 640-647, https://doi.org/10.1529/biophysj.103.036749.

Kupec, J., Dvorackova, M., Rudolva, S., Rucika, J. and Kolemaznik, K. (2002), "Deproteination of chrome waste by washings and enzymatic hydrolysis", J Am Leather Chem Assoc, 97, 349-54.

Mannucci, A., Munz, G., Mori, G. and Lubello, C. (2010), "Anaerobic treatment of vegetable tannery wastewaters: a review", Desalination, 264, 1-8, https://doi.org/10.1016/j.desal.2010.07.021.

Mella, B., Glanert, A.C. and Gutterres, M. (2016), "Removal of chromium from tanning wastewater by chemical precipitation and electrocoagulation", J. Soc. Leather Technol. Chem., 100, 55-61

Nallathambi Gunaseelan, V. (1997), "Anaerobic digestion of biomass for methane production: a review", Biomass Bioenergy, 13, 83-114, https://doi.org/10.1016/S0961-9534(97)00020-2.

Parry, D.A.D. and North, A.C.T. (1998), "Hard keratin intermediate filament chains: substructure of the Nand C-terminal domains and the predicted structure and function of the C-terminal domains of type 1 and type 2 chains", J. Struct. Biol., 122, 67-75, https://doi.org/10.1006/jsbi.1998.3967.

Paul, T., Halder, S.K., Das, A., Bera, S., Maity, C., Mandal, A., Das, P.S., Mohapatra, P.K.D., Pati, B.R. and Mondal, K.C. (2013), "Exploitation of Chicken Feather Waste as a Plant Growth Promoting Agent Using Keratinase Producing Novel Isolate Paenibacillus woosongensis TKB2", Biocat. Agricul. Biotechnol., 2, 50-57, https://doi.org/10.1016/j.bcab.2012.10.001.

Paul, T., Jana, A., Mandal, A.K., Mandal, A., Das Mohpatra, P.K. and Mondal, K.C. (2016), "Bacterial keratinolytic protease, imminent starter for NextGen leather and detergent industries", Sustainable Chemistry and Pharmacy, 3, 8-22, https://doi.org/10.1016/j.scp.2016.01.001.

Paul, T., Mandal, A., Mandal, S.M., Ghosh, K., Mandal, A., Halder, S.K., Das, A., Maji, S.K., Kati, A., Das Mohapatra, P.K., Pati, B.R. and Mondal, K.C. (2015), "Enzymatic Hydrolyzed Feather Peptide, a Welcoming Drug for Multiple-Antibiotic-Resistant Staphylococcus aureus: Structural Analysis and Characterization", Appl. Biochem. Biotech., 175, 3371-3386, https://doi.org/10.1007/s12010-015-1509-2.

Piccin, J.S., Gomes, C.S., Mella, B. and Gutterres, M. (2016), "Color removal from real leather dyeing effluent using tannery waste as an adsorbent", J. Environ. Chem. Eng., 4, 1061-1067, https://doi.org/10.1016/j.jece.2016.01.010.

Pillai, P. and Archana, G. (2012), "A novel process for biodegradation and effective utilization of chrome shavings, a solid waste generated in tanneries, using chromium resistant Bacillus subtilis P13", Process Biochemistry, 47, 2116-2122, https://doi.org/10.1016/j.procbio.2012.07.030.

Scano, E.A., Asquer, C., Pistis, A., Ortu, L., Demontis, V. and Cocco, D. (2014), "Biogas from anaerobic digestion of fruit and vegetable wastes: Experimental results on pilot - scale and 7 preliminary performance evaluation of a full-scale power plant", Energy Conversion and Management, 77, 22-30 https://doi.org/10.1016/j.enconman.2013.09.004

Schnürer, A. and Jarvis, Å. (2010), "Microbiological handbook for biogas plants", Swedish Waste Management U2009:03 Swedish Gas Centre Report 207.

Thacker, U., Parikh, R., Shouche, Y. and Madamwar, D. (2006), "Hexavalent chromium reduction by Providencia sp.”, Process Biochem, 41, 1332-7, https://doi.org/10.1016/j.procbio.2006.01.006.

Valenti, G., Arcidiacono, A. and Nieto Ruiz, J.A. (2016), "Assessment of membrane plants for biogas upgrading to biomethane at zero methane emission", Biomass and Bioenergy, 85, 35-47, https://doi.org/10.1016/j.biombioe.2015.11.020.

Yi, Z., Jia, Z., Fuqing, X. and Yebo, L. (2014), "Pretratament of lignocellulosic biomass for enhanced biogas Review", Progress in Energy and Combustion Science, 42, 35-53, https://doi.org/10.1016/j.pecs.2014.01.001.

Zupancic, G.Z. and Grilc, V. (2012), "Anaerobic treatment and biogas production from organic waste", in: Management of organic waste, Edited by Kumar S. and Bharti A., InTech, ISBN 978-953-307-925-7. 


\title{
ISOLATION AND CHARACTERIZATION OF FUNGAL AND BACTERIAL
} PROTEOLYTIC STRAINS FROM CHROME SHAVINGS

\author{
MARIANA FERDES ${ }^{1}$, RODICA ROXANA CONSTANTINESCU ${ }^{2}$ \\ ${ }^{1}$ University Politehnica Bucharest, Romania, marianaferdes@yahoo.com \\ ${ }^{2}$ INCDTP - Division Leather and Footwear Research Institute, Bucharest, Romania, \\ rodica.roxana@yahoo.com
}

\begin{abstract}
The chrome shavings waste obtained as a result of the leather finishing process accumulates in a large volume in tanneries and represent a major problem for the environment. This waste are particularly resistant to attack of microorganisms, due to the significant concentration of chromium and are thus difficult to degrade. In this study, chrome shavings were analyzed microbiologically by determining the total number of germs and the number of yeasts and molds on specific culture media. Several bacterial and fungal strains were isolated from the cultures in Petri dishes, after the growth of the colonies. These strains were characterized in terms of the production of proteolytic enzymes, by a method of screening on the media with casein, which allows the determination of proteolytic indices of microorganisms. As a result of the tests performed, five bacterial strains probably belonging to the genus Bacillus and two fungal strains from the genera Penicillium and Cladosporium were selected.
\end{abstract}

Keywords: chrome shavings, proteolytic bacteria and fungi

\section{INTRODUCTION}

Industries of leather-manufacturing or tanneries are highly polluting and generate significant solid and liquid wastes and obnoxious smell due to the degradation of proteinaceous components of waste (Pillai and Archana, 2012). In addition, these activities release gases like $\mathrm{H}_{2} \mathrm{~S}, \mathrm{NH}_{3}$ and $\mathrm{CO}_{2}$ (Thanikaivelan et al., 2004).

The main form of solid tanned waste, constituting about $10 \%$ of the total weight of raw materials processed, is chromium and protein in the form of "chrome shavings" (CRS), which are small, thin pieces of leather generated during the hide shaving operation and represent high value protein-based waste (John Sundar et al., 2011). Chromium is added to raw hide during leather manufacture to produce chromium tanned leather. This is done to prevent microbial degradation and produce a more durable product.

The traditional practice of disposal of chrome shavings is by landfilling, but lately there are restrictions on the disposal of chromium bearing waste in many parts of the world. The existence of valuable proteins in this waste necessitates alternative waste management strategies (John Sundar et al., 2011). In the environment, the chromium exists in many oxidation forms, ranging from $\mathrm{Cr} 2+$ to $\mathrm{Cr} 6+$, but the most stable and common states are trivalent, $\mathrm{Cr}(\mathrm{III})$ and hexavalent, $\mathrm{Cr}(\mathrm{VI})$ species (Fendorf, 1995; Dhala et al., 2013). Cr(IIl) has a high potential for environmental contamination, especially of aquifers and surface water, but it has a lower biological toxicity than $\mathrm{Cr}(\mathrm{VI})$, Hexavalent chromium has a high toxicity, because of its easy diffusion across the cell membrane in prokaryotic and eukaryotic organisms and subsequent $\mathrm{Cr}(\mathrm{VI})$ reduction in cells, which gives free radicals that may directly cause DNA alterations as well as toxic effects (Arslan et al., 1987; Kadiiska et al., 1994; Liu et al., 1995).

In the treatment of waste from tanning industries various physico-chemical (Lofrano et al., 2013) and biological systems have been used (Durai and Rajasimman, 2011; Fernandez et al., 2019), but the biological methods have low operational cost and high efficiency in the removal of contaminants (Delgado, 2009).

https://doi.org/10.24264/icams-2020.II.9 
The biotechnology and green chemistry, represent sustainable activities to remediate pollutants by eco-friendly approaches through bioremediation, waste reuse and/or byproduct recovery (Rao et al., 2002). Microbiological and enzymatic treatment of CRS has been used to recover chromium and protein from CRS. Alkaline proteases like alcalase and combination of trypsin and esterase are used in the preparation of soluble collagen hydrolysate from CRS called chrome cake (Kupec et al., 2002). Along with enzymes, the microbial management of industrial or other wastes for the removal of hazardous compounds can be achieved through degradation, biosorption or bioconversion to less toxic forms (Zouboulis et al., 2003; Feng et al., 2011).

An attractive and less expensive alternative for efficient disposal of leather industry waste, especially protein waste is the biological treatment with enzyme-producing strains of bacteria and fungi ((Pillai and Archana, 2012). The used microbial populations should have the ability to grow in highly concentrated chromium containing environment (Thacker et al., 2006). Although there are not many reports on microbial degradation of CRS, some studies have shown the possibility of selecting and using hydrolytic microorganisms. For example, Katsifas et al. (2004) obtained a fungal strain of Aspergillus carbonarius, that is able to degrade CRS in SSF (solid state fermentation) process and is tolerant to the presence of chromium. This fungal strain should be used in the treatment of waste from tanning industries for the disposal of CRS.

Recently, several proteolytic bacteria of the genus Bacillus have been tested for waste treatment in the leather industry, due to their properties for the synthesis of hydrolytic enzymes, especially collagenases and keratinases, as well as resistance to environmental factors. Pillai et al. isolated a hot spring bacterium, called Bacillus subtilis $\mathrm{P} 13$, that is able to effectively degrade and grow using chrome shavings (Pillai and Archana, 2012). Due to the high resistance of some compounds to biodegradation, bioagumentation strategies have been studied, (Chen et al., 2017; Dahiya and Venkata Mohan, 2016; Nzila et al., 2016). Bioaugmentation involves the addition of microorganisms that have the ability to biodegrade recalcitrant molecules in a polluted environment (Nzila et al., 2016; Fernendez et al., 2019).

Circular economy (CE) model has been tested as a new way of raw materials, water and energy consumption reduction in the leather industry. This model was suggested for reducing, reusing, recycling and recovering of the tannery effluents in leather processing to the different operation processes (Kanagaraj et al., 2015).

\section{MATERIALS AND METHODS}

The chrome shavings waste comes from the process of hide shaving from the tannery SC PIELOREX SA Jilava, Ilfov county.

The process of shaving is performed to obtain a uniform thickness of the skin, and due to its low specific weight, a large volume of waste accumulates in tanneries.

The chrom shavings were analyzed for microbial load. The total number of mesophilic aerobic germs and the number of yeasts and molds were determined by cultural methods. In order to determine the total number of germs, the sterilized Plate Count Agar culture medium was used and poured into Petri dishes containing $0.1-0.2$ grams of sample and, after homogenization, the samples were incubated at $30^{\circ} \mathrm{C}$. The colony counting was performed after $48-72$.

The number of yeasts and molds was performed using Potato Dextrose Agar culture medium supplemented with chloramphenicol. The dishes were incubated at $25^{\circ} \mathrm{C}$, and the colonies were counted after 5-7 days.

The results represent the average of 3 determinations. 
Several species were isolated from the colonies using an inoculation needle, and the microorganisms were grown in test tubes on Nutrient Agar for bacteria and Potato Dextrose Agar for fungi. The cultures were stored at $4{ }^{\circ} \mathrm{C}$ until use.

The proteolytic activity of isolated microorganisms was determined by a semiquantitative screening method in Petri dishes, on an agar medium containing $0.25 \%$ casein as sole carbon source. Due to the hydrolysis of casein (which causes opacification of the agar medium), a transparent area appears around the colonies producing proteolytic enzymes.

After the development of the colonies ( 3 days for bacteria and 5 days for molds) the diameters of the colonies and of hydrolysis zone were measured. The proteolytic index was determined as the ratio between the 2 diameters:

$$
\mathrm{I}_{\mathrm{P}}=\frac{\text { Diameter of hydrolysis zone }}{\text { Diameter of colony }}
$$

\section{RESULTS AND DISCUSSION}

The results obtained showed that on this type of waste there is a significant number of microorganisms, probably most in sporulated form, such as sporogenic bacteria of the genus Bacillus and several fungi. These microorganisms were able to withstand the presence of chromium in CRS and to germinate and grow in Petri dishes with autoclaved CRS samples (for 15 minutes at $121^{\circ} \mathrm{C}$ ). The appearance of Petri dishes after the growth of microorganisms is shown in figure 1.
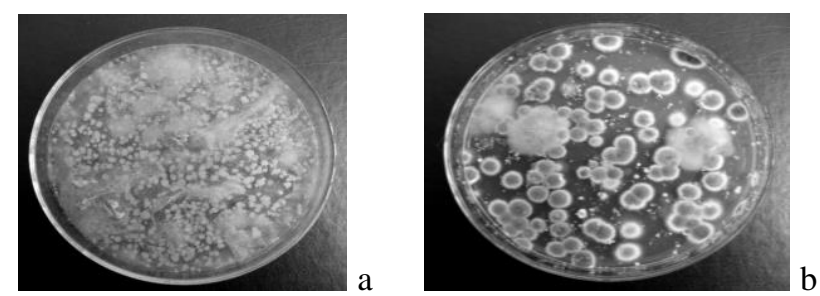

Figure 1. Determination of a) total germs number and b) fungi number in Petri dishes

The average value of number of total germs was $3 \times 10^{3} \mathrm{CFU} \cdot \mathrm{g}^{-1}$, while the number of fungi was smaller, $5.5 \times 10^{2} \mathrm{CFU} \cdot \mathrm{g}^{-1}$. On Petri dishes, we can see bacterial white colonies with a diameter of a few millimeters, shiny or rough, with irregular margins, flat profile, and odorless.

In Petri dishes containing Potato Dextrose Agar as a culture medium for fungi, three major types of colonies were observed. The most numerous were the green-blue colonies, with white edges, fluffy appearance, and characteristic odor. The microscopic preparations show thin hyphae, hyaline and reproductive structures specific to the genus Penicillium (figure 2c). Other, less numerous, gray-beige colonies belong to the genus Cladosporium, as can be seen under a microscope (figure 2b). The third type of colonies, large, gray, with a pasty appearance, belong to the coenocytic molds of the genus Mucor. They appear on the microscope slides as being made up of thicker hyphae, some of them with black sporangia with sporangiospores (figure $2 \mathrm{a}$ ). 

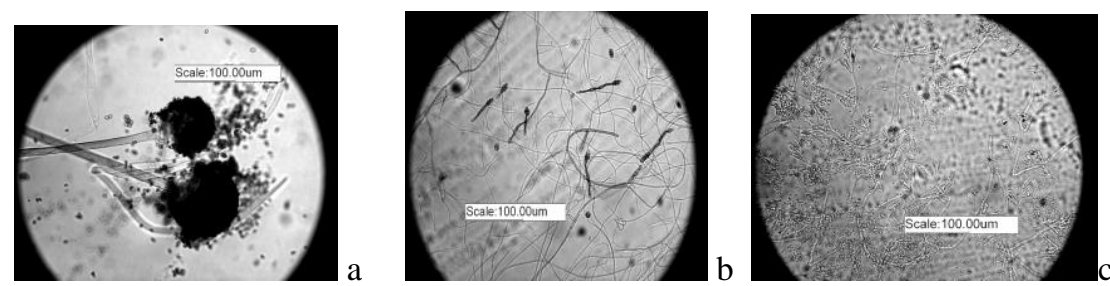

Figure 2. Microscopic aspect of a) Mucor sp., b) Cladosporium sp., c) Penicillium sp.

\section{Microorganisms Producing Proteolytic Enzymes}

The selection of the colonies that produce proteolytic enzymes was performed by observing the hydrolysis of casein in the culture medium and measuring the diameter of transparent lysis area. The proteolytic colonies were transferred in tubes and kept at $4{ }^{\circ} \mathrm{C}$ until use. The aspect of proteolytic colonies in Petri dishes are shown in figure 3 and 4 . This test method is a semi-quantitative one, and the proteolytic activity will have to be analyzed after this selection, by more accurate methods. The action of microorganisms could be tested directly, by cultivating selected microorganisms in culture media containing chrome shavings or other materials as the sole source of nutrients.
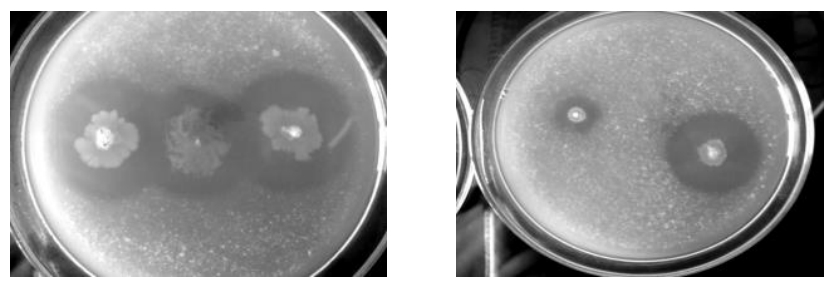

Figure 3. Proteolytic bacterial colonies grown on casein agar medium
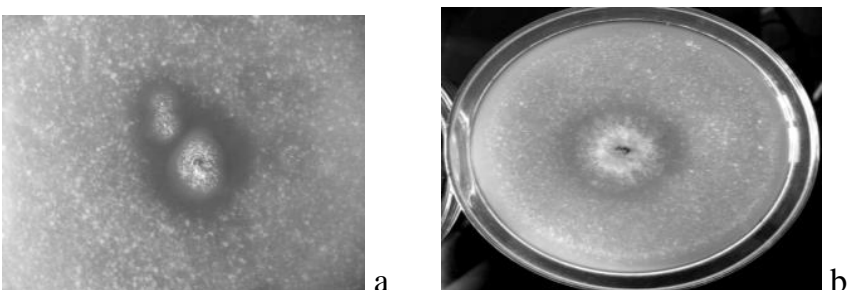

Figure 4. Proteolytic Penicillium (a) and Cladosporium (b) colonies on casein agar medium

The values of the proteolytic indices are shown in table 1 . With the exception of the Mucor colonies, all other selected species had considerable proteolytic activity, with indices between 1.6 and 3.3, and were isolated, cultivated and preserved for future research.

Table 1. Proteolytic indices of selected bacteria and fungi

\begin{tabular}{ccccc}
\hline Microorganism & Bacteria/Fungi & $\begin{array}{c}\text { Time, } \\
\text { hours }\end{array}$ & $\begin{array}{c}\text { Diameter of } \\
\text { hydrolysis zone, cm }\end{array}$ & $\begin{array}{c}\text { Proteolytic } \\
\text { index }\end{array}$ \\
\hline B1 & Bacteria & 72 & 3 & 3.3 \\
B2 & Bacteria & 72 & 3 & 1.6 \\
\hline
\end{tabular}

https://doi.org/10.24264/icams-2020.II.9 


\begin{tabular}{ccccc}
\hline Microorganism & Bacteria/Fungi & $\begin{array}{c}\text { Time, } \\
\text { hours }\end{array}$ & $\begin{array}{c}\text { Diameter of } \\
\text { hydrolysis zone, cm }\end{array}$ & $\begin{array}{c}\text { Proteolytic } \\
\text { index }\end{array}$ \\
\hline B3 & Bacteria & 72 & 3.4 & 2.2 \\
B4 & Bacteria & 72 & 3 & 3.3 \\
B5 & Bacteria & 72 & 3.5 & 2.3 \\
Penicillium sp. & Fungi & 96 & 2.5 & 2.5 \\
Cladosporium sp. & Fungi & 96 & 3.6 & 1.6 \\
Mucor sp. & Fungi & 72 & 0 & No proteolytic \\
& & & & activity \\
\hline
\end{tabular}

Numerous other studies have been conducted on this subject, which attest to the importance of microorganisms in the biodegradation process of CRS. Various microbial species producing proteolytic enzymes have been isolated, able to survive in the presence of chromium ions and transforming the protein substrate into peptides and amino acids (Chen et al., 2012; Desta et al., 2014; Liang et al., 2016; Ma et al., 2018). The research has resulted in significant results on the importance of using single microorganisms or in microbial communities, and also the effect of bioaugmentation on prokaryotic microbial community structure (Sul et al., 2016). It has been shown that the microbial community of wastewater treatment systems is affected by various factors such as influent composition, operational parameters, and environmental conditions (Chen et al., 2017; Gao et al., 2016; Niu et al., 2016).

\section{CONCLUSION}

The use of chromium-resistant proteolytic microorganisms represents an alternative of interest for the degradation process of chromed leather waste. The selection of microorganisms is the first step in degrading this waste into peptides and amino acids that can be used for other purposes, such as biogas production by codigestion.

The waste represented by chrome shavings was analyzed microbiologically in terms of the total number of germs and the number of yeasts and molds. The obtained results showed that the samples contained $3 \times 10^{3} \mathrm{CFU} \cdot \mathrm{g}^{-1}$ total aerobic microorganisms and 550 $\mathrm{CFU} \cdot \mathrm{g}^{-1}$ fungi, probably in sporulated form.

Of these microorganisms that resisted in the presence of chromium, several randomly selected colonies were isolated, belonging to the group of bacteria and fungi.

The selected bacteria and fungi were tested for the production of proteolytic enzymes by a semi-quantitative method, on a casein culture medium. These microorganisms showed proteolytic indices between 0 and 3.3, and will be tested later for enzyme production and hydrolysis of chromed leather waste.

5 bacterial strains probably belonging to the genus Bacillus and two fungal strains of the genera Penicillium and Cladosporium, which had significant proteolytic activity, were kept for further testing.

The microorganisms are therefore a permanent source of biodegrading agents, by virtue of its tolerance to chromium and capability to utilize thermally processed collagen present in the chrome shavings as sole protein source.

\section{Acknowledgements}

This project is funded by the Ministry of Research and Innovation within Program 1 - Development of the national R\&D system, Subprogram 1.2 - Institutional Performance - RDI excellence funding projects, Contract no 6 PFE/16.10.2018 


\section{REFERENCES}

Arslan, P., Beltrame, M. and Tomasi, A. (1987), "Intracellular chromium reduction", Biochem. Biophys. Acta, 931(1), 10-15, https://doi.org/10.1016/0167-4889(87)90044-9.

Chen, Y., Lan, S., Wang, L., Dong, S., Zhou, H., Tan, Z. and Li, X. (2017), “A review: driving factors and regulation strategies of microbial community structure and dynamics in wastewater treatment systems", Chemosphere, 174, 173-182, https://doi.org/10.1016/j.chemosphere.2017.01.129.

Dahiya, S. and Venkata Mohan, S. (2016), "Strategic design of synthetic consortium with embedded wastewater treatment potential: deciphering the competence of isolates from diverse microbiome", Front. Environ. Sci., 4, 1-15, https://doi.org/10.3389/fenvs.2016.00030.

Delgado, A.G. (2009), "Management of Microbial Communities to Improve Growth of ChloroetheneRespiring Dehalococcoides", Ph.D. Dissertation, Arizona State University, USA.

Dhala, B., Thatoib, H.N., Dasc, N.N. and Pandeya, B.D. (2013), "Chemical and microbial remediation of hexavalent chromium from contaminated soil and mining/metallurgical solid waste: A review", Journal of Hazardous Materials, 250-251, 272-291, https://doi.org/10.1016/j.jhazmat.2013.01.048.

Durai, G. and Rajasimman, M. (2011), "Biological treatment of tannery wastewater - A review", J. Environ. Sci. Technol., 4, 1-17, https://doi.org/10.3923/jest.2011.1.17.

Fendorf, S.E. (1995), "Surface reactions of chromium in soils and waters", Geoderma, 67 (1-2), 55-71, https://doi.org/10.1016/0016-7061(94)00062-F.

Feng, C., Zeng, G., Huang, D., Hu, S., Zhao, M., Lai, C., Huang, C., Wei, Z. and Li, N. (2011), "Effect of ligninolytic enzymes on lignin degradation and carbon utilization during lignocellulosic waste composting", Process Biochem, 46, 1515-20, https://doi.org/10.1016/j.procbio.2011.01.038.

Fernandez, M., Pereira, P.P., Agostini, E. and González, P.S. (2019), "How the bacterial community of a tannery effluent responds to bioaugmentation with the consortium SFC 500-1. Impact of environmental variables", Journal of Environmental Management, 247, 46-56, https://doi.org/10.1016/j.jenvman.2019.06.055.

John Sundar, V., Raghavrao, J., Muralidharan, C. and Mandal, A.B. (2011), "Recovery and utilization of chromium-tanned proteinous wastes of leather making: a review", Crit Rev Environ Sci Technol, 41, 2048-75, https://doi.org/10.1080/10643389.2010.497434.

Kadiiska, M.B., Xiang, Q.H. and Mason, R.P. (1994), "In vivo free radical generation by chromium (VI): an electron spin resonance spin-trapping investigation", Chem. Res. Toxicol., 7, 800-805, https://doi.org/10.1021/tx00042a013.

Kanagaraj, J., Senthilvelan, T., Panda, R.C. and Kavitha, S. (2015), "Eco-friendly waste management strategies for greener environment towards sustainable development in leather industry: a comprehensive review", Journal of Cleaner Production, 89, 1-17, https://doi.org/10.1016/j.jclepro.2014.11.013.

Katsifas, E.A., Giannoutsou, E., Lambraki, M., Barla, M. and Karagouni, A.D. (2004), "Chromium recycling of tannery waste through microbial fermentation", J Ind Microbiol Biotechnol, 31, 57-62, https://doi.org/10.1007/s10295-004-0115-z.

Kupec, J., Dvorackova, M., Rudolva, S., Rucika, J. and Kolemaznik, K. (2002), "Deproteination of chrome waste by washings and enzymatic hydrolysis", J Am Leather Chem Assoc, 97, 349-54.

Liu, K.J., Jiang, J.J., Shi, X.L., Gabrys, H., Walczak, T. and Swartz, H.M. (1995), "Low frequency EPR study of chromium (V) formation from chromium (VI) in living plants", Biochem. Biophys. Res. Commun. 206, 829-834, https://doi.org/10.1006/bbrc.1995.1118

Lofrano, G., Meriç, S., Zengin, G.E. and Orhon, D. (2013), "Chemical and biological treatment technologies for leather tannery chemicals and wastewaters: a review", Sci. Total Environ., 461-462, 265-281, https://doi.org/10.1016/j.scitotenv.2013.05.004.

Nzila, A., Razzak, S.A. and Zhu, J. (2016), "Bioaugmentation: an emerging strategy of industrial wastewater treatment for reuse and discharge", Int. J. Environ. Res. Public Health, 13(9), 846, https://doi.org/10.3390/ijerph13090846.

Pillai, P. and Archana, G. (2012), "A novel process for biodegradation and effective utilization of chrome shavings, a solid waste generated in tanneries, using chromium resistant Bacillus subtilis P13", Process Biochemistry, 47, 2116-2122, https://doi.org/10.1016/j.procbio.2012.07.030

Rao, J.R., Thanikavelan, P., Sreeram, K.J. and Nair, B.U. (2002), "Green route for the utilization of chrome shavings (chromium-containing solid waste) in tanning industry", Environ Sci Technol, 36, 1372-6, https://doi.org/10.1021/es015635s.

Thacker, U., Parikh, R., Shouche, Y. and Madamwar, D. (2006), "Hexavalent chromium reduction by Providencia sp.”, Process Biochem, 41, 1332-7, https://doi.org/10.1016/j.procbio.2006.01.006.

Thanikaivelan, P., Rao, J.R., Nair, B.U. and Ramasami, T. (2004), "Progress and recent trends in biotechnological methods for leather processing", Trends Biotechnol, 22, 181-8, https://doi.org/10.1016/j.tibtech.2004.02.008.

Zouboulis, A.I., Loukidou, M.X. and Matis, K.A. (2003), "Biosorption of toxic metals from aqueous solutions by bacteria strains isolated from metal-polluted soils", Process Biochem, 39, 909-16, https://doi.org/10.1016/S0032-9592(03)00200-0.

https://doi.org/10.24264/icams-2020.II.9 
OLIPO-WET OLIVE POMACE, A NEW RENEWABLE SOURCE FOR LEATHER RETANNING

\author{
CARMEN GAIDAU ${ }^{1}$, MARIA STANCA $^{1,2 *}$, DEMETRA SIMION ${ }^{1}$, \\ OLGA NICULESCU $^{1}$, COSMIN-ANDREI ALEXE ${ }^{1}$, CONCEPCIO CASAS ${ }^{3,4}$, \\ ANNA BACARDIT ${ }^{3}$, STOICA TONEA $^{5}$, GABRIELA PAUN ${ }^{6}$ \\ ${ }^{1}$ INCDTP - Leather and Footwear Research Institute Division, 93, Ion Minulescu St, 3, 031215 , \\ Bucharest, Romania \\ ${ }^{2}$ Doctoral School of Faculty of Applied Chemistry and Materials Science, Department of Science \\ and Engineering of Oxide Materials and Nanomaterials, University Politehnica of Bucharest, \\ Romania, maria.stanca@icpi.ro \\ ${ }^{3}$ Universitat de Lleida (UdL), A3 Leather Innovation Center Pla de la Massa, 8, 08700 Igualada, \\ Spain \\ ${ }^{4}$ Miret y Compañia S.A., 08700, concepcio.casas@udl.cat \\ ${ }^{5}$ SC Taro Comimpex SRL, Int. Giurgiului, 28A, Jilava, Ilfov, 077120, Romania, \\ tarocomimpex@yahoo.com \\ ${ }^{6}$ National Institute for Research and Development of Biological Sciences, Center of Bioanalysis, \\ 296 Splaiul Independentei, Bucharest 6, 060031, Romania, gpaunroman@ gmail.com
}

\begin{abstract}
The aim of OLIPO project is to find suitable extraction methods for an important waste of olive oil production, wet olive pomace, in view of reclaiming it as tanning and retanning material, alternative to petroleum origin materials. The total volume of wet olive pomace in Mediterranean countries where olive crops are traditional is about $80 \%$ of processed olives and is the result of a two-phase continuous extraction process. Wet olive pomace is rich in polyphenolic compounds, fats, tannins, non-tannins, possible to be extracted, concentrated, chemical processed in view of developing a new tanning product. The use of new renewable materials from oil industry as biobased tanning material for leather industry represents an important step in lowering carbon footprint of both sectors and complies with circular economy principles. The antioxidant and antimicrobial properties of olive oil pomace can be exploited in view of increasing the efficiency of the new product. The paper presents the characterisation of four kinds of wet olive pomace wastes, water and water-organic solvent extracts as tanning materials in order to select the methods for a new tanning material elaboration and testing on leathers in retanning processes.
\end{abstract}

Keywords: wet olive pomace, polyphenols, extraction methods.

\title{
INTRODUCTION
}

The olive oil industry and leather industry have in common the processing of valuable agriculture products with low yields (Araújo et al., 2015; Ludvik and Buljan, 2000) and releasing of important biomass quantities with high potential to be recovered and recycled. Solid olive mill waste is mainly generated in two-phase extraction processes and it is rich in organic matters with phytotoxic potential for the environment. The reevaluation and the finding of efficient reclaiming solutions for wet olive pomace waste represents an actual requirement; moreover, Europe target for circular economy (European Commission, 2014) is very ambitious and proposes by 2025 to not landfill the biodegradable and recyclable waste. OLIPO project target is to find new synergies in olive oil industry waste processing in view of valuable tanning components recovery and ecological sound products implementation in leather industry. The olive oil is extracted only by mechanical methods (Souilem et al., 2017) by fruits crushing and malaxation, followed by oil separation through pressure or centrifugation. Traditional discontinuous process uses the extraction of olive oil by pressure and generates pomace 
and olive milling wastewater (OMWW), meanwhile the modern processes in three and two phases use centrifugation and generate OMWW (Souilem et al., 2017). Around $98 \%$ of phenolics are released in olive oil processing by-products (Araújo et al., 2015) and represent a valuable component, able to replace synthesis preservatives with potential application in medical, food or cosmetic products. The composition of OMWW is very complex (Souilem et al., 2017) and contains fat and oils, proteins, amino acids (glutamic acid, proline), sugars (glucose, mannitol, glucose, sucrose, galactose), cellulose, hemicelluloses, lignin, phosphorous, phenolic compounds, potassium, calcium and other metals $(\mathrm{Pb}, \mathrm{Cd}, \mathrm{Fe}, \mathrm{Zn}, \mathrm{Mn}, \mathrm{Mg}, \mathrm{Na})$. The acidity of OMWW is between 4 and 6 and is due to the malic, citric, tartric, succinic or oxalic acids and the value depends on fruit variety, maturity time and storage conditions. The possibility to use OMWW in hide soaking and tanning processes showed similar performances in collagen crosslinking antioxidant and antibacterial as commercial vegetable tanning materials (Franceschi et al., 2018). The olive mill solid waste in paste or cake form is composed from 50-56.8\% moisture, 3.8-4.6\% fats and oils, $3.4-2.8 \%$ proteins, $0.9-0.8 \%$ sugars, $17.3-14.5 \%$ cellulose, $6.6-7.9 \%$ hemicelluloses, $1.4-1.7 \%$ ash, 8.5-10.2\% lignin, 0.4-0.5 N, 0.04-0.05\% $\mathrm{P}_{2} \mathrm{O}_{3}, 0.3-0.4 \% \mathrm{~K}_{2} \mathrm{O}, 0.3-0.4 \% \mathrm{CaO}$ and 25-29\% C (Souilem et al., 2017). Phenols represent the most valuable component of olive oil waste and the efficient use or extraction methods are not implemented at industrial level. The composition of phenol compounds can be classified as low molecular weight (tyrosol, hydroxyl tyrosol, oleuropein, apigenin, luteolin, p-coumaric acid, ferulic acid, syringic acid, protocatechuic acid etc.) and high molecular weight (tannins, anthocyanins, catechol-metaninic polymers etc.). The content of hydrolysable tannins and condensed tannins of extractable polyphenols from olive pomace was found to be $5.4 \%$ and $4.9 \%$, respectively (Speroni et al., 2019). The affinity of oleuropein (glycosylated seco-iridoid) or hydrolyzed oleuropein for collagen crosslinking was demonstrated on collagen films (Antunes et al., 2008) or in leather processing with commercial products (wet-green ${ }^{\circledR}$ OBE tanning agent) originated from olive leaves. The aim of this paper is the characterization of wet olive pomace waste as biobased resource for tanning materials extraction and formulation.

\section{EXPERIMENTAL}

\section{Materials and Methods}

\section{Wet Olive Pomace Waste}

Four types of olive waste (Table 1) originated from 2-phase extraction process, byproducts of the olive pomace (Arbequina, Palomar and Agro Igualada) and from 3phase extraction process (Polpa d'oliva), in dry condition were received from different Spanish olive oil companies. In table 1 the aspect of 4 kinds of olive waste in dry state as they were received and after grinding with a coffee grinder are presented.

Table 1. Olive waste from 2- and 3-phase extraction processes of olive oil

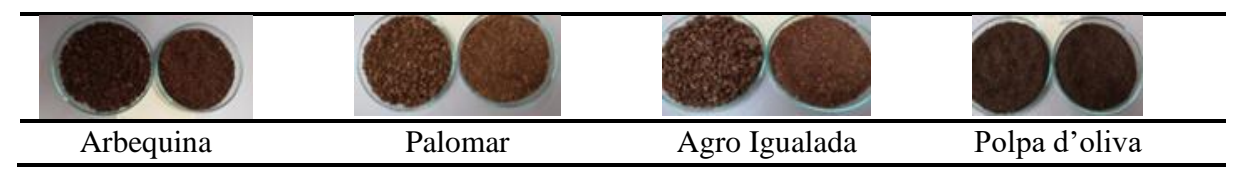

https://doi.org/10.24264/icams-2020.II.10 


\section{Olive Pomace Waste Characterization}

The characterization of olive solid waste was performed according to standardized methods for tanning materials: dry substance (SR EN ISO 4684:2006), ash (SR EN ISO 4047:2002), total nitrogen and protein content using 6.25 conversion factor (SR EN 5397: 1996), extractible substances (SR EN ISO 4048: 2018), total residuum, total soluble substances, non tannins, tannins (shaking method), insoluble substances, tanning power, binding power (SR 1883: 2008) and pH (STAS 86193/3: 1990). The total phenols were analyzed following Folin-Ciocalteu method (Singleton et al., 1999). To obtain the phenols content $15 \mathrm{~g}$ of olive waste sample were magnetically stirred for 24 hours in $60 \mathrm{~mL}$ solution of $80 \%$ methanol. After stirring, the samples were ultrasounded for one hour and then filtered on Whatman paper. The UV-VIS spectra (JASCO V550) were recorded at $\lambda=740 \mathrm{~nm}$ on a calibration curve made with gallic acid.

ATR-FTIR spectroscopy was performed on solid extracts in order to compare the chemical profile of different olive waste products and to understand the differences in their tanning properties by using a Jasco FT-IR 400 equipment from JASCO, Metertech.

\section{Extraction Methods}

The extraction methods were based on the variation of different conditions in aqueous and alcohol medium, at $55^{\circ} \mathrm{C}$ or by ultrasound (Elmo ultrasound bath, $280 \mathrm{~W}$ ) and are presented in Table 2 for ground Polpa d'oliva.

Table 2. Extraction methods for olive waste

\begin{tabular}{cl}
\hline Sample & \multicolumn{1}{c}{ Extraction conditions } \\
\hline E1 & water at $\mathrm{pH}=2,1: 40(\mathrm{w} / \mathrm{w}), 1 \mathrm{~h}$ ultrasound. \\
E2 & water at $\mathrm{pH}=2,1: 40(\mathrm{w} / \mathrm{w}), 4 \mathrm{~h}$ at $55^{\circ} \mathrm{C}$. \\
E3 & water, $1: 40(\mathrm{w} / \mathrm{w}), 1 \mathrm{~h}$ ultrasound. \\
E4 & water, $1: 40(\mathrm{w} / \mathrm{w}), 4 \mathrm{~h}$ at $55^{\circ} \mathrm{C}$. \\
E5 & water: ethanol $=1: 1(\mathrm{v} / \mathrm{v}), \mathrm{pH}=2,1: 40(\mathrm{w} / \mathrm{w}), 1 \mathrm{~h}$ ultrasound. \\
E6 & water: ethanol $=1: 1(\mathrm{v} / \mathrm{v}), \mathrm{pH}=2,1: 40(\mathrm{w} / \mathrm{w}), 4 \mathrm{~h}$ at $55^{\circ} \mathrm{C}$. \\
E7 & water: ethanol $=1: 1(\mathrm{v} / \mathrm{v}), 1: 40(\mathrm{w} / \mathrm{w}), 1 \mathrm{~h}$ ultrasound. \\
E8 & water: ethanol $=1: 1(\mathrm{v} / \mathrm{v}), 1: 40(\mathrm{w} / \mathrm{w}), 4 \mathrm{~h}$ at $55^{\circ} \mathrm{C}$. \\
E9 & water: methanol $=1: 1(\mathrm{v} / \mathrm{v}), \mathrm{pH}=2,1: 40(\mathrm{w} / \mathrm{w}), 1 \mathrm{~h}$ ultrasound. \\
E10 & water: methanol $=1: 1(\mathrm{v} / \mathrm{v}), \mathrm{pH}=2,1: 40(\mathrm{w} / \mathrm{w}), 4 \mathrm{~h}$ at $55^{\circ} \mathrm{C}$. \\
E11 & water: methanol $=1: 1(\mathrm{v} / \mathrm{v}), 1: 40(\mathrm{w} / \mathrm{w}), 1 \mathrm{~h}$ ultrasound. \\
E12 & water: methanol $=1: 1(\mathrm{v} / \mathrm{v}), 1: 40(\mathrm{w} / \mathrm{w}), 4 \mathrm{~h}$ at $55^{\circ} \mathrm{C}$. \\
\hline
\end{tabular}

\section{RESULTS AND DISCUSSIONS}

\section{Olive Waste Characterization}

The analyses of solid olive mill waste are presented in table 3 and show that the moisture is higher for Palomar (13.92\%) as compared to Polpa d'oliva with $7.76 \%$ volatile matters, values which are lower than other solid olive waste with 19-27\% moisture (Souilem et al., 2017). The ash and protein contents are higher for Polpa d'oliva as compared to the other 3 olive wastes meanwhile the extractible substances are the highest in Arbequina waste. The $\mathrm{pH}$ variation is between 5.05 and 6.15, in the range of reported olive waste $\mathrm{pH}$ values (Souilem et al., 2017). The tanning power has the values which are not in agreement with total phenol content (table 4), probably due to 
the low molecular phenol content of Polpa d'oliva waste and lower solubility. The tanning power was ranked as follows: Arbequina > Palomar > Agro Igualada > Polpa d'oliva. The binding power was not detectable due to the low concentration of the soluble substances in the analytical solution prepared directly from the solid wastes. From table 4 it can be seen that the phenols concentration extracted with methanol is highest in Polpa d'oliva and very similar in Arbequina and Palomar, all values being in the range of the stated values of 0.4-2.4 $\mathrm{g}$ GAE/100 $\mathrm{g}$ pomace for solid olive mill wastes (Demeche et al., 2013).

The phenols content of analytical solutions and non tannin solutions shows that the Arbequina water extracted phenols have the highest affinity to hide powder as compared to the other extracts and confirms the potential low molecular weight phenol content of Polpa d'oliva waste.

Table 3. Physical-chemical characteristics of solid olive waste

\begin{tabular}{lcccc}
\hline Characteristics & \multicolumn{3}{c}{ Solid olive waste } \\
& Arbequina & Palomar & $\begin{array}{c}\text { Agro } \\
\text { Igualada }\end{array}$ & $\begin{array}{c}\text { Polpa } \\
\text { d'oliva }\end{array}$ \\
\hline Dry substance, \%, & 89.31 & 86.08 & 90.24 & 92.24 \\
Ash, \% & 2.65 & 1.74 & 2.40 & 6.18 \\
Total nitrogen, \% & 0.76 & 0.70 & 1.17 & 2.23 \\
Protein, \% & 4.75 & 4.38 & 7.31 & 13.93 \\
Extractible substances \% & 14.48 & 10.90 & 11.71 & 7.92 \\
Total residuum, \%, & 29.49 & 34.73 & 25.00 & 20.72 \\
Total soluble substances, \% & 18.54 & 9.31 & 14.22 & 13.98 \\
Non tannin, \% & 15.14 & 6.88 & 12.06 & 11.94 \\
Tannin, \% & 3.40 & 2.43 & 2.16 & 1.77 \\
Insoluble substance, \% & 10.95 & 25.42 & 10.78 & 8.51 \\
pH (1:10), pH units & 5.10 & 5.05 & 5.52 & 6.15 \\
Tanning power, \% & 3.10 & 1.77 & 1.97 & 1.52 \\
Binding power, \% & nd & nd & nd & nd \\
\hline
\end{tabular}

Table 4. Total phenols in methanol (1), analytical water solution (2) extracts and in non tannins (3) of olive waste

\begin{tabular}{lccc}
\hline \multicolumn{1}{c}{ Sample } & \multicolumn{3}{c}{ Phenols, } \\
& mg GAE/g olive waste \\
& 1 & 2 & 3 \\
\hline Arbequina & 12.69 & 11.5 & 4.23 \\
Palomar & 12.57 & 7.50 & 3.56 \\
Agro Igualada & 6.74 & 9.62 & 8.31 \\
Polpa d'oliva & 19.02 & 13.96 & 4.38 \\
\hline
\end{tabular}

The main components of olive waste (Fig.1) can be recognized from the main functional groups vibrations (Hamed et al., 2005; Erdogan et al., 2015): polyphenols, alcohols and carboxylic compounds (3258-3311.18 $\left.\mathrm{cm}^{-1}\right)$, cellulose (2922.59-2925.48 $\mathrm{cm}^{-1}$ and 2853.15-2854.43 $\mathrm{cm}^{-1}$ ), hemicelluloses in Agro Igualada and Arbequina (1740$\left.1743 \mathrm{~cm}^{-1}\right)$, oleuropein $\left(1022.05-1078 \mathrm{~cm}^{-1}\right)$, proteins $\left(1631.48-1637.27 \mathrm{~cm}^{-1}\right.$ and 1598 $\mathrm{cm}^{-1}$ ), phenols $\left(1371.14-1375.96 \mathrm{~cm}^{-1}\right)$ and $B$-glycoside links $\left(1151-1156.12 \mathrm{~cm}^{-1}\right)$. 
The absorption intensity at polyphenol wavelength was recorded for Arbequina waste product as the most intense, compared to the others, which is in agreement with tanning affinity.

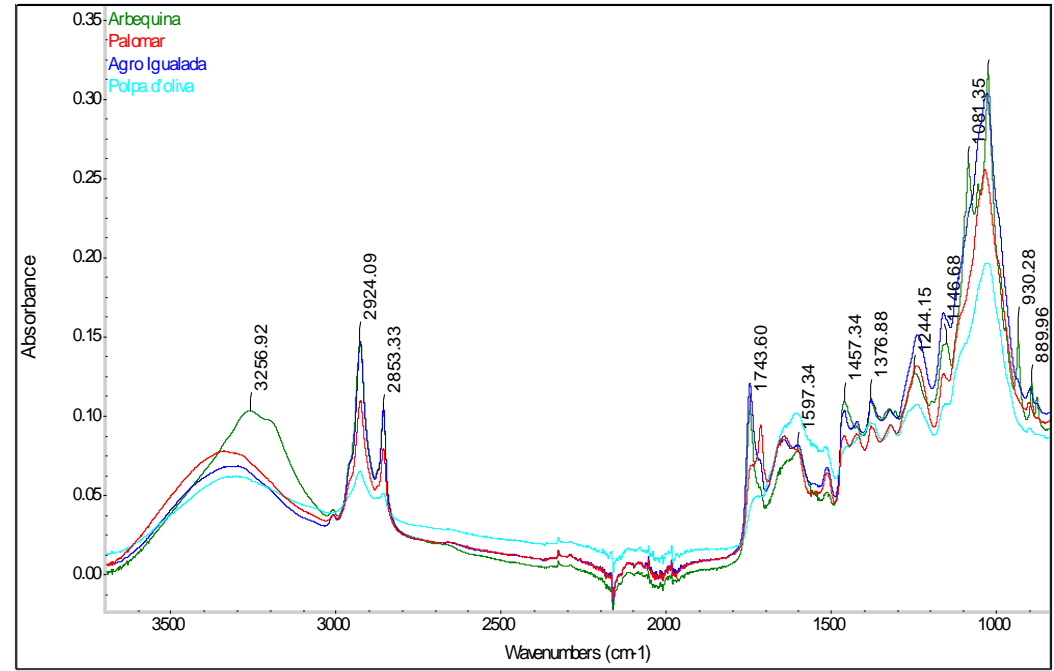

Figure 1. ATR-FTIR of solid olive waste: 1- Palomar, 2- Polpa d'oliva, 3- Agro Igualada, 4- Arbequina

\section{Olive Waste Extracts Characterization}

The total phenols of tested extracted methods performed on Polpa d'oliva waste (Table 2) are presented in Figure 2 and allowed to conclude that the extraction in acidulated water and assisted by ultrasound was the most efficient (15.32 mg GAE/g pomace). Similar phenol concentrations, between 7.72-9.42 mg GAE/g pomace, were extracted in acidulated water or in water heated at $55^{\circ} \mathrm{C}$ for 4 hours, or in water by ultrasound for 1 hour (Figure 2).

Further research will be carried out for high molecular weight phenols identification (tannins, anthocyans, catechol-metaninic polymers etc. (Speroni et al., 2019)) in correlation to extraction methods and tanning properties.

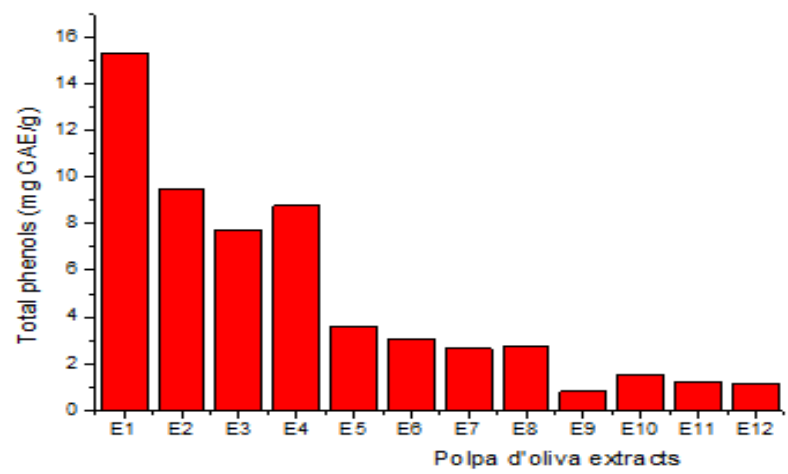

Figure 2. Total phenols content of Polpa d'oliva waste extracts

https://doi.org/10.24264/icams-2020.II.10 


\section{CONCLUSIONS}

OLIPO project aims are to find the suitable synergies for circular economy between olive oil and leather industries by exploiting the valuable polyphenol content of wet pomace wastes. Four solid olive pomace wastes were characterized from the tanning properties point of view. The experimental on extraction methods showed that the acidic conditions and ultrasound treatment are the most suitable for polyphenol solubilization. Further research will be focused on tanning and retanning experiments for a renewable product development.

\section{Acknowledgements}

The works were supported by a grant of the Romanian National Authority for Science Research and Innovation, CCCDI-UEFISCDI, project numbers 144 and 145/2020, ERANET-MANUNET-OLIPO-1 and 2, within PNCDI III and by the Operational Program Human Capital of the Ministry of European Funds through the Financial Agreement 51668/2019, SMIS 124705.

\section{REFERENCES}

Antunes, A.P.M., Attenburrow, G., Covington, A.D. and Ding, J. (2008), "Utilisation of oleuropein as a crosslinking agent in collagenic film", J Leather Sci, 2, 1-12.

Araújo, M., Pimentel, F., Alves, R.C. and Oliveira, M.B.P.P. (2015), "Phenolic compounds from olive mill wastes: health effects, analytical approach and application as food antioxidants", Trends Food Sci Technol, https://doi.org/10.1016/j.tifs.2015.06.010.

Demeche, S., Nadour, M., Larroche, C., Moulti-Mati, F. and Michaud, P. (2013), "Olive mill wastes: biochemical characterization and valorization strategies", Proc Biochem, 48, 10, 1532-1552, https://doi.org/10.1016/j.procbio.2013.07.010.

Erdogan, I., Demir, M. and Bayraktov, O. (2015), "Olive Leaf Extract as Crosslinking Agent for the Preparation of Electrospun Zein Fibers", J Appl Polym Sci, https://doi.org/10.1002/app.41338.

European Commission (2014), Towards a circular economy: A zero waste programme for Europe, Document 52014DC0398R(01)

Franceschi, M., Pacchi, G. and Maraviglia, M. (2018), "Use of olive mill waste waters in the leather industry", WO 2018/025210A1.

Hamed, O.A., Jodeh, S., Al-Hajj, N., Hamed, E.M., Abo Obeid, A. and Fouad, Y. (2005), "Cellulose acetate from biomass waste of olive industry", J Wood Sci, 61, 45-53, https://doi.org/10.1007/s10086-014-1442-y.

Ludvik, J. and Buljan, J. (2000), “Chrome management in the tanyard”, US/RAS/92/120/11-51, UNIDO,

Singleton, V.L., Orthofer, R. and Lamuela-Raventós, R.M. (1999), "Analysis of total phenols and other oxidation substrates and antioxidants by means of Folin-Ciocalteu reagent", Methods in Enzymology, 299, 152-178, https://doi.org/10.1016/S0076-6879(99)99017-1, ISBN 9780121822002.

Souilem, S., El-Abbassi, A., Kiai, H., Hafidi, A., Sayadi, S. and Galanakis, C.M. (2017), "Olive oil production sector: Environmental effects and sustainability challenges”, in: Olive Mill Waste, Oxford: Academic Press, 1-28, https://doi.org/10.1016/B978-0-12-805314-0.00001-7.

Speroni, C.S., Stiebe, J., Guera, D.R., Beutinger Bender, A.B., Ballus, C.A., dos Santos, D.R., Dal Pont Morisso, F., da Silva, L.P. and Emanuelli, T. (2019), "Micronization and gralunometric fractionation improve polyphenol content and antioxidant capacity of olive pomace", Ind Crops Prod, 137, 347-355, https://doi.org/10.1016/j.indcrop.2019.05.005. 


\title{
EVALUATION OF COMBINATION TANNING AND NATURAL FINISHING
} ON SHEEP LEATHER WITH Uncaria Gambir Roxb EXTRACT

\author{
GRESY GRIYANITASARI ${ }^{1}$, EMILIANA KASMUDJIASTUTI ${ }^{1}$, RAGIL YULIATMO ${ }^{2}$, \\ MOHAMMAD ZAINAL ABIDIN ${ }^{3 *}$ \\ ${ }^{1}$ Center for Leather, Rubber and Plastics, Ministry of Industry, Jl. Sokonandi No. 9, Yogyakarta, \\ 55166, Indonesia \\ ${ }^{2}$ Department of Leather Processing Technology, Polytechnic of Leather Technology, Yogyakarta, \\ 55188, Indonesia \\ ${ }^{3}$ Laboratory of Leather, Waste, and Animal By-products Technology, Department of Animal \\ Product Technology, Faculty of Animal Science, University of Gadjah Mada, Bulaksumur, \\ Yogyakarta, 55281, Indonesia; * corresponding author: m.zainal.abidin@ugm.ac.id
}

\begin{abstract}
The discovery of natural local resources for tanning and coloring agents are growing dramatically to sustain a cleaner leather manufacturing technology. Gambir (Uncaria gambir Roxb) has been found potential as tannin and dyestuffs for leather and textile industry. Therefore, to explore these issues, a combination tanning and finishing system were evaluated to observe the efficiency of this extract on leather processing. A combination tanning system based on chrome or glutaraldehyde - gambir and followed by gambir as coloring agent with different concentrations (100 - 200 parts/L) have been applied. In this FTIR (Fourier-transform infrared spectroscopy) analysis of gambir extract showed the presence of phenolic hydroxyl group. Our results revealed that chrome - gambir and glutaraldehyde - gambir finished leather product with 150 parts/L of gambir extract showed excellent rub fastness, color fastness to perspiration and washing. Most of the result values obtained were generally at satisfactory levels which were between $3 / 4$ and 5 fastness values. Visual evaluation reported that chrome-gambir finished leather product with 150 parts/L of gambir extract exhibited better uniformity of colour fastness.
\end{abstract}

Keywords: combination tanning, natural finishing, Uncaria gambir Roxb

\section{INTRODUCTION}

The leather tannery industry is one of the oldest industries in the world, in which need developing to meet requirements of the consumer. The various consumers and agencies expect the industry to limit the use of the hazardous materials. Tanning and leather finishing process is the critical step in leather manufacture. Mainly, chrome is used as tanning agents, in which have a negative impact for environment and health (Adiguzel-Zengin et al., 2017; China et al., 2020). While, finishing leather is the last step in whole process of leather manufacture on determination of the leather appearance (Ariram and Madhan, 2020, Sathish et al., 2016). Thus, in the conventional leather making, tanning process produced chrome in form sodium dichromate, and leather finishing use chrome in azo dyes formed azo-chromophore (Dixit et al., 2015).

Recently, eco-friendly tanning using plants and glutaraldehyde is the promising alternative for finishing leather process. Generally, this step consists of base coat and top coat, in which the base coat aims to give color effect in the leather. The colorant material can be categorized as a dyes and pigments (Covington and Wise, 2020).

Nowadays, the various consumers and agencies require the industry to limit the use of azo dyes in the manufacture of leather, and there is a rising awareness of the environment leads to the expanding demand of natural material and products (Tamil Selvi et al., 2013). Plants are the main natural resource to replace pigment for leather coloring purposes. Each species of the plant has its natural colorant (Gong et al., 2019). 


\section{Evaluation of Combination Tanning and Natural Finishing on Sheep Leather with Uncaria gambir Roxb Extract}

Tamil Selvi et al. (2013) reported that chrome-tanned leather was dyed and finished by a natural dye extract showed better coloring properties.

Gambir (Uncaria gambir Roxb) is one of the typical plants from West Sumatera, Indonesia that known as a vegetable tannin (Maier et al., 2017) and is also usually used for textile dyes (Morakotjinda \& Nitayaphat, 2016). However, the application of gambir extract for tanning agents and dyestuff of leather have not been explored yet. Thus, the objective of this study is to investigate the fixation of dyes to differently tanned-leather and to evaluate its employability in the process of natural dyeing. This approach will involve a measuring various colors and fastness that leather gains when applied with gambir through the use of different its concentration.

\section{EXPERIMENTAL}

\section{Material and Instrument}

Natural source of dyeing agent in this study were Uncaria gambir Roxb which procured from CV. Rasdi \& Co., West Sumatera, Indonesia. Pickled sheepskins were obtained from local tanneries in Yogyakarta, Indonesia and all chemicals of experiment were commercial grades. FT-IR ATR measurement was carried out on a Perkin Elmer instrument (Spectrum one, wavelength range $500-4.500 \mathrm{~cm}^{-1}$ ) to place the functional groups present in the gambir extract. Rub fastness test was determined according to (ISO, 2012), while colour fastness to perspiration and washing was determined according to (BSI, 1990).

\section{Gambir Extraction}

Gambir was used as a natural tanning and dyeing agent in the finishing process. After procured the gambir, sample was then ground using grinder machine Retsch Gmbh 5657 HAAN type SK1 and filtered manually using a sieve. Five hundred grams of ground gambir was extracted with $1500 \mathrm{~mL}$ of water in the Memmert water bath for 5 hours and stirred occasionally. Subsequent extract of dye solution was filtered and concentrated under vacuum filtration.

\section{Leather Tanning Procedure}

Six samples of sheepskin were treated with two different tanning combination: glutaraldehyde-gambir and chrome-gambir. Then, the finishing leathers were applied with a various level of gambir extract as natural dyes.

Table 1. Sample identification

\begin{tabular}{ccc}
\hline $\begin{array}{c}\text { Sample } \\
\text { identification }\end{array}$ & Tanning agent & $\begin{array}{c}\text { Quantity of gambir for } \\
\text { finishing (parts/L) }\end{array}$ \\
\hline GG100 & glutaraldehyde - gambir & 100 \\
GG150 & glutaraldehyde - gambir & 150 \\
GG200 & glutaraldehyde - gambir & 200 \\
CG100 & chrome - gambir & 100 \\
CG150 & chrome - gambir & 150 \\
CG200 & chrome - gambir & 200 \\
\hline
\end{tabular}


Table 2. Formulation of the tanning process

\begin{tabular}{llcc}
\hline Process & Product & $\%$ & Duration (min) \\
\hline \multirow{4}{*}{ Repickle } & Salt & 8 & 10 \\
& Water & 100 & \\
& Sodatan SB & 2 & 30 \\
Pretanning & Sodatan TSN & 2 & 30 \\
Tanning & Tannit LSW & 0,5 & 30 \\
& Chrome/glutaraldehyde & 6 & 60 \\
& Baking Soda (NaHCO 3$)$ & $0,5-2$ & $3 \times 15$ \\
Drained, aged, and & Gambir extract & 25 & 6 x (30 Ø 15) \\
Retanning & Acrylic Syntan & 6 & 60 \\
& $40^{\circ} \mathrm{C}$ water & 70 & \\
Fatliquoring & Synthetic fatliquor & 16 & \\
& $40^{\circ} \mathrm{C}$ water & 40 & 120 \\
Fixating & Synthetic fatliquor & 16 & \\
Antifungal & Formic acid (HCOOH) & 1,5 & $2 \times 30$ \\
Masking & Antifungal agent & 0,05 & 30 \\
\hline
\end{tabular}

\section{Leather Finishing Procedure}

The finishing procedure of two steps: basecoat and topcoat. For the base coat, the gambir extract was varied at 100, 150, and 250 parts/L (Table 2).

Table 3. Basecoat formulation

\begin{tabular}{lccc}
\hline \multicolumn{1}{c}{ Materials } & \multicolumn{3}{c}{ Quantity (g/L) } \\
& 100 & 150 & 200 \\
\hline Medium soft acrylic (RA 193) & 150 & 150 & 150 \\
Soft acrylic (RA 1) & 100 & 100 & 100 \\
Soft Uretane (Resin Uretane) & 50 & 50 & 50 \\
Wax filler & 35 & 35 & 35 \\
Penetrator & 15 & 15 & 15 \\
Gambir (Dyes) & 100 & 150 & 200 \\
Water & 550 & 500 & 450 \\
\hline
\end{tabular}

To protect the base coat and improve the properties of leather, the leather was treated using lacquer water, water, and KS water for the top coat. The formulation of topcoat was presented at Table 4.

Table 4. Topcoat formulation

\begin{tabular}{cc}
\hline Materials & Quantity $(\mathrm{g} / \mathrm{L})$ \\
\hline Lacquer water & 500 \\
Water & 350 \\
KS water & 150 \\
\hline
\end{tabular}




\section{RESULTS AND DISCUSSION}

\section{FT-IR}

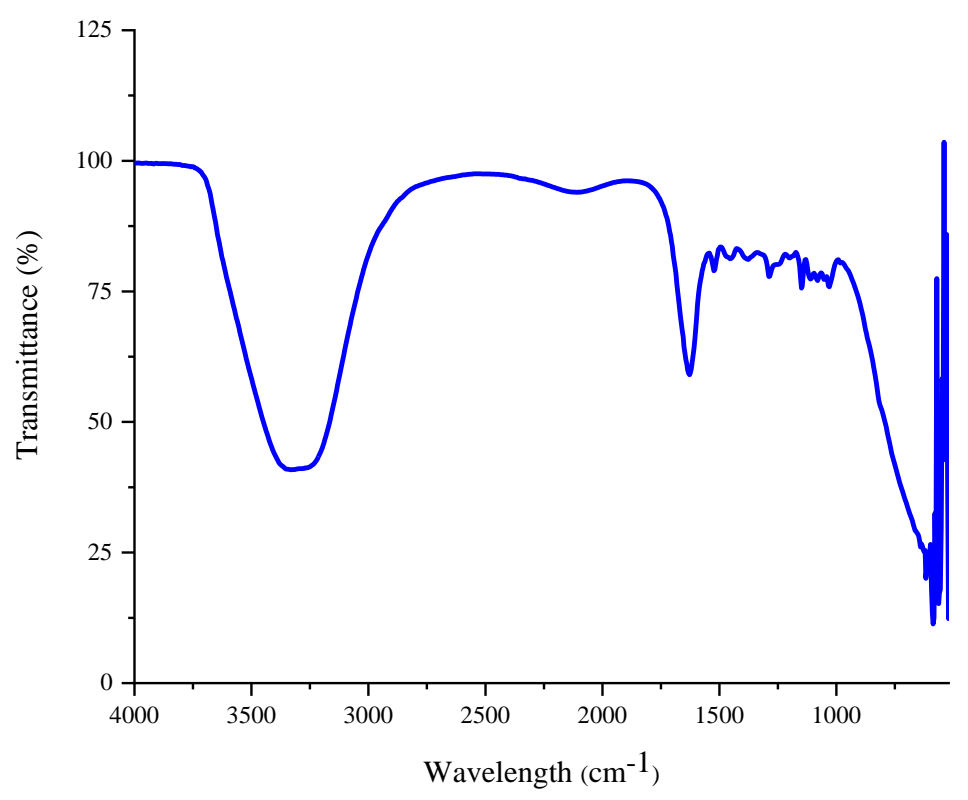

Figure 1. FTIR Spectra of gambir extract

The FTIR Spectra of gambir extract can be seen in Fig. 1. In the spectra, the broad band that falls in the range of $3700-3200 \mathrm{~cm}^{-1}$ is attributed to the fundamental stretching vibration of $-\mathrm{OH}$ groups. The peak at $1631 \mathrm{~cm}^{-1}$ is associated with the aromatic $\mathrm{C}=\mathrm{O}$ stretching. Further, the peak at $1288 \mathrm{~cm}^{-1}$ corresponds to the phenol $\mathrm{C}-\mathrm{O}$, while at $1053 \mathrm{~cm}^{-1}$ is stretching vibration of C-O-C. In general, FTIR spectra for gambir extract indicated that gambir extract containing an anthocyanin, in which the core component of natural dyeing.

\section{Rub Fastness}

Wet and dry colour fastnesses of leathers analyzed on the grain sides to rubbing are comparatively shown in Table 5. The higher quantity of gambir extract showed the low value of wet rub fastness of glutaraldehyde-gambir finished leather. Whereas, chromegambir tanned leather finished at 200 parts/L gambir extract also obtained lower wet rub fastness value than at 150 parts/L. Various quantity of gambir extract resulted in excellent rub fastness with no variation (4/5 at dry basis) on glutaraldehyde-gambir finished leather. Meanwhile, 100 parts/L of gambir extract has the highest dry rub fastness for chrome-gambir finished leather.

The rate of 4/5 on dry basis implies that leather has good to very good fastness, while the rate of 5 showed very good fastness on the finished leather (Çolak and 
Ortafidan, 2016; Berhanu \& Ratnapandian, 2017). It was observed that the higher parts of gambir in the finishing process did not increase the wet and dry rubbing fastness. The addition of 200 parts/L gambir extract on base coating resulted in lower wet and dry basis rub fastness. However, the drying rubbing fastness of the leather proved to have the same or higher values compared to wet rubbing fastness. Leather rub fastness could be affected by a combination of pigment, resin, and binder used for base coat (Sundar et al., 2006).

Table 5. Dry and wet rub fastness test results

\begin{tabular}{ccccccc}
\hline Rub fastness & \multicolumn{3}{c}{ Glutaraldehyde - Gambir } & \multicolumn{3}{c}{ Chrome - Gambir } \\
& GG 100 & GG 150 & GG 200 & CG 100 & CG 150 & CG 200 \\
\hline Wet & 4 & 4 & $3 / 4$ & $3 / 4$ & 4 & $3 / 4$ \\
Dry & $4 / 5$ & $4 / 5$ & $4 / 5$ & 5 & $4 / 5$ & $4 / 5$ \\
\hline
\end{tabular}

\section{Colour Fastness to Perspiration}

The leather colour fastness to perspiration in all combinations were separately analyzed on the grain sides and are shown comparatively in Table 6. The staining to each type of fiber (acetate, cotton, polyamide, polyester, acrylic, and wool) and the colour change in the experimental piece were assessed. This method was analyzed using a Grey scale and rated from 1-5, where the value close to 5 means that leather has excellent fastness properties (Prakash, et al., 2016). In Table 6. presented the values of colour fastness of different tanned and finished leather with varied gambir extract in terms of acetate, cotton, polyamide, polyester, acrylic, and wool.

Table 6. Colour fastness to perspiration test result

\begin{tabular}{ccccccc}
\hline Colour fastness & \multicolumn{3}{c}{ Glutaraldehyde - Gambir } & \multicolumn{3}{c}{ Chrome - Gambir } \\
to perspiration & GG 100 & GG 150 & GG 200 & CG 100 & CG 150 & CG 200 \\
\hline Acetat & 4 & $4 / 5$ & $3 / 4$ & $3 / 4$ & 5 & $3 / 4$ \\
Cotton & 3 & $3 / 4$ & 3 & $3 / 4$ & 4 & 3 \\
Polyamide & $3 / 4$ & $4 / 5$ & $3 / 4$ & 4 & $4 / 5$ & 4 \\
Polyester & $3 / 4$ & 4 & 3 & $3 / 4$ & $4 / 5$ & $3 / 4$ \\
Acrylic & 4 & 4 & 3 & $3 / 4$ & 5 & $3 / 4$ \\
Wool & 3 & 4 & 3 & $3 / 4$ & 4 & $3 / 4$ \\
\hline
\end{tabular}

Thus, examination of Table 6 revealed that the fastness of chrome-gambir tanned leather are slightly better compared to glutaraldehyde-gambir finished leather. It also showed that the concentration of gambir (150 part/L) exhibited better colour fastness. Overall, the result of colour fastness to perspiration samples were quite good value (not less than 3), especially at concentration of Gambir 150 parts/L.

\section{Colour Fastness to Washing}

This colour fastness to washing test could determine the colour resistance of leather samples to washing under domestic conditions. The staining materials were similar with colour fastness to perspiration. Before washing and drying, samples were agitated in a neutral of synthetic solution. The result of colour fastness to washing test samples are shown in Table 7. Compared with each material samples, chrome-gambir tanned leather at 150 parts/L produced slightly higher value of colour fastness to wool. However, all of 


\section{Evaluation of Combination Tanning and Natural Finishing on Sheep Leather with Uncaria gambir Roxb Extract}

finished leather with glutaraldehyde-gambir and chrome-gambir showed excellent colour fastness to washing value.

Table 7. Colour fastness to washing test result

\begin{tabular}{ccccccc}
\hline Wash fastness & GG 100 & GG 150 & GG 200 & CG 100 & CG 150 & CG 200 \\
\hline Acetat & 5 & 5 & 5 & 5 & 5 & 5 \\
Cotton & $4 / 5$ & $4 / 5$ & $4 / 5$ & $4 / 5$ & $4 / 5$ & $4 / 5$ \\
Polyamide & 5 & 5 & 5 & 5 & 5 & 5 \\
Polyester & 5 & 5 & 5 & 5 & 5 & 5 \\
Acrylic & 5 & 5 & 5 & 5 & 5 & 5 \\
Wool & $4 / 5$ & $4 / 5$ & $4 / 5$ & $4 / 5$ & 5 & $4 / 5$ \\
\hline
\end{tabular}

\section{Visual Assessment}

The visual assessment of finished leather showed different unique colour as Figure 2. The higher proportion of gambir extract on base coating resulted the darker colour of chrome-gambir finished leather. The use of 200 parts/L of gambir extract showed a better colour uniformity for both glutaraldehyde-gambir and chrome-gambir finished leather, while finished leather at low concentration of gambir was less consistency. At the similar quantity of gambir extract, the chrome-gambir finished leather produced a slightly darker colour than glutaraldehyde-gambir tanned leather.

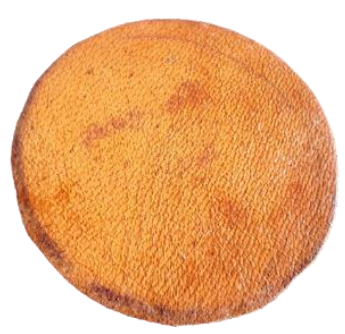

GG100

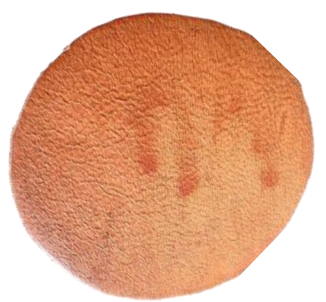

CG100

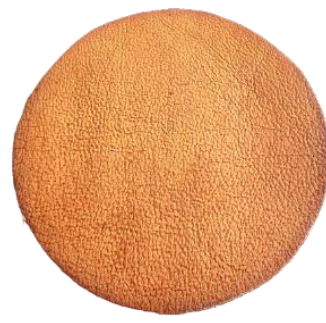

GG150

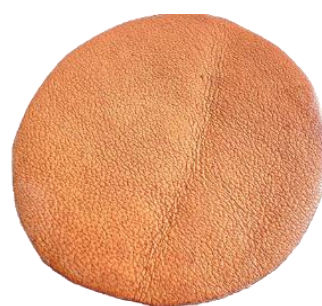

CG150

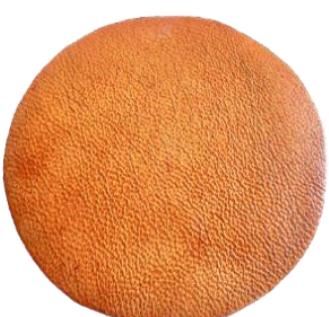

GG200

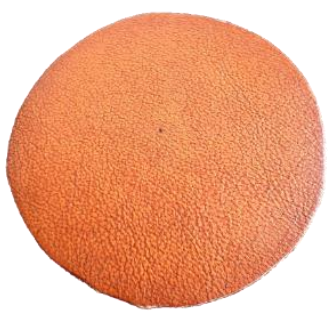

CG200

Figure 2. Photograph samples of finished leather

On the other hand, at 100 parts/L of gambir extract generated less uniform colour. Because of the concentration of gambir is dilute, the penetration of dye to leather could not as good as concentrated dyes $(200 \mathrm{~g} / \mathrm{L})$. The concentrated dye provided the finished leather was darker. 


\section{CONCLUSIONS}

One hundred fifty parts/L of gambir extract at base coating (finishing stage) were conclude as optimum concentration for the finished leather, resulted the value of the rub fastness, colour fastness to perspiration, and colour fastness to washing are excellent. Here, Uncaria Gambir Roxb (Gambir) provides an alternative of natural dyeing for finishing leather and support for eco-benign leather finishing process. However, further exploring about natural local resources for tanning and dyeing agents in Indonesia is on progress.

\section{REFERENCES}

Adiguzel-Zengin, A.C., Zengin, G., Kilicarislan-Ozkan, C., Dandar, U. and Kilic, E. (2017), "Characterization and application of Acacia nilotica L. as an alternative vegetable tanning agent for leather processing", Fresenius Environmental Bulletin, 26(12), 7319-7326.

Ariram, N. and Madhan, B. (2020), "Development of bio-acceptable leather using bagasse", Journal of Cleaner Production, 250. https://doi.org/10.1016/j.jclepro.2019.119441.

Aslan, A. (2013), "Improving the dyeing properties of vegetable tanned leathers using chitosan formate", Ekoloji, 35(86), 26-35, https://doi.org/10.5053/ekoloji.2013.864.

Berhanu, T. and Ratnapandian, S. (2017), "Extraction and Optimization of Natural Dye from Hambo Hambo (Cassia singueana) Plant Used for Coloration of Tanned Leather Materials", Advances in Materials Science and Engineering, https://doi.org/10.1155/2017/7516409.

British Standard Institution (1990), "BS 1006:1990: Methods of test for colour fastness of textiles and leather".

China, C.R., Hilonga, A., Nyandoro, S.S., Schroepfer, M., Kanth, S.V., Meyer, M. and Njau, K.N. (2020), "Suitability of selected vegetable tannins traditionally used in leather making in Tanzania", Journal of Cleaner Production, 251, 119687, https://doi.org/10.1016/j.jclepro.2019.119687.

Çolak, S.M. and Ortafidan, T. (2016), "Ecofriendly dyeing process with enzymes", Proceedings of the $6^{\text {th }}$ International Conference on Advanced Materials and Systems, https://doi.org/10.24264/icams-2016.iii.3.

Covington, A.D. and Wis, W.R. (2020), Tanning Chemistry. The Science of Leather, $2^{\text {nd }}$ Edition, Royal Society of Chemistry, London.

Dixit, S., Yadav, A., Dwivedi, P.D. and Das, M. (2015), “Toxic hazards of leather industry and technologies to combat threat: A review", Journal of Cleaner Production, 87(C), 39-49, https://doi.org/10.1016/j.jclepro.2014.10.017.

Gong, K., Pan, Y., Rather, L.J., Wang, W., Zhou, Q., Zhang, T. and Li, Q. (2019), "Natural pigment during flora leaf senescence and its application in dyeing and UV protection finish of silk and wool--a case study of Cinnamomum Camphora", Dyes and Pigments, 166, 114-121, https://doi.org/10.1016/j.dyepig.2019.03.037.

ISO (International Organization for Standardization) (2012), "ISO 20433:2012 (IULTCS/IUF 452): Leather -Test for colour fastness -- colour fastness to crocking",

Maier, M., Oelbermann, A.L., Renner, M. and Weidner, E. (2017), "Screening of European medicinal herbs on their tannin content-New potential tanning agents for the leather industry", Industrial Crops and Products, 99, 19-26, https://doi.org/10.1016/j.indcrop.2017.01.033.

Morakotjinda, P. and Nitayaphat, W. (2016), "Dyeing properties and color fastness of cotton fabrics dyed with uncaria gambir extract by the padding techniques", Key Engineering Materials, https://doi.org/10.4028/www.scientific.net/KEM.675-676.505.

Prakash, A., Aravindhan, R., Fathima, N. and Raghava-Rao, J. (2016), "Dyeing of chamois leather using water soluble sulphur dyes", Journal of the American Leather Chemists Association, 111(10), 383-388.

Sathish, M., Madhan, B., Sreeram, K.J., Raghava Rao, J. and Nair, B.U. (2016), "Alternative carrier medium for sustainable leather manufacturing - A review and perspective", Journal of Cleaner Production, 112, 49-58, https://doi.org/10.1016/j.jclepro.2015.06.118.

Sundar, S., Vijayalakshmi, N., Gupta, S., Rajaram, R. and Radhakrishnan, G. (2006), "Aqueous dispersions of polyurethane-polyvinyl pyridine cationomers and their application as binder in base coat for leather finishing", Progress in Organic Coatings, 56(2-3), 178-184, https://doi.org/10.1016/j.porgcoat.2006.04.001.

Tamil Selvi, A., Aravindhan, R., Madhan, B. and Raghava Rao, J. (2013), "Studies on the application of natural dye extract from Bixa orellana seeds for dyeing and finishing of leather", Industrial Crops and Products, https://doi.org/10.1016/j.indcrop.2012.07.015.

Zengin, G., Sardroudi, S.P., Bitlisli, B.O. and Adiguzel Zengin, A.C. (2016), "Effect of various tanning processes on characteristics of lining leathers", 6th International Conference on Advanced Materials and Systems (ICAMS), 435-440, https://doi.org/10.24264/icams-2016.III.21.

https://doi.org/10.24264/icams-2020.II.11 
Evaluation of Combination Tanning and Natural Finishing on Sheep Leather with Uncaria gambir Roxb Extract

https://doi.org/10.24264/icams-2020.II.11 
BOD \& COD REDUCTION FROM TEXTILE WASTEWATER USING BIOAUGMENTED HDPE CARRIERS

\author{
OVIDIU IORDACHE ${ }^{1}$, IOANA CORINA MOGA ${ }^{2}$, ELENA CORNELIA MITRAN ${ }^{1}$, IRINA \\ SANDULACHE $^{1}$, MARIA MEMECICA ${ }^{1}$, LUCIA OANA SECAREANU ${ }^{1}$, CRISTINA LITE $^{1}$, \\ ELENA PERDUM ${ }^{1}$ \\ ${ }^{I}$ National R\&D Institute for Textile and Leather, Lucretiu Patrascanu 16, Bucharest, Romania, \\ office@incdtp.ro \\ ${ }^{2}$ DFR Systems SRL, Drumul Taberei 45, Bucharest, Romania,dfr@dfr.ro
}

\begin{abstract}
Textile wastewater effluents are considered one of the most polluting sources, among all industrial sectors, in terms of both effluent volume and composition, with high BOD and COD values. Biochemical Oxygen Demand (BOD) represents the amount of oxygen consumed by bacteria and other microorganisms in decomposing organic matter under aerobic conditions. Chemical Oxygen Demand (COD) represents the measurement of the oxygen required to oxidize soluble and particulate organic matter in water. The main goal of the present study was the investigation in reduction of both BOD and COD concentrations, in a textile wastewater source, using bioaugmented MBBR specific HDPE carriers (composition: 5\% talc, 7\% cellulose and 88\% HighDensity-Polyethylene). The HDPE carriers were bio-augmented in an experimental laboratory installation with five fungi microbial strains (either as a mix or individual strain): 3 own microbial isolates (from decaying wood source) and 2 collection strains, namely Cerioporus squamosus (Basidiomycota phylum) and Fusarium oxysporum (Ascomycota phylum). Results showed a reduction rate of COD value of $53.45 \%$, of HDPE carriers bio-augmented in the experimental laboratory installation (mix inoculation), and BOD reduction rates between $28 \%$ (carriers bioaugmented with isolate \#2) and 61\% (carriers bio-augmented with Cerioporus squamosus strain).
\end{abstract}

Keywords: BOD, COD, MBBR

\title{
INTRODUCTION
}

Biochemical Oxygen Demand (BOD) and Chemical Oxygen Demand (COD) are general indicators of water quality. The biochemical consumption of oxygen represents the amount of oxygen consumed by microorganisms, in a time interval, for the biochemical decomposition until mineralization of the organic substances contained in the water. Chemical oxygen consumption (COD) analysis is designed to measure the maximum amount of oxygen that can be consumed by organic matter in a given water sample. This is important, because when organic pollutants are discharged into the aquatic environment, it will normally take up dissolved oxygen during its subsequent degradation thus reducing the amount of oxygen available for the respiration of fish and other aquatic life (Cazaudehore et al., 2019). Chemical oxygen consumption is an important parameter for water quality because, similar to biochemical oxygen consumption (CBO), it provides an index to assess the effect that discharged wastewater will have on the environment (Jouanneau et al., 2013). Higher CCO levels mean a higher amount of oxidizable organic compounds in the sample, which will reduce the level of dissolved oxygen (OD). A reduction in dissolved oxygen can lead to anaerobic conditions, which are detrimental to aquatic life forms. Often, COD analysis is used to estimate BOD (Biological Oxygen Demand) values, between these 2 indicators existing strong correlations (Zhu et al., 2018). 


\section{MATERIALS AND METHODS}

\section{Treatment Installation and Wastewater Source}

Wastewater treatment was carried out on a previously developed laboratory treatment installation, designed for High Density Polyethylene carriers bioaugmentation, which allows continuous media aeration (Figure 1).

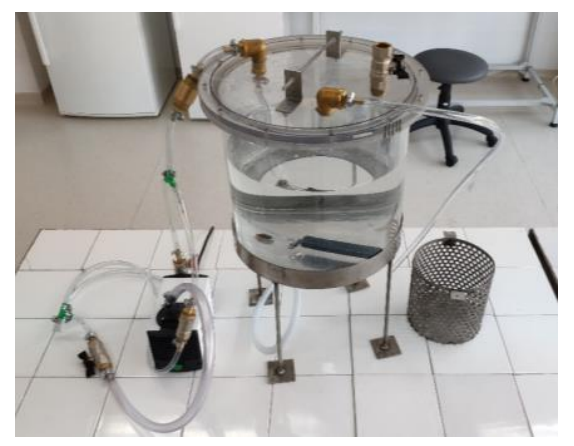

Figure 1. Experimental installation

To demonstrate the efficiency of the experimental installation, samples of wastewaters were taken from the wastewater storage basin of INCDTP Bucharest, water resulted from specific technological processes, without any applied processing step.

\section{HDPE Carriers Bio-Augmentation}

Polymeric carriers with 5\% talc, 7\% cellulose and 88\% HDPE composition were used in bio-functionalization experiments in the experimental treatment installation. Bio-augmentation experiments were carried with five microbial strains, three decaying wood isolates (T1, T2 and T3), and two collection isolates: Cerioporus squamosus and Fusarium oxysporum. Preliminary bio-augmentation of the HDPE carriers was carried out on each batch, in a volume of $12 \mathrm{~L}$ (final volume), with the addition of liquid medium based on potato extract and dextrose. In case of COD analysis, the carriers were functionalized with a volume of $500 \mathrm{~mL}$ of mix inoculum from all 5 selected strains $(100 \mathrm{~mL}$ of inoculum for each strain). For BOD analysis, the carriers bioaugmentation process was performed individually on each of the five selected microbial strains. The preliminary bio-augmentation process was carried out for 20 days, at $28^{\circ} \mathrm{C}$, with continuous aeration, in a Lovibond thermoreactor.

\section{BOD Method}

BOD analysis was carried out on a BOD Direct (Hach Lange) equipment, at 5 days. The analysis is performed in sealed bottles, in which the incubation is performed at the specified temperature for 5 days. Dissolved oxygen is measured initially and after incubation, and BOD value is calculated from the difference between the initial and final DO (Kolár et al., 2005). Because the initial DO is determined shortly after dilution, all oxygen uptake that occurs after this measurement is included in the CBO measurement. To ensure adequate biological activity, the $\mathrm{pH}$ of the water was corrected in the range of 6.5-7 (with $\mathrm{NaOH} / \mathrm{HCl}$ ). Due to the very small section of the bottle 
opening, the previously functionalized polymeric supports, with each strain, were sectioned into 2, and a number of $\sim 20$ carriers were added to each BOD bottle (Figure 2 ), over which a volume of $300 \mathrm{~mL}$ of wastewater was poured. The process was carried out for 5 days, with incubation at $20^{\circ} \mathrm{C}$, and continuous stirring, in Lovibond thermoreactor.

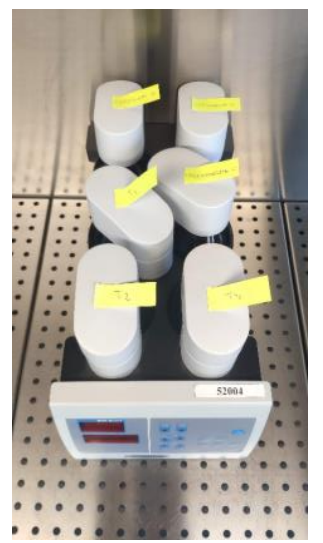

BOD equipment

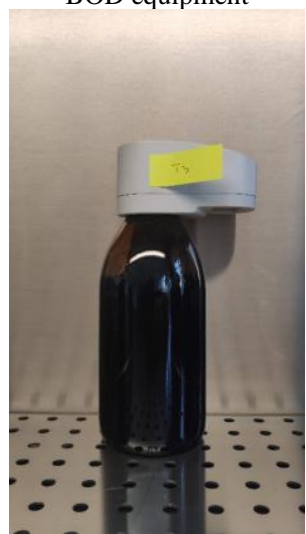

T3 strain

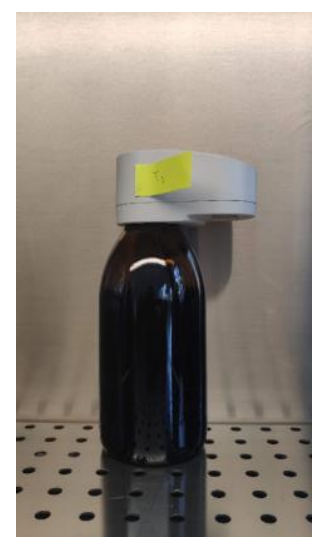

T1 strain

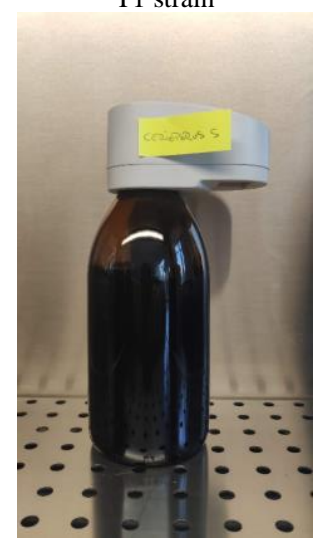

Cerioporus squamosus

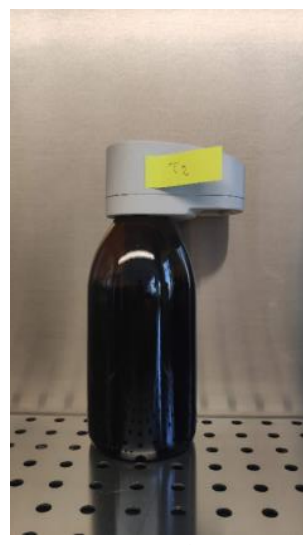

T2 strain

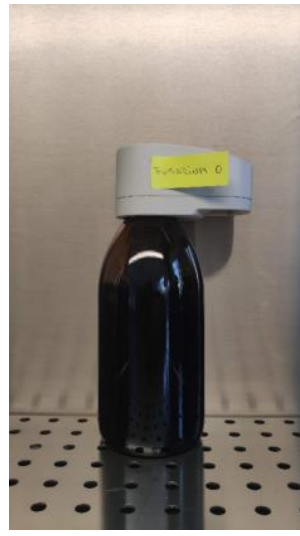

Fusarium oxysporum

Figure 2. BOD bottles with DO counter

Aerobic biodegradation consists in the oxidation of biological organic matter. During this process, the organic matter is transformed by microorganisms into microbial biomass, produced during the biodegradation reaction, assessed according to the following equation:

$$
\mathrm{X}_{0}+\mathrm{S}+\mathrm{O}_{2} \stackrel{N, P, M N}{\longrightarrow} X_{f}+T_{p}+\mathrm{CO}_{2}+\mathrm{H}_{2} \mathrm{O}
$$

where: $\mathrm{X}_{0}$ : initial biomass; $\mathrm{S}$ : organic carbon source; $\mathrm{O}_{2}$ : oxygen; $\mathrm{N}$ : nitrogen source; $\mathrm{P}$ : phosphorous source; $\mathrm{MN}$ : mineral nutrients; $\mathrm{X}_{\mathrm{f}}$ : final biomass; $\mathrm{T}_{\mathrm{p}}$ : products obtained following biodegradation.

For assessment of biochemical oxygen consumption, an incubation period of 5 days was established at a temperature of $20^{\circ} \mathrm{C}$ and initial $\mathrm{CBO}_{5}$ was noted. The biochemical 
oxygen consumption after 5 days was considered the difference obtained between the determination of dissolved oxygen from the initial sample and that after five days from incubation. Biochemical oxygen consumption $\left(\mathrm{CBO}_{5}\right)$ is the amount of oxygen, expressed in $\mathrm{mg} / \mathrm{L}$, required for the oxidation of organic substances in water by the microorganisms after 5 days of incubation.

\section{COD Method}

After the bio-functionalization period, the entire treatment media was evacuated, leaving only the polymeric supports in the reaction vessel. The installation was again loaded with $12 \mathrm{~L}$ of wastewater. The process was carried out for 14 days, at $28^{\circ} \mathrm{C}$, with continuous aeration, in Lovibond thermoreactor. COD values were detected at the beginning of the experiment (T0) and after the end of the experiment (T14), and the result was expressed as a percentage reduction. COD analysis was carried out according to SR ISO 6060, which implies the boiling with reflux for a certain duration, of the water samples mixed with mercury sulphate (III), with a known volume of potassium dichromate, in the presence of a silver catalyst in a strongly acidic environment (sulfuric acid), so that part of the potassium dichromate is reduced by the oxidizable materials present. The excess potassium dichromate was titrated with iron (III) sulphate and ammonium solution. The COD value was calculated from the reduced amount of potassium dichromate. For the boiling stage with reflux, a C.O.D. thermoreactor was used, namely ECO6, Velp Scientifica type, with a temperature set to $200^{\circ} \mathrm{C}$.

All reagents used were of known analytical quality:

1. Sulfuric acid $(\rho=1,84 \mathrm{~g} / \mathrm{mL}) . c\left(\mathrm{H}_{2} \mathrm{SO}_{4}\right)=4 \mathrm{~mol} / \mathrm{L}$;

2. Silver sulphate $\left(\mathrm{Ag}_{2} \mathrm{SO}_{4}\right)$;

3. Potassium dichromate, reference standard solution. $\mathrm{c}\left(\mathrm{K}_{2} \mathrm{Cr}_{2} \mathrm{O}_{7}\right)=0.040 \mathrm{~mol} / \mathrm{L}$;

4. Iron (II) sulphate and ammonium, titrated solution. $\mathrm{c}\left[\left(\mathrm{NH}_{4}\right)_{2} \mathrm{Fe}\left(\mathrm{SO}_{4}\right)_{2} * 6 \mathrm{H} 2_{0}\right]=$ $0.12 \mathrm{~mol} / \mathrm{L}$;

5. Ferroin (as an indicator solution).

The chemical oxygen consumption (COD) expressed in milligrams oxygen per liter is calculated according to the formula:

$\mathrm{COD}(\mathrm{mg} / \mathrm{L})=\left[800 \mathrm{c}\left(\mathrm{V}_{1}-\mathrm{V}_{2}\right)\right] / \mathrm{V}_{0}$

where: $\mathrm{c}=$ concentration of the amount of substance of iron (II) sulphate and ammonium solution; $\mathrm{V}_{0}=$ the volume of the sample to be analyzed, before dilution (if performed), in milliliters; $\mathrm{V}_{1}=$ volume of iron (II) sulphate and ammonium solution, used for titration of the control sample, in milliliters; $V_{2}=$ volume of iron (II) sulphate and ammonium solution, used for titration of the sample to be analyzed, in milliliters; $8000=$ molar mass of $1 / 2 \mathrm{O}_{2}$, in milligrams per liter.

\section{RESULTS AND DISCUSSIONS}

A BOD meter was used to measure the initial concentration of dissolved oxygen $(\mathrm{mg} / \mathrm{L})$ in each sample container. After five days, the final dissolved oxygen concentration was measured. The concentration of CBO was expressed as difference between initial one and after the incubation, the percentage reduction values of the CBO values for each set of functionalized polymeric supports being highlighted in Figure 3.

https://doi.org/10.24264/icams-2020.II.12 


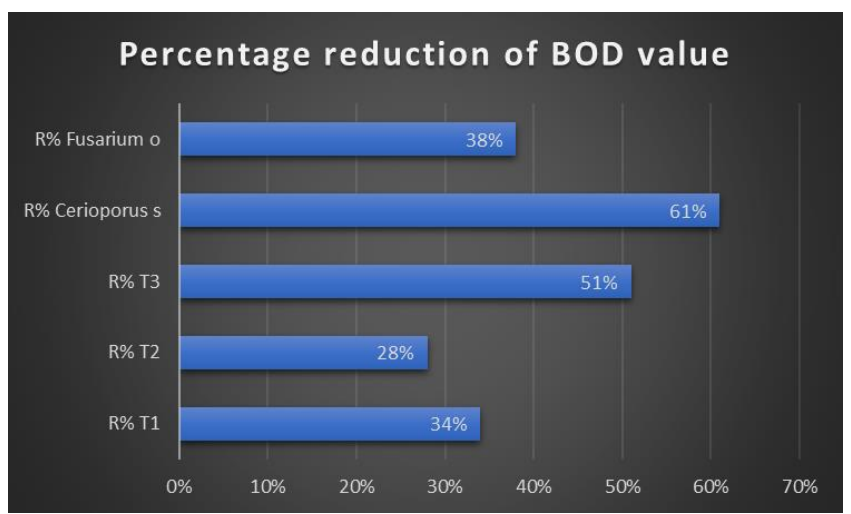

Figure 3. CBO percentage reductions for each strain

The highest reduction rate of $\mathrm{CBO}$ value, at 5 days, can be observed on polymeric carriers functionalized with Cerioporus squamosus strain (WRF strain - White Rot Fungi), with $61 \%$, and at the opposite pole, the T2 isolate showed the lowest rate of percentage reduction, respectively $28 \%$.

COD analysis is a general indicator of the water quality, measuring the capacity of dissolved oxygen depletion, in the samples contaminated with organic matter (Zhang et al., 2017). Specifically, the analysis determines the equivalent amount of oxygen required for chemical oxidation of organic compounds in water. The results highlighted an initial COD value of $61.075 \mathrm{mg} / \mathrm{L}$. Following the treatment, in the experimental installation, a reduction of the COD value of $53.45 \%(32.644 \mathrm{mg} / \mathrm{L})$ was observed (Figure 4).
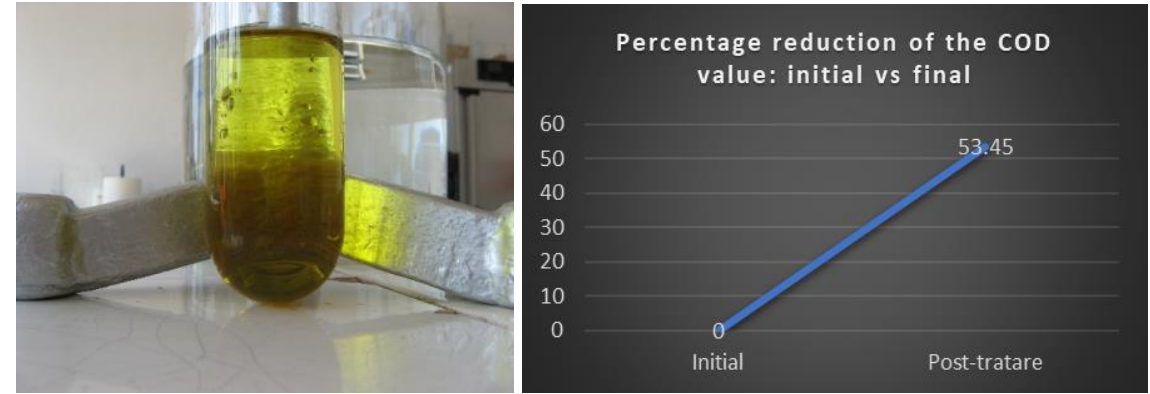

Figure 4. Percentage reduction of the COD value

Following microbial treatment, with bio-augmented HDPE carriers, a satisfactory reduction of the $\mathrm{COD}$ value could be observed, also taking into consideration the possibility of a high bacterial load in the wastewater, which can have a real inhibitory effect on fungal populations. 


\section{CONCLUSIONS}

Present study aimed the exploitation of novel wastewater treatment technique, consisting of bio-augmentation of HDPE structures with either singular fungal strains or mix of strains (representatives of both Ascomycota and Basidiomycota phyla).

Based on a previous microbial screening, regarding ability to colonize HDPE structures, five microbial strains were selected, representing the phyla Ascomycota and Basidiomycota: own isolates T1, T2 and T3, and two collection strains, respectively Cerioporus squamosus and Fusarium oxysporum. Experiments were performed to reduce the values of both Chemical Oxygen Consumption (COD) in the experimental installation, on polymeric supports functionalized with the Cerioporus squamosus strain. The obtained results showed good rates of reduction of COD values (percentage reduction of 53.45\%). At the same time, the installation was tested for the functionalization of 5 batches of polymeric supports, with the five selected strains, and the BOD analysis was performed at 5 days, highlighting rates of reduction of BOD value between $28 \%$ (isolated T2) and $61 \%$ (Cerioporus squamosus). Results obtained show promising applicability of artificial bio-augmented HDPE carriers for treatment of wastewater (Yang et al., 2009).

\section{Acknowledgments}

This work was supported by a grant of the Romanian National Authority for Scientific Research and Innovation, CCCDI - UEFISCDI, project number COFUNDMANUNET III-FUNCELL, within PNCDI III.

\section{REFERENCES}

Cazaudehore, G., Schraauwers, B., Peyrelasse, C., Lagnet, C. and Monlau, F. (2019), "Determination of chemical oxygen demand of agricultural wastes by combining acid hydrolysis and commercial COD kit analysis", Journal of Environmental Management, 250, 15 November 2019, 109464, https://doi.org/10.1016/j.jenvman.2019.109464.

Jouanneau, S., Recoules, L., Durand, M.J., Boukabache, A., Picot, V., Primault, Y., Lakel, A., Sengelin, M., Barillon, B. and Thouand, G. (2013), "Methods for assessing biochemical oxygen demand (BOD): A review", Water Research, 49C(1), 62-82, https://doi.org/10.1016/j.watres.2013.10.066.

Kolář, L., Kužel, S., Hanušová, A., Gergel, J., Ledvina, R., Šindelářová, M., Silovská, S. and Štindl, P. (2005), "The use of Spectroquant Merck BOD photometric test to evaluate the stability of organic matters in soil”, Plant Soil Environment, 51, 46-51, https://doi.org/10.17221/3554-PSE.

Yang, Q., Li, C., Li, H., Li, Y. and Yu, N. (2009), "Degradation of synthetic reactive azo dyes and treatment of textile wastewater by a fungi consortium reactor", Biochemical Engineering Journal, 43(3), 15 March 2009, 225-230, https://doi.org/10.1016/j.bej.2008.10.002.

Zhang, F., Xuea, H., Ma, X., Wang, H. (2017), "Grey Prediction Model for the Chemical Oxygen Demand Emissions in Industrial Waste Water: An Empirical Analysis of China”, Procedia Engineering, 174, 827 834, https://doi.org/10.1016/j.proeng.2017.01.229.

Zhu, J.J., Kang, L. and Anderson, P.R. (2018), "Predicting influent biochemical oxygen demand: Balancing energy demand and risk management", Water Research, 128, 1 January 2018, 304-313, https://doi.org/10.1016/j.watres.2017.10.053 
EFFECT OF ESSENTIAL OILS ON SOME PATHOGENS THAT CAUSE ECZEMA

\author{
MERYEM KARAÇAM, DURMUŞ ALPASLAN KAYA* \\ Mustafa Kemal University, Faculty of Agriculture, Department of Field Crops, \\ dak1976@msn.com
}

\begin{abstract}
In this study, the antimicrobial activity of essential oils obtained from Thymbra spicata L., Lavandula angustifolia Mill. and Myrtus communis L. on the pathogens causing eczema Staphylococcus aureus (ATCC 29213), Staphylococcus epidermidis (ATCC 12228), Escheria coli (ATCC 25922), Acinetobacter baumannii (ATCC 43498), Pseudomonas aeruginosa (ATCC 27853 ) ve Candida albicans (ATCC 90028) were investigated. The MIC and MBC values of the essential oils used in the study against the pathogens causing eczema were determined. As a result of the results obtained, antimicrobial activity of plant essential oils used in the study on test microorganisms was determined. Among the essential oils, it was found that the most effective essential oil was thyme followed by the lavender.
\end{abstract}

Keywords: Essential oil, Eczema, GC-MS, Antimicrobial

\title{
INTRODUCTION
}

Turkey; It has a rich vegetation due to its geographical location, climate and wide area. It also contains many medicinal and aromatic plants (Faydaoğlu ve Sürücüoğlu, 2011). Medicinal and aromatic plants are used as a drug in traditional and modern medicine to prevent, cure or maintain health (Anonim, 2012). Essential oils are obtained from various parts of the plant such as flowers, buds, seeds, leaves, branches, wood, fruit and roots, and approximately $1 / 3$ of nearly 300 plant families growing in nature contain essential oil (Anonim, 2013). Although the mechanism of action of essential oils varies according to their active ingredients, they have antimicrobial, carminative, coloretic, sedative, diuretic, antispasmodic effects (Maksimovic ve ark., 2005).

Thymbra spicata L. is widely grown in the Aegean, Mediterranean and Southeastern Anatolia Regions. In bush form, it grows up to $15-50 \mathrm{~cm}$. Flowering stems are ascending or steep and sometimes very branched. Lavandula angustifolia Mill. is a perennial plant with an average of $50 \mathrm{~cm}$ in semi-shrub form, growing up to a maximum of $1 \mathrm{~m}$, with grayish green leaves, blue colored and fragrant flowers (Ceylan, 1996). Myrtus communis L., can be generally short and rarely 1-3 m long, especially in coastal areas where the Mediterranean climate prevails (Oğur, 1994). Perennial, evergreen, bush-shaped flowers are white in color, fruits are multi-seeded, blackish purple in color (İlçim and Diğrak, 1998).

Bacteria and yeast cause some diseases in the skin. The most common of these diseases is eczema. It is a psychosomatic skin disease that occurs for various reasons and is seen with symptoms such as redness, swelling, vesicles and itching on the skin. Gram-positive bacteria such as Staphylococcus aureus, Staphylococcus epidermidis, Escheria coli, Acinetobacter baumanii, Pseudomonas aeruginosa and Candida albicans pathogens cause eczema. While $S$. aureus is common in nature, $S$. epidermidis disease is found in many parts of the body, especially human skin and upper respiratory mucosa (Gülbandılar, 2009; Eryılmaz ve Gürpınar, 2017). While P. aeruginosa is found in nature, A. baumanii is found in hospital environment, E. coli are common bacteria found in intestines (Enoch et al., 2009; Torlak, 2011). C. albicans, a yeast type fungus, is found in various parts of the body (Acarkan, 2014).

https://doi.org/10.24264/icams-2020.II.13 
Antibiotics are widely used in the treatment of eczema. The progressive increase in side effects caused by the use of synthetic origin substances in the treatment of diseases, the resistance of organisms against synthetic substances, and the gradual restriction of synthetic drugs have led people to seek alternative solutions in the treatment of the disease.

In this study, the effects of T. spicata L., L. angustifolia Mill. and M. communis L. essential oils on some pathogens (S. aureus, S. epidermidis, E. coli, A. baumanii, $P$. aeruginosa and $C$. albicans) causing eczema were investigated in vitro.

\section{MATERIALS AND METHODS}

T. spicata, L. angustifolia and M. communis essential oils were used as material in the study. The antimicrobial activities of these essential oils against $S$. aureus (ATCC 29213), S. epidermidis (ATCC 12228), E. coli (ATCC 25922), A. baumannii (ATCC 43498), P. aeruginosa (ATCC 27853) and C. albicans (ATCC 90028). pathogens were investigated. Antimicrobial activity studies were conducted in Hatay Mustafa Kemal University. Activity studies of essential oils were carried out by tube dilution method. The group without essential oil was selected as the negative control, and gentamicin and fluconazole were used as the drug control. Dimethyl sulfoxide (DMSO) of 1\% was used to dissolve essential oils in the medium. Mueller-Hinton Broth was used for bacteria in the tube dilution method for the production of microorganisms and Saboraud Dextroz Broth for $C$. albicans. It has been determined by controlled experiments that $1 \%$ concentration of DMSO is non-toxic on the growth of microorganisms.

\section{Obtaining Essential Oils}

The essential oils used in the study were obtained from the leaves of $T$. spicata and M. communis plants naturally found in the flora of Hatay. And L. angustifolia essential oil was collected from plants that were previously cultivated in Hatay Mustafa Kemal University in full blooming period and obtained by water distillation method from leaves and herbs of plants.

\section{Determination of Essential Oil Components}

The determination of essential oil components was carried out under the following conditions with the Thermo Scientific ISQ Single Quadrupole model GC device. TRFAME MS model, 60 m length column was used. Helium (99.9\%) was used as the carrier gas at a flow rate of $1 \mathrm{~mL} / \mathrm{min}$. Ionization 22 energy was set at $70 \mathrm{eV}$, mass range $\mathrm{m} / \mathrm{z}$ 1.2-1200 amu. Scan Mode was used for data collection. The MS transfer line temperature is $250{ }^{\circ} \mathrm{C}$, the MS ionization temperature is $220{ }^{\circ} \mathrm{C}$, the injection port temperature is $220^{\circ} \mathrm{C}$, the column temperature is $50{ }^{\circ} \mathrm{C}$ at the beginning and has been increased to $220^{\circ} \mathrm{C}$ with a temperature increase rate of $3{ }^{\circ} \mathrm{C} / \mathrm{min}$. The structure of each compound was identified with the Xcalibur program using mass spectra (Wiley 9).

\section{Antimicrobial Activity Tests}

Microorganism colonies taken with the loop were suspended in Phosphate Buffered Saline (PBS), which is a phosphate buffer solution. $1 \times 10^{8}$ bacteria $/ \mathrm{ml}$ compared to $\mathrm{Mc}$ Farland turbidity tube no 0.5 ; C. albicans dilution was prepared to be $1 \times 10^{5}$ and these 
dilutions were used as inoculum. The determination of the antimicrobial activities of essential oils was evaluated in accordance with the National Committee for Clinical Laboratory Standards (NCCLS) criteria. Tested bacterial and fungal microbial strains were suspended in PBS (phosphate buffered water) to McFarland 0.5, and those containing bacterial strains Mueller-Hinton agar and Sabouraud Dextrose agar for $C$. albicans were inoculated on plates. In the study, dimethyl sulfoxide (DMSO) was used as a solvent to dissolve essential oils. Non-toxic concentration (1\%) of the selected concentration of DMSO on microorganisms was used. The concentrations of the essential oils tested in the study $(10.24,5.12,2.56,1.28,0.64,0.32,0.16,0.08,0.04$ and $0.02 \mu \mathrm{g} / \mathrm{ml}$ ) were used. DMSO was used as negative control. Amikacin, gentamicin, and nystatin were used as reference drugs for gram-positive anti-bacterial activity, gramnegative anti-bacterial activity, and antifungal activity, respectively. All microorganism plates were incubated at $37{ }^{\circ} \mathrm{C}$ and the results were evaluated after $24^{\text {th }}$ hour of incubation for bacteria and after $48^{\text {th }}$ hour of incubation for C. albicans. Essential oil concentrations that inhibit apparent growth were considered to be minimum inhibitory concentrations (MICs). In addition, minimal bactericidal and fungicidal concentrations were determined by seeding on Mueller Hinton agar and Saboraud Dexroz agar from the next dilutions with the final concentration without visible growth.

\section{RESULTS AND DISCUSSION}

\section{Essential Oil Rates and Components of Plants Used in the Study}

According to the results obtained, thyme essential oil ratio was $3.00 \%$, lavender essential oil ratio was $2.90 \%$, and the essential oil ratio obtained from myrtle leaves was $1.25 \%$. When we examine the essential oil components of the thyme, the highest component was determined as Carvacrol with $55.30 \%$, followed by o-Cymene with $13.51 \%$ and-Terpinene with $12.30 \%$. When the components of the essential oils of the lavender were examined, it was found that the highest component was Linalool with $18.03 \%$, followed by $\alpha$-Bisabolol with $17.44 \%$ and Linalyl acetate with $8.76 \%$. When we examine the main components of the essential oils of the myrtle, the essential oil components were determined as $33.80 \%$ Eucalyptol, $25.42 \% \alpha$-Pinene and $10.75 \%$ Linalool, respectively.

\section{Effectiveness of Essential Oils on S. aureus}

The effectiveness of thyme, lavender and myrtle essential oils on S. aureus bacteria was examined in terms of MIC values, it was determined that thyme essential oil inhibited bacterial growth at $0.02 \mu \mathrm{g} / \mathrm{ml}$, lavender essential oil at $0.32 \mu \mathrm{g} / \mathrm{ml}$, and myrtle essential oil at $0.64 \mu \mathrm{g} / \mathrm{ml}$. When the effectiveness of thyme, lavender, myrtle essential oils on S. aureus bacteria was examined in terms of MBK values, it was determined that thyme essential oil showed a bactericidal effect at $0.04 \mu \mathrm{g} / \mathrm{ml}$, lavender essential oil at $0.64 \mu \mathrm{g} / \mathrm{ml}$, and myrtle essential oil at $1.28 \mu \mathrm{g} / \mathrm{ml}$.

\section{Effectiveness of Essential Oils on S. epidermidis}

The effectiveness of thyme, lavender and myrtle essential oils on S. epidermidis was examined in terms of MIC values, $0.02 \mu \mathrm{g} / \mathrm{ml}$ of thyme essential oil, 0.16 of lavender essential oil. It has been determined that $\mu \mathrm{g} / \mathrm{ml}$, myrtle essential oil inhibits bacterial growth at $0.32 \mu \mathrm{g} / \mathrm{ml}$. When the effectiveness of thyme, lavender and myrtle essential 
oils on S. epidermidis was examined in terms of MBC values, it was determined that thyme essential oil showed a bactericidal effect at $0.04 \mu \mathrm{g} / \mathrm{ml}$, lavender essential oil at $0.32 \mu \mathrm{g} / \mathrm{ml}$, and myrtle essential oil at $0.64 \mu \mathrm{g} / \mathrm{ml}$.

Table 1. Efficacy concentrations of essential oils against $S$. aureus

\begin{tabular}{ccccccc}
\hline $\begin{array}{c}\text { Essential Oil } \\
\text { Concentration } \\
(\mu \mathrm{l} / \mathrm{ml})\end{array}$ & \multicolumn{2}{c}{ T. spicata } & \multicolumn{2}{c}{ L. angustifolia } & \multicolumn{2}{c}{ M. communis } \\
0.01 & MIC & MBC & MIC & MBC & MIC & MBC \\
0.02 & + & + & + & + & + & + \\
0.04 & - & + & + & + & + & + \\
0.08 & - & - & + & + & + & + \\
0.16 & - & - & + & + & + & + \\
0.32 & - & - & + & + & + & + \\
0.64 & - & - & - & + & + & + \\
1.28 & - & - & - & - & - & + \\
\hline +: There is reproduction, -: There is no reproduction & & &
\end{tabular}

Table 2. Efficacy concentrations of essential oils against $S$. epidermidis

\begin{tabular}{ccccccc}
\hline $\begin{array}{c}\text { Essential Oil } \\
\begin{array}{c}\text { Concentration } \\
(\mu \mathrm{l} / \mathrm{ml})\end{array}\end{array}$ & \multicolumn{2}{c}{ T. spicata } & \multicolumn{2}{c}{ L. angustifolia } & \multicolumn{2}{c}{ M. communis } \\
\hline 0.01 & MIC & MBC & MIC & MBC & MIC & MBC \\
0.02 & + & + & + & + & + & + \\
0.04 & - & + & + & + & + & + \\
0.08 & - & - & + & + & + & + \\
0.16 & - & - & + & + & + & + \\
0.32 & - & - & - & + & + & + \\
0,64 & - & - & - & - & - & + \\
\hline +: There is reproduction, -: There is no reproduction & & &
\end{tabular}

\section{Effectiveness of Essential Oils on $E$. coli}

The effectiveness of thyme, lavender and myrtle essential oils on E. coli was examined in terms of MIC values, it was determined that thyme and lavender essential oils inhibit bacterial growth at $0.32 \mu \mathrm{g} / \mathrm{ml}$ and myrtle essential oil at $0.64 \mu \mathrm{g} / \mathrm{ml}$. When the effectiveness of thyme, lavender and myrtle essential oils on E. coli was examined in terms of MBC values, it was determined that thyme and lavender essential oils showed a bactericidal effect at $0.64 \mu \mathrm{g} / \mathrm{ml}$ and myrtle essential oil at $1.28 \mu \mathrm{g} / \mathrm{ml}$.

\section{Effectiveness of Essential Oils on A. baumannii}

The efficiency of thyme, lavender and myrtle essential oils on A. baumannii was examined in terms of MIC values, it was determined that thyme essential oil inhibits bacterial growth at $0.02 \mu \mathrm{g} / \mathrm{ml}$, lavender essential oil at $0.32 \mu \mathrm{g} / \mathrm{ml}$, and myrtle essential oil at $0.64 \mu \mathrm{g} / \mathrm{ml}$. When the effectiveness of thyme, lavender and myrtle essential oils on A. baumannii was examined in terms of MBK values, it was determined that thyme essential oil had a bactericidal effect at $0.04 \mu \mathrm{g} / \mathrm{ml}$, lavender essential oil at $0.64 \mu \mathrm{g} / \mathrm{ml}$, and myrtle essential oil at $1.28 \mu \mathrm{g} / \mathrm{ml}$. 
Table 3. Efficacy concentrations of essential oils against $E$. coli

\begin{tabular}{ccccccc}
\hline $\begin{array}{c}\text { Essential Oil } \\
\text { Concentration } \\
(\mu \mathrm{l} / \mathrm{ml})\end{array}$ & \multicolumn{2}{c}{$T$. spicata } & \multicolumn{2}{c}{ L. angustifolia } & \multicolumn{2}{c}{ M. communis } \\
\hline 0.01 & $\mathrm{MIC}$ & $\mathrm{MBC}$ & $\mathrm{MIC}$ & $\mathrm{MBC}$ & $\mathrm{MIC}$ & $\mathrm{MBC}$ \\
0.02 & + & + & + & + & + & + \\
0.04 & + & + & + & + & + & + \\
0.08 & + & + & + & + & + & + \\
0.16 & + & + & + & + & + & + \\
0.32 & + & + & + & + & + & + \\
0.64 & - & + & - & + & + & + \\
1.28 & - & - & - & - & - & + \\
\hline +: There is reproduction, -: There is no reproduction & & &
\end{tabular}

Table 4. Efficacy concentrations of essential oils against A. baumannii

\begin{tabular}{ccccccc}
\hline $\begin{array}{c}\text { Essential Oil } \\
\text { Concentration }\end{array}$ & \multicolumn{2}{c}{ T. spicata } & \multicolumn{2}{c}{ L. angustifolia } & \multicolumn{2}{c}{ M. communis } \\
$(\mu \mathrm{l} / \mathrm{ml})$ & MIC & MBC & MIC & MBC & MIC & MBC \\
\hline 0.01 & + & + & + & + & + & + \\
0.02 & + & + & + & + & + & + \\
0.04 & + & + & + & + & + & + \\
0.08 & + & + & + & + & + & + \\
0.16 & + & + & + & + & + & + \\
0.32 & - & + & - & + & + & + \\
0.64 & - & - & - & - & - & + \\
1.28 & - & - & - & - & - & - \\
\hline +: There is reproduction, -: There is no reproduction & & &
\end{tabular}

\section{Effectiveness of Essential Oils on $P$. aeruginosa}

The effectiveness of thyme, lavender and myrtle essential oils on $P$. aeruginosa was examined in terms of MIC values; It was determined that thyme essential oil inhibits bacterial growth at $0.32 \mu \mathrm{g} / \mathrm{ml}$, lavender essential oil at $10.24 \mu \mathrm{g} / \mathrm{ml}$, and myrtle essential oil at $5.12 \mu \mathrm{g} / \mathrm{ml}$. When thyme, lavender and myrtle communis essential oils were examined in terms of MBC values on P. aeruginosa, it was determined that thyme essential oil had a bactericidal effect at $0.64 \mu \mathrm{g} / \mathrm{ml}$, lavender essential oil at 20.48 $\mu \mathrm{g} / \mathrm{ml}$, and myrtle essential oil at $10.24 \mu \mathrm{g} / \mathrm{ml}$.

\section{Effectiveness of Essential Oils on $C$. albicans}

The effectiveness of thyme, lavender and myrtle essential oils on C. albicans was examined in terms of MIC values, it was determined that thyme essential oil inhibits fungus growth at $0.04 \mu \mathrm{g} / \mathrm{ml}$, lavender essential oil at $2.56 \mu \mathrm{g} / \mathrm{ml}$ and myrtle essential oil at $0.16 \mu \mathrm{g} / \mathrm{ml}$. When thyme, lavender and myrtle essential oils were examined in terms of MFC values on $C$. albicans, it was determined that thyme essential oil had a fungicidal effect at $0.04 \mu \mathrm{g} / \mathrm{ml}$, lavender essential oil at $5.12 \mu \mathrm{g} / \mathrm{ml}$, and myrtle essential oil at $0.32 \mu \mathrm{g} / \mathrm{ml}$. 
Effect of Essential Oils on Some Pathogens that Cause Eczema

Table 5. Efficacy concentrations of essential oils against $P$. aeruginosa

\begin{tabular}{ccccccc}
\hline $\begin{array}{c}\text { Essential Oil } \\
\text { Concentration } \\
(\mu \mathrm{l} / \mathrm{ml})\end{array}$ & \multicolumn{2}{c}{ T. spicata } & \multicolumn{2}{c}{ L. angustifolia } & \multicolumn{2}{c}{ M. communis } \\
\hline 0.16 & MIC & MBC & MIC & MBC & MIC & MBC \\
0.32 & + & + & + & + & + & + \\
0.64 & + & + & + & + & + & + \\
1.28 & + & + & + & + & + & + \\
2.56 & + & + & + & + & + & + \\
5.12 & + & + & + & + & + & + \\
10.24 & - & + & + & + & - & + \\
20.48 & - & - & - & + & - & - \\
\hline +: There is reproduction, -: There is no reproduction & & &
\end{tabular}

Table 6. Efficacy concentrations of essential oils against $C$. albicans

\begin{tabular}{ccccccc}
\hline $\begin{array}{c}\text { Essential Oil } \\
\text { Concentration } \\
(\mu \mathrm{l} / \mathrm{ml})\end{array}$ & \multicolumn{2}{c}{ T. spicata } & \multicolumn{2}{c}{ L. angustifolia } & \multicolumn{2}{c}{ M. communis } \\
\hline 0.01 & MIC & MFC & MIC & MFC & MIC & MFC \\
0.02 & + & + & + & + & + & + \\
0.04 & + & + & + & + & + & + \\
0.08 & - & - & + & + & + & + \\
0.16 & - & - & + & + & + & + \\
0.32 & - & - & + & + & - & + \\
0.64 & - & - & + & + & - & - \\
1.28 & - & - & + & + & - & - \\
2.56 & - & - & + & + & - & - \\
5.12 & - & - & - & + & - & - \\
\hline There is reproduction, -: There is no reproduction & & & - \\
\hline T: & - & - & - & - & - &
\end{tabular}

\section{REFERENCES}

Anonymous, 2019. Türkiye İstatistik Kurumu datas. http://www.tuik.gov.tr/

Baytop,T.,1984.Türkiye'de Bitkiler ile Tedavi (Geçmişte ve Bugün). İstanbul Üniversitesi Eczacılık Fakültesi Yayınları, no:3255, page 211-212.

Gün, M., 2012 Kutsal Tohum (Nigella sativa): Çörek Otunun İyileştirici Etkisine İlişkin Bazı Bilgiler, Lokman Hekim Journal 2012; 2 (1): 43-46 https://dergipark.org.tr/tr/download/article-file/643364.

Karaman, A., 1999. Çörek otunda (Nigella damascena) farklı ekim zamanlarının tohum verimi ve kaliteye etkisi üzerinde bir araştırma. Çukurova Üniversitesi Fen Bilimleri Enstitüsü Yüksek Lisans Tezi, Adana. 


\title{
OIL GLANDS NUMBER AND OIL GLANDS DIAMETERS OF Thymbra spicata
} var. spicata L. LEAVES

\author{
DURMUŞ ALPASLAN KAYA*, FILIZ AYANOĞLU \\ Mustafa Kemal University, Faculty of Agriculture, Department of Field Crops, \\ dak1976@msn.com
}

\begin{abstract}
In the study, a genetic pool was created with plant samples taken from the locations where the Thymbra spicata var. spicata L. plant, which is culturally and economically important, grows densely in the province of Hatay. Plants were propagated with cuttings taken from these single plants. 213 plants were collected from 68 different locations for the genetic pool. The leaves of the plants in this gene pool were examined in terms of the number of essential oil glands per unit area and the diameter of the essential oil glands. The number of glands per unit area in plant leaves showed a wide variation and ranged from 5.61 to 56.04 pieces $/ \mathrm{mm}^{2}$. The diameters of the oil glands varied between $75.40-112.86 \mu \mathrm{m}$ and the average diameter was determined as $94.09 \mu \mathrm{m}$ In the study, it was determined that some plants with low essential oil ratios such as Z144 and Z158 also have low oil glands number and oil glands diameter. It would be appropriate to evaluate these values obtained as preliminary knowledge together with future ontogenetic variability studies.
\end{abstract}

Keywords: Thymbra spicata var. spicata L, essential oil gland.

\section{INTRODUCTION}

The plant species that make up plant formations do not show the same characteristics everywhere. With the emergence of local differences arising from climate, soil and geomorphological features, the plant species that make up the plant communities diversify. The region, which includes many mountains, plateaus and valley areas, especially Amanos mountains, is in a very rich position in terms of biological resources. In addition, there is a rich biological diversity with the amount of rainfall and high relative humidity in the summer months (Çakan and Byfield, 2005; Vural and Aytaç 2005; Avc1, 2005). There are plant species that are naturally found in this region, almost all of which are collected from nature or traded for various purposes. Among these, Thymbra spicata var. spicata L. comes first.

Thyme species are most export products made of medicinal and aromatic plants in Turkey (Özgüven ve ark. 2005). Flora of Turkey has four species of this plant is located naturally which are Thymbra spicata var. spicata, Thymbra spicata var. intricata, Thymbra sintenisii var. sintenisii and Thymbra sintenisii var. isaurica (Davis, 1982; Başer, 2002). Thymbra spicata var. spicata L., which is called sater, zahter or blackhead thyme (Baytop, 1994) naturally grows intensively in the Eastern Mediterranean region, especially in the province of Hatay. The plants collected from nature are mostly consumed fresh traditionally in the region. In addition, it is dried and used as a spice and tea, and its essential oil, which is rich in carvacrol, is also used for different purposes. T. spicata $\mathrm{L}$. is a plant that naturally grows intensively in Mediterranean countries and in the Mediterranean region of Turkey, especially in Hatay province.

Although there are many studies on the morphology, anatomy, amount of essential oil, components and antimicrobial properties of the thymbra plant, studies on agriculture are very limited in Tukey (Doğan et al., 1987; Hancı et al., 2003; Özel et al., 2003; Baydar et al., 2004; Erken, 2005; Özcan et al., 2008). Inan et al. (2011), in their study on Thymbra spicata L., which they collected from Adiyaman region, determined that the amount of essential oil changed in different harvest times and that the highest rate of 
essential oil was obtained from plants harvested during the flowering period. Kiz1 (2013) made evaluations on 28 different populations collected from Southeast Anatolia and the Mediterranean region and determined that there were significant differences between the populations. Carvacrol is the most abundant component as the main component (Başer, 2002). But Hanc1 et al. (2003) stated that this species also has thymol-containing chemotypes.

Although standardization is important in all other agricultural products, it is even more important for medicinal plants. Because products that do not meet certain quality standards cannot be traded. Ensuring standardization in agricultural products is possible by growing standard products. This can only be achieved through variety breeding. Breeding studies are long-term but must be done. First of all, the genetic material we have should be well known and its genetic potentials should be revealed. In this study, the number of glands per unit area and the size of the oil glands in the leaves of the thymbra grown in different locations in the flora of Hatay, and the infrastructure for future single plant selection improvement. is intended to be created. For this purpose, a genetic pool was created for $T$. spicata L. plant with plant samples selected from different locations where the plants are densely grown and these plants were examined in terms of oil glands.

\section{MATERIALS AND METHODS}

Survey studies were carried out in areas where Thymbra spicata L. plant spread in Hatay province and collection studies were carried out in these areas. As a result of the studies 213 single plants were selected from 68 different locations and were examined in terms of the number of oil glands per unit area and the size of oil glands. Plants were selected primarily different, well developed and healthy ones according to the single plant selection method. The oil cells of the Thymbra spicata L. species are found in sessile glandular hairs (Davis, 1982). In the study, the number of oil glands and the size of oil glands for each individual plant were determined. Later, plants propagated with cuttings taken from these plants were planted in the collection garden.

\section{Number of Oil Glands per Unit Area $\left(\mathrm{pcs} / \mathrm{mm}^{2}\right)$}

JEOL brand JSM-5500LV model SEM (Scanning Electron Microscope, X 300.000) electron microscope was used at Science Research and Application Center in Mustafa Kemal University. In each sample, the number of oil glands per unit area on 4 leaves (4 replication) of the plant was determined in terms of units/mm2 and averaged. The largest middle part of the leaf samples was used in counting and measurements made in SEM.

\section{Average Oil Gland Diameter $(\mu \mathrm{m})$}

In the imaging performed to determine the number of oil glands per unit area, the diameter of 5 oil glands of these 4 leaves was determined for each plant and the average was obtained. A total of 20 measurements were made for each plant. 


\section{RESULTS AND DISCUSSION}

\section{Number of Oil Glands per Unit Area $\left(\mathrm{pcs} / \mathrm{mm}^{2}\right)$}

As a result of the counts and measurements made on the SEM (Scanning Electron Microscope) images on the leaves of thymbra plants collected from the flora of Hatay, the number of essential oil glands per $\mathrm{mm}^{2}$ was determined and given in Table 1 . The average number of oil glands of ecotypes in the experiment was $18.74 \mathrm{pcs} / \mathrm{mm}^{2}$. The lowest oil gland numbers were obtained from the ecotypes Z158 with $5.61 \mathrm{pcs} / \mathrm{mm}^{2}$ and Z144 with $5.89 \mathrm{pcs} / \mathrm{mm}^{2}$. The highest numbers of oil glands were obtained from ecotypes Z98 (56.04 $\left.\mathrm{pcs} / \mathrm{mm}^{2}\right), \mathrm{Z} 131\left(44.57 \mathrm{pcs} / \mathrm{mm}^{2}\right)$ and Z102 (41.59 $\left.\mathrm{pcs} / \mathrm{mm}^{2}\right)$ respectively.

Table 1. The number of oil glands of T. spicata var. spicata L. leaves (pcs $/ \mathrm{mm}^{2}$ )

\begin{tabular}{|c|c|c|c|c|c|c|c|c|c|}
\hline Code & Glands & Code & Glands & Code & Glands & Code & Glands & Code & Glands \\
\hline $\mathrm{Z1}$ & 20.23 & $\mathrm{Z} 44$ & 17.43 & $\mathrm{Z} 87$ & 14.76 & Z130 & 16.18 & Z173 & 16.67 \\
\hline $\mathrm{Z} 2$ & 9.26 & $\mathrm{Z} 45$ & 28.44 & Z88 & 16.17 & Z131 & 44.57 & Z174 & 21.04 \\
\hline $\mathrm{Z3}$ & 26.63 & Z46 & 11.13 & Z89 & 15.39 & Z132 & 9.81 & Z175 & 20.17 \\
\hline $\mathrm{Z} 4$ & 15.91 & $\mathrm{Z} 47$ & 16.32 & Z90 & 19.25 & Z133 & 15.90 & Z176 & 19.75 \\
\hline $\mathrm{Z} 5$ & 16.41 & Z48 & 15.87 & Z91 & 24.24 & Z134 & 10.09 & Z177 & 18.05 \\
\hline Z6 & 28.11 & Z49 & 16.57 & Z92 & 22.10 & Z135 & 20.31 & Z178 & 19.87 \\
\hline $\mathrm{Z7}$ & 22.63 & $\mathrm{Z} 50$ & 17.28 & Z93 & 21.69 & Z136 & 9.05 & Z179 & 21.07 \\
\hline Z8 & 16.57 & Z51 & 16.34 & Z94 & 22.33 & Z137 & 11.97 & Z180 & 20.19 \\
\hline Z9 & 7.82 & $\mathrm{Z} 52$ & 14.62 & Z95 & 28.08 & Z138 & 12.78 & Z181 & 18.74 \\
\hline Z10 & 17.13 & Z53 & 25.27 & Z96 & 23.79 & Z139 & 19.47 & Z182 & 20.14 \\
\hline Z11 & 19.46 & $\mathrm{Z} 54$ & 25.43 & Z97 & 31.10 & $\mathrm{Z} 140$ & 22.02 & Z183 & 20.78 \\
\hline Z12 & 17.88 & $\mathrm{Z} 55$ & 13.41 & Z98 & 56.04 & Z141 & 16.41 & Z184 & 19.84 \\
\hline Z13 & 12.46 & Z56 & 19.84 & Z99 & 14.98 & Z142 & 10.12 & Z185 & 18.96 \\
\hline Z14 & 18.66 & $\mathrm{Z} 57$ & 23.37 & Z100 & 15.47 & $\mathrm{Z} 143$ & 22.75 & Z186 & 19.32 \\
\hline Z15 & 17.34 & $\mathrm{Z} 58$ & 10.04 & Z101 & 16.32 & Z144 & 5.89 & Z187 & 21.04 \\
\hline Z16 & 12.26 & Z59 & 30.40 & Z102 & 41.59 & Z145 & 9.98 & Z188 & 20.78 \\
\hline Z17 & 19.32 & Z60 & 12.48 & Z103 & 9.65 & Z146 & 22.40 & Z189 & 19.43 \\
\hline Z18 & 15.24 & Z61 & 18.58 & Z104 & 28.38 & $\mathrm{Z} 147$ & 16.70 & Z190 & 17.54 \\
\hline Z19 & 12.92 & Z62 & 19.48 & Z105 & 15.87 & Z148 & 13.36 & Z191 & 16.35 \\
\hline $\mathrm{Z} 20$ & 15.32 & Z63 & 18.98 & Z106 & 11.99 & Z149 & 36.86 & Z192 & 19.03 \\
\hline Z21 & 24.98 & Z64 & 27.03 & Z107 & 12.56 & $\mathrm{Z} 150$ & 6.82 & Z193 & 16.79 \\
\hline $\mathrm{Z} 22$ & 14.20 & Z65 & 21.02 & Z108 & 9.62 & Z151 & 18.27 & Z194 & 21.08 \\
\hline Z23 & 20.10 & Z66 & 10.73 & Z109 & 23.16 & Z152 & 15.73 & Z195 & 22.05 \\
\hline Z24 & 14.78 & Z67 & 18.87 & Z110 & 14.36 & Z153 & 36.54 & Z196 & 19.56 \\
\hline Z25 & 10.30 & Z68 & 15.10 & Z111 & 10.55 & Z154 & 28.66 & Z197 & 18.96 \\
\hline Z26 & 20.53 & Z99 & 22.59 & $\mathrm{Z} 112$ & 10.96 & Z155 & 12.19 & Z198 & 18.61 \\
\hline Z27 & 19.12 & $\mathrm{Z70}$ & 16.54 & Z113 & 16.07 & Z156 & 22.14 & Z199 & 18.97 \\
\hline Z28 & 18.65 & Z71 & 17.02 & Z114 & 26.43 & Z157 & 19.78 & Z200 & 20.14 \\
\hline Z29 & 30.30 & $\mathrm{Z72}$ & 21.75 & Z115 & 22.12 & Z158 & 5.61 & Z201 & 21.59 \\
\hline $\mathrm{Z} 30$ & 29.42 & $\mathrm{Z73}$ & 20.74 & Z116 & 12.02 & Z159 & 19.28 & Z202 & 22.03 \\
\hline Z31 & 22.10 & $\mathrm{Z74}$ & 19.18 & Z117 & 18.02 & $\mathrm{Z} 160$ & 10.88 & Z203 & 16.57 \\
\hline $\mathrm{Z} 32$ & 20.11 & $\mathrm{Z75}$ & 16.84 & Z118 & 17.45 & Z161 & 18.11 & Z204 & 20.45 \\
\hline Z33 & 19.17 & Z76 & 10.12 & Z119 & 17.68 & Z162 & 19.81 & Z205 & 19.52 \\
\hline Z34 & 18.66 & Z77 & 19.19 & Z120 & 16.37 & Z163 & 22.14 & Z206 & 17.51 \\
\hline Z35 & 18.94 & Z78 & 21.05 & Z121 & 12.94 & Z164 & 21.94 & Z207 & 16.29 \\
\hline Z36 & 11.79 & Z79 & 20.12 & Z122 & 17.65 & Z165 & 17.89 & Z208 & 18.97 \\
\hline Z37 & 22.02 & Z80 & 19.01 & Z123 & 27.21 & Z166 & 6.34 & Z209 & 21.04 \\
\hline Z38 & 13.95 & Z81 & 11.73 & Z124 & 28.85 & Z167 & 8.21 & $\mathrm{Z} 210$ & 20.10 \\
\hline Z39 & 11.87 & Z82 & 17.92 & $\mathrm{Z} 125$ & 20.95 & Z168 & 19.62 & $\mathrm{Z} 211$ & 19.87 \\
\hline $\mathrm{Z} 40$ & 11.04 & Z83 & 24.57 & Z126 & 28.84 & Z169 & 15.79 & Z212 & 19.10 \\
\hline Z41 & 33.35 & Z84 & 12.49 & Z127 & 12.62 & $\mathrm{Z} 170$ & 18.04 & Z213 & 19.12 \\
\hline $\mathrm{Z} 42$ & 14.52 & Z85 & 21.15 & Z128 & 20.04 & Z171 & 17.93 & & \\
\hline Z43 & 30.91 & Z86 & 13.2 & Z129 & 19.87 & $\mathrm{Z} 172$ & 18.97 & & \\
\hline
\end{tabular}

Min: 5.61; Max: 56.04; Average: 18.74; Std. deviation: 6.45; Coef. of variation: 34,41

https://doi.org/10.24264/icams-2020.II.14 


\section{Average Oil Gland Diameter $(\mu \mathrm{m})$}

In the study, measurements made for each individual thymbra plant collected and the average diameter was determined as $\mu \mathrm{m}$ and given in Table 2 . The diameters of the oil glands of the collected thymbra varied between 75,40-112,86 $\mu \mathrm{m}$. The highest average diameter values were obtained from plants with code number Z17 $(112.86 \mu \mathrm{m})$, Z31 $(106.57 \mu \mathrm{m})$ and Z141 $(105.25 \mu \mathrm{m})$, respectively. In the experiment, the lowest mean diameter values were obtained from ecotypes Z158 $(75.40 \mu \mathrm{m}), \mathrm{Z} 52(80.29 \mu \mathrm{m})$, Z137 $(81.60 \mu \mathrm{m})$ and Z144 $(82.20 \mu \mathrm{m})$. The diameter of an oil glands in the thymbra leaves was determined to be 94.09 on average.

Table 2. The average oil gland diameters of $T$. spicata var. spicata L. leaves $(\mu \mathrm{m})$

\begin{tabular}{|c|c|c|c|c|c|c|c|c|c|}
\hline Code & Gland. & Code & Glands & Code & Glands & Code & Glands & Code & Glands \\
\hline $\mathrm{Z1}$ & 99.75 & Z44 & 99.76 & $\mathrm{Z87}$ & 92.22 & $\mathrm{Z} 130$ & 93.00 & Z173 & 104.86 \\
\hline $\mathrm{Z} 2$ & 88.44 & Z45 & 99.71 & Z88 & 101.75 & Z131 & 97.00 & Z174 & 87.80 \\
\hline $\mathrm{Z3}$ & 103.25 & Z46 & 96.22 & Z89 & 99.60 & Z132 & 88.22 & Z175 & 95.73 \\
\hline $\mathrm{Z} 4$ & 83.80 & Z47 & 91.72 & Z90 & 89.15 & Z133 & 92.00 & Z176 & 94.10 \\
\hline $\mathrm{Z} 5$ & 86.86 & Z48 & 99.89 & Z91 & 94.00 & Z134 & 84.50 & Z177 & 88.20 \\
\hline Z6 & 89.56 & Z49 & 98.12 & Z92 & 91.25 & Z135 & 91.12 & Z178 & 87.75 \\
\hline $\mathrm{Z7}$ & 88.24 & $\mathrm{Z} 50$ & 100.00 & Z93 & 90.00 & Z136 & 84.90 & Z179 & 98.98 \\
\hline Z8 & 96.12 & $\mathrm{Z} 51$ & 97.40 & Z94 & 97.14 & Z137 & 81.60 & Z180 & 99.70 \\
\hline $\mathrm{Z9}$ & 93.20 & Z52 & 80.29 & Z95 & 88.86 & Z138 & 93.17 & Z181 & 94.58 \\
\hline $\mathrm{Z} 10$ & 92.22 & Z53 & 98.25 & Z96 & 94.50 & Z139 & 95.10 & Z182 & 98.20 \\
\hline Z11 & 90.00 & $\mathrm{Z} 54$ & 91.80 & Z97 & 98.57 & Z140 & 94.00 & Z183 & 94.58 \\
\hline $\mathrm{Z} 12$ & 98.45 & Z55 & 91.43 & Z98 & 101.59 & Z141 & 105.25 & Z184 & 99.72 \\
\hline Z13 & 96.26 & Z56 & 94.44 & Z99 & 95.25 & Z142 & 97.64 & Z185 & 91.43 \\
\hline Z14 & 100.80 & $\mathrm{Z} 57$ & 94.75 & $\mathrm{Z} 100$ & 91.60 & Z143 & 84.57 & Z186 & 96.40 \\
\hline Z15 & 98.50 & Z58 & 84.75 & Z101 & 89.13 & Z144 & 82.20 & Z187 & 95.18 \\
\hline Z16 & 96.75 & Z59 & 96.00 & Z102 & 97.14 & Z145 & 88.89 & Z188 & 99.20 \\
\hline Z17 & 112.86 & Z60 & 94.00 & Z103 & 92.00 & Z146 & 86.25 & Z189 & 90.22 \\
\hline Z18 & 96.64 & Z61 & 93.25 & Z104 & 104.29 & Z147 & 88.60 & Z190 & 94.00 \\
\hline Z19 & 92.60 & Z62 & 90.76 & Z105 & 89.00 & Z148 & 90.31 & Z191 & 85.90 \\
\hline $\mathrm{Z} 20$ & 90.76 & Z63 & 90.13 & Z106 & 96.80 & Z149 & 97.14 & Z192 & 84.60 \\
\hline $\mathrm{Z} 21$ & 99.75 & Z64 & 91.00 & Z107 & 94.20 & Z150 & 85.40 & Z193 & 95.17 \\
\hline $\mathrm{Z} 22$ & 96.60 & Z65 & 90.56 & Z108 & 84.50 & Z151 & 99.43 & Z194 & 93.00 \\
\hline $\mathrm{Z} 23$ & 95.75 & Z66 & 98.50 & Z109 & 99.14 & Z152 & 99.25 & Z195 & 88.57 \\
\hline $\mathrm{Z} 24$ & 90.12 & Z67 & 94.68 & Z110 & 96.32 & Z153 & 98.57 & Z196 & 83.20 \\
\hline $\mathrm{Z} 25$ & 100.22 & Z68 & 80.75 & Z111 & 99.74 & Z154 & 101.43 & Z197 & 88.98 \\
\hline Z26 & 92.60 & Z99 & 92.57 & Z112 & 85.80 & Z155 & 98.20 & Z198 & 86.25 \\
\hline $\mathrm{Z} 27$ & 82.76 & $\mathrm{Z70}$ & 96.74 & Z113 & 102.00 & Z156 & 88.16 & Z199 & 88.60 \\
\hline Z28 & 84.10 & Z71 & 90.00 & Z114 & 100.86 & Z157 & 84.12 & $\mathrm{Z} 200$ & 90.31 \\
\hline Z29 & 94.75 & $\mathrm{Z} 72$ & 96.32 & Z115 & 93.75 & Z158 & 75.40 & Z201 & 97.14 \\
\hline Z30 & 96.86 & Z73 & 86.66 & Z116 & 95.00 & Z159 & 104.00 & Z202 & 86.40 \\
\hline Z31 & 106.57 & Z74 & 84.88 & Z117 & 92.66 & Z160 & 94.25 & Z203 & 99.43 \\
\hline Z32 & 84.14 & Z75 & 96.76 & Z118 & 90.60 & Z161 & 93.11 & Z204 & 99.25 \\
\hline Z33 & 87.75 & Z76 & 93.80 & Z119 & 88.30 & Z162 & 100.75 & Z205 & 98.57 \\
\hline Z34 & 90.75 & Z77 & 96.25 & Z120 & 86.00 & Z163 & 89.80 & Z206 & 98.20 \\
\hline Z35 & 96.13 & Z78 & 94.18 & Z121 & 85.75 & Z164 & 104.29 & Z207 & 88.40 \\
\hline Z36 & 91.11 & Z79 & 93.27 & Z122 & 96.98 & Z165 & 96.90 & Z208 & 94.25 \\
\hline Z37 & 96.50 & Z80 & 86.45 & Z123 & 96.57 & Z166 & 85.71 & Z209 & 93.11 \\
\hline Z38 & 96.60 & Z81 & 77.60 & Z124 & 92.57 & Z167 & 89.25 & $\mathrm{Z} 210$ & 88.71 \\
\hline Z39 & 93.40 & Z82 & 87.78 & $\mathrm{Z} 125$ & 99.00 & Z168 & 100.61 & $\mathrm{Z} 211$ & 89.25 \\
\hline $\mathrm{Z} 40$ & 102.40 & Z83 & 93.71 & Z126 & 99.71 & Z169 & 87.75 & $\mathrm{Z} 212$ & 87.75 \\
\hline Z41 & 96.29 & Z84 & 99.00 & Z127 & 89.43 & Z170 & 101.67 & Z213 & 97.67 \\
\hline $\mathrm{Z} 42$ & 96.64 & $\mathrm{Z} 85$ & 88.25 & Z128 & 96.00 & $\mathrm{Z} 171$ & 97.67 & & \\
\hline Z43 & 94.86 & Z86 & 92.25 & Z129 & 86.22 & Z172 & 88.87 & & \\
\hline
\end{tabular}

https://doi.org/10.24264/icams-2020.II.14 

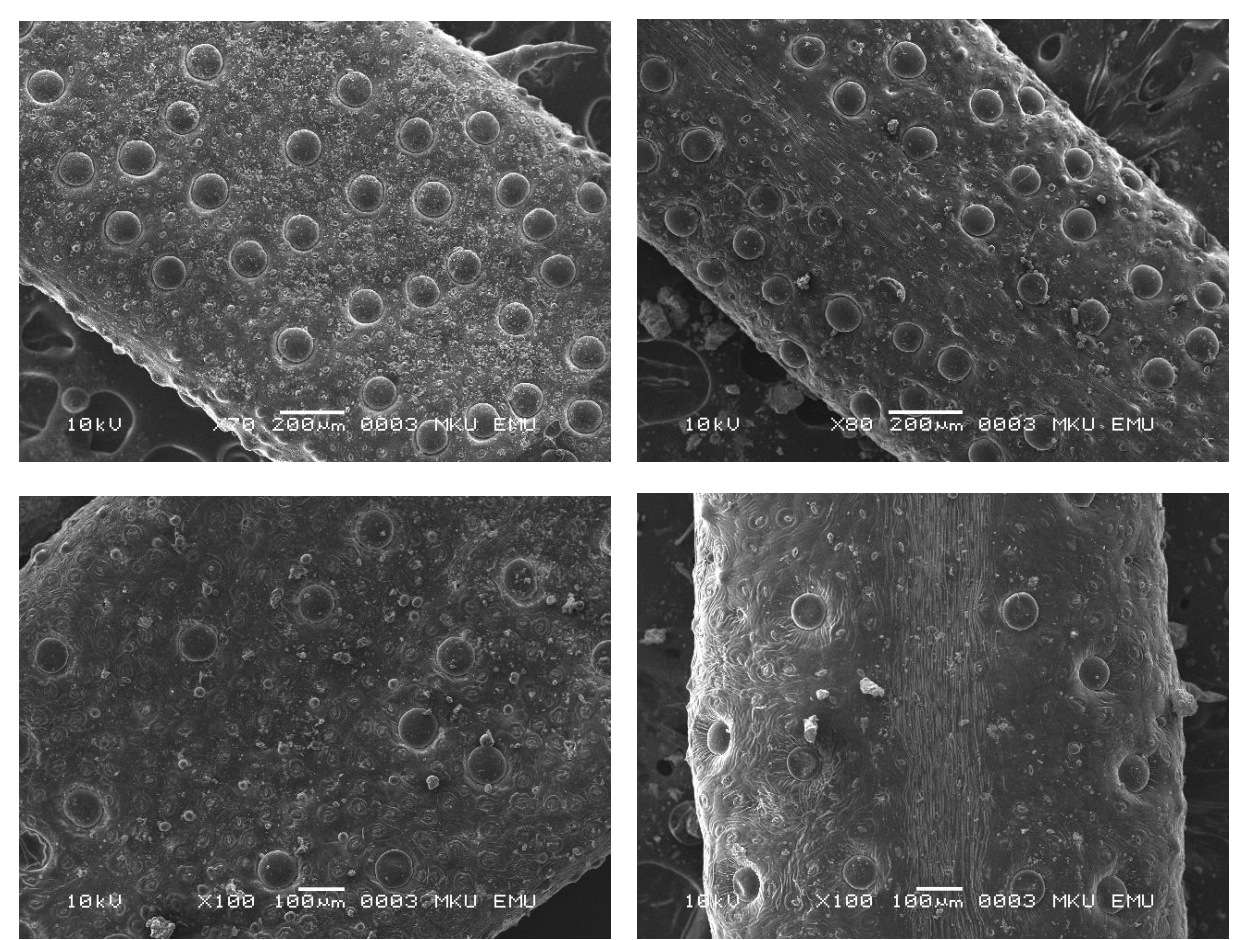

Figure 1. SEM images of some thymbra leaves with the plant code number Z17, Z92, Z144 and Z158, respectively

\section{CONCLUSION}

In the study, first of all, 213 different, well-developed and healthy plants were selected from 68 different locations by single plant selection method. The thymbra plants collected were examined in terms of the number of oil glands per unit area and oil glands diameters in their leaves. It was determined that some plants with low essential oil ratios such as Z144 and Z158 also have low oil grease number and oil gud diameter. It would be appropriate to evaluate these values obtained as preliminary knowledge together with future ontogenetic variability studies.

\section{Acknowledgements}

This study was supported by the Scientific and Technological Research Council of Turkey (TUBITAK-Project No:2140691).

\section{REFERENCES}

Avcı, M. (2005), “Çeşitlilik ve endemizm açısından Türkiye'nin bitki örtüsü”, İstanbul Üniv. Edebiyat Fakültesi Coğrafya Dergisi, (13), 27-55.

Başer, K.H.C. (2002), "Aromatic biodiversity among the flowering plant taxa of Turkey", Pure Appl. Chem., 74(4), 527-545, https://doi.org/10.1351/pac200274040527.

Baydar, H., Sağdiç, O., Özkan, G. and Karadoğan, T. (2004), "Antibacterial activity and composition of essential oils from Origanum, Thymbra and Satureja species with commercial importance in Turkey", Food Control, 15, 169-172, https://doi.org/10.1016/S0956-7135(03)00028-8. 


\section{Oil Glands Number and Oil Glands Diameters of Thymbra spicata var. spicata L. Leaves}

Baytop (1994), Türkçe Bitki Adları Sözlüğü, Ankara: Atatürk Kültür, Dil ve Tarih Yüksek Kurumu Türk Til Kurumu Yayınları, No: 578, P. 168.

Çakan, H. and Byfield, A. (2005), "Amanos dağları" Türkiye'nin 122 önemli bitki alanı (Ed: N.Özhatay, A. Byfield ve S. Atay) WWF Türkiye doğal hayatı koruma vakfi yayını, s:254-257 İstanbul.

Davis, P.H. (1982), Flora of Turkey and the east Aegeans Íslands, Vol. 7. Edinburg University Press.

Doğan, A., Bayrak, A. and Akgül, A. (1987), "Thymol/carvacrol containing Labiatae species and volatile composition of essential oil of Thymbra spicata", Glda, Say1: 6, 359-362.

Ekim, T., Koyuncu, M., Vural, M., Duman, H., Aytaç, Z. and Adıgüzel, N. (2000), Türkiye Bitkileri Kirmızı Kitabı (Ĕgrelti ve Tohumlu Bitkiler), Türkiye Tabiatını Koruma Derneği, Van 100. Yıl Üniv. s:246. Barışcan Ofset. Ankara.

Erken, S. (2005), "Morphological and anatomical studies on Thymbra sintenisii Bornm \&Aznav. (Labiateae)", Turk. J. Bot., 29,389-397.

Hancı Sonsuzer, S., Şahin, S. and Yılmaz, L. (2003), "Isolation of volatile oil from thyme (Thymbra spicata) by steam distillation", Nahrung/Food, 47(4), 252-255, https://doi.org/10.1002/food.200390059.

İnan, M., Kırpık, M., Kaya, D.A. and Kırıcı, S. (2011), "Effect of harvest time on essential oil composition of Thymbra spicata L. Growing in flora of Adiyaman", Advances in Environmental Biology, 5, pp.356-358.

Kizll, S. (2013), "Selection of A clones from Thymbra spicata var. spicata by clonal selection method", Industrial Crops and Products, 41, 1-9, https://doi.org/10.1016/j.indcrop.2012.04.013.

Özcan, M.M., Ünver, A., Uçar, T. and Arslan, D. (2008), "Mineral content of some herbs and herbal teas by infusion and decoction", Food Chemistry, 106, 1120-1127, https://doi.org/10.1016/j.foodchem.2007.07.042.

Özel, M.Z., Göğüş, F. and Lewis, A.C. (2003), "Subcritical water extraction of essential oils from Thymbra spicata", Food chemistry, 82, 381-386, https://doi.org/10.1016/S0308-8146(02)00558-7.

Özgüven, M., Sekin, S., Gürbüz, B., Şekeroğlu, N., Ayanoğlu, F. and Ekren, S. (2005), Tütün, Tıbbi ve Aromatik Bitkiler Üretimi ve Ticareti. Türkiye Ziraat Mühendisliği VI. Teknik Kongresi. 481-501.

Saraç, A. and Tunç, I. (1995), "Residual toxicity and repellency of essential oils to stored-product insets. Rucktandstoxizitat und abstossende wirkung von atherischen ole auf vorratsschadliche insekten", Zeitschrift-fur-Pflanzenkrankheiten und Pflanzenschutz, 102(4), 429-434.

Vural, M. and Aytaç, Z. (2005), "The Flora of Erciyes Dağı (Kayseri, Turkey)”, Tur. J. of Bot., 29, 185-236. 
COLLAGEN MATRICES FROM LEATHER INDUSTRY WASTES FOR BIOMEDICAL APPLICATION

\author{
LESIA MAISTRENKO $^{1}$, OLGA IUNGIN ${ }^{1}$, OLEKSII SAVCHUK ${ }^{2}$, OLENA OKHMAT ${ }^{1}$ \\ ${ }^{1}$ Kyiv National University of Technologies and Design, 2, Nemyrovycha-Danchenka Str., Kyiv, \\ 01011,Ukraine, maystrenko.la@knutd.edu.ua,olga.suslova11@gmail.com \\ ${ }^{2}$ Educational and Scientific Center «Institute of Biology and Medicine» Taras Shevchenko \\ National University of Kyiv, 64/13, Volodymyrska Str., Kyiv, 01601, Ukraine
}

\begin{abstract}
Modern biomedical science is challenged to develop new wound healing drugs. The collagencontaining wastes of leather industry could be the rich source of collagen products for further use in biomedical science. The aim of this research was to find the best source of collagen between limed pelt, delimed pelt and fleshings of cattle hides, and to prepare it for the use as a matrix for further microbiological studies. Collagen was extracted with $0.5 \mathrm{M}$ acetic acid and $5 \mathrm{mM}$ EDTA. The purity of the extracted collagen was checked by gel-electophoresis (SDS-PAGE). The rate of growth and crystal violet assay of laboratory strains ( $S$. aureus, $P$. aeruginosa) were used for microbiological evaluation of obtained collagen matrices. The delimed pelt provided the highest concentration of collagen and the greatest volume of collagen products. All obtained collagen products were applicable as matrices for microbial cells growth. The applicability of collagen products from leather industry wastes for biomedical studies in Ukraine was shown.
\end{abstract}

Keywords: Collagen, biomedical application, leather industry wastes.

\title{
INTRODUCTION
}

Collagen can be extracted from different species of animals as it is usually obtained from by-products of slaughter. The main sources of collagen are the skin, tendons, cartilage and bones. Some studies were focused on obtaining collagen from such animal sources as fish and birds, as an alternative to bovine collagen (cattle) due to the risk of zoonosis, and as an alternative to pig-derived collagen for use in countries with restrictions because of ethical or religious reasons.

In the pharmaceutical and biomedical fields collagen is used as microparticles, injectable dispersions, ophthalmic shields, drug delivery systems, skin substitutes, blood vessels, and human ligaments. This is due to its characteristics such as weak antigenicity, ability to attach to cells, biodegradability and biocompatibility. Type I collagen is considered the gold standard for tissue engineering due to its high biocompatibility. It is used as the main matrix for the cell culture system. Collagenbased biomaterials, such as injection matrices, scaffolds for bone regeneration etc. are widely used (Parenteau-Bareil et al., 2010). In modern medicine, scaffolding based on collagen plays a vital role. It helps in the reconstruction of cartilage and bones. During vascular and cardiac reconstruction, patients are successfully treated with collagen in the form of tissue engineered vessels. Collagen-based bandages are used in the form of sponges, films and powders for wounds or burns, surgical sutures, for urogenital disorders, corneal defects, study of nerve migration, in dentistry, bone transplantation, arthritis and obesity (Sanz-Herrera and Reina-Romo, 2011). Collagen has a variety of applications in cardiology (heart valve), dermatology (for skin replacement, soft tissue augmentation, skin engineering), surgery (as a hemostatic agent, in wound healing and dressing, nerve repair, blood vessel prostheses), orthopedics (tendon, bone and ligament repair, cartilage reconstruction), ophthalmology (horn grafts, contact lenses), urology (hemodialysis membranes, sphincter restoration) (Rose and Oreffo, 2002). 
Only fully or partially soluble collagen can be used in the production of matrices, powders, sponges, fibers or threads for tissue engineering. The distribution of molar mass, structure and composition, as well as functional properties of collagen depend on the extraction process and conditions of the raw material (Prestes, 2013). So it is needed to get the proper extraction parameters for each type of raw material to obtain the best characteristics of the extracted collagen. Due to the extraordinary diversity of tissues and types of collagen, it is difficult to develop a standard method of its extraction. The number of covalent intermolecular interactions in the structure of collagen increases over time and often determines the almost complete insolubility of the obtained product (Mocan et al., 2011). It is required to remove numerous covalent intra- and intermolecular cross-links primarily including lysine and hydroxylysine residues, ether bonds, and other polysaccharide bonds for collagen extraction.

\section{OBJECTIVES OF THE STUDY}

The objects of this study were collagen samples obtained from leather production waste of the footwear uppers from raw materials of the cattle tannery "Chinbar" (Kyiv, Ukraine). To find the best option, different types of waste were selected such as limed pelt after trimming, delimed pelt after trimming, limed fleshings.

\section{Chemical Analysis of Leather Production Waste}

It was determined the content of the main components in the waste samples: moisture, minerals, total Nitrogen and calcium hydroxide (Table 1).

Table 1. Chemical composition of leather production wastes

\begin{tabular}{lccc}
\hline Indicator & limed pelt & delimed pelt & fleshings \\
\hline Mass fraction, \%: & & & \\
moisture & 82.1 & 80.4 & 84.2 \\
minerals * & 10.7 & 3.3 & 31.7 \\
total Nitrogen* & 14.3 & 15.0 & 5.4 \\
calcium hydroxide* & 2.6 & 0.4 & 6.7 \\
\hline
\end{tabular}

Note: * on absolutely dry matter

The increased content of minerals and calcium hydroxide in the limed pelt and limed fleshings samples can be explained by the peculiarities of the technological process of leather production, which involves the processing of raw materials in a calcium hydroxide concentrated solution. As the main purpose of the deliming process is the extraction of calcium hydroxide from the dermis, the content of minerals and calcium hydroxide in the delimed pelt after trimming was 3.2-9.6 and 6.5-16.8 times lower, respectively. The lowest content of total Nitrogen, which indirectly indicated the amount of collagen in the structure, was found in the limed fleshings samples.

\section{METHODS}

The content of moisture, minerals and total Nitrogen in the waste of leather production was determined by standard methods ISO 1666:1996, ISO 3593:1981, ISO 
5397:1984. The concentration of collagen in the solution was determined by biuret method on Spectrophotometer ULAB 102, China (540 nm).

\section{Method of Collagen Extraction}

The method of (Savchuk et al., 2017) with changes was chosen as a basis.

\section{First Extraction}

1. Deliming process with an ammonium sulfate consumption of $3 \%$ by weight of the samples, duration $1 \mathrm{~h}, 38-40{ }^{\circ} \mathrm{C}$ (only to obtain samples of delimed pelt).

2. Samples grinding to the size of $3 \times 3 \mathrm{~mm}$.

3. Weighing.

4. Washing with water at $20{ }^{\circ} \mathrm{C}$ for 45 minutes with water change every 15 minutes.

5 . Deposition of non-collagen proteins with $10 \%$ sodium chloride solution, duration $1 \mathrm{~h}$ of shaking at $20^{\circ} \mathrm{C}$, then $22 \mathrm{~h}$ of rest at $4{ }^{\circ} \mathrm{C}$ and again $1 \mathrm{~h}$ of shaking at $20^{\circ} \mathrm{C}$.

6. Washing the samples with distilled water to $\mathrm{pH}=6.5$.

7. Extraction of collagen with $0.5 \mathrm{M}$ acetic acid solution in the presence of $5 \mathrm{~mm}$ EDTA in a ratio of $1: 10$ (weight : volume), $2 \mathrm{~h}$ of shaking at $20^{\circ} \mathrm{C}, 20 \mathrm{~h}$ of rest at $4{ }^{\circ} \mathrm{C}$ and again $2 \mathrm{~h}$ of shaking at $20^{\circ} \mathrm{C}$.

8. Filtering through a paper filter. The samples were used for the 2 nd extraction.

9. Precipitation of collagen from the filtrate with dry sodium chloride (with a final concentration of $\mathrm{NaCl} 1 \mathrm{M}$ ) for $24 \mathrm{~h}$ at $4{ }^{\circ} \mathrm{C}$.

10. Centrifugation for $30 \mathrm{~min}$ at $3000 \mathrm{rpm}$

11. Dissolution of precipitated collagen in the least amount of $0.5 \mathrm{M}$ acetic acid.

12. Re-precipitation of collagen with dry sodium chloride (with a final concentration of $\mathrm{NaCl} 0.9 \mathrm{M}$ ) for $24 \mathrm{~h}$ at $4{ }^{\circ} \mathrm{C}$.

13. Centrifugation for $30 \mathrm{~min}$ at $3000 \mathrm{rpm}$

14. Dissolution of precipitated collagen in the smallest amount of $0.1 \mathrm{M}$ acetic acid.

\section{Second Extraction}

Collagen samples remaining after the $1^{\text {st }}$ extraction (item 8) were processed according to items $7-14$.

\section{Third Extraction}

Collagen samples remaining after the $2^{\text {nd }}$ extraction (item 8) were processed according to items 7-14.

\section{Estimation of Extracted Collagen and Collagen Peptides by Sodium Dodecyl Sulfate Polyacrylamide Gel Electrophoresis (SDS-PAGE)}

SDS-PAGE was done as described by the (Laemmli, 1970) using $4 \%$ (w/v) stacking gel, $6 \%(\mathrm{w} / \mathrm{v})$ separating gel for estimation of purity of extracted collagen and $18 \%$ (w/v) separating gel for collagen peptides. SDS-PAGE was performed using MiniProtean Tetra System (Bio Rad, USA) at $19 \mathrm{~mA}$ for stacking and $36 \mathrm{~mA}$ for separating gels. After electrophoresis, the gels were stained with $2.5 \%$ coomassie brilliant blue R250 in $10 \%(\mathrm{v} / \mathrm{v})$ ethanol, $10 \%(\mathrm{v} / \mathrm{v})$ acetic acid, $15 \%(\mathrm{v} / \mathrm{v})$ isopropanol and the background of the gel was destained with $7 \%$ (v/v) acetic acid for $30 \mathrm{~min}$. 


\section{Microbial Assay}

Collagen samples after extraction and washing with distilled water (for $\mathrm{pH} 5.5$ ) in sterile conditions was used as matrices for bacterial cell growth. Laboratory strains and hospital isolates of Staphylococcus aureus and Pseudomonas aeruginosa were used. The OD and attachment rate were evaluated with microtiter plate assay $(630 \mathrm{~nm})$ and crystal violet assay $(570 \mathrm{~nm})$ on Spectrophotometer UV-Vis, Russia (O'Toole, 2011).

\section{RESULTS}

The main criteria for evaluating the extraction efficiency of collagen obtained from collagen-containing wastes from the leather industry was the amount of extracted collagen, which was determined by the protein content in the solution after extraction and subsequent dissolution in acetic acid. Collagen was re-extracted twice from the supernatant obtained after the first and second extraction stages to determine the economic feasibility of re-extraction. The changes in $\mathrm{pH}$ of the solutions at different stages of extraction are presented (Table 2).

Table 2. The $\mathrm{pH}$ values of collagen-containing solutions

\begin{tabular}{lccc}
\hline Extraction method item & limed pelt & delimed pelt & fleshings \\
\hline it. 4 after $15 \mathrm{~min}$ & 10.55 & 6.80 & 7.95 \\
it. 4 after 30 min & 7.85 & 6.40 & 6.60 \\
it. 4 after 45 min & 6.50 & 5.90 & 6.40 \\
it. 5 & 8.00 & 7.50 & 9.80 \\
it. 14 & 2.60 & 2.60 & 2.80 \\
\hline
\end{tabular}

Data on the collagen amount obtained after the first and further extractions from different leather waste were presented in Table 3.

Table 3. Collagen amount obtained after extraction

\begin{tabular}{lccc}
\hline & $\begin{array}{c}\text { limed } \\
\text { pelt }\end{array}$ & $\begin{array}{c}\text { delimed } \\
\text { pelt }\end{array}$ & fleshings \\
\hline The amount of total Nitrogen after the $1^{\text {st }}$ extraction, mg & 7.6 & 50.4 & 3.3 \\
The amount of total Nitrogen after the $2^{\mathrm{d}}$ extraction, mg & 39.2 & 71.4 & 11.5 \\
The amount of total Nitrogen after the $3^{\mathrm{d}}$ extraction, mg & 3.3 & 1.0 & 0.5 \\
The total yield of total Nitrogen after three extractions, $\%$ & 2.79 & 5.97 & 2.60 \\
The amount of collagen after the $1^{\text {st }}$ extraction, mg & 10.1 & 25.3 & 3.0 \\
The amount of collagen after the $2^{\text {nd }}$ extraction, mg & 5.0 & 11.4 & 1.4 \\
The amount of collagen after the $3^{\text {nd }}$ extraction, mg & 2.2 & 4.7 & 0.6 \\
\hline
\end{tabular}

The largest amount of collagen was extracted from samples of delimed pelt. It was found that the third extraction was not efficient due to the low amount of extracted collagen in all samples. The less amount of collagen in limed pelt and fleshings could be explained by the fact that part of the acetic acid from the solution was spent on neutralizing the calcium hydroxide excess found in the samples of these groups. The fleshings samples were also characterized by a lower initial content of total Nitrogen (Table 1). 
It is known that type I collagen consists of two or more different chains (heterotrimers): usually two alpha-1, one alpha-2 chain with a similar molecular weight of about 100-110 kDa and the structure of the beta component (Kimura et al., 1987). It was shown that extracted collagen consisted of both $\alpha 1$ and $\alpha 2$ chains (Fig.1). Thus, based on the SDS-PAGE results collagen extracted from the waste of the leather industry belongs to type I collagen.

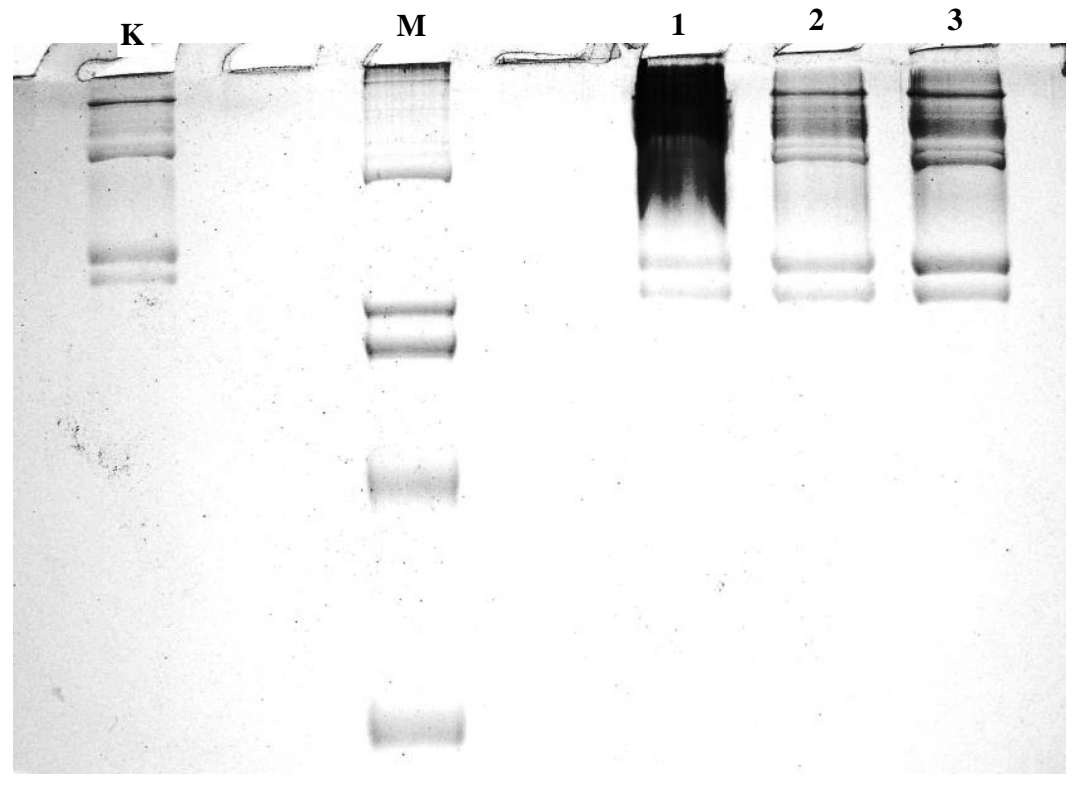

Figure 1. SDS-PAGE pattern of collagen extracted from waste of leather industry on $6 \%$ separating gel. Lane $K$ - control collagen (Savchuk et al., 2017), $M$ - high molecular weight (MW, kDa) protein markers;

1 - limed pelt, 2 - delimed pelt, 3 - fleshings

After microbiological studies it was found that the optical density of $S$. aureus strains was two times less while the attachment was two times higher in the presence of collagen samples. This tendency was also kept in case of $P$. aeruginosa with the only difference - the indexes were about $30 \%$. The highest bacterial growth and attachment were observed on the collagen matrices from $1^{\text {st }}$ extraction samples of delimed pelt and limed pelt. The $2^{\text {nd }}$ extraction samples were about $25-30 \%$ slightly less effective. According to studied parameters the first choice collagen matrices for bacterial growth was collagen from delimed pelt, the second choice matrices - collagen from limed pelt.

\section{CONCLUSIONS}

Collagen-containing leather industry wastes could be the great source of collagen for biomedical application. The greatest amount of collagen was obtained from delimed pelt after the first and second extractions. Such kind of collagen was classified as type I and pure. It was also the perfect matrix for microbial cell grow which could be the evidence of its matching for further using in skin wound treatment. 


\section{Collagen Matrices from Leather Industry Wastes for Biomedical Application}

\section{REFERENCES}

Bhaskar, N., Modi, V.K., Govindaraju, K., Radha, C. and Lalitha, R.G. (2007), "Utilization of meat industry by products: protein hydrolysate from sheep visceral mass", Bioresource Technology, 98(2), 388-394, https://doi.org/10.1016/j.biortech.2005.12.017

Kimura, S., Ohno, Y. and Miyauchi, Y. (1987), "Fish skin type I collagen: wide distribution of an alpha 3 subunit in teleosts", Comparative Biochemistry and Physiology, 88(1), 27-34, https://doi.org/10.1016/0305-0491(87)90074-5.

Laemmli, U.K. (1970), "Cleavage of Structural Proteins during the Assembly of the Head of Bacteriophage T4", Nature, 227, 680-685, https://doi.org/10.1038/227680a0.

Mocan, E., Tagadiuc, O. and Nacu,V. (2011), "Aspects of Collagen Isolation Procedure”, Clinical Research Studies, 2(320), 3-5.

Nollet, L.M.L. and Toldra, F. (2011), Handbook of Analysis of Edible Animal By-Products, CRC Press, Gent, Belgium, https://doi.org/10.1201/b10785.

O'Toole, G.A. (2011), "Microtiter dish biofilm formation assay", Journal of visualized experiments, (47), 2437, https://doi.org/10.3791/2437.

Parenteau-Bareil, R., Gauvin, R. and Berthod F. (2010), “Collagen-Based Biomaterials for Tissue Engineering Applications", Materials, 3(3), 1863-1887, https://doi.org/10.3390/ma3031863.

Prestes, R.C. (2013), “Colageno e seus derivados: caracteristicas e aplicagoes em produtos carneos”, Revista Unopar Cientffica Ciencias Biologicas e da Saüde, 15(1), 65-74, https://doi.org/10.17921/2447$8938.2013 \mathrm{v} 15 \mathrm{n} 1 \mathrm{p} \% 25 \mathrm{p}$.

Rose, F.R. and Oreffo, R.O. (2002), "Bone tissue engineering: hope vs hype", Biochem Biophys Res Commun, 292, 1-7, https://doi.org/10.1006/bbrc.2002.6519.

Sanz-Herrera, J.A. and Reina-Romo, E. (2011), "Cell-Biomaterial Mechanical Interaction in the Framework of Tissue Engineering”, Int. J. Mol. Sci., 12, 8217- 8244, https://doi.org/10.3390/ijms12118217.

Savchuk, O., Raksha, N., Ostapchenko, L., Mokrousova, O. and Andreyeva, O. (2017), "Extraction and Characterization of Collagen Obtained from Collagen-Containing Wastes of the Leather Industry", Solid State Phenomena, 267, 172-176, https://doi.org/10.4028/www.scientific.net/SSP.267.172. 


\title{
DESIGN AND EVALUATION OF \\ DOXYCYCLINE/COLLAGEN/CHONDROITIN SULFATE DELIVERY SYSTEMS USED FOR CARTILAGE REGENERATION
}

\author{
MARIA-MINODORA MARIN ${ }^{1,2}$, MĂDĂLINA GEORGIANA ALBU KAYA ${ }^{2 *}$, MIHAELA \\ VIOLETA GHICA ${ }^{3}$, ELENA DĂNILA ${ }^{1}$, GHEORGHE COARA ${ }^{2}$, LĂCRĂMIOARA POPA ${ }^{3}$, \\ CIPRIAN CHELARU ${ }^{2}$, DURMUȘ ALPASLAN KAYA ${ }^{4}$, VALENTINA ANUȚA ${ }^{3}$, \\ CRISTINA-ELENA DINU-PÎRVU ${ }^{3}$, IOAN CRISTESCU ${ }^{5}$ \\ ${ }^{1}$ University Politehnica of Bucharest, Faculty of Applied Chemistry and Materials Science, 1-7 \\ Gheorghe Polizu Str., 011061, Bucharest, Romania \\ ${ }^{2}$ National Research and Development Institute for Textile and Leather, Division Leather and \\ Footwear Research Institute, Collagen Department, 93 Ion Minulescu Str., 031215, Bucharest, \\ Romania; albu_mada@yahoo.com \\ 3 "Carol Davila" University of Medicine and Pharmacy, Faculty of Pharmacy, Physical and \\ Colloidal Chemistry Department, 6 Traian Vuia Str., 020956, Bucharest, Romania, \\ ${ }^{4}$ Mustafa Kemal University, Faculty of Agriculture, Department of Field Crops, 31034, Antakya- \\ Hatay, Turkey \\ ${ }^{5}$ Carol Davila" University of Medicine and Pharmacy, Faculty of Medicine, Department of \\ Orthopaedy, 8 Bulevardul Eroilor Sanitari, 050474, Bucharest, Romania
}

\begin{abstract}
Cartilage damage is difficult to self-heal due to an avascular microenvironment and distinct mechanical properties. These features are a challenge in designing a cartilaginous tissue with repairing effect without producing any local infections. Thus, a biodegradable scaffold in which the drug can be incorporated is preferable. Drug delivery systems based on collagen sponges have progressively become remarkable biomaterials for different medical applications. The aim of this work was to design and characterize some collagen/chondroitin sulfate supports with doxycycline for cartilage tissue regeneration. The doxycycline should prevent the development of potential infections. Collagen, chondroitin sulfate and doxycycline gels were cross-linked with different concentrations of glutaraldehyde and then freeze-dried in order to obtain collagen matrices. The structural characteristics for the new synthesized biomaterials were firstly assessed by infrared spectroscopy (FT-IR), and scaffolds morphology was then evaluated by optical microscopy and water uptake. The enzymatic biodegradation was also performed. Also, the sponges surface properties were quantified through contact angle. The in vitro doxycycline kinetics release was performed with a dissolution equipment and the release mechanism was investigated. The obtained results recommend these new scaffolds based on doxycycline/collagen/chondroitin sulfate as a promising approach for the treatment of cartilage problems.
\end{abstract}

Keywords: collagen, doxycycline, chondroitin sulfate

\section{INTRODUCTION}

The cartilage tissue is powerless to self-repair and restore due to the absence of nerves, blood vessels and lymphatic tissues, which makes the treatment more difficult (Huang et al., 2016). These features are a challenge in designing a cartilaginous tissue with repairing effect without producing any local infections. Thus, a biodegradable scaffold in which the drug can be incorporated is preferable (Song et al., 2017; Huey et al., 2012). Collagen, a natural biomaterial, represents the main protein from the human organism: skin, bone, cartilage, organs, blood vessels, ligaments and tendons (Miao et al., 2018). Collagen exhibits excellent properties, such as low antigenicity, gradual biodegradability, good biocompatibility and optimal cell proliferation (Lan et al., 2019). Drug delivery systems based on collagen sponges have progressively become remarkable biomaterials for different medical applications (Vikash et al., 2013). 
Chondroitin sulfate (CS), a fundamental component of extracellular matrix in conjunctive tissue (cartilage, bones etc.), is a glycosaminoglycan which helps the formation of aggrecans in the native cartilage (Bang et al., 2018). Collagen and chondroitin sulfate can be great candidates for the design of cartilage tissue engineering in controlling cellular performances (Zhang et al., 2011; Cao et al., 2008). Doxycycline hyclate $(\mathrm{DH})$ represents a tetracycline antibiotic with a strong broad-spectrum activity against aerobic microorganisms and it is usually used in the treatment of the infections (Raval et al., 2014). Thus, the aim of this work was to design and characterize some collagen/chondroitin sulfate supports with doxycycline, for cartilage tissue regeneration.

\section{MATERIALS AND METHODS}

\section{Materials and Preparation of the Scaffolds}

The type II collagen was extracted from bovine cartilage using technology currently available at the National Research and Development Institute for Textile and Leather, Division Leather and Footwear Research Institute - Collagen Department. Doxycycline hyclate and chondroitin sulfate were purchased from Sigma-Aldrich, China. Sodium hydroxide and hydrochloric acid were of analytical grade. Type I collagenase obtained from Clostridium histolyticum was purchased from Sigma-Aldrich, Germany and glutaraldehyde (GA) from Merck, Germany.

The concentration of each collagen gel was adjusted at $1.5 \%$ and $7.4 \mathrm{pH}$ using $1 \mathrm{M}$ sodium hydroxide. $0.2 \% \mathrm{DH}$ and different concentration of CS were added to collagen gel $(\mathrm{w} / \mathrm{v})$, and then the collagen gels were cross-linked with $0.0025 \%$ GA as Table 1 presents. The samples were coded as follows: $\mathrm{M}-1 \div \mathrm{M}-5$.

Table 1. Composition of the obtained samples

\begin{tabular}{ccc}
\hline Sample & Doxy, $\%$ & CS, $\%$ \\
\hline M-1 & 0 & 0 \\
M-2 & 0.2 & 0.05 \\
M-3 & 0.2 & 0.10 \\
M-4 & 0.2 & 0.15 \\
M-5 & 0 & 0.05 \\
\hline
\end{tabular}

\section{FT-IR Analysis}

FT-IR spectral measurements were recorded by a Jasco FT-IR 4200 spectrophotometer. All the spectra were recorded at the following parameters: spectral range $4000-600 \mathrm{~cm}^{-1}$, resolution $4 \mathrm{~cm}^{-1}$ with 30 acquisitions per each sample.

\section{Optical Microscopy Analysis}

The morphology of the designed sponges was carried out using a LEICA optical microscope model S8AP0, with increase power of 20-160x.

\section{Contact Angle Evaluation}

The sponges surface wettability was assessed with CAM 101 (KSV Instruments), using the pendant drop dynamic method, as reported in our previous works (Ghica et al., 2013; Popa et al., 2013). 


\section{Water Uptake}

In order to evaluate the water absorption, the obtained samples were immersed in ultrapure water. At timetabled intervals, the samples were weighed. The experiment was done in triplicate. The water uptake was calculated using the equation: $\%$ Water uptake $=\frac{W_{t}-W_{d}}{W_{d}} \times 100$

where $\mathrm{W}_{\mathrm{d}}$ is the weight of the dry samples and $\mathrm{W}_{\mathrm{t}}$ is the weight of the swollen sample at immersion time $\mathrm{t}$.

\section{Degradation Studies}

Enzymatic degradation of sponges was investigated by measuring the weight loss depending on contact time to collagenase solution. At specific intervals, the swollen scaffolds were weighed. The percentage of scaffold degradation was determined by the following equation:

$$
\% \text { Weight loss }=\frac{W_{i}-W_{t}}{W_{t}} \times 100
$$

where $\mathrm{W}_{\mathrm{i}}$ is the initial weight and $\mathrm{W}_{\mathrm{t}}$ is the weight after time $\mathrm{t}$.

\section{Drug Release Kinetics Analysis}

In vitro drug release from collagen spongious matrices was carried out using a "sandwich" device adapted to a paddle dissolution equipment, as described in our previous studies (Albu et al., 2010). The concentration of doxycycline hyclate released in the medium (phosphate buffer $\mathrm{pH}$ 7.4) at different time intervals was monitored by UV spectroscopy and the cumulative released drug percentage was evaluated.

\section{RESULTS AND DISCUSSION}

The collagen gels with compositions according to Table 1 were freeze-dried and spongious matrices were obtained and characterized.

From the FT-IR spectra (Figure 1) the characteristic bands from collagen it can be observed: amide A, B, I, II and III for all the samples (Albu, 2011).

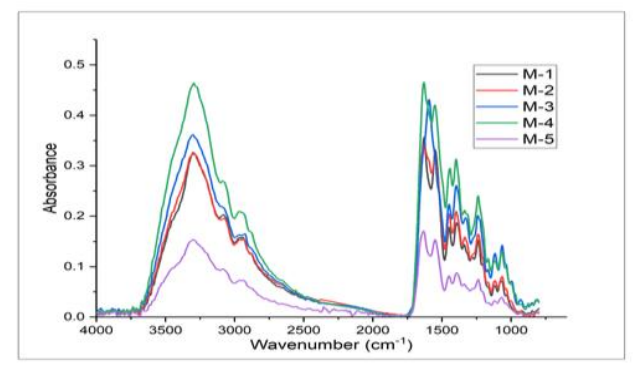

Figure 1. FT-IR spectra
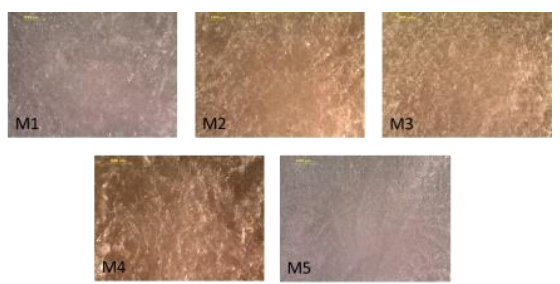

Figure 2. Optical microscopy (20X)

There are no significant changes when doxycycline hyclate and different concentration of chondroitin sulfate were added to collagen gel.

The optical results for the obtained samples are present in Figure 2. Optical images have shown for all the samples a highly porous structure with interconnected pores presenting various dimensions and shapes which is beneficial to the cell adhesion and proliferation.

https://doi.org/10.24264/icams-2020.II.16 


\section{Design and Evaluation of Doxycycline/Collagen/Chondroitin Sulfate Delivery Systems Used for Cartilage Regeneration}

The spongious matrices surface wettability was expressed through contact angle $\left(\mathrm{CA}^{\circ}\right)$ value. The drop shape (Figure 3$)$, monitored with a digital camera, was mathematically described by the Young equation (eq. 3):

$\gamma_{S G}=\gamma_{S L}+\gamma_{L G} \cdot \cos \theta$

where $\gamma_{S G}$ is the interfacial tension S/G, $\gamma_{S L}-$ the interfacial tension S/L, $\gamma_{L G}-$ the superficial tension $\mathrm{L} / \mathrm{G}$ and $\theta-$ the contact angle.

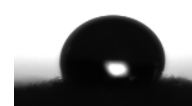

a) $89.03^{\circ} \pm 2.01$

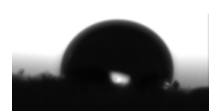

b) $69.58^{\circ} \pm 1.06$

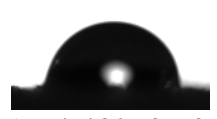

c) $74.48^{\circ} \pm 0.60$

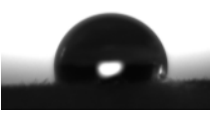

d) $82.24^{\circ} \pm 2.49$

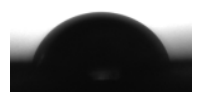

e) $55.64^{\circ} \pm 2.85$

Figure 3. Images of the drop shape for the spongious matrices and the corresponding contact angle values: a) M-1; b) M-2; c) M-3; d) M-4; e) M-5

As it can be noticed in Figure 3, the contact angle values are smaller than $90^{\circ}$, with values between 55.64 and $89.03^{\circ}$, indicating an adequate hydrophilicity of the sponges surface and consequently a good wetting ability by the biologic fluids at the application site. The $\mathrm{CA}$ is strongly influenced by the sponges formulation. Thus, the highest value of CA was recorded for the sample M-1 without drug and chondroitin sulfate, and with a high collagen concentration. The addition of CS $(0.05 \%)$ in the sponge formulation (M-5) lead to the smallest CA, the decrease being about 1.6 times. It seems that the CS presence produced a more porous structure of the sponge surface allowing a better wetting. On the other hand, for the spongious matrix with the same concentration of CS $(0.05 \%)$, but with $0.2 \%$ doxycycline hyclate (M-2) an increase of CA about 1.25 times was observed. These results are in line with our previous studies which highlighted that doxycycline has a cross-linking effect (Albu et al., 2009), inducing in this way a decrease of sponge surface hydrophilicity. Concerning the sponges M-2 $\div$ M4 with the same concentration of biopolymer, drug and cross-linking agent, the increase of CS amount determined an increase of CA, more obvious for higher concentration $(0.15 \%)$.

The water uptake for the studied samples is presented as kinetics during 24 hours in Figure 4. In Figure 4 it can be observed the water absorption during a 24 hours period for the studied samples. All the samples exhibited a high water uptake, this being in concordance with contact angle studies. The maximum amount of water was absorbed by M-5 sample which presented the best hydrophilicity.

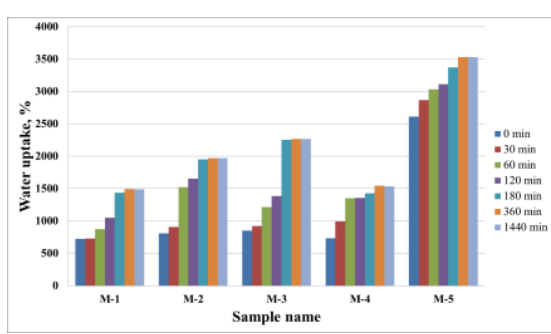

Figure 4. Water uptake during 24 hours for spongious forms

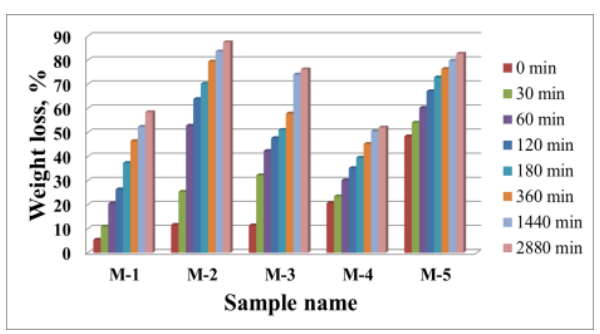

Figure 5. Enzymatic degradation over 48 hours for spongious forms

The degradation results (Figure 5) indicated an excellent biodegradation for all the samples during the $48 \mathrm{~h}$ time interval. The samples with slower degradation rate were M-4 and M-1 with approximately 50\% and 60\% weight loss in 48 hours. 
The in vitro doxycycline kinetic profiles from spongious matrices were recorded as drug cumulative released percentage as a function of time (Figure 6).

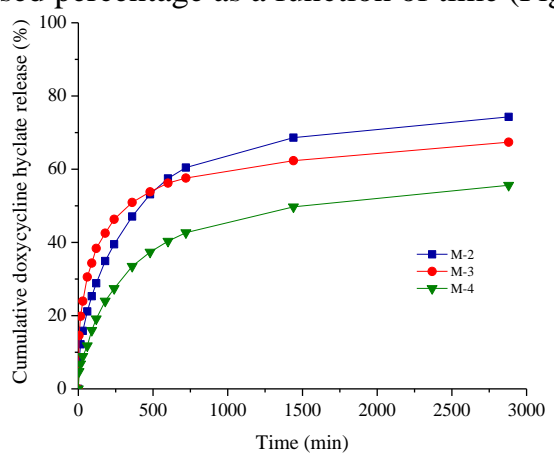

Figure 6. Cumulative release patterns of doxycycline hyclate from collagen spongious matrices as a function of time

The kinetic profiles are similar, with an initial burst release in the first 2 hours, followed by a gradual and prolonged drug delivery over the next 48 hours. The most pronounced rapid release effect was recorded for the M-3 sponge with a medium concentration of chondroitin sulfate $(38.37 \%)$, followed by the M-2 sponge $(28.85 \%)$ with the smallest concentration of CS, while the less evident burst effect was reported for the M-4 sponge (19.13\%). The cumulative doxycycline hyclate released percentage after $48 \mathrm{~h}$ has varied between 55.62 (M-4) and 74.29 (M-2) (Table 2). This long-term antibiotic release provides a local and protective antibacterial effect over a longer period of time necessary for tissue repair.

To set up the drug mass transfer mechanism, the kinetic data were fitted to the Power law model (equation 4 and its particular case, Higuchi model $(n=0.5)$, the corresponding correlation coefficients $(\mathrm{R})$ values being listed in Table 2:

$\frac{m_{t}}{m_{\infty}}=k \cdot t^{n}$

where $m_{t} / m_{\infty}$ represents the fraction of drug released at time $t, k-$ the kinetic constant, $n$ - the release exponent characteristic for the drug release mechanism.

The highest values for the correlation coefficients were recorded for the Power law model, indicating a non-Fickian mechanism for drug release from collagen spongious matrices, in line with our previous studies (Ghica et al., 2013). The release exponent and kinetic constant values specific to the Power law model are given in Table 2.

Table 2. Correlation coefficients for the Power law and Higuchi models; parameter values specific to the Power law model; percentage of drug released

\begin{tabular}{cccccc}
\hline $\begin{array}{c}\text { Spongious } \\
\text { matrices }\end{array}$ & $\begin{array}{c}\text { R Higuchi } \\
\text { model }\end{array}$ & $\begin{array}{c}\mathrm{R} \\
\text { Power Law model }\end{array}$ & $\begin{array}{c}\text { Release } \\
\text { exponent, } \mathrm{n}\end{array}$ & $\begin{array}{c}\text { Kinetic constant, } \\
\mathrm{k}\left(1 / \mathrm{min}^{\mathrm{n}}\right)\end{array}$ & $\begin{array}{c}\text { Drug } \\
\text { Released (\%) }\end{array}$ \\
\hline M-2 & 0.9402 & 0.9806 & 0.31 & 0.069 & 74.29 \\
M-3 & 0.8849 & 0.9850 & 0.22 & 0.133 & 67.38 \\
M-4 & 0.9524 & 0.9801 & 0.34 & 0.039 & 55.62 \\
\hline
\end{tabular}

\section{CONCLUSIONS}

All the designed sponges presented adequate goniometric, morphological and biological properties. The drug release patterns presented a biphasic shape, targeted

https://doi.org/10.24264/icams-2020.II.16 


\section{Design and Evaluation of Doxycycline/Collagen/Chondroitin Sulfate Delivery Systems Used for Cartilage Regeneration}

both to prevent and to control local infection at affected tissue, providing a protective antibacterial effect over the required period to favor long-term healing. The results obtained recommend these new scaffolds based on doxycycline/collagen/chondroitin sulfate as a promising approach for the treatment of cartilage problems.

\section{Acknowledgements}

The work has been funded by the Operational Programme Human Capital of the Ministry of European Funds through the Financial Agreement 51668/09.07.2019, SMIS code 124705 . The study was also financially supported by the Ministry of Research and Innovation, Program 1: Development of National System of Research-Development, Subprogram 1.2: institutional performance projects for funding the excellence in RDI, contract no 6PFE/16.10.2018 and contract no PN 19170302.

\section{REFERENCES}

Albu, M.G. (2011), Collagen Gels and Matrices for Biomedical Applications, Lambert Academic Publishing, Saarbrücken.

Albu, M.G., Ghica, M.V., Leca, M., Popa, L., Borlescu, C., Cremenescu, E., Giurginca, M. and Trandafir, V. (2010), "Doxycycline Delivery from Collagen Matrices Crosslinked with Tannic Acid", Molecular Crystals and Liquid Crystals, 523, 97/[669]-105/[677], https://doi.org/10.1080/15421401003724159.

Albu, M.G., Ghica, M.V., Popa, L., Leca, M. and Trandafir, V. (2009), "Kinetics of In Vitro Release of Doxycycline Hyclate from Collagen Hydrogels", Revue Roumaine de Chimie, 54(5), 373-379.

Bang, S., Jung, U-W. and Noh, I. (2018), "Synthesis and Biocompatibility Characterizations of in Situ Chondroitin Sulfate-Gelatin Hydrogel for Tissue Engineering”, Tissue Engineering and Regenerative Medicine, 15(1), 25-35, https://doi.org/10.1007/s13770-017-0089-3.

Cao, H. and Xu, S.Y. (2008), "EDC/NHS-crosslinked type II collagen-chondroitin sulfate scaffold: characterization and in vitro evaluation", Journal of Materials Science, 19(2), 567-575, https://doi.org/10.1007/s10856-007-3281-5.

Chak, V., Kumar, D. and Visht, S. (2013), A Review on Collagen Based Drug Delivery Systems, Internationa Journal of Pharmacy Teaching \& Practices, 4(4), 811-820.

Ghica, M.V., Albu, M.G., Popa, L. and Moisescu, Ș. (2013), "Response Surface Methodology and Taguch Approach to Assess the Combined Effect of Formulation Factors on Minocycline Delivery from Collagen Sponges", Pharmazie, 68(5), 340-348.

Huang, B.J., Hu, J.C. and Athanasiou, K.A. (2016), "Cell-based tissue engineering strategies used in the clinical repair of articular cartilage", Biomaterials, 98, 1-22, https://doi.org/10.1016/j.biomaterials.2016.04.018.

Huey, D.J., Hu, J.C. and Athanasiou K.A. (2012), "Unlike bone, cartilage regeneration remains elusive", Science, 338, 917-921, https://doi.org/10.1126/science.1222454.

Li, L., Yu, F., Zheng, L., Wang, R., Yan, W., Wang, Z., Xu, J., Wu, J., Shi, D., Zhu, L., Wang, X., Jiang, Q. (2019), "Natural hydrogels for cartilage regeneration: Modification, preparation and application", Journal of Orthopaedic Translation, 17, 26-41, https://doi.org/10.1016/j.jot.2018.09.003.

Miao, Z., Lu, Z., Wu, H., Liu, H., Li, M., Lei, D., Zheng, L. and Zhao, J. (2018), "Collagen, agarose, alginate and matrigel hydrogels as cell substrates for culture of chondrocytes in vitro: a comparative study", Journal of Cellular Biochemistry, 119, 7924-7933, https://doi.org/10.1002/jcb.26411.

Popa, L., Ghica, M.V., Albu, M.G., Orţan, A. and Dinu-Pîrvu, C.E. (2013), "Hysteresis of Contact Angle. Dynamic Wettability Studies of Collagen and Doxycycline Porous Matrices Crosslinked with Tannic Acid", Digest Journal of Nanomaterials and Biostructures, 8(3), 937-943.

Raval, J.P., Naik, D.R., Amin, K.A. and Patel, P.S. (2014), "Controlled-release and antibacterial studies of doxycycline-loaded poly (e-caprolactone) microspheres", Journal of Saudi Chemical Society, 18, 566573, https://doi.org/10.1016/j.jscs.2011.11.004.

Song, X., Zhu, C., Fan, D., Mi, Y., Li, X., Fu, R., Duan, Z., Wang, Y and Feng, R. (2017), "A novel humanlike collagen hydrogel scaffold with porous structure and sponge-like properties", Polymers, 9, 638 https://doi.org/10.3390/polym9120638.

Zhang, L., Li, K.F., Xiao, W.Q., Zheng, L., Xiao, Y.M., Fan, H.S. and Zhang, X.D. (2011), "Preparation of collagen-chondroitin sulfate-hyaluronic acid hybrid hydrogel scaffolds and cell compatibility in vitro", Carbohydrate Polymers, 84(1), 118-125, https://doi.org/10.1016/j.carbpol.2010.11.009. 
OBTAINING OF HIGH DENSITY CARBON MATERIALS BY COKE SINTERING RESULTING FROM HEAT TREATMENT OF TAR FOR APPLICATIONS IN SENSORS MANUFACTURE

\author{
MARIA-ROXANA MARINESCU ${ }^{1,2}$, ELENA DAVID $^{3}$, LIVIU-DANIEL GHICULESCU $^{4}$ \\ ${ }^{1}$ University Politehnica of Bucharest, Romania \\ ${ }^{2}$ National Institute for Research and Development in Microtechnologies, IMT-Bucharest, \\ Voluntari, Romania, roxana.marinescu@imt.ro \\ ${ }^{3}$ National Institute for Cryogenic and Isotopic Technologies, 4 Uzinei st.; P.O. Raureni, P.O. Box \\ 7, elena.david@icsi.ro \\ 4 "Politehnica” University of Bucharest, Machine Building Technology, 313 Splaiul \\ Independentei, sector6, Bucharest, Romania,daniel.ghiculescu@upb.ro
}

\begin{abstract}
In this research, high-density and high-strength carbonized carbon blocks were manufactured by coke sintering resulted from heat treatments of biomass pyrolysis tar. First, the molecular weight distribution of the tar was controlled through a pressurized heat treatment at $365^{\circ} \mathrm{C}$ and then this heattreated tar was treated using a delayed coking system to obtain the self-sintering coke. Finally, carbon blocks were molded from the self-sintering coke and carbonized at $1100^{\circ} \mathrm{C}$ for $2 \mathrm{~h}$. Through rapid decomposition of the high molecular weight compounds in the tar at $360^{\circ} \mathrm{C}$, the molecular weight distribution of tar was confirmed to be controllable by this treatment stage. During carbonization was observed a swelling in carbon blocks manufactured that contain more than $15 \mathrm{wt} \%$ of volatile matter from $150-500^{\circ} \mathrm{C}$. The optimum conditions of the two heat treatments stage were established to be $310^{\circ} \mathrm{C}$ for $3 \mathrm{~h}$ and $500^{\circ} \mathrm{C}$ for $1.5 \mathrm{~h}$. The highest density and highest strength of the carbonized carbon blocks manufactured were $1.44 \mathrm{~g} / \mathrm{cm}^{3}$ and $68.7 \mathrm{MPa}$, respectively.
\end{abstract}

Keywords: high density carbon; biomass tar; heat treatment; coke sintering.

\title{
INTRODUCTION
}

Various kinds of carbon materials such as blocks, powders, emulsions are widely used as important components in the modern industry. For example, carbon materials are employed as biomedical substances, materials used in chemical sensors manufacture for applications in optoelectronics or in medical devices, electronic stuff, aerospace items, and so one (Delport and Badenhorst, 2016; Xiao et al., 2016). In the early stage of the carbon industry, tar, pitch, naphtha and coke were developed as raw materials for the manufacture of graphite powder (Choi et al., 2017; Mearz et al., 2018). With the accelerated development of carbon industries, the manufacturing and processing techniques for artificial graphite blocks have been developed precisely and efficiently (Xiao et al., 2016; Lee, Kang and Roh, 2015).

The processes for the graphite manufacturing include the following steps: (i) The process of coking to eliminate the volatile matter, (ii) The grinding and sieving steps for particle sizing, (iii) The mixing of coke with the binder to prevent the phenomenon of swelling, (iv) Block formation of different forms and dimensions, following of calcination, (v) Repeating the steps of impregnation, drying and calcination to obtain a high density material with high strength, and (vi) Graphitization of the resulted material from before step (Du et al., 2010; Chen et al., 2012; Shen et al., 2015). In this processes the important factors are the coke types and the mixing ratio of coke and binder, but at the same time, to obtain high density blocks of graphite, the steps of impregnation, drying and calcination need to be repeated, and this represents a critical problem because to repeat the impregnation and calcination processes can increase the manufacturing costs and time. In order to eliminate these deficiencies, many studies were achieved to investigate other advanced methods, such as using of mesophase 
powder (Li et al., 2011; Zhao et al., 2013; Cheng et al., 2015), mesoporous carbon microbeads (MCMB) (Fang et al., 2015; Shen et al., 2011), self-sintering coke (Kocaefe et al., 2016), or adding a sintering agent (Shen et al., 2013).

In this research, high density and high strength carbonized carbon blocks were obtained by coke sintering resulted from heat treatments of biomass pyrolysis tar. First, the molecular weight distribution of the tar was controlled through a pressurized heat treatment at $365^{\circ} \mathrm{C}$ and then this heat-treated tar was treated using a delayed coking system to obtain the self-sintering coke. Finally, carbon blocks were molded from the self-sintering coke and carbonized at $1100^{\circ} \mathrm{C}$ for $2 \mathrm{~h}$.

\section{EXPERIMENTAL PART}

\section{Material and Methods}

For obtaining of high density carbon block, it was used as raw material the biomass tar, resulted from residual biomass pyrolysis, so it is described in our previous research (David, 2013). The biomass tar was mixed with tetrahydrofuran $(99.9 \%$ from SigmaAldrich) in a 1:1 ratio, and then the mixture was refined by a pressure filtering process. Then, the biomass tar was separated of tetrahydrofuran in a rotary evaporator, by increasing the temperature from room temperature to $75^{\circ} \mathrm{C}$. Table 1 contains the elemental analysis, insolubility and ash content of the refined biomass tar.

Table 1. The elemental analysis, insolubility and ash content of the refined biomass tar

\begin{tabular}{|c|c|c|c|c|c|c|c|}
\hline \multicolumn{5}{|c|}{ Ultimate analysis (wt.\%) } & \multicolumn{3}{|c|}{ Proximate analysis $(\%)$} \\
\hline $\mathrm{C}$ & $\mathrm{H}$ & $\mathrm{N}$ & $\mathrm{S}$ & $\mathrm{O}$ & TI & QI & Ash \\
\hline 91,75 & 5,18 & 1,71 & 0,67 & 0,09 & 5,78 & - & 0,10 \\
\hline
\end{tabular}

The scheme of manufacture method to obtain the carbonized carbon block by heat treatments is presented in Figure 1.

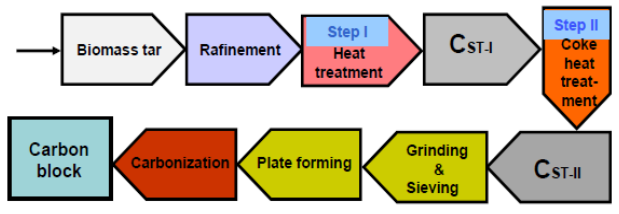

Figure 1. The scheme of heat treatments in two steps for manufacture the carbon block from biomass tar

The refined biomass tar was first heated at temperatures of 310,330 and $365^{\circ} \mathrm{C}$ for 1 and $3 \mathrm{~h}$ in the pressurized system (the pressure was increased to about $6,9,12$, and 15 bar, depending on the temperature, respectively). Second, the resulted heat treated tar from stage I ( $\left.\mathrm{C}_{\text {ST-I }}\right)$ was once again heated to $500^{\circ} \mathrm{C}$ in the coking system (2nd stage treatment) to produce coke ( $\left.\mathrm{C}_{\mathrm{ST}-\mathrm{II}}\right)$. The resulted coke from stage II was grinded, sieved to under $75 \mathrm{~mm}$ in size, and then extruded into small plates with the dimensions of $12 \times 12 \times 3 \mathrm{~mm}$ and $30 \times 15 \times 3 \mathrm{~mm}$ using a cold high pressure press $(280 \mathrm{bar})$. These rectangular plates were each prepared three times according to the manufacture conditions and finally, the raw carbon blocks were carbonized at $1100^{\circ} \mathrm{C}$ for $1 \mathrm{~h}$ to $3 \mathrm{~h}$ at the heating rate of $2^{\circ} \mathrm{C} / \mathrm{min}$. As obtained carbonized carbon blocks were analyzed to establish hardness, bending strength and surface texture. 


\section{Analytical Tools Used}

The proximate and ultimate analysis of the refined biomass tar, $\mathrm{C}_{\text {ST-I }}$ and $\mathrm{C}_{\text {ST-II }}$ samples was made using a FLASH-2000 Elemental Analyzer. The insolubility was determined based on ASTM D-2318 and ASTM D-4072, by quinolone insolubility (QI) and toluene insolubility, respectively. Thermogravimetric analysis was performed using TGA, STA 409 PC, Netzsch, Germany, analyzer and the weight loss was examined at a rate of $10^{\circ} \mathrm{C} / \mathrm{min}$ at $900^{\circ} \mathrm{C}$ under a $\mathrm{N}_{2}$ flow of $15 \mathrm{ml} / \mathrm{min}$. The image of the carbon block surface was obtained by scanning electron microscopy (SEM, using Jeol JSM-7000, JEOL Ltd., Japan). The flexural strengths of carbon blocks were determined using a universal testing machine (WL2100, WITHLAB, Korea) based on the ASTM C-1161.

\section{RESULTS AND DISCUSSION}

The content of carbon, hydrogen, nitrogen and sulfur into $\mathrm{C}_{\mathrm{ST} \text {-I }}$ samples prepared under various temperature conditions and times are presented in Table 2.

Table 2. Elemental analysis of $\mathrm{C}_{\text {ST-I }}$ samples prepared under various heating temperatures and different times

\begin{tabular}{llllll}
\hline Conditions for Stage I & C(wt.\%) & $\mathrm{H}($ wt.\%) & $\mathrm{N}($ wt.\%) & $\mathrm{S}($ wt.\%) & C/H ratio \\
\hline $310^{\circ} \mathrm{C} ; 1 \mathrm{~h}$ & 90.83 & 5.64 & 0.62 & 0.31 & 1.34 \\
$330^{\circ} \mathrm{C} ; 1 \mathrm{~h}$ & 91.78 & 5.68 & 0.75 & 0.35 & 1.35 \\
$365^{\circ} \mathrm{C} ; 1 \mathrm{~h}$ & 91.96 & 5.58 & 0.87 & 0.41 & 1.37 \\
$310^{\circ} \mathrm{C} ; 2 \mathrm{~h}$ & 91.53 & 5.54 & 0.71 & 0.38 & 1.38 \\
$330^{\circ} \mathrm{C} ; 2 \mathrm{~h}$ & 91.65 & 5.49 & 0.79 & 0.46 & 1.39 \\
$365^{\circ} \mathrm{C} ; 2 \mathrm{~h}$ & 91.79 & 5.42 & 0.83 & 0.47 & 1.41 \\
$310^{\circ} \mathrm{C} ; 3 \mathrm{~h}$ & 92.73 & 5.43 & 0.74 & 0.49 & 1.42 \\
$330^{\circ} \mathrm{C} ; 3 \mathrm{~h}$ & 92.89 & 5.33 & 0.81 & 0.53 & 1.45 \\
$365^{\circ} \mathrm{C} ; 3 \mathrm{~h}$ & 93.04 & 5.61 & 0.93 & 0.54 & 1.38 \\
\hline
\end{tabular}

With the increasing of reaction temperature and reaction time, the $\mathrm{C} / \mathrm{H}$ ratio increased. The $\mathrm{C} / \mathrm{H}$ ration for sample treated at $310^{\circ} \mathrm{C}$ for $3 \mathrm{~h}$ was higher than that samples treated at $310^{\circ} \mathrm{C}$ for $2 \mathrm{~h}$ and $1 \mathrm{~h}$, respectively. On the other hand, the $\mathrm{C} / \mathrm{H}$ ration of sample treated at $330^{\circ} \mathrm{C}$ for $3 \mathrm{~h}$ was higher than that of samples treated at $310^{\circ} \mathrm{C}$ for $2 \mathrm{~h}$ and $1 \mathrm{~h}$ respectively. These results suggest that the compounds with low molecular weights were decomposed or polymerized into polycyclic aromatic hydrocarbons (PAHs), having higher molecular weight. However, so can be seen in table 2 , the $\mathrm{C} / \mathrm{H}$ ratio of the sample treated at $330^{\circ} \mathrm{C}$ for $3 \mathrm{~h}$ was higher than that of the sample treated at $365^{\circ} \mathrm{C}$ for the same time $(3 \mathrm{~h})$ and this results indicate that the aromatic bond was fast broken at the temperature of $365^{\circ} \mathrm{C}$ for $3 \mathrm{~h}$ under a pressure around 15 bar (Im et al., 2017). Additionally, it can be demonstrated that the distribution of the molecular weights of the compounds in the refined biomass tar can be controlled by the 1 st step of treatment. The stage II of treatment was performed at temperature of $500^{\circ} \mathrm{C}$ for 1 to $3 \mathrm{~h}$.

\section{Analytical Tools Used Morphological and Mechanical Characteristics of Sintered Carbon Blocks}

The weight loss was measured by the thermogravimetric analyses of the $\mathrm{C}_{\mathrm{ST}-\mathrm{II}}$ samples. A phenomenon of swelling was observed for the carbon blocks manufactured at $330^{\circ} \mathrm{C}$ and $365^{\circ} \mathrm{C}$ for $1 \mathrm{~h}$, they contained over $15 \mathrm{wt} \%$ volatile matter. It was observed that the carbon blocks with less than $15 \mathrm{wt} \%$ volatile matter kept their rectangular form after carbonization. The samples treated in first stage at $310^{\circ} \mathrm{C} ; 330^{\circ} \mathrm{C} ; 365^{\circ} \mathrm{C}$ for $3 \mathrm{~h}$ 
and which were treated for $1.5 \mathrm{~h}$ in the stage II, exhibited a weight loss of less than $15 \mathrm{wt} . \%$ and shrinkage of volume less than $20 \mathrm{wt} \%$.

Table 3 present that optimal conditions for the 2 nd step of treatment to obtain the high density carbon block and this are $500^{\circ} \mathrm{C}$ for $1.5 \mathrm{~h}$. Thus, $\mathrm{C}_{310-3-1.5}$ has exhibited an apparent density of $1.44 \mathrm{~g} / \mathrm{cm}^{3}$, which was higher than the reported density of approximately $1.40 \mathrm{~g} / \mathrm{cm}^{3}$ (Zhou and McGinn, 2006). Also, $C_{310-3-1.5}$ showed the best mechanical properties having a flexural strength of 68.7 $\mathrm{MPa}$.

Table 3. Characteristics of the carbon blocks resulted after the second stage of treatment (temperature treatment for stage II of treatment $500^{\circ} \mathrm{C}$ )

\begin{tabular}{lcrrrrrr}
\hline $\begin{array}{l}\text { Sample } \\
* \mathrm{C}_{\mathrm{x}-\mathrm{y}-\mathrm{z}}\end{array}$ & \multicolumn{9}{c}{ Teight loss ratio (wt.\%) } & \multicolumn{2}{c}{$\begin{array}{l}\text { Vol.shrinkage } \\
\text { Tlex.stength } \\
(\%)\end{array}$} & $\begin{array}{l}\text { Density } \\
\left(\mathrm{g} / \mathrm{cm}^{3}\right)\end{array}$ & \begin{tabular}{c}
$(\mathrm{MPa})$ \\
\hline $\mathrm{C}_{310-1-1.5}$
\end{tabular} & 23.54 & 11.52 & 2.26 & 9.87 & 35.68 & 1.35 & 28.3 \\
$\mathrm{C}_{330-1-1.5} \mathrm{C}$ & 18.21 & 7.18 & 2.05 & 8.72 & 25.27 & 1.22 & 4.7 \\
$\mathrm{C}_{365-1-1.5}$ & 20.68 & 15.25 & 2.26 & 2.85 & 21.28 & 1.14 & 2.8 \\
$\mathrm{C}_{310-3-1.5}$ & 24.36 & 11.56 & 2.83 & 9.74 & 40.32 & 1.44 & 68.7 \\
$\mathrm{C}_{330-1-1.5}$ & 23.52 & 10.68 & 3.01 & 9.61 & 33.58 & 1.33 & 28.3 \\
$\mathrm{C}_{365-1-1.5}$ & - & 18.22 & 2.46 & - & - & - & - \\
$\mathrm{C}_{330-3-3}$ & 14.24 & 6.14 & 1.77 & 6.44 & 29.75 & 1.32 & 29.2 \\
\hline
\end{tabular}

${ }^{*} \mathrm{C}_{\mathrm{x}-\mathrm{y}-\mathrm{z}}$ denotes: $\mathrm{x}$-temperature value in the first stage of treatment; $\mathrm{y}$-time of treatment in the first stage; $\mathrm{z}$ - time of treatment in second stage.

The cracks containing the size around 100-500 $\mu \mathrm{m}$ were found in $\mathrm{C}_{365-3-1}$, as is shown in Figure 2a. $\mathrm{C}_{310-1-1.5}$ presented swelling phenomena, as showed in Figure 2b. For $\mathrm{C}_{365-3-1.5}$ carbonized sample, the rectangular plate of the original shape disappeared completely.
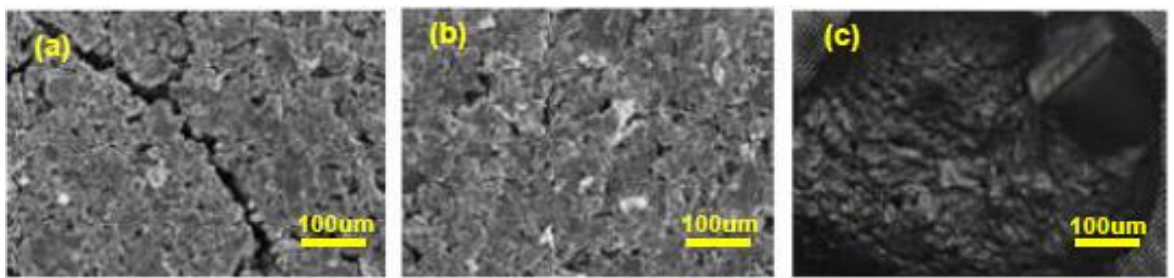

Figure 2. The change appeared in carbon block after carbonization:(a)-SEM image for $\mathrm{C}_{365-1-1.5}$; (b)-SEM image for $\mathrm{C}_{310-1-1.5}$; (c) - SEM image for $\mathrm{C}_{365-3-1.5}$

The SEM images of surfaces for carbon blocks carbonized at $1100^{\circ} \mathrm{C}$ are presented in Figure 3. In Figure 3(a, c and d), the particle forms are quite difficult to notice. In Figure 3(b, e and f), can be observed small cracks between different particles. Also, an important quantity of particle cracks between 100 and $500 \mathrm{~mm}$ can be observed in Figures 2(a) and 3(c).

The results for the weight loss in the carbonization process showed that the samples range of treatment temperatures can be divided into three ranges, by dividing the treatment temperatures. $\mathrm{C}_{310-1-1.5}$ and $\mathrm{C}_{310-3-1.5}$ had a significant difference in the amount of weight loss in the range temperature of $500^{\circ} \mathrm{C}-650^{\circ} \mathrm{C}$, and $\mathrm{C}_{310-3-1.5}$ and $\mathrm{C}_{330-3-1.5}$ differ in their weight losses in the temperature range of $150^{\circ} \mathrm{C}-500^{\circ} \mathrm{C}$. The amount of weight loss from $150^{\circ} \mathrm{C}$ to $650^{\circ} \mathrm{C}$ showed to be a main factor that affects the mechanical bending strength. The small cracks between coke particles, reduce mechanical properties, and were more pronounced for the samples when the volatile matter at 500$650^{\circ} \mathrm{C}$ were smaller, as it can be seen in Figure 3. 

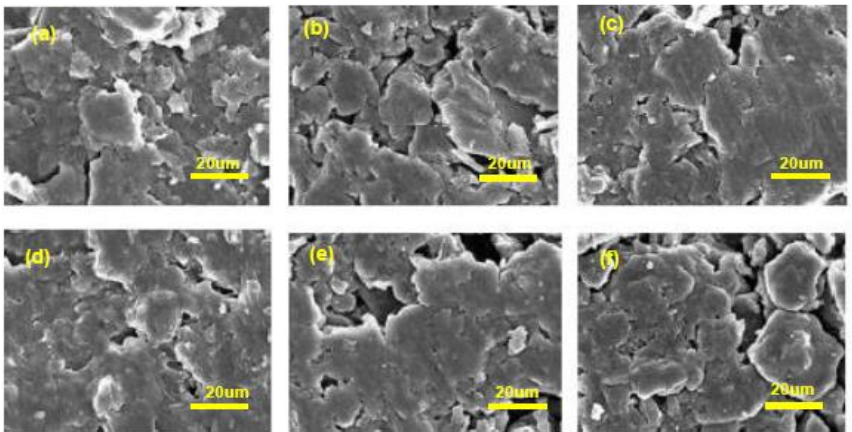

Figure 3. SEM images of carbon blocks carbonized at $1100^{\circ} \mathrm{C}$ : (a)- $\mathrm{C}_{310-1-1.5}$; (b)- $\mathrm{C}_{330}$ ${ }_{1-1.5}$; (c)- $\mathrm{C}_{365-1-1.5}$; (d)- $\mathrm{C}_{310-1-1.5}$; (e)- $\mathrm{C}_{330-3-1.5}$; and (f)- $\mathrm{C}_{330-3-3}$;

However, comparing the results for $\mathrm{C}_{330-1-1.5}$ and $\mathrm{C}_{330-3-3}$ in Table 2, the flexural strength of $\mathrm{C}_{330-3-3}$ is better. This result maybe is due to the occurrence of small cracks that could reduce the flexural strength for carbon blocks when the volatile matter is evaporated in the temperature range of $650-1100^{\circ} \mathrm{C}$ and the degree of volume shrinkage decreased, these results being in agreement with other studies (Cheng et al., 2015; Cheng et al., 2013).

Figure 4 shows the optimum conditions for the first stage of treatment to manufacture high density carbon blocks based on the weight losses and volume shrinkages of $\mathrm{C}_{310-1-1.5}, \mathrm{C}_{330-1-1.5}$ and $\mathrm{C}_{365-1-1.5}$ samples.

Also, it can be observed that a decrease in the the volume shrinkage took place when the reaction temperature for the first stage treatment has increased. In addition, the weight loss ratio of the $\mathrm{C}_{365-1-1.5}$ sample was greater than that of $\mathrm{C}_{330-1-1.5}$, and this is due to the decomposition of high molecular weight compounds at a reaction temperature above $330{ }^{\circ} \mathrm{C}$. According to this analysis, it can be concluded that the amount of high molecular weight produced in the stage I of treatment lead to decreasing of the volume shrinkage, the density and the mechanical properties.

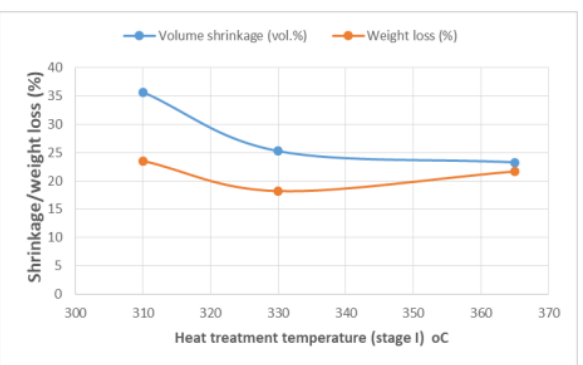

Figure 4. Effect of the heat treatment temperature on volume shrinkage and weight loss during first stage of treatment $\left(\mathrm{C}_{310-1-1.5}, \mathrm{C}_{330-1-1.5}\right.$ and $\mathrm{C}_{365-1-1.5}$ samples $)$

\section{CONCLUSIONS}

The high density carbon blocks were developed by coke sintering of the biomass tar. As the reaction temperature increased during the first stage of treatment, the $\mathrm{C} / \mathrm{H}$ ratio increased. The low molecular weight compounds of the $\mathrm{C}_{\mathrm{ST} \text {-Is }}$ increased if the reaction temperature was above $330^{\circ} \mathrm{C}$. All of the sintering coke obtained by the two stage of heat treatment starting from biomass tar present a uniform mesophase structure. The carbon blocks containing over $15 \mathrm{wt} \%$ volatile matter at $150-500^{\circ} \mathrm{C}$, were swollen. In addition, the small cracks in the carbonized carbon blocks containing less than $2 \mathrm{wt} \%$ 
volatile matter at $500-650^{\circ} \mathrm{C}$ were observed. The weight loss, from 150 to $650^{\circ} \mathrm{C}$ during carbonization process, was due to active volume shrinkage, resulting sample with a high density. The amount of weight loss from $650-1100^{\circ} \mathrm{C}$ during the carbonization process was found to be a key factor that reduced the mechanical properties. Thus, the optimum conditions of the first stage and the second stage of heat treatment were confirmed to be $310^{\circ} \mathrm{C}$ for $3 \mathrm{~h}$ and $500^{\circ} \mathrm{C}$ for $1.5 \mathrm{~h}$, respectively.

\section{Acknowledgements}

The financial support for this research was provided by the Ministry of Education and Research from Romania, Project no. PN 191103 01/2019-2022 and by the European Social Fund from the Sectoral Operational Programme Human Capital 2014 2020, through the Financial Agreement with the title "Scholarships for entrepreneurial education among doctoral students and postdoctoral researchers (Be Antreprenor!)", Contract no. 51680/09.07.2019 - SMIS code: 124539.

\section{REFERENCES}

Chen, X. et al. (2012), "The thermal and mechanical properties of graphite foam/epoxy resin composites", Mater. Des., 40, 497-501, https://doi.org/10.1016/j.matdes.2012.04.026.

Cheng, Y. et al. (2013), "In situ preparation and mechanical properties of CNTs/MCMBs composites", Comp. Part B, (47), 290-297, https://doi.org/10.1016/j.compositesb.2012.11.009.

Cheng, Y. et al. (2015), "Preparation and characterization of mesophase pitch via Co-carbonization of waste polyethylene/petroleum pitch", J. Mater. Sci. Technol., 31, 857-863, https://doi.org/10.1016/j.jmst.2015.07.010.

Choi, P.R. et al. (2017), "High-energy-density activated carbon electrode for organic electric-double-layercapacitor using carbonized petroleum pitch", Carbon Lett, (22), 70-80.

Delport, M.R. and Badenhorst H. (2016), "Production of a self-adhering mesophase powder from anthracene oil for low pressure forming of graphite artefacts", J. Mater. Sci., 51, 6309-6318, https://doi.org/10.1007/s10853-016-9927-2.

David, E. (2013), "Valorization of residual biomass by thermochemical processing", Journal of Analytical and Applied Pyrolysis, 104, 260-268, https://doi.org/10.1016/j.jaap.2013.07.007.

Du, C. et al. (2010), "The preparation technique optimization of epoxy/compressed expanded graphite composite bipolar plates for proton exchange membrane fuel cells", J. Power Sources, (195), 5312-531, https://doi.org/10.1016/j.jpowsour.2010.03.005.

Fang, M.-D. et al. (2015), "Improving the sintering behavior of mesocarbon-microbeads for the manufacture of high quality carbon products using a joint promoter comprising carbon black and glycidyl methacrylate", Mater. Chem. Phys., 149, 400-404, https://doi.org/10.1016/j.matchemphys.2014.10.035.

Im, U.-S. et al. (2017), "Effects of two-stage heat treatment on delayed coke and study of their surface texture characteristics", JOM, (69), 2460-2466, https://doi.org/10.1007/s11837-016-2104-x.

Kocaefe, D. et al. (2016), "Review of different techniques to study the interactions between coke and pitch in anode manufacturing”, in: B.A. Sadler (Ed.), Light Metals 2013, Springer International Publishing, Cham, 1045-1050, https://doi.org/10.1007/978-3-319-65136-1_176.

Lee, S.-M., Kang, D.-S. and Roh, J.-S. (2015), "Bulk graphite: materials and manufacturing process", Carbon Lett, (16), 135-146, https://doi.org/10.5714/CL.2015.16.3.135.

Li, H. et al. (2011), "Effects of air oxidation on mesophase pitch-based carbon/carbon composites", Carbon, (49), 1416-1422, https://doi.org/10.1016/j.carbon.2010.12.009.

Mearz, B. et al. (2018), "Mesoscopic structure features in synthetic graphite", Mater. Des., 142, 268-278, https://doi.org/10.1016/j.matdes.2018.01.038.

Shen, K. et al. (2011), "Effect of oxidative stabilization on the sintering of mesocarbon microbeads and a study of their carbonization", Carbon, (49), 3200-3211, https://doi.org/10.1016/j.carbon.2011.03.044.

Shen, K. et al. (2013), "The use of asphalt emulsions as a binder for the preparation of polycrystalline graphite", Carbon, (58), 238-241, https://doi.org/10.1016/j.carbon.2013.02.025.

Shen, K., et al. (2015), "Advantages of natural microcrystalline graphite filler over petroleum coke in isotropic graphite preparation”, Carbon, (90), 197-206, https://doi.org/10.1016/j.carbon.2015.03.068.

Xiao, J., et al. (2016), "Effect of high temperature pyrolysis on the structure and properties of coal and petroleum coke", J. Anal. Appl. Pyrolysis, 117, 64-71, https://doi.org/10.1016/j.jaap.2015.12.015.

Zhao, Y. et al. (2013), "Microstructure and thermal/mechanical properties of short carbon fiber-reinforced natural graphite flake composites with mesophase pitch as the binder", Carbon, (53), 313-320, https://doi.org/10.1016/j.carbon.2012.11.013.

Zhou, C. and McGinn, P.J. (2006), "The effect of oxygen on the processing of mesocarbon microbeads to high-density carbon”, Carbon, (44), 1673-1681, https://doi.org/10.1016/j.carbon.2006.01.004. 


\title{
THE VARIATION OF ESSENTIAL OIL AND CARVACROL CONTENTS OF
} NATIVE GROWN Thymbra spicata var. spicata $L$.

\author{
KAZIM MAVI'1 ${ }^{1}$ DURMUŞ ALPASLAN KAYA2*, MUSA TÜRKMEN ${ }^{2}$, FILIZ AYANOĞLU ${ }^{2}$ \\ ${ }^{1}$ Hatay Mustafa Kemal University, Faculty of Agriculture, Department of Horticulture, Hatay \\ Turkey \\ ${ }^{2}$ Hatay Mustafa Kemal University, Faculty of Agriculture, Department of Field Crops, Hatay \\ Turkey,*dak1976@msn.com
}

\begin{abstract}
In the study, it is aimed to create breeding lines of thyme (Thymbra spicata L.), which is important both culturally and economically, by selecting a single plant among the plants grown in different regions in Hatay. For this purpose, a genetic pool was created for Thymbra spicata L. plant in the plant samples taken from the locations where the plants are densely grown, and these plants were examined in terms of leaf characteristics, number of oil glands per unit area, oil gud size and essential oil components. Plants were propagated and preserved with cuttings taken from these single plants. In this study, which includes the pre-selection stage, 213 plants from 68 different locations were determined in the province of Hatay. The essential oil ratios of the plants varied between $0.70 \%$ and $3.90 \%$ and showed a wide variation. The rate of carvacrol, which is the main component of the essential oil of the thyme plant, was between $28.12 \%$ and $78.48 \%$. Plants with code number Z14, Z3, Z25, Z38, Z77, Z104, Z35 and Z43 with an essential oil ratio of 3.5\% and above and plants with code number Z167, Z165 and Z64 with a high carvacrol ratio were selected to be used in future breeding studies.
\end{abstract}

Keywords: Thymbra spicata L, essential oil, carvacrol content

\section{INTRODUCTION}

Turkey is a rich country in terms of plant genetic resources, and it is stated that there are near 12000 plant taxa (species, subspecies, variety) with the studies done in recent years (Avc1, 2005; Erik and Tarıkahya, 2004). However, more studies are needed to make a complete inventory of this richness of plant genetic resources and to bring these plants to the economy (Ekim et al., 2000).

Thyme is the most exported plant among medicinal and aromatic plants in Turkey (Ozguven et al., 2005). Thymbra spicata var. spicata L, (zahter) a species of thymbra, it grows naturally and intensively in Mediterranean countries and Turkey. However, the agricultural cultivation of the Zahter is not much done in Turkey and the plants collected mostly from nature and used.

Although Zahter collected from nature are mostly consumed in the region and in the domestic market, but their essential oil and spice are also exported. In addition to its traditional use as fresh in the region, the plants are dried and used as spice and tea, the essential oil of the plants is also used for different purposes. In particular, the use of fresh tip shoots is increasing day by day. The dried leaves of the plant are used as a spice in almost all dishes (soups, meat dishes, fish, herbed cottage cheese, pastries, etc.) for various purposes.

Like many other medicinal and aromatic plants, Zahter is used for various stomach aches and ailments diabetes and colds due to its antimicrobial and antiseptic properties (Hanc1 et al., 2003). In a study by Baydar et al. (2004) investigating the composition and antibacterial effects of essential oils of Origanum, Thymbra and Satureja species, it was determined that the most active antibacterial effect was the essential oil of the Thymbra spicata species. 
Saraç and Tunç (1995) determined that some essential oils have toxic and repellent effects, and that the essential oil of Thymbra spicata, has a repellent effect against the adults of the rice lice pest (Sitophilus oryzae). The importance and use of the Zahter is increasing day by day due to the increasing as use of the traditional food and the characteristics of its essential oil. As a matter of fact, many researchers have made researches on the cultivation of plants (Tonçer and Kız1l, 2005; İnan et al, 2011). The morphology, anatomy of zahter and the amount of essential oil content and components and antimicrobial properties of these compounds have also been studied by many researchers in Turkey (Doğan et al., 1987; Hanc1 et al., 2003; Özel et al., 2003; Baydar et al., 2004; Erken, 2005; Özcan et al., 2008).

There is not much research on the breeding of Zahter plant. In this study, it was aimed to create breeding lines by selecting single plant selection among plants grown naturally in different regions of Hatay province and to select high quality chemotypes and agrotypes with essential oil yield.

\section{MATERIAL AND METHODS}

Survey and collection studies of the Zahter (Thymbra spicata L.) plant were carried out. As a result of these studies, 213 single plants were selected and reproduced from 68 different locations and examined in terms of some characteristics. Healthy, differentlooking and highly qualified individuals were selected in the pre-selection study. Since it is widely used for fresh consumption in the region, especially dense and large-leaved, highly branched types were also selected for this purpose during selection.

Clonal reproduction was achieved by taking cuttings from the selected plants. 1 part peat, 1 part soil and 1 part perlite were used as rooting medium in rooting tables. 1000 ppm IBA (Indole-3-butyric acid) was applied to the cuttings for 5 seconds. All of the rooted cuttings (213 plants) were planted at a distance of $50 \times 70 \mathrm{~cm}$ in the collection garden and kept under protection.

The essential oil content of each plant was determined and essential oil components were also analyzed in GCMS and those with high carvacrol content were determined.

Leaf density: Each selected plant was evaluated in three groups as observation, sparse, middle and frequent.

Essential oil ratio (\%): It was determined according to European pharmacopoeia with Neo-clavenger in $20 \mathrm{~g}$ sample taken from each plant. In the study, plant samples were distilled for three hours.

Essential oil components (\%): It was determined by GC-MS in the essential oil samples obtained from each plant. Essential oil composition analysis was determined by using "Thermo Scientific ISQ Single Quadrupole" gas chromatography and mass spectrum in the Medical and Aromatic Plants Laboratory of Mustafa Kemal University, Faculty of Agriculture, Department of Field Crops. A TR-5MS (5\% Phenyl Polysilphenylene-siloxane, $0.25 \mathrm{~mm}$ x $30 \mathrm{~m}$ i.d, film thickness 0.25 ) column was used. Helium at $99.9 \%$ purity $1 \mathrm{~mL} / \mathrm{min}$ was used as carrier gas. The ionization energy is 70 $\mathrm{eV}$, the mass range is $1.2-1100 \mathrm{amu}$, the MS transfer line temperature is $250^{\circ} \mathrm{C}$, the ion source temperature is $220^{\circ} \mathrm{C}$, and the injection block temperature is $220^{\circ} \mathrm{C}$. The samples were injected at a split ratio of 250 . The injection amount was set as $1 \mu \mathrm{l}$ and the furnace temperature was adjusted to increase from $50^{\circ} \mathrm{C}$ to $220^{\circ} \mathrm{C}$ by $3^{\circ} \mathrm{C} / \mathrm{min}$. As a result of the analysis, each component was automatically identified by comparing the library mass spectra (Wiley and NIST) using Xcalibur software. Retention indices were 
calculated using standard n-alkane homologous solutions C8-C20 (Fluka, product code: 04070) and C21-C40 (Fluka, product code: 04071). Analysis of each sample on GC-MS takes 56 minutes.

\section{RESEARCH AND DISCUSSION}

\section{Leaf Density}

Each selected plant was evaluated in three groups as observation, sparse, middle and frequent and shown at Figure 1. It has been determined that frequent leaves common in general. Of the 213 plants, 138 of them have frequent leaves, 72 of them have medium density and 3 of them have sparse leaves. Leaf density is especially important in fresh consumption. In the locally consumed thyme salad, the ones with dense leaves are more preferred. For this reason, the density of leaves is important.

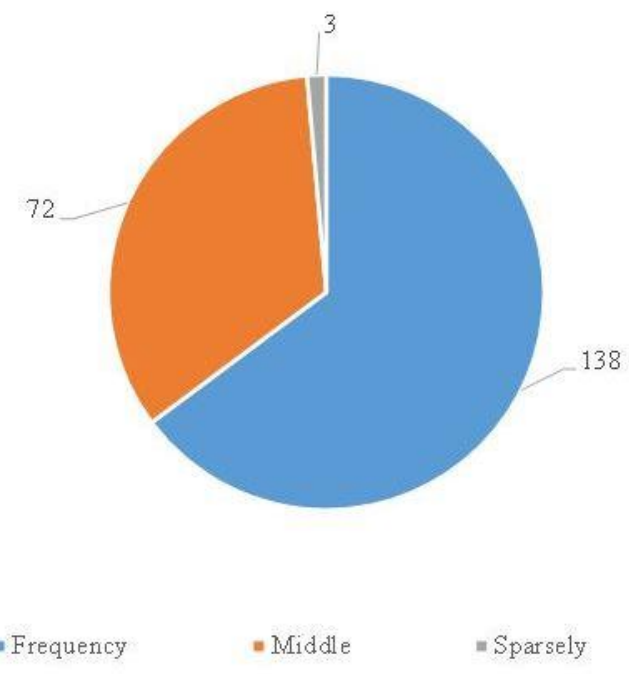

Figure 1. The distribution of leaf density of thyme genotypes

\section{Essential Oil Ratio (\%)}

The essential oil ratios obtained as a result of the distillation of the leaves of 213 Thymbra spicata plants collected from the flora of Hatay were determined and given in Table 1. According to the results, the essential oil ratios of selected thyme plants varied between $0.70 \%$ and $3.90 \%$. The values obtained have shown that the variation is greater by expanding the minimum and maximum values $1-3.4 \%$ given in the literature (Başer, 2002) in both directions. This situation reveals that the thymbra plants in the flora of Hatay show great differences in terms of essential oil ratios. The large variation requires the selection of types with a high rate of essential oils to be used in variety development. Among the collected thymbra plants, the varieties with code numbers Z14, Z3, Z25, Z38, Z77, Z104, Z35 and Z43, which are high in essential oil (over $3.50 \%$ ), were selected to be used in future development studies. 
The Variation of Essential Oil and Carvacrol Contents of Native Grown Thymbra spicata var. spicata L.

Table 1. The essential oil contents of leaves of collected $T$. spicata $\mathrm{L}$. ecotypes

\begin{tabular}{|c|c|c|c|c|c|c|c|c|c|}
\hline Code & $\begin{array}{c}\text { Essential } \\
\text { oil } \\
\%\end{array}$ & Code & $\begin{array}{c}\text { Essential } \\
\text { oil } \\
\%\end{array}$ & Code & $\begin{array}{c}\text { Essential } \\
\text { oil } \\
\%\end{array}$ & Code & $\begin{array}{c}\text { Essential } \\
\text { oil } \\
\%\end{array}$ & Code & $\begin{array}{c}\text { Essential } \\
\text { oil } \\
\%\end{array}$ \\
\hline $\mathrm{Z1}$ & 2,43 & Z44 & 3,66 & $\mathrm{Z} 87$ & 2,50 & $\mathrm{Z} 130$ & 2,33 & $\mathrm{Z173}$ & 2,50 \\
\hline $\mathrm{Z} 2$ & 2,75 & $\mathrm{Z} 45$ & 2,97 & Z88 & 2,50 & Z131 & 3,26 & $\mathrm{Z} 174$ & 2,03 \\
\hline $\mathrm{Z3}$ & 3,83 & Z46 & 2,66 & Z89 & 2,50 & Z132 & 1,78 & Z175 & 2,33 \\
\hline $\mathrm{Z4}$ & 2,66 & $\mathrm{Z} 47$ & 2,03 & $\mathrm{Z} 90$ & 1,59 & Z133 & 1,50 & Z176 & 1,85 \\
\hline $\mathrm{Z} 5$ & 3,00 & Z48 & 2,50 & Z91 & 2,50 & Z134 & 1,74 & Z177 & 1,78 \\
\hline Z6 & 3,53 & Z49 & 3,00 & Z92 & 2,06 & Z135 & 2,86 & Z178 & 3,23 \\
\hline $\mathrm{Z7}$ & 2,95 & $\mathrm{Z} 50$ & 2,25 & Z93 & 1,00 & Z136 & 1,56 & Z179 & 2,71 \\
\hline Z8 & 2,27 & Z51 & 3,40 & Z94 & 2,66 & Z137 & 2,00 & $\mathrm{Z} 180$ & 1,80 \\
\hline $\mathrm{Z9}$ & 3,33 & $\mathrm{Z} 52$ & 2,89 & Z95 & 2,29 & Z138 & 1,66 & Z181 & 1,50 \\
\hline Z10 & 2,23 & Z53 & 3,00 & Z96 & 2,00 & Z139 & 3,26 & Z182 & 2,08 \\
\hline Z11 & 2,83 & $\mathrm{Z} 54$ & 3,66 & Z97 & 0,83 & $\mathrm{Z} 140$ & 1,80 & Z183 & 3,23 \\
\hline $\mathrm{Z} 12$ & 2,40 & $\mathrm{Z} 55$ & 2,50 & Z98 & 0,83 & Z141 & 1,87 & Z184 & 1,66 \\
\hline Z13 & 1,92 & Z56 & 2,86 & Z99 & 2,15 & Z142 & 3,86 & Z185 & 2,00 \\
\hline Z14 & 3,90 & Z57 & 2,60 & $\mathrm{Z} 100$ & 1,85 & Z143 & 1,87 & Z186 & 1,20 \\
\hline $\mathrm{Z} 15$ & 3,13 & Z58 & 2,26 & Z101 & 2,66 & Z144 & 0,70 & Z187 & 2,66 \\
\hline Z16 & 2,43 & Z59 & 3,33 & Z102 & 1,50 & Z145 & 1,30 & Z188 & 3,02 \\
\hline Z17 & 2,83 & Z60 & 2,66 & Z103 & 2,71 & Z146 & 0,83 & Z189 & 1,80 \\
\hline Z18 & 3,23 & Z61 & 2,60 & Z104 & 3,63 & Z147 & 2,03 & Z190 & 3,26 \\
\hline Z19 & 2,72 & Z62 & 2,16 & Z105 & 2,20 & Z148 & 2,25 & Z191 & 2,03 \\
\hline $\mathrm{Z} 20$ & 2,02 & Z63 & 3,86 & Z106 & 2,00 & Z149 & 2,38 & Z192 & 2,75 \\
\hline Z21 & 2,50 & Z64 & 3,00 & Z107 & 1,75 & $\mathrm{Z} 150$ & 2,00 & Z193 & 1,30 \\
\hline $\mathrm{Z} 22$ & 2,50 & Z65 & 2,66 & Z108 & 1,50 & Z151 & 1,33 & Z194 & 3,02 \\
\hline $\mathrm{Z} 23$ & 2,53 & Z66 & 2,14 & Z109 & 1,42 & Z152 & 2,00 & Z195 & 2,89 \\
\hline Z24 & 2,66 & Z67 & 2,00 & Z110 & 3,12 & Z153 & 1,52 & Z196 & 3,37 \\
\hline $\mathrm{Z} 25$ & 3,83 & Z68 & 2,66 & $\mathrm{Z} 111$ & 2,66 & Z154 & 2,50 & Z197 & 1,74 \\
\hline Z26 & 2,35 & Z69 & 3,26 & Z112 & 1,87 & Z155 & 2,15 & Z198 & 2,00 \\
\hline $\mathrm{Z} 27$ & 3,00 & $\mathrm{Z70}$ & 3,13 & Z113 & 2,00 & Z156 & 2,90 & Z199 & 1,80 \\
\hline Z28 & 3,27 & $\mathrm{Z71}$ & 3,65 & Z114 & 1,78 & Z157 & 2,75 & Z200 & 3,36 \\
\hline Z29 & 2,50 & $\mathrm{Z72}$ & 3,20 & Z115 & 1,00 & Z158 & 1,75 & Z201 & 2,03 \\
\hline $\mathrm{Z} 30$ & 3,23 & $\mathrm{Z73}$ & 2,70 & Z116 & 2,08 & Z159 & 1,75 & Z202 & 2,25 \\
\hline Z31 & 2,00 & Z74 & 2,96 & Z117 & 1,85 & Z160 & 2,25 & Z203 & 2,97 \\
\hline $\mathrm{Z} 32$ & 3,26 & $\mathrm{Z75}$ & 2,26 & Z118 & 1,50 & Z161 & 2,06 & Z204 & 1,80 \\
\hline Z33 & 3,40 & $\mathrm{Z76}$ & 1,90 & Z119 & 1,87 & $\mathrm{Z} 162$ & 2,00 & $\mathrm{Z} 205$ & 1,76 \\
\hline Z34 & 2,66 & Z77 & 3,73 & Z120 & 1,20 & Z163 & 1,75 & Z206 & 2,66 \\
\hline $\mathrm{Z} 35$ & 3,53 & $\mathrm{Z78}$ & 2,54 & Z121 & 3,00 & Z164 & 1,61 & Z207 & 1,87 \\
\hline Z36 & 3,46 & Z79 & 3,33 & $\mathrm{Z} 122$ & 2,50 & Z165 & 3,02 & Z208 & 3,70 \\
\hline Z37 & 2,71 & $\mathrm{Z} 80$ & 2,05 & Z123 & 0,92 & Z166 & 2,75 & Z209 & 3,26 \\
\hline Z38 & 3,76 & Z81 & 3,16 & Z124 & 1,30 & Z167 & 1,30 & $\mathrm{Z} 210$ & 2,16 \\
\hline Z39 & 3,33 & Z82 & 3,66 & $\mathrm{Z} 125$ & 2,75 & Z168 & 2,62 & $\mathrm{Z} 211$ & 2,50 \\
\hline $\mathrm{Z} 40$ & 2,75 & Z83 & 1,66 & Z126 & 1,00 & Z169 & 2,50 & $\mathrm{Z} 212$ & 1,85 \\
\hline Z41 & 3,33 & Z84 & 2,76 & Z127 & 1,75 & $\mathrm{Z} 170$ & 1,50 & Z213 & 2,90 \\
\hline $\mathrm{Z} 42$ & 2,40 & Z85 & 1,88 & Z128 & 1,91 & Z171 & 2,35 & & \\
\hline Z43 & 3,50 & Z86 & 3,00 & Z129 & 3,33 & Z172 & 1,60 & & \\
\hline
\end{tabular}

Min: 0.70; Max: 3.90; Average: 2.42; Std. deviation: 0.72; Coef. of variation: 29.53

\section{Essential Oil Components (\%)}

The essential oil components obtained as a result of GC/MS analyzes in the study are given in Table 2, respectively $(\geq 1 \%)$. The main component of thyme herbs is carvacrol. However, a wide variation has been detected among ecotypes in terms of carvacrol content. The carvacrol contents of the thyme plants varied between $28.12 \%$ (Z104, Z142) and 78.48\% (Z167) and the average carvacrol ratio was $48.68 \%$ (Table 2). 
ICAMS $2020-8^{\text {th }}$ International Conference on Advanced Materials and Systems

The prominent types in terms of carvacrol ratio were determined as Z167 (78.48\%), Z165 (77.98\%) and Z64 (72.54\%).

Table 2. Carvacrol contents of the thymbra genotypes (\%)

\begin{tabular}{|c|c|c|c|c|c|c|c|c|c|}
\hline Code & $\begin{array}{c}\text { Carvacrol } \\
\%\end{array}$ & Code & $\begin{array}{c}\text { Carvacrol } \\
\%\end{array}$ & Code & $\begin{array}{c}\text { Carvacrol } \\
\%\end{array}$ & Code & $\begin{array}{c}\text { Carvacrol } \\
\%\end{array}$ & Code & $\begin{array}{c}\text { Carvacrol } \\
\%\end{array}$ \\
\hline Z1 & 55,66 & Z44 & 56,25 & Z87 & 49,00 & Z130 & 30,32 & Z173 & 67,08 \\
\hline $\mathrm{Z} 2$ & 49,95 & $\mathrm{Z} 45$ & 51,83 & Z88 & 59,77 & Z131 & 44,49 & Z174 & 49,02 \\
\hline $\mathrm{Z3}$ & 54,37 & Z46 & 42,87 & Z89 & 39,54 & Z132 & 50,28 & $\mathrm{Z} 175$ & 39,13 \\
\hline $\mathrm{Z4}$ & 54,50 & Z47 & 59,88 & Z90 & 40,08 & Z133 & 40,40 & Z176 & 48,05 \\
\hline $\mathrm{Z} 5$ & 59,25 & Z48 & 45,52 & Z91 & 38,51 & Z134 & 35,63 & Z177 & 47,76 \\
\hline Z6 & 48,61 & Z49 & 56,38 & Z92 & 45,07 & Z135 & 47,58 & Z178 & 56,63 \\
\hline $\mathrm{Z7}$ & 50,52 & $\mathrm{Z} 50$ & 47,87 & Z93 & 49,61 & Z136 & 41,89 & Z179 & 39,32 \\
\hline Z8 & 55,66 & Z51 & 49,11 & Z94 & 49,17 & Z137 & 43,45 & Z180 & 40,92 \\
\hline Z9 & 52,41 & $\mathrm{Z} 52$ & 50,41 & Z95 & 41,63 & Z138 & 42,94 & Z181 & 57,80 \\
\hline Z10 & 54,49 & Z53 & 54,49 & Z96 & 52,33 & Z139 & 41,63 & Z182 & 37,82 \\
\hline Z11 & 47,81 & Z54 & 51,24 & Z97 & 32,71 & Z140 & 46,44 & Z183 & 47,84 \\
\hline Z12 & 49,65 & Z55 & 53,67 & Z98 & 51,62 & Z141 & 47,00 & Z184 & 53,77 \\
\hline Z13 & 59,66 & Z56 & 32,20 & Z99 & 66,77 & $\mathrm{Z} 142$ & 28,12 & Z185 & 56,64 \\
\hline Z14 & 49,80 & Z57 & 47,83 & $\mathrm{Z100}$ & 46,83 & Z143 & 39,48 & Z186 & 60,23 \\
\hline Z15 & 51,05 & Z58 & 49,02 & Z101 & 49,01 & Z144 & 52,44 & Z187 & 44,74 \\
\hline Z16 & 46,30 & Z59 & 47,32 & Z102 & 33,52 & Z145 & 34,92 & Z188 & 44,28 \\
\hline Z17 & 58,18 & Z60 & 44,37 & Z103 & 56,25 & Z146 & 43,10 & Z189 & 51,60 \\
\hline Z18 & 56,03 & Z61 & 61,76 & Z104 & 28,12 & Z147 & 41,13 & Z190 & 39,63 \\
\hline Z19 & 51,02 & Z62 & 50,90 & Z105 & 56,58 & Z148 & 43,74 & Z191 & 38,39 \\
\hline $\mathrm{Z} 20$ & 56,18 & Z63 & 52,02 & Z106 & 28,84 & Z149 & 56,96 & Z192 & 42,80 \\
\hline Z21 & 56,39 & Z64 & 72,54 & Z107 & 40,07 & Z150 & 30,32 & Z193 & 59,10 \\
\hline $\mathrm{Z} 22$ & 48,17 & Z65 & 47,50 & Z108 & 66,57 & Z151 & 42,96 & Z194 & 54,05 \\
\hline Z23 & 31,28 & Z66 & 52,35 & Z109 & 55,63 & Z152 & 49,23 & Z195 & 64,09 \\
\hline Z24 & 59,64 & Z67 & 73,66 & $\mathrm{Z} 110$ & 48,85 & Z153 & 43,79 & Z196 & 36,36 \\
\hline $\mathrm{Z} 25$ & 44,74 & Z68 & 48,85 & Z111 & 36,39 & Z154 & 54,41 & Z197 & 45,79 \\
\hline Z26 & 59,16 & Z69 & 53,94 & Z112 & 37,11 & Z155 & 37,68 & Z198 & 50,53 \\
\hline $\mathrm{Z} 27$ & 53,49 & $\mathrm{Z70}$ & 51,39 & Z113 & 52,16 & Z156 & 59,96 & Z199 & 38,13 \\
\hline Z28 & 54,84 & Z71 & 52,52 & Z114 & 39,10 & Z157 & 41,89 & Z200 & 31,37 \\
\hline Z29 & 45,60 & $\mathrm{Z} 72$ & 53,95 & Z115 & 64,85 & Z158 & 41,52 & Z201 & 39,59 \\
\hline $\mathrm{Z} 30$ & 49,00 & $\mathrm{Z73}$ & 58,74 & Z116 & 38,28 & Z159 & 44,53 & Z202 & 48,31 \\
\hline Z31 & 54,74 & $\mathrm{Z74}$ & 32,94 & Z117 & 53,94 & Z160 & 38,72 & Z203 & 45,66 \\
\hline Z32 & 47,92 & $\mathrm{Z75}$ & 61,76 & Z118 & 58,74 & Z161 & 46,16 & Z204 & 51,42 \\
\hline Z33 & 57,00 & Z76 & 45,76 & Z119 & 32,87 & Z162 & 41,40 & Z205 & 48,93 \\
\hline Z34 & 41,02 & $\mathrm{Z77}$ & 58,74 & $\mathrm{Z} 120$ & 37,70 & Z163 & 47,00 & Z206 & 49,33 \\
\hline Z35 & 46,30 & Z78 & 29,79 & Z121 & 44,35 & Z164 & 69,89 & Z207 & 46,13 \\
\hline Z36 & 53,15 & Z79 & 32,87 & Z122 & 40,05 & Z165 & 77,98 & Z208 & 51,23 \\
\hline Z37 & 44,26 & $\mathrm{Z} 80$ & 49,91 & $\mathrm{Z} 123$ & 50,02 & Z166 & 38,83 & Z209 & 50,28 \\
\hline Z38 & 44,05 & Z81 & 47,98 & Z124 & 54,83 & $\mathrm{Z} 167$ & 78,48 & $\mathrm{Z} 210$ & 70,53 \\
\hline Z39 & 49,01 & Z82 & 45,87 & $\mathrm{Z} 125$ & 41,21 & Z168 & 68,29 & Z211 & 34,60 \\
\hline $\mathrm{Z} 40$ & 47,94 & Z83 & 47,58 & Z126 & 41,64 & Z169 & 57,38 & Z212 & 48,26 \\
\hline Z41 & 51,28 & Z84 & 46,12 & $\mathrm{Z} 127$ & 44,23 & $\mathrm{Z} 170$ & 63,00 & $\mathrm{Z} 213$ & 51,15 \\
\hline $\mathrm{Z} 42$ & 59,64 & Z85 & 52,10 & Z128 & 43,56 & Z171 & 54,37 & & \\
\hline Z43 & 49,35 & Z86 & 44,15 & Z129 & 47,98 & $\mathrm{Z} 172$ & 51,39 & & \\
\hline
\end{tabular}

Generally, p-Cymene is seen as the second component. Even in some ecotypes, pCymene values were obtained almost similar to carvacrol. In addition, some ecotypes contain thymol with carvacrol. Z23 (18.18\%), Z41 (16.88\%), Z56 (20.75\%), Z74 (21.51\%), Z79 (7.41\%), Z119 (8.56\%), Z130 (16.00\%) and Z150 (18.21\%) code numbered ecotypes contain thymol at specified rates. The results obtained are in agreement with the literature (Başer et al., 1996, Hanc1 et al., 2003).

https://doi.org/10.24264/icams-2020.II.18 


\section{The Variation of Essential Oil and Carvacrol Contents of Native Grown Thymbra spicata var. spicata L.}

The zahtar that were collected were examined in terms of leaf density, essential oil ratio and essential oil components. Among the zahtar collected in the study, the plants with code number Z14, Z3, Z25, Z38, Z77, Z104, Z35 and Z43, which are high in essential oil (over $3.50 \%$ ), were selected. The prominent types in terms of carvacrol ratio were determined and selected as Z167 (78.48\%), Z165 (77.98\%) and Z64 $(72.54 \%)$. In the study, it was also determined that some plants with low essential oil content such as Z144 and Z158 also have low oil glands number and oil glands diameter. It would be appropriate to evaluate these values obtained as preliminary knowledge together with future ontogenetic variability studies.

\section{REFERENCES}

Avcı, M. (2005), “Çeşitlilik ve endemizm açısından Türkiye'nin bitki örtüsü”, İstanbul Üniv. Edebiyat Fakültesi Coğrafya Dergisi, (13), 27-55.

Başer, K.H.C. (2002), "Aromatic biodiversity among the flowering plant taxa of Turkey", Pure Appl. Chem., 74(4), 527-545, https://doi.org/10.1351/pac200274040527.

Başer, K.H.C., Ermin, N., Özek, T., Demirçakmak, B., Tümen, G. and Duman, H. (1996), "Essential Oils of Thymbra sintenisii Bornm. et Aznav. subsp. isaurica P.H. Davis and Origanum leptocladum Boiss.", Journal of Essential Oil Research, 8(6), 675-676, https://doi.org/10.1080/10412905.1996.9701040.

Baydar, H., Sağdiç, O., Özkan, G. and Karadoğan, T. (2004), “Antibacterial activity and composition of essential oils from Origanum, Thymbra and Satureja species with commercial importance in Turkey", Food Control, 15, 169-172, https://doi.org/10.1016/S0956-7135(03)00028-8.

Doğan, A., Bayrak, A. and Akgül, A. (1987), "Thymol/carvacrol containing Labiatae species and volatile composition of essential oil of Thymbra spicata", Gida, Sayı: 6, 359-362.

Ekim, T., Koyuncu, M., Vural, M., Duman, H., Aytaç, Z. and Adıgüzel, N. (2000), Türkiye Bitkileri Kırmızı Kitabı (Ĕgrelti ve Tohumlu Bitkiler), Türkiye Tabiatını Koruma Derneği, Van 100. Yıl Üniv. s:246. Barışcan Ofset. Ankara.

Erik, S. and Tarıkahya, B. (2004), Türkiye Florası üzerine, Kebikeç, 17, 139-163.

Erken, S. (2005), "Morphological and anatomical studies on Thymbra sintenisii Bornm \&Aznav. (Labiateae)", Turk. J. Bot., 29,389-397.

Hancı Sonsuzer, S., Şahin, S. and Yılmaz, L. (2003), "Isolation of volatile oil from thyme (Thymbra spicata) by steam distillation", Nahrung/Food, 47(4), 252-255, https://doi.org/10.1002/food.200390059.

İnan, M., Kırpık, M., Kaya, D.A. and Kırıcı, S. (2011), "Effect of harvest time on essential oil composition of Thymbra spicata L. Growing in flora of Adiyaman”, Advances in Environmental Biology, 5, pp.356-358.

Özcan, M.M., Ünver, A., Uçar, T. and Arslan, D. (2008), "Mineral content of some herbs and herbal teas by infusion and decoction", Food Chemistry, 106, 1120-1127, https://doi.org/10.1016/j.foodchem.2007.07.042.

Özel, M.Z., Gögüş, F. and Lewis, A.C. (2003), "Subcritical water extraction of essential oils from Thymbra spicata", Food chemistry, 82, 381-386, https://doi.org/10.1016/S0308-8146(02)00558-7.

Özgüven, M., Sekin, S., Gürbüz, B., Şekeroğlu, N., Ayanoğlu, F. and Ekren, S. (2005), Tütün, Tıbbi ve Aromatik Bitkiler Üretimi ve Ticareti. Türkiye Ziraat Mühendisliği VI. Teknik Kongresi. 481-501.

Saraç, A. and Tunç, I. (1995), "Residual toxicity and repellency of essential oils to stored-product insets. Rucktandstoxizitat und abstossende wirkung von atherischen ole auf vorratsschadliche insekten", Zeitschrift-fur-Pflanzenkrankheiten und Pflanzenschutz, 102(4), 429-434.

Tonçer, Ö. and Kızıl, S. (2005), "Determination of yield and yield components in wild thyme (Thymbra spicata var. spicata) as influenced by development stages", Hort. Sci. (Prague), 32(3), 100-103, https://doi.org/10.17221/3773-HORTSCI. 


\title{
COMPLEXES BASED ON COLLAGEN AND KERATIN FOR APPLICATIONS
} IN AGRICULTURE

\author{
MIHAELA-DOINA NICULESCU ${ }^{1}$, CRISTINA EMANUELA ENASCUTA $^{2,3}$, \\ MARIA STANCA ${ }^{1,4}$, CARMEN CORNELIA GAIDAU $^{1}$, COSMIN ALEXE $^{1}$, \\ MIHAI GIDEA ${ }^{5}$, MARIUS BECHERITU ${ }^{6}$ \\ ${ }^{1}$ INCDTP-Leather and Footwear Research Institute Division, 93 Ion Minulescu Street, Bucharest, \\ Romania, mihaelaniculescu59@yahoo.com \\ ${ }^{2}$ National Institute for Research \& Development in Chemistry and Petrochemistry ICECHIM, \\ Bucharest, Romania, cristina.enascuta@gmail.com \\ ${ }^{3}$ Doctoral School of Faculty of Applied Chemistry and Materials Science, Department of \\ Chemical and Biochemical Engineering, University Politehnica of Bucharest, Romania \\ ${ }^{4}$ Doctoral School of Faculty of Applied Chemistry and Materials Science, Department of Science \\ and Engineering of Oxide Materials and Nanomaterials, University Politehnica of Bucharest, \\ Romania \\ ${ }^{5}$ University of Agronomic Science and Veterinary Medicine, 59, Marasti St, sector 1, Bucharest, \\ Romania,gideam@yahoo.com \\ ${ }^{6}$ SC Probstdorfer Saatzucht Romania SRL, 20 Siriului St, sector 1, Bucharest, Romania, \\ marius.becheritu@probstdorfer.ro
}

\begin{abstract}
In the circular economy context, the use of proteins from collagen and keratin by-products of leather industry to obtain products for agriculture serves to reduce the carbon footprint generated from industry by reducing the amount of chemical synthesis products administered in agricultural technologies. This paper presents complexes based on collagen and keratin extracts obtained from by-products of the leather industry and their characterization. Thermal and chemical-enzymatic hydrolysis of semi-processed leather and degreased wool by-products was performed for protein extraction. Complexes were obtained by addition and crosslinking with active principles and vegetable tannins to collagen and keratin extracts. The characterization of complexes was performed based on the results of analytical investigations by physico-chemical methods: volumetry, potentiometry, IR spectroscopy, Dynamic Light Scattering and Texture Analysis. It has been found that collagen and keratin extracts contain sufficient proportions of small and medium size components size, of the order of 1-100 nm and of 100-1000 nm, specific for free amino acids and small oligopeptides with a role in bio stimulating seed germination, but also contain large size components, over $1000 \mathrm{~nm}$, in considerable proportions, which provide the adhesive and filmforming properties, with a role in foliar application and retarded release of amino acids.
\end{abstract}

Keywords: by-products, proteinic complexes, texture analysis.

\section{INTRODUCTION}

The use of protein extracts as a source of nitrogen for biostimulation, plant nutrition and protection, is a constant concern in research and in recent years there have been important results in this direction.

Unlike inorganic nitrogen-based fertilizers that are used predominantly in intensive agriculture, the main advantages of protein extracts administered in crops are their ecological relationship with plants and soil, but especially the systemic effects of biostimulation and protection under stress. A major advantage is the availability of proteins as secondary resources in aquaculture, food industry, leather industry (Li et al., 2020; Hukmi et al., 2018; Lavinska et al., 2017).

Protein extracts are a valuable source of nitrogen (nutrients) for agriculture, which can be used by both root and foliar application. The root application favors the release of the organic fraction in the soil, preventing dehydration due to the high rate of absorption 
through the roots, and by foliar application the nutritional role is amplified by the role of vehicle for microelements. Protein compounds offer long-lasting fertilizing effects compared to inorganic fertilizers, and by functionalizing with various plant extracts, protective effects on crops can be induced against pests (fungi and insects).

The intelligent use of secondary protein resources for plant nutrition, increasing soil fertility and reducing the carbon footprint, is an ecological alternative to synthetic materials and promoting sustainable agriculture in the context of the circular economy.

This paper presents complexes based on collagen and keratin extracts obtained from by-products of the leather industry and their characterization. The collagen and keratin extracts from the leather processing by-products were used to obtain complexes products with specific properties for the agricultural field, with effects on germination, nutrition and plant protection in various stages of vegetation. Also, the protein complexes properties can be exploited in other applications (Matyasovsky et al., 2017; Ranjithkumar et al., 2019), in accordance with the current trends of circular economy development.

\section{EXPERIMENTAL PART}

\section{Materials}

The bovine leather by-products and wool by-products from the Leather and Footwear Research Institute Division, Romania, for collagen and keratin extraction, as gelatin with average molecular weight over $30 \mathrm{kDa}$ and collagen and keratin hydrolysate with average molecular weight below $15 \mathrm{kDa}$.

Formic acid $80 \%$ from SC CHIMOPAR TRADING SRL, for pH corrections.

Ammonia solution $25 \%$ p.a, anhydrous sodium carbonate p.a from Chimreactiv SRL and ethoxylated alkyl non-ionic detergent from Borron SE for wool degreasing.

Hydrated calcium oxide p.a from SC Cristal R Chim SRL for leather and wool by-products hydrolysis.

Vegetable tara tannin from Leather Quimica SLU was used as powder with volatile matter content 8.6 and tannin content $39 \%$ to obtain tannin extract.

Glycerol were products of SC Chimopar SA Romania.

\section{Procedures}

Bovine gelatin was obtained by thermal hydrolysis of semi-processed leather byproducts at $70^{\circ} \mathrm{C}$ temperature and $\mathrm{pH} 4.0$.

The collagen hydrolysate was obtained by chemical-enzymatic hydrolysis at $60^{\circ} \mathrm{C}$ temperature and $\mathrm{pH}$ 8.0-8.5.

The keratin hydrolysate was obtained by chemical hydrolysis at $80^{\circ} \mathrm{C}$ temperature for 24-28 hours.

The Tara tannin extract (with 5\% dry substance, $4 \%$ tanning substances) was obtained by hydrolyzing the Tara powder in water at a temperature of $60-80^{\circ} \mathrm{C}$ for a period of $1-2$ hours, centrifugation and vacuum filtration on cellulosic membranes (Gaidau et al., 2014).

The complexes based on collagen or collagen and keratin were made by continuously stirring the gelatin with collagen or keratin hydrolysate additivatedcrosslinked with glycerol and tannin extract at $50-60^{\circ} \mathrm{C}$ for $40-90$ minutes.

\section{Analytical Methods}

The collagen and keratin extracts and their complexes were analysed by gravimetric methods, dry substance (SR EN ISO 4684:2006) and total ash (SR EN ISO 4047:2002), 
by volumetric methods, in terms of total nitrogen and protein substance (SR ISO 5397:1996), aminic nitrogen (ICPI protocol) by potentiometric method for $\mathrm{pH}$ measurement (SR EN ISO 4045:2008).

Dynamic Light Scattering was used for size particle determination and distribution by ZetaSizer device Nano ZS (Malvern, UK).

Texture tests of complexes based on collagen and keratin were carried out using a TEX'AN texture analyser.

IR spectroscopy was used for structural analysis by FT/IR-4200 (Jasco) with ATR device equipped.

\section{RESULTS AND DISCUSSIONS}

To obtain collagen and keratin complexes, the following protein extracts were prepared: GA gelatin, WH collagen hydrolysate, KH keratin hydrolysate, with the chemical characteristics presented in Table 1.

Table 1. Characteristics of protein extracts

\begin{tabular}{lcccc}
\hline \multicolumn{1}{c}{ Characteristics } & MU & Gelatin GB & \multicolumn{2}{c}{ Hydrolysates } \\
& & & WH & KH \\
\hline Dry substance & $\%$ & 14.52 & - & - \\
Volatile matter & $\%$ & - & 10.11 & 8.50 \\
Total ash & $\%$ & 0.20 & 12.58 & 12.28 \\
Total nitrogen & $\%$ & 16.41 & 12.08 & 12.55 \\
Protein substance & $\%$ & 92.67 & 67.89 & 76.04 \\
Amino nitrogen & $\%$ & 0.50 & 1.16 & 0.70 \\
pH analytical solution & - & 4.42 & 8.90 & 7.75 \\
\hline
\end{tabular}

The analysis of collagen and keratin hydrolysates by Dynamic Light Scattering (DLS) presented in Figure 1, (i) for WH collagen hydrolysate and (ii) for KH keratin hydrolysate, highlights the existence of small peptide fragments, in the specific "nano" field in this case for free amino acids and oligopeptides.

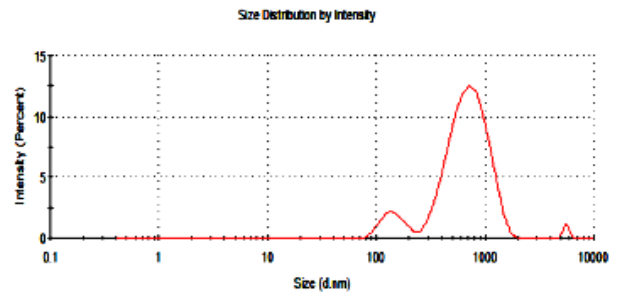

(i)

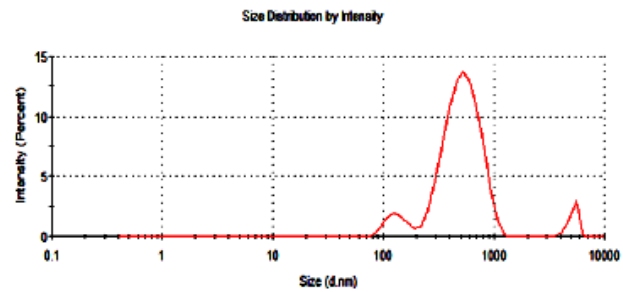

(ii)

Figure 1. Particle size in protein hydrolysates

The particle size distribution in collagen and keratin hydrolysates is shown in Table 2:

Table 2. Particle size distribution in protein hydrolysates

\begin{tabular}{lccc}
\hline \multicolumn{1}{c}{ Sample } & \multicolumn{2}{c}{ Particle size share } \\
& $10-100 \mathrm{~nm}$ & $100-1000 \mathrm{~nm}$ & $1000-10000 \mathrm{~nm}$ \\
\hline Collagen hydrolysate, WH & 0.5 & 83.6 & 15.9 \\
Keratin hydrolysate, KH & 0.7 & 93.5 & 5.8 \\
\hline
\end{tabular}

https://doi.org/10.24264/icams-2020.II.19 
DLS analysis reveals the existence of a higher percentage of particles below 1000 $\mathrm{nm}$ in collagen hydrolysate, being consistent with the higher content of amino nitrogen, which indicates a lower average molecular weight of collagen hydrolysate compared to keratin hydrolysate.

Gelatin and hydrolysates combined to form two types of complex gels, one based on collagen, GW and one based on collagen-keratin, GK, plasticized with glycerol and crosslinked with vegetable tannin extract. The chemical characteristics of the gels are presented in Table 3.

Table 3. Characteristics of protein gels

\begin{tabular}{lccc}
\hline \multicolumn{1}{c}{ Characteristics } & MU & GW & GK \\
\hline Dry substance & $\%$ & 32.48 & 29.39 \\
Total ash & $\%$ & 2.71 & 4.05 \\
Total nitrogen & $\%$ & 9.76 & 10.04 \\
Protein substance & $\%$ & 54.85 & 56.42 \\
Amino nitrogen & $\%$ & 0.67 & 0.48 \\
pH analytical solution & - & 5.76 & 5.39 \\
\hline
\end{tabular}

The gels strength of proteinic complexes formed by cross-linking and additivation was studied in comparison with gelatin in standard conditions by Direct Compression (CD) test. The results of the analysis are shown in Figure 2, for gelatin (a), collagen complex (b) and collagen-keratin complex (c).

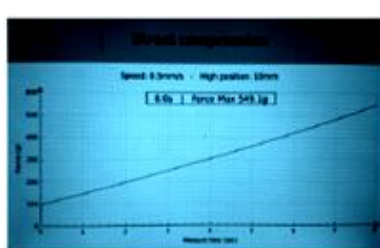

(a) $\mathrm{GB}$

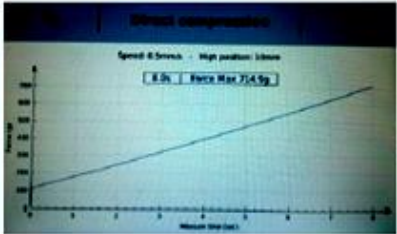

(b) GW

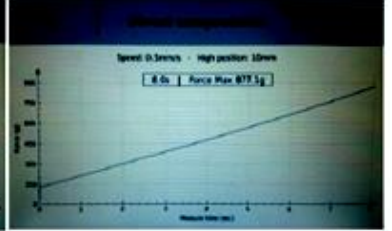

(c) GK

Figure 2. The comparative gels strength

It is found that the addition of gelatin with hydrolysates, and crosslinking lead to gels with a significantly higher strength, the largest increase being recorded by the gel additivated with keratin. The increase of the gelatin strength by additivation and crosslinking is the consequence of bond formation and new compounds that consolidate the newly formed structures.

The complete textural analysis of collagen and keratin based complexes for agricultural applications, whether they are plant or soil fertilizers, support bands for very small seeds, biodegradable packaging, etc. is done by Compression-RelaxationTraction (CRT) tests. By CRT test three consecutive phases are carried out: compression followed by a relaxation phase without movement where the reaction force (elastic thrust) of the sample is measured, then the probe is lifted while the fluid's traction force is measured, indicating its adhesiveness.

CRT tests measure the consistency, elasticity and adhesiveness and enable relevant parameters to be selected to define a product's texture, which will be related to its hardness, cohesion, and adhesiveness or free-running nature. 
Figure 3 shows the complete textural analysis of gelatin and collagen and keratin based complexes.

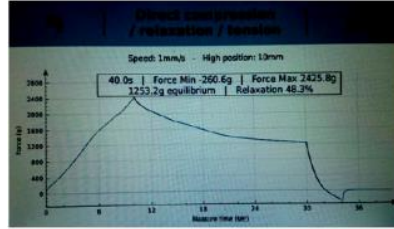

(A) GB

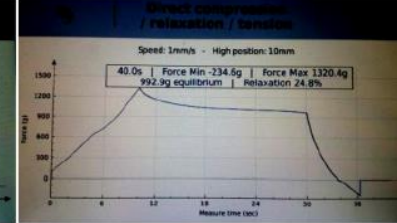

(B) GW

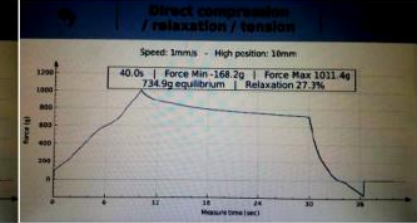

(C) GK

Figure 3. The Compression-Relaxation-Traction tests

The three phases can be identified on the curve Force =f(time): Fmax, which is the product's consistency in terms of defined compression (probe and distance); \% Relaxation, which is inversely proportional to the product's elasticity; Fmin, which reflects the traction or adhesion force of the product on the probe when it is brought out of the sample. The results of CRT tests show that the addition-crosslinking of gelatin causes a decrease in consistency but a pronounced increase in the elasticity of the gels, compared to the control gelatin, as well as a lower adhesiveness to the probe, without noticing significant differences between the two gels, only differences from gelatin.

According to response curve and the quantified values of these parameters, different textures of products can be compared and ranked.

The physical properties of the protein complexes are the consequence of the structural changes occurred following the crosslinking of the protein extracts with Tara tanning agent, highlighted by the IR spectral analysis. IR spectra show the spectral analysis of PW (in I) and PK (in II) films, formed by GW and GK gels, respectively, compared to gelatin (GB) and collagen hydrolysates (WH in I) and keratin (KH in II), respectively. IR spectra are presented in Figure 4.
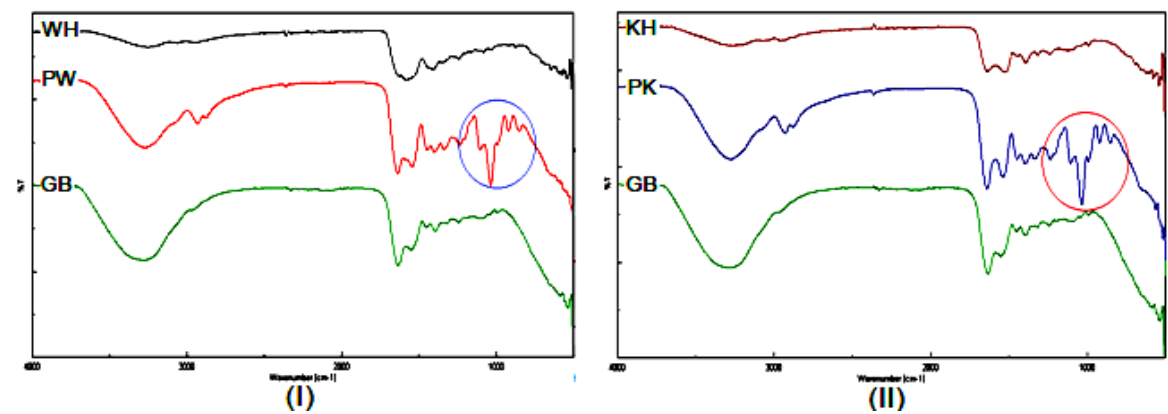

Figure 4. IR spectral analysis of proteinic complexes

The comparative analysis reveals that, in the spectra of the films formed by crosslinking the combinations of collagen extracts and combinations of collagen and keratin extracts with Tara tanning agent, there are missing peaks compared to hydrolysates, from the characteristic band $2600-3100 \mathrm{~cm}^{-1}$ specific to free amino acids and from band $1230-1260 \mathrm{~cm}^{-1}$ also specific to amino acids, while new peaks appear, non-existent in gelatin and hydrolysates, in the characteristic band $724-1174 \mathrm{~cm}^{-1}$. The common peaks in the films have frequencies and intensities slightly modified compared 
to those existing in gelatin and hydrolysates. Changes in crosslinking conditions can be attributed to the recombination of proteins of different sizes and the formation of larger peptide chains, specific to film-forming materials.

Testing complexes based on collagen and keratin to cover rapeseed as an outer layer of coating and monitoring of rapeseed crops in which seeds coated in this manner were used, led to superior results compared to control crops, established with conventionally treated seeds. There was a significant increase in germination compared to the control, as well as a better subsequent development of the plants. The overall development of the plants, both of the aerial part and of the underground part, was constant and much better highlighted than that of the untreated control.

\section{CONCLUSIONS}

The addition of collagen or keratin hydrolysates into gelatin provides a content of free amino acids and small oligopeptides with a role in bio stimulating seed germination. Crosslinking of collagen and keratin extracts with Tara tanning agent improves the film-forming properties and elasticity of the protein complexes. Collagen and keratin extracts are an alternative to the replacement of synthetic amino acids used to stimulate germination and plant development.

\section{Acknowledgements}

This work was supported by a grant of the Romanian National Authority for Scientific Research and Innovation CCCDI - UEFISCDI, project no. PN-III-P3-3.5EUK-2016-0029/COLL-RAPE, a grant of Core Program, project no. PN 19.17.01.02, and by the Operational Programme Human Capital of the Ministry of European Funds through the Financial Agreement 51668/09.07.2019, SMIS code 124705; and the article is funded by the Ministry of Research and Innovation within Program 1 - Development of the national RD system, Subprogram 1.2 - Institutional Performance - RDI excellence funding projects, Contract no. 6 PFE/16.10.2018.

\section{REFERENCES}

Gaidau, C., Simion, D., Niculescu, M.D., Paun, G., Popescu, M., Bacardit, A., Casas, C. (2014), “Tara Tannin Extract Improvement. Part I: Extraction and Concentration Through Membranary Filtration Techniques” Rev. Chim.-Bucharest, 65(8), 929-933

Hukmi, N. M. M. and Sarbon, N. M. (2018), "Isolation and characterization of acid soluble collagen (ASC) and pepsin soluble collagen (PSC) extracted from silver catfish (Pangasius sp.) skin”, International Food Research Journal, 25(6), 2601-2607.

Lawinska, K., Gendaszewska, D., Grzesiak, E., Jagiello, J. and Obraniak, A. (2017), "Coating of leguminosarum seeds with collagen hydrolyzates from tanning waste", Przemysl Chemiczny, 96(11), 1877-1880, 10.15199/62.2017.11.24.

Li, P.H., Lu, W.C., Chan, Y.J., Ko, W.C., Jung, C.C., Duynh, T.H., Ji, Y.X. (2020), "Extraction and characterization of collagen from sea cucumber (holothuria cinerascens) and its potential application in moisturizing cosmetics", Aquaculture, 515, 734590, 10.1016/J.Aquaculture.2019.734590.

Matyasovsky, J., Jurkovic, P., Skultety, J., et al. (2017), "Collagen and keratin as environmentally friendly natural polymers for modification of wood adhesives", in: Gatecka A., Wood-Science-Economy 2nd International Scientific Conference Proceedings, Wood Technology Institute, Poznan, Poland, 73.

Ranjithkumar, A., Sundar, V.J., Rose, C., Muralidharan, C. (2019), “Amino Acid Enriched Proteinous Wastes: Recovery and Reuse in Leather Making" Waste and Biomass Valorization, online, 10.1007/s12649-019-00912-6.

https://doi.org/10.24264/icams-2020.II.19 
ECOLOGICAL APPROACHES FOR PROTECTING AND PERFUMING NATURAL SHEEPSKIN FUR

\author{
OLGA NICULESCU ${ }^{1}$, CARMEN GAIDAU ${ }^{1}$, ELENA BADEA ${ }^{1,2}$, LUCRETIA MIU ${ }^{1}$, DANA \\ GURAU $^{1}$, MARIANA DANIELA BERECHET ${ }^{1}$ \\ ${ }^{1}$ INCDTP - Division: Leather and Footwear Research Institute, 93 Ion Minulescu, 031215 \\ Bucharest, Romania, o_niculescu@yahoo.com \\ ${ }^{2}$ University of Craiova, Faculty of Sciences, Department of Chemistry, Calea Bucureşti 107 I, \\ 200585, Craiova, Romania
}

\begin{abstract}
Fur and leather have been among the first materials used for clothing and bodily decoration. It is known that Homo sapiens and Homo neanderthalensis used fur clothing. Even though the invention of inexpensive synthetic textiles for insulating clothing led to fur clothing falling out of fashion, fur is still worn in most cool climates around the world such due to its superior warmth and durability. In addition, a huge number of furs exists in the ethnography and anthropology museums around the world. The storage and conservation of furs, old and modern, is still challenging for both conservators and population because most commercial products are highly toxic for humans and environment. We therefore tried to control or limit the damage caused by external factors and insects by using green finishing and maintenance treatments. It is known that essential oils, known for their special perfume, can be used to repel insects. Mint, cedar, lavender oils were hence tested to treat sheepskin furs as a final finishing operation. In addition, the use of new products based on natural oils, ethyl alcohol, nonionogenic surfactants from the class of polyethoxylated fatty acids and of polyethylene glycols, and cationic surfactants (quaternary ammonium salts) were tested and proved they contribute to both perfuming and improving the resistance of furs to moths.
\end{abstract}

Keywords: green conservation, fur, finish \& maintenance

\title{
INTRODUCTION
}

In order to prevent the emergence and growth of microorganisms, biocides are used in various stages of leather processing, improving resistance to biological attack and preventing deterioration of mechanical and chemical properties of leathers and furs.

Some biocides used in the leather industry have a certain toxicity, and are prohibited by the directives in force (Directive 2010/75/EU).

The storage and conservation of furs, old and modern, is still challenging for both conservators and population because most commercial products are highly toxic for humans and environment.

Essential oils are very concentrated in active chemical elements (aliphatic and aromatic hydrocarbons, alcohols, aldehydes, esters etc.) and have various properties: they are antiseptic, antibacterial etc. (European Pharmacopeia, 2005).

It is known that essential oils, known for their special perfume, can be used to repel insects (Niculescu et al., 2015; Niculescu et al., 2019).

Mint, cedar, lavender oils were tested to treat sheepskin furs as a final finishing operation. In addition, the use of new products based on natural oils, ethyl alcohol, nonionogenic surfactants from the class of polyethoxylated fatty acids and of polyethylene glycols, and cationic surfactants (quaternary ammonium salts) were tested and proved they contribute to both perfuming and improving the resistance of furs to moths.

The paper presents methods of obtaining the furs fragrant and moth-resistant furs, can be used for preservation of modern and old furs in the museums of ethnography and anthropology. 
Ecological Approaches for Protecting and Perfuming Natural Sheepskin Fur

\section{EXPERIMENTAL}

\section{Materials}

Mint oil (Adams, Romania), containing menthol - $40.16 \%$, L-menthone $-23.90 \%$, I-menthone $-14.89 \%$, D-limonene $-8.65 \%$, menthyl acetate $-4.39 \%$ etc.

Cedar essential oil (Solaris Plant, Bucharest), containing thujopsene $-37.36 \%$, cedrenol $-20.90 \%$ cedrene $-20.14 \%$ and cuparene $-9.58 \%$.

Lavender oil (Adams, Romania), containing linalool - 36.59\%, linalyl acetate $35.76 \%$, alpha - terpineol $-7.63 \%$, lavandulyl acetate $-2.98 \%$, caryophylene $-2.96 \%$.

Ethanol (Chemical Company, Germany), colorless liquid, boiling point $78.37^{\circ} \mathrm{C}$, density $-0.79 \mathrm{~g} / \mathrm{cm}^{3}$

Nonionic emulsifier - lauryl alcohol ethoxylated with 7 moles of ethylene oxide (Elton Corporation, Romania), density $-0.97 \mathrm{~g} / \mathrm{cm}^{3}$ at $40^{\circ} \mathrm{C}, \mathrm{pH}(10 \%$ solution $)-7-8$.

Polyethylene Glycol 400 (Merck, Germany), density $-1.15 \mathrm{~g} / \mathrm{cm}^{3}$ at $20^{\circ} \mathrm{C}$, flash point > $200^{\circ} \mathrm{C} ; \mathrm{pH}(10 \%$ solution $)-5-7$; melting point $-5^{\circ} \mathrm{C}$, ignition temperature $360^{\circ} \mathrm{C}$.

Hexadecyl-trimethyl ammonium bromide (Merck, Germany), water solubility of $3 \mathrm{~g} / \mathrm{L}, \mathrm{pH}(10 \%$ solution $)-5-7$, melting point $237-243^{\circ} \mathrm{C}$, hygroscopic.

Product P-MCL based on essential oils (mint, cedar, lavender): dry substance - 19$21 \%, \mathrm{pH}(10 \%$ solution $)-4.5-5.0$, density $-0.890-0.900 \mathrm{~g} / \mathrm{cm}^{3}$.

Sheepskins tanned with syntans based on phenolsulphonic acids and aromatic oxisulfones (INCDTP - Division Leather and Footwear Research Institute Bucharest, Romania).

\section{Methods}

Synthesis of materials based on plant extracts for treatmentation of furs was conducted in a glass flask using a heating and homogenization installation (Velp) and an ultrasonic bath (Elmasonic S $15 \mathrm{H}$ ).

Analysis of the essential oils was carried out by using Gas Chromatography Mass Spectrometry Analysis - Agilent 7890 A GC System equipped with Agilent 5795 C MS, and HP-5 MS (0.25 mm x $30 \mathrm{~m}$ i.d., film thickness 0.25$)$.

Attenuated Total Reflectance Fourier transform infrared spectroscopy measurements were run with a Jasco instrument (model 4200), in the following conditions: wavenumber range $-600-4000 \mathrm{~cm}^{-1}$; data pitch $-0.964233 \mathrm{~cm}^{-1}$.

Chemical characteristics of products based on essential oils were determined according to the following standards: dry substance (\%) - SR EN ISO 4684:2006; pH SR-EN ISO 4098: 2006.

Chemical and mechanical characteristics of furs were determined according to the following standards: volatile matter \% - SR EN ISO 4684:2012, extractible substances $\%$ - SR EN ISO 4048:2002, ash \% - SR EN ISO 4047:2002, shrinkage temperatures $\left({ }^{\circ} \mathrm{C}\right)$ - SR EN ISO 3380:2003, the longitudinal and transverse tensile strength - SR EN ISO 3376:2012.

\section{Obtaining the Product Based on Essential Oils}

The following components were added to the mixing vessel: $20 \%$ mint essential oil, $20 \%$ cedar essential oil and $20 \%$ lavender essential oil, $10 \%$ ethyl alcohol, $10 \%$ lauric 
alcohol ethoxylate with seven moles of ethylene oxide, $10 \%$ polyethylene glycol 400 , $1 \%$ hexadecyltrimethylammonium bromide and deionized water. Components were homogenised using a mechanical stirrer, on an electrically heated installation, at the temperature of $30-35^{\circ} \mathrm{C}$, for $15-20 \mathrm{~min}$. In order to homogenise components, an ultrasound bath was used, in which the glass flask was inserted, at the temperature of $25^{\circ} \mathrm{C}$, for 10 minutes. The products obtained were marked P-MCL.

\section{RESULTS AND DISCUSSION}

\section{Characterisation of Components Used to Obtain P-MCL Product}

Mint, cedar and lavender essential oils used to obtain product P-MCL were analysed using GC-MS. Identification of compounds in their composition is presented in Tables 1-3.

Table 1. Identification of organic compounds in the mint essential oil by GC-MS

\begin{tabular}{cccc}
\hline No. & RT & Amount, $\%$ & Compounds \\
\hline 1 & 17.99 & 8.65 & D-limonene \\
2 & 25.33 & 23.90 & L-menthone \\
3 & 25.93 & 14.89 & I-menthone \\
4 & 26.79 & 40.16 & Menthol \\
\hline
\end{tabular}

Table 2. Identification of organic compounds in the cedar essential oil by GC-MS

\begin{tabular}{cccc}
\hline No. & RT & Amount, $\%$ & Compounds \\
\hline 1 & 36.85 & 20.14 & Cedrene \\
2 & 37.67 & 37.36 & Thujopsene \\
3 & 40.76 & 9.58 & Cuparene \\
4 & 45.12 & 20.90 & Cedrenol \\
\hline
\end{tabular}

Table 3. Identification of compounds in the lavender essential oil by GC-MS

\begin{tabular}{cccc}
\hline No. & RT & Amount, $\%$ & Compounds \\
\hline 1 & 21.54 & 36.59 & Linalool \\
2 & 25.32 & 7.63 & Alpha -Terpineol \\
3 & 28.62 & 35.76 & Linalyl acetate \\
4 & 29.98 & 2.98 & Lavandulyl acetate \\
\hline
\end{tabular}

\section{FT-IR Characterization of Components Used and the Obtained Product}

Figure 1 presents the spectral characteristics of mint (MIN), cedar (CED), lavender (LAV) essential oils and of product (P-MCL).

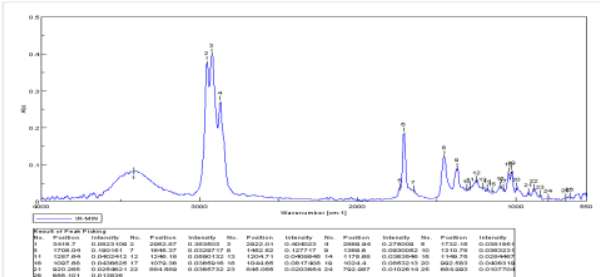

a - The FTIR spectrum of MIN

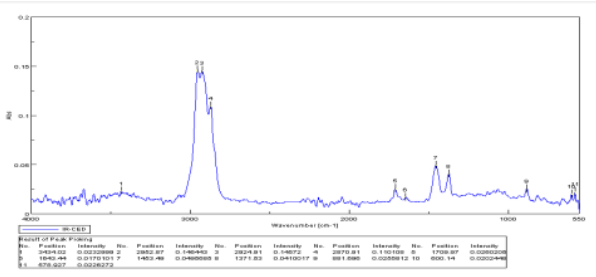

b - The FTIR spectrum of CED

https://doi.org/10.24264/icams-2020.II.20 
Ecological Approaches for Protecting and Perfuming Natural Sheepskin Fur

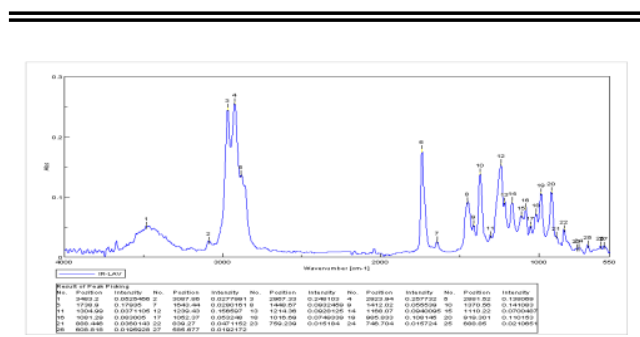

c - The FTIR spectrum of LAV

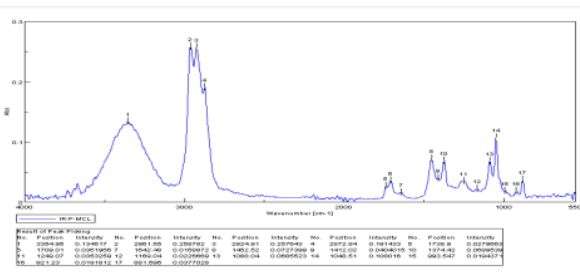

$\mathrm{d}$ - The FTIR spectrum of P-MCL

Figure 1. The FTIR spectra of mint (MIN), cedar (CED), lavender (LAV) essential oils and of product (P-MCL)

The main bands of product P-MCL are (Fig. 1d): $3354 \mathrm{~cm}^{-1}$ - indicating the presence of hydroxyl groups, $2961 \mathrm{~cm}^{-1}, 2924 \mathrm{~cm}^{-1}$ and $2872 \mathrm{~cm}^{-1}-$ indicating the presence of aliphatic $\mathrm{CH}_{2}$ groups, $1738 \mathrm{~cm}^{-1}$ and $1709 \mathrm{~cm}^{-1}$ - indicating the presence of $\mathrm{C}=\mathrm{O}$ group from ester, $1088 \mathrm{~cm}^{-1}, 1048 \mathrm{~cm}^{-1}$ and $881 \mathrm{~cm}^{-1}$ given by the C-O group from ether.

The bands with similar wavelengths are found in the FTIR spectra of the essential oils of mint, cedar and lavender (Fig. 1a-c), which are part of the composition of the PMCL perfume product.

\section{Physical-Chemical Characteristics of the Product Based on Essential Oils}

The physical-chemical characterization of new P-MCL product, based on essential oils, ethyl alcohol, non-ionogenic surfactants from the category of polyethoxylated fatty alcohols and polyethylene glycols and water: it is a yellowish white fluid, homogenous, with $19-21 \%$ dry substance, $\mathrm{pH}-4.5-5.0$, density $-0.890-0.900 \mathrm{~g} / \mathrm{cm}^{3}$.

\section{FT-IR Characterization of Obtained Fur Assortments}

Figure 2 presents the spectral characteristics of the untreated fur with P-MCL, F1 (control sample), (Fig. 2a), compared with those of the treated ones (with P-MCL product) F2, F2(1), F2(2) and F2(3), (Fig. 2b-e). Figure 2f presents superposed IR spectra of treated fur samples with P-MCL product, F2, F2(1), F2(2) and F2(3), which have different intensities, depending on the amount of P-MCL product used.

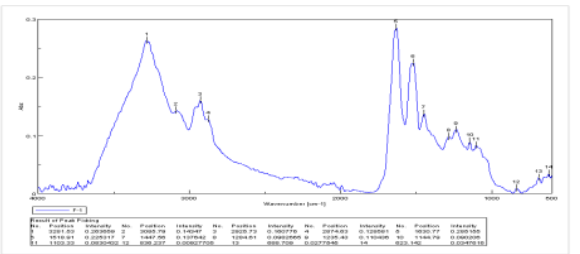

a - The FTIR spectrum of F1

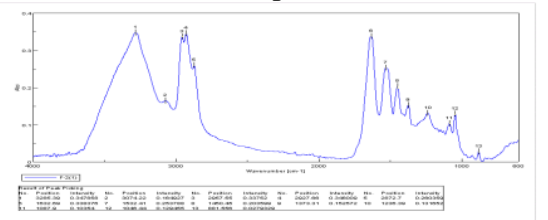

c - The FTIR spectrum of F2(1)

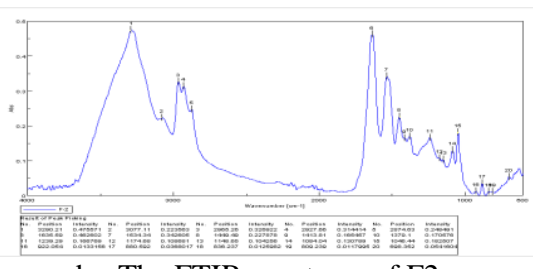

b - The FTIR spectrum of F2

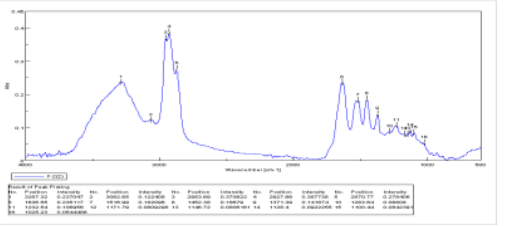

d - The FTIR spectrum of F2(2)

https://doi.org/10.24264/icams-2020.II.20 


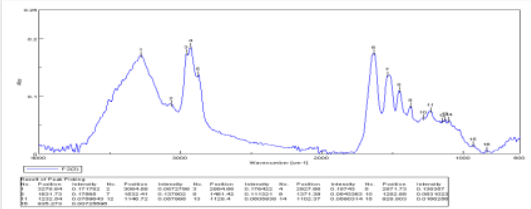

e - The FTIR spectrum of F2(3)

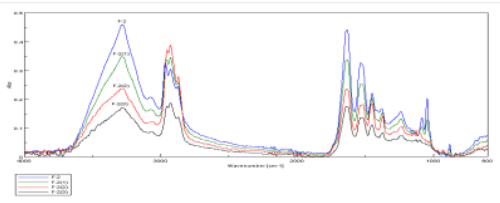

f - The superposed FTIR spectra of F2, F2(1), F2(2), F2(3)

Figure 2. The FTIR spectra of samples F1, F2, F2(1), F2(2), F2(3)

The main spectral bands of the F1 fur sample are found in the following regions: 2925 and $2874 \mathrm{~cm}^{-1}$ (-CH3, -CH2-), $1630 \mathrm{~cm}^{-1}$ (-OC-N), $1518 \mathrm{~cm}^{-1}(\mathrm{NH}), 1447 \mathrm{~cm}^{-1}$ (CH), 1284 and $1235 \mathrm{~cm}^{-1}$ (NH-CO), 1144 and $1103 \mathrm{~cm}^{-1}$ (CO) (Fig. 2a).

The main spectral bands of the F2 fur sample, treated with the product P-MCL, are found in the following regions: 2965 and $2927 \mathrm{~cm}^{-1}(-\mathrm{CH} 3,-\mathrm{CH} 2-), 1636$ and $1634 \mathrm{~cm}^{-1}$, characteristic for the groups $\mathrm{C}=\mathrm{O}$ and $-\mathrm{OC}-\mathrm{N}, 1440 \mathrm{~cm}^{-1}(\mathrm{CH}), 1239 \mathrm{~cm}^{-1}(\mathrm{NH}-\mathrm{CO})$ (Fig. 2b).

Modification of the F2 fur sample spectral bands was observed at the absorption peaks of approximately 1413 and $1379 \mathrm{~cm}^{-1}$ (indicating the presence of $\mathrm{C}-\mathrm{OH}$ group from alcohol), 1084, 1048 and $881 \mathrm{~cm}^{-1}$ (given by the CO group from ether); they are slightly modified and of lower intensities, but are present in the P-MCL product. The above differences between the IR spectra of the treated and untreated fur can be considered as a proof of the presence of the P-MCL product.

\section{Characterization of Furs by Physical-Chemical and Physical-Mechanical Analyses}

The values of the physical-chemical characteristics of the furs are comparable to the values set by the standards for sheep furskins intended for clothing (volatile dermal matter $12.20-12.80 \%$ and volatile wool matter $10.20-12.60 \%$, extractable dermal substances $9.50-11.70 \%$ and wool extracts $0.60-0.90 \%$, ash $3.50-3.90 \%$, $\mathrm{pH}$ of aqueous extract, 4-4.5. Values of shrinkage temperatures for sheep furskins are lower $\left(70-75^{\circ} \mathrm{C}\right)$ than those of sheep furs processed with basic chromium salts (approx. $80^{\circ} \mathrm{C}$ ).

The longitudinal tensile strength tests resulted in a value of $250-350 \mathrm{~N} / \mathrm{mm}$, compared to the standard for the sheep furskins tanned with chromium salts for clothing, which are of $\min .110 \mathrm{~N} / \mathrm{mm}$, and the transverse tensile strength values are $200-250 \mathrm{~N} / \mathrm{mm}$, compared to the values given in the standard for sheep furskins tanned with chromium salts for clothing, which are of min. $80 \mathrm{~N} / \mathrm{mm}$.

\section{Characterisation of Obtained Fragrant Fur Assortments}

The obtained P-MCL product can be applied to Merinos sheep fur (free of metals). In order to obtain the perfuming effect, the sheep furs were finished with a wetting solution for the final treatment of the furs, using the P-MCL product in different proportions, as follows:

- Sample F2 treated with a solution containing $300 \mathrm{~mL} / \mathrm{L}$ P-MCL product, $3 \mathrm{~mL} / \mathrm{L}$ nonionic emulsifier and deionized water (at a temperature of $30^{\circ} \mathrm{C}$ );

- Sample F2 (1) treated with a solution containing $250 \mathrm{~mL} / \mathrm{L}$ P-MCL product, 2.5 $\mathrm{mL} / \mathrm{L}$ nonionic emulsifier and deionized water (at a temperature of $30^{\circ} \mathrm{C}$ );

- Sample F2 (2) treated with a solution containing $200 \mathrm{~mL} / \mathrm{L}$ P-MCL product, 2 $\mathrm{mL} / \mathrm{L}$ nonionic emulsifier and deionized water (at a temperature of $30^{\circ} \mathrm{C}$ ); 
- Sample F2 (3) treated with a solution containing $100 \mathrm{~mL} / \mathrm{L}$ P-MCL product, 1 $\mathrm{mL} / \mathrm{L}$ nonionic emulsifier and deionized water (at a temperature of $30^{\circ} \mathrm{C}$ ).

The wetting solution is spread evenly over the hair coating, using thick plastic brushes. After depositing the solution, it is uniformized by brushing with plush pad (avoiding staining the dermis with the wetting solution). The operation is repeated twice.

Fur articles treated with a solution containing $300 \mathrm{~mL} / \mathrm{L} \mathrm{P-MCL}$ are more effective (fragrance lasts for 10-15 days).

\section{CONCLUSIONS}

- Sheepskins were tanned (free of metals) with syntans based on phenolsulphonic acids and aromatic oxisulfones, and treated with fragrance products based on vegetable extracts with insecticidal action (mint, cedar, lavender).

- The P-MCL product can be used to treat the surface of finished sheep furskins (free of metals) for perfuming and improving the resistance of furs to moths.

- The fragrant and moth-resistant furs can be used for preservation of modern and old furs in the museums of ethnography and anthropology.

\section{Acknowledgements}

These works were carried out with the support of Nucleus Program, TEX-PELVISION 2022, 4N/8.02.2019/Act ad.1/2019, with the support of the Ministry of Research and Innovation, project number PN 19170201 /2020, and within Program 1 - Development of the national RD system, Subprogram 1.2-Institutional PerformanceRDI excellence funding projects, Contract no. 6PFE / 16.10.2018.

\section{REFERENCES}

Council of Europe (2005), European Pharmacopeia, vol. II, ESCOP Strasbourg.

Niculescu, O., Leca, M., Moldovan, Z. and Deselnicu, D.C. (2015), "Research on obtaining products for fragrance and biological protection of natural leathers and furs", Revista de Chimie (Bucharest), 66(12), 1956.

Niculescu, O., Tonea, R.A. and Tonea, S. (2019), "Insecticidal and perfuming composition for the treatment of natural furs and natural fur articles", OSIM Patent, no. 130692/2019.

*** (2010), Directive 2010/75/EU of the European Parliament and of the Council of 24 November 2010 on industrial emissions (Integrated Pollution Prevention and Control). 
SPECIAL EFFECT FINISH FOR BOOKBINDING LEATHER

\author{
OLGA NICULESCU ${ }^{1}$, CARMEN GAIDAU ${ }^{1}$, ELENA BADEA ${ }^{1,2}$, LUCRETIA MIU $^{1}$, DANA \\ GURAU $^{1}$, DEMETRA SIMION ${ }^{1}$ \\ ${ }^{1}$ Leather Research Department, National Research \& Development Institute for Textiles and \\ Leather, ICPI Division, Ion Minulescu 93, 031215 Bucharest, Romania \\ ${ }^{2}$ Department of Chemistry, Faculty of Sciences, University of Craiova, Calea București 107 I, \\ 200585, Craiova, Romania
}

\begin{abstract}
The art of bookbinding requires not only skills in the old craft of bookbinding but also materials that can transform a simple book into a high-quality artistic product. Due to its unique properties, leather still remains the first-choice material in the case of art and archival bindings. However, the long-term durability of modern leather is not known since there is little commercial interest in long periods of durability and the market of leather for art, design and archival purposes is very small. It is worth noting that deterioration is influenced by the manufacturing technology, and especially by the chemical ingredients used in the various steps of leather making, from dehairing to tanning and finishing. It is therefore very likely that modern and contemporary artworks made of/with modern leather undergo faster degradation than ancient and medieval artworks. Thus, leather finishing is very important for both artistic and sustainable points of view. In fact, finishes with special effects such as antique, bicolour, printed, cracked, waxy are highly sought for vegetable tanned leather used for artistic and luxury bookbinding, archival bookbinding and restoration purposes. The evolving leather finishing technology of chrome-free leather (i.e. vegetable tanned leather) has enabled us to protect and improve the quality, look and feel of leather and to make it suitable for contemporary art bindery.
\end{abstract}

Keywords: durable, finish, bookbinding leather

\title{
INTRODUCTION
}

Due to its unique properties, leather still remains the first-choice material in the case of art and archival bindings. Ancient and medieval works of art, but also modern and contemporary works of art made of/with modern leather can suffer a certain degradation over time.

Finishing the skin is very important both artistically and sustainably, and aims to beautify, obtain a pleasant gloss and touch, cover defects, form a protective surface layer during use, as well as improve resistance to external factors (light, friction, scratches, water) of objects made from leather.

The finishing is carried out by spraying or with the help of ecological roller finishing machines, applying on the dermal support of some dispersed systems, in the composition of which the following auxiliary materials are used: pigments, binders, natural waxes and synthetics, preservatives, plasticizers, thickeners, fillers, odorants, penetrants, solvents (Lange, 1982; Heidemann, 1994).

Binders are film-forming macromolecular compounds used in all stages of finishing to give leather flexibility, adhesion and resistance to wear and to external factors. The composition of leather surface finishing systems includes acrylic, polyurethane, butadiene and nitrocellulose binders. Depending on molar masses and hardness, binders are used in various finishing coats (basecoat or dressing) to obtain the desired finish.

The types of finishes differ in terms of the working procedures or the effects obtained. By varying the components or working methods, even the same finishing process can lead to different gloss, matting or coating effects (Niculescu et al., 2015a; 2015b; 2015c; Niculescu and Mendea, 2019). Thus, finished natural leathers can be made by modern means, imitating ancient natural leathers.

https://doi.org/10.24264/icams-2020.II.21 
For bookbinding, leathers can be used with contrasting, antique, cracked, wrinkled, pleated, polished, printed, glossy, matte, pearlescent effect, with a silky, waxed, aniline, semianiline touch, in a wide range of colors, finished with materials which provide the desired characteristics of the finished product, for artistic and luxury bookbinding, archival bookbinding and restoration purposes.

The paper presents the surface leather finishing technologies that can be used for bookbinding leather with special effect finish. The auxiliary finishing materials selected, both for the basecoat and for the final finishing coat, as well as the working procedures used, led to obtaining assortments of natural leather finished with antique and cracked effect, which can imitate old, used leathers for valuable book covers.

\section{EXPERIMENTAL}

\section{Materials}

- Roda-Cryl 87, Roda-pure 302, Roda Wax MONO, Roda-Pur 5011, Roda lacquer 93, Roda feel KTA 950, Roda Casicolor Ochre, Roda Casicolor Brown $\mathrm{R}$ (Triderma, Romania).

- $\quad$ Eukesol OL Grund AB-1, LURON Glanz E, LURON Top, Eukesolar (STHAL, Holland).

- The crust bovine (calves) leathers natural grain assortments, vegetable tanned and wet finished by retanning, and fatliquoring (1.2-1.4 mm thick) (INCDTP Division Leather and Footwear Research Institute Bucharest, Romania).

\section{Methods}

Optical microscopy images were captured using a Leica stereomicroscope S8AP0 model with optic fiber cold light source, L2, with three levels of intensity, and 20X magnification for the finished leather surface.

Finished leathers were artificially aged and tested by colorimetric measurements were performed using a MINOLTA spectrophotometer (CM 2002), with light impulses from a xenon lamp with $0.8 \mathrm{~cm}$ aperture. Light reflection is focused on a silicon photo diode with wavelengths between 400 and $700 \mathrm{~nm}\left(10 \mathrm{~nm}\right.$ steps) and $\mathrm{L}^{*} \mathrm{a} * \mathrm{~b} *$ values (chromatic coordinates: brightness, red/green and yellow/blue), according to the CIE LAB system. Parameters of colour difference between aged samples using IT1, IT2, and IL methods $(\mathrm{T})$ and unaged ones $(\mathrm{R})$ were calculated using the following equations:

$\Delta \mathrm{a}^{*}=\mathrm{a}^{*} \mathrm{~T}-\mathrm{a}{ }_{\mathrm{R}}$

$\Delta \mathrm{b}^{*}=\mathrm{b}^{*} \mathrm{~T}-\mathrm{b}^{*} \mathrm{R}$

$\Delta \mathrm{L}^{*}=\mathrm{L}^{*} \mathrm{~T}-\mathrm{L}{ }_{\mathrm{R}}$

Chromatic deviation or shift $\Delta \mathrm{E}^{*}$ was calculated with equation: $\Delta \mathrm{E}^{*}=\left[\Delta \mathrm{a}^{* 2}+\Delta \mathrm{b}^{* 2}+\Delta \mathrm{L}^{* 2}\right]^{1 / 2}$

\section{Elaboration of Dry Finishing Technologies for Natural Leathers for Bookbinding}

Dry finishing technologies have been developed for natural grain coloured bovine hides with contrasting, antique and cracked effect (Triderma, 2019; STAHL, 2019).

The framework technology for dry finishing of bovine leather into natural grain box assortments for bookbinding, with contrasting effect, marked P1(P), with antique effect, 
marked $\mathrm{P} 2(\mathrm{P})$ and with cracked effect, marked $\mathrm{P} 3(\mathrm{P})$ and polyurethane final dressing, is presented in Tables 1-3.

Table 1. Framework technology for dry finishing of bovine leathers into natural grain box with contrasting effect

\begin{tabular}{ll}
\hline Operation & \multicolumn{1}{c}{ Dispersion composition/application method } \\
\hline Application of & 80-100 g/L pigment paste (Roda Casicolor Ochre) \\
dispersion I & $30-50 \mathrm{~g} / \mathrm{L}$ aqueous wax emulsion (Roda wax MONO) \\
(basecoat) & 250 g/L acrylic binder (Roda-cryl 87) \\
& $600-640 \mathrm{~g} / \mathrm{L}$ water \\
& Application by spraying (2 passes dispersion I) \\
Intermediate & In hydraulic press with the mirror or fog plate, parameters: \\
pressing & - temperature $-50-60^{\circ} \mathrm{C}$; pressure $-50-100$ atm \\
Application of & 30-50 g/L metalcomplex dye (Roda Brown $\mathrm{H})$ \\
dispersion II & $30-50 \mathrm{~g} / \mathrm{L}$ ethanol \\
& $250 \mathrm{~g} / \mathrm{L}$ acrylic binder (Roda-cryl 87) \\
& $650-690 \mathrm{~g} / \mathrm{L}$ water \\
& Application by spraying (2 passes dispersion I) \\
& The skins are wrinkled before applying dispersion II \\
Application of & $700 \mathrm{~g} / \mathrm{L}$ aqueous polyurethane dispersion (Roda pur 5011) \\
final dressing & 20 g/L aqueous wax emulsion for handle (Roda feel KTA 950) \\
(fixing) & $280 \mathrm{~g} / \mathrm{L}$ water \\
& Application by spraying (2 passes final dressing) \\
& In hydraulic press with the mirror plate, parameters: \\
Final pressing & - temperature $-70-80^{\circ} \mathrm{C} ;$ pressure $-50-100$ atm. \\
\hline
\end{tabular}

Table 2. Framework technology for dry finishing of bovine leathers into natural grain box with antique effect

\begin{tabular}{ll}
\hline Operation & \multicolumn{1}{c}{ Dispersion composition/application method } \\
\hline Application of & $60-80 \mathrm{~g} / \mathrm{L}$ pigment paste (Roda Casicolor Ochre) \\
dispersion I & $30-50 \mathrm{~g} / \mathrm{L}$ aqueous wax emulsion (Roda wax MONO) \\
(basecoat) & $200 \mathrm{~g} / \mathrm{L}$ acrylic binder (Roda-cryl 87) \\
& $100 \mathrm{~g} / \mathrm{L}$ polyurethane binder (Roda-pure 302) \\
& $570-610 \mathrm{~g} / \mathrm{L}$ water \\
& Application by spraying (2 passes dispersion I) \\
Intermediate & In hydraulic press with the mirror or fog plate, parameters: \\
pressing & temperature $-50-60^{\circ} \mathrm{C}$; pressure $-50-100$ atm \\
Application of & $80-100 \mathrm{~g} / \mathrm{L}$ pigment paste (Roda Casicolor Brown) \\
dispersion II & $30-50 \mathrm{~g} / \mathrm{L}$ aqueous wax emulsion (Roda wax MONO) \\
& $100 \mathrm{~g} / \mathrm{L}$ acrylic binder (Roda-cryl 87) \\
& $100 \mathrm{~g} / \mathrm{L}$ polyurethane binder (Roda-pure 302) \\
& $650-690$ g/L water \\
Application by spraying (2 passes dispersion II) \\
Apumming & It is executed in the drum, 2-4 hours \\
final dressing & $700 \mathrm{~g} / \mathrm{L}$ aqueous polyurethane dispersion (Roda pur 5011) \\
(fixing) & $20 \mathrm{~g} / \mathrm{L}$ aqueous wax emulsion for handle (Roda feel KTA 950) \\
& $280 \mathrm{~g} / \mathrm{L}$ water \\
Final pressing & Application by spraying (2 passes final dressing) \\
& In hydraulic press with the mirror plate, parameters: \\
& - temperature - 70-80 ${ }^{\circ}$ C; pressure - 50-100 atm. \\
\hline
\end{tabular}


Table 3. Framework technology for dry finishing of bovine leathers into natural grain box with cracked effect

\begin{tabular}{ll}
\hline Operation & \multicolumn{1}{c}{ Dispersion composition/application method } \\
\hline Application of & $150 \mathrm{~g} / \mathrm{L}$ cationic oil (Eukesol OLGrund AB-1) \\
dispersion I & $850 \mathrm{~g} / \mathrm{L}$ water \\
(basecoat) & Very good drying \\
Application of & $50 \mathrm{~g} / \mathrm{L}$ metal complex dye (Eukesolar) \\
dispersion II & 200-250 g/L protein binder (LURON Glanz E) \\
& $350-400 \mathrm{~g} / \mathrm{L}$ protein binder (LURON Top) \\
& $300-400 \mathrm{~g} / \mathrm{L}$ water \\
& Application by spraying (2 passes dispersion II) \\
Intermediate & In hydraulic press with the mirror plate, parameters: \\
pressing & - temperature $-90^{\circ} \mathrm{C}$; pressure -100 atm \\
Staking & It is executed on the Mollisa staking machine (2 passes) \\
Application of & Application by spraying (2 passes dispersion II) \\
dispersion II & In hydraulic press with the mirror plate, parameters: \\
Intermediate & temperature $-90^{\circ} \mathrm{C} ;$ pressure -100 atm \\
pressing & \\
Drumming & It is executed in the drum, 2-4 hours \\
Application of & $700 \mathrm{~g} / \mathrm{L}$ aqueous polyurethane dispersion (Roda pur 5011) \\
final dressing I & $300 \mathrm{~g} / \mathrm{L}$ water \\
(fixing) & Application by spraying (2 passes final dressing) \\
Final pressing & In hydraulic press with the mirror plate, parameters: \\
& temperature $-80^{\circ} \mathrm{C} ;$ pressure -100 atm \\
Application of & $700 \mathrm{~g} / \mathrm{L}$ aqueous polyurethane dispersion (Roda pur 5011) \\
final dressing II & $20 \mathrm{~g} / \mathrm{L}$ aqueous silicone oil emulsion (Roda feel KTA 950) \\
(fixing) & $280 \mathrm{~g} / \mathrm{L}$ water \\
& Application by spraying (2 passes final dressing) \\
\hline
\end{tabular}

Application of the final dressing was performed in two variants: $\mathrm{P}$ - polyurethane (Roda pur 5011) and N - nitrocellulose (Roda lac 93). Finished leathers for bookbinding using the technologies (application by spraying) for dry finishing presented in Tables 1-3, and nitrocellulose final dressing, with contrasting effect, were marked $\mathrm{P} 1(\mathrm{~N})$, with antique effect, marked P2(N) and with cracked effect, marked P3(N).

Finished natural leathers for bookbinding can be made by applying dispersed systems on the surface of crust leathers, and with the help of modern ecological finishing machines with rollers, which have certain designs, thus obtaining special effects.

\section{Testing Artificially Aged Finished Leather}

Finished leathers were artificially aged and tested according to ISO 17228/2006 standard. Mechanical characteristics of finished natural grain box assortments in the same variants but artificially aged were determined. The following abbreviations were used:

- IT1 - leather aged at $50^{\circ} \mathrm{C}$ for 7 days;

- IT2 - leather aged at $70^{\circ} \mathrm{C}$ for 7 days;

- IL - leather aged with artificial light (Xenotest) for 7 days. 


\section{RESULTS AND DISCUSSION}

\section{Optical Microscopy Analysis of Obtained Leather Assortments}

Optical microscopy images were recorded for bovine hides into natural grain box with contrasting effect, marked P1(P), antique effect, marked P2(P) and cracked effect, marked $\mathrm{P} 3(\mathrm{P})$ and polyurethane final dressing are presented in figure 1 .

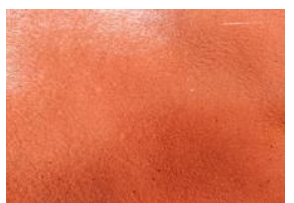

$\mathrm{P} 1(\mathrm{P})$

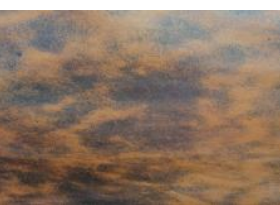

$\mathrm{P} 2(\mathrm{P})$

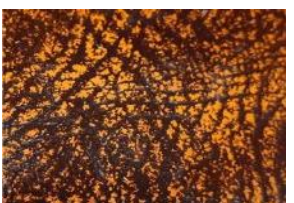

$\mathrm{P} 3(\mathrm{P})$

Figure 1. Optical images recorded for the finished natural grain box bovine leather with contrasting, antique and cracked effect

Assortments of bovine (calves) finished using in the final dressing, aqueous polyurethane dispersion, have a matte appearance, and those finished with aqueous nitrocellulose emulsion, in the final dressing, have a glossy appearance.

\section{Characterization of Finishing Leathers by Colorimetric Method}

Finished leathers were artificially aged and tested according to the CIE LAB system. Chromatic characteristics of natural grain box leather samples P1(P)-P3(P) and P1(N)P3(N) non-aged and aged using the methods IT1, IT2 and IL, are given in Table 4.

Table 4. Values of colorimetric parameters for finished natural grain box leather samples

\begin{tabular}{cccccc}
\hline Sample & CIE L* & CIE a* & CIE b* $^{*}$ & CIE C* & CIE H* $^{*}$ \\
\hline P1(P) & 45.38 & 25.54 & 26.72 & 35.69 & 49.49 \\
P2(P) & 45.67 & 25.61 & 26.98 & 35.95 & 49.73 \\
P3(P) & 45.42 & 24.83 & 25.73 & 25.73 & 49.19 \\
P1(N) & 44.89 & 24.35 & 26.47 & 34.78 & 50.75 \\
P2(N) & 45.20 & 24.48 & 26.60 & 34.88 & 50.74 \\
P3(N) & 45.92 & 24.46 & 26.36 & 34.68 & 50.45 \\
\hline
\end{tabular}

Variation of colorimetric parameters for finished natural leather samples P1(P)P3(P) and P1(N)-P3(N), aged using IT1, IT2 and IL methods is shown in Table 5.

Table 5. Variation of colorimetric parameters for finished aged natural grain box leather samples using IT1, IT2, and IL methods

\begin{tabular}{cccccc}
\hline Sample & MI & $\Delta \mathrm{L}^{*}$ & $\Delta \mathrm{a}^{*}$ & $\Delta \mathrm{b}^{*}$ & $\Delta \mathrm{E}^{*}$ \\
\hline P1(P) & IT1 & -0.30 & -0.02 & -0.08 & 0.33 \\
& IT2 & -0.32 & -0.36 & -0.48 & 0.65 \\
& IL & -0.38 & -0.86 & -0.88 & 1.29 \\
P2(P) & IT1 & -0.28 & -0.15 & -0.09 & 0.28 \\
& IT2 & -0.29 & -0.04 & -0.42 & 0.55 \\
& IL & -0.30 & -0.32 & -0.07 & 0.86 \\
P3(P) & IT1 & -0.24 & -0.44 & -0.08 & 0.26 \\
& IT2 & -0.20 & -0.06 & -0.20 & 0.42 \\
\hline
\end{tabular}

https://doi.org/10.24264/icams-2020.II.21 
Special Effect Finish for Bookbinding Leather

\begin{tabular}{cccccc}
\hline \hline & & & & \\
\hline Sample & MI & $\Delta \mathrm{L}^{*}$ & $\Delta \mathrm{a}^{*}$ & $\Delta \mathrm{b}^{*}$ & $\Delta \mathrm{E}^{*}$ \\
\hline \multirow{2}{*}{ P1(N) } & IL & -0.19 & -0.09 & -0.08 & 0.24 \\
& IT1 & 0.66 & 0.56 & 0.79 & 1.02 \\
& IT2 & 0.78 & 0.39 & 0.87 & 1.28 \\
& IL & 0.27 & 0.28 & 0.36 & 0.62 \\
& IT1 & 0.54 & 0.45 & 0.72 & 1.32 \\
& IT2 & 0.68 & 0.29 & 0.88 & 1.12 \\
& IL & 0.22 & 0.21 & 0.30 & 0.74 \\
& IT1 & 0.86 & 0.63 & 0.65 & 1.25 \\
& IT2 & 0.72 & 0.48 & 0.74 & 1.18 \\
& IL & 0.46 & 0.35 & 0.36 & 0.59 \\
\hline
\end{tabular}

After irradiation and thermal treatment, samples P1(N)-P3(N) have positive values for brightness $\left(\Delta \mathrm{L}^{*}>0\right)$, i.e. they have lighter (brighter) colours than samples $\mathrm{P} 1(\mathrm{P})$ $\mathrm{P} 3(\mathrm{P})$, that has a negative value for brightness $\left(\Delta \mathrm{L}^{*}<0\right)$, indicating the positive influence of polyurethane dressing on fastness to light of leathers compared to the nitrocellulose dressing, on the colour.

\section{CONCLUSIONS}

- The leathers with special effect finish (contrasting antique and cracked effect), can be used for artistic and luxury bookbinding, archival bookbinding and restoration purposes.

- Thermal and artificial light ageing change colorimetric characteristics (CIE $\left.\mathrm{L}^{*} \mathrm{a}^{*} \mathrm{~b}^{*}\right)$ compared to those of unaged samples, depending on the ageing method, leather assortment and type of final dressing. The highest values for fastness to light after artificial light ageing were those of leathers finished with polyurethane dressing and the lowest, those finished with nitrocellulose dressing.

\section{Acknowledgements}

These works were carried out with the support of Nucleus Program, TEX-PELVISION 2022, 4N/8.02.2019/Act ad.1/2019, with the support of the Ministry of Research and Innovation, project number PN 19170201 /2020, and within Program 1 - Development of the national RD system, Subprogram 1.2-Institutional PerformanceRDI excellence funding projects, Contract no. 6PFE/16.10.2018.

\section{REFERENCES}

Heidemann, E. (1994), Fundamentals of Leather Manufacturing, Eduard Roether KG Druckerei und Verlag, Darmstadt.

Lange, J. (1982), Leather Finishing, Leather library vol. V, Umschau, Frankfurt.

Niculescu, O., Leca, M., Moldovan, Z., Chelaru, C. and Gurău, D., (2015a), Obtaining and characterizing of an ecologic wax emulsions for finishing natural leathers and furs, Revista de Chimie (Bucharest), 66(8), 1173-1176

Niculescu, O., Leca, M., Staicu, T., Micutz, M. and Moldovan, Z., Chelaru, C. and Berechet, D.M., (2015b), Obtaining and characterizing of some ecologic pigment pastes for finishing natural leathers and furs, Revista de Chimie (Bucharest), 66(9), 1278-1281.

Niculescu, O., Moldovan, Z., Leca, M., Chelaru, C. and Alexandrescu, L., (2015c), Characteristics of natural leather surface finished with some ecofriendly mixtures of polymeric aqueous dispersions, J. Polymer Eng., 35(6), 463-470.

Niculescu, O. and Mendea, F. (2019), Inorganic pigment pastes for natural leather finishing, Patent OSIM A/130001/2019.

https://doi.org/10.24264/icams-2020.II.21 
NEW NANOSTRUCTURED COMPOSITE OBTAINED BY INNOVATIVE TECHNOLOGIES

\author{
DEMETRA SIMION $^{1 *}$, CARMEN GAIDĂU $^{1}$, JIANZHONG MA² $^{2}$ ZHANG WENBO ${ }^{2}$ \\ ${ }^{1}$ INCDTP - Division Leather and Footwear Institute (ICPI), 93 Minulescu Ion Str., 031215, \\ Bucharest, Romania, demetra.simion@yahoo.com \\ ${ }^{2}$ Shaanxi University of Science and Technology Division Shaanxi Collaborative Innovation \\ Center of Industrial Auxiliary Chemistry and Technology, Xi'an 710021, China
}

\begin{abstract}
The paper is focused on obtaining a new nanostructured composite by innovative technologies based on: fibrillar cellulose/titanium dioxide/surfactants (bolaform- dodecandioyl-diglycine and surfactant classic-collagen hydrolysate)/ethanol/water, for improved surface properties. Innovation consists in technologies for obtaining new nanostructured composites, solubilisation/compatibilisation of their component substances for the conditioning of supports processed with the film created by evaporation of the emulsion nanocomposites. Fibrillar cellulose/titanium dioxide nanocomposites have been stabilized with bolaform surfactants in a 1:1 ratio of ethanol/water solvents in order to increase the uniformity of titanium dioxide shell nanocomposites. Nanostructured "cauliflower"like composites developed as a result of biopolymer-surfactant interactions for fibrillar cellulose/titanium dioxide/bolaform couple in ethanol/water system are reported by SEM microscopy. The analysis by FTIR-ATR spectroscopy of bolaform, fibrillar cellulose and dynamic light scattering of 2 types of nanocomposites emulsions (with bolaform and classic surfactant emulsions) were reported. The new nanocomposites could provide the hybrid film with increased mechanical resistance to water and heat. Supports processing with the composite film improve wet/dry friction resistance, water resistance and tensile strength. Environmentally-friendly supports with smart multifunctional features are obtained for various applications.
\end{abstract}

Keywords: hybrid nanocomposites, fibrillar cellulose/titanium dioxide/surfactants, innovative technologies, improved surface properties

\title{
INTRODUCTION
}

A new nanostructured composite was created by innovative technologies based on fibrillar cellulose/titanium dioxide/(bolaform-dodecandioyl-diglycine and classic-collagen hydrolysate)/ethanol/water, for different applications (leather or plastics industry) (Kang, S.M. et al., 2006). The trend is to develop new materials with multiple functions and added value, with low environmental impact and without harmful emissions of pollutants that can affect the climate balance (Jing, S. et al., 2007). This paper brings a solution to avoid environmental pollution and meets the increasing demands for an advanced economy by capitalizing on the cellulose-based by-products from the wood industry which are currently less exploited resources (Trandafir, V. et al., 2008). The high potential of using cellulose as a material for various applications: in membrane technologies for pollutant absorption, in pharmacy, etc. opens the way to obtaining new added value materials (hybrid nanocomposites) (Simion, D. et al., 2009). The interaction of classic surfactants (amphiphilic molecules with a single hydrocarbon chain and a hydrophilic head group) with biopolymers in aqueous medium results in the formation of different association structures (Niculescu, M.D. et al., 2013). There are various morphologies of biopolymer-surfactant association complexes depending on the molecular structure of the biopolymer and surfactant, on the nature of interaction forces between solvents and surfactant or biopolymer (Xu, Q. et al., 2013). Bolaamphiphiles have the ability to self-organize at water/air or water/solvent interfaces. In solution they can selfassemble in structures such as core-shell, fibers, ribbons, tubules, planar monolayers and multilayers, depending on various factors (Ma, J. et al., 2013). Compared with classic tensides, the introduction of a second hydrophilic group to the bolaamphiphiles increases their water 
solubility and the critical micellar concentration, and decreases the aggregation number (Ma, J. et al., 2007). pH is a basic parameter which influences the association structures (Gaidău, C. et al., 2007). As an example, it is mentioned the $\mathrm{pH}$-sensitive structural transformation for a peptidic bolaform. The bolaforms might be used as template in the synthesis of nanocomposites, developing nanotubes, fabrication of nanostructures assemblies with many applications. A special class of nanoarchitectures is represented by multi sheets nanostructures organized as flower-like assemblies (Ma, J. et al., 2008). Such nanoarchitectures were reported, where nanosheets stack layer-by-layer onto multisheets architectures like flower petals. That hierarchical architectures had properties that made them appropriate for preparation of superhydrophobic surfaces.

\section{EXPERIMENTAL}

\section{Materials and Methods}

\section{Materials}

For obtaining of new nanostructured compsites the following materials have been used: titanium dioxide from Sigma-Aldrich (nanoparticles, molecular weight $79.87 \mathrm{~g} / \mathrm{mol}$, particle size: $200 \mathrm{~nm}$ ); tenside based on hydrolysates of collagen HCN6CV. Collagen extract - HCN6CV used as a tenside due to its specific properties was obtained by alkaline-enzymatic hydrolysis of leather waste, quality filtration and vacuum concentration in a 3:1 ratio; dodecandioyl-diglycine from Sigma-Aldrich; fibrillar cellulose from SERVA Feinbiochemica GmbH \& Co. Crust leathers processed in Leather Research Department were used for finishing trials with new nanocomposites.

\section{Nanocomposites Preparation}

A number of 10 samples of fibrillar cellulose/surfactant (classic or bolaamphiphile)/ titanium dioxide/ethanol/water were prepared in the following working conditions: waterethanol solvents at ratio $1: 1$, temperature $=60^{\circ} \mathrm{C}$ at 30 minutes with fibrillar cellulose$\mathrm{c}=1 \%$; titanium dioxide-c=0.2\%, fig.1.; samples 1, 2: fibrillar cellulose/classic surfactant/titanium dioxide/ethanol/water with classical surfactant concentration 1-c=1\%; 2-c $=2 \%$; samples 3-10: fibrillar cellulose/bolamphiphile/titanium dioxide/ethanol/water with the concentration of bolaform $3-\mathrm{c}=1 \% ; 4-\mathrm{c}=2 \% ; 5-\mathrm{c}=3 \% ; 6-\mathrm{c}=4 \% ; 7-\mathrm{c}=5 \% ; 8$ $\mathrm{c}=6 \% ; \mathbf{9}-\mathrm{c}=7 \% ; \mathbf{1 0}-\mathrm{c}=8 \%$.

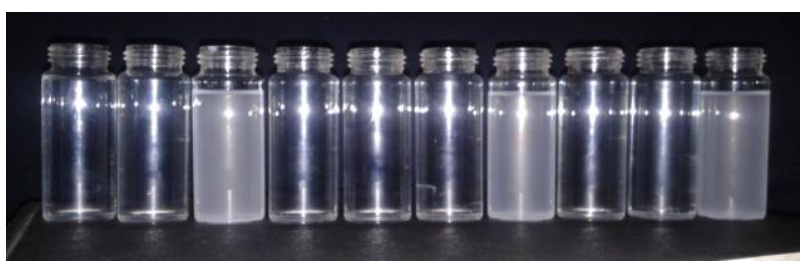

Figure 1. Photographic images of 10 samples of nanostructured composites

The "cauliflower"-like architectures were observed only for bolaform (samples $\mathbf{3 ,} \mathbf{7}$, 10) and not for the classic surfactant. 


\section{Nanocomposite Characterisation}

The characterisation techniques used in this paper consist in infrared spectral analyses (FT/IR-ATR Jasco, spectrophotometer, model 4200), electronic scanning microscopy (SEM QUANTA 200”, FEI) and dynamic light scattering tests (Zetasizer Nano-ZS, MALVERN, UK) with measuring range between $0.3 \mathrm{~nm}-10.0$ microns and zeta potential determination with an accuracy of $+/-2 \%$.

\section{Leather Surface Finishing with New Nanocomposite and Characterisation}

The classical technologies were performed (Table 1) by spraying different composites for waterproofing (Recipe C3), for base and for top coat layers, adding the new structured "cauliflower" emulsion in base coat (Recipe C2) or in top coat (Recipes C1 and C1_1). The difference between recipe $\mathrm{C} 1$ and $\mathrm{C} 1$ 1 was the concentration of "cauliflower" emulsion, from $\mathrm{x} \%$ to $2 \mathrm{x} \%$ in the top coat layer.

Table 1. Different compositions for leather surface covering with new "cauliflower" emulsion

\begin{tabular}{|c|c|c|c|c|c|c|}
\hline \multirow[t]{2}{*}{ Materials } & \multirow{2}{*}{$\begin{array}{c}\text { Waterproofing } \\
\text { coat } \\
\text { New } \\
\text { "cauliflower" } \\
\text { composite } \\
\text { emulsion }\end{array}$} & \multicolumn{3}{|c|}{ Base coat } & \multicolumn{2}{|c|}{ Top coat } \\
\hline & & $\begin{array}{c}\text { Compact } \\
\text { acrylic } \\
\text { binder }\end{array}$ & $\begin{array}{c}\text { Pigment } \\
\text { paste }\end{array}$ & $\begin{array}{c}\text { New } \\
\text { "cauliflower" } \\
\text { composite } \\
\text { emulsion }\end{array}$ & $\begin{array}{l}\text { Nitrocel- } \\
\text { lulose } \\
\text { lacquer } \\
\text { emulsion }\end{array}$ & $\begin{array}{c}\text { New } \\
\text { "cauliflower" } \\
\text { composite } \\
\text { emulsion }\end{array}$ \\
\hline Control & - & $\mathrm{x}$ & $\mathrm{x}$ & - & $\mathrm{X}$ & - \\
\hline Recipe C1 & - & $\mathrm{x}$ & $\mathrm{x}$ & - & $\mathrm{x}$ & $\mathrm{x}$ \\
\hline Recipe C1_1 & - & $\mathrm{x}$ & $\mathrm{x}$ & - & $\mathrm{x}$ & $\mathrm{xx}$ \\
\hline Recipe $\mathrm{C} \overline{2}$ & - & $\mathrm{x}$ & $\mathrm{x}$ & $\mathrm{x}$ & $\mathrm{x}$ & $\mathrm{x}$ \\
\hline Recipe C3 & $\mathrm{x}$ & $\mathrm{x}$ & $\mathrm{x}$ & $\mathrm{x}$ & $\mathrm{x}$ & $\mathrm{x}$ \\
\hline
\end{tabular}

Sheepskin leathers covered with selected nano and micro structured "cauliflower"-like emulsion composite were analysed for resistance to abrasion (SR EN ISO 13520:2003) and for rubbing resistance at dry and wet rub cycles (SR EN ICO 11640:2013) as compared to the control sample covered with the same recipe but without the new composite.

\section{RESULTS AND DISCUSSION}

Structured nanocomposites are formed and the properties derive from the surfactants used, as well as the conditions and working parameters. This phenomenon is controlled by the concentration of biopolymer, bolaform (dodecandioyl-diglycine), titanium dioxide, the hydrophilic nature of biopolymer, ethanol/water ratio, $\mathrm{pH}=4.5$.

There are various morphologies of biopolymer-surfactant association complexes depending on the molecular structure of the biopolymer and surfactant, on the nature of interaction forces between solvents and surfactant or biopolymer.

FTIR-ATR analyses (Table 2) showed that in surfactant/cellulose microemulsion systems not only physical associations are expected by means of hydrogen bonds, but also esterification reactions are possible with a different chemical structure. 
New Nanostructured Composite Obtained by Innovative Technologies

Table 2. Characteristic vibration bands $\left(\mathrm{cm}^{-1}\right)$ in FT/IR-ATR spectra of surfactant and biopolymer

\begin{tabular}{|c|c|c|}
\hline $\begin{array}{c}\text { Bolaamphiphile: } \\
\text { Dodecandioyl-diglycine; } \mathrm{n} \text {, } \\
{\left[\mathrm{cm}^{-1}\right]}\end{array}$ & $\begin{array}{l}\text { Fibrillar } \\
\text { cellulose; } \\
\mathrm{n},\left[\mathrm{cm}^{-1}\right]\end{array}$ & Assignments \\
\hline & 3342 & OH stretching \\
\hline 3303 & & NH stretching \\
\hline 2915 & 2894 & $\mathrm{CH}_{2}$ symmetrical and asymmetrical \\
\hline 2847 & & stretching \\
\hline & & $\mathrm{C}=\mathrm{O}$ stretching \\
\hline 1634 & 1642 & amide $\mathrm{I}$ band, $\mathrm{C}=\mathrm{O}$ stretching \\
\hline 1547 & & amide II band, NH deformation \\
\hline 679 & 667 & $-\left(\mathrm{CH}_{2}\right)_{n^{-}}, \mathrm{n}>3$ deformation \\
\hline
\end{tabular}

The SEM micrograph of fibrillar cellulose is presented in Figure 2.

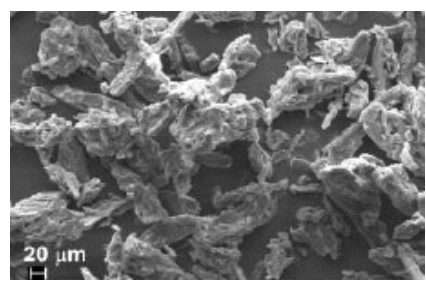

Figure 2. SEM micrograph of fibrillar cellulose

The association complexes with "cauliflower"-like morphologies begin to appear at high concentration of bolaform at a certain ethanol/water/titanium dioxide ratio, above critical concentration and for $\mathrm{pH}=4.5$, as seen in Fig. 3. The nanosheet microstructures built as "cauliflower"-like morphologies are the result of interaction of cellulose/titanium dioxide with glycine-based bolaamphiphile. Although bolaform and cellulose are not aromatic compounds (no $\pi-\pi$ stacking interactions), as in the case of aniline, they succeed in creating "cauliflower"-like structures in a fibrillar cellulose/titanium dioxide/(bolaform-dodecandioyl-diglycine)/ethanol/water system.
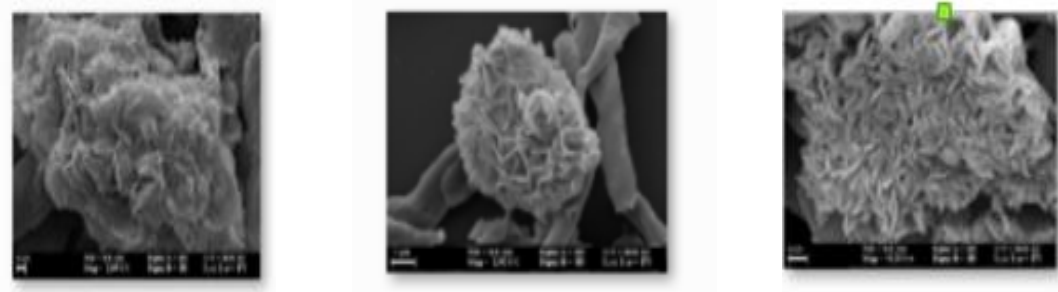

Figure 3. SEM micrographs of "cauliflower"-like morphology in fibrillar cellulose/titanium dioxide/(bolaform-dodecandioyl-diglycine)/ethanol/water composites

Efforts in leather processing aim at beneficial effects on the environment with zero waste and low energy consumption. "Cauliflower"-like association nanostructures composites developed as the result of biopolymer-surfactant interactions for fibrillar cellulose/titanium dioxide/bolaamphiphile couple in ethanol/water system are reported. 
The morphology of "cauliflower"-like association complexes is noticed only in the case of dodecandioyl-diglycine bolaamphiphile as compared with classic single chain surfactant-collagen hydrolysate. This phenomenon is controlled by the concentration of: biopolymer (fibrillar cellulose), bolaform (dodecandioyl-diglycine), titanium dioxide, the hydrophilic nature of biopolymer, ethanol/water ratio, $\mathrm{pH}$.

Dynamic light scattering test showed that all three types of nanocomposites are nano and microstructured. The fibrillar cellulose/bolaform/titanium dioxide/solvents nanocomposites have sizes ranging between $40 \mathrm{~nm}, 220 \mathrm{~nm}$ and $5000 \mathrm{~nm}$ without stirring and sizes of $80 \mathrm{~nm}$ and $400 \mathrm{~nm}$ after 10 minutes of mechanical stirring. The recorded zeta potential was of $-13.5 \mathrm{mV}$.

In view of testing the micro and nano "cauliflower" emulsion functionality, sample 3 from Fig. 1 was selected for quality improvement of leather surface finishing (Fig. 4) due to the special architecture and zeta potential value which can generate resistance to mechanical stress and stability in film forming polymers.

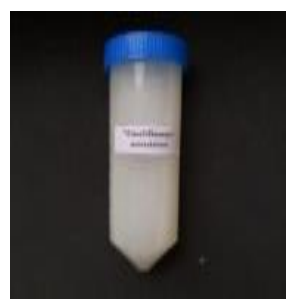

Figure 4. "Cauliflower" emulsion composite based on fibrillar cellulose/titanium dioxide/bolaform- dodecandioyl-diglycine - sample 3 from Fig. 1

\section{Leather Surface Properties Improvement by Using Nano and Micro Structured "Cauliflower"-like Emulsion Composite}

The leather surfaces covered with different recipes of finishing composites as compared to the control samples (without structured "cauliflower"-like emulsion composite) are presented in Fig. 5a). The aspect of leather surface covered with "cauliflower"-like emulsion composite showed the ability to cover imperfections and improve the final classification of leather quality as compared to control samples.

The main physical characteristics of new leather surface finishing showed resistance to wet rub and to abrasion as compared to control sample. In Fig. 5b) the control sample after the abrasion test shows cracks and the sample $\mathrm{C}_{-} \_1$ surface is undamaged. In Table 3 it can be seen that the sample $\mathrm{C} 1 \_1$ has slightly better resistance to wet rubbing and substantial improvement of abrasion resistance.
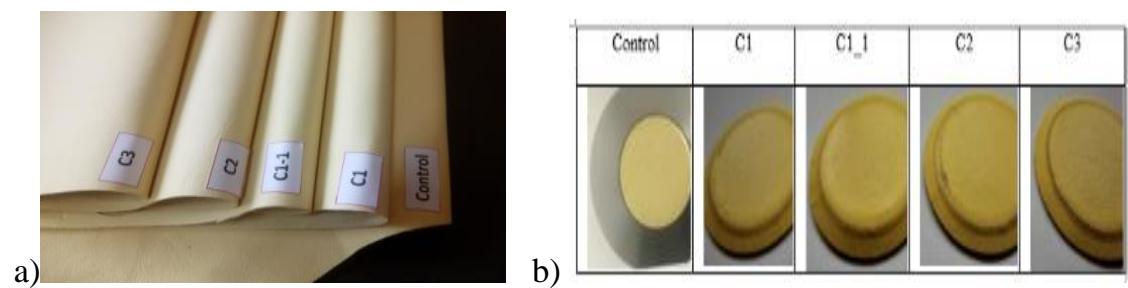

Figure 5. a) Leathers covered with different compositions of finishing layers with "cauliflower" emulsion as compared to control sample (without emulsion); b) abrasion tests 
New Nanostructured Composite Obtained by Innovative Technologies

Table 3. Physical tests of leather surface covered with finishing composites with new "cauliflower" emulsion

\begin{tabular}{cccccc}
\hline Characteristics & $\mathrm{M}$ & $\mathrm{C} 1$ & $\mathrm{C} 1 \_1$ & $\mathrm{C} 2$ & $\mathrm{C} 3$ \\
\hline $\begin{array}{c}\text { Resistance to 50 wet } \\
\text { rubbing, cycles }\end{array}$ & $5 / 4-5$ & $5 / 4-5$ & $5 / 5$ & $4-5 / 4$ & $4 / 4-5$ \\
$\begin{array}{c}\text { Resistance to abrasion, } \\
\text { no of revolutions }\end{array}$ & 25600 & 25600 & 51200 & 25600 & 25600 \\
\hline
\end{tabular}

The main advantages of new finishing coat is the quality improvement of leather classification and durability due to the better covering power, wet rubbing and abrasion resistance provided by the new micro and nano structured "cauliflower" emulsion. The research is in progress regarding the identification of new properties of leather surface finished with the new smart structured emulsion.

\section{CONCLUSIONS}

The successful preparation of cellulose/titanium dioxide/surfactant composites with "cauliflower"-like morphology for leather finishing was presented. The analyses results showed that rubbing and abrasion resistance can be improved for more durable leather goods manufacture in the spirit of European ecological label principles.

\section{Acknowledgments}

These works were supported by Romanian Ministry of Research and Innovation and CCCDI-UEFISCDI, under bilateral cooperation project Romania-China_HNAN, within PNCD III, project no.18/2018; project number PN-III-P2.1-PTE-2019-0655, contract 5PTE, BIOTEHKER, and 4PERFORM-TEX-PEL project.

\section{REFERENCES}

Gaidău, C. et al. (2007), "Contribution to characterization of a new organic tanning material", Revista de Chimie, 58(8), 768-77.

Jing, S. et al. (2007), "Synthesis and characterization of Ag/polypyrrole nanocomposites based on silver nanoparticles colloid", Materia Lett, 61, 4528-4530, https://doi.org/10.1016/j.matlet.2007.02.045.

Kang, S.M. et al. (2006), "Biomimetic approach to the formation of gold nanoparticle/silica core/shell structures and subsequent bioconjugation", Nanotechnology, 17, 4719-4735, https://doi.org/10.1088/09574484/17/18/032.

Ma, J. et al. (2007), "Polyacrylate/silica nanocomposite materials prepared by sol-gel process", European Polymer Journal, 43, 4169-4177, https://doi.org/10.1016/j.eurpolymj.2007.06.051.

Ma, J. et al. (2008), "Plasticity studies on leather retanned with various types of acrylic polymers", Journal of the American Leather Chemists Association, 103(11), 363-369, SCI:374YD, EI:20085111791317.

$\mathrm{Ma}$, J. et al. (2013), "Synthesis and biological response of casein-based silica nano-composite film for drug delivery system", Colloids and Surfaces B: Biointerfaces, 111, 257-263, https://doi.org/10.1016/j.colsurfb.2013.06.011.

Niculescu, M.D. et al. (2013), Patent: "Process for extracting proteins from chrome leather wastes and reduceing the average molecular weight for collagen hydrolysates", HO, No.3/41 of 30.04.2013.

Simion, D. et al. (2009), Patent: "Bolaamphiphilic material with ester structure and the process for obtaining it", No. 122542/2009, OSIM, Part 1, No. 541/08.08.2007.

Trandafir, V. et al. (2008), Patent: "Bioactive compounds and its obtaining process", No.121670/28.02.2008, OSIM, Part 1, No.541/08.08.2007.

$\mathrm{Xu}$, Q. et al. (2013), "Bio-based core-shell casein-based silica nano-composite latex by double-in situ polymerization: Synthesis, characterization and mechanism", Chemical Engineering Journal, 228, 281-289, https://doi.org/10.1016/j.cej.2013.04.079.

https://doi.org/10.24264/icams-2020.II.22 


\title{
INFLUENCE OF TYPE OF PRE-HAIR TREATMENT FROM DIFFERENT TYPES OF ANIMAL SOURCES ON THE DEGREE OF HYDROLYSIS OF KERATIN
}

\author{
DARINA ZHELEVA, MARGARITA KOLEVA \\ University of Chemical Technology and Metallurgy, Department of Textile and Leather, Sofia, \\ Bulgaria; e-mail: darinajeleva@abv.bg
}

\begin{abstract}
Keratin biomaterials have many different advantages over other biomolecules. A number of techniques have been studied to prepare keratin hydrolysates. Many of them use strong reagents and the processes take place under very drastic conditions. The present study focuses on the following aspects: producing keratin hydrolysates from various animal sources; application of various methods for extraction; comparison of the type of treatment over the degree of hydrolysis. Sheep wool samples were used, respectively native and alkaline pre-treated and samples of goat hair, respectively native and enzyme pre-treated. The methods used for the hydrolysis of keratin materials are: 1) by sulfotolysis with sodium pyrosulfate and urea; 2) with thioglycolic acid and 3) with sodium hydroxide. The obtained hydrolysates were characterized by qualitative reactions, spectrophotometric and FTIR analysis. It was found that the samples from one and the same animal source show very different properties and different degrees of hydrolysis. The highest degree of hydrolysis was achieved for the pre-treated samples. It was proved that the method of hydrolysis with $\mathrm{NaOH}$ is the most appropriate for sheep wool and to a much greater extent for the alkaline treated wool than for the native. The reducing agent: sodium pyrosulfate and urea is the most appropriate for enzyme pre-treated samples of goat skin. Therefore, pre-treatment of animal hair samples facilitates the hydrolysis process and makes it easier to break disulfide bonds. The disadvantage of proteins, and in particular keratins, is the difference in the structure of macromolecules, which are obtained from different animal sources. Therefore, this requires a specific approach to the hydrolysis of keratin from each individual animal source.
\end{abstract}

Keywords: keratin, hydrolysates, analyzes

\section{INTRODUCTION}

Keratin is of considerable interest as a new product for use in the pharmaceutical, medical, cosmetic and biotechnology industries. Keratin is a healthy, insoluble biomaterial that can play a fundamental structural role in many biological systems (Rouse and Van Dyke, 2010). The amino acid composition changes depending on the type of keratin. The presence of a significant amount of cystine in keratin explains the presence of another type of cross-covalent bond between the main polypeptide chains, namely the disulfide bond $\mathrm{S}-\mathrm{S}$-, due to which keratin is very resistant to various physicochemical effects. Upon hydrolysis or reduction of the disulfide bond, free sulfhydryl groups - $\mathrm{SH}$ are formed. They can participate in exchange reactions with the disulfide bonds. As a result, the position of the disulfide bond in the keratin macromolecule may be altered, i.e. from transverse interchain to intrachain and vice versa (Pesheva, 1982):

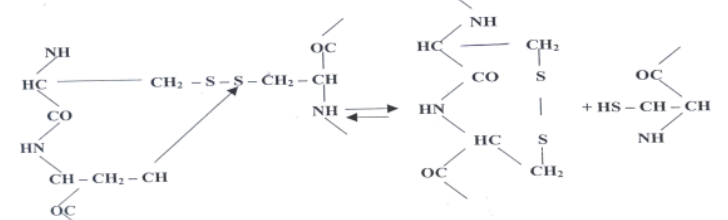

This leads to the destruction of keratin, and the degree of destruction depends on the type of pre-treatment and the conditions under which it is carried out.

Disulfide bonds are relatively resistant to acids. The bases act on all bonds in keratin. At high temperatures and high hydroxide concentrations, the peptide bonds in the major molecular chains are attacked. The disulfide bond in keratin is the most vulnerable place in its treatment with alkalis.

https://doi.org/10.24264/icams-2020.II.23 
The action of reducing agents on keratin leads to significant changes in some of its physicochemical and mechanical properties, which is used to ennoble the hair cover (Pesheva and Papazyan, 1990). The reduction of keratin is associated primarily with the destruction of disulfide bonds and the addition of new groups at the sites of released valences. The type of the newly formed group depends on the type of reducing agent and the process conditions.

The unhairing processes in leather production is carried out using sodium sulfide, which reduces the disulfide bond and results in a destruction of the hair due to the destruction of the cross-bridges between polypeptide chains of keratin as follows:

$$
\mathrm{R}-\mathrm{S}-\mathrm{S}-\mathrm{R}_{1}+2 \mathrm{Na}_{2} \mathrm{~S} \rightarrow \mathrm{R}-\mathrm{SNa}+\mathrm{R}_{1}-\mathrm{SNa}+\mathrm{Na}_{2} \mathrm{~S}_{2}
$$

Thioglycolic acid and its salts have a strong reducing effect on the disulfide bond:

$$
\mathrm{R}-\mathrm{S}-\mathrm{S}-\mathrm{R}+2 \mathrm{HS}-\mathrm{CH}_{2}-\mathrm{COOH} \rightarrow 2 \mathrm{R}-\mathrm{SH}+2 \mathrm{~S}-\mathrm{CH}_{2}-\mathrm{COOH}
$$

This reaction proceeds very rapidly in an alkaline medium, for example in $0.5 \mathrm{M}$ sodium thioglycolate at $30^{\circ} \mathrm{C}$ and $\mathrm{pH}=12$, at which the sheep's wool dissolves completely in 3 hours.

Research on the hydrolysis of keratins has been going on for many years. Some studies rely on long-term acid hydrolysis with concentrated sulfuric acid, other methods are carried out using reducing agents - thioglycolic acid, sodium cyanide, sodium sulfide, others use hydroxides - $\mathrm{NaOH}, \mathrm{KOH}, \mathrm{Ca}(\mathrm{OH}) 2$.There are also studies with the use of enzymes to denature keratin. All these methods have been known for many years, but the application of most of them is extremely difficult. During the hydrolysis, in addition to breaking both types of bonds (disulfide and peptide), the resulting structure of keratin hydrolysates is different from the structure of keratin protein (Mokrejs et al., 2011; Krejci et al., 2011; Yin et al., 2013; Cardamone et al., 2009; Cardamone, 2010; Saravanan et al., 2013; Hikima and Nonomura, 2008; Rivalcola and Martinez, 2011; Aluigi and Tonetti, 2011; Zoccola et al., 2009; Xing et al., 2011; Yang et al., 2007; Gupta et al., 2012).

The production and use of keratin hydrolysates of waste products from various industries is a topical issue. Many researchers have investigations in this field. Hydrolysis is performed under drastic conditions and aggressive reagents. The optimization of hydrolysis methods is of great importance for environmental protection, as well as for obtaining energy-saving technologies in this area.

The present study focuses on the following aspects:

- Producing keratin hydrolysates from various animal sources;

- Application of different methods for extraction;

- Comparison of the type of treatment over the degree of hydrolysis.

\section{MATERIALS AND METHODS}

In our research on the production of keratin hydrolysates, samples of goat skin coat and sheep wool were used, respectively in native form and pre-treated. Enzyme pre-treated hair cover was obtained after enzyme unhairing of goat skins. The wool, which is lime-sulphide treated, is obtained after the process of unhairing of sheepskins under certain conditions.

\section{Methods for Hydrolysis of Keratin-Containing Samples}

\section{Hydrolysis with Sodium Pyrosulphate (Metabisulphate)}

Keratin is extracted from wool by sulfitolysis with sodium metabisulfate (Aluigi and Tonetti, 2011). Approximately $5 \mathrm{~g}$ of the cleaned and conditioned fibers are treated with $100 \mathrm{~mL}$ of a solution containing urea $(8 \mathrm{M})$, sodium pyrosulphate $\left(\mathrm{Na}_{2} \mathrm{~S}_{2} \mathrm{O}_{5}\right)(0.5 \mathrm{M})$. The treatment was continued until $\mathrm{pH} 6.5$ with $\mathrm{NaOH}(5 \mathrm{~N})$ by stirring during $2 \mathrm{~h}$ at $65^{\circ} \mathrm{C}$. 
Hydrolysis with Thioglycolic Acid

The samples were hydrolysed in an aqueous solution of $0.5 \mathrm{M}$ thioglycolic acid under heating at $30^{\circ} \mathrm{C}$ for 6 hours in a water bath (Gupta et al., 2012).

Hydrolysis with Sodium Hydroxide

Cleaned and washed bird feathers are cut (Saravanan et al., 2013). They were then dissolved in $5 \% \mathrm{NaOH}$ solution for 4 hours at $40^{\circ} \mathrm{C}$. The resulting solution was dialyzed and precipitated with concentrated $\mathrm{HCl}$ at $\mathrm{pH} 4.2$.

\section{Methods for Analysis of Protein Substances}

\section{Biuret Method}

Qualitative and quantitative determination of protein can be performed by the biuret method (Jotova and Dobrev, 2000).

\section{Spectrophotometric Method}

The solutions are poured in a cuvette with a width of $10 \mathrm{~mm}$ at the wavelength $\lambda=$ $540 \mathrm{~nm}$. The apparatus used is a type JENWAY 6300 Spectrophotometer.

\section{Methods for Determination of Sulfur-Containing Amino Acids}

They are typical for proteins containing cystine and cysteine (Jotova and Dobrev, 2000).

\section{Nitroprusside Reaction}

$0.5 \mathrm{~mL}$ protein solution is mixed with $0.5 \mathrm{~mL}$ of $10 \% \mathrm{NaOH}$ solution and heated during 3 mins. After cooling, is added 2-3 drops of sodium nitroprusside. A reddishbrown color appears.

\section{Fol's Reaction}

A solution of $\mathrm{NaOH}$ was gradually added to $1 \mathrm{~mL}$ of lead acetate solution until the precipitate of lead hydroxide formed dissolved. Then add $0,5-1,0 \mathrm{~mL}$ of the tested protein solution and heat until a black color appears.

\section{Methods for Using Infrared Spectroscopy (FTIR)}

Infrared spectroscopy is a method of molecular absorption spectroscopy (Andreev, 2010). The analysis was performed using a Bruker Tensor 27 Spectrometer with a scanning speed of $10 \mathrm{kHz}$. The spectrum was recorded using an MCT detector (64 scans and $1 \mathrm{~cm}^{-1}$ resolution).

\section{EXPERIMENTAL}

Wool and goat hair samples are washed, degreased and conditioned. Each sample of the two types of hair cover is finely chopped and weighed to calculate the parameters of the obtained hydrolysates. The following methods were used:

1) hydrolysis with thioglycolic acid and urea;

2) sulphitolysis with sodium pyrosulphate and urea;

3) hydrolysis with $5 \% \mathrm{NaOH}$. 
Influence of Type of Pre-Hair Treatment from Different Types of Animal Sources on the Degree of Hydrolysis of Keratin

\section{Hydrolysis of Keratin Products from Sheep Wool}

Table 1. Results of the analysis of keratin hydrolysates from wool

\begin{tabular}{|c|c|c|c|c|}
\hline $\begin{array}{l}\text { Type of sample } \\
\text { /Wool/ }\end{array}$ & $\begin{array}{l}\text { Method of } \\
\text { hydrolysis }\end{array}$ & Biuret reaction & $\begin{array}{l}\text { Nitroprusside } \\
\text { reaction }\end{array}$ & Fol's reaction \\
\hline 1. Native wool & $\begin{array}{l}\text { thioglycolic } \\
\text { acid and urea }\end{array}$ & $\begin{array}{l}\text { Orange-yellow } \\
\text { coloring }\end{array}$ & $\begin{array}{l}\text { Does not give } \\
\text { coloring }\end{array}$ & $\begin{array}{c}\text { Black } \\
\text { coloring }\end{array}$ \\
\hline $\begin{array}{l}\text { 2. Lime-sulfide } \\
\text { pre-treated wool }\end{array}$ & $\begin{array}{l}\text { urea and } \\
\text { sodium } \\
\text { pyrosulfate }\end{array}$ & $\begin{array}{l}\text { Light violet } \\
\text { coloring } \\
A=0,167\end{array}$ & $\begin{array}{l}\text { Does not give } \\
\text { coloring }\end{array}$ & $\begin{array}{l}\text { Does not give } \\
\text { coloring }\end{array}$ \\
\hline 3. Native wool & $5 \% \mathrm{NaOH}$ & $\begin{array}{l}\text { Light violet } \\
\text { coloring } \\
\mathrm{A}=0,154\end{array}$ & $\begin{array}{l}\text { Slight brown } \\
\text { and then } \\
\text { disappears }\end{array}$ & $\begin{array}{l}\text { Does not give } \\
\text { coloring }\end{array}$ \\
\hline $\begin{array}{l}\text { 4. Lime-sulfide } \\
\text { pre-treated wool }\end{array}$ & $5 \% \mathrm{NaOH}$ & $\begin{array}{l}\text { Violet coloring } \\
\qquad \mathrm{A}=0,568\end{array}$ & Red coloring & $\begin{array}{l}\text { Does not give } \\
\text { coloring }\end{array}$ \\
\hline
\end{tabular}

In the spectra of keratin hydrolysates from the wool samples, the absorption bands for Amide III (related to the vibrational states of $\mathrm{C}-\mathrm{N}$ and N-H) and Amide I (Figures 2 , 3 and 4) were observed. Absorption band at 1000-1025 $\mathrm{cm}^{-1}$ indicates the presence of cysteine residues or sulfur-containing amino acids, and at $630-625 \mathrm{~cm}^{-1}$ indicates the presence of S-S bonds. Rupture of the disulfide bonds was observed in the lime-sulfide pre-treated wool (Fig.2 and Fig.4), as well as in the hydrolysis of the native wool with sodium hydroxide (Fig.3), in contrast to the hydrolysis of the native wool with thioglycolic acid (Fig.1). It is known that under the action of bases, hydrolysis of the disulfide bond initially occurs. FTIR observations correlate with the qualitative and quantitative methods described above.

The hydrolyzing effect of the three methods was compared, as well as the influence of the preliminary chemical treatment of the wool. Sodium hydroxide has the strongest hydrolyzing effect on both native and lime-sulfide pre-treated wool. Pre-treatment of the wool during unhairing process with calcium hydroxide and sodium sulfide significantly facilitates hydrolysis. This is due to the preliminary breaking of part of the disulfide bridges during alkaline treatment. This is confirmed by the literature. The presence of keratin protein was confirmed quantitatively and qualitatively by biuret reaction and photometrically. The infrared spectra also confirmed the extent to which disulfide and peptide bonds are depleted depending on the intensity of the adsorption bands at the respective wavelengths.

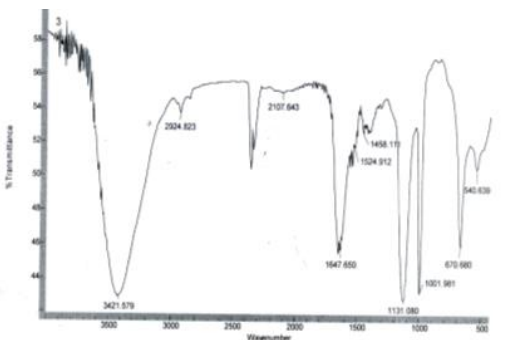

Figure 1. FTIR of native wool (thioglycolic acid and urea)

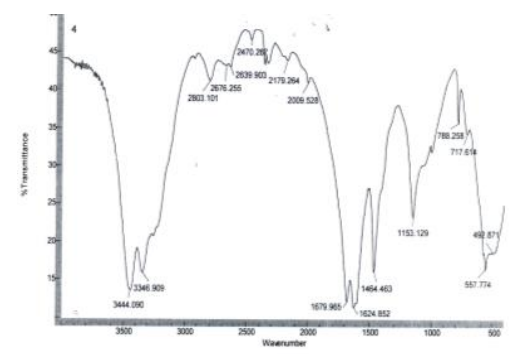

Figure 2. FTIR of lime sulfide treated wool (Na pyrosulfate and urea) 


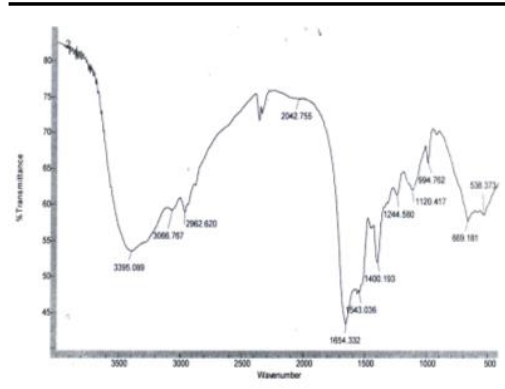

Figure 3. FTIR of native wool $(\mathrm{NaOH})$

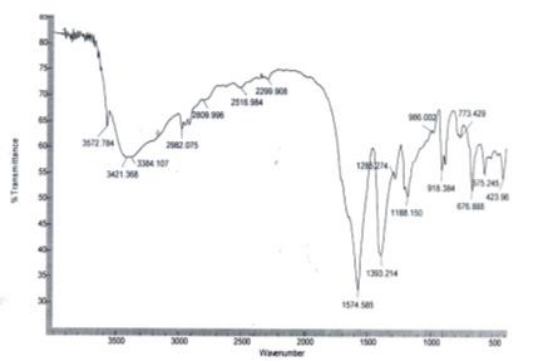

Figure 4. FTIR of lime sulfide treated wool $(\mathrm{NaOH})$

\section{Hydrolysis of Keratin Products from the Hair Cover of Goat Skins}

Table 2. Results of the analysis of keratin hydrolysates from the hair of goat skins

\begin{tabular}{llccc}
\hline $\begin{array}{l}\text { Type of sample } \\
\text { /goat hair / }\end{array}$ & \multicolumn{1}{c}{$\begin{array}{c}\text { Method of } \\
\text { hydrolysis }\end{array}$} & Biuret reaction & $\begin{array}{c}\text { Nitroprusside } \\
\text { reaction }\end{array}$ & Fol's reaction \\
\hline 1. Native & thioglycolic acid & Pale pink color & Yellow & Black \\
& and urea & $\mathrm{A}=0,045$ & coloring & coloring \\
2. Enzyme treated & urea and sodium & Violet coloring & Red coloring & $\begin{array}{c}\text { Does not give } \\
\text { coloring }\end{array}$ \\
& pyrosulfate & $\mathrm{A}=0,434$ & & Black \\
3. Native & $5 \% \mathrm{NaOH}$ & Light purple & Yellow & coloring \\
\hline
\end{tabular}

Regarding the keratin hydrolysate obtained from the enzyme pre-treated goat hair, the Fol's reaction and the Nitroprusside reaction showed that there is a rupture of the disulfide bonds and the presence of free - $\mathrm{SH}$ groups, while the results in the samples from the native hair coat showed the exact opposite, there are still disulfide bonds. This method has once again proved that pre-enzymatic treatment has played a significant role in the breaking of disulfide bridges.

The spectral analyzes show the rupture of the disulfide bonds in the sample from enzyme pre-treated goat hair (Fig.5) in contrast to the hydrolysates from native hair with thioglycolic acid (Fig.6).

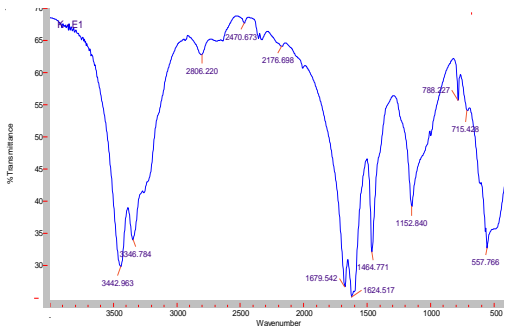

Figure 5. FTIR of enzyme pre-treated goat hair (Na pyrosulfate and urea)

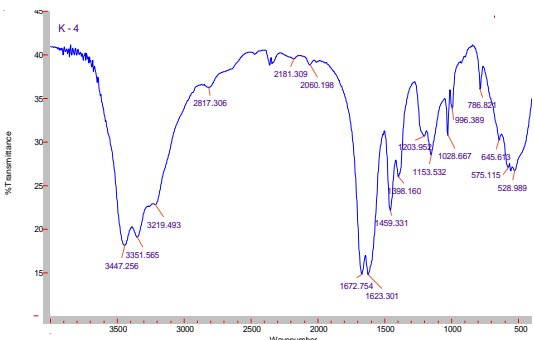

Figure 6. FTIR of native goat hair (thioglycolic acid and urea) 


\section{CONCLUSIONS}

It was found that the samples from one and the same animal source show very different properties and different degrees of hydrolysis. The highest degree of hydrolysis was achieved for the pre-treated samples. It was proved that the method of hydrolysis with $\mathrm{NaOH}$ is the most appropriate for sheep wool and to a much greater extent for the alkaline treated wool than for the native. The reducing agent: sodium pyrosulfate and urea is the most appropriate for enzyme pre-treated samples of goat hair. Therefore, pre-treatment of animal hair samples facilitates the hydrolysis process and makes it easier to break disulfide bonds. The data from the FTIR analysis completely correlate with the quantitative and qualitative analyzes and show the degree of rupture of the disulfide bonds depending on the intensity of the adsorption bands.

It was observed that the hydrolysis processes proceed more easily in sheep wool samples than in goat hair samples. Lime sulfide treatment is a more suitable option for weakening peptide and disulfide bonds, which is a widespread method in the leather industry for unhairing. Therefore, waste from tanneries is a suitable material for the hydrolysis of keratin.

\section{REFERENCES}

Aluigi, A. and Tonetti, C. (2011), “Adsorption of cooper (II) ions by keratin / PA6 blend nanofibers", Eur. Polym. J., (47), 1756-1764, https://doi.org/10.1016/j.eurpolymj.2011.06.009.

Andreev, G. (2010), Molecular spectroscopy, University ed., p.72 (in Bulgarian)

Cardamone, J. (2010), "Investigation the microstructure of keratin extracted from wool: Peptide sequence (MALDI-TOF/TOF) and protein conformation (FTIR)", J. Mol. Structure, 969, 97-105, https://doi.org/10.1016/j.molstruc.2010.01.048.

Cardamone, J., Nunez, A., Garcia, R. and Ramos, M. (2009), “Characterizing Wool Keratin”, Research Letter in Mater. Sci., https://doi.org/10.1155/2009/147175.

Gupta, A., Kamarudin, N., Kee, C. and Yunus, R. (2012), "Extraction of Keratin Protein from Chicken Feather, J. Chem. Chem. Eng., 6, 732-737.

Hikima, T. and Nonomura, Y. (2008), "Powderization of Wool Keratin by Alkali Hydrolysis in Higher Alcohol /Water Binary Systems", Chemistry Letters, 37(3), 338-339, https://doi.org/10.1246/cl.2008.338.

Jotova, L. and Dobrev, I. (2000), Practicum in biochemistry part I, Diagnosis Press (in Bulgarian).

Krejci, O., Mokrejs, P. and Sukop, S. (2011), "Preparation and Characterization of Keratin Hydrolysates", Math. Methods and Tech. in Eng. and Env. Sci., ISBN: 978-1-61804-046-6, p.308.

Mokrejs, P., Krejci, O. and Svoboda, P. (2011), "Producing Keratin Hydrolysates from Sheep Wool", Oriental J. of Chemistry, 27(4), 1303-1309.

Pesheva, M. (1982), Chemistry and Physics of raw leather and tanning substances, Sofia (in Bulgarian).

Pesheva, M. and Papazyan, L. (1990), Fundamentals of leather and fur production, Sofia (in Bulgarian).

Rivalcola, V. and Martinez, A. (2011), "Removal of Hexavalent Chromium from Water by Polyurethane/ Keratin Hybrid Membranes", Water Air Soil Pollut. J., 218, 557-571, https://doi.org/10.1007/s11270-010-0668-6.

Rouse, J. and Van Dyke, M. (2010), “A Review of Keratin-Based Materials for Biomedical Applications Material J., 3, 999-1014, https://doi.org/10.3390/ma3020999.

Saravanan, S., Sameera, D., Moorthi, A. and Sevamurugan, N. (2013), "Chitosan scaffolds containing chicken keratin nanoparticles for bone tissue engineering”, Inter. J. Biol. Macromol., 62, 481-486, https://doi.org/10.1016/j.ijbiomac.2013.09.034.

Xing, Z., Yuan, J., Chae, W. and Kang, I., (2011), "Keratin Nanofibres as a Biomaterial”, 2010 Inter. Conf. on Nanotechnology and Biosensors IPCBEE, 2, 120-124.

Yang, X., Lin, Z. and Shuxun, C. (2007), "Effect of concentration of wool keratin on the rebuilding of disulfur bond", Chinese Science Bulletin, 52(20), 2876-2879, https://doi.org/10.1007/s11434-007-0418-3.

Yin, X., Li, F., He, Y., Wang, Y. and Wang, R. (2013), "Study of effective extraction of chicken feather keratins and their films for controlling drug release", Biomaterials Sci. J., 5, 528-536, https://doi.org/10.1039/c3bm00158j.

Zoccola, M., Aluigi, A. and Tonin, C. (2009), "Characterization of keratin biomass from butchery and wool industry wastes", J. Mol. Structure, 938, 35-40, https://doi.org/10.1016/j.molstruc.2009.08.036. 


\section{III.}

\section{INNOVATIVE SYSTEMS, \\ TECHNOLOGIES \\ AND QUALITY \\ MANAGEMENT}


SYNTHESIS OF POLYURETHANES WITH LOW VOLATILE ORGANIC COMPOUNDS CONTENT FOR UPHOLSTERY AND AUTOMOTIVE ARTICLES

\author{
ANNA BACARDIT, MIREIA CONDE, SÍLVIA SOROLLA, CONCEPCIÓ CASAS, \\ LLUÍS OLLÉ
}

A3 Leather Innovation Center. Escola Politècnica Superior (EPS). Departament d'Informàtica i Enginyeria Industrial. Universitat de LLeida (UdL). Pla de la Massa,8. 08700 Igualada, Spain, e-mail: anna.bacardit@udl.cat

\begin{abstract}
The manufacture of upholstery and automotive articles is linked to the release of Volatile Organic Compounds (hereinafter VOCs) during their manufacture, which have short and long-term effects on the health of users and the environment. In the leather sector, around $40 \mathrm{~kg}$ of VOCs are generated per $1000 \mathrm{~kg}$ of raw skin. This research work has focused on the synthesis of new and more sustainable urethane-based polymers that, in turn, allow the quality requirements of the finish to be met, which vary depending on the leather article manufactured. The main objective of the study is to minimize the content of VOCs in the different aliphatic polyurethanes synthesized in a pilot-scale reactor, making small modifications to the synthesis formulations. The synthesis route developed is based on the preparation of polymers of ionomeric polyurethanes and their subsequent dispersion in water. In the synthesis processes developed, the content of coalescing solvents and neutralizing agents, which directly contribute to the concentration of VOCs of the urethane polymers, is eliminated and / or minimized as much as possible. The new urethane-based polymers obtained have been analyzed according to the parameters of $\mathrm{pH}$, viscosity, density and percentage of solids in the resin. Likewise, organoleptic tests (color, transparency, hardness, touch and tacking) and physical tests (tensile strength, water absorption, hardness and color change at $100^{\circ} \mathrm{C}$ for 24 hours) have been carried out on the film corresponding to each synthesized polyurethane resin. These products will be introduced in finishing formulations designed to obtain high-performance upholstery and automotive leather with minimal impact in terms of VOC content at the pilot level. Tests of fastness and physical resistance have been carried out to evaluate the performance of these leathers.
\end{abstract}

Keywords: VOCs, resin, film, finishing.

\title{
INTRODUCTION
}

This work details the synthesis procedures for new more sustainable polyurethane polymers, and more specifically, aliphatic polyurethanes of the polyester, polyether, polycarbonate and polyether-polyester blends.

Polyurethanes are polymers or macromolecules formed from the catalyzed reaction between a polyisocyanate and a polyol. The simple reaction between an alcohol and an isocyanate produces the urethane (or carbamate).

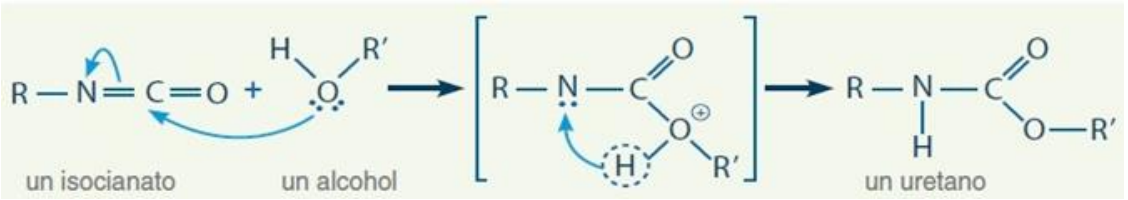

Figure 1. Urethane formation reaction

Conventional polyurethanes are not compatible with water, for this reason some modifications are necessary in the production process in order to allow the generation of an aqueous dispersion. For the production of the aqueous polyurethane dispersion, two differentiated stages are carried out: 1) formation of pre-polymer chains, either in mass or in solution; and, 2) dispersion of the prepolymer in water. (Szycher, 2012; Noble, 1997). 
The synthesis process carried out must be careful and strict, starting from completely dry material and using a nitrogen atmosphere in the reactor throughout the process. The variables to reduce VOCs are based on the elimination of coalescing solvents in the formation of pre-polymer chains and on the minimization of neutralizing agents in the process of dispersing the pre-polymer in water.

Water-based polyurethanes have gained commercial relevance in recent years. The main reason for this fact is the growing concern for the environment regarding solvents and volatile organic compounds (VOCs) which are emitted into the atmosphere, causing the deterioration of the ozone layer, acid rain and a possible chemical imbalance in the ecosphere of the Earth. The secondary reasons are related to the economic costs derived from the consumption of raw material (substitution of solvents for water) and the scope of benefits equivalent to those provided by solvent-based polyurethanes (Szycher, 2012).

\section{MATERIALS AND METHODS}

\section{Materials}

The raw materials for the synthesis of polyurethanes are: N, N-Diethylethanamine; 2,2-Bis (hydroxymethyl) propionic acid; Hexanedioic acid 1,6-bis (2-methylpropyl) ester; 3- isocyanatomethyl-3,5,5-trimethylcyclohexyl isocyanate; Dipropylene Glycol Dimethyl Ether; Hydroxy compound, polyester oligomer containing: Dimethyl adipate, Dimethyl glutarate and Dimethyl succinate; Neopentyl glycol butanediol polyadipate; Polyol polypropylene linear ether; Polyol polypropylene linear ether; Polycarbonate diols based on 1-Hexanediol; Polycarbonate diols based on 1-Hexanediol; Tin dibutyl dilaurate; 1,2-benzisothiazolin-3-one solution in dipropylene glycol and water.

There are two glass reactors tailor made by the V. Forné Mechanical Workshops, with a volumetric capacity of $5 \mathrm{~L}$, arranged in a chain. The first reactor has a double cover heated by means of oil in a thermal bath. This thermal bath regulates the temperature of the reactor. The thermal bath temperature adjustment systems do not allow to exceed $150^{\circ} \mathrm{C}$.

The first reactor is connected to a nitrogen flow that allows an inert medium to be obtained inside and that allows the achievement of the pre-polymer safely and thoroughly.

The first reactor is located in a higher position than the second, in order to take advantage of the gravity effect to transfer the contents of the first reactor to the second. Both reactors have an adjustable stirring system.

\section{Synthesis Methods}

The synthesis of eight polyurethanes with the functional groups shown in Table 1 have been carried out.

Table 1. Synthesized polyurethane type

\begin{tabular}{ll}
\hline \multicolumn{1}{c}{ Polyurethane type } & \multicolumn{1}{c}{ Nomenclature } \\
\hline & PES NV001 \\
Polyester (PES) & PES NV002 \\
& PES NV003 \\
& PES NV004 \\
Polyether (PET) & PET NV005 \\
Polyester-Polyether (PES-PET) & PES-PET-NV006 \\
Polycarbonate (PC) & PC NV007 \\
& PC NV008 \\
\hline
\end{tabular}

https://doi.org/10.24264/icams-2020.III.1 
After the synthesis of each pre-polymer, the following phases of the polymer synthesis process were carried out: neutralization, elongation of the polymer and aqueous dispersion.

\section{Film Resin Test Methods}

For the characterization of the emulsions resulting from each synthesis process, the following chemical tests have been carried out: $\mathrm{pH}$, density $(\mathrm{g} / \mathrm{mL})$, percentage of solids (\%) and viscosity (s). For a more exhaustive characterization of the different polymers generated, the film for each one of them has been elaborated, taking into account the percentage of solids obtained in the characterization of the emulsion. To create a film layer that allows the subsequent characterization of the material formed, plastic material molds are used in which a certain amount of each polymer is allowed to dry at $30-35^{\circ} \mathrm{C}$ until the formation of a film layer is observed.

To evaluate the new synthesized resins, organoleptic tests are carried out: color, degree of transparency, hardness and tacking, and physical tests according to the standards:

-ISO 3376 (tensile strength);

-ISO 5403-1 (water absorption for 1 hour);

-ISO 868 (hardness of plastics and ebonite);

-ISO 17228 (color change at $100^{\circ} \mathrm{C}$ for 24 hours).

\section{Application of the New Synthesized Resins}

The aim of this part of the work is to evaluate the fastness and physical resistance that these polyester and polyether resins provide once sealed to the leather surface and to analyze whether the partial and total substitution of DMM for DBE 3 is reflected in any way in the leather finish. A finishing formula consisting of a base coat and a top coat is used. A black color has been selected as the pigment to more clearly visualize the differences in the transfer of color in the tests of rub fastness, flex strength and adhesion of the finish.

The base coat integrates each of the synthesized resins. Finally, the same top formulation has been applied to all leathers to provide a pleasant touch. The physical and fastness tests selected for the comparison of the finishes made with the different synthesized resins have been the following:

-ISO 5402-1 (dry / wet flex strength);

-ISO 11640 (dry / wet rub strength);

-ISO 15700 (water drop absorption);

-ISO 11644 (dry / wet finish adhesion).

\section{RESULTS}

\section{Results of the Synthesized Urethane Emulsions}

Table 2 shows the values obtained in the different tests carried out on the resins in emulsion state.

Table 2. Results obtained from the analysis of polyester polyurethane resins

\begin{tabular}{lcccc}
\hline & PES-NV001 & PES-NV002 & PES-NV003 & EPES- NV004 \\
\hline $\mathrm{pH}$ & 10.0 & 7.3 & 7.1 & 7.2 \\
Viscosity (s) & 14 & 18 & 15 & 140 \\
Density (g/mL) & 1.01 & 1.01 & 1.02 & 0.97 \\
Solids (\%) & 29.9 & 31.6 & 29.9 & 33.4 \\
\hline
\end{tabular}

https://doi.org/10.24264/icams-2020.III.1 
Synthesis of Polyurethanes with Low Volatile Organic Compounds Content for Upholstery and Automotive Articles

\begin{tabular}{lcccc}
\hline \hline & & & & \\
\hline & PET- NV005 & NV006 & PC-NV007 & PC- NV008 \\
\hline $\mathrm{pH}$ & 10.6 & 7.2 & 10.6 & 8.3 \\
Viscosity (s) & 18 & 19 & 14 & 18 \\
Density (g/mL) & 1.01 & 1.03 & 1.02 & 1.00 \\
Solids (\%) & 22 & 41.8 & 27.9 & 29.9 \\
\hline
\end{tabular}

Upon completion of the synthesis process, the resulting polyurethanes should include the following specifications: percentage of solids (30 $\pm 2 \%$ in PES, PET and PC; $40 \pm 2 \%$ in PES-PET) and $\mathrm{pH}(7.8-8.8)$. $\mathrm{pH}$ values higher than 8.8 denote an excess of TEA in the neutralization carried out in the second reactor, while values lower than 7.8 denote a lack of the same product for neutralization. Regarding the value of the percentage of solids, most of the resins meet this specification, with the exception of the PET-NV005 emulsion which presents a much lower value. The viscosity parameter marks an obvious difference from the PES-NV004 emulsion, with a Ford Cup number 4 value of 140 seconds much higher than the rest of the tested resins. The density of all the resins oscillates between 0.97 and $1.03 \mathrm{~g} / \mathrm{mL}$, presenting the minimum value the PES-NV004 emulsion.

\section{Results of the Study of the Films}

The resulting films have the same solid content, which makes it possible to compare the results of the organoleptic tests (color, degree of transparency, hardness and tacking) and of the physical tests carried out on the formed polymers or resins.

The results of the organoleptic parameters of the resins in the film-forming state are shown in Table 3.

Table 3. Results of the organoleptic analysis of the resin film

\begin{tabular}{|c|c|c|c|c|c|c|c|c|}
\hline Parameter & $\begin{array}{l}\text { PES- } \\
\text { NV001 }\end{array}$ & $\begin{array}{c}\text { PES- } \\
\text { NV002 }\end{array}$ & $\begin{array}{l}\text { PES- } \\
\text { NV003 }\end{array}$ & $\begin{array}{l}\text { PES- } \\
\text { NV004 }\end{array}$ & $\begin{array}{l}\text { PET- } \\
\text { NV005 }\end{array}$ & $\begin{array}{c}\text { PES- } \\
\text { PET- } \\
\text { NV006 }\end{array}$ & $\begin{array}{c}\text { PC- } \\
\text { NV007 }\end{array}$ & $\begin{array}{c}\text { PC- } \\
\text { NV008 }\end{array}$ \\
\hline Color & $\begin{array}{l}\text { Color- } \\
\text { less }\end{array}$ & $\begin{array}{l}\text { Color- } \\
\text { less }\end{array}$ & $\begin{array}{l}\text { Yellow- } \\
\text { ish }\end{array}$ & White & White & $\begin{array}{l}\text { Color- } \\
\text { less }\end{array}$ & White & White \\
\hline $\begin{array}{l}\text { Transparency } \\
\text { degree }\end{array}$ & $\begin{array}{l}\text { Trans- } \\
\text { parent }\end{array}$ & $\begin{array}{l}\text { Trans- } \\
\text { parent }\end{array}$ & $\begin{array}{l}\text { Semi } \\
\text { opaque }\end{array}$ & $\begin{array}{l}\text { Semi } \\
\text { trans- } \\
\text { parent }\end{array}$ & $\begin{array}{l}\text { Semi } \\
\text { trans- } \\
\text { parent }\end{array}$ & $\begin{array}{l}\text { Trans- } \\
\text { parent }\end{array}$ & $\begin{array}{c}\text { Semi } \\
\text { opaque }\end{array}$ & $\begin{array}{c}\text { Semi } \\
\text { opaque }\end{array}$ \\
\hline Hardness & $\begin{array}{l}\text { Very } \\
\text { hard }\end{array}$ & $\begin{array}{l}\text { Very } \\
\text { hard }\end{array}$ & $\begin{array}{l}\text { Very } \\
\text { hard }\end{array}$ & Hard & Very soft & Hard & $\begin{array}{l}\text { Very } \\
\text { hard }\end{array}$ & Hard \\
\hline \multirow[t]{2}{*}{ Tacking } & No & No & Yes & No & No & No & No & No \\
\hline & $\begin{array}{l}\text { Film } \\
\text { PES- } \\
\text { NV001 }\end{array}$ & $\begin{array}{l}\text { Film } \\
\text { PES- } \\
\text { NV002 }\end{array}$ & $\begin{array}{l}\text { Film } \\
\text { PES- } \\
\text { NV003 }\end{array}$ & $\begin{array}{l}\text { Film PES- } \\
\text { NV004 }\end{array}$ & $\begin{array}{l}\text { Film PET- } \\
\text { NV005 }\end{array}$ & $\begin{array}{l}\text { Film PES- } \\
\text { PET-NV006 }\end{array}$ & $\begin{array}{l}\text { Film } \\
\text { PC- } \\
\text { NV007 }\end{array}$ & $\begin{array}{l}\text { Film } \\
\text { PC- } \\
\text { NV008 }\end{array}$ \\
\hline Tensile strength & & & & & & & & \\
\hline$-\operatorname{Load}(\mathrm{N})$ & 7.3 & 5.1 & (1) & 2.2 & 41.0 & 5.6 & (1) & (1) \\
\hline $\begin{array}{l}\text {-Elongation mm } \\
\text { Water }\end{array}$ & 254.9 & 223.6 & (1) & 671.6 & 520.5 & 520.8 & (1) & (1) \\
\hline $\begin{array}{l}\text { absorption }(\%) / \\
1 \text { hour }\end{array}$ & 55.1 & 27.2 & 11.5 & 6.2 & 90.0 & 6.6 & (1) & 9.3 \\
\hline Hardness $\left({ }^{0} \mathrm{ShA}\right)$ & 92 & 93 & 92 & 78 & 19.2 & 77 & (1) & 65 \\
\hline $\begin{array}{l}\text { Color change at } \\
100^{\circ} \mathrm{C} / 24 \text { hours }\end{array}$ & 5 & 5 & 3 & 5 & $4 / 5$ & 5 & (1) & 4 \\
\hline
\end{tabular}

(1) It is impossible to punch out and therefore makes it impossible to perform any test

All polyester polyurethane films are very hard and all of them except PES-NV003 do not present tacking. At the level of transparency, the first two (PES-NV001 and PES NV002) are transparent, the PES-NV004 film is semi-transparent while the PES-NV003 film is semi-opaque. Polyether polyurethane resin film (PET-NV005) is softer than 
polyester urethane resin films. Likewise, it is characterized by its white color, the absence of tacking and high water absorbance. The PES-PET-NV006 film turns out to be hard and solid to light, but it is not the most resistant. The PC-NV007 film is impossible to analyze as it fragments and is not consistent. The PC-NV008 film is white, semi-opaque, without tacking and, in addition, softer than polyester urethane resins or mixed (PES-PET).

\section{Application of the New Synthesized Resins}

After applying polyester polyurethane and polyether polyurethane on the leather surface, the leather was left to rest for 24 hours before starting the physical tests mentioned in the previous section.

The finished leathers present a similar appearance between them. At an organoleptic level, it can be pointed out that the leather finished with the PET-NV005 base has some tacking, while the rest of the leather shows the silicone touch of the top finishing layer applied.

The results obtained in the physical tests can be seen in Table 4 .

Table 4. Results of the finishing tests

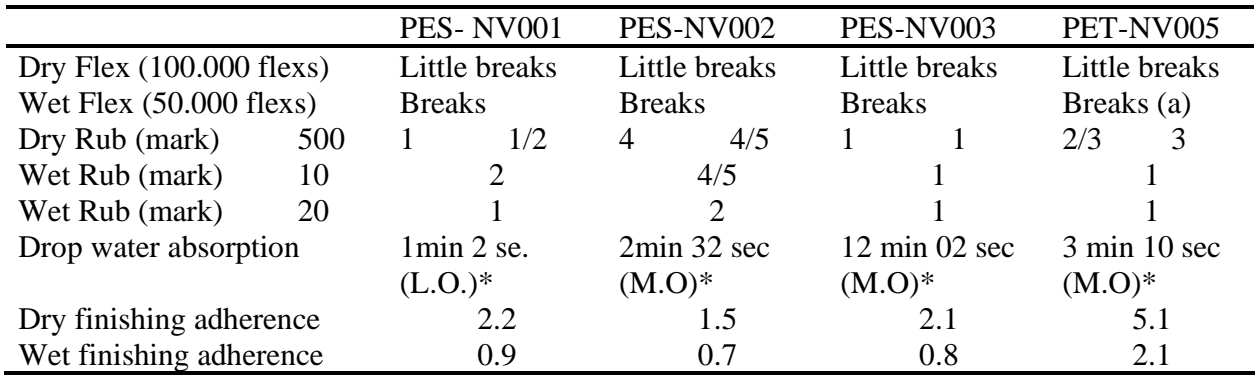

*(L.O.) Slight darkening

*(M.O.) Moderate darkening

According to the results observed in Table 13, it is observed that after the dry flexion test, all samples present small ruptures, which are increased if they are tested in wet. In the case of leathers with PET-NV005 in the base coat, the finish peeling off. Regarding dry rubbing, PES-NV002 has a high rubbing fastness value. The rest of the synthesized resins applied have a low rub fastness value. The fastness to the drop of water is conditioned by the study area of the leather, but in general terms none of the resins reaches the 30 minutes established in the test. Furthermore, darkening occurs in all resins, light in the case of PES-NV002 resin and moderate in the rest. In either case, loss of gloss occurs. Synthesized polyol ester resins have lower finish adhesion values compared to polyol ether based polyurethane resin.

\section{CONCLUSIONS}

In this work, new finishing products of the aliphatic polyurethane type in aqueous dispersion have been developed. Certain variables, specifically, the hydroxylated compounds (polyether oligomers and polyester oligomers and polycarbonates) and the type of solvent (DMM and DBE-3) have been modified in order to obtain minimal or no volatile substances.

The new polymers obtained have been analyzed according to the parameters of $\mathrm{pH}$, viscosity, density and percentage of solids. In general terms, most resins have a low viscosity, less than 20 seconds measured with a Ford cup, with the exception of the 
PET-NV005 sample. Polyester polyurethane resins have a solids percentage of around $30 \%$. The polyether polyurethane resin has a $\mathrm{pH}$ greater than 8.8 . This fact is due to incomplete prepolymer transport in the dispersion reactor, as well as an excessive addition of triethylamine. As a consequence, the percentage of solids is around $20 \%$ and the resin gives off an odor of the products added in the second reactor.

Finally, a polycarbonate polyurethane type synthesis was carried out, which resulted in polycarbonate urethane resin (PC-NV007). During the filtration of the same previous packaging, a carbonic cloud was observed when contacting the dispersed resin with the air. This synthesis has not been satisfactory as a consequence of the incompatibility of monomers in the prepolymerization reactor.

In the synthesis of polycarbonate urethane resin (PC-NV008), a single polycarbonate polyol monomer has been used. In organoleptic terms, the PC-NV008 resin is similar to the results of the other resins, that is of polyester or polyether origin.

For reasons of fastness and high content of VOCs, the study with PES-NV001 resin was refused. The best results of the physical tests were obtained in the leathers finished with the PES-NV002 resin. A possible future way of study would consist in the application of this resin combined with other commercial resins, as well as its application in different layers of the finish. Not to be underestimated is PES-NV003 urethane resin synthesized using a lower volatile solvent in the prepolymerization process. Polyether urethane resin (PET-NV005) will also be studied in greater detail due to the absence of solvent in the process of obtaining said resin.

\section{Acknowledgements}

This research work has been developed within the framework of the NoVOCs project (RTC-2016-4575-5) within the Collaboration Challenges program (2016-2018) approved by the Ministry of Economy and Competitiveness of Spain. We appreciate the collaboration of Curtidos Aqualata, S.A as leader of the project.

\section{REFERENCES}

Aqdas, N., Khalid, M., Zia, M., Zuber, S., Tabasum, A. and Fawad, Z. (2016), "Bio-based polyurethane: An efficient and environment friendly coating systems: A review", Progress in Organic Coatings, 91, 25-32, https://doi.org/10.1016/j.porgcoat.2015.11.018.

Bacardit, A., Cobos, M., Borràs, E. and Ollé, Ll. (2014), "Epoxi resin cross-linked binders in aqueous finishing", Journal of the Society of Leather Technologists and Chemists, 94(1), 1-8.

Darroman, E., Durand, N., Boutevin, B. and Caillol, S. (2016), "Improved cardanol derived epoxy coatings", Progress in Organic Coatings, 91, 9-16, https://doi.org/10.1016/j.porgcoat.2015.11.012.

Institute for Prospective Technological Studies, Sustainable Production and Consumption Unit European IPPC Bureau (2013), "Best Available Techniques (BAT) Reference Document for the Tanning of Hides and Skins", Industrial Emissions Directive 2010/75/EU (Integrated Pollution Prevention and Control), Joint Research Centre.

Kleban, M. (2014), "VOC-free leathers: A moving target", World Leather, June-July, 21-25.

Niculescu, O., Moldovan, Z., Leca, M., Chelaru, C., Alexandrescu, L. and Aboul-Enein, H.Y. (2015), "Characteristics of natural leather finished with some eco-friendly mixtures of polymeric aqueous dispersions", J. Polym. Eng, 35-5, 463 - 470, https://doi.org/10.1515/polyeng-2014-0286.

Noble, K.-L. (1997), "Waterborne polyurethanes", Progress in Organic Coatings, 32(1-4), 131-136, https://doi.org/10.1016/S0300-9440(97)00071-4.

Ollé, Ll., Bou, J., Shendrik, A. and Bacardit, A. (2013), "Sustainable solvent-free finishing of patent leather using carbonyl-functional resins", Journal of Cleaner Production, 65(15), 590-594, https://doi.org/10.1016/j.jclepro.2013.07.058.

Szycher, M. (2012), Szycher's Handbook of Poliurethanes, Ed. CRC Press, ISBN 9781439839584.

Thébault, M., Pizzy, A., Essawy, H.A., Barhoum, A. and Van Assche, G. (2014), "Isocyanate free condensed tanninbased polyurethanes", European Polymer Journal, 67, https://doi.org/10.1016/j.eurpolymj.2014.10.022.

United Nations Industrial Development Organization (UNIDO) (2015), "The framework for sustainable leather manufacture".

*** https://www.fullquimica.com/2013/01/formacion-de-los-polimeros-sinteticos_31.html

https://doi.org/10.24264/icams-2020.III.1 
THE IMPACT OF SUPPLY CHAIN INTEGRATION ON OPERATIONAL PERFORMANCE THROUGH RESILIENCE UNDER COVID-19 PANDEMIC

\author{
MAHMOUD BARAKAT ${ }^{1}$, AHMED ALI $^{1,2}$, ISLAM ABDELBARY ${ }^{1}$, MAI HAROUN $^{1}$ \\ ${ }^{1}$ Arab Academy for Science Technology and Maritime Transport, College of International \\ transport and Logistics, Egypt, e-mail: m.ramadan47@gmail.com \\ ${ }^{2}$ Universität Duisburg-Essen Fakultät für Ingenieurwissenschaften Duisburg, Nordrhein- \\ Westfalen, Germany, e-mail: Ahmed.Ali.14@stud.uni-due.de
}

\begin{abstract}
COVID-19 has now unleashed a global supply chain crisis across a huge number of organizations, stemming from a lack of understanding and flexibility of the multiple layers of their global supply chains. In addition to the lack of efficient management through unpredicted events and occasions. Drawing on extended resources based view and resilience theory, this paper attempts to shed the light on the mediating role of resilience between supply chain integration and organizational performance represented in quality, cost and delivery performance during the COVID-19 pandemic. 224 questionnaires were collected and analyzed through process macro technique proposed by Andrew Hayes to test the mediating role of resilience. The results indicated that resilience can significantly mediate the relationship between supply chain integration and quality, cost and delivery performance. Therefore, this paper contributes to both extended resources based view and resilience theory. As it empathize on how organizations can acquire a unique bundle of resources through integration, which will allow them to maintain a desirable level of performance during market disruption through building resilience. The results will practically guide organizations to invest in building resilience in order to be able to cope with unexpected events that disrupts the business environment such as COVID19.
\end{abstract}

Keywords: Supply chain integration, supply chain resilience, operational performance

\title{
INTRODUCTION
}

Supply chain integration (SCI) one of the collaboration types that make managers meet the new challenges of the global competitive environment and the unexpected crises, as SCM is a strategic concept for increasing the collaboration between the chain members within the value chain (Jonsson et al., 2011). The integration between supply chain members whether the upstream or downstream is considered the key to improving the companies' performance and added value to the network (Ataseven and Nair, 2017). However, with the appearance of COVID-19 pandemic, the interaction between supply chain members was disrupted (Inoue and Todo, 2020). This research focuses on the importance of resilience in mediating the relationship between SCI and operational performance represented in quality performance (OPQ), cost performance (OPC) and delivery performance (OPD) during COVID-19.

\section{LITERATURE REVIEW AND HYPOTHESIS DEVELOPMENT}

Based on the extended resource-based prospect, organizations need to acquire resources through forming alliances in order to enhance performance (Mishra et al., 2018). This means that SCI can help the organization gain the needed resources for maintaining competitive advantage (Xu et al., 2014). SCI plays an important role in creating value, which leads to performance improvements (Ataseven and Nair, 2017). Flynn et al. (2010) confirmed that SCI is an effective technique that improves the performance of an organization. Lo and Yeung (2006) clarified that SCI significantly impacts operational performance. On the other hand, Soares et al. (2017) found that 
supply chain integration can improve operational performance significantly. Operational performance is considered one of the critical factors that determine the competitive advantage of a company or a supply chain in a specific time, moreover it affects business performance measures such as market share and customer satisfaction (Li et al., 2006).

Drawing on resilience theory, organizations need to resume normal operations quickly through building resilience in order to recover from disruptive events such as COVID-19 pandemic (Koronis and Ponis, 2018). As it negatively affected supply chains integrations (Inoue and Todo, 2020). Resilience is an important enabler of Supply chain performance because building resilience prevents disruptive shocks from occurring and assists in establishing and maintaining acceptable levels of performance (Jüttner and Maklan, 2011).

Based on the above arguments, this paper argued that SCI could lead to an increase in operational performance through resilience. Thus, the research hypothesis is formulated as:

Resilience mediates the relationship between SCI and operational performance (quality, cost and delivery).

Figure 1 shows the hypothesized relationship.

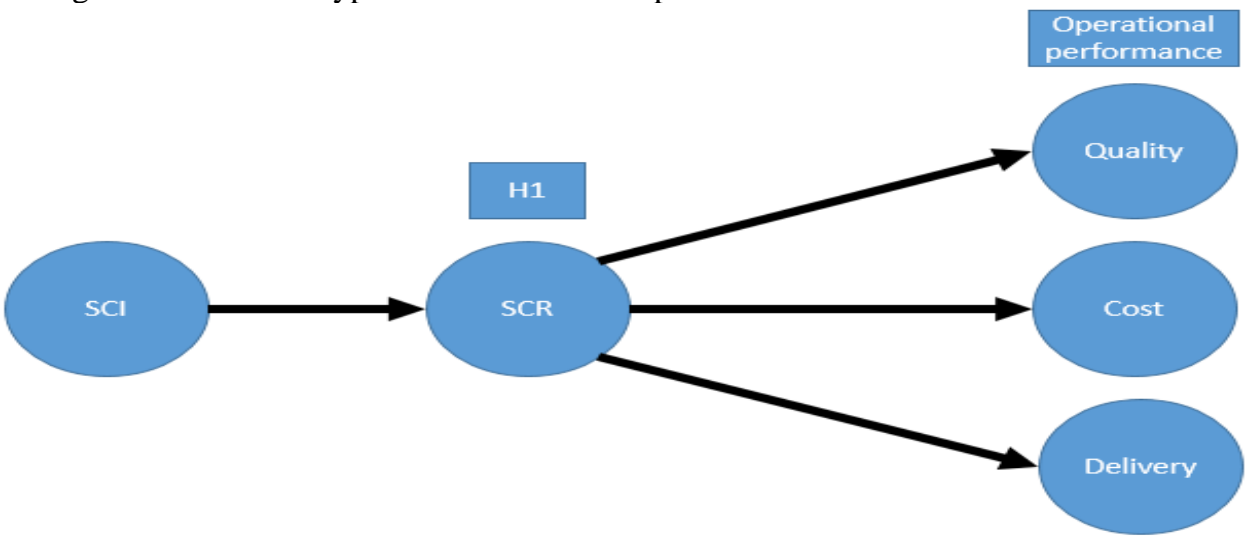

Figure 1. Research model

\section{METHODOLOGY}

The quantitative approach of the study was based on survey methods. The questionnaire was divided into four main sections, general information, supply chain integration, supply chain resilience and operational performance represented in quality, cost and delivery. All scales were adapted from previous research, SCI was adapted from The impact of asymmetry on performance. SCR scales were adapted from Supply chain capabilities, risks, and resilience. Operational performance scales (quality, cost and delivery) were adapted from (Phan et al., 2019). Scales was originally in English a back-translation process was conducted to ensure the accuracy of the translation. The questionnaire was distributed in the Egyptian market, participants were mainly working at a senior level position. Sample characteristics are illustrated in table 1. 
ICAMS $2020-8^{\text {th }}$ International Conference on Advanced Materials and Systems

Table 1. Sample characteristics

\begin{tabular}{llcc}
\hline Characteristics & Criteria & Frequency & Percentage \\
\hline Size & Large & 33 & 14.7 \\
& Medium & 71 & 31.7 \\
& Small & 55 & 24.6 \\
& Very small & 65 & 29 \\
Location of the firm & Cairo & 97 & 43.3 \\
& Alexandria & 109 & 48.7 \\
Core activity & Other & 18 & 8 \\
& Industrial & 97 & 43.3 \\
Years of experience on the senior position & Service sector & 58 & 25.9 \\
& Commercial/ trader & 69 & 30.8 \\
& Less than 1 year & 80 & 10 \\
& From 1 year to 2 year & 157 & 19 \\
& From 3 years to 4 years & 145 & 18 \\
& From 5 years to 6 years & 193 & 24 \\
& More than 6 years & 20 & 3 \\
\hline
\end{tabular}

Pretest was conducted to ensure face and content validity. Followed by a pilot testing with 105 participants in order to further ensure the validity and reliability of the data collection instrument. Factor loadings, average variance extracted, composite reliability were calculated using exploratory factor analysis. In addition, Cronbach's alpha was also calculated to ensure scales of reliability. Finally, the square root of the average variance extracted to ensure discriminant validity (Fornell and Larcker, 1981). Tables 2 and 3 show the results of the pilot study, which clearly indicates that all indicators are above their cutoff points (Hair et al., 1998).

Table 2. Results of the pilot study

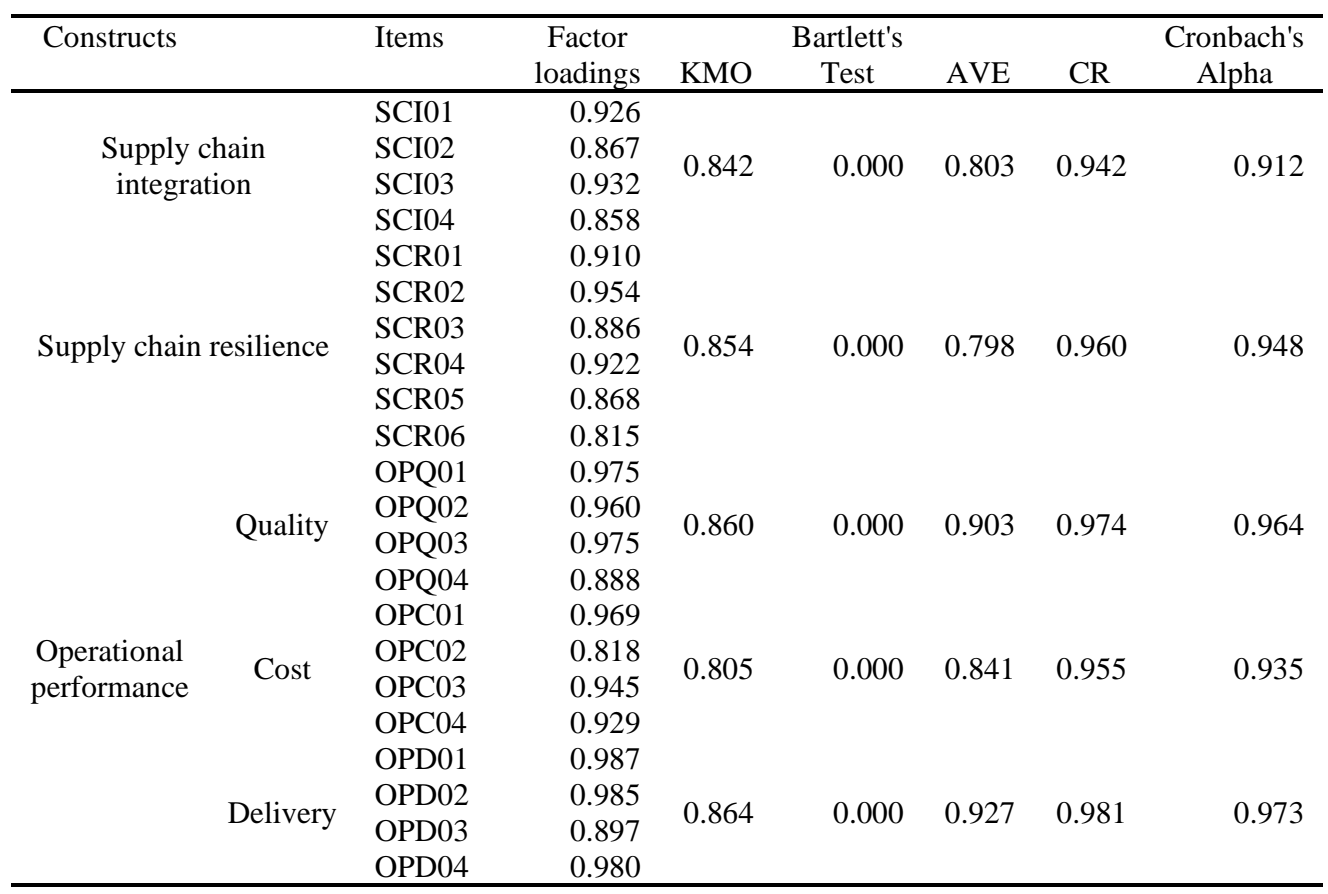

https://doi.org/10.24264/icams-2020.III.2 
Table 3. Discriminant validity of the pilot study

\begin{tabular}{lcccccc}
\hline & SCI & SCR & OPQ & OPC & OPD & SQR-AVE \\
\hline SCI & 1.00 & 0.67 & 0.12 & 0.56 & 0.01 & 0.90 \\
SCR & 0.67 & 1.00 & 0.23 & 0.48 & 0.17 & 0.89 \\
OPQ & 0.12 & 0.23 & 1.00 & -0.08 & 0.03 & 0.95 \\
OPC & 0.56 & 0.48 & -0.08 & 1.00 & -0.20 & 0.92 \\
OPD & 0.01 & 0.17 & 0.03 & -0.20 & 1.00 & 0.96 \\
SQR-AVE & 0.90 & 0.89 & 0.95 & 0.92 & 0.96 & \\
\hline
\end{tabular}

Regarding main study hypothesis testing, confirmatory factor analysis using AMOS was conducted to ensure validity and reliability on the 224 questionnaire collected. Based on the results illustrated in appendix B, all goodness of fit model criteria for all constructs were adequate. In addition, average variance extracted, the square root of average variance extracted when compared to the correlation matrix, factor loadings, composite reliability and Cronbach's Alpha shows ensures the validity and reliability of the questionnaire.

The developed hypothesis was tested through conducting mediation analysis using the macro process by (Preacher and Hayes, 2008). As the results are shown in Table 4 that there is no zero between the upper and lower boundaries, it can be concluded that resilience is significantly mediating the relationship between SCI and quality performance, SCI and cost performance and SCI and delivery performance. This concludes that the developed hypothesis is accepted.

Table 4. Mediation analysis

\begin{tabular}{lrrr}
\hline Indirect paths & BootLLCI & BootULCI & Significance \\
\hline SCI -> SCR -> OPQ & 0.1808 & 0.3972 & Sig \\
SCI -> SCR -> OPC & 0.1467 & 0.3353 & Sig \\
SCI -> SCR -> OPD & 0.0805 & 0.3231 & Sig \\
\hline
\end{tabular}

\section{CONCLUSION}

This study focused on illustrating the mediating role of resilience between SCI and operational performance during COVID-19 pandemic. The results showed that resilience can significantly mediate the impact of SCI on the three operational performance dimensions; quality, cost and delivery. This will theoretically contribute to resilience theory and extended resources-based view as it shows the importance of enhancing adaptability through integration to enhance performance under COVID-19. In addition, the results of this study will practically help organizations adapt to market disruptions such as COVID-19. As it shows that supply chain integration can enable organizations to have access to unique resources that will allow them to enhance resilience and eventually achieve higher performance. In other words, instead of focusing on enhancing performance organizations must take advantage of their integration to build resilience in order to thrive in a dynamic business environment. This will eventually allow them to gain a competitive edge through maintaining highperformance levels during market disruption. 


\section{REFERENCES}

Ataseven, C. and Nair, A. (2017), "Assessment of supply chain integration and performance relationships: A meta-analytic investigation of the literature", International Journal of Production Economics, 185, 252265, https://doi.org/10.1016/j.ijpe.2017.01.007.

Flynn, B.B., Huo, B. and Zhao, X. (2010), "The impact of supply chain integration on performance: A contingency and configuration approach", Journal of Operations Management, 28(1), 58-71, https://doi.org/10.1016/j.jom.2009.06.001

Fornell, C. and Larcker, D.F. (1981), "Evaluating Structural Equation Models with Unobservable Variables and Measurement Error ", Journal of Marketing Research, 18(1), 39-50, https://doi.org/10.2307/3151312.

Hair, J.F., Black, W.C., Babin, B.J., Anderson, R.E. and Tatham, R.L. (1998), Multivariate data analysis (Vol. 5), Prentice hall Upper Saddle River, NJ.

Inoue, H. and Todo, Y. (2020), "The propagation of the economic impact through supply chains: The case of a mega-city lockdown against the spread of COVID-19", available at SSRN 3564898, https://doi.org/10.2139/ssrn.3564898.

Jonsson, P., Andersson, D., Boon-itt, S. and Wong, C.Y. (2011), "The moderating effects of technological and demand uncertainties on the relationship between supply chain integration and customer delivery performance", International Journal of Physical Distribution \& Logistics Management, 41(3), 253-276. https://doi.org/10.1108/09600031111123787.

Jüttner, U. and Maklan, S. (2011), "Supply chain resilience in the global financial crisis: An empirical study", Supply Chain Management, 16(4), 246-259, https://doi.org/10.1108/13598541111139062.

Koronis, E. and Ponis, S. (2018), "Better than before: the resilient organization in crisis mode", Journal of Business Strategy, https://doi.org/10.1108/JBS-10-2016-0124.

Li, S., Ragu-Nathan, B., Ragu-Nathan, T. and Rao, S.S. (2006), "The impact of supply chain management practices on competitive advantage and organizational performance”, Omega, 34(2), 107-124, https://doi.org/10.1016/j.omega.2004.08.002.

Lo, V.H. and Yeung, A. (2006), "Managing quality effectively in supply chain: a preliminary study", Supply Chain Management: An International Journal, https://doi.org/10.1108/13598540610662103.

Mishra, D., Luo, Z., Hazen, B., Hassini, E. and Foropon, C. (2018), "Organizational capabilities that enable big data and predictive analytics diffusion and organizational performance ", Management Decision, https://doi.org/10.1108/MD-03-2018-0324.

Phan, A.C., Nguyen, H.A., Trieu, P.D., Nguyen, H.T. and Matsui, Y. (2019), "Impact of supply chain quality management practices on operational performance: empirical evidence from manufacturing companies in Vietnam”, Supply Chain Management: An International Journal, https://doi.org/10.1108/SCM-12-20180445 .

Preacher, K.J. and Hayes, A.F. (2008), "Asymptotic and resampling strategies for assessing and comparing indirect effects in multiple mediator models", Behavior research methods, 40(3), 879-891, https://doi.org/10.3758/BRM.40.3.879.

Soares, A., Soltani, E. and Liao, Y.-Y. (2017), "The influence of supply chain quality management practices on quality performance: an empirical investigation", Supply Chain Management: An International Journal, https://doi.org/10.1108/SCM-08-2016-0286.

$\mathrm{Xu}$, D., Huo, B. and Sun, L. (2014), "Relationships between intra-organizational resources, supply chain integration and business performance", Industrial Management \& Data Systems, https://doi.org/10.1108/IMDS-05-2014-0156. 


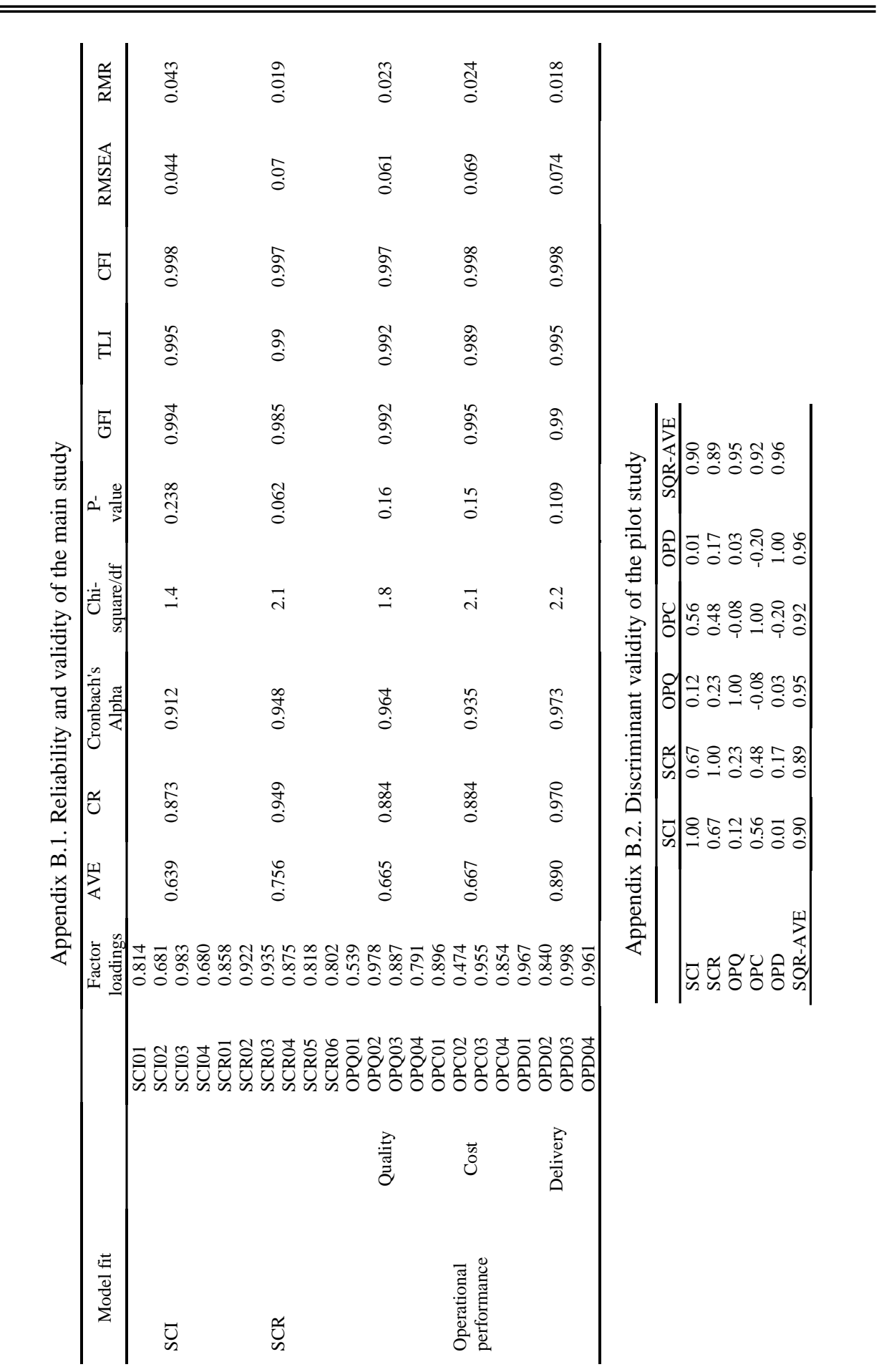

https://doi.org/10.24264/icams-2020.III.2 


\title{
INVESTIGATING THE IMPACT OF INTERNET OF THINGS ON EGYPTIAN
} PORTS SUSTAINABILITY

\author{
ENGY BAYOUMI ${ }^{1}$, SARA ELGAZZAR ${ }^{1}$, MATEVZ OBRECHT ${ }^{2}$ \\ ${ }^{1}$ College of International Transport and Logistics at Arab Academy for Science, Technology and \\ Maritime Transport, Alexandria,Egypt,engyosman48@gmail.com,sara.elgazzar@aast.edu \\ ${ }^{2}$ Department of Managing Logistics and Supply Chain, Faculty of Logistics, University of \\ Maribor, MariborskaCesta 7,3000 Celje,Slovenia, matevz.obrecht@um.si
}

\begin{abstract}
There are increasing concerns on the environmental impact of port operations which resulted in pressing global issues such as adopting sustainable technologies to reduce environmental harmful impacts on ports. From the sustainability perspective, a port should manage and balance three aspects which are economic, social, and environmental impacts. Using Internet of things (IoT) technology could minimize the environmental harmful impacts on ports and increase their sustainability. This paper aims at investigating the impact of applying IoT technology on Egyptian ports' sustainability. A semi structured interview was conducted with a representative sample of different stakeholders (government, port authority, freight forwarders, importers and exporters), upon which the main challenges and obstacles to adopt IoT technology in the Egyptian ports were identified. The paper concluded with suggestions and recommendations to help practitioners to consider the applicability of IoT technology as a sustainability driver in the Egyptian ports.
\end{abstract}

Keywords: port sustainability, Internet of things, Egyptian ports

\section{INTRODUCTION}

Environmental issues such as scarcity of resources, environmental pollution, global warming and decrease in biological diversity cause harming to the ecological balance. These ecological problems are increasing continuously to the extent that enforced governments, organizations and individuals to take precautions in environmental matters to maintain sustainability. International and national laws have recently been more restricted with organizations contributing to environmental problems and developed procedures in order to enforce them to change their production processes and supply chains to be more green and environmental friendly (Çankaya and Sezen, 2019).

The fourth industrial revolution has a tremendous impact on the digitalized environment and the environmental sustainability. Internet of things (IoT) is considered one of the core technologies related to the fourth industrial revolution. It facilitates all the supply chain operations to be more efficient and green with low costs on the long term and with higher quality products and services (Kayikci, 2018). It provides many applications in the fields of smart ports, smart transportation, smart energy, waste management and smart logistics (Ahmed, 2017).

Port traditional operations (such as cargo handling, loading and discharging, waste management) could cause environmental pollutions such as leakage, waste emissions and noise. Automating operations in ports by using technologies such as IoT could increase sustainability and reduce emissions (Figueiredo et al., 2017).

The purpose of this research is to investigate the impact of applying IoT on ports' sustainability. The paper starts in section two with review of previous studies to show the importance of IoT and the main barriers that may hinder its applicability. Semistructured interview methodology was presented and conducted in sections three and four respectively to examine the benefits of IoT and its applicability in The Egyptian 
ports. Finally the paper concluded in section five by proposing suggestions to facilitate IoT adoptability in The Egyptian ports.

\section{LITERATURE REVIEW}

The fourth industrial revolution is the era of automation and digitalization. The fourth industrial revolution tools such as Internet of Things (IoT) will increase transparency, enhance efficiency, and reduce the inefficient use of time (Adeniran, 2018). The application of IoT and the connected devices is increasing each year (Jović, 2019).

Internet of Things (IoT) is "a sophisticated technology with several applications and services in everyday life and in every field. IoT aims at penetrating our everyday environment and its objects, linking the physical world to the digital world and allowing people and devices to be connected anytime, anywhere, with anything and with anyone". IoT solutions can be applied to all fields and environments such as transportation and logistics field which includes sophisticated devices and applications for tracking, RFIDs in warehouses and assisted driving devices (Lampropoulos et al., 2019).

Ports are very complex industry because of their nature, offered services and a wide range of environmental issues such as harmful emissions, waste and noise production and pollution (Hakam, 2015). They are consisting of different companies, dealing with different activities and offering a wide range of services. Seaports are notably known to be very polluting industry. However, they have many opportunities for reducing emissions and pollutions (Broesterhuizen et al., 2012). Sustainability in the port industry is of growing source of fear for port authorities, policy makers, port users and local communities; which makes sustainability a one of the important concepts for port industry (Özispa and Arabelen, 2018).

With the development of the IoT, a growing area in academic research focuses on smart ports. They find some implications on how to enable intelligence of the port and they highlighted the principles of creativity in the smart sustainable development of port areas (Siror et al., 2011). In addition, there are key performance indicators guiding the evaluation of smart ports, involving the energy utilization rate, application of energy saving and low carbon technology, and the carbon intensity of port equipment, showing the challenges and obstacles in transformation into smart seaports (Ferretti and Schiavone, 2016).

An automated port consists of the sophisticated logistics solutions to enhance containers management, traffic flows, terminal and parking slot capacities. IoT enable to share information between terminal staffs and ship's crew about the ship capacity, the number of containers, the duration of stopover at port, etc. (Belfkih et al., 2017).

Moreover, IoT can help port workers to take the right planning decisions which improve the time of load and unload of containers and reduce the risk of damage. Also, using wireless sensors, fixed on the containers, enable data collection about containers before load and unload cargo to improve the containers management in the terminals and move them to the designated places, which reduces unnecessary moves, logistics time and costs. It also allows tracking containers of dangerous goods and facilitates the accesses control device by customs. IoT technology can also reduce the number of empty containers which enhance the terminal storage capacities (Dong et al., 2013). 
From the sustainability perspective, a port should manage and balance three aspects which are economic, social, and environmental impacts. From economic perspective, IoT provides real time monitoring and maximising the economic performance by increasing the port operations efficiency. Moreover, IoT will result in cost efficiency in the long run as all processes will be automated. The impact of applying IoT on social sustainability is mainly contributing to the enhancement and the monitoring of people's quality of life by supporting port activities to satisfy socio-economic priorities such as employment opportunities with safety precautions, education for employees and communities, and improving social stability of the area surrounding ports (Narula, 2014). While environmental sustainability can be achieved by applying IoT through minimising the negative impacts caused by a wide range of operational and shipping activities within the ports such as energy and resources used. So, this will minimize wastes and hence, all port practices will be green (Lim et al., 2018).

As indicated in the previous discussion, the automated ports that utilize IoT technology in their operations possesses many advantages especially in saving labour costs, improving the operation efficiency and economic benefits, reducing energy consumption, improving the level of safe operation, and promoting the image of the port and even the image of the city (Minh et al., 2012). According to estimations, the automated ports can save at least $25 \%$ more energy and reduce $15 \%$ more carbon emissions than the traditional ports (Yang et al., 2018).

Despite the benefits of IoT and its advantages, there are problems regarding the use of this technology such as security, privacy, and legal aspects. Keeping security and privacy in IoT devices must be a fundamental priority. Users need to trust that IoT devices and related data services are secure from attacks. Low secured IoT devices can expose user data to theft by leaving data streams inadequately protected. Accordingly, a collaborative approach to security will be needed to develop effective and appropriate solutions to IoT security challenges (Shakara et al., 2017).

There are also other barriers to implement IoT, such as high cost of implementation. IoT applications require designing IoT devices with centralized cloud-based business model. This requirement is very expensive and it would take years to cover its expenses, and then generate revenues. Also the lack of knowledge to users about implementing IoT could be challenging and time cost, and effort consuming (Bandyopadhyay and Sen, 2011). Moreover, lack of infrastructure is one of the greatest barriers that hinder the application of IoT. In addition, lack of universally agreed-upon standards is another problem to adopt IoT technology (Joshi, 2018).

Egypt has a strategic geographical location at the crossroads of Europe, Africa and Asia. Egypt lies between two major seas: the Mediterranean Sea, which provides a route to Europe and North America, and the Red Sea, which continues on to the Indian Ocean. In addition, the two seas are linked by the Suez Canal which is rendered as a major trade hub between Europe and Asia (Hafez and Madney, 2020).

Egyptian ports includes 15 commercial ports; 2 ports in Alexandria (Alexandria port and El-Dekheila port), 6 ports in Red Sea Ports Authority (Suez port, Hurghada port, Safaga port, Sharm El-Sheikh port, Nuwaiba port, Petroleum Dock port), 6 ports in Suez Canal Zone (Port-Said port, East Port-Said port, Sokhna port, El-Tour port, Arish port, Adabiya port), and Damietta port. Moreover, Egypt has 33 specialized ports in mining, petroleum, tourist and fishing. These ports are characterized by their strategic geographical locations and the availability of promising investment projects at the coming years and hence, this increases their competitive advantage (Maritime Transport Sector, 2020).

https://doi.org/10.24264/icams-2020.III.3 
Although the potentiality of Egyptian ports to lead worldwide, till now most of the Egyptian ports' operations are un-automated, this could be of a great importance to implement IoT applications in the Egyptian ports' operations.

This section highlighted the importance of IoT implementation in ports operations as well as its barriers and challenges, while showing the vital role it could play to contribute to the Egyptian ports competitiveness and sustainability. The next two sections will illustrate the methodology used in this paper to investigate the current situation of Egyptian ports concerning the automation procedures and IoT applicability, followed by suggestions to speed up and enable IoT applicability in the Egyptian ports operations.

\section{METHODOLOGY}

The research used a qualitative data collection strategy through conducting semi structured interviews to investigate IoT impacts on Egyptian ports and the main obstacles that may hinder its application in order to conclude with suggestions to overcome these obstacles. The semi structured interviews were conducted with a sample from the Egyptian port industry representing different stakeholders (Egyptian government, port authority, freight forwarders, importers and exporters) in order to obtain comprehensive discussion and analysis. First, the interview questions were developed based on the extensive literature review illustrated in the previous section. Then, pilot interviews were carried out with two operators at the Egyptian ports to get new unforeseen ideas about IoT implementation in Egyptian ports and verify the interview questions. Finally, a semi structured interview was conducted with five interviewees representing the main stakeholders in Egyptian port sector. The interview design includes 6 questions discussing: the impact of IoT on the 3 pillars of sustainability (environmental, economic and social), the barriers and challenges that hinder the application of IoT applications in the Egyptian ports and how to overcome them, and the interviewees suggestions to facilitate IoT application in the Egyptian ports.

\section{DISCUSSION OF RESULTS}

\section{Evaluation of the Impact of IoT on Environmental Sustainability in Egyptian Ports}

The interviewees believe that applying IoT could help in reducing emissions of using traditional equipment and vessel emissions due to lack of automation. For instance, if all containers on board the vessels were connected by sensors, the time of vessel arrival would be more accurate and hence, congestion in ports would decrease which will lead to less emissions. Also it will lead to less human interventions as they stated that the main reasons for environmental pollution are human and documents. The interviewees gave an example of Covid-19 and how it showed complications to handle ports operations in the traditional way with documents and un-automated operations. While if IoT was implemented, it would help in the continuity of the port operations without human intervention and without pollution. 


\section{Evaluation of the Impact of IoT on the Social and the Economic Aspects}

The interviewees agreed on implementing IoT in Egyptian ports would help in the social and the economic development. For the social impacts, IoT will reduce human congestion at the port as they commented that in Egyptian ports there are many customers supervise their shipments by themselves which lead to useless human congestions in ports. While IoT and automated systems could help them finish and track their shipments remotely, and hence, safety and security to them will increase.

For the economic impacts, the international trade in Egypt will increase because the operation itself will be more accurate, efficient and with less mistakes. Accordingly, the revenues of the authority will increase and thus the competitive advantage of the Egyptian ports will improve. The interviewees gave an example about port of Port-Said in Egypt as it is considered as an automated port compared to other ports in Egypt and customers prefer to receive their shipments from it because the process of receiving their shipment is easier and automated.

\section{The Barriers Hinder the Application of IoT in Egypt}

The interviewees summarize the barriers of applying IoT in Egyptian ports in 3 main barriers. Firstly, the high cost of implementation in the short run. IoT implementation will cost a lot in the short run, but when looking for the long run, it will cover its costs by generating huge amount of revenues. The second barrier is the lack of knowledge to many operators and it would be challenging and time-consuming to train them, in addition to the resistance to change. The third barrier is custom clearance in Egypt because it is $100 \%$ un-automated and it is the first process that customers do when they receive their shipment. The interviewees argued that the custom clearance workers will not be willing to change their process because IoT applications and systems are not unified and not standardized so many conflicts will happen.

\section{Suggested Tools that Could Be Used to Solve the Lack of Knowledge Barrier}

The interviewees stated that operators in Egypt are willing to do anything in order to get the business done. Once they learn something, they just follow it. So, if they are well prepared, by giving them appropriate seminars or on the job training, this will lead to the required capacity building. On the other hand, the interviewees commented that drivers and old operators might refuse to change their operations.

\section{The Influence of Privacy and Security Concerns on Applying IoT}

The interviewees believe that security concerns are a big issue because operators will not feel safe giving any information to any person to put on a device or an application. Also, if someone hack the sensors or the applications, it would be a problem because the port operations will be delayed and hence, a huge demurrage will be paid. Moreover, the operators and customers personal information will be hacked. Therefore in order to build such application, a well set plan on how legally it will be controlled is a must.

\section{Suggestions to Implement IoT in the Egyptian Ports}

The interviewees recommend to apply IoT in Egyptian ports partially as a pilot on one or two operations first (such as in cargo handling and transportation processes 
inside the port) in order to assess the results, and get the operators familiar with these technologies. Once the operators will witness the benefits of IoT applications especially in terms of the revenues that they will gain on the long run and the positive environmental impacts, this will encourage them to extent IoT applications to all port operations.

Another point they raised as a main trigger to speed up the implantation of IoT is considering the security aspects of the platform and data bases to gain the trust of the operators and other stakeholders. Moreover they said that Egypt has to unify the rules of transport operations and custom clearance in order to facilitate the implement of IoT.

\section{CONCLUSION}

IoT and digitization tools could provide seaports better monitoring to achieve sustainability and reduce the negative environmental, social and economic impacts. However, there are some risks and barriers could face the implementation of IoT such as the privacy concerns, the unified rules and the lack of knowledge from all parties in the port industry.

The paper provided a procedure to investigate the impact of applying IoT on the 3 pillars of sustainability and to suggest solutions to facilitate its applicability which can be adopted by other researchers in other countries. The interviews findings provided suggestions and insights that can help practitioners and decision makers in the Egyptian port industry to apply IoT technology as a sustainability driver in the Egyptian ports.

Further research can investigate how to solve the obstacles of IoT implementations identified in this research. Also a comparative research method can be employed to compare between countries already applied IoT technology and applications in their ports and Egypt in order to identify the gap analysis and lesson learned to shape a road map for Egyptian ports towards IoT implementation.

\section{REFERENCES}

Adeniran, A.O. (2018), "Impacts of the Fourth Industrial Revolution on Transportation in the Developing Nations”, International Educational Scientific Research Journal, 2(11), 56-60.

Ahmed, E.S. (2017), "Internet of Things Applications, Challenges and Related Future Technologies", World Scientific News International Journal, 67(2), 126-148, eISSN 2392-2192, 21, available online at www.worldscientificnews.com.

Bandyopadhyay, D. and Sen, J. (2011), "Internet of Things: Applications and Challenges in Technology and Standardization”, Wireless Personal Communications, 58, 49-69, https://doi.org/10.1007/s11277-0110288-5.

Belfkih, A., Duvallet, C. and Sadeg, B. (2017), "The Internet of Things for Smart Ports Application to the Port of Le Havre", Proceedings of IPaSPort 2017, 15-16.

Broesterhuizen, E., Vellinga, T., Docters van Leeuwen, L., Zwakhals, J.W., Taneja, P. and Nijdam, M (2012), "Sustainability as a procurement criterion for port investments", Third International Engineering Systems Symposium CESUN 2012, Rotterdam, The Netherlands.

Çankaya, S.Y. and Sezen, B. (2019), "Effects of green supply chain management practices on sustainability performance", Journal of Manufacturing Technology Management, 30(1), 98-121, https://doi.org/10.1108/JMTM-03-2018-0099.

Dong, X., Xiong, G., Li, Y., Guo, X. and Lv, Y. (2013), "Intelligent Ports Based on Internet of Things", Proceedings of 2013 IEEE International Conference on Service Operations and Logistics, and Informatics, https://doi.org/10.1109/SOLI.2013.6611428.

Ferretti, M. and Schiavone, F. (2016), "Internet of Things and business processes redesign in seaports. The case of Hamburg”, Business Process Management Journal, 22(2), https://doi.org/10.1108/BPMJ-052015-0079.

https://doi.org/10.24264/icams-2020.III.3 
Figueiredo, A.P., Carvalhal, R.D., Hoeflich, S., Figueiredo, L., Pereira, S.L. and Dias, E.M. (2017), "Port operation - increase of automated systems, decline of workforce jobs?", Recent Advances in Computer Science, 259-266.

Hafez, R.M. and Madney, I. (2020), "Suez Canal Region as an economic hub in Egypt location analysis for the mass real estate appraisal process", HBRC Journal, 16(1), 59-75, https://doi.org/10.1080/16874048.2020.1734347.

Hakam, M.H. (2015), "Nordic Container Port Sustainability Performance-A Conceptual Intelligent Framework", Journal of Service science and Management, 8(1), 14-23, https://doi.org/10.4236/jssm.2015.81002.

Joshi, N. (2018), "Due to these 5 Barriers, companies hesitate to adopt IoT", retrieved from https://www.allerin.com/blog/due-to-these-5-barriers-companies-hesitate-to-adopt-iot.

Jović, M., Tijan, E., Aksentijević, S. and Čišić, D. (2019), “An Overview of Security Challenges of Seaport IoT Systems", 42nd International Convention on Information and Communication Technology, Electronics and Microelectronics (MIPRO), 1571-1576, https://doi.org/10.23919/MIPRO.2019.8757206.

Kayikci, Y. (2018), "Sustainability impact of digitization in logistics", 15th Global Conference on Sustainable Manufacturing (p. 8), Beykoz, Istanbul, Turkey, Procedia Manufacturing, 21, 782-789, https://doi.org/10.1016/j.promfg.2018.02.184.

Lampropoulos, G., Siakas, K. and Anastasiadis, T. (2019), "Internet of Things in the Context of Industry 4.0: An Overview", International Journal of Entrepreneurial Knowledge, 7(1), 4-19, https://doi.org/10.37335/ijek.v7i1.84.

Lim, S., Pettit, S., Abouarghoub, W. and Beresford, A. (2019), "Port sustainability and performance: a systematic literature review", Transportation Research Part D: Transport and Environment, 72, 47-64, https://doi.org/10.1016/j.trd.2019.04.009.

Maritime Transport Sector (2020), retrieved from Maritime transport sector (MTS): http://www.mts.gov.eg/en.

Minh, L.H., Yassine, A. and Riadh, M. (2012), "Scheduling of lifting vehicle and quay crane in automated port container terminals", Intern. J. Intelligent Inform. Database Syst, 6(6), 516-531, https://doi.org/10.1504/IJIIDS.2012.050092.

Narula, K. (2014), "Emerging Trends in the Shipping Industry-Transitioning Towards Sustainability", Maritime Affairs: Journal of the National Maritime Foundation of India, 10(1), 113-138, https://doi.org/10.1080/09733159.2014.928473.

Özispa, N. and Arabelen, G. (2018), "Sustainability issues in ports: content analysis and review of the literature (1987- 2017)", SHS Web of Conferences, 58, 01022 https://doi.org/10.1051/shsconf/20185801022.

Shakara, A.H., Hasan, M.T. and Akter, N. (2017), "Solutions of common challenges in IoT", IOSR Journal of Computer Engineering, 19(5), 57-65, https://doi.org/10.9790/0661-1905055765.

Siror, J.K., Huanye, S. and Dong, W. (2011), "RFID based model for an intelligent port", Comput. Ind, 62(8 9), 795-810, https://doi.org/10.1016/j.compind.2011.08.004.

Yang, Y., Zhong, M., Yao, H., Yu, F., Fu, X. and Postolache, O. (2018), "Internet of things for smart ports: Technologies and challenges", IEEE Instrumentation \& Measurement Magazine, 21(1), 34-43, https://doi.org/10.1109/MIM.2018.8278808. 
Investigating the Impact of Internet of Things on Egyptian Ports Sustainability

https://doi.org/10.24264/icams-2020.III.3 
ANALYSIS OF THE BAKERY INDUSTRY STRATEGIC GROUPS IN ROMANIA

\author{
DANA CORINA DESELNICU ${ }^{1}$, MIHAELA-RAMONA BULBOACĂ², DAN DUMITRIU ${ }^{1}$, \\ LAURENTIA ALEXANDRESCU ${ }^{3}$ \\ ${ }^{1}$ University Politehnica of Bucharest, 313 Splaiul Independentei, sector 6, Bucharest, Romania \\ dana.deselnicu@upb.ro, dan.dumitriu@upb.ro \\ ${ }^{2}$ Vel Pitar S.A., 3-5 Emil Racovita Street, sector 4, Bucharest, Romania, \\ ramona.bulboaca@velpitar.ro \\ ${ }^{3}$ INCDTP - Division Leather and Footwear Research Institute, 93 Ion Minulescu, Bucharest, \\ Romania
}

\begin{abstract}
The bakery industry is one with as many challenges as any other FMCG (Fast Moving Consumer Goods) industry. In this sense, at the basis of each strategic decision, in order to improve competitiveness, on a constantly moving market, is the knowledge of competitors, the actions taken by them but also the innovation processes in which they are engaged. This paper aims to analyze the strategic groups formed in the bakery industry in Romania in terms of competitiveness. The paper begins with the analysis of the bakery industry, based on the parameters identified in the literature, continues with the investigation of strategic groups of the analyzed industry, according to the number of products sold and the geographical coverage of its players. Some of the paper results include a series of possible solutions and decisions that each company could apply, considering this segmentation. Analyzing the strategic groups of the bakery industry in Romania using current data and innovative analysis methods. The results of the study revealed a map of the strategic groups formed in the bakery industry, useful for companies in order to establish future strategic directions for maintaining or increasing their competitive position in the market in which they operate.

Keywords: strategic groups, bakery industry, competitiveness.
\end{abstract}

\title{
INTRODUCTION
}

The year of changes, as it is considered 2020 by more and more economic analysts, brings a lot of changes in the bakery industry in Romania, where all the players in the last years have been in decline due to the low consumption of bread. According to recent studies (GfK Romania, 2019), the consumption decreased from 9.8 kilos to around 8.2 kilo monthly per each Romanian resident. Thus, starting from the changes of the consumption habit in the pandemic context of this year, the companies found themselves in the situation to take quick decisions to change strategies at the company level, strategies adapted to current needs and in a continuous movement of the market.

The bakery industry is one with as many challenges as any other FMCG (Fast Moving Consumer Goods) industry. In this regard, the basis of each strategic decisions to improve competitiveness on a constant moving market is knowing the competitors, their undertaken actions and also the innovation processes they are engaged in. An industry dominated by the traditional white unpacked bread until few years ago, started to reinvent itself, as the consumers tastes have become more refined. This is how, although white bread stays on top of consumers' preferences, other types of bread became more popular, such as types of artisanal bread, rye or seeds bread, due to the healthy trends. Consumers are more concerned about what they eat, meaning they want to eat healthy and tasty products, and the producers had to adapt to their changing tastes. 
The paper aims to analyze the current framework where the bakery industry is, its main players and how they form strategic groups, as well as illustrating the way that they compete in the analyzed industry.

\section{THE STRUCTURAL ANALYSIS OF THE BAKERY INDUSTRY}

The structure of an industry has a strong influence in determining the rules of the competitive game, influencing the potential strategies for the companies that carry out their activity in that field. The essence of formulating a strategy is to link companies to their environment. Because the environment of companies is a fairly broad term, the key aspect is given by the industry or industries in which they compete. The industry, in this paper, will be defined as all the companies that produce close products, easily to substitute in final customers buying decision.

The intensity of competition in an industry is not a game of chances. Its roots are in the basic economic structure and exceeds by far current competitors' behavior (Deselnicu et al., 2008). How it is a competition in an industry depends on the five basic competitive forces illustrated by M. Porter, presented in Figure 1:

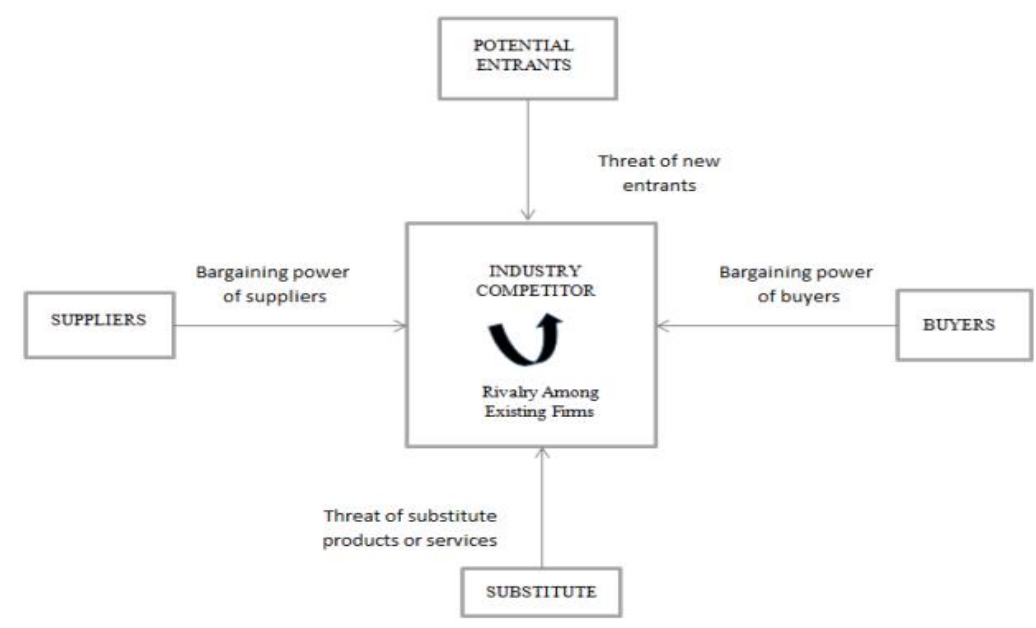

Figure 1. Forces driving the industry competition. Source: Porter, M.E. (1998), p.4.

The collective strength of the forces illustrated in figure 1 determines the profit potential of an industry. Not all industries have the same level of potential and attractiveness. The purpose of the competitive strategy for a company in an industry, in general, is to find a position in which it can best respond to the competitive forces, in the sense of defending themselves from them or influencing them for their own purposes. The set of 5 forces determines the intensity of competition and the profitability of the industry on which they act, and companies must determine very well the forces that act most strongly on their fields of activity, so as to formulate the most appropriate strategies (Popescu, 2017). 
The bakery industry in Romania is extremely fragmented in terms of the fact that there are many small competitors, bakeries that operate on the specifics of local markets. For this reason, the authors decided to analyze the competitive situation for the first eleven competitors, which have the strongest impact at national, whose turnover (CA) in 2019 totaled represents approximately 25\% of industry's sales, industry estimated by experts at 6.5 billion RON.

In order to familiarize with the size of the analyzed competitors, table 1 was prepared, showing the status of the 11 main players in the bakery industry, considering the turnover of year 2019 (Ministry of Public Finance, 2020), their main characteristic, the approximate number of products marketed, their dominant strategy and number of production units/logistics units, owned by each one:

Table 1. Presentation of the main competitors in the bakery industry, in Romania

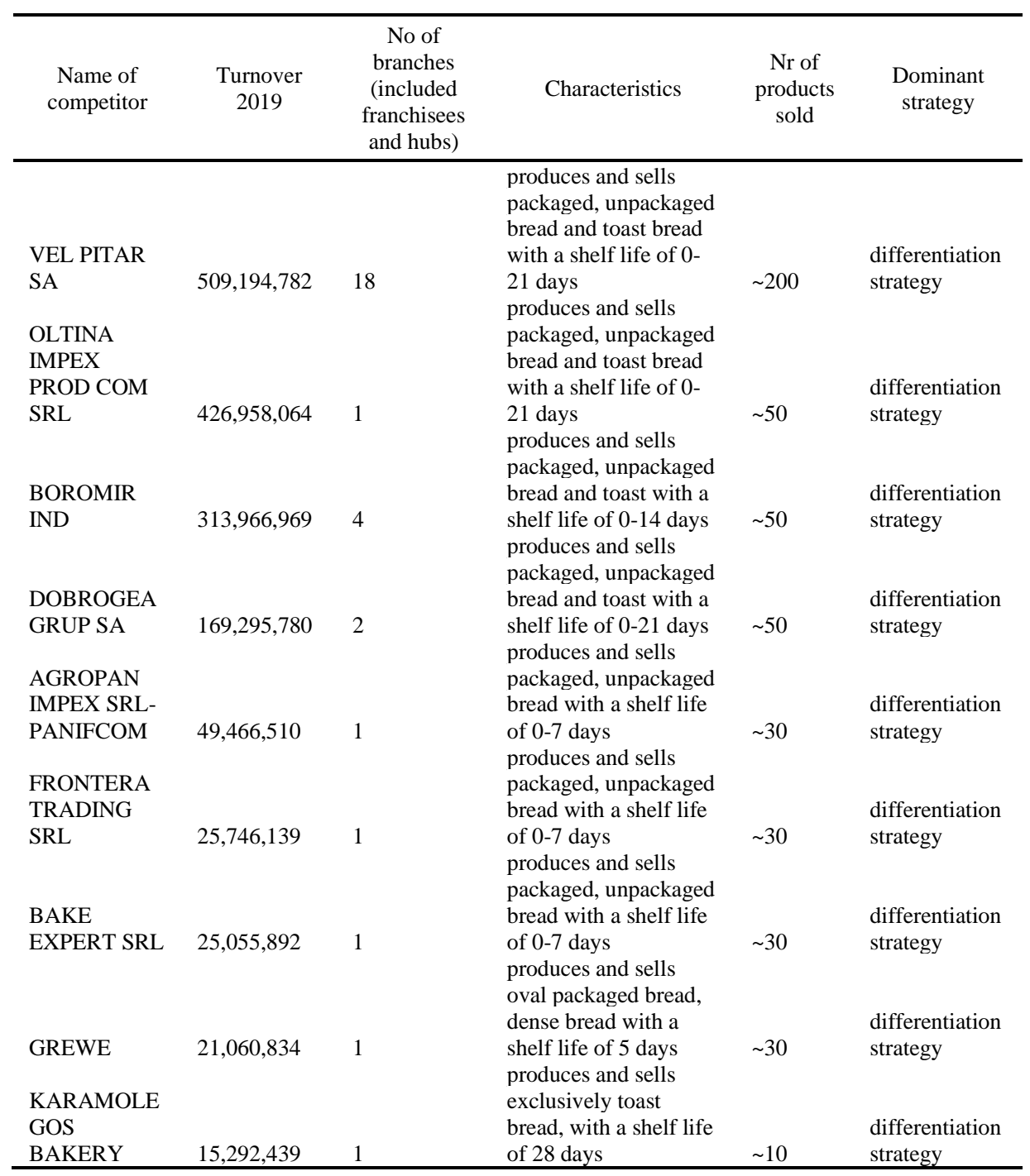

https://doi.org/10.24264/icams-2020.III.4 
Analysis of the Bakery Industry Strategic Groups in Romania

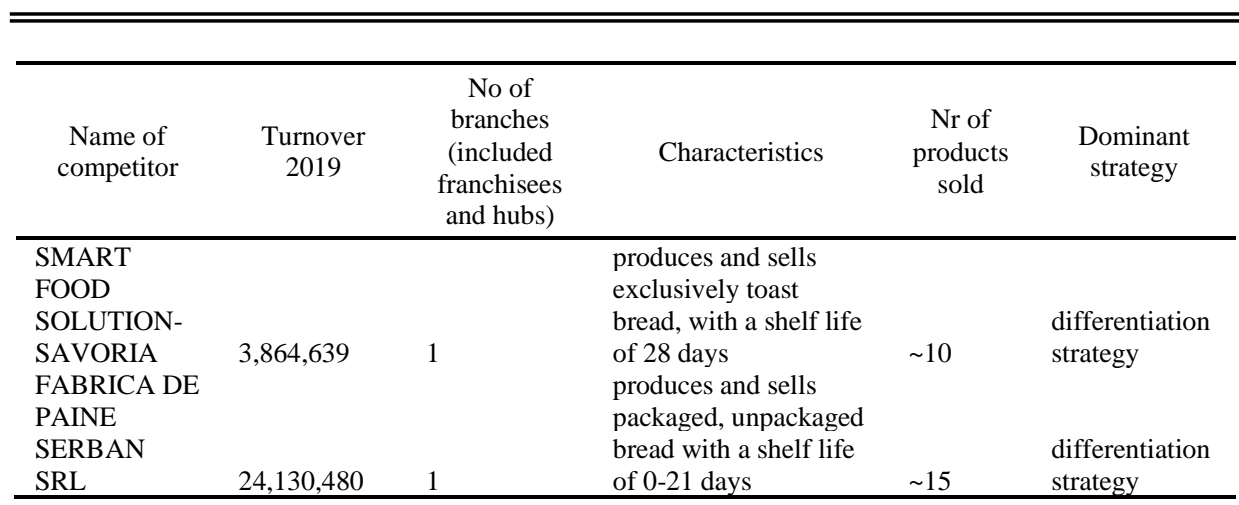

Continuing the process of analyzing the competitive situation in the bakery industry, figure 2 was made, to illustrate the map of production units for competitors already presented in table 1:

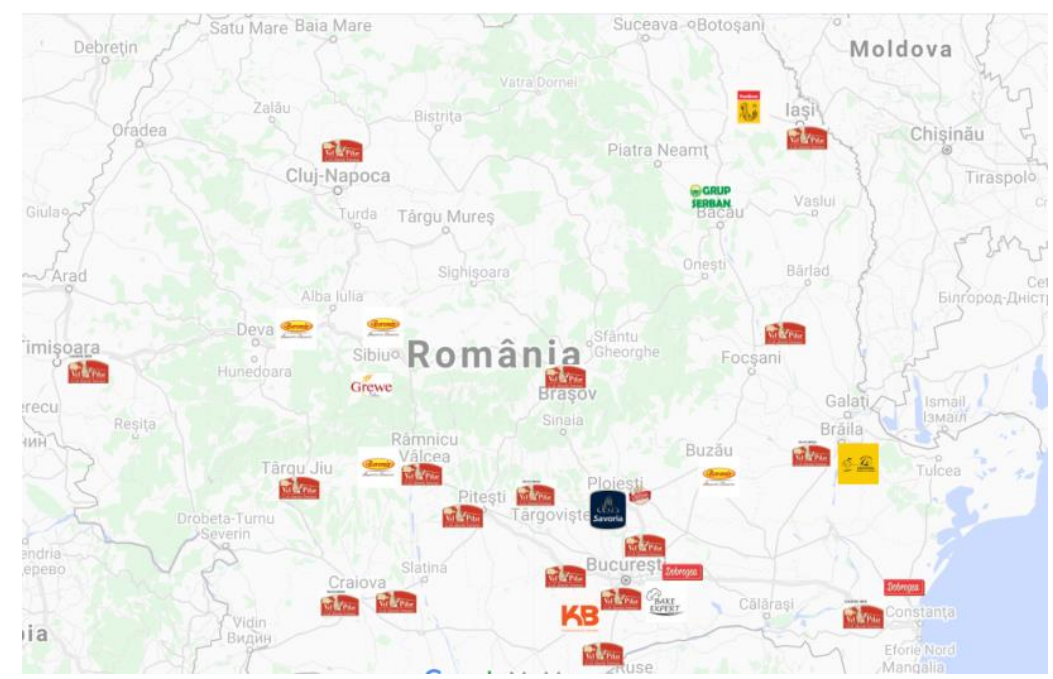

Figure 2. Map of factories and hubs of the main competitors in the bakery industry, in Romania. Source: created by the authors.

In this map, one can easily observe the fact that the strongest area of Romania is the South, where are the headquarters for the most important of the 11 competitors and where there are the largest units of production in the industry.

Analyzing the bakery industry in light of the 5 strengths, research of the authors have revealed that the most dominant forces for this industry are threat of substitute products and potential entrants. The first of them is important because for the moment, the star product in this industry is unpacked white bread, a very easy product to be substituted by the end consumers.

The second important force protects the biggest players, because the start-up costs for a company in this industry are very high, if that company really wants to be a significant player. The last-mentioned force affects or protects each one of the eleven, in different proportions - for example: Vel Pitar is protected by new entrants because of 
the scale economies, Oltina and Dobrogea Grup, also Boromir benefit of a vertically integrated business (regarding the acquisition of main raw materials - that is flour).

\section{THE MAP OF STRATEGIC GROUPS}

In his books, M. Porter (1998) defines a strategic group, in short, as being a framework all the competing companies on a market, whose behavior is similar. The criteria on which companies that compete in a market and that belong to a strategic group are identified, refer to their behavior resulting from similar approaches to market mechanisms. These criteria refer mainly to: targeting the same segment of consumers; satisfying the needs of consumers by offering products with similar main characteristics; use of the same distribution channels; offering products that are at a similar level as quality/price ratio (Russu, 1999).

Analyzing the way of forming strategic groups in the Romanian bakery industry, using the analytical tool called "map of strategic groups", launched in the literature by M. Porter, resulted four strategic groups. These groups were formed by considering two strategic dimensions, namely geographical coverage and the number of products marketed by the main players of this industry:

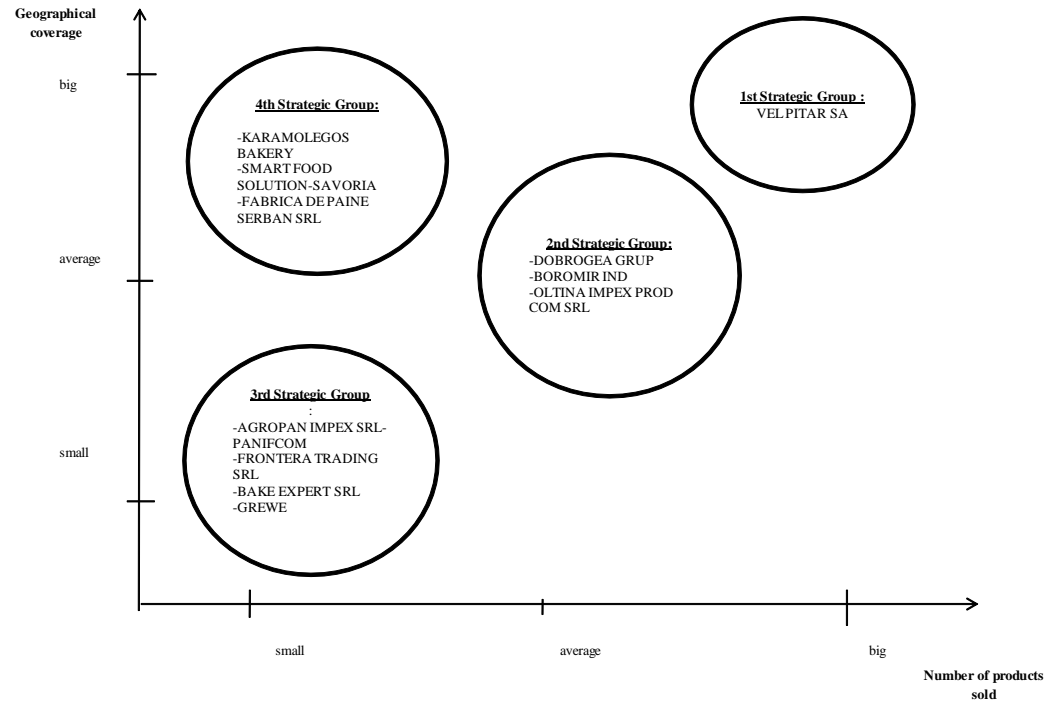

Figure 3. Map of Strategic Groups in the bakery industry. Source: created by the authors

From the picture of the strategic groups formed in the analyzed industry, Vel Pitar stands out by far, as being the strongest competitor in the industry, from the analyzed points of view. Its main followers seems to be those from Boromir and Dobrogea Group and in secondary, the ones from Oltina (thanks to the private label projects, especially for toast bread). The other strategic groups, even if they were trying to adopt the same strategy, do not have the resources or capabilities of those three mentioned above, in order to succeed in causing them problems. The third group has more local players, with tradition in their markets: they market products in big cities, but, with some products, they also target the largest market in the country, the Bucharest one. The fourth group focuses on secure contracts with IKA (International Key Accounts), adopts the 
centralized delivery method at the logistics platforms of large retailers, which limits them in product distribution.

\section{CONCLUSIONS}

This analysis model is a new one for the Romanian bakery industry, an industry rather seldom analyzed, but one with a huge potential for selling value-added products, helped, in this particular moment, by the economic context, namely, changing consumption habits and growing consumers preferences for packaged bread. This can be explained by the sense of security given by packaged bread for consumption.

The two axes that make up the functional model for analyzing strategic groups can be adapted and interpreted considering many more of strategic dimensions. Such dimensions can be: the model is a dynamic one, which can be used as a starting point for any competitive analysis, for any company regardless the industry; it is also an important model based on which a company's strategies can be adopted or changed, depending of the moment in which the industry is, the dimensions analyzed, but also of the purpose for which this functional model of mapping competitors using a map of strategic group is used.

\section{REFERENCES}

Deselnicu, D.C., Albu L., Swiger, J.A. and Cioponea, I. (2008), "Competitiveness and Innovation in Romanian Enterprises: A Case Study", Proceedings of the 2nd International Conference on Advanced Materials and Systems, ICAMS 2008, 545-550, Ed. CERTEX, 23-24 October, Bucharest.

GfK, ROMPAN (2019), Market Study: "Understanding the flour products market", available at https://artaalba.ro/piata-painii-intre-traditie-si-inovatie/

Ministry of Public Finance (2020), "Indicators from the annual financial statements", available at https://www.mfinante.gov.ro/infocodfiscal.html

Popescu, C.A. (2017), Strategic Management, Politehnica Press, Bucharest.

Porter, M.E. (1998), Competitive Strategy - Techniques for analyzing industries and competitors, The Free Press, New York.

Russu, C. (1999), Strategic Management, All Beck, Bucharest. 


\title{
APPLICATION OF THE "SIX SIGMA" METHOD FOR THE ANALYSIS OF THE IMPROVEMENT OF THE ENVIRONMENTAL AIR QUALITY \\ PARAMETERS AT THE MUNICIPALITY OF BUCHAREST, BY MONITORING THE POLLUTANCES OF NOx POLLUTANTS
}

\author{
ION DURBACĂ, NICOLETA SPOREA*, DANA-CLAUDIA FARCAȘ-FLAMAROPOL, \\ ELENA SURDU \\ "Politehnica" University of Bucharest, Spl. Independenței street, 313, Bucharest, Romania \\ e-mail: ion.durbaca@yahoo.com,nsporea@yahoo.com, claudia.flamaropol@gmail.com, \\ elena.surdu@yahoo.com
}

\begin{abstract}
This paper analyzes the improvement of ambient air quality indicators by monitoring the $\mathrm{NO}_{x}$ concentration in one of the most polluted areas of Bucharest, using the statistical method "SIX SIGMA" $(6 \sigma)$. By applying the methodology of this statistical approach, the aim is to reduce nonconformities within the specified limits (according to the standards and legislative norms in force) and respectively, to ensure maximum efficiency $(99,99 \%)$, equivalent to a yield of 3.4 defects per million opportunities (DPMO). As high concentrations of air pollutants have a major impact on human health, the most harmful effect has been found to be caused by nitrogen dioxide $\left(\mathrm{NO}_{2}\right)$, mainly from ground-level ozone. Using the " $\sigma \sigma$ " method, the optimal solutions for eliminating non-conformities and implicitly for reducing the $\mathrm{NO}_{2}$ concentration and ensuring the improvement of the ambient air quality can be identified.
\end{abstract}

Keywords: Six Sigma, air quality, air pollutant, efficiency.

\section{INTRODUCTORY CONSIDERATIONS}

At present, air pollution has proven to be one of the greatest dangers from an environmental, human health and ecosystem perspective, attracting negative effects not only locally or nationally, but also at European and global level (European Environment Agency).

At the level of large urban agglomerations, including the city of Bucharest, one of the major pollutants is road transport, as the main responsible for the production of nitrogen oxides $\left(\mathrm{NO}_{\mathrm{x}}\right.$ - extremely harmful pollutants), but also suspended particles (PM) (Brue, 2002).

The Great Metropolis - Bucharest is one of the most polluted cities in Europe and is in the process of infringing with the European Environment Commission for constant exceedances of the quantities of fine moving particles in the air (PM10, PM2.5), but also for the inability to monitor air quality according to the law.

Currently, the National Air Quality Monitoring Network (RNMCA) includes 148 continuous air quality monitoring stations, equipped with automatic equipment for measuring the concentrations of the main air pollutants: sulfur dioxide $\left(\mathrm{SO}_{2}\right)$, nitrogen oxides $\left(\mathrm{NO}_{\mathrm{x}}\right)$, carbon monoxide $(\mathrm{CO})$, ozone $\left(\mathrm{O}_{3}\right)$, suspended particles (PM10 and $\mathrm{PM} 2.5)$ etc. The air quality in each monitoring station is represented by suggestive quality indices, established based on the values of the concentrations of the main air pollutants measured (Bejan et al., 2009; Alpopi, 2008; Vişan et al., 2000; Crișan et al., 2017; Kifor, 2006).

Due to the identification by measurement of high concentrations of nitrogen dioxide $\left(\mathrm{NO}_{2}\right)$ in the six existing monitoring stations in Bucharest, the statistical approach "Six Sigma" was addressed in order to analyze the improvement of ambient air quality parameters by including preventive and corrective measures to reduce the $\mathrm{NO}_{2}$.

https://doi.org/10.24264/icams-2020.III.5 
Application of the New Statistical Approach "Six Sigma" for the Analysis of the Improvement of the Air Quality Parameters at the Level of Bucharest, by Monitoring the $\mathrm{NO}_{x}$ Concentrations from the Emissions of Air Pollutants

\section{DEFINITION OF THE STATISTICAL METHOD "SIX SIGMA"}

The novelty of this statistical method "Six Sigma", typical of quality engineering, is the possibility of its application at the level of many organizations, regardless of their specifics (Moțoiu, 1994).

So, starting with the emergence of the concept of "zero defects", which was the starting point in the creation of the statistical method "Six Sigma" for Motorola in 1986 by Bill Smith and then continuing with its application to other top companies (General Electric, Honeywell International, ABB, Lockheed Martin, Polaroid, Sony, Honda, American Express and Solectron), the application of this statistical approach has been launched in many organizations around the world, most of which can prove the role of a pivotal element of the "Six Sigma" method to their success (Pande, 2008; Pande et al., 2009; Durbacă and Sporea, 2012; Durbacă, 2015).

By using the results of concrete assessments of quality characteristics specific to the concentration of nitrogen dioxide $\left(\mathrm{NO}_{2}\right)$ measured in the six existing monitoring stations in Bucharest, the paper aims as a methodology specific to the statistical approach Six Sigma (" $6 \sigma$ "), lead implicitly to improvements in the performance, efficiency and quality of the ambient air, as well as to the reduction of defects / nonconformities corresponding to the values measured within the specified limits (Lower limit of specification, LIS $=15 \mu \mathrm{g} / \mathrm{m}^{3}$ and Upper limit of specification, LSS $=40$ $\mu \mathrm{g} / \mathrm{m}^{3}$ ), according to the provisions of Law no. 104 of June 15, 2011.

In Figure 1, the performances of the " $6 \sigma$ " method are highlighted graphically, by comparison with the " $3 \sigma$ " method, taking into account the standard deviation " $\sigma$ " which represents the basic metric in the statistical analysis of the data of some evaluated / measured characteristics, respectively the value of the variable showing the distribution of the output characteristic of the process.

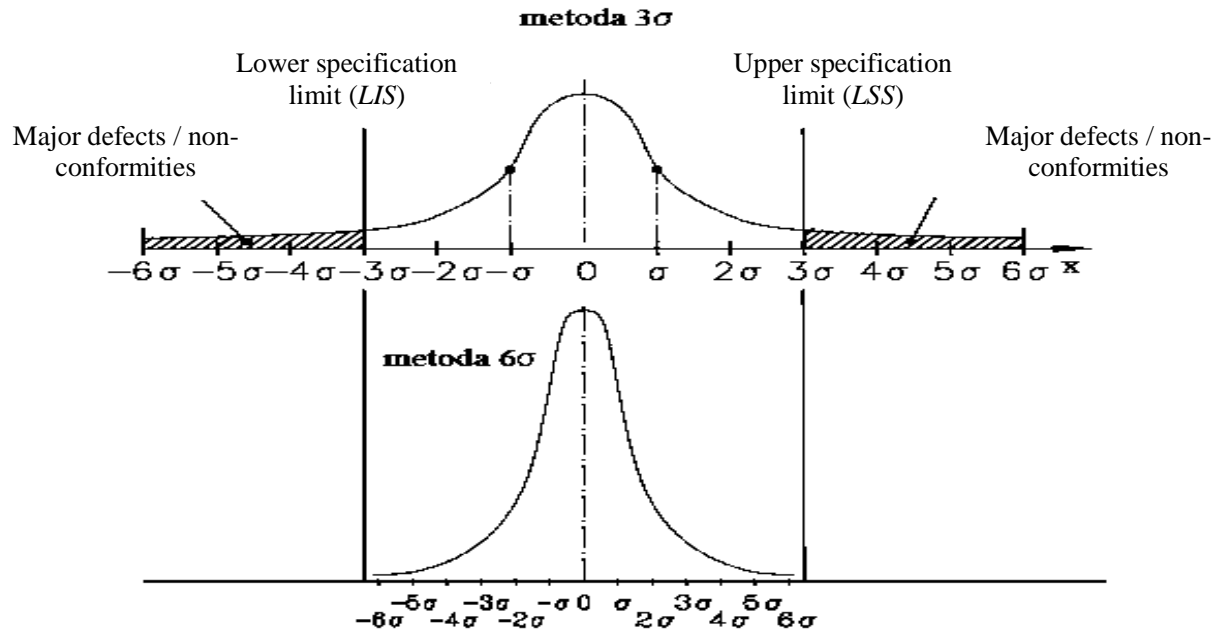

Figure 1. Performance of the "Six Sigma" method (Durbacă and Sporea, 2012)

A higher value for sigma $(\sigma)$ indicates a more stable process, with a lower risk for defective events (rejects / major nonconformities) and lower costs, respectively. 


\section{DESCRIPTION OF THE APPLICATION}

The step-by-step approach of the specific phases of the "Six Sigma" method (DMAIC) is considered: Defining opportunities / quality problems; Measuring current performance levels; Analysis of the causes underlying the non-conformities / defects; Improving performance; Control performance (Durbacă and Sporea, 2012).

To obtain the best results, an analysis of the daily values of $\mathrm{NO}_{2}$ concentrations $\left[\mu \mathrm{g} / \mathrm{m}^{3}\right]$ was performed measured at the six continuous air quality monitoring stations in Bucharest (S1 - Lacul Morii; S2 - Titan; S3 - Mihai Bravu; S4 - Berceni; S5 - Camp Road; S6 - Military Circle) for a period of 20 calendar days (12.08.2020 - 31.08.2020).

Following the brainstorming analysis at a meeting of a group of specialists in the field, it was decided that the daily values of $\mathrm{NO}_{2}$ concentrations $\left[\mu \mathrm{g} / \mathrm{m}^{3}\right]$ measured at station S3 - Mihai Bravu, having the highest values, to be subjected to the application of the Six Sigma methodology.

To represent the probability density graph in the case of a normal distribution (Gauss) the results corresponding to the strategy for accurately detecting non-compliant $\mathrm{NO}_{2}$ concentrations $\left[\mu \mathrm{g} / \mathrm{m}^{3}\right]$, of their quantification and elimination, it is necessary to go through the following methodological procedure (Durbacă and Sporea, 2012):

a) ascending order of the string of 20 measured values $X_{i}(i=1, \ldots \ldots, 20)$ of the quality characteristic / $\mathrm{NO}_{2}$ concentration $\left[\mu \mathrm{g} / \mathrm{m}^{3}\right]$, thus:

26,$29 ; 30,04 ; 34,93 ; 37,09 ; 37,18 ; 37,23 ; 38,30 ; 38,41 ; 38,69 ; 44,18 ; 45,70 ; 48,72$; 56,$38 ; 57,28 ; 58,94 ; 60,10 ; 61,10 ; 62,19 ; 64,15 ; 69,02$.

b) notation of the minimum value $\mathrm{X}_{\min }$ and respectively, maximum $\mathrm{X}_{\max }$ of the ascending ordered string:

$X_{\min }=26,29\left[\mu \mathrm{g} / \mathrm{m}^{3}\right] ; X_{\max }=69,02\left[\mu \mathrm{g} / \mathrm{m}^{3}\right]$.

c) determining the amplitude of the string:

$R=X_{\max }-X_{\min }=69,02-26,29=42,73\left[\mu \mathrm{g} / \mathrm{m}^{3}\right]$.

d) determining the number of classes $K$ of the sequence of ordered values, with Sturges formula (Durbacă and Sporea, 2012):

$K=1+3,22 \lg n=1+3,22 \lg 20 \approx 5$.

e) determining the amplitude of the class, $\delta$ :

$\delta=R / K=42,73 / 5=8,54$.

f) completing the ordered values according to the previously determined classes, in Table 1:

Table 1. Values ordered by classes

\begin{tabular}{ccccc}
\hline K1 & K2 & K3 & K4 & K5 \\
\hline 26,29 & 37,18 & 38,69 & 56,38 & 61,10 \\
30,04 & 37,23 & 44,18 & 57,28 & 62,19 \\
34,93 & 38,30 & 45,70 & 58,94 & 64,15 \\
37,09 & 38,41 & 48,72 & 60,10 & 69,02 \\
\hline
\end{tabular}

g) determining the grouping intervals corresponding to the value classes and respectively, average value $\bar{X}$ (v. Table 2 ): 
Application of the New Statistical Approach "Six Sigma" for the Analysis of the Improvement of the Air Quality Parameters at the Level of Bucharest, by Monitoring the $\mathrm{NO}_{x}$ Concentrations from the Emissions of Air Pollutants

Table 2. Grouping intervals corresponding to value

\begin{tabular}{cccccccc}
\hline No. & $\begin{array}{c}\text { Grouping } \\
\text { interval }\end{array}$ & Centered & \multicolumn{4}{c}{ Frequency } \\
& $x_{i} \div x_{i+j}$ & $\boldsymbol{x}_{\boldsymbol{i c}}$ & $\boldsymbol{a}_{\boldsymbol{i}}$ & $\boldsymbol{f}_{\boldsymbol{i}}$ & $\boldsymbol{A}_{\boldsymbol{i}}$ & $\boldsymbol{F}_{\boldsymbol{i}}$ & $\boldsymbol{X}_{\boldsymbol{c}} \boldsymbol{f}_{\boldsymbol{i}} \boldsymbol{.}$ \\
\hline 1 & $26,29 \div 34,83$ & 30,56 & 2 & 0,10 & 2 & 0,10 & 3,056 \\
2 & $34,93 \div 43,47$ & 39,20 & 7 & 0,35 & 9 & 0,45 & 13,720 \\
3 & $44,18 \div 52,72$ & 48,45 & 3 & 0,15 & 12 & 0,60 & 7,267 \\
4 & $56,38 \div 64,92$ & 60,65 & 7 & 0,35 & 19 & 0,95 & 21,227 \\
5 & $69,09 \div 69,02$ & 0 & 1 & 1,05 & $\mathbf{2 0}$ & $\mathbf{1 , 0 0}$ & 0,000 \\
& $\Sigma$ & & $\mathbf{2 0}$ & $\mathbf{1 , 0 0}$ & - & - & $\mathbf{4 5 , 2 7}$ \\
\hline
\end{tabular}

h) determination of the mean square error $\sigma$, with the relation:

$\sigma=\sqrt{\frac{\sum_{i=1}^{n}\left(X_{i}-\bar{X}\right)^{2}}{n-1}}=12,806$

i) determining the limits of specifications $\backslash$ (lower, LIS* and upper, $L S S^{*}$ ) corresponding to the actual level of performance $6 \sigma=76,836$ :

$L I S^{*}=\bar{X}-3 \sigma=45,27-3 \cdot 12,806=6,852$;

$L S S^{*}=\bar{X}+3 \sigma=45,27+3 \cdot 12,806=83,688$.

j) the representation of the normal distribution curve, related to the values specific to the quantification of the maximum performance (corresponding to level $6 \sigma$, equivalent to 3,4 defects per 1 million opportunities), via the normal Gauss distribution function, $f(x)$ and the MathCAD program (see Figure 2).

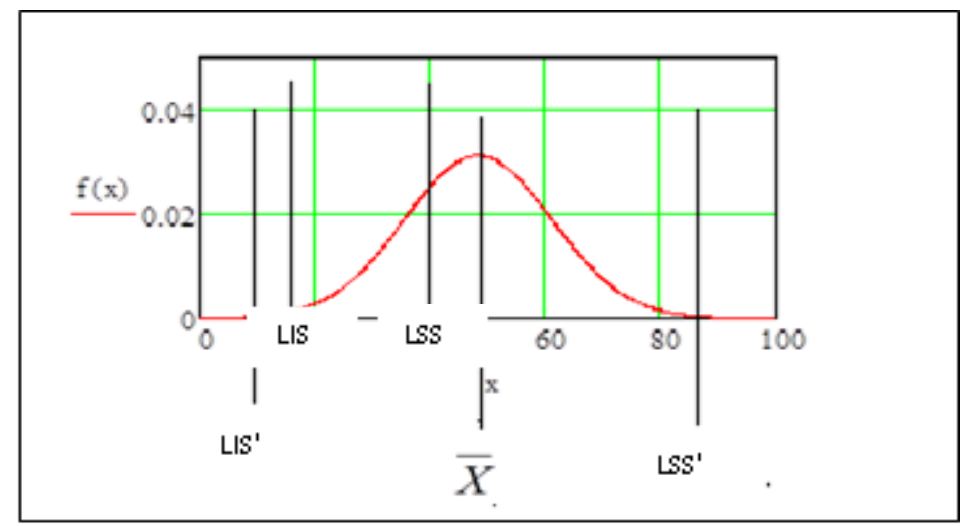

Figure 2. Gauss normal distribution curve

According to the graphical representation of the function $f(x)$ in Figure 2, corresponding to the distribution of the measured values of the analyzed quality characteristic, in relation to their average value, it follows that the graphical distribution of the "Gauss's bell" is between the limits of specifications (LIS* and LSS*) previously determined with relationships (5) and (6). Such a distribution characterizes a real level of maximum performance " $6 \sigma$ ”, equivalent to 3,4 defects per 1 million opportunities

https://doi.org/10.24264/icams-2020.III.5 


\section{RESULTS AND DISCUSSIONS}

Considering that in the analyzed case the condition is fulfilled: $6 \cdot \sigma>\mathrm{R}$, this would correspond to the need to improve the quality and, respectively, to take measures to help to rigorously frame the values of $\mathrm{NO}_{2}$ concentrations between the limits of the LIS and LSS specifications and respectively, eliminating values that do not fall within these limits. But nevertheless, the graphical representation of the normal Gaussian distribution function $f(x)$, in Figure 2 above, confirms the permissiveness of extending the range of measured values between the specification limits $L I S^{*}$ and $L S S^{*}$, which attests to the achievement of the efficiency index of 3,4 defective parts per million opportunities (DPMO), corresponding to an efficiency of $99,9997 \%$ and synonymous with the successful completion of the quality improvement project.

From the calculation of the capability index of the quality improvement process, $\mathrm{C}_{p}$ results (Durbacă, 2017):

$C_{p}^{*}=\frac{\text { LSS } * \text { LIS } *}{6 \sigma}=1,00$.

In this case, for which $C_{p} *<1,33$, the improvement process may become efficient under certain conditions, but careful supervision is required (Durbacă, 2017).

\section{CONCLUSIONS}

By applying the statistical method "Six Sigma" at the level of any organization regardless of the activity profile, it is possible to respond to the purpose function, defined by the improvement of the quality of the analyzed entities. Although it is a method based on mathematical statistics, "Six Sigma" does not offer a difficult tool to use and thus represents, a guaranteed success for such organizations that aim to achieve outstanding results and ensure superior levels of performance.

Therefore, the "Six Sigma" statistical method is equally addressed to all organizations for improving performance, efficiency and quality of entities (products, processes, services, resources, environmental factors, etc.), as well as to reduce defects / non-conformities within the specified limits, by ensuring maximum stability and efficiency.

\section{REFERENCES}

Alpopi, Cr. (2008), "The effects of urban agglomeration on the environment, in the magazine", Economia series Management, 11(2), 12-20.

Bejan, M., Rusu, T. and Bălan, I. (2009), "The effects of air pollution due to car transport activity", AGIR Bulletin, 4, October-December, 192 - 199.

Brue, Gr. (2002), Six Sigma for Managers, McGraw-Hill, New York.

Crișan, H.G., Crișan, O.A., Lakatos, E.S. and Scurtu, I.L. (2017), "Aspects regarding the effects of vehicle pollution on the environment - Case study", XVII International - multidisciplinary Conference "Professor Dorin Pavel - founder of Romanian hydropower", Sebeș.

Durbacă, I. (2015), "New Statistical Approach "Six Sigma" as a Solution for Improving Plastic Quality Products", Materiale Plastice, 52(1), 43-47.

Durbacă, I. (2017), "Engineering and quality control «Six Sigma»", Guide to applied works - For students" use, Printech Publishing House, Bucharest, ISBN 978-606-23-0728-8, 142 pages, CNCSIS Code 54, BN Quota - 62.

Durbacă, I. and Sporea, N. (2012), Engineering, management and quality assurance, Bucharest, Printech Publishing House.

Kifor, C.V. (2006), Quality engineering. Improving 6 Sigma, "Lucian Blaga” University of Sibiu Publishing House.

https://doi.org/10.24264/icams-2020.III.5 
Application of the New Statistical Approach "Six Sigma" for the Analysis of the Improvement of the Air Quality Parameters at the Level of Bucharest, by Monitoring the $\mathrm{NO}_{x}$ Concentrations from the Emissions of Air Pollutants

Moțoiu, R. (1994), Quality Engineering, Chiminform Data S.A. Publishing House, Bucharest.

Pande, S.P. (2008), The Six Sigma Leader. How Top Executives Will Prevail in the 21st Century, McGrawHill Professional.

Pande, S.P., Neuman, P.R. and Cavanagh, R.R. (2009), "Six Sigma”, How GE, Motorola and other top companies improve their performance, ALL Publishing House, Bucharest.

Vişan, S., Angelescu, A. and Alpopi, C. (2000), Environment. Pollution and protection, Bucharest, Economic Publishing House.

*** European Environment Agency, “Air pollution”, https://www.eea.europa.eu/themes/air, Date las accessed: 30.06 .2020 


\title{
FACTORS AFFECTING STARTUPS SURVIVAL IN THE MENA REGION IN
} THE PRESENCE OF COVID19

\author{
NERMIN GOHAR ${ }^{1}$, HANY HANNA ${ }^{1,2}$, MAI H. HAROUN ${ }^{1}$ \\ ${ }^{1}$ Logistics of Supply Chain Department, College of International Transport and Logistics, Arab \\ Academy for Science, Technology and Maritime Transport, AASTMT, Alexandria, Egypt, e-mail: \\ hany.hanna@strath.ac.uk \\ ${ }^{2}$ University, Strathclyde College of Engineering, DMEM, Glasgow, UK
}

\begin{abstract}
A great percentage of the world economy is driven by entrepreneurs, start-ups and Small - and Medium - sized Enterprises (SMEs), as they are the driving forces of economic stability. This paper attempts to gain a full understanding of the environment in which entrepreneurs and SMEs operate; the so-called entrepreneurial ecosystem in MENA region to gather and collect information related to the needs examination on promoting entrepreneurial important criteria with the focus on Specific Region. The methodology proposed follows a qualitative approach using semi-structured interviews directed to owners and managers of startups in the MENA region. The process will be to observe, analyze, visualize the entrepreneurial ecosystem, including surrounding criteria's and factors that effects the startup survival. The proposed ecosystem is then 'mapped' to enable to see the gaps and constrains in the ecosystem in the MENA region, and to devise the most relevant measures to energize the ecosystem in MENA region. The proposed methodology and conceptual frame work not only will look at traditional indicators as business environment and investment climate, but also focus on creating optimal ecosystem that is well structured and ready for any unexpected events or occasions, like COVID19, which might have its impact on startups.
\end{abstract}

Keywords: Entrepreneurs in MENA, Entrepreneurial ecosystem, startups MENA region

\section{INTRODUCTION}

Entrepreneur is one of the most important inputs in the economic development of the country. Entrepreneurial competence makes all the difference in the rate of economic growth through creation of utility and generation of employment. Success of entrepreneurial activity, in any country, depends on a number of factors such as affluent natural resources, good infrastructure, availability of skilled labor, availability of adequate finance, wide market, advanced technology.

Government Policies in favor of entrepreneurs, talent, skill and ambitions of the entrepreneurs. This fact had been widely realized and accepted in the world today for the development of nations and it is an urgent need to find, nourish and develop competent entrepreneurship, if they want to achieve quick industrial development. Therefore, the paper investigates different factors affecting survival of entrepreneurial startups in the presence of the COVID19 event. The paper is divided into five sections; the first section is the current introduction, the second section presents the literature review for this research, the third section discusses the methodology followed for this research, the fourth section introduces the main research findings and finally, the fifth section presents the conclusion derived from this research.

\section{LITERATURE REVIEW}

This section discusses previous studies related to the entrepreneurial ecosystem, as well as startups survival criteria. 


\section{Entrepreneurial Ecosystem}

As a multidisciplinary field of study (Parker, 2009) that involves economics, management, social science and anthropology (Ahmed and Seymour, 2008), there exists several definitions of entrepreneurship, each carries a slightly different perspective and scope. The 1997 Organization on Economic Cooperation and Development (OECD) survey defined entrepreneurship as "The dynamic process of identifying economic opportunities and acting upon them by developing producing and selling goods and services". Therefore, entrepreneurship was considered to be as an engine of economic growth and it is related to a combination of several determinants such as education levels, business climate and legal and political conditions (Alvarez et al., 2014).

Additionally, based on the systems approach by Acs et al. (2014), entrepreneurship is an action undertaken and driven by agents on the basis of incentives. It provides civilization with an enormous amount of goods and services and enhances the growth of social welfare. Furthermore, the main importance of entrepreneurship is the creation of job opportunities, innovation, and improve the economy. In addition, entrepreneurship is central to achieving those national objectives for several reasons for instance, Entrepreneurship improves productivity, spurs innovation and creates jobs.

To realize growth and innovation, the ecosystem must function well for entrepreneurs. Such an "entrepreneurial ecosystem" is an interactive network of actors who influence each other and the chances of survival of a venture creator and his company in a region or country (Stam, 2016). In addition, the competencies of a business owner, networks, formal institutions, human capital and culture, new knowledge, and financing are also crucial for value creation from entrepreneurship. The challenge is to let all these elements complement each other in such a way that together, they provide more value. That is what characterizes a well-functioning ecosystem. Hence, the Entrepreneurship Ecosystem is therefore complex. So, what is essential is not only data at the macroeconomic level but also at the individual level.

Furthermore, Coordination among the various actors for an adequate functioning of the startup ecosystem is one of top priorities that needs to be resolved in order to create and maintain a well-functioning environment for startups and new businesses. Until recently, there was no relevant institution (ministry) fully dedicated to innovation and entrepreneurship that would be entirely responsible for regulating such ecosystem and bridging actors towards an effective synergy. Other organizations had to intensively engage in coordinating close cooperation among the actors, since the Ministry of Innovation and Entrepreneurship has been recently established.

Micro, small and medium-sized enterprises (MSME) are considered the backbone of local and global businesses. Globally, it constitutes around $90 \%$ of all firms and around $60-70 \%$ of total employment. In Developing countries, the MSME contribute to $99 \%$ of private enterprises according to recent research. Thus, developing countries usually view MSMEs as a dynamic force for sustained economic growth and job creation.

\section{Startups Survival Criteria}

The Middle East and North Africa (MENA) region is vast, rapidly transforming and heterogeneous. Since 2011, the region has experienced an eruption of conflict in several Arab countries. Startup companies support economic development through growing on the market and thus generating economic growth and employment opportunities for the country.

https://doi.org/10.24264/icams-2020.III.6 
A startup, as such, represents a newly emerged business venture that has the intention of developing a feasible business model in order to meet the needs of a society by creating a virtuous cycle that derives constant improvement through innovative solutions. A startup therefore, fits best in developing economies whose main objective is to reduce poverty and generate sustainable wealth through innovative solutions that are able to solve industry-wide problems. Hence the startups in MENA regions need a specific ecosystem and criteria to support its survival and existence. Besides, it is also essential to understand the problems facing small business development in MENA region because they are significantly different and unique from those being faced in developed countries (Okpara and Wynn, 2007). The Problems facing the growth and survival of Startups can be generally classified as follows:

- Regulatory Administrative: Boundaries for entry

- Market Conditions: Competition

- Access to finance and fund: lack of capital and investments

- Knowledge creation and diffusion: Technological operations

Another acknowledged factors which negatively affect small business development include corruption, poor infrastructure, poor location, failure to conduct basic market research, and the economy (Tushabomwe-Kazooba, 2006; Mambula, 2002). Therefore, we must understand and notice that, lacking of the previous criteria and influencing factors the startups cannot easily survive and maintain. Also, very specified criteria that must be available in the ecosystem to help startups in the MENA region to survive and cope with the dynamic growth within starts up, thereby increasing effectiveness and efficiency in the economy.

Recently a further shortcoming, during the pandemic of (COVID-19) crisis, start-ups have continued to play a critical role for economies. Some innovative young firms have reacted fast and flexibly to the pandemic, and have been critical in helping many countries shift towards fully-digital work, education, and health services, and have provided innovations in medical goods and services. At that time marked by significant economic uncertainty and with their revenues affected by containment measures and significant drop in demand, start-ups may become even more financially fragile and will need support for their short-term liquidity needs, critical for their survival. Hence, this un expected event proofs that a healthy ecosystem contains the survival elements to provides actual business support.

\section{RESEARCH METHODOLOGY}

This research follows the qualitative approach, as a semi-structured interview was designed to explore the factors affecting startups survival in the presence of COVID19. The interview was directed to owners and managers of startups in the MENA region, where a number of 30 responses were collected. Respondents include males and females from the age group of 30 to 45 years old who belong to different countries of the MENA region. Analysis was done using NVIVO, version 12, by first describing the approach to interview analysis, then identifying the interviews procedures, and finally, identifying the coding process followed in the analysis process. Therefore, data was extracted in the form of codes and nodes. 


\section{FINDINGS AND DISCUSSION}

This section discusses the main results of the qualitative analysis of the in-depth interview conducted with managers and owners of startups in the MENA region. Examining the data inductively allowed the researcher to organize the data into concepts and elements that link them together. Breaking down the data gives the way to the understanding of the data and explain the emerging patterns. Then the researcher is able to bring together bits of theory from the interpretation of these patterns. The final themes that emerge from the content analysis establish answers for the research.

The interview is divided into sets of semi structured questions that are related to the factors affecting startups survival. Based on the observed themes, there are some important factors obtained, which are: Government Policies, Barrier of Entry, Access to finance and fund, and Technological operations. They are described in details, according to their rank of importance to respondents of interview, as follows:

\section{Theme 1: Technological Operations}

Almost all respondents agree that technology becomes the most important factor for their business. They claim that, in the presence of COVID19, technology could help in reducing fixed cost and even some variable cost that they bear. One evidential quote is "After COVID19, we rely on teleworking and we have all our meetings online. I become able to reduce rental cost by relying on virtual work".

\section{Theme 2: Financing}

Majority of respondents agree that having enough fund is very important, as they might need to pay salaries to employees, while there is no income, in order to avoid losing those employees. One evidential quote is "At the first instance of COVID19, I was obliged to give long vacation to employees, with normal salaries. So, you should have enough financing to face unexpected events, otherwise, you will fail".

\section{Theme 3: Government Policies}

Majority of respondents agree that government policies is a major factor that influence their business. One evidential quote is "I was obliged to close my business during COVID19 by government regulation, which causes my failure!".

\section{Theme 4: Market Conditions}

Majority of respondents agree that market conditions had been dramatically changing in the presence of COVID19. One evidential quote is "Only necessary products find their ways in the market during COVID19, while luxurious ones are present in the market only through online channels".

Other themes that evolve were regulatory administrative, Turnover, Innovation, education, but they come in the rank after the above-mentioned themes.

\section{CONCLUSION}

There are several factors that affect startups in the MENA region nowadays, which could be listed as follows: Access to finance and fund, market conditions, regulatory administrative, Government policies, Turnover, Innovation and education. These factors 
were showing up with different importance to startups before COVID19. After such event, technological operations become the most superior factor to these ones for startups. A great attention should be devoted to technology when starting your business, as it could be the best way of communication with employees as well as customers.

\section{REFERENCES}

Abu Bakar, A.R., Ahmad, S.Z., Wright, N.S. and Skoko, H. (2008), "The propensity to business startup: Evidence from Global Entrepreneurship Monitor (GEM) data in Saudi Arabia", Journal of Entrepreneurship in Emerging Economies, 9(3), 263-285, https://doi.org/10.1108/JEEE-11-2016-0049.

Acs, Z.J., Autio, E. and Szerb, L. (2014), "National system of entrepreneurship: Measurement issues and policy implications", Research policy, 43, https://doi.org/10.1016/j.respol.2013.08.016.

Ahmad, N. and Seymour, R.G. (2008), "Defining Entrepreneurial Activity: Definitions Supporting Frameworks for Data Collection", OECD Statistics Working Paper, 5-6.

Alvarez et al. (2014), "Building Understanding of Smart City Initiatives", Lecture Notes in Computer Science, $7443,40-53$.

Mambula, C. (2002), "Perceptions of SME Growth Constraints in Nigeria", Journal of Small Business Management, 40(1), 58-65, https://doi.org/10.1111/1540-627X.00039.

Okpara, J.O. and Wynn, P. (2007), "Information Technology and the challenges of Economic Development", in: African E-Markets Information and Economic Development, Economic Commission for Africa.

Parker, D. (2009), “The magnitude and causes of agglomeration economies", Journal of Regional Science, 50(1), 203-219, https://doi.org/10.1111/j.1467-9787.2009.00657.x.

Stam, E. (2015), "Entrepreneurial ecosystems and regional policy: a sympathetic critique", European Planning Studies, 23(9), 1759-1769, https://doi.org/10.1080/09654313.2015.1061484.

Tushabomwe-Kazooba, C. (2006), "Causes of Small Business Failure in Uganda: A Case Study from Bushenyi and Mbarara Towns", African Studies Quarterly, 8(4).

*** (2018), "For Entrepreneurship and Innovation Ecosystem Leaders: A Step-by-Step Country Master Plan to Create and Drive a Healthy Early Stage Investment. Market for Your Country/Region, http://wbaforum.org/for-entrepreneurshipinnovation-ecosystem-leaders/index.html. 
Factors Affecting Startups Survival in the MENA Region in the Presence of COVID19 


\title{
DEVELOPING AN E-BUSINESS SYSTEM TO IMPROVE THE DOWNSTREAM PHARMACEUTICAL SUPPLY CHAIN (A STUDY ON THE EGYPTIAN MARKET)
}

\author{
SANDRA HADDAD \\ College of International Transport and Logistics - Arab Academy for Science, Technology and \\ Maritime Transport,Egypt, Sandra.haddad@aast.edu, sandra.haddad16@gmail.com
}

\begin{abstract}
Pharmaceutical products are considered sensitive products that require a well-managed distribution channel as they impact human lives. The aim of this research is to investigate the applicability of improving the downstream of the pharmaceutical supply chain (distribution channel) in Egypt through developing an Ebusiness system. The study has adopted a deductive approach. Qualitative and quantitative methodologies were followed respectively. Qualitative in-depth interviews were conducted to get a better understanding of the situation in Egypt followed by a structured survey to test and verify the significance and the relation of the extracted variables using statistical tools. This resulted in highlighting significant variables impacting the relationship between pharmaceutical retailers (pharmacies) and consumers and the applicability of introducing an e-business retailing application that improves downstream chain performance by facilitating transactions i.e. easily locating the required medicine, matching consumers and retailers and spotting the inventory level in the distribution channel for better management solutions.
\end{abstract}

Keywords: case study, distribution, downstream supply chain, pharmaceutical products

\section{INTRODUCTION}

Humans everywhere and everyday are attached to common products affecting their health; these are pharmaceutical products. Pharmaceutical products are counted as the most sensitive products, they require an efficient distribution channels, and they can affect people and their health if they are not distributed in an effective way. The changing nature of distribution and the reduced wholesaler models, are beginning to have a significant impact in some countries. In principle, changes in the distribution model should make the process of delivering medicines from factory gates to the patient bed-side more efficient and cost-effective (Kanavos et al., 2011). Yet, there seem to be some concerns about the availability of medicines in the markets. Different stakeholders (manufacturers, wholesalers, retailers) of the supply chain have different perspectives about incentives and disincentives in this process. From patients/consumers side access to medicines is a vital factor (WHO, 2017). Supply chain of this type of products is critical especially in Egypt, according to the shortage of medicines in the market and the economical inflation attacking the country. Thus, this research attempts to find a solution to enhance the downstream pharmaceutical supply chain in Egypt through an electronic system supported by an application to ease locating the required medicine, match consumers and pharmacies possessing the required medicine and spotting the inventory level in the distribution channel for better management solutions.

\section{LITERATURE REVIEW}

The pharmaceutical industry supply chain covers drug research, development, manufacture; distribution and application through a range of healthcare services, together with all the ancillary businesses that help these different stages function effectively. The

https://doi.org/10.24264/icams-2020.III.7 
Developing an E-business System to Improve the Downstream Pharmaceutical Supply Chain (A Study on the Egyptian Market)

pharmaceutical and healthcare industry is extremely complex because it involves so many markets, products, processes and intermediaries. It is also globally heavily regulated and used by everyone in life. Changes in any area impact upon the others and environmental factors such as regulatory change, pricing, or actions by competing bodies, influence the whole supply chain in ways that are not easily understood or effectively managed. Distribution usually gets delegated to third-party logistics and wholesalers in this industry and falls behind when it comes to channel management in comparison to other industries. This means that only a limited amount of information about patients' demand and product flow that is shared with supply chain partners (Xie and Breen, 2012).

\section{Challenges in Pharmaceutical Supply Chain}

The slow growth for the industry had also been a major challenge in the recent past. Innovation rates in the industry have shown considerable decline because of the long time spent in the development of new drugs. Drug prices rise as high as 65 percent than the acceptable international standards in under-developed countries. In addition to the low availability of cheap medicines in the market. Insufficient funding, inability to forecast accurately, lack of incentives for maintaining stocks, inefficient distribution systems and conversion of prescriptions for private resale have encouraged the low accessibility of cheap medicines that are essential for the primary healthcare sector (WHO, 2017). Singh et al. (2016) concentrated on risks and uncertainties related to the recovery of pharmaceutical drugs such as timing, quality, quantity and variety of returns; estimation of operation and cost-related parameters for reverse supply networks; customer behavior and preferences; decisions for product returns; and cost of coordination along the reverse supply chain. Pharmaceutical supply chain is observed as more complex because it demands the active role of different stakeholders such as pharmaceutical manufacturers, wholesalers, distributors, customers, information service providers and regulatory agencies. Given these complexities, the pharmaceutical supply chain lacks sufficient research work. It is also felt that because of lack of research, the pharmaceutical sector in developing countries is unable to contribute significantly in global markets (Bhakoo and Chan, 2011; Singh et al., 2016).

\section{Case of Egypt}

The domestic pharmaceutical industry in Egypt is strong, with a presence of around 120 pharmaceutical companies, of which less than 10 are multinationals with local production branches. There are 17 private sector companies in the industry, together with 9 multinational pharmaceutical players. The pharmaceutical companies operating in Egypt come under three categories: public sector companies, private sector Egyptian companies, and multinational companies. Sales of pharmaceutical products consist of generic drugs, over-the-counter (OTC) medicines, and patented drugs (Ngage Consulting, 2017). Although, Egypt is the largest drug producer and consumer in the Middle East and Africa region in terms of volume, the market is fragmented and distribution is unplanned and scattered i.e. ineffective supply chain management practices. Over three-quarters of the local market are controlled by the private sector. One-third of the pharmaceutical market is controlled by the five largest companies. Low labor costs and a large pool of highly trained pharmacists, engineers, and skilled

https://doi.org/10.24264/icams-2020.III.7 
technicians. Local production of finished pharmaceuticals represents some $90 \%$ of domestic consumption. Demand for pharmaceutical products is much higher than supply, nevertheless, imports are limited to APIs - raw materials, as well as patented and difficult-to-produce pharmaceuticals (BMI, 2018; GAFI, 2019).

\section{Effect of Technology on Pharmaceutical Supply Chain}

Digitization possesses a huge potential to assist pharmaceutical companies the mentioned challenges. Digitization has proved its benefits in many industries as companies are enhancing operations through enabling smart, decentralized production via intelligent factories, integrated IT systems, the Internet of Things, and flexible, highly integrated manufacturing systems. Pharmaceutical industry supply chain operations can be significantly transformed via digitization which can lead to improving processes. The use of technology will lead to improved distribution of system and can serve as an effective marketing instrument as online pharmacies are making medicines and pharmaceutical products directly available to patients which is especially necessary nowadays (Ehrhardt, 2016; Nagy et al., 2018).

\section{METHODOLGY}

In this research multiple methods of data collection instruments are being used to raise the level of the research accuracy and precision. As a matter of fact, combining research methods and using triangulation techniques is encouraged. Amaratunga (2002) explained that Triangulation is about combing several methods (e.g. qualitative and quantitative) in the study of a specific subject.

Thus, this research method combines qualitative and quantitative research methods and analysis. The first data collection phase followed a qualitative approach by conducting semistructured interview with a leading pharmaceutical company manager and a number of semistructured interviews with different pharmacies' owners and patients in Alexandria in order to investigate the market situation and challenges facing the pharmaceutical supply chain in Egypt. After reviewing literature and the results of the qualitative phase a few variables were extracted (independent variables) to measure their effect on the applicability of the electronic ordering and matching application (dependent) by both consumers/patients and pharmacies' owners. Thus, hypotheses were proposed for each variable followed by the second data collection phase using quantitative techniques of collection ana analysis by conducting two structured surveys with pharmacists and patients. Then data was analyzed by running a multiple regression model using the statistical tool SPSS. The following are the developed hypothesis:

\section{Pharmacies' Hypotheses}

$\mathrm{H}_{1}$. There is a significant positive relationship between safety and the applicability of the e-business system.

$\mathrm{H}_{2}$ : There is a significant positive relationship between ease of use of the system and the applicability of the e-business system.

$\mathrm{H}_{3}$ : There is a significant negative relationship between price for running this system and the applicability of the e-business system.

https://doi.org/10.24264/icams-2020.III.7 
Developing an E-business System to Improve the Downstream Pharmaceutical Supply Chain (A Study on the Egyptian Market)

$\mathrm{H}_{4}$ : There is a significant positive relationship between realized profit and the applicability of the e-business system.

\section{Patients' Hypotheses}

$\mathrm{H}_{5}$ : there is a significant positive relationship between safety and the applicability of the e- business system.

$\mathrm{H}_{6}$ : there is a significant positive relationship between frequency of ordering and the applicability of the e- business system.

$\mathrm{H}_{7}$ : there is a significant positive relationship between the realized benefit and the applicability of the e- business system.

$\mathrm{H}_{8}$ : there is a significant negative relationship between price of this service and the applicability of the e- business system.

$\mathrm{H}_{9}$ : there is a significant positive relationship between the availability and the applicability of the e- business system.

The following section will discuss and analyze the results of the qualitative and quantitative phases.

\section{DISCUSSION AND ANALYSIS}

This study aims to test the applicability of the new E- business system that can be used to improve the downstream of the pharmaceutical supply chain in Egypt through developing an E-business system. The study firstly conducts semi-structured interviews to verify literature extracted variables that are assumed to have an effect on the implementation of an e-business solution. It also investigates the current pharmaceutical supply chain condition in the Egyptian market. The second phase follows more structured research techniques. The primary data that was collected from the semi-structured interview and the variables that were extracted from the literature are tested using quantitative technique to validate the outcomes of the qualitative phase and to verify the derived hypotheses.

\section{Qualitative Results and Analysis}

This section discusses the results of the analysis of the semi-structured interview with a leading pharmaceutical products' manufacturer, pharmacist and patients. The results are summarized as follows:

There is a major problem concerning the geographical distribution as products are not equally distributed which effects the availability of products in the pharmacies. Focus of the e-system is Egypt should be on the downstream of the pharmaceutical supply chain specially the retailers and the consumers and to exclude manufacturers. Pharmaceutical demand in Egypt is much higher than supply which makes the system somehow ineffective to manufacturers.

From retailer perspective the e-business system needs to be easy in its usage so anyone can use. Accordingly, the ease of use is supported by the interviews. The interviews also revealed the intention of the pharmacies' owners to use the application as long it will help them maximize profit by increasing the number of orders, thus, their market share. In addition, this phase uncovered the need to integrated a swapping function in the application

https://doi.org/10.24264/icams-2020.III.7 
to enable pharmacies to swap stocks or trade among themselves to overcome uneven geographical distribution i.e. excess in one medicine at a pharmacy and shortage of the same medicine at another pharmacy in another location. This service will reduce their loss and accordingly will maximize their profit. Maximizing profit is the second variable that is verified by the pharmacists as it affects the applicability of this e-system.

From consumers perspective the e-business system can especially help consumers with frequent orders. This application will enable them to save much time which supports another independent variable, namely, frequency of ordering medicine by the customer. Availability of the medicines in one of the pharmacies nearby the customer's location is another important variable stressed by patients. The application shall guide the customers to the pharmacy that has the required medicine which will encourage them to use this application.

\section{Quantitative Results and Analysis}

The variables that were extracted from the literature and supported in the qualitative phase were quantitively tested using the statical tool SPSS to investigate the significance of the relationship between these extracted variables on the applicability of the e-business system by both pharmacies and consumers and to verify the derived hypotheses.

\section{Pharmacies Regression Model Results and Analysis}

This study undertook a linear regression model using the statistical tool SPSS for the following pharmacies model:

$\mathrm{APP}=\alpha+\beta_{1} \mathrm{Saf}+\beta_{2}$ Eas $+\beta_{3}$ Pri $+\beta_{4}$ Prof

where APP: refers to the applicability of the new E-business system measured, Saf: safety, Eas: ease of use, Pri: price, Prof: profit.

After running the linear regression using the previous equation the following results are reached. Table 1 shows the result of regression model and the coefficient of every variable and its level of significance on the dependent variable.

Table 1. betas' Coefficients and p- value of each variable of pharmacies

\begin{tabular}{ccc}
\hline Variables & Beta & Sig \\
\hline Safety & .123 & .194 \\
Ease of use & .227 & $.030^{* *}$ \\
Price & -.024 & .792 \\
Profit & .302 & $.003^{*}$ \\
\hline *significant at 0.01 & & \\
$* *$ significant at 0.05 & & \\
$* * *$ significant at 0.1 & & \\
$\mathrm{APP}=\alpha+0.123 \mathrm{Saf}+0.030$ Eas +0.792 Pri+0.003Prof &
\end{tabular}

Safety: The coefficient of the safety is 0.123 which means that there is a positive relation between the independent variable (safety) and the dependent variable (applicability), however, it was not significant which leads to rejecting hypothesis $\mathrm{H} 1$. 
Developing an E-business System to Improve the Downstream Pharmaceutical Supply Chain (A Study on the Egyptian Market)

Ease of use: The coefficient of the safety is 0.227 which mean that there is a significant positive relation between the independent variable (ease of use) and the dependent variable (applicability), which is similar to the result extracted from the semi-structured interviews and leads to accepting the second hypothesis H2. These results mean that it is very critical for the pharmacies that the new E-business system to be simple and not sophisticated.

Price The coefficient of the price is -0.024 which mean there is a negative insignificant relationship between the Price paid by the pharmacies to run the application and the dependent variable (applicability), which is justifiable as the majority of the pharmacies are already offering delivery services bear extra fees this service and increasing their costs in an attempt to increase their market share. Accordingly, this confirms the negative relationship and justifies its insignificance as pharmacies can accept additional fees for running this Ebusiness system. This leads to rejecting hypothesis $\mathrm{H} 3$.

Profit: The coefficient of the profit is 0.302 which means that there is a significant positive relationship between the independent variable (Profit) and the dependent variable (applicability) these results were the same as what was hypothesized in the hypothesis $\mathrm{H} 4$, similar to the result extracted from the semi structured interviews. This indicates that the target of these pharmacies is to maximize their profit, accordingly the fourth hypothesis $\mathrm{H} 4$ is accepted.

Thus, for the pharmacies' hypotheses H2, H4 are accepted and hypotheses H1, H3 are rejected.

\section{Customers' Regression Model Results and Analysis}

The following part shows the results of the regression model for the customer's questionnaire.

This study undertook a following linear regression model using the statistical tool SPSS for the following customers' model:

APP $=\alpha+\beta_{1}$ Saf $+\beta_{2}$ Freq $+\beta_{3}$ Ben $+\beta_{4}$ Pri $+\beta_{5}$ Avail $+\beta_{6}$ beha

where APP refers to applicability of the new E-business system, Saf: safety, freq: frequency, Ben: ease of use, Pri: price is measured, Avail: Availability, Beha: Behavior.

After running the linear regression using the previous equation the following results are reached. Table 2 demonstrates the results of the regression model and the coefficient of every variable and its level of significance on the dependent variable.

Table 2. betas' Coefficients and p- value of each variable of the customer

\begin{tabular}{ccc}
\hline Variable & Beta & Sig \\
\hline 'Safety & .209 & $.062^{* * *}$ \\
Frequency & .051 & .635 \\
Benefit & .030 & .792 \\
Price & -.046 & .658 \\
Availability & .278 & $.009^{*}$ \\
Behavior & .051 & .632 \\
\hline
\end{tabular}

*significant at 0.01

**significant at 0.05

$* * *$ significant at 0.1

https://doi.org/10.24264/icams-2020.III.7 
$\mathrm{APP}=\alpha+0.209 \mathrm{Saf}+0.51$ Freq $+0.030 \mathrm{Ben}+0.046 \mathrm{Pri}+0.278$ Avail +0.051 beha

Safety: The coefficient of the safety is 0.209 which means there is a significant positive relation between the independent variable (safety) and the dependent variable (applicability) which the same as what was hypothesized in the hypothesis H5.It's significance level indicates that it is important for customers to feel safe while using the E-business system, accordingly the hypothesis $\mathrm{H} 5$ is accepted.

Frequency: The coefficient of Frequency is 0.051 which indicates an insignificant positive relation between the independent variable Frequently of the customers' orders and the dependent variable (applicability) which contrasts the result of the semi structured interview and previous scholarly work. However, the significance might be due to survey sample as more than $85 \%$ of the customers who took the questionnaire are less than 35 years old, which means that they have good health and do not order medicines frequently. Nevertheless, H6 is rejected.

Benefit: The coefficient of the benefit is 0.30 which shows an insignificant positive relationship between the expected benefit from using the e-system and the dependent variable (applicability) which again contradicts with literature and the qualitative results and leads to rejecting H7. This result can be again linked to the age demographics of the surveyed sample as since the customers are young and do not order medicine frequently then they are not expecting extra benefit from using the E-business system to order their medicines.

Price: The coefficient of the price is -0.046 which means that there is an insignificant negative relationship between the Price paid by customers to use this service and the dependent variable (applicability) which leads to rejecting H8. Referring to the demographics of the sample which shows that more than $65 \%$ of the customers enjoy a high household income, it explains why the price variable is not significant from the customer point of view.

Availability: The coefficient of the availability is 0.278 which means there is a significant positive relationship between the independent variable (Availability) and the dependent variable (applicability) which confirms hypothesis H9 as was also supported in the semi-structured interviews. This led to accepting hypothesis $\mathrm{H} 9$.

Behavior: The coefficient of the behavior is 0.051 which means that there is an insignificant positive relation between the (customers' attitude) in buying medicines and the dependent variable (applicability). Although a significant positive relationship was stated in hypothesis $\mathrm{H} 10$ and confirmed by scholarly work, $\mathrm{H} 10$ is rejected which might be due to the reason that the majority of the customers who took the questionnaires were males do not usually perform the shopping act or behavior like females in Egypt.

Accordingly, hypotheses $\mathrm{H} 5$ and $\mathrm{H} 9$ are accepted while hypotheses $\mathrm{H} 6, \mathrm{H} 7, \mathrm{H} 8$, and $\mathrm{H} 10$ are rejected because of their weak significance level.

\section{CONCLUSION}

This study aims to enhance the Egyptian downstream pharmaceutical supply chain through introducing an e-business system that facilitates locating medicines in order to overcome availability and unequal geographical distribution issues. Also, the e-system shall help pharmacies swap their stocks to improve geographical distribution of the medicines. Triangulation of qualitative and quantitative data collection methods and analysis were

https://doi.org/10.24264/icams-2020.III.7 


\section{Developing an E-business System to Improve the Downstream Pharmaceutical Supply Chain (A Study on the Egyptian Market)}

followed to investigate the situation in the Egyptian market and examine the variables that might influence the applicability of the proposed e-business system. The variables extracted from the literature and supported in the semi-structured interviews were Benefits, Safety, Behavior, Price, Frequency, Availability, Ease of use and Profit. The variables used in the pharmacies model were Safety, Ease of use, Price, and Profit. The significant variables from the pharmacies' perspective were Ease of use at 0.05 significance level and Profit at 0.01 significant level. On the other hand, the customer model variables were Safety, Frequency, Benefits, Price, Availability and Behavior. The results show that the significant variables according to customers were Safety at 0.1 significant level and Availability at 0.01 significant level. Thus, according to the qualitative and quantitative analysis, it seems that an e-business system that helps patients locate the required medicine easily without the physical search effort and which is safe and easy to use, can be considered applicable to patients in the Egyptian market. Moreover, the e-business system appears to be beneficial to retailers/ pharmacies who want to increase the market share, reach more customers, thus, maximize profit and also want reduce their losses through being enabled to swap stocks with other pharmacies via an easy to use e-platform.

\section{REFERENCES}

Amaratunga, D., Baldry, D., Sarshar, M. and Newton, R. (2002), "Quantitative and qualitative research in the built environment: application of "mixed" research approach", Work Study, 51(1), 1731, https://doi.org/10.1108/00438020210415488.

Bhakoo, V. and Chan, C. (2011), "Collaborative implementation of e-business processes within the health-care supply chain: the Monash Pharmacy Project”, Supply Chain Management: An International Journal, 16(3), 184-193, https://doi.org/10.1108/13598541111127173.

BMI research (2019), Egypt Pharmaceuticals and Healthcare Report [online], available at: https://nationsemergentes.org/wp-content/uploads/2019/02/egypt-pharmaceutical-survey.pdf, accessed 15 August 2020.

Ehrhardt, M. (2016), "The Digital Game-Changer", Pharma Manufacturing [online], available at: https://www.pharmamanufacturing.com/articles/2016/the-digital-game-changer/

GAFI (2019), "Invest in Egypt", Cairo: General authority for Investment and Free Zones.

Kanavos, P., Schurer, W. and Vogler, S. (2011), "The pharmaceutical distribution chain in the European Union: structure and impact on pharmaceutical prices", European Commission, Brussels, Belgium [online], available at: http://eprints.lse.ac.uk/51051/1/Kanavos_pharmaceutical_distribution_chain_2007.pdf

Nagy, J., Oláh, J., Erdei, E., Máté, D. and Popp, J. (2018), “The Role and Impact of Industry 4.0 and the Internet of Things on the Business Strategy of the Value Chain-The Case of Hungary", Sustainability, 10, 3491, https://doi.org/10.3390/su10103491.

Ngage Consulting (2017), "Egypt's Pharmaceutical Sector Following Bold Economic Reforms: Challenges and Opportunities" [online], available at: https://www.ngage-consulting.com/downloads/Pharmaceutical_ PDF_Final_Version_K_and_A.pdf

Singh, R.K., Kumar, R., Singh, P.K. (2016), "Strategic issues in pharmaceutical supply chains: a review", International Journal of Pharmaceutical and Healthcare, 10(3), 234-157, https://doi.org/10.1108/IJPHM-102015-0050.

WHO (2017), "Global Surveillance and Monitoring System for Substandard and Falsified Medical Products" [online] available at: file:///C:/Users/Sandra/Downloads/9789241513425-eng.pdf, Accessed 20 August 2020, https://doi.org/10.1108/13598541211212195.

Xie, Y. and Breen, L. (2012), "Greening community pharmaceutical supply chain in UK: a cross boundary approach”, Supply Chain Management: An International Journal, 17(1), 4053(14), https://doi.org/10.1108/13598541211212195.

https://doi.org/10.24264/icams-2020.III.7 
THE ROLE OF INTERNET OF THINGS ON INTELLIGENT TRANSPORT SYSTEM: A TRAFFIC OPTIMIZATION MODEL

\author{
ISLAM SALEH HASSANIN \\ Arab Academy for Science, Technology and Maritime Transport, Faculty of International \\ Transport and Logistics, Alexandria, Egypt, islam.saleh@aast.edu
}

\begin{abstract}
The road traffic congestion has become an excessive problem and a great pressure on both the governmental and social aspects especially in megacities. Hence, developing control systems that administrate the traffic flows is a vigorous solution, which can solve the problem of traffic congestion especially in megacities that should convert their services to be smart. The Intelligent Transport System (ITS) one of the Internet of Things (IoT) applications that provides a group of pioneer schemes for handling the challenges of traffic congestion. Traditionally, sensor-based structures have been used for gathering traffic information, but the coverage, cost, and real-time matters have remained unexplained. Thus, this paper aims at proposing a new model named Traffic Flow Optimization Model (TFOM) applied in Alexandria as a case study, which is considered a cost-effective and easily maintainable traffic congestion minimization method. It combines Infra-Red (IR) and active Radio Frequency Identification (RFID) technologies. TFOM is designed to minimize traffic congestion, given reroute options to the users, taken decisions regarding congestion, and finally it could be an initial step to help future predictions in linear megacities.
\end{abstract}

Keywords: Intelligent Transport System (ITS), Internet of Things (IoT), Traffic Optimization Models

\title{
INTRODUCTION
}

The economic growth and the increase of population with a limited traffic area cause numerous traffic congestion in megacities, which is one of the strategic difficulties in various countries around the world (Sutandi, 2020). Over the decades, many novel ideas and tools have been introduced related to the various issues regarding traffic control (Sharif et al., 2019). However, developing counties such as Egypt face serious challenges that need to suggest novel ideas to optimize traffic controlling. As the technologies of smart cities develop rapidly such as the booming of the Internet of Things (IoT) devices (Nagy and Simon, 2018). Therefore, Egypt should convert its megacities such as Alexandria to be smart to overcome the overpopulation problem that reflected on road traffic.

Smart world is the main vision of IoT as the sensing technologies and smart components will be spread dominantly (Lu et al., 2018; Al-Turjman and Lemayian, 2020). One of the IoT applications is called Intelligent Transport System (ITS) (Muthuramalingam et al., 2019), which is a transportation system that also makes use of Information and Communication Technology (ICT) to clarify and minimize transportation and congestion problems. Therefore, recently many megacities develop and invest in ITS on a large scale to be smart (Chen et al., 2016; Turner and Uludag, 2016). Thus, this paper proposes a new model named Traffic Flow Optimization Model (TFOM) to be available in all types of vehicles with high accuracy and low price, which is designed to optimize traffic control, depending on IoT broad concept while using ITS applications, considering Alexandria "the second megacity in Egypt" as a case study to apply such model.

In megacities especially in developing countries, on the one hand, the traffic flow can be smoothly achieved by the support of vehicle driver with appropriate reroute 
recommendations as the drivers become uncertain about which alternative route will be the better to follow 'even if received congestion warning' that could be a source of disorganized traffic flow (Aldegheishem et al., 2018). Hence, the main aim of this model is to design a promising congestion detection scheme that offers sensible timely warnings to the drivers about potential traffic situations and provides proper reroute recommendations accordingly. On the other hand, there are variable patterns in future traffic volumes (Ezzat et al., 2014). Therefore, an accurate data collection methodology should be planned wisely to generate a reliable estimate of traffic flows and dissipation rates as in Egypt case current population is around 101 million and Alexandria population is about 5.4 million according to Egyptian Central Agency for Public Mobilization and Statistics (CAPMAS 2020), and the total number of registered vehicles according to the last statistics available in CAPMAS in 2017 were about 9.9 million vehicles.

Nowadays, in Egypt, forming vehicular ad hoc networks (VANET) would be possible as all vehicles will be equipped with novel technologies that make them capable to communicate with other adjoining vehicles (Adly, 2020). To monitor the traffic violations and accidents, Egypt's interior ministry has launched a novel technology to controlling its traffic system by sticking fixed electronic tags on the car windshields, which contains a SIM card that read by special infrared devices. It contains all the vehicle's data that allowing authorities to track violations, licence expiration and traffic congestion. Those smart tags will be one of the components for applying the proposed model.

\section{METHODOLOGY}

\section{Preface}

A traffic system is a dynamic system that is distinguished by several dynamic variables. Demand outlines change from a period to another during the day (Ezzat et al., 2014). In Alexandria, the day could include five periods as shown in Table 1, which defines the rush and non-rush times. Those classifications are described according to the author experience and the surveillance via Google map of Alexandria. Also, they could be different in the summer season as Alexandria is a coastal city, which is very crowded in summer.

Table 1. The characteristic of day periods classifications

\begin{tabular}{ccc}
\hline Periods & Timing & Rush/ Non-rush \\
\hline First & From 07:00 to 10:00 & Rush \\
Second & From 10:00 to $12: 00$ & Non-rush \\
Third & From 12:00 to 16:00 & Rush \\
Fourth & From 16:00 to 20:00 & Non-rush \\
Fifth & From 20:00 to 23:00 & Rush \\
\hline
\end{tabular}

Two kinds of traffic congestion are recognized, the first one is recurring traffic congestion, which takes place in the same area during the same time every day as it is presented in Table 1. Secondly, the non-recurring traffic congestion that arises randomly like an unplanned event such as accidents, vacations, or summer time. This non-recurring can summon an unexpected traffic volume boost. Handling of non- 
recurring traffic congestion is serious compared to the recurring kind because it needs real-time traffic information and evaluation with appropriate traffic controlling decisions (Nellore and Hancke, 2016). The model proposed in this paper works on two traffic congestion types the recurring traffic and non/recurring congestion as TFOM did not work on a time basis but it works on all vehicles along the road.

Traffic Flow Optimization Model (TFOM) uses both infrared (IR) and Radio Frequency Identification (RFID) technology. As TFOM cannot rely on IR only as it always needs line-of-sight to work properly and it is not effective for long distances. Therefore, TFOM merges RFID as it can pass through obstacles and work over much longer distances, hence, TFOM model can be a solution for congestion as well as covering the main aim of the Egyptian government which minimizing accidents and monitoring violations.

The vehicle data will be saved in the smart stickers for further processing and decision making to help in keeping traffic records for further use as in Egypt there are no yet traffic records, as keeping traffic records will help in the future prediction. At the meantime, Egypt starts tracking vehicles with the usage of GPS that have some potential errors, which may be returned particularly in megacities with high-rise buildings, multipath issues, few visible satellites, the possibility of missing data (Basyoni, 2016). In addition, a GPS sensor can only track one vehicle at a time. Therefore, comprehensive statistics (for example, the density of the traffic flow or the number of vehicles), which can only be estimated relying on the number of available moving sensors in the area (Nagy and Simon, 2018). Thus, the main reasons behind employing TFOM model for RFID is that it is considered fixed position sensor and the biggest advantage of traditional fixed position sensors is that they are accurate data sources and detecting all vehicles moving within their ranges.

\section{TFOM Initialization}

\section{TFOM Assumptions}

The following assumptions are generated to facilitate the flow of the proposed model:

1. For homogenous congestion measurements, RFID tags should be attached parallel with the smart stickers to ensure that TFOM model is recording all the data.

2. Annually, with the vehicles licence renewal, all the traffic data and information will be saved in the smart stickers for further processing, to observe the traffic history of each vehicle that help traffic department to predict the whole traffic system in the city.

\section{TFOM Setup Phase}

1. IR setup: The Egyptian government already setup IR technology in all renewing license or new vehicles and force other vehicles to attach by the end of October 2020.

2. RFID setup: The system depends on the manageability and reliability of wireless data communication. The 'unit system' can measure the traffic congestion of a single part of the road. And next is the steps regarding TFOM RFID setup:

i. One active RFID tag to be kept in all type of vehicles attached on the car number plate. 
ii. One wireless router $(\mathrm{R})$ and one wireless coordinator $(\mathrm{C})$ (both acting as RFID readers) to be installed at the roadside (for examples, on Lamppost), as it is recommended by reference (Mandal et al., 2011) the distance between $\mathrm{R}$ and $\mathrm{C}$ around $200 \mathrm{~m}$ to calculate the Safety Distance (SD) and average Waiting Time (WT) of vehicles at the road. The goal is to implement a system that would measure the SD between two vehicles moving on the same lane to measure the lane velocity and the average WT.

iii. Two Global System for Mobile Communications (GSM) modems (which is a special modem type that works with a SIM card, and runs by a mobile operator, just like a mobile phone. A new available version that supports SMS and capable of using smartphones, as those devices for both receiving and sending SMS and/or MMS messages). One of them will be settled with the coordinator and the other one will be in central monitoring station for wireless data transmission between gateway and software monitoring system (Mandal et al., 2011).

3. Placement of devices

i. $\quad 100$ meters away should be the distance between the coordinator and the router, as routers work as readers as mentioned before so they must be placed before the coordinator that recording the absolute occurrence time of events, which is called timestamps.

ii. The computer system that works as a central admin, which is connected to a GSM modem. In practice, the communicating range of an RF system is limited by the interference of the signal delivered directly from emitter to reader, and the one reflected by the ground plane. For ranges more than a considered distance, these two signals cancel each other out, and quickly the strength of the received signal will be decreased. An approximation to the suitable range of a RF transmitting system is given by equation (1), Where $\lambda$ is the wavelength of the RF signal, and $h_{T}$ and $h_{R}$ are respectively the heights of the emitter and the receiver (Perez et al., 2010).

$$
d_{T}=2 \pi h_{T} h_{R} / \lambda
$$

\section{Execution Phase}

To trace the congestion for a road, RFID readers will be used to receive signals from active RFID tags attached to the vehicles. The processes will be explained in the following points:

1. Tags incessantly transmit RF beacons. Then the central station collects the trip time when the vehicle attached with RFID tag moves across the router (the distance between the coordinator and the router), and determine the lane velocity, waiting time, and safety distance to measure possible congestion. The coordinator determines the vehicular trip time by using equation (2):

$\mathrm{Tt}=\mathrm{t}_{2}-\mathrm{t}_{1}$

The coordinator will send SMS to the central admin that contains trip time and tag ID. The SMS will be distributed by the software, extracts the trip time, tag ID and its current location. From the trip time, the lane speed can be computed through equation (3), where $\mathrm{d}$ is the distance between the router and the coordinator: $\mathrm{v}=\mathrm{Tt} / \mathrm{d}$ 
Depending on the user's definition of congestion level, the three levels of congestion (low, medium and high) will be pre-configured by the central station (Mandal et al., 2011).

2. Then the router forwards this data to its connected coordinator. If normal lane velocity has occurred but one vehicle waits longer than the other vehicles on the same lane, then a site of the incident is detected (non-recurring traffic congestion), thus this information is sent directly using SMS to the nearest emergency center to take action. The Central station will check if the vehicle waiting time is longer and lane velocity is normal at the same place regularly then it might help in minimizing accidents.

3. Then waiting time will be calculated, suppose the coordinator is continually receiving tag beacons from a vehicle. Accordingly, the coordinator will send special SMSs informing the central station which calculates the time until it leaves the coordinator range (Mandal et al., 2011). If the velocity is under 60 $\mathrm{km} / \mathrm{h}$ (which is the normal speed limit of the main roads in Alexandria), or the waiting time is more than 0.1 (the time by minutes that the vehicle crossing the distance between $\mathrm{C}$ and $\mathrm{R}$ ). The Central station software specifies the congestion level according to lane velocity and waiting time calculations.

4. The coordinator sends this information to the central station and to the vehicle driver in the form of SMS. If the vehicle lasts under the coordinator, the coordinator senses the tag and continuously updates the central admin. Accordingly, central station sends SMS to the upcoming vehicles appear in its range to notify them about congestion and also to give rerouting options, and computes the waiting time till the tag remains at the vicinity of the coordinator.
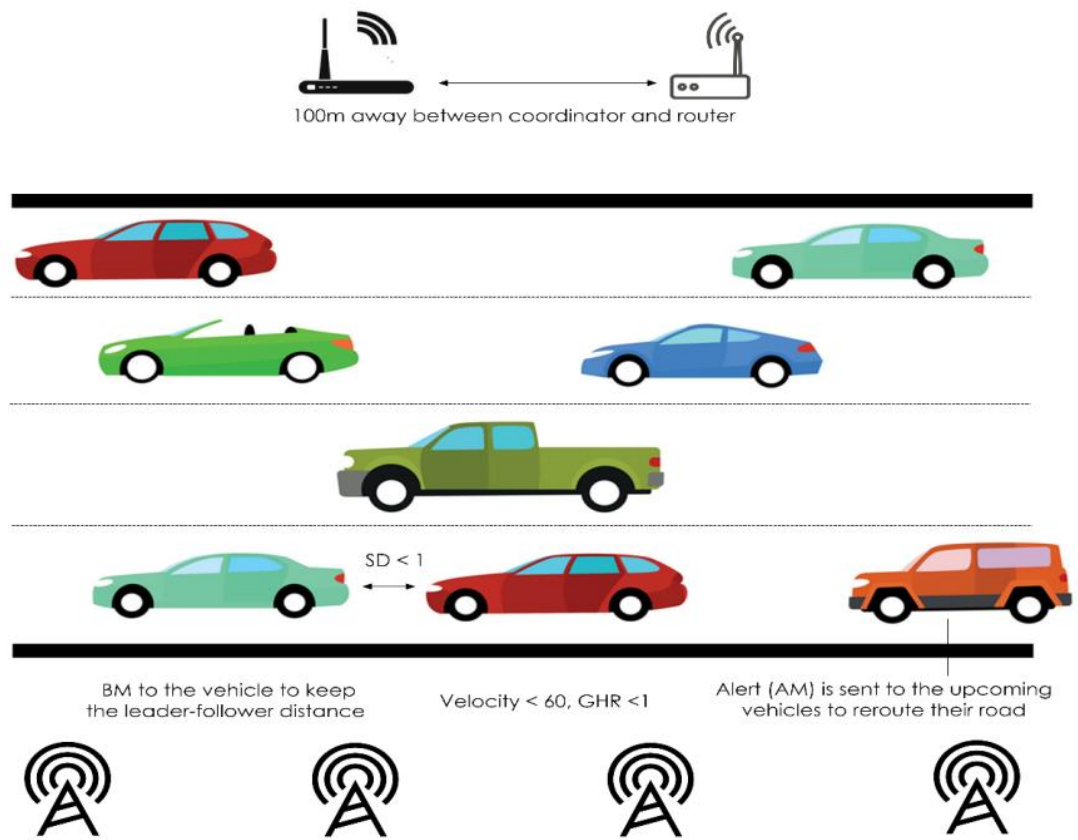

Figure 1. TFOM model techniques 


\section{CONCLUSION}

In high population city like Alexandria, traffic prediction is an advantage as connected devices (sensors and tags) will make it easier to shorten the travel time, or it might in the future help in finding a solution for the crowded areas like downtown. Therefore, the proposed model suggested in this paper might be fitting with the main roads in Alexandria such as El-Gaiesh, El-Horrya, Ring roads to detect the predicted bottlenecks spots and find a smart solution before the consignation occurred. As TFOM is considered a cost-effective scheme so it is reasonable to be afforded by municipalities and the maintenance strategy could be administered and monitored by them.

Prospective researches could be addressing such issues that related to smart cities especially converting megacities to facilitate its provided services and enhance the quality of life. It is recommended that the research community should highlight the role of IoT and its applications in the conversion processes using appropriate methodologies, highlighting the effect of BDA in different aspects. In addition, the need for more novel methods and mechanizes to be developed in order to sustain traffic flows that could be positively affecting economic, environmental, and social aspects.

\section{REFERENCES}

Adly, A.S. (2020), "Integrating Vehicular Technologies Within the IoT Environment: in Connected Vehicles in the Internet of Things.", Springer, p. 85-100, https://doi.org/10.1007/978-3-030-36167-9_4.

Aldegheishem, A. et al. (2018), "Smart road traffic accidents reduction strategy based on intelligent transportation systems (tars)", Sensors, 18(7), 1983-2006, https://doi.org/10.3390/s18071983.

Al-Turjman, F. and Lemayian, J.P. (2020), "Intelligence, security, and vehicular sensor networks in internet of things (IoT)-enabled smart-cities: An overview", Computers \& Electrical Engineering, 87, 106776.

Basyoni, Y. (2016), "A Traffic Datacenter Based on Available GPS Data A Case Study for Egypt", Sustainable Building Conference - SB16, Cairo.

Chen, Y., Ardila-Gomez, A., and Frame, G. (2016), "Achieving energy savings by intelligent transportation systems investments in the context of smart cities", World Bank, https://doi.org/10.1596/24740.

Ezzat, A.A. et al. (2014), "Optimization using simulation of traffic light signal timings.", in Proceedings of the International Conference on Industrial Engineering and Operations Management Bali, Indonesia.

Lu, Y., Papagiannidis, S., and Alamanos, E. (2018), "Internet of Things: A systematic review of the business literature from the user and organisational perspectives", Technological Forecasting and Social Change, 136, p. 285-297, https://doi.org/10.1016/j.techfore.2018.01.022.

Mandal, K. et al. (2011), "Road traffic congestion monitoring and measurement using active RFID and GSM technology", in: $14^{\text {th }}$ International IEEE Conference on Intelligent Transportation Systems (ITSC), https://doi.org/10.1109/ITSC.2011.6082954.

Muthuramalingam, S. et al. (2019), "IoT based intelligent transportation system (IoT-ITS) for globa perspective: a case study", in Internet of Things and Big Data Analytics for Smart Generation, Springer, p. 279-300, https://doi.org/10.1007/978-3-030-04203-5_13.

Nagy, A.M. and Simon, V. (2018), "Survey on traffic prediction in smart cities.", Pervasive and Mobile Computing, 50, 148-163, https://doi.org/10.1016/j.pmcj.2018.07.004.

Nellore, K. and Hancke, G. (2016), "A survey on urban traffic management system using wireless sensor networks", Sensors, 16(2), p. 157-182, https://doi.org/10.3390/s16020157.

Pérez, J. et al. (2010), "An RFID-based intelligent vehicle speed controller using active traffic signals", Sensors, 10(6), 5872-5887, https://doi.org/10.3390/s100605872.

Sharif, A., Li, J.P., and Sharif, M.I. (2019), "Internet of Things network cognition and traffic management system.", Cluster Computing, 22(6), 13209-13217, https://doi.org/10.1007/s10586-018-1722-1.

Sutandi, A.C. (2020), Advanced traffic control systems: Performance evaluation in a developing country, LAP Lambert Academic Publishing.

Turner, S.W. and Uludag, S. (2016), "Intelligent transportation as the key enabler of smart cities", in IEEE/IFIP Network Operations and Management Symposium - NOMS, Istanbul, Turkey. 


\title{
RESEARCH ON CONSUMER PORTRAITS OF OFFLINE FAST FASHION
} SHOE STORES BASED ON IOT SMART HARDWARE

\author{
KEYU HOU ${ }^{1}$, SHUNXUN LI ${ }^{1}$, JINGJING LI ${ }^{1}$, WEI XU ${ }^{2}$, RUI WANG ${ }^{3}$, JIN ZHOU ${ }^{1,2^{*}}$ \\ ${ }^{1}$ Sichuan University, College of Biomass Science and Engineering, National Engineering \\ Research Center of Clean Technology in Leather Industry, Section of Chengdu No. 24 Southern \\ Yihuan, 610065, Chengdu, China,625368881@qq.com \\ ${ }^{2}$ Science Lab, Zhejiang Red Dragonfly Footwear Co., LTD., Zhejiang Province, Wenzhou, \\ 325100,Wenzhou, China, zijscu@scu.edu.cn \\ ${ }^{3}$ Chengdu Institute of Product Quality Inspection Co. Ltd, 610000, Chengdu, China \\ wangruicd@sina.com
}

\begin{abstract}
There is a close relationship between consumer's portrait and product's attributes. Consumer portraits are usually obtained from information provided by consumers or by using data analysis of computer vision technology when they were in store. However, there was few concerns on the product's attribute which was a critical factor affecting consumer portrait establishment. Therefore, the purpose of this study was to establish IoT based product attributes' data collection system then to use this system to portrait consumers behaviors. Firstly, we used our own developed smart hardware to collect consumers' attention data on products of fast fashion shoe stores. Then the product attention index was obtained by combining sales data, and the quantitative attributes of the products with the highest attention index were analyzed, including the age, style and price. At last, improved the TOFA model to make it suitable for the conversion analysis of product attributes to consumer portraits. The results showed that there were core hedonic middle-aged consumer groups and potential thrifty youth consumer groups in the store, and the styles of shoes tend to be fashionable and casual. The conclusion was that the new model can effectively analyze the core consumer portraits of shoe stores and provide strategies for shoe store positioning and supply.
\end{abstract}

Keywords: IoT (The Internet of Things), Fast fashion shoe store, Consumer portrait

\section{INTRODUCTION}

As we all know, fast fashion imitates the current fashion trends of luxury brands and keeps up with fashion trends with the advantages of low prices and rapid design (Joy et al., 2012), production, and launch (Tokatli, 2008). From the relevant research reports of fast fashion, we found that fast fashion brands can respond to supply and demand matching through rapid production, reducing the risk of slow sales or shortages (Cachon and Swinney, 2011). By proposing the complexity of the fast fashion supply chain concept, it could quickly respond to the existing supply chain management model (Barnes and Lea-Greenwood, 2006). Through forward-looking pricing, discount strategies could be selected in advance to adjust seasonal inventory balance and increase potential profits (Aviv and Pazgal, 2008). Through inventory forecasting, the inventory allocation problem of all stores in the retail network could be solved (Caro and Gallien, 2010). Therefore, fast fashion is fast, flexible, forward-looking and strategic. It is important to study fast fashion products, achieve rapid response to demand, and reduce the information asymmetry of supply and demand.

Nicosia (Nicosia, 1966) proposed that the consumer behavior pattern consists of four aspects: the generation of consumer attitudes, the investigation and evaluation of products by consumers, the effective decision-making behavior of consumers, and the formation of consumer experience. Consumer behavior firstly originated from the first acquaintance (Hsieh et al., 2010, Di Sorrentino et al., 2016), and then the customer's cognitive process was triggered by interest, which ultimately lead to the solidification of 
decision-making and experience. We summarize the process by which consumers are interested in products as attention behavior. Generosi et al. (2018) adopted face recognition technology and introduced an expression tracking system to collect consumer attention emotions. Liu et al. (2015) developed a system that captures the characteristics of the customer's arm to track consumer attention behavior. There was also research to establish regional product attribute data (Yoshida and Koiso, 2010), and used RFID and infrared equipment to collect the attention-to-hand rate of a single shelf area (Chen, 2014). Due to the short product life cycle $\mathrm{f}$ in fast fashion store (Hou et al., 2019) and high cost, these results were limited to discussion.

Research on consumer portraits can accurately tailor information for consumers (Palmer, 2010), and the research on consumer portraits was mainly carried out on three indicators: age (Hervé and Mullet, 2009), consumer values (Kahle et al., 1986), and consumer preferences (Choi et al., 2010). Consumer portraits can change with market changes. It was important to grasp the core age groups in the market and made marketing strategies for them (Dychtwald and Gable, 1990). The consumer values and consumer preferences would be diversified due to individual differences in consumers. We can use the attributes of the shoe product (Wang, 2014) to quantify the consumer values of the consumers who bought it (the price reflected the frugal or hedonistic) and consumer preference (product style). The TOFA (Traditionalism, Optimism, Financing and Advance) model proposed by Kahle et al. (1992) introduced the fashion index style (S) to measure the consumer preference between fashion and tradition; and the cost index risk (R) was introduced to measure the consumer values between thrift and hedonic. The current studies have not considered age, consumer preferences and consumer values together into the evaluation of consumer portraits. Thus, this paper improved the TOFA model and considered the age of consumers in the parameters describing consumer portraits.

The purpose of this study was to obtain the core consumer profile by analyzing the attributes of the core product. We mainly developed a set of intelligent hardware that used RFID technology to collect the number of times each product in the store has been visited. The system constructed offline store consumer behavior detection and consumer portrait analysis models. Through analyzing product attributes, we can construct consumer portraits by product preferences. Thereby, the characteristics of the core customer group of the store were determined. Therefore, the store can achieve precise marketing in the short product cycle and increase profits.

\section{METHOD}

\section{Intelligent Hardware Design}

The basic principle of attention data collection: we installed product heat sensors on footwear products, when customers pick up the shoes, the sensor emits vibration frequency to collect data. Through the analysis of specific RFID algorithms, the number of concerns of the product was obtained.

The wireless acquisition device used in this study was characterized by its miniaturization $(2 \mathrm{~cm} \times 3 \mathrm{~cm} \times 0.4 \mathrm{~cm})$ and convenient use. As shown in Figure 1, the data collection of the product was received wirelessly. The pairing devices used to directly hung on the corresponding shoes, and recorded the number of attentions by

https://doi.org/10.24264/icams-2020.III.9 
shock sensing, which optimized the defect that the receipt cannot be accurately collected due to the limited distance of RFID.
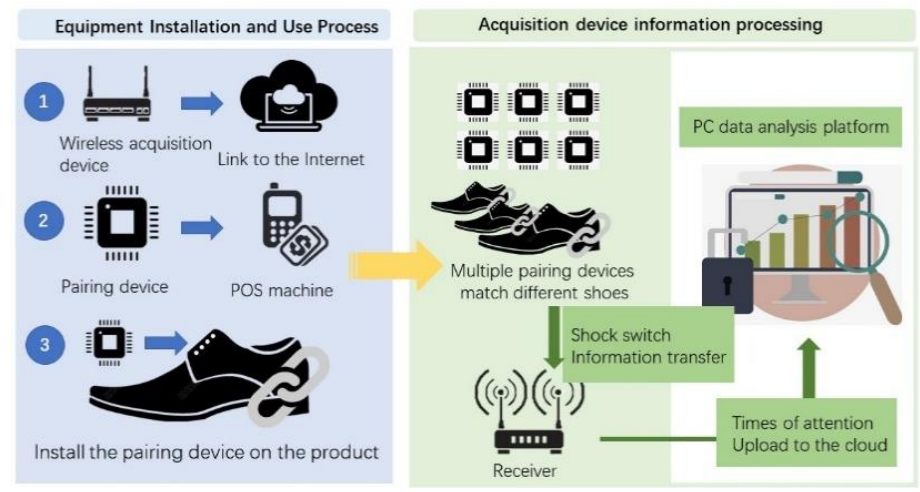

Figure 1. Equipment installation and data collection process

\section{Data Collection}

Firstly, we selected a flagship store of a shoe brand in China, set up smart store system hardware for the target store, and built a data collection platform. Then, the brand branch company gave a list of products for the season, which included attribute information such as shoe model number (SKU), style, price, product age, etc. And we entered the product information into the data platform. Finally, we collected the attention and sales data of shoes in the spring and summer (from March 2019 to August 2019) of the store.

\section{Model Building}

This study proposed to apply the product age $(A)$ factor to the traditional TOFA model (Kahle et al., 1992), so that the improved model can also be used to describe the age positioning of store consumers (as shown in Figure 2-a).

To fill in the product's style, age, and price into the three-dimensional coordinate system, it was necessary to deal with the three in quantitative rules. The style (6 types of styles were shown in Figure 2-b) denoted as $S \in\{-3,-2 、-1,1,2,3\}$; As for ages, 20 represented under 20 years old, 30 represented 21 to 30 years old and so on, which denoted as $A \in\{20 、 30 、 40 、 50 、 60\}$; The zero point of the price was the average price of all products in the store, and the corresponding value was the sample product price minus the average price, denoted as $R \in(-\infty,+\infty)$. 


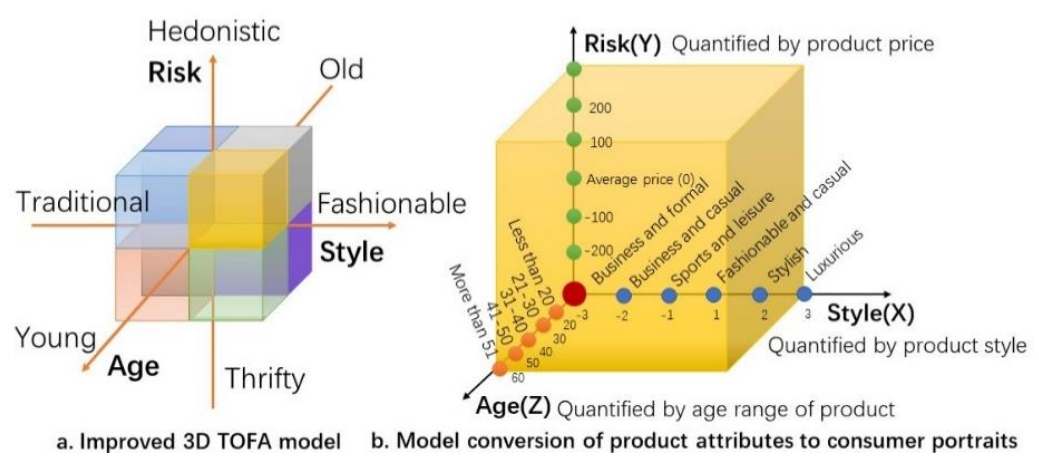

Figure 2. Improved model of relationship between product attributes and consumer profile

\section{Data Processing}

The store's core products can reflect the shopping preferences of the store's core consumer groups. In this study, we selected the core products through the ranking of the comprehensive index of product attention and sales.

Firstly, we separately standardized the collected product attention numbers and sales numbers. Assuming that the number of attention were $\mathrm{x}_{1}, \mathrm{x}_{2}, \cdots, \mathrm{x}_{\mathrm{n}}$, the number of sales were $\mathrm{y}_{1}, \mathrm{y}_{2}, \cdots, \mathrm{y}_{\mathrm{n}}$. The formulas for obtaining attention index $(X)$ and sales index $(Y)$ were as follows:

$X=\frac{x_{i}-\min _{1 \leq j \leq n}\left\{x_{j}\right\}}{\max _{1 \leq j \leq n}\left\{x_{j}\right\}-\min _{1 \leq j \leq n}\left\{x_{j}\right\}}$

$Y=\frac{y_{i}{ }^{-} \min _{1 \leq j \leq n}\left\{y_{j}\right\}}{\max _{1 \leq j \leq n}\left\{y_{j}\right\}-\min _{1 \leq j \leq n}\left\{y_{j}\right\}}$

Then, the comprehensive index $(C)$ of the core product was obtained by the attention index $(X)$ and the sales index $(Y)$ :

$C=0.5 \times(X+Y$

Finally, the top 50 products with the highest comprehensive index were selected as the main research samples. We filled in the applicable age, price and style of the core products into the three-dimensional coordinate system to make a scatter diagram. Through the distribution of scattered points, the characteristics of consumers' age, consumption level and preferences are obtained. The portrait of the store's core consumer groups was also obtained through the pairwise comparison between the three factors. 


\section{RESULTS}
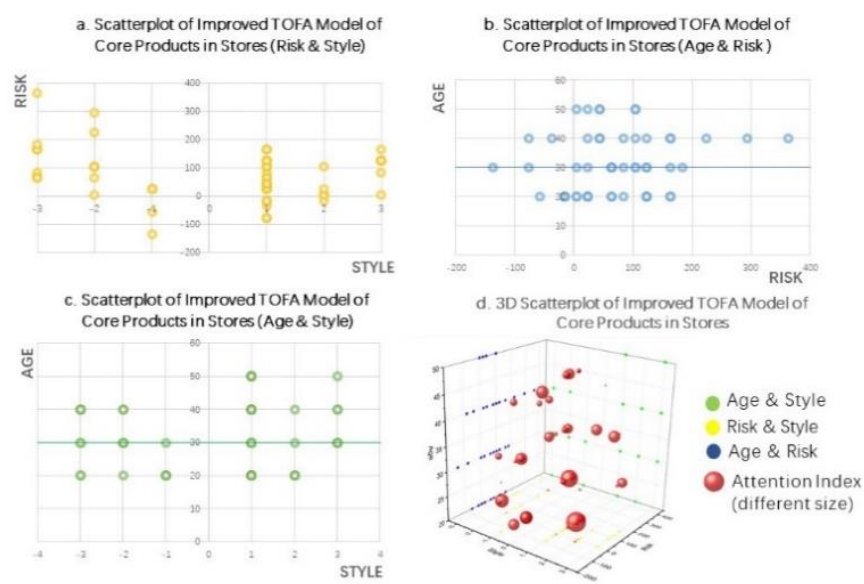

Figure 3. Scatter plot results of core products and consumer portraits

From the basic distribution of the scatter diagram in the Figure3-a, 3-b, 3-c, it can be seen intuitively that the core product style of the store was fashionable and casual, and the age of the products was mainly concentrated in the 31-40 years old. More than half of the product prices were about 100 yuan (about 375.32 yuan) higher than the average store price. From the improved TOFA three-dimensional model (as shown in Figure 3d), the shoes with high attention index (the higher the index, the larger the shape) were mainly concentrated in the 21-30 years old. The price were slightly lower than the average price, and the style of shoes were also fashionable and casual.

We can further analyze the consumer portrait. The core consumer group in the store was mainly middle-aged, whose consumption level was higher than the average price of the products in the store. They preferred fashionable and casual style shoes, and thier consumption values were biased towards hedonic. There were potential thrifty young consumers whose consumption level was close to the average price of products in stores, and their also preferred fashionable and casual shoes.

\section{DISCUSSION}

This study designed a set of consumer attention data collection system to collect shoe products data for a Chinese brand's fast fashion offline store. We used the improved TOFA model to analyze the attributes (style, price, and applicable age) of the store's core products, and converted the results into a description of the store's core consumer profile, including consumer age, consumption level and preferences.

From the results of consumer portraits, we obtained the actual core consumer group portraits and potential consumer group portraits in the store. Both of them prefer fashionable and casual style shoes, but there were certain differences in age and consumer values. The age of potential consumers was lower than the age of actual core consumers. We suggested that the shoes production can be younger in product transformation. Potential consumers tended to be diligent and thrifty in their consumption values, which can indicate that the main factor for potential consumers not 


\section{Research on Consumer Portraits of Offline Fast Fashion Shoe Stores Based on IoT Smart Hardware}

to buy products was that the price was higher than expected. Stores can appropriately lower the price of products for this group of people to promote sales.

The advantage of the attention behavior collection system was that it can track the attention degree of a single product. Compared with computer vision technology to collect behavioral data from a consumer's perspective (Rosa, 2015), this system simplified the analysis process and reduces equipment costs. Chen et al. (Chen, 2014) used RFID and infrared equipment to analyze the number of concerns of the entire shelf or store area. Our advantage was that we can collect the attention data of specific products without being affected by display adjustments. So as to achieve rapid response, precise marketing, and meet the needs of fast fashion product data conversion.

This study also has certain limitations. In terms of equipment wear and tear, because the pairing device used disposable button batteries, the batteries need to be replaced regularly (about half a year). In terms of model analysis, due to the limited number of shoes in stores, the number of core product samples is relatively small. In future research, we plan to apply this technology to different formats stores to compare the differences in core consumer's portrait in stores.

\section{CONCLUSION}

The consumer attention data collection system can effectively collect attention data of specific individual products. The improved new model can effectively analyze the core consumer profile of shoe stores, and provide strategies for shoe store customer positioning and supply.

\section{REFERENCES}

Aviv, Y. and Pazgal, A. (2008), "Optimal pricing of seasonal products in the presence of forward-looking consumers", Manufacturing \& Service Operations Management, 10(3), 339-359, https://doi.org/10.1287/msom.1070.0183.

Barnes, L. and Lea-Greenwood, G. (2006), "Fast fashioning the supply chain: shaping the research agenda", Journal of Fashion Marketing and Management: An International Journal, 10(3), 259-271, https://doi.org/10.1108/13612020610679259.

Cachon, G.P. and Swinney, R. (2011), "The value of fast fashion: Quick response, enhanced design, and strategic consumer behavior", $\quad$ Management Science, 57(4), 778-795, https://doi.org/10.1287/mnsc.1100.1303.

Caro, F. and Gallien, J. (2010), "Inventory management of a fast-fashion retail network" Operations Research, 58(2), 257-273, https://doi.org/10.1287/opre.1090.0698.

Chen, C.-C. (2014), "RFID-based intelligent shopping environment: a comprehensive evaluation framework with neural computing approach", Neural Computing and Applications, 25(7-8), https://doi.org/10.1007/s00521-014-1652-7.

Choi, T.-M., Liu, N., Liu, S.-C., Mak, J. and To, Y.-T. (2010), "Fast fashion brand extensions: An empirical study of consumer preferences", Journal of Brand Management, 17(7), 472-487, https://doi.org/10.1057/bm.2010.8.

Di Sorrentino, E.P., Woelbert, E. and Sala, S. (2016), "Consumers and their behavior: state of the art in behavioral science supporting use phase modeling in LCA and eco-design", The International Journal of Life Cycle Assessment, 21(2), 237-251, https://doi.org/10.1007/s11367-015-1016-2.

Dychtwald, K. and Gable, G. (1990), "Portrait of a changing consumer", Business Horizons, 33(1), 62-74, https://doi.org/10.1016/0007-6813(90)90073-K.

Generosi, A., Ceccacci, S. and Mengoni, M. (2018), "A deep learning-based system to track and analyze customer behavior in retail store", Paper presented at the 2018 IEEE 8th International Conference on Consumer Electronics-Berlin (ICCE-Berlin), https://doi.org/10.1109/ICCE-Berlin.2018.8576169.

Hervé, C. and Mullet, E. (2009), "Age and factors influencing consumer behavior", International Journal of Consumer Studies, 33(3), 302-308, https://doi.org/10.1111/j.1470-6431.2009.00743.x. 
Hou, K., He, J., Liang, L., Li, J. and Zhou, J. (2019), "Bass-modeling for fast fashion lady's shoes based on consumer behavior". Leather and Footwear Journal, 3, 167-176, https://doi.org/10.24264/lfj.19.3.1.

Hsieh, L.-W., Yang, S.-C., Chen, M.-Y. and Tung, I.-W. (2010), "Multiple Groups Confirmatory Factor Analysis of Exploratory Consumer Buying Behavior in Athletic Footwear Inventory", Asian Sports Management Review, 4(1), 44-64.

Jackson, V., Stoel, L. and Brantley, A. (2010), "Mall attributes and shopping value: Differences by gender and generational cohort", Journal of Retailing and Consumer Services, 18(1), https://doi.org/10.1016/j.jretconser.2010.08.002.

Joy, A., Sherry, J.F., Venkatesh, A., Wang, J. and Chan, R. (2012), "Fast Fashion, Sustainability, and the Ethical Appeal of Luxury Brands", Fashion Theory, 16(3), 273-295, Dhttps://doi.org/10.2752/175174112X13340749707123.

Kahle, L.R., Beatty, S.E. and Homer, P. (1986), “Alternative measurement approaches to consumer values: the list of values (LOV) and values and life style (VALS)", Journal of consumer research, 13(3), 405409, https://doi.org/10.1086/209079.

Kahle, L.R., Liu, R. and Watkins, H. (1992), "Psychographic variation across United States geographic regions", ACR North American Advances.

Liu, J., Gu, Y., \& Kamijo, S. (2015), "Customer behavior recognition in retail store from surveillance camera", Paper presented at the 2015 IEEE International Symposium on Multimedia (ISM), https://doi.org/10.1109/ISM.2015.52.

Nicosia, F.M. (1966), "Consumer decision processes; marketing and advertising implications", Journal of Marketing Research, 5(3), 334, https://doi.org/10.2307/3150361.

Palmer, E.L. (2010), “Consumer Research". The Corsini Encyclopedia of Psychology, 1-2, https://doi.org/10.1002/9780470479216.corpsy0224

Rosa, P. (2015), "What do your eyes say? Bridging eye movements to consumer behavior", International Journal of Psychological Research, 8(2), 90-103, https://doi.org/10.21500/20112084.1513.

Tokatli, N. (2008), "Global sourcing: insights from the global clothing industry - the case of Zara, a fast fashion retailer", Journal of economic Geography, 8(1), 21-38, https://doi.org/10.1093/jeg/lbm035.

Wang, Y. (2014), "Consumers' purchase intentions of shoes: theory of planned behavior and desired attributes", International Journal of Marketing Studies, 6(4), 50, https://doi.org/10.5539/ijms.v6n4p50.

Yoshida, T. and Koiso, T. (2010), "System and method for customer behavior movement frequency prediction in a store", in: Google Patents. 
Research on Consumer Portraits of Offline Fast Fashion Shoe Stores Based on IoT Smart Hardware 
INVESTIGATING THE IMPACT OF COVID-19 ON MARITIME SUPPLY CHAIN SUSTAINABILITY AND TECHNOLOGY: A REVIEW

\author{
JILAN KESHTA ${ }^{1}$, HEBATALLAH ELMESMARY ${ }^{2}$, MATEVŽ OBRECHT ${ }^{3}$ \\ ${ }^{1}$ Arab Academy for Science, Technology, and Maritime Transport, Egypt, Jilan9a@yahoo.com \\ ${ }^{2}$ Arab Academy for Science, Technology, and Maritime Transport, College of International \\ Transport and Logistics, Logistics and Supply Chain Management Department, Egypt, \\ hebaelmesmary@aast.edu \\ ${ }^{3}$ Department of Managing Logistics and Supply Chain, Faculty of Logistics, University of \\ Maribor, Mariborska Cesta 7, 3000 Celje, Slovenia, matevz.obrecht@um.si
}

\begin{abstract}
Despite the increasing interest toward sustainability issues in supply chain over the academic and practitioners' perspectives, a comprehensive and updated assessment of the existing literature is still negotiated. Epidemic outbreaks are a unique case of supply chain (SC) risks which is particularly characterized by using a long-time period interruption lifestyle, and extreme uncertainty. As international transport stands at the front of trade and is mainly dependent on travel and human interaction, the shipping industry has been affected substantially from the epidemic of COVID-19 directly and indirectly. This paper represents a review that aims to investigate the impact of COVID-19 on the sustainability of maritime supply chains in general and also in selected geographical areas in terms of the regulations and restrictions imposed by specific organizations in some countries as well as the effect on the supply chain sustainability. In addition, the paper highlights the recent technologies that are currently applied in maritime supply chains and how they can be employed as proposed solutions to facilitate the supply chain flow, overcome the negative effect caused by the COVID-19, and provide further managerial insights to cope with this situation in the most efficient and effective way.
\end{abstract}

Keywords: Maritime Supply Chain Sustainability, Maritime Supply Chain Technology, Coronavirus/COVID-19.

\title{
INTRODUCTION
}

A supply chain is a network of various activities, people, entities, resources, and information. This network is between a company and its suppliers to manufacture and distribute a certain product or service to the end user. The supply chain includes the steps taken to move the product or service from its original state to the final consumer (Kenton, 2020). Sustainability is the future, understanding the level of social, economic and environmental effect and viability that suppliers and customers have. Sustainability is not only going green and being environmentally friendly. It also influences the whole manufacturing process, from where raw materials are obtained, to the processes within the factory, to the consumption and possible recyclability of the product or service (Grimshaw, 2019).

Organizations should track their success in a sustainable manner. This monitoring will require proper setting on Key Performance Indicator (KPI). Measuring success in these terms has a huge impact on the performance of organizations and makes tracking and keeping up to date in the motion easier. KPIs can be classified as financial and nonfinancial KPIs, which differ from one industry to another. Hence it can be difficult to compare KPIs. Procurement, warehousing, production, and transportation have their own defined KPIs for measuring sustainable efficiency (Smith \& Van Der Heijden, 2017).

https://doi.org/10.24264/icams-2020.III.10 


\section{SUSTAINABILITY IN MARITIME SUPPLY CHAIN}

"Sustainability" refers to studying how the natural systems purpose remain various and construct everything it needs for the ecology to remain in stability. Sustainability and sustainable development emphases on balancing the competing needs - the need to move forward economically and technologically, and the needs to protect the environments where we live. Sustainability has become a main priority in the plan and operation of supply chains in the twenty-first century. This emphasis on sustainability allows a supply chain to better set out more environmentally aware customers while improving supply chain performance (Chopra and Meindl, 2016).

Sustainable Supply Chain Management (SSCM) is a holistic view of supply chain activities, which have an impact on the environmental, social, financial and legal features of a supply chain's parts. Defining property SSCM as a group of social control practices that cover all the subsequent environmental effect as an essential consideration of all ranges across every product's overall chain; and a multi-disciplinary standpoint, surrounding the whole life-cycle of the product (Gupta and Palsule-Desai, 2011).

Maritime Supply Chains breaks the maritime chain into components, persistently bearing on them to the overall integrated supply chain. As maritime transport is a crucial linkage of the global supply chains, there are trade-offs to be made with other links, including the choice of port and transshipment as well as hinterland services. Managing maritime supply chains examines how such chains can be affected through exogenous developments and events, such as structural changes in worldwide trade, evolutions in the international fleet, and national and international maritime policymaking (Sys, 2020). Many transportation and logistics issues are controlled via maritime link. The complete SC to keep these things to do going requires robust support from materials such as fuel, spare components as these need to be managed and organized. It is also significant to manage the information flow. In addition, cash flow is required to be systematized through internet or enabling mechanisms such as ecommerce portals. Maritime SCM is a self-discipline that needs to be set in its deserved important position as regards its significance in global financial system and the sizable sea size it covers throughout continents (Deshmukh, 2014).

\section{THE IMPACT OF CORONAVIRUS COVID-19 ON MARITIME SUPPLY CHAIN SUSTAINABILITY}

Epidemic outbreaks are a unique case of supply chain (SC) risks which is distinctively distinguished by using a long-time period disruption lifestyle, and excessive uncertainty. The maritime industry is taking part in an important role within the short-run emergency response to the pandemic, by smoothing the transport of important commodities and merchandise, therefore sustaining jobs, international trade, and international economy. And discussing how to magnify sustainability and resilience of ports and maritime transport during and after the pandemic. The epidemic outbreak effect on the SC overall performance as opposed to an upstream disruption length or the speed of epidemic propagation. Other vital components are lead-time, lick of epidemic propagation, and the upstream and downstream disruption intervals within the SC (Kuhlman and Farrington, 2020).

The outbreak of COVID-19 has affected international transport and the shipping industry directly and indirectly. Operations of shipping companies and relevant industries, including ports, terminals, etc., have been affected due to personnel having been guided to 
abstain from traveling or announcing to work. Decreased command for commodities and raw material, and thus need for shipment, has reduced freight rates. Numerous shipping companies have commenced warning about decreased visibility of earnings and weak future earnings effects.

Genuinely, there do not appear to be any parts of the shipping enterprise that to this point have been proof against COVID-19. The shipping legal profession has been pouring over charter parties and investigating the probability of whether or not COVID19 can represent force majeure, a factor with innumerable consequence to the charter marketplace. Supply chains and logistics as well were affected; for instance, the Chinese trucking industry has collapsed too, because the government has enforced travel limits, which prevents packing containers-for-export from reaching the loading dock, and containers-for-import stay piling on the dock awaiting discharging vessels (Kuo, 2020).

For the reason that freight marketplace for dry bulk vessels and offshore drilling assets has already been weak for some time, it'll not be sudden seeing COVID-19 motivating for bankruptcy protection during the year by a few financially insecure businesses in these sectors (Kuo, 2020).

\section{IMPACT OF COVID-19 PANDEMIC ON SUSTAINABLE SHIPPING SC WORLDWIDE}

Production and shipping is one part of the SC that was affected by COVID-19. Due to the COVID-19 pandemic lockdown and procedures taken. In the meantime, China manufactured half of the world's face masks and shipped them to different countries. However, the shipping industry is responsible for $3 \%$ of greenhouse gas emissions and has traditionally used cheap, polluting fuel, but new standards in the industry are forcing it to clean up its act as it still has a major environmental impact (Queiroz et al., 2020).

Another major part affected is the crew. Airline and port restrictions in most of the countries have made it almost impossible for crew members to urge home if the governments don't construct particular measures. The safe return of the crew from the vessels would necessitate the collaborative efforts of the governmental agencies, the crew manning agency, and the owners. Finally regarding legal disputes, if the cargo is nonessential cargo it cannot be moved to the ports for the duration of national lockdown. Furthermore, before the vessel can take on cargo, it should be cleared by the port authorities, in the pandemic-affected countries the process of vetting the crew may additionally take time, and this delay will fall on the ship-owner as an alternative than on the charter (Ivanov, 2020).

Table 1. Impact of Covid-19 on the maritime SC sustainability over different geographical areas

\footnotetext{
Geographical Area: Balkans - Country: Bulgaria - Organization: The Bulgarian Maritime Administration

Regulations and Restrictions:

- Observance of the instructions of the Paris Memorandum of Understanding on Port State Control relating to COVID-19.

- The whole available information about these ships, such as: last port (s) of call; crew nationality; any recent shifts in ports; any symptoms of COVID-19 on board; any instructions from the health authorities regarding the ship/crew. Based on the collected information, the regional coordinators assess the risk to the ship and if they determine a ship to be inspected, appoint the inspector(s) to carry out the inspection.
}

https://doi.org/10.24264/icams-2020.III.10 
- During the on board inspection, inspector(s) shall wear appropriate personal protective clothing and Equipment and observe the distance as these are stipulated in the orders of the Minister of Health and the instructions of the health authorities.

Effect on supply chain sustainability:

- Local value chains will gain priority and ensuring a local source of responsible supplies will become a new contingency norm for sustainable supply chains.

Geographical Area: Europe - Country: Luxemburg - Organization: Ministry of the Economy Maritime Administration

Regulations and Restrictions:

- Ministry of Infrastructure and Transport Italian Coast Guard Headquarters

- Extension of the validity of seafarer documents beyond their expiry date

- Extension of statutory certificates, surveys, audits and flag state inspections

Effect on supply chain sustainability:

- Showing that many corporate supply chains are vulnerable; needing more disinfection.

Geographical Area: Scandinavian - Country: Norway - Organization: The Norwegian Authorities

Regulations and Restrictions:

- As a partial reporting of the Maritime Declaration of Health, all vessels are required to confirm through SafeSeaNet Norway that there is no detection or suspected infection on-board.

- Specific functionality based on NCA reporting and information systems has been developed to collect data and analyses the effects of the COVID-19 measures on sea traffic.

Effect on supply chain sustainability:

- Efforts to unlock low-carbon growth in the maritime sector, the Norwegian government is supporting this process through the Green Coastal Shipping Programme, which brings industries and state departments together to implement a new maritime strategy.

Geographical Area: Central Europe - Country: Poland - Organization: Ministry of Maritime Economy and Inland Navigation Norwegian Authorities

Regulations and Restrictions:

- Traffic at the maritime border has not been suspended.

- Cargo vessels operations are carried as usual. Ferry traffic for heavy goods vehicles operate as usually.

- All passengers of the ships are subjected to sanitary control, consisting of measuring the body temperature and filling out the location card.

- All persons, with some exceptions, returning to the territory of the Republic of Poland, including the ship's passengers have to undergo a 14-day quarantine.

Effect on supply chain sustainability:

- Sustainable Development Goals and ensure that people have high awareness of health precautions.

- Investing more in technology solutions and working closely with their IT software and hardware suppliers to develop more automated supply chains that rely less on cross borders.

Geographical Area: South-eastern Europe - Country: Romania - Organization: The Romanian Authorities

Regulations and Restrictions:

- After descending from the ship, the protective equipment is collected in bags and incinerated by the waste collection company with which the respective institutions have a contract.

- Limiting contact with other essential crew members (master, watch officer, helmsman)

- Keeping as much as possible the documents taken from the ship (pilot's vouchers) in a separate envelope in the pilot's bag (content that can be disinfected after getting on board).

Effect on supply chain sustainability:

- To support sustainable local production and supply of critical medicines, medical supplies and food in all nations.

- Green recovery plan that enables supply chains to integrate more sustainable and ethical practices.

- Operate within legal limits and comply with agreed-upon contractual requirements; addressing 


\section{COVID-19 AND MARITIME SUPPLY CHAIN TECHNOLOGIES}

COVID-19 makes the world recognize how greatly we depend on the interactions between humans to make things work. Businesses that are demanding labor, such as retailing, warehousing, manufacturing and logistics are the worst affected. COVID-19 has strongly pushed to rollout the utilization of robots and research on robotics. In recent weeks, robots have been employed to sterilize and sanitize areas and to deliver things to those in quarantine (Xiao and Fan, 2020).

Cash might transfer the virus, so central banks in China, US and South Korea have employed several measures to ensure banknotes are clean before they are being exchanged. Currently, contactless digital payments, either in the form of cards or ewallets, are the suggested payment method to prevent the spread of COVID-19. Digital payments for even container customs and customs clearances service enable people to deal online to finish and continue working without any delays or affecting each other (Xiao and Fan, 2020).

Principal technologies of the Fourth Industrial Revolution, such as Cloud Computing; as its power to serve through daily sales data rapidly; shifting patterns quickly and forecast demand capacity with machine teaching algorithms and show these insights with predictive analytics. Internet-of-Things ("IoT"), Big Data and blockchain are gearing maritime SC failures revealed by Covid-19 and constructing a more resilient supply chain management system for the future by means of improving the accuracy of statistics and catalyzing records sharing. Blockchain additionally permit leaders to amplify the benefits and reduce the risks of the technology. SC resilience depends on transparency, trust, and unity. This can be enhanced through implementing the technologies of blockchain which provide a "shared truth" (May, 2020).

Port authorities in many countries have requested personnel to work from home. Remote work is supported by technologies such as virtual private networks (VPNs), virtual meetings, voice over internet protocols (VoIPs), work collaboration tool, cloud technology, and even facial realization technologies to maintain the privacy of the home (Guttman, 2020).

\section{CONCLUSION}

To conclude, the goal of the research is to find out the impact of Coronavirus/COVID19 and on maritime supply chains sustainability. This was done through conducting the review through identification of research scope, selecting relevant studies, assessing the quality of selected studies, extracting data, synthesizing the selected relevant studies and compromising sources. Literature was gathered on the basis of selected keywords (Sustainability, Maritime Supply Chain etc.) that were identified and used to online databases search. The sources were selected to be sufficient to address the topic and provide an evaluation for staring at and predicting each non-permanent and long-term effect of epidemic outbreaks on the maritime SCs alongside with managerial insights during COVID-19 situation. 


\section{REFERENCES}

Chopra, S. and Meindl, P. (2016), Supply Chain Management: Strategy, Planning, and Operation, 6th Edition, Pearson, Managerial Economics, Decision Sciences and Operations, available at: https://www.scholars.northwestern.edu/en/publications/supply-chain-management-strategy-planning-andoperation-2.

Deshmukh, S G. (2014), Re: How prominent and important is the role of the maritime supply chain in logistics and supply chain operations? Retrieved from: https://www.researchgate.net/post/How_prominent_and_important_is_the_role_of_the_maritime_supply _chain_in_logistics_and_supply_chain_operations/534a3462d5a3f2f4308b45b5/citation/download.

Grimshaw, J. (2019), "Lean Supply Solutions - Innovative Supply Chain Solutions", www.leansupplysolutions.com/author/lsstomk/.

Gupta, S. and Palsule-Desai, O.D. (2011), "Sustainable supply chain management: Review and research opportunities", IIMB Management Review, 23(4), 234-245, https://doi.org/10.1016/j.iimb.2011.09.002.

Guttman, C. (2020), "How Technology Helps Connect People \& Reduce Social Isolation." The Forecast by Nutanix, April, www.nutanix.com/theforecastbynutanix/technology/how-technology-helps-connectpeople-reduce-social-isolation.

Ivanov, D. (2020), "Predicting the impacts of epidemic outbreaks on global supply chains: A simulation-based analysis on the coronavirus outbreak (COVID-19/SARS-CoV-2) case", Transportation research. Part E, Logistics and transportation review, 136, 101922, https://doi.org/10.1016/j.tre.2020.101922.

Kuhlman, T. and Farrington, J. (2010), "What is Sustainability?", Sustainability, 2(11), 3436-3448, https://doi.org/10.3390/su2113436.

Kuo, M.A. (2020), “COVID-19: Impact on Global Shipping and China's Economy. Insights from Basil M. Karatzas", The Diplomat, available at: https://thediplomat.com/2020/03/covid-19-impact-on-globalshipping-and-chinas-economy/.

May, A. (2020), "Blockchain to Tackle Supply Chain Failures Exposed by COVID-19 and Boost Economic Recovery", World Economic Forum, April, www.weforum.org/press/2020/04/blockchain-to-tacklesupply-chain-failures-exposed-by-covid-19-and-boost-economic-recovery/.

Queiroz, M.M., Ivaov, D., A. Dolgui and Wamba, S.F. (2020), "Impacts of epidemic outbreaks on supply chains: mapping a research agenda amid the COVID-19 pandemic through a structured literature review", Ann Oper Res, https://doi.org/10.1007/s10479-020-03685-7.

Smith, S. and Van Der Heijden, H. (2017), "Analysts' evaluation of KPI usefulness, standardization and assurance", Journal of Applied Accounting Research, 18(1), 63-86, https://doi.org/10.1108/JAAR-062015-0058.

Sys, C. (2020), "Masterclass on Maritime Supply Chains", University of Antwerp, Apr. 2020, www.uantwerpen.be/en/centres/c-mat/programme/specialisation-courses/maritime-supply-chains/.

World Shipping Council (2020), "International Organizations", www.worldshipping.org/additionalresources/international-organizations.

Xiao, Y. and Fan, Z. (2020), "10 technology trends to watch in the COVID-19 pandemic", World Economic Forum, available at: https://www.weforum.org/agenda/2020/04/10-technology-trends-coronaviruscovid19-pandemic-robotics-telehealth/. 
APPLICATION OF OPENPOSE ALGORITHM TO DETECT CONSUMER BEHAVIOR IN STORE

\author{
JINGJING LI ${ }^{1}$, JIE ZENG $^{1}$, KEYU HOU $^{1}$, JIN ZHOU $^{1,2 *}$, RUI WANG ${ }^{3}$ \\ ${ }^{1}$ Sichuan University, College of Biomass Science and Engineering, National Engineering \\ Research Center of Clean Technology in Leather Industry, Section of Chengdu No. 24 Southern \\ Yihuan, 610065, Chengdu, China, 625368881@qq.com \\ ${ }^{2}$ Science Lab, Zhejiang Red Dragonfly Footwear Co., LTD., Zhejiang Province, Wenzhou, \\ 325100, Wenzhou, China, zij_scu@scu.edu.cn \\ ${ }^{3}$ Chengdu Institute of Product Quality Inspection Co. Ltd, 610000, Chengdu, China, \\ wangruicd@sina.com
}

\begin{abstract}
Due to the importance of offline consumer behavior, more and more people had begun to study consumer behavior in store. In offline consumer behavior research, the application of video analysis technology was the most direct and convenient. Recognizing human posture was a key technology in video analysis. The OpenPose algorithm was one of the advantageous technologies that could accurately recognize multi-person poses in different environments in real time, so we used it innovatively to study consumer behavior in store. We hope to develop the potential of this application in the research of consumer behavior in store in the footwear retail industry by the technical advantages of the OpenPose algorithm. In our study, we first used an OpenPose algorithm to estimate multi-person pose and detection behavior, and then processed and recognized the videos collected in the store. We collected a week's surveillance video of a Red Dragonfly offline store from July 10 to July 16,2020 in China. The specific process was to calibrate the area in the selected camera screen, then the algorithm performs identification and detection, and finally output in-store consumption Behavioral data. Our research results not only verified the feasibility of this application in offline retailing stores, but the data results also indicated that consumers tend to enter the store from the right, staying concentrated in the middle and back of the store. These results may be affected by the store space, product display, and staff guidance and reception.
\end{abstract}

Keywords: OpenPose; Footwear; Consumer Behavior.

\title{
INTRODUCTION
}

Online retail had been hit hardly by offline retail, but it could not replace offline shopping (Chen, 2020), especially in footwear retailing industry (Levin et al., 2003). Consumers had to go to footwear retailing stores to experience the service and fit the shoe so as to buy a suitable product (Ferreira, 2015). Consumer behavior digital could provide brands valuable information, this information could provide consumers with better service and experience. So convenient and accurate digital information collection methods had been widely studied by people (Frontoni et al., 2013, Marginean et al., 2019).

There were two main digital information collection methods (Mancini et al., 2013), one was RFID technology, another one was video analysis technology (Chen, 2014). Marina Kholod et al. (Jung and Kwon, 2011) used RFID technology to collect consumer behavior in store and realize information identification of footwear products. Emanuele Frontoni et al. (2013) used RGBD video analysis technology to track consumer behavior in store, they successfully studied the interaction between customers and products on the shelf.

In these methods, video analysis technology (Mancini et al., 2013) was a more appropriate method for digital information collection. The store monitoring camera (Liciotti et al., 2014) was a common devices in store and it could be directly applied in 
body pose estimation (Frontoni et al., 2013), and recognizing video, predicting human posture was an extremely important part of it. The application of artificial intelligence realized the development from identifying a single person to multiple in different environments and track (Cao et al., 2018). There were mainly two mainstream multiperson recognition models, one was to detect multiple people first and then estimated each person's pose, which had high accuracy but low processing efficiency (Li et al., 2018), such as AlphaPose; another one was to detect the joint points first, and then determined which person each joint belongs to, such as OpenPose (Cao et al., 2018). OpenPose could capture and recognize human body postures and movements in real time, it has advantages in in-store consumer behavior research. Because it was mainly used in the recognition of human skeleton points and gesture recognition (Osokin, 2018), including fitness (Noori et al., 2019), action collection (Li et al., 2018), etc. These above studies still had shortcomings, there was no actual application of these algorithms to the footwear and garment retail industry, and they also lack planning and long-term applications.

Therefore, the purpose of this research was first to establish in-store consumer behavior detection algorithm based on OpenPose to verify the feasibility of this new method, and then processed the data after identifying the video of the store to get the data result of in-store consumer behavior.

\section{METHOD}

\section{Target}

We used OpenPose algorithm deviced an application to process surveillance video of a shoe store, which was a store of Red Dragonfly in Chengdu. Red Dragonfly was as well as a well-known shoe company in China. And we collected videos of this store from July 10 th to July 16 th, the daily video time was 8 am to $10 \mathrm{pm}$.

\section{Technology Architecture}

\section{Algorithm Technology}

The openpose algorithm in this research was responsible for recognizing the video datas taken by the camera and determining the consumer behavior in the store. The application process of the algorithm was to first use the 2-way convolutional neural network VGG-19-branch, then predicted the 2D vector of the key bone connection mode, and then used greedy reasoning to analyze the human body pose from the confidence map. Finally it would output the coordinates of each individual and each joint independent point. These data were the result of this research

In order to ensure the reliability of data identification, we cattied out a preliminary actual verification of the accuracy of the method first. The video clipped in the same period were selected for three times of identification to obtain the results respectively, then the completeness and usability of the data were checked by comparing the manual counting results. If the video recognition result was unstable, judged the video data this time as invalid and retest until the result was stable. 


\section{Processing the Undetected Frames with Median Filter}

Due to the occlusion of human joints and the defects of the OpenPose algorithm, some joints may not be detected in certain frames, and median filter was performed on the joints coordinates of these frames which appeared as both $X$ and $Y$ coordinates are 0 . Set $\operatorname{Pos} X(k)$ and $\operatorname{Pos} Y(k)$ represent the $x$ and $y$ coordinates at kth time respectively which were not be detected. Median filtering process was as follows:

$$
\begin{aligned}
& \operatorname{Pos} X(k)=\frac{\operatorname{Pos} X(k-1)+\operatorname{Pos} X(k+n)}{2}(k \neq \text { firstand } k \neq \text { end }) \\
& \operatorname{Pos} Y(k)=\frac{\operatorname{Pos} Y(k-1)+\operatorname{Pos} Y(k+n)}{2}(k \neq \text { firstand } k \neq \text { end })
\end{aligned}
$$

Among them, $\operatorname{Pos} X(k+n)$ and $\operatorname{Pos} Y(k+n)$ were the first points whose $X$ and $Y$ coordinates were not 0 after time $k$.

\section{Digital FIR Low-Pass Filter Designed}

In order to remove high-frequency jitter, it was necessary to perform low-pass filtering on the obtained continuous coordinate data. People walking frequency did not exceed 2-3 $\mathrm{Hz}$, so set the passband frequency to $0 \mathrm{~Hz} \leq f_{p}<6 \mathrm{~Hz}$. The 17-order FIR filter designed by MATLAB R2019b was used to low-pass filter the coordinates of each joint.

\section{Data Processing}

\section{Consumer Action Judgment}

The criteria for determining consumer behavior in this study were: 1) When someone entered the entrance area, it was marked as in-store passenger flow; 2) When someone stood in the consumer's attention area for more than 5 seconds, it was marked as having an intention to shoe in that area; 3) When someone stood in the consumer's attention area and raised his hand to take the shoes, it was marked as having an intention to shoe in the area; the algorithm counted the number of foucs people in each area and facilitated further analysis.

Regarding the calibration method of the sales area in the camera equipment image. There were three areas in the screen: entrance, product display and consumer attention. The consumer's attention area was manually calibrated, it was a frame with the same width as the product showcase and a length of $80 \mathrm{~cm}$.In this study, the 5 areas in front of 5 stations were calibrated, as shown in the following figure:
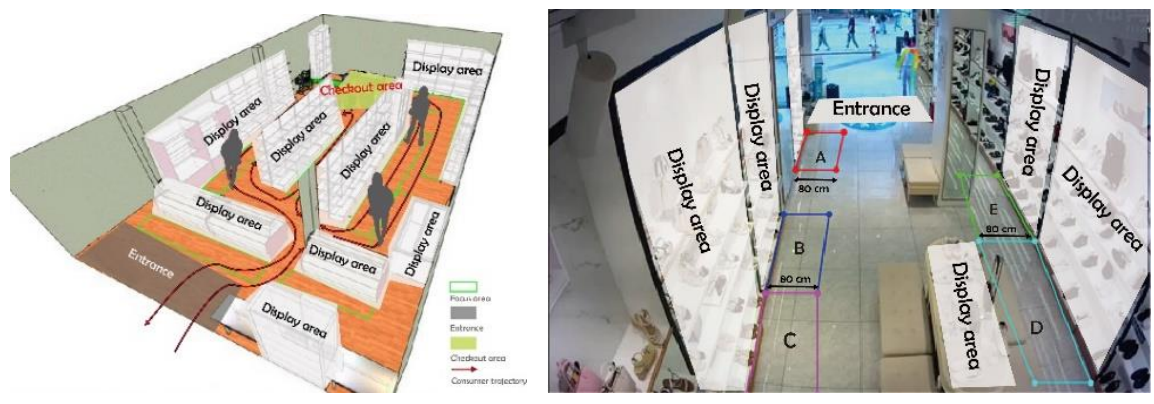

Figure 1. Schematic of the area calibration of the monitoring screen in the study

https://doi.org/10.24264/icams-2020.III.11 


\section{Passenger Flow-Attention on Conversion Rate Calculation}

Divided the number of video attentions into five categories, and accumulated the effective attention in each area according to the period, so as to obtain the number of consumer attention $A$ of the products in each area in stores. Determined the number of attention $A$ and the number of passengers $P$ in the corresponding period according to the following formula:

$A=\sum_{i=1}^{1} a i$

$P=\sum_{i=1}^{1} p i$

Among them, the formula represents the number of follow-ups within the set time period $I$, the number of follow-ups per day was $f_{\mathrm{i}}$, the number of passenger flows was $p_{\mathrm{i}}, i$ represented the serial number of each day, $i=1,2,3, \ldots$, and the corresponding date serial number. From the number of follow-up times $A$ and the number of in-store visitors $P$ in the period, then we could obtain passenger flow-following conversion rate $T$. Determined the attention conversion rate $T$ during the period according to the following formula:

$$
T=\frac{A}{P} \%
$$

\section{RESULTS}

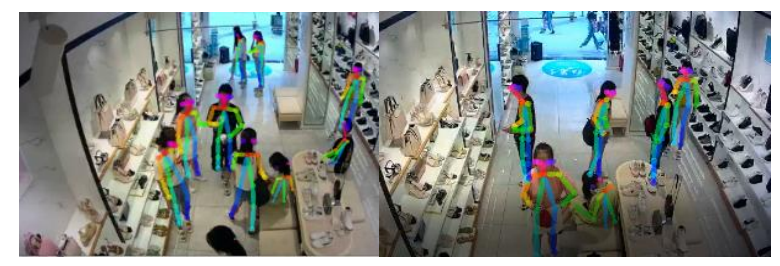

Figure 2. The OpenPose human body gesture recognition screen in the study

We applied this algorithm in the experiment to get the recognition result graph shown in Figure 2. This method could better detect and display all the key points of the human posture in each area of the video, and successfully output the data results. According to the data in the collection period, we obtained the results in Table 1.

Table 1. In-store consumer behavior data analysis results

\begin{tabular}{|c|c|c|c|c|c|}
\hline Category & \multicolumn{3}{|c|}{ Passenger Flow } & \multicolumn{2}{|c|}{ Attention } \\
\hline Daily average & \multicolumn{3}{|c|}{1765} & \multicolumn{2}{|c|}{1302} \\
\hline $\begin{array}{l}\text { Passenger flow-attention on } \\
\text { conversion rate }\end{array}$ & & & $73.76 \%$ & & \\
\hline Aera & A & B & $\mathrm{C}$ & $\mathrm{D}$ & $\mathrm{E}$ \\
\hline $\begin{array}{l}\text { Passenger flow-attention on } \\
\text { conversion rate (Daily average) }\end{array}$ & $2.2 \%$ & $20.7 \%$ & $41.2 \%$ & $8.4 \%$ & $1.2 \%$ \\
\hline
\end{tabular}

First, the overall customer flow conversion rate could be optimized. Secondly, in the conversion rate of each area in stores, area $\mathrm{C}$ was the highest at $41.2 \%$, and area $\mathrm{A}$ and E were extremely low. In terms of the overall distribution of consumers entering the 
store, consumers preferred to enter the store on their own right. After entering the store, their trajectories were concentrated on the right side of the store, gathered in area B and $\mathrm{C}$; Compared to area $\mathrm{A}$ and $\mathrm{E}$, they were more concentrated in the middle and rear of the store, such as area B, C, and D.

\section{DISCUSSION}

In this research, we innovatively applied the OpenPose algorithm in offline retail to identify consumer behavior in store. Experiments were conducted in a real environment, it also objectively verified the reliability of the application in the store.After processing the surveillance video datas, we finally successfully obtained the data result of in-store consumer behavior.

From the application's successful verification, compared with RFID and other video analysis technology to study consumer behavior in store, the advantages of the technology used in our study were high efficiency, high accuracy, cost reduction, and reduction of human and material resources. Zhe Cao et al. (2018) also verified that.

The in-stoe consumer behavior data result showed the trajectory trend of consumers entering the store to the right, middle and back. It was verified the finding of GroeppelKlein \& Bartmann (2007), they also suggested that customers were used to entering the store from the right and then starting to search for products. The reason for this was related to the spatial layout of the store. In one hand, customers would avoid the notwide locations and the location of the cashier counter (Ebster, 2011); On the other hand, it may be related to the display of men's and women's shoes and shop assistant (Alexander et al., 2002). Because who entered the store were mainly women, the sales staff could leadfemale customers to area B and C directly, as we could find from the picture, the styles of sandals in these areas were more suitable for the current season. The data results were also convenient for the store staff to judge the location of more attention based on this situation, and summarized the hot products and the display area in the store, so they could updat the layout of the store in time (Frontoni et al., 2013).

However, there were still some problems in this study: First, when the human body was overlapped or occluded, the single camera device collection may get less accurate video results. Secondly, the accuracy of algorithm recognition could be enhanced.

Therefore, in the future, we can increase the number of cameras in store to improve the accuracy of video results. Secondly, we can improve the speed of algorithm recognition and response, associate relevant product information when recognizing behavior, and add analysis of regional matching products to the results. It can also combine the observation and analysis of facial recognition technology to obtain more detailed information about the crowd that produces a specific behavior. Overall, the technology has a wide range of potential for consumer behavior research in the retail industry.

\section{CONCLUSION}

We proved this algorithm was a convenient and accurate method that could be applied to offline retailing stores for a long time and on a large scale. It could give the store more accurate consumer behavior data analysis so it can be more widely used in in store consumer behavior research. 


\section{Application of OpenPose Algorithms to Detect Consumer Behavior in Store}

\section{REFERENCES}

Alexander, G.M., Packard, M.G. and Peterson, B.S. (2002), "Sex and spatial position effects on object location memory following intentional learning of object identities", Neuropsychologia, 40(8), 15161522, https://doi.org/10.1016/S0028-3932(01)00215-9.

Cao, Z., Hidalgo, G., Simon, T., Wei, S.-E. and Sheikh, Y. (2018), “OpenPose: realtime multi-person 2D pose estimation using Part Affinity Fields", Paper presented at the IEEE Conference on Computer Vision and Pattern Recognition, 7291-7299, https://doi.org/10.1109/CVPR.2017.143.

Chen, C.-C. (2014), "RFID-based intelligent shopping environment: a comprehensive evaluation framework with neural computing approach", Neural Computing and Applications, 25(7-8), https://doi.org/10.1007/s00521-014-1652-7.

Chen, Y. (2020), "How the Internet Affects the Development of Retail Industry", Paper presented at the International Conference on Applications and Techniques in Cyber Security and Intelligence, https://doi.org/10.1007/978-3-030-53980-1_141.

Ebster, C. (2011), Store design and visual merchandising: creating store space that encourages buying, Business Expert Press, NY, https://doi.org/10.4128/9781606490952.

Ferreira, J.P.S. (2015), “Online shopping behavior in offline retail stores: strategic value for companies?" Universidade Católica Portuguesa, 11(4).

Frontoni, E., Raspa, P., Mancini, A., Zingaretti, P. and Placidi, V. (2013), "Customers' activity recognition in intelligent retail environments", Paper presented at the International Conference on Image Analysis and Processing, https://doi.org/10.1007/978-3-642-41190-8_55.

Jung, I.-C. and Kwon, Y.S. (2011), "Grocery customer behavior analysis using RFID-based shopping paths data", World Academy of Science, Engineering and Technology, 59, https://doi.org/10.5281/zenodo.1081169.

Levin, A.M., Levin, I.R. and Heath, C.E. (2003), "Product category dependent consumer preferences for online and offline shopping features and their influence on multi-channel retail alliances", J. Electron. Commerce Res, 4(3), 85-93.

Li, B., Zou, J., Wang, L., Li, X., Li, Y., Lei, R. and Sun, S. (2018), "The Overview of Multi-person Pose Estimation Method", Paper presented at the International Conference on Signal and Information Processing, Networking and Computers, https://doi.org/10.1007/978-981-13-7123-3_70.

Liciotti, D., Zingaretti, P. and Placidi, V. (2014), "An automatic analysis of shoppers behaviour using a distributed rgb-d cameras system", Paper presented at the 2014 IEEE/ASME 10th International Conference on Mechatronic and Embedded Systems and Applications (MESA), https://doi.org/10.1109/MESA.2014.6935617.

Mancini, A., Frontoni, E., Zingaretti, P. and Placidi, V. (2013), "Smart vision system for shelf analysis in intelligent retail environments", Paper presented at the International Design Engineering Technical Conferences and Computers and Information in Engineering Conference, https://doi.org/10.1115/DETC2013-12317.

Marginean, A., Brehar, R. and Negru, M. (2019), "Understanding pedestrian behaviour with pose estimation and recurrent networks", Paper presented at the 2019 6th International Symposium on Electrical and Electronics Engineering (ISEEE), https://doi.org/10.1109/ISEEE48094.2019.9136126.

Noori, F.M., Wallace, B., Uddin, M.Z. and Torresen, J. (2019), "A robust human activity recognition approach using openpose, motion features, and deep recurrent neural network", Paper presented at the Scandinavian Conference on Image Analysis, https://doi.org/10.1007/978-3-030-20205-7_25.

Osokin, D. (2018), "Real-time 2D multi-person pose estimation on CPU: Lightweight OpenPose", arXiv preprint arXiv:1811.12004, Cornell University, NY, https://doi.org/10.5220/0007555407440748. 
QUALITY MANAGEMENT AND FOOTWEAR VALUE CHAIN. A CASE STUDY OF FIBALCO

\author{
ALEXANDRA LUCA, ALINA IOVAN DRAGOMIR \\ "Gheorghe Asachi" Technical University of Iasi, Faculty of Industrial Design and Business \\ Management, 28 D. Mangeron, Iasi, Romania, alexandra.luca@tuiasi.ro; \\ adragomir@tex.tuiasi.ro
}

\begin{abstract}
In the complicated economic context, will survive only companies who can obtain recognizable products, with higher quality and who are economically efficient by reducing losses of any kind. An example of good practices is represented by SC Fibalco SRL, a footwear company from Craiova, Romania, which emphasizes the development strategy of the Quality Management and the value chain analysis. Fibalco's success is explained by the fact that the value chain analysis is a component of the quality management and the responsibility is distributed pyramidally starting from each employee to the top management. One of the chains in Fibalco's value chain is Industrialization of the new product, which is an element of originality and makes the difference between the theoretical and the practical approaches from the perspective of launching a new product on the market. Due to the Industrialization of the new product chain, in order to make it efficiently reproducible, many risks of failure are eliminated from the design phase, especially those related to the technical execution of the product. The result is a "clean" footwear product with clear lines without unnecessary sophistication. This paper demonstrates that the implementation of quality management and a complex value chain adapted to the company needs leads to brand value and loyalty among consumers.
\end{abstract}

Keywords: footwear, value chain, quality management.

\title{
INTRODUCTION
}

In the current economic situation, marked by a crisis of overproduction, the Romanian footwear manufacturers are facing big challenges related to the market access of the products on one hand and to their sale on the other.

In this complicated economic context, only those who can obtain recognizable products, with higher quality and who are economically efficient by reducing losses of any kind, will survive.

An example of good practices is represented by SC Fibalco SRL a footwear company from Craiova, Romania, which emphasizes the development strategy of the Quality Management and the value chain analysis. Although these two are most often seen and implemented separately and independently, Fibalco's management has integrated and correlated them in the company's development strategy, in order to increase business performance. The experience of this company shows us that understanding the dynamics of the value chain and the importance of quality, will generate positive effects on business performance.

\section{Quality Management}

After the Second World War, two important forces appeared with an impact on the concept of quality: the Japanese revolution in quality and the awareness of the importance of quality in customer perception. These two forces have led to a change in mentality towards quality.

Over time, the literature has revealed various definitions and approaches to the concept of quality and the quality management. The most well-known definitions of quality, offered by quality gurus are: Dr. W.E. Deming defines quality as "The efficient 
production of the quality that the market expects"; Crosby P.B. (1979) considers that "Quality is conformance to customer requirements"; Feigenbaum A.V. (1961) affirms that "Quality is what the customer says it is"; Dr. Taguchi G. (1986) "Quality is the loss that a product costs to the society after being shipped to the customer". Another definition is offered by Dr. Juran J.: "Quality is the fitness of use" (Bisgaard S., 2008, Juran J., 1967). Prof. Ishikawa K. is considered one of the "parents" of quality and total quality control (TQC). He believes that TQL is responsible for every element of the company that studies, practices and participates in quality control (Kumar P. et al. 2016). According to ISO 9001:2000 (Quality Management Systems, 2000-12) the quality is "degree to which a set of inherent characteristics (distinguishing features) fulfils requirements".

\section{Value Chain}

The concept of value chain appeared in the early '80s as the total of activities necessary to obtain a product. The value chain concept was developed by Porter M.E. in 1985 as a management concept that describes a company as a conglomeration of tasks (activities) divided into main tasks and support tasks.

This approach aims to improve the company's performance, to create added value for the company and the customer and also to reduce costs by eliminating unprofitable activities. Over the years, the value chain has evolved by integrating activities that increase added value and take into account the "buyer's voice".

\section{CASE STUDY}

SC Fibalco SRL is a footwear company, 100\% Romanian, with an experience of over 20 years in the field, specialized in the production of snickers.

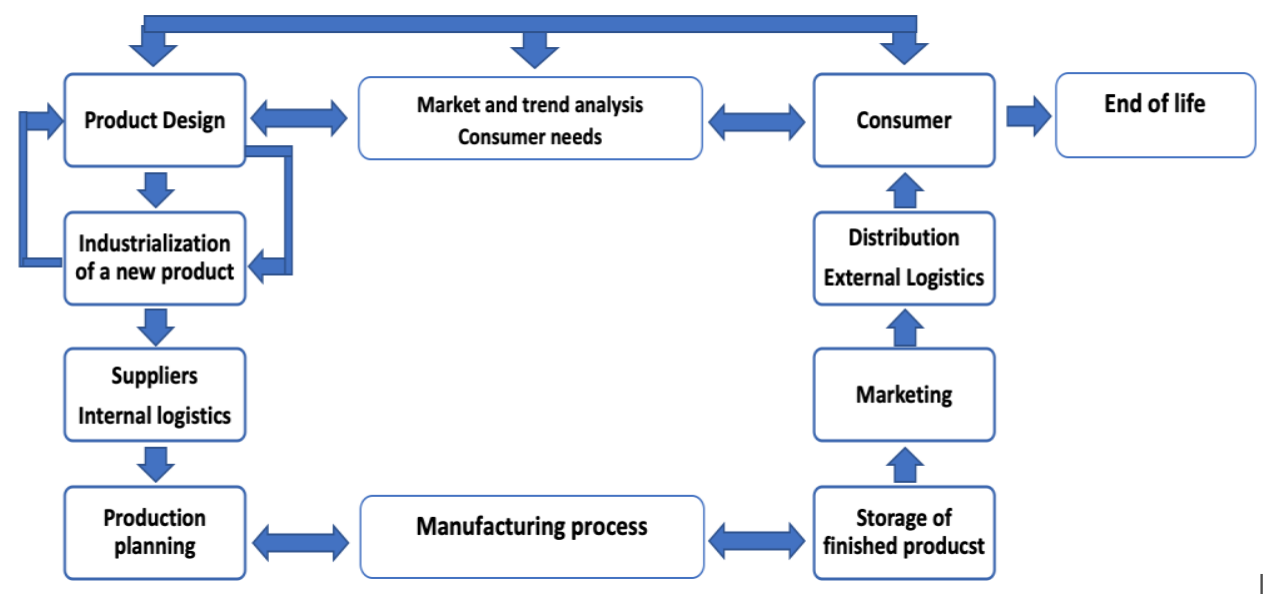

Figure 1. Fibalco's footwear value chain

In their management concept they have developed and included their own value chain adapted to the consumer needs and their technological resources. In the figure 1 is presented Fibalco's footwear value chain.

https://doi.org/10.24264/icams-2020.III.12 
The footwear value chain proposed by Fibalco, is a value chain in a closed loop that focuses on the consumer (it starts with the consumer needs and ends with consumer use of the product)

\section{Market and Trend Analysis and Defining Consumer Needs}

In this stage, the market research is performed by analyzing the offer of direct competitors (those who sell the same products, with the same quality at the same prices) but also of the potential ones (those who produce the same products with better / worse quality at higher or lower prices). In this phase is established the targeted audience from a geographical, demographic and financial point of view and the habits and frequency of purchase for this segment. In order to accomplish this activity, marketing tools such as physical or online surveys, questionnaires, in-store interviews, participation at fairs and exhibitions are used.

A macro-trend is the "wellness" boom - it appears in the context that includes the crisis of the global health systems, the choice to adopt a healthy lifestyle and the rejection of unrealistic esthetic ideals.

To meet the market needs, the company Fibalco with the experience gained in collaboration with brands from Italy and the Netherlands, created its own brand L'Escarpe.

\section{Product Design}

The design of a sneaker is a very complex operation and follows a precise process, it has specific functions and features. This stage includes stylistic proposals, making the model sketch, the pattern model, choosing the last, the soles, the materials and accessories. Another important step is to calculate the price of the product after the sample is made.

\section{Industrialization of a New Product to Make it Efficiently Reproducible}

This link of the value chain elaborated by Fibalco represents an element of originality and makes the difference between the theoretical and the practical thinking from the perspective of launching a new product on the market.

The activities that subscribe to this stage have in view: the elaboration of the technical documentation, the elaboration of the manufacturing process, as well as the establishment of the quality standard of the product whose sample was made in the previous stage. These are compared with the existing equipment in the factory, with the stocks of available materials and with the supply possibilities.

The execution times of some operations are analyzed and proposals / modifications are made in order to streamline the activity and to reduce costs.

\section{Supply Chain and Internal Logistics}

The activity at the level of this link has several main components: the management of the relationship with the suppliers, the management of the inputs of raw materials and materials, the management of the stocks, the management of the waste and the coordination of the internal transport. 


\section{Planning Production}

Planning production is an important stage that covers many activities such as: to respect delivery times, quality and safety at work, ensuring the availability of production equipment, ensuring the synchronization of material and manufacturing flows, ensuring the training and proper development of staff, and also the coordination of the occupational health and safety process.

\section{Manufacturing Process}

The production process is structured on 3 workshops corresponding to the operations of cutting, joining and lasting the product. The 3 workshops have a delimitation without walls to facilitate communication between them. For each operation, a technical file is elaborated, which includes information regarding the setting parameters of the operation and the way of accomplishing them in images and text. The production process ends with the packaging, in the L'Escarpe packaging box.

\section{Storage of Finished Products}

The product boxes are stored in the finished product warehouse, considering the easy access to them.

\section{Marketing and External Logistics}

These two components are especially important in order to ensure the success of the company.
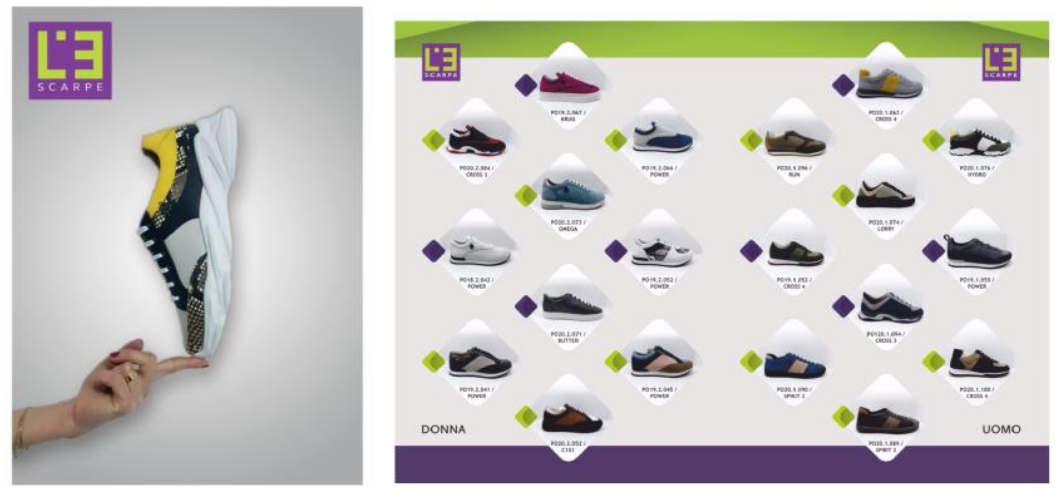

Figure 2. Posters and flyers of Fibalco

The marketing component includes all the actions necessary to transform the ordinary consumer into a consumer promotion vector. To achieve this, promotional activities are carried out through online advertising, the use of social networks, invitations in the network, the creation of posters, flyers and presentation catalogs sent to loyal and targeted customers. In the figure 2 are presented some posters and flyers.

The marketing component also deals with the impact analysis of the promotion actions and of the position of the product on the market. Figure 3 presents the impact analysis of Fibalco's online advertising.

https://doi.org/10.24264/icams-2020.III.12 


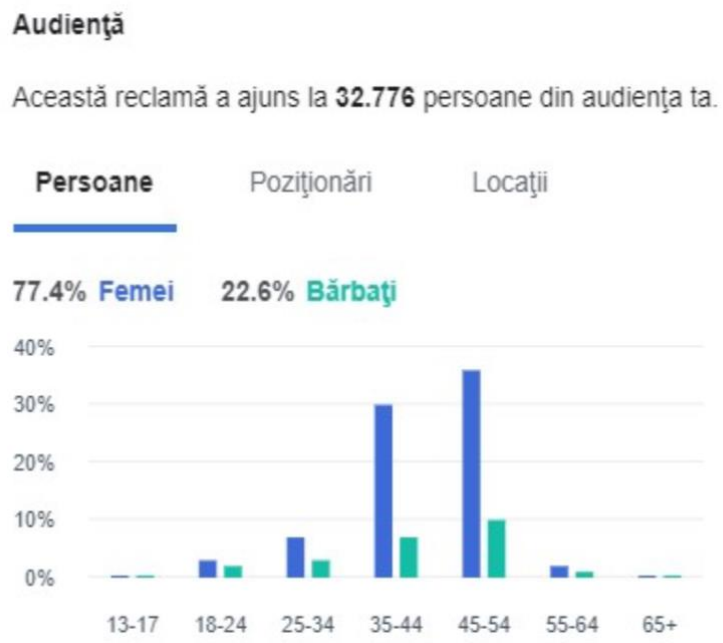

Figure 3. Impact analysis of online advertising

Online advertising was made through social media and the results shows that most of the people interested are women between age of 35 and 54. The analysis shows that over $99 \%$ of potential customers have seen online ads/catalogs using the mobile application. The brand is present in all the historical provinces of the country and highlights the potential growth of the company.

The external logistics component includes finding sales agents to propose products to potential buyers and to promote the brand, but also the delivery and organization of transport to warehouses, stores or consumer.

\section{Final Consumer}

SC Fibalco SRL sells the products in its own stores, both physically and online, but also to other specialized stores. The final consumer is the one who really determines the success or not of a product. The consumer's perception can be influenced by advertising campaigns, testimonials, fashion, sales level but also after-sales services.

A condition of success is the creation of an identity that makes the product recognizable.

\section{End of Life}

This activity is positioned outside the closed loop of the value chain and falls under the responsibility of the final consumer. This situation happened in almost $99 \%$ of footwear manufacturers around the world, except for a few very large companies, which can afford seasonal campaigns such as buy back or collection of used footwear.

Without the involvement of the authorities and programs to support producers in order to be encouraged to receive used footwear, which will then be taken over and processed by specialized companies, the used footwear will continue to reach landfills.

The value chain developed by Fibalco has not explicitly assigned a link for quality control activities. This is explained by the fact that the value chain analysis is a component of the quality management, and the quality control is distributed 
pyramidally and is the responsibility of each employee in each department, of each workshop or service manager.

\section{CONCLUSIONS}

The company's success is based on the fact that they are realistic about the company's development projects, preferring small but safe steps that provide stability and trust to employees and partners. They do not risk in unrealistic projects, being rather traditionalists but permanently open and receptive to the new.

The advantages of implementing the value chain in the company strategy was: an efficient programming of the production, an efficient distribution of the products only in the places where is demand and in the necessary quantities which leads to a stock reduction of finished products.

Due to the Industrialization of the new product chain, in order to make it efficiently reproducible, many risks of failure are eliminated from the design phase, especially those related to the technical execution of the product. The result is a "clean" footwear product with clear lines without unnecessary sophistication.

The implementation of quality management, by assuming the goal of "zero defects" and sharing responsibilities related to quality and quality control to all employees of the company, gives them an advantage over the competitor.

\section{Acknowledgements}

This work has been carried out as part of a Master programme (Product Development of Footwear and Leather Goods) at Faculty of Industrial Design and Business Management, Iasi, Romania. Acknowledgements are also due to Liviu Budică, the manager of SC Fibalco SRL.

\section{REFERENCES}

Bisgaard, S. (2008), “Quality Management and Juran's Legacy”, Quality Engineering, 20(4), 390401, https://doi.org/10.1080/08982110802317398.

Chandrupatla, T.R. (2009), Quality and Reliability in Engineering, Rowan University, New Jersey, ISBN: 978052151522.

Crosby, P. (1979), Quality is free: the art of making quality certain, New York: McGraw-Hill, ISBN 0-07014512-1.

Deming, E.W. (2000), Out of the Crisis, Massachusetts Institute of Technology, by permission of the MIT Press.

Feigenbaum, A.V. (1961), Total Quality Control, New York, McGraw-Hill, OCLC 250573852.

Ishikawa, K. (1968), Guide to Quality Control, Tokyo: Asian Productivity Organization.

ISO 9001:2000, Quality Management Systems, (2000-12), https://www.iso.org/standard/21823.html.

Juran, J. (1967), Management of Quality Control, New York, New York, OCLC 66818686.

Kumar Pradeep, M., Raju, N.V.S. and Satish, K.M.V. (2016), "Quality of quality definition - An analysis", International Journal of Scientific Engineering and Technology, 5(3), https://doi.org/10.17950/ijset/v5s3/304.

Porter, M.E. (1985), Competitive Advantage: Creating and Sustaining Superior Performance, New York: Simon and Schuster, ISBN 9781416595847.

Taguchi, G. (1986), Introduction to Quality Engineering, White Plains, NY: Asian Productivity Organization, UNIPUB.

Taguchi, G. (1988), “The Development of Quality Engineering”, ASI Journal, 1, 1(1-4).

https://doi.org/10.24264/icams-2020.III.12 


\title{
FACTORS INFLUENCING THE PURCHASING DECISIONS OF LOW EMISSION CARS: COMPARING STUDY BETWEEN EGYPT AND SLOVENIA
}

\author{
ALAA OTHMAN $^{1}$, AHMED DABEES ${ }^{1}$, BAHER RAHMA ${ }^{1}$, MATJAZ KNEZ ${ }^{2}$ \\ ${ }^{1}$ College of international Transport\& Logistics at Arab Academy for Science, Technology and \\ Maritime Transport, Alexandria, Egypt, Alaa.abomousa@gmail.com, \\ Ahmed_dabees@hotmail.com,baherrahma@gmail.com \\ ${ }^{2}$ University of Maribor, Faculty of Logistics, Slovenia, Matjaz.knez@um.si
}

\begin{abstract}
This paper provides a study about the factors influencing the purchasing of low emission vehicles. In order to achieve the objectives of the paper, and in the light of the pool of literature and availability of data, the authors relied on qualitative methods to offers a comparison between Egypt as a developing country and Slovenia as a developed country, through analysing a survey that involves an Egyptian sample and Slovenian samples, it also studies the effect of different push and pull methods on different buyers in order to help the governments as well as the manufacturers to understand the most significant factors that affects the purchasing behaviour of LEV in the future. The results of this paper show the important vehicle performance factors, financial considerations and Environmental considerations along with the gender and age of the consumer and show that consumers are more interested in the total price of the car than in different taxes.
\end{abstract}

Keywords: Low emission vehicles, purchasing behaviour, developed and developing countries

\section{INTRODUCTION}

Despite the contextual differences between developed and developing countries, there is an agreed opinion from researchers in both regions that the green technologies, especially in the area of green transport, are interesting for policy makers, vehicle producers, customers and energy suppliers. Many stakeholders from public and private sector are devoting a lot of effort to identify customer behaviour for future enhancements in development of their green products and strategies (El-Dorghamy, 2014; Knez et al., 2014). The predictions of the International Energy Agency (2017) estimate that world energy demand from 2005 to 2030 will increase by approximately $52 \%$, while forecast of World Energy Council estimate that energy demand will double by 2050, which is similar to IEA's prediction (Obrecht and Denac, 2011).

Environmental Performance Index (EPI) rankings ranks countries on how close they are to established environment policy goals and indicate how well governments are controlling or dealing with pollution against the range of environmental pressures that every country faces. The 2018 (EPI) ranks 180 countries on 24 performance indicators across ten issue categories covering environmental health and ecosystem vitality. This report declared that Slovenia currently ranks 34th rank while Egypt currently ranks 66th (EPI, 2018). The automotive sector in Egypt has a vital contribution in the Egyptian economic growth. It is consequently necessary to achieve a balance between the environmental and economic drives of policy makers in order to be assessed in terms of its environmental, economic, and social impact based on Cost-Benefit Analysis (CBA) that also take into consideration the external costs and external benefits together with the other common economic indicators (El-Dorghamy, 2014).

In Slovenia the issue of air pollution is largely high, linked especially to $\mathrm{PM}_{10}$ which are caused by road transport, especially in urban centres with heavy traffic in Ljubljana as well as emissions from heating appliances and industrial sources in Zasavje and Celje. Measurements of $\mathrm{PM}_{10}$ indicate an exceeding of the limit values across the whole of Slovenia, and especially in winter in the inner areas (Slovenian Environment Agency, 2019). 
Current energy import dependency in the EU and in Slovenia stands at approximately $50 \%$. Energy specialties have forecasted that the EU and Slovenia as a member state in it will change over to a sustainable energy industry by 2046, considering that there are no significant oil reserves, and they highly depending on importing oil which can lead to economic and socio-political risk.

Egypt is considerably the largest consumer of oil and natural gas in Africa, showing $22 \%$ of petroleum and other liquids of African total consumption and $37 \%$ of its dry natural gas consumption. Egypt face a low variety of oil importers as the major portion of the Egyptian imported oil ( 4.9 million tons), imports only from three suppliers: Kuwait, Iraq and Oman (Atlam and Rapiea, 2016). Minister of Environment Khaled Fahmy appeals that the government is working on reducing vehicle consumes by switching recent cars with low emission electric ones and he stated that 15 electric buses were put forward in Alexandria in 2018. Nevertheless, it would require charging stations, parking lots, and energy sources to supply the cars, but it would be more economical than gasoline in any other car, as well as there is a huge difference between the technology compared to their counterparts in gasoline (Awad, 2018). According to Director General of Brilliance-Bavarian auto group and Ellithy group company, the electric car has two methods of charging. First, is the (Home shipping) in which the Charging time lost by 220 volts' charger is from 2.30 hours 3.30 hours in case of battery $0 \%$. Second, is the "External freight points" or "shipping stations" which become "available" significantly in Cairo and the governorates of Egypt and desert roads. As well as, High-speed superchargers are installed nowadays to recharge the battery in 30 minutes to facilitate travel. In addition, it does not need maintenance like any other car as the electric motor does not need oils for cooling and does not have any belts or pouches or filters and does not produce any heat which is environmentally friendly.

\section{METHODOLOGY}

The study presented in this paper is a comparative research study. A comparative approach was chosen to investigate the similarities and differences in the factors influencing the purchasing of low emission decisions between developing and developed countries. For the developed country, Slovenia was selected as it is the green heart of Europe; it has abundant natural features placed between the Mediterranean, Central and Southeast Europe. In addition, Slovenia has achieved numerous development goals that helps in raising the level of economy, better outcomes in social and environmental improvement. For the developing country, Egypt has been selected as its one of the largest economies in the Arab world and home to one of the fastest growing middle classes in the Middle East and North Africa region. In additions, Egypt opened up new possibilities with more economic intelligent, social inclusive, environmental responsibility as well as with many challenges.

For the primary data collection, a pre-structured questionnaire was distributed in Slovenia then Egypt. We processed the collected empirical data by using SPSS 18. First, a descriptive statistical analysis was carried out for each of the two countries' samples. In the second step, significant differences in mean of vehicle performance factors, financial considerations and Environmental considerations along with the gender and age of the consumer between Egypt and Slovenia were investigated. We follow a sequential mixed methodology, with the qualitative phase following a descriptive phase. A mixed methods study combines both qualitative and quantitative analysis for the purpose of better understanding an issue, the survey targeted 1086 samples from Egypt and 681 samples from Slovenia whose current opinion about relevant vehicle performance aspects and financial factors for vehicle purchasing decisions was studied. 
In addition, secondary data was collected with compilation method from books, online references and periodicals and specialized journals in sustainability, various scientific and professional papers, researches and project reports focused on the research topic at hand.

\section{DISCUSSION OF RESULTS}

The study was designed to reveal the underlying factors that affect the purchasing habits of people. The results reveal new perspective of purchasers, and indicate which factors are the most important for the purchase of a LEV. Results display the important vehicle performance factors, financial considerations and Environmental considerations. Results also show the reasons beyond future decision to buy a lower emission car as well as the gender and age distribution between different segments of consumers. Bar charts are used to show different results.

Figure 1 displays the important vehicle performance factors with respect to males and females in Egypt versus Slovenia, it could be noted that Luggage/storage space is more important for women, while, Body shape (e.g. hatchback, saloon, estate), Mileage (if you buy a used car), Acceleration time, Fuel type, and Model of vehicle (e.g. Golf, Clio) are more important for men. However, in Slovenia, the results indicated that there are some differences between male and female population, especially when examining safety features, acceleration and fuel type. Safety is more important for women; acceleration and fuel type are more important for men. The interesting point shown is that women find safety more important than men, even though they cause much fewer traffic accidents. The Two non-financial factors are crucial when deciding on a car purchase - 1: "overall condition and mileage of vehicle (when buying a used car)", and - 2: "safety features", other very important factors are: vehicle size (exterior), style/appearance/colour, body shape (e.g. hatchback, coupe, etc.) and fuel type.
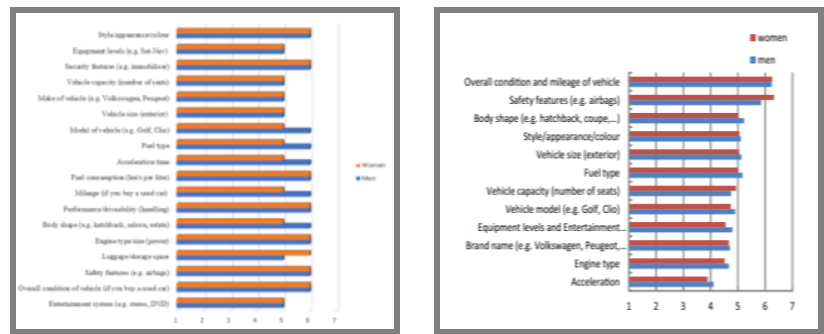

Figure 1. Important Vehicle Performance Factors in Egypt versus Slovenia

Figure 2 displays the important financial considerations with respect to males and females in Egypt versus Slovenia, it could be noted that Maintenance/repair costs, Insurance group for vehicle, and Annual road tax Fuel economy (How much fuel it uses per $\mathrm{km}$ ) are more important for women. On the other hand, in Slovenia, most important thing seems to be the total price of the vehicle. Second feature, also very important, is fuel economy. Especially now when gas prices are high and still increasing, information on fuel consumption is crucial. People also put emphasis on repair costs and on the value/money ratio. Two features that are less important are "trade-in value" (how much money you get when you sell your vehicle) and "annual road tax". 

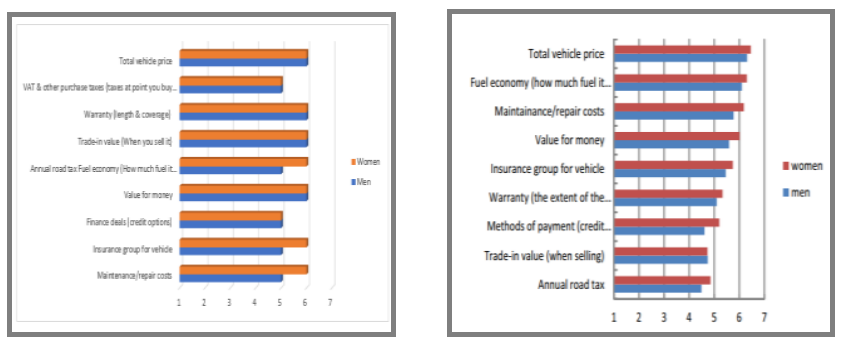

Figure 2. Important Financial Considerations

Figure 3 presents the important Environmental Considerations in Egypt and Slovenia. The results from Egypt indicate that there is no difference between Women and Men in Emissions of $\mathrm{CO}_{2}$ and other greenhouse gases. However, it could be noted that Emissions of other air pollutants, and Vehicle noise are more important for women. In Slovenia, they scored high grades on the question about $\mathrm{CO}_{2}$ and other emissions. Moreover, they are also very attentive about vehicle noise.

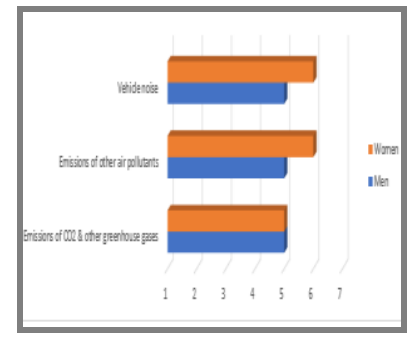

Figure 3. Important Environmental Considerations
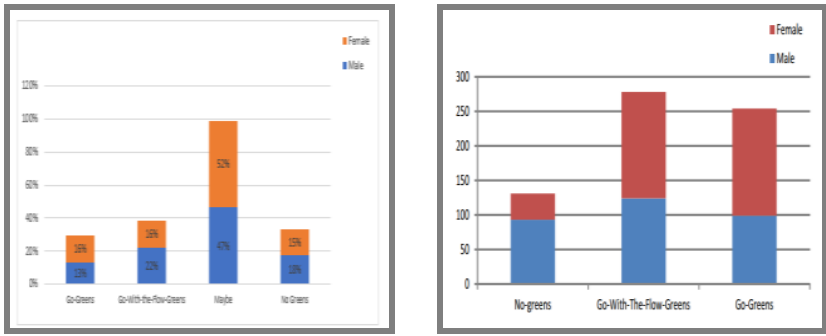

Figure 4. Ratio between Males and Females in different Consumers Segments

The results obtained for ratio between males and females in different segments of consumers. The Egyptians show that only $52 \%$ of all females are in the "Maybe" group, followed by both "Go-With-The-Flow-Greens" and "Go-Greens" as they got 16\%, and finally is "No Green" group as it got $15 \%$. For Male respondents, the highest group is the "Maybe", as it got 47\% followed by "Go-With-The-Flow-Greens" group with a percentage of $22 \%$, is the third place is "No Green" group with $18 \%$, finally, "No Green" group with only $13 \%$. While the Slovenians show that only $11 \%$ of all women are in the-No-green- group and the remaining $89 \%$ are a part of the other two groups. Men are more equally distributed through all three groups $(29.4 \%$ in No-Greens, $39.2 \%$ 
in the Go-With-The-Flow-Greens and 31.3\% in the Go-Greens). The study shows that there are considerably more men than women in the "No-Green" group.
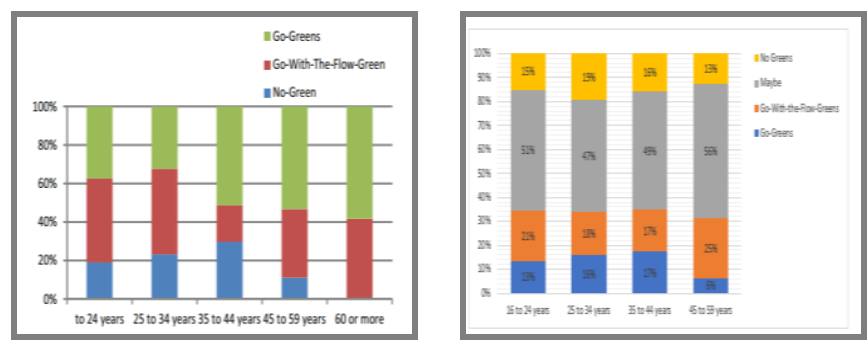

Figure 5. Ratio between Age Groups in different Consumers Segments

For Egypt, the results obtained for ratio between different age groups in different segments of consumers show that most chosen group is "Maybe" group, in every age group. Also, it could be noted that "Go Green" is decreasing with age. For Slovenia, only $11.1 \%$ of population above 44 can be defined as No-Greens. The ratio of GoGreens is increasing with age. It is also very interesting that we did not find any people above 60 years who would classify as No-Greens.

In Egypt, the respondents were asked about petrol and diesel prices, too. Ten percent of study participants were already seriously thinking about buying a car running on alternative fuels (e.g. biodiesel, bio alcohol, hydrogen and electricity). If gas prices increased by $30 \%, 60 \%$ of respondents would start thinking about buying a car which is powered by an alternative fuel. And If gas prices increased by $50 \%, 90 \%$ of respondents would start thinking about buying a car which is powered by an alternative fuel.

In Slovenia, the respondents were asked about petrol and diesel prices, too. Ten percent of study participants were already seriously thinking about buying a car running on alternative fuels (e.g. biodiesel, bio alcohol, hydrogen and electricity). If gas prices increased by 30\% (to EUR 1.96 per litre for petrol, and to EUR 1.80 EUR per litre for diesel), 58\% of respondents would start thinking about buying a car which is powered by an alternative fuel (30\% increase of petrol prices over several years is not an unlikely situation). And If gas prices increased by $40 \%, 80 \%$ of respondents would start thinking about buying a car which is powered by an alternative fuel.

\section{CONCLUSION}

Developed and developing countries needs to significantly accelerate growth toward higher efficiency, more de-carbonization, greater fuel diversity and lower emission of pollutants to manage the consequences of growing consumption and demand for commercial forms of energy. Egyptian and Slovenian respondents do not differ significantly regarding their perceptions in vehicle performance factors. With respect to financial cost, insurance cost, total price of the vehicle and fuel economy are the most important factors in both countries, besides, emissions of air pollutants are very important for Egyptian women, while Vehicle noise was observant in Slovenia.

Egyptian still not have the full awareness of LEV since it's a new concept for them however they have a positive attitude toward LEVs, even if they are not sure about buying one in the near future. On the other side in Slovenia, the biggest group is the Go-With-TheFlow-Greens with $39.2 \%$, while in Egypt a percentage of $19 \%$ is with that flow. Research demonstrate that there are considerably more men than women in the "No-Green" group, also it could be noted that "Go Green" is decreasing with age in Egypt unlike Slovenia it is 
increasing with age. The statistical testing revealed that when consumers are purchasing a car, they are more concerned with the total price of the car than in different taxes, more than $30 \%$ of Egyptian respondents would be seriously thinking about purchasing an electric car if prices decreased by $30 \%$, as well as more than one half $(59 \%)$ of Slovenian respondents would be seriously thinking about purchasing an electric car, if prices decreased by $30 \%$, Furthermore, the effect of different push and pull methods on different purchasers was also studied. In Egypt, the most influential measure is paying more VAT on higher emission cars. While in Slovenia, the most influential measure is 'Vehicle scrappage scheme'. Finally, the results illustrate the main influential factors between two different geographical areas and emphasize on the purchasing behaviour of consumers for LEV in future to help governments and manufacturers understand the attractive factors in different countries. Slovenian and Egyptian government must be aware that if they want to increase interest in purchasing LEVs, it should combine both pull and push factors, Push factor gives incentives to prospective users not to buy a car, provide rebates on VAT, vehicle registration fee and motor insurance premiums based on carbon emissions consumption and, at the same time pulls them to the low carbon modes public and non-motorized transport. Slovenian and Egyptian car industry should be aware that car drivers are more acquainted with information about fuel economy than information about a car's environmental influences (e.g. carbon emissions). Most people do not actually know the meaning of "grams of $\mathrm{CO}_{2}$ per $100 \mathrm{~km}$ " especially in Egypt and the amount of money they could save by buying a LEV.

Our research findings have few limitations. In order to obtain comparable data our methodology had to be wider and to enlarge the scale regarding population. Further limitations that we are aware that opinion and related preferences of people might change over time, and age of our respondents is an intervening variable.

\section{REFERENCES}

Atlam, B.A. and Rapiea, A.M. (2016), “Assessing the Future of Energy Security in Egypt", International Journal of Energy Economics and Policy, 6(4), 684-700.

Awad, M. (2018), Here Is Egypt's Plan to Halve its Air Pollution Rate by 2023, retrieved from http://cairoscene.com/Buzz/ministry-environment-reduce-pollution.

El-Dorghamy, A. (2014), "Fuel Economy and $\mathrm{CO}_{2}$ Emissions of Light-Duty Vehicles in Egypt", Centre for Environment and Development in the Arab Region and Europe, available at: https://www.globalfueleconomy.org/media/461049/me-and-wa_gfei_egypt_report_draft.pdf.

International Energy Agency (2017), "Global shifts in the energy system”, retrieved from https://www.iea.org

Knez, M., Jereb, B. and Obrecht, M. (2014), "Factors influencing the purchasing decisions of low emission cars: A study of Slovenia", Transportation Research Part D - Transport and Environment, https://doi.org/10.1016/J.TRD.2014.05.007.

Mostafa, A.R., Hegazi, A.H., El-Gayar, M.Sh. and Andersson, J.T. (2008), "Source characterization and the environmental impact of urban street dusts from Egypt based on hydrocarbon distributions", Fuel, 88, 95-104, https://doi.org/10.1016/j.fuel.2008.08.006.

Obrecht, M. and Denac, M. (2011), "Biogas - a sustainable energy source: new possibilities and measures for Slovenia", Journal of Energy Technology, 4(5), 1-10.

Obrecht, M. and Denac, M. (2018), "Forecast of Sustainable Energy Development in Slovenia", Journal of Energy Technology, 11(1), 57-68.

Slovenian Environment Agency (2019), “Air pollution”, retrieved from: http://www.arso.gov.si/en/soer/air_pollution.html.

Tawfik, F. (2018), "Cairo is world's second most polluted city: WHO”, retrieved from Egypt Independent: https://ww.egyptindependent.com/cairo-is-worlds-second-most-polluted-city-who/

*** (2018), “2018 Environmental Performance Index, Global metrics for the environment: Ranking country performance on high-priority environmental issues", Yale Center for Environmental Law \& Policy, Yale University, Center for International Earth Science Information Network, Columbia University. 


\title{
HORSE PAWS AS RAW MATERIAL FOR FUR INDUSTRY
}

\author{
VERA RADNAEVA ${ }^{1}$, DMITRY SHALBUEV ${ }^{1}$, NIKOLAY SOVETKIN ${ }^{1}$, KHURELSUKH $^{2}$ \\ GAANBAATAR $^{2}$, SOLONGO KHOSBAYAR ${ }^{2}$, SUVDA DASHDORJ ${ }^{2}$, VICTORIY ALEKHINA ${ }^{1}$ \\ ${ }^{1}$ East Siberia State University of Technology and Management, Ulan-Ude, Russia, \\ shalbuevd@mail.ru \\ ${ }^{2}$ Company Khorol Collection, Ulaanbaatar, Mongolia
}

\begin{abstract}
Horse skin is used for processing various types of skin. However, paws of horse skin are not used as fur raw material. Usually they are burned or sent to landfills and may cause infectious diseases. It is possible to minimize negative impact on the environment by converting this waste into fur raw material. In cold regions of Russia high fur boots made of cattle and deer paws are very popular. The aim of the research is to study the possibility of using paws of horse skin as raw material for fur industry. Processing of horse paws based on well-known leather and fur processing technologies lead to semi-finished product characterized by increased stiffness and uneven properties on different skin parts. Such semi-finished product was not suitable for high fur boots manufacture. The aim of the research is to work out a new technology and study chemical and physico-mechanical properties. In the work various treatment options for horse paws and their properties are investigated: moisture content, amount of minerals and chromium oxide, $\mathrm{pH}$ of the aqueous extract, tensile strength, elongation at a voltage of $10 \mathrm{MPa}$, and stiffness are determined. The possibility of transferring horse paws from municipal solid waste into fur raw material is shown.
\end{abstract}

Key words: horse paws, technology, properties

\section{INTRODUCTION}

The Mongolian horse is a native breed of Mongolian horses. It is assumed that the breed has remained practically unchanged since the time of Genghis Khan. The nomads living in the traditional Mongolian style contain 4,2 million animals, which (data from 2019) exceeds the population of the country. In Mongolia, horses live outdoors all year round, with temperatures ranging from $30^{\circ} \mathrm{C}\left(86^{\circ} \mathrm{F}\right)$ in summer to $-40^{\circ} \mathrm{C}\left(-40^{\circ} \mathrm{F}\right)$ in winter (https://ru.wikipedia.org/wiki/Монгольская_лошадь). They graze and look for food on their own, serve as riding and transport animals, and are used both for the daily work of nomads and at races (Figure 1).
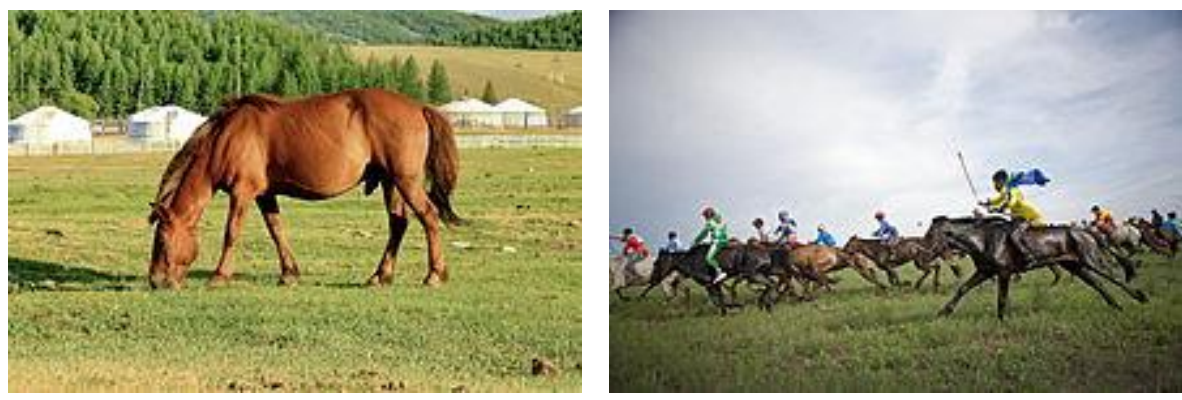

Figure 1. Mongolian horse (Equus ferus caballus)

In theory, the Mongolian horse is the basis for many other Asian horse breeds, including the Tuva, Akhal-Teke, Yunan, Japanese, and Jeju horse breeds. The national drink airag is produced from mare's milk. Some animals are slaughtered for meat. The 
skins of adult and semi-mature horses are considered leather raw materials. The skins of suckling foals and premature foals are classified as fur raw material. In contrast to the skins of cattle, horse skins are uneven in thickness (the front is thin, the khaz is thickened). The unevenness of horse skins in thickness is due to the fact that the forepart has a large number of sweat and sebaceous glands, rather thin fibers and bundles, and loose ligature. The significant difference between the forepart and the "khaz" causes the division of horse skins into parts, launching them into production in separate batches and processing them using various methods.

The border of dividing a horse hide into two parts is considered to be a hairline that runs perpendicular to the back line and is formed as a result of hair growth on the back in one direction and on the croup in the other direction (http://www.otkani.ru/leathercommodity/rawhide/6.html). In terms of histological structure, horse skins differ somewhat from cattle skins. The thickness of the epidermis is $2-3 \%$ of the total thickness of the dermis. The papillary layer of the dermis occupies over $30 \%$ of the entire thickness of the dermis and has a loose structure, since it contains numerous hair follicles. The reticular layer in horse skins is less developed than in cattle skins. Thin fibers form loose weaves over the entire area of the skin and only in the spiegel are they rather dense. The sweat and sebaceous glands are highly developed, and in the floors they form, as it were, a continuous layer, which reduces the strength of the attachment of the reticular and papillary layers in the skin.

In Russia (Sakha-Yakutia, Buryatia, Tyva, Kalmykia) and Mongolia, there is a traditional method of waste-free technology for processing horse products, including horse skins. Horse skins, as well as cattle skins, are used for the manufacture of various types of leather: "khaz" - for the production of sole leather, the forepart - for the manufacture of upper leather and Russia calf. They have a beautiful and delicate graining. The pullet hide, that is, the skins of young horses weighing from 5 to $10 \mathrm{~kg}$, with an area of 120-200 $\mathrm{dm}^{2}$, are mainly directed to obtain fur products (foal-sklizok) or on chrome-tanned and lacquered leather (foal). However, horse paws are not processed. Usually they are burned or sent to landfills, where, as a result of the combined action of abiotic and biotic factors, they undergo rotting. Putrefactive processes create conditions for the development of pathogenic microflora, which can contribute to the emergence of infectious diseases. It is possible to minimize the negative impact on the environment by processing this waste into a fur semi-finished product that can be used for sewing fur shoes (Shalbuev, 2018).

The production of paws from the skins of Mongolian horses according to the wellknown technologies of processing paws from the skins of cattle with the preservation of the hairline led to the production of a semi-finished product, characterized by increased rigidity, uneven properties of the topographic areas. Such a semi-finished product was not suitable for the manufacture of fur boots. One of the reasons that impede the use of horse paws is the lack of studies on the influence of processing parameters on the chemical and physical-mechanical properties of the leather tissue of the paws.

The aim of this work is to study the possibility of using horse paws as raw materials for the fur industry. To achieve this goal, it is necessary to investigate the influence of technological parameters on the chemical and physical-mechanical properties of horse paws.

https://doi.org/10.24264/icams-2020.III.14 


\section{OBJECTS AND METHODS}

The main objects of the study were undressed leather, dry salting skin and dressed paws from the skins of Mongolian horses. Horse paws preserved by dry salting were processed according to the scheme: soaking - pickling - neutralization - tanning - drying - finishing.

Enzymes are one of the well-known preparations allow to give softness to the skin tissue. It was of interest to study the effect of enzymes used at the stage of pickling paws from the skins of Mongolian horses on the physical and mechanical properties of the cut paws in order to obtain soft skin tissue. The following enzymes with proteolytic properties were used as enzyme preparations:

Enzym PKL from Lederplus (Spain) is an acidic (moderate) enzyme for processing fur, which is active at $\mathrm{pH}$ 3,0-6,0. Enzyme activity depends on the $\mathrm{pH}$ of the medium. It is white powder. It has a high penetrating power into the structure of leather tissue. It allows you to combine softening and pickling in one operation, dissolves and flushes out all ballast proteins from the leather tissue, providing faster penetration of chemical materials into the structure of the leather tissue (Sovenkin and Suleymanov, 2016).

Enzymacid ESP (Spain) stabilized cross-linked enzyme molecules have increased stability in an acidic environment (www.ericsonlab.ru/catalog/face_care/enzymacid/).

Betazim TK (Russia) has a high penetrating ability and facilitates the cleaning of the collagen structure from carbohydrates, lipids and soluble proteins, contributing to better penetration and distribution of acid throughout the entire area of the skins. It can work in an acidic environment in the $\mathrm{pH}$ range $2,8-3,0$. Using it in a pickle with a flow rate of $1-2 \mathrm{~g} / \mathrm{dm}^{3}$ makes it possible to increase the softness and plasticity of the skins, significantly reduce their weight, and also improve the uniformity of tanning (https://betachem.ru/pickling/betazim-tk).

The treatment of the paws of Mongolian horses was carried out according to five variants, differing in the composition of the pickle solution. Pickel solution in all variants contained sodium chloride in an amount of $50 \mathrm{~g} / \mathrm{dm}^{3}$, formic acid $5 \mathrm{~cm}^{3} / \mathrm{dm}^{3}$, Betol H-3 $-0,5 \mathrm{~cm}^{3} / \mathrm{dm}^{3}$. As an enzyme preparation, we used: an acidic enzyme for the treatment of fur brand Enzym PKL from Lederplus (Spain) - variant 1, Enzymacid ESP (Spain) - variant 2, Betazim TK (Russia) - variant 3. Variants 4 and 5 did not contain enzymes, but only acids of a different chemical nature: formic acid $5-10 \mathrm{~cm}^{3} / \mathrm{dm}^{3}$ option 4, formic acid $\left(5-10 \mathrm{~cm}^{3} / \mathrm{dm}^{3}\right)$ and sulfuric acid - option 5. It was assumed that enzymes, having a high penetrating ability, will help cleanse the collagen structure from carbohydrates, lipids and soluble proteins, thereby improving the penetration and distribution of acid over the entire area of the hides. Tanning was carried out with chromium (III) compounds.

Regulatory documents according to which the quality of leather fabric of horse paws was assessed are presented in GOST 4661-76 "Dressed fur sheepskins" (GOST 4661-76 - technical specification, 2002). The obtained values, characterizing the quality of the leather tissue of the paws of Mongolian horses, were compared with the normative data of TS - 205 RSFSR 17.203 - 79 "Camus dressed". The choice of these regulatory documents is due to the lack of regulated methods for determining the chemical and physical-mechanical properties of dressed horse paws. The rigidity of the leather tissue of the horse paws was determined according to the method developed at the Department of "Leather and Fur Technology Water Resources and Commodity Research" ESSUTM (Sovetkin and Dumnov, 1988). 


\section{RESULTS AND DISCUSSION}

The conclusion about the suitability of the horse paws made from the skins of Mongolian horses for sewing winter boots of high fur boots is made on the basis of a comprehensive analysis of physical, mechanical and chemical properties, as well as trial sewing of high fur boots. It was noted above about the increased rigidity of the leather tissue of the dressed horse paws when using the technique for processing paws from cattle skins.

Table 1 shows the indicators of the chemical composition of the paws from the skins of Mongolian horses of the different variants.

Table 1. Indicators of the properties of paws from the skins of Mongolian horses of different variants

\begin{tabular}{|c|c|c|c|c|c|c|}
\hline \multirow[t]{2}{*}{ №/№ } & \multirow[t]{2}{*}{ Variant } & \multicolumn{3}{|c|}{$\begin{array}{c}\text { Mass fraction, \% (absolutely dry } \\
\text { substance) }\end{array}$} & \multirow{2}{*}{$\begin{array}{c}\mathrm{pH} \\
\text { water } \\
\text { extract }\end{array}$} & \multirow[t]{2}{*}{$\begin{array}{l}\text { Shrinkage, } \\
{ }^{\circ} \mathrm{C}\end{array}$} \\
\hline & & moisture & $\begin{array}{c}\text { mineral } \\
\text { substances }\end{array}$ & $\begin{array}{l}\text { chromium } \\
\text { oxide }\end{array}$ & & \\
\hline 1 & Enzym PKL & $13,3 \pm 0,1$ & $14,7 \pm 0,2$ & $4,5 \pm 0,1$ & $3,3 \pm 0,1$ & $93 \pm 4$ \\
\hline 2 & $\begin{array}{l}\text { Enzymacid } \\
\text { ESP }\end{array}$ & $12,3 \pm 0,2$ & $14,8 \pm 0,3$ & $4,8 \pm 0,1$ & $3,2 \pm 0,1$ & $96 \pm 2$ \\
\hline 3 & Betazim TK & $12,4 \pm 0,2$ & $14,5 \pm 0,1$ & $4,7 \pm 0,1$ & $3,1 \pm 0,1$ & $97 \pm 1$ \\
\hline 4 & Formic Acid & $12,2 \pm 0,1$ & $14,9 \pm 2,2$ & $3,9 \pm 0,1$ & $2,9 \pm 0,1$ & $98 \pm 1$ \\
\hline 5 & $\begin{array}{c}\text { Formic Acid } \\
\text { and Sulfuric } \\
\text { Acid }\end{array}$ & $12,5 \pm 0,3$ & $15,7 \pm 0,3$ & $4,3 \pm 0,2$ & $3,1 \pm 0,1$ & $97 \pm 3$ \\
\hline $\begin{array}{l}\text { Normative } \\
\text { document }\end{array}$ & $\begin{array}{c}\text { TS - } 205 \\
\text { RSFSR } \\
17.203-79 \\
\text { "Camus } \\
\text { dressed" }\end{array}$ & $10-14$ & None & None & $3,5-7,0$ & $>63$ \\
\hline
\end{tabular}

The choice of TS - 205 RSFSR 17.203 - 79 "Camus dressed" as a normative document is due to the fact that at the moment in Russia this document is the only one that regulates these indicators. As can be seen from Table 1, the moisture content of the dressed paws is within the limits allowed by TS 205 RSFSR 17.203 -79 It should be noted that the moisture content of all leather and fur products is usually regulated in the range of $12-14 \%$ or $12-16 \%$, therefore, according to this indicator, the paws made from horse skins correspond to the accepted standards.

Analysis of the data obtained showed that the composition of the pickle bath does not have a significant effect on the content of minerals and chromium oxide in leather tissue, while the mass fraction of chromium oxide is in the range of $3,9-4,5 \%$, and mineral substances $-14,5-15,7 \%$. The data in Table 1 show the high heat resistance of the samples of the paws for almost all treatment options $\left(93-98^{\circ} \mathrm{C}\right)$, which indicates a strong bond of chromium compounds with the functional groups of the protein. It should be noted that for «kamus» made from reindeer skins, the lower limit of heat resistance of leather fabric is $63^{\circ} \mathrm{C}$, which is considered acceptable for sewing winter shoes.

Therefore, despite the lack of normative data on the mass fraction of chromium oxide and, knowing about the known correlation between this indicator and the shrinkage temperature, the value of this indicator can be considered sufficient to obtain 
high-quality products. From the data in Table 1 , it can be seen that the $\mathrm{pH}$ of the aqueous extract for the prototypes is somewhat lower than the regulated value. The lowest $\mathrm{pH}$ was observed for paws pickled with formic acid. The obtained data on the $\mathrm{pH}$ of the aqueous extract indicate the need to correct the technological parameters at the tanning-neutralization.

The results presented in Table 1 showed no significant difference in chemical properties for horse paws isolated using different enzymes, formic acid and its mixture with sulfuric.

Table 2 shows the results of the influence of the composition of the pickle on the physico-mechanical properties of the paws from the skins of Mongolian horses.

Table 2. Results of physical and mechanical tests of Mongolian horse paws of different variants

\begin{tabular}{|c|c|c|c|c|c|c|}
\hline \multirow[b]{2}{*}{ Data } & \multicolumn{5}{|c|}{ Variant } & \multirow{2}{*}{$\begin{array}{l}\text { TS - } 205 \\
\text { RSFSR } \\
17.203-79 \\
\text { "Camus } \\
\text { dressed" }\end{array}$} \\
\hline & $\begin{array}{l}\text { Enzym } \\
\text { PKL }\end{array}$ & $\begin{array}{l}\text { Enzymacid } \\
\text { ESP }\end{array}$ & $\begin{array}{c}\text { Betazim } \\
\text { TK }\end{array}$ & $\begin{array}{c}\text { Formic } \\
\text { acid }\end{array}$ & $\begin{array}{l}\text { Formic } \\
\text { Acid and } \\
\text { Sulfuric } \\
\text { Acid }\end{array}$ & \\
\hline Stiffness, H & 1,8 & 1,8 & 1,7 & 1,8 & 1,6 & - \\
\hline $\begin{array}{l}\text { Elongation at a stress } \\
\text { of } 10 \mathrm{MPa}, \%\end{array}$ & 67,2 & 60,7 & 64,4 & 67,2 & 65,3 & - \\
\hline $\begin{array}{l}\text { Tensile strength, } \\
\mathrm{MPa}\end{array}$ & 17,8 & 19,6 & 18,7 & 17,8 & 23,3 & - \\
\hline $\begin{array}{l}\text { Elongation at break, } \\
\%\end{array}$ & 93,0 & 89,0 & 90,7 & 95,6 & 96,3 & - \\
\hline
\end{tabular}

The results of physical and mechanical tests showed that the paws were distinguished by high strength - tensile strength ranged from 17,8 to $23,3 \mathrm{MPa}$, elongation at break was $89,0-96,3 \%$, elongation at a stress of $10 \mathrm{MPa} 64,4-67,2 \%$. At the same time, it should be noted that the rigidity of all samples was practically the same. The organoleptic assessment of the stiffness of the paws showed the need to improve the method for determining the stiffness.

Table 3 shows the data of organoleptic evaluation of paws horse of the different variants. A score of 1 was given to samples with the minimum stiffness, and a score of 5 was the samples with the maximum stiffness.

Table 3. Organoleptic evaluation of horse paws of the different variants

\begin{tabular}{ccc}
\hline Variant & Score & Stiffness, H \\
\hline 1 & 1 & 1,76 \\
2 & 3 & 2,79 \\
3 & 2 & 2,29 \\
4 & 4 & 3,02 \\
\hline
\end{tabular}

Comparison of the results of the organoleptic evaluation of the cut paws from the skins of Mongolian horses with the stiffness indicators determined on the device showed a correlation relationship - the correlation coefficient is 0,98 .

Analyzing the results of physical and mechanical tests and the chemical properties of the cut paws from the skins of Mongolian horses, it was concluded that they can be used for sewing winter shoes (Figure 2). 

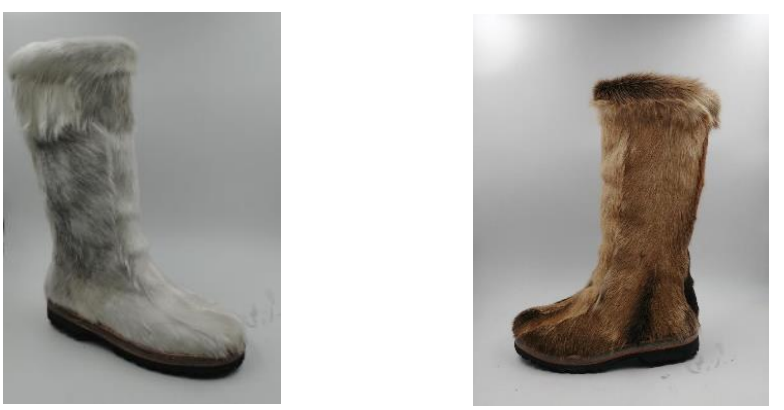

Figure 2. Winter boots from paws of the Mongolian horse

Thus, the new fur material obtained from the paws of the skins of Mongolian horses had optimal functional and technological properties, was soft and flexible, which makes it possible to recommend it for "untov" - winter fur boots.

\section{CONCLUSION}

1. The possibility of using horse paws as a raw material for the fur industry is shown.

2. It was revealed that the chemical and physical-mechanical properties of the paws from the skins of Mongolian horses have optimal functional and technological properties, which determines their suitability and manufacturability when sewing winter high fur boots.

3. It has been established that dressed paws from the skins of Mongolian horses are a new material for sewing winter shoes, and the raw materials can be transferred from solid household waste to the category of raw materials for fur industry.

\section{REFERENCES}

GOST 4661-76 (2002), "Dressed fur sheepskins. Technical specifications." - M.: Publishing house of standards, $11 \mathrm{p}$.

Shalbuev, Dm., Tumurova, T., Radnaeva, V. and Manieva, V. (2018), “On destruction of fatty substances by prokaryotic organisms isolated from fur production waste water.”, International Journal of Civil Engineering and Technology (IJCIET), 9(11), 1918-1926

Sovenkin, N.V. and Suleymanov, R.G. (2016), "Study the influence of enzymes in the pickle on consumer properties sheepskin", in Proceeding of the XII International scientific and practical conference: Leather and fur in the XXI century: technology, quality, ecology, education. - Ulan-Ude: ESSUTM Publishing House, P. 397-401.

Sovetkin, N.V. and Dumnov, V.S. (1988), RU Patent 1395983, IPC GO1N 3/42. Publ. 05/15/1988 "Device for determining the stiffness of elastic materials".

*** https://betachem.ru/pickling/betazim-tk/ (Date 08/25/2020)

*** https://ru.wikipedia.org/wiki/Монгольская_лошадь (Date 08/19/2020) Mongolian Horse URL

*** https://www.ericsonlab.ru/catalog/face_care/enzymacid/ (Date 08/25/2020)

*** http://www.otkani.ru/leathercommodity/ rawhide/6.html (Date 08/19/2020) Characteristics of raw hides. Use of horse hides. 
ICAMS $2020-8^{\text {th }}$ International Conference on Advanced Materials and Systems

\title{
INNOVATIVE ECOLOGICAL PROCESSES WITH RECOVERY OF CHEMICALS AND WATER FOR REUSE IN LEATHER SECTOR - AN ECONOMICAL AND SUSTAINABLE APPROACH
}

\author{
SENGODA GOUNDER RAJAMANI
}

Chairman, Asian International Union of Environment Commission (AIUE), No.18/45, South Beach Avenue, MRC Nagar, Chennai - 600 028, India, Mobile: + 91 - 98400 63210. E-mail: dr.s.rajamani@gmail.com

\begin{abstract}
The effluent discharged from conventional process in textile dyeing and tanneries are unable to meet some of the discharge parameters such as Total Dissolved Solids (TDS) in the existing physiochemical \& biological treatment units. In addition to TDS management the control of volatile solids in hazardous category sludge is also becoming a necessity. To overcome these challenges faced by tanneries in the world leather, improved cleaner production, segregation of saline soak liquor and separate treatment, modified chrome recovery system and recovery of chromium \& sodium chloride salt in the form of powder and quality water with TDS less than $500 \mathrm{mg} / \mathrm{l}$ for reuse by tanneries have been developed for field application. Physiochemical treatment is converted into total biological treatment with sulphide oxidation using enzyme and biomass which resulted in 50\% reduction in sludge generation. The secondary treated effluent and supernatant from chrome recovery system are processed with membrane units for recovery of high saline stream and quality salt for reuse in pickling process and other industrial requirement. These developments are being implemented at field level for cluster of nearly 400 tanneries in India which is first of its kind in the world.
\end{abstract}

Keywords: Cleaner production, Water \& Chrome recovery, TDS control

\section{INTRODUCTION}

The tanneries in World Leather Sector process about 17 million tones of hides \& skins per year. Only less than $20 \%$ of fresh hides and skins are processed without applying salt and more than 8-10million tones of salt mainly in the form of sodium chloride is applied for curing. They are transported, stored and processed in a period of 2-6 months. The entire salt applied is discharged as waste in the effluent as dissolved solids, causes environmental challenges due to increase in salinity, depletion of quality water resources and transfer of non-degradable pollutants such as salt from one region to other region in the world.

With a view address the environmental challenges, technological developments such as (i) Advanced process control and cleaner production, (ii) Segregation of Spent chrome stream and adoption of improved chrome recovery system by recovering chromium in the form of cake and power, (iii) Segregation of saline stream with high TDS around 20000-30000mg/L from soak liquor, separate treatment and recovery of quality salt and water for reuse by adopting ZLD system, (iv) Improved biological treatment system with mild chemical usage for reduced sludge generation, (iv) Advanced tertiary treatment system for the application of Reverse Osmosis (RO) system for recovery of water. Recent applied $R \& D$ on the sustainable development in cleaner leather production, environmental protection techniques with focus on saving of energy and chemical by converting the physiochemical treatment into total biological treatment, water-recovery for reuse, quality salt recovery for reuse, etc. are explained in this paper. 
Innovative Ecological Processes with Recovery of Chemicals and Water for Reuse in Leather Sector - An Economical and Sustainable Approach

\section{SEPARATE TREAMTENT OF SOAK STREAM FOR RECOVERY OF QUALITY SALT AND WATER}

Due to inherent quality of industrial wastewater such as textile dyeing units, tanneries etc., the conventional treatment plants are unable to meet the prescribed TDS level of 2100 $\mathrm{mg} / \mathrm{l}$ in the treated effluent. In addition to TDS management the control of volatile solids in hazardous category sludge is also becoming a necessity. For control of salinity, sludge and viable management of TDS with recovery of quality water and salt from wastewater, the required treatment steps are (i) Cleaner production and other viable process control in tanneries, (ii) Segregation of saline soak liquor, spent chrome liquor for separate treatment, (iii) Improved two stage biological treatment systems with better efficiency in BOD and COD removal, (iv) Minimum usage of chemicals in the treatment process and reduction in sludge generation, (v) Reduction in TDS level in the mixed stream and (vi) Tertiary treatment of the low saline mixed stream and integration of treated tannery effluent with treated domestic sewage wherever feasible for TDS management.

The availability of domestic sewage is limited in many locations for dilution/mixing with treated tannery effluent for TDS management. The viable plan of segregation of soak liquor, separate treatment and recovery of quality salt will be helpful in reduce the TDS level in the mixed stream and scope for adoption of dilution / mixing with available treated domestic sewage.

The segregated soak liquor generated from presoaking and main soaking is taken to the CETPs through separate pipe line and after primary and secondary treatment units, membrane system is adopted for recovery of water and quality of saline stream for reuse in pickling. The balance treated saline stream is evaporated in the multiple effect evaporator and quality salt ( $98 \%$ purity) is recovered for reuse without any difficulty. In addition to recovery and reuse of quality water by the industry, the additional benefits are savings in chemical usage in the tanning process and reduction in pollution load in the effluent.

The segregated chrome stream is taken for Centralized Chrome Recovery System (CCRS) for recovery of chromium in the form of chromium cake. In the improved chrome recovery system, the time required in the chrome recovery process is reduced from $16 \mathrm{hrs}$ to less than $8 \mathrm{hrs}$. By avoiding the soak stream and supernatant from the CCRS to the main composite stream, the TDS level will be reduced from the level of about $15000 \mathrm{mg} / \mathrm{l}$ to $8000 \mathrm{mg} / \mathrm{l}$.

\section{IMPROVED COMMON CHORME RECOVERY SYSTEM}

The basic concept, design and development of improved Common Chrome Recovery System (CCRS) comprises of the following:

- Segregation of spent chrome liquor and collection in separate tank, transportation through tankers mounted on trucks with GPS and vacuum pumps

- Separate collection tanks with screen chamber near CCRS for discharge of spent chrome liquor from the tankers

- Transfer of spent chrome liquor from the collection tank by pumping to the main reactor for chrome precipitation by using suitable Alkali chemical dosing

- Decanting of supernatant, clarification and distribute in tanneries for pickling with alternative option of recovery of water using UF\&RO units installed for saline soak treatment system and recovery of reusable salt using the MEE system

https://doi.org/10.24264/icams-2020.III.15 
ICAMS $2020-8^{\text {th }}$ International Conference on Advanced Materials and Systems

- Dewatering of Chromium Hydroxide slurry and making it in the form of cake and powder. Further process of making chromium cake/powder in to Basic Chromium Sulphate (BCS) for reuse in tanneries.

The process flow diagram of segregation and collection of three streams viz. (i) Saline Soak liquor, (ii) Spent Chrome liquor and (ii) Composite stream with low TDS and separate treatment is shown below:

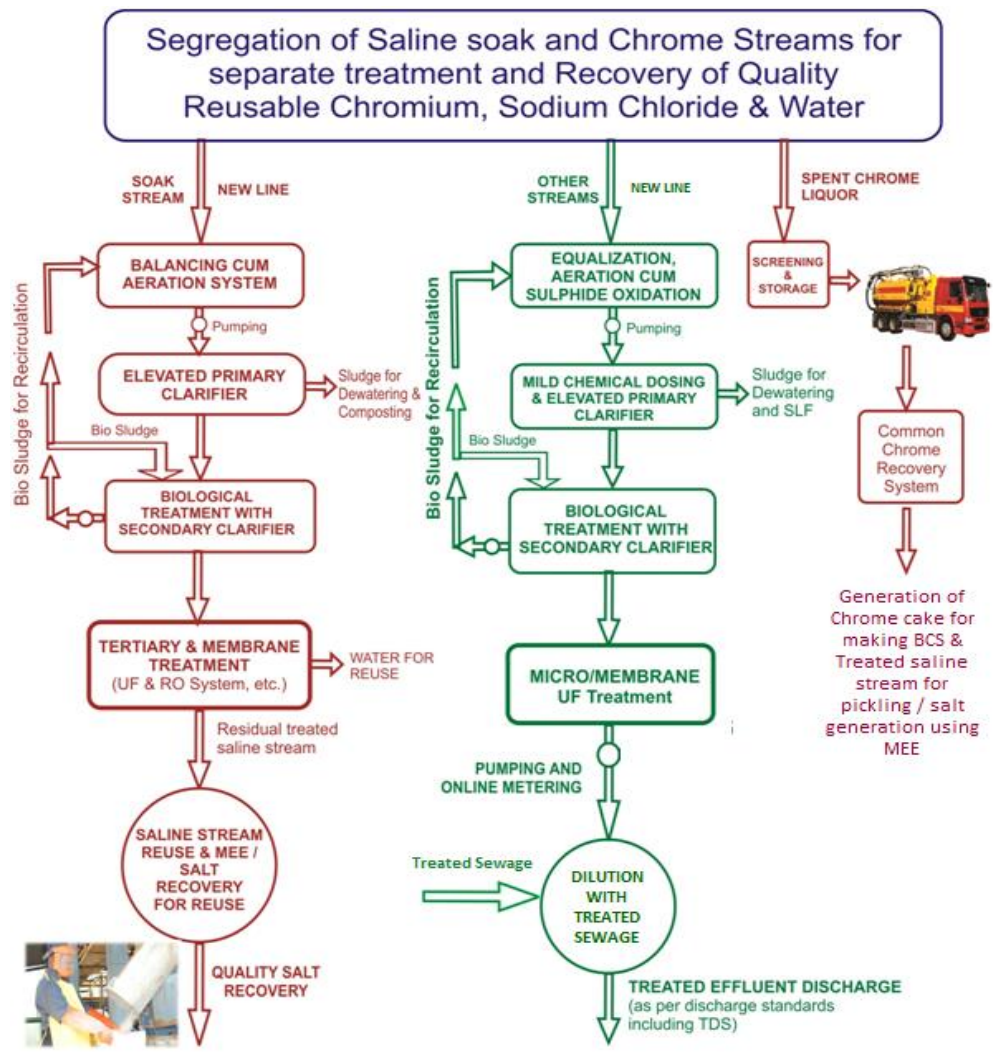

Figure 1. Treatment process of Soak saline, Spent chrome and Composite low saline streams

\section{Chrome Precipitation in Main Reactor Using Suitable Alkali Chemical}

The equalized spent chrome liquor from the collection tank is pumped to the Reaction tank, provided with a slow speed agitator. The alkali solution shall be prepared in the Alkali preparation tank by mixing alkali and water. The Alkali dosing feed pump shall draw the Alkali solution from the alkali preparation tank to the Reaction tank.

The alkali dosage is regulated by the $\mathrm{pH}$ analyzer/transmitter with integrated controller installed in the Reaction tank. Depending on the $\mathrm{pH}$ value in the reaction tank the Alkali solution feed pump speed will be varied to control the reaction. The agitator in the Reaction tank will ensure the proper mixing of the spent chrome liquor and the alkali solution. The chromium present in the chrome liquor will be quantitatively precipitated as chromium hydroxide by increasing the $\mathrm{pH}$.

The Chemical reaction of a typical chrome recovery process is:

https://doi.org/10.24264/icams-2020.III.15 
Innovative Ecological Processes with Recovery of Chemicals and Water for Reuse in Leather Sector - An Economical and Sustainable Approach

$\mathrm{Cr} 2(\mathrm{SO} 4) 3+6 \mathrm{NaOH} \rightarrow 2 \mathrm{Cr}(\mathrm{OH}) 3+3 \mathrm{Na} 2 \mathrm{SO} 4$

\section{Separation of Chrome Slurry and Supernatant from the Main Reactor}

In about 3-4 hours the chromium precipitates and settles as chromium hydroxide slurry in the bottom of the main reactor. The supernatants account for about 70 to $80 \%$ of the volume in the Reaction tank. After settling the chrome slurry has to be separated from supernatant by decanting the supernatant by providing proper arrangement. Chrome slurry from the bottom of the reaction tank shall be discharged by gravity into the collection tank in the form of chromium hydroxide.

\section{Dewatering of Chromium Hydroxide Slurry \& Making it in the Form of Cake}

A series of Filter press with feed tank shall be provided to dewater the chromium hydroxide slurry and convert into chromium hydroxide cake. The solid concentration of the chromium hydroxide cake shall be about $30 \%$. An agitator shall be provided in the each of the filter press feed tank for proper feed in to the filter press. The Filter press feed pump shall draw the chrome hydroxide precipitate from the filter press feed tank and pump it to the filter press. The water passes through the filter clothes and chromium hydroxide is retained. This shall be repeated till the filtration cycle is completed. The filtrate is collected in the supernatant collection tank and then taken for further process and reuse.

At the end of the filtration cycle, the filter press is opened and chromium hydroxide cake is collected in a separate tray, shifted to storage yard to be provided adjacent to CCRS and kept for further dry and stored in anticorrosive polythene bags. The chromium hydroxide cake is further processed by authorized vendors / BCS manufacturers and reused in the member industries for recycle.

The overall schematic diagram of the improved chrome recovery system is given below:

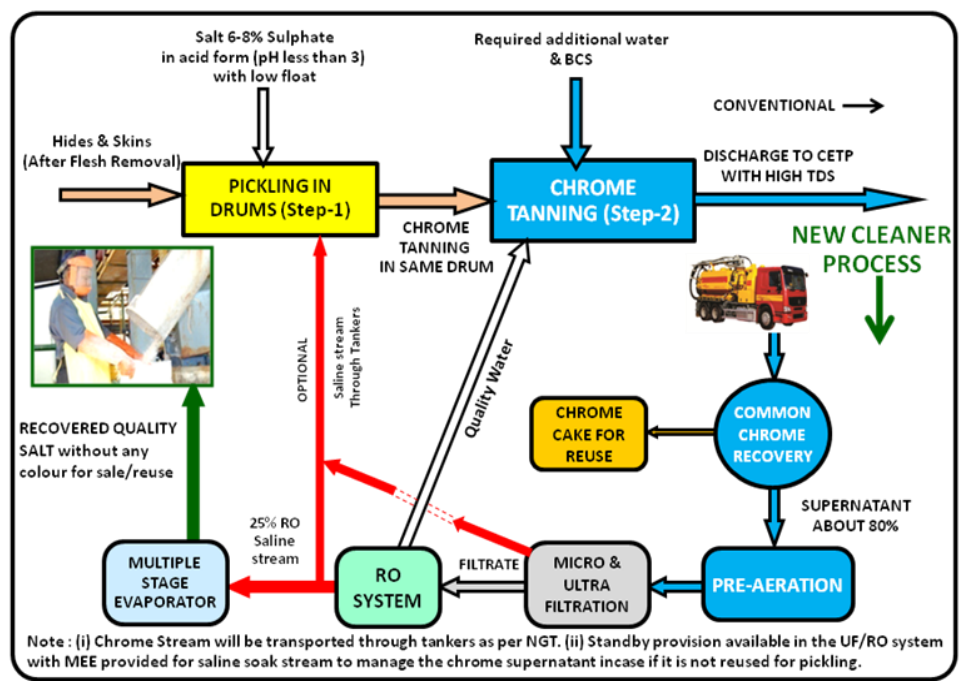

Figure 2. Chrome Stream Treatment, Recovery \& Reuse 
ICAMS $2020-8^{\text {th }}$ International Conference on Advanced Materials and Systems

Integration of Equalization cum Mixing System with Biological Treatment for Sulphide Oxidation

The effluent is collected in equalization cum mixing system, pumped to the primary clarifier, mixed with high dosing of chemicals such as lime alum, etc. The conventional system adopted in most of the CETPs in India could not reduce the sulphide level in the physiochemical treatment and the sludge accumulation in the equalization tank is one of the major problems. The COD reduction to the prescribed level (i.e. 250mg/l) in the final treated effluent could not be met by some of the CETPs adopting conventional physiochemical and biological treatment. The performances of the aerobic biological treatment system with limited detention time are not satisfactory and unable to produce required quality effluent.

With a view to oxidize the sulphide present in the effluent, control the sludge settling in the equalization tank and to minimize the chemical usage the equalization system has been upgraded with increased detention time, increased depth and usage of new type of aspirators integrated with compressor. The residual excess biosludge from secondary clarifier is pumped to the equalization tank which is helpful in biological oxidation process and to reduce the chemical dosage in the first stage clarifier.

The primary clarifier units are also upgraded by providing elevated clarifiers with minimum required chemical dosing. This improved system is performing better in terms of sludge settling, withdrawal and dewatering.

The improved aeration system with jet aspirator has been successfully adopted in many CETPs in Tamilnadu and proposed to be implemented in more CETPs. The sustainable alternatives to total ZLD system for single combined stream have been developed and are being introduced in upgradation of CETPs in Uttar Pradesh and other States. It is also estimated that nearly $80 \%$ capacity of the wastewater from Indian Leather Sector will be treated by adopting cleaner technologies, segregation of streams and separate treatment, integration with treated domestic sewage, etc. In this circumstance for long term sustainability of the CETPs which adopted ZLD for single combined streams, the concept of separate treatment of saline streams with recovery of quality reusable salt, cleaner productions, etc. may have to be followed. UNIDO in its recent technical publications on environment and effluent treatment for World Leather sector clarifies the limitations of ZLD system and emphasize the segregated stream treatment aspects.

\section{CONCLUSION}

The conventional effluent treatment systems are being upgraded by segregating the saline soak stream with separate treatment, adoption of UF \& RO and Multiple Effect Evaporators (MEE) with recovery of quality salt for reuse. About $200 \mathrm{~kg}$ of quality salt (sodium chloride) is recovered from the effluent discharged during the process of each $\&$ every tone of hides \& skins. The physiochemical treatment is converted into total biological treatment system to reduce sludge generation by $50 \%$, achieving the pollution control discharge standards and clarity in treated effluent. Upgradation of CETPs with Improved Cleaner Production Process, Centralized Chrome Recovery and Reuse systems, integrated treatment with treated domestic sewage for sustainable TDS management with financial support from National and International organization in India and other countries. These technological developments and upgradation of CETPs

https://doi.org/10.24264/icams-2020.III.15 
Innovative Ecological Processes with Recovery of Chemicals and Water for Reuse in Leather Sector - An Economical and Sustainable Approach

are being implemented in many locations covering more than 700 tanneries in India with financial outlay of more than 150 million US dollars.

\section{Acknowledgements}

Contributions of National Mission for Clean Ganga (NMCG), Department for Promotion of Industry and Internal Trade (DPIIT), National Green Tribunal (NGT), Banthar Industrial Pollution Control Company (BIPCC), Unnao Tanneries Pollution Control Company (UTPCC), IL\&FS Cluster Development Company, Asian International Union Environment (AIUE) Commission, Asian International Forum and others commission members from various countries, IULTCS, UNIDO, European Union including Italy, Spain, Netherlands and other Countries such as China, Romania, Turkey and Russian Federation, New Zealand are acknowledged. Leather Industry Associations and Common Effluent Treatment Plants (CETP) specifically Pallavaram, Dindigul, Madhavaram, Jajmau CETPs in India are acknowledged.

\section{REFERENCES}

Rajamani, S. (2015), "Novel Industrial Wastewater Treatment Integrated with Recovery of Water and Salt under Zero Liquid Discharge Concept", The Pacific Basin Consortium for Environment and Health, University of Indonesia, City of Depok, West Java, https://doi.org/10.1515/reveh-2016-0006.

Rajamani, S. (2016), "Innovative Environmental Technologies including Water Recovery for Reuse from Tannery and Industrial Wastewater - Indian and Asian Scenario", Proceedings of the 6th International Conference on Advanced Materials and Systems (ICAMS 2016), Bucharest, Romania, https://doi.org/10.24264/icams-2016.IV.13.

Rajamani, S. (2018), "Sustainable Environmental Technologies Integrated with Cleaner Production - Recent developments in World Leather Sector", XIVth International Scientific-Practical Conference, Ulan-Ude, Russia.

Rajamani, S. (2018), "Viable Environmental Technologies integrated with Cleaner Production - Sustainable Options for Global Leather Sector", Innovative solutions for sustainable development of textile and leather industry - International Science Conference 2018, Oradea, Romania

Rajamani, S. (2019), "Innovative Technologies on Cleaner Production and Waste Management in Tanneries", International Leather Engineering Congress Innovative Aspects for Leather Industry, Izmir, Turkey.

Rajamani, S. (2020), "Sustainable ZLD System by Adopting Centralized Treatment of Segregated Streams for Recovery of Reusable Quality Chemical, Salt \& Water - First of its kind in India \& Asia”, Leather News India Journal. 


\title{
THEORETICAL AND PRACTICAL ASPECTS OF THE DESIGN PHASE FOR
} A SINGLE SKIN TEXTILE WING

\author{
ADRIAN SALISTEAN, CARMEN MIHAI, DOINA TOMA, SABINA OLARU \\ INCDTP - National R\&D Institute for Textile and Leather, Lucretiu Patrascanu No.16, Bucharest \\ -Romania,office@incdtp.ro
}

\begin{abstract}
This paper depicts the early phase in the research development for an integrated support system tailored for emergency response actions and remote sensing. The support system is envisioned as an integrated Unmanned Aerial System (UAS) system that consists of one or more ultralight multifunctional aerial units with a configuration that can be adapted to the nature of the intervention: monitoring, mapping, observation and logistics etc. These aerial units comprise of para-motor type UAVs that use textile paraglider wings of a special design. The paper summarizes the basic materials used in the construction of parachutes, as well as it depicts the design phase for the main material used on the wing construction. Starting from wing airfoil and materials selection, a design phase is ongoing for a single sail paraglider wing that can meet the operational demands for emergency response situations. The wing is designed mainly to have an easy handling and to have a predictable deployment at all times. The entire system and the aerial units are designed with increased modularity in order to be tailored for specific operational requirements of the intervention. A numerical model is under development and rigorous testing to validate the theoretical aspects and the design choices.
\end{abstract}

Keywords: Unmanned Aerial System (UAS), Single Sail Paraglider, Technical Textiles.

\section{INTRODUCTION}

The laws of mechanics and aerodynamics apply to the performance and stress analysis of parachute systems. However, the textile fabrics used in parachute construction have distinctly different mechanical and environmental characteristics than metals or composites.

This paper depicts the early phase in the research development for an integrated support system tailored for emergency response actions and remote sensing. In this phase we try to develop a fabric that is tailored for use in the manufacturing process of a paraglider type wing design (Knache, 1992) that utilizes a single skin construction (Poynter, 1984) and solid reinforcements in the sewing for shape stability.

In order to achieve this we used as a baseline several commercial fabrics and tried to determine the best combination of yarn, weave and finishing method in order to best suite our paraglider wing. Please keep in mind that this is a preliminary work and is subject to change if the prototype performances will not fall within the projected limits.

\section{MATERIALS AND METHODS}

In order to establish a baseline for the fabric characteristics several readily available fabrics were analysed. The fabrics used in the testing were selected so they cover a wide array of parachute types.

Therefore we selected as material one (S1), a fabric commonly used in paraglider manufacturing. This fabric is a rather heavy fabric having polyurethane and silicone coating for UV protection.

The second material (S2) is a fabric used in most of the Ram-Air parachutes available today. It's a light fabric with polyurethane coating for zero air permeability.

The third material (S3) is a fabric with similar structure as S2 but without polyurethane coating. This fabric is only calendered and it is commonly referred to as 
F111 type fabric. This type of fabric has some air permeability therefore is mainly used in reserve ram-air parachutes or partially on the intrados side of main parachutes.

Testing of the tear resistance of the samples was done on the Tinius Olsen Dynamometer H5KT dynamometer (Figure 1). The device is designed to test a wide range of materials (yarns, fabrics, leather) for traction, flexion, and assembly strength (made by sewing, thermofusion, etc.).

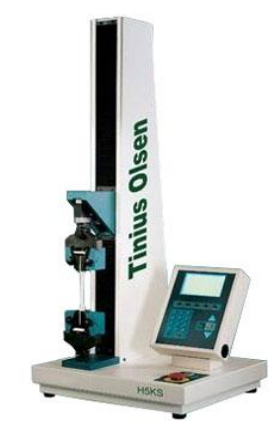

Figure 1. H5KT dynamometer

Further on we extracted yarns from the fabrics in order to determine the yarn characteristics. The values of the structural parameters of the fabrics (air permeability, mass, thickness, etc.) were used in conjunction with the extracted yarn test results to determine the multivariate regression equations in which the independent variables were considered the breaking strengths in warp and weft (Figure 2). In this figure on $\mathrm{x}$-axis we have the displacement of the clamping device, in $\mathrm{mm}$. We notice a very inconsistent reading, as if the yarn is partially slipping, compared with the clean regular Nylon 6.6 yarn. We suspect this to be because of the residual polyurethane coating present on the extracted yarns. S3 sample, that was not coated, had smaller reading spikes. A statistical smoothing of the readings puts the breaking strength of the extracted yarns roughly on a value that is double than that of the regular Nylon 6.6 yarn. This is the tell-tale sign that we are dealing with HT Nylon 6.6 yarns. At the time of the testing we did not have stocked HT Nylon 6.6 to make a direct comparison.
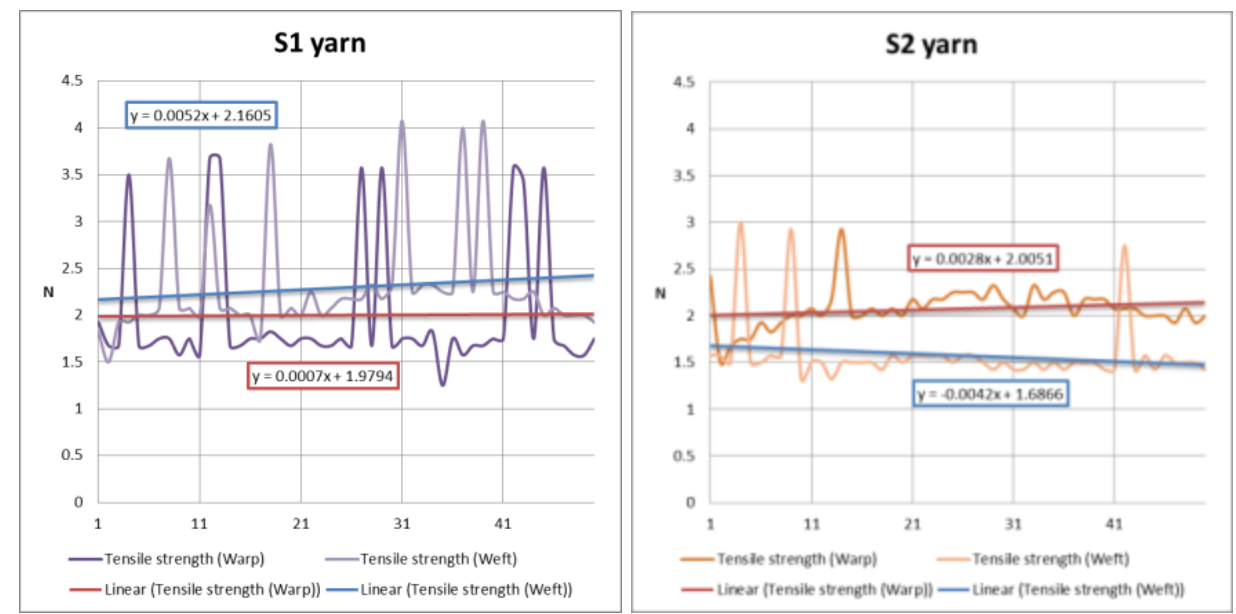

https://doi.org/10.24264/icams-2020.III.16 

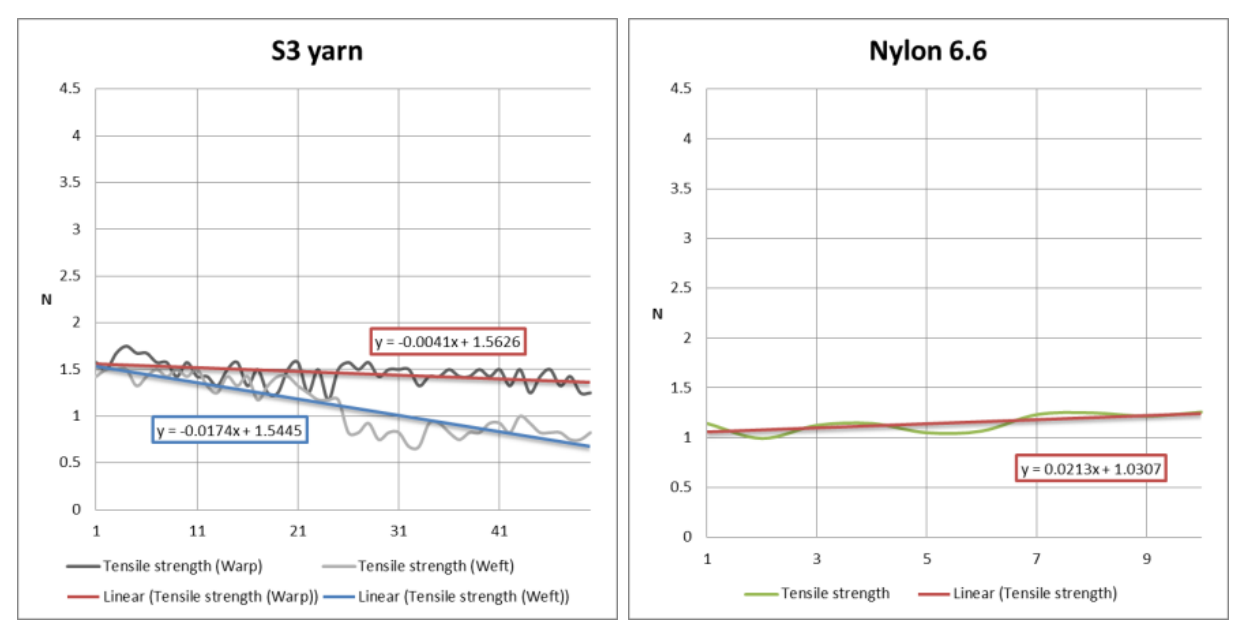

Figure 2. Breaking strength and trend line for each analysed yarn

\section{RESULTS AND DISCUSSION}

Further on we try to assess the strength transfer coefficient (Cristian et al., 2012) given mathematically as:

$$
C=\frac{T f_{2}}{T f_{1}}
$$

where $\mathrm{Tf}_{1}$ - yarn tenacity before its integration in fabric expressed in $\mathrm{N} / \mathrm{Tex}$ calculated with the equation:

$T f_{1}=\frac{F_{b k g(t)}}{\operatorname{Tex}}$

$\mathrm{Tf}_{2}$ is theoretical yarn tenacity after its integration in woven structure, including the influence of the weave structure/finishing treatments and is expressed also in N/Tex:

$T f_{2}=\frac{F_{b k g(t)}}{P \times b \times T e x}$

The strength transfer coefficient $\mathrm{C}$ for the given samples has the following values:

- $\quad$ S1 sample: Warp 1.14; Weft 0.97;

- $\quad$ 22 sample: Warp 0.78; Weft 1.03;

- $\quad$ S2 sample: Warp 1.16; Weft 1.56.

Closer these coefficients are from unity the more linear is the transfer rate, above one means the existing woven structure and treatment strengthens the yarn properties. From this we observed S1 and S3 structures to be superior in this regard.

One of the most important properties for these fabrics is the air permeability (Buyuk et al., 2019) and we tried to reduce this by catering several aspects:

- Yarn torsion of the two systems;

- The use of specially designed connections like ripstop or double ripstop type, with a binding segment of maximum two which interrupt the tendency of the wires of one system to slide towards the wires of the other system (not recommended to use the connections D2 / 1, R2 / 1, R1 / 2 or P2 / 2).

https://doi.org/10.24264/icams-2020.III.16 
Theoretical and Practical Aspects of the Design Phase for a Single Skin Textile Wing

- Finishing treatment, polyurethane coating.

Two woven types of fabrics were developed accordingly to the following weave diagrams and general characteristics:

- Yarn fiber composition: $100 \%$ PA6.6HT;

- Yarn linear density: 30 den/32 f;

- Yarn count warp: 495 threads $/ 10 \mathrm{~cm}$;

- V1 Yarn count weft: 504 threads/10 cm (Figure 3);

- V2 Yarn count weft: 508 threads/10 cm (Figure 4).

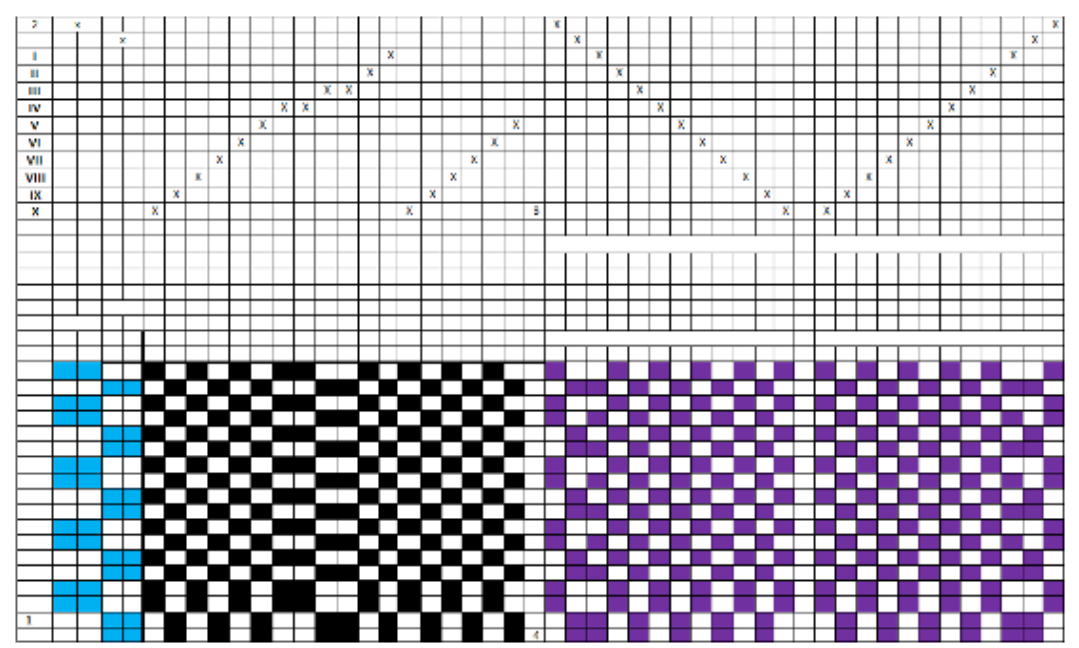

Figure 3. Programming card for weave structure V1 (Ripstop weave)

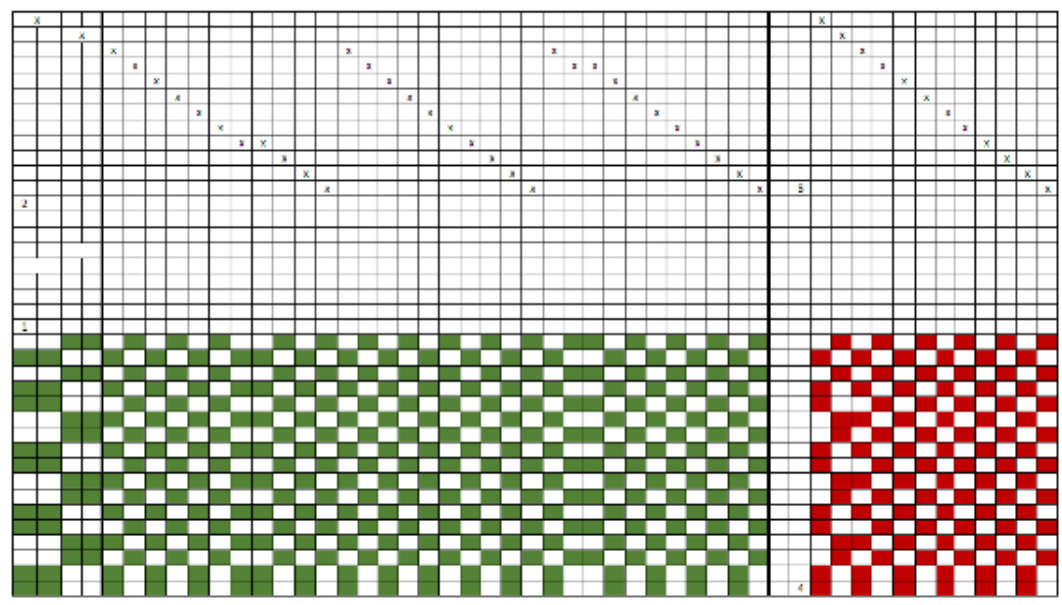

Figure 4. Programming card for weave structure V2 (Double ripstop weave)

Four fabric variants were developed as follows:

- a fabric with ripstop connection (V1 and V3) and

- $\quad$ another double-ripstop (V2 and V4).

https://doi.org/10.24264/icams-2020.III.16 
Each connection variant was made in two finishing variants:

- $\quad$ calendering (V1 and V2) and

- polyurethane coating (V3 and V4) thus resulting in four variants of finished fabrics.

Table 1. Finished fabrics test results

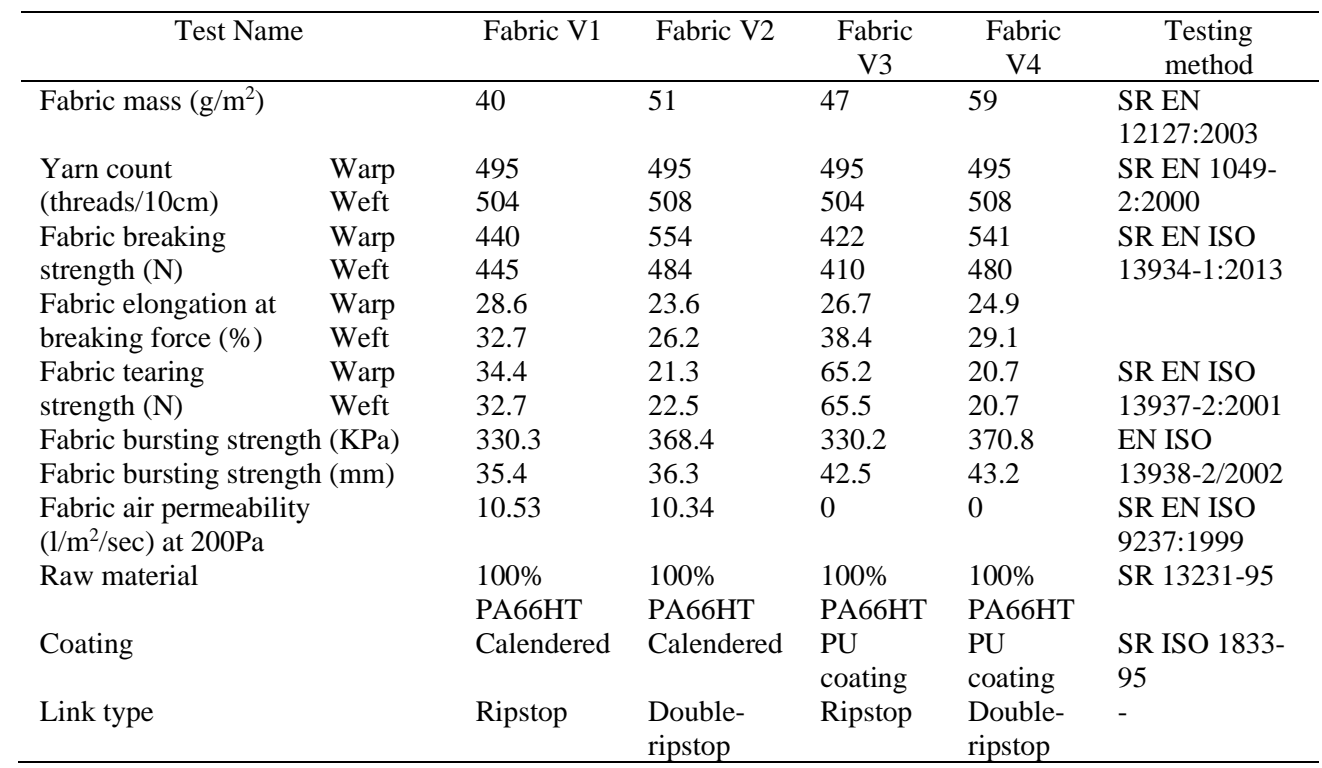

\section{CONCLUSIONS}

The fabric breaking strength is in line with the breaking strength of the yarn, this validates the testing methods and yarn extraction method. A strength transfer coefficient greater than one means the woven structure has higher theoretical tenacity than all the yarns combined. This means that the calendred fabric S3 woven structure amplifies better the yarn tenacity than coated fabrics; however the S1 fabric is not far behind and has way better breaking strength, lower elongation and also lower air permeability, probably because of the double-ripstop structure.

The highest yarn elongation of S2 influences in an interesting way the tearing behaviour and tearing strength results. The S2 fabric gets the highest tearing resistance due to this but is not necessarily the correct one since the fabric torn incompletely. Some threads remained in structure and influenced the results.

Due to the nature of the single sail wing, the amount of fabric used in the manufacture is almost halved therefore the fabric can be a little heavier and also can have a less than perfect air permeability because the shape is maintained by several rigid members. Thus we conclude that the fabric must use yarn of high tenacity Nylon 66 ; then make use of the rip-stop weave link and polyurethane coating.

The fabric variants obtained were tested and these conclusions were drawn:

- Regarding the air permeability, the most performing variants were the coated ones (V3 and V4)

- Considering the specific mass, the lightest fabric is the V1 variant.

- Considering the breaking resistances, all variants are in the same performance class 
but with significantly higher values in the case of double-ripstop variants V2 and V4. However, increased tear strength is observed in the case of the V3 variant, this is due to the tearing mode which opposes the propagation of the rupture. This type of tearing behaviour is presented by both V1 and V3.

- Further testing is required to decide if the fabric can be functionalized with hydrophobic (Toma et al., 2018) properties in order to expand the operational capabilities of the UAV for rainy weather or with applied heating elements (Buhu et al., 2019) for use on sub-zero temperatures or high altitude flying.

- Analysing the results and given the desirable tearing behaviour of the V3 variant, we choose this working variant for the UAV textile structure prototype manufacturing in the next stages of system design.

\section{REFERENCES}

Buhu, L., Negru, D., Loghin, E.C. and Buhu, A. (2019), “Analysis of tensile properties for conductive textile yarns", Industria Textila, 70(2), 116-119, https://doi.org/10.35530/IT.070.02.1517.

Buyuk, F.M., Adnan, M., Antonin, H. and Karel, A. (2019), "Theoretical model: analysing theoretically the air flow through car seatfoam material”, Industria Textila, 70(4), 324-330, https://doi.org/10.35530/IT.070.04.1559B.

Cristian, I., Nauman, S., Boussu, F. and Koncar, V. (2012), “A Study of Strength Transfer from tow to Textile Composite Using Different Reinforcement Architectures”, in: Appl. Compos. Mater., 19(3-4), 447-458, https://doi.org/10.1007/s10443-011-9215-x.

Knache, T.W. (1992), "Parachute Recovery Systems - Design Manual”, Para Publishing, Santa Barbara, California, 230-231

Poynter, D. (1984), The Parachute Manual - A Technical Treatise on Aerodynamic Decelerators, vol. 2, Santa Barbara, California, 184-185.

Toma, D., Chirila, L., Popescu, A., Chirila, C. and Iordache, O. (2018), "Multifunctional finishing treatments applied on textiles for protection of emergency personnel", Industria Textila, 69(5), 357-362, https://doi.org/10.35530/IT.069.05.1585. 


\section{IV.}

\section{ECOLOGICAL PROCESSES FOR CIRCULAR AND NEUTRAL ECONOMY}




\title{
DEVELOPMENT AND CHARACTERIZATION OF BIODEGRADABLE POLYMERIC COMPOSITES BASED ON BUTADIENE-CO-ACRYLONITRILE RUBBER AND FUNCTIONALIZED POST-CONSUMER WOOD WASTE
}

\author{
LAURENTIA ALEXANDRESCU, MIHAI GEORGESCU, MARIA SÖNMEZ, MIHAELA \\ NITUICA, DANIELA STELESCU
}

INCDTP - Division Leather and Footwear Research Institute, 93 Ion Minulescu, 031215, Bucharest, Romania, laurentia.alexandrescu@icpi.ro

\begin{abstract}
In a circular economy, the value of products and materials is maintained as much as possible; waste and resource use are kept to a minimum, and resources do not leave the economic flow once they reach the end of their life, but are reused and create further value. The paper presents the obtaining and characterization of a composite based on butadiene-co-acrylonitrile rubber continuous phase, where are added post-consumer recycled wood particles, with dimensions of $500 \mathrm{~nm}$ - discontinuous phase, and ingredients, such as: active fillers, plasticizers, vulcanizing agents, antioxidants. Wood waste acts as a filling material which leads to the biodegradability of the composite and the decrease in density. The small size of the waste particles and their functionalization with potassium oleate leads to the formation of bonds between the matrix and the particles so that the physical-mechanical characteristics of the composite correspond to the requirements of the products obtained from it.
\end{abstract}

Keywords: biodegradability, polymeric composite, post-consumption, green footwear

\section{INTRODUCTION}

The problem of waste that pollutes the environment has been addressed over the years by several methods: depollution (landfilling, burial, composting, burning), recovery by reuse and/or energy recycling (incineration) and/or mechanical and/or chemical (pyrolysis, gasification, hydrolysis etc.) (Sienkiewicz et al., 2012; JATMA, 2010). The integrated concept of elastomeric waste management, along with these methods, also includes the possibilities of reducing the quantities by using them in biodegradable polymeric materials. Recycling and the use of renewable natural resources offer a new dimension in the discovery of new materials. Recently, special attention has been paid to the development of composites with polymer matrix reinforced with natural fibers instead of conventional composites reinforced with inorganic fibers (glass, carbon, etc.). The development of environmentally friendly "green" materials is conferred by the biodegradability of these natural materials (from various sources), low weight, low cost, high availability, high specific strength compared to glass or carbon fibers, as well as the possibility of adapting existing equipment to processors for mass production (ETRMA, 2011). Composites reinforced with natural fibers are used in a variety of structural applications such as aerospace, automotive components/parts, sports or recreational equipment, craft and office products, equipment, etc. (WBCSD, 2011; RMA, 2011; Naik and Singh, 1991). Wood waste is a set of products and materials whose origin comes from all stages of the wood industry, from logging to the manufacture of finished products. Also, the scrap wood (boxes, crates, pallets) represents a significant quantity (Turku et al., 2017).

Polymer composites are systems that consist of one or more discontinued phases, dispersed in a continuous phase. Thus, at least two different materials, which are completely immiscible, are mixed to form a composite. Additives such as compatibilizers, plasticizers, pigments, temperature stabilizers and UV radiation, nanoparticles are also frequently added in order to improve certain properties. The type

https://doi.org/10.24264/icams-2020.IV.1 
and geometry of the discontinuous phase give the composite optimized properties, such as high specific strength, rigidity and hardness, low specific weight, etc. (Navarro et al., 2004).

The properties of composite materials depend on the compatibility method. For this purpose, the outer surface of the nanoparticles can be functioned with different agents, the most common functionalizing agents being organo-functional siloxanes or sodium oleate. Functionalizing agents are used to improve the adhesion between the polymer matrix and nanoparticles, protect surfaces from internal stresses that can cause cracks, stabilize the interface layer, improve wetting and increase hydrophobicity. In this paper, a composite based on butadiene-co-acrylonitrile-rubber - continuous phase is obtained, in which post-consumer wood waste particles with dimensions of $500 \mathrm{~nm}$ are introduced - discontinuous phase, and ingredients, such as: active fillers, plasticizers, vulcanizing agents, antioxidants.

\section{EXPERIMENTAL}

\section{Materials}

Materials used to obtain the biodegradable polymeric composites were: butadieneco-acrylonitrile with 35\% nitrile, from SAFIC ALCAN; stearin - flakes, white color, molecular weight $284,48 \mathrm{~g} / \mathrm{mol}$; zinc oxide, microparticles, white powder, precipitate 93-95\%; silicon dioxide, molecular mass 60,08 g/mol, white color, particle size $<0,5$ $\mathrm{mm}$; precipitate chalk - white powder, molecular weight 100.09; polyethylene glycol, slightly yellow, $\mathrm{pH}: 5-7$, density: $1.080 \mathrm{~g} / \mathrm{cm}^{3}$; mineral oil, colorless liquid, density: $1.0982 \mathrm{~g} / \mathrm{cm}^{3} ; N$-Isopropyl-N'-phenyl-1,4-phenylenediamine, brown flat granules, molar mass: $226,317 \mathrm{~g} / \mathrm{mol}$, density: $1.04 \mathrm{~g} / \mathrm{cm}^{3}$; sulfur, vulcanization agent (fine yellow powder, melting point: $115^{\circ} \mathrm{C}$ ); tetramethylthiuram disulfide - vulcanization agent (density $1.40 \mathrm{~g} / \mathrm{cm}^{3}$ ), melting point $<146^{\circ} \mathrm{C}$, an ultrafast curing accelerator); all ingredients are from Bayer company.

Wood waste was collected from the manufacture and repair of wooden furniture, cryogenically ground at $12000 \mathrm{rpm}$ for $15 \mathrm{~s}$ and screened through a $500 \mathrm{~nm}$ mesh screen.

\section{Method}

The functionalization of wood waste with potassium oleate was achieved by mixing with a mechanical stirrer with 80 rotations $/$ min with heat, at a temperature of $80^{\circ} \mathrm{C}$ for 8 hours. The ratio between wood waste and potassium oleate - $50 \%$.

\section{Obtaining the Composites}

The rubber is plasticized for $1 \mathrm{~min}$ and $30 \mathrm{~s}$, speed 40 rotations $/ \mathrm{min}$ and $45^{\circ} \mathrm{C}$, mechanically mixed in a Brabender Plasti-Corder PLE-360, then the fillers, plasticizers and wood waste are added, for 4 minutes and $30 \mathrm{~s}$ at 20 rotations $/ \mathrm{min}$ and $45^{\circ} \mathrm{C}$. Homogenized for 2 minutes, speed 60 rotations $/ \mathrm{min}$ and $80-100^{\circ} \mathrm{C}$. The total processing times was 8 minutes. Table 1 shows tested formulations. The mixture is removed from the mixer and finished on the electric roller, by adding the antioxidant and vulcanizing agents. The processing parameters are as following: temperature $23-30^{\circ} \mathrm{C}$, friction of the rollers 1:2, and 50 rotations/min, for $5 \mathrm{~min}$ and homogenization for another $2 \mathrm{~min}$. 
Table 1. Polymeric composites based on polybutadiene-co-acrylonitrile rubber compounded with elastomers functionalized with potassium oleate

\begin{tabular}{|c|c|c|c|c|c|c|}
\hline Ingredients / Symbol & M.U. & B0 & BL1 & BL2 & BL3 & BL4 \\
\hline \multicolumn{7}{|c|}{ Processing on Brabender } \\
\hline $\begin{array}{l}\text { butadiene-co-acrylonitrile } \\
\text { rubber }\end{array}$ & $\mathrm{g}$ & 190 & 190 & 190 & 190 & 190 \\
\hline stearin & $\mathrm{g}$ & 2.28 & 2.28 & 2.28 & 2.28 & 2.28 \\
\hline zinc oxide & $\mathrm{g}$ & 9.5 & 9.5 & 9.5 & 9.5 & 9.5 \\
\hline precipitated chalk & $\mathrm{g}$ & 47.5 & 47.5 & 47.5 & 47.5 & 9.5 \\
\hline silicon dioxide & $\mathrm{g}$ & 57 & 38 & 19 & 0 & 0 \\
\hline PEG 4000 & $\mathrm{~g}$ & 7.6 & 7.6 & 7.6 & 7.6 & 7.6 \\
\hline functionalized Wood waste & $\mathrm{g}$ & 0 & 19 & 38 & 57 & 95 \\
\hline mineral oil & $\mathrm{g}$ & 19 & 19 & 19 & 19 & 19 \\
\hline \multicolumn{7}{|c|}{ Processing on Roller } \\
\hline sulfur & g & 2.85 & 2.85 & 2.85 & 2.85 & 2.85 \\
\hline antioxidant IPPD & g & 5.7 & 5.7 & 5.7 & 5.7 & 5.7 \\
\hline accelerator Th & $\mathrm{g}$ & 1.14 & 1.14 & 1.14 & 1.14 & 1.14 \\
\hline
\end{tabular}

The Brabender mixing diagrams, Figure 1, show that in the first section (A-B) which lasts 1'30" at $40 \mathrm{rpm}$, the elastomer is added into the mixer and therefore the torque initially increases to A. The first loading peak, A, corresponds to the introduction of elastomers. As the torque increases, so does the temperature due to friction. The torque begins to decrease near A to B, mainly due to the homogenization and plasticization of the elastomer, as well as due to the increase in temperature caused by the shear force. Then the other ingredients are introduced and the rotation speed is reduced to $20 \mathrm{rpm}$ for 4'30", and the mixer chamber is kept open. Between point B and point X, the torque begins to increase due to the incorporation of the ingredients, but also as a result of compaction and reinforcement of elastomers. After incorporating the fillers and other ingredients, the second loading peak, $X$, is observed when a maximum torque point appears. The torque starts to decrease, indicating the homogenization of the mixture. Then the homogenization of the compounds occurs for $2^{\prime}$ at $60 \mathrm{rpm}$, with the mixer chamber closed. As a result, a maximum value of torque is obtained due to the compaction and homogenization of the rubber mixture. This is generally followed by a decrease in the value of the torque, which indicates both homogenization of the mixture and increase in the temperature due to friction at a higher rotational speed $(60 \mathrm{rpm})$.

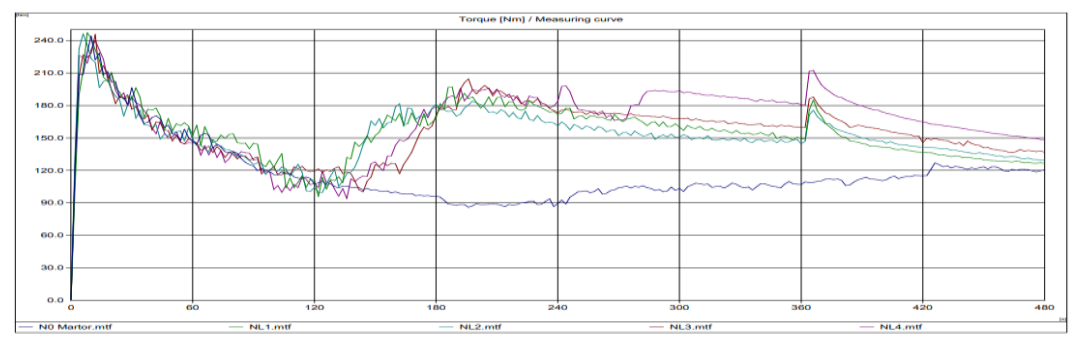

Figure 1. Torque variation vs. the time, recorded at Plasti-Corderul Brabender, when obtaining the rubber composites

It is observed that the control sample has one maximum torque peak, which occurs when the rubber is plasticized. The other two, associated with adding fillers and 
especially wood waste, do not show, instead a slight increase is observed when the mixture is homogenized.

The compounds were then compression-molded (using an electrically heated laboratory press) to obtain a sheet about $2 \mathrm{~mm}$ thick. Press parameters: preheating 3 min.; pressing 4 min.; cooling 13 min.; pressure $300 \mathrm{kN}$; temperature $165^{\circ} \mathrm{C}$. The material was then cooled down to room temperature under the same pressure. The specimens were die-cut from the compression molded sheet and used for testing after 24 hours of storage at room temperature.

\section{Testing Methods}

1. Tensile strength tests of the samples were carried out according to SR ISO 37:2012 using a Schopper Tensile Testing machine 1445, at a constant crosshead speed of $500 \pm 5 \mathrm{~mm} / \mathrm{min}$.

2. Hardness of the samples was measured by Shore "D" Durometer according to SR ISO 7619-1:2011.

3. Abrasion resistance was carried out according to ISO 4649/2010, the cylinder method, using a pressure of $10 \mathrm{~N}$. Abrasion resistance was expressed by relative volume loss in relation to calibrated abrasive paper. A wearing tester with abrasive cloth having granulation of 212-80 mm (PE 80). The samples used were obtained from rolled blends and pressed into sheets, then cutting with a rotating die and have cylindrical shape, with a diameter of $16 \mathrm{~mm}$ and height of min. $6 \mathrm{~mm}$.

4. FT-IR spectroscopy was done using the FT-IR 4200 JASCO, Herschel series instrument, equipped with ATR having diamond crystal and sapphire head within the spectrometric range $2000-530 \mathrm{~cm}^{-1}$.

\section{RESULTS AND DISCUSSION}

The polymer structures obtained, in initial state and after accelerated ageing were characterized in terms of their physical-mechanical properties, and results are presented in table 2. Analyzing the values of physical-mechanical tests reveals the following:

Hardness of the control sample is $57^{\circ} \mathrm{Sh} \mathrm{A}$, when adding the functionalized wood waste increases proportionally with the amount of waste used in the mixture, up to $62^{\circ} \mathrm{Sh} \mathrm{A}$. This is demonstrated by the fact that the hardness increases with the amount of fillers in the mixture.

The tensile strength decreases compared to the control sample, $8.16 \mathrm{~N} / \mathrm{mm}^{2}$, and proportionally to the amount of wood waste introduced into the composite, reaching $1.03 \mathrm{~N} / \mathrm{mm}^{2}$ for the composite with $50 \%$ functionalized wood waste.

Elasticity does not vary compared with control sample.

Table 2. Physical-mechanical characteristics of the composites

\begin{tabular}{llllll}
\hline $\begin{array}{l}\text { Physical mechanical- } \\
\text { characteristics }\end{array}$ & \multicolumn{7}{c}{ B0 } & BL1 & \multicolumn{2}{c}{ BYMBOL } & BL3 & BL4 \\
\hline Hardness, ${ }^{\circ}$ Sh A & 57 & 57 & 58 & 60 & 62 \\
Elasticity, $\%$ & 22 & 22 & 22 & 22 & 22 \\
Modulus 100\%, N/mm ${ }^{2}$ & 0.96 & 1.01 & 1.11 & 0.83 & 0.89 \\
Modulus 300\%, N/mm ${ }^{2}$ & 1.80 & 1.59 & 1.69 & & \\
Tensile strength, N/mm & 8.16 & 6.79 & 2.38 & 1.0 & 1.03 \\
Elongation at break, \% & 660 & 740 & 460 & 280 & 260 \\
\hline
\end{tabular}

https://doi.org/10.24264/icams-2020.IV.1 


\begin{tabular}{llllll}
\hline Residual elongation, \% & 24 & 28 & 20 & 20 & 14 \\
Tear strength, N/mm & 24.13 & 21.92 & 16.42 & 10.33 & 13.71 \\
Specific weight, $\mathrm{g} / \mathrm{cm}^{3}$ & 1.22 & 1.21 & 1.18 & 1.16 & 1.01 \\
Abrasion resistance, $\mathrm{mm}^{3}$ & 142.98 & 181.74 & 180.82 & 264.81 & 400.25 \\
\hline
\end{tabular}

Elongation at break, similar to tensile strength, decreases from $660 \%$ of the control sample, proportional to the amount of wood waste, down to $260 \%$ for the composite with $50 \%$ wood waste in the composition. Similar values are obtained for residual elongation and tear strength.

Density decreases proportional to the amount of wood waste, from $1.22 \mathrm{~g} / \mathrm{cm}^{3}$ control sample to 1.01 in the BL4 sample with $50 \%$ wood waste.

The abrasion increases exponentially with the proportion of wood waste added, thus the control sample has a value of $142.98 \mathrm{~mm}^{3}$, the BL1 sample with $10 \%$ wood waste has the value of $181.74 \mathrm{~mm}^{3}$, and the BL4 sample with $50 \%$ wood waste reaches 400.25 $\mathrm{mm}^{3}$. It was observed that composites with $10-20 \%$ wood waste have values that fall within the standardized requirements for abrasion, namely $200 \mathrm{~mm}^{3}$.

FT-IT spectroscopy. IR spectrum represents the radiant energy absorption curve in the IR domain by the sample molecule, depending on the wave length or radiation frequency. The infrared domain of the electromagnetic radiation is between 0.8 and 200 $\mu \mathrm{m}$. IR domain for usual organic chemistry is between 2.5 and $25 \mu \mathrm{m}$. The structural determinations were carried out on an IR molecular absorption spectrometer with double beam, in the range of 4000-600 $\mathrm{cm}^{-1}$, using 4200 FT-IR equipped with ATR diamond crystal and sapphire head. The solid state samples were set in the ATR and the equipment recorded the transmittance spectra of the sample and then compared it with the background spectra previously recorded. The recorded spectra of the samples were compared with the pure elastomer spectrum. After the tests were carried out, the following were found:

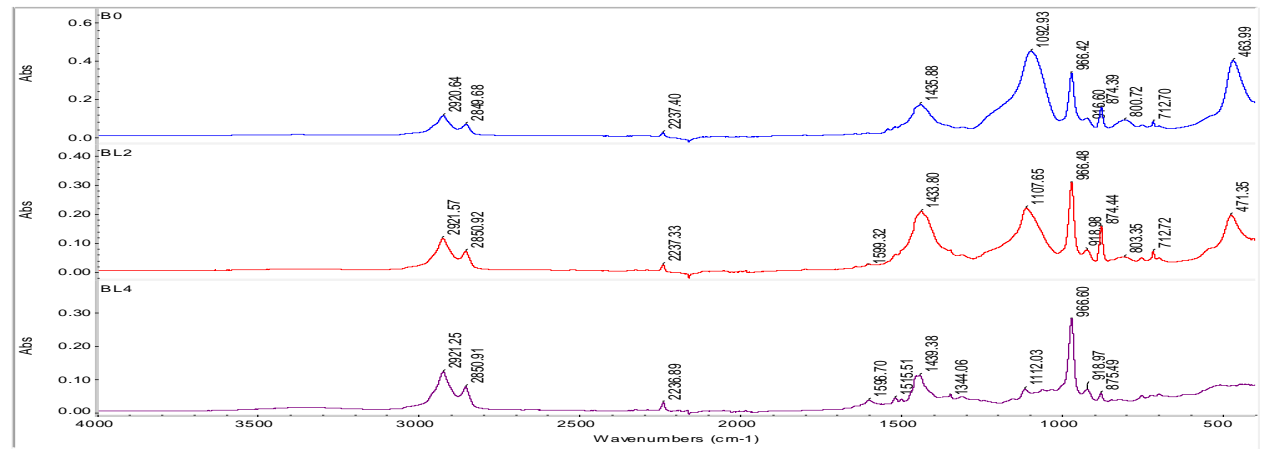

Figure 2. FTIR spectra of composites based on butadiene-co-acrylonitrile rubber / potassium oleate functionalized wood waste

In the case of butadiene-co-acrylonitrile rubber/wood waste treated with potassium oleate composite, in addition to the band's characteristic of the treated waste (especially at $\left.\sim 1596,1515 \mathrm{~cm}^{-1}\right)$ the characteristic band of silica $\left(1090-1100 \mathrm{~cm}^{-1}, \sim 800 \mathrm{~cm}^{-1}, 460\right.$ $\left.470 \mathrm{~cm}^{-1}\right)$, calcium carbonate $\left(\sim 874,712 \mathrm{~cm}^{-1}\right)$ and rubber, can also be observed. Moreover, the presence of wood waste can be proved in BL4 by the fact that the area $1000-1100 \mathrm{~cm}^{-1}$ contains several overlapping strips including the $\mathrm{C}-\mathrm{O}$ band from approximately $1027 \mathrm{~cm}^{-1}$ of the untreated wood waste. In $\mathrm{BL} 2$ due to the presence of $\mathrm{SiO}_{2}$ 
there is a merging of the bands, one characteristic of silica between $1000-1100 \mathrm{~cm}^{-1}$ and the C-OH strips from the wood waste, which leads to an asymmetry of the peak.

\section{CONCLUSION}

The paper presents the study of the new biodegradable polymeric composites, based on wood waste nanoparticles functionalized with potassium oleate dispersed in the butadiene-co-acrylonitrile elastomer matrix. Wood waste (fillers), by dispersing them in the elastomeric matrix, led to a biodegradable polymeric material, with less performance characteristics, which still meets the requirements of the profile standards, but the composite with $50 \%$ wood waste. The specific weight decreases in proportion to the amount of wood waste used to process the composites, which leads to the weight loss of the products. The materials are adapted for applications in "green" biodegradable footwear, with short life after use.

\section{Acknowledgements}

This paper is funded by the Ministry of Research and Innovation within Program $1-$ Development of the national RD system, Subprogram 1.2 - Institutional Performance RDI excellence funding projects, Contract no. 6PFE/2018-PERFORM-TEX-PEL, the LIFE program in the frame of LIFEGREENSHOES 4 ALL (LIFE17ENV/PT/000337) project and PN $19 \quad 17 \quad 01 \quad 03 / 2019$ project: "Biodegradable composites from technological and post-consumption polymeric wastes by designing and applying $4 \mathrm{R}$ eco-innovative technologies (4R-ECO-MAT)".

\section{REFERENCES}

ETRMA - European Tyre \& Rubber Manufacturers’ Association (Belgium) (2010), “A Valuable Resource with Growing Potential”, 2010 edition, http://www.etrma.org/default.asp (May 2011)

JATMA - The Japan Automobile Tyre Manufacturers Association NC (Japan) (2010), "Tyre Industry of Japan 2010”, Report downloaded from: http://www.jatma.or.jp/english/media/ (05.2011).

Naik, T.R. and Singh, S.S. (1991), "Utilization of Discarded Tires as Construction Materials for Transportation Facilities", Report No. CBU-1991-02, UWM Center for By-Products Utilization. University of Wisconsin-Milwaukee, Milwaukee, p. 16.

Navarro, F.J., Partal, P., Martínez-Boza, F. and Gallegos, C. (2004), "Thermo-rheological behaviour and storage stability of ground tire rubber-modified bitumens", Fuel, 83, 2041-2049, https://doi.org/10.1016/j.fuel.2004.04.003.

RMA - Rubber Manufacturers Association (USA) (2009), "Scrap Tire Markets in the United States 9th Biennial Report”, Report downloaded from: http://www.rma.org/scrap_tires/ (May 2011)

Sienkiewicz, M., Kucinska-Lipka, J., Janik, H. and Balas, A. (2012), "Progress in used tyres management in the European Union: A review", Waste Management, 32, 1742-1751, https://doi.org/10.1016/j.wasman.2012.05.010.

Turku, I., Keskisaari, A., Kärki, T., Puurtinen, A. and Marttila, P. (2017), "Characterization of wood plastic composites manufactured from recycled plastic blends", Composite Structures, 161 469-476, https://doi.org/10.1016/j.compstruct.2016.11.073.

WBCSD - World Business Council for Sustainable Development (Switzerland) (2008), "The Report Managing End-of-Life Tyres", Report downloaded from: http://www.wbcsd.org/templates/Template WBCSD5/layout.asp?type=p\&MenuId=MTYwNg\&doOpen=1\&ClickMenu=LeftMenu (05. 2011). 


\title{
SUSTAINABLE PRODUCTS IN THE LEATHER INDUSTRY
}

\author{
OLGA BALLUS ${ }^{1}$, ANNA BACARDIT ${ }^{2}$ \\ ${ }^{1}$ Cromogenia Units S.A, C/ Feixa Llarga 1-3 Pol. Ind. Zona Franca, 08040 Barcelona, \\ oballus@cromogenia.com \\ ${ }^{2}$ Departament d'Informàtica i Enginyeria Industrial, Universitat de Lleida, Campus Universitari \\ d'Igualada, Av. Pla de la Mass 8,08700Igualada,anna.bacardit@udl.cat
}

\begin{abstract}
Protecting the environment is one of the three objectives of sustainability. One way to achieve this is to preserve natural resources by using renewable or residual raw materials. These products have a shorter lifespan and a lower carbon footprint, are highly biodegradable, and are therefore considered to be sustainable products. In this paper, three retanning agents and two oils classified as sustainable products were studied. First, biobased carbon content (an indicator of renewable raw material content) was determined. Then, the physical and organoleptic properties of the leathers treated with each product (degree of softness, firmness and fullness) were evaluated. The COD of residual baths was also determined in oils. The products presented in this paper meet the sustainability requirements, i.e., high renewable raw material content, short lifespan, and low carbon footprint. In addition, these products show high fixation and therefore have a low COD in residual baths, thus also contributing to their sustainability.
\end{abstract}

Keywords: Sustainable products, bio-based products, leather industry

\section{INTRODUCTION}

The transformation of an animal's skin into leather is known as a tanning process. This process includes a series of physical-chemical transformations that turn the skin into a durable and usable material. This process is divided into four main stages: beamhouse, tanning, retanning and finishing. In the beamhouse stage, the skin is hydrated and the hair and epidermis are removed. Then, leather is tanned, generally with chromium salts (wet blue) and to a lesser extent with aldehydes and/or vegetable extracts (wet white), to give stability to the collagen. The retanning stage also includes dyeing and fatliquoring and at this stage the properties and characteristics of the tanned leather are modified to obtain different types of articles. Finally, the finishing stage consists of applying surface treatments to the leather to achieve different properties such as gloss or physical resistances.

Sustainability in the leather industry is an important factor that has gained relevance in recent years. The definition of sustainability proposed in the Bruntland (Finkbeiner et al., 2010) report implies, among other things, maintaining a balance between the resources used and the waste dumped. In other words, avoiding the depletion of nonrenewable resources and avoiding the overexploitation of renewable resources, as well as reducing the discharge of pollutants to the environment.

Leather is a by-product of the meat and dairy industry, and if it is not transformed into leather, this by-product becomes a problematic waste, among other things due to the high volume (currently 10 million tons of leather are processed per year) (Buljan and Král', 2019). Hides and skins are therefore renewable resources and are considered sustainable products that contribute to the circular economy. However, the tanning process uses a high amount of water and chemicals that can affect both the environment and people's health. This must be studied in depth in order to contribute to the sustainability of the tanning process.

Currently most of the products used in the tanning process come from petroleum chemistry. Petroleum is a non-renewable resource and its treatment and use have a high contribution to climate change (Okkerse and Van Bekkum, 1999) and therefore great efforts are being devoted to the search for cleaner and more sustainable alternatives. 
In this work, various products used in the retanning and fatliquoring stage are proposed that contribute to the improvement of the sustainability of the leather. The products must meet the following characteristics: they must come from renewable resources thus they will have improved biodegradability (European Commission, 2009; http://www.biobasedinprocurement.eu, 2017), reduced danger to humans and the environment, highly effective and low COD in wastewater, and they do not contain restricted substances. For this, two acrylic-based and one phenolic-based retanning products have been designed in which part of the acrylic/phenolic base have been replaced by biopolymers from renewable resources: proteins and polysaccharides and two oils based on natural raw materials: fish and soy derivatives.

\section{EXPERIMENTAL}

Three retanning agents (two acrylic-based and one phenolic-based) and two fatliquoring agents were studied (Table 1). All products contain raw material from renewable resources.

Table 1. Products, nature, active matter

\begin{tabular}{lcc}
\hline Product & Nature & Active matter \\
\hline PRODUCT ST-AA & Acrylic & $27 \%$ \\
PRODUCT ST-A2P & Acrylic & $30 \%$ \\
PRODUCT ST-MP & Phenolic & $95 \%$ \\
PRODUCT ST-AW & Oil of natural origin & $90 \%$ \\
PRODUCT ST-AF & Oil of natural origin & $70 \%$ \\
\hline
\end{tabular}

\section{Biobased Content Analysis}

Percentage of biobased carbon was determined by ASTM D6866-18 Method B (AMS) to determine the raw material derived from renewable resources. The reference standard for C14 measurements is NIST 4990C.

\section{Leather Application}

Hides were divided into $40 \times 100 \mathrm{~cm}$ pieces for each product.

Wet blue tanned leathers of Spanish origin split at 1.4-1.5 mm were used to evaluate the retanning agents. Retanning products were applied at $5 \%$ after leather neutralization at $\mathrm{pH}$ 5.5. Fatliquoring and dyeing were performed according to a standard formulation. Wet blue tanned leathers of Spanish origin split at $1.0-1.1 \mathrm{~mm}$ were used to evaluate fatliquoring agents. Leathers were neutralized at pH 6.5 and fatliquored by applying $10 \%$ of the oil in a separate bath. Retanning and dyeing were performed according to standard formulation.

\section{Bath and Hide Determinations}

Chemical oxygen demand (COD) of the residual baths was determined. Analysis were performed with $1-1500 \mathrm{mg} / \mathrm{L}$ vials heated under reflux for 2 hours at $150^{\circ} \mathrm{C}$, and COD was measured with an Aqualytic AL100 spectrophotometer.

After retanning, color intensity and physical properties were determined. The degree of softness was determined according to IUP 36, thickness according to IUP 4 and firmness by SATRA PM 36 scale. In order to assess the dyeing properties of the different products used, dyeing intensity was measured with a Color Data Spectraflash SF-30 colorimeter. 
RETANNING AGENTS

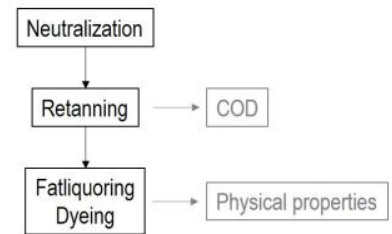

FATLIQUORING AGENTS

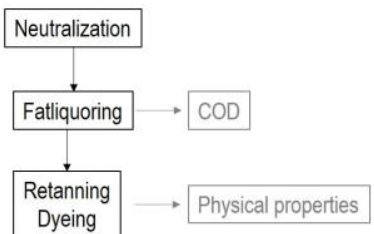

Figure 1. Scheme of application formulation

\section{RESULTS AND DISCUSSION}

\section{Products}

\section{Acrylic Polymers}

Acrylic polymers are widely used as retanning agents. These products provide high firmness, they are very well fix to the leather and have good fastnesses. Acrylic resins are polymers from the acrylic acid, and the standard products are $100 \%$ derived from petroleum. To improve the sustainability of these products, biopolymers have been reacted with the acrylic resins. The synthesis is based on a radical polymerization of the acrylic acid with biopolymers (BP) derived from proteins and polysaccharides. Reaction is shown in Figure 2.

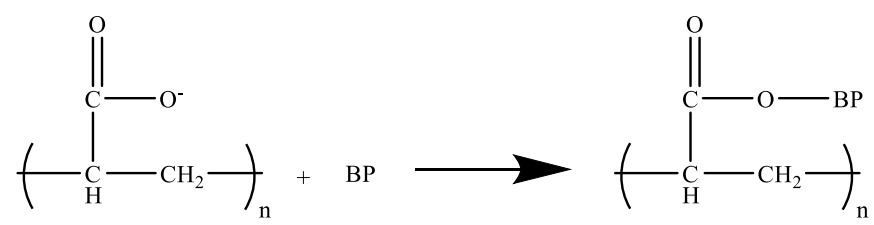

Figure 2. Scheme of application formulation

\section{Phenolic Polymer}

Phenolic polymers are used in the retanning stage to increase leather fullness, among other properties. These products are obtained trough a condensation reaction of phenol and formaldehyde. Basic structure of these polymers is shown in Figure 3.

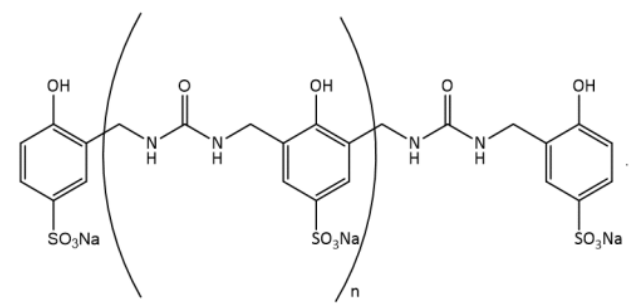

Figure 3. Phenolic retanning polymer structure

These products are $100 \%$ petroleum derivatives and they are considered non sustainable products. To improve its sustainability, biopolymers from different origins can be added during the condensation phase. In this work, lignin and protein derivatives 
were added during the condensation stage so they can form polymeric bonds and a biopolymer of high molecular weight is obtained.

\section{Fatliquoring Agents}

Fatliquoring agents can come from petroleum derivatives such as mineral oils or sulphochlorinated paraffins. They can also come from natural fat (animal and vegetal) and are mainly form by triglycerides. Triglycerides are treated first with oxygen and with bisulfite to obtain water soluble products. Sulphitation reaction is shown in Figure 4.<smiles>CCc1cc([N+](=O)[O-])ccc1O</smiles>

Figure 4. Sulphitation reaction of triglycerides

Fatliquoring products are usually combinations of both products, mineral and synthetic. In this work we developed two fatliquoring agents with high biobased carbon content that can give similar characteristics as the standard products: PRODUCT STAW and PRODUCT ST-AF.

\section{Renewable Source Determination}

Carbon-14 analysis is used to determine the percentage of biobased carbon in a product, that is, the carbon from renewable animal or plant resources.

Results are reported as \% biobased carbon. A value of $100 \%$ biobased would indicate that all carbon content comes from renewable sources (plants or animals byproducts) and a value of $0 \%$ would point that all of the carbon of the sample was derived from non-renewable sources (petrochemicals, coal and other fossil sources). Values between these two proportions would mean that there is a mixture, where the higher the percentage, the greater the proportion of renewable raw material in the sample and therefore higher sustainability of the product.

The percentage of biobased carbon of the retanning agents is showed in Figure 5. RETANAL ST-MP has the highest value of C14 content followed by RETANAL STA2P and RETANAL ST-AA.
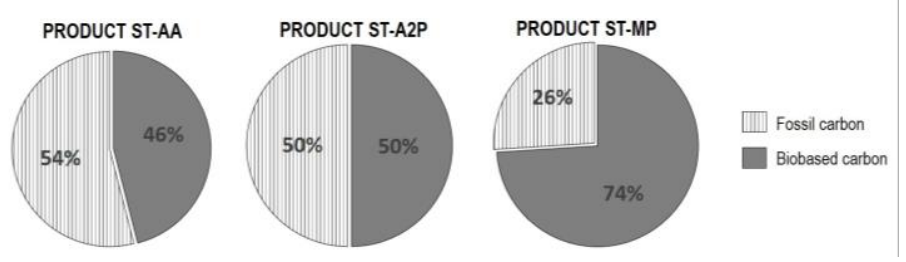

Figure 5. Bio-based carbon content of retanning agents

The percentage of biobased carbon of fatliquoring agents are shown in Figure 6. In this case, the two products show that most of the carbon contained in the product is biobased, that is, from sustainable sources. 

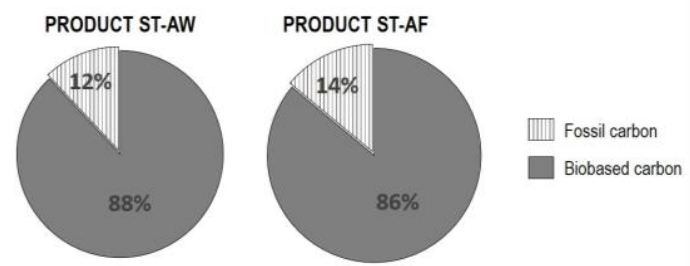

Figure 6. Bio-based carbon content of fatliquoring agents

\section{Assessment of Properties}

Depending on the final leather article (upholstery, garment, etc) different products are selected. Standard processes always have a combination of retanning agents from different nature (acrylic, phenolic, sulfones, vegetables, etc) as well as oils of different nature (animal or vegetable origin, sulfited, sulphated, etc) in order to provide the best characteristics to the leather such as fullness, softness, physical resistances, etc.

To evaluate the performance of the retanning agents, a comparison of the sustainable products was made with respect to a reference sample (non-renewable acrylic and phenolic product). COD of the baths were analyzed at the end of the retanning stage to evaluate the fixation of the products and different properties such as thickness, softness firmness and dyeing intensity were assessed after fatliquoring and dyeing.

Table 2 shows the results of COD and physical properties expressed as a percentage variation of sustainable products versus non-renewable products.

Table 2. COD and physical properties

\begin{tabular}{lcccc}
\hline Product & COD & Softness & Thickness & Firmness \\
\hline PRODUCT ST-AA & $-20 \%$ & $+30 \%$ & $0 \%$ & $0 \%$ \\
PRODUCT ST-A2P & $-30 \%$ & $-25 \%$ & $-20 \%$ & $-15 \%$ \\
PRODUCT ST-MP & $-20 \%$ & $+20 \%$ & $-15 \%$ & $-20 \%$ \\
\hline
\end{tabular}

In all cases, there is a decrease in COD values for sustainable products. Softness is improved (except for PRODUCT ST-A2P), but thickness and firmness are slightly worse for PRODUCT ST-A2P and PRODUCT ST-MP. PRODUCT ST-AA improves all characteristics.

COD values of sustainable oils are compared versus a lecithin-based oil as a reference. Table 3 shows the results as a percentage variation of the sustainable fatliquors versus the reference.

Table 3. COD and physical properties

\begin{tabular}{lcccc}
\hline Product & COD & Softness & Thickness & Firmness \\
\hline PRODUCT ST-AW & $-60 \%$ & $+10 \%$ & $0 \%$ & $0 \%$ \\
PRODUCT ST-AF & $-25 \%$ & $+25 \%$ & $-10 \%$ & $-15 \%$ \\
\hline
\end{tabular}

There is a reduction in COD values of sustainable products, which indicates that products are well fixed in the leather and baths contain less pollutants.

Softness is improved for both products PRODUCT ST-AW and PRODUCT ST-AF with respect to the reference product, but firmness is similar for PRODUCT ST-AW and slightly lower for PRODUCT ST-AF. 
Dyeing intensity was measured by colorimeter and color levelness was evaluated organoleptically. Both characteristics are expressed in a 1-5 scale (in ascending order). Results for all products are shown in Table 4.

Table 4. Color intensity and color levelness

\begin{tabular}{lcc}
\hline Product & Intensity & Levelness \\
\hline PRODUCT ST-AA & 3 & 5 \\
PRODUCT ST-A2P & 5 & 4 \\
Acrylic standard & 2 & 5 \\
PRODUCT ST-MP & 4 & 5 \\
Phenolic standard & 2 & 5 \\
PRODUCT ST-AW & 2 & 4 \\
PRODUCT ST-AF & 4 & 3 \\
Standard fatliquor & 2 & 4 \\
\hline
\end{tabular}

PRODUCT ST-AA and PRODUCT ST-A2P improve color intensity and provide similar color levelness as the standard acrylic product.

PRODUCT ST-MP provides similar color levelness as the phenolic standard and better dyeing intensity.

PRODUCT ST-AW has similar characteristics as its reference regarding dyeing intensity and color levelness, while PRODUCT ST-AF improves color intensity and reduces color levelness.

\section{CONCLUSIONS}

Several retanning and fatliquoring products with sustainable characteristics have been developed. These products meet the following characteristics: they have a high percentage of biobased carbon $(\geq 50 \%)$, which indicates that the product comes mainly from renewable sources, they have higher fixation (lower COD in wastewater than standard products) and they do not contain restricted substances. Compared to standard products, sustainable products provide similar characteristics to the leather.

\section{REFERENCES}

Adzet, J.M. (1985), Química Técnica de Tenería. Igualada: Romanyà/Valls.

Buljan, J. and Král', I. (2019), "The Framework for Sustainable Leather Manufacture Second Edition," 27, https://leatherpanel.org.

European Commission (2009), "Taking Bio-Based from Promise to Market - Measures to Promote the Market Introduction of Innovative Bio-Based Products," 28, https://doi.org/10.2769/34881.

Finkbeiner, M., Schau, E.M., Lehmann, A. and Traverso, M. (2010), "Towards Life Cycle Sustainability Assessment", Sustainability, 2(10), 3309-22. https://doi.org/10.3390/su2103309.

Harris, J.M. (2003), "Sustainability and Sustainable Development", International Society for Ecological Economics, February: 1-12, available at: http://isecoeco.org/pdf/susdev.pdf.

Khan, M.A. (1995), "Sustainable Development: The Key Concepts, Issues and Implications. Keynote Paper given at the International Sustainable Development Research Conference, 27-29 March 1995, Manchester, UK", Sustainable Development, 3(2), 63-69, https://doi.org/10.1002/sd.3460030203.

Okkerse, C. and Van Bekkum, H. (1999), "From Fossil to Green", Green Chemistry, 1(2), 107-14 https://doi.org/10.1039/a809539f.

Sutton, P. (2004), “A Perspective on Environmental Sustainability?”, A paper for the Victorian Commissioner for Environmental Sustainability, available at: http://www.green-innovations.asn.au/APerspective-on-Environmental-Sustainability.pdf

Zimmerman, J.B., Anastas, P.T., Erythropel, H.C. and Leitner, W. (2020), "Designing for a Green Chemistry Future", Science, 367(6476), 397-400, https://doi.org/10.1126/science.aay3060.

*** (2017), "Bio-Based Products in Procurement How to Consider Innovative Bio-Based Products in Procurement.", July, http://www.biobasedinprocurement.eu/.

https://doi.org/10.24264/icams-2020.IV.3 


\author{
RODICA ROXANA CONSTANTINESCU, GABRIEL ZAINESCU, IULIA CANIOLA \\ INCDTP - Division: Leather and Footwear Research Institute, 93 Ion Minulescu str., 031215, \\ Bucharest, Romania e-mail: icpi@icpi.ro
}

\begin{abstract}
The area of interest is the synthesis and study of properties of new types of hydrogels made from pelt waste, in order to recover waste from tanneries. The complex aspects related to protein projects in the leather industry are addressed by accurately determining a chemical composition, a skin designer and a different possibility of recovery and claiming a value, the use of biotechnology. The complex aspects related to protein waste in the leather industry are addressed by accurately determining the chemical composition of leather waste and the different possibilities of recovery and recycling using biotechnology. The technologies used in order to obtain a smart hydrogel based on collagen and natural polymers are non-polluting and waste-free. An important aspect to note is that the smart hydrogel is obtained through an almost identical technological process to the one used for medical collagen. An extensive study of the potential for reuse and recycling of leather protein waste in ecological conditions by developing innovative procedures for obtaining an NPK collagen matrix to be used successfully as smart fertilizer for modifying nutrient-poor soils. Hydrogels with collagen structure are characterized by a high-performance instrumental analysis system (FT-IR-ATR, SEM, EDAX, etc.).
\end{abstract}

Keywords: pelt waste, collagen hydrolysate, smart fertilizers

\title{
INTRODUCTION
}

Leather is one of the important sectors globally, nonetheless, the process of conversion of raw hides or skins into non-putrescible leather involves usage of large volume of water, chemicals and mechanical processes to remove unwanted components so as to meet the quality standards of leather. The leather manufacture process, in turn, generates a variety of solid wastes and stream of wastewater (Bodalo and Gomez, 2007).

The leather processing includes unit operations viz. Pretanning, tanning, post tanning and finishing. It is reported that about $1000 \mathrm{~kg}$ of wet salted hides when processed would yield only $200 \mathrm{~kg}$ of leather and about $700 \mathrm{~kg}$ of solid wastes are generated during the conversion (Chen et al., 2008). The solid wastes generated from the leather industry can be classified into untanned and tanned wastes. The raw trimmings, fleshing wastes (green and limed) and keratin wastes fall into the untanned wastes category (Collivignarelli et al., 1994). Currently the leather industry has to face very high costs to treat and eliminate waste. Worldwide research on leather recycling is directed towards obtaining protein composites by biochemical treatments using microorganism enzymes and obtaining protein hydrolysates and protein binders with different uses. Indeed, leather, even in the form of waste, is a valuable protein source for many areas: automotive industry, agriculture, pharmaceuticals, cosmetics, etc. Organic biopolymers are an important source of raw materials for agriculture, as protein waste composition provides sufficient elements to improve the composition of poor and degraded soils, and plants can benefit from elements such as nitrogen, calcium, magnesium, sodium, potassium (Nogueira et al., 2010).

The European Union (EU) "circular economy" aims to maintain the value of products, materials and resources for as long as possible, minimizing the generation of waste by recycling and reusing them (Eurostat, 2019). 
A way to valorize the untreated hide and skin waste is production of threedimensional molecular network named, hydrogels, by cross-linking of proteins hydrolyzed with copolymers based on polyacrylamide, polyvinyl alcohol, oligo oxyethylene methacrylate, acrylic acid, maleic acid, cellulose, starch, gum, that form three-dimensional molecular networks. The hydrogels enriched with nutrient $\mathrm{C}, \mathrm{N}, \mathrm{P}, \mathrm{K}$ can be used as amendments in agriculture for degraded soils (Puoci et al., 2008; Ramli et al., 2015).

The hydrolyzed collagen represents a high solubility product that could be used in food industry as food supplement or in cosmetic industry for skin care products (Yazaky et al., 2017). By functionalization of collagen hydrolysate with encapsulated nutrients a product with applications as biofertilizers could be obtained (Zainescu et al., 2017).

Collagen hydrolysate added to synthetic polymers could improve the biodegradability of plastic materials (Azeem et al., 2014), and also collagen basedmatrices could be successfully cross-linked in order to modify the mechanical properties or the biodegradation rates (Puccini et al., 2010).

This paper presents exploratory research as a starting point to obtain new polymeric complex products - multicomponent - called hydrogels, by processing organic waste with applications in agriculture (Katime et al., 2004).

Obtaining hydrogels with collagen structure by pelt waste hydrolysis with applications in agriculture is a novelty, given that collagen is used only in medicine (Cabeza et al., 1999).

Multicomponent absorbent hydrogel-type networks are next generation materials, with three-dimensional structure and high swelling capacity. The applications of these materials are diversifying, in recent years entering the fields of agriculture, food, pharmaceuticals, electrical devices and electronics, environmental protection and biomaterials (Haiyang et al., 2018). Hydrogels have a distinct three-dimensional structure, and although they have a high water content, hydrogels are water-insoluble due to the crosslinked (physical or chemical) structure of the steric or crystalline linkages. When the hydrogel is in contact with the aqueous solution, there is a swelling thereof.

\section{MATERIALS AND METHODS}

In this study, limed hide waste (treated with $1.5 \%$ sodium sulfide, $1.3 \%$ sodium hydrosulfide and 2,5\% calcium hydroxide) was used. Raw hide contains (based on dry weight) $55-70 \%$ protein, 0.6 to $9 \%$ fat, $20-50 \%$ ash and less than $7 \%$ water.

The waste came from fleshing and trimming cattle hides (weight category $40 \mathrm{~kg}$ ) from SC Pielorex tannery in Jilava, Ilfov County, Romania.

The technological process for obtaining smart hydrogel by pelt waste hydrolysis. An innovative process is proposed for treating rawhide waste by protein waste hydrolysis in acid or alkaline medium, to obtain a proteinaceous smart biopolymer which, in combination with other polymers (polyacrylamide, acrylic acid, maleic acid, cellulose, starch, etc.) can be used in agriculture as hydrogels with controlled release of nutrients.

The proposed technological process for obtaining protein hydrogel includes the following:

An amount of $6000 \mathrm{~g}$ of pelt waste is washed with water at a temperature of $20-25^{\circ} \mathrm{C}$ in a drum for 20-30 minutes (as it is strongly alkaline), hide waste is then ground using a special grinder (with double knives), yielding a pasty homogenous mass - protein biopolymer.

https://doi.org/10.24264/icams-2020.IV.4 
The protein biopolymer is introduced together with $3.5-5 \%$ dipotassium hydrogen phosphate (which helps to improve the nutritional properties by the addition of phosphorus and potassium) in an autoclave equipped with heating jacket and agitator.

The mixture is stirred for $60-120 \mathrm{~min}$ at $75-85^{\circ} \mathrm{C}$. Then to this mixture an amount of $1-$ $1.5 \%$ boric acid is added and the mixture is removed from the autoclave in plastic drums.

Depending on the fertilizer particle structure, the resulting hydrogel may form the matrix where the fertilizer is embedded or the coating for the solid fertilizer (mono- or multi-layered particles).

\section{RESULTS AND DISCUSSIONS}

A hydrogel is defined as a polymer network which has the property of absorbing large amounts of solvent causing macroscopic changes in the dimensions of the polymer. The most important property of hydrogels is their degree of swelling as well as dissolution and gradual release of water and nutrients needed for plant growth.

\section{Characterisation of Compounded Hydrogel}

Physical chemical characterization of smart hydrogel is given in Table 1, where for the samples studied the following codes have been allocated:

$\mathrm{HC}$ - collagen hydrolysate,

SHC - collagen hydrolysate with nutrients encapsulated.

Table 1. Chemical analysis of tested fertilizers

\begin{tabular}{cccccc}
\hline No & $\begin{array}{c}\text { Fertilizer } \\
\text { Parameter }\end{array}$ & UM & HC & SHC & Method of analysis \\
& Dry substance & $\%$ & 24.15 & 60.18 & SR EN ISO 4684: 2006 \\
\hline 1 & Ash & $\%$ & 2.35 & 21.36 & SR EN ISO 4047: 2002 \\
2 & Total nitrogen & $\%$ & 10.36 & 12.55 & SR ISO 5397: 1996 \\
3 & Soluble phosphorous, $\mathrm{P}_{2} \mathrm{O}_{5}$ & $\%$ & - & 6.75 & SR EN 15959: 2012 \\
4 & Soluble potassium, $\mathrm{K}_{2} \mathrm{O}$ & $\%$ & - & 10.62 & SR ISO 5397: 1996 \\
5 & Total organic carbon, TOC & $\%$ & 46.2 & 45.2 & SR EN 13137/2005 \\
6 & $\mathrm{pH}$ & units & 6.70 & 7.20 & STAS 8619/3-1990 \\
7 & & & &
\end{tabular}

Total phosphorus $\left(\mathrm{P}_{2} \mathrm{O}_{5}\right)$, total potassium $\left(\mathrm{K}_{2} \mathrm{O}\right)$ and total sodium $\left(\mathrm{Na}_{2} \mathrm{O}\right)$ - were analyzed by extraction through wet mineralization with sulfonated per chloric mixture:

- $\mathrm{K}_{2} \mathrm{O}$ and $\mathrm{Na}_{2} \mathrm{O}$ were determined by atomic emission spectrophotometry;

- $\mathrm{P}_{2} \mathrm{O}_{5}$ was determined by molecular absorption spectrophotometry;

- Nt -mineralization and distillation by the Kjeldahl method.

Collagen hydrogels with encapsulated nutrients obtained are delivered in a dry state, packed in polyethylene bags accompanied by instructions for use.

This experimental model can establish a technology for converting pelt waste into collagen hydrogels with encapsulated nutrients, which can be used as fertilizers in agriculture (especially in horticulture).

The hydrogels were analyzed in terms of structure and elemental composition using modern instrumental methods, namely SEM-EDAX electron microscopy. Examination of collagen hydrogel samples with encapsulated nutrients by SEM EDAX shows the 
appearance of crosslinking centres and the formation of collagen-starch or collagenpolyacrylamide compounds can be seen.

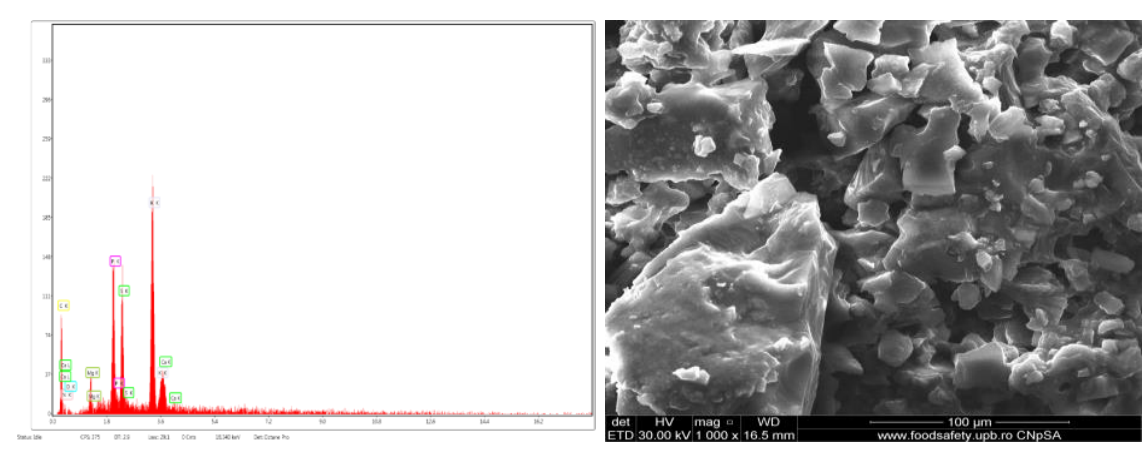

Figure 1. Elemental analysis by electron microscopy of smart hydrogel

SEM-EDAX micrographs of collagenic hydrogels with encapsulated nutrients for soil fertilization, showing the fibrillar collagenic structure with nutrient crystals phosphorus, potassium, magnesium, etc., are shown in Figure 1.

In conclusion, this work can establish a technology for converting pelt waste into collagen hydrogels with encapsulated nutrients, which can be used as fertilizers in agriculture, to remedy degraded soils.

Soil conditioning consists in improving the physical properties by using substances of various origins, known in the specialized literature as soil conditioners.

To highlight the structural changes in the process of hydrolysis and interaction with various synthetic polymers, attenuated total reflectance spectrophotometer FT/IR-ATR, Perkin Elmer, USA, was used.

Molecular absorption spectrometry in the infrared IR is based on vibration-rotation transitions that occur at the molecular level by absorbing infrared radiation. From IR spectra chemical bonds and the molecular structure of organic compounds may be identified. The bands specific for collagen are similar to those of other proteins. The IR spectrum shows bands of the amide I, II and III at about 1660, 1550 and $1240 \mathrm{~cm}^{-1}$, respectively.

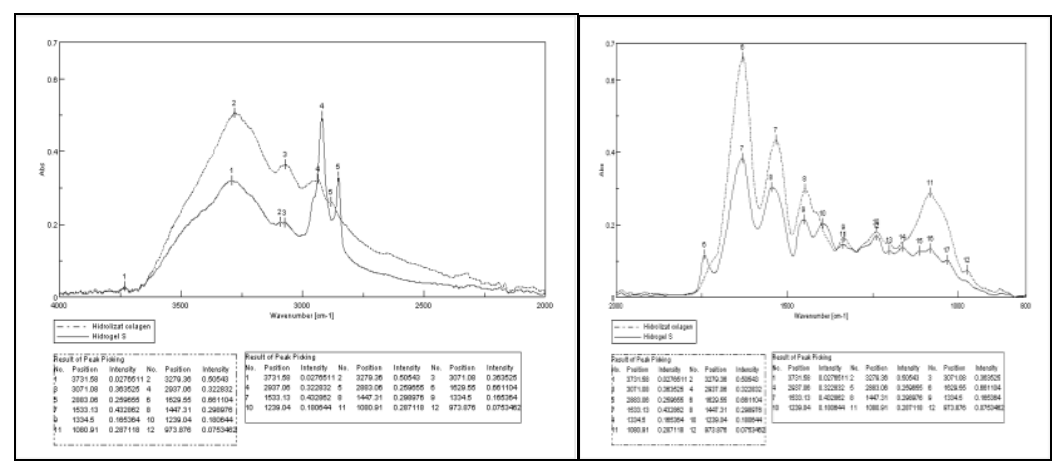

Figure 2. IR spectrum of the hydrogel, collagen/smart hydrogel 
Hydroxyl groups and the hydrogen bonds are recorded between 3600 and $3100 \mathrm{~cm}^{-1}$. According to the spectral assignments, in the case of collagen hydrolysate, bands were observed corresponding to amide groups $\left(v_{\mathrm{C}=0} 1645 \mathrm{~cm}^{-1}\right.$ to $\delta_{\mathrm{NH}}$ and $\left.v_{\mathrm{C}-\mathrm{N}} 1556 \mathrm{~cm}^{-1}\right)$.

Also, the characteristic signals of $\mathrm{NH}$ groups are present at $1338 \mathrm{~cm}^{-1}$. OH groups in units of hydroxyproline, are signalled at $1082 \mathrm{~cm}^{-1}$. Therefore, the bands at 1660 to $1550 \mathrm{~cm}^{-1}$, by their position and absorbance, give information on the degree of degradation. Hydrolysis of the chain is marked by changes in the band from 3450-3200 $\mathrm{cm}^{-1}$ region that tends to broaden and change its transmittance; at the same time, the band at $1660 \mathrm{~cm}^{-1}$ increases in intensity, because its structure includes a -OH component, and the band at $1550 \mathrm{~cm}^{-1}$ decreases in intensity. In the collagen hydrolysates, some of the $\mathrm{OH}$ groups are replaced with methoxide groups, $\left(\mathrm{CH}_{3}\right)$, which attenuate the hydrogen bonds and decrease the crystallinity of the collagen while increasing the water solubility.

\section{CONCLUSIONS}

A comprehensive study is presented on recycling hide waste resulted from leather industry by capitalising their valuable protein components to obtain efficient collagenbased hydrolysates as smart fertilizers for poor soils amelioration. Hydrogels as controlled fertilizer release systems in agriculture have major advantages in that they

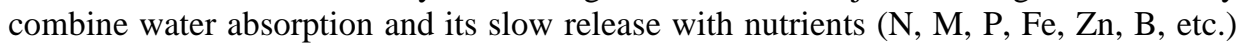
necessary for plant growth. The fertilizer quality is mainly conferred by the content in NPK nutrients available for plant growth and their leaching in soil solutions.

A framework technology was established for obtaining hydrogels with collagen structure from pelt waste.

\section{Acknowledgement}

This project is funded by the Ministry of Research and Innovation within Program 1 - Development of the national R\&D system, Subprogram 1.2 - Institutional Performance - RDI excellence funding projects, Contract no 6 PFE/16.10.2018

\section{REFERENCES}

Azeem, B. et al. (2014), "Review on materials and methods to produce controlled release coated urea fertilizer" Journal of Controlled Release, 11-21, https://doi.org/10.1016/j.jconrel.2014.02.020.

Bódalo, A. and Gómez, J.L. (2007), "Study of the evaporation process of saline waste from the tanning industry", Waste Manag, 25, 467-474, https://doi.org/10.1177/0734242X07079869.

Cabeza, L.F. et al. (1999), "Isolation of protein products from chromium-containing leather waste using two consecutive enzymes and purification of final chromium product: pilot plant studies", J. Soc. Leather Technol. Chem., 83, 14-19.

Chen, C. et al. (2008), "Development of comprehensive utilization of leather solid waste", Leather Sci. Eng., 18, 27-33.

Collivignarelli, C. et al. (1984), "Waste recovery in the tanning industry". Waste Manag. Res., 2, 265-278, https://doi.org/10.1177/0734242X8400200130.

Eurostat (2019), "Circular economy in the EU, Record recycling rates and use of recycled materials in the EU”, 39/2019, available at: https://ec.europa.eu/eurostat/documents/2995521/9629294/8-04032019-BPEN.pdf/295c2302-4ed1-45b9-af86-96d1bbb7acb1.

Haiyang, Y. et al. (2018), "Synthesis and self-assembly of multiple-responsive magnetic nanogels", Polymers for Advanced Technologies, 30(2), 312-319, https://doi.org/10.1002/pat.4467.

Katime, I. et al. (2004), Los materiales inteligentes de este milenio: Los hidrogeles macromoleculares. Síntesis, propiedades y aplicaciones, Servicio Editorial de la Universidad del País Vasco, España.

https://doi.org/10.24264/icams-2020.IV.4 


\section{Smart Biopolymers from Protein Wastes Used in Agriculture}

Nogueira, F.G.E. et al. (2010), "Incorporation of mineral phosphorus and potassium on leather waste (collagen): A new $\mathrm{N}_{\text {collagen }} \mathrm{PK}$-fertilizer with slow liberation”, Journal of Hazardous Materials, 176374 380, https://doi.org/10.1016/j.jhazmat.2009.11.040.

Puccini, M. et al. (2015), "Utilization of starch films plasticized with urea as fertilizer for improvement of plant growth", Chemical Engineering Transactions, 43, 7005-7010.

Puoci, F. et al. (2008), "Polymer in Agriculture: a Review", American Journal of Agricultural and Biological Sciences, 3(1), 299-314, https://doi.org/10.3844/ajabssp.2008.299.314.

Ramli, N.K. et al. (2015), "A comprehensive review on biodegradable polymers and their blends used in controlled release fertilizer processes", Rev Chem Eng, 31(1), 69-95, https://doi.org/10.1515/revce-20140021

Yazaki, M. et al. (2017), "Oral Ingestion of Collagen Hydrolysate Leads to the Transportation of Highly Concentrated Gly-Pro-Hyp and Its Hydrolyzed Form of Pro-Hyp into the Bloodstream and Skin", $J$. Agric. Food Chem., 65, 2315-2322, https://doi.org/10.1021/acs.jafc.6b05679.

Zainescu, G. and Constantinescu, R.R. (2017), "Smart hydrogels with collagen structure made of pelt waste", Rev. Chim. (Bucharest), 68(2), 393-395, https://doi.org/10.37358/RC.17.2.5461. 


\title{
COMPARATIVE ANALYSIS OF SUSTAINABLE DEVELOPMENT
} INDICATORS AT E.U. AND ROMANIAN LEVEL

\author{
DANA CORINA DESELNICU ${ }^{1}$, BEATRICE ALEXANDRA ALEXANDRESCU ${ }^{2}$ \\ ${ }^{1}$ University Politehnica of Bucharest, Romania, d_deselnicu@yahoo.com \\ ${ }^{2}$ Leaders Foundation, Bucharest, Romania
}

\begin{abstract}
Sustainable development aims for a better future for us and future generations. To follow it, the 2030 Agenda for Sustainable Development was developed, which proposes, through its 17 objectives, the intervention directions. The 2030 Agenda was adopted by both Romania and the EU. Each objective is represented by a set of indicators. The objective of the paper is the comparative analysis of the most important indicators of sustainable development in EU and Romania. In this paper, the main indicators of sustainable development at EU level were analyzed. Subsequently, the same indicators were investigated in Romania, to perform a comparative analysis. The research used secondary data. Romania's declared objective is to be a regional leader in implementing Sustainable Development goals. Based on the results obtained on data available in 2020, a set of recommendations was developed to reach the proposed target for 2030. Analyzing the sustainable development indicators for EU and Romania against the new EU 2030 Agenda. The paper provides an overview of the Sustainable development of Romania. Its main indicators are compared against the values at EU level, resulting in relevant recommendations that can be implemented to meet the goals of EU 2030 Agenda.
\end{abstract}

Keywords: EU 2030 agenda, economic growth, sustainability indicators

\section{INTRODUCTION}

The official definition of sustainable development was first developed in 1987 through the Brundtland Report. Sustainable development is a way for people to use resources without the planet being left without resources for decades, centuries, or even millennia. This means growing without damaging or harming the environment. The term used by the Brundtland Commission is sustainable development that "meets the needs of the present and also compromises the ability of future generations to meet their own needs" (Brundtland, 1987).

Sustainable development is based on three pillars:

1. Environmental sustainability refers to the ecological component that must be found in every initiative by protecting biodiversity;

2. Economic sustainability or sustainable development in economic terms involves methods of economic diversification. These can be, access to services and environmental protection that are necessary to ensure the success and sustainability of development (Ukaga et al., 2019);

3. Socio-political sustainability presupposes, on the one hand, healthy socio-political relations that allow the development of all states, based on partnership and mutual support.

In 2015, at a meeting of the United Nations General Assembly in New York, a historic document was adopted: the 2030 Agenda for Sustainable Development. Through its 17 goals, this document seeks to achieve a better future not only for us but also for the next generation. Built on the three pillars of sustainable development economic, social and environmental - the 2030 Agenda was also adopted by Romania and the European Union. This strategy translates the Agenda to the realities of Romania today.

https://doi.org/10.24264/icams-2020.IV.5 


\section{The European Union Perspective on Sustainable Development}

The concept of sustainable development in the European Union was introduced into the Strategy for an Enlarged Europe 2006. This strategy was part of a unified and coherent strategic vision with the general objective of continuously improving the quality of life for present and future generations (Freeman, 2010). Its goal was to create sustainable communities capable of managing and using resources efficiently and of exploiting the ecological and social potential of the economy to ensure prosperity, environmental protection, and social cohesion (Hák et al., 2016).

On 22 November 2016, the European Commission published "Next Steps for a Sustainable European Future.” This document presents the European Union's response to the 2030 Agenda, confirming the need to integrate the Sustainable Development Goals into the European policy framework while highlighting the European Commission's current priorities (Mohieldin, 2017). The document also presents an evaluation of the Union's current situation and identifies the most relevant concerns regarding sustainability. The European Union declared itself in favor of a sustainable development that will ensure "a life of dignity for all within the Planet's limits that reconciles economic prosperity and efficiency, peaceful societies, social inclusion, and environmental responsibility." The EU Council's conclusions in "A sustainable future for Europe: the EU response to the 2030 Agenda for Sustainable Development," adopted on 20 June 2017, represents the political document adopted by the member states of the EU regarding the implementation of the 2030 Agenda for Sustainable Development. The EU response to the 2030 Agenda is to integrate the 17 SDGs into its public policies to support the global effort to build a sustainable future.

\section{The Romanian Perspective on Sustainable Development}

In Romania, as a member state of the European Union, in 1997 the National Center for Sustainable Development was created, under the auspices of the Romanian Academy, which over time managed to become the most authorized voice in civil society in the field of developing proposals and strategies for development (Deselnicu et al., 2017). The purpose of the National Center for Sustainable Development is to identify Romania's sustainable development priorities and achieve them through concrete projects at national and local levels.

To achieve sustainable development in Romania, and, by extension, to meet the goals of the 2030 Agenda, together with the European Union's commitments regarding the 2030 Agenda, this Strategy is built around the citizen and the needs of future generations. Romania's Sustainable Development Strategy is based on the premise that sustainable development requires a mindset which, once adopted by the citizen, will help create a more equitable society defined by balance and solidarity, and the ability to cope with the changes brought about by current global, regional and national challenges, including a declining population. The state's concern for its citizens, and the citizens' respect for public institutions, for their peers, for moral values, and cultural and ethnic diversity will lead to a sustainable society.

The 2030 Agenda includes a set of 17 Sustainable Development Goals (SDGs) and an action plan for the next 15 years, to eradicate extreme poverty, combat inequality, injustice and protect the planet by 2030 (Kanie et al., 2017). The 17 Sustainable Development Goals (SDGs) and their related 169 targets, which are at the heart of the UN's 2030 Agenda for Sustainable Development, provide a new policy framework 
worldwide towards ending all forms of poverty, fighting inequalities, and tackling climate change while ensuring that no one is left behind (United Nations, 2020).

\section{Comparative Analysis of Sustainable Development Indicators}

In this paper, two of the 17 objectives of sustainable development have been chosen:

Responsible consumption and production (SDG 12) calls for action on all fronts: adoption of sustainable practices and sustainability reporting by businesses; promotion of sustainable procurement practices and rationalization inefficient fossil-fuel subsidies by policy-makers; environmentally-aware lifestyles of consumers; development of new technologies and production and consumption methods by researchers and scientists and others.

Decent work and economic growth (SDG 8) recognizes the importance of sustained economic growth and high levels of economic productivity for the creation of well-paid quality jobs and the achievement of global prosperity. SDG 8 calls for providing opportunities for full and productive employment and decent work for all while eradicating forced labor, human trafficking, and child labor and promoting labor rights and safe and secure working environments.

Every SDG has many indicators of which are analyzed. For this paper there were chosen two representative indicators for each goal. SGD 12 Responsible consumption and production - comparatively analyzed indicators: Energy Productivity and Resource productivity, and domestic material consumption. The values of the indicators in Romania were compared with the average of the European states (Figure 1):

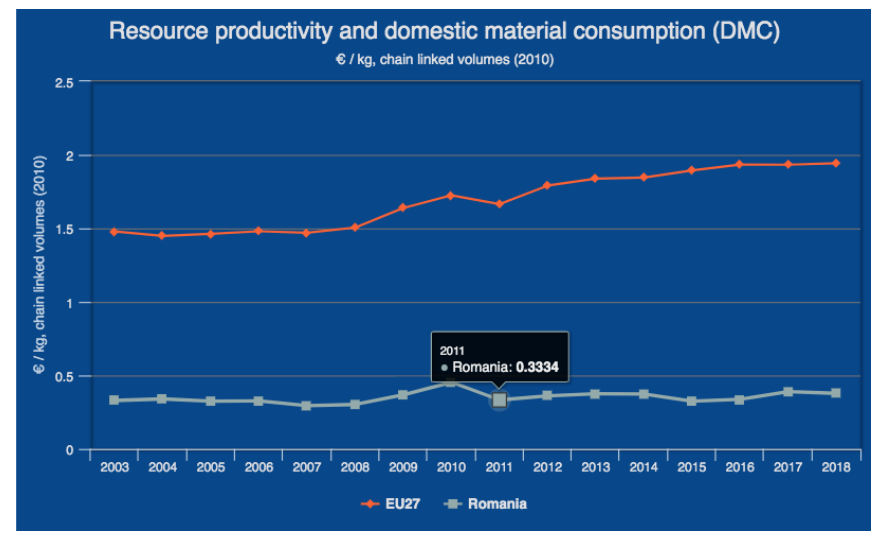

Figure 1. Resource productivity and domestic material consumption comparison between EU 27 and Romania. Source: Eurostat, 2020

There is a major difference in the level of the average indicator in the $27 \mathrm{EU}$ states and Romania's level. Resource productivity varies greatly from one Member State to another. It depends to a large extent on the structure of national economies and the size and structure of their international trade. Usually, open industrial economies consume more resources because they import large quantities of raw materials, which are then exported as finished products. Service-based economies, on the other hand, tend to generate GDP from activities that consume fewer raw materials, such as financial services, tourism, the arts and recreation, healthcare, and public administration. Therefore, service-based savings appear to be more efficient because they consume 
fewer material resources per euro of production. In the case of Romania, a low and constant level of productivity can be observed (Figure 2):

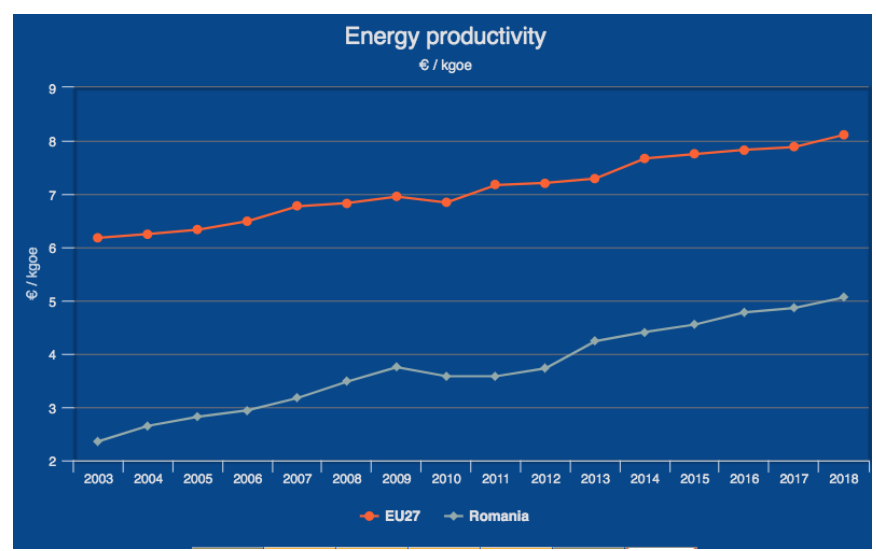

Figure 2. Energy productivity, comparison between EU 27 and Romania.

Source: Eurostat, 2020

Making progress towards a more resource-efficient economy also means reducing energy consumption at all stages of the energy chain, from production to final consumption. This means providing more services for the same energy input or the same services for lower energy input. This is the indicator that has a constant and positive evolution. Romania follows the ascending trend, achieving, as can be seen, considerable progress from 2003 to 2018, with a faster growth rate than the EU 27 (Figure 3):

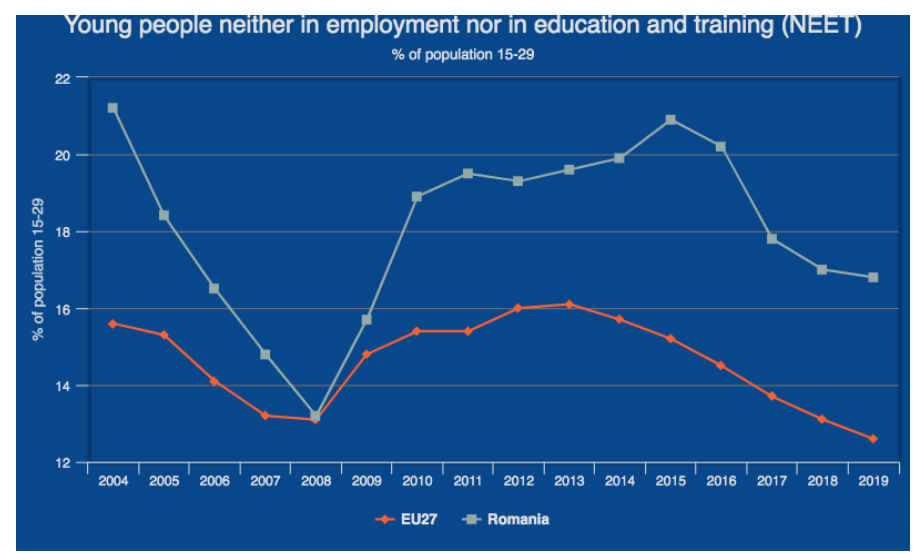

Figure 3. Young people neither in employment nor in education and training (NEET), comparison between EU 27 and Romania. Source: Eurostat, 2020

The transition of young people from education to work is hampered by specific difficulties. As a result, there are relatively low employment rates, a high unemployment rate, and high rates of young people who are not employed and do not 
attend any education or training (NEET) program. It can be noticed that Romania has a higher percentage within this indicator than the EU 27 average. According to the data, the percentage is decreasing starting with 2015 , when a maximum of $21 \%$ of the total population analyzed was reached, although in 2019, the difference between the EU27 and Romania average is 5 percent (Figure 4):

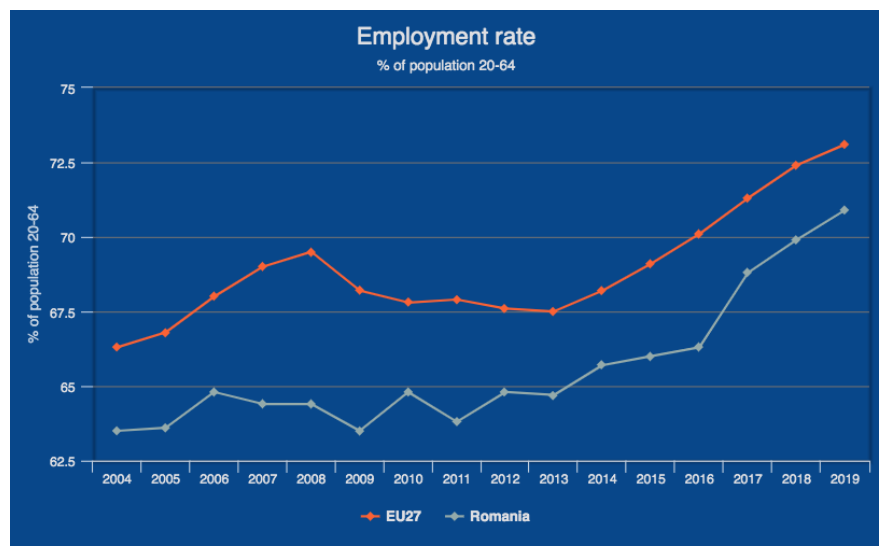

Figure 4. Employment rate, comparison between EU 27 and Romania.

Source: Eurostat

This indicator reached historic highs in 2019, according to data provided by Eurostat (2020). Romania has also reached a historic percentage. The difference between the EU 27 average and Romania is $3 \%$. These results are very important, being a relevant economic indicator for analysis. As in the case of other indicators, there can be observed large differences between countries. Romania has the highest percentage, followed by Sweden $(82.6 \%)$, while the lowest percentage is held by Greece $(59.7 \%)$.

\section{RESULTS}

In this paper, four very important sustainability indicators were analyzed, related to the two selected objectives. Following the analysis, it was found that Romania is following a positive trend and the continuous improvement of the indicators, as it happens at EU level. A low level of the two indicators related to SGD 12 - Responsible consumption and production - can be observed. The low level of Resource productivity and domestic material consumption represents an effect of the type of Romanian economy, in which the services industry has a much lower share than the European average. In parallel, positive results are observed within SDG 8 - Decent work and economic growth.

\section{Recommendations to Reach the Proposed Target for 2030}

The following set of recommendations was suggested in order for Romania to reach its proposed target in terms of sustainable development for the year 2030:

- Development of public-private partnership by increasing the number of education classes in the dual system; 
- Initiation of basic vocational training programs in correlation with the requirements of the labor market, focusing on young or socially vulnerable segments of the population;

- Elaboration and implementation of a coherent program for the development of tourism, including agrotourism, generator of jobs and considerable income in areas where the employment rate is low;

- Development of the legislative framework for the support of green housing;

- The transition to the circular economy by changing the mentality through education, changing consumer behavior and developing financial mechanisms to support the transition period;

Reduction of food waste along the entire production-transport-processingmarketing-consumption route, from farm harvesting to final waste disposal;

- Popularization and promotion of sustainable production and consumption models through information campaigns for the general public and efforts to expand these good practices in school and extracurricular educational programs;

- $\quad$ Encourage companies, especially large and transnational companies, to adopt sustainable practices and integrate sustainability information into the reporting cycle.

\section{CONCLUSION}

In the present paper, following the analysis, it was discovered that Romania makes sustained progress every year on each objective and indicator. It is observed that it follows the EU trend and has real chances to become a regional leader in implementing the 2030 Sustainability Agenda. To become a regional leader in implementing the 2030 Sustainability Agenda, it is necessary to involve all actors in achieving the objectives. The involvement of every citizen is imperative in carrying out this strategy. It takes the formation of critical mass, the moment when these principles will be learned by most citizens, to achieve the change we want. In this way, we can be optimistic about achieving the proposed objectives.

\section{REFERENCES}

Brundtland, G.H. (1987), Report of the World Commission on Environment and Development - Our Common Future, UN, available at: www.sustainabledevelopment.un.org.

Deselnicu, D.C., Militaru, G., Deselnicu, V., Zăinescu, A.G. and Albu, L. (2017), "Sustainable Development in the Frame of the 7th Environment Action Programme", Proceedings of the International Conference on Advanced Materials and Systems (ICAMS 2018), 557-562, https://doi.org/10.24264/icams-2018.XI.3.

Freeman, E. (2010), Strategic Management: A Stakeholder Approach, Cambridge University Press, Cambridge, https://doi.org/10.1017/CBO9781139192675.

Hák, T., Janoušková, S. and Moldan, B. (2016), "Sustainable development goals: A need for relevant indicators", Ecological Indicators, 60, 565-573, https://doi.org/10.1016/j.ecolind.2015.08.003.

Kanie, N. and Biermann, F. (2017), Governing through goals; sustainable development goals as governance innovation, MIT Press, Cambridge, https://doi.org/10.7551/mitpress/10894.001.0001.

Mohieldin, M. (2017), The sustainable development goals and private sector opportunities, EAFIT University of Medellin, available at: www.pubworks.worldbank.org.

Ukaga, U., Maser., C. and Reichenbach, M. (2019), Sustainable development: principles, frameworks, and case studies, CRC Press, Florida.

United Nations (2020), Transforming our world: the 2030 Agenda for Sustainable Development, available at: https://sustainabledevelopment.un.org/post2015/transformingourworld. 


\title{
LIFE GREENSHOES4ALL - FOOTWEAR ENVIRONMENTAL FOOTPRINT
}

\author{
MARIA JOSÉ FERREIRA, VERA V. PINTO, PATRÍCIA COSTA \\ CTCP - Portuguese Footwear Research and Technological Centre, Rua de Fundões - Devesa \\ Velha, 3700-121, São João da Madeira, Portugal, mjose.ferreira@ctcp.pt
}

\begin{abstract}
One important step towards sustainability in footwear industries is to measure and tune the environmental impact a product makes throughout its life cycle. By performing a product's life cycle assessment (LCA), the footwear value chain can produce footwear more responsibly, economically and in an eco-friendly way by addressing the three pillars of sustainability. LIFEGreenShoes4All teams are conducting LCA studies in representative footwear models ranging from fashion to safety footwear, with uppers in leather or textiles; midsoles in polyurethane foam (PU) or ethyl vinyl acetate (EVA); soles in vulcanized rubber, thermoplastic rubber (TR), EVA and others. These studies make possible to identify and quantify the most relevant life cycle stages, contributing to the most relevant impact categories (e. g, climate change, resource use), helping companies on the definition of how their footwear environmental performance may be improved. Considering the results, the higher contributors are materials and components, followed by waste from manufacturing and end-of-life. The creative design phase plays a significant role in footwear life cycle sustainability impact. The implementation of ecodesign on the product conception is crucial to reduce the Product Environmental Footprint (PEF). LIFEGreenShoes4All is being developed by 9 partners AMF, APICCAPS, ATLANTA, CEC, CTCP, EVATHINK, ICPI, INESCOP, FICE and PESTOS (https://www.greenshoes4all.eu/)
\end{abstract}

Keywords: Footwear, Product Environmental Footprint, Ecodesign

\section{BACKGROUND}

Nowadays, there is a proliferation of so called 'eco' labels or schemes, and misleading 'green' claims. Consumers find this confusing and footwear manufacturers that want to produce 'green products' find it hard to differentiate their products from these and need the right tools to do. Life Cycle Assessment (LCA) standards may be too flexible to ensure comparability of results. In this respect, Product Environmental Footprint (PEF) could play an important role.

The LIFE GreenShoes4All project is designed to support the implementation of a Product Environmental Footprint evaluation methodology, to help companies involved in the footwear business to measure the environmental performance of their products. The PEF methodology envisages to introduce several improvements compared to other existing LCA methods including, namely, clear identification of the potential environmental impact categories to be looked at, data sets and minimum data quality requirements.

LIFE GreenShoes4All project and metrics also encompasses supply chain activities from materials and products industrial production through the product use and waste management. The project will propose more tangible targets on raw materials selection, products ecodesign and manufacturing, waste polymers recycling (rubber, thermoplastic and EVA), and measures to reduce greenhouse gas emissions.

The partnership involved includes research organisations, footwear associations and manufacturers of materials, components and footwear from Belgium, Portugal, Romania, and Spain, all working to achieve these objectives. In 2020, the project opened to new EU stakeholders aiming to apply the draft PEF methodology and ecodesign approaches to footwear products and all are welcome onboard. Due to its 


\section{Life GreenShoes4All - Footwear Product Environmental Footprint}

importance this paper is dedicated to a product's life cycle evaluation using the draft PEF methodology.

\section{EXPERIMENTAL}

\section{Materials}

Five shoe models presenting aesthetics, touch, and general properties usual in casual ladies' and man's shoes were selected and the impact of feasible modifications studied. Table 1 presents generically the models, describes the main materials and components studied and details the functional unit defined to perform the PEF/LCA studies. 1-year lifetime was assumed.

Table 1. Shoe models description and functional unit

\begin{tabular}{|c|c|c|}
\hline Model & Description & Functional Unit \\
\hline Sandal & $\begin{array}{l}\text { Leather upper } \\
\text {. Leather lining } \\
\text {. TR outsole } \\
\text {. Leather upper } \\
\text {. Polyester lining } \\
\text {. TR outsole } \\
\text {. Leather upper } \\
\text {. Polyester lining } \\
\text {. PU foam outsole }\end{array}$ & $\begin{array}{l}\text { One pair of sandals, size } 37 \text {, packed. } \\
\text { Total weight: } 0,922 \mathrm{~kg} \text {. } \\
\text { Pair of shoes: } 0,628 \mathrm{~kg} \text {. } \\
\text { One pair of sandals, size } 37 \text {, packed. } \\
\text { Total weight: } 0,922 \mathrm{~kg} \text {. } \\
\text { Pair of shoes: } 0,628 \mathrm{~kg} \text {. } \\
\text { One pair of sandals, size } 37 \text {, packed. } \\
\text { Total weight: } 0,692 \mathrm{~kg} \text {. } \\
\text { Pair of shoes: } 0,397 \mathrm{~kg} \text {. }\end{array}$ \\
\hline Low boot & $\begin{array}{l}\text { Leather upper } \\
\text { Polyester lining } \\
\text { TR outsole } \\
\text {. Leather upper } \\
\text { Polyester lining } \\
\text {. PU foam }\end{array}$ & $\begin{array}{l}\text { One pair of boots, size } 37 \text {, packed. } \\
\text { Total weight: } 1,119 \mathrm{~kg} \text {. } \\
\text { Pair of shoes: } 0,825 \mathrm{~kg} \text {. } \\
\text { One pair of boots, size } 37 \text {, packed. } \\
\text { Total weight: } 0,929 \mathrm{~kg} \text {. } \\
\text { Pair of shoes: } 0,635 \mathrm{~kg} \text {. }\end{array}$ \\
\hline Medium boot & $\begin{array}{l}\text { Leather upper } \\
\text { Cotton lining } \\
\text { TR outsole } \\
\text {. Leather upper } \\
\text { Cotton lining } \\
\text { PU foam }\end{array}$ & $\begin{array}{l}\text { One pair of boots, size } 37 \text {, packed. } \\
\text { Total weight: } 1,213 \mathrm{~kg} \text {. } \\
\text { Pair of shoes: } 0,918 \mathrm{~kg} \text {. } \\
\text { One pair of boots, size } 37 \text {, packed. } \\
\text { Total weight: } 0,939 \mathrm{~kg} \text {. } \\
\text { Pair of shoes: } 0,644 \mathrm{~kg} \text {. }\end{array}$ \\
\hline High Boot & $\begin{array}{l}\text { Leather upper } \\
\text {. Polyester lining } \\
\text { TR outsole } \\
\text {. Leather upper } \\
\text {. Polyester lining } \\
\text {. PU foam } \\
\text {. Leather and synthetic } \\
\text { upper. Polyester lining } \\
\text {. PU foam }\end{array}$ & $\begin{array}{l}\text { One pair of boots, size } 37 \text {, packed. } \\
\text { Total weight: } 1,532 \mathrm{~kg} \text {. } \\
\text { Pair of shoes: } 0,993 \mathrm{~kg} \text {. } \\
\text { One pair of boots, size } 37 \text {, packed. } \\
\text { Total weight: } 1,285 \mathrm{~kg} \text {. } \\
\text { Pair of shoes: } 0,746 \mathrm{~kg} \text {. } \\
\text { One pair of boots, size } 37 \text {, packed. } \\
\text { Total weight: } 1,285 \mathrm{~kg} \text {. } \\
\text { Pair of shoes: } 0,746 \mathrm{~kg}\end{array}$ \\
\hline Men casual shoe & $\begin{array}{l}\text { Leather upper } \\
\text { EVA outsole }\end{array}$ & $\begin{array}{l}\text { One pair of shoes, size } 42, \text { packed. } \\
\text { Total weight: } 1,081 \mathrm{~kg} \text {. } \\
\text { Pair of shoes: } 0,803 \mathrm{~kg} \text {. }\end{array}$ \\
\hline
\end{tabular}




\section{PEF Method}

A PEF study is essentially a standardised LCA study aiming to ensure that environmental information is comparable and reliable and can be used confidently. During a product's life cycle assessment, environmental impacts are evaluated with a holistic view of environmental interactions that covers a range of activities, from the extraction of raw materials from the Earth, the production and distribution of energy, through the upstream and downstream processes associated with materials and products production and end-of-life (EoL).

A Footwear PEF/LCA study includes essentially five steps.

1. Define the functional unit, e.g. 'one pair of shoes including packaging' (Table 1).

2. Define the system boundary, e.g. 'cradle-to-grave system boundary'. Figure 1 shows a simplified cradle-to-grave system boundary.

3. Collect primary data, namely, the composition and weight of materials and the components employed for the manufacture of the shoes, water and energy consumption, production waste, annual total production, suppliers, and retailers' locations, and EoL.

4. Select appropriate secondary data, PEF data sets, modelling, and tools.

5. Insert data and calculate values for the impact categories defined.

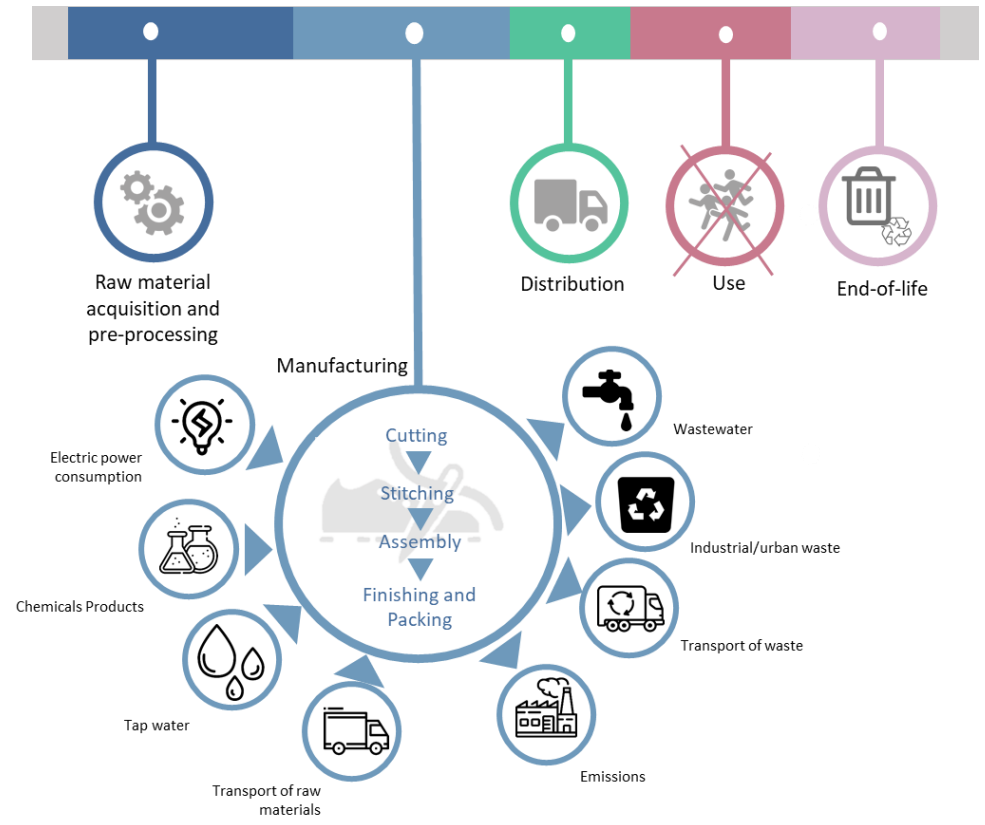

Figure 1. Life cycle stages included in the PEF/LCA study

In this study specific primary data was collected during visits to the companies and other information provided by them. Additionally, the application of alternative materials was theoretically evaluated. Since the use phase of footwear is usually insignificant its impact was not considered. Regarding secondary data, Ecoinvent database v3.3 and other data sets were used. The software used to model the data was OpenLCA 1.9. The impact categories were calculated using the EU EF method (adapted). 


\section{Life GreenShoes4All - Footwear Product Environmental Footprint}

\section{RESULTS AND DISCUSSION}

The applied footwear PEF method assesses 16 impact categories (Table 2), covering, namely, climate change, acid rain, human and ecotoxicity, and particulate matter as well as impacts due to the use of water, land, and resources.

Table 2. PEF Impact Categories, including indicators and units.

\begin{tabular}{|c|c|}
\hline Impact Categories & Indicator [Unit] \\
\hline Climate change & Global Warming Potential $\left[\mathrm{kg} \mathrm{CO}_{2} \mathrm{eq}\right]$ \\
\hline Ozone depletion & Ozone Depletion Potential [kg CFC-11eq] \\
\hline Human toxicity, cancer effects & Comparative Toxic Unit for humans [CTUh] \\
\hline Human toxicity, non-cancer effects & Comparative Toxic Unit for humans [CTUh] \\
\hline $\begin{array}{l}\text { Particulate matter, respiratory } \\
\text { inorganics }\end{array}$ & $\begin{array}{l}\text { Human health effects associated with exposure to } \\
\text { PM2.5 [Disease incidences] }\end{array}$ \\
\hline Ionizing radiation, human health & Human exposure efficiency relative to $\mathrm{U}[\mathrm{kBq} \mathrm{U}]$ \\
\hline $\begin{array}{l}\text { Photochemical ozone formation, } \\
\text { human health }\end{array}$ & $\begin{array}{l}\text { Tropospheric ozone concentration increase } \\
\text { [kg NMVOC eq] }\end{array}$ \\
\hline Acidification & Accumulated Exceedance (AE) $[\mathrm{mol} \mathrm{H}+$ eq] \\
\hline Eutrophication, terrestrial & Accumulated Exceedance (AE) [mol H+ eq] \\
\hline Eutrophication, aquatic freshwater & Fraction of nutrients to freshwater $(\mathrm{P})[\mathrm{kg} \mathrm{P}$ eq] \\
\hline Eutrophication, aquatic marine & Fraction of nutrients to marine $(\mathrm{N})[\mathrm{kg} \mathrm{N}$ eq] \\
\hline Ecotoxicity freshwater & Comparative Toxic Unit for ecosystems [CTUe] \\
\hline Land use & Soil quality index [Dimensionless*] \\
\hline Water use & User deprivation potential [kg world eq. deprived] \\
\hline Resource use, minerals and metals & Abiotic resource depletion $[\mathrm{kg} \mathrm{Sb} \mathrm{eq}]$ \\
\hline Resource use, fossils/energy carriers & Abiotic resource depletion - fossil fuels [MJ] \\
\hline
\end{tabular}

Figure 2 presents the most relevant impact categories determined based on the normalised and weighted results for a cumulative contribution of $84 \%$ of the total. The other 9 impact categories totalize $16 \%$ of the total.

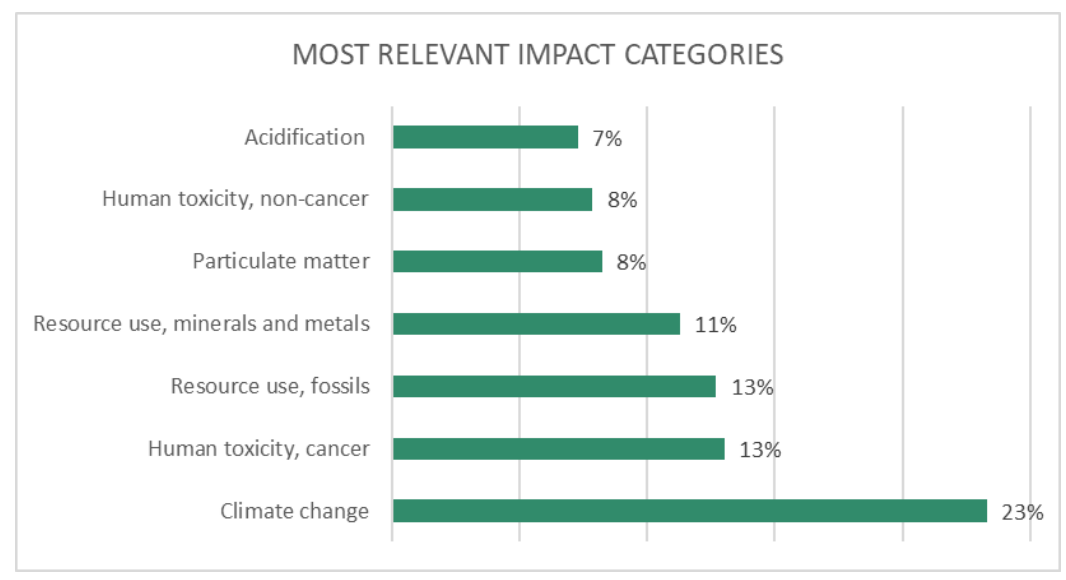

Figure 2. Most relevant impact categories calculated based on sandal 
Among these, as "Climate Change" is the most relevant impact category, and usually the more well-known, was chosen to present and discuss the environmental impact of the shoe models. Table 3 presents the results of the Climate Change impact category, Global Warming Potential indicator (GWP100), in $\mathrm{kg} \mathrm{CO}_{2}$ eq, calculated for each pair of footwear. Analysing the values of $\mathrm{kg} \mathrm{CO}_{2}$ eq obtained for the ladies' shoes, in general, an increase of the amount of materials (mass) used, results in an increase of footwear EF. Additionally, the compositions of the materials and components are relevant for the EF of footwear products. These conclusions are corroborated by the $\mathrm{kg}$ $\mathrm{CO}_{2}$ eq obtained for man's shoes, because despite the higher size, the use of specific materials results in an EF similar to some ladies' footwear.

Table 3. Impact on climate change as GWP100

\begin{tabular}{lc}
\hline \multicolumn{1}{c}{ Model } & $\mathrm{Kg} \mathrm{CO} 2 \mathrm{eq}$ \\
\hline Sandal & $6-9$ \\
Low boot & $13-15$ \\
Medium boot & $13-16$ \\
High boot & $14-19$ \\
Men casual shoe & $13-15$ \\
\hline
\end{tabular}

Figure 3 shows the contribution of each life cycle stage to the Climate Change impact category, for each shoe model type. The results indicate that materials selection and pre-processing (including, raw materials, components, adhesives, and packaging) is the most relevant life cycle stage, representing around (75 to 90) \% of the total GWP100 in $\mathrm{kg} \mathrm{CO}_{2}$ eq. The heavier components, upper and outsole, are the main EF contributors, representing on average about (70 to 90$) \%$, depending on their mass and composition. Manufacturing (including namely electricity and production waste) and EoL represent, respectively, around (4 to 18) \% and (5 to 9) $\% \mathrm{~kg} \mathrm{CO}_{2}$ eq of the total GWP100. These range of results are related with the production processes and type of models. Distribution account for less than $2 \%$ of the total GWP100 in $\mathrm{kg} \mathrm{CO}_{2}$ eq.

Based on these results is clear that reducing the mass of materials, selecting lower environmental footprint (EF) materials, and reducing the waste generated during the manufacturing of footwear will have a great effect of the reduction of footwear EF. To this end, as most of the environmental impact is decided during the design and development phase of footwear products, the implementation of ecodesign approaches will bring several benefits to footwear business companies. To reduce its PEF, a company's plan could include the following actions:

1. Design its footwear to reduce the number of different materials used.

2. Preferably select light biobased, recycled, or recyclable materials and components from local suppliers.

3. Apply efficient digitalised production processes with low energy consumption and reduced emissions and wastes.

4. Make footwear lighter and durable, easier to repair, and/or recycle.

To extend the use stage of the product, minimise the consumption of resources and the impact at EoL is important to consider designing for disassembly, recycling, reuse, or recovery; biodegradability or compostability, among other approaches. 


\section{Life GreenShoes4All - Footwear Product Environmental Footprint}

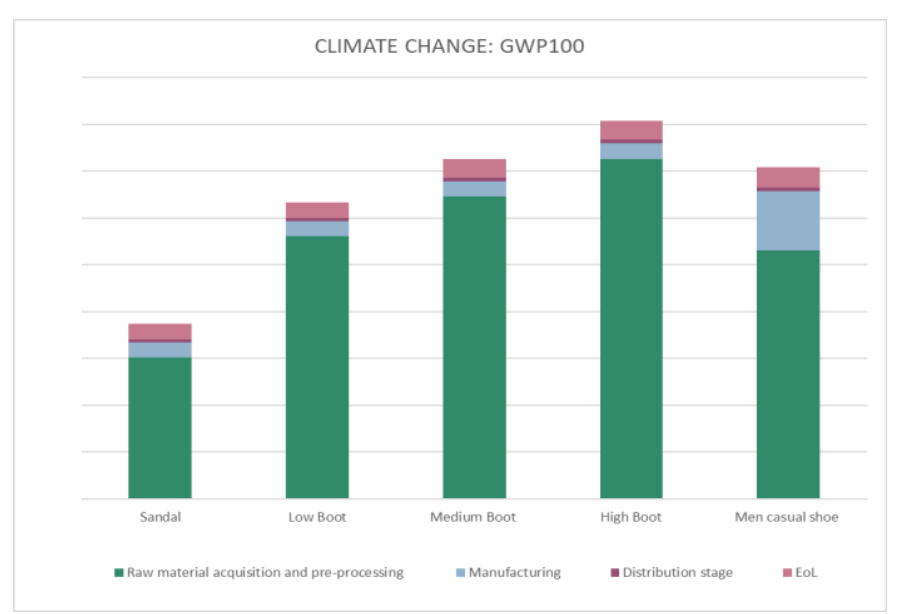

Figure 3. Contribution of each life cycle stage to the climate change impact category, using the GWP100 indicator $\left(\mathrm{Kg} \mathrm{CO}_{2} \mathrm{eq}\right)$

\section{CONCLUSIONS}

This study involves the evaluation and comparison of environmental footprint of five footwear models based on PEF method. The results indicate that for the "Climate Change" impact category, raw material acquisition and pre-processing is the most relevant life cycle stage. The reduction of footwear environmental footprint can be achieved by implementing several measures throughout the entire life cycle, namely, careful selection of materials and components (selection of lower impact raw materials), reduction of materials amount (mass), reduction of waste generation by implementing more efficient production processes (e.g., more efficient cutting process), buy local materials and use more efficient means of transport, among others. Companies will benefit from the implementation of ecodesign in the development of footwear products, since it is at this stage that the product concept, raw materials, as well as production process are defined. Additionally, adopting circular business models, aiming for the efficient use of natural resources powered by renewable energy, will add true value and environmental differentiation to footwear. Using PEF/LCA, these and other actions may be evaluated and tuned, enabling sustainable choices to be made and promoting the creation, production and use of 'truly green shoes' in a circular economy.

\section{Acknowledgements}

CTCP, the Portuguese Footwear Technology and Research Centre, thanks the support of the European project LIFE GreenShoel4All (LIFE17 ENV/PT/000337).

\section{REFERENCES}

Zampori, L. and Pant, R. (2019), "Suggestions for updating the Product Environmental Footprint (PEF) method", EUR 19682 EN, Publications Office of the European Union, Luxembourg, ISBN 978-92-7600654-1, https://doi.org/10.2760/424613, JRC115959.

*** (2015), thinkstep on Behalf of Sustainable Apparel Coalition, "First Draft Product Environmental Footprint Category Rules (PEFCR)", Rev. 0.1.

*** (2017), PEFCR Guidance document, "Guidance for the development of Product Environmental Footprint Category Rules (PEFCR)”, version 6.3, December 2017.

https://doi.org/10.24264/icams-2020.IV.6 
DESIGNING AND OBTAINING WOOD WASTE AND CHLOROPRENE RUBBER-BASED COMPOSITES

\author{
MIHAI GEORGESCU, MARIA SÖNMEZ, LAURENȚIA ALEXANDRESCU, MIHAELA \\ NIŢUICĂ, MARIA DANIELA STELESCU, DANA GURĂU \\ INCDTP - Division Leather and Footwear Research Institute, 93, Ion Minulescu St., 031215, \\ Bucharest, Romania, mihai.georgescu@icpi.ro
}

\begin{abstract}
The aim of this paper is to obtain and study the properties of wood waste reinforced elastomer composites with various fibre contents (10-50 wt $)$. The composite is based on chloroprene rubber, and added post-consumer recycled wood particles, with dimensions of $500 \mathrm{~nm}$ eco-reinforcing material, and active fillers, plasticizers, vulcanizing agents, antioxidants. In order to enhance the compatibility and their level of interaction, the wood waste was finely ground (cryogenic mill) and functionalized with potassium oleate. Wood waste acts as a filling material which leads to the biodegradability of the composite and the decrease in density. Tensile, tear strength, elasticity, hardness, abrasion resistance, melt flow index and morphological study (FT-IR) of those composites were examined in order to determine the viability in various applications domains.
\end{abstract}

Keywords: composite, wood waste, eco material.

\title{
INTRODUCTION
}

The waste management concept of integrating various materials into a composite, along with various recycling methods, offer new possibilities of reducing the footprint and amounts of wastes. Using those wastes in polymeric materials biodegradable composites can be obtained. Recycling and the use of renewable natural resources offer a new dimension in the discovery of new materials. Nowadays, special attention has been paid to the development of composites with polymer matrix reinforced with natural fibers.

The problem of waste has been addressed over time by several methods: depollution, consumption reduction, waste recovery, recycling, reusing.

The use of natural fibers as a reinforcing agent in the composites (Chaudemanche $e t$ al., 2018; Brostow et al., 2016) is gaining new momentum.

Types of natural fibers are:

- $\quad$ wood fibers (wood waste);

- leather fibers (leather waste);

- textile fibers (textile wastes, cloth scraps)

Wood waste is a set of products and materials whose origin comes from all stages of the wood industry, from logging to the manufacture of finished products. Also, the scrap wood (boxes, crates, pallets) represents a significant quantity (Turku et al., 2018; Najafi, 2013; Piao et al., 2014).

The main sources of wood waste are: logging: bark, sawdust, thin wood (small); wood processing industry (cutting, carpentry, furniture factories, parquet): chips, sawdust, scrap; scrap: construction wood, railway crosses, pallets, formwork wood, etc.

Polymer composites are materials that consist of two or more components that are connected, or chemically bind, and offer better properties due to their synergies. Thus, at least two different materials, which are completely immiscible, are mixed to form a composite. Additives such as compatibilizers, plasticizers, pigments, UV radiation stabilizers, nanoparticles are also frequently added in order to improve certain properties. The type and geometry of the discontinuous phase give the composite optimized properties, such as high specific strength, rigidity and hardness, low specific weight, etc. (Navarro et al., 2004).

In this research the composite is based on chloroprene rubber, and added post-consumer recycled wood particles, with dimensions of $500 \mathrm{~nm}$ eco-reinforcing material, and active 
fillers, such as plasticizers, vulcanizing agents, antioxidants. In order to enhance the compatibility and their level of interaction, the wood waste was finely ground (cryogenic mill) and functionalized with potassium oleate. Wood waste acting as a filling material which leads to the biodegradability of the composite and the decrease in density.

\section{MATERIALS AND METHODS}

\section{Materials}

Materials used to obtain the composites were: chloroprene rubber, from SAFIC ALCAN; stearin - powder, white colour, molecular weight 284,48 g/mol; zinc oxide, microparticles, white powder, precipitate $93-95 \%$; magnesium oxide, microparticles, fine powder, precipitate $93-95 \%$, silicon dioxide, molecular mass 60,08 g/mol, white colour, particle size < 0,5 mm; chlorinated paraffin - solid state, powder; $N$-Isopropyl- $N$ '-phenyl1,4-phenylenediamine, brown flat granules, molar mass: 226,317 g/mol, density: 1.04 $\mathrm{g} / \mathrm{cm}^{3}$; sulfur, vulcanization agent (fine yellow powder, melting point: $115^{\circ} \mathrm{C}$ ); tetramethylthiuram disulfide - vulcanization agent (density $1.40 \mathrm{~g} / \mathrm{cm}^{3}$ ), melting point $<146^{\circ} \mathrm{C}$, an ultrafast curing accelerator); all ingredients are from Bayer company.

Wood waste was collected from the manufacture of wooden furniture, cryogenically ground at $12.000 \mathrm{rpm}$ for $15 \mathrm{~s}$ and screened through a $500 \mathrm{~nm}$ mesh screen.

\section{Method}

\section{Functionalization}

The functionalization of wood waste with potassium oleate was achieved by mixing with a mechanical stirrer with 80 rotations/min with heat, at a temperature of $80^{\circ} \mathrm{C}$ for 8 hours. The ratio between wood waste and potassium oleate $-50 \%$.

\section{Composites Processing}

The rubber is plasticized for $90 \mathrm{~s}$, speed $40 \mathrm{rpm}$ and $45^{\circ} \mathrm{C}$, mechanically mixed in a Brabender Plasti-Corder PLE-360. The other ingredients like fillers, plasticizers and wood waste are slowly added, in $4 \mathrm{~min} 30 \mathrm{~s}$, twin screw speed set to 20rpm and temperature rise to $90^{\circ} \mathrm{C}$. Compound homogenization for another 2 minutes, speed 60 $\mathrm{rpm}$ and temperature reach over $100^{\circ} \mathrm{C}$. The total processing times was 8 minutes. Table 1 shows tested formulations. The mixture is extracted from the mixer chamber and finished on the electric roller, by adding the antioxidant and vulcanizing agents. The processing parameters are as following: temperature $23-30^{\circ} \mathrm{C}$, friction of the rollers $1: 2$, and 50 rotations/min, for $5 \mathrm{~min}$ and homogenization for another $2 \mathrm{~min}$.

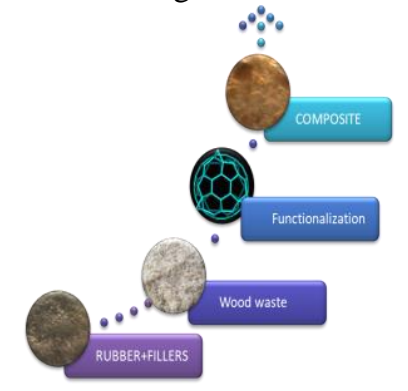

Figure 1. Processing stages 
Table 1 shows the formulations of compound based on chloroprene rubber, with semi-active - $\mathrm{MgO}$ and $\mathrm{ZnO}$ white mineral fillers, and flame retardant materials such as chlorinated paraffin, stearin.

This recipe was adapted to have a higher ecological component, by adding functionalized waste in different amounts, respectively 10, 20, 30, 50\% waste compared to the amount of elastomer. The waste used is wood (CL1-CL4).

Table 1. Polymer composites based on polychloroprene rubber compounded with wood waste

\begin{tabular}{|c|c|c|c|c|c|c|}
\hline Ingredients / Symbol & M.U. & $\mathrm{CO}$ & CL1 & CL2 & CL3 & CL4 \\
\hline \multicolumn{7}{|c|}{ Processing on Brabender } \\
\hline Chloroprene Rubber & $\mathrm{g}$ & 190 & 190 & 190 & 190 & 190 \\
\hline Stearin & $\mathrm{g}$ & 2.28 & 2.28 & 2.28 & 2.28 & 2.28 \\
\hline Zinc oxide & $\mathrm{g}$ & 9.5 & 9.5 & 9.5 & 9.5 & 9.5 \\
\hline Magnesium oxide & $\mathrm{g}$ & 7.6 & 7.6 & 7.6 & 7.6 & 7.6 \\
\hline Silicon dioxide & $\mathrm{g}$ & 57 & 38 & 19 & 0 & 0 \\
\hline Chlorinated paraffin & $g$ & 57 & 57 & 57 & 57 & 19 \\
\hline functionalized Wood waste & $\mathrm{g}$ & 0 & 19 & 38 & 57 & 95 \\
\hline Antioxidant IPPD & $\mathrm{g}$ & 5.7 & 5.7 & 5.7 & 5.7 & 5.7 \\
\hline \multicolumn{7}{|c|}{ Processing on Roller } \\
\hline Sulfur & $\mathrm{g}$ & 2.85 & 2.85 & 2.85 & 2.85 & 2.85 \\
\hline Accelerator & $\mathrm{g}$ & 2.28 & 2.28 & 2.28 & 2.28 & 2.28 \\
\hline
\end{tabular}

\section{RESULTS AND DISCUSSIONS}

It can be seen in figure 2, that the torque in the first part which lasts 90 secs at 40 $\mathrm{rpm}$, the elastomer is added into the mixer and therefore the torque increases initially.

The first loading peak, corresponds to the introduction of elastomers. As the torque increases, so does the temperature due to friction.

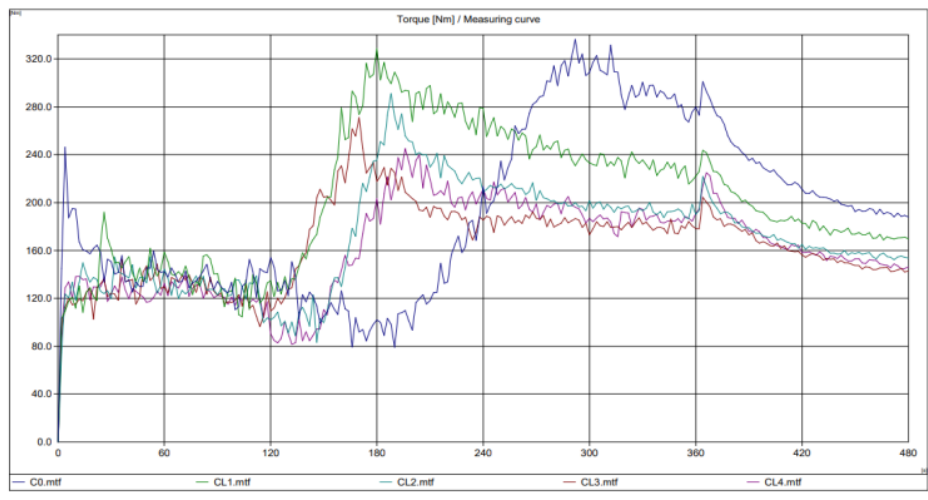

Figure 2. Brabender mixing diagram for composites C0-CL4

After the loading peak is reached, the torque begins to decrease, mainly due to the homogenization and plasticization of the elastomer, as well as due to the increase in temperature due to shear.

Then the other ingredients are added and the speed is reduced to $20 \mathrm{rpm}$ for $4^{\prime}: 300^{\prime \prime}$. 
After $90 \mathrm{Sec}$ first part, the torque begins to increase due to the incorporation of the ingredients, but also as a result of elastomer reinforcement and energy transfer.

After incorporating the fillers and other ingredients, the second loading peak, is observed when a maximum torque is achieved.

The torque starts to decrease, indicating the homogenization of the mixture.

Then the homogenization of the compounds takes place for $120 \mathrm{sec}$ at $60 \mathrm{rpm}$.

As a result, a maximum value of torque is obtained due to the compaction and homogenization of the rubber mixture.

This is generally followed by a decrease in the value of the torque, which indicates both the homogenization of the mixture and the increase of the mixture temperature due to the friction at a higher rotation speed $(60 \mathrm{rpm})$.

\section{FTIR Spectroscopy}

IR spectrum represents the radiant energy absorption curve in the IR domain by the sample molecule, depending on the wave length or radiation frequency. The infrared domain of the electromagnetic radiation is between 0.8 and $200 \mu \mathrm{m}$. IR domain for usual organic chemistry is between 2.5 and $25 \mu \mathrm{m}$. The structural determinations were carried out on an IR molecular absorption spectrometer with double beam, in the range of 4000-600 cm-1, using 4200 FT-IR equipped with ATR diamond crystal and sapphire head. The solid state samples were set in the ATR and the equipment recorded the transmittance spectra of the sample and then compared it with the background spectra previously recorded. The recorded spectra of the samples were compared with the pure elastomer spectrum. After the tests were carried out, the following were found:

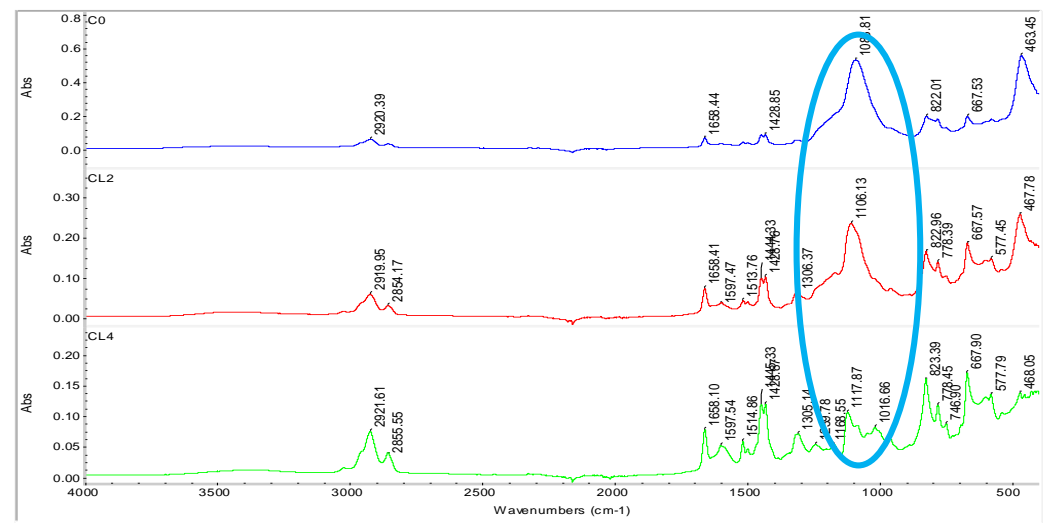

Figure 3. FTIR spectra of composites based on chloroprene rubber/wood waste treated with potassium oleate

In the case of chloroprene rubber/wood waste treated with potassium oleate composite, in addition to the band's characteristic of the treated waste (especially at $\left.1596,1515 \mathrm{~cm}^{-1}\right)$ the characteristic band of silica $\left(1090-1100 \mathrm{~cm}^{-1}, \sim 800 \mathrm{~cm}^{-1}, 460\right.$ $\left.470 \mathrm{~cm}^{-1}\right)$, calcium carbonate $\left(\sim 874,712 \mathrm{~cm}^{-1}\right)$ and rubber, can also be observed. Moreover, the presence of wood waste can be proved in CL4 by the fact that the area 1000-1100 $\mathrm{cm}^{-1}$ contains several overlapping strips including the $\mathrm{C}-\mathrm{O}$ band from approximately $1027 \mathrm{~cm}^{-1}$ of the untreated wood waste. In CL2 due to the presence of $\mathrm{SiO}_{2}$ 
there is a merging of the bands, one characteristic of silica between $1000-1100 \mathrm{~cm}^{-1}$ and the $\mathrm{C}-\mathrm{OH}$ strips from the wood waste, which leads to an asymmetry of the peak.

The physical-mechanical characteristics of the composites - CL series (based on chloroprene rubber and wood waste) are shown in table 3.

Table 3. Physical mechanical-characteristics

\begin{tabular}{|c|c|c|c|c|}
\hline \multirow[t]{2}{*}{ Physical mechanical-characteristics } & \multicolumn{4}{|c|}{ Symbol } \\
\hline & CL1 & CL2 & CL3 & CL4 \\
\hline Hardness, ${ }^{\circ} \mathrm{Sh} \mathrm{A}$ & 60 & 57 & 62 & 72 \\
\hline Elasticity, \% & 16 & 18 & 18 & 26 \\
\hline Tensile strength, $\mathrm{N} / \mathrm{mm}^{2}$ & 12.6 & 7.83 & 6.43 & 5.87 \\
\hline Elongation at break, \% & 640 & 600 & 560 & 580 \\
\hline Remanent elongation, \% & 28 & 28 & 36 & 34 \\
\hline Tear strength, $\mathrm{N} / \mathrm{mm}$ & 49.5 & 45.5 & 37.5 & 30 \\
\hline Specific weight, $\mathrm{g} / \mathrm{cm}^{3}$ & 1.4 & 1.39 & 1.36 & 1.29 \\
\hline Abrasion resistance, $\mathrm{mm}^{3}$ & 187.28 & 189.86 & 185.41 & 219.46 \\
\hline
\end{tabular}

Hardness of the control sample is $60^{\circ} \mathrm{Sh} \mathrm{A}$, when adding the functionalized wood waste increases proportionally with the amount of waste used in the mixture, up to $72^{\circ} \mathrm{Sh} \mathrm{A}$. This is demonstrated by the fact that the hardness increases with the amount of fillers in the mixture.

The tensile strength decreases compared to the control sample, $12.6 \mathrm{~N} / \mathrm{mm}^{2}$, and proportionally to the amount of wood waste introduced into the composite, reaching $5.87 \mathrm{~N} / \mathrm{mm}^{2}$ for the composite with $50 \%$ functionalized wood waste.

Elasticity does vary compared with control sample.

Elongation at break, similar to tensile strength, decreases, from $640 \%$ of the control sample, proportional to the amount of wood waste, down to $580 \%$ for the composite with $50 \%$ wood waste in the composition. Similar values are obtained for remanent elongation and tear strength.

Specific weight decreases proportional to the amount of wood waste, from $1.4 \mathrm{~g} / \mathrm{cm}^{3}$ control sample to 1.29 in the CL4 sample with $50 \%$ wood waste.

The abrasion increases exponentially with the proportion of wood waste added; thus, the control sample has a value of $187.28 \mathrm{~mm}^{3}$, the CL1 sample with $10 \%$ wood waste has the value of $189.86 \mathrm{~mm}^{3}$, and the CL4 sample with $50 \%$ wood waste reaches $219.46 \mathrm{~mm}^{3}$. It was observed that composites with $10-20 \%$ wood waste have values that fall within the standardized requirements for abrasion, namely $200 \mathrm{~mm}^{3}$.

\section{CONCLUSIONS}

Composites based on Chloroprene rubber and semi-active fillers compounded with functionalized post-consumption wood waste were obtained.

$>$ Hardness increase up to $72^{\circ} \mathrm{ShA}$ and the elasticity has variable values and are uneven.

The values of the tensile and the tearing strength decrease as the silicone dioxide is replaced with the wood waste.

$>$ Elongation at break has good values, over $550 \%$.

$>$ Abrasion resistance increases in samples with wood wastes.

The density of the mixtures decreases as the amount of wood waste increases and replace other components with heavier density.

> Properties like hardness, and increased, indicating that the composite is becoming more rigid and due to the replacement of other components by wood waste, it became lighter. 
Thus, the use of post-consumption wood waste in elastomeric composites could contribute to sustainable development in a near future.

\section{Acknowledgements}

This paper is funded by the Ministry of Research and Innovation within Program $1-$ Development of the national RD system, Subprogram 1.2 - Institutional Performance RDI excellence funding projects, Contract no. 6PFE/2018-PERFORM-TEX-PEL, the LIFE program in the frame of LIFEGREENSHOES 4 ALL (LIFE17ENV/PT/000337) project and PN 191701 03/2019 project: "Biodegradable composites from technological and post-consumption polymeric wastes by designing and applying $4 \mathrm{R}$ eco-innovative technologies (4R-ECO-MAT)".

\section{REFERENCES}

Brostow, W., Datashvili, T., Jiang, P. and Miller, H. (2016), "Recycled HDPE reinforced with sol-gel silica modified wood sawdust”, European Polymer Journal, 76, 28-39, https://doi.org/10.1016/j.eurpolymj.2016.01.015.

Chaudemanche, S., Perrot, A., Pimbert, S., Lecompte, T. and Faure, F. (2018), "Properties of an industrial extruded HDPE-WPC: The effect of the size distribution of wood flour particles", Construction and Building Materials, 162, 543-552.

Najafi, S.K. (2013), "Use of recycled plastics in wood plastic composites - A review", Waste Manag, 33(9), 1898-1905, https://doi.org/10.1016/j.wasman.2013.05.017.

Navarro, F.J., Partal, P., Martínez-Boza, F. and Gallegos, C. (2004), "Thermo-rheological behaviour and storage stability of ground tire rubber-modified bitumens", Fuel, 83, 2041-2049, https://doi.org/10.1016/j.fuel.2004.04.003.

Piao, C., Cai, Z., Stark, N.M., Montezun, C.J. (2014), "Dimensional stability of wood-plastic composites reinforced with potassium methyl siliconate modified fiber and sawdust made from beetle-killed trees", Eur J Wood Prod, 72, 165-176, https://doi.org/10.1007/s00107-013-0736-x.

Turku, I., Karki, T. and Puurtinen, A. (2018), "Durability of wood plastic composites manufactured from recycled plastic", Heliyon, 4, e00559, https://doi.org/10.1016/j.heliyon.2018.e00559. 
DEVELOPING INTEGRATED GREEN SUPPLY CHAIN DRIVERS AND BARRIERS FRAMEWORK FOR GREEN SUPPLY CHAIN ADOPTION, MENA REGION

\author{
HANY HANNA $^{1,2}$, PAUL XIROUCHAKIS ${ }^{3}$, ATHANASIOS RENTIZELAS ${ }^{4}$ \\ ${ }^{1}$ Strathclyde University, College of Engineering, DMEM, Glasgow, UK, hany.hanna@strath.ac.uk \\ ${ }^{2} A A S T$, College of International Transport and Logistics, LSCM, Egypt, hany.ayaad@aast.edu \\ ${ }^{3}$ Strathclyde University, College of Engineering, DMEM, Glasgow, UK, \\ paul.xirouchakis@strath.ac.uk \\ ${ }^{4}$ National Technical University of Athens, School of Mechanical Engineering, Greece, \\ arent@mail.ntua.gr
}

\begin{abstract}
Recently, corporate environmental practice such as Green Supply Chain Management (GSCM) and green innovation appears as a novel organized environmental practice for manufacturing companies to handle the increasing environmental issues. The aim of this paper is to assess the level of adoption of GSCM and green practices through proposing framework. A total of 123 responses from industrial sector of the Middle East companies were collected from mail questionnaire. The results showed industrial sector of the Middle East manufacturing companies are in trial adoption stage for both GSCM practices. Internal environmental management practice of GSCM practices are relatively high adopted in industrial sector. Meanwhile, most of all green innovation practices are heavily implemented in MENA region companies. This paper empirically attempts to provide understanding and new insights for sustainability management area where GSCM and green innovation practices are important to improve organizational environmental performance, which can directly offer great benefits for both researchers and practitioners.
\end{abstract}

Keywords: Green Supply Chain Management (GSCM), green practices, organizational environmental performance, barriers, drivers.

\title{
INTRODUCTION
}

The integration of environmental concerns and organizational performance started gaining attention over the recent decades. Greening the supply chain becomes authoritative command in developed countries but most of Middle East countries do not adopt the idea of greening the supply chain. Green supply chain is a concept that combines green procurement, environmental management of manufacturing materials, environmental circulation (i.e. any external change for the company), marketing, and reverse logistics.

As Kawasaki et al. (2012) is one of the researchers of the MENA region (including the Middle East and North Africa regions) residents and is involved in the logistics sector through the work field, an analysis of the situation of the green supply chain management process in the MENA region was introduced. Besides, the enablers and drivers of the green supply chain management process are observed and an evidence was presented for the applicable enablers and drivers. MENA is an abbreviation used for the region of Middle East and North Africa. The countries of the MENA region are located geographically on two continents, Asia and Africa. The Arabic language is the most common spoken language in the region except for Iran, Turkey, and Israel. Middle East and North Africa (MENA) region are characterized by absence of a vibrant private sector, poor trade performance, social and political instability, GDP positive but volatile, scarcity of water resources, agriculture is not a dominant economic activity in most countries (O'Sullivan et al., 2011). Economic development has been widely affected by these factors.

The researcher chooses this region as it has its own nature. The MENA region is well known with its oil resources and large size of population that make the human resources available in the region. Despite the presence of such resources, the countries 


\section{Developing Integrated Green Supply Chain Drivers and Barriers Framework for Green Supply Chain Adoption, MENA Region}

of the MENA region are not well utilizing such resources in their industries and they are misusing these resources in several ways that make the region not adopting green processes. Therefore, the purpose of this study is to explore the green supply chain management process drivers and barriers within the context of the industrial sector of the Middle East. It also develops a framework for the green supply chain management process that relates the supplier with the firm practices according to the existing market pressure, which may lead to specific supply chain management practices.

\section{LITERATURE}

\section{Supply Chain Management Process}

Numerous definitions were given to the supply chain. Supply chain is the management of flow of goods and services that transformed raw material to final goods and it is the achievement for economic, social, environmental aims for institutions to develop the economic performance for any company in long term being and its supply chain (Carter and Rogers, 2008). Furthermore, it is an active process that contains of materials, funds and information and their continuous movement among a number of functional areas within and between chain members (Ahi and Searcy, 2012). Sustainable Supply Chain Management (SSCM) can be identified as the construction of optional and volitional collaboration and integration of supply chains partners and parties by all means and aspects such as: environmental, economic and social. All of those considerations should work with the main aspect and key of an interorganizational business system. Supply chain is considered with the product process that is starting from the initial processing of raw materials till delivery to the end-user. Thus, achieving development of sustainability requires a solid step towards adopting supply chain management system (Ahi and Searcy, 2012).

\section{Integrated Green Supply Chain Management Process}

GSCM is important for raising the awareness of the environment in the last few decades. Many organizations reacted to green issues by directing green principles to their companies like using environmentally friendly raw materials, reducing the usage of petroleum power in addition to using recycled papers for packaging, and recycling of electronic wastes. Because of strategic driving forces and pressures from different stakeholders, firms are embracing green supply chain management (GSCM) practices to spread out environmental sustainability objectives to suppliers (Laari et al., 2017). The green supply chain conceptually covers the whole process from having the raw materials that consists of less environmental harmful factors till the finished product; each supply chain consists of many separate companies, and they are linked by the role of each company in achieving the needs of the consumer, and through this process supply chain also includes transports, warehouses, retailers and the customers themselves. There is an impact from the suppliers, customers and management through the supply chain as to make the expansion more sustainable for the future cooperation. Some firms being driven by their top management and others are being driven by external influences, like stakeholder stress or customers' demands, so here organizations could suffer from barriers and drivers to application of sustainable supply chain management.

Full supply chain integration means much more than simply managing the movement of materials and resources and addressing logistical issues thereof. Rather, supply chain integration means an acknowledgement that production stages of the supply chain must exchange data, and analytics and time-sensitive information in realtime with other points in the supply network. Full supply chain integration means much 
more than simply managing the movement of materials and resources and addressing logistical issues thereof. Rather, supply chain integration means an acknowledgement that production stages of the supply chain must exchange data, and analytics and timesensitive information in real-time with other points in the supply network.

\section{Green Supply Chain Management Process Application in the Industrial Sector}

It was found by several studies that linked between the environmental practices which is related to GSCM are industrial ecology, industrial symbiosis. In addition, it was found that eco-design, life cycle analysis, product stewardship, extended producer responsibility, and environmental management systems (EMS) are much related to the common environmental policies and procedures of GSCM. All these practices targets removing and alleviating the environmental effects of organization procedures on the environment. According to (Oyo et al., 2014) Study, the idea of cost reduction to assist and one of aims that is created by the development of co-operative and integration between suppliers and encouraging life-cycle is considered one of the critical and core aspects that drive individual firm for applying GSCM. Although the importance and requirement for environmental awareness, there is a slow enactment of GSCM between intuitions and transform this awareness into practice in the Chinese manufacturing industry. GSCM has performed various studies especially in the industrial sector. One of the studies discovered that there are many organizations are practicing internal environment management and resource recovery. However, they are not that active with green supply chain in total. Suppliers' assessment is a critical function within supply chain management. Green supplier assessment is also necessary for sustainable supply chain management. Supplier assessment is considered as not just supplier selection but other phases of evaluation and aspects of management. It refers to the process of evaluating and approving potential suppliers by qualitative and quantitative assessment.

The purpose is to ensure a portfolio of best in class suppliers is available for use. It is also applied to current suppliers to assess their performance for the purposes of decreasing costs and risks, increasing quality and driving continuous improvement.

\section{Green Supply Chain Management Process in a Developing Country and Middle East}

Kappa (2013) study that is an empirical study in Pakistan for investigation the performance of GSCM and its network has been undertaken. It found that there are links (leadership, institutional pressure, internal green practices, external green collaboration and green and economic performance in GSCM network) that are needed for examining the relationship between GSCM and its impacts on the economic and green performance of developing countries. GSCM is being recognizably practiced in large organizations more than small organizations. Therefore, large companies are considered a diffusion mechanism to help collaborations with other countries to adopt GSCM. For instance, in India $80 \%$ of the manufacturing is a collaboration between small and medium organizations and large organizations. One of the recent studies on Middle East is the one applied by Younis et al. (2016) study that adopted green supply chain management (GSCM) practices was achieved and its impacts on corporate performance (CP). This study implemented consequences of applying GSCM practices on dimensions of the CP. Younis et al., (2016) study integrated research model to examine the relation between the four dimensions of GSCM (eco-design, green purchasing, environmental cooperation and reverse logistics) on four dimensions of CP (operational performance, environmental performance, social performance and environmental performance) with controlling firm size, firm age and environment management system certification. Younis et al. (2016) study depended on collecting data from 117 industrial companies in United Arab Emirates (UAE) through questionnaires and analyzed these data. The results of Younis et al. (2016) study found that GSCM practice had impact on 


\section{Developing Integrated Green Supply Chain Drivers and Barriers Framework for Green Supply Chain Adoption, MENA Region}

CP dimensions but, the four GSCM practices had no impact on the dimensions of the $\mathrm{CP}$ which are environmental performance, green purchasing and environmental cooperation were found to have a significant impact on the operational performance.

\section{Barriers and Enablers to Green Supply Chain Management Process}

One of the main and important enhancement of the organizational philosophy is green supply chain management as it is used as one of tool that helps in diminishing the environmental risk. Legislations and environmental regulations, the competitors' pressures', meeting the demand of the global market, which required high level of environmental adoption among their imported products and one of leading drivers that enforce organizations to execute and implement GSCM is the raise of awareness of consumers toward green products. Finally, it could be stated that all of these drivers are the main elements that are leading organizations to implement GSCM (Zhu and Geng, 2013). Barriers in GSCM must be deflected to accomplish an environmentally sound supply chain in construction despite of the existence of public awareness, absence of learning about natural effects notwithstanding, poor duty by top administration and absence of legitimate implementation by the legislature, while absence of resources, absence of maintainable practices in the association's vision and mission. In addition to, absence of market for recyclable materials, absence of data sharing between construction firms and suppliers and absence of interest were additionally distinguished as barriers. Accordingly, implementation of GSCM plays vital role in organizations at both environmental and organizational levels (Gandhi et al., 2015). This framework below which is developed by the author is representing a concept of an integrative GSCD framework that has been constructed which arranges all elements identified in the literature review as well as the relations between them. The framework is based on the typical green supply chain practices and producers. The starting point is represented by drivers and barriers. Also the framework represents the different dimensions (Environmental, organizational, and technological) which influence the firms and supply chain practices which have impact on green supply chain adoption where drivers and barriers are highlighted through the research besides the upstream and downstream engagement in enchasing and improving the GSC practices adoption. This framework helps to reduce the complexity and limit the number of potential alternatives.

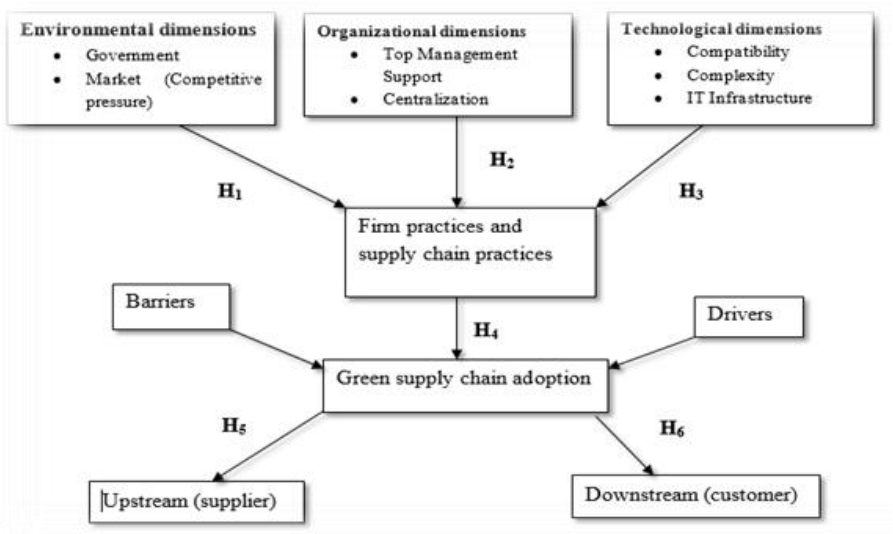

Figure 1. A proposed GSC Framework adoption - Source (Author) 
The drivers and barriers of green supply chain management have been classified into six alternate torrents (internal, external, customers, competition, market and suppliers). There are many obstructions facing GSCM implementation. Capital investment was considered as the most important barrier that industrial ecology faced. After capital investment is the shortage of trained personnel, current legislation, and company policies. Some small-medium enterprises (SMEs) believed that a financial cost would be added due to the better implementation of environment practice and this cost cannot push through customers therefore it is considered as an obstruction to implementation.

Therefore, these barriers to implementation of GSCM in Middle East industrial sector are: Lack of IT Implementation; Resistance to Technology Advancement Adoption; Lack of Organization Encouragement; Poor Quality of Human Resources; Market Competition and Uncertainty; Lack of Government Support System; Lack of Implementing Green Practices; Lack of Top Management Commitment; Cost Implications; Supplier Reluctance to Change towards GSCM and Unawareness of customers. All of these barriers having impacts on all green practices and increase the environmental disruption.

\section{METHODOLOGY}

For the sake of this research, the current research will follow the qualitative methods approach, where a semi-structured interview for the qualitative approach is designed. The target populations are Potential Experts, and Managers. Managers of the industrial sector in the Middle East are considered as the unit of analysis for this study. A number of 10 interviews were handled with managers of different SMEs in the industrial sectors to explore the drivers and barriers they face to achieve green supply chain management process. In the qualitative phase, data was analyzed into generative themes, which were cascaded to categories and codes. Data collected from interviews was analyzed by applying the content analysis using the NVIVO software package.

\section{RESULTS AND FINDINGS}

This section describes the qualitative analysis of the data. The findings of the study will be presented in this chapter with the purpose to introduce a qualitative analysis of the GSCM themes, categories and codes. Under the sample of this study and according to the responses received from the participants during an interview. The study findings had been found and illustrated in the following sections; each section represents a GSCM theme. Finally, a conclusion of this chapter's main findings and results.

\section{Barriers}

This section illustrates the findings regarding the prevailing Barriers and its categories; Outsourcing category with its codes; [Lack of Government Support, Complexity, reflects technological complexity usage through data transformation and Practices of Suppliers], Technology category with its codes; [Fear of Failure, Lack of new technology, and Lack of Materials], Knowledge category with its codes; [Lack of Environmental Knowledge, Lack of awareness about reverse logistics, and Perception of "out-of-responsibility" zone], Financial category with its codes; [Financial Constraints, High cost of Investment cost is high to implement green practices like eco design, green manufacturing, and Cost of switching to a new system], finally the Involvement and Support category and their codes; [Lack of training courses, Lack of customer awareness, Lack of top management involvement, and Poor supplier commitment]. Figure 1 shows in detail the importance of each code of the Outsourcing

https://doi.org/10.24264/icams-2020.IV.9 


\section{Developing Integrated Green Supply Chain Drivers and Barriers Framework for Green Supply Chain Adoption, MENA Region}

category. As shown Lack of Government Support code received "8" responses, while, Practices of Suppliers code received "7" responses. Finally, Complexity code received the lowest responses, "6" responses which means that Lack of Government Support code is relatively the most important factor to the Outsourcing category.

First the Technology category. As shown Lack of new technology lack of IT implementation is an important barrier to achieve efficient GSCM code received " 8 " responses, while, Lack of Materials Industries have pressure of lowering the prices at the cost of environment for their survival code received "7" responses. Finally, Fear of Failure code received the lowest responses, "6" responses which means that Lack of new technology code is relatively the most important factor to the Technology category. Second, shows in detail the importance of each code of the Knowledge category. As shown Lack of Environmental Knowledge code received "9" responses, while, Lack of awareness about reverse logistics code received "7" responses. Finally, Perception of "out-of-responsibility" zone code received the lowest responses, "5" responses which means that Lack of Environmental Knowledge code is relatively the most important factor to the Knowledge category. Third, shows in detail the importance of each code of the financial category. As shown Cost of switching to new system code received "9" responses, while, Financial Constraints code received "7" responses. Finally, High cost of Investment cost is high to implement green practices like eco design, green manufacturing received the lowest responses, "6" responses which means that Cost of switching to new system code is relatively the most important factor to the Financial category. Fourth, shows in detail the importance of each code of the Involvement and Support category. As shown Cost of Lack of top management involvement code received " 8 " responses, while, Poor supplier commitment code received "7" responses, then Lack of customer awareness code received " 6 " responses. Finally, Lack of training courses code received the lowest responses with "5" responses which means that Lack of top management involvement code is relatively the most important factor to the Involvement and Support category.

Finally the fifth category, shows the Barriers' categories. Which could be observed that Involvement and Support category ranked the highest in importance to the Barriers as it received " 26 " responses, while Financial category ranked the second in importance as it received " 23 " responses, the lowest responses was for both of the Outsourcing Technology, and Knowledge categories which they received " 21 " responses. Besides shows the Barriers' categories. Which could be observed that Involvement and Support category ranked the highest in importance to the Barriers as it received "26" responses, while Financial category ranked the second in importance as it received " 23 " responses, the lowest responses was for both of the Outsourcing Technology, and Knowledge categories which they received " 21 " responses.

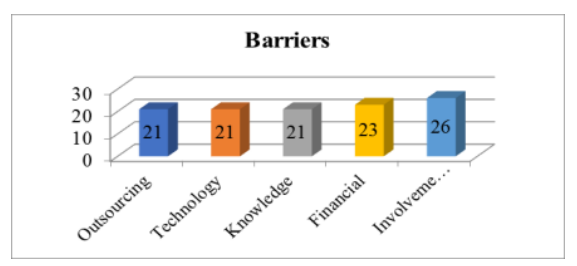

Figure 3. Barriers

\section{Drivers}

This section illustrates the findings regarding the prevailing Drivers and its categories; Government category with its codes; [CEO Initiative, Government Requirement, and 
International Requirement], Managerial category with its codes; [Waste Management, Top Management, ISO 50001, and Company Policy], Economic Benefits category with its codes; [Long Term Cost, Cost Reduction, and Health \& Safety]. Figure 7 shows in detail the importance of each code of the Government category. As shown CEO Initiative code received " 10 " responses, while, both of Government Requirement, and International Requirement codes received equally " 9 " responses. This means that CEO Initiative code is relatively the most important factor to the Government category. In detail the importance of each code of the Managerial category. As shown Top Management code received "10" responses, while, ISO 50001 code received "9" responses.

Finally, both of Waste Management, and Company Policy codes received the lowest responses with equally " 8 " responses which mean that Top Management code is relatively the most important factor to the Managerial category. The importance of each code of the Economic Benefit category. As shown, both of Long Terms Cost, and Cost Reduction codes are equally received "10" responses, while, Health \& Safety code received "9" responses which means that both of Long Terms Cost, and Cost Reduction codes are relatively the most important factor to the Economic Benefit category. Last Figure shows the Drivers' categories. Which could be observed that Managerial category ranked the highest in importance to the Drivers as it received " 35 " responses, while Economic Benefit category ranked the second in importance as it received " 29 " responses, the lowest responses was for the Government category as it received " 28 " responses.

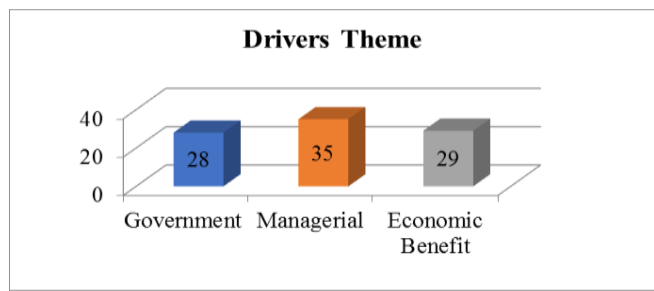

Figure 4. Drivers

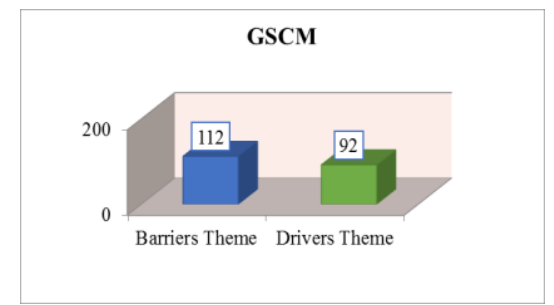

Figure 5. Shows the overall model for GSCM themes (GSCM)

\section{CONCLUSION}

This research includes many objectives that differ between defining the significance of GSCM, identifying the barriers and drivers of GSC implementation, determine the effectiveness of barriers on GSC adoption, and develop a GSC Framework which is stated in the literature review part is to enhance GSCM adoption, and verify and validate the Framework in the industrial sector. Therefore, all steps followed in this research, including literature review, selecting the research method, targeting population and sample study, and finally analyzing data, mainly serve these referred objectives. Testing Drivers and Barriers from the semi-structured interview reached the same results. Shown by the reviewed literature. It could be noted for the Barriers that Involvement and Support 


\section{Developing Integrated Green Supply Chain Drivers and Barriers Framework for Green Supply Chain Adoption, MENA Region}

category ranked the highest in importance to the Barriers theme as it received " 26 " responses, while Financial category ranked the second in importance as it received " 23 " responses, the lowest responses was for both of the Outsourcing Technology, and Knowledge categories which they received " 21 " responses. Moreover, according to the Drivers it could be noted that Managerial category ranked the highest in importance to the Drivers theme as it received "35" responses, while Economic Benefit category ranked the second in importance as it received "29" responses, the lowest responses was for the Government category as it received " 28 " responses.

\section{REFERENCES}

Ahi, P. and Searcy, C. (2015), "An analysis of metrics used to measure performance in green and sustainable supply chains", Journal of Cleaner Production, 86, 360-377, https://doi.org/10.1016/j.jclepro.2014.08.005.

Al-Ghwayeen, W.S. and Abdallah, A.B. (2018), "Green supply chain management and export performance: The mediating role of environmental performance", Journal of Manufacturing Technology Management, 29(7), 1233-1252, https://doi.org/10.1108/JMTM-03-2018-0079.

Ansari, Z.N. and Kant, R. (2017), "A state-of-art literature review reflecting 15 years of focus on sustainable supply chain management”, Journal of cleaner production, 142, 2524-2543, https://doi.org/10.1016/j.jclepro.2016.11.023.

Carter, C.R. and Rogers, D.S. (2008), “A framework of sustainable supply chain management: moving toward new theory", International journal of physical distribution \& logistics management, 38(5), 360-387, https://doi.org/10.1108/09600030810882816.

Dhull, S. and Narwal, M. (2016), "Drivers and barriers in green supply chain management adaptation: A state-ofart review", Uncertain Supply Chain Management, 4(1), 61-76, https://doi.org/10.5267/j.uscm.2015.7.003.

Diabat, A. and Govindan, K. (2011), "An analysis of the drivers affecting the implementation of green supply chain management”, Resources, Conservation and Recycling, 55(6), 659-667, https://doi.org/10.1016/j.resconrec.2010.12.002.

El Saadany, A.M.A., Jaber, M.Y. and Bonney, M. (2011), "Environmental performance measures for supply chains", Management Research Review, 34(11), 1202-1221, https://doi.org/10.1108/01409171111178756.

Elbarky, S. and Elzarka, S. (2015), “A green supply chain management migration model based on challenges faced in Egypt", in: 2015 International Conference on Industrial Engineering and Operations Management (IEOM), 1-8, https://doi.org/10.1109/IEOM.2015.7228093.

Govindan, K., Kaliyan, M., Kannan, D. and Haq, A.N. (2014), "Barriers analysis for green supply chain management implementation in Indian industries using analytic hierarchy process", International Journal of Production Economics, 147, 555-568, https://doi.org/10.1016/j.ijpe.2013.08.018.

Gurel, O., Acar, A.Z., Onden, I. and Gumus, I. (2015), "Determinants of the green supplier selection", Procedia-Social and Behavioral Sciences, 181, 131-139, https://doi.org/10.1016/j.sbspro.2015.04.874.

Laari, S., Töyli, J. and Ojala, L. (2017), "Supply chain perspective on competitive strategies and green supply chain management strategies", Journal of cleaner production, 141, 1303-1315, https://doi.org/10.1016/j.jclepro.2016.09.114.

Laari, S., Töyli, J., Solakivi, T. and Ojala, L. (2016), "Firm performance and customer-driven green supply chain management", Journal of cleaner production, 112, 1960-1970, https://doi.org/10.1016/j.jclepro.2015.06.150.

Large, R.O. and Thomsen, C.G. (2011), "Drivers of green supply management performance: Evidence from Germany", Journal of Purchasing and Supply Management, 17(3), 176-184, https://doi.org/10.1016/j.pursup.2011.04.006.

O'Sullivan, A., Rey, M.E. and Mendez, J.G. (2011), "Opportunities and Challenges in the MENA Region", Arab world competitiveness report, 2012, 42-67.

Vijayvargy, L., Thakkar, J. and Agarwal, G. (2017), "Green supply chain management practices and performance: the role of firm-size for emerging economies" Journal of Manufacturing Technology Management, 28(3), 299-323, https://doi.org/10.1108/JMTM-09-2016-0123.

Younis, H., Sundarakani, B. and Vel, P. (2016), "The impact of implementing green supply chain management practices on corporate performance", Competitiveness Review, 26(3), 216-245, https://doi.org/10.1108/CR-04-2015-0024.

Zailani, S., Jeyaraman, K., Vengadasan, G. and Premkumar, R. (2012), "Sustainable supply chain management (SSCM) in Malaysia: A survey", International Journal of Production Economics, 140(1), 330-340, https://doi.org/10.1016/j.ijpe.2012.02.008.

Zhu, Q. and Geng, Y. (2013), "Drivers and barriers of extended supply chain practices for energy saving and emission reduction among Chinese manufacturers", Journal of Cleaner Production, 40, 6-12, https://doi.org/10.1016/j.jclepro.2010.09.017. 


\author{
KAROLIS KUBILIUS, VIOLETA VALEIKIENE, VIRGILIJUS VALEIKA \\ Kaunas University of Technology, Department of Polymer Chemistry and Technology, Radvilenu \\ pl. 19, 50254, Kaunas, Lithuania, virgilijus.valeika@ktu.lt
}

\begin{abstract}
Chrome shavings are posing a pollution threat. An alkali-enzymatic hydrolysis method was utilized to get collagen hydrolysate $(\mathrm{CH})$ as possible constituent for leather finishing formulations. The new enzyme preparation Vilzim PRO Conc was exploited for the hydrolysis. The dependence of $\mathrm{CH}$ properties on conditions of hydrolysis was explored. The direct addition $5 \% \mathrm{CH}$ into finishing compositions increases tensile strength and relative elongation of films obtained from the compositions. Further increase of the collagen hydrolysate content in the films leads to worse mechanical properties of the films.
\end{abstract}

Keywords: leather, chromed shavings, collagen hydrolysate, coating.

\title{
INTRODUCTION
}

Chromium has been used as primary tannage for many leathers for over 100 years (Musa and Gasmelseed, 2013). Despite the fact that many chrome-free technologies were developed (Plavan et al., 2009), nevertheless chromium is considered as the best mineral-tanning. Unfortunately, the chromium gets into leather production wastes like shavings, cuttings, dust and a removal of the chromium from them is very complicated.

Chrome shavings are formed during shaving, which is performed to give the leather the desired thickness. Chrome shavings, one of the major proteinous solid wastes of leather industry are posing a pollution threat (Shakilanishi et al., 2017). Nearly $0.8 \times 10^{6} \mathrm{t}$ of chrome shavings are produced from leather industries annually (Rao et al., 2002).

The most common method of disposal for chromed leather shavings is depositing them in landfill (Brown et al., 1996). Unfortunately, there is a risk that trivalent chromium in landfills can oxidize to a more dangerous form.

The next often proposed technological solution to the problem of waste shavings utilization is the production of secondary or artificial leathers designed for footwear elements, fancy goods or non-woven fabrics as substrates for leather-like materials (Prepiorkowska et al., 2007). Another trend (Prepiorkowska et al., 2007) of utilization consists in detanning to recover chromium (III) compounds and processing the recovered collagen into gelatin, adhesives or protein hydrolysate (fodders, modified polymers, film-forming agents). Of course, various products can be obtained from the chromed waste shavings but usually the application of such products as fodders, fertilizers, cosmetic preparations are limited by chromium present in the products (Chaudhary and Pati, 2016). The residues of chromium have less importance when the products are used as fillers for rubber or are reused for leather processing.

Usually, removal of chromium from chromed shavings takes place after detanning of the waste. Depending on a kind of the applied detanning agent, three fundamental means of chromium removal are known chemical and enzymatic methods (Pati et al., 2014). Usually, chemical and enzymatic methods are combined. Firstly, the removal of chromium by chemical materials is carried out and, after that, the enzymatic hydrolysis of treated shavings is executed (Adeoye et al., 2014). The alkali-enzyme two step hydrolysis methods are commonly utilized for improved protein recovery efficiency. Sasia et al. (2019) studied a method of treatment which involves a first-step 
denaturation and degradation with alkali followed by inoculation with bating enzyme. Accordingly, hydrolysis using conventional bating enzyme could offer a low-cost alternative for the reuse of chrome-tanned shaving solid waste. Pati et al. (2013) investigated protein extraction by protease mixed with a-amylase and found that there was significant change in the protein extraction by protease in the presence of aamylase.

Probably, the best decision is to apply the hydrolysates for the processing of leather again. Zarlok et al. (2015) demonstrated that $\mathrm{CH}$ produced from waste chromiumtanned hide by means of acid hydrolysis in the finishing mixes improved the hygienic properties of finished leather. Pahlawan et al. (2019) hydrolysed the scraps with $\mathrm{NaOH}$ in $90^{\circ} \mathrm{C}$ and the hydrolysis resulted in two forms of substance, liquid and solid. The liquid substance, as protein binder has the potential to replace the common binder. It is important to know the quality of leather finished using the protein binder.

The aim of the current research was to explore the possibility to use the $\mathrm{CH}$ obtained from chromed shavings as constituent of composition for leather finishing.

\section{EXPERIMENTAL}

\section{Materials}

All chemical materials used for experiments were of analytical grade. Proteolytic enzyme preparation (EP) Vilzim PRO Conc "Baltijos enzymai" (Lithuania) having proteolytic activity at $\mathrm{pH} 11$ and temperature $50^{\circ} \mathrm{C}-1400$ units/g. Chromed shavings from calf leather obtained from tannery "Kedainiu oda" (Lithuania) contained 3.7\% $\mathrm{Cr}_{2} \mathrm{O}_{3}$ were used for hydrolysis. Acrylic resin RA-2312 ("Stahl", Netherlands); polyurethane acrylate mix polimerisate PU-Binder 5954 ("DLH LEDERTECHNIC", Austria) and polyurethane mix Filler 150 ("EstCo", Slovakia) were used for coating films formation.

\section{Procedure}

Hydrolysis of the shavings was carried out according to the method described by Cantera et al. (1994): $10 \mathrm{~g}$ of shavings; $2 \%$ (\% here and further from mass of shavings) $\mathrm{Ca}(\mathrm{OH})_{2}$; distilled water $(1200 \%)$ and mixing for 30 minutes; $\mathrm{NaOH} 10 \%$ and mixing 2 hours; EP Vilzim PRO Conc and mixing 6 hours. Temperature of the treatment $50^{\circ} \mathrm{C}$. After treatment, the liquid hydrolysate was filtrated from solid part.

\section{Analysis and Testing}

The enzymatic activity of EP was determined according to Anson method (Standard USSR, 1988). The content of chromium in shavings and CH was determined according standard (Standard ISO, 2007). Dynamic viscosity of $\mathrm{CH}$ was determined using Ubbelohde viscometer. The tensile strength and elongation of films were determined using dynamometer Zwick/Roell BDO-FBO.5TH ("ZwickRoell GmbH\&Co" Germany). 


\section{RESULTS AND DISCUSSION}

The exploration of the activity of the EP Vilzim PRO Conc has shown that optimal conditions for action of the $\mathrm{EP}$ are: $\mathrm{pH} 11$ and $50^{\circ} \mathrm{C}$ (activity 1420 units per gram).

The next step was to establish an influence of EP on properties of $\mathrm{CH}$. Accordingly, various amounts of EP were used in the hydrolysis process: 0 (control), 2, 4 and 6\%. (Table 1).

Table 1. Properties of collagen hydrolysate dependently on enzyme preparation Vilzim PRO Conc amount used for hydrolysis.

\begin{tabular}{cccc}
\hline $\begin{array}{c}\text { Enzyme preparation } \\
\text { amount, \% }\end{array}$ & \multicolumn{4}{c}{$\begin{array}{c}\text { Collagen hydrolysate qualitative indexes } \\
\mathrm{Cr}_{2} \mathrm{O}_{3} \text { concentration, } \\
\mathrm{mg} / \mathrm{l}\end{array}$} & $\begin{array}{c}\text { Nitrogen content, } \mathrm{gynamic} \text { viscosity, } \\
\mathrm{Pa} \cdot \mathrm{s}\end{array}$ \\
\hline 0 & 9.3 & 0.74 & - \\
2 & 5.4 & 0.75 & 2.1 \\
4 & 4.8 & 0.79 & 1.6 \\
6 & 8.8 & 0.69 & 1.4 \\
\hline
\end{tabular}

Assessment of the presented data allows conclusion that $4 \%$ EP is appropriate amount to obtain qualitative $\mathrm{CH}$.

After hydrolysis according to conditions described in the Experimental the properties of the obtained liquid $\mathrm{CH}$ were determined: amount of $\mathrm{Cr}_{2} \mathrm{O}_{3} 3 \mathrm{mg} / \mathrm{l}$; dynamic viscosity $1.49 \mathrm{~Pa} \cdot \mathrm{s}$.

The $\mathrm{CH}$ was mixed with other film forming materials used in leather industry in various proportions, films from mixtures obtained and mechanical properties of the films evaluated. The results are presented in Table 2 and Table 3.

Table 2. Properties of films formed from first composition mixtures.

\begin{tabular}{cccccc}
\hline CH & \multicolumn{2}{c}{ Composition, $\%$} & & \multicolumn{2}{c}{ Mechanical properties } \\
RA-2312 & $\begin{array}{c}\text { PU-Binder } \\
5954\end{array}$ & Water & $\begin{array}{c}\text { Tensile strength, } \\
\text { N/mm } \text { Relative elongation, }^{2}\end{array}$ \\
\hline 0 & 12 & 23 & 65 & 6.36 & 463 \\
5 & 12 & 23 & 60 & 9.15 & 482 \\
10 & 12 & 23 & 50 & 9.6 & 444 \\
21 & 12 & 23 & 44 & 10.6 & 385 \\
\hline
\end{tabular}

The addition 5\% of $\mathrm{CH}$ into the first composition (Table 1) improves the tensile strength of the film. Further increasing of $\mathrm{CH}$ amount in the composition leads to decreasing of relative elongation of the film.

Table 3. Properties of films formed from second composition mixtures.

\begin{tabular}{ccccccc}
\hline CH & RA-2312 & $\begin{array}{c}\text { Composition, \% } \\
\text { PU-Binder } \\
5954\end{array}$ & $\begin{array}{c}\text { Filler } \\
150\end{array}$ & Water & $\begin{array}{c}\text { Mechanical properties } \\
\text { Tensile strength, } \\
\text { N/mm }\end{array}$ & $\begin{array}{c}\text { Relative } \\
\text { elongation, \% }\end{array}$ \\
\hline 0 & 11 & 22 & 6 & 61 & 4.44 & 582 \\
5 & 11 & 22 & 6 & 56 & 5.27 & 645 \\
9 & 11 & 22 & 6 & 52 & 4.17 & 508 \\
18 & 11 & 22 & 6 & 43 & 4.22 & 529 \\
\hline
\end{tabular}


The investigation of the properties of films obtained using the second composition (Table 2) shows similar results: the addition of $5 \%$ of $\mathrm{CH}$ into composition improves mechanical properties of the film.

\section{CONCLUSIONS}

The collagen hydrolysate obtained from chromed shavings after alkali and enzymatic hydrolysis using enzyme preparation Vilzim PRO Conc is suitable as constituent for leather finishing coatings. The addition 5\% collagen hydrolysate into finishing compositions increases tensile strength and relative elongation of films obtained from the compositions. Further increase of the collagen hydrolysate content in the films leads to their worse mechanical properties.

\section{REFERENCES}

Adeoye, D.T., Adeyi, O.A., Kathir, I.B. and Ejila, A. (2014), "De-chroming of Chrome Tanned Leather Solid Waste using Modified alkaline Hydrolysis Process.”, International Journal of Engineering Research and Technology, 3, 620-623.

Brown, E.M., Taylor, M.M. and Marmer, W.N. (1996), "Production and potential uses of co-products from solid tannery waste", Journal of the American Leather Chemists Association, 91, 270-276

Cantera, C.S., Greco, C.A., De Giusti, M. and Bereciartua, R. (1994), "Dechroming of shavings. Part 1. Enzymic Alkaline Treatment. Study of Variables", Das Leder, 11, 265-270.

Chaudhary, R. and Pati, A. (2016), "Poultry feed based on protein hydrolysate derived from chrome-tanned leather solid waste: Creating value from waste", Environmental Science and Pollution Research, 23, 8120-8124, https://doi.org/10.1007/s11356-016-6302-4.

Musa, A.E. and Gasmelseed, G.A. (2013), "Semi-Chrome Upper Leather from Rural Goat Vegetable Tanned Crust", Journal of Applied and Industrial Sciences, 1, 43-48.

Pahlawan, I.F., Sutyasmi, S. and Griyanitasari, G. (2019), "Hydrolysis of leather shavings waste for protein binder", Book Series: IOP Conference Series-Earth and Environmental Science, 230, Article Number: 012083, https://doi.org/10.1088/1755-1315/230/1/012083.

Pati, A., Chaudhary, R. and Subramani, S. (2013), "Biochemical method for extraction and reuse of protein and chromium from chrome leather shavings: A waste to wealth approach", Journal of the American Leather Chemists Association, 108, 365-372.

Pati, A., Chaudhary, R. and Subramani, S. (2014), "A review on management of chrome-tanned leather shavings: A holistic paradigm to combat the environmental issues", Environmental Science and Pollution Research, 21, 11266-11282, https://doi.org/10.1007/s11356-016-6302-4.

Plavan, V., Valeika, V., Kovtunenko, O., Širvaityte, J. (2009), "THPS Pretreatment before Tanning (Chrome or Non-chrome)", Journal of the Society of Leather Technologists and Chemists, 93, 186-192.

Prepiorkowska, A., Chronska, K. and Zaborski, M. (2007), "Chrome-tanned leather shavings as a filler of butadiene-acrylonitrile rubber", Journal of Hazardous Materials, 141, 252-257, https://doi.org/10.1016/j.jhazmat.2006.06.136.

Rao, J.R., Thanikaivelan, P., Sreeram, K.J. and Nair, B.U. (2002), "Green route for the utilization of chrome shavings (chromium-containing solid waste) in tanning industry", Environmental science \& technology, 36, 1372-1376, https://doi.org/10.1021/es015635s.

Sasia, A.A., Sang, P. and Onyuka, A. (2019), "Recovery of Collagen Hydrolysate from Chrome Leather Shaving Tannery Waste through Two-Step Hydrolysis using Magnesium Oxide and Bating Enzyme", Journal of the Society of Leather Technologists and Chemists, 103, 80-84.

Shakilanishi, S., Babu, N.K.C. and Shanthi, C. (2017), "Exploration of chrome shaving hydrolysate as substrate for production of dehairing protease by Bacillus cereus VITSN04 for use in cleaner leather production", Journal of Cleaner Production, 149, 797-804, https://doi.org/10.1016/j.jclepro.2017.02.139.

Standard USSR: GOST 20264-88. Enzyme preparations. Methods of determination of proteolytic activity.

Standard ISO 5398-1:2007. Leather - Chemical determination of chromic oxide content - Part 1: Quantification by titration.

Zarlok, J., Smiechowski, K. and Kowalska, M. (2015), "Hygienic Properties of Leather Finished with Formulations Containing Collagen Hydrolysate Obtained by Acid Hydrolysis", Journal of the Society of Leather Technologists and Chemists, 99, 297-301. 
COMPARATIVE ANALYSIS OF CARBON DIOXIDE METHANATION TECHNOLOGIES FOR LOW CARBON SOCIETY DEVELOPMENT

\author{
GHEORGHE LAZAROIU ${ }^{1}$, DANA-ALEXANDRA CIUPAGEANU ${ }^{1}$, LUCIAN MIHAESCU ${ }^{2}$, \\ RODICA-MANUELA GRIGORIU ${ }^{1}$ \\ ${ }^{1}$ Power Engineering Faculty, University Politehnica of Bucharest, Splaiul Independentei 313, \\ 060042, Romania, e-mails: glazaroiu@yahoo.com,dana_ciupageanu@yahoo.com, \\ manuela.grigoriu.1968@gmail.com \\ ${ }^{2}$ Mechanics and Mechatronics Faculty, University Politehnica of Bucharest, Splaiul \\ Independentei 313,060042,Romania,e-mail: Imihaescu@caz.mecen.pub.ro
}

\begin{abstract}
Conversion technologies able to transform renewable energy sources (RES) based electricity in gaseous fuels, which can be stored over long timeframes, represent a key focus point considering the low carbon society development. Thus, Power-to-Gas technologies emerge as a viable solution to mitigate the variability of RES power generation, enabling spatial and temporal balancing of production vs. demand mismatches. An additional benefit in this context is brought by the decarbonization facilities, employing polluting carbon dioxide $\left(\mathrm{CO}_{2}\right)$ emissions and RES-based electricity to produce synthetic natural gas with high methane $\left(\mathrm{CH}_{4}\right)$ concentration. The fuel obtained can be stored or injected in the gas distribution infrastructure, becoming a clean energy vector. This paper investigates the functional parameters of such technologies, aiming to comparatively analyze their suitability for further integration in hybrid and ecofriendly energy systems. Given the stability of $\mathrm{CO}_{2}$ molecule, a catalyst must be used to overcome the methanation reaction kinetics limitations. Therefore, the required conditions (in terms of pressure and temperature) for $\mathrm{CO}_{2}$ methanation reaction unfolding are analyzed first. Further, $\mathrm{CO}_{2}$ conversion rate and $\mathrm{CH}_{4}$ selectivity are investigated in order to provide a detailed comparison of available technologies in the field, addressing moreover the particularities of catalyst preparation processes. It is found that Nickel (Ni) based catalysts are performing well, achieving good performances even at atmospheric pressure and low temperatures. It is remarkable that, within a $[300,500]^{\circ} \mathrm{C}$ temperature range, Ni-based catalysts enable a $\mathrm{CO}_{2}$ conversion rate over $78 \%$ with a $\mathrm{CH}_{4}$ selectivity of up to $100 \%$. Last, integration perspectives and benefits are discussed, highlighting the crucial importance of the results presented in this paper.
\end{abstract}

Keywords: Carbon dioxide methanation; Decarbonization; Low carbon society.

\title{
INTRODUCTION
}

Electrification (based on renewable energy sources - RES) of energy intensive economy sectors, such as transportation, is identified as a solution to mitigate harmful emissions, therefore effective incentives must be carefully designed in this regard (Duic and Rosen, 2014). However, including progressively higher shares of strongly variable energy sources (wind, photovoltaic, wave energy, etc.) entails dealing with supply continuity issues (Eltigani and Masri, 2015). Therefore, in order to enable a slick transition towards environmentally friendly power supply also for the conventional generation, the operating traditional plants have to be upgraded. More in detail, they should comprise decarbonization technologies, facilities with improved combustion efficiency and employ alternative fuels with low carbon footprint (Balcu et al., 2019). Further, flexibility requirements (evaluated based on the mismatch between the energy demand and the continuous supply and the boundaries of the confidence interval limits) have to be satisfied to increase the high reliability of supply (Soares et al., 2017).

Technologies for the conversion of electricity into long-term gas-to-gas (P2G) energy agents play a key role in the contemporary development of low-carbon energy systems (Gallo et al., 2016). P2G is a viable solution for storing highly variable 
renewable energy in the medium and long term, thus ensuring the satisfaction of time imbalances between energy production and demand in the current uncertain context (Lazaroiu and Ciupageanu, 2019). The basic principle of P2G is represented in Figure 1 and consists in the production of a combustible gas (which can be stored or injected in the distribution network) using:

- renewable energy for obtaining hydrogen $\left(\mathrm{H}_{2}\right)$ by electrolysis;

- an additional source of carbon dioxide $\left(\mathrm{CO}_{2}\right)$ for the production of Synthetic Natural Gas (SNG), with a high content of methane $\left(\mathrm{CH}_{4}\right)$, in the methanation reaction.

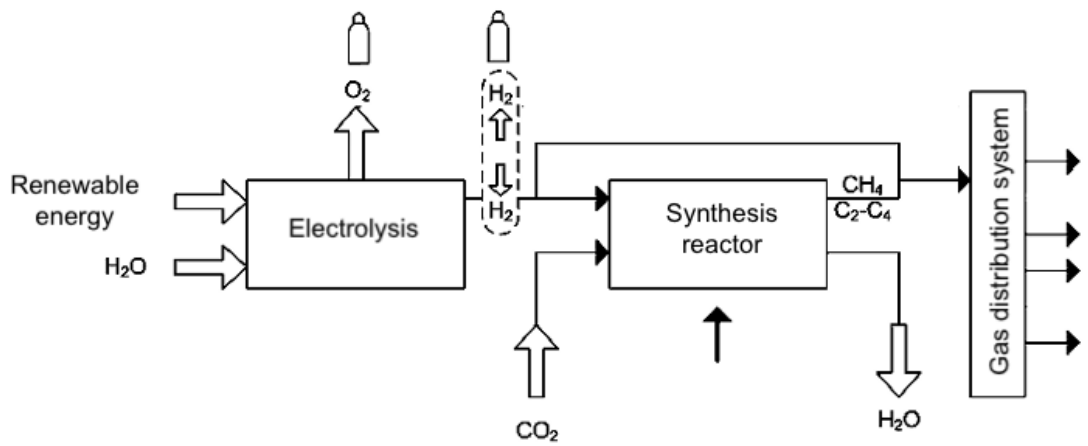

Figure 1. P2G system basic layout

It is highlighted that methanation decarbonation is technically feasible through a combination of technologies (methanation reactor, electrolyzer for $\mathrm{H}_{2}$ production, etc.). As a result, the production of $\mathrm{CH}_{4}$ in decarbonation processes is not currently economically competitive with conventional production technology if the use of atmospheric $\mathrm{CO}_{2}$ is intended. A possible more accessible and implementable solution in a shorter time is by "reusing" $\mathrm{CO}_{2}$ emitted by polluting installations. This concept falls within the field of carbon capture and use technologies, which can eliminate $\mathrm{CO}_{2}$ emissions generated in the operation of various polluting plants (such as conventional power plants). Therefore, the functionality of the technology discussed in this paper shows a huge research potential, being yet less investigated and exploited.

\section{CARBON DIOXIDE METHANATION TECHNOLOGY}

\section{Basic Concept}

Given the stability of the $\mathrm{CO}_{2}$ molecule, obtaining $\mathrm{CH}_{4}$ based on it requires the use of a catalyst to overcome the kinetic limitations of the methanation reaction (1), which have high selectivity relative to $\mathrm{CH}_{4}$ formation and be active at relatively low temperatures (Ghaib et al., 2016).

$\mathrm{CO}_{2}+4 \mathrm{H}_{2} \stackrel{\text { catalyst }}{\longleftrightarrow} \mathrm{CH}_{4}+2 \mathrm{H}_{2} \mathrm{O} \quad \Delta \mathrm{H}=-165 \mathrm{~kJ} / \mathrm{mol}$

It is noticeable that there are two variants through which the $\mathrm{CO}_{2}$ methanation reaction can develop, namely the thermochemical path (showing superior performance) or electrochemical approach (Wang and Gong, 2011). More in detail, catalytic 
methanation of $\mathrm{CO}_{2}$ by the Sabatier thermochemical method requires temperatures and pressures in the range, respectively, and is based on the use of metal catalysts in fixed bed or mobile reactors of specific construction. Regarding the properties of the catalyst, it is preferred to use powders, which ensure the intensification of mass and heat transfer, low pressure losses in the column and a better controllability of the reaction parameters (Castellani et al., 2017).

It is remarked that two important criteria in the catalyst selection and methanation tank design are $\mathrm{CO}_{2}$ conversion rates and selectivity of $\mathrm{CH}_{4}$ formation. Theoretically, for the thermochemical technology, the optimal thermal range, where both criteria reach high values, is in the range of low temperatures. However, the default caloric intake of the reaction can lead to increasing these temperatures (Stangeland, 2017).

In reference to the reaction temperature, it should be emphasized that values above $550^{\circ} \mathrm{C}$ should be avoided, as at such values the catalyst risks to be deactivated through sintering. On the other hand, according to le Chatelier's principle, methanation reaction development is favored at high pressures (Younas et al., 2016). Analyses presented in the literature show that, for temperatures in the range $[200 ; 500]^{\circ} \mathrm{C}$ and pressures above the value of $10 \mathrm{bar}$, the $\mathrm{CO}_{2}$ conversion rate exceeds $90 \%$.

Moreover, it was observed how highly reactive metal catalysts (Nickel - Ni or Ruthenium - $\mathrm{Ru}$ ) causes almost exclusively the production of $\mathrm{CH}_{4}$, while those less reactive (Palladium - Pd, Platinum - Pt, Rhodium - Rh, Molybdenum - Mo or Gold $\mathrm{Au})$ generate by-products such as carbon monoxide $(\mathrm{CO})$ or methanol $\left(\mathrm{CH}_{3} \mathrm{OH}\right)$. Therefore, Ni-based catalysts represent a viable option for $\mathrm{CO}_{2}$ methanation, both in terms of good chemical performance and affordable costs (Wang and Gong, 2011; Stangeland, 2017).

\section{Catalyst Preparation}

The preparation of the catalyst has a very important role in the initiation and unfolding of the methanation reaction. The technique used to combine the metal with the support material affects the crystalline structure of the resulting product, its dispersion in the reactor and the catalytic activity in general (Park and McFarland, 2009). Regarding the catalyst preparation techniques, there are the following variants (Younas et al., 2016):

- Sol-Gel: a solid colloidal porous structure is formed from alkaline metal oxide molecules, nitrites or sulfites. Generally, for the methanation catalytic reaction, this metal catalyst is generated by combining metal salts with the base metal.

- Synthesis of micro-emulsions produces catalysts with large contact surface and very good dispersion of the metal phase, which improves the methanation reaction.

- Capillary impregnation: used to obtain heterogeneous catalysts. In principle, the active metal is dissolved in an aqueous or organic solution, with which the support material is impregnated (by absorption).

- Double impregnation: involves two steps, namely impregnation of the support (inorganic) with an organic reagent and, after drying, impregnation with an ionic solution of the active metal.

- Precipitation / deposition.

The following evaluations are conducted to compare the optimal conditions under which a Ni-based catalyst (in particular Catalyst $1-\mathrm{Ni} / \mathrm{Al}_{2} \mathrm{O}_{3}$ and Catalyst $2-\mathrm{Ni} / \mathrm{Al}$ hydrotalcite) allows to obtain the maximum performance in the catalytic reaction of 
$\mathrm{CO}_{2}$ methanation at atmospheric pressure in a fixed bed reactor. For the first option, the support is obtained by the Sol-Gel method and subsequently impregnated sequentially. For the second solution considered, the catalyst is obtained by co-precipitation in an alkaline solution. According to Figure 2, it is highlighted that:

- for $\mathrm{Ni} / \mathrm{Al}_{2} \mathrm{O}_{3}$ (Catalyst 1 ), the optimal temperature range for which the $\mathrm{CO}_{2}$ conversion rate reaches the maximum value (78\%) is between;

- for $\mathrm{Ni} / \mathrm{Al}$ hydrotalcite (Catalyst 2), the $\mathrm{CO}_{2}$ conversion rate increases linearly with temperature, being higher than $90 \%$ over the whole temperature range taken into account.

It is remarked that both solutions reach $100 \%$ selectivity for $\mathrm{CH}_{4}$ formation.
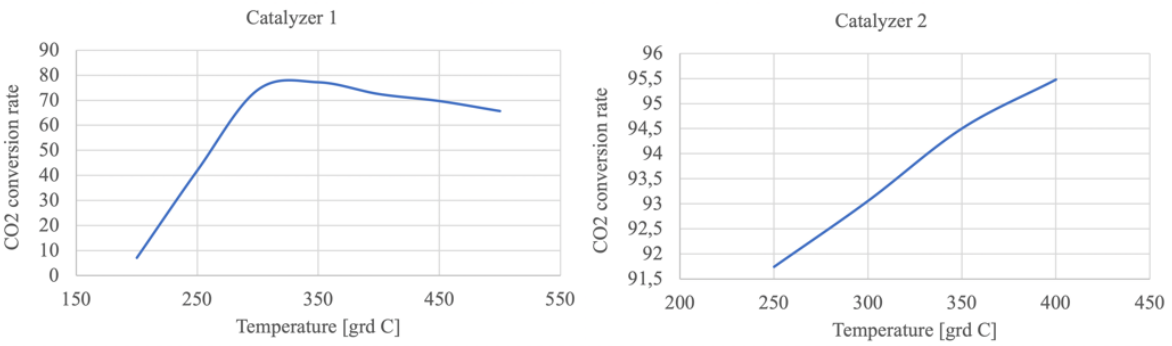

Figure 2. Reaction conditions comparison

\section{INTEGRATION PERSPECTIVES IN ENERGY SYSTEMS}

In addition, the advantages of integrating such a facility into more complex architectures that include an integrative sectoral approach are also discussed. One of the advantages of storing energy in the form of $\mathrm{CH}_{4}$ (compared to storing it in the form of $\mathrm{H}_{2}$ ) is that there are no quantitative (but only qualitative) restrictions on $\mathrm{CH}_{4}$ delivered to the gas distribution network. If the SNG obtained in the methanation process does not have a sufficiently high $\mathrm{CH}_{4}$ content, then further purification must be carried out for use in energy or transport applications (Blanco et al., 2018). The disadvantage is the higher investment in equipment. In order to design a technically and economically advantageous solution, the current or feasible possibilities must be weighed in the near future for the use of $\mathrm{CH}_{4}$ and $\mathrm{H}_{2}$ (Salomone et al., 2018).

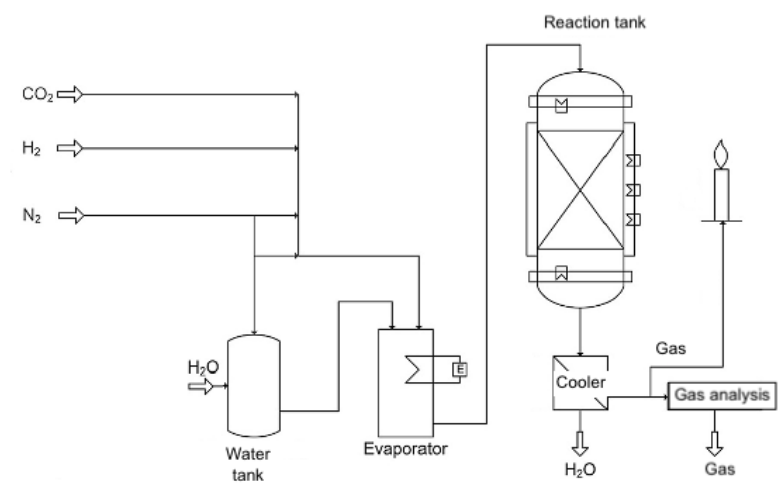

Figure 3. Integration of methanation facility 
A possible integration solution of $\mathrm{CO}_{2}$ methanation facilities in the current energy framework is by connecting them to the exhaust of diesel generators, employed as UPS (Uninterruptible Power Supply). It is feasible to partially supply the diesel group fuel flow (up to $80 \%$ (Balcu et al., 2019)) by feeding the flue gas at the exhaust of a diesel group to the methanation unit. The amount of $\mathrm{CH}_{4}$ that can be obtained represent a cleaner alternative to diesel fuel (based on equations (2) and (3)).

$\mathrm{CH}_{4}+2 \mathrm{O}_{2} \rightarrow \mathrm{CO}_{2}+2 \mathrm{H}_{2} \mathrm{O}+Q_{\text {methane }}$

$\mathrm{C}_{16} \mathrm{H}_{34}+\frac{49}{2} \mathrm{O}_{2} \rightarrow 16 \mathrm{CO}_{2}+17 \mathrm{H}_{2} \mathrm{O}+Q_{\text {diesel }}$

As a consequence of different heating values, it is demonstrated in Balcu et al. (2019) that the fuel flow can be reduced with approximately $16 \%$, while the emissions are brought down by up to $27 \%$.

\section{CONCLUSIONS}

In a framework highly oriented towards innovation and new technology development, intensive research is necessary to enable market penetration and wide diffusion of cutting-edge solutions. Improved and highly efficient technologies are still needed to achieve acceptable levels of emissions while ensuring mitigation of RES related effects on power systems behavior. In high-penetration renewable energy systems, the variability of electricity generation sources must be mitigated, in order to enable their optimal exploitation. For this purpose, they have to comprise flexibility resources (such as storage devices) additional fully controllable generating units (diesel groups, for instance) and, sometimes, flexible demand. In this environmentally restricted and increasingly uncertain power generation context, the paper addresses the selection of catalysts for a methanation tank possibly coupled to a diesel generator, considering a further innovative and integrative approach within hybrid energy systems. It is highlighted the need to establish a compromise solution, affordable and able to ensure high values of $\mathrm{CO}_{2}$ conversion rate and selectivity in the formation of $\mathrm{CH}_{4}$, under conditions of pressure and temperature that involve low energy consumption.

\section{Acknowledgments}

This work was supported by a grant of the Romanian Ministry of Research and Innovation, CCCDI-UEFISCDI, project number PN-III-P2-2.1-PED- 2019-2350, within PNCDI III, contract number 322PED/2020.

\section{REFERENCES}

Balcu, I. et al. (2019), "Decarbonization of Low Power Applications through Methanation Facilities Integration," in: IEEE PES Innovative Smart Grid Technologies Europe - ISGT-Europe, Presented, https://doi.org/10.1109/ISGTEurope.2019.8905506.

Blanco, H., Nijs, W., Ruf, J., and Faaij, A. (2018), "Potential of Power-to-Methane in the EU energy transition to a low carbon system using cost optimization", Appl. Energy, 232, 323-340, https://doi.org/10.1016/j.apenergy.2018.08.027.

Castellani, B. et al. (2017), "Experimental investigation on $\mathrm{CO}_{2}$ methanation process for solar energy storage compared to $\mathrm{CO}_{2}$-based methanol synthesis", Energies, 10(7), 1-13, https://doi.org/10.3390/en10070855.

Duić, N. and Rosen, M.A. (2014), "Sustainable development of energy systems", Energy Convers. Manag., 87, 1057-1062, https://doi.org/10.1016/j.enconman.2014.08.046.

Eltigani, D. and Masri, S. (2015), "Challenges of integrating renewable energy sources to smart grids: A

https://doi.org/10.24264/icams-2020.IV.11 


\section{Comparative Analysis of Carbon Dioxide Methanation Technologies for Low Carbon Society Development}

review”, Renew. Sustain. Energy Rev., 52, 770-780, https://doi.org/10.1016/j.rser.2015.07.140.

Gallo, A.B., Simões-Moreira, J.R., Costa, H.K.M., Santos, M.M., and Moutinho dos Santos, E. (2016), "Energy storage in the energy transition context: A technology review", Renewable and Sustainable Energy Reviews, 65, 800-822, https://doi.org/10.1016/j.rser.2016.07.028.

Ghaib, K., Nitz, K., and Ben-Fares, F.-Z. (2016), "Chemical Methanation of $\mathrm{CO}_{2}$ : A Review", ChemBioEng Rev., 3(6), 266-275, https://doi.org/10.1002/cben.201600022.

Lazaroiu, G. and Ciupageanu, D. (2019), "Multi-criteria decision making in sustainable renewable energy systems", in 19th International Multidisciplinary Scientific Geoconference SGEM2019, 19(4.1), 569-576, https://doi.org/10.5593/sgem2019/4.1/S17.042.

Park, J. and McFarland, E. (2009), "A highly dispersed $\mathrm{Pd}-\mathrm{Mg} / \mathrm{SiO}_{2}$ catalyst active for methanation of $\mathrm{CO}_{2}$ ", J. Catal., 266(1), 92-97, https://doi.org/10.1016/j.jcat.2009.05.018.

Salomone, F., Giglio, E., Ferrero, D., Santarelli, M., Pirone, R., and Bensaid, S. (2018), “Techno-economic modelling of a Power-to-Gas system based on SOEC electrolysis and $\mathrm{CO}_{2}$ methanation in a RES-based electric grid", Chem. Eng. J., 1-20, https://doi.org/10.1016/j.cej.2018.10.170.

Soares, J., Fotouhi Ghazvini, M.A., Borges, N., and Vale, Z. (2017), “A stochastic model for energy resources management considering demand response in smart grids", Electr. Power Syst. Res., 143, 599-610, https://doi.org/10.1016/j.epsr.2016.10.056.

Stangeland, K., Kalai, D., Li, H. and Yu, Z. (2017), " $\mathrm{CO}_{2}$ Methanation: The Effect of Catalysts and Reaction Conditions”, Energy Procedia, 105(1876), 2022-2027, https://doi.org/10.1016/j.egypro.2017.03.577.

Wang, W. and Gong, J. (2011), "Methanation of carbon dioxide: An overview", Front. Chem. Eng. China, 5(1), 2-10, https://doi.org/10.1007/s11705-010-0528-3.

Younas, M., Loong Kong, L., Bashir, M.J.K., Nadeem, H., Shehzad, A., and Sethupathi, S. (2016), "Recent Advancements, Fundamental Challenges, and Opportunities in Catalytic Methanation of $\mathrm{CO}_{2}$ ", Energy and Fuels, 30(11), 8815-8831, https://doi.org/10.1021/acs.energyfuels.6b01723. 

IN THE BIOECONOMY FRAMEWORK

\author{
GHEORGHE LAZAROIU ${ }^{1}$, LUCIAN MIHAESCU ${ }^{2}$, RODICA-MANUELA GRIGORIU ${ }^{1}$, \\ DANA-ALEXANDRA CIUPAGEANU ${ }^{1}$, IULIA SIMION ${ }^{1}$ \\ ${ }^{1}$ Power Engineering Faculty, University Politehnica of Bucharest, Splaiul Independentei 313, \\ 060042, Romania, e-mails: glazaroiu@yahoo.com, manuela.grigoriu.1968@gmail.com 2 \\ dana_ciupageanu@yahoo.com, julia.simion@gmail.com \\ ${ }^{2}$ Mechanics and Mechatronics Faculty, University Politehnica of Bucharest, Splaiul \\ Independentei 313,060042,Romania,e-mail: Imihaescu@caz.mecen.pub.ro
}

\begin{abstract}
In order to ensure EU's transition to a climate-neutral energy environment, in accordance with the Paris Agreement, enhanced energy efficiency of waste utilization emerges as an important tool to achieve carbon neutrality goals. Several technologies for renewable waste treatment are investigated lately, researches worldwide focusing on exploiting their energy potential and diminishing the environmental impact. It is remarkable that, solid renewable waste is suitable to supply in particular grate or layer combustion plants. This energy valorization solution reached the technical maturity, experimentally and numerically proven. Further, to support regional development incentives implementation, local utilization of different wastes is strongly encouraged. Considering the fairly uniform territorial spread of poultry farms in Romania, this paper presents a case study aiming to provide a sustainable solution for bird waste management and local energy recovery from it, avoiding significant additional costs, as well as storage and transportation issues. The energy independence level is assessed in two scenarios. To this regard, the energy consumption of a real poultry production hall of $910 \mathrm{~m}^{2}$ (located in Giurgiu County, having 4650 birds/operating cycle, with a poultry waste flow of $558 \mathrm{~kg}$ waste /day) is taken into account. The first scenario analyzes the disposal (for energy recovery purposes) of poultry waste as an individual raw material, while the second scenario investigates a mixture of poultry waste and agricultural biomass residues. It is demonstrated that the electricity and heating requirements of the hall can be partially satisfied in the first scenario and fully in the second one. Therefore, the multi-waste management concept investigated in this paper represents a sustainable solution to reduce industry's carbon footprint, answering multiple requirements in the environmentally friendly energy sector development.
\end{abstract}

Keywords: multi-waste; poultry industry; regional development.

\title{
INTRODUCTION
}

\section{Regulatory Framework}

Following energy demand continuous growth, overlapped with traditional resources availability limitations, the European Commission elaborated an action plan for circular economy unfolding, complying also with the 2030 Agenda for Sustainable Development targets (Directive 2008/98/EC, 2008). In reference to waste management policies, it is remarked that they mainly pursue avoiding and reducing not only their rate of generation, but also the potential environmental impact issued by their toxicity. The most popular methods available to diminish the amount of waste are (Klemeš et al., 2019):

- Waste reduction at source;

- Alleviating the waste flows in different sectors by implementing the best available practices;

- Waste valorization by reuse, recycle and energy recovery; 
- Waste disposal through standardized incineration methods and warehouse storage.

EU legislation regarding waste management imposes different treatment approaches depending on the sustainability level of the regarded waste fount. The highest priority is given to prevention and recycling, as evident also in Figure 1 (Law 211/2011). In order to integrate waste management targets in a low carbon society and support circular economy development, it is necessary to promote innovation and investments in new waste treatment capacity while mitigating the losses determined by the standardization shortcomings (Closing the loop - An EU action plan COM, 2015). As a consequence, waste-to-energy conversion technologies are thoroughly investigated lately, researchers worldwide focusing on sustainable solutions, with high potential in the circular economy framework (Klemeš et al., 2019).

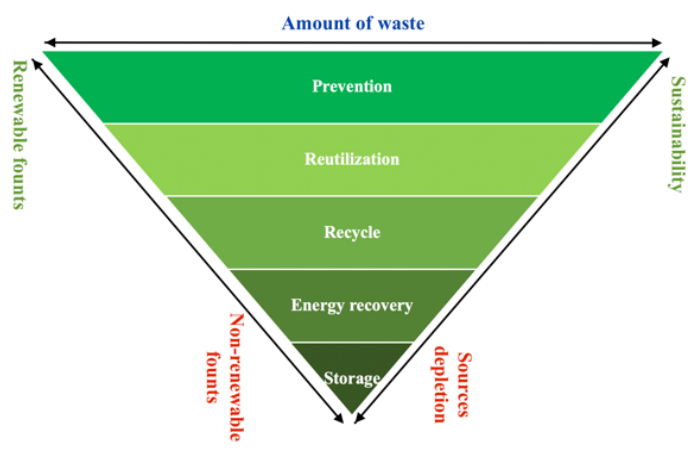

Figure 1. Waste management hierarchy.

Depending on the type of waste, achieving the best outcome in terms of environmental impact starts from the priority order given in Figure 1. Further, considering technical feasibility, ecologic aspects and economic viability the treatment approach is selected (Krajačić et al., 2016).

\section{Waste-to-Energy Conversion Technologies}

A key step enabling the transition towards circular economy is to lay down a set of ranked priorities that reduce the environmental impact, while achieving the optimal resource efficiency in waste prevention and management (Duić and Rosen, 2014). The following waste treatment technologies emerge as having top integration potential:

- co-incineration of waste in combustion plants (e.g. power plants, cement and lime production facilities);

- waste incineration in dedicated facilities;

- $\quad$ anaerobic digestion of biodegradable waste;

- $\quad$ production of waste-derived solid, liquid or gaseous fuels;

- other processes including indirect incineration following a pyrolysis or gasification step.

It is highlighted that classification of these technologies according to the ranking in Figure 1 takes into account many criteria and is actually complex. For instance, wasteto-energy processes such as anaerobic digestion resulting in the production of biogas 
and digestate are regarded as a recycling operation. However, waste incineration with limited energy recovery is considered disposal.

Considering the poultry industry development, poultry manure became lately an important issue to be addressed. Land application may be sometimes restricted by logistical limitations or over-application risks (Sarfaraz et al., 2020). The energy recovery approach applied to the poultry industry waste encourages poultry producers to integrate it within their own facilities, cutting the outsourcing related expenses. Furthermore, biochar (secondary product in the energy recovery process) employment as fertilizer can absorb excess nutrients if applied properly, even if the environmental risks associated are similar to straight poultry litter landfill spreading (Hidalgo et al., 2019).

Anaerobic digestion of poultry manure is used for biogas (with over $50 \% \mathrm{CH}_{4}$ content) production. This resulting fuel can be further employed in heat and electricity generation facilities. The remaining digestate could be used as crop fertilizer, but high ammonia concentration in the raw waste and residual bacteria in the digestate represent an important concern (Kelleher et al., 2002).

Direct combustion is not a very effective waste-to-energy conversion technology using poultry manure, because of its high moisture content (which represents a drawback is exceeds 10\%) (Mihaescu et al., 2019). However, dry poultry litter has good calorific values. Additional concerns are linked to the ammonia, potassium and sodium fractions, posing problems not only in the actual burning process, but also from the harmful emissions perspective (Lazaroiu et al., 2018). Other important factors influencing combustion efficiency refer to fuel supply chain, moisture content and temperature of the poultry litter, as well as the duration of outdoor storage. Investigations reported in the literature estimate a net electricity production through poultry manure direct combustion in the range 0.75 to $1.15 \mathrm{kWh} / \mathrm{kg}_{\text {manure }}$ (Cavalaglio et al., 2018).

Another technology employed for poultry manure treatment is pyrolysis. In this process, the waste is heated up to high temperatures, in an environment lacking oxygen. The direct products (biogas or biofuel) are suitable for electricity generation, while the secondary biochar can be used as fertilizer (containing high phosphorus and potassium contents per mass unit, enabling a net zero economic value in the bioeconomy framework) (Mihaescu et al., 2018). Although both direct products issue greenhouse gases during combustion, the resulting environmental impact is much lower compared to fossil fuels, due to net $\mathrm{CO}_{2}$ neutrality. Although pyrolysis is a feasible solution, both from environmental and technical points of view, the mass processing infrastructure for medium to large scale applications is not yet available (Hadroug et al., 2019).

\section{Aims of Research}

This paper introduces a multi-waste management concept, representing a sustainable solution to reduce industry's carbon footprint, answering multiple requirements in the environmentally friendly energy sector development. More in detail, the paper presents a case study aiming to provide a sustainable local poultry waste management solution by means of energy recovery approaches. The energy independence level is assessed in two scenarios. To this regard, the energy consumption of a real poultry production hall of $910 \mathrm{~m}^{2}$ (located in Giurgiu County, having 4650 birds/operating cycle, with a poultry waste flow of $558 \mathrm{~kg}$ waste/day) is taken into account. The first scenario analyzes the disposal (for energy recovery purposes) of poultry waste as an individual raw material, 
while the second scenario investigates a mixture of poultry waste and agricultural biomass residues. It is demonstrated that the electricity and heating requirements of the hall can be partially satisfied in the first scenario and fully in the second one.

\section{WASTE CHARACTERISTICS}

From the point of view of the classification of waste used experimentally, poultry manure and / or by-products of crops and forests, depending on its nature it is from the category of household and assimilated waste, and depending on its origin it is from the category of agricultural and food waste, organic waste, which requires collection procedures and particular treatments.

In the analysis of the optimal waste treatment chain with energy recovery, the results obtained from the environmental impact analysis, the energy analysis and the economic analysis, respectively, will be taken into account.

As a result of increased demand, poultry production has expanded in Romania, creating the need to properly manage wastes generated by this industry. The most common method over time has been to apply poultry manure to the field as nutrients.

Based on the large amount of poultry manure generated, we have advanced a case for the construction of a small power plant, which will be supplied exclusively with farm-generated poultry manure (scenario 1 ), or farm-generated poultry manure mixed with forest biomass (scenario 2).

Analysis of the properties of raw chicken manure, pre-dried and mixed with wood pellets confirms that the drying process is necessary for the self-sustaining combustibility of chicken manure. The use of mixtures should significantly facilitate the thermal transformation of chicken manure, regardless of the form of animal waste. The heating value of the samples is evaluated based on the elemental composition, according to equation (1) (Mihaescu et al., 2019):

$\mathrm{Hi}=2.336 \cdot[145 \cdot C+610 \cdot(H-0.125 \cdot O)+40 \cdot S+10 \cdot N][\mathrm{kJ} / \mathrm{kg}]$

where $C, H, O, S$ and $N$ represent the wight percentage of each element.

As expected, the biomass composition differs significantly. Wood pellets have higher organic matter and a lower ash content, resulting in a higher calorific value than that calculated for chicken manure. The results are listed in Table 1.

Table 1. Elemental analysis results

\begin{tabular}{ccccccc}
\hline & $\mathrm{C}$ & $\mathrm{H}$ & $\mathrm{O}$ & $\mathrm{S}$ & $\mathrm{N}$ & $\mathrm{H}^{\mathrm{i}}[\mathrm{kJ} / \mathrm{kg}]$ \\
\hline $\begin{array}{c}\text { Poultry } \\
\text { manure }\end{array}$ & 35.81 & 1.72 & 58.53 & 0.08 & 3.86 & 4252.78 \\
$\begin{array}{c}\text { Wood } \\
\text { pellets }\end{array}$ & 52.45 & 6.81 & 40.55 & 0.04 & 0.15 & 20254.32 \\
\hline
\end{tabular}

\section{ENERGY CONSUMPTION OF A POULTRY HALL}

The bird farm Buturugeni, Giurgiu (Figure 2) is considered in this study. It is located in the southern region of Romania, where solar irradiation reaches $1400 \mathrm{kWh}^{2}$.year, corresponding to $160 \mathrm{~W} / \mathrm{m}^{2}$ incident direct radiation (https://solargis.com/maps-and-gisdata). The poultry farm considered in this study consists of 18 meat poultry production 
halls identical in terms of their sizing. The assessment presented here is made for an individual hall, but the results can be conveniently multiplied to include more, providing relevant information about the energy independence capability of the entire farm.

The specific production parameters vary depending on the age of the birds, from $32.5^{\circ} \mathrm{C}$ and $50-70 \%$ relative humidity, to $21^{\circ} \mathrm{C}$ and $60-70 \%$ relative humidity. it is considered a herd of 4650 birds/operating cycle. The heat flow generated by the birds is of $0.12 \mathrm{~kW} / \mathrm{bird}$, and the manure flow reaches $M=558 \mathrm{~kg} /$ day.
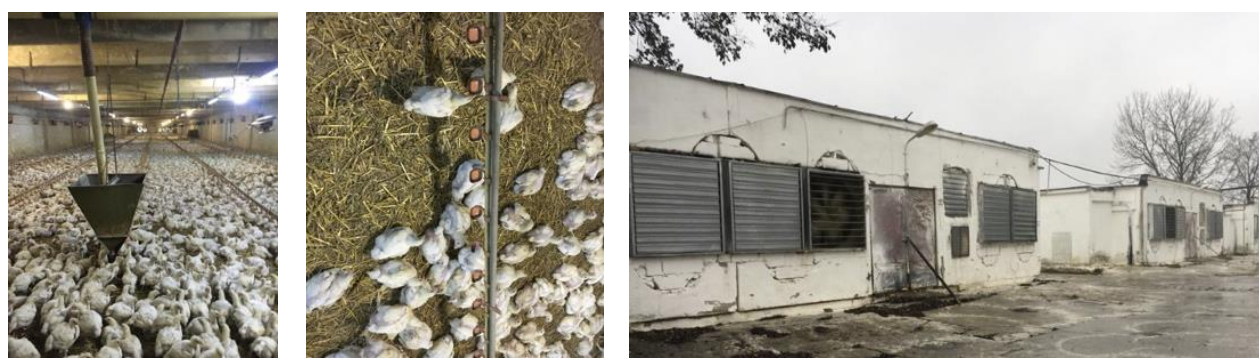

Figure 2. Details of Buturugeni poultry farm

\section{Poultry Hall Geometry}

The geometric characteristics of each hall are the following: Length: $\mathrm{L}=76.9 \mathrm{~m}$; Width: $\mathrm{W}=11.84 \mathrm{~m}$; Height: $\mathrm{H}=3.8 \mathrm{~m}$; Roof surface: $A_{\text {roof }}=1138.12 \mathrm{~m}^{2}$. The surface of the side walls is determined as: $A_{\text {walls }}=674.42 \mathrm{~m}^{2}$.

\section{Electricity Consumers}

Each hall is illuminated by 26 fluorescent tubes, with a power of $58 \mathrm{~W} /$ fluorescent tube. Heating, ventilation and other requirements are satisfied as follows:

- 2 radiators, with a rated power of $12 \mathrm{~kW} /$ radiant;

- 4 motors of $0.55 \mathrm{~kW} /$ motor, with reducer and power supply sensor, ensure the feeding of the bird supply coils with fodder;

- 1 motor $1.5 \mathrm{~kW}$ for feeding the outer hopper;

- 6 fans/hall, of $35 \mathrm{~m}^{3} / \mathrm{h}$ per fan, with a rated power of $1.5 \mathrm{~kW} / \mathrm{fan}$;

- 1 variable front fan/hall, with a power of $0.55 \mathrm{~kW}$.

\section{RESULTS AND DISCUSSIONS}

Based on the geometry characteristics and electricity load and consumption data, the inlet heat flows ( 1 - radiative flux, 2 - ventilation airflow, 3 - birds body heat, 4 internal misting flow), the heat losses and accumulated heat are determined. Then, considering the manure flow, the possible amount of energy to be generated in both scenarios (taking into account a global waste-to-electricity conversion efficiency of 0.4 ) is evaluated.

In scenario 1 , the energy independence level ensured by exploiting the exclusively the poultry manure reaches $25 \%$. Regarding scenario 2 , a sensitivity analysis is performed in reference to the share of biomass (having the properties in Table 1). According to Figure 3 it is noticeable that a full energy independence level is achieved 
for a biomass share of $80 \%$. Depending on the biomass supply conditions, a tradeoff between the energy independence level and biomass costs has to be accepted.

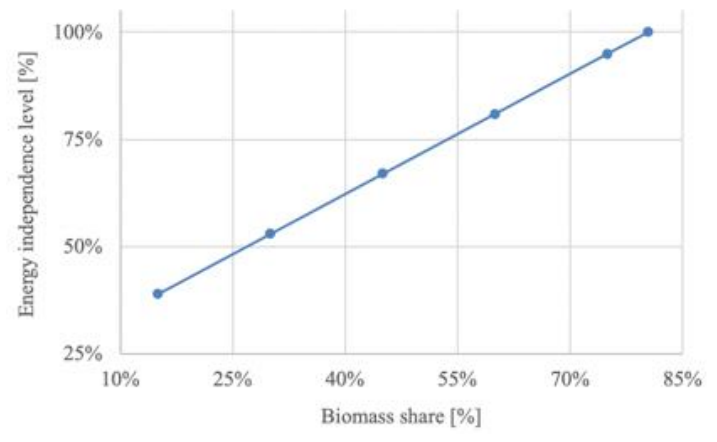

Figure 3. Sensitivity analysis in reference to the biomass share in scenario 2

\section{CONCLUSIONS}

In the field of zootechnical production, waste recovery is required by the extension of concentration and specialization by species and products. Continuous reduction of specific consumptions and improvement of production technologies, storage and treatment of zootechnical residues are the main trends in the field. Agriculture can become the main user of all livestock waste, thus ensuring a renewable energy supply while providing high quality production and protecting the environment. In conclusion, energy recovery in a multi-waste approach is a viable solution to reduce industrial energy consumption, but also to optimize the costs of managing and disposing of wastes. Specifically, the energy independence level can be increased from $25 \%$ up to $100 \%$ by mixing the poultry manure with a higher calorific value biomass residue.

\section{Acknowledgments}

This work was supported by a grant from the Romanian Ministry of Research and Innovation, CCCDI-UEFISCDI, project number PN-III-P1-1.2-PCCDI-20170404/31PCCD/2018, within PNCDI.

\section{REFERENCES}

Cavalaglio, G., Coccia, V., Cotana, F., Gelosia, M., Nicolini, A., and Petrozzi, A. (2018), "Energy from poultry waste: An Aspen Plus-based approach to the thermo-chemical processes," Waste Manag., 73 , 496-503, https://doi.org/10.1016/j.wasman.2017.05.037.

Duić, N. and Rosen, M.A. (2014), "Sustainable development of energy systems", Energy Convers. Manag., 87, 1057-1062, https://doi.org/10.1016/j.enconman.2014.08.046.

European Commission (2008), "Directive 2008/98/EC of The European Parliament and of the Council of 19 November 2008 on Waste and repealing certain Directives".

Hadroug, S. et al. (2019), "Pyrolysis process as a sustainable management option of poultry manure: Characterization of the derived biochars and assessment of their nutrient release capacities," Water (Switzerland), 11(11), 1-18, https://doi.org/10.3390/w11112271.

Hidalgo, D., Martín-Marroquín, J.M., and Corona, F. (2019), “A multi-waste management concept as a basis towards a circular economy model", Renew. Sustain. Energy Rev., 111, 481-489, https://doi.org/10.1016/j.rser.2019.05.048.

Kelleher, B.P., Leahy, J.J., Henihan, A.M., O’Dwyer, T.F., Sutton, D., and Leahy, M.J. (2002), “Advances in poultry litter disposal technology - A review”, Bioresour. Technol., 83(1), 27-36,

https://doi.org/10.24264/icams-2020.IV.12 
https://doi.org/10.1016/S0960-8524(01)00133-X.

Klemeš, J.J., Varbanov, P.S., Walmsley, T.G., and Foley, A. (2019), "Process Integration and Circular Economy for Renewable and Sustainable Energy Systems", Renew. Sustain. Energy Rev., 116(October), https://doi.org/10.1016/j.rser.2019.109435.

Krajačić, G., Duić, N., Vujanović, M., Kılkış, Ş., Rosen, M.A., and Al-Nimr, M.A. (2016), "Sustainable development of energy, water and environment systems for future energy technologies and concepts", Energy Conversion and Management, 125, 1-14, https://doi.org/10.1016/j.enconman.2016.08.050.

Lazaroiu, G. et al. (2018), "Experimental Investigations of Innovative Biomass Energy Harnessing Solutions", Energies, 11(12), 3469, https://doi.org/10.3390/en11123469.

Mihaescu, L., Lazaroiu, G., Pisa, I., Ciupageanu, D.-A., and Berbece, V. (2018), "Energy valorization of poultry manure through products resulted in a pyrolysis process", in: 18th International Multidisciplinary Scientific GeoConference SGEM2018, 349-355, https://doi.org/10.5593/sgem2018/4.1/S17.046.

Mihaescu, L., Lazaroiu, G., Pisa, I., Negreanu, G.-P., Berbece, V., and. Ciupageanu, D.-A. (2019), "Thermal conditions required for poultry manure combustion in furnace boilers", in: 19th International Multidisciplinary Scientific Geoconference SGEM2019, 19(4.1), 569-576, https://doi.org/10.5593/sgem2019/4.1/S17.073.

Sarfaraz, Q. et al. (2020), "Characterization and carbon mineralization of biochars produced from different animal manures and plant residues", Sci. Rep., 10(1), 1-9, https://doi.org/10.1038/s41598-020-57987-8.

Solargis, Solar resource maps and GIS data for 200+ countries, https://solargis.com/maps-and-gis-data.

*** (2011), "Waste regime", Romanian Law 211/2011.

*** (2015), "Closing the loop - An EU action plan for the circular economy, $\operatorname{COM(2015)~} 614$ final. A circular economy is one in which the value of products, materials and resources is maintained for as long as possible, minimising waste and resource use." 
Efficient Poultry Industry Waste Management Approach in the Bioeconomy Framework

https://doi.org/10.24264/icams-2020.IV.12 


\title{
BIODEGRADABLE POLYMERIC COMPOSITES BASED ON EPDM RUBBER
} AND FUNCTIONALIZED ELASTOMERIC WASTE

\author{
MIHAELA (VILSAN) NITUICA ${ }^{1}$, LAURENTIA ALEXANDRESCU ${ }^{1}$, MIHAI GEORGESCU $^{1}$, \\ MARIA SONMEZ ${ }^{1}$, MARIA DANIELA STELESCU ${ }^{1}$, DANA GURAU ${ }^{1}$, CARMEN \\ CURUTIU $^{2}$, STEFANIA STOLERIU ${ }^{3}$ \\ ${ }^{1}$ INCDTP - Division Leather and Footwear Research Institute, 93 Ion Minulescu St., district 3 , \\ Bucharest,icpi@icpi.ro,mihaela.nituica@icpi.ro \\ ${ }^{2}$ University of Bucharest, Faculty of Biology, 1-3 Portocalelor St., 6th district, 060101, \\ Bucharest, Romania \\ ${ }^{3}$ University Politehnica of Bucharest, Faculty of Applied Chemistry and Materials Science, 1 \\ Polizu Str., 011061, Bucharest, Romania
}

\begin{abstract}
Elastomeric and plastic materials are used in different sectors of the European Union, and their recycling and reuse is still at a low level, compared to other types of waste (paper, glass, etc.). By approaching an efficient global strategy related to waste management, it is possible to make the transition to a circular economy with low $\mathrm{CO} 2$ emissions, offering the population a cleaner and safer environment. Due to the transformation of waste by various methods into new value-added products, we can say that their life cycle contributes to the efficiency of the economy and to the reduction of the negative impact on the environment. Research can make a difference in preventing the generation of technological and post-consumer polymeric waste by making biodegradable polymer composites that are harmless to the environment and ecosystem. The biodegradable polymer composites based on EPDM elastomer and rubber waste (rubber powder) were made on equipment specific to elastomers and characterized rheologically and physicallymechanically according to the standards in force.
\end{abstract}

Keywords: polymeric materials, elastomeric waste, characterization, EPDM

\section{INTRODUCTION}

An important part of the source of pollution with $\mathrm{CO}_{2}$ and other harmful elements originates from the industries producing and processing elastomers and plastics. The direct and indirect harmful effects due to these industries, as well as the materials used as raw materials and finished products can be mitigated by developing reusable technologies and environmentally friendly materials (optimized by their longer lifespan, through a much longer cycle large, compared to the current one - up to 4 cycles of reuse in production) (Plastic Recyclers Europe, 2015; United Nations Environment Programme, 2015).

By making biodegradable polymer composites based on elastomers compounded with technological and post-consumer polymeric waste using appropriate technologies, without them having a negative impact on the ecosystem for a long time, the products obtained will be viable both in terms of high performance, economic and ecological properties (Nituica et al., 2018; Plastics, The Facts, 2017; Regulation (EC) No. 1013/2006; Fan et al., 2019).

The development of new biodegradable polymeric materials based on EPDM rubber compounded with elastomeric waste (rubber powder) through various vulcanization systems, lead to the greening of processing technology by eliminating pollutants released during the vulcanization operation and of course waste elimination, by the possibility of reintroducing it into the production process, without negatively influencing the quality of products to protect the human factor and natural resources, increasing the sustainability of both the current population and future generations 
(Stelescu, 2011; Roucoules et al., 2007; Stelescu et al., 2020). All these meet the current quality and aesthetic requirements, for obtaining components designed for footwear and elastomeric parts without special characteristics by injection, as well as by mold pressing. This paper describes their development and testing by methods of obtaining and characterization using equipment specific to elastomers (Alexandrescu $e t$ al., 2019).

\section{EXPERIMENTAL PROCEDURE}

\section{Materials}

Materials used to obtained the antibacterial composites were: (1) EPDM, ethylenepropylene-diene terpolymer rubber, specific gravity -0.872 , Mooney viscosity -60 $\mathrm{MU}$, ethylene content $-67.5 \mathrm{wt} \%$, ethylidene norbornene (EBN) contents $-5.0 \mathrm{wt} \%$; (2) ST, stearin, granules, white color, molecular weight $284,48 \mathrm{~g} / \mathrm{mol}$, dynamic viscosity $9,87 \mathrm{mPa} . \mathrm{s}$ at $70^{\circ} \mathrm{C}$, volumetric weight approx. $400-500 \mathrm{~kg} / \mathrm{m}^{3}$; (3) $\mathbf{Z n O}$, zinc oxide, microparticles: white powder, precipitate $93-95 \%$, density $-5.5 \mathrm{~g} / \mathrm{cm}$, specific surface - 45-55 m²/g; (4) $\mathrm{SiO}_{2}$ - silicon dioxide, molecular mass $60,08 \mathrm{~g} / \mathrm{mol}$, white color, volumetric weight approx. $200-1.430 \mathrm{~kg} / \mathrm{m}^{3}$, particle size $<0,5 \mathrm{~mm}$; (5) $\mathbf{C a C O}_{3}$, chalk precipitate white powder, molecular weight 100.09; (6) elastomeric waste, obtained by cryogenic grinding at $10000 \mathrm{rpm}$ for $15 \mathrm{~s}$ and screened through a $1 \mathrm{~mm}$ mesh screen; (7) PEG 4000, polyethylene glycol, slightly yellow or white flakes, $\mathrm{pH}$ : 57 (1\% APA), density: $1.080 \mathrm{~g} / \mathrm{cm}^{3}$, dynamic viscosity: $310 \mathrm{mPa} . \mathrm{s}$; (8) DOF, dioctylphthalate, colorless liquid, density: $0.982 \mathrm{~g} / \mathrm{cm}^{3}$; (9) IPPD, N-Isopropyl-N'phenyl-1,4-phenylenediamine, brown flat granules, molar mass: $226,317 \mathrm{~g} / \mathrm{mol}$, density: $1.04 \mathrm{~g} / \mathrm{cm}^{3}$; (10) $\mathbf{S}$, sulphur, vulcanization agent (fine yellow powder, insoluble in water, melting point: $115^{\circ} \mathrm{C}$, faint odor); (11) $\mathbf{T H}$, tetramethylthiuram disulfidecuring agent (density $1.40 \mathrm{~g} / \mathrm{cm}$, melting point $<146^{\circ} \mathrm{C}$, an ultrafast curing accelerator); (12) M, 2 - mercaptobenzothiazole, curing agent (slow curing accelerator, molar mass: $167.25 \mathrm{~g} / \mathrm{mol}$, assay $97 \%$, density $1.19 \mathrm{~g} / \mathrm{cm}$ ).

\section{Preparation and Characterization of Biodegradable Polymer Composites}

Biodegradable polymeric composites based on EPDM rubber and functionalized elastomeric waste were made by mixing technique on a Plasti-Corder Brabender Mixer (Table 1), with the possibility of adjusting the temperature and mixing speed, by strictly observing the order of introduction of the ingredients according to the recipe in Table 1. Vulcanization accelerators were added on a laboratory roller, of $1 \mathrm{~kg}$ mixing capacity, according to the working method in Table 1.

The obtained biodegradable polymer composite recipes are then rheologically tested on a Monsanto 100S Rheometer, to establish the optimal vulcanization time and processing in the electric press (at the preset pressure and temperature parameters). The sample is closed tightly in a cavity of the device, at a controlled temperature, which surrounds a rotor with oscillations at a frequency of $1.67 \mathrm{~Hz}(100 \mathrm{cpm})$, so the output data correlates with the degree of vulcanization depending on the vulcanization time. To determine vulcanization characteristics for Monsanto rheometer, the working temperature was $165^{\circ} \mathrm{C}$ and time of $24^{\prime}$.

To characterize the biodegradable polymer composites, plates were pressed in the electric laboratory press, TP 600, at specific parameters, by means of compression 
method, between its platters, Table 2. After conditioning the plates for 24 hours at ambient temperature they are subjected to determinations.

Biodegradable polymeric composites based on EPDM and elastomeric waste were characterized in terms of rheological and physical-mechanical characteristics by appropriate techniques.

The biodegradable polymeric composites were tested in compliance with the rheological and physical-mechanical standards in effect: determination of vulcanization characteristics - SR ISO 3417; hardness ${ }^{\circ} \mathrm{ShA}$ - ISO 48-4:2018; elasticity \%, ISO 4662:2017; tensile strength, modulus, N/ $\mathrm{mm}^{2}$ - SR ISO 37-2020; tear strength, $\mathrm{N} / \mathrm{mm}-$ ISO 34-1:2015; elongation at break, $\mathrm{N} / \mathrm{mm}^{2}$ - SR ISO 37-2020; density, $\mathrm{g} / \mathrm{cm}^{3}-$ ISO 2781:2018, normal condition.

Table 1. Formulation of biodegradable compounds based on EPDM rubber and wood waste

\begin{tabular}{|c|c|c|c|c|c|c|}
\hline Symbol & $\mathrm{MU}$ & S0 & $\mathrm{SC}_{1}$ & $\mathrm{SC}_{2}$ & $\mathrm{SC}_{3}$ & $\mathrm{SC}_{4}$ \\
\hline \multicolumn{7}{|c|}{$\begin{array}{l}\text { Processing on Plasti-Corder Brabender Mixer, capacity } 350 \mathrm{~g} \text {, following the order in Table } 1 \\
\text { plasticizing EPDM for } 1{ }^{\prime} 30^{\prime}, 40 \mathrm{rpm} \text {, at } 45^{\circ} \mathrm{C} \text {; all ingredients (without vulcanizing agents) } \\
\text { mixing time }-4{ }^{\prime} 30^{\prime},, 20 \mathrm{rpm} \text {, at } 45^{\circ} \mathrm{C} \text {; homogenization time }-2^{\prime}, 60 \mathrm{rpm} \text {, at } 60^{\circ}-145^{\circ} \mathrm{C} \text {; tota } \\
\text { mixing time }-8 \text { '. }\end{array}$} \\
\hline EPDM & $\mathrm{g}$ & 190 & 190 & 190 & 190 & 190 \\
\hline Stearin & $\mathrm{g}$ & 2.85 & 2.85 & 2.85 & 2.85 & 2.85 \\
\hline $\mathrm{ZnO}$ & $\mathrm{g}$ & 9.5 & 9.5 & 9.5 & 9.5 & 9.5 \\
\hline $\mathrm{SiO}_{2}$ & $\mathrm{~g}$ & 19 & 38 & 19 & - & - \\
\hline $\mathrm{CaCO}_{3}$ & $\mathrm{~g}$ & 76 & 47.5 & 47.5 & 47.5 & 9.5 \\
\hline Elastomeric waste & g & - & 19 & 38 & 57 & 95 \\
\hline PEG4000 & $\begin{array}{l}8 \\
\mathrm{~g}\end{array}$ & 7.6 & 7.6 & 7.6 & 7.6 & 7.6 \\
\hline DOF & $\mathrm{g}$ & 19 & 19 & 19 & 19 & 19 \\
\hline IPPD & $\mathrm{g}$ & 5.7 & 5.7 & 5.7 & 5.7 & 5.7 \\
\hline \multicolumn{7}{|c|}{$\begin{array}{l}\text { Processing on laboratory electric roller, water cooling; mixing at } 20^{\circ}-30^{\circ} \mathrm{C} \text { temperature, } 50 \\
\text { rpm, addition and mixing time between 4' to } 8 \text { ', continuing mixing for maximum } 2 \text { ', the } \\
\text { mixture obtained is in the form of a } 3 \mathrm{~mm} \text { thick sheet. }\end{array}$} \\
\hline Sulphur & $\mathrm{g}$ & 1.9 & 1.9 & 1.9 & 1.9 & 1.9 \\
\hline M & $\mathrm{g}$ & 2.28 & 2.28 & 2.28 & 2.28 & 2.28 \\
\hline Th & $\mathrm{g}$ & 1.14 & 1.14 & 1.14 & 1.14 & 1.14 \\
\hline
\end{tabular}

Table 2. Vulcanization parameters on electric press, TP 600, for biodegradable polymeric composites, $\mathrm{S} 0, \mathrm{SC}_{1}-\mathrm{SC}_{4}$

\begin{tabular}{lccccc}
\hline Vulcanization parameters & & \multicolumn{3}{c}{ Symbol } \\
& $\mathrm{S} 0$ & $\mathrm{SC}_{1}$ & $\mathrm{SC}_{2}$ & $\mathrm{SC}_{3}$ & $\mathrm{SC}_{4}$ \\
\hline Vulcanization temperature & $165^{\circ} \mathrm{C}$ & $165^{\circ} \mathrm{C}$ & $165^{\circ} \mathrm{C}$ & $165^{\circ} \mathrm{C}$ & $165^{\circ} \mathrm{C}$ \\
Vulcanization time & $6^{\prime}$ & $6^{\prime}$ & $6^{\prime}$ & $6^{\prime}$ & $6^{\prime}$ \\
Cooling time & $10^{\prime}$ & $10^{\prime}$ & $10^{\prime}$ & $10^{\prime}$ & $10^{\prime}$ \\
Pressing force & $300 \mathrm{kN}$ & $300 \mathrm{kN}$ & $300 \mathrm{kN}$ & $300 \mathrm{kN}$ & $300 \mathrm{kN}$ \\
Cooling temperature & $45^{\circ} \mathrm{C}$ & $45^{\circ} \mathrm{C}$ & $45^{\circ} \mathrm{C}$ & $45^{\circ} \mathrm{C}$ & $45^{\circ} \mathrm{C}$ \\
\hline
\end{tabular}




\section{RESULTS AND DISCUSSION}

The biodegradable polymeric composites based on EPDM rubber and elastomeric waste (rubber powder) were characterized from a rheological and physical-mechanical point of view.

\section{Rheological Characterization of Biodegradable Polymeric Composites}

The rheological characteristics (Table 3) for the biodegradable polymeric composites based on EPDM rubber and rubber powder (elastomeric waste) were determined using the Monsanto rheometer, at $\mathrm{T}=165^{\circ} \mathrm{C}$. By replacing the amount of active batch, $\mathrm{SiO}_{2}$ with elastomeric waste functionalized with potassium oleate, from the interpretation of the interregistered rheological characteristics the following are observed:

- minimum torque - ML, increases by a maximum of $28 \%$, and the maximum torque $(\mathrm{MH})$ decreases by a maximum of $48 \%$, so that the variation of the torque $(\Delta \mathrm{M})$ decreases by a maximum of $54 \%$ as the amount of elastomeric waste increases, to the detriment of the amount of $\mathrm{SiO}_{2}$, indicating a slight increase in the rigidity of the mixtures in the unvulcanized state and a decrease in the rigidity of the rubber mixtures in the vulcanized state;

- because the vulcanization system used in the mixing process is based on sulphur and semi-efficient vulcanization accelerators, for all samples there is NO reversal phenomenon, which is specific to vulcanized mixtures by the classical method (Figure 1), indicating a good behavior of mixtures at high temperatures or accelerated aging;

- scorching time $\left(\mathrm{ts}_{2}\right)$ decreases as the amount of rubber powder increases and the amount of active charge decreases, and the optimal vulcanization time shows a slight increase by replacing $\mathrm{SiO}_{2}$ with elastomeric waste functionalized with potassium oleate (rubber powder);

- comparing sample $\mathrm{SC}_{3}$ with sample $\mathrm{SC}_{4}$, it is observed that by replacing $20 \mathrm{phr}$ of calcium carbonate, which is inactive batch, with rubber powder, there is a decrease of $\mathrm{MH}$ and $\Delta \mathrm{M}$, by 11 , respectively $20 \%$ and increases of $4 \%, 14 \%$, $12 \%$ and $5 \%$ of $\mathrm{ML}, \mathrm{ts}_{2}, \mathrm{t}_{50}$ and $\mathrm{t}_{90}$, respectively.

Table 3. Rheological characteristics for biodegradable polymeric composites, $\mathrm{S} 0, \mathrm{SC}_{1}-$ $\mathrm{SC}_{4}$

\begin{tabular}{cccccc}
\hline Rheological characteristics, & \multicolumn{5}{c}{ Symbol } \\
$\mathrm{T}=165^{\circ} \mathrm{C}$ & $\mathrm{S} 0$ & $\mathrm{SC}_{1}$ & $\mathrm{SC}_{2}$ & $\mathrm{SC}_{3}$ & $\mathrm{SC}_{4}$ \\
\hline ML: minimum torque $(\mathrm{dNm})$ & 11.1 & & 14.2 & 14.1 & 14.7 \\
MH: maximum torque $(\mathrm{dNm})$ & 66.4 & 51.16 & 38.4 & 34.1 \\
$\Delta \mathrm{M}=$ MH-ML $(\mathrm{dNm})$ & 53.3 & 36.9 & 24.3 & 19.4 \\
ts2: scorching time (min) & 0.8 & 0.77 & 1.17 & 1.33 \\
t50: (min) & 1.52 & 1.49 & 2.22 & 2.48 \\
t90: optimal vulcanization time (min) & 4.7 & 7.42 & 8.09 & 8.52 \\
\hline
\end{tabular}


ICAMS $2020-8^{\text {th }}$ International Conference on Advanced Materials and Systems

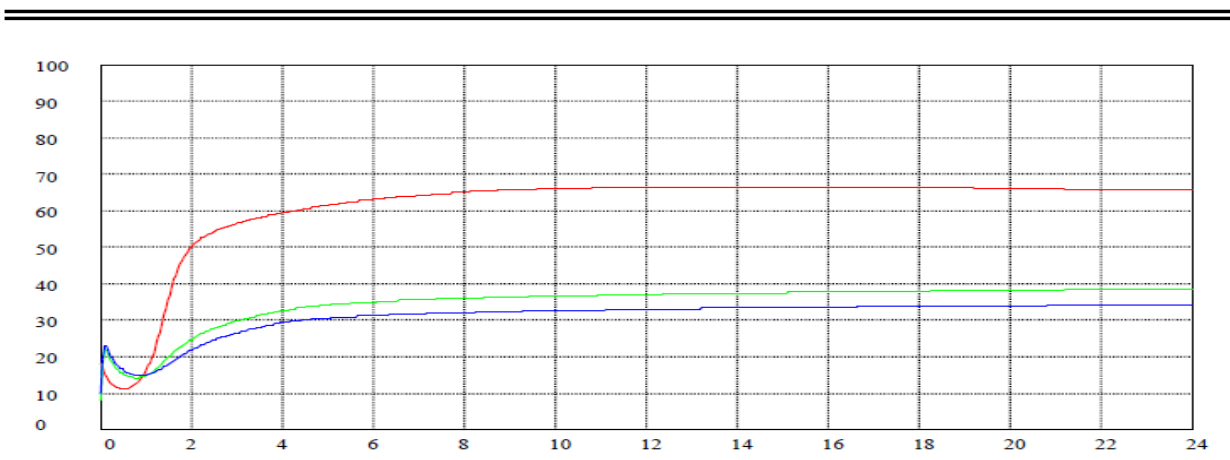

Figure 1. Rheogram recorded on Monsanto Rheometer for samples - S0 (red), $\mathrm{SC}_{2}$ (green), $\mathrm{SC}_{3}$ (blue)

\section{Physical-Mechanical Characterization of Biodegradable Polymeric Composites}

The biodegradable polymeric composites based on EPDM rubber and functionalized elastomeric waste (rubber waste) are subjected to physical-mechanical determinations. After stabilization at room temperature for 24 hours the following were recorded, Table 4 :

Table 4. Physical-mechanical characterisation of biodegradable polymeric composites based on EPDM rubber and functionalized elastomeric waste

\begin{tabular}{|c|c|c|c|c|c|}
\hline Symbol & S0 & $\mathrm{SC}_{1}$ & $\mathrm{SC}_{2}$ & $\mathrm{SC}_{3}$ & \\
\hline \multicolumn{5}{|c|}{ Physical-mechanical characterization: normal condition } & \\
\hline Hardness, ${ }^{\circ} \mathrm{Sh} \mathrm{A}$ & 50 & 54 & 49 & 47 & 46 \\
\hline Elasticity, \% & 32 & 34 & 28 & 32 & 30 \\
\hline Modulus $100, \%$ & 0.89 & 0.91 & 0.75 & 0.83 & 0.68 \\
\hline Modulus 300, \% & 1.47 & 1.3 & 1.04 & 0.85 & 0.81 \\
\hline Tensile strength, $\mathrm{N} / \mathrm{mm}^{2}$ & 2.06 & 3.51 & 1.4 & 0.99 & 0.81 \\
\hline Elongation at break, \% & 420 & 580 & 500 & 500 & 560 \\
\hline Tear strength, $\mathrm{N} / \mathrm{mm}^{2}$ & 10.37 & 16.6 & 11.03 & 8.6 & 7.53 \\
\hline Density, $\mathrm{g} / \mathrm{cm}^{3}$ & 1.14 & 1.12 & 1.1 & 1.07 & 1.0 \\
\hline
\end{tabular}

From the data recorded for biodegradable polymeric composites based on ethylenepropylene-terpolymer rubber (EPDM) and rubber powder (elastomeric waste functionalized with potassium oleate), it is observed that by replacing the amount of active filler $-\mathrm{SiO}_{2}$ with functionalized elastomeric waste, the physical-mechanical characteristics of the sample are modified as follows:

* the hardness varies with $\pm 5^{\circ} \mathrm{ShA}$, and the elasticity presents a non-uniform variation of $\pm 13 \%$;

* the values of tensile strength, tear strength, elongation at break and modulus increase as the active filler is replaced with the elastomeric waste functionalized with potassium oleate, presenting a maximum point for the $\mathrm{SC}_{1}$ sample;

the density of the samples decreases as the amount of powder increases.

Comparing sample $\mathrm{SC}_{3}$ with $\mathrm{SC}_{4}$, it is observed that by replacing $20 \mathrm{phr}$ of calcium carbonate with rubber powder there is a decrease in hardness, modulus, tear strength, and an increase in elongation at break. The results obtained are due primarily to the composition and morphology of the mixture obtained. 


\section{Biodegradable Polymeric Composites Based on EPDM Rubber and Functionalized Elastomeric Waste}

\section{CONCLUSIONS}

The biodegradable polymeric composites based on EPDM rubber and functionalized elastomeric waste (rubber waste) are tested in compliance to rheological and physicalmechanical determinations, on equipment specific to elastomer determinations (normal condition).

The rheological characteristics for the biodegradable polymeric composites based on EPDM rubber and rubber powder were performed to establish the optimal vulcanization and processing times in the electric press.

Comparing sample $\mathrm{SC}_{3}$ with $\mathrm{SC}_{4}$, it is observed that by replacing $20 \mathrm{phr}$ of calcium carbonate with rubber powder there is a decrease in hardness, modulus, tear strength, and an increase in elongation at break. The results obtained are due primarily to the composition and morphology of the mixture obtained

The obtained biodegradable polymeric composites based on elastomeric rubber (EPDM rubber) and elastomeric waste (rubber powder) are used in the processing of general-purpose footwear and elastomeric parts without special characteristics.

\section{Acknowledgements}

This research was financed by ANCSI through PN 1917 01 03/2019 project: "Biodegradable composites from technological and post-consumption polymeric wastes by designing and applying 4R eco-innovative technologies (4R-ECO-MAT)", and 6PFE/2018 - PERFORM-TEX-PEL.

\section{REFERENCES}

Alexandrescu, L., Deselnicu, V., Sonmez, M., Georgescu, M., Nituica, M., Zainescu, G., Deselnicu, D.C. and Pang, X. (2019), "Biodegradable polymer composite based on recycled polyurethane and finished leather waste", IOP Conf. Series: Earth and Environmental Science, 401, https://doi.org/10.1088/17551315/401/1/012006

Fan, Q., Ma, J. and Xu, Q. (2019), "Insights into functional polymer-based organic-inorganic nanocomposites as leather finishes", Journal of Leather Science and Engineering, 1(3), https://doi.org/10.1186/s42825019-0005-9.

Nituică (Vîlsan), M., Meghea, A., Gurău, D. and Stelescu, M.D. (2018), "PP/EPDM Polymeric Compound Dynamically Cured Compatibilized and Reinforced with Nanoclay”, Plastic Materials, 53(3), 335-339, https://doi.org/10.37358/MP.18.3.5024.

Plastic Recyclers Europe (2015), Increased EU Plastics Recycling Targets: Environmental, Economic and Social Impact Assessment - Final Report, Deloitte, available at: https://www.plasticsrecyclers.eu/plasticsrecyclers-publications.

Plastics, The Facts (2017), “An Analysis of European Plastics Production, Demand and Waste Data. Plastics Europe", available online: the facts 2017 FINAL for website one page.pdf (accessed on 5 December 2018), https://www.plasticseurope.org/application/files/5715/1717/4180/Plastics

Roucoules, V., Siffer, F., Ponche, A., Egurrola, U. and Vallat, M.F. (2007), "Strengthening the Junction between EPDM and Aluminium Substrate via Plasma Polymerisation", The Journal of Adhesion, 83(10), 875-895, https://doi.org/10.1080/00218460701699732.

Stelescu, M.D. (2011), Thermoplastic elastomers based on ethylene-propylene rubber (EPDM), which can be used in the footwear industry (in Romanian), Performantica Press, Iasi, ISBN: 978-973-730-809-2.

Stelescu, M.D., Manaila, E., Nituica, M. and Georgescu, M. (2020), "New materials based on Ethylene Propylene Diene Terpolymerand Hemp Fibres Obtained by Green Reactive Processing", Materials, 13, 2067, https://doi.org/10.3390/ma13092067.

United Nations Environment Programme (2015), Global Waste Management Outlook, available at: https://www.unenvironment.org/resources/report/global-waste-management-outlook.

*** (2006), Regulation (EC) No 1013/2006 of the European Parliament and of the Council of 14 June 2006 on shipments of waste. 
DEVELOPMENT AND CHARACTERIZATION OF BIODEGRADABLE COMPOUND BASED ON EPDM AND WOOD WASTE

\author{
MIHAELA (VILSAN) NITUICA ${ }^{1}$, LAURENTIA ALEXANDRESCU ${ }^{1}$, MIHAI GEORGESCU $^{1}$, \\ MARIA SONMEZ ${ }^{1}$, MARIA DANIELA STELESCU ${ }^{1}$, DANA GURAU ${ }^{1}$, CARMEN $^{2}$ \\ CURUTIU $^{2}$, STEFANIA STOLERIU ${ }^{3}$ \\ ${ }^{1}$ INCDTP - Division Leather and Footwear Research Institute, 93 Ion Minulescu St., district 3 , \\ Bucharest,icpi@icpi.ro,mihaela.nituica@icpi.ro \\ ${ }^{2}$ University of Bucharest, Faculty of Biology, 1-3 Portocalelor St., 6th district, 060101, \\ Bucharest, Romania \\ ${ }^{3}$ University Politehnica of Bucharest, Faculty of Applied Chemistry and Materials Science, 1 \\ Polizu Str., 011061, Bucharest, Romania
}

\begin{abstract}
In the European Union, the potential for recycling technological and post-consumer polymeric waste is untapped. Their recycling and reuse are very low, compared to other types of waste such as glass, paper, etc., and the rates of storage, even of incineration, is very high in terms of percentage. Therefore, by reusing them, but also making use of new advanced technologies, we can contribute to improving the quality of products, and to environmental protection by recycling waste, protecting human health by eliminating toxins during their incineration, but also increasing turnover for global economic agents. Thus, this paper presents the obtaining and characterization of an antibacterial compound based on EPDM elastomer and wood waste (sawdust). The antibacterial compound is characterized from a physical-mechanical and structural point of view (FT-IR), all according to standards in force.
\end{abstract}

Keywords: polymeric materials, wood waste, characterization, EPDM

\title{
INTRODUCTION
}

Worldwide, and in Europe especially, millions of tons of waste are generated every year from the textile industry, from the wood processing industry and not only. Reuse and recycling of waste are real options to reduce the amount of waste and thus their impact on the environment, as provided by Directive 2008/98/EC (Plastics, The Facts, 2017). About $30 \%$ of this waste, even less, is collected for recycling. A large part of this is stored in improper conditions, and incineration has seen an alarming increase in the last decade. Thus, it has been globally estimated that by generating and burning only plastic waste and those from the wood industry, approximately 600 million tons of $\mathrm{CO}_{2}$ are generated (Plastics, The Facts, 2017). Wood waste is classified by European standards as common waste, not hazardous or toxic to the environment. The transformation of waste (cryogenically ground and functionalized) into new valueadded products will lead to remarkable improvements in the life cycle of raw materials and sustainable use of this waste, contributing to increasing sustainability, improving eco-efficiency and economic efficiency and reducing the "pressure" of environmental waste (Alexandrescu et al., 2019; Zhu et al., 2017; Fan et al., 2019).

The compounding of elastomers and wastes, in the presence of vulcanization systems, led to biodegradable mixtures with good properties for the footwear industry and for elastomeric parts without special characteristics (Nituica et al., 2018). Vulcanization of compounds has a major impact on the final properties of products, representing an important property, and in order to obtain biodegradable composites, current trends are the use of natural materials (wood, protein fibers, etc.) and vulcanized rubber as a reinforcing material (Roucoules et al., 2007; Stelescu, 2011; Stelescu et al., 2020). 


\section{EXPERIMENTAL PROCEDURE}

\section{Materials}

The following materials were used: (1) ethylene-propylene-diene (EPDM), terpolymer rubber, specific gravity -0.872 , Mooney viscosity - 60 MU, ethylene content - $67.5 \mathrm{wt} \%$, ethylidene norbornene (EBN) contents - $5.0 \mathrm{wt} \%$; (2) Stearin (ST), granules, white color, molecular weight $284,48 \mathrm{~g} / \mathrm{mol}$, dynamic viscosity 9,87 mPa.s at $70^{\circ} \mathrm{C}$, volumetric weight approx. $400-500 \mathrm{~kg} / \mathrm{m}^{3}$; (3) Zinc oxide $(\mathbf{Z n O})$, microparticles: white powder, precipitate $93-95 \%$, density $-5.5 \mathrm{~g} / \mathrm{cm}$, specific surface $45-55 \mathrm{~m}^{2} / \mathrm{g}$ ); (4) chalk (CaCO3 precipitate) - white powder, molecular weight 100.09); (5) wood waste (sawdust), obtained by cryogenic grinding at $10000 \mathrm{rpm}$ for $15 \mathrm{~s}$ and screened through a $1 \mathrm{~mm}$ mesh screen; (6) silicon dioxide $\left(\mathbf{S i \mathbf { O } _ { 2 }}\right)$, molecular mass 60,08 $\mathrm{g} / \mathrm{mol}$, white color, volumetric weight approx. $200-1.430 \mathrm{~kg} / \mathrm{m}^{3}$, particle size $<0,5$ mm; (7) Polyethylene Glycol (PEG 4000), slightly yellow or white flakes, pH: 5-7(1\% APA), density: $1.080 \mathrm{~g} / \mathrm{cm}^{3}$, dynamic viscosity: $310 \mathrm{mPa} . \mathrm{s}$; (8) Dioctylphthalate (DOF), colorless liquid, density: $0.982 \mathrm{~g} / \mathrm{cm}^{3}$; (9) N-Isopropyl-N'-phenyl-1,4-phenylenediamine (IPPD), brown flat granules, molar mass: $226,317 \mathrm{~g} / \mathrm{mol}$, density: $1.04 \mathrm{~g} / \mathrm{cm}^{3} ;$ (10) sulphur (S), vulcanization agent (fine yellow powder, insoluble in water, melting point: $115^{\circ} \mathrm{C}$, faint odor); (11) tetramethylthiuram disulfide (TH) - curing agent (density 1.40 $\mathrm{g} / \mathrm{cm}$, melting point $<146^{\circ} \mathrm{C}$, an ultrafast curing accelerator); (12) 2mercaptobenzothiazole $(\mathbf{M})$ - curing agent (slow curing accelerator, molar mass: $167.25 \mathrm{~g} / \mathrm{mol}$, assay $97 \%$, density $1.19 \mathrm{~g} / \mathrm{cm}$.

\section{Procedure}

The biodegradable compounds based on EPDM elastomer, wood waste (sawdust), $\mathrm{CaCO}_{3}, \mathrm{SiO}_{2}, \mathrm{ZnO}$, antioxidants, sulfur, Th, $\mathrm{M}$ and plasticizers were made on a PlastiCorder Brabender Mixer, in strict compliance with the order of introduction of the ingredients, Table 2. After the mixing process, the recipes were completed with accelerators on a laboratory roller, $1 \mathrm{~kg}$ capacity, water cooling. The making of the mixtures on the Plasti-Corder Brabender mixer is presented in Table 1.

Table 1. Working method using Brabender mixer

\begin{tabular}{|c|c|c|c|}
\hline $\begin{array}{c}\text { The order of introduction } \\
\text { of the ingredients }\end{array}$ & $\begin{array}{l}\text { Mixing time } \\
\text { (minutes) }\end{array}$ & Mixing speed & $\begin{array}{c}\text { Temperature, } \\
{ }^{\circ} \mathrm{C} \\
\end{array}$ \\
\hline EPDM rubber & 1'30”' & $40 \mathrm{rpm}$ & $45^{\circ} \mathrm{C}$ \\
\hline $\begin{array}{l}\text { Ingredients (without } \\
\text { vulcanizing agents) }\end{array}$ & $4^{\prime} 30^{\prime \prime}$ & $20 \mathrm{rpm}$ & \\
\hline Homogenization time & $2^{\prime}$ & $60 \mathrm{rpm}$ & $60-145^{\circ} \mathrm{C}$ \\
\hline TOTAL & 8, & $20-60 \mathrm{rpm}$ & $45^{\circ} \mathrm{C}-145^{\circ} \mathrm{C}$ \\
\hline
\end{tabular}

Vulcanizing agents are added on the laboratory electric roller, at temperatures of 23$30^{\circ} \mathrm{C}, 50 \mathrm{rpm}$, and the working method is as follows: dosing of raw materials; the mixture is plasticized between the rollers; the vulcanizing agents are introduced according to the recipe, Table 1; addition and mixing time 5-10 minutes; after adding the vulcanization accelerators, mixing is continued for 1-2 minutes for a good homogenization. The mixture is then removed in the form of a $2-3 \mathrm{~mm}$ thick sheet. 
For the characterization the obtained polymer composite is added in the molds, using the electrically heated press, TP 600 , by means of compression method, between its platters, at a temperature of $165^{\circ} \mathrm{C}$ and $300 \mathrm{KN}$ pressure for 6 to 13 minutes actual forming in the press and 10 minutes cooling with water.

Table 2. Formulation of biodegradable compounds based on EPDM rubber and wood waste

\begin{tabular}{lcccccc}
\hline Symbol & MU & S0 & $\mathrm{SL}_{1}$ & $\mathrm{SL}_{2}$ & $\mathrm{SL}_{3}$ & $\mathrm{SL}_{4}$ \\
\hline EPDM & $\mathrm{g}$ & 190 & 190 & 190 & 190 & 190 \\
Stearin & $\mathrm{g}$ & 2.85 & 2.85 & 2.85 & 2.85 & 2.85 \\
$\mathrm{ZnO}$ & $\mathrm{g}$ & 9.5 & 9.5 & 9.5 & 9.5 & 9.5 \\
$\mathrm{SiO}_{2}$ & $\mathrm{~g}$ & 19 & 38 & 19 & 0 & 0 \\
$\mathrm{CaCO}_{3}$ & $\mathrm{~g}$ & 76 & 47.5 & 47.5 & 47.5 & 9.5 \\
Wood Waste & $\mathrm{g}$ & - & 19 & 38 & 57 & 95 \\
$\mathrm{PEG} 4000$ & $\mathrm{~g}$ & 7.6 & 7.6 & 7.6 & 7.6 & 7.6 \\
$\mathrm{DOF}$ & $\mathrm{g}$ & 19 & 19 & 19 & 19 & 19 \\
$\mathrm{IPPD}$ & $\mathrm{g}$ & 5.7 & 5.7 & 5.7 & 5.7 & 5.7 \\
$\mathrm{Sulphur}$ & $\mathrm{g}$ & 1.9 & 1.9 & 1.9 & 1.9 & 1.9 \\
$\mathrm{M}$ & $\mathrm{g}$ & 2.28 & 2.28 & 2.28 & 2.28 & 2.28 \\
Th & $\mathrm{g}$ & 1.14 & 1.14 & 1.14 & 1.14 & 1.14 \\
\hline
\end{tabular}

\section{Characterization of Biodegradable Compounds}

The testing of biodegradable polymeric compounds based on EPDM rubber and wood waste was performed in terms of physical-mechanical and structural characterization (FT-IR) by appropriate techniques.

The biodegradable compounds were tested in compliance with the physicalmechanical standards in effect: ${ }^{\circ}$ ShA hardness - ISO 48-4:2018; elasticity \%, ISO 4662:2017; tensile strength, modulus, N/mm² - SR ISO 37-2020; tear strength, N/mm ISO 34-1:2015; elongation at break, N/mm² - SR ISO 37-2020; Abrasion, $\mathrm{mm}^{3}$, SR ISO 4649/2010, normal condition.

FT-IR spectral determinations were performed with a double beam IR molecular absorption spectrometer, in the range $4000-400 \mathrm{~cm}^{-1}$, using the FT-IR Thermo Nicolet iS 50, equipped with ATR with diamond crystal.

\section{RESULTS AND DISCUSSION}

The obtained biodegradable compounds were physically-mechanically and structurally characterized according to the standards in force.

\section{Physical-Mechanical Characterization of Biodegradable Compounds}

After stabilization at room temperature for 24 hours, the biodegradable compound specimens based on EPDM rubber and sawdust (wood waste) are subjected to physicalmechanical determinations, Table 3. 
Development and Characterization of Biodegradable Compound Based on EPDM and Wood Waste

Table 3. Physical-mechanical characterization of biodegradable compounds based on EPDM rubber and wood waste

\begin{tabular}{lccccc}
\hline Symbol & S0 & SL $_{1}$ & SL $_{2}$ & SL $_{3}$ & SL4 \\
& Physical-mechanical characterization: normal condition & & \\
\hline Hardness, ${ }^{\circ}$ Sh A & 50 & 61 & 62 & 63 & 61 \\
Elasticity, \% & 32 & 34 & 36 & 34 & 32 \\
Modulus 100, \% & 0.89 & 1.2 & 1.03 & 0.89 & 0,75 \\
Modulus 300, \% & 1.47 & 1.8 & - & - & - \\
Tensile strength, N/mm & & 2.62 & 1.5 & 1.04 & 0.90 \\
Elongation at break, \% & 2.06 & 440 & 300 & 180 & 240 \\
Tear strength, N/mm & 420 & 18.05 & 12.76 & 7.76 & 12 \\
Abrasion resistance, mm $^{3}$ & 10.37 & 144.78 & 282.13 & 395.4 & 431.38 \\
\hline
\end{tabular}

As the amount of $\mathrm{SiO}_{2}$ in the mixtures is replaced, it is observed that the hardness of the compounds increases from $61-63^{\circ} \mathrm{ShA}$. The increase is determined by the hardness of the sawdust, as well as by the bonds that are formed during processing between the EPDM elastomer and the filler. Elasticity presents a small and non-uniform variation. The values of tensile strength, tear strength, elongation at break and modulus increase as the active filler is replaced with wood waste, reach a maximum point for the $\mathrm{SL}_{1}$ sample, and then decrease; this phenomenon may be due to larger particle sizes of wood and powder, respectively, compared to those of the active filler of silicon dioxide. The abrasion resistance in the case of these mixtures made with wood waste increases by approximately $9 \%$.

Comparing the 2 specimens that do not contain silicon dioxide, $\mathrm{SL}_{3}$ and $\mathrm{SL}_{4}$, it is observed that by replacing $20 \mathrm{phr}$ (parts) of calcium carbonate (inactive filler) with wood waste, there is a decrease of hardness by approximately $2^{\circ} \mathrm{ShA}$; also the values of tensile strength increase by $13 \%$ and of course in the case of the abrasion resistance an increase of $9 \%$ is observed. Elongation at break and tear strength also increase. The changes in characteristics are not significant, compared to the changes observed by replacing the silicon dioxide active filler.

\section{FT-IR Spectrometric Analysis}

The spectra for biodegradable compounds based on EPDM elastomer and wood waste are shown in Figures 1 and 2. The bands related to the stretching vibrations are a function of those obtained in the reference spectra for EPDM rubber and those of simple wood waste treated with potassium oleate. The vibration attributes obtained for the nonvulcanized EPDM elastomer are shown in Table 4.

Table 4. Vibration assignments and IR frequencies of unvulcanized EPDM

\begin{tabular}{llll}
\hline Sample code & Frequency & Intensity & Vibration assignment \\
\hline EPDM & 1463,71 & 0,1755108 & $\left(\mathrm{CH}_{2}\right) \mathrm{CH}_{3}$ asymmetric \\
& 1375,96 & 0,0827913 & $\mathrm{CH}_{3}$ symmetric \\
& 721,247 & 0,0512599 & $\left(\mathrm{CH}_{2}\right)$ crystallinity \\
\hline
\end{tabular}

The FTIR spectrum of untreated wood waste (Figure 1) shows the adsorption bands in the region $3339.55,2901.48$ and $1735.06 \mathrm{~cm}^{-1}$ due to the stretching vibrations of the $\mathrm{O}-\mathrm{H}, \mathrm{C}-\mathrm{H}$ and $\mathrm{C}=\mathrm{O}$ bonds. These adsorption bands are due to the hydroxyl groups in cellulose, the carbonyl group of acetyl ester in hemicellulose and the - $\mathrm{CHO}$ groups in lignin. The bands from $1601.17 \mathrm{~cm}^{-1}$ and $1508.58 \mathrm{~cm}^{-1}$ are due to $\mathrm{C}=\mathrm{C}$ bonds in the 
aromatic skeleton of lignin. The band corresponding to the peak at $1422.04 \mathrm{~cm}^{-1}$ is due to the deformation of lignin $\mathrm{C}-\mathrm{H}$ bonds and the band from $1263.31 \mathrm{~cm}^{-1}$ represents the stretching vibration of C-O bonds from lignin, while the one from $1027.01 \mathrm{~cm}^{-1}$ is due to the stretching of $\mathrm{C}-\mathrm{O}$ and $\mathrm{C}-\mathrm{C}$ bonds of the ring from cellulose and hemicellulose.

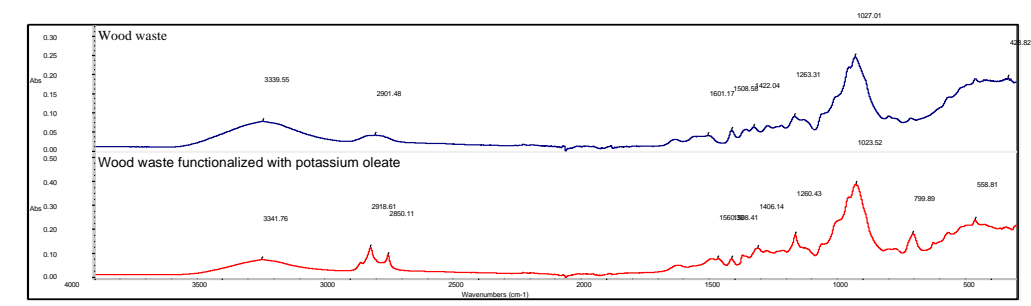

Figure 1. FTIR spectra of untreated wood waste and wood waste treated with potassium oleate

In the case of wood waste treated with potassium oleate, Figure 1, the band associated with $-\mathrm{OH}$ groups moved to the value of $3341.76 \mathrm{~cm}^{-1}$, and the band corresponding to C-H groups moved to $2918.61 \mathrm{~cm}^{-1}$ compared to the values obtained for the untreated wood waste. Moreover, the peak corresponding to the carbonyl groups $\mathrm{C}=\mathrm{O}$ moved to the value of $1722.9 \mathrm{~cm}^{-1}$ in the case of treated wood waste, because the ester bonds in hemicellulose were broken following the chemical treatment. The band from $1560.39 \mathrm{~cm}^{-1}$ comes from the -COO- group from oleate, which demonstrates that the process of modifying wood waste has taken place.

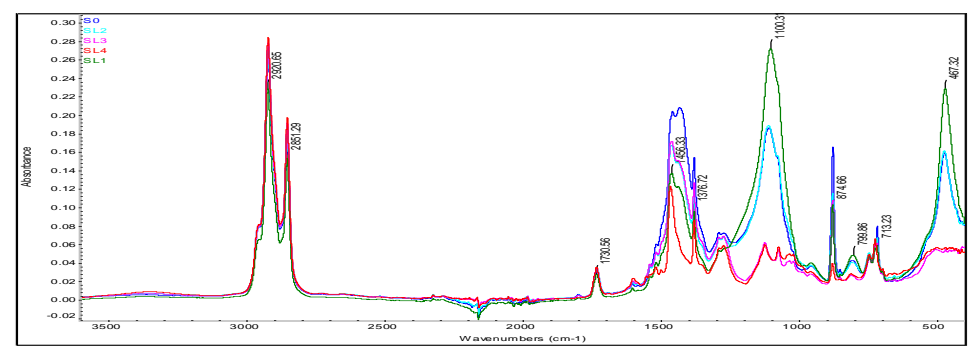

Figure 2. FTIR spectra of biodegradable compounds based on EPDM rubber and wood waste treated with potassium oleate, $\mathrm{S} 0, \mathrm{SL}_{1}, \mathrm{SL}_{2}, \mathrm{SL}_{3}, \mathrm{SL}_{4}$

In the case of EPDM potassium oleate-treated wood waste samples, Figure 2, the bands from silica, calcium carbonate, wood waste (sawdust) and EPDM rubber can be identified; their relative intensities vary depending on the percentage existing in mixtures. Thus, for a better identification of the presence of wood, the three spectra related to $\mathrm{SO}$ mixtures (control sample with the highest percentage of $\mathrm{CaCO}_{3}-40 \%$, $10 \% \mathrm{SiO}_{2}$, but without treated wood waste), the $\mathrm{SL}_{2}$ mixture containing $20 \% \mathrm{SiO}_{2}$, $20 \%$ treated wood waste and $25 \% \mathrm{CaCO}_{3}$, and $\mathrm{SL}_{4}$ mixture containing $50 \%$ treated wood waste, $5 \% \mathrm{CaCO}_{3}$ and $0 \% \mathrm{SiO}_{2}$ were overlapped. Thus, in $\mathrm{SL}_{2}$ one can easily see the $\mathrm{SiO}_{2}$ peak centered at about $1107 \mathrm{~cm}^{-1}$, and calcium carbonate at about 1427 , 874 and $713 \mathrm{~cm}^{-1}$. For the $\mathrm{SL}_{4}$ sample, the $1107 \mathrm{~cm}^{-1}$ band characteristic of silica disappears, and the band characteristic of calcium carbonate decreases significantly, due to the considerable reduction of the percentage of $\mathrm{CaCO}_{3}$. The wood waste, due to the low intensity peaks can be highlighted at about $1030 \mathrm{~cm}^{-1}$ as peaks

https://doi.org/10.24264/icams-2020.IV.14 
superimposed over the peaks characteristic of the basic compositions. The band from approximately $1730 \mathrm{~cm}^{-1}$ can be assigned to $\mathrm{C}=\mathrm{O}$ groups from the plasticizer used and DOF, its relative intensity being similar in all mixtures, because the amount introduced in all mixtures remained constant at $10 \%$.

\section{CONCLUSIONS}

The testing of biodegradable polymeric compounds based on EPDM rubber and wood waste was performed in terms of physical-mechanical and FT-IR characterization according to the standards in force after stabilization of room temperature for $24 \mathrm{~h}$.

From the comparison of the specimens that do not contain silicon dioxide, $\mathrm{SL}_{3}$ and $\mathrm{SL}_{4}$, it is observed that by replacing $20 \mathrm{phr}$ of calcium carbonate with wood waste, there is a decrease of hardness and an increase in tensile strength and abrasion. The changes in characteristics are not significant, compared to the changes observed by replacing the silicon dioxide active filler.

From the FT-IR spectra of the EPDM/potassium oleate-treated wood waste samples, the bands from silica, calcium carbonate, wood waste and EPDM rubber can be identified, their relative intensities varying depending on the percentage present in the mixtures.

The biodegradable compounds obtained are used in the processing of generalpurpose footwear and elastomeric parts without special characteristics.

\section{Acknowledgements}

This research was financed by ANCSI through PN 19- $170103 / 2019$ project: "Biodegradable composites from technological and post-consumption polymeric wastes by designing and applying 4R eco-innovative technologies (4R-ECO-MAT)", and 6PFE/2018 - PERFORM-TEX-PEL.

\section{REFERENCES}

Alexandrescu, L., Deselnicu, V., Sonmez, M., Georgescu, M., Nituica, M., Zainescu, G., Deselnicu, D.C. and Pang, X. (2019), "Biodegradable polymer composite based on recycled polyurethane and finished leather waste", IOP Conf. Series: Earth and Environmental Science, 401, https://doi.org/10.1088/1755$1315 / 401 / 1 / 012006$

Fan, Q., Ma, J. and Xu, Q. (2019), "Insights into functional polymer-based organic-inorganic nanocomposites as leather finishes", Journal of Leather Science and Engineering, 1(3), https://doi.org/10.1186/s42825019-0005-9.

Nițică (Vîlsan), M., Meghea, A., Gurău, D. and Stelescu, M.D. (2018), "PP/EPDM Polymeric Compound Dynamically Cured Compatibilized and Reinforced with Nanoclay”, Plastic Materials, 53(3), 335-339, https://doi.org/10.37358/MP.18.3.5024.

Plastics, The Facts (2017), "An Analysis of European Plastics Production, Demand and Waste Data. Plastics Europe”, available online: the_facts_2017_FINAL_for_website_one_page.pdf (accessed on 5 December 2018), https://www.plasticseurope.org/application/files/5715/1717/4180/Plastics

Roucoules, V., Siffer, F., Ponche, A., Egurrola, U. and Vallat, M.F. (2007), "Strengthening the Junction Between EPDM and Aluminium Substrate via Plasma Polymerisation”, The Journal of Adhesion, 83(10), 875-895, https://doi.org/10.1080/00218460701699732.

Stelescu, M.D. (2011), Thermoplastic elastomers based on ethylene-propylene rubber (EPDM), which can be used in the footwear industry (in Romanian), Performantica Press, Iasi, ISBN: 978-973-730-809-2.

Stelescu, M.D., Manaila, E., Nituica, M. and Georgescu, M. (2020), "New materials based on Ethylene Propylene Diene Terpolymerand Hemp Fibres Obtained by Green Reactive Processing", Materials, 13 2067, https://doi.org/10.3390/ma13092067.

Zhu, G., Xing, X., Wang, J. and Zhang, X. (2017), "Effect of acid and hydrothermal treatments on the dye adsorption properties of biomass-derived activated carbon", $J$ Mater Sci, 52, 7664-7676, https://doi.org/10.1007/s10853-017-1055-0.

https://doi.org/10.24264/icams-2020.IV.14 
CIRCULAR PRODUCT DESIGN ASSESSMENT APPLIED TO CLOTHING PRODUCTS

\author{
SABINA OLARU, IONELA BADEA \\ National Research and Development Institute for Textiles and Leather, 16, Lucretiu Patrascanu \\ street, Bucharest, Romania,email: sabina.olaru@incdtp.ro,ionela.badea@incdtp.ro
}

\begin{abstract}
One of the major Strategic Innovation Theme and corresponding Research Priority for the next years is Circular Economy and Resource Efficiency, according to Euratex. Recently, the European Commission launched the new "Industrial Strategy for a globally competitive, green and digital Europe", that will help deliver on three key priorities: maintaining European industry's global competitiveness and a level playing field, at home and globally, making Europe climate-neutral by 2050 and shaping Europe's digital future. In this context, innovation and market potential of the European textile and clothing industry involve the frequent use of the terms "Circular Economy". Forward, the sector will operate according to a globalised and efficient circular economic model which maximises the use of local resources, exploits advanced manufacturing techniques and engages in cross-sectorial collaborations and strategic clusters. Although the benefits of the circular economy are fairly well understood, in reality there are few industrial examples of companies that have implemented a circular economy paradigm. Circular product design provides long-term sustainability performance for products, by applying the principle of "designing out waste". This paper presents the application of circular product design assessment for clothing, by using two practical tools to assess products' circularity: HotSpot Mapping and Circularity Calculator (developed by Delft University of Technology, Netherlands). Generally, clothing products need major redesign to fit the circular economy, so it is essential to assess the potential of the various circular strategies such as Reuse, Repair, Remanufacture and Recycle.
\end{abstract}

Keywords: circular economy, clothing, design, circular strategies

\title{
INTRODUCTION
}

According to the document "Towards a 4th Industrial Revolution of Textiles and Clothing - A Strategic Innovation and Research Agenda for the European Textile and Clothing Industry" developed by EURATEX and European Technology Platform, the first seeds of this 4th Industrial Revolution are currently being sown in the European textile and clothing industry (Euratex, 2016). One of the major Strategic Innovation Theme and corresponding Research Priority for the next years is Circular Economy and Resource Efficiency, including the research priorities:

- Novel flexible process technologies to save water, energy and chemicals;

- High-tech textile recycling for circular economy concepts;

- Sustainable substitutes for hazardous or restricted textile processing chemicals or bio-chemistry based textile processing;

- Bio-refinery concepts using European biomass or waste for textile fibers;

- Greater use of EU-origin natural fibers.

Recently, the European Commission launched the new "Industrial Strategy for a globally competitive, green and digital Europe", that will help deliver on three key priorities: maintaining European industry's global competitiveness and a level playing field, at home and globally, making Europe climate-neutral by 2050 and shaping Europe's digital future (EC, 2020).

In this context, innovation and market potential of the European textile and clothing industry involve the frequent use of the terms "Circular Economy". Forward, the sector will operate according to a globalised and efficient circular economic model which 
maximises the use of local resources, exploits advanced manufacturing techniques and engages in cross-sectorial collaborations and strategic clusters (EC, 2016).

Although the benefits of the circular economy are fairly well understood, in reality there are few industrial examples of companies that have implemented a circular economy paradigm (Parida et al., 2019). Furthermore, the demand-based redesign activities can help an organization to earn a profit (Paras et al., 2019).

\section{PRINCIPLES OF CIRCULAR PRODUCT DESIGN}

Circular product design provides long-term sustainability performance for products, by applying the principle of "designing out waste": fitting the product in a circular economy where the value of the product, its components and materials are maintained and not wasted (TUDelft, 2020). Circular product design provides solutions for understanding the product development processes for fashion to rethink, reuse or upcycle the waste in the production stage (Cuc and Tripa, 2018).

This paper presents the application of circular product design assessment for clothing, by using two practical tools to assess products' circularity: HotSpot Mapping and Circularity Calculator (developed by Delft University of Technology, Netherlands).

The selected product is a quilted jacket for women composes by main material doubled with a thermal layer/padding and the lining, made from synthetic textile material (polyester). The fasteners are plastic zippers for the front component and also for the pockets. The upper pockets are covers with flaps that fasten with metal staples. Also, little metal staples are used in order to strengthen the termination waist line pockets, on shoulders line, on the back and on the hips line (Figure 1).

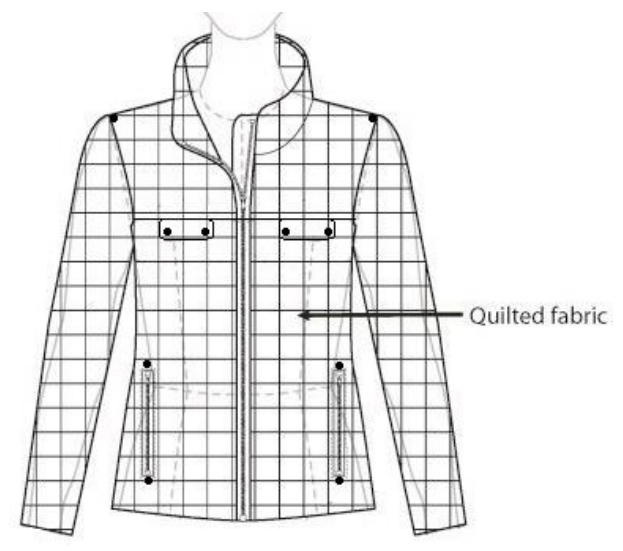

Figure 1. Selected clothing product for circular product design assessment

\section{Gentle Dismantle}

The disassembly took around an hour for the woman jacket using $10 \mathrm{~cm}$ and $25 \mathrm{~cm}$ shears/scissors and included the take off the lining, the labels, the trimmings and the collar, then the front zipper, the collar, the sleeves, the back and the front (Figure 2). The hardest part was to detach the stitches in the hip line and the metal staples. 


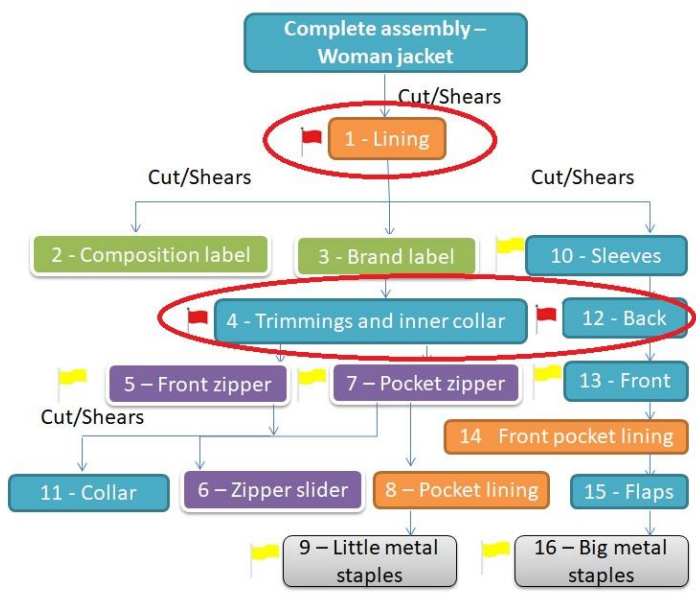

Figure 2. Disassembly map of the selected clothing product

\section{HOTSPOT MAPING (HSM)}

This tool was used to analyze the product architecture through the dismantling of the product, and mapping the key properties of all components, such as material, weight, ease of disassembly, likelihood to break, and more (Flipsen et al., 2020). The tool spotted the critical points in the product architecture related to key circularity metrics (Figure 3):

$>$ Lining was prioritised because of the time to cut the assemblies with the main material, especially in the hip line and sleeves line were the stiches were reinforced with a tape made for the same main material.

$>$ Trimmings and inner collar were prioritised also for the time of disassembly. One edge of these components is attached to the zipper by multiple stiches.

$>$ Back was prioritised on environment and economic impact. It gathers a larger mass of material made from synthetic fibres (polyester).

These 3 hotspots actually are inter-related and focus the functionality of the product. They were prioritized because of the time to detach but the resistance of the stiches is very important for the durability of the jacket during wearing. The quilted back doubled with the thermal layer assures the protection against external factors like cold, rain or wind.

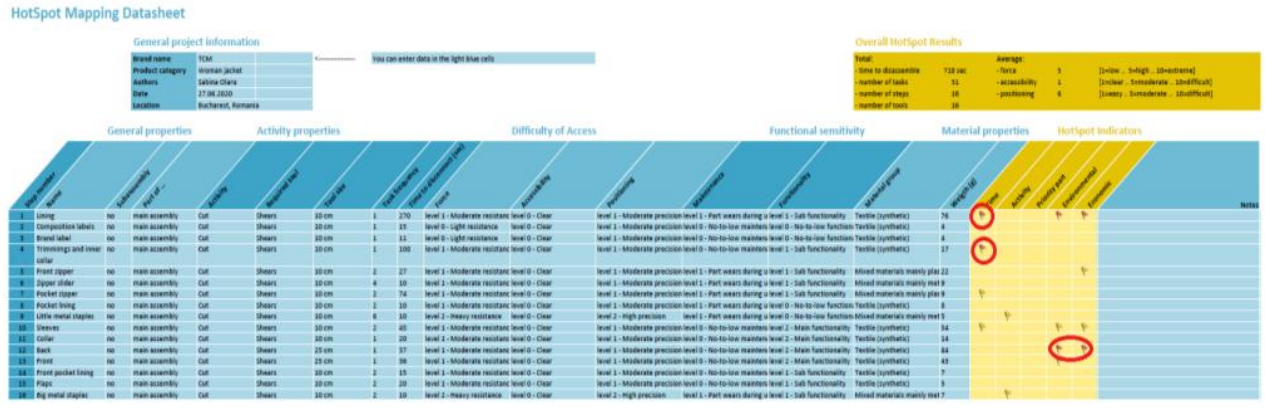

Figure 3. Hotspot maping of the selected clothing product 


\section{CIRCULARITY CALCULATOR}

The tool was used to quantify key circularity metrics and develop circular scenarios based on the data from the early stages of product development. These metrics include resource circularity and potential value capture for different circular scenarios (IDEAL\&CO Explore, 2020).

Generally, clothing products need major redesign to fit the circular economy, so it is essential to assess the potential of the various circular strategies such as Reuse, Repair, Remanufacture and Recycle.

\section{Linear Scenario}

The linear scenario is actually not circular but recycled materials are used for the metal staples and that means there is a loss of $33.4 \mathrm{k} €$ in total (Figure 4).

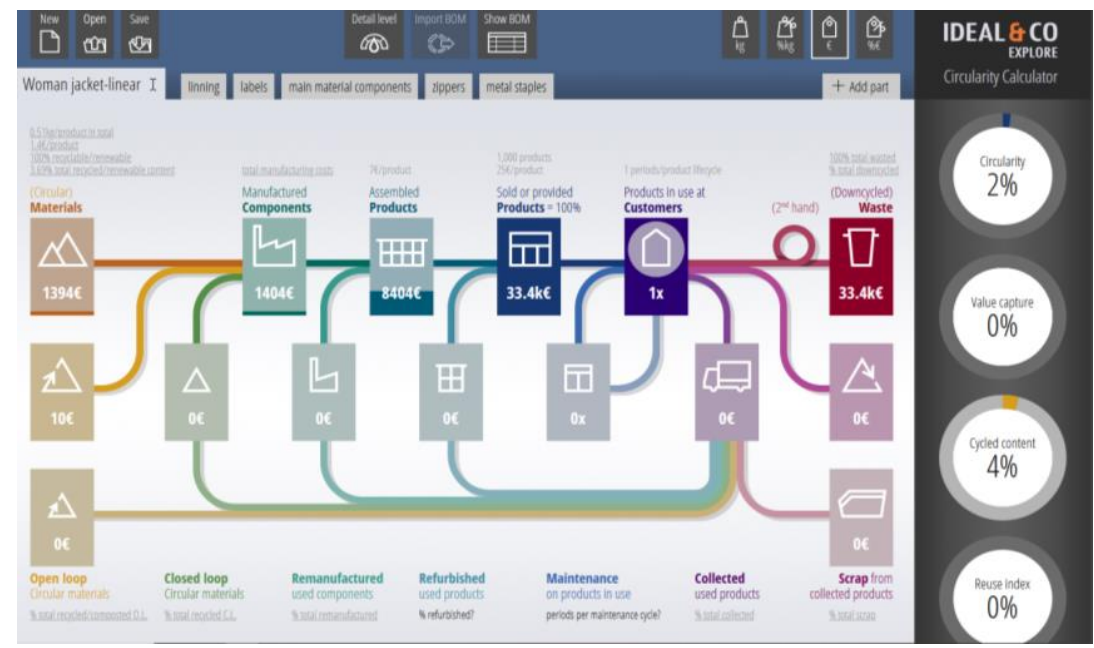

Figure 4. Linear scenario of the selected clothing product

\section{Circular Scenarios}

The disassembly of the jacket is a destructive one so it cannot be easily reassembled. And this is the general case of clothing products. In the redesign the trimming strategy can be applied by rethinking the use of stiches for lining assembly with reversible fasteners like Velcro band, zippers or unconventional (activated by heat) so the disassembly can be done very easy.

In the first circular scenario, the collected used products were assumed to $40 \%$ after applying offers of free shipment and a buy-back programme. In this case, the circularity increases to $36 \%$ and the value capture to $8 \%$.

\section{Refurbish}

In the refurbish scenario, it was assumed that $80 \%$ of the collected used products can be refurbished and reused. In this case, resource circularity increases to $37 \%$ and the value capture to $8 \%$ (Figure 5). 


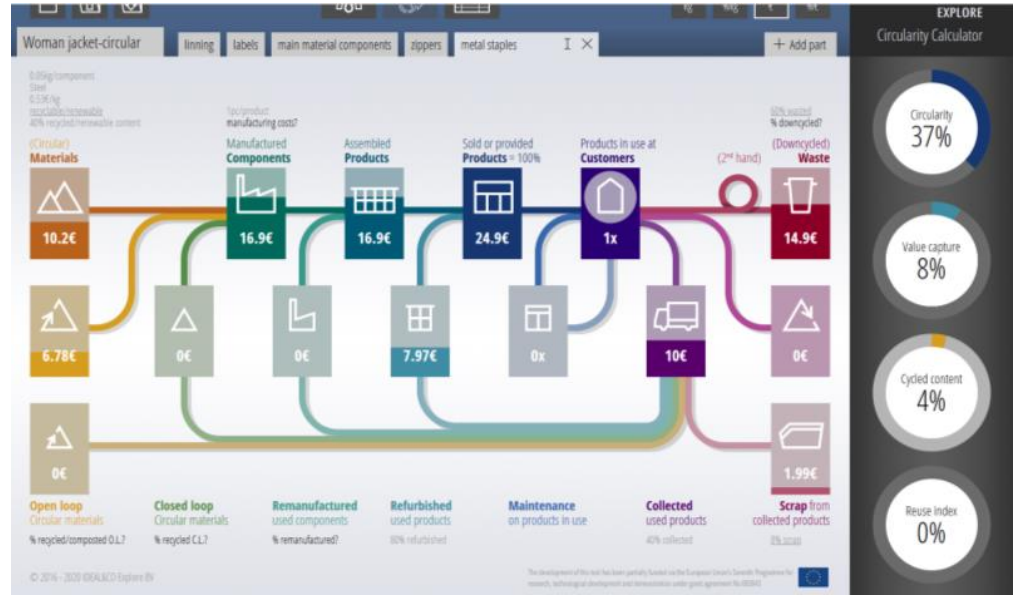

Figure 5. Refurbish scenario of the selected clothing product

Recycling

The recycling scenario increases the circularity to $66 \%$ and the cycled content to $94 \%$. In the same time, the value capture decreases to $2 \%$. The assumption in this scenario is that all the polyester materials are $100 \%$ recycled (figure 6).

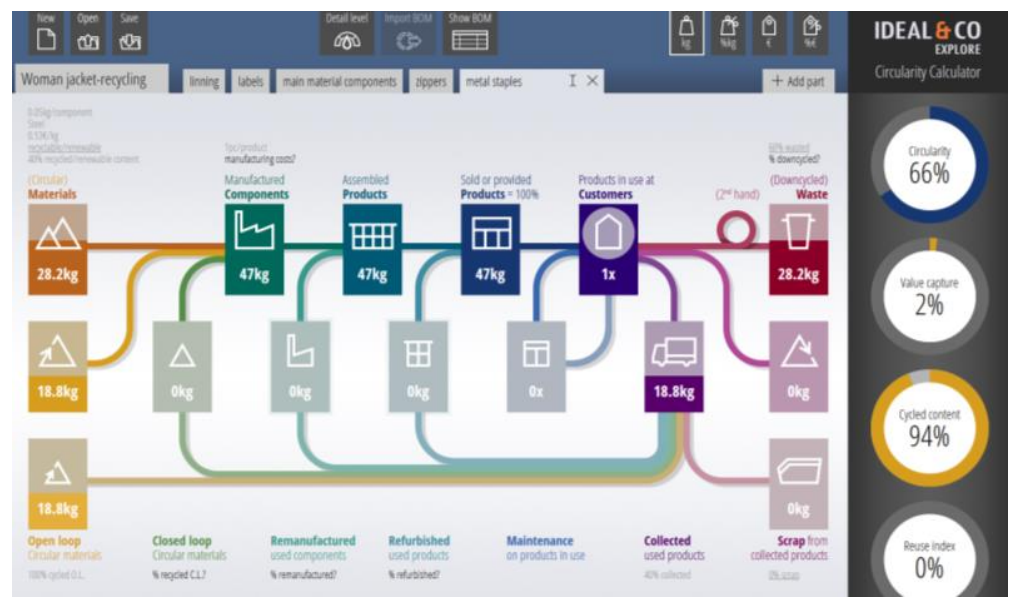

Figure 6. Recycling scenario of the selected clothing product

\section{CONCLUSIONS}

By using these two tools, substantiated criteria and facts were obtained to decide which are the most promising circular design strategies for the particular context. The tools pointed to the most significant contributors to the effective implementation of circular strategies.

The most promising circular scenario is the recycling, by using recycled materials and also recycled products after use in $40 \%$. The business model is a circular and sustainable one, and the changes in the product architecture/design the trimming https://doi.org/10.24264/icams-2020.IV.15 
strategy can be applied by rethinking the use of stiches for lining assembly with reversible fasteners like Velcro band or zippers so the disassembly can be done very easy.

The opportunities are indeed not using virgin raw materials wherever possible. The bottleneck will be the collection of the products since the bay-back strategies are not so user friendly nowadays.

\section{REFERENCES}

Cuc, S. and Tripa, S. (2018), "Redesign and upcycling -a solution for the competitiveness of small and medium-sized enterprises in the clothing industry", Industria Textila, 69(1), 31-36, https://doi.org/10.35530/IT.069.01.1417.

European Commission - Joint Research Centre (2016), Industrial Landscape Vision Study 2025, Brussels.

European Commission (2020), Press release: Making Europe's businesses future-ready: A new Industrial Strategy for a globally competitive, green and digital Europe, Brussels, https://ec.europa.eu/commission/presscorner/detail/en/fs_20_425.

Flipsen, B., Bakker, C. and de Pauw, I. (2020), "Hotspot Mapping for product disassembly; a circular product assessment method", Conference of Electronics Goes Green 2020+, Berlin, September 1, 2020.

IDEAL\&CO Explore (2020), "Circularity Calculator", Software available via www.circularitycalculator.com.

Paras, M.K., Curteza, A., Pal, R., Chen, Y. and Wang, L. (2019), "A Romanian case study of clothes and accessories upcycling”, Industria Textila, 70(3), 285-290, https://doi.org/10.35530/IT.070.03.1500.

Parida, V., Burströmc, T., Visnjicd, I. and Wincenta, J. (2019), "Orchestrating industrial ecosystem in circular economy: A two-stage transformation model for large manufacturing companies", Journal of Business Research, 101, 715-725, https://doi.org/10.1016/j.jbusres.2019.01.006.

Textile ETP (2016), Towards a 4th Industrial Revolution of Textiles and Clothing. A Strategic Innovation and Research Agenda for the European Textile and Clothing Industry, EURATEX, Brussels, Belgium.

TUDelft (2020), Circular Product Design Assessment, https://online-learning.tudelft.nl/courses/circularproduct-design-assessment/ 
WOOL PROCESSING OUTCOMES AND OPPORTUNITIES

\author{
MIRCEA CONSTANTIN OPRIȘ ${ }^{1}$, DANA CORINA DESELNICU ${ }^{2}$ \\ ${ }^{1} A \& D$ Pharma SRL, Romania, oprismircea0@gmail.com \\ ${ }^{2}$ University Politehnica of Bucharest, 313 Splaiul Independentei, Romania, \\ d_deselnicu@yahoo.com
}

\begin{abstract}
This paper provides an analysis of what happens with the sheep wool in Romania. Unfortunately, I found out that most of this "gold mine" is going to export, being processed, and then imported back for a much bigger price. The processing of sheep wool is not a very complicated process. There are processing lines that can take the sheep wool and by the end of the line, you get lanolin and fibers. Besides the very high margin that can be obtained from this type of business, motivation came from the idea of using our country resources at the maximum and try to have some products made in Romania. The main objective of this paper is to analyze the outcomes of a sheep wool processing line and the market in Romania. Sheep wool is a very precious raw material that can provide profit by processing it, having significant margins.
\end{abstract}

Keywords: wool processing, wool market, profit margin

\title{
INTRODUCTION
}

\section{Wool}

Wool is possibly the oldest fiber known to humans. It absolutely was one among the first fibers to be spun into yarn and woven into a material. Wool mostly comes from sheep but also alpacas, camels, and goats. Australia, Eastern Europe, New Zealand, and China are major wool producers. The American woolen industry began within the Massachusetts settlements in 1630, where each household was required by law to provide wool cloth.

\section{Lanolin}

Lanolin is extracted in the first stage of the sheep wool processing where it is washed (scouring) and then found in the residue. A typical high-purity grade of lanolin is composed predominantly of long-chain waxy esters (approximately 97\% by weight) the remainder being lanolin alcohols, lanolin acids, and lanolin hydrocarbons.

An estimated 15000 different types of lanolin esters are present in lanolin, resulting from combinations between the 200 or so different lanolin acids and the 100 or so different lanolin alcohols identified so far. For this reason, the industry has a precise segmentation, depending on the use of lanolin.

Among other applications of lanolin, the most common of them are detailed below:

- It is often utilized in protective baby skin treatment and for sore nipples in breastfeeding mothers. It helps healing the skin and also gives decent hydration.

- Lanolin is used commercially in many industrial products starting from rustproof coatings to lubricants. Some sailors use lanolin to form slippery surfaces on their propellers and stern gear to which barnacles cannot adhere. The water-repellent properties make it valuable as a lubricant grease where corrosion would rather be an issue.

- Anhydrous liquid lanolin, combined with parabens, has been utilized in trials as artificial tears to treat dry and/or tired eye feeling. 
- Anhydrous lanolin is additionally used as a lubricant for brass instrument tuning slides.

- Lanolin may also be restored to woolen garments to create them water and dirt repellent, like for cloth diaper covers.

- Lanolin is usually employed by people on continuous positive airway pressure therapy to scale back irritation with masks, particularly nasal pillow masks that may often create sore spots within the nostrils.

- Lanolin may be a popular additive to mustache wax, particularly "extra-firm" varieties.

- Lanolin, when mixed with ingredients like neat's-foot oil, beeswax and glycerin (glycerol), is used in various leather treatments, as an example in some saddle soaps and in leather care products.

Crude lanolin constitutes about $15 \%$ of the weight of freshly wool. The wool from one Merino sheep will produce about 250 of recoverable wool grease. This number can be obtained only from the Merino breed and has the highest lanolin in it.

\section{Wool Fibers}

Wool today is prized for its beauty and sturdiness. It's still the prime choice for highquality business suits, warm sweaters, and premium carpets.

Most of the wool (72.8\%) is used in apparel, while the rest of it in home furnishing, industrial uses, and other statistically insignificant small percentages.

The most important use of wool is in apparel coats, jackets, suits, dresses, skirts, slacks made up of woven fabrics of varying weights, and knitted fabrics.

In the home furnishing area, the major use of wool is in carpets and rugs where wool is used more, cover to the carpets, and warm in the rugs. Blends of different synthetic fibers with wool for suiting materials are increasingly important. This results in fabrics that are more appropriate in warmer conditions. Polyester is the most important fiber used in blending with wool.

As well-known already, the wool fiber is a very high performance one in the textile industry. It is largely used in professional risk takers and extreme sports athletes to protect them in any type of environmental condition. Wool can provide the following advantages:

- Thermo and moisture management: wool clothing can retain or release heat and moisture very well.

- Odor management: Wool clothing can reduce the amount of sweat on the body

- $\quad$ Resilience: wool fibers have a very high resistance on bending, up to 20.000 times, and also can stretch more than 30 percent of its own original size and shape.

Wool is very commonly used in newborns and baby clothing textiles. Some studies show the link between wool and wellbeing. This type of material assures the right body temperature. This happens because of its protein-based fiber made of keratin. Other materials do not have this ability. They also play an important role in the skin property of breathing, controlling humidity. 


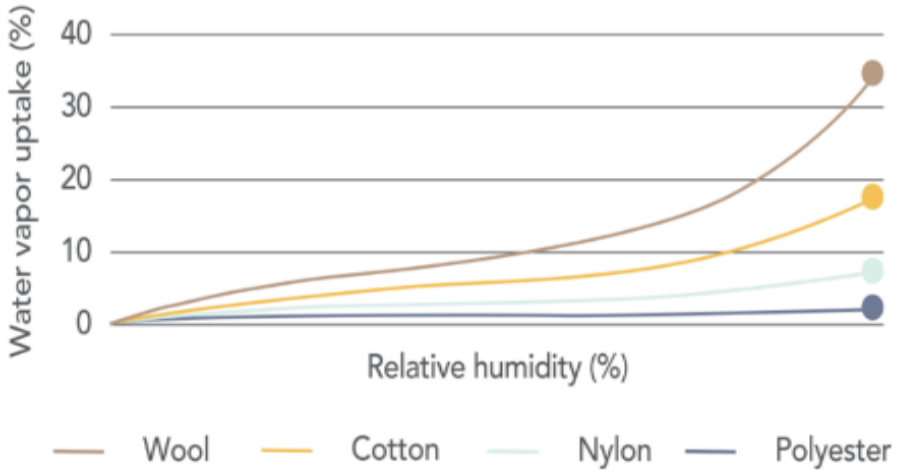

Figure 1. Moisture uptake of wool and other common fibers

As shown in Figure 1, wool has better water vapor uptake which leads to a higher grade of comfort to the user. This characteristic leads to better control of humidity which makes it a better choice for increased comfort of the textile.

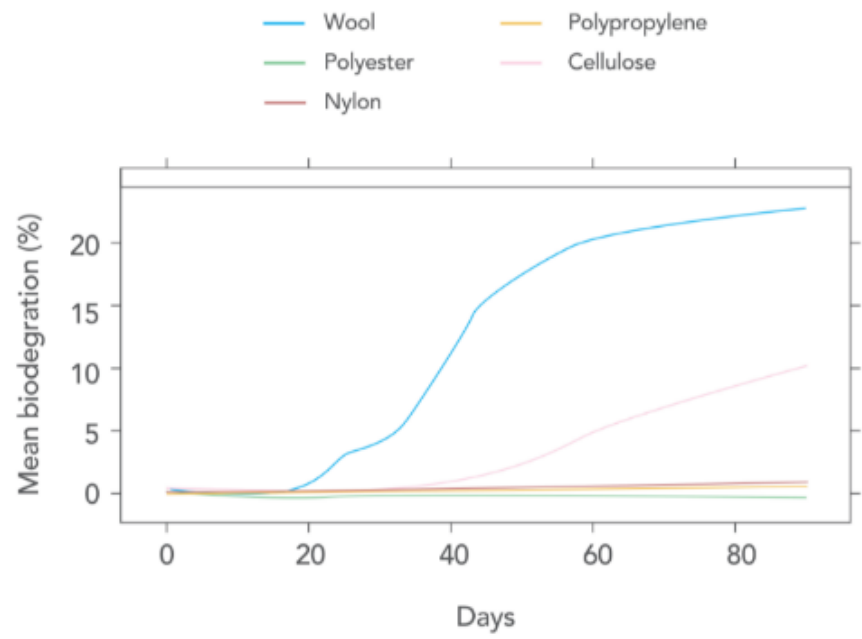

Figure. 2. The time needed for biodegradation for different types of material

Another big advantage of this type of material, as presented in Figure 2, is the fact that it is ecofriendly. It is considered biodegradable. Microplastic materials are one major source and they occur in the wastewater used for laundry. They then reach the seawater. Studies prove that the wool fiber starts decomposing after 21 days only in seawater.

Time required for textile materials of biodegradation:

- Cotton shirt requires 2-5 months;

- Wool sock requires 1-5 years;

https://doi.org/10.24264/icams-2020.IV.16 
- $\quad$ Nylon fabric requires $30-40$ years;

- Leather requires 50 years;

- Rubber boot sole requires 50-80 years;

- Disposable diaper requires 450 years.

\section{INDUSTRY INSIGHTS}

The global lanolin market size was valued at USD 222.0 million in 2018 and is projected to expand at a Compound Annual Growth Rate of 6.0\% from 2019 to 2025 . Growth in end-use industries, like pharmaceuticals, personal care \& cosmetics, and baby care products, is that the key factor driving the market. aside from cosmetics or pharmaceuticals, lanolin features a wide scope of applications in various industries. Over the years, research has led to an increased application in technical applications, like lubricants and therefore the production of anti-corrosion paints for ferrous materials.

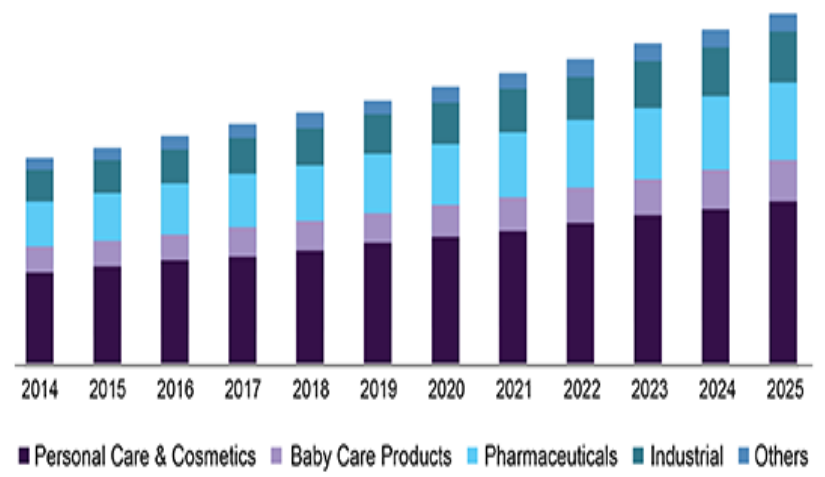

Figure 3. North America lanolin market demand, by application 2014 to 2025 (tons)

As shown in Figure 3, the demand of lanolin has increased year by year and it is expected to have the same ascending trend up to 2025. The demand was segmented, and it was observed that the main application of lanolin was in personal care and cosmetics, of course, as a base material for these products.

\section{REGIONAL INSIGHTS AND MARKET SEGMENTATION}

Asia Pacific is anticipated to be the biggest and fastest-growing regional market. Rapidly expanding end-use industries, growing population, and increasing consumer awareness regarding bio-based and natural products are expected to drive the regional market over the forecast period. Rising expenditure on mass cosmetics and personal care products thanks to improving economic conditions in emerging countries like India, Vietnam, Thailand, and Indonesia will boost the expansion further. Similarly, the growing prevalence of lifestyle-related diseases is augmenting the demand for natural medicines, with lower or negligible adverse effects.

According to official reports of the Agriculture government in Romania, there are around 15 million living sheep and each one provides on average $6 \mathrm{~kg}$ of wool. The two 
biggest competitors are handling only about $40 \%$ of this amount, while the rest of it is wasted.

Yet wool comprised only $1.26 \%$ of the world fiber market of $90 \mathrm{M}$ tons in 2013 , according to the planet survey on textiles and nonwovens, The Fiber Year 2014, published by The Fiber Year Consulting. Approximately $60 \%$ of world wool production goes into apparel; with knits comprising 59\%, and wovens $40 \%$, according to wool marketing organization Australian Wool Innovation (AWI).

The retail value of wool apparel sold is around US\$80bn annually. With the worth of wool often averaging five to seven times as much as the price of cotton, wool is very much a premium product, increasingly confined to niche markets. In an era of artificial synthetics and fast fashion, has wool lost its relevance for the apparel business? Or is wool making a comeback as a 21 st-century fiber, driven by improved aesthetics, performance aspects, and sustainability credentials?

The truth is that only a few of us actually understand wool in the context of textile fibers, and why wool is so unique. This report will examine the place of wool fiber in the textile value chain, addressing its decline in popularity through the second half of the 20th century, researching the explanations for its current comeback, and assessing future prospects for the wool industry.

Based on application, the lanolin market has been segmented into personal care \& cosmetics, baby care products, pharmaceuticals, industrial, and others, like sports goods and humectants. In terms of revenue, the personal care $\&$ cosmetics segment accounted for $44.63 \%$ of the worldwide market share in 2018. the product has high moisturizing properties, thus features a tremendous demand in a form of cosmetics, additionally as baby care products.

While a number of the characteristics of wool are often altered through biotechnology of sheep, most of the modifications of design are implemented during the manufacturing of the fabric. Wool is blended with any number of natural or synthetic fibers, and various finishes and coverings may be applied.

\section{CONCLUSIONS}

In conclusion, even though the fibers are altered for the final using purpose, the sheep wool is used as presented, then mixed with other constituents. The best quality comes from the Merino breed, which can be found a lot in Romania. Mixing the fibers does not affect the business since it is used as presented for mixing afterward. In the last years, more and more attention was focus on eco-friendly materials. Wool fibers are the best compared in the industry. Other than wool fibers, there are many other exploitation opportunities of the sheep wool, like, lanolin, vitamin D3. Predictions for sheep wool industry are favorable with a $6 \%$ growth yearly, until 2025 which shows that the wool industry.

\section{REFERENCES}

Benisek., L, Harnett., P.R. and Palin., M.J. (1987), "The influence of fiber and fabric type on thermophysiological comfort", Melliand Textilberichte, 68, 878-888.

Brown, R.M. (1994), "The Microbial Degradation of Wool in the Marine Environment", thesis for the degree of Master of Science in Microbiology, University of Canterbury, New Zealand.

Collie, S.R. and Johnson, N.A.G. (1998), The benefits of wearing wool rather than man-made fiber garments, Lincoln, Christchurch, New Zealand, WRONZ.

Cone, L.G. (2009), “Minimize Heat Stress”, Chilton's Industrial Safety and Hygiene News, 43(4), 39.

https://doi.org/10.24264/icams-2020.IV.16 


\section{Wool Processing Outcomes and Opportunities}

Eunomia (2016), "Plastics in the Marine Environment", Eunomia Research \& Consulting Ltd, Bristol UK, June 2016, https://www.eunomia.co.uk/reports-tools/plastics-in-the-marine-environment/

Grand View Research (2019), "Lanolin Market Size, Share \& Trends Analysis Report by Application (Personal Care \& Cosmetics, Pharmaceuticals), by Derivative (Lanolin Alcohol, Cholestrin, Lanosterol), by Region, and Segment Forecasts, 2019 - 2025", https://www.grandviewresearch.com/industryanalysis/lanolin-market.

Mayo Clinic, Sweating and body odor symptoms \& causes, https://www.mayoclinic.org/diseasesconditions/sweating-and-body-odor/symptoms-causes/syc-20353895 (accessed 27/11/2018).

National Oceanic and Atmospheric Administration Marine Debris Program, US Department of Commerce

Wood, E. (2009), Tangling with wool, A resource book of information and activities about wool and textiles, AgResearch.

*** https://iwto.org/resources/statistics/ 
REWEART - A 100\% REAL CIRCULAR ECONOMY MANUFACTURING PROCESS FOR VEGAN-ORGANIC-RECYCLED FOOTWEAR

\author{
MIRELA PANTAZI-BAJENARU ${ }^{1}$, ENRIQUE MONTIEL PARRENO $^{2}$, TRAIAN FOIASI ${ }^{1}$, \\ DANA GURAU ${ }^{1}$ \\ ${ }^{1}$ INCDTP - Division: Leather and Footwear Research Institute, 93 Ion Minulescu St., Bucharest, \\ Romania, email: icpi@icpi.ro \\ ${ }^{2}$ VESICA PISCIS FOOTWEAR, S.L.
}

\begin{abstract}
The negative impact on the environment caused by the current production of over 23,000 million pairs of shoes each year, either due to the consumption of huge quantities of raw materials or due to the thousands of tons of waste generated, is a sufficient reason to conceive a new type of shoe making process. The predominant manufacturing methods and the many components that the shoe contains make it impossible to recycle, due to the high complexity involved in separating materials. Once they reach the end of their life cycle, they inevitably end up in a landfill, generating a large amount of waste. REWEART is a footwear manufacturing project that is capable of producing sustainable goods based on a circular economy, co-financed by the European Commission through the LIFE program. REWEART aims to make vegan shoes with accessories made from recycled and organic materials, with $90 \%$ less energy and almost zero waste. REWEART wants to provide a tool to avoid the generation of waste in footwear consumption and to offer the footwear industry an alternative model of circular production to reduce energy consumption and to stop the destructive and unsustainable production of footwear.
\end{abstract}

Keywords: vegan, organic, recycled footwear.

\title{
INTRODUCTION
}

REWEART is a LIFE project co-financed by the European Commission that aims to develop a new business model to create new $100 \%$ recycled, organic footwear.

Project duration: 01.09.2018-30.06.2021.

\section{AIMS \& OBJECTIVES}

- The REWEART project aims to demonstrate a new model of footwear production in order to reduce the consumption of resources and waste in the textile and footwear sectors;

- The REWEART project aims to guarantee the transferability and replicability of footwear production;

- The project also aims to provide tools to the public administration for the evaluation of footwear manufacturing policies and strategies;

- Another objective of the REWEART project is to increase the awareness and support of the footwear sector;

- It also aims to identify and involve all relevant stakeholders related to footwear production issues.

\section{CONSORTIUM}

The consortium was created based on the combination of different environments, experience and expertise of the partners, includes all the skills, recognized expertise and competences needed to achieve all aspects of the work program. The consortium includes 6 institutions from 3 countries.

https://doi.org/10.24264/icams-2020.IV.17 
There is a mixture of institutions with different profiles, capacities and complementary competences suitable for the development of the work program (companies, research centers).

Most of the partners have experience in implementing transnational projects, which must accomplish all aspects of the project work program and complement each other in terms of their role in the project. In this way, the project provides for a stable partnership.

\section{PROJECT PARTNERS}

$\checkmark$ INCDTP - Division: Leather and Footwear Research Institute (ICPI) (Romania) Coordinator;

$\checkmark$ ATEVAL (Spain);

$\checkmark$ FERRE AGRUPACIÓN, S.A. (Spain);

$\checkmark$ HILATURAS FERRE, S.A. (Spain);

$\checkmark$ MUSTANG, S.R.L. (Italy);

$\checkmark$ VESICA PISCIS FOOTWEAR, S.L. (Spain).

\section{ACTIONS AND MEANS INVOLVED}

The project includes a set of implementation activities that will materialize the project objectives, supported by training, management, quality and dissemination activities that will ensure the wide distribution of its results even after the end of the project.

Actions and means to achieve the objectives are organized as follow:

- Implementation actions encompass actions to enable transferability and replicability by means of technical courses to public administration and professionals and guidelines to support the identification and the "know-how" to implement the suitable technique in each case. The main demonstration activities are: complete engineering design of REWEART system starting with the selection of basic components of the garment recycling process; building of a pilot on vegan, recycledorganic shoes; implementation of a REWEART platform and ICT tools; recycling of soling materials, mainly rubber for outsoles and playgrounds; produce footwear maker replication of the model of organic-vegan/recycled footwear.

- Monitoring actions include different methodologies to measure the environmental, social and economic impacts of the project.

- Public awareness and dissemination actions (D) include activities to involve all relevant stakeholders.

- Results will be disseminated by using project website and collaborators and other networking tools (Facebook, Twitter). Peer reviewed international publications will be also provided. Participation at national and international conferences and events will be foreseen. Specific dissemination material will be designed, produced and distributed to all relevant stakeholders and general public. The dissemination actions encompass an itinerary with information panels about the techniques and expected results. A Post LIFE communication plan is considered where commitments acquired by the partners regarding the continuation of the project will be included.

- The coordination of actions and involved partners is crucial in this project due to the interdependences among the proposed actions.

https://doi.org/10.24264/icams-2020.IV.17 


\section{EXPECTED RESULTS}

The REWEART project will demonstrate the concept of complete recycling of footwear and clothing properly and their reuse as components for the creation of new items.

All materials used for project trials will be organic, chemicals free and animal free, so we want to achieve a product $100 \%$ ORGANIC, VEGAN and RECYCLED, achieving modest sales of 30.000 pairs during the first year after project end.

The following innovative results will be obtained through the REWEART project:

$>$ Creating a service-oriented framework that is willing to produce footwear based on "Eco-Design";

$>$ Methods and processes for the development of recyclable or reusable products (i.e. new design and assembly methods based on modularity and which allow easy disassembly);

$>$ Methods for assessing recyclability, taking into account resource consumption, waste generation and cost impact;

$>$ ICT tool to support advanced 3D configuration of products;

$>$ Development of comprehensive, innovative and practical "Eco-Design" decisionmaking applications, for better informed consumers.

\section{ACTIVITIES AND RESULTS}

\section{Specifications and Component Selection}

Phase 1 - The basic models were designed and the components were selected. 30 models were initially evaluated and subsequently modified (Fig. 1). It has been established that the outer sole would be made of LATEX.

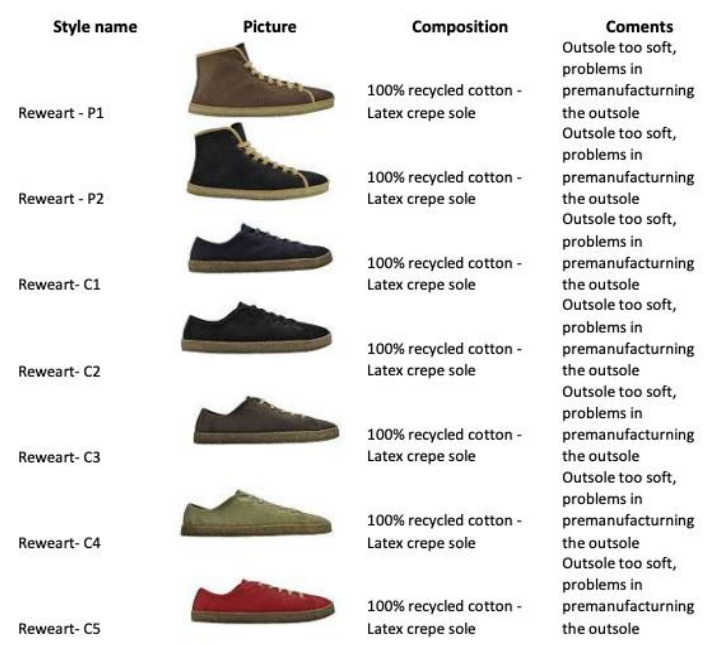

Figure 1. Basic models

Phase 2 - Two types of "helmet" soles were chosen (one white and one green) made of SBR + EVA (styrene-butadiene copolymer + ethylene-vinyl acetate copolymer). Both cause problems in that they contain stearic acid of animal origin as a vulcanizing 
agent. The tests performed at ICPI show that the green sole is more sustainable. It has up to $70 \%$ recycled rubber and synthetic rubber.

Phase 3 - Research and tests on the composition of the soles.

Phase 4 - In this phase, life cycle analysis information is collected, each component is weighed for size number 42: lining, upper, laces, yarns, sole, insole, adhesive, box. Also, the composition of each component is established.

\section{PILOT STATION Initiation Demonstration}

REWEWART manufacturing process proposed for the vegan shoes, is based on the STROBEL lasting system, which consist on the attachment of a non-woven textile to the upper by stitching. After this, the assembly of the upper material to the sole is done by stitching, with minimum use of adhesive, water based anyway.

With this approach, we propose a prototype line consisting on a cutting machine, two/three types of stitching machines (upper and lining assembly and side stitching) and then just a lasting station by hand and an upper-sole joining with pressure and final stitching.

The PILOT STATION for manufacturing footwear was developed (Fig. 2).
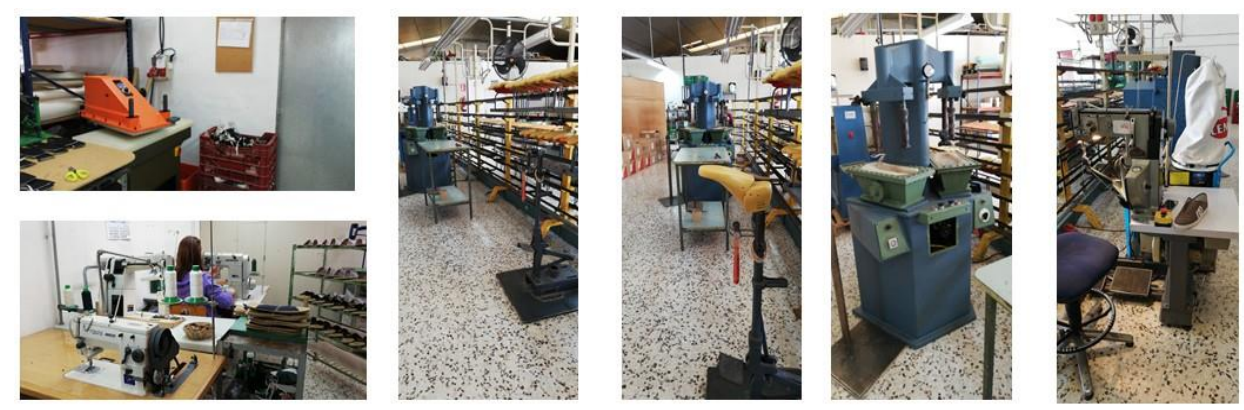

Figure 2. PILOT STATION

\section{Demonstration and Validation of the Pilot Installation for Recycling Clothes and New Yarns}

Yarns were developed from recovered fibers from technological waste, waste from the population, whether or not mixed with conventional or organic virgin cotton fiber, for which a presence of less than $5 \%$ was accepted and other unspecified fibers was taken over by partners with textile yarn processing profile.

The selection of yarn variants, within the project, was made taking into account 2 vectors of textile yarn processing requirements:

- requirements known per se, of the project, in accordance with the specific objectives,

- specific requirements for the production of textile components of footwear products.

Various fabrics were developed (Fig. 3, Fig. 4) that are still in testing: tensile strength and elongation, tear strength, abrasion/ friction resistance, pilling effect, seam strength, air permeability, resistance on surface hanging, fibrous composition of the woven fabrics.

https://doi.org/10.24264/icams-2020.IV.17 

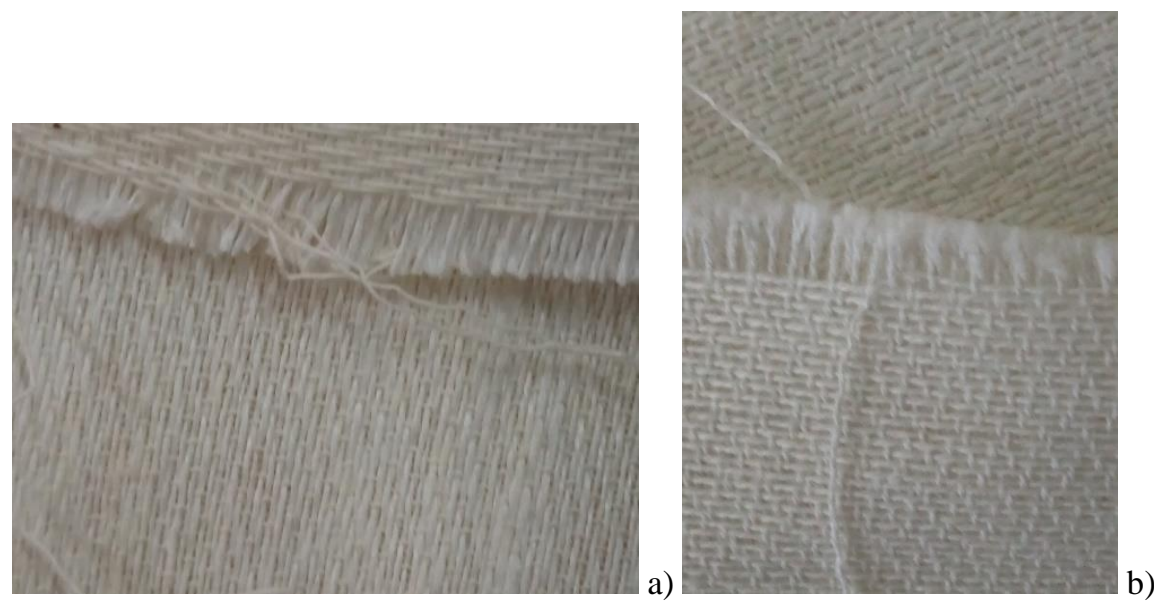

Figure 3. Woven fabrics - semi-doubled weft type: a) the warp is $100 \%$ cotton fibers and the wefts are $100 \%$ cotton and $100 \%$ linen; b) the warp is $98 \%$ cotton fibers / $2 \%$ regenerated cellulose fibers and the wefts are $100 \%$ linen fibers and $98 \%$ cotton fibers / $2 \%$ regenerated cellulose fibers

These structures are meant to be 2 in 1, namely upper side of the shoe (linen weft) and the lining layer (cotton or cotton/regenerated cellulose type weft).

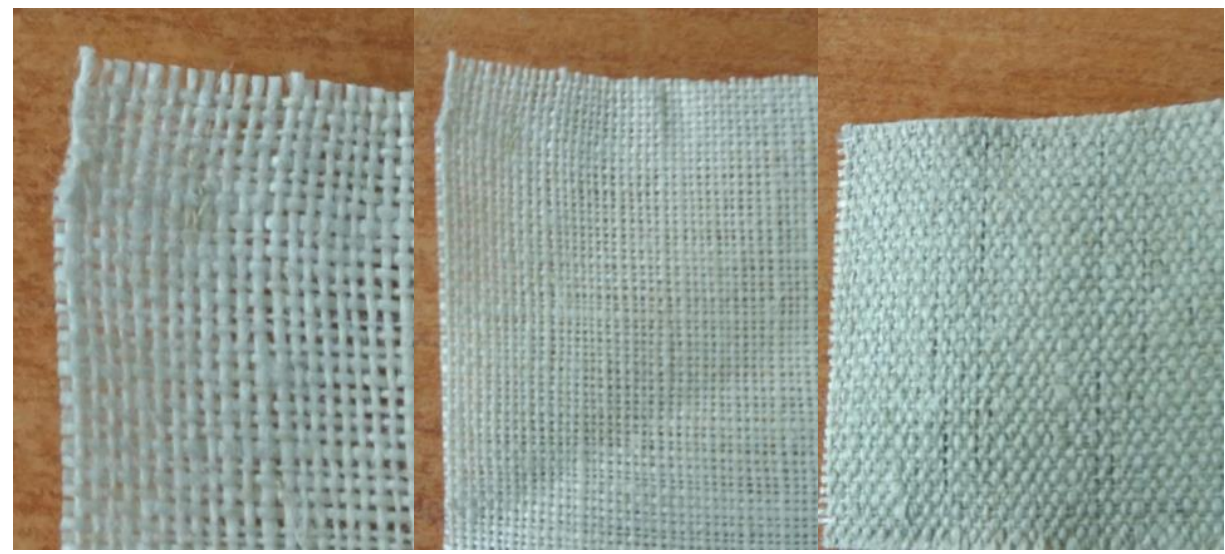

Figure 4. Woven fabric with $100 \%$ linen fibers 
Demonstration and Validation of the Unit for Recycled Vegan Footwear

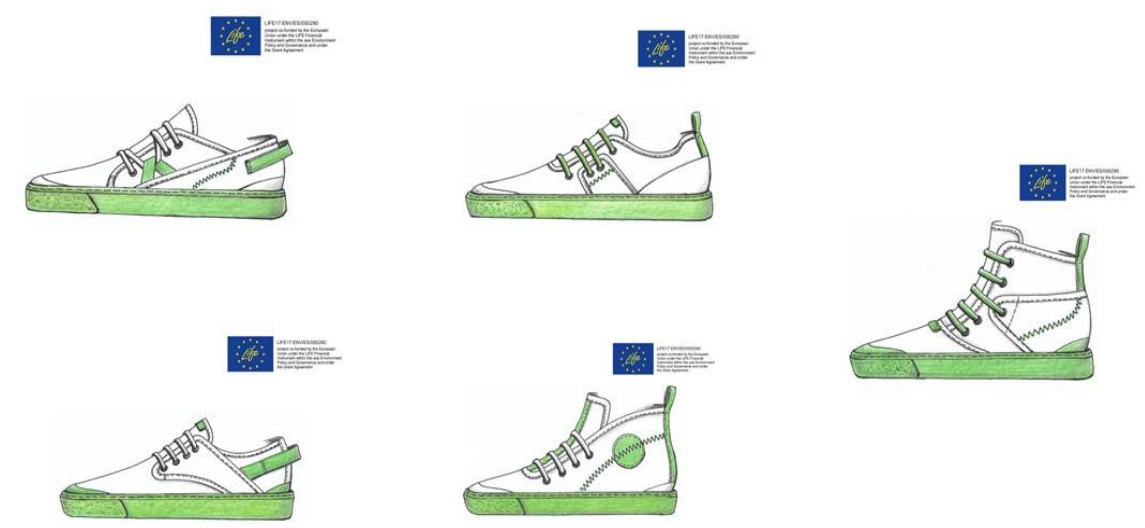

Figure 5. Shoes designed at ICPI

\section{Future Activities}

(i) INCDTP makes textile materials woven with yarn from HIFESA and tests these materials in order to verify the characteristics necessary for the production of footwear;

(ii) ICPI will make a composite based on natural rubber compounded with wood waste. From these mixtures plates will be obtained for the physical-mechanical characterization. The physical-mechanical tests will also be performed on the finished soles made by the partners.

(iii) ICPI will verify and evaluate the footwear prototypes by wear tests, by questionnaires, by testing the finished product.

(iv) ICPI will support the project with LCA using its own tools, with data provided by VESICA.

\section{CONCLUSIONS}

Upon completion of the project, the following will be obtained: (a) the complete design of the REWEART system starting with the selection of the basic components of the clothing recycling process; (b) the development of a pilot station for vegan, recycled and organic footwear; (c) implementation of a REWEART platform and ICT tools; (d) recycling of sole materials, mainly rubber for the sole; (e) creating a bio-vegan/recycled footwear model.

\section{Acknowledgements}

The REWEART project has received funding from the European Union's LIFE Program under the grant agreement number LIFE17 ENV/ES/000290.

\section{REFERENCES}

\footnotetext{
*** www.life-reweart.eu
}

https://doi.org/10.24264/icams-2020.IV.17 


\title{
COMPARATIVE LIFE CYCLE ASSESSMENT STUDY FOR FABRIC BASED
} ELECTROMAGNETIC SHIELDING

\author{
ION RAZVAN RADULESCU ${ }^{1}$, EMILIA VISILEANU ${ }^{1}$, RAZVAN SCARLAT ${ }^{1}$, \\ CATALIN CONSTANTIN ${ }^{2}$, BOGDANA MITU ${ }^{2}$ \\ ${ }^{1}$ INCDTP - Bucharest, Str. L. Patrascanu 16, 030508 Bucharest, Romania, \\ razvan.radulescu@incdtp.ro,e.visileanu@incdtp.ro, razvan.scarlat@incdtp.ro \\ ${ }^{2}$ INFLPR - Bucharest, Str. Atomistilor 409, 077125 Bucharest, Romania, \\ catalin.constantin@inflpr.ro,mitu.bogdana@inflpr.ro
}

\begin{abstract}
Life Cycle Assessment (LCA) studies represent the scientific approach for elaborating modern policies and supporting management decisions in the field of Sustainable Production and Consumption. The goal of many LCA studies undertaken for research are related to an exhaustive comparison of a modern, innovative product or process with respect to an initial, conventional one. This paper deals with such an approach for fabric based electromagnetic shielding. Electrically conductive textile fabrics are used in applications of electromagnetic shielding. Two basic types of technology for imparting electro- conductive properties to textiles are available, namely: insertion of conductive yarns in the fabric structure and coating with conductive layers. Magnetron plasma coating is a modern technology for achieving thin metallic layers on fabrics. Therefore, we focused the LCA study to the comparison between cotton woven fabrics with inserted conductive yarns out of stainless steel in warp and weft direction and cotton fabrics coated with thin layers of copper by magnetron plasma laboratory equipment. Functional unit of the comparative study was one square meter of EM textile shield with $5.2 \mathrm{~dB}$ at $1 \mathrm{GHz}$. A modelling of the fabric with inserted conductive yarns was performed in order to reach same shielding effectiveness at a certain frequency, as in the case of the coated fabric. Inventory data was collected for the fabric with conductive yarns from the textile company SC Majutex SRL, while for the plasma coated fabric from INFLPR. Impact assessment was performed by INCDTP, by using the LCA software SimaPro7 and the data basis EcoInvent 3.0. Interpretation of results shows that weaving of conductive yarns has a smaller impact on the environment than magnetron plasma coating using laboratory equipment, in a ratio of 1:2. This fact is explained by the industrial process of weaving as compared to laboratory process of coating, whereas brings the idea that upon utilization of industrial magnetron equipment for coating one may achieve in the end better environmental impact due to the process optimization for large area plasma processing.
\end{abstract}

Keywords: LCA, EMI shielding, fabrics

\section{INTRODUCTION}

Electromagnetic (EM) shielding is especially relevant in nowadays radiation polluted environment (Paul, 2006). This is a field belonging to Electromagnetic Compatibility (Schwab and Kuerner, 2013). Textile materials with electrical conduction properties are suitable for EM shielding due to their flexibility, low weight and good mechanical resistance as well as due to the tailored design for end user requirements property (Neruda and Vojtech, 2018; Radulescu et al., 2018a; Radulescu et al., 2018b). There are two types of technologies for imparting electric conductive properties to textile materials: insertion of conductive yarns in the fabric structure and coating with conductive layers (Ziaja and Jaroszewski, 2011). Modern coating technologies, such as magnetron plasma coating are recently used to achieve textile electromagnetic shields (Koprowska et al., 2004).

Life Cycle Assessment (LCA) is an instrument to quantify the impact on the environment for a specific product or process (Kloepffer, 2014). LCA studies are used to foster decisions for implementing new technologies on management level of SMEs (Wolf et al., 2010). LCA studies may be conducted for various reasons, such as: 
benchmarking of various products, identifying Key Environment Performance Indicators (KEPI), motivating green acquisitions or comparative assessment between a modern and a conventional technology. Latter reason is used mainly for research, to prove the environmentally friendly character of a modern technology.

Several LCA studies were accomplished in the scientific literature for textile products and processes. Research was accomplished on LCA studies for textile raw materials, namely cotton, polyester, nylon, acryl and elastane within a benchmarking study (Van der Velden et al., 2014). Research was directed towards LCA for various treatment processes on textiles, such as the fireproof treatment of fabrics and its environment impact reduction by eco-path disposal treatment (Pesnel and Perwuelz, 2011). Three different methods of recycling PES trousers (chemical/ mechanical and energy recovery) were analyzed in (Yasin et al., 2018). Moreover, attention was focused on LCA for smart and e-textiles with conductive fibers made of conjugated polymers, carbon nanotubes, graphene, polymer blend or nanocomposite (Lund et al., 2018). Ecodesign in case of smart textiles plays an important role: a comparative LCA study for eco-designed and original smart textiles products was achieved in (Van der Velden et al., 2015). End-of-life and recycling management of the textile chain was analyzed by LCA in countries, such as Finland (Dahlbo et al., 2017) and Denmark (Koligkioni et al., 2018). A review for the overall impact of nanomaterials on the environment was performed in (Miseljic and Olsen, 2014).

Main aim of this paper is to conduct a comparatively LCA study for electromagnetic shields made of woven fabrics with conductive yarns and shields made of plasma coated fabrics, in order to analyze the environmentally friendly character of plasma processing.

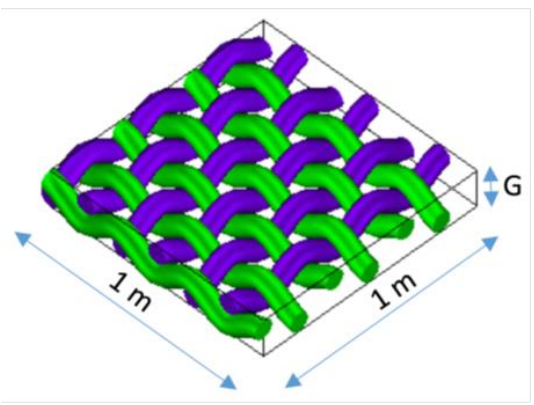

Figure 1. Woven fabric with inserted stainless steel yarns

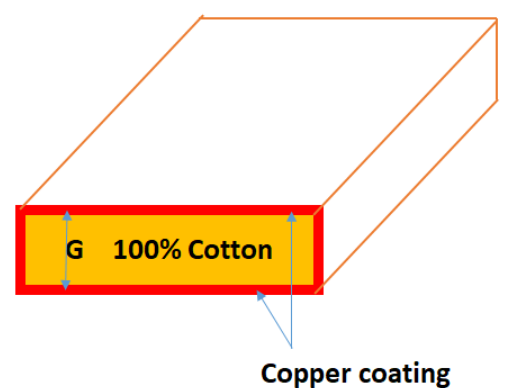

Figure 2. Woven fabric with Copper coating

Secondary aim is to evidence the improved environment performance of a modern magnetron sputtering plasma equipment with deposition rate of $100 \mathrm{~nm} / \mathrm{min}$ compared to an initial plasma equipment with deposition rate of $9 \mathrm{~nm} / \mathrm{min}$. 


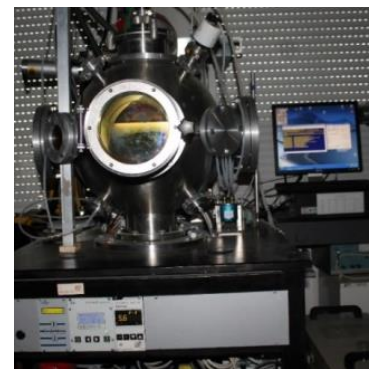

Figure 3. Modern magnetron plasma equipment

The LCA study was conducted according to the standard ISO 14040 on four phases: Goal and Scope definition, Life Cycle Inventory (LCI), Life Cycle Impact Assessment (LCIA) and Interpretation. It tackled the recommendations of the ILCD Handbook. The LCA study is a cradle-to-gate study. Following LCI data was applied with following limitations:

- LCI data applied: consume of raw materials, natural resources, auxiliary chemical substances, electrical energy on equipment and emissions to air;

- Limitations: consume of heat (gas / coal), as equivalent for both types of processes, distribution, storage and recycling of materials.

The method EcoIndicator99 (E) was used for the study with the impact categories: carcinogens, respiratory organics, respiratory inorganics, climate change, radiation, ozone layer, ecotoxicity, acidification / eutrophication, land use, minerals, fossil fuels. These impact categories may be divided in three groups: Humans health, Quality of ecosystem and Resource depletion.

\section{MODELLING OF THE WOVEN FABRIC STRUCTURE}

The functional unit of the electromagnetic shield was set to 1 sqm of textile fabric with $5.2 \mathrm{~dB}$ at $1 \mathrm{GHz}$. The electromagnetic shielding effectiveness (EMSE) of both types of textile shields was determined experimentally by TEM cell, according to the standard ASTM ES-07:

- The plasma coated cotton fabric with Copper on both sides of the material with the thickness of $t=400 \mathrm{~nm}$ had an EMSE of $5.2 \mathrm{~dB}$ at $1 \mathrm{GHz}$

- The cotton fabric with inserted conductive stainless steel yarns and a grid with the distance $\mathrm{a}=4 \mathrm{~mm}$ had an EMSE of $24 \mathrm{~dB}$ at $1 \mathrm{GHz}$

The distance of the grid formed by conductive yarns was modelled according to analytical relations (Neruda and Vojtech, 2018), in order the woven fabric with stainless steel yarns should have same EMSE of $5.2 \mathrm{~dB}$ at $1 \mathrm{GHz}$, as the plasma coated fabric.

The following analytic relations were used for calculation of the grid distance:

$$
E M S E=20 \log \left(\frac{\lambda / 2}{a \sqrt{\pi}}\right)
$$

$a=\frac{\lambda}{2 \sqrt{\pi}} \times 10^{-\frac{E M S E}{20}}$

with following notations:

$E M S E$ = electromagnetic shielding effectiveness, in this case EMSE was set to $5.2 \mathrm{~dB}$

https://doi.org/10.24264/icams-2020.IV.18 
$\lambda$ - wave length of the incident EM radiation, in this case frequency $=1 \mathrm{GHz}$ and $\lambda=30$ $\mathrm{cm}$ $a$ - distance of the grid formed by the conductive yarns of the woven fabric

As such, the distance of the grid formed by conductive yarns was enlarged, by keeping same fabric density and achieving a corresponding lower content of stainless steel per sqm of fabric.

Figure 4 shows the Matlab diagram for relation (2) - the distance of the grid for the frequency domain of 0.1-10 GHz in logarithmic scale.

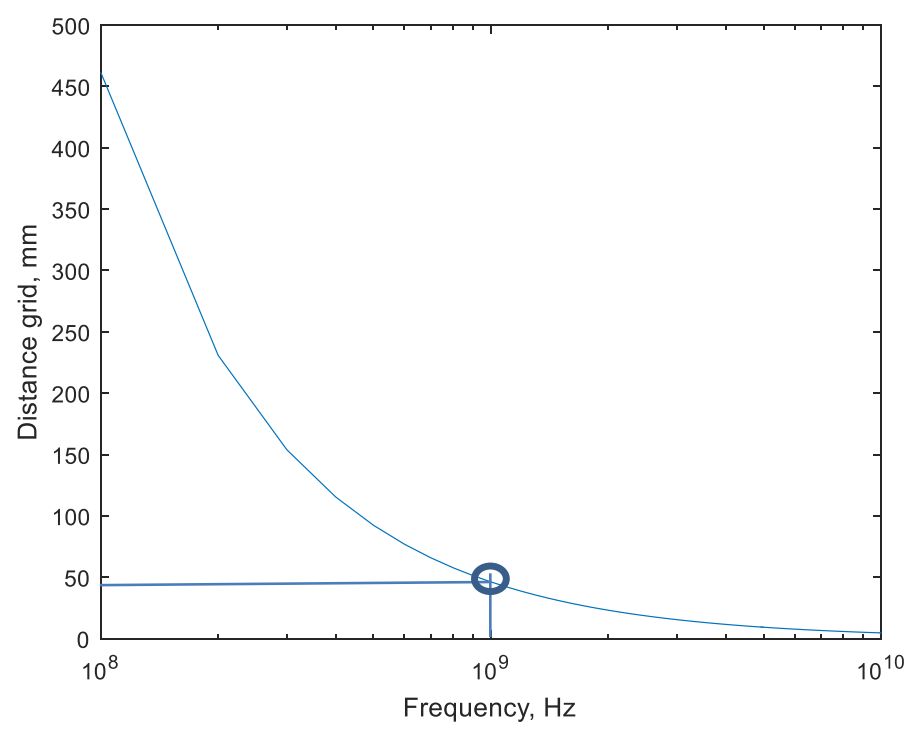

Figure 4. Distance of grid related to frequency for $\mathrm{EMSE}=5.2 \mathrm{~dB}$

According to calculation it resulted a modelled distance of $\mathrm{a}=46 \mathrm{~mm}$ between conductive yarns, by keeping same fabric density and yarn fineness, namely fabric structure: warp fabric density $=180$ yarns $/ 10 \mathrm{~cm}$, weft density $=170$ yarns $/ 10 \mathrm{~cm}$ with yarn fineness Nm50/2 both for cotton and stainless steel yarns.

\section{LCI, LCIA AND INTERPRETATION}

The following LCI data were considered for the cotton fabrics with inserted stainless steel yarns:

- Electric energy consumption for spinning the stainless-steel yarn with mean values from the literature (Van der Velden et al., 2014);

- $\quad$ Electric energy consumption for spinning of cotton (EcoInvent3.0 data base)

- Electric energy consumption for cotton weaving (data from industrial company SC Majutex SRL)

- Raw material consumption of cotton and stainless steel (according to the fabric's specific mass of $143 \mathrm{~g} / \mathrm{sqm})$

- $\quad$ Emissions of heat into the air (EcoInvent3.0 data base) 
Figure 5 shows within a weighting diagram the environmental impact with respect to eleven categories (impact related to a common reference), for the woven fabric with inserted stainless steel yarns.

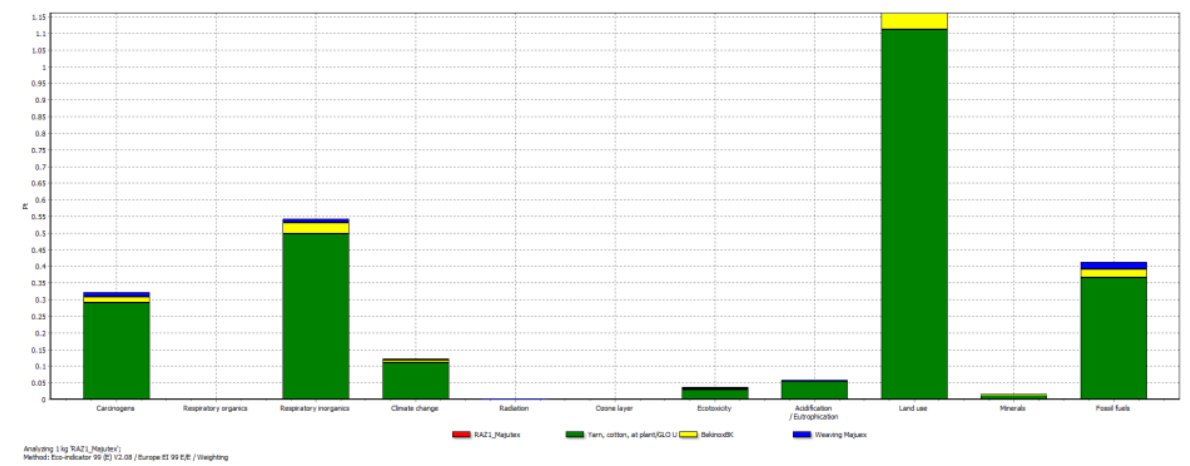

Figure 5. Weighting diagram for woven fabric with conductive yarns

Land use is the most addressed impact category, a fact explained by cultivation of cotton raw material. Respiratory organics and carcinogens may be explained by the emissions to air of the weaving process. Fossil fuels impact category is addressed by electric energy consumption.

The following LCI data were considered for the copper coated cotton fabrics:

- Electric energy consumption for plasma equipment, including vacuum pumps and pressure control systems, mass flow controllers, RF generator and matching box, PC (data from INFLPR);

- Raw material consumption: cotton fabric, copper, argon (data from Majutex and INFLPR);

- Emissions into the air: argon (data from INFLPR).

Figure 6 shows the environmental impact within a weighting diagram upon the same categories as previous, for the copper plasma coated woven fabric.

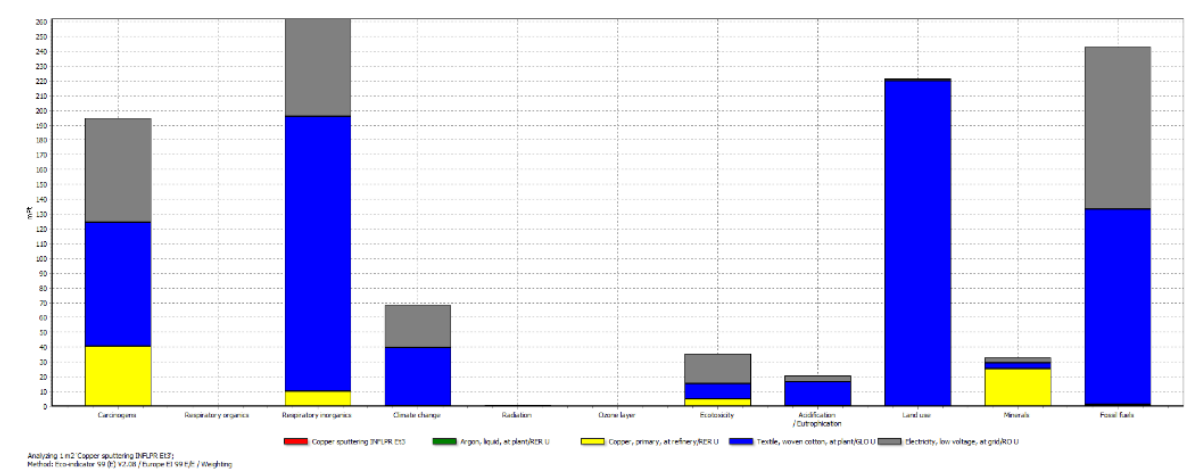

Figure 6. Weighting diagram for plasma coated fabric

Figure 6 evidenced that the most addressed impact categories are those related to respiratory inorganics in conjunction with emissions of Argon, Fossil fuels because of 
electric energy consumption and Land use originating from cotton cultivation for the raw fabric. Figure 7 shows the comparative single score diagram between the impact of the woven fabrics with conductive yarns and the plasma coated fabric.

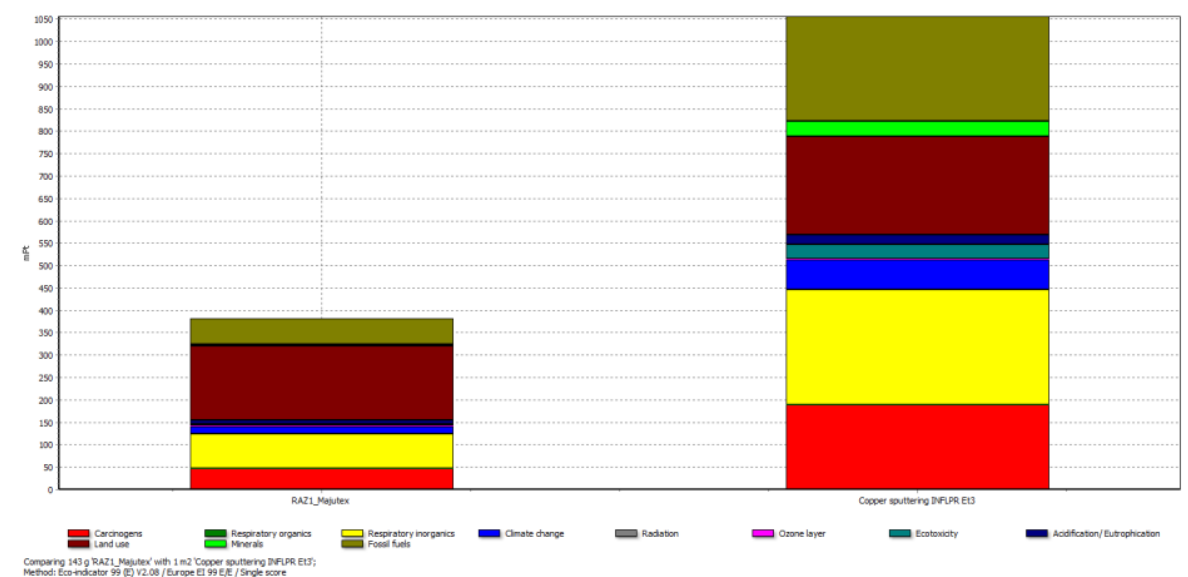

Figure 7. Comparative single score diagram for woven fabrics with conductive yarns (left) and plasma coated fabrics (right) - Fabric mass $=143 \mathrm{~g} / \mathrm{sqm}$

The overall impact indicator of the plasma coated fabrics is of $1050 \mathrm{mPt}$ while the indicator of fabrics with conductive yarns is of $380 \mathrm{mPt}$. The ratio is of $1: 2.76$, which means impact of plasma coated fabrics is 2.76 times higher than impact of woven fabrics with conductive yarns.

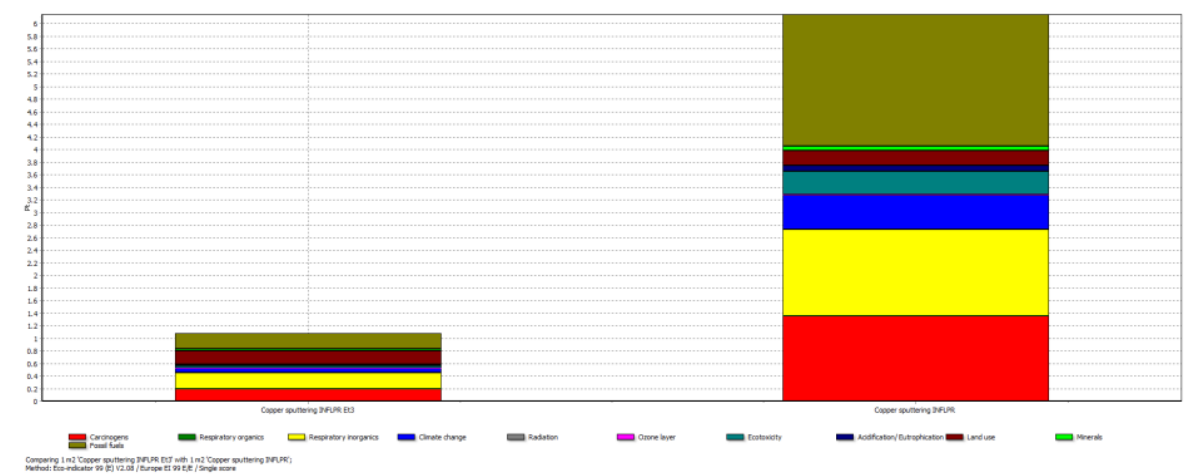

Figure 8. Comparative single score diagram for plasma coated fabrics with modern equipment (left) and conventional equipment (right)

Figure 8 is a single score diagram showing the impact for the modern plasma equipment with deposition rate of Copper $100 \mathrm{~nm} / \mathrm{min}$ and the conventional plasma equipment with deposition rate of $9 \mathrm{~nm} / \mathrm{min}$. The reduced impact in case of the modern equipment is mainly due to savings in energy consumption for shorter process time, as well as the implementation of recirculation for water cooling system. The overall impact 
indicator for the modern plasma equipment is $1.05 \mathrm{Pt}$, while for the conventional plasma equipment is $6.2 \mathrm{Pt}$. This indicates a ratio of 1:6 between the impact of coating by the two types of plasma equipment.

\section{CONCLUSION}

An industrial process of weaving was comparatively assessed with a laboratory process of plasma coating for production of textile electromagnetic shields. Plasma coating with metals (Copper) is an advanced technique of rendering electroconductive properties to fabrics and has multiple advantages: the nanometer coating enables a good flexibility of the fabric, thickness of coating may be precisely designed and adapted to end-user requirements. Method EcoIndicator $99 \mathrm{E}$ was applied and related impact diagrams were generated within software SIMAPRO7.

LCA was conducted by considering main consumption data during production stage - Cradle-to-gate study. LCI data covered consumption of electric energy, raw materials gas and emissions to air. The impact on the environment was according to single score diagram 2.76 times higher in case of plasma coating compared to weaving of conductive yarns. Same functional unit was ensured by modelling the fabric with inserted conductive yarns: 1 sqm of textile shield with $5.2 \mathrm{~dB}$ at $1 \mathrm{GHz}$.

As such, the LCA study has an indicative character in comparison of weaving and plasma coating. LCAs on conductive fabrics are in early stage of research, while this study is tackling the modern technology of plasma coating.

\section{Acknowledgment}

Research work for this study was supported by a grant of CCDI-UEFISCDI project number COFUND -MANUNET III - TexEMFiRe, contract 28/2018.

Publishing has been funded by Ministry of Research and Innovation, by Program $1-$ Development of the national system for research-development, Subprogram 1.2 Institutional performance - projects for funding excellence in RDI, contract no. 6PFE from 16.10.2018.

\section{REFERENCES}

Dahlbo, H., Aalto, K., et al. (2017), "Increasing textile circulation-Consequences and requirements", Sustainable Production and Consumption, 44-57, https://doi.org/10.1016/j.spc.2016.06.005.

Kloepffer, W. (editor) (2014), Background and Future Prospects in Life Cycle Assessment, Springer, Dordrecht, ISSN 2214-3513, https://doi.org/10.1007/978-94-017-8697-3.

Koligkioni, A., Parajuly, K., et al. (2018), "Environmental assessment of end-of-life textiles in Denmark", Procedia CIRP, 69, 962 - 967, https://doi.org/10.1016/j.procir.2017.11.090.

Koprowska, J. et al. (2004), "New type textiles with shielding properties", Fibres \& Textiles in Eastern Europe, 12, 39-42.

Lund, A., Van der Velden, N., Persson, N-K. et al. (2018), "Electrically conducting fibres for e-textiles: An open playground for conjugated polymers and carbon nanomaterials", Materials Science \& Engineering $R, 126,1-29$, https://doi.org/10.1016/j.mser.2018.03.001

Miseljic, M. and Olsen, S. (2014), "Life-cycle assessment of engineered nanomaterials: a literature review of assessment status", J Nanopart Res, 16, 2427, https://doi.org/10.1007/s11051-014-2427-x.

Neruda, M. and Vojtech, L. (2018), "Electromagnetic shielding effectiveness of woven fabrics with high electrical conductivity: complete derivation and verification of analytical model", MDPI - Materials, 11, 1657, https://doi.org/10.3390/ma11091657.

Paul, C. (2006), Introduction to Electromagnetic Compatibility, WILEY Interscience, ISBN-13: 978-0-47175500-5.

https://doi.org/10.24264/icams-2020.IV.18 


\section{Comparative Life Cycle Assessment Study for Fabric Based Electromagnetic Shielding}

Pesnel, S. and Perwuelz, A. (2011), "LCA: a decision-making tool for recycling process in textile industry", https://docplayer.net/24614249-Lca-a-decision-making-tool-for-recycling-processes-in-textile-industry.html

Radulescu, I.-R., Costea, M., et al. (2018a), "Web application for predicting the shielding effectiveness of woven fabrics with conductive yarns", U.P.B. Sci. Bull., Series C, C(4), ISSN 2286-3540.

Radulescu, I.-R., Surdu, L. et al. (2018b), "Modelling and testing the electromagnetic near field shielding effectiveness achieved by woven fabrics with conductive yarn", Industria Textila, 3, 169-176, https://doi.org/10.35530/IT.069.03.1508.

Schwab, A. and Kuerner, W. (2013), Electromagnetic Compatibility, AGIR Publishing House, ISBN: 978 973-720-359-5.

Van der Velden, N., Patel, M. et al. (2014), "LCA benchmarking study on textiles made of cotton, polyester nylon, acryl or elastane", Int. J. Life Cycle Assess, 19, 331-356, https://doi.org/10.1007/s11367-013-0626-9.

Van der Velden, N.M., Kuusk, K. and Koehler, A. (2015), "Life cycle assessment and eco-design of smart textiles: The importance of material selection demonstrated through e-textile product redesign", Materials and Design, 84, 313-324, https://doi.org/10.1016/j.matdes.2015.06.129.

Wolf, M.-A., Pant, R. et al. (2010), ILCD Handbook - The International reference Life Cycle Data system, Joint Research Centre, ISBN 978-92-79-19092-6, https://doi.org/10.2788/38479.

Yasin, S., Behary, N. and Perwuelz, A. (2018), "Life cycle assessment of flame retardant cotton textiles with optimized end-of-life phase", Journal of Cleaner Production, 172, 1080-1088, https://doi.org/10.1016/j.jclepro.2017.10.198

Ziaja, J. and Jaroszewski, M. (2011), "EMI shielding using composite materials with plasma layers", in Vitaliy Zhurbenko (ed.), Electromagnetic Waves, InTechOpen https://doi.org/10.5772/16553. 

MATERIALS FOR A SUSTAINABLE FOOTWEAR INDUSTRY

\author{
CARLOS RUZAFA-SILVESTRE, PILAR CARBONELL-BLASCO, ELENA ORGILÉS- \\ CALPENA, FRANCISCA ARÁN AÍS \\ INESCOP, Footwear Technological Centre, 03600 Elda, Alicante, Spain, cruzafa@inescop.es
}

\begin{abstract}
In this paper INESCOP proposes the improvement of the bonding of footwear soling materials using the low-pressure plasma surface treatment as a non-polluting and resource-efficient technology by means of adhesive bonds, with a reactive hot melt polyurethane adhesive, as a more sustainable alternative to current chemical surface treatments such as halogenation. More precisely, low-pressure plasma is capable of cleaning and removing all impurities, such as oxides, oils and fats on material surface. Then, it is activated by producing new chemicals species on the top layer of the substrate. Thus, the materials' surface acquires new surface functionalities, improving the compatibility adhesive-substrate and, therefore their adhesion properties. Furthermore, in this work the surface modifications produced in these materials of different polymeric nature have been optimised to increase their roughness, wettability, adhesive properties, etc., and have been validated through various experimental characterisation techniques. As a result, the samples treated with plasma meet the adhesion requirements for footwear materials. As a result, low-pressure plasma treatment has desmonstrated to be a green, alternative, and sustainable technology in line with European policies on circular economy, which enhances material surface properties by improving the adhesion bonding process.
\end{abstract}

Keywords: bonding, sustainability, wettability

\title{
INTRODUCTION
}

The appearance of new materials for the manufacture of footwear and components, especially in the production of soles are mainly polymeric materials, which present excellent mechanical and aesthetical properties, but due to their low surface energy they show poor adherence to other components of footwear presents adhesion difficulties (Orgilés-Calpena et al., 2019).

Currently the footwear industry is looking for environmentally sustainable alternatives that are already used in other sectors such as plasma technology. This is because until now to obtain strong and durable adhesive bonds, surface treatments with solvents and dangerous chemicals have been used that can suppose risks to the health of workers and the environment (Orgilés-Calpena et al., 2018).

Plasma is considered the fourth state of matter, since it is a gas that is supplied with a continuous energy charge, ionizing it and electrically charging its particles. This process originates the so-called "plasma", which acquires a series of properties that do not exist in the other states of matter, being able to interact with the substrates with which it comes into contact. Thanks to the properties of plasma, it can produce different effects such as an ultra-surface cleaning, activation surface and surface etching of materials. This surface treatment can clean and remove all impurities, such as oxides and fats. Once the surface has been cleaned, it is activated by the production of new chemicals species of the gas introduced, which are attached to the top layer of the substrate. Then, the surface of the material acquires a new surface function, thereby improving the compatibility of the adhesive with the substrate and their adhesion performance (Tyczkowski et al., 2005; Kapica et al., 2019; Mandolfino et al., 2019).

Therefore, this paper focuses on the study of the improvement of the adhesion with plasma technology in representative polymeric materials of the footwear industry, such 
as thermoplastic polyurethane (TPU) and ethylene-vinyl-acetate based materials (EVA). Specifically the low-pressure plasma techonology, whose effectiveness has been optimized by varing plasma processing parameters and analysed with different charaterization experimental techinques.

\section{EXPERIMENTAL}

\section{Materials}

In this study, materials of different polymeric nature have been used as representative soling materials of the footwear industry. On the one hand, a thermoplastic polyurethane (TPU) and an ethylene-vinyl-acetate (EVA) have been used as adherents, and as an upper material, chrome-tanned leather split. On the other hand, a reactive polyurethane hot-melt adhesive has been used.

\section{Low-Pressure Plasma Technology}

To generate the low pressure plasma, a Tetra 30 plasma system has been used. The treatment is carried out in a chamber in which the samples are introduced and the vacuum process is carried out at $15 \mathrm{~Pa}$. Then, a flow gas is introduced at a pressure of $30 \mathrm{~Pa}$, and in this case, oxygen at a mass flow of $500 \mathrm{~cm}^{3} / \mathrm{min}$. The gas enters the chamber through a high frequency electric field of $13.56 \mathrm{MHz}$ and $300 \mathrm{~W}$ of maximum power $(100 \%)$. The chemical radicals originated in this discharge react with the material's surface. In addition, plasma operating conditions, such as the exposure time range, have been configured between ( 30 to $300 \mathrm{~s}$ ), and plasma power (from 50 to $100 \%)$.

\section{Characterization Techniques}

The effectiveness of the plasma treatment and chemical modification on the material's surface has been evaluated by different experimental techniques such as: Fourier Transformed Infrared Spectroscopy (FTIR), Scanning Electron Microscopy (SEM), contact angle measurements (EN 828:2013) and surface energy measurement (Owens and Wendt, 1969). The adhesion performance before and after plasma treatment has been evaluated by the T-peel strength test of upper-soling joints obtained with the reactive hot melt polyurethane adhesive (EN 1392:2007; EN 15307:2015).

\section{RESULTS AND DISCUSSION}

In Figure 1, the infrared spectra obtained with and without the low pressure oxygen plasma treatment can be observed. The non-treated TPU and EVA samples show the most characteristic bands of both materials. According to the spectra obtained with plasma treatment, no significant chemical modifications were evidenced with respect to the untreated samples. (Enciso et al., 2017; Sundryal et al., 2020). 


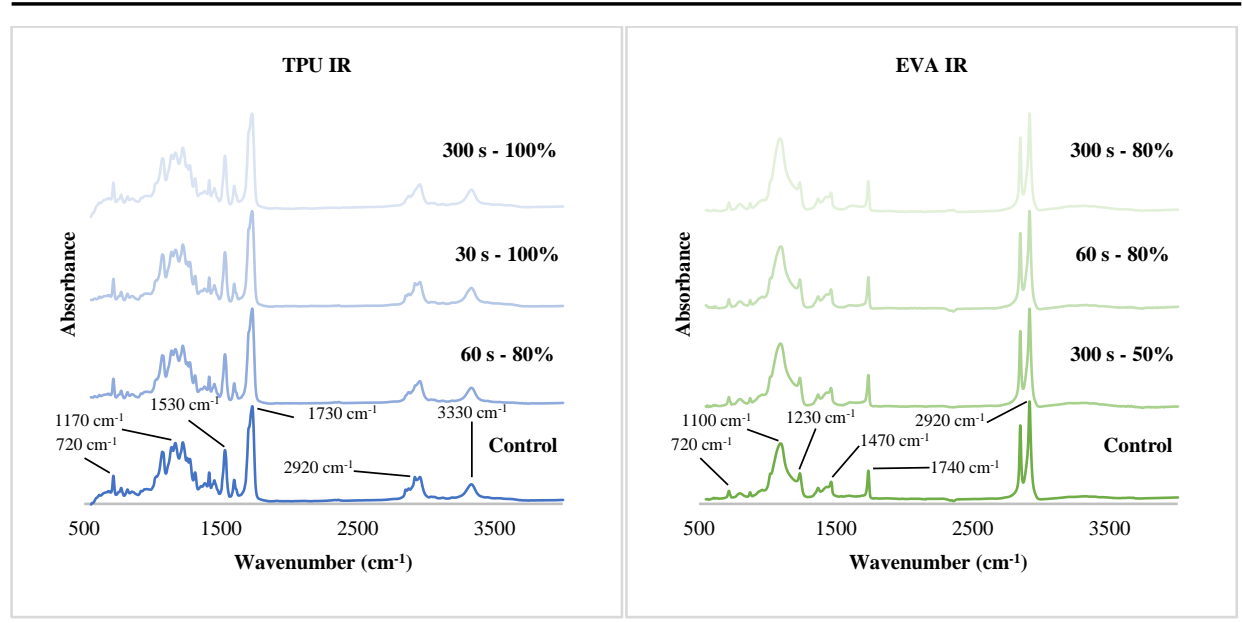

Figure 1. Infrared spectra of TPU and EVA with and without plasma treatment

The surface modifications produced on TPU and EVA surface by the different lowpressure plasma treatment conditions were analysed by scanning electron microscopy are shown in Figure 2. On the one hand, in the case of TPU, it is observed that the untreated sample surface is completely smooth and clean but in the plasma-treated samples, some roughness has been originated on the surface, making it less uniform. On the other hand, the untreated EVA surface shows a high porosity, which decreases after applying the plasma treatments that close the surface's pores and increase its roughness. In both materials, these effects might involve an increasement of the mechanical adhesion caused by an increased surface contact area regardless of the different plasma treatments applied (Tyczkowski et al., 2005).

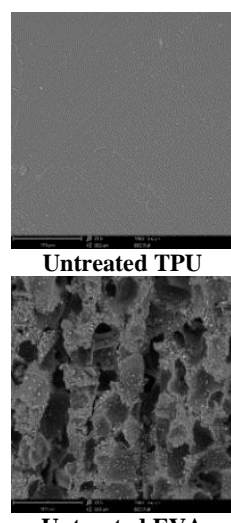

Untreated EVA

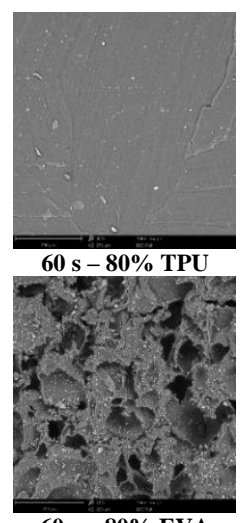

$60 \mathrm{~s}-80 \%$ EVA
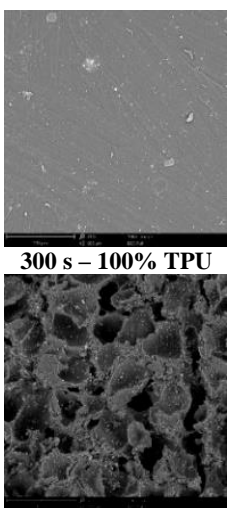

$300 \mathrm{~s}-\mathbf{5 0 \%}$ EVA

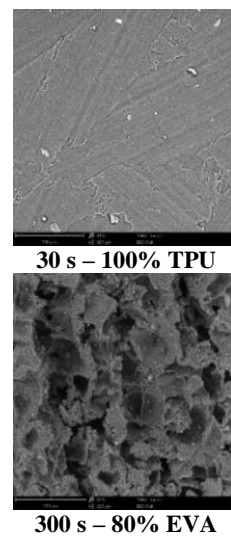

Figure 2. SEM images of non-treated and plasma treated samples of TPU and EVA

Figure 3 shows the contact angle values of the plasma-treated and untreated materials samples. The unmodified samples show high contact angle values in both materials, especially with liquid water, which means that the surface has low polarity and wettability, therefore a low surface energy. After oxygen plasma treatment, the contact angles measured using water as reference liquid are reduced due to the fact that 
the polarity of the surface increases and slightly increases simultaneously the contact angle values obtained using the non-polar liquid diiodomethane (DIM) as reference liquid. This chemical and morphological effect in the polarity of the materials increases their wettability (Kapica et al., 2019; Mandolfino et al., 2019; Monzó-Pérez, 2015; Chen et al., 2017).

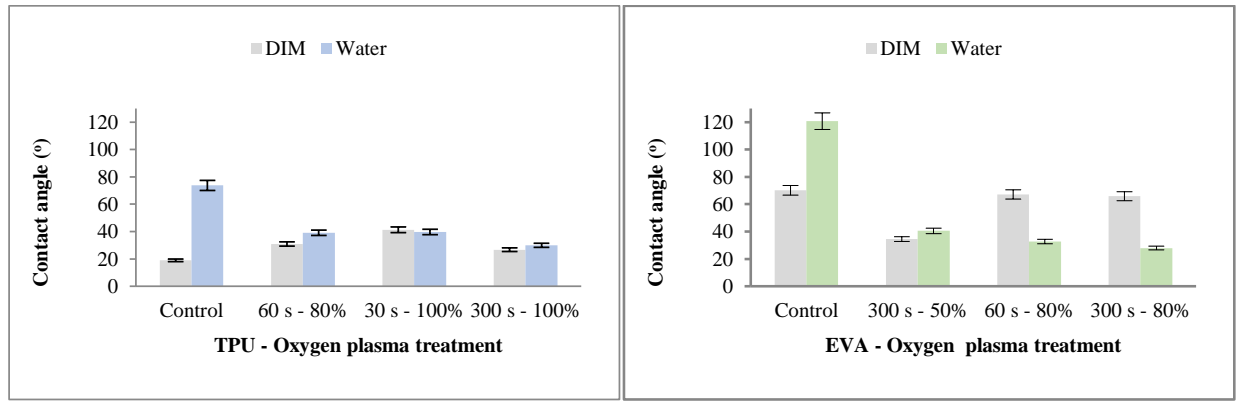

Figure 3. Contact angle values obtained from materials studied.

According to the results obtained from the contact angles, the surface energy of each material can be calculated. This is shown in Figure 4 according to Owens-Wendt method. In both untreated materials, low surface energy values are obtained, in which the polar component is not appreciated. This indicates that it is a non-polar surface. Plasma treatment increases the surface energy values, whose dispersive component is decreased and polar component is increased in all the treatments. The chemical modification produced on the materials surface is translated into an increase in the polarity, wettability and compatibility of the substrates with the adhesive, particularly in the treatments in which the both components, nonpolar and dispersive ara balanced, such as $30 \mathrm{~s}-100 \%$ and $300 \mathrm{~s}-50 \%$ for TPU and EVA, due to the need for a balance of polarity on the surface (Yáñez-Pacios and Martín-Martínez, 2018; Ngoc and Thai, 2017; Arán-Aís et al., 2020).
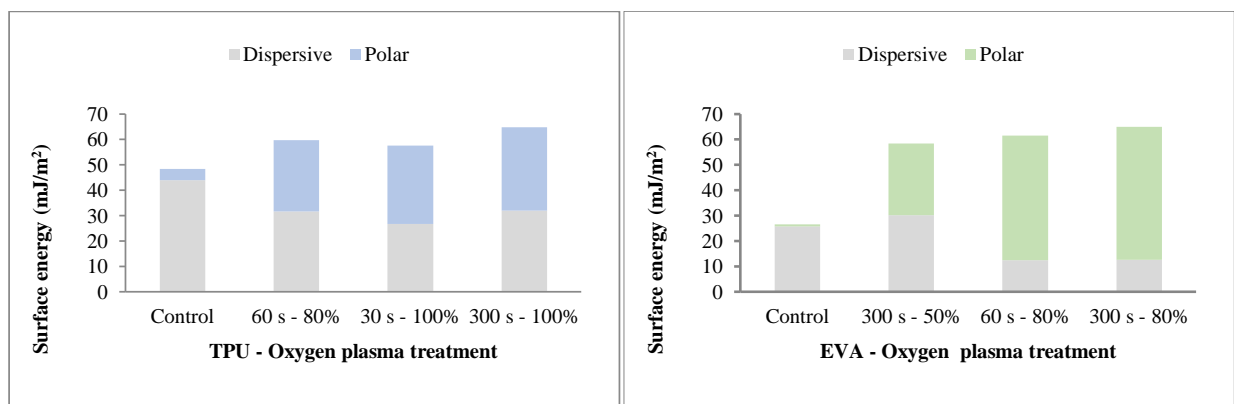

Figure 4. Surface energy values obtained from materials studied

The effect of oxygen plasma treatment on the adhesion properties of the considered materials, TPU and EVA respectively, was evaluated by means of T-peel strength assays using split leather/reactive polyurethane adhesive/plasma treated and untreated 
TPU or EVA joints. Before joint formation, EVA and split leather samples were duly roughened.

Figure 5 shows the T-peel strength values obtained after $72 \mathrm{~h}$ of the joints formation. The effect of low-pressure plasma treatment on the bonding properties of treated bonds caused a noticeable increase in the adhesion compared to control samples. This improvement of the adhesion properties can be atributed to both an increase in surface roughness the increase in surface energy, respectively. According to the results, the highest value obtained in TPU bonds corresponds to the lowest exposure time ( $30 \mathrm{~s})$ and the highest power $(100 \%)$. In addition, in EVA bonds is observed that the best result corresponds to the highest exposure time (300 s) and the lowest power (50\%). Both plasma treatments described indicate that these parameters increase the low-pressure plasma effectiveness, which is also verified in the case of EVA rubber by a change of the failure type from a mixture of adhesion failure and superficial exfoliation (A2/S2) to superficial exfoliation (100S2), indicating increased bond strength adhesive-substrate. Otherwise, with TPU, the type of adhesion failure (A2) is the same with and without plasma, therefore, it is not modified due to the nature of the material that is more resistant, however, there is a marked increase in the bond strength (Tyczkowski et al., 2005; Kapica et al., 2019).

It should be noted that all cases in which oxygen plasma treatments have been applied improve the adhesion of the bonds studied and exceed the minimum quality requirements demanded for footwear according to standard tests (EN 15307:2015; Arán-Aís et al., 2020).

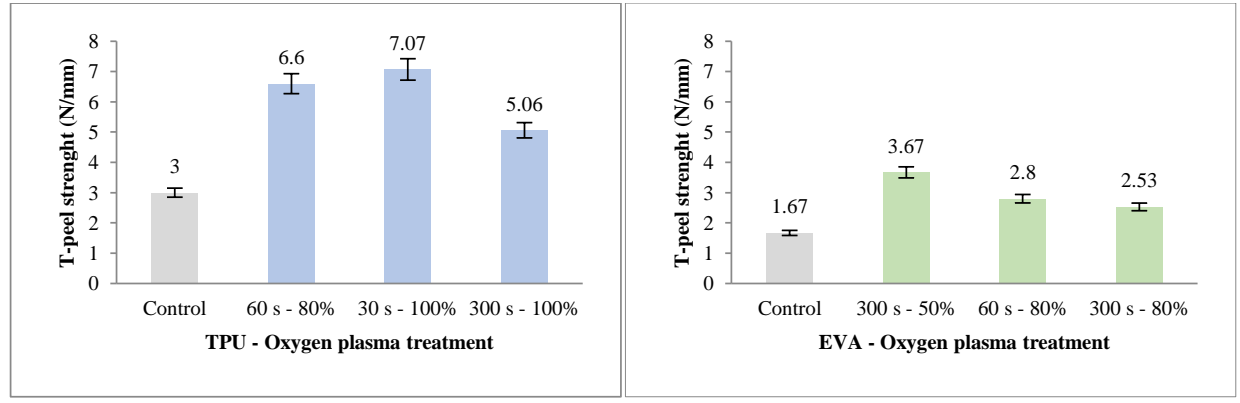

Figure 5. Comparative graphs of T-peel strength values of leather/HMPUR adherive/TPU and EVA joints after $72 \mathrm{~h}$ with and without oxygen plasma treatment. Failure type: A2: adhesive failure of the TPU. S2: superficial exfoliation of the EVA.

\section{CONCLUSIONS}

According to the obtained results with the applicacion of the different low-pressure plasma surface treatments on the TPU and EVA. It is concluded that when there is an increase in roughness, there is a decrease in the contact angles that results in an increase in the surface energy, which is mainly due to the increase in polar component rather than the dispersive one. Consequently, leading to an improvement in the final adhesion properties of the considered adherents, that allows fulfill minimum requirements for the upper-to-sole joints for footwear applications.

In short, plasma technology is an innovative technology and its implementation in the bonding processes of the upper-soling joint in footwear will contribute to the 
development of a sustainable footwear industry in line with the circular economy and the European sustainability policies, such as the Green Deal, as well as the achievement of the sustainable development goals.

\section{Acknowledgements}

This work was funded by the Valencian Institute for Business Competitiveness (IVACE) of Generalitat Valenciana, and the European Regional Development Fund (FEDER), through the R\&D projects PLASMADHESION II (IMDEEA/2019/23).

\section{REFERENCES}

Arán-Aís, F., Ruzafa-Silvestre, C., Carbonell-Blasco, P., Pérez-Limiñana, M.A. and Orgilés-Calpena, E. (2020), "Sustainable adhesives and adhesion processes for the footwear industry", $1^{\text {st }}$ International Conference on Industrial Applications of Adhesives 2020, Madeira, Portugal.

Chen, W., Zhou, X., Zhang, X., Bian, J., Shim, S., Nguyen, T., Chen, M., and Wan, J. (2017), "Fast enhancement on hydrophobicity of poplar wood surface using low-pressures dielectric barrier discharges (DBS) plasma", Applied Surface Science, 407, 412-417, https://doi.org/10.1016/j.apsusc.2017.02.048.

EN 1392:2007. Adhesives for leather and materials for footwear. Solvent-based and dispersion adhesives. Test methods to measure bond strength under specific conditions.

EN 15307:2015. Adhesives for leather and footwear materials. Upper-sole joints. Minimum resistance.

EN 828:2013. Adhesives. Wettability determination by measurement of the contact angle and critical surface tension of the solid surface.

Enciso, B., Abenojar, J. and Martínez, M.A. (2017), "Influence of plasma treatment on the adhesion between a polymeric matrix and natural fibres", Cellulose, 24, 1791-1801, https://doi.org/10.1007/s10570-0171209-X

Kapica, R., Tyczkowski, J., Balcerzak, J., Makowski, M., Sielski, J. and Worwa, E. (2019), "Enhancing adhesive joints between commercial rubber (SBS) and polyurethane by low pressure plasma surface modification", Int. J. Adhes. Adhes., 95, 102415. https://doi.org/10.1016/j.ijadhadh.2019.102415.

Mandolfino, C., Lertora, E., Gambaro, C. and Pizzorni, M. (2019), "Functionalization of Neutral Polypropylene by Using Low Pressure Plasma Treatment: Effects on Surface Characteristics and Adhesion Properties", Polym., 11, 202, https://doi.org/10.3390/polym11020202.

Monzó-Pérez, M.R. (2015), "Investigación de la influencia del tipo de gas utilizado en el plasma atmosférico, en la modificación superficial del polipropileno", $\mathrm{PhD}$ thesis, Polythenic University of Valencia, Valencia, Spain.

Ngoc, T. and Thai, D. (2017), "Shoes surface bonding by cold plasma technology", International Conference on System Science and Engineering (ICSSE), Ho Chi Minh City, 681-684, https://doi.org/10.1109/ICSSE.2017.8030962.

Orgilés-Calpena, E., Arán-Aís, F., Torró-Palau, A.M and Martínez-Sánchez M.A. (2019), “Adhesives in the Footwear industry: A Critical Review", Rev. Adhes. Adhes. 7(1), 69-91, https://doi.org/10.7569/RAA.2019.097303

Orgilés-Calpena, E, Arán-Aís, F., Torró-Palau, A.M. and Orgilés-Barceló, C. (2018), "New adhesive technologies in the footwear industry", in: A. Pizzi and K.L. Mittal (eds.), Handbook of Adhesive Technology, $3^{\text {rd }}$ Edition, Boca Raton, FL:CRC Press, 603-618.

Owens, D.K. and Wendt, R.C. (1969), "Estimation of the surface free energy of polymers", J. Appl. Plym. Sci, 13(8), 1741-1747.

Sundryal, P., Bhattacharya, S. and Pandey, M. (2020), "Plasma-assisted surface alteration of industrial polymers for improved adhesive bonding", Int. J. Adhes. Adhes., 102626, https://doi.org/10.1016/j.ijadhadh.2020.102626.

Tyczkowski, J., Krawczyk, I. and Wozniak, B. (2005), "Plasma-Surface Modification of Styrene-Butadiene Elastomers for Improved Adhesion”, in: d'Angostino, R, Favia, P, Oehr, C and Wertheimer, MR (eds). Plasma processes and polymers, 1st edition, WILEYVCH Verlag GmbH \& Co. KGaA, Weinheim, $233-$ 252, https://doi.org/10.1002/3527605584.ch18.

Yáñez-Pacios, A.J. and Martín-Martínez, J.M. (2018), "Improved Surface and Adhesion Propoerties of WoodPolyethylene Composite by Treatment with Argon-Oxygen Low Pressure Plasma”, Plasma Chem Plasma Process, 38, 871-886, https://doi.org/10.1007/s11090-018-9899-x. 
INNOVATIVE TOOL FOR THE CIRCULAR DESIGN OF TECHNICAL TEXTILES

\author{
TEODOR SÂRBU, ANGELA DOROGAN, CRISTINA GROSU, CRISTINA ELENA STROE \\ National Research \& Development Institute for Textiles and Leather, 16 Lucretiu Patrascanu \\ street, Bucharest, Romania, teodor.sarbu@incdtp.ro,angela.dorogan@incdtp.ro, \\ cristina.grosu@incdtp.ro,cristina.stroe@incdtp.ro
}

\begin{abstract}
Our planet is going through political, economic, social and ecological crisis, which are constantly feeding each other. Human activities driven by a rapidly growing global population, unsustainable economic growth, technological innovations, but also inappropriate production practices and consumption models- create increasing pressures on ecosystems and natural resources. Neither the social nor the ecological crisis can't be overpassed without changing the way of our economic system works and which involves the manner how innovative transformations take place. In this context, it is mandatory to use design as a strategy consist of people to understand the basic principles of design: user orientation, empathy, mental and physical process, future orientation, visual approach, cocreativity, interconnection of complex systems, continuous testing and iteration. The circular design is an innovative tool for implementing the circular economy whose main purpose is: "to connect all material flows, integrating them in a circular process, which ensures efficient consumption of resources and minimizes the amount of resulting waste". The paper presents a practical example of using an interactive map, owned by Delft University of Technology (Netherlands), applied as a technical analysis tool, in order to determine the reuse potential of a technical product components, specifically a laptop bag for transporting personal IT equipment.
\end{abstract}

Keywords: interactive map, circular design, technical textiles

\title{
INTRODUCTION
}

Europe must transform its economic model from a pattern of growth such as "obtaining, manufacturing, using, disposing" - a linear model that starts from the premise that resources are abundant, available and cheap to eliminate - to a pattern that favors the reuse, repair, reconditioning and recycling of existing materials and products.
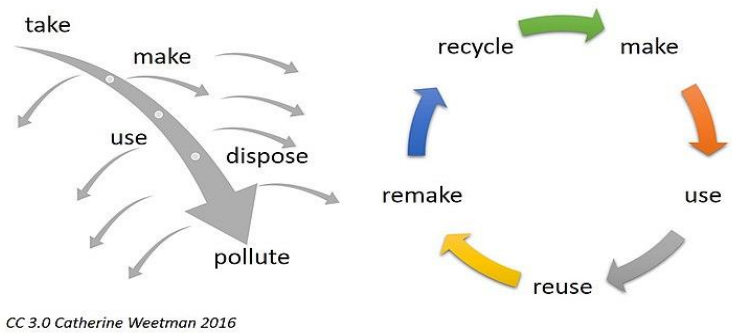

Figure 1. Linear economy versus circular economy (Weetman, 2016)

The implementation of the concept of circular economy is essential for maintaining and increasing the competitiveness of the European Union (EC, 2015), requiring the promotion of political and legal measures to support the recycling and reuse of waste in productive processes (EC, 2010). The estimated impact of the implementation of the circular economy at European level will be manifested simultaneously on four levels (Rizos et al., 2017; EEA, 2016): 
- in economic terms, by increasing competitiveness, supporting innovative initiatives;

- social, through integration and social cohesion, increasing the quality of life, mutations of consumer behavior, creating new jobs;

- environment, by reducing the negative and irreversible effects on the climate and the environment;

- efficient use of resources, reducing the dependence of the European economy on imports of raw materials.

Increasing resource efficiency is based on six key concepts: circular economy, waste hierarchy, increasing responsibility in production, industrial symbiosis, new business models (Ecoprofit, 2014).

In the process of achieving a European circular economy, SMEs and social enterprises have been recognized as particularly important (EC, 2014). Among the barriers that can prevent the implementation of "circular" and "green" economy practices (Rizos et al., 2015) can be mentioned distinct groups of barriers that refer to: organizational culture and management style, the need for financial investments for the implementation of sustainable solutions; Lack of adequate government support / legislation, lack of information, lack of internal technical skills, insufficient support from suppliers and consumers, high level of bureaucracy in monitoring and reporting data on the performance of SMEs in the field of circular economy.

Synthesizing the barriers encountered of companies in the implementation of the principles of the circular economy can be mentioned the following categories (Ritzén and Sandström, 2017): financial, structural, operational, attitudinal, technological.

The specialized literature indicates working tools that can be a support for the activity of specialists for the implementation of the concept of circular economy.

\section{MATERIALS AND METHODS}

The method used, "Hotspot Mapping" (Flipsen et al., 2020), combines the disassembly of a product with the recording of all the steps necessary to reach the most critical parts of the product architecture. The method is unique in that it includes both economic and environmental value for each component. Critical parts are parts with a high failure rate or need for maintenance and / or with a high economic and environmental value, which should be easily accessible with little effort to enable efficient recovery processes. The ease of disassembly of a product is determined by factors that help or prevent the disconnection of critical parts from the rest of the product. A component part of a product with a high economic value or an impact on the built-in environment should be easily accessible for recycling loops. There are five indicators: the time required to disconnect the components, the difficulty of access, the priority elements, the impact on the environment and the economically valuable parts, which show the criticality of the part or activity involved.

The object of study was a product from the group of technical textiles, respectively a bag made mainly of textile elements, a product that may be a distinct element of a system of work equipment for various fields of use.

In order to detect the critical parts and the difficult activities involved, all the stages were recorded and aspects related to the functionality and wear rate of the components and the composition of the material were identified.

Steps: 
- Disassembly of the product without damaging the component parts;

- Completing the calculation sheet to identify the component parts after disassembly;

- Optionally, a product redesign can be made for greater disassembly ease.

\section{RESULTS}

The component parts and the activities undertaken for the disassembly of the product were identified respectively: the time to disassemble a component part, the difficulty of the activity in question, the priority of the part due to failure or replacement rate, environmental impact, built-in economic value.

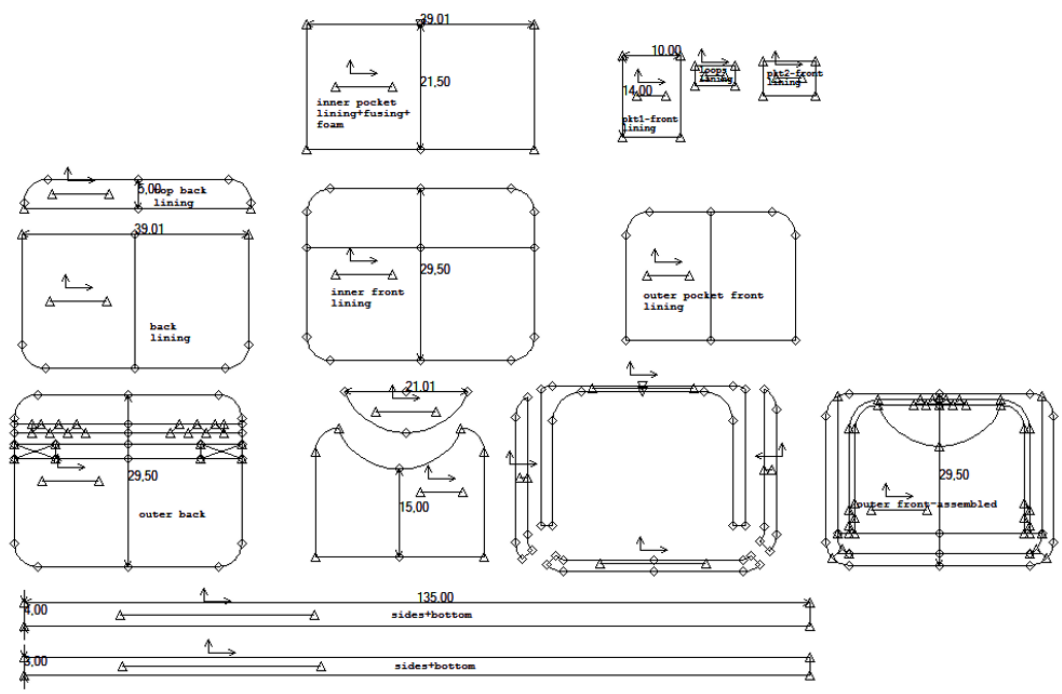

Figure 2. Component parts of the bag - scheme

The interactive map (Table 1), highlights each step taken in the disassembly process of the product, in the centralization of its component parts, as well as in the characterization / hierarchy according to different parameters.

From the analysis of the included data, the following aspects can be mentioned:

- the disassembly operation, registered the greatest degree of difficulty, the time necessary to undo the seams of the textile elements being longer than in the case of the other operations specific to the analysis (normal aspect considering that the object of study is a technical product).

- the structure of the product is mostly made of textile elements, which can be reused through mechanical recycling or up-cycling operations.

- the number of component elements is in correlation with the product architecture determined by the functionality requirements, as well as by the creative capacity of the specialist.

- the symbolism of the characterization indicators from an economic point of view and of the protection of the environment allows the hierarchy of the component elements.

https://doi.org/10.24264/icams-2020.IV.20 
Table 1. Interactive map - summary table

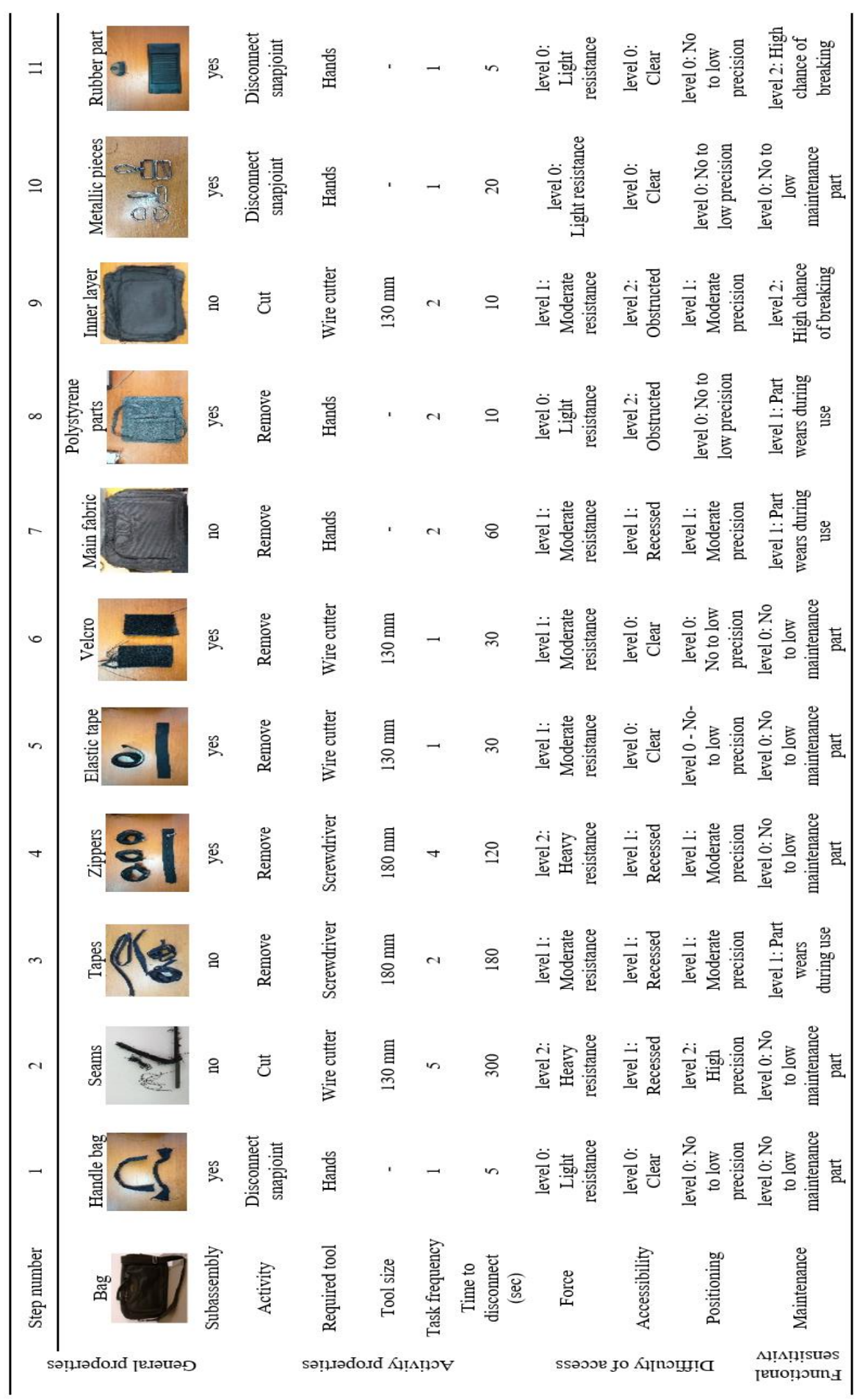

https://doi.org/10.24264/icams-2020.IV.20 


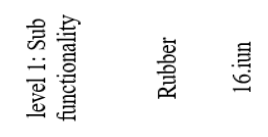

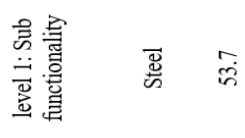

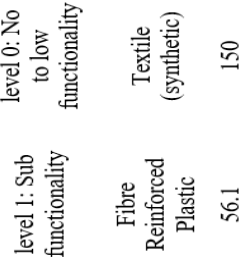

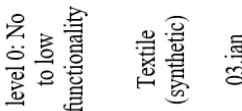

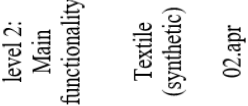

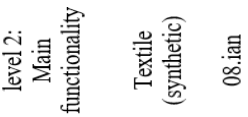

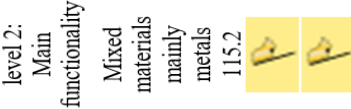

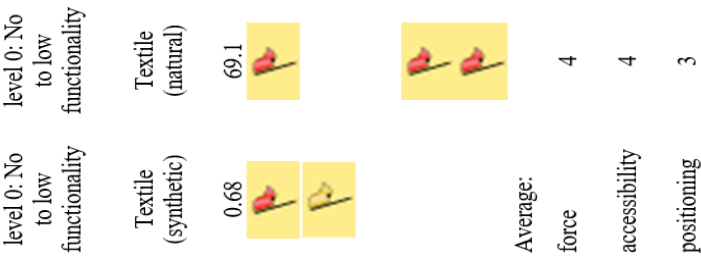

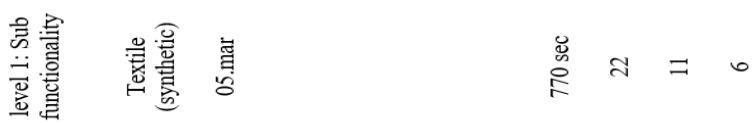

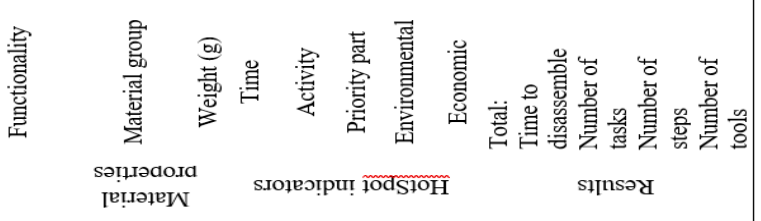

https://doi.org/10.24264/icams-2020.IV.20 


\section{CONCLUSIONS}

- We are the generations that face most acutely the reduction of natural resources and the negative effects of the impact of human activities on the environment, and this fact forces us to change both our way of thinking and the way we relate to the environment and economic and social activities.

- The textile sector is one of the basic pillars of the sustainable development of the $\mathrm{EU}$ and at the same time the second largest polluter of the environment, with a fast pace of change which requires new ideas for future technologies, products and markets.

- A new economy of textile products is distributive by design which requires work tools specific to the application of the concept of circular economy both in the stage of professional training and in the production process.

\section{Acknowledgments}

This work was supported by a grant of the Romanian Research and Innovation Ministry, through Sectorial Plan, contract no. 1PS/2019.

\section{REFERENCES}

Ecoprofit (2014), Schimbarea societatii industriale; eficienta resurselor, http://ecoprofit.ro/economiacirculara-schimbarea-societatii-industriale-eficienta-resurselor/

European Commission (2010), "Being wise with the waste. The EU's approach to waste management", Brussels: European Commission.

European Commission (2014), "Green action plan for SMEs, Enabling SMEs to turn environmental challenges into business opportunities", Communication from the Commission to the European Parliament, the Council, the European economic and Social Committee and the Committee of the Regions, com (2014) 440 final.

European Commission (2015), "Closing the loop: An EU action plan for the Circular Economy".

European Environment Agency (2016), "Circular economy in Europe, Developing the knowledge base", Luxembourg: European Environment Agency, available at: https://www.eea.europa.eu/publications/circulareconomy-in-europe.

Flipsen, B., Bakker, C. and de Pauw, I. (2020), "Hotspot Mapping for product disassembly; a circular product assessment method", Conference of Electronics Goes Green 2020+, Berlin, September 1, 2020.

Ritzén, S. and Sandström, G.O. (2017), "Barriers to the Circular Economy - integration of perspectives and domains", Procedia CIRP, 64, 7-12, https://doi.org/10.1016/j.procir.2017.03.005.

Rizos, V., Behrens, A., Kafyeke, T., Hirschnitz-Garbers, M. and Ioannou, A. (2015), The circular economy: barriers and opportunities for SMEs, available at: https://www.ceps.eu/publications/circular-economybarriers-and-opportunities-smes.

Rizos, V., Tuokko, K. and Behrens, A. (2017), "The Circular Economy: A review of definitions, processes and impacts", CEPS Working Document, available at: https://www.ceps.eu/publications/circulareconomy-review-definitions-processes-and-impacts.

Weetman, C. (2016), "Linear versus circular; The circular economy as a new economic model", https://cvoucher.com/circular-economy-vs-linear-economy/ 
TREATMENT OF HIDE LIMING WASTEWATER BY CARBON DIOXIDE

\author{
AUKSĖ ŠLIBURYTE், VIRGILIJUS VALEIKA \\ Kaunas University of Technology, Department of Polymer Chemistry and Technology, Radvilenu \\ pl. 19, 50254, Kaunas, Lithuania, virgilijus.valeika@ktu.lt
}

\begin{abstract}
Results of the investigation of hide liming process wastewater treatment by carbon dioxide are presented in a paper. Comparison of the wastewater characteristics before and after the treatment by carbon dioxide was carried out. It was attempted to regenerate sodium sulphide using three different solutions: $10 \%$ solution of sodium carbonate and $5 \%$ or $10 \%$ solution of sodium hydroxide. The kinetic of sodium sulphide concentration, general alkalinity and $\mathrm{pH}$ was established. The solutions with the regenerated sodium sulphide were explored for unhairing of hide. The solution of $10 \%$ sodium hydroxide with regenerated sulphides was the mostly suitable for this aim. The properties of unhaired pelt were determined and assessed.
\end{abstract}

Keywords: leather, liming, carbon dioxide, sodium sulphide, regeneration.

\title{
INTRODUCTION
}

The leather industry is one of the most environmentally polluting branches of industry, especially due to its wastewaters. Huge amounts of lime sludge and total solids formation are the main drawbacks of lime (Thanikaivelan et al., 2001). Herewith, the cleaning of unhairing solutions, polluted with lime, sulphides and the products of protein degradation, remains very difficult and expensive (Sirvaityte et al., 2016).

Despite the fact that numerous unhairing methods were developed trying to avoid the use of lime (Thanikaivelan et al., 2001; Munz and Sonnleitner, 2005; Valeika et al., 2000). or even both lime and sulphide (Andrioli and Gutterres, 2014; Khandelwal et al., 2014), the use of the lime-sulphide process remains the most commonly applied method of unhairing and opening up of the derma in the leather industry (Sirvaityte et al., 2016).

The attempts were done to use the liming solution repeatedly. Faki et al. (2018), report that recycling of the spent unhairing-liming liquor can safely be applied in tanneries without affecting the quality of produced leathers. Nazer et al. (2006) developed method which allowed for four times reuse of the unhairing-liming liquor without visibly affecting the quality of the final product of leather. Unfortunately, the repeated use of liming solutions is not adapted in practise.

Other way of utilization of the used liming solution is just to process it before cleaning getting chemicals, which can be reused for various purposes as well as in leather industry. The study of Venkatakrishnan et al. (2019) depicts an innovative approach to reduce pollutant and recover calcite $\left(\mathrm{CaCO}_{3}\right)$ from theliming process waste by carbon dioxide $\left(\mathrm{CO}_{2}\right)$ fixation. The results indicated rover $>95 \%$ of calcite $\left(\mathrm{CaCO}_{3}\right)$ recovery from synthetic lime wastewater followed by $85-90 \%$ from lime sludge product and relime sludge product under optimized conditions.

Unfortunately, the used liming solution is a complicated system containing not only calcium hydroxide but sodium sulphide/hydrosulphide, hair and hide-skin proteins degradation products as well. Accordingly, the interaction of $\mathrm{CO}_{2}$ with the used liming solution containing conventional ingredients should not be as simple as with pure calcium hydroxide solution. 
The presented research had aim to investigate the treatment of the used liming solution with gaseous $\mathrm{CO}_{2}$ and assess the possibilities to reuse the regenerated sulphides for leather processing.

\section{EXPERIMENTAL}

\section{Materials}

All chemical materials used for experiments were of analytical grade.

The hide liming-unhairing wastewater (HLW) used for experiments was obtained after unhairing according to the method: $\mathrm{H}_{2} \mathrm{O} 40 \%$, temperature $20-22^{\circ} \mathrm{C}, \mathrm{Ca}(\mathrm{OH})_{2}$ $2.3 \%, \mathrm{Na}_{2} \mathrm{~S}(100 \%) 1.2 \%, 1 \mathrm{~h}$ run continuously, $\mathrm{Ca}(\mathrm{OH})_{2} 2.3 \%, 1 \mathrm{~h}$ run continuously, $\mathrm{H}_{2} \mathrm{O} 100 \%, 2 \mathrm{~h}$ run continuously, later 5 min. every $3 \mathrm{~h}$, total duration 24 hours. A sample of soaked cattle hide was used in the liming-unhairing process.

\section{Equipment}

The equipment (Fig.1) used for the experiments consisted of balloon (1) with gaseous $\mathrm{CO}_{2}$, gas flow meter (2) and three Drechsel's bottles with $100 \mathrm{ml}$ of HLW (3) and with solutions for catching of hydrogen sulphide: $100 \mathrm{ml}$ of sodium hydroxide solution (4 and 5) or sodium carbonate solution (in such case also 4 and 5).

The rate of $\mathrm{CO}_{2}$ flow was $60 \mathrm{~g}$ per hour.

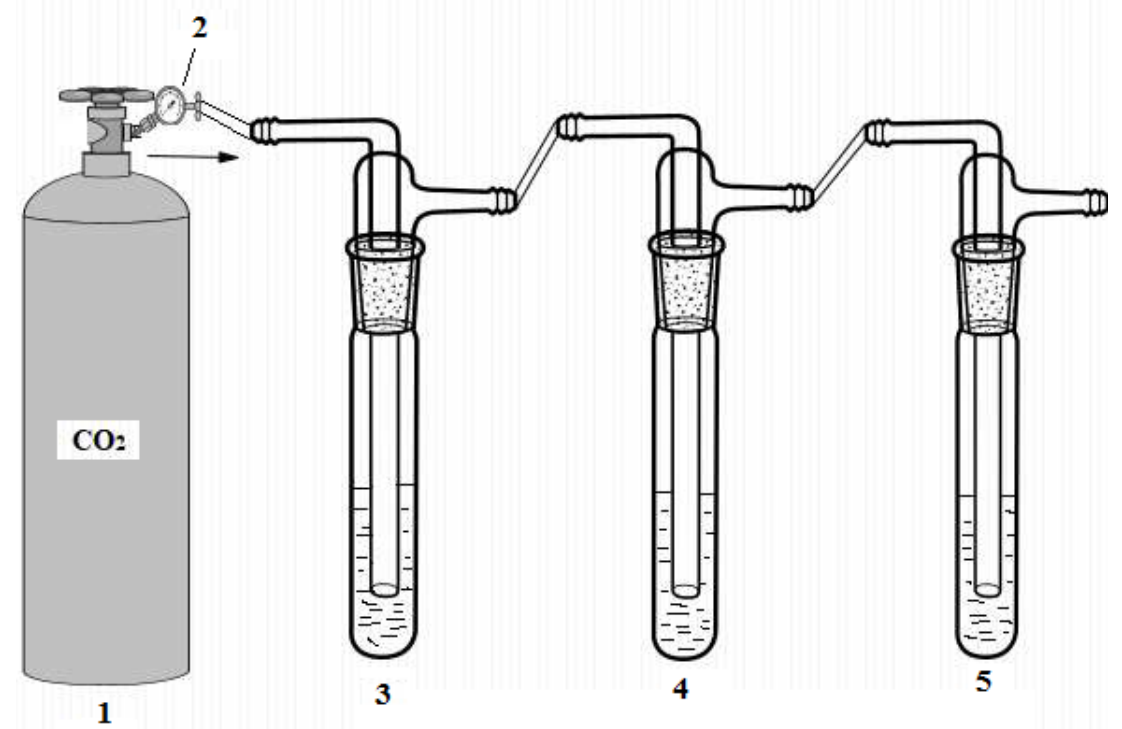

Figure 1. Equipment for treatment of unhairing-liming solution with gaseous $\mathrm{CO}_{2}$

\section{Analysis Methods}

The concentrations of calcium hydroxide, sodium sulphide and total amount of nitrogen in solutions were determined according to methods described in literature (Golovteeva et al., 1982). 
Total alkalinity was determined by titration of solutions with hydrochloric acid using methyl orange as indicator.

The amounts of soluble and insoluble materials in solutions were established by gravimetric method weighing sediments after filtration (insoluble materials) or dry residue after drying of filtrate.

The amount of collagen proteins removed was estimated from the amount of hydroxyproline in the treatment solution. The amount of hydroxyproline was determined using a photo colorimetric method (Zaides et al., 1964).

\section{RESULTS AND DISCUSSION}

The first step was to evaluate the amount of $\mathrm{CO}_{2}$ necessary for complete removal of sulphides from HLW. Accordingly, the $\mathrm{CO}_{2}$ had been blown (60 g per h) through the HLW and the blowing was continued till all sulphides were completely removed from the HLW. It was reached during 7 hours, hence, $420 \mathrm{~g}$ of $\mathrm{CO}_{2}$ were absorbed. The characteristics of the treated HLW are presented in Table 1.

Table 1. Characteristics of hide liming-unhairing wastewater before and after treatment with gaseous $\mathrm{CO}_{2}$

\begin{tabular}{|c|c|c|}
\hline \multirow[t]{2}{*}{ Characteristic } & \multicolumn{2}{|c|}{$\begin{array}{l}\text { Values of characteristics' of hide liming-unhairing } \\
\text { wastewater }\end{array}$} \\
\hline & before treatment & after treatment \\
\hline $\mathrm{pH}$ & 12.6 & 6.7 \\
\hline $\mathrm{Na}_{2} \mathrm{~S}, \mathrm{~g} / \mathrm{l}$ & 5.75 & 0 \\
\hline $\mathrm{Ca}(\mathrm{OH})_{2}, \mathrm{~g} / 1$ & 12.8 & 0 \\
\hline Total nitrogen in filtrated solution, $\mathrm{g} / \mathrm{l}$ & 1.47 & 1.08 \\
\hline Soluble materials, $\mathrm{g} / \mathrm{l}$ & 28.4 & 25.7 \\
\hline Insoluble materials, $\mathrm{g} / \mathrm{l}$ & 16.3 & 26.5 \\
\hline
\end{tabular}

The possible chemical reactions between $\mathrm{CO}_{2}$ and components of the HLW can be as follows:

$$
\begin{gathered}
\mathrm{Ca}(\mathrm{OH})_{2}+2 \mathrm{CO}_{2} \rightarrow \mathrm{Ca}\left(\mathrm{HCO}_{3}\right)_{2} \\
\mathrm{Na}_{2} \mathrm{~S}+\mathrm{CO}_{2}+\mathrm{H}_{2} \mathrm{O} \rightarrow \mathrm{H}_{2} \mathrm{~S}+\mathrm{Na}_{2} \mathrm{CO}_{3} \\
\mathrm{Na}_{2} \mathrm{CO}_{3}+\mathrm{CO}_{2}+\mathrm{H}_{2} \mathrm{O} \rightarrow 2 \mathrm{NaHCO}_{3}
\end{gathered}
$$

The HLW have become acidic during the treatment, and, probably, calcium bicarbonate is formed because it predominates within the $\mathrm{pH}$ range 6.36-10.25 (Wikipedia, 2020). Sodium sulphide converts into hydrogen sulphide, which is blown out from the HLW. Later, the formed sodium carbonate converts into sodium bicarbonate.

Therefore, $\mathrm{CO}_{2}$ can be applied for the neutralization of HLW but the formed poisonous hydrogen sulphide can not be released into environment in such form.

Afterwards, the conversion of hydrogen sulphide into sodium sulphide using solutions of $10 \%$ sodium carbonate and $5 \%$ or $10 \%$ sodium hydroxide has been explored. After formation of hydrogen sulphide in Drechsel bottle (3) with HLW, the mixture of $\mathrm{H}_{2} \mathrm{~S}$ and $\mathrm{CO}_{2}$ is pushed firstly through Drechsel bottle (4) and secondly, may be, through Drechsel bottle (5) (see Fig. 1).

During such process the $\mathrm{pH}$ of first catching solution decreases significantly (Fig. 2): down to $7.8-7.9$ for $10 \%$ sodium carbonate and $5 \%$ sodium hydroxide solutions and 
down to 8.9 for $10 \%$ sodium hydroxide solution. The possible reactions should be as follows:

$$
\begin{gathered}
\mathrm{H}_{2} \mathrm{~S}+\mathrm{Na}_{2} \mathrm{CO}_{3} \rightarrow \mathrm{Na}_{2} \mathrm{~S}+\mathrm{CO}_{2}+\mathrm{H}_{2} \mathrm{O} \\
\mathrm{H}_{2} \mathrm{~S}+2 \mathrm{NaOH} \rightarrow \mathrm{Na}_{2} \mathrm{~S}+2 \mathrm{H}_{2} \mathrm{O}
\end{gathered}
$$

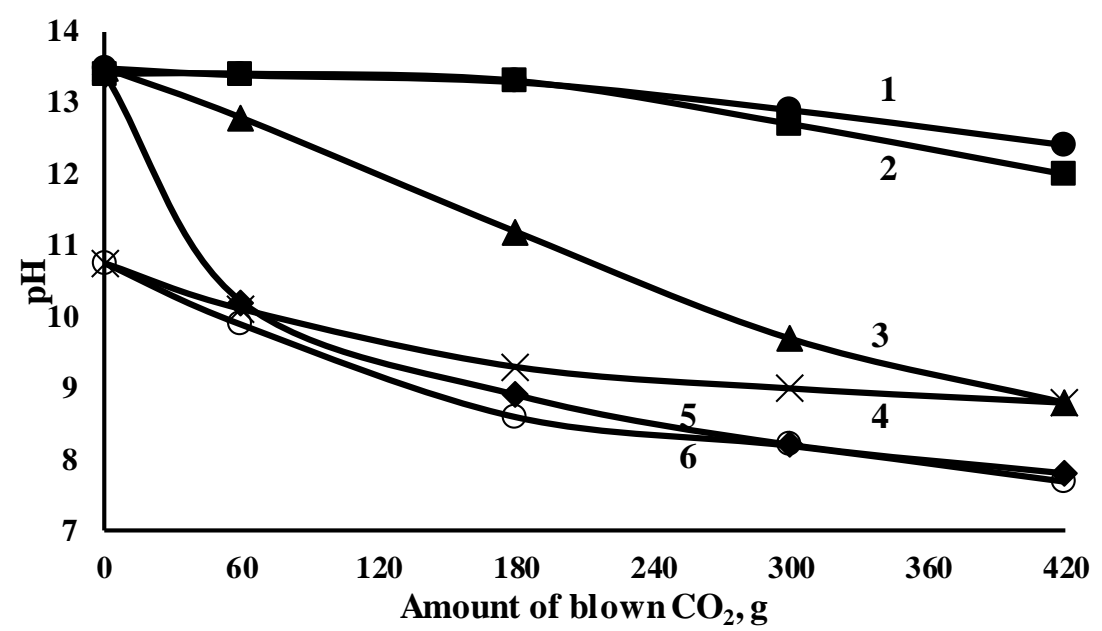

Figure 2. $\mathrm{pH}$ of solution for catching of hydrogen sulphide dependence on blown $\mathrm{CO}_{2}$ amount (solution and Drechsel bottle number: $1-10 \% \mathrm{NaOH}, 5 ; \mathbf{2}-5 \% \mathrm{NaOH}, 5 ; 3-$ $\left.10 \% \mathrm{NaOH}, 4 ; \mathbf{4}-10 \% \mathrm{Na}_{2} \mathrm{CO}_{3}, 5 ; \mathbf{5}-5 \% \mathrm{NaOH}, 4 ; \mathbf{6}-10 \% \mathrm{Na}_{2} \mathrm{CO}_{3}, 4\right)$

The changes of regenerated $\mathrm{Na} 2 \mathrm{~S}$ in catching solutions are presented in Fig. 3.

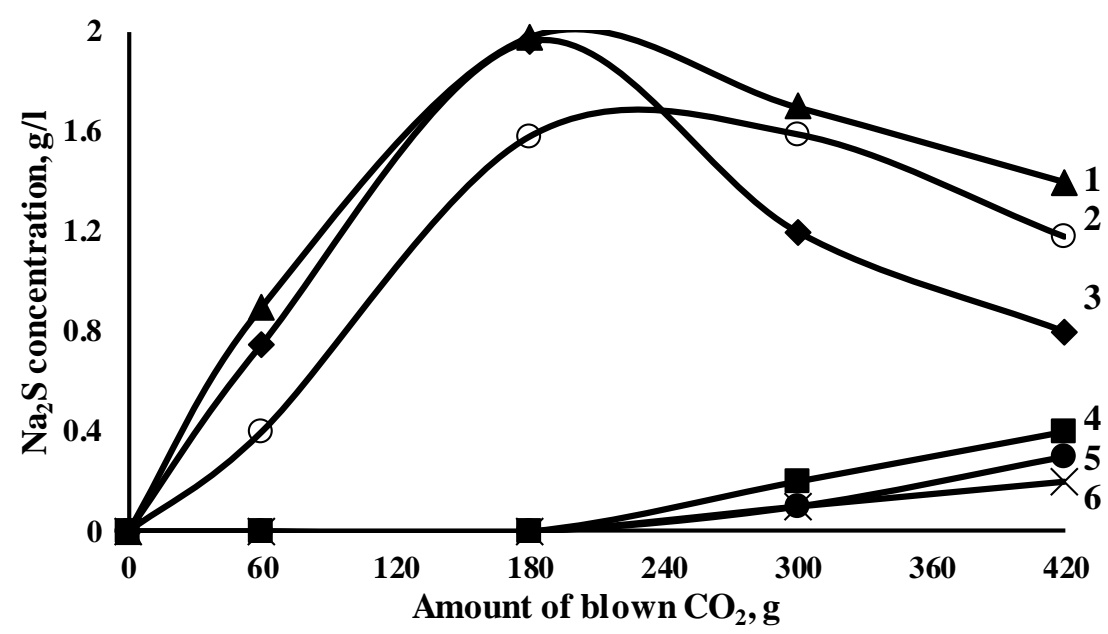

Figure 3. $\mathrm{Na}_{2} \mathrm{~S}$ concentration in solution for catching of hydrogen sulphide dependence on blown $\mathrm{CO}_{2}$ amount (solution and Drechsel bottle number: $\mathbf{1}-10 \% \mathrm{NaOH}, 4 ; 2-$

$10 \% \mathrm{Na}_{2} \mathrm{CO}_{3}, 4 ; \mathbf{3}-5 \% \mathrm{NaOH}, 4 ; \mathbf{4}-5 \% \mathrm{NaOH}, 5 ; \mathbf{5}-10 \% \mathrm{NaOH}, 5 ; \mathbf{6}-10 \%$ $\left.\mathrm{Na}_{2} \mathrm{CO}_{3}, 5\right)$ 
Therefore, the situation is very complicated: due to the decrease of $\mathrm{pH}$ of catching solutions (Fig. 2), the formed $\mathrm{Na}_{2} \mathrm{~S}$ begins again to convert into $\mathrm{H}_{2} \mathrm{~S}$. In the case of $\mathrm{Na}_{2} \mathrm{CO}_{3}$, additional $\mathrm{CO}_{2}$ releases, mixes with new formed $\mathrm{H}_{2} \mathrm{~S}$ and runs into second bottle with the same catching solution and process continues. The similar situation we have in the bottles with sodium hydroxide, just the excess of $\mathrm{CO}_{2}$ forms again $\mathrm{H}_{2} \mathrm{~S}$ and begins to push it through second bottle containing the same $\mathrm{NaOH}$ solution. Theoretically, such a process can be infinitive if blown $\mathrm{CO}_{2}$ for a very long time using many solutions for the conversion of $\mathrm{H}_{2} \mathrm{~S}$ to $\mathrm{Na}_{2} \mathrm{~S}$.

The former $10 \% \mathrm{NaOH}$ solution (solution $\mathrm{A}$ ) with regenerated $\mathrm{Na}_{2} \mathrm{~S}$ was taken after $\mathrm{CO}_{2}$ blowing during 3 hours, when the concentration of $\mathrm{Na}_{2} \mathrm{~S}$ was highest (about $2 \mathrm{~g} / \mathrm{l}$ ) and used for lime free unhairing of soaked cattle hide sample.

Parameters of unhairing-derma structure opening up are presented in Table 2. After process, the obtained pelt and unhairing-derma opening solution were analysed (Table 3 ).

Table 2. Parameters of hide unhairing-opening up of derma structure

\begin{tabular}{|c|c|c|c|}
\hline \multicolumn{4}{|c|}{ Methods of hide unhairing-derma opening up process } \\
\hline Control & $1^{\text {st }}$ experimental & $2^{\text {nd }}$ experimental & $3^{\text {rd }}$ experimental \\
\hline $\begin{array}{l}\mathrm{H}_{2} \mathrm{O} 100 \% \\
\mathrm{NaOH}_{2} \% \\
\mathrm{Na}_{2} \mathrm{~S} 12 \mathrm{~g} / \mathrm{l} \\
\mathrm{NaCl} 5 \%\end{array}$ & $\begin{array}{l}\mathrm{H}_{2} \mathrm{O} 74 \% \text {; } \\
\text { Solution A } 26 \% \text {; } \\
\mathrm{Na}_{2} \mathrm{~S} \text { adjusted to } 12 \mathrm{~g} / \mathrm{l} \text {; } \\
\mathrm{NaCl} 5 \% \text {. }\end{array}$ & $\begin{array}{l}\mathrm{H}_{2} \mathrm{O} 87 \% ; \\
\text { Solution } \mathrm{A} 13 \% ; \\
\mathrm{NaOH} 1 \% ; \\
\mathrm{Na}_{2} \mathrm{~S} \text { adjusted to } 12 \mathrm{~g} / \mathrm{l} ; \\
\mathrm{NaCl}-5 \% \text {. }\end{array}$ & $\begin{array}{l}\mathrm{H}_{2} \mathrm{O} 93.5 \% ; \\
\mathrm{A} \text { tirpalas } 6.5 \% \text {; } \\
\mathrm{NaOH} 1.5 \% \text {; } \\
\mathrm{Na}_{2} \mathrm{~S} \text { adjusted to } 12 \mathrm{~g} / \mathrm{l} \text {; } \\
\mathrm{NaCl} 5 \% \text {. }\end{array}$ \\
\hline
\end{tabular}

Notes: 1) Temperature $20-22^{\circ} \mathrm{C}, 4$ hours run continuously, later 5 min. every 3 hours, total duration 24 hours. 2) $\%$ are based on hide weight.

Table 3. Characteristics of pelt and unhairing-opening up of derma structure solution after process carried out adding $\mathrm{NaOH}$ solution with regenerated sulphides

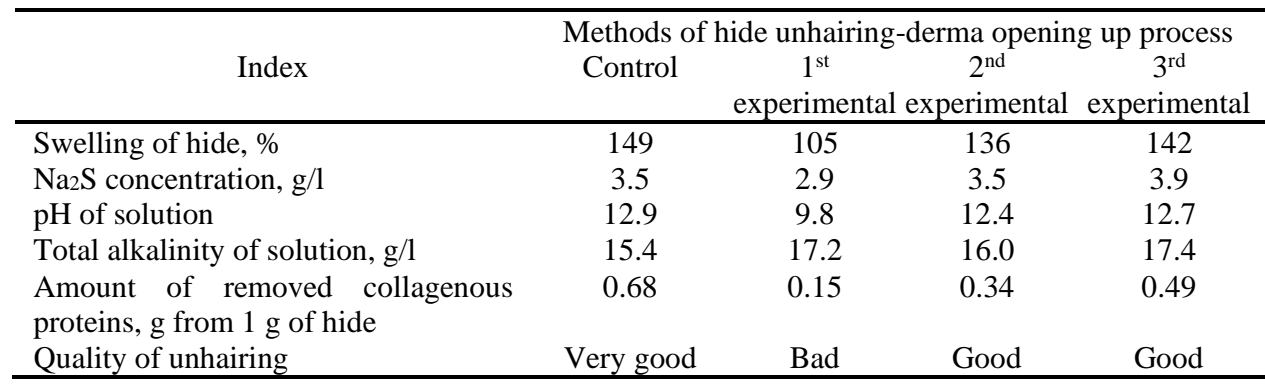

The mostly qualitative pelt is obtained when unhairing according to control process parameters. The least effect on pelt and worst unhairing-opening up of derma structure was obtained replacing all necessary $\mathrm{NaOH}$ by $26 \%$ of Solution A. Relatively good results were reached using $2^{\text {nd }}$ and $3^{\text {rd }}$ experimental methods.

It can be assumed that despite the fact that the total alkalinity is high in all cases, the addition of a larger amount of the former $\mathrm{NaOH}$ solution with regenerated sulphides at the same time leads to a higher amount of $\mathrm{NaHCO}_{3}$ (which forms parallel with $\mathrm{Na}_{2} \mathrm{~S}$ ) entering the unhairing solution. Accordingly, the $\mathrm{NaHCO}_{3}$ as weak base stops the degradation of hair and opening up of derma structure. 


\section{CONCLUSIONS}

It was found that when blowing $\mathrm{CO}_{2}$ at a flow rate $(60 \mathrm{~g} / \mathrm{h})$ through $100 \mathrm{ml}$ of liming-unhairing wastewater containing $5.75 \mathrm{~g} / \mathrm{l}$ sulphides and $12.88 \mathrm{~g} / \mathrm{l}$ calcium hydroxide, no sulphides and calcium hydroxide remained after 7 hours.

Regeneration of sodium sulphide has been found to take place best in a $10 \%$ sodium hydroxide solution into which hydrogen sulphide enters at a flow of $60 \mathrm{~g} / \mathrm{h} \mathrm{CO}_{2}$ through liming-unhairing wastewater. The concentration of regenerated $\mathrm{Na}_{2} \mathrm{~S}$ was highest after 3 h. when reached about $2 \mathrm{~g} / \mathrm{l}$ and the total alkalinity remained high.

The worst regeneration of sodium sulphide is on in $10 \%$ sodium carbonate solution. Maximum sulphide concentration was not higher than $1.6 \mathrm{~g} / \mathrm{l}$ and the $\mathrm{pH}$ of this solution decreases rapidly as sodium carbonate is converted to sodium bicarbonate.

In any case, the neutralization of the liming-unhairing wastewater and the regeneration of sulphides is a very complex process in which a wide variety of chemical reactions take place. In addition, such a process takes a long time and requires a relatively large amount of $\mathrm{CO}_{2}$.

Unhairing-opening up of derma structure was best performed using a $10 \%$ sodium hydroxide solution with regenerated sulphides to replace some of the sodium hydroxide.

\section{REFERENCES}

Andrioli, E. and Gutterres, M. (2014), “Associated use of enzymes and hydrogen peroxide for cowhide hair removal", Journal of the American Leather Chemists Association, 109, 41-48.

Faki, F.E., Musa, A.E., Gasmelseed, G.A and Elamin, I.H. (2018), "Recycling of Spent Unhairing-Liming Liquor", International Journal of Engineering and Applied Sciences, 5, 12-15.

Golovteeva, E., Kutsidi, A. and Sankin, L. (1982), Laboratornyj praktikum po khimiyi i tekhnologiyi kozhy $i$ mekha, Legkaiya i Pischevaiya Prom. Moscow (Russian).

Khandelwal, H.B., More, S.V., Kalal, K.M. and Laxman, R.S. (2014), "Eco-friendly enzymatic dehairing of skins and hides by C-brefeldianus protease", Clean Technologies and Environmental Policy, 17, 393-405, https://doi.org/10.1007/s10098-014-0791-y.

Munz, K.H. and Sonnleitner, R. (2005), "Application of soluble silicates in leather production", Journal of the American Leather Chemists Association, 100, 66-75.

Nazer, D.W., Al-Sa'ed, R.M. and Siebel, M.A. (2006), "Reducing the environmental impact of the unhairingliming process in the leather tanning industry", Journal of Cleaner Production, 14, 65-74, https://doi.org/10.1016/j.jclepro.2005.04.002.

Sirvaityte, J., Beleska, K. and Valeika, V. (2016), "Lime Free Unhairing: Sodium Aluminate as an Alternative Towards a Cleaner Process", Journal of the American Leather Chemists Association, 111, 406-412.

Thanikaivelan, P., Rao, J.R., Nair, B.U. and Ramasami, T. (2001), "Approach towards zero discharge tanning: Exploration of $\mathrm{NaOH}$ based opening up method", Journal of the American Leather Chemists Association, 96, 222-233.

Valeika, V., Balciuniene, J., Beleska, K. and Valeikiene, V. (2000), "Lime-free unhairing: Part 3 Influence of unhairing method on chemical, physical and mechanical properties of pelt and leather", Journal of the Society of Leather Technologists and Chemists, 84, 165-169.

Venkatakrishnan, B., Sandhya, K.V., Abinandan, S., Vedaraman, N. and Velappan, K.C. (2019), "Fixation of carbon dioxide and optimization of liming process waste produced in tanneries using response surface methodology", Journal of Cleaner Production, 209 855-861, https://doi.org/10.1016/j.jclepro.2018.10.275.

Zaides, A., Mikhailov, A. and Pushenko, O. (1964), "Modiphitsirovanyi method opredeleniya oksiprolina", Biokhimiya, 1, 5.

****Calcium bicarbonate", Wikipedia internet page: https://en.wikipedia.org/wiki/Calcium_bicarbonate. 
DEVELOPMENT OF ELASTO-PLASTIC ECO-NANO-MATERIALS FOR FOOTWEAR INDUSTRY

\author{
MARIA DANIELA STELESCU ${ }^{1}$, DANIELA IOANA CONSTANTIN ${ }^{2}$, MARIA SONMEZ ${ }^{1}$, \\ LAURENTIA ALEXANDRESCU ${ }^{1}$, MIHAELA NITUICA ${ }^{1}$, MIHAI GEORGESCU ${ }^{1}$ \\ ${ }^{1}$ INCDTP - Division Leather and Footwear Research Institute, 93, Ion Minulescu St., 031215, \\ Bucharest, Romania,0213235060, maria.stelescu@icpi.ro \\ ${ }^{2}$ Punto Suola Rom SRL, str. Str. Baicului, nr. 80, 021784, Bucharest, Romania, tel. 0212524792, \\ puntosuolarom@yahoo.com
}

\begin{abstract}
The paper refers to the obtaining of new types of eco-nano elasto-plastic materials with highperformance characteristics based on ethylene-propylene-terpolymer rubber (EPDM), high-density polyethylene (HDPE), plasticized starch and organically modified montmorillonite (OMMT). The new materials were obtained by the technique of dynamic vulcanization and melt intercalation in a Plasti-Corder Brabender internal mixer, at $80 \mathrm{rpm}$ and a temperature of $170^{\circ} \mathrm{C}$. The influence of using the OMMT type nanofiller and the plasticized starch filler on the characteristics was observed. The new materials have a melt flow index of over $12 \mathrm{~g} / 10 \mathrm{~min}$ at $180^{\circ} \mathrm{C}$ for a force of 10 $\mathrm{kg}$, which allows injection processing - an ecological method of processing polymeric materials. The samples show very good physical-mechanical characteristics both in the normal state and after accelerated aging at 168 hours at $170^{\circ} \mathrm{C}$ (tensile strength over $16 \mathrm{~N} / \mathrm{mm}^{2}$, tear strength over 102 $\mathrm{N} / \mathrm{mm}$, hardness $55-59^{\circ} \mathrm{ShD}$, elasticity over $30 \%$, etc.). The materials show high values of abrasion resistance (below $30 \mathrm{~mm}^{3}$ ), and very good results for mass and volume variation after 22 hours at $23^{\circ} \mathrm{C}$ in: water, acids and concentrated bases. These characteristics are due both to the composition of the new materials and to the obtaining technology. For evaluating the structural modification, analysis of the FT-IR spectral of the samples was carried out. The new materials can be used in different fields such as: in the footwear industry (soles, heels and plates), safety equipment (boots, etc), obtaining gaskets, hoses, technical rubber products for cars etc.
\end{abstract}

Keywords: eco-nano-materials, EPDM, OMMT, plastified starch.

\title{
INTRODUCTION
}

In recent years, much research has been done on the development of starch-based products, as an ecological alternative to the use of polymeric materials obtained by petroleum synthesis (Carvalho et al., 2003). Based on these, we set out to create new eco-materials for the footwear industry, made by incorporating starch into the synthetic polymer matrix. Because starch cannot be processed in the melt as it is, because it degrades before melting, it was plasticized with glycerin to lower the melting temperature below the degradation temperature (Nafchi et al., 2013). In order to improve some chemical characteristics (such as: resistance to water, to chemical agents, etc.) and physical-mechanical (tensile and tear strength, etc.), the aim was to obtain nanocomposites by introducing in the mixture a layered clay - organic modified montmorillonite (OMMT). Obtaining these nanocomposites was carried out by the "melt intercalation" method, which is an ecological method of obtaining a nanocomposite, and leads to significant improvements of the properties (Stelescu, 2011). At the same time, the dynamic vulcanization technique was used - the crosslinking of the elastomer in the melt of a thermoplastic polymer in dynamic conditions when dynamically vulcanized thermoplastic elastomers (TPV) are obtained (Mirci, 2005). Dynamic crosslinking was performed with small amounts of vulcanizing agents such as alkylphenolic resins. In this way, the new materials can be processed by the method of injection or extrusion, eliminating the classic vulcanization operation with sulfur and organic accelerators, which is highly consuming electricity and which 
releases toxic gases (nitrosamines - carcinogens). At the same time, the technological flow is simplified and the total processing time corresponding to obtaining the finished products is significantly reduced.

\section{MATERIALS AND METHODS}

\section{Materials}

The materials used to obtain eco-nano materials were: (a) ethylene-propyleneterpolymer rubber (EPDM) Nordel rubber 3745P (ethylene content 70\%, density 0.88 $\mathrm{g} / \mathrm{cm}^{3}$ ), from Dow Chemical Company; (b) thermoplastic polymer - high density polyethylene (HDPE) Hostalen GC 7260 (density $0.962 \mathrm{~g} / \mathrm{cm}^{3}$ ) from Lyondell Basell Industries Holdings; (c) compatibility agent chlor-butyl rubber Butyl (IIR-Cl) CB 1240 DISP IIR-Cl (1.25\% Chlorine, density $0.92 \mathrm{~g} / \mathrm{cm}^{3}$ ) produced by Lanxess; (d) soluble starch obtained from potatoes produced by Lach-Ner; (e) starch plasticizer - glycerin (free acidity $0.02 \%$, density $1.26 \mathrm{~g} / \mathrm{cm}^{3}$ ), produced by S.C. Chimreactiv S.R.L.; (f) organic modified montmorillonite (OMMT) - Nanoclay I 31.PS (chemically modified montmorillonite layered clay with 0.5 - 5\% propyl-amino-trietoxysilane and 15-35\% octadecylamine, particle size below 20 microns), from Sigma Aldrich; (g) antioxidant pentaerythritol tetrakis(3-(3,5-di-tert-butyl-4-hydroxyphenyl)propionate Irganox 1010 (98\% active ingredient), (h) vulcanize agent - heat-reactive resin SP-1055 - based on octylphenol-formaldehyde containing methylol groups (bromine content 4\%, methylol content $11 \%$, density $1.05 \mathrm{~g} / \mathrm{cm}^{3}$ ), (i) zinc oxide I grade quality ( $99 \%$ purity).

\section{Obtaining Mixtures and Plates for Characteristic Analysis}

The mixtures were obtained according to the formulations presented in Table 1, using an internal Brabender mixer, at $175^{\circ} \mathrm{C}$ and $\max 80 \mathrm{rpm}$. The ingredients order was: HDPE - melts about 2', and then EPDM and IIR-Cl elastomers are added in approx. 2', add and incorporate the resin in 1', and then add the other ingredients. Homogenize for 2' and remove from the mixer. Prior to use, the starch was dried at $100^{\circ} \mathrm{C}$ for 8 hours and then plasticized with glycerin in a 2:1 mass ratio with continuous stirring at $70^{\circ} \mathrm{C}$ until a homogeneous mixture was obtained.

Table 1. Blend formulations

\begin{tabular}{lccccc}
\hline Ingredients, g & \multicolumn{5}{c}{ Sample Codes } \\
& 1 & 2 & 3 & 4 & 5 \\
\hline EPDM Nordel 3745D & 75 & 75 & 70 & 70 & 70 \\
HDPE Hostalen GC7260 & 175 & 175 & 175 & 175 & 175 \\
IIR-Cl & 0 & 0 & 5 & 5 & 5 \\
Zinc oxide & 3.75 & 3.75 & 3.75 & 3.75 & 3.75 \\
Irganox 1010 & 2.5 & 2.5 & 2.5 & 2.5 & 2.5 \\
Resin SP 1055 & - & 7.5 & 7.5 & 7.5 & 7.5 \\
OMMT I 31 & - & - & - & 7.5 & 7.5 \\
Starch plasticized with glycerine & - & - & - & - & 30 \\
\hline
\end{tabular}

The semi-finished products obtained in the form of rubber mixtures, are modeled in plates for determining the characteristics using molds and vulcanizing presses. The processing parameters for the press were: preheating time 2' at a temperature of 
$170^{\circ} \mathrm{C} ;$ molding time $5^{\prime}$ at $170^{\circ} \mathrm{C}$, and $300 \mathrm{kN}$ pressing force; cooling time $8^{\prime}$ until reach $45^{\circ} \mathrm{C}$ and $300 \mathrm{kN}$ pressing force.

\section{Specimen Characterization}

After conditioning for 24 hours at room temperature, the plates were subjected to physical-mechanical determinations. Tensile strength and tearing strength tests were carried out with a Schopper1445 strength tester with testing speed $500 \mathrm{~mm} / \mathrm{min}$, using dumb-bell shaped specimens according to ISO 37/2020 and angular test pieces (Type II) according to EN 12771/2003, respectively. Hardness was measured by using a hardness tester according to ISO 48-4/2018 using 6-mm thick samples. Elasticity was evaluated with a Schob test machine using 6-mm thick samples, according to ISO 4662/2017.

Accelerated ageing evaluation was carried out according to ISO 188/2001 using the hot air oven method. Test duration was of $168 \mathrm{~h}$ and temperature of $70 \pm 1^{\circ} \mathrm{C}$.

Melt flow index (MFI) of the thermoplastic materials was measured by means of an extrusion plastometer (capillary rheometer - Melt Flow Index - Haake), at $180^{\circ} \mathrm{C}$ and a $10 \mathrm{~kg}$ force was employed according to ISO 1133/2003.

The densities of elastomer samples were measured according to ISO 2781/2011.

Determining abrasion resistance was carried out according to ISO 4649/2017 the cylinder method, using a force of $10 \mathrm{~N}$. Abrasion resistance was expressed by relative volume loss in relation to calibrated abrasive paper. The samples used were obtained from rolled mixtures and pressed, by cutting with a rotating die and have cylindrical shape, with a diameter of $16 \mathrm{~mm}$ and height of min. $6 \mathrm{~mm}$.

In order to determine the action of various mediums, volume and mass variation was monitored using the volumetric and gravimetric methods according to ISO 1817/2015. Immersion time was $22 \pm 0.25 \mathrm{~h}$. The samples used had a volume of $1-3 \mathrm{~cm}^{3}$ and a uniform thickness of $2 \pm 0.2 \mathrm{~mm}$.

Fourier Transform Infrared Spectroscopy (FTIR) spectra of all the samples were obtained using Nicolet iS50 FT-IR spectrophotometer in the wave number ranging from $400 \mathrm{~cm}^{-1}$ to $4000 \mathrm{~cm}^{-1}$.

\section{RESULTS AND DISCUSSIONS}

\section{Melt Flow Index (MFI)}

All samples show very good values of the MFI (Table 2), showing a good process ability by the injection method. Compared to the control sample (sample 1), the MFI value decreases by introducing the crosslinking agent (resin) - see sample 2 , as a result of the crosslinking of the elastomer in the plastomer melt. The decrease is even greater with the addition of the chlor-butyl rubber (II-Cl) compatibilizer (see sample 3), showing that it has significantly improved the yield of elastomer crosslinking reactions in the presence of phenolic resin, due to improved phase compatibility. The melt flow index increases by the addition of nanofiller (see sample 4), indicating the change in the morphology of the mixture in the molten state due to the formation of the nanocomposite. The MFI value improves at sample 5 compared to sample 4, because it also contains plasticizer from plasticized starch. 


\section{The Density of the Mixtures}

The density of the mixtures (Table 2) increases from the control sample (sample 1) to the samples with phenolic resin (samples 2-5) and the samples with nanofiller (samples 4-5), indicating both the modification of the morphology with the formation of the micro / nanocomposite, and the introduction of dense ingredients (such as glycerin, resin or OMMT).

\section{Abrasion Resistance}

Abrasion resistance has high performance values, below $30 \mathrm{~mm}^{3}$ for all samples due to the very good wear values of HDPE - the polymer matrix of these (nano) composites (Table 2)

Table 2. Characteristics of blends

\begin{tabular}{|c|c|c|c|c|c|}
\hline \multirow[t]{2}{*}{ Characteristics } & \multicolumn{5}{|c|}{ Sample Codes } \\
\hline & 1 & 2 & 3 & 4 & 5 \\
\hline $\mathrm{MFI} 180^{\circ} \mathrm{C}$ and $10 \mathrm{~kg}$ force, $\mathrm{g} / 10^{\prime}$ & 21.6 & 16.6 & 5.59 & 12.5 & 19.5 \\
\hline Density, $\mathrm{g} / \mathrm{cm}^{3}$ & 0.95 & 0.96 & 0.96 & 0.97 & 1.00 \\
\hline Abrasion resistance, $\mathrm{mm}^{3}$ & 27.03 & 20.71 & 20.81 & 26.54 & 28.88 \\
\hline \multicolumn{6}{|l|}{ Medium action analysis, $22 \mathrm{~h} \times 23^{\circ} \mathrm{C}$} \\
\hline \multicolumn{6}{|l|}{ Immersion in distilled water } \\
\hline - Mass variation, $\%$ & +0.22 & +1.61 & +0.03 & +0.12 & +0.43 \\
\hline - Volume variation, $\%$ & +0.50 & +1.98 & +0.03 & +0.53 & +0.60 \\
\hline \multicolumn{6}{|l|}{ Immersion in sol. $\mathrm{H}_{2} \mathrm{SO}_{4}, 70 \%$} \\
\hline - $\quad$ Mass variation, $\%$ & +0.47 & -0.07 & +0.01 & -0.09 & +0.18 \\
\hline - Volume variation, $\%$ & +0.62 & -0.16 & +0.29 & +0.18 & -8.32 \\
\hline \multicolumn{6}{|l|}{ Immersion in sol. $\mathrm{NaOH} 50 \%$} \\
\hline - Mass variation, $\%$ & +1.06 & +0.3 & +0.28 & +0.01 & -0.03 \\
\hline - Volume variation, $\%$ & -7.43 & -0.16 & +0.03 & +0.13 & +1.12 \\
\hline \multicolumn{6}{|l|}{ Immersion in toluene } \\
\hline - $\quad$ Mass variation, $\%$ & +29.00 & +25.04 & +26.04 & +29.47 & +30.90 \\
\hline - Volume variation, $\%$ & +33.56 & +26.50 & +27.61 & +33.82 & +38.83 \\
\hline \multicolumn{6}{|c|}{ Physical-mechanical properties - normal state } \\
\hline o Hardness, ${ }^{\circ} \mathrm{ShA}$ & 99 & 99 & 99 & 98 & 99 \\
\hline Hardness, ${ }^{\circ} \mathrm{ShD}$ & 57 & 57 & 58 & 58 & 59 \\
\hline Elasticity, \% & 52 & 53 & 52 & 48 & 30 \\
\hline Tensile strength, $\mathrm{N} / \mathrm{mm}^{2}$ & 18.20 & 17.67 & 16.04 & 16.76 & 16.85 \\
\hline Elongation at break, \% & 80 & 80 & 120 & 100 & 80 \\
\hline Residual elongation, \% & 4 & 8 & 28 & 20 & 40 \\
\hline o Tear strength, N/mm & 107 & 119 & 130 & 121 & 102 \\
\hline \multicolumn{6}{|c|}{ Physical-mechanical properties after accelerated ageing $168 \mathrm{~h}$ at $170^{\circ} \mathrm{C}$} \\
\hline ○ Hardness, ${ }^{\circ} \mathrm{ShA}$ & 99 & 99 & 99 & 99 & 99 \\
\hline Hardness, ${ }^{\circ} \mathrm{ShD}$ & 58 & 57 & 56 & 56 & 55 \\
\hline Elasticity, \% & 42 & 50 & 53 & 52 & 36 \\
\hline Tensile strength, $\mathrm{N} / \mathrm{mm}^{2}$ & 21.62 & 18.32 & 16.49 & 16.39 & 16.82 \\
\hline Elongation at break, \% & 80 & 100 & 100 & 100 & 80 \\
\hline Residual elongation, $\%$ & 4 & 36 & 36 & 36 & 20 \\
\hline Tear strength, $\mathrm{N} / \mathrm{mm}$ & 106 & 115 & 141 & 127 & 105 \\
\hline
\end{tabular}




\section{Medium Action Analysis}

Medium action analysis, $22 \mathrm{~h} \times 23^{\circ} \mathrm{C}$ - the samples show small variations of mass and volume after immersion in distilled water, sulfuric acid $70 \%$, sodium hydroxide $50 \%$ because EPDM rubber materials with and without HDPE have a very good stability to the action of chemical agents. Because EPDM rubber is not resistant to toluene, the samples are inflated after immersion for 22 hours in toluene and increase its mass and volume. According to Table 2, there is an improvement in this feature by crosslinking the rubber and a slight increase in the addition of charge, behavior observed by other researchers (Stelescu, 2011).

\section{Physical-Mechanical Properties in Normal State and After Accelerated Aging $\left(168 \mathrm{~h}\right.$ at $\left.70^{\circ} \mathrm{C}\right)$}

The analysis of the physical-mechanical properties in normal state and after accelerated aging (Table 2) shows the following: - hardness has high values (98$99^{\circ} \mathrm{ShA}$, respectively $55-59^{\circ} \mathrm{ShD}$ ) and varies with $\max \pm 1^{\circ} \mathrm{ShA}$, respectively with \pm $4^{\circ} \mathrm{ShD}$ after accelerated aging; $\bullet$ the elasticity shows high values for samples 1-4 (42$53 \%$ ) and shows a decrease to the value of $30-36 \%$ by introducing the plasticized starch in the mixture; - both hardness and elasticity are surface properties and are strongly influenced by the characteristics of the thermoplastic matrix, namely HDPE, respectively HDPE and plasticized starch; • the values of tensile strength are high, over $16 \mathrm{~N} / \mathrm{mm}^{2}$ for all samples, and show variations between $+18.8 \%$ and $-2.4 \%$ after accelerated aging; - elongation at break has small values, of $80-120 \%$, and the remanent elongation has values of $4-40 \%$; $\bullet$ the phenomenon of forming a neck specific to plastics was observed; $\bullet$ tear resistance is very good $(102-141 \mathrm{~N} / \mathrm{mm})$, with small variations after accelerated aging (between $+8.5 \%$ and $-3.4 \%$ ); $\bullet$ there is an increase in tear strength and elongation at break due to dynamic crosslinking with resin, respectively as a result of the introduction of OMMT; $\bullet$ the addition of plasticized starch did not lead to a decrease in characteristics.

\section{Fourier Transform Infrared Spectroscopy (FTIR) Analysis}

Figures 1-6 present the infrared spectra obtained in the range of $450-3000 \mathrm{~cm}^{-1}$ of the control blend (sample 1), plasticized starch and sample 5. Control sample spectrum (sample 1) includes $\mathrm{C}-\mathrm{H}$ stretching vibration $\left(2914,53 \mathrm{~cm}^{-1}\right.$ and $\left.2847,59 \mathrm{~cm}^{-1}\right), \mathrm{CH}_{2}$ bending and rocking vibrations $\left(1472 \mathrm{~cm}^{-1}, 1462 \mathrm{~cm}^{-1}\right.$ and $\left.719-730 \mathrm{~cm}^{-1}\right)$, specific bands of EPDM and HDPE (Craciun et al., 2020).
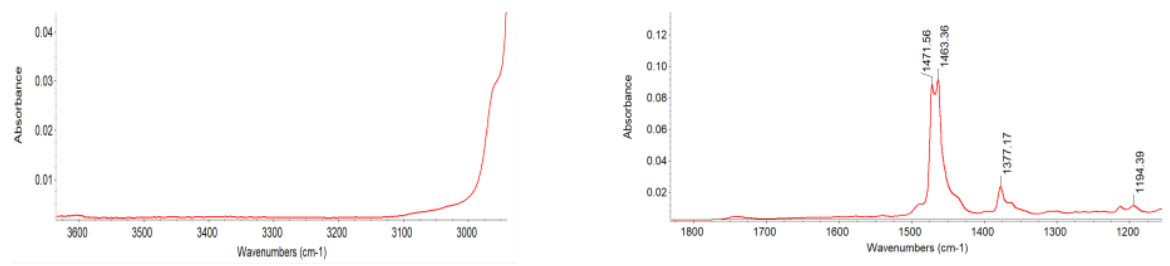

Figure 1. FTIR spectra of Sample 1 (Control) for $3000-3650 \mathrm{~cm}^{-1}$ and $1150-750 \mathrm{~cm}^{-1}$ 


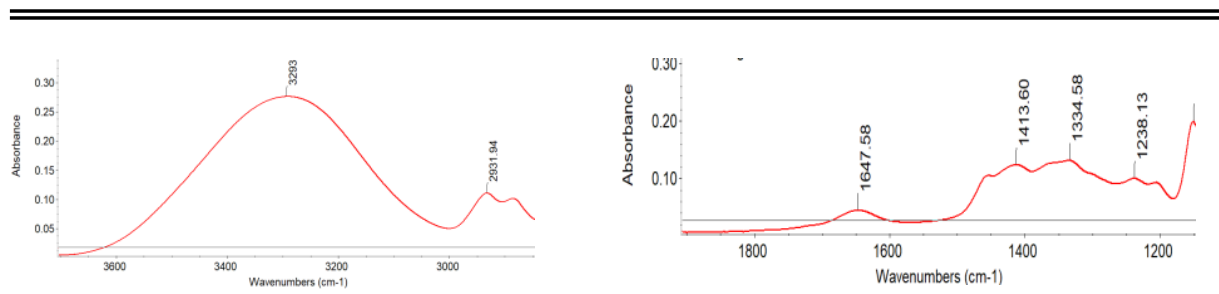

Figure 2. FTIR spectra of plasticized starch for $3000-3650 \mathrm{~cm}^{-1}$ and $1150-1750 \mathrm{~cm}^{-1}$
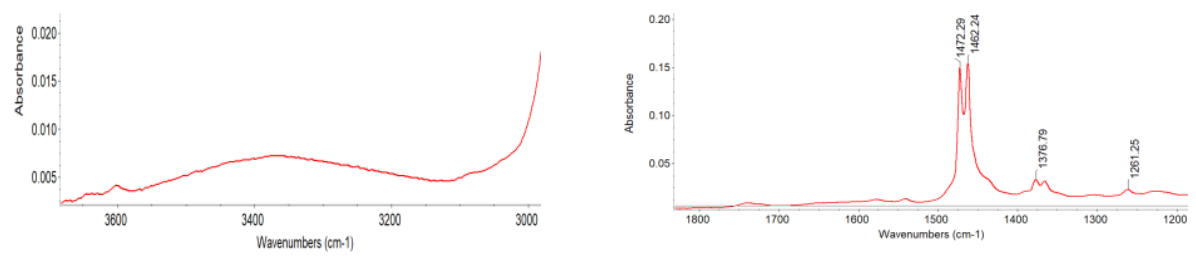

Figure 3. FTIR spectra of Sample 5 for $3000-3650 \mathrm{~cm}^{-1}$ (left) and $1150-1750 \mathrm{~cm}^{-1}$ (right)

The FTIR spectra of starch typically shows bands at $2870-2950 \mathrm{~cm}^{-1}(\mathrm{C}-\mathrm{H}$ stretching), $1100-1150 \mathrm{~cm}^{-1}$ (C-O, C-C and C-O-H stretching) and $1100-900 \mathrm{~cm}^{-1}$ (C$\mathrm{O}-\mathrm{H}$ bending). Overlapping are, the plasticizer-glycerin specific bands, such as the absorption bands from 3000-3700 $\mathrm{cm}^{-1}$ specific for hydrogen bonds, the absorption bands from the region $1200-1000 \mathrm{~cm}^{-1}$ due to $\mathrm{C}-\mathrm{O}, \mathrm{C}-\mathrm{C}, \mathrm{C}-\mathrm{OH}$ stretching and $\mathrm{C}-\mathrm{OH}$ bending (Warren et al., 2016). In the spectrum of sample 5, in addition to the control sample, new bands appear due to the plasticized starch, the addition of OMMT and dynamic crosslinking using phenolic resin. Thus, it can observe the absorption bands of the stretching vibration of - $\mathrm{OH}$ from the phenolic resin at $3600 \mathrm{~cm}^{-1}$ and the hydrogen bonds formed by dynamic crosslinking at 3340-3380 $\mathrm{cm}^{-1}$ (Van Duin and Souphanthong, 1995). The band observed at 3600-3640 $\mathrm{cm}^{-1}$ can also be attributed to the structural water of OMMT crystal lattices, and the bands from $1027.83 \mathrm{~cm}^{-1}, 803.41$ $\mathrm{cm}^{-1}$ and $464.64 \mathrm{~cm}^{-1}$ to the vibration absorptions of $\mathrm{Si}-\mathrm{O}-\mathrm{Si}$ and $\mathrm{Si}-\mathrm{O}-\mathrm{Al}$ groups in OMMT crystal lattice (Lü et al., 2006).

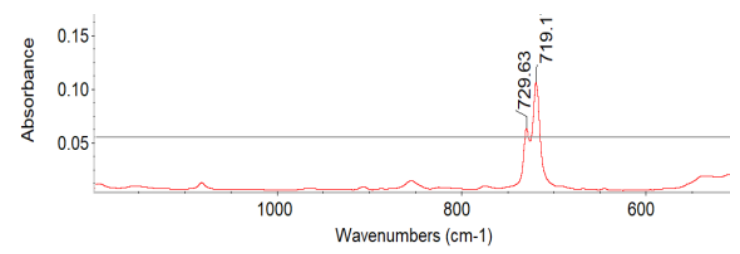

Figure 4. FTIR spectra of Sample 1 for $400-1150 \mathrm{~cm}^{-1}$

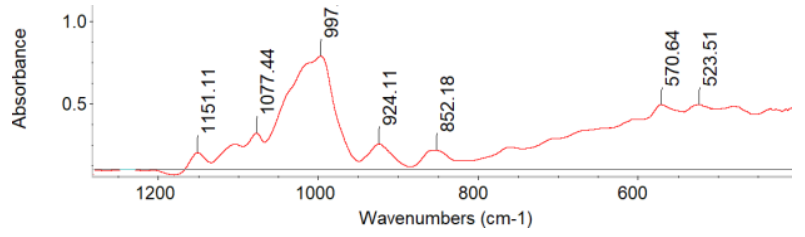

Figure 5. FTIR spectra of plasticized starch for $400-1150 \mathrm{~cm}^{-1}$

https://doi.org/10.24264/icams-2020.IV.22 


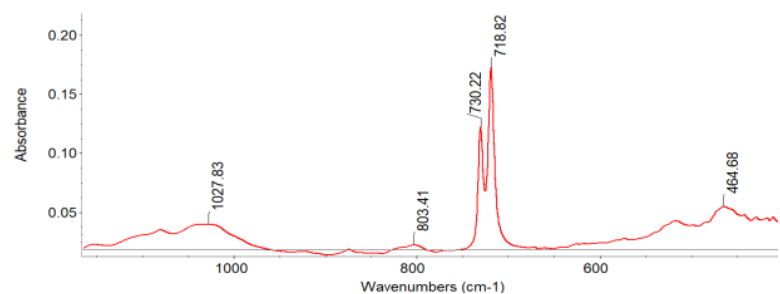

Figure 6. FTIR spectra of Sample 5 for $400-1150 \mathrm{~cm}^{-1}$

\section{CONCLUSIONS}

The new types of elastic-plastic materials for the footwear industry based on EPDM rubber and HDPE, reinforced with plasticized starch and OMMT, were obtained using the latest technologies in the field, namely: the dynamic vulcanization technique and the melt interleaving method. Due to the composition and the processing technology, materials with very good properties were obtained (very good resistance to concentrated acids and bases, very good values of abrasion resistance, tensile and tear strength, etc.). The materials have MFI values over $3 \mathrm{~g} / 10$ ' and can be processed by injection method.

\section{Acknowledgements}

This research was funded by the Romanian Ministry of Education and Research through Contract No. 184CI/2018 and Contract No. 6PFE/2018-PERFORM-TEX-PEL.

\section{REFERENCES}

Carvalho, A.J.F., Job, A.E., Alves, N., Curvelo, A.A.S. and Gandini, A. (2003), "Thermoplastic starch/natural rubber blends", Carbohydrate Polymers, 53(1), 95-99, https://doi.org/10.1016/S0144-8617(03)00005-5.

Craciun, G., Manaila, E., Ighigeanu, D. and Stelescu, M.D. (2020), “A Method to Improve the Characteristics of EPDM Rubber Based Eco-Composites with Electron Beam”, Polymers, 12(1), 215, https://doi.org/10.3390/polym12010215.

Lü, W., Zhao, G. and Xue, Z. (2006), "Preparation and characterization of wood/montmorillonite nanocomposites", For. Stud. China, 8(1), 35-40, https://doi.org/10.1007/s11632-006-0007-6.

Mirci, L.E. (2005), Thermoplastic Elastomers, Publisher Ed. Art Press and Ed. Augusta, Timişoara.

Nafchi, A.M., Moradpour, M., Saeidi, M. and Alias, A.K. (2013), "Thermoplastic starches: Properties, challenges, and prospects", Starch/Starke, 65, 61-72, https://doi.org/10.1002/star.201200201.

Stelescu, M.D. (2011), High-performance thermoplastic elastomers based on ethylene-propylene terpolymer rubber (EPDM) which can be used in the footwear industry, Publisher Ed. Performantica, Iasi.

Van Duin, M. and Souphanthong, A. (1995), "The Chemistry of Phenol-Formaldehyde Resin Vulcanization of EPDM: Part I. Evidence for Methylene Crosslinks”, Rubber Chemistry and Technology, 68(5), 717-727, https://doi.org/10.5254/1.3538768.

Warren, F.J., Gidley, M.J. and Flanagan, B.M. (2016), "Infrared spectroscopy as a tool to characterise starch ordered structure - a joint FTIR-ATR, NMR, XRD and DSC study", Carbohydrate Polymers, 139(30), 35-42, https://doi.org/10.1016/j.carbpol.2015.11.066. 
Development of Elasto-Plastic Eco-Nano-Materials for Footwear Industry

https://doi.org/10.24264/icams-2020.IV.22 


\title{
ELASTOMERIC NANOMATERIALS BASED ON NATURAL RUBBER FOR THE FOOD INDUSTRY
}

\author{
MARIA-DANIELA STELESCU ${ }^{1}$, MIHAI GEORGESCU ${ }^{1}$, MARIA SONMEZ ${ }^{1}$, MIHAELA \\ NITUICA $^{1}$, ADRIANA STEFAN ${ }^{2}$ \\ ${ }^{1}$ INCDTP - Division: Leather and Footwear Research Institute, 93, Ion Minulescu, 031215, \\ Bucharest, Romania, maria.stelescu@icpi.ro \\ ${ }^{2}$ National Institute of Aerospace Research "Elie Carafoli”, 220 Iuliu Maniu Blv., 061126, \\ Bucharest, Romania, stefan.adriana@incas.ro
}

\begin{abstract}
This paper presents the obtaining and characterization of new elastomeric nanocomposites based on natural rubber reinforced with plasticized starch, precipitated silica and layered clay, for obtaining consumer goods for the food industry. Obtaining nanocomposites was carried out by the technique of mixing and melt interleaving. The mixtures were vulcanized in the press, at high temperatures, using peroxides as vulcanizing agents, and triallyl cyanurate as vulcanizing coagent. In order to obtain products with improved characteristics, the influence of the amount of modified organic montmorillonite layered clay (OMMT) Nanomer I31PS and the adhesion promoter between mineral filler and polymer - bis-[3-(triethoxysilyl)-propyl]-tetrasulfane (TEPS) on the characteristics of the mixtures, was analysed. The rheological characteristics of the samples show an increase of the minimum torque at the increase in the amount of OMMT type nanofiller and a decrease in the optimal vulcanization time by adding the adhesion promoter between the rubber and the filler. An improvement of the mechanical characteristics of the samples was observed at the introduction of both OMMT and TEPS. These changes may be due to both the nanofiller reinforcement effect and the changes in the morphology of the mixture. The samples showed a good behaviour after immersion in different environments specific to the food industry (water, ethyl alcohol, $10 \%$ glucose solution, $0.9 \%$ sodium chloride solution and sunflower oil). SEM analyses indicate that the starch particles, together with the other ingredients of the mixture, are quasi uniform distributed in the elastomer matrix. Several superficial microcracks are observed, on the surface of the analysed material, without structural discontinuities or other defects.
\end{abstract}

Keywords: elastomeric nanocomposites, plasticized starch, OMMT

\section{INTRODUCTION}

Currently, in the production of elastomeric materials, the goal is to replace inorganic fillers (which are generally toxic) with different types of natural fibers or organic fillers. (Carvalho et al., 2003; Shey et al., 2006). These materials can be used for various applications, such as: NR / coconut fiber composites - used for Mercedes A-class seats, Cordenka cellulose fibers are used to reinforce tires, starch is added as a rubber mixture filler used in obtaining the tires for Ford Fiesta etc. (Shinoj et al., 2011; Mohammed et al., 2015; Wu et al., 2006). In general, the use of organic fillers as a filler in the polymer matrix offers several advantages over conventional inorganic ones, especially in terms of biodegradability and non-toxicity, but has inadequate adhesion to the elastomeric matrix, has low water resistance and dimensional stability (Wang et al., 2009; Wu et al., 2006). To remedy these problems, in this paper was used a small amount of organic modified montmorillonite layered clay (OMMT) nanofiller and an adhesion promoter bis-[3-(triethoxysilyl)-propyl]-tetrasulfane (TEPS) type, between mineral filler and polymer, to improve physical-mechanical characteristics and behaviour to various mediums for an elastomeric composite based on natural rubber reinforced with plasticized starch and precipitated silica. 


\section{MATERIALS AND METHODS}

\section{Materials}

The following materials were used for rubber mixtures: natural rubber (NR) Crep from Sangtvon Rubber Ltd, starch - produced by Lach-Ner - soluble potato starch, glycerine from SC Chimreactiv SRL, organic modified montmorillonite layered clay Nanomer I31PS produced by Nanocor, amorphous precipitated silica - BM30 Egesil, antioxidant 2,2,4 trimethyl 1,1, dihydroquinone TMQ , polyethyleneglycol PEG 4000, adhesion promoter between mineral filler and polymer, bis-[3-(triethoxysilyl)-propyl]tetrasulfane Luvomaxx TESPT DL50/L (50\% active substance), benzoyl peroxide Luperox A75, di(2-tert-butylperoxyisopropyl)benzene Perkadox 14-40B (40\% active substance), triallyl cyanurate Vulcofac TAC - 70 (70\% active substance).

\section{Methods}

\section{Specimen Obtaining}

The starch is dried at $80^{\circ} \mathrm{C}$ for 24 hours and then plasticized by mixing 2 parts starch with one part glycerine for $7-10$ minutes at $70^{\circ} \mathrm{C}$ and $50-100$ rotations per minute until a homogeneous mixture is obtained. For an efficient compounding, the mixtures based on natural rubber, plasticized starch, precipitated silica, and OMMT were obtained by the mixing technique on an internal Brabender type mixer. The processing parameters were: rotation speed $12-80 \mathrm{rpm}$, temperature $45-110^{\circ} \mathrm{C}$, total time $12^{\prime}$. The compositions of the rubber mixtures expressed in parts per 100 parts rubber (phr) are shown in Table 1. Vulcanizing agents were incorporated into the mixture on a laboratory roller with a heatingcooling system, at a temperature of $\max 90^{\circ} \mathrm{C}$ for $4^{\prime}$ and a 1: 1 friction. After homogenization for 3', the mixtures were removed from the roller in the form of sheets with a thickness of about $2 \mathrm{~mm}$. From these sheets, the test specimens were obtained for characterizing the material, by the compression method using a hydraulic press. Processing parameters: $165^{\circ} \mathrm{C}, 300 \mathrm{kN}$ press force, $8^{\prime}$ cooling time until it reaches a temperature of $35^{\circ} \mathrm{C}$ and the optimum curing time $\left(\mathrm{t}_{90}\right)$ is determined using the Monsanto rheometer.

Table 1. Formulation of rubber mixtures

\begin{tabular}{lccccc}
\hline Ingredients & \multicolumn{5}{c}{ Mixture symbol } \\
& S0 & S3 & S3t & S6 & S6t \\
\hline Internal mixer & & & & & \\
Natural rubber (g) & 100 & 100 & 100 & 100 & 100 \\
Starch plasticized with glycerine $(\mathrm{g})$ & 30 & 30 & 30 & 30 & 30 \\
Egesil BM30 (g) & 20 & 20 & 20 & 20 & 20 \\
PEG 4000 (g) & 3 & 3 & 3 & 3 & 3 \\
Antioxidant TMQ (g) & 1 & 1 & 1 & 1 & 1 \\
TESPT DL 50/L (g) & - & - & 3 & - & 3 \\
OMMT I31PS (g) & 0 & 3 & 3 & 6 & 6 \\
Laboratory two-roll mill machine & & & & & \\
Luperox A75 (g) & 3 & 3 & 3 & 3 & 3 \\
Perkadox 14-40B (g) & 6 & 6 & 6 & 6 & 6 \\
Vulcofac TAC (g) & 4 & 4 & 4 & 4 & 4 \\
\hline
\end{tabular}

\section{Specimen Characterization}

\section{Curing Characteristics}

Curing characteristics were determined by an oscillating disk rheometer (Monsanto), at $165^{\circ} \mathrm{C}$ for $30 \mathrm{~min}$, according to the ISO $3417 / 2008$. Delta torque or extent of 
crosslinking is the maximum torque (MH) minus the minimum torque (ML). Optimum cure time $\left(\mathrm{t}_{90}\right)$ is the time to reach $90 \%$ of the delta torque above minimum.

\section{Physical-Mechanical Characteristics}

Tensile strength and tearing strength tests were carried out with a Schopper strength tester with testing speed $500 \mathrm{~mm} / \mathrm{min}$, using dumb-bell shaped specimens according to ISO 37/2012 and angular test pieces (Type II) according to EN 12771/2003, respectively. Hardness was measured by using a hardness tester according to ISO 7619 1/2011 using 6-mm thick samples. Elasticity (rebound resilience) was evaluated with a Schob test machine using 6-mm thick samples, according to ISO 4662/2009.

\section{Swell Determination}

Swell Determination was performed according to ISO 1817/2015 and the action of the following liquids was tested: water, ethyl alcohol, $10 \%$ glucose solution, $0.9 \%$ sodium chloride solution and sunflower oil. The specimens of known weight, $\mathrm{m}_{0}$, were immersed in various solvents in test bottles and kept at room temperature for 22 hours. After immersion the samples were taken out from the solvents and the wet surfaces were quickly dried using a tissue paper and re-weighted, $\mathrm{m}_{\mathrm{i}}$. To calculate the percentage change in mass $\Delta \mathrm{m}_{100}$ the following formula was used:

$\Delta m_{100}=\frac{m_{i}-m_{0}}{m_{0}} \times 100$

where $m_{0}$ is the initial mass of the test piece and $m_{i}$ is the mass of the test piece after immersion.

To calculate the percentage change in volume $\Delta \mathrm{V}_{100}$ the following formula was used:

$\Delta V_{100}=\left(\frac{m_{i}-m_{i, w}+m_{s, w}}{m_{0}-m_{0, w}+m_{s, w}}-1\right) x 100$

where:

- $\quad \mathrm{m}_{0, \mathrm{w}}$ is the initial mass of the test piece (plus sinker if used) in water;

- $\quad \mathrm{m}_{\mathrm{i}, \mathrm{w}}$ is the mass of the test piece (plus sinker if used) after immersion in water;

- $\quad \mathrm{m}_{\mathrm{s}, \mathrm{w}}$ is the mass of the sinker, if used, in water.

The result was reported as the median value for the three test pieces.

\section{SEM and EDAX Analysis}

Scanning Electron Microscopy (SEM) and Energy Dispersive X-ray (EDAX) analysis were carried out by Quanta 250 electronic microscope. SEM analysis was performed on a square shaped sample of $10 \times 10 \mathrm{~mm}$, with a magnification between $100 \div 10.000$. EDAX analyse was used to determine the elemental composition of the materials.

\section{RESULTS AND DISCUSSIONS}

\section{Rheological Characteristics}

The rheological characteristics of the mixtures are presented in Table no. 2. It is observed that with the introduction of OMMT, there is an increase in the minimum torque, showing an increase in the rigidity of the unvulcanized material. The optimum vulcanization time increases for mixtures containing nanofillers and decreases for the mixtures containing promoter adhesion between mineral filler and polymer. This shows an increase in vulcanization rate due to improved filler dispersion in the elastomeric matrix. 
Elastomeric Nanomaterials Based on Natural Rubber for the Food Industry

Table 2. Rheological characteristics of blends

\begin{tabular}{lccccc}
\hline Rheological characteristics & \multicolumn{5}{c}{ Mixture symbol } \\
& S0 & S3 & S3t & S6 & S6t \\
\hline The minimum torque $(\mathrm{ML})(\mathrm{dNm})$ & 7.8 & 12.7 & 13.6 & 12.6 & 13.9 \\
The maximum torque $(\mathrm{MH})(\mathrm{dNm})$ & 74.3 & 74.7 & 74.3 & 72.2 & 74.6 \\
$\Delta \mathrm{M}=\mathrm{MH}-\mathrm{ML}(\mathrm{dNm})$ & 67 & 62 & 60.7 & 59.6 & 60.7 \\
Optimum cure time $\left(\mathrm{t}_{90}\right)($ minute) & 8.6 & 13.08 & 9 & 11.05 & 10.87 \\
\hline
\end{tabular}

\section{Physical-Mechanical Characteristics}

Physical-mechanical characteristics of obtained samples are presented in Table 3. Hardness increase with $1-6^{\circ} \mathrm{Sh}$ A by adding OMMT into mixtures, as a result of reinforcing the mixtures, as the amount of filler increases. Elasticity and elongation at break vary unevenly and have very good values for all samples, over $38 \%$ and over $500 \%$, respectively. Improvements are observed in the modulus, tensile, and tear strength for samples with different amounts of nanofiller, and which also contain an adhesion promoter between rubber and filler, namely S3t and S6t mixtures. These mixtures presented the best characteristics and were selected to be tested and observe the effect of different mediums used in the food industry on them.

Table 3. Physical-mechanical properties of blends

\begin{tabular}{lccccc}
\hline Physical-mechanical properties & \multicolumn{5}{c}{ Mixture symbol } \\
& S0 & S3 & S3t & S6 & S6t \\
\hline Hardness, ${ }^{0}$ Sh A & 56 & 62 & 57 & 57 & 59 \\
Elasticity, \% & 40 & 46 & 42 & 38 & 39 \\
Modulus $100 \%, \mathrm{~N} / \mathrm{mm}^{2}$ & 0.89 & 1.3 & 1.1 & 1.1 & 1.2 \\
Modulus 300\%, N/ mm ${ }^{2}$ & 1.9 & 2.6 & 2.3 & 2.2 & 2.45 \\
Tensile strength, N/mm ${ }^{2}$ & 8.9 & 8 & 10 & 10 & 11 \\
Elongation at break, \% & 580 & 500 & 560 & 600 & 590 \\
Tear strength, N/mm & 23 & 24 & 25.5 & 21 & 26 \\
\hline
\end{tabular}

\section{Analysis of the Variation of Mass and Volume in Different Liquids}

The analysis of the variation of mass and volume in different liquids specific to the food industry was performed at room temperature for $22 \mathrm{~h}$. From the results obtained (presented in Table no. 4), it is observed that for immersions in liquids containing a large amount of water (distilled water, $10 \%$ glucose solution and $0.9 \% \mathrm{NaCl}$ solution) or in ethyl alcohol, good values were obtained for both analyzed mixtures: less than 9.24\% mass variation and less than $15.9 \%$ volume variation. It is known that natural rubber is water-resistant (hydrophobic) but starch is hydrophilic - absorbs water (Stelescu et al., 2017). The good results obtained are due both to the use in mixtures of the OMMT nanofiller, and to the fact that the starch is embedded in the elastomeric matrix, according to the morphological analyses presented in section 3.4, and does not come into contact with the immersion medium except at the sample surface. Similar aspects have been presented by other existing studies in the literature (Manaila et al., 2018). The variation of mass and volume when immersed in sunflower oil has higher values and decreases with increasing amount of OMMT, and the obtained values (below $14 \%$, respectively below $24.1 \%$ ) allow the use of new materials to produce elastomeric consumer goods for the food industry. 
Table 4. Variation of mass and volume after immersion $22 \mathrm{~h}$ in different mediums

\begin{tabular}{lcccc}
\hline Immersion medium & \multicolumn{2}{c}{ Mixture S3t } & \multicolumn{2}{c}{ Mixture S6t } \\
& $\Delta$ mass, $\%$ & $\Delta$ volume, $\%$ & $\Delta$ mass, $\%$ & $\Delta$ volume, $\%$ \\
\hline Distilled water & 9.24 & 15.9 & 7.74 & 13.5 \\
Ethyl alcohol & 6.39 & 11.1 & 6.8 & 11.9 \\
10\% glucose solution & 7.68 & 12.8 & 7.15 & 12.4 \\
$0.9 \%$ NaCl solution & 9.04 & 15.07 & 7.48 & 12.9 \\
Sunflower oil & 14 & 24.1 & 12.5 & 21.7 \\
\hline
\end{tabular}

\section{SEM and EDAX Analyses}

The phase morphology of some rubber mixtures depends on the composition of the mixture, the processing technology, method, the interfacial stresses between the phases, etc. All microscopic images were registered in the fracture area of samples. The surface morphology of the S6t sample shown in Figure 1 shows that the starch particles have a typical granular shape about $5-60 \mu \mathrm{m}$ in size. These, together with the other ingredients of the mixture, are uniformly dispersed in the elastomer matrix. This quasi-uniform distribution led to good physical and mechanical properties. Several superficial microcracks are observed on the surface of the analysed material, without structural discontinuities or other defects. The EDAX spectra for the S6t sample (Figure 2) confirm the presence of $\mathrm{Al}(1.95 \% \mathrm{w})$ and $\mathrm{Si}(4.97 \% \mathrm{w})$ from OMMT, a high amount of oxygen $(21.73 \% \mathrm{w})$ which comes from plasticized starch, the basic element being $\mathrm{C}$ $(71.3 \% \mathrm{w})$, which exists in both elastomer and plasticized starch.
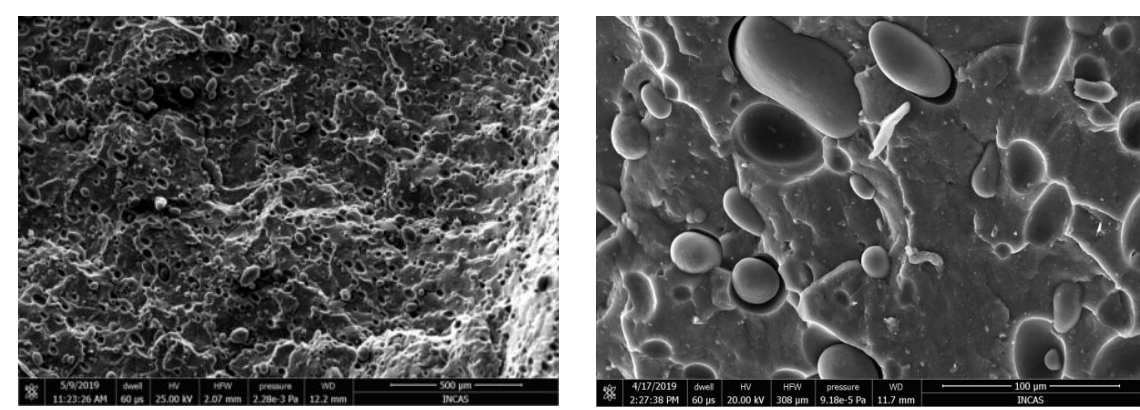

Figure 1. SEM micrographs of S6t sample

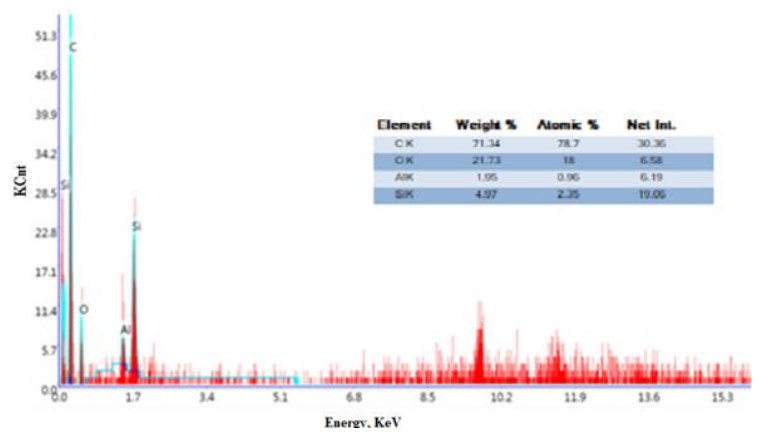

Figure. 2. EDAX spectrum for 6St sample 


\section{CONCLUSIONS}

From the data presented it can be concluded that by introducing small quantities of OMMT in composites based on natural rubber, plasticized starch and precipitated silica, the following occurs: (1) an increase in the minimum torque, showing an increase in the rigidity of the unvulcanized material with increase of filler amount, (2) increase of hardness by $1-6^{\circ} \mathrm{ShA}$ and an improvement in $100 \%$ and $300 \%$ elongation modulus.

By adding the adhesion promoter between the rubber and the filler, an improvement of tear and tensile strength is observed and a decrease of the optimal vulcanization time. These changes may be due to both the nanofiller reinforcement effect and the changes in the morphology of the mixture.

Samples S3t and S6t show a very good behavior after immersion 22 hours at $23^{\circ} \mathrm{C}$ in: distilled water, ethyl alcohol, $10 \%$ glucose solution and $0.9 \% \mathrm{NaCl}$ solution, due to the polymer matrix of natural rubber is hydrophobic.

Obtaining good physical-mechanical and chemical properties is determined both by the composition of the mixtures and by the technology and methods used in processing the mixtures that led to the obtaining of materials in which the starch particles and other ingredients of the mixture have a quasi-uniform distribution in elastomer matrix (according to SEM and EDAX analyses), without structural discontinuities or other defects.

New elastomeric nanomaterials can be used to obtain rubber consumer goods for the food, pharmaceutical, the footwear industry etc.

\section{Acknowledgements}

This research was funded by the Romanian Ministry of Education and Research through Nucleu Project PN 16340101 Contract No. 26N/2016, Sectoral Project Contract No. 3PSI/2019 and Contract No. 6PFE/2018-PERFORM-TEX-PEL.

\section{REFERENCES}

Carvalho, A.J.F., Job, A.E., Alves, N., Curvelo, A.A.S. and Gandini, A. (2003), "Thermoplastic starch/natura rubber blends", Carbohydrate Polymers, 53(1), 95-99, https://doi.org/10.1016/S0144-8617(03)00005-5.

Manaila, E., Stelescu, M.D. and Craciun, G., (2018), "Degradation Studies Realized on Natural Rubber and Plasticized Potato Starch Based Eco-Composites Obtained by Peroxide Cross-Linking", International Journal of Molecular Sciences, 19, https://doi.org/10.3390/ijms19102862.

Mohammed, L., Ansari, M.N.M., Pua, G., Jawaid, M. and Islam, M.S. (2015), “A Review on Natural Fiber Reinforced Polymer Composite and Its Applications", International Journal of Polymer Science, Article ID 243947, https://doi.org/10.1155/2015/243947.

Shey, J., Imam, S.H., Glenn, G.M. and Orts, W.J. (2006), "Properties of baked starch foam with natural rubber latex", Industrial Crops and Products, 24(1), 34-40, https://doi.org/10.1016/j.indcrop.2005.12.001.

Shinoj, S., Visvanathan, R., Panigrahi, S. and Kochubabu, M. (2011), "Oil palm fiber (OPF) and its composites: a review", Industrial Crops and Products, 33(1), 7-22, https://doi.org/10.1016/j.indcrop.2010.09.009.

Stelescu, M.D., Mănăilă, E., Sönmez, M. and Niţuică, M. (2017), "Characteristics of Polymer Composites Based on Natural Rubber”, Leather and Footwear Journal, 17(3), 147-154, https://doi.org/10.24264/lfj.17.3.4.

Wang, Z.F., Peng, Z., Li, S.D., Lin, H., Zhang, K.X., She, X.D. and Fu, X. (2009), "The impact of esterification on the properties of starch/natural rubber composite", Composites Science and Technology, 69(11-12), 1797-1803, https://doi.org/10.1016/j.compscitech.2009.04.018.

Wu, Y.P., Qi, Q., Liang, G.-H. and Zhang, L.-Q. (2006), "A strategy to prepare high performance starch/rubber composites: In situ modification during latex compounding process", Carbohydrate Polymers, Vol. 65(1), 109-113, https://doi.org/10.1016/j.carbpol.2005.12.031. 
V.

\section{CREATIVE INDUSTRIES AND CULTURAL HERITAGE}


INNOVATIVE AND SUSTAINABLE MODELS IN THE ECODESIGN OF GREEN-VEGAN FOOTWEAR

\author{
TRAIAN FOIASI, MIRELA PANTAZI-BAJENARU \\ INCDTP - Division: Leather and Footwear Research Institute, 93 Ion Minulescu St., Bucharest, \\ Romania, email: icpi@icpi.ro
}

\begin{abstract}
The ECODESIGN concept, which has appeared since the early 1960s, plays a fundamental role in the life cycle of a fashion product, from design, production, service life to recycling. The main vectors of ECODESIGN are guided in several directions: the selection of materials and their impact on production - non-toxic natural fiber materials will be used, recyclable rubber, all obtained with low energy consumption; designing models and making samples - these will be produced from material obtained by recycling used textile fibers with a high durability and low weight; production - the aim is to optimize the production process, replacing toxic auxiliary materials with natural ones (natural rubber, water-based adhesives), identification and elimination of toxic emissions, as well as the use of unconventional, solar and wind energy; packaging - it will be made of recycled vegetable fibers and will be designed in such a way as to protect the product as well as possible without damaging it. All these vectors will contribute to extending the "life" of the product, offering comfort, durability and eco-efficiency.
\end{abstract}

Keywords: VEGAN-DESIGN, recycling, fashion.

\title{
INTRODUCTION
}

Globalization and online sales are a reality of these times. Markets and sales spaces have been reinvented, and consumers are paying much more attention to fashion, style and are reorienting towards ECO products. An important role in this approach is played by ECODESIGN, starting with the discovery of the latest materials up to design, lifespan, recycling (Dufrene, 2016).

The ECODESIGN concept has appeared since the early 1960s and plays a fundamental role in the life cycle of a fashion product, from design to production, service life, and recycling, in other words DESIGN - PRE-PRODUCTION, END OF PRODUCT LIFE (Stefano and Ferreira, 2013).

The main vectors of ECODESIGN go in several directions:

- Selection of materials and their impact on production;

Non-toxic, used, natural fiber materials, and recyclable rubber will be used, all obtained with low energy consumption.

- Designing models and making samples;

These will be produced from material obtained by recycling used textile fibers with a high durability and low weight. Also, the other components must use the same production elements with low costs.

- Production;

The aim will be to optimize the production process, replace toxic auxiliary materials with natural ones (natural rubber, water-based adhesives), identify and eliminate toxic emissions, as well as use unconventional, solar and wind energy.

- Packaging.

These will be produced from recycled vegetable fibers and will be designed in such a way as to protect the product as well as possible without damaging it.

All these vectors will contribute to extending the "life" of the product by providing comfort, durability and eco-efficiency (Laruccia and Garcia, 2015). 


\section{SELECTION OF MATERIALS}

Designers and all the factors involved in the development of an ECO product must find the newest materials that can be recycled as easily as possible, with low energy consumption. Natural fibers come first to the attention of designers. The fabrics from recycled cotton, linen, hemp, to which are added those made of pineapple or palm fiber, from certified crops respecting the international directives are the main components of an Eco fashion product. In addition, materials obtained from synthetic yarns after recycling plastic containers can be used. They will be combined with biodegradable polyamide and natural fibers (cotton, hemp). The accessories will in turn be made of plastic recovered from the oceans in combination with mother of pearl (shells) and wood fiber.

\section{DESIGN OF MODELS AND MAKING SAMPLES}

A first aspect that is required in the design of models is the identification of eco materials with high durability and low costs in the recycling and production process (Guerra Ashton, 2018). The design in terms of volumes and colors will focus on the predictions of fashion specialists. Vegetable pigments will give dynamism and color to natural fibers.

Sketches of ideas, and then the use of 2D and 3D CAD design systems will reduce work time in the different phases of product development.

\section{SKETCHES OF IDEAS GENERATING NEW AESTHETIC CONCEPTS IN ECO-DESIGN}

In order to create footwear that meets the growing demand for market-specific niches, in accordance with sustainability and circular economy criteria that combine elements of recyclability, organic use, non-use of animal products (leather) and natural components, it is necessary to design idea sketches.

The proposed models (Fig. 1) are based on the STROBEL circular system, which consists in attaching a non-woven textile material to the upper through sewing. After that, the assembly of the upper material on the sole is done by seams, with a minimal use of a water-based adhesive.

The "helmet" type sole from SBR + EVA (styrene-butadiene copolymer + ethylenevinyl acetate copolymer) was chosen. It has up to $70 \%$ recycled rubber and synthetic rubber. 

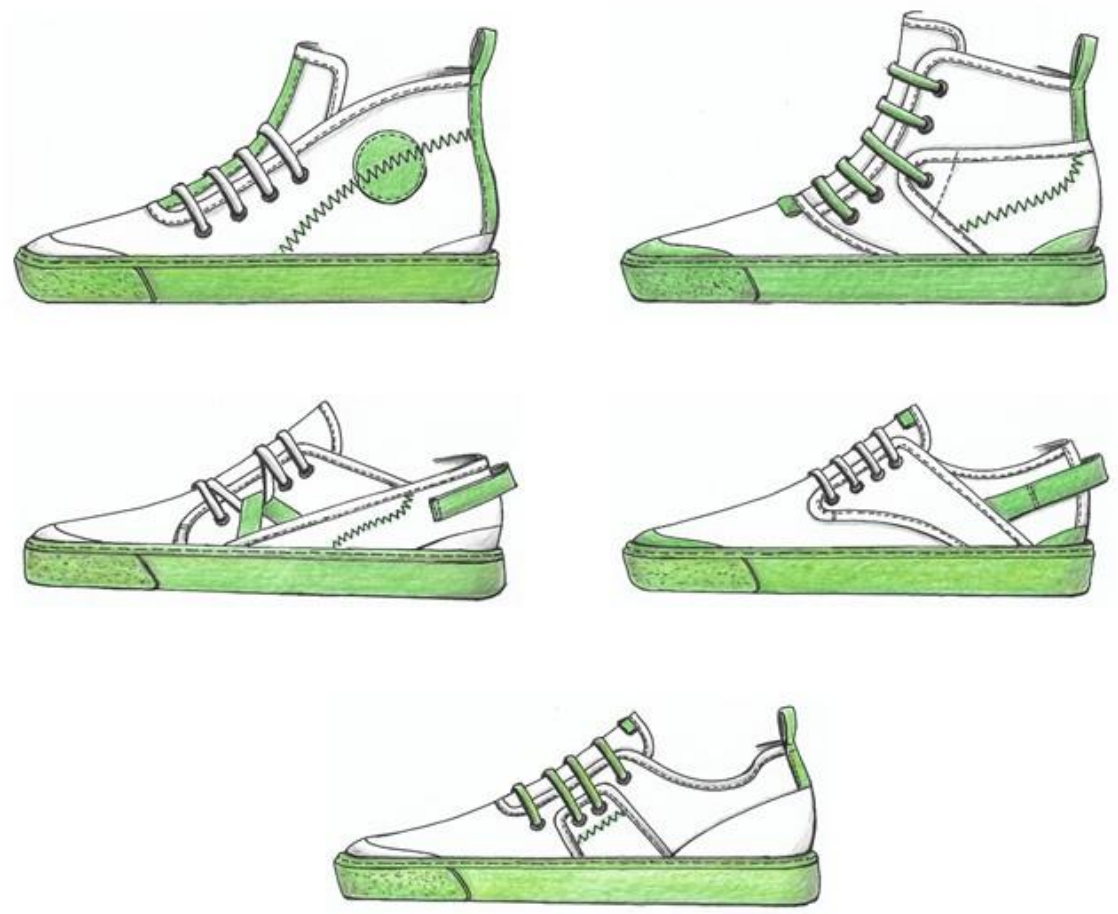

Figure 1. Model sketches

\section{PRODUCTION}

An important role in the organization of production will be the elimination of toxic components and their replacement with natural ones. The products presented in Figure 2 are exclusively "vegan" with elements of vegetable origin only in their composition.

The upper ensemble of the shoes is made of textile material obtained by interweaving hemp and linen yarns.

The materials are dyed using vegetable tannins (turmeric, dandelion, beetroot).

Also, unconventional materials are used resulting from the weaving of textile strips obtained by recycling used clothing.

Crepe soles made from natural rubber are $100 \%$ ecological and recyclable. They are very resistant to abrasion, water, acidic, alkaline, and salty solutions and are very elastic and flexible. In the process of calendering natural rubber, all operations are environmentally friendly. No dust, vapors are produced, and the residues are reused in the production cycle.

Cork also comes to the attention of vegan shoe manufacturers. It is used in the manufacture of anatomical and very light soles, as well as pure cellulose insoles, giving stability and torsional resistance.

Water-based adhesives in turn help eliminate toxic emissions. 

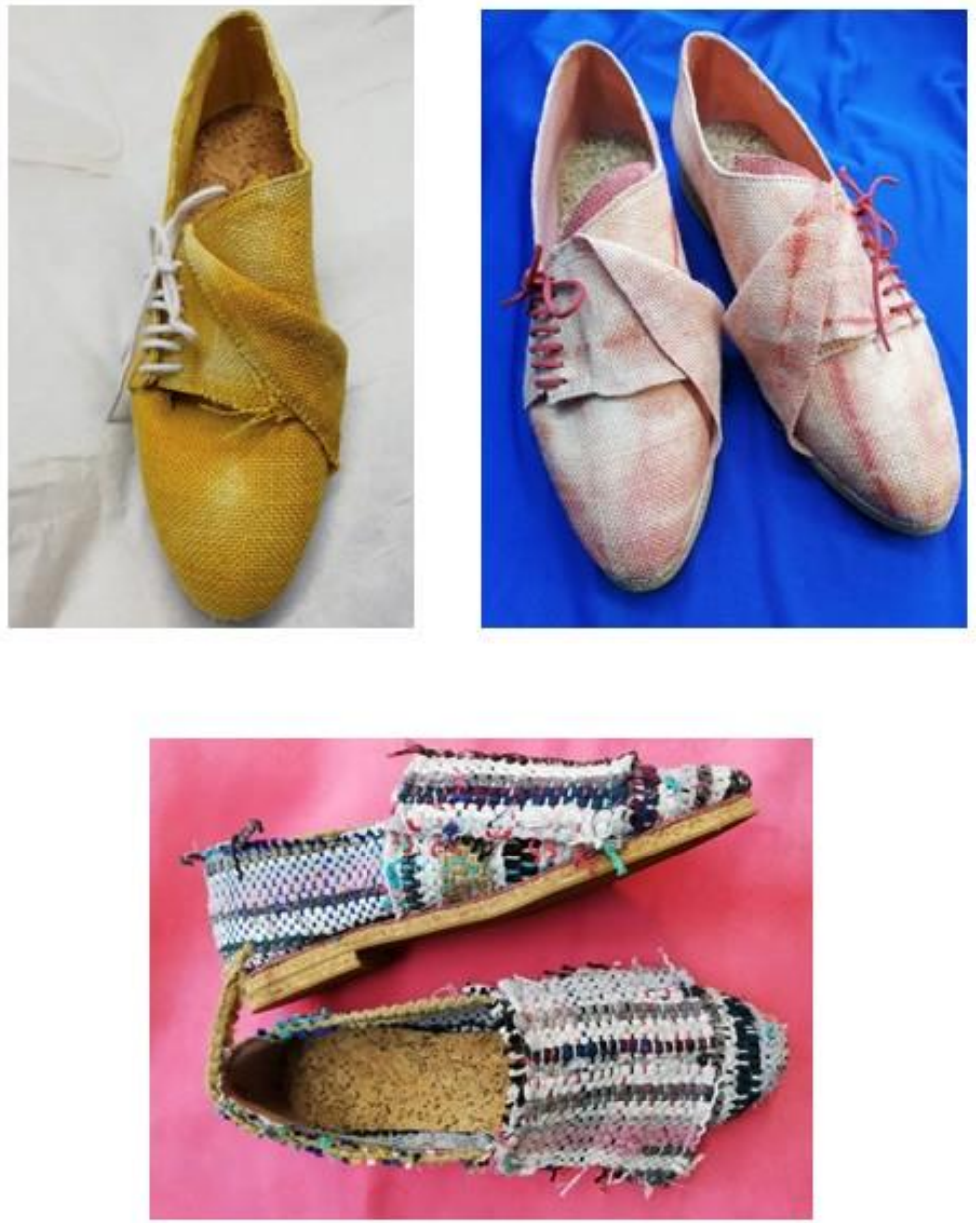

Figure 2. "Vegan" shoes

\section{PACKAGING}

Their design will primarily aim to give the shoe a firm protection so as not to damage the product. When they are made, plant-based materials obtained exclusively from their recycling will take precedence. The packaging will be inscribed with vegetable dyes, green being the color that will signal to the consumer that it is an ecological product.

\section{CONCLUSIONS}

All the listed factors are of the primary importance in the eco-design of the footwear and not only. The use of natural, renewable and recyclable resources, materials, ecodesign, production and distribution, consumption duration, unconventional energies will be found in the concept of circular economy, sustainability and eco-efficiency. 
Acknowledgements

This study was funded by the Minister of Research and Innovation through the Program 1 - Development of the National Research and Development System, Subprogram 1.2 - Institutional Performance - RDI excellence funding projects, Contract no. 6PFE/16.10.2018.

\section{REFERENCES}

Dufrene, M. (2016), “A methodological framework to support integrated ecodesign for companies: requirements and conceptualization towards a software platform", p. 15-18, available at https://hal.archives-ouvertes.fr/tel-01267717.

Guerra Ashton, E. (2018), "Analysis of footwear development from the design perspective: Reduction in solid waste generation", Strategic Design Research Journal, 11(1), 2-8, https://doi.org/10.4013/sdrj.2018.111.01.

Laruccia, M.M. and Garcia, M.G. (2015), "Uma Análise da Percepção e da Utilização de Práticas de Ecodesign nas Empresas”, BBR, Braz. Bus. Rev. (Port. ed., Online), Vitória, 12(3), Art. 1, p. 1-16.

Stefano, N.M. and Ferreira, A.R. (2013), "Ecodesign referencial teórico e analise de conteúdo: proposta inicial para estudos futuros", Estudos EM Design, 21(2). 
Innovative and Sustainable Models in the Ecodesign of Green-Vegan Footwear

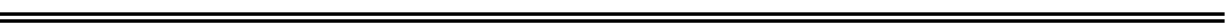

https://doi.org/10.24264/icams-2020.V.1 


\title{
INVESTIGATION OF ENERGY RESOURCES AND GAS DISCOVERIES IN THE EASTERN MEDITERRANEAN REGION: THE CASE OF PEOPLE'S EXPECTATIONS AND SOCIAL IMPACTS IN EGYPT
}

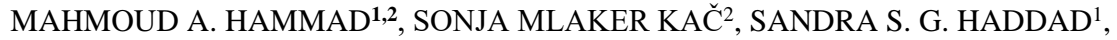 \\ HABIBA S. EL ROUBY ${ }^{1}$ \\ ${ }^{1}$ Arab Academy for Science, Technology and Maritime Transport/College of International \\ Transport and Logistics, Alexandria,Egypt,m.hammad@aast.edu, sandra.haddad@aast.edu, \\ habiba.roubi@aast.edu \\ ${ }^{2}$ University of Maribor/ Faculty of Logistics, Celje, Slovenia, e-mail: sonja.mlaker@um.si
}

\begin{abstract}
This study discusses the expected social impacts due to the recent offshore gas findings and development in the Eastern Mediterranean region on human communities in Alexandrian in the North of Egypt. A sample of 401 respondents of ordinary people who are living in the Alexandria governorate were sampled for the study using a convenience non-random sampling approach. The study showed most people are somewhat familiar with the ongoing gas discoveries in the Mediterranean Sea. The study also revealed that the people in Egypt have relatively high expectations from these gas discoveries. The ordinary people in Egypt expected that such discoveries of gas would have many positive and negative social impacts side by side. They expected severa social benefits of gas finds such as: the contribution to the diversification of the economy, infrastructural development, expanding social services, improvement of the standard of living, business and investment opportunities, employment.
\end{abstract}

Keywords: Oil and gas discoveries, people's expectations, social impacts

\section{INTRODUCTION}

Energy plays a vital role in the economy of every country and its importance can be seen in all aspects of our life (Ambituuni et al., 2015). Gas or natural gas is being used for several purposes such as heating energy, power or electricity, fuel for engines or transport fuel, and the chemical feedstock as well as an energy source for making a commodity that needs large energy requirements in its manufacture (Thomas and Dawe, 2003). Egypt is considered one of the important players in the Mediterranean region. It is the largest non-OPEC (Organization of the Petroleum Exporting Countries) oil producer in Africa (Hegazy, 2015) as well as it owns one of the most highly developed liquefied natural gas (LNG) and export infrastructures in the Eastern Mediterranean (Tsakiris et al., 2018). This study aims to identify the potential positive and negative social implications on the Egyptians' life as a result of gas findings in the Mediterranean Sea. Thus, the study is structured around three main objectives, namely: 1) to highlight the main social impacts of gas explorations on Egyptians, 2) to identify the social benefits of gas exploration on the Egyptian people, 3) to identify the social drawbacks/challenges of gas exploration on the Egyptian people.

\section{THEORETICAL BACKGROUND}

In order to realize the objectives of this study literature was reviewed and the three following theories, on the impact of gas explorations on social, economic and political implications, were studied. 


\section{Resource Curse Theory}

According to Adusah-Karikari (2012) resource curse theory aims at explaining the correlation between the exploitation of natural resources in the social, economic and political implications for the nations that host them. The theory posits that countries endowed with natural resources, like fossil fuels and minerals, are more likely to experience poor development outcomes because of underlying political and institutional issues, such as authoritarianism and neo-patrimonial and rentier politics.

\section{Dutch Disease}

Dutch disease is a term coined after the negative effects of the North Sea oil discoveries in the Netherlands (1959) (Meadors, 2013; Tadeo, 2016) and it explains the loss of competitiveness in the non-resource sectors (non-booming goods) through the eminent appreciation of the exchange rate (Boughanem, 2014; Mahbob, 2017; Mawejje, 2019; Swilling, 2019) and switch of capital and labor to the booming mineral sector.

\section{Gas Discoveries and Citizens' Expectations}

According to Bategeka et al. (2008), citizens' expectation exists in different forms and may have different orientations whether positive or negative. Likewise, in the case of oil and gas discovery, the positive expectations are represented the real hopes that the precious resources will deliver considerable social, economic and infrastructural improvement.

\section{Social Impacts of Gas Exploitation}

Akakpo (2015) defines social impacts as the changes occur in communities or to individuals as a result of an externally-induced change, result in changing people live, work, play, relate to one another, organize to meet their needs, and generally cope as members of society. These social impacts are both negative and positive.

The following section will present the research methodology by which the aim of the study shall be realized.

\section{MATERIAL AND METHODS}

\section{Population and Sample}

The target population is the people who live in Alexandria governorate, Egypt and they were all the strata of society of Alexandria. In determining the sample- frame, the basic criterion was people who are only living in Alexandria. According to the Central Agency for Public Mobilization and Statistics (CAPMAS) and official reports, the total number of populations in Alexandria is about 5,380,000. Out of the target population, a sample size of minimum 384 respondents was selected to answer the questionnaires using a convenience non-random or non-probability sampling method.

\section{Data Collection and Analysis}

The quantitative approach was applied in the study; this is through conducting structured questionnaires in order to collect the primary data. The questionnaire was divided into two sections: first section, the level of awareness and the expectations of Egyptian citizens about the research topic and the role of the state to manage and control gas resources were measured. The construct of this section was adapted from the previous works of Paulson E. 
Tadeo (2016), Choumert-Nkolo (2018), Omar (2018). Second section contains of two subsections: Firstly, part one reflected the expected social benefits from natural gas explorations in the Mediterranean Sea on Egyptian people's life. Secondly, part two measured the prospective social challenges on the ordinary people in Egypt from natural gas explorations in the Mediterranean Sea. The second section was measured by using a seven-point Likert Scale anchored from 1= strongly disagree to $7=$ strongly agree. The construct of the second section was adapted from previous literature (Akakpo, 2015; Omar, 2018). The questionnaire-collected data were coded and analyzed using the statistical package for social sciences (SPSS) software, version 25.

\section{DISCUSSION}

\section{Perceptions and Expectations of the Respondents from Gas Operations and Discoveries}

Regarding the level of awareness, familiarity and the expectations of Egyptian citizens about gas resources and discoveries in the Mediterranean Sea. The results show that about $17 \%$ of the respondents have broad knowledge about these gas discoveries, but, most of them have average knowledge with $64 \%$, and $37 \%$ with limited knowledge. The optimists of the Egyptians see that there would be significant and positive effects on Egypt after the latest gas discoveries at $(84 \%)$, while a few of pessimists argue that these discoveries negative effects at (4\%), and the rest (12\%) said that no effect or change would happen at all. Nevertheless, $69 \%$ of them strongly support these gas explorations, $29 \%$ are neutral, and the opponents represent 2\%. This is because almost of people in Egypt (82\%) believe that gas resources are boon and gift from God, not indignation or curse. From their point of view, $35 \%$ of the Egyptians expected that the revenues or returns resulting from the explorations of gas in the Mediterranean would be "distributed evenly/equally", while $30 \%$ see that it may distribute according to "the population density", and "the distribution to the poor and poorest regions" was predicted by $27 \%$, finally "distribution to the areas where gas was discovered" at $8 \%$. Moreover, the respondents expected that the main sector in which such revenues should be invested or spent on is "education" with the highest answer (49\%), followed respectively by living standards $(21 \%)$, health services (10\%), economic development $(8 \%)$, infrastructure $(6 \%)$, national security $(3 \%)$, saving gas revenues $(2 \%)$, while, the least answer was "environment protection" as no one has chosen it. Furthermore, the ordinary people in Egypt expect that the Mediterranean gas may create some developments and improvements in many fields of their life. In which they respectively prioritized these improvements as follows; living standards or welfare, employment opportunities, healthcare service, infrastructure, social support (symbiosis), educational opportunities, the subsidy of goods or services, and public security by $(37 \%),(18 \%),(13 \%)$, $(11 \%),(9 \%),(6 \%),(2 \%)$, and $(2 \%)$. Hence, $37 \%$ agree that it will increase the standard of living and luxury level as the highest answer, whereas, the least answer was both support for goods or services and public security at $(2 \%)$.

\section{Level of State Involvement}

On the other hand, concerning the role and involvement of the Egyptian state in managing and controlling natural resources like oil and gas, about $61 \%$ of respondents see that the state has effective involvement and plays an essential role in managing such resources, while 20\% see the opposite, and 19\% don't have an idea. Although most of them expect that the state or government has the ability to manage and govern the revenue generated from new discoveries of gas in a "somewhat good" way at $(63 \%)$, "good" with $(27 \%)$ and "bad" way at $(10 \%)$. Additionally, respondents disagree that developing 
natural gas resources in Egypt will not lose the country's competitiveness in other sectors (non-gas related sectors) such as agriculture, services and manufacturing by (58\%), while $(19 \%)$ agree upon this.

\section{The Social Perceptions and Positive Social Impacts of the Mediterranean Gas Discoveries}

It could be observed that the majority of the sample anticipates achieving major positive effects on all social levels from gas exploration and in high degrees. Also, it was noted that the diversification of the economy had the most agreed answer with $(90 \%)$, while, the least answer is freeing the state from dependence on donors with (78\%).

\section{The Drawbacks and Negative Social Impacts of the Mediterranean Gas Discoveries}

The increased cost of living and increase in prices of goods and materials had the most agreed expectation with $(73 \%)$, whereas, the lowest expected impact is the wars and conflicts whether internal or external $(57 \%)$.

\section{Further Inferential Analyses Using Tests of Differences (T-test and ANOVA)}

Table 1. T-test for differences according to employment sector, gender and family position

\begin{tabular}{lllllll}
\hline & \multicolumn{2}{l}{ Level of awareness } & \multicolumn{2}{l}{ Social benefits } & \multicolumn{2}{l}{ Social challenges } \\
\hline 1. Gender & Male & Female & Male & Female & Male & Female \\
& $\mathrm{N}=263$ & $\mathrm{~N}=138$ & $\mathrm{~N}=263$ & $\mathrm{~N}=138$ & $\mathrm{~N}=263$ & $\mathrm{~N}=138$ \\
Mean & 2.5399 & 2.1957 & 5.6236 & 6.1087 & 3.0304 & 2.7174 \\
P-value & $\mathbf{. 0 0 0}$ & & $\mathbf{. 0 0 0}$ & & $\mathbf{. 5 9 1}$ & \\
2. You are an employee? & Gov. & private & Gov. & private & Gov. & private \\
& sector & sector & sector & sector & sector & sector \\
& $\mathrm{N}=20$ & $\mathrm{~N}=381$ & $\mathrm{~N}=20$ & $\mathrm{~N}=381$ & $\mathrm{~N}=20$ & $\mathrm{~N}=381$ \\
Mean & 2.2500 & 2.4304 & 6.2500 & 5.7664 & 2.2500 & 2.9580 \\
P-value & $\mathbf{0 0 0}$ & & $\mathbf{. 0 1 9}$ & & $\mathbf{. 0 1 3}$ & \\
3. Are you the head of the & $\mathrm{Yes}$ & $\mathrm{No}$ & $\mathrm{Yes}$ & $\mathrm{No}$ & $\mathrm{Yes}$ & $\mathrm{No}$ \\
family? & $\mathrm{N}=211$ & $\mathrm{~N}=190$ & $\mathrm{~N}=211$ & $\mathrm{~N}=190$ & $\mathrm{~N}=211$ & $\mathrm{~N}=190$ \\
Mean & 2.4929 & 2.3421 & 5.7299 & 5.8579 & 3.1327 & 2.6895 \\
P-value & $\mathbf{. 0 0 0}$ & & $\mathbf{. 0 4 6}$ & & $\mathbf{. 0 0 3}$ & \\
\hline
\end{tabular}

Table 1 shows in social challenges an insignificant difference with different gender groups, as the corresponding p-value is greater than 0.05 . Also, there is a significant difference in awareness level and social benefits and challenges since the corresponding $\mathrm{p}$-values are less than 0.05 .

Table 2. ANOVA Test for educational level

\begin{tabular}{cccccccc}
\hline \multirow{2}{*}{ Educational level } & \multirow{2}{*}{$\mathrm{N}$} & \multicolumn{2}{c}{ Level of awareness } & \multicolumn{2}{c}{ Social benefits } & \multicolumn{2}{c}{ Social challenges } \\
& & mean & P-value & mean & P-value & mean & P-value \\
\hline Primary & 6 & 3.0000 & & 7.0000 & & 1.0000 & \\
High school & 34 & 2.1471 & & 5.8529 & & 3.1176 & \\
University degree & 244 & 2.4713 & & 5.6926 & .001 & 3.0369 & .008 \\
Post graduate & 80 & 2.5375 & .000 & 5.6750 & & 2.8625 & \\
Nothing & 37 & 2.0000 & & 6.4324 & & 2.4324 & \\
Total & 401 & 2.4214 & & 5.7905 & & 2.9227 & \\
\hline
\end{tabular}


Table 2 demonstrated a significant difference in awareness level and social benefits and challenges because the corresponding p-values are all less than 0.05.

Table 3. ANOVA Test for differences according to job or social statuses

\begin{tabular}{cccccccc}
\hline \multirow{2}{*}{ Job/social status } & \multirow{2}{*}{$\mathrm{N}$} & \multicolumn{2}{c}{ Level of awareness } & \multicolumn{2}{c}{ Social benefits } & \multicolumn{2}{c}{ Social challenges } \\
& & mean & P-value & mean & P-value & mean & P-value \\
\hline Employee & 194 & 2.597 & & 5.582 & & 2.886 & \\
Unemployed & 13 & 2.38 & & 6.000 & & 2.846 & \\
Retired & 39 & 2.256 & & 5.179 & & 3.974 & \\
Student & 67 & 2.358 & .000 & 6.089 & .001 & 2.895 & .008 \\
House wife & 80 & 2.175 & & 6.287 & & 2.725 & \\
Unable to work & 8 & 2.000 & & 6.000 & & 1.000 & \\
Total & 401 & 2.421 & & 5.790 & & 2.9227 & \\
\hline
\end{tabular}

The table proves that there is a significant difference in the level of awareness as well as social benefits and challenges. i.e. the corresponding p-values are all less than 0.05 .

Table 4. ANOVA Test for differences according to level of social welfare

\begin{tabular}{cccccccc}
\hline Level of social & \multirow{2}{*}{$\mathrm{N}$} & \multicolumn{2}{c}{ Level of awareness } & \multicolumn{2}{c}{ Social benefits } & \multicolumn{2}{c}{ Social challenges } \\
welfare & & mean & P-value & mean & P-value & mean & P-value \\
\hline Poor family & 42 & 2.00 & & 6.30 & & 2.45 & \\
Fairly average family & 349 & 2.46 & .000 & 5.73 & .015 & 3.03 & .000 \\
Rich family & 10 & 2.50 & & 5.50 & & 1.00 & \\
Total & 401 & 2.42 & & 5.79 & & 2.92 & \\
\hline
\end{tabular}

Table 4 shows that there is a significant difference of level of awareness, social benefits and social challenges, as the corresponding p-values are all less than 0.05 .

Table 5. ANOVA test for differences according to the main source of income

\begin{tabular}{|c|c|c|c|c|c|c|c|}
\hline \multirow{2}{*}{$\begin{array}{l}\text { The main source of } \\
\text { income }\end{array}$} & \multirow{2}{*}{$\mathrm{N}$} & \multicolumn{2}{|c|}{ Level of awareness } & \multicolumn{2}{|c|}{ Social benefits } & \multicolumn{2}{|c|}{ Social challenges } \\
\hline & & mean & $\mathrm{P}$-value & mean & P-value & mean & P-value \\
\hline Salary & 209 & 2.42 & & 5.99 & & 2.86 & \\
\hline Bank interest & 5 & 2.00 & & 4.00 & & 1.00 & \\
\hline Pension (retirement) & 92 & 2.48 & & 5.06 & & 3.73 & \\
\hline $\begin{array}{l}\text { Income through the } \\
\text { business }\end{array}$ & 31 & 2.58 & .029 & 5.32 & .000 & 3.35 & .000 \\
\hline $\begin{array}{l}\text { Income source } \\
\text { through a property }\end{array}$ & 13 & 2.38 & & 7.00 & & 1.61 & \\
\hline Another source & 51 & 2.25 & & 6.43 & & 1.94 & \\
\hline Total & 401 & 2.42 & & 5.79 & & 2.92 & \\
\hline
\end{tabular}

At the end, ANOVA test for the difference in research variables according to different groups of the main source of income demonstrated that there is a significant difference in level of awareness, social benefits and social challenges, as the corresponding p-values are all less than 0.05 . 


\section{CONCLUSION}

This study assessed the expectations of the ordinary people in Egypt, and also examined the level of their awareness regarding recent gas discoveries in the Mediterranean region. The study used the questionnaires to examine the expected social opportunities and risks offered by the discovery of natural gas in Egypt's Alexandria governorate. The paper argued that the discovery of gas is followed by both positive and negative impacts on the social level. The expected positive impacts from gas discoveries in the Mediterranean from Egyptians' point of view could include; the contribution to the diversification of the economy, infrastructural development, expanding social services, improvement of the standard of living, business and investment opportunities, employment. Whereas, the negative social impacts might include; high cost of living, social vices, corruption, inequality, and wars. Nevertheless, the expected rates of occurrence of the positive impacts are higher than the frequents of the occurrence of the negative impacts. But that is not all, concerning the role and involvement of the Egyptian state in managing and controlling oil and gas resources, most of people see that the state has effective involvement and plays an essential role in managing such resources. Also, the majority of them expect that the government is fairly has the ability to manage and govern the revenue generated from new discoveries of gas, as well as these new gas resources will not lose the country's competitiveness in other sectors.

\section{REFERENCES}

Adusah-Karikari, A. (2012), "Black gold in Ghana: Changing Livelihoods for Women in Communities Affected by Oil Production", The Extractive Industries and Society, 2, 24-32, https://doi.org/10.1016/j.exis.2014.10.006.

Ambituuni, A., Amezaga, J. and Emeseh, E. (2015), "Analysis of Safety and Environmental Regulations for Downstream Petroleum Industry Operations in Nigeria: Problems and Prospects", Environmental Development, 9, 43-60, https://doi.org/10.1016/j.envdev.2013.12.002.

Bategeka, L., Kiiza, J. and Ssewanyana, S. (2008), "Oil Discovery in Uganda: Managing Expectations", Economic Policy Reseach Centre and Makerere University.

Boughanem, L.N. (2014), "The Curse of Natural Resources: A Vulnerability Assessment for Lebanon", Master's Thesis, American University of Beirut.

Hegazy, K. (2015), "Egypt's Energy Sector: Regional Cooperation Outlook and Prospects of Furthering Engagement with the Energy Charter", Brussels, Belgium, Energy Charter Secretariat.

Mahbob, M. (2017), “The Impact of the Oil Sector and Political Conflicts on the Libyan Economy”, Master's Thesis, Western Illinois University.

Mawejje, J. (2019), “The Oil Discovery in Uganda's Albertine Region: Local Expectations, Involvement, and Impacts", The Extractive Industries and Society, 6, 129-135, https://doi.org/10.1016/j.exis.2018.09.007.

Meadors, L. (2013), "Israel's Newfound Petroleum Wealth: A Critique of the "Resource Curse", Syracuse University Honors Program Capstone Projects (37), Syracuse University.

Swilling, R.A. (2019), "Investigating Sino-Africa relations: Exploring Investment and Governance regimes in the Sino-Ugandan Oil relations", Master's Thesis, Stellenbosch University.

Tadeo, P.E. (2016), “A Comparative Study of Oil Resource Management in Norway and Nigeria: Lessons for Kenya", Master's Thesis, University of Nairobi.

Thomas, S. and Dawe, R.A. (2003), "Review of Ways to Transport Natural Gas energy from Countries Which Do Not Need the Gas for Domestic Use”, Energy, 2003(28), 1461-1477, https://doi.org/10.1016/S03605442(03)00124-5.

Tsakiris, T., Ulgen, S. and Han, A.K. (2018), "Gas Developments in the Eastern Mediterranean: Trigger or Obstacle for EU-Turkey Cooperation?”, FEUTURE Online Paper No. 22, W. Wessels and N. Tocci, FEUTURE. 
THE INFLUENCE OF $\mathrm{TIO}_{2}$ AND N-TIO 2 NANOPOWDERS IN NATURAL LEATHER FINISHING FOR HERITAGE OR MODERN BINDING

\author{
MADALINA IGNAT ${ }^{1}$, LUCRETIA MIU ${ }^{1}$, EMANUEL HADIMBU ${ }^{1}$, CLAUDIU ȘENDREA $^{1}$, \\ MARIA-CRISTINA MICU ${ }^{1}$, SIMONA MARIA PĂUNESCU ${ }^{1}$, IULIA MARIA CANIOLA ${ }^{1}$, \\ ELENA BADEA ${ }^{1,2}$ \\ ${ }^{1}$ INCDTP - Leather and Footwear Research Institute (ICPI) Division, Bucharest, \\ Romania, madalina.fleancu@yahoo.com \\ ${ }^{2}$ Department of Chemistry, Faculty of Sciences, University of Craiova, Craiova, \\ Romania
}

\begin{abstract}
The methods for processing raw hides over time, have been numerous: smoke, fat, fermented milk, egg, volcanic soils, plants (shells, fruits, leaves) and so on. The books binding with leather has its origins in the Orient and are known various types of goatskin and calfskin, tanned with sumac finished marbled (sapphire), in black (Moroccan), by floating and dyeing in various colors (Cordoba leathers). Preoccupations regarding the assurance of optimal characteristics for the binding leathers, respectively the durability of the leathers for the restoration of the patrimony objects or of the modern binding were of the most topicality in the last decades, abroad and also in our country. The paper describes the finishing of vegetable and alum tanned leather samples that have been functionalized with titanium dioxide $\left(\mathrm{TiO}_{2}\right)$ or nitrogen-doped titanium dioxide $(\mathrm{N}$ $\mathrm{TiO}_{2}$ ) nanopowders, in different concentrations. To simulate soiling in real conditions, four types of soiling agents were applied: tea, coffee, beetroot extract and pen paste. The samples were exposed to irradiation in a photoreactor with various light sources for up to 192 hours. The evaluation of the photocatalytic degradation was performed by the CIELab technique.
\end{abstract}

Keywords: preserving heritage objects, nanopowders, leather finishing

\title{
INTRODUCTION
}

Leather processing was the first biotechnology developed by mankind, raised to the art level by combining this craft with tradition, skill, talent, beauty and usefulness. In the history of humanity, leather and fur objects have had many fundamental meanings related to the development of society (social distinction, mysticism, aesthetics, defense against enemies) in addition to the basic function, as protection against temperature differences (clothing, footwear, tents, bellows etc., for domestic, common, military use etc.). The first documentary attestation - Sumerians, 2700 BC (Delort, 1993), and on the territory of our country is attested the processing of skins in clay tablet no. 3 from Tartaria, dated 2900 BC, in which animal skins are rendered in the manner of Egyptian ideograms.

Over time, leather processing has gained importance for history, art, but especially for science (Higham, 1999). The methods of processing raw hides were numerous: smoke, fats, fermented milk, egg, volcanic soils, plants (shells, fruits, leaves etc.), urine, excrement or combinations thereof (Bravo, 1964; Deselnicu et al., 1984). This first biotechnology developed by mankind, remained almost unchanged until the end of the nineteenth century, when tanning with $\mathrm{Cr}$ salts came into use.

The book binding in leather has its origins in the Orient in the same time with paper, which being fragile had to be protected by a resistant material, such as leather. The most important types of leathers used for book binding were those of goat and calf, tanned with sumac and finished marbled (saphian), in black (marochin), by floating and dyeing in various colors (Cordoba leathers). Information regarding the making of binding leathers up to the 17th century is described in the encyclopedias of De La Lande and 
Diderot (Thomson, 2000). The leather for book binding had to be smooth, resistant to bending and pressing, thin, vegetable tanned, finished specifically for book covers.

Concerns regarding the identification of optimal characteristics for binding leathers and the elaboration of technical specifications to responsibly ensure the durability of leathers for the restoration of heritage objects have been the most topical in recent decades abroad, but also in our country (Larsen, 1994; Larsen, 1997; Miu et al., 2007; Project CRAFT No BRST-CT98-5535; Project PNCDI-Partnerships PN 91012/2007).

The variety of degradation phenomena that affect works of art, reflects the wide range of materials that have been used by mankind since the beginning. Therefore, both conservatives and scientists are involved in finding effective solutions to counteract aging processes due to the action of light, temperature, relative humidity and microorganisms, chemical degradation and physical erosion or anthropogenic causes such as industrial pollution, vandalism or simple artifact handling.

In the field of preservation, the application of dispersed solid particles is preferable to aqueous solutions or organic solvents for many reasons.

In the last decade, nanostructured materials have been of great interest as catalysts for other applications due to their unique textural and structural characteristics. The most studied materials were metal oxides, such as $\mathrm{TiO}_{2}, \mathrm{SnO}_{2}, \mathrm{VO}_{2}$ and $\mathrm{ZnO}$.

Nanomaterials are an innovative research direction for the leather industry due to their high potential of replacing potentially toxic volatile organic materials and their ability to develop smart properties. Owing to its many advantages mainly involving most stable and active naturally occurring photocatalyst, $\mathrm{TiO}_{2}$ is, so far, seen as the best catalytic material for degradation of various soiling agents.

\section{RESULTS AND DISCUSSION}

Assortments of natural leather were obtained through ecological tanning processes, with specific characteristics of art and heritage book covers.
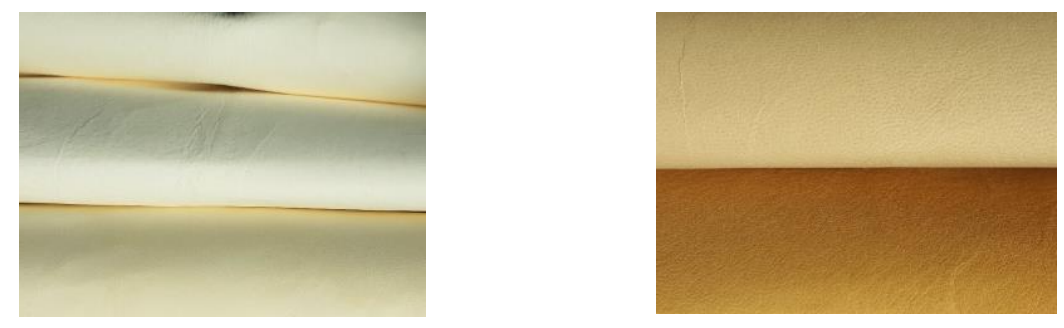

Figure 1. Leathers tanned with $\mathrm{Al}$ salts (left) and vegetable tanning extracts (right)

The leather samples were then functionalized with titanium dioxide $\left(\mathrm{TiO}_{2}\right)$ or nitrogen-doped titanium dioxide $\left(\mathrm{N}-\mathrm{TiO}_{2}\right)$ nanopowders, in various concentrations.

In order to determine the nanoparticles size and the stability of the dispersions, measurements were performed using the Dynamic Light Scattering (DLS) technique and the Zetasizer Nano ZS equipment. 


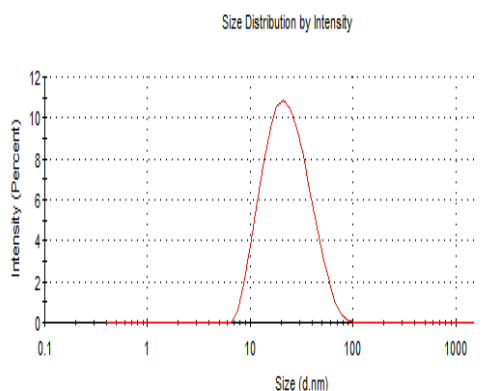

Zeta Potentilaldistibution

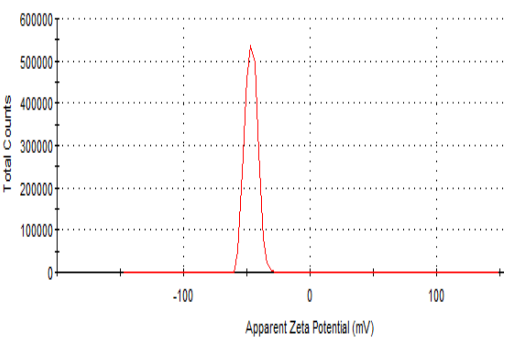

Figure 2. Size distribution (left) and Zeta potential (right) for simple $\mathrm{TiO}_{2}$ nanoparticles
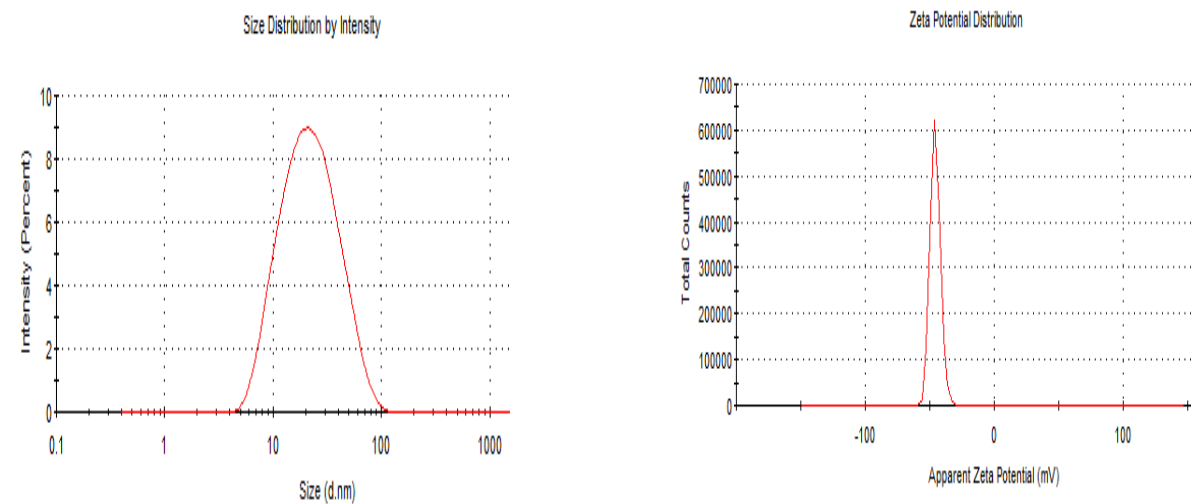

Figure 3. Size distribution (left) and Zeta potential (right) for $\mathrm{N}$-doped $\mathrm{TiO}_{2}$ nanoparticles

$\mathrm{TiO}_{2}$ nanoparticles have sizes of approximately $25 \mathrm{~nm}$, those doped with $\mathrm{N}$ of $30 \mathrm{~nm}$ and the Zeta potential is $-45 \mathrm{mV}$ for simple nanoparticles and $-47 \mathrm{mV}$ for doped ones, which suggests a high stability of the samples.

To simulate dirt in real conditions, 4 types of soiling agents were applied onto leather surfaces: tea (t), coffee (c) and beetroot extract (b), which were applied to the skin in the form of drops $(0.01 \mathrm{~mL})$ and pen paste $(\mathrm{p})$, applied as a hatch.

Depending on the type of applied treatment, the samples were exposed to irradiation in a photoreactor (Labtech) with different light sources, as follows: $\mathrm{TiO}_{2}$-treated leathers were exposed to UV light provided by Osram L BL UVA 8W / 78 G5 lamps and the leathers treated with $\mathrm{N}-\mathrm{TiO}_{2}$, due to the known efficiency of this photocatalyst in these conditions, were exposed to visible light, using Osram L 8W / 756 Cool Daylight lamps.

The leather samples were exposed for 192 hours, performing 4 measurements, initial, after 48, 96 hours and at the end.

In the Tables below are presented the results, before and after irradiation. 
The Influence of $\mathrm{TiO}_{2}$ and $\mathrm{N}-\mathrm{TiO}_{2}$ Nanopowders in Natural Leather Finishing for Heritage or Modern Binding

Table 1. Photographic images of leather samples treated with $\mathrm{N}-\mathrm{TiO}_{2}$ and exposed to Vis irradiation

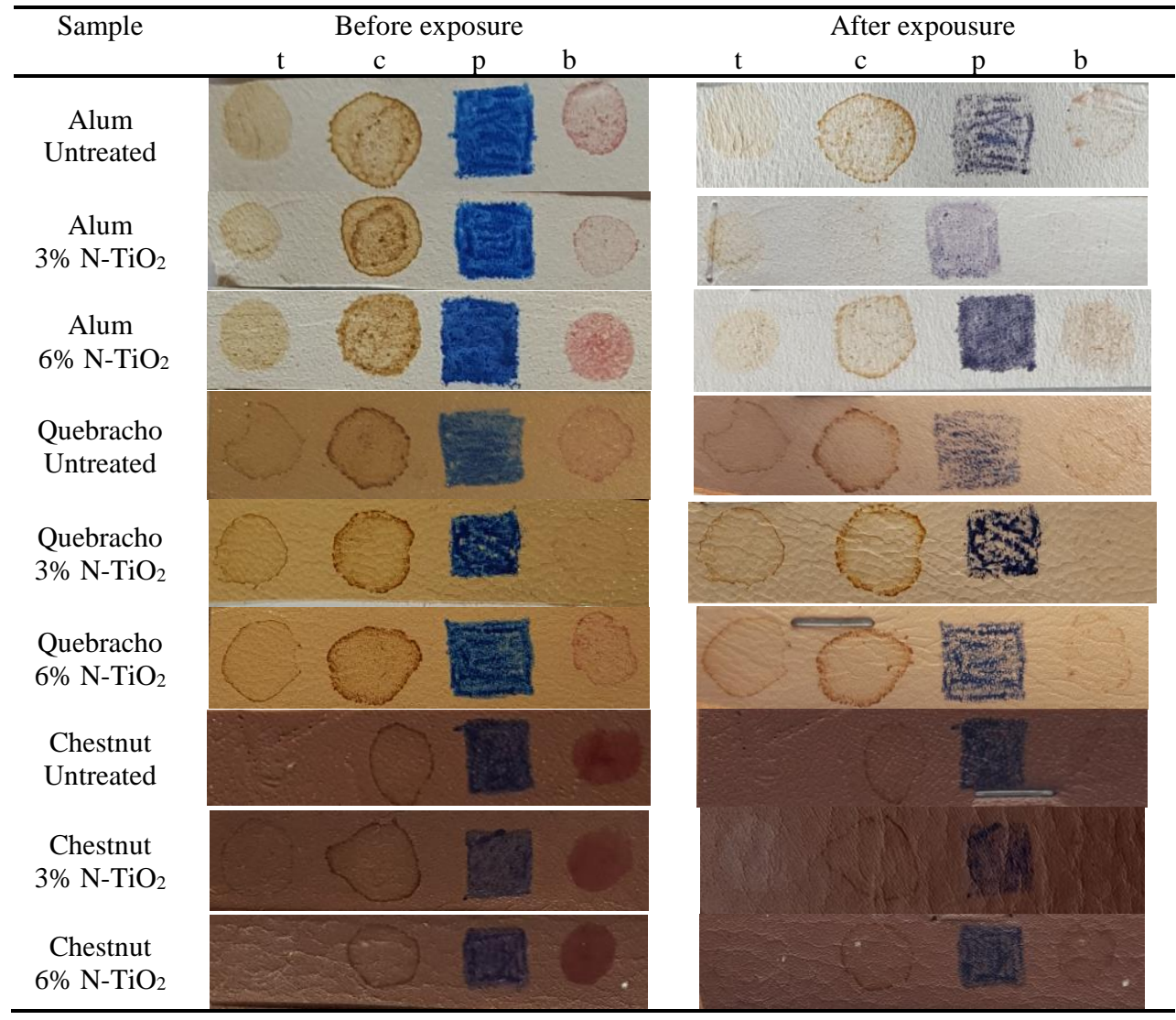

Vis lighting irradiation for 192 hours determine the photodegradation of all 4 types of soiling agents from the surface of functionalized leathers, as can be seen in Table 1.

Table 2. Photographic images of leather samples treated with $\mathrm{TiO}_{2}$ and exposed to UV irradiation

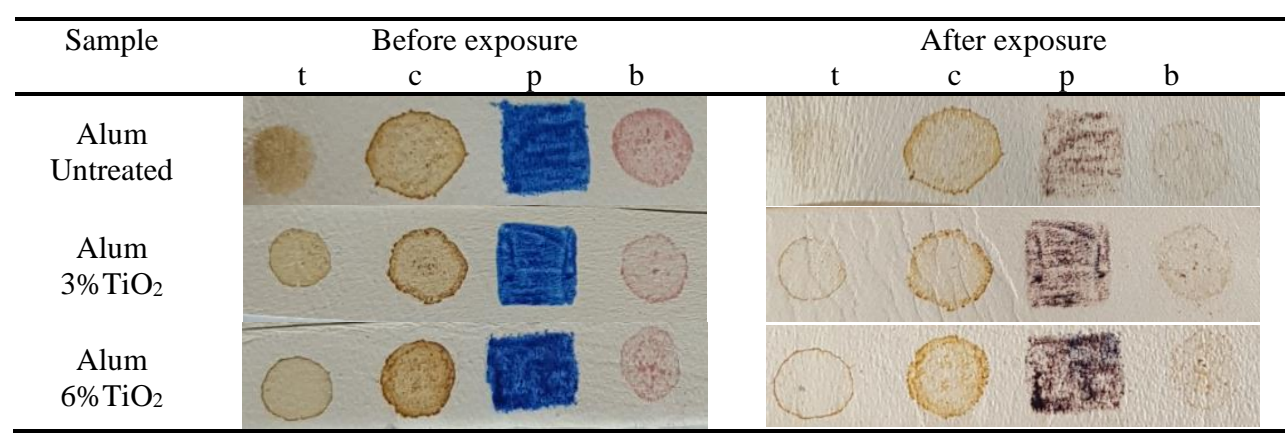

https://doi.org/10.24264/icams-2020.V.3 


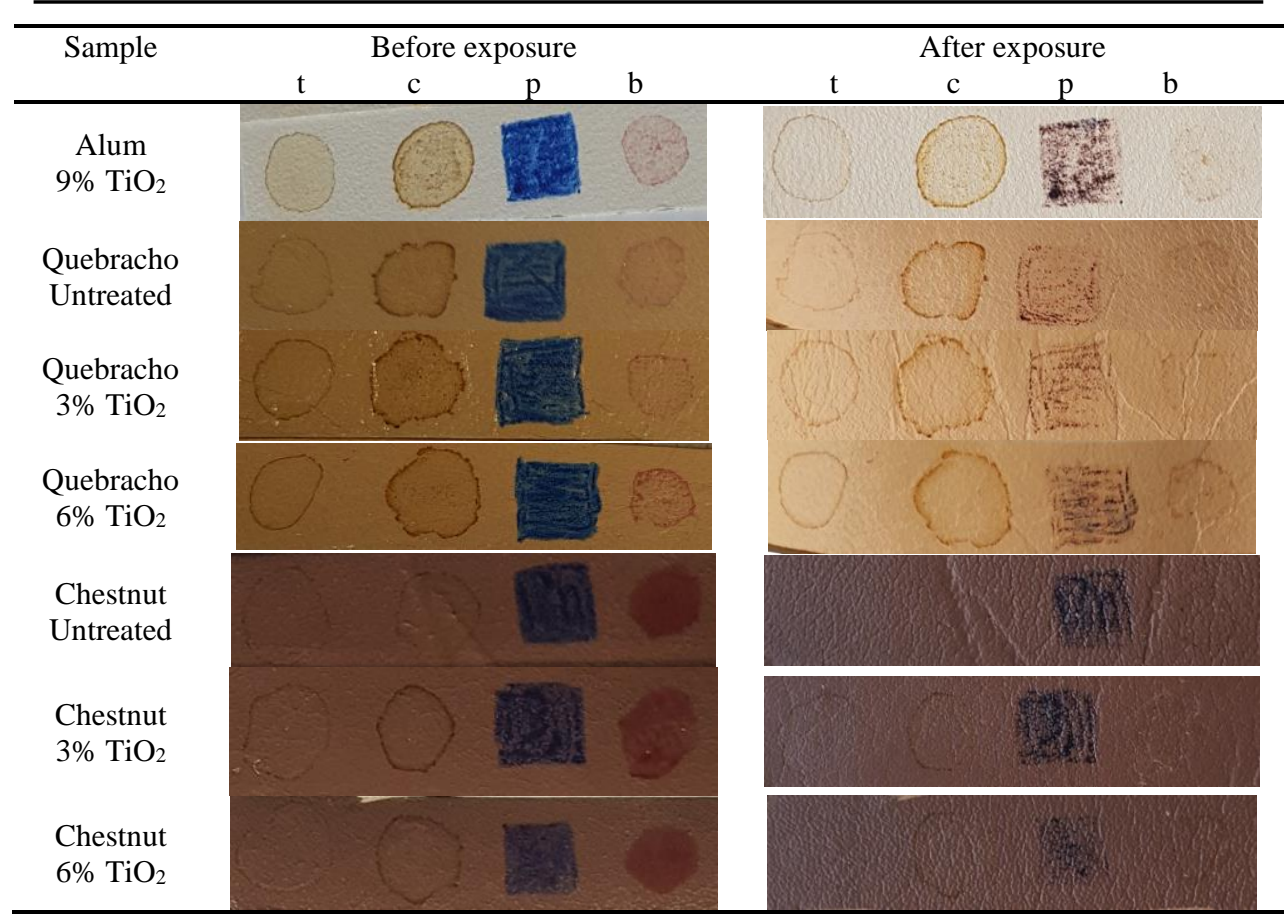

It can be observed that after 192 hours of UV irradiation, all 4 types of contaminants have undergone color changes, which suggests the efficiency of photocatalysts.

The evaluation of the photocatalytic degradation of the dyes was performed by the CIELab technique with a Datacolor Check II spectrophotometer, following the color change by recording the parameters $\mathrm{L} *$ (brightness), $\mathrm{a} *$ (red-green), $\mathrm{b} *$ (yellow-blue), $\mathrm{C} *$ (chrome) and $\mathrm{h} *$ (hue angle). To demonstrate dyes degradation, the parameter $\mathrm{L}$ was selected, which measures the brightness of a color from completely opaque (0) to completely transparent (100), which best highlights the color variation.

From both photographic images and CIELab analysis, can be observed the efficiency of the $\mathrm{N}-\mathrm{TiO}_{2}$ photocatalysts, following the irradiation with Vis light. The best results were obtained as follows:

$>$ in the case of tea stains, for the leather sample tanned with Quebracho and functionalized with $3 \% \mathrm{~N}-\mathrm{TiO}_{2}$ nanoparticles, where the $\mathrm{L}$ parameter varies from 68 to 74, which suggests the degradation of the dye;

$>$ for coffee stains, for leather samples tanned with Alum and functionalized with $3 \% \mathrm{~N}-\mathrm{TiO}_{2}$ nanoparticles; $\mathrm{L}$ parameter varies from 78 to 94 ;

$>$ the pen stain was mostly discolored in the case of the Alum tanned leather sample and functionalized with $3 \% \mathrm{~N}-\mathrm{TiO}_{2}$ nanoparticles; the $\mathrm{L}$ parameter varies from 51 to 80 ;

$>$ for beet extract stain, for the leather sample tanned with Alum and functionalized with $6 \% \mathrm{~N}-\mathrm{TiO}_{2}$ nanoparticles, the $\mathrm{L}$ parameter varies from 84 to 87 .

Simple $\mathrm{TiO}_{2}$ nanoparticles have also been shown to be effective in the case of UV irradiation, an aspect that results from both photographic images and CIELab analysis.

For each type of soiling agent, the best results were obtained as follows: 
$>\quad$ in the case of tea stains, for the leather tanned with Alum and functionalized with $3 \% \mathrm{TiO}_{2}$ nanoparticles, the $\mathrm{L}$ parameter varies from 88 to 94 ;

$>$ for coffee stains, in the case of the leather tanned with Alum and functionalized with $9 \% \mathrm{TiO}_{2}$ nanoparticles, where the parameter $\mathrm{L}$ varies from 80 to 92;

$>$ pen stain was discolored mostly in the case of Alum tanned leather sample and functionalized with $9 \% \mathrm{TiO}_{2}$ nanoparticles; $\mathrm{L}$ parameter varies from 48 to 67 ;

$>$ for beet extract stain, in the case of the sample tanned with Chestnut and functionalized with $6 \% \mathrm{TiO}_{2}$ nanoparticles, where the parameter $\mathrm{L}$ varies from 41 to 43 .

\section{CONCLUSIONS}

New doped nanoparticles with improved photocatalytic properties for heritage leather treatment were developed. $\mathrm{TiO}_{2}$ dispersions characterized by dynamic light scattering technique indicate that these are very stable and well dispersed.

The experimental results evidenced the photocatalytic activity of the new synthesized $\mathrm{TiO}_{2}$ nanoparticles, both in UV and visible light. The photocatalytic properties were confirmed by colorimetric measurements for the spots applied to the treated leather surface exposed to UV and visible light irradiation.

The obtained results suggest that $\mathrm{TiO}_{2}$ nanoparticles are a good candidate for the treatment of leathers for art and heritage book covers.

\section{Acknowledgement}

This work was supported by a grant of the Romanian Ministry of Research and Innovation, CCCDI - UEFISCDI, project number PN-III-P1-1.2-PCCDI-20170878/NR. 55PCCDI/2018, within PNCDI III.

\section{REFERENCES}

Bravo, A.G. (1964), Storia del cuoio e dell'arte conciaria, Assoc. Italiana dei Chimici del Cuoio.

Delort, R., (1993), L'Histoire de la Fourrure de l'Antiquité à nos jours, Lazarus.

Deselnicu, M., Olteanu, S. and Teodorescu, V. (1984), History of Leather Processing on the Romanian Territory (in Romanian), Technical Publishing House.

Higham, R.D. (1999), “The Tanner's Privilege - A Historical Review of the Tanner's Status in Different Societies", Journal of the Society of Leather Technologists and Chemists, 83, 25.

Larsen, R. (1994), "STREP Leather Project Evaluation of the Correlation between Natural and artificial Ageing of Vegetable Tanned Leathers", Final Report.

Larsen, R. (1997), "ENVIRONMENT Leather Project Deterioration and Conservation of Vegetable Tanned Leathers", Final Report.

Miu, L. et al. (2007), Patent RO122098 - Process for making natural leather for patrimony book binding.

Thomson, R. (2000), "Bookbinding leather; yesterday, today and perhaps tomorrow", Journal of the Society of Leather Technologists and Chemists, 85, 66.

*** Project CRAFT No BRST-CT98-5535 - "The development of Archival Quality of Leather".

*** Project PNCDI-Partnerships PN 91012/2007, Sustainable materials and technologies for conservation and restoration of heritage objects made of leather for the viability of cultural heritage at EU level, PelRestauro. 


\title{
PRODUCT DEVELOPMENT OF THE LEATHER GOOD USING WALTON'S
} MATRIX

\author{
ALINA IOVAN DRAGOMIR, ALEXANDRA LUCA \\ "Gheorghe Asachi" Technical University of Iasi, Faculty of Industrial Design and Business \\ Management, 28 D. Mangeron, Iasi, Romania, adragomir@tex.tuiasi.ro, \\ alexandra.luca@tuiasi.ro
}

\begin{abstract}
One of the most popular activities included in creative industries in Romania is leather goods craft. Nowadays the consumer needs are very high that's why the companies are facing many challenges and will resist on the market only those who will be the first to launch a certain product or surprise the market. In this paper was used Walton's matrix in order to identify a product that will provide a high profit and is useful for developing production strategies and the long-term development plan of the company's portfolio. This method was applied on a leather good product for women, made from leather. The opinions of the customers about the product, as well as the problems identified by them are very important for the development team in order to obtain new improved products. Modular matrix helps to obtain a technological design model from the design phase. The main advantage of the matrix is the fact that the development is focused on the module without having an impact on the relationships with the other parts. The modular matrix was developed using DSMMatrix program.
\end{abstract}

Keywords: leather goods, Walton matrix, product development

\section{THEORETICAL ASPECTS}

One of the most popular activities included in creative industries in Romania is leather goods craft.

The leather goods market has grown steadily worldwide, because the demand for various products has increased and also consumer needs. In this context, the companies are facing many challenges and will resist on the market only those who will be the first to launch a certain product or surprise the market. As the launch of an absolutely new product is very difficult, the most realistic market strategy remains to surprise the consumer by proposing a well-known product that has an element of novelty. In order to achieve this, an analysis of the company's product portfolio is proposed, and each product is distributed in one of Walton's matrix boxes (Walton, 2008), depending on the market share of each and the growth potential of the market.

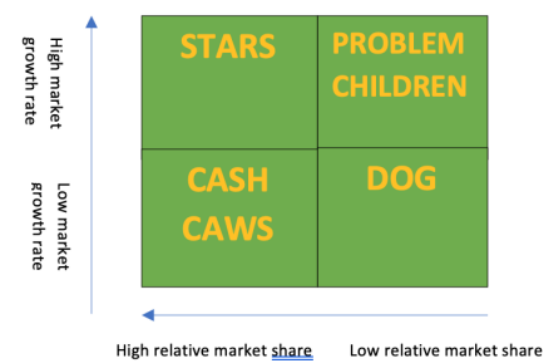

Figure 1. Walton's matrix (adapted of Walton G., 2008) 
The products in the Star category requires a high level of investment but are the products that bring the highest profit. If the market growth potential decreases, these products will fall into the Cash Cows category and investments will be lower.

The products in the Problem Children category have a small market share but operates in a growing market. Products in this category consume a lot of resources but the profit made is insignificant. This product can be passed to Cash Cows or Dogs.

The products in the Cash Cows category have a large market share, so they are wellknown products that do not require new investment. At the same time, it addresses a saturated market segment with low growth potential.

The Dogs category includes those products that also have a reduced market share but also address to a market segment without growth potential. It is a difficult task to move these products to another category.

Despite some shortcomings, the major advantages of the portfolio matrix are that: it helps to identify a product that will provide a high profit and is useful for developing production strategies and the long-term development plan of the company's portfolio.

\section{PRACTICAL ASPECTS}

\section{Applying the Method in Case of Developing a New Leather Goods Product}

An example of applying this method is starting from a product positioned in Walton's portfolio matrix in the Dog box. This products from the company's portfolio have low relative market share and low growth rate.

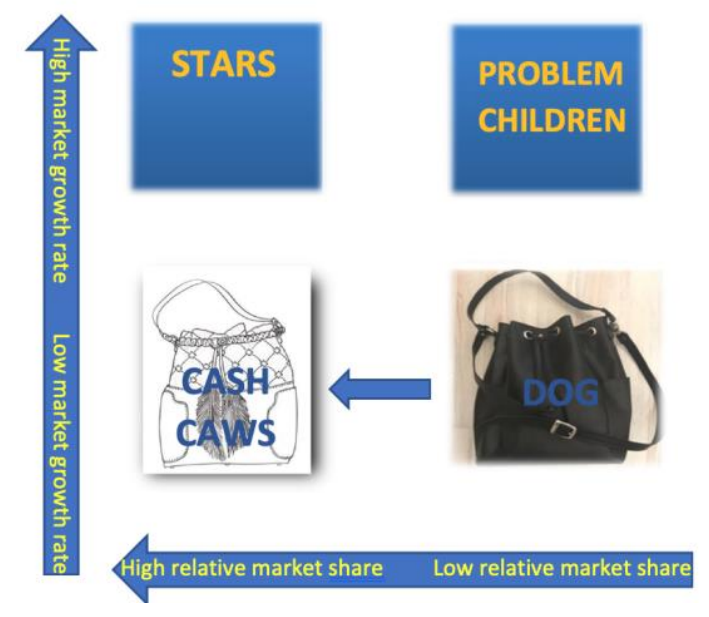

Figure 2. Walton's matrix for the studied product

The aim is to move the product to another box in the matrix. The most profitable solution, because it does not require very large investments, is to move to Cash cows, the product remains on a market with low growth potential but will satisfy as many consumer requirements as possible, so that will have a high relative market share.

The leather good product under analysis is a women's bag, bucket style, made from natural leather, and the product body is divided in the front, back and bottom. The 
product has two outer pockets, without locking system, with an adjustable shoulder strap and a smaller strap. The product closes by tightening a lace.

The opinions of the clients about this product, as well as the problems identified by them are very important for the development team. The most important consumer requirements, retained by the product development team are:

- large space

- many compartments

- hidden anti-theft pocket

- handsfree

- modern look, fresh.

The directions for development and improvement are established as follows:

-adding incorporeal attributes -modification and / or addition of

(concept, product names) decorative elements

-adding functional elements -repositioning on another niche

-dimensional change

To give the product a modern look, the concept chosen for the collection is ALEGRIA, inspired from the show with the same name, by Cirque Du Soleil. Alegria represents a struggle to preserve the power and energy of youth, with a decadent baroque aesthetic, in a nostalgic atmosphere and with a chromatic palette in warm, bright colors. Each product of the collection will have a name such as: joy, happiness.

\section{Functional Diversification}

For this purpose, a modular analysis of the initial product was made. For all the component parts, the relations and connections between them were established. The study implies a detailed knowledge of the technological elements of manufacturing process of the product. Table 1 presents the components of the model and the relationships between them.

Table 1. The components of the model and the relationships between them

\begin{tabular}{|c|c|c|c|c|c|}
\hline ID & Part name & Relationship & Part name & Relationship & ID \\
\hline 1 & Bottom & 2,3 & Side pocket & $14,11,12$ & 13 \\
\hline 2 & Front & $1,3,4,5,9,6,24$ & Piping & $11,12,13$ & 14 \\
\hline 3 & Back & $1,2,4,5,6,9,24$ & $\begin{array}{l}\text { Welt pocket } \\
\text { (bound) }\end{array}$ & $10,17,16$ & 15 \\
\hline 4 & $\begin{array}{l}\text { Right side } \\
\text { outer pocket }\end{array}$ & 2,3 & Bound pocket & $15,17,10$ & 16 \\
\hline 5 & $\begin{array}{l}\text { Left side } \\
\text { outer pocket }\end{array}$ & 2,3 & Zipper & $10,16,15$ & 17 \\
\hline 6 & Staple & $2,3,9,7$ & Handle & 22,24 & 18 \\
\hline 7 & Lace & 6,8 & $\begin{array}{l}\text { Adjustable small } \\
\text { strap }\end{array}$ & 23,21 & 19 \\
\hline 8 & Stopper & 7 & $\begin{array}{l}\text { Adjustable large } \\
\text { strap }\end{array}$ & 23,21 & 20 \\
\hline 9 & Lining Trim & $2,3,10,11,12,6$ & Buckle & 19,20 & 21 \\
\hline 10 & $\begin{array}{l}\text { Back side } \\
\text { lining }\end{array}$ & $11,12,9,15,16,17$ & Ring & 18,24 & 22 \\
\hline 11 & $\begin{array}{l}\text { Right front } \\
\text { side lining }\end{array}$ & $9,10,12,14,13$ & Hardware & 19,20 & 23 \\
\hline 12 & $\begin{array}{l}\text { Left front } \\
\text { side lining }\end{array}$ & $9,10,11,13,14$ & Tab & $2,3,22,18$ & 24 \\
\hline
\end{tabular}

https://doi.org/10.24264/icams-2020.V.4 
Based on the relationships established between the landmarks, a matrix (figure 3) is elaborated where the black dots highlight the connections between the elements.

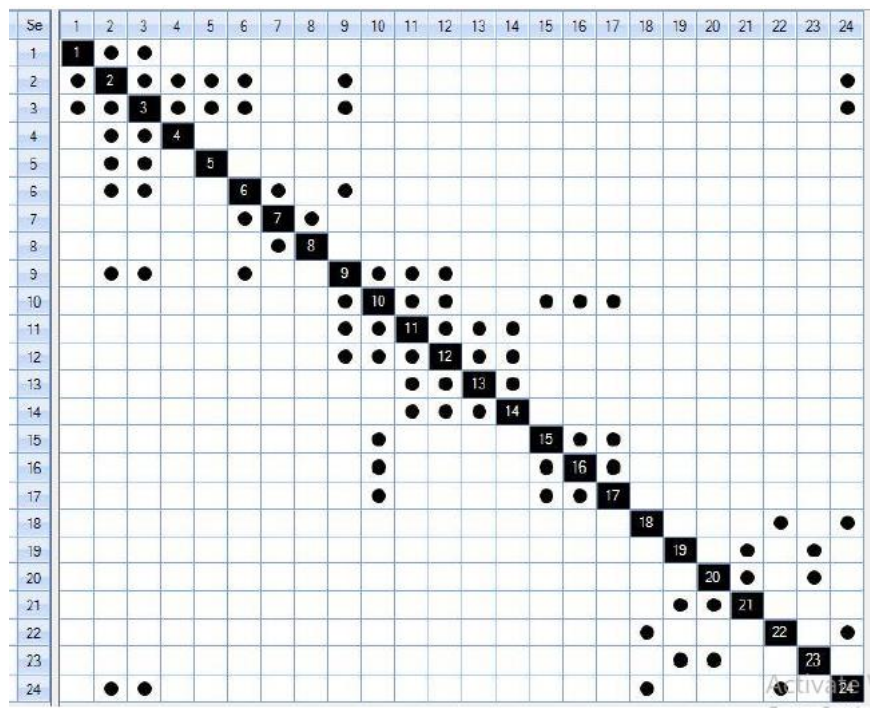

Figure 3. Initial matrix for Bucket basic model

To transform the initial structural matrix from the previous figure, the DSMMatrix program was used, thus obtaining a rearrangement of the rows, so that the dots corresponding to the relationships between the elements are grouped as close as possible to the diagonal. Therefore, a technological design model is obtained from the design stage of the product.

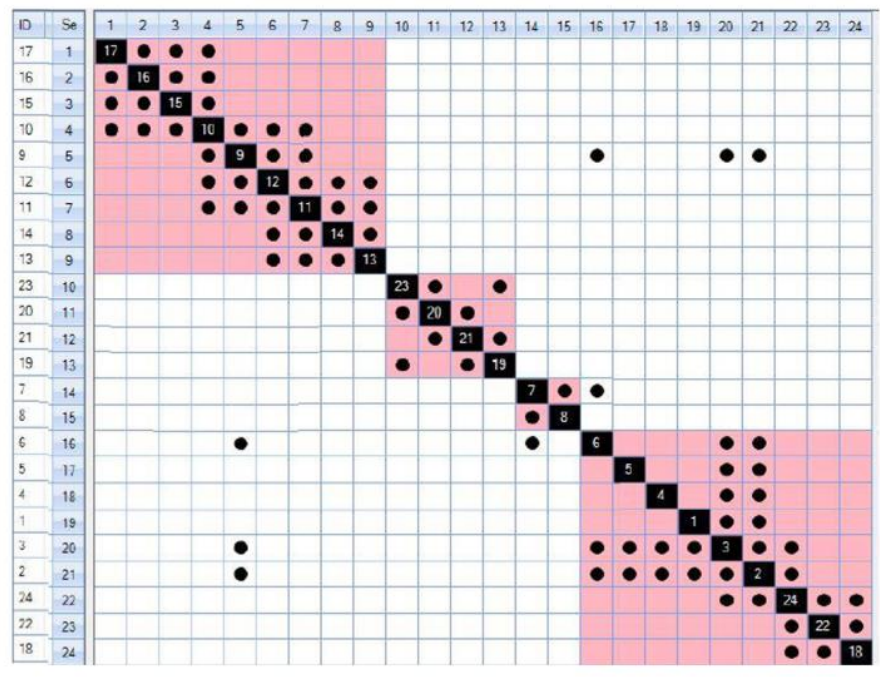

Figure 4. Modular matrix for Bucket basic model 
By segmenting and arranging the initial matrix, 4 modules are obtained (figure 4 ):

Module 1: inner subassembly, lining

Module 2: support system

Module 3: lace with stopper

Module 4: body (front, back, bottom)

All 4 modules are useful in the diversification and improvement phase. In order to obtain a collection, modifications can be made on 1,2 or all the modules or combinations between them.

For exemplification, 2 modules were chosen, respectively 1 and 4, which were distributed on the lines and columns of a matrix. At the intersection of each line with each column resulted in a new variant of the initial model.

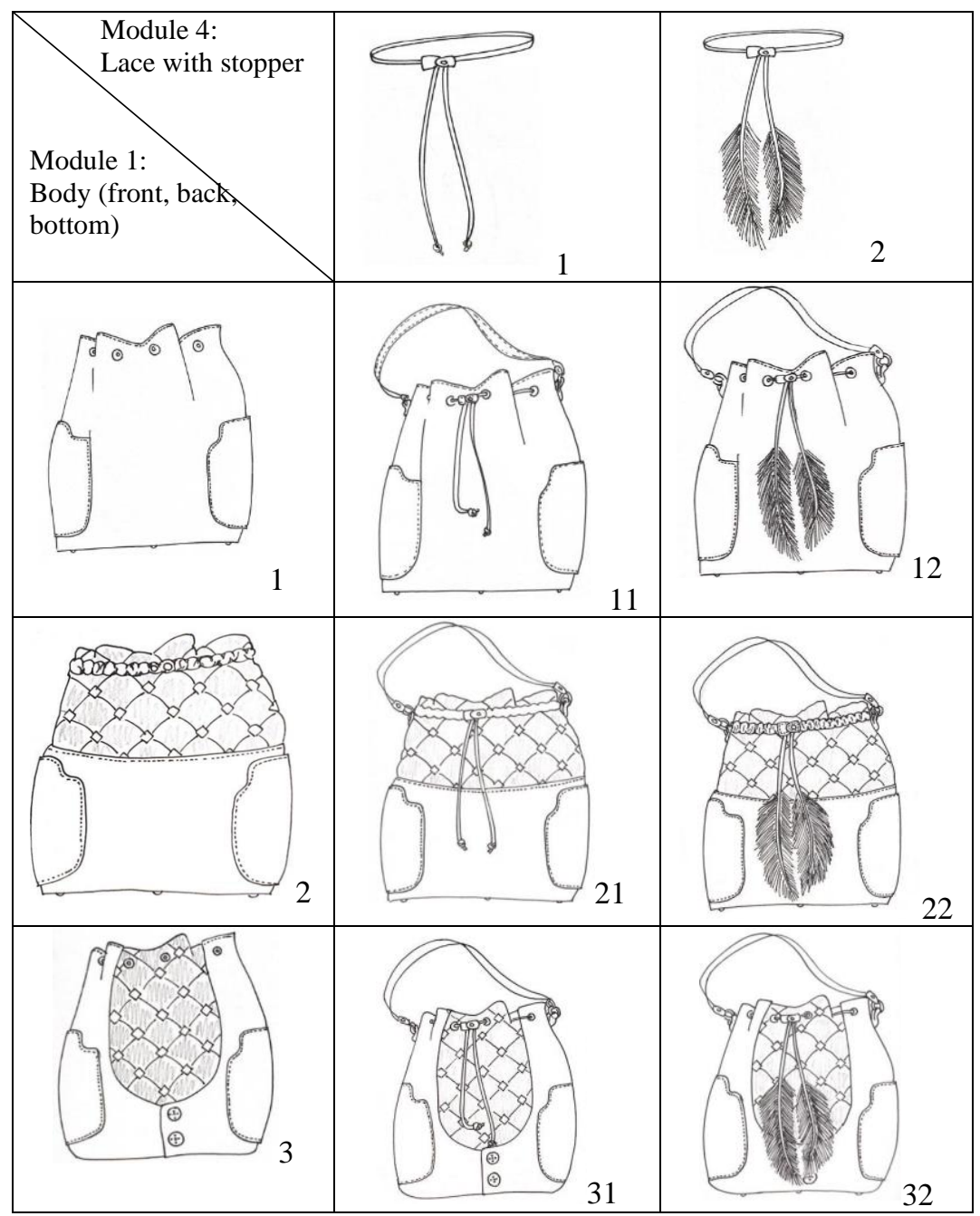

Figure 5. The matrix of the Bucket bag family models 
Figure 5 presents the Bucket bag family obtaining 6 different models with the same functionality without significant changes in the technological and manufacturing process.

\section{CONCLUSIONS}

Walton's matrix is a useful instrument for managing the product portfolio of a company. Also, it helps to decide which product can be maintained, removed or improved. This involve all the departments and the development strategy of the company.

Modular matrix helps to obtain a technological design model from the design phase. The main advantage of the matrix is the fact that the development is focused on the module without having an impact on the relationships with the other parts.

The product development includes 2 components: functional and esthetic. For the analyzed product it was presented the functional development. Development stage should be complete with an esthetic analyze included the chromatic pallet trends according to Lineapelle (International Leather Fair).

\section{Acknowledgements}

This work has been carried out as part of a Master programme (Product Development of Footwear and Leather Goods) at Faculty of Industrial Design and Business Management, Iasi, Romania. Acknowledgements are also due to Yolanda Blazquez Lopez.

\section{REFERENCES}

Gurbuz, E. (2018), “Theory of new product development and its application”, in: Sonyel Oflazoglu (ed.), Marketing, https://doi.org/10.5772/intechopen.74527.

Harnagea, F. (2000), Structure and technology of leather goods (in Romanian), Ed. Cermi, Iași.

Mihai, A. and Pastina, M. (2009), Methods used in conceptualization and development of footwear (in Romanian), Ed. Performantica, Iasi.

Walton, G. (2008), "Theory research and practice in library management 2: The balanced product portfolio", Library Management, 28(4/5), 262-268, https://doi.org/10.1108/01435120710744218. 


\title{
ASPECTS REGARDING THE EFFECTIVENESS OF VOLATILE OILS OF THE TANACETUM VULGARE KIND IN THE CONSERVATION OF HERITAGE OBJECTS
}

\author{
LUCRETIA MIU ${ }^{1}$, RODICA-ROXANA CONSTANTINESCU ${ }^{1}$, ALPASLAN DURMUŞ \\ KAYA $^{2}$, EMANUEL HADIMBU ${ }^{1}$, CLAUDIU ȘENDREA ${ }^{1}$, MARIA-CRISTINA MICU, \\ SIMONA-MARIA PĂUNESCU ${ }^{1}$, IULIA MARIA CANIOLA ${ }^{1}$, ELENA BADEA ${ }^{1,3^{*}}$ \\ ${ }^{1}$ INCDTP - Division Leather and Footwear Research Institute, Bucharest, Romania \\ ${ }^{2}$ Hatay Mustafa Kemal University, Faculty of Agriculture, Field Crops Department, 31034, \\ Antakya-Hatay, Turkey \\ ${ }^{3}$ Department of Chemistry, Faculty of Sciences, University of Craiova, Craiova, Romania, \\ elena.badea@unito.it
}

\begin{abstract}
The paper refers to a composition with antifungal and antibacterial effect in order to test new materials for preserving heritage objects on collagen support with bactericidal/antifungal role, essential oils-based from Vetrice (Tanacetum vulgare, Compositae family), having antifungal and antibacterial properties. The biocidal effect of plant extracts is due to the presence of constituent agents, such as alcohols, ethers, phenols, aldehydes, ketones, which makes them extremely effective against a wide range of microbial strains. The essential oil was obtained by boiling the plants through continuous hydrodistillation with Clevenger refrigerant. The testing of the antimicrobial efficiency of the plant extracts was performed on two strains of pathogenic fungi, respectively Trichophyton interdigitale and Epidermophyton floccosum. The microbial inoculum was mixed with the plant extract from various sources (leaves, flowers and mixed flower-leaves). All extracts were tested in duplicate according to the specific test standards, and the results were expressed as a mean percentage and logarithmic reduction between the readings on the two Petri plates corresponding to each sample. To quantify the antimicrobial efficacy, the degree of microbial and logarithmic reduction of each sample was calculated, relative to the initial cell concentration. The results of antimicrobial tests showed a high antifungal character of the extracts obtained from flowers, leaves and mixed flower-leaves.
\end{abstract}

Keywords: preserving heritage objects, antifungal and antibacterial effect

\section{INTRODUCTION}

The paper refers to a study on the antifungal and antibacterial effect of Tanacetum vulgare essential oil in the curative and preventive conservation of heritage objects on collagen support.

Animal hide, but also tanned leather, due to its structure and chemical composition, which includes a large amount of proteins (collagen, elastin, albumin), mucopolysaccharides, water, fat and mineral salts is an excellent culture medium for the development of biological factors-microorganisms (bacteria, molds), which damage both the surface and the structure of the skin.

Since ancient times, essential oils have been widely used for bactericidal, fungicidal, insecticidal, medicinal and cosmetic applications. They are currently used in the pharmaceutical, sanitary, cosmetic, agricultural and food industries. Plant-derived essential oils have great potential as antimicrobial agents against a wide range of pathogens. The biocidal effect of plant extracts is due to the presence of constituent agents, such as alcohols, ethers, phenols, aldehydes, ketones, which makes them extremely effective against a wide range of microbial strains. Fast and sensitive detection of dermatophytes is very helpful because most superficial fungal infections are caused by this group of fungi (Kupsch et al., 2016; Garg et al., 2009). Their exact 
identification is essential for choosing the most appropriate antimicrobial treatment and for tracking the sources of infection, thus helping to avoid reinfection (Jung et al., 2009).

Various emollient and conservation compositions based on lanolin, hoof oil, beeswax and hexane are known, with cedar oil as an antifungal preservative [British Museum dressing]. Research is also known on the use of lavender oil to preserve and protect the atmosphere in archives and libraries that are effective against insects and molds. It is known that in libraries and warehouses, collagen-based objects are subject to the attack of mold and bacteria due to variable temperature conditions, degradation of skin structure, changes in acidity.

Tanacetum vulgare (family Asteraceae), popularly called Vetrice is a species native to Europe (up to one meter tall, strong chrysanthemum-like scent, specific, dark green, with beautiful intense yellow flowers), but widespread also in the spontaneous flora of Asia and North America. This plant has been known since ancient times as a therapeutic remedy, being cultivated in gardens, having uses in medicine (antibacterial, antiseptic, anti-inflammatory) but also in cosmetics, food (spice), obtaining perfumes, insecticides, preservatives, all these properties being obtained due to its composition rich in bioactive components such as polyphenols, flavonoids and tannins. The basic chemical composition of the volatile oil from Tanacetum vulgare is complex, depending on the morpho-anatomical part of the plant (leaves or flowers; the stem has no volatile oils), climate (temperature, amount of precipitation) of soil, altitude, etc. For the extraction process, the plant was separated into flowers and leaves, the extractions being performed separately for each of them with the help of a Clevenger extractor. There are numerous studies on the composition of essential oils extracted from Tanacetum vulgare and which highlight the potential of their use especially in medicine, pharmacy and cosmetics (Muresan, 2015; Boucher et al., 2017; Kumar et al., 2016; Ulukanli et al., 2017; Marian et al., 2013).

The effectiveness of the active principles extracted Tanacetum vulgare has been studied on Epidermophyton and Trichophyton which are fungi that cause skin diseases in animals and humans. Conventional methods for identifying these fungi are quick and simple, but they are qualitatively comparable to molecular methods (Grumbt et al., 2013). Both strains were selected due to their enzymatic structure.

Trichophyton interdigitale is an anthropophilic fungus that is a common cause of tinea pedis, especially vesicular type, tinea corporis, and sometimes superficial nail invasion. Trichophyton interdigitale is also responsible for the condition called "athlete's foot" that affects the skin between the toes (Wisselink et al., 2011).

Epidermophyton floccosum is an anthropophilic dermatophyte with a worldwide distribution and one of the most common causes of dermatophytosis in healthy individuals. It infects the skin (tinea corporis, tinea cruris, tinea pedis) and nails (onychomycosis). Infection is limited to the stratum corneum of the epidermis because the microorganism does not have the ability to penetrate viable tissues of the immunocompetent host (Gueho et al., 2015).

The use of conservation products aims to lubricate the fibers of tanned leather, with a role in reducing fragility and the tendency to break, preventing them from sticking to repeated drying. The products offer softness, suppleness and reduce water absorption. The role of the products used in the conservation of heritage leather is to surround the fibrillar elements of the tanned leather, so as to give it the softness and elasticity 
necessary for a proper use, and also to offer protection in case of fungicidal and bactericidal attacks.

\title{
EXPERIMENTAL PART
}

\begin{abstract}
Materials
In order to test new materials for the conservation of heritage objects on collagenic support with bactericidal / antifungal role, essential oils were obtained from Vetrice (Tanacetum vulgare, fam. Compositae), a perennial plant that sows itself, having antifungal and antibacterial properties. The chopped plant, which can be dried or green, has been extracted in a Clevenger refrigerant. Essential oils are mixtures of 5, 10 to tens and even a hundred compounds. In an essential oil each compound has specific biological properties and participates in the overall harmonization of the odor and specific activity (antimicrobial, antifungal, regenerative, antibacterial) of the mixture. Extracts of essential oils with various origins were tested, as follows: Flower extract, with F notations; Leaf extract, with L notations; Mixed flower and leaf extract, with $\mathrm{M}$ notations. Each extract was tested in 2 concentrations, the dilutions being made in paraffin oil, respectively: $1 \%$ and $3 \%$;
\end{abstract}

\section{Method}

Testing of the extracts was performed in a 1: 1 ratio with the microbial inoculum, for a final solution volume of $1 \mathrm{~mL}(500 \mu \mathrm{L}$ extract as such or diluted $+500 \mu \mathrm{L}$ microbial inoculum). Fresh cultures were obtained from each strain, by growing in nutritious Czapek-Dox broth, at $28{ }^{\circ} \mathrm{C}$ for 14 days, representing the stock culture. For testing, two decimal dilutions in paraffin oil $\left(10^{-2}\right)$ were made from each culture and the cell concentration in the inoculum used was $9.8 \times 10^{3} \mathrm{UFC} / \mathrm{mL}$ (Colony Forming Units) for Trichophyton interdigitale (with $\mathrm{Ti}$ notations). and $8.92 \times 10^{3} \mathrm{UFC} / \mathrm{mL}$ for Epidermophyton floccosum (with Ef notations).

The initial cell concentration was previously determined by decimal dilutions $\left(10^{-4}\right)$ in sterile deionized water, and from the last dilution, for each strain, $100 \mu \mathrm{L}$ were taken and spread on Czapek-Dox agarized nutrient medium. The counts on the plate were performed after $24 \mathrm{~h}$ of incubation, these being kept as a reference for the cellular developments in the control sample from the sample set (taken as the sample performed in paraffin oil). Thus, plates with cell density similar to that of dilution $10^{-4}$ were considered to have similar UFC values $\left(9.8 \times 10^{3} \mathrm{UFC} / \mathrm{mL}\right.$-Trichophyton interdigitale and $8.92 \times 10^{3}$ UFC / mL-Epidermophyton floccosum). This method was chosen due to the very low dilution $\left(10^{-2}\right)$ performed in the case of the inoculum that was tested on extracts, respectively of a high cellular concentration, which makes it extremely difficult to count filamentous fungal colonies in plates with abundant development.

The experiments on extracts were performed in Eppendorf tubes, previously sterilized at $121{ }^{\circ} \mathrm{C}$, for $15 \mathrm{~min}$. The extracts were not sterilized, in order to avoid the volatilization of their active principles. The microbial inoculum was mixed with the plant extract, from various origins (flowers, leaves and mixed flower leaves), both the microbial inoculum and the extract having constant volumes $(500 \mu \mathrm{L})$, only the concentration of extract and paraffin oil varying in these $500 \mu \mathrm{L}$. The microbial inoculum solution was prepared in paraffin oil to rule out its possible antimicrobial activity on microbial cells. 


\section{RESULTS AND DISCUSSIONS}

All extracts were tested in duplicate according to the specific test standards, and the results were expressed as a mean percentage and logarithmic reduction between the readings on the two Petri plates corresponding to each sample. Plate counts were performed after $24 \mathrm{~h}$ incubation to detect colony-forming cell units. Pictures of the Petri dishes were taken after $48 \mathrm{~h}$ incubation. To quantify the antimicrobial efficacy, the degree of microbial and logarithmic reduction of each sample was calculated, relative to the initial cell concentration (Table 1).

Table 1. Antifungal test results

\begin{tabular}{|c|c|c|c|c|c|}
\hline Sample & Petri Image & Mean 2 plates & $* \mathrm{R} \%$ & $* * \log _{10}$ red. & Source \\
\hline \multicolumn{6}{|c|}{ Trichophyton interdigitale } \\
\hline M & & $\begin{array}{c}(9800+9800) / 2 \\
\mathrm{M}=9800 \text { colonies } \\
=> \\
9,8 \times 10^{3} \mathrm{UFC} / \mathrm{mL}\end{array}$ & - & - & $\begin{array}{c}\text { Untreated } \\
\text { Sample }\end{array}$ \\
\hline 1 & & $\begin{array}{c}(13+13) / 2 \\
\mathrm{M}=130 \text { colonies } \\
=> \\
13 \times 10^{1} \mathrm{UFC} / \mathrm{mL}\end{array}$ & 95,55 & 1,88 & $1 \% \mathrm{FTi}$ \\
\hline 2 & & $\begin{array}{c}(14+14) / 2 \\
\mathrm{M}=140 \text { colonies } \\
=> \\
14 \times 10^{1} \mathrm{UFC} / \mathrm{mL}\end{array}$ & 95,5 & 1,85 & $1 \% L T i$ \\
\hline 3 & & $\begin{array}{c}(19+20) / 2 \\
\mathrm{M}=1950 \text { colonies } \\
=> \\
19,5 \times 10^{3} \mathrm{UFC} / \mathrm{mL}\end{array}$ & 92,3 & 1,7 & $1 \% M T i$ \\
\hline 4 & & $\begin{array}{c}(3+4) / 2 \\
\mathrm{M}=35 \text { colonies }=> \\
3,5 \times 10^{1} \mathrm{UFC} / \mathrm{mL}\end{array}$ & 99,65 & 1,45 & $3 \% \mathrm{FTi}$ \\
\hline 5 & & $\begin{array}{c}(0+1) / 2 \\
\mathrm{M}=5 \text { colonii } \\
=> \\
5 \mathrm{UFC} / \mathrm{mL}\end{array}$ & 100 & 3,26 & $3 \% L T i$ \\
\hline 6 & & $\begin{array}{c}(1+1) / 2 \\
\mathrm{M}=10 \text { colonies } \\
\Rightarrow \\
1 \times 10^{1} \mathrm{UFC} / \mathrm{mL}\end{array}$ & 99,9 & 2,99 & $3 \% M T i$ \\
\hline
\end{tabular}


ICAMS $2020-8^{\text {th }}$ International Conference on Advanced Materials and Systems

\begin{tabular}{|c|c|c|c|c|c|}
\hline Sample & Petri Image & Mean 2 plates & $* \mathrm{R} \%$ & *** $\log _{10}$ red. & Source \\
\hline \multicolumn{6}{|c|}{ Epidermophyton floccosum } \\
\hline M & & $\begin{array}{c}(8920+8920) / 2 \\
\mathrm{M}=8920 \text { colonies } \\
\Rightarrow> \\
8,92 \times 10^{3} \mathrm{UFC} / \mathrm{mL}\end{array}$ & & & $\begin{array}{c}\text { Untreated } \\
\text { Sample }\end{array}$ \\
\hline 7 & & $\begin{array}{c}(1+1) / 2 \\
\mathrm{M}=10 \text { colonies }=> \\
1 \times 10^{1} \mathrm{UFC} / \mathrm{mL}\end{array}$ & 99,9 & 2.95 & $1 \% \mathrm{FEf}$ \\
\hline 8 & & $\begin{array}{c}(12+14) / 2 \\
\mathrm{M}=130 \text { colonies }=> \\
13 \times 10^{2} \mathrm{UFC} / \mathrm{mL}\end{array}$ & 95,5 & 1,84 & $1 \% L E f$ \\
\hline 9 & & $\begin{array}{c}(14+16) / 2 \\
\mathrm{M}=\text { colonies } \Rightarrow \\
15 \times 10^{2} \mathrm{UFC} / \mathrm{mL}\end{array}$ & 95,55 & 1,78 & $1 \% M E f$ \\
\hline 10 & & $\begin{array}{c}(2+2) / 2 \\
\mathrm{M}=2 \text { colonies }=> \\
2 \times 10^{1} \mathrm{UFC} / \mathrm{mL}\end{array}$ & 99,77 & 2.65 & $3 \%$ FEf \\
\hline 11 & & $\begin{array}{c}(1+1) / 2 \\
\mathrm{M}=10 \text { colonies } \\
\Rightarrow> \\
1 \times 10^{1} \mathrm{UFC} / \mathrm{mL}\end{array}$ & 99,9 & 2,95 & $3 \%$ LEf \\
\hline 12 & $*$ & $\begin{array}{c}(8+9) / 2 \\
\mathrm{M}=125 \text { colonies }=> \\
12,5 \times 10^{1} \mathrm{UFC} / \mathrm{mL}\end{array}$ & 99,85 & 1,86 & $3 \% M E f$ \\
\hline
\end{tabular}

* Antibacterial activity ratio $(R) \quad R=\frac{C_{\mathrm{t}}-T_{\mathrm{t}}}{C_{\mathrm{t}}} X 100 \% ; C_{t}=$ is the average number of colonies of two control samples after $24 \mathrm{~h}$ or the specified incubation period, expressed as CFU/ml; $T_{t}=$ is the average number of colonies of two test samples after $24 \mathrm{~h}$ or the specified incubation period, expressed as CFU/ml. The percentage reduction can be interpreted in logarithmic reduction, as follows: $90 \%$ reduction $=1 \log$ reduction $(1,000,000$ cells reduced to 100,000 represents $1 \log$ reduction); $99 \%$ reduction $=2 \log$ reduction $(1,000,000$ cells reduced to 10,000 represents $2 \log$ reduction); $99.9 \%$ reduction $=3 \log$ reduction $(1,000,000$ cells reduced to 1000 represents $3 \log$ reduction); $99.99 \%$ reduction $=4 \log$ reduction $(1,000,000$ cells reduced to 100 represents $4 \log$ reduction)

** Antibacterial activity value (A), $A=\lg C_{\mathrm{t}}-\lg T_{\mathrm{t}} \quad$ where $A$ - is the antibacterial activity value; $\lg C_{\mathrm{t}}$ is the average number of colonies of the two control samples after incubation, 
expressed as $\mathrm{CFU} / \mathrm{ml} ; \lg T_{\mathrm{t}}$ is the average number of colonies of the two control samples immediately after incubation, expressed as $\mathrm{CFU} / \mathrm{ml}$.

\section{CONCLUSIONS}

The results of antimicrobial tests presented a strong antifungal character of the extracts obtained from flowers, leaves and mixed flower leaves. The extracts obtained from the leaf showed antimicrobial activity at a concentration $3 \%$ better than $1 \%$, this being confirmed on both strains. At the same time, it is observed that the sample with $3 \%$ leaf extract (L) has the same influence depending on the strain tested, Trichophyton interdigitale and Epidermophyton floccosum. At the same time, it is highlighted that the entire antifungal character is due to the extracts, and not to the paraffin oil, which does not inhibit the degree of cell viability.

\section{Acknowledgements}

This work was supported by a grant of the Romanian Ministry of Research and Innovation, CCCDI - UEFISCDI, project number PN-III-P1-1.2-PCCDI-20170878/NR. 55PCCDI/2018, within PNCDI III.

\section{REFERENCES}

Boucher, M.-A. et al. (2017), "Anti-Inflammatory, Antioxidant, Antibiotic, and Cytotoxic Activities of Tanacetum vulgare L. Essential Oil and Its Constituents", Medicines, 4(34), https://doi.org/10.3390/medicines4020034.

Garg, J. et. al (2009), "Rapid detection of dermatophytes from skin and hair", BMC Res Notes, https://doi.org/10.1186/1756-0500-2-60.

Grumbt, M. et al. (2013), "Keratin degradation by dermatophytes relies on cysteine dioxygenase and a sulfite efflux pump", J. Invest. Dermatol., 133(6), 1550-1555, https://doi.org/10.1038/jid.2013.41.

Gueho, E. et al. (2015), "Existence of ornamentations on macroconidia and hyphae of Epidermophyton floccosum", Annales de l'Institut Pasteur / Microbiologie, 136(2), Supplement B, 195-203, https://doi.org/10.1016/S0769-2609(85)80044-2.

Jung, S. et al. (2009), "Epidermophyton fungal keratitis following laser-assisted subepithelial keratectomy", Journal of Cataract \& Refractive Surgery, 35(12), 2157-2160, https://doi.org/10.1016/j.jcrs.2009.06.035.

Kumar, V. et al. (2016), "Chemical composition and antibacterial activity of essential oils of Tanacetum", Int. J. Cuur. Microbial. Appl. Sci., 5, 836-884, https://doi.org/10.20546/ijcmas.2016.510.091.

Kupsch, C. et al. (2016), "The agony of choice in dermatophyte diagnostics-performance of different molecular tests and culture in the detection of Trichophyton rubrum and Trichophyton interdigitale", Clinical Microbiology and Infection, 22(8), https://doi.org/10.1016/j.cmi.2016.05.015.

Marian, E. et al. (2013), "Evaluation of antimicrobial activity of some types of inclusion complexes of Erythromycin with $\beta$-cyclodextrin on Staphylococcus aureus", Farmacia, 61(3), 518-525.

Muresan, M.L. (2015), "Antimicrobial effects of the ethanolic extracts and essential oils of Tanacetum vulgare L. from Romania", Food Technol., 19, 75-80, https://doi.org/10.1515/aucft-2015-0016.

Ulukanli, Z. et al. (2017), "Essential oil constituents of Tanacetum: Antimicrobial and phytotoxic activities", J. Food Qual., https://doi.org/10.1155/2017/6214896.

Wisselink, G.J. et al. (2011), "Trapped in keratin; a comparison of dermatophyte detection in nail, skin and hair samples directly from clinical samples using culture and real-time PCR", Journal of Microbiological Methods, 85(1), 62-66, https://doi.org/10.1016/j.mimet.2011.01.023. 

AND PARCHMENT FOR MUSEUM PURPOSES

\author{
LUCREȚIA MIU ${ }^{1}$, MARIANA GIURGINCA ${ }^{2}$, IULIA-MARIA CANIOLA ${ }^{1}$, MARIA- \\ CRISTINA MICU ${ }^{1}$, SIMONA-MARIA PĂUNESCU ${ }^{1}$, CLAUDIU ȘENDREA ${ }^{1}$, \\ ELENA BADEA ${ }^{1,3}$ \\ ${ }^{1}$ INCDTP - Leather and Footwear Research Institute (ICPI) Division, Bucharest, Romania \\ ${ }^{2} i$-CON Association - Science and cultural heritage in connection (http://i-con-org.ro/) \\ ${ }^{3}$ Department of Chemistry, Faculty of Sciences, University of Craiova, Craiova, Romania, \\ elena.badea@unito.it
}

\begin{abstract}
Cultural heritage represents a national treasure evolving from the culture and spirituality of people. Therefore, it must be continuously recovered, protected and developed by all generations as priceless heritage. Referring to the Romanian cultural heritage items that are placed in restoration, at present, some works have been done for partial or complete replacing of degraded materials, both as the result of ageing under atmospheric and biologic agents. The aim of this study is testing different samples of new leather and parchment under UV radiation in the 220-380nm domain, in order to simulate the accelerated destruction in photo-oxidative conditions. The following materials have been studied: book binding leather obtained from calf hide, tanned by different systems (vegetable or vegetable and chromium) and parchment obtained from different kinds of animals. The evolution of the chromatic characteristics (luminosity, shade and chroma) at different exposure time was quantified by the diffuse reflectance technique in visible domain range and CIE - Lab software. Based on the chromatic stability after UV exposure, the most stable types of leather and parchment have been selected for further applications in the restoration process of historical manuscripts and books' covers.
\end{abstract}

Keywords: chromatic investigations, parchment, leather

\title{
INTRODUCTION
}

A very important category from the museum cultural heritage is represented by leather and parchment objects, which lead to precious historical information. Such kind of objects are book bindings, manuscripts, charters, belts, arm covers, maps, sword sheaths, etc.

In order to perform this study, some of the most representative Romanian cultural heritage objects (Fig. 1 and 2), which are made out of leather and parchment, were taken into consideration: a Romanic Greek Evangelic book, an old printing by the Metropolitan Theodosie, from 1693, a Bucharest Bible from 1688, an old printing by Serban Cantacuzino, an Evangelic from 1697, an old printing by Antim Ivireanu, a Penticostarion from 1742, a document on parchment by Simion Moghila, from 1601 and a document on parchment by Mihail Racovita, from 1731.

The restoration of cultural heritage objects from leather or parchment involves, in many cases, the replacement of the irremediable destroyed parts with new ones. The manufacturing process of new pieces of leather and parchment for museum purpose assumes the fulfilment of some specific characteristics, adequate for each cultural heritage object. Two of the most important features of new collagen-based materials (leather and parchment) are represented by the structural compatibility and durability. In order to obtain the structural compatibility, the manufacturing process of new leather and parchment must be very similar to the old one, in which natural auxiliary materials, vegetable extracts and special handmade operations were used. 


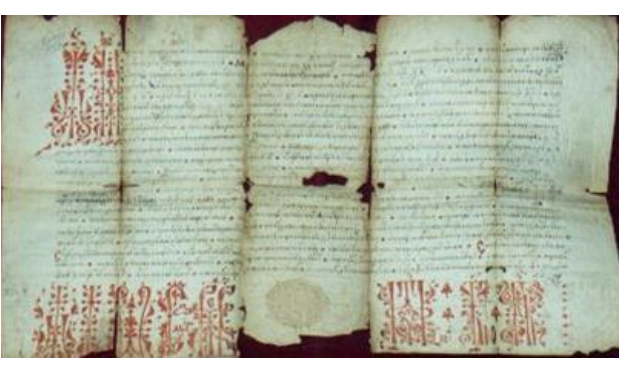

Figure 1. Document on parchment by Simion Moghila 1601

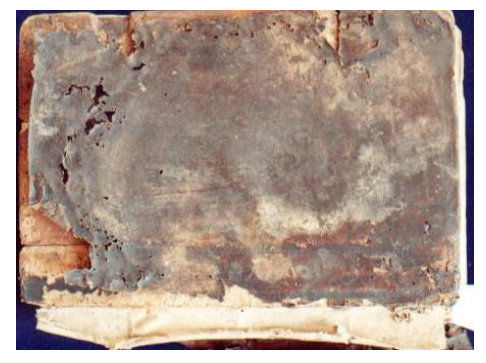

Figure 2. Old printing by Serban Cantacuzino, Evanghelic from 1697

Regarding the assessment of leather and parchment durability, it is obtained by simulating some artificial ageing processes, followed by a series of chemical, physicalmechanical and instrumental analyses.

The aim of our paper is to present an objective instrumental procedure meant to evaluate the durability characteristics of newly manufactured leather and parchment for further use in the interest of museums.

\section{MATERIALS AND METHOD}

In order to produce new leather and parchment items for museum purpose, we tested different types of technologies and chemical auxiliary materials, especially selected to fulfill the specific characteristics required for such materials. The main characteristics that leather and parchment must have for museum restoration purpose are the following: structural compatibility with the restoration cultural heritage object, physicalmechanical strength and color preservation on artificial ageing.

Among all newly manufactured leather and parchment samples, only a few were selected by means of physical-mechanical and chemical tests. From this point of view, the most resistant types of leather and parchment were:

- leathers tanned with: chestnut (1Ch), chestnut and chromium $(2 \mathrm{Ch} / \mathrm{Cr})$, oak $(1 \mathrm{O})$, oak and chromium $(2 \mathrm{O} / \mathrm{Cr})$, mimosa $(1 \mathrm{M})$, mimosa and chromium $(2 \mathrm{M} / \mathrm{Cr})$, quebracho (1Q), quebracho and chromium (2Q/Cr);

- $\quad$ parchment obtained from lambskin, unhaired with enzymes (PL1), sulphide and lime (PL2), unhaired with lime (PL3); parchment obtained from calfskin, unhaired with lime (PC1) and parchment obtained from goatskin, unhaired with lime (PC2).

In order to assess the durability of leather and parchment, different types of experiments have been proposed for simulating the environmental conditions, which contribute to oxidative, hydrolytic or thermal degradation (STEP Leather Project, 19911994; ENVIRONMENT Leather Project; Chahine; 1995; SMT Programme; IDAP Project, 2001).

In our previous works (Sendrea et al., 2017; Miu et al., 2002a; Miu et al., 2002b; Meghea et al., 2002), different kinds of artificial ageing have been performed by simulating the thermal degradation in cyclic exposure times (at $70{ }^{\circ} \mathrm{C}, 168$ hours - cycle I, 336 hours - cycle II and 504 hours - cycle III) followed by physical, mechanical, chemical, instrumental analyses (DTA, DSC, UV-VIS-NIR, IR). Another type of artificial ageing that was performed implied artificial light conditions using a Xenotest device. 
In order to establish a reliable method of evaluation of new leather and parchment materials manufactured for museum purposes, we have also tested the thermal-photo oxidative degradation under UV radiation in the $220-380 \mathrm{~nm}$ domain, in a special room, at $50-70{ }^{\circ} \mathrm{C}$, in oxidative conditions.

The color change of samples was assessed by measuring the chromatic characteristics like luminosity, shade and chroma by the diffuse reflectance technique in the UV-VIS-NIR range (spectrometer V 570, JASCO). The main chromatic characteristics were processed with CIE-Lab software. This technique can also allow the evaluation and selection of different kinds of treatments for leather and parchment maintenance.

\section{RESULTS AND DISCUSSION}

Tables 1 and 3 show the chromatic characteristics obtained for the initial samples of leather and parchment and for the same samples, but after 250 hours of exposure in photo-oxidative conditions. In tables 2 and 4, the chromatic characteristics are again presented, but this time, they are expressed as the differences between the final and the initial state.

The chromatic modification of leather and parchment's surface under thermal, UV or artificial light conditions represents the result of a predominantly oxidative (ENVIRONMENT Leather Project; Meghea et al., 2002) degradation of proteins by generation of ketone groups (Miu et al., 2002b) and simulates in a satisfactory way the natural ageing process of collagen-based materials. The artificial ageing in thermal photo-oxidative conditions leads to the modification of the main chromatic and organoleptic characteristics (softness, touch) of the early-mentioned materials.

By combining the chromatic characteristics measured by diffuse reflectance technique, CIE-Lab software, and organoleptic assessment of leather and parchment exposed at UV radiation (Herascu et al., 2008; Plavan et al., 2010), it was possible to select the suitable samples for restoration purpose as follows: $2 \mathrm{Ch} / \mathrm{Cr}, 1 \mathrm{O}, 1 \mathrm{Q}$ - leather samples, and PC2 - parchment sample, as the most resistant and appropriate materials for restoration propose.

Table 1. Chromatic characteristics for leather samples exposed to UV radiation

\begin{tabular}{ccccccc}
\hline \multirow{2}{*}{$\begin{array}{c}\text { Sample symbol and } \\
\text { type of tannage }\end{array}$} & \multicolumn{5}{c}{ Chromatic characteristics } \\
& initial & $250 \mathrm{~h}$ & initial & $250 \mathrm{~h}$ & initial & 250h \\
\hline $1 \mathrm{Ch}$ & 38.96 & 42.96 & 16.19 & 20.69 & 64.38 & 71.69 \\
$2 \mathrm{Ch} / \mathrm{Cr}$ & 31.97 & 43.13 & 10.35 & 11.35 & 52.10 & 61.02 \\
$1 \mathrm{O}$ & 45.04 & 38.96 & 15.68 & 16.20 & 70.64 & 64.38 \\
$2 \mathrm{O} / \mathrm{Cr}$ & 29.83 & 32.74 & 6.41 & 8.06 & 45.88 & 60.36 \\
$1 \mathrm{M}$ & 55.78 & 46.77 & 16.00 & 25.10 & 65.86 & 61.18 \\
$2 \mathrm{M} / \mathrm{Cr}$ & 49.92 & 45.44 & 15.20 & 20.58 & 58.26 & 60.10 \\
$1 \mathrm{Q}$ & 55.82 & 49.97 & 21.82 & 23.46 & 64.70 & 61.00 \\
$2 \mathrm{Q} / \mathrm{Cr}$ & 45.72 & 40.37 & 20.80 & 19.19 & 58.10 & 56.00 \\
\hline
\end{tabular}


Chromatic Assessment of Newly Manufactured Leather and Parchment for Museum Purposes

Table 2. Modification of chromatic characteristics of leather samples exposed at UV radiation $(\%)$

\begin{tabular}{|c|c|c|c|c|c|}
\hline \multirow[b]{2}{*}{ Sample } & \multicolumn{5}{|c|}{ Modification of chromatic characteristics } \\
\hline & Initial sample & $\begin{array}{l}\text { After UV } \\
\text { exposure }\end{array}$ & $\mathrm{L}^{*}$ & $\mathrm{C}^{*}$ & $h_{a b}$ \\
\hline $1 \mathrm{Ch}$ & & & +10.3 & +27.7 & +11.1 \\
\hline $2 \mathrm{Ch} / \mathrm{Cr}$ & & & +6.8 & +9.7 & +17.1 \\
\hline 10 & & & -13.8 & +3.3 & -8.8 \\
\hline $2 \mathrm{O} / \mathrm{Cr}$ & & & +9.7 & +25.7 & +31.6 \\
\hline $1 \mathrm{M}$ & & & -16.2 & +56.9 & +7.1 \\
\hline $2 \mathrm{M} / \mathrm{Cr}$ & & & -9.1 & +35.5 & +3.2 \\
\hline $1 \mathrm{Q}$ & & & -10.4 & +7.5 & -5.7 \\
\hline $2 \mathrm{Q} / \mathrm{Cr}$ & & & -11.7 & -7.8 & -3.6 \\
\hline
\end{tabular}

Table 3. Chromatic characteristics for parchment samples exposed at UV radiation

\begin{tabular}{ccccccc}
\hline \multirow{2}{*}{ Sample } & \multicolumn{3}{c}{ Chromatic characteristics } \\
& initial & L* & \multicolumn{2}{c}{$\mathrm{C}^{*}$} & \multicolumn{2}{c}{$\mathrm{h}_{\mathrm{ab}}$} \\
& 72.90 & 72.37 & 26.01 & 31.50 & 88.21 & 83.58 \\
PL1 & 72.46 & 82.06 & 14.70 & 25.74 & 81.08 & 86.93 \\
PL2 & 68.46 & 83.17 & 3.90 & 10.90 & 77.22 & 87.38 \\
PL3 & 76.61 & 88.81 & 7.30 & 9.76 & 75.73 & 87.48 \\
PC1 & 82.40 & 88.86 & 14.24 & 17.92 & 80.18 & 89.84 \\
PC2 & 80.26 & &
\end{tabular}

https://doi.org/10.24264/icams-2020.V.6 
Table 4. Modification of chromatic characteristics of parchment samples exposed at UV radiation $(\%)$

\begin{tabular}{|c|c|c|c|c|c|}
\hline \multirow[b]{2}{*}{ Sample } & \multicolumn{5}{|c|}{ Modification of chromatic characteristics } \\
\hline & Initial sample & $\begin{array}{l}\text { After UV } \\
\text { exposure }\end{array}$ & $\mathrm{L}^{*}$ & $\mathrm{C}^{*}$ & $\mathrm{~h}_{\mathrm{ab}}$ \\
\hline PL1 & & & +10.3 & +27.7 & +11.1 \\
\hline PL2 & & & +6.8 & +9.7 & +17.1 \\
\hline PL3 & & & +9.7 & +25.7 & +31.6 \\
\hline PC1 & & & -13.8 & +3.3 & -8.8 \\
\hline $\mathrm{PC} 2$ & & & -16.2 & +56.9 & +7.1 \\
\hline
\end{tabular}

\section{CONCLUSIONS}

After testing many methods, the quantitative chromatic characterization of leather and parchment samples under simulated conditions of accelerated thermal photooxidative degradation represents the most valuable one for selecting the adequate type of leather or parchment materials for museum restoration use.

In addition, it was demonstrated that the thermal photo-oxidative deterioration simulates the natural ageing process of leather and parchment through protein deterioration, creation of ketone groups and modification of the color characteristics.

\section{REFERENCES}

Chahine, C. (1995), "Study on the stability of leather trated with PEG", ICOM Committee for conservation. Conservation of Leathercrafts and Related Objects Symposium, Amsterdam, 3-5 April.

ENVIRONMENT Leather Project: Deterioration and Conservation of Vegetable Tanned Leather, (Contract NO EV5V-CT 94-0514), Protection and conservation of European cultural heritage, Research Report No. 6.

Herascu, N., Simileanu, M. and Radvan, R. (2008), "Color changes in the artwork materials aged by UV radiation", Romanian Reports in Physics, 60(1), 95-103.

IDAP Project (2001), A Parchment Damage Assessment Programme, www.idap-parchment.dk.

Meghea, A., Giurginca, M. and Miu, L. (2002), "Spectral Methods for Physical-Chemical Characterization of Historic Leather and Parchment", 5th Conference Cultural Heritage Research, Cracow, Poland.

Miu, L., Bratulescu, V., Niculescu, O., Gaidau, C. and Meghea, A. (2002a), "Scientific Consideration on Preserving and Restoring Leather Based Object from Romanian Museums", 5th Conference Cultural Heritage Research, Cracow, Poland.

Miu, L., Gaidau, C., Niculescu, O., Bocu, V. and Meghea, A. (2002b), "Manufacturing of Vegetal Tanned Leathers and Parchments Used for Restoration Purposes“, 5th Conference Cultural Heritage Research, May, Cracow, Poland.

https://doi.org/10.24264/icams-2020.V.6 


\section{Chromatic Assessment of Newly Manufactured Leather and Parchment for}

Museum Purposes

Plavan, V., Giurginca, M., Budrugeac, P., Vilsan, M. and Miu, L. (2010), "Evaluation of the physico-chemical characteristics of leather samples of some historical objects from kiev", Rev. Chim., 61, 627.

Sendrea, C., Miu, L., Crudu, M. and Badea, E. (2017), "The Influence of New Preservation Products on Vegetable Tanned Leather for Heritage Object Restoration", Leather and Footwear Journal, 17, 9-16, https://doi.org/10.24264/lfj.17.1.2.

SMT Programme, Normalization, Measures and Tests (96-99), «Micromethodes pour l'analyse du parchemin ».

STEP Leather Project (1991-1994), Evaluation of the Correlation between Natural and Artificial Ageing of Vegetable Tanned Leather and Determination of Parameters for Standardization of an Artificial Ageing Method. 


\author{
LUCREȚIA MIU ${ }^{1}$, SIMONA-MARIA PĂUNESCU ${ }^{1}$, MARIA-CRISTINA MICU ${ }^{1}$, IULIA- \\ MARIA CANIOLA ${ }^{1}$, MĂDĂLINA IGNAT ${ }^{1}$, CLAUDIU ŞENDREA $^{1}$, ELENA BADEA $^{1,2}$ \\ ${ }^{1}$ National Research \& Development Institute for Textiles and Leather, ICPI Division, Ion \\ Minulescu 93, 031215, Bucharest, Romania \\ ${ }^{2}$ Department of Chemistry, Faculty of Sciences, University of Craiova, Calea București 107 I, \\ 200585, Craiova, Romania
}

\begin{abstract}
Leather is a complex material mostly consisting of a matrix of collagen, chemically stabilized by various tannins. This matrix, sooner or later undergoes alterations as a consequence of interactions between their structure and environment. A comprehensive study based on multiple chemical and physico-mechanical standard tests regarding leather samples which were artificially aged from 7 to 112 days has been made at $70^{\circ} \mathrm{C}$. The behavior in artificial aging of calf leather samples tanned at pilot level with two different vegetal tannins, mimosa and quebracho, were investigated due to its's similarity to the natural degradation of historical leather samples. Physico-mechanical characteristics of historical leather can be corelated with the high impact of degree of deterioration even though there are no standard regulations. To be able to choose the proper way to achieve compatibility with an appropriate material in the restoration-conservation process, multiple sample characteristic must be known. The condition of historical leather can be assessed by a series of simple visual and physical examinations which determine the flexibility, strength and coherency of the fibers and then correlate these assessments with the condition of leather as determined by various chemical and physical-chemical analyses. Therefore, the following chemical standard tests were made: volatile substances, shrinkage temperature, extractable substances, total soluble substances and the following physico-mechanical tests: tensile strength, elongation at breaking and tear resistance.
\end{abstract}

Keywords: artificially aged leather, physico-mechanical and chemical analysis, deterioration.

\title{
INTRODUCTION
}

The term tannin is used for a number of compounds widely used in the plant regna. Tannins have the property of turning raw skin into tanned leather. Tanning substances consist of fairly large molecules with a high molecular weight, although it is debatable whether they can be considered as macromolecular substances that can be fragmented. (Chiriță and Chiriță, 1999).

New and artificially aged leathers are complex systems, due to the number of components introduced in the processing. For this reason, their analysis must take into account the fact that it is a system consisting polymer matrix (collagen) to which are added solid and liquid substances dispersed or dissolved in the substrate and which, in different stages of processing, interact between each other. (Miu et al., 2007; Sendrea et al., 2017).

In addition, due to the intervention of environmental (temperature, humidity, light, pollution), biological (bacteria, fungi, rodents, insects), and chemical (acids, bases, oxygen, ozone, ultraviolet radiation) factors, many structural changes happen and they must be known from a scientific point of view, in order to achieve compatibility with an appropriate material in the restoration-conservation process (Sebestyén et al., 2015).

In museums there are a multitude of leather heritage objects in various degrees of damage. To restore these artifacts, museums need leathers processed according to traditional methods from the International Conservation and Restauration Standards (ENVIRONMENT Leather Project; Meghea et al., 2002; Badea et al., 2012).

https://doi.org/10.24264/icams-2020.V.7 


\section{METHODS \& ANALYSIS}

For this study, calf hides were processed at pilot level in INCDTP-ICPI. The technological process included the specific operations of the usual leather processing. The tanning of the leather was done with $15 \%$ vegetable tanning extracts of mimosa and quebracho.

The main chemical characteristics are presented in Table 1.

Table 1. Chemical characteristics of new leathers

\begin{tabular}{cccccc}
\hline Sample code [tanning] & $\begin{array}{c}\text { Humidity, } \\
\%\end{array}$ & $\begin{array}{c}\text { Ash, } \\
\%\end{array}$ & $\begin{array}{c}\text { Extractable } \\
\text { substances, \% }\end{array}$ & $\begin{array}{c}\text { Dermal } \\
\text { substance, \% }\end{array}$ & $\begin{array}{c}\text { Organic soluble } \\
\text { substances, \% }\end{array}$ \\
\hline VM [mimosa] & 14.1 & 0.8 & 9.6 & 66.8 & 4.2 \\
VQ [quebracho] & 14.8 & 1.2 & 8.7 & 67.1 & 5.8 \\
\hline
\end{tabular}

The samples of newly processed leathers, artificially aged, were analyzed according to the actual standards specific to vegetable and/or tanned leathers.

It is found that all the determined characteristics fall within the admissible limits, including the $\mathrm{pH}$ of the aqueous extract, which has values in the range 4.1-5.8.

Aging behavior tries to simulate a degradation over time, similar to the natural degradation of heritage leather. According to numerous studies performed on various skin types, the temperature of $70^{\circ} \mathrm{C}$ was selected as the most suitable for performing accelerated aging. For this purpose, VM and VQ were selected to be aged for 7-112 days at $70^{\circ} \mathrm{C}$ (Table 2$)$.

Table 2. Tanned leathers with tanned vegetable extracts subjected to artificial aging

\begin{tabular}{ccc}
\hline No. & Sample code & Sample, aging time \\
\hline 1 & VM_0 (initial) & Calf leather tanned with mimosa, not subject to aging \\
2 & VM_7 & Calf leather tanned with mimosa, aging 7 days \\
3 & VM_14 & Calf leather tanned with mimosa, aging 14 days \\
4 & VM_21 & Calf leather tanned with mimosa, aging 21 days \\
5 & VM_28 & Calf leather tanned with mimosa, aging 28 days \\
6 & VM_56 & Calf leather tanned with mimosa, aging 56 days \\
7 & VM_112 & Calf leather tanned with mimosa, aging 112 days \\
8 & VQ_0 (initial) & Calf leather tanned with quebracho, not subject to aging \\
9 & VQ_7 & Calf leather tanned with quebracho, aging 7 days \\
10 & VQ_14 & Calf leather tanned with quebracho, aging 14 days \\
11 & VQ_21 & Calf leather tanned with quebracho, aging 21 days \\
12 & VQ_28 & Calf leather tanned with quebracho, aging 28 days \\
13 & VQ_56 & Calf leather tanned with quebracho, aging 56 days \\
14 & VQ_112 & Calf leather tanned with quebracho, aging 112 days \\
\hline
\end{tabular}

For the aged leather samples, the chemical characteristics which may suffer changes due to the accelerated aging process were determined, such as: volatiles, extractable substances, free fatty acids, total soluble substances, mineral soluble substances, organic soluble substances and shrinkage temperature. The values obtained for the samples of calf leather (VM, VQ) tanned with vegetable tannins are shown in Fig.1 and Fig. 2.

Physico-chemical characteristics of aged samples, compared to the initial ones: 

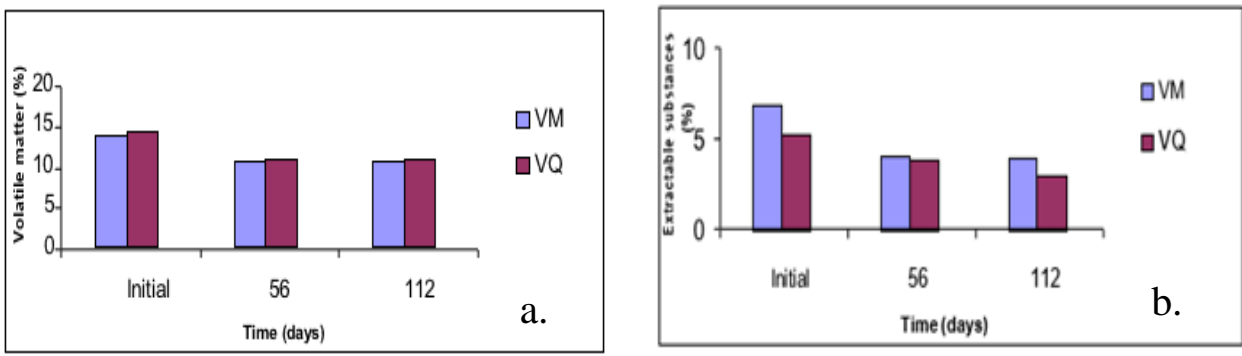

Figure 1. Variation of volatile compounds (a.) and extractable substances (b.)
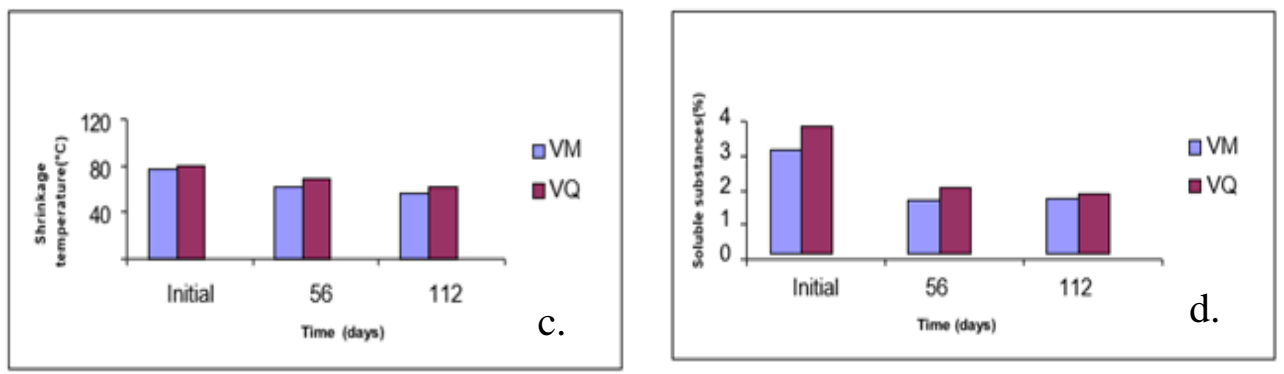

Figure 2. Shrinkage temperature variation (c.) and variation of total soluble substances (d.)

Comparing the values of the chemical characteristics obtained for the samples of tanned calf leather with those obtained for the leathers aged at 56 days and 112 days, significant decreases in the content of volatiles and extractable substances can be observed, and the most affected is the shrinkage temperature, which shows a very accelerated decrease in the treatment of 112 days of aging.

Decreasing the content of extractable substances during accelerated aging leads to impairment of physical and mechanical characteristics (traction, tearing).

Also, the decrease of the content of total soluble substances in aged leathers denotes the involvement of tanning and lubricating materials by their binding in interfibrillary networks with the impairment of hydrogen or covalent bonds, which leads to decreased leather strength properties.

\section{PHYSICAL-MECHANICAL ANALYSIS}

The samples were analyzed from a physical-mechanical point of view, and the results are presented in Table 3.

Table 3. The main physical-mechanical characteristics of new leathers

\begin{tabular}{ccccc}
\hline Sample code [tannin] & Sample code & $\begin{array}{c}\text { Tensile strength at } \\
\text { tearing, N/mm }{ }^{2}\end{array}$ & $\begin{array}{c}\text { Elongation at } \\
\text { breaking, } \%\end{array}$ & $\begin{array}{c}\text { Tear resistance, } \\
\mathrm{N} / \mathrm{mm}\end{array}$ \\
\hline VM [mimosa] & $\mathrm{VM}$ & 20.9 & 53 & 86.7 \\
VQ [quebracho] & $\mathrm{VQ}$ & 16.6 & 53 & 102.6 \\
\hline
\end{tabular}

Dimensional change, tensile strength $\left(\mathrm{N} / \mathrm{mm}^{2}\right)$, elongation at breaking $(\%)$ and tear resistance $(\mathrm{N} / \mathrm{mm})$ were determined. Examination of the data (Figures 3 and 4 ) shows:

https://doi.org/10.24264/icams-2020.V.7 
dimensional change, \%: after 1-6 cycles (Figure 3) at 7 days there are no changes, and at the end of the experiment there are accentuated dimensional changes especially for the surface of the specimen (contraction of up to $8 \%$ at 112 days in the VM test) and its thickness (dimensional increase, maximum $3 \%$ for the VM sample).
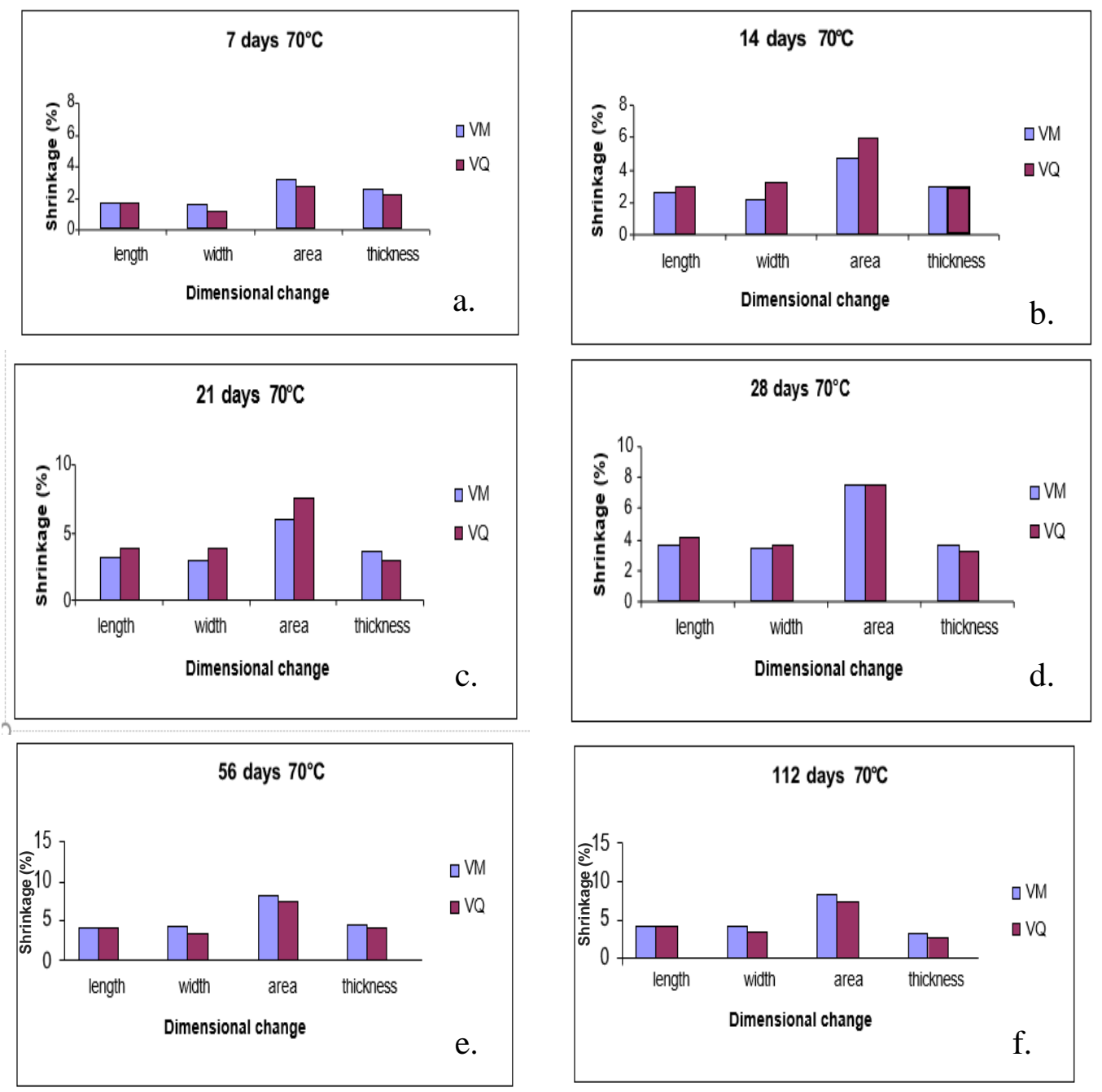

Figure 3. Dimensional changes of aged leather samples (a.-f.)

the tensile behavior at break $\left(\mathrm{N} / \mathrm{mm}^{2}\right)$, elongation at break $(\%)$ and tear $(\mathrm{N} / \mathrm{mm})$, after $1-6$ cycles of accelerated aging in the oven at $70^{\circ} \mathrm{C}$ is shown in Figure 4. 


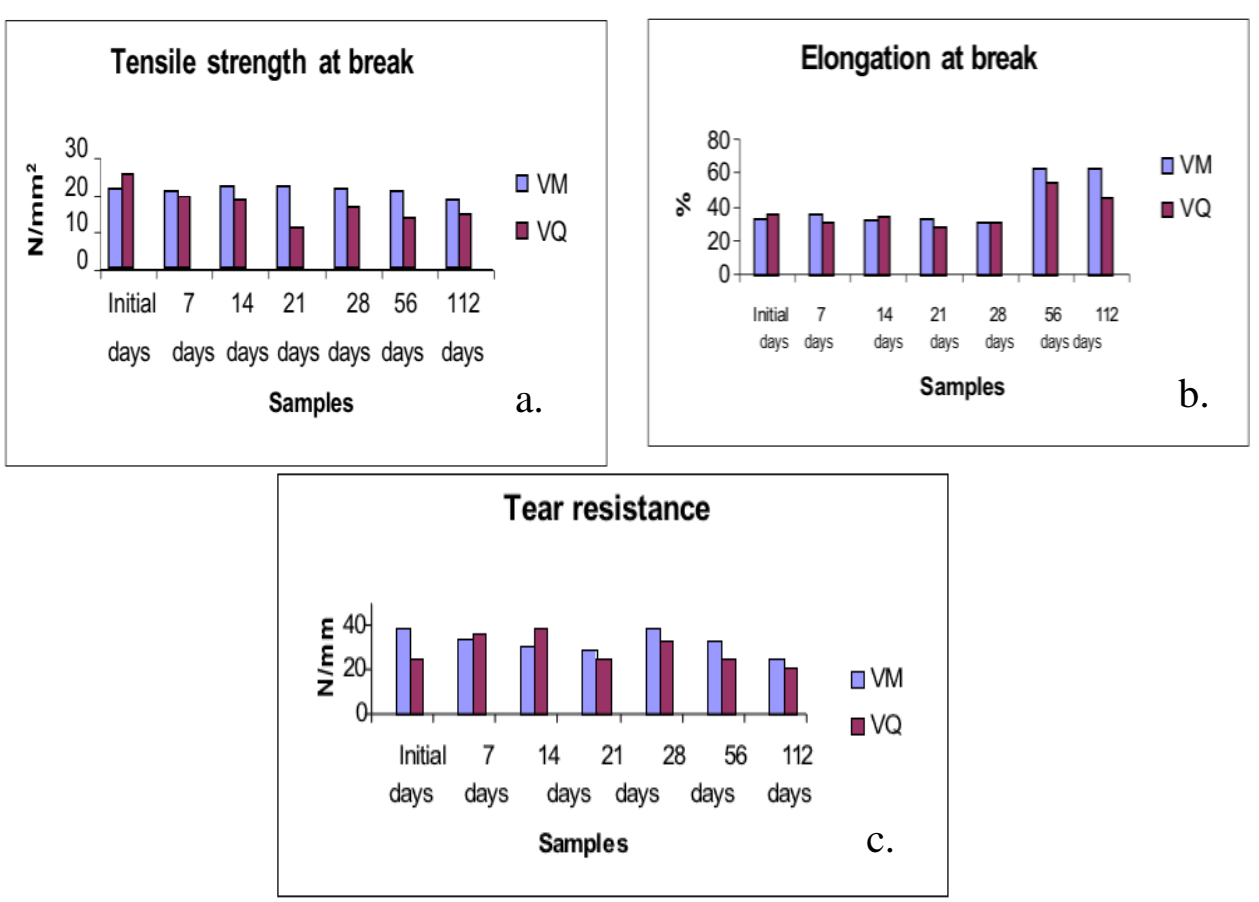

Figure 4. Physical-mechanical characteristics of calfskin (a.-c.)

According to the presented data, regarding the tensile strength, for the VM test an almost constant behavior is observed during the six aging cycles. The VQ sample registers a decrease of the resistance compared to the initial sample during the period $7-$ 21 days (of $11-25 \%$ ), and in the interval 28-112 days a minimal fluctuation is observed.

The elongation at breaking of the VM sample has an almost constant behavior over the 28 days, while at 56 days and 112 days it showed an increase of up to $61 \%$. A similar behavior with the VM sample can be observed in the case of the VQ sample.

The tear strength of the samples shows some fluctuations throughout the period, without significant losses.

\section{CONCLUSIONS}

The same types of samples were subjected to accelerated aging for 7-112 days, at $70^{\circ} \mathrm{C}$, and the samples of tanned leather with plant extracts of mimosa and quebracho did not have considerable different behaviors during the aging process, in terms of chemical analysis or physical-mechanical resistance. From the presented data result a certain scattering of the values obtained due to the fact that for the historical leathers there is no regulation of the physical-mechanical characteristics. This diversity of values can be attributed both to the complex technologies applied and to the structural (native) variety of calfskin.

Both types of leather correspond to restauration activity. 
Acknowledgement

This work was supported by a grant of the Romanian Ministry of Research and Innovation, CCCDI - UEFISCDI, project number PN-III-P1-1.2-PCCDI-20170878/NR. 55PCCDI/2018, within PNCDI III.

\section{REFERENCES}

Badea, E., Sommer, D., Axelsson, K., Larsen, R., Kuryshev, A., Miu, L. and Della, G. (2012), "Damage Ranking in Historical Parchments: From Microscopic Study to Collagen Denaturation Assessment by Micro DSC", E-Preservation Science, 9, pp. 97-109.

Chiriță, Gh. and Chiriță M., (1999), Treaty of Leather and Fur Chemistry and Technology (in Romanian), Gheorghe Asachi, Iasi.

ENVIRONMENT Leather Project: Deterioration and Conservation of Vegetable Tanned Leather, (Contract NO EV5V- CT 94-0514), Protection and conservation of European cultural heritage, Research Report No 6.

Meghea, A., Giurginca, M. and Miu, L. (2002), "Spectral Methods for Physical-Chemical Characterization of Historic Leather and Parchment”, 5th Conference Cultural Heritage Research, pp. 923-928.

Miu, L., Giurginca, M., Budrugeac, P., Carsote, C. and Badea, E. (2007), Medieval Documents on Parchment: Evaluation and Investigation, CERTEX.

Sebestyén, Z., Czégény, Z., Badea, E., Carsote, C., Şendrea, C., Barta-Rajnai, E., Bozi, J., Miu, L. and Emma, J. (2015), "Thermal characterization of new, artificially aged and historical leather and parchment", Journal of Analytical and Applied Pyrolysis, Elsevier, 115, pp. 419-427, https://doi.org/10.1016/j.jaap.2015.08.022

Sendrea, C., Miu, L., Crudu, M. and Badea, E. (2017), "The Influence of New Preservation Products on Vegetable Tanned Leather for Heritage Object Restoration", Leather and Footwear Journal, 17, pp. 9-16, https://doi.org/10.24264/lfj.17.1.2. 
START-UP INVESTMENT FOR A SHEEP WOOL PROCESSING LINE

\author{
MIRCEA CONSTANTIN OPRIȘ ${ }^{1}$, DANA CORINA DESELNICU ${ }^{2}$ \\ ${ }^{1}$ A\&D Pharma SRL, Romania, oprismircea0@gmail.com \\ ${ }^{2}$ University Politehnica of Bucharest, 313 Splaiul Independentei,d_deselnicu@yahoo.com
}

\begin{abstract}
Sheep wool has been a resource we had access to for a very long time and it is widely used on large scale. It has remarkable properties of which people can take benefit from in many ways. After processing the sheep wool by using special machinery, it provides various applications in different industries such as pharmaceuticals, cosmetics, textiles, and fabrics. The main objective of this research is the analysis of a sheep wool processing line and the estimated start-up investment for this type of business in Romania. In this paper, the wool processing line was depicted, the necessary equipment was analyzed, and the total cost of investment was calculated, in order to conclude on the feasibility of the investment. The results of this research paper are taking into consideration the full value of the sheep wool and the profit that can be generated by processing it, as well as providing relevant data regarding time and costs of starting a business in Romania, analyzing the sustainability and profitability of the raw material that can be found in Romania. Sheep wool is a high potency raw material for multiple industries, and it can provide a big margin for obtaining profit by processing it
\end{abstract}

Keywords: sheep wool processing line, start-up investment, profitability

\title{
INTRODUCTION
}

Sheep wool has various applications in different industries like pharmaceuticals, cosmetics, textiles, fabrics, and so on. Lanolin has a lot of use in the pharmaceutical and cosmetics industry. It states as raw material for some of the products found in these industries. It is also a precursor of vitamin $\mathrm{D}$, which is very important in an equilibrated lifestyle and has a lot of benefits on the human body. Moreover, studies show that Romanians are mostly in a deficit of this vitamin. Wool fibers are used in the textile industry for clothing, decorations, special equipment required for different environmental situations.

\section{Wool, Lanolin and Vitamin D3, Fibers}

Wool

Wool is produced by follicles which are small cells located in the skin. These follicles are located in the upper layer of the skin called the epidermis and push down into the second skin layer called the dermis as the wool fibers grow. Follicles can be classed as either primary or secondary follicles. From here on we can talk about the types, quantity, and quality of the wool. Processing the wool includes two main stages:

- Sheep shearing is that the process by which the woolen fleece of a sheep is cut off. After shearing, the wool is separated into four main categories: fleece (which makes up the vast bulk), broken, bellies, and locks. The standard of fleeces is set by a method referred to as wool classing, whereby a professional person called a wool classer groups wool of comparable gradings together to maximize the return for the farmer or sheep owner.

- Before the wool will be used for commercial purposes, it must be scoured, a process of cleaning the greasy wool. Scouring will be done as simple as washing in warm water or as complicated as a process using special detergent and other chemical substances in specialized equipment. Wool straightaway from a sheep will be cataloged as "greasy wool" or "wool in the grease", deposits a high level of lanolin, also because of 
the sheep's dead skin and sweat residue, and customarily also contains pesticides and matter from the animal's environment. the rest beside the lanolin and clean wool must be eliminated.

\section{Lanolin}

Lanolin also called wool wax or wool grease is a wax secreted by the sebaceous glands of wool-bearing animals. The lanolin that can be useful for humans comes from domestic breeds that are raised specifically for their wool. Historically, many pharmacopeias have referred to lanolin as wool fat; however, as lanolin lacks glycerides, it is not a true fat. Lanolin primarily consists of sterol esters instead.

Examples of common applications of lanolin:

- Lanolin and its many derivatives are used extensively in both personal care (e.g., high-value cosmetics, facial cosmetics, lip products) and health care sectors like topical liniments. Lanolin is additionally found in lubricants, rust-preventive coatings, blacking, and other commercial products.

- Lanolin is commonly used as a material for producing vitamin D3 using irradiation. this is often the sole natural source of vitamin D3. The other on the market presents some form of chemical synthetization.

\section{Vitamin D3}

Cholecalciferol also referred to as vitamin D3 is a sort of vitamin D that is made by the human skin, found in some foods, and brought as a dietary supplement. It is used to treat and forestall D deficiency and associated diseases, including rickets.

It is produced by the ultraviolet irradiation of 7-dehydrocholesterol extracted from lanolin found in sheep's wool. Cholesterol is extracted from wool fat and wool wax alcohols obtained from the cleaning of wool after shearing. The cholesterol undergoes a four-step process to create 7-dehydrocholesterol, the identical compound that is produced within the skin of animals. The 7-dehydrocholesterol is then irradiated with UV. Some unwanted isomers are formed during irradiation, but these are removed by various techniques, leaving a resin which melts at room temperature and typically includes a potency of 25,000,000 to 30,000,000 International Units per gram.

\section{Wool Fibers}

Of the most important apparel fibers, wool is the most reusable and recyclable fiber on Earth. The eco-credentials of wool are enhanced by its long service life and suitability to be recycled to new textiles for clothing, resilient upholstery, or products that call on its natural resistance to fire and temperature extremes. Other than premium next-to-skin apparel, the wool may be utilized in industrial applications like thermal and acoustic insulation or in pads to soak up oil spills.

At the disposal stage, natural fibers like wool reduce the impact of the textile industry on pollution and landfill build-up. In warm, moist conditions like in soil, wool biodegrades rapidly through the action of fungi and bacteria to essential elements (i.e. Nitrogen and Sulphur) for the expansion of organisms as a part of natural carbon and nutrient cycles. 


\section{THE MANUFACTURING PROCESS OF WOOL}

The process behind the clean wool starts from the raw material and follows the next steps:

1. Shearing: Sheep shearing is the process of cutting wool fleece from Sheep.

Each sheep is sheared once a year. Sheep are sheared in all seasons, but spring is usually preferred for this process.

2. Sorting: In sorting, wool is split into four sections of various quality fibers (fleece, broken, bellies, and locks). the most effective quality of wool comes from the shoulders and side of the sheep which are used for clothing. The lower quality comes from the underside of the legs and is used for carpet making.

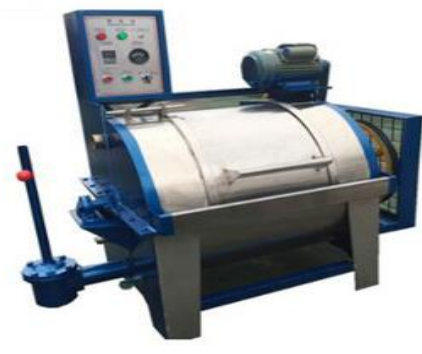

Figure 1. Scouring machine

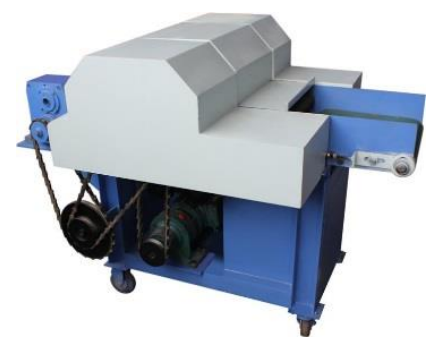

Figure 2. Carding machine

3. Scouring: is a process of cleaning the greasy wool because it contains a high level of lanolin, the sheep's dead skin, sweat residue, pesticides, and matter from the animal's environment. It is a water bath contains alkaline, soda ash, and soap. The rollers of the cleaning machines press the surplus water from the wool, but the wool isn't allowed to dry completely. After this process, wool is processed with oil to make it easier to manage.

4. Carding wool: At this stage, the fibers are separated then assembled again into a loose rope (sliver) by removing short fibers and replacing them with long parallel fibers. The combing machine consists of one large roller and smaller ones surrounding it. All cylinders are covered with small metal teeth, and when the wool reaches more on the teeth becomes finer.

5. Spinning: The thread is made by spinning the fibers together to make the strand. The plexus is woven with two or three other threads. Because the wool fibers hold tight one another, it's easy to expand and spindle the wool into yarns. Woolen yarns are often spun on any number of spinning machines. After this stage, spun threads are wrapped around rollers or commercial drums.

6. Weaving: The woolen threads are woven into the material. in this industry, two basic sorts of weaves are used, the plain and also the twill weaves. the foremost common method is the plain weaving which supplies soft surface textiles. The twill method gives more beautiful and more precise wool textiles, but in turn, it's more expensive. 


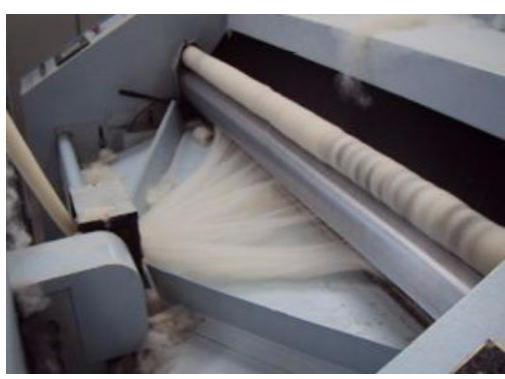

Figure 3. Weaving machine

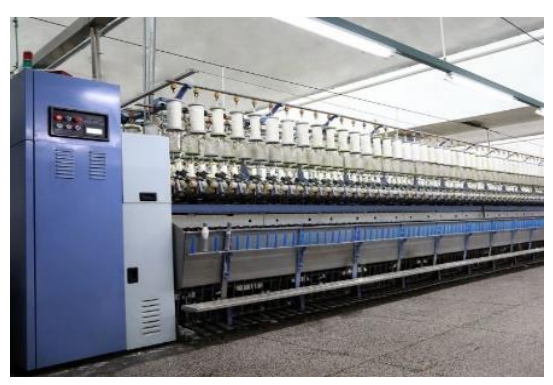

Figure 4. Spinning machine

7. Finishing: After weaving, wool fabrics are subjected to a series of ultimate processing including Fulling or tucking is a step within the woolen clothing industry that involves cleaning the fabric to eliminate oils, dirt, and other impurities, and make them thicker. Crabbing is a process that ensures that the material expands or is relaxed as necessary and equips the thickness of the material. Cribbing prevents the formation of wrinkles or uneven contraction. Although wool fibers are often dyed before the carding (combing) process, dyeing may be done after weaving the wool into the material.

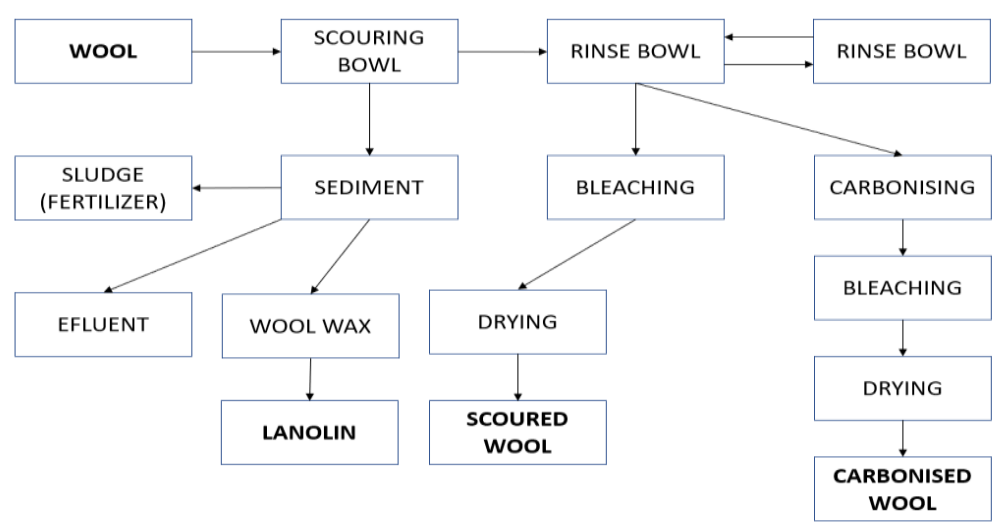

Figure 5. Sheep wool processing line phases

Increasing demand from the pharmaceuticals and healthcare sectors as a result of the rising preference for non-toxic and chemical-free products will drive the market growth further. The support of local governments through funds and tax exemption for the development of lanolin associated ancillary industries is predicted to enrich the expansion during this sector. The program designed to assist aspiring business owners during the startup and formation stages of their enterprise. The main objective is to significantly reduce the high first-year failure rate of startup businesses in the region. A program like described before in Romania could be the Start-Up Nation.

The program can found your business idea concept, based on analysis of the market, feasibility, chances of success. The budget they can allocate for your idea its 44.000 EUR, non-refundable money with the condition that the organization to work at least 3 years and have the minimum number of employees as established on the project. 
After a short analysis regarding creating a new business in Romania, I evaluated the times required for every step and to count the number of procedures that need to be done. I used very accurate 2020 data and I could extract the steps which would be involved in.

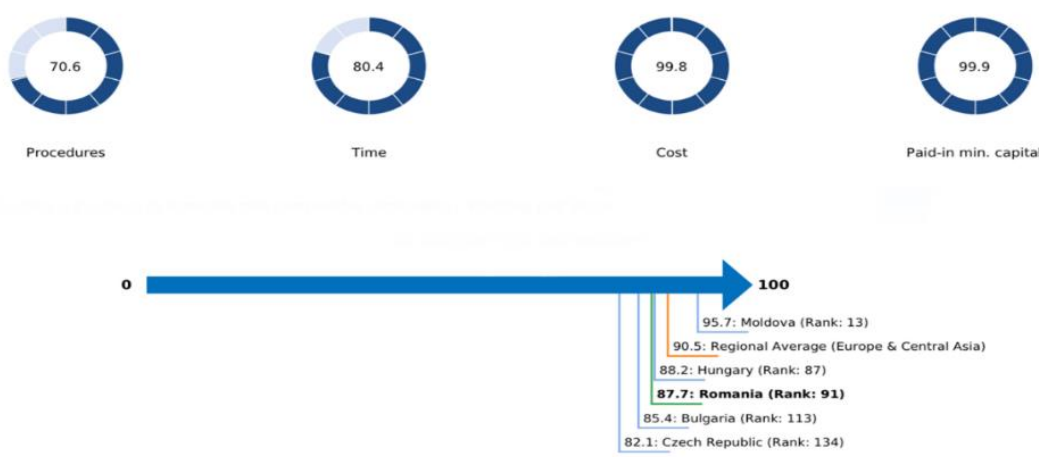

Figure 6. Starting a business in Romania compared to other group members

An overall score of 87.7 out of 100 for starting a business was attributed to Romania. This number is ranking us in the 91st position in the group. It consists of 6 main procedures, 20 days, and has a pretty small cost. The business opportunity, described in a matter of investments and revenues, shows that profit can be obtained in a three-year period.

Table 1. Financial projection and feasibility analysis

\begin{tabular}{|c|c|}
\hline Investments & Price (EUR) \\
\hline Starting the business & 5000 \\
\hline Acquisition of machinery and maintenance & 84000 \\
\hline Service car & 10000 \\
\hline Administrative costs & 4700 \\
\hline Total & 103700 \\
\hline \multicolumn{2}{|l|}{ Revenues (NET) } \\
\hline Clean wool & 40000 \\
\hline Lanolin & 40000 \\
\hline Wool fiber & 20000 \\
\hline Vitamin $\mathrm{d} 3$ & 10000 \\
\hline Total & 110000 \\
\hline
\end{tabular}

\section{CONCLUSIONS}

The business plan is projected on a 3 year period. In this time the purpose is to cover all the initial costs. Despite the mean annual growth rate of a company is $10 \%$, this company will have more than that. Responsible for this is the fact that in the second and third years from its beginning the processing line will have one more product as an outcome from the same raw material. The profit is planned to be negative in the first year and start on a positive trend in the second year, respectively the third year.

The results of this research confirm that wool is a very high potential raw material for a lot of industries. Lanolin, vitamin D3, and wool fibers can be obtained by processing sheep wool. Lanolin can be used in the pharmaceutical and cosmetic industry while 
vitamin D3, obtained by putting lanolin under UV radiation would have a major benefit for human healthcare. The wool fibers are a premium type of material in the textile industry. Accordingly to the financial prediction, the investment will be returned at the last of the third year. If the business plan is accepted and funded by the start-up program, after the 3-year period, the profit should be around 110000 EUR before taxes.

\section{REFERENCES}

Laitala, K., Klepp, I. and Henry, B. (2018), "Does use matter? Comparison of Environmental Impacts of Clothing Based on Fiber Type", Sustainability, 10, 2524, https://doi.org/10.3390/su10072524.

*** http://www.doingbusiness.org/ /media/WBG/DoingBusiness/Documents/Profiles/Country/ROM.pdf

$* * *$ http://www.madehow.com/Volume-1/Wool.html\#ixzz5powBdHWW

*** https://en.wikipedia.org/wiki/Merino\#Wool\&qualities

*** https://en.wikiversity.org/wiki/Processing_of_wool

*** https://start-up.ro/articole/start-up-nation/ 
DESIGN AND MANUFACTURE OF PRODUCTS WITH ROMANIAN CULTURAL IDENTITY

\author{
MIRELA PANTAZI-BAJENARU, TRAIAN FOIASI \\ INCDTP - Division: Leather and Footwear Research Institute, 93 Ion Minulescu St., Bucharest, \\ Romania, email: icpi@icpi.ro
}

\begin{abstract}
In the current context, research on cultural identity unfolds as an uninterrupted process of modeling and remodeling, constituting the link between past, present and future. The issue of cultural identity has become an increasingly important topic in recent years, especially in the European context, due to the need to legitimize European cultural identity as a link between European citizens and as a unifying principle of different cultural heritage. The current processes of social and economic globalization provide major challenges on the world market of consumeroriented production sectors. The increase of competition in the market, as well as consumers' exigency, calls for the manufacture of innovative products in the field of footwear as well. Thus, the approached topic appeared as a necessity to make footwear products with innovative design, in order to sustainably develop the competitiveness of companies through the strategic development of footwear production. The research that is the object of this article consists in the creative reinterpretation of footwear, creating a new product concept with Romanian cultural identity. The aim is, on the one hand, to identify and analyze the product concept and, on the other hand, to develop the product with the help of creative industries, highlighting cultural identity.
\end{abstract}

Keywords: footwear, cultural identity, heritage.

\title{
INTRODUCTION
}

In the work "Romanian identity in the context of trend modernity", Constantin Schifirnet mentions that "national identity returns as a problem of maximum relevance in countries integrated in the European Union, obviously interested in reinventing their national identity" (Schifirnet, 2009).

In this context, research on cultural identity takes place as an uninterrupted process of modeling and remodeling, constituting the link between past, present and future. (Brubaker and Cooper, 2000). The issue of cultural identity has become an increasingly important topic in recent years, especially in the European context, due to the need to legitimize European cultural identity as a link between European citizens and as a unifying principle of different cultural heritage (Kjær and Palsbro, 2008; Antonsich, 2008).

The current processes of social and economic globalization offer major challenges on the world market of consumer-oriented production sectors. The increase of the competition on the market, as well as that of the consumers' exigency, calls for the manufacture of innovative products in the field of footwear as well.

To meet such a global challenge of competition, new competitive strategies must be designed and developed based on new consumer-focused product capabilities and new industrial paradigms, especially in the footwear sector, characterized by rapid market dynamics and a large number of SMEs.

Thus, the approached topic appeared as a necessity to create footwear products with innovative design, in order to sustainably develop the competitiveness of companies through the strategic development of footwear production.

The main objective of this research is to develop a collection of shoes at a high standard of quality, inspired by national heritage, helping to trigger a real change in which we appreciate, protect and promote our heritage, shaping the personality and daily life of wearers. 
The innovative character of the research consists in the elaboration of the finished product with cultural identity and the creation of a high-class concept-collection, with the help of Romanian cultural instruments.

The research consists in the creative reinterpretation of footwear, developing a new product concept with a Romanian cultural identity. Basically, the research aims, on the one hand, to identify and analyze the product concept and on the other hand, to develop the product with the help of creative industries, highlighting cultural identity.

\section{METHODOLOGY}

The research is structured in two main stages:

- documentary research on the symbols of Romanian culture, in order to make the cultural tools useful for the concept of footwear, as well as the models and types of footwear specific to each region of the country;

- development, by creating prototypes, which will be made with Romanian cultural tools, specific to each region of the country, through new design concept technologies, with scientific know-how of product creation, incorporating the experience and competence of design research staff.

The identification and analysis of the Romanian patrimony objects and of the geographical areas from which they come are the first steps taken in the vertical and horizontal development of the first creative elements that will lead to the development of a concept collection with roots in the national patrimony.

\section{Selection of Materials and Accessories}

The design of footwear deals not only with the image of the product and the image elements of the elaborated stylistic concept but also with the identification and selection of materials and accessories that will lead to the manufacture of the finished object. Choosing the material and accessories is a key step in any design process because it is the crucial decision in making a product. Improper choice of material or accessory can lead not only to a failed product, but also to an inadequate cost. The selection of materials and accessories is an activity that establishes the technological process, adapted to the selected material and accessory, in order to meet the requirements of the new product. The texture of the materials and accessories leads to the recomposition of the heritage object, in new forms, with impact in contemporaneity.

\section{Creating a Collection of Shoe Models by Transposing the Heritage Object into Modernity}

The decoding of images and their transposition into the sketches that prefigure the finished product go through a whole process of emotional and pragmatic conception:

- atmosphere sketches are the first to refer to the emotional space of the designer;

- the image sketches lead to the reception of the product in the imaging space;

- the sketches of ideas express a cultural communication through image and its decoding, through key words, which lead to graphic and technical expression.

The conceived object is transposed into component parts and patterns of idea sketches. Footwear design involves a complex process of ideation, heritage identification, conceptualization, design, graphic design, advertising, illustration. All these factors lead to the creation of the finished product with cultural identity and of a 
high-class concept-collection. Original, internationally competitive footwear products are designed, with a major socio-economic impact on the consumer.

\section{RESULTS}

\section{Bibliographic Study on the Symbols of Romanian Culture}

All the manifestations expressed, spiritually or materially, are subordinated to almost immutable codes, sacredly observed by all members of an ethnic group. Tradition is a complex of highly resistant chains of habits. In some specialized studies, it is claimed that, at the level of traditional culture, there is an ornamental "grammar" and a chromatic one.

We set out to establish the defining characteristics of the traditional Romanian culture, based on the presence of the component elements within the symbolic series, to which we added their frequency and area of circulation. A bibliographic study on the symbols of Romanian culture was made.

\section{Technical Documentation Containing Model Sketches}

Making model sketches is essential for the implementation to be correct and to obtain a finished product exactly as it was conceived by the designer. The technical sketch of the shoe model shows the upper and lower assemblies of the shoe (Fig. 1-5), the characteristics of the model, the destination, the materials as well as the colors of the component parts.

The design of the footwear not only deals with the image of the product and the image elements of the elaborated stylistic concept but also with the identification and selection of materials and accessories that will lead to the manufacture of the finished object. Choosing the material and accessories is a key step in a design process because it is the crucial decision in making a product. Improper choice of material or accessory can lead not only to a failed product, but also to an inadequate cost.

The selection of materials and accessories is an activity that helps establish the technological process, adapted to the selected material and accessory, in order to meet the requirements of the new product. The texture of the materials and accessories led to the recomposition of the heritage object, in new forms, with an impact on contemporaneity.

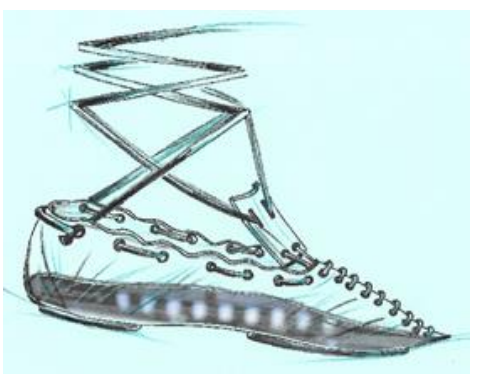

Figure 1. Peasant sandal inspired from Oltenia region

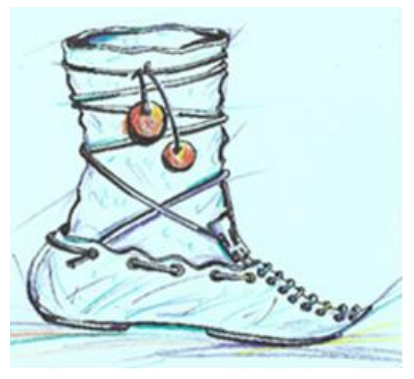

Figure 2. Children's peasant sandal - inspired from Oltenia region 


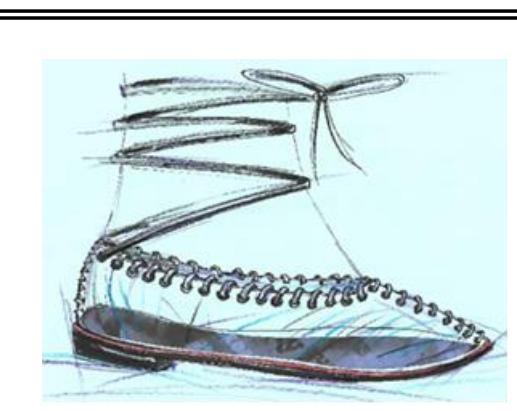

Figure 3. Peasant sandal inspired from Muntenia region

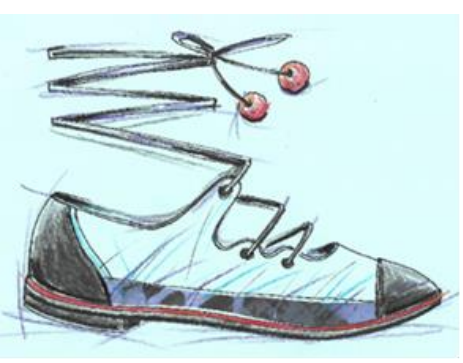

Figure 4. Peasant sandal inspired from Mehedinti region

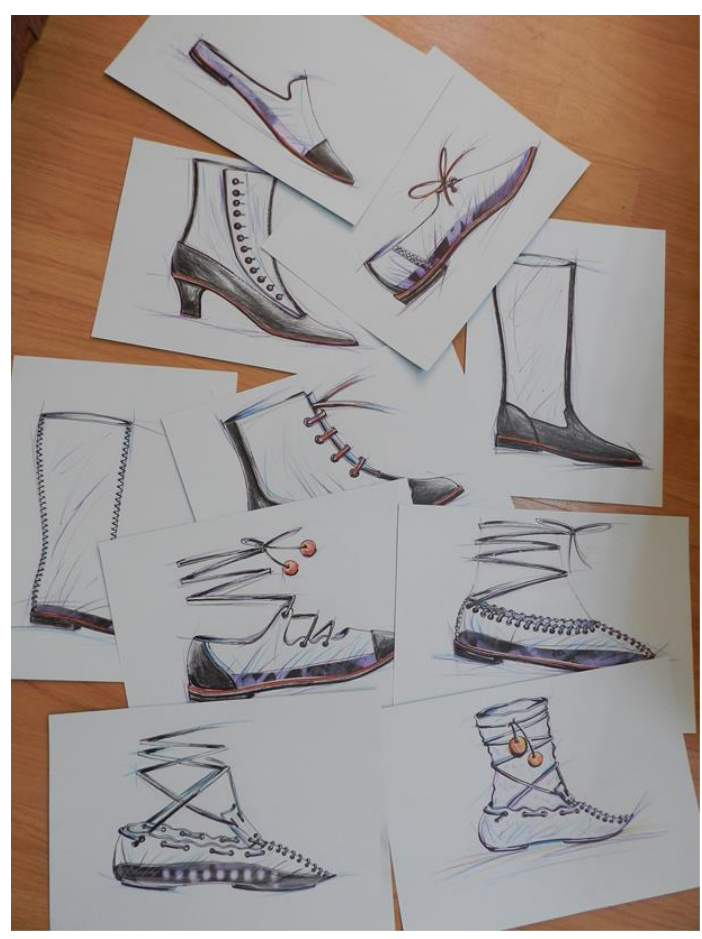

Figure 5. Model sketches

\section{Technical Documentation regarding the Design of Footwear Models (Standard Copy, Footwear Patterns, Materials, Technology, etc.)}

The technical documentation regarding the design of footwear models entails making the standard copy of the footwear, the patterns of the component parts of the footwear, the choice of materials, as well as the technological manufacturing process.

The last is a three-dimensional object that must be copied and transformed without distortion into a 2D shape (the surface of the shoe unfolded), thus obtaining a copy. Therefore, a copy is a flat representation of the dorsal surface of the last.

The stages of obtaining the patterns were: 
- Making the standard copy of the shoe - The standard copy represents the average of the unfolded outer and inner surfaces of the last and is the most important "pattern" when designing the shoe.

- Drawing the baselines - On the standard copy, placed in a reference system, the baselines are drawn. These are the traces of transverse profile planes that section the foot through a series of important anatomical points.

- Drawing the 3D model directly on the last - To draw the 3D network and the model lines on the last, the following steps must be followed: marking the metatarsophalangeal joints I and V; drawing the toe line; marking the height of the quarters at the back; drawing the upper line of the quarters; marking the instep point; drawing the instep line; drawing pattern lines.

- Obtaining patterns - each pattern is separated, sewing allowances, signs and all technical details of the parts are marked and patterns are obtained for: vamp, tongue, quarter, counter, eyelet reinforcement, collar, etc., depending on the model (Fig. 6).

Conventional (natural leather) and unconventional materials (transparent PVC foil) were used to create the collection. The shoes were manufactured using the glued sole system.
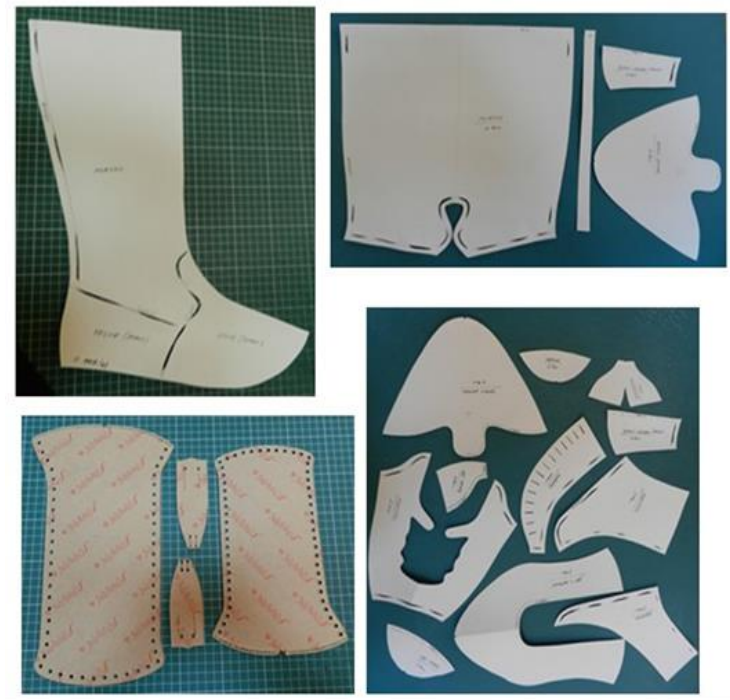

Figure 6. Footwear patterns

\section{Collection of Footwear Models}

The high-class concept-collection contains 20 footwear models and was created by transposing the heritage object into modernity, according to Figure 7. 


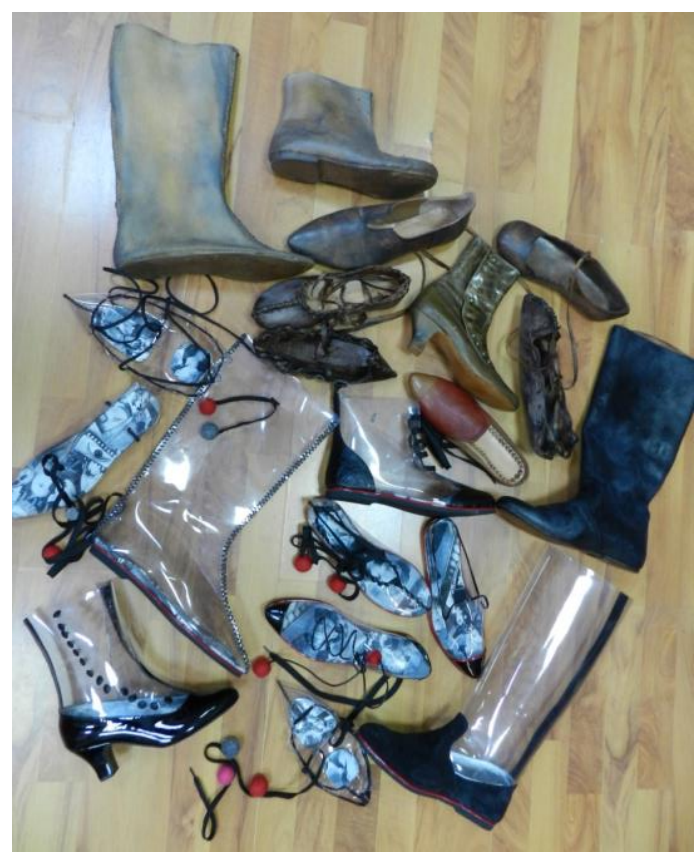

Figure 7. Collection of footwear models

\section{CONCLUSIONS}

The research consisted in the creative reinterpretation of footwear, creating a new product concept with Romanian cultural identity. Basically, the aim was, on the one hand, to identify and analyze the product concept and, on the other hand, to develop the product with the help of creative industries, highlighting the cultural identity. Footwear design involves a complex process of ideation, heritage identification, conceptualization, design, graphic design, advertising, illustration. All these factors lead to the elaboration of the finished product with cultural identity and of a high-class concept-collection.

\section{Acknowledgements}

This study was funded by the Ministry of Research and Innovation through the Program 1 - Development of the National R\&D System, Subprogram 1.2 - Institutional Performance - RDI excellence funding projects, Contract no. 6PFE/16.10.2018.

\section{REFERENCES}

Antonsich, M. (2008), "European attachment and meanings of Europe. A qualitative study in the EU-15", Political Geography, 27, p. 691, https://doi.org/10.1016/j.polgeo.2008.07.004.

Brubaker, R. and Cooper, F. (2000), "Beyond Identity", Theory and Society, 29, 1, p. 2, https://doi.org/10.1023/A:1007068714468.

Kjær, A.L. and Palsbro, L. (2008), "National Identity and Law in the Context of European Integration: The Case of Denmark", Discourse Society, Vol 19 (5), p. 600, https://doi.org/10.1177/0957926508092245.

Schifirnet, C. (2009), "Romanian identity in the context of trend modernity" (in Romanian), Revista Română de Sociologie, new series, year XX, no. 5-6, p. 461-480, Bucharest.

https://doi.org/10.24264/icams-2020.V.9 
DESIGN THINKING IN PRODUCT DEVELOPMENT - CASE STUDY: LEATHER LIBRARY

\author{
MARLENA POP \\ INCDTP - Division: Leather and Footwear Research Institute, Bucharest, Romania \\ pop_marlen@yahoo.ca
}

\begin{abstract}
Called "design thinking", this human-centered method is the most advanced way to meet the requirements of clients or target groups. In the elaboration of the Leather Library, this method was experimented and verified, proving its efficiency in the development of creativity, problem solving and accountability of designers.
\end{abstract}

Keywords: design thinking methodology, Leather Library project, design students

\title{
INTRODUCTION
}

The material library is an object, a library of materials, but it is also a repository of knowledge of materials in a certain scientific, industrial, design, artistic field, etc. Basically, the material library is an archive of specialized materials, a cataloged sample tool of scientific work in different fields of economics. The Leather Library project aims to design this library of materials, unique in the country, as a specialized modular display, using the "Design Thinking" methodology. This design method, generated as a phrase by Herbert A. Simon (1996), a renowned specialist in the sciences of knowledge, since 1969, has been rediscovered and used as a design method by Stanford professors and popularized by Ideo in recent years, as a method of business development. Using this method, the design process is no longer linear, but turns into an iterative, spatial and empathic process, being centered in each stage on the requirements of the person who will use that product (Image 1). In order to verify the "design thinking" method in the design practice, a case study was created, the design of the leather material library, as a modular sample, together with the students of the European Politechnique Student program of UPB in collaboration with INCDTP - Division ICPI. The group of students consists of French, British and Romanian students.

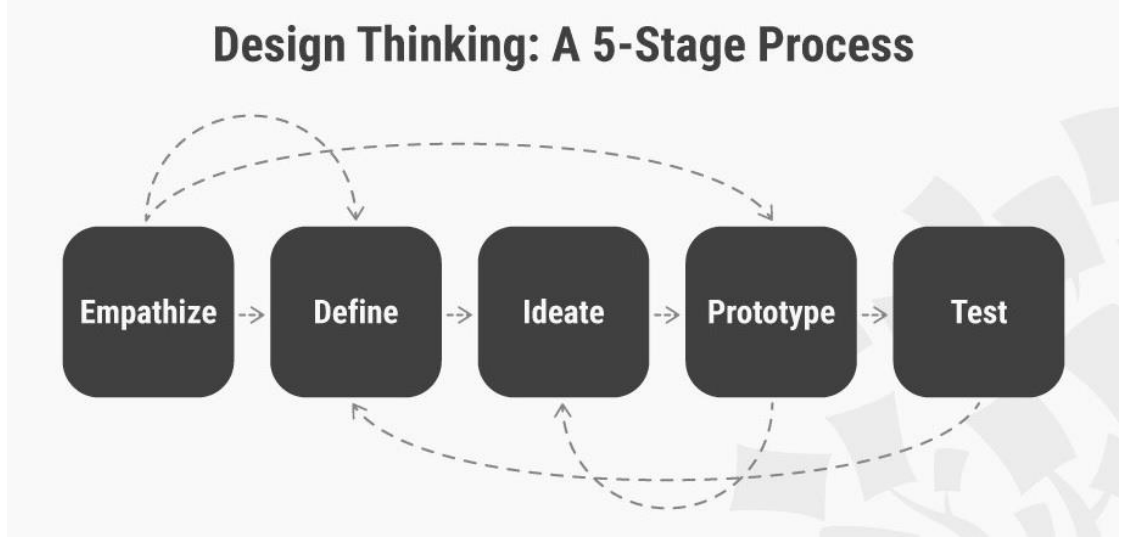

Image 1. The design process through the Design Thinking method (Interaction Design Foundation, 2019) 


\section{CASE STUDY - LEATHER LIBRARY/ DESIGN THINKING METHOD}

The case study solves a deeply specific research situation, such as this challenge, of making a leather material library. That is why all the stages of the "design thinking" method were observed in the development of the design process, as follows:

\section{Empathy - The First Stage of the Design Thinking Method}

- Understanding design thinking and tools to stimulate individual and team creativity;

- Visiting a real material library, the one at Nod Maker and understanding its role in design, architecture and engineering;

- Online documentation: Specialized libraries in different fields, from UK, France, USA;

- Questions about the structure of the subject - Understanding the functions of this design product / service - A first impression, intuitive perception, without prejudices, of the library of materials.

\section{Defining the Problem - The Second Stage}

- Identification of project objectives: Identifying the design requirements of an object called the material library;

- Functional requirements: The material library must be an archive of knowledge of the field, by means of the technical sheet of material characteristics and tangible samples;

- Requirements for use: the material library must be functional, with easy access to both written information and samples, to allow the storage of several samples of material;

- Technical requirements: a body easy to handle and access, made of light but resistant material;

- Aesthetic requirements: the structure of the spatial body of the material library must have the best design, so as to respond to both the functions and the uniqueness of the samples and the particular aesthetic expressiveness of its object.

\section{Ideation / Conceptualization / Elaboration of Sketches According to the Accumulated Information}

- Knowledge of intellectual work tools: Open mind and brainstorming;

- Ideation requirement: Develop an intuitive sketch of the product - Leather Library;

- Issuing an incipient concept / developing the concept;

- Establishing roles in the team, based on the Ideation;

- Iteration and finalization of the design concept.

In order to develop a balanced accountability of design students in the project team, based on their first intuitive ideas (Image 2), each of them received a role for a specific conceptualization, as follows: project manager, 3D design manager, quality manager, environmental design manager and marketing manager. 


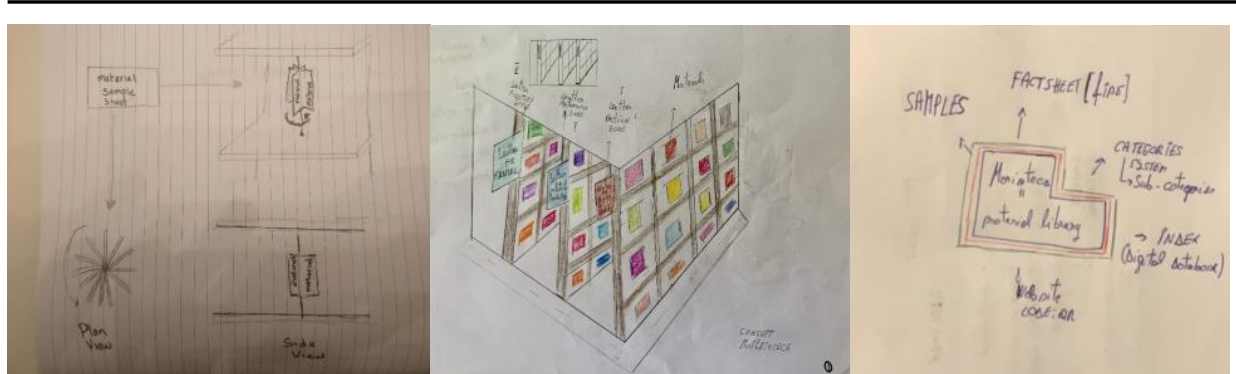

Image 2. Primary ideation, intuitive sketches

The Ideation process consisted of:

- two brainstorming sessions to define product functions;

- ideation process: intuitive idea sketches, not conceptual;

- drafting the initial specifications of the project;

- the method of the conceptual mix for defining the product concept.

Iteration is the most important creative stage that leads to the clear conceptualization of the ideas of a project and the elaboration of the final concept. According to the students' sketches, J.'s idea, a rotating cylindrical body, combined with D.'s boxes, received the most votes from the team. Elaboration of the final Leather Library concept will consist of seven vertical modules: the module will be called "Soldier", authors: J., D., L. (Image 3).
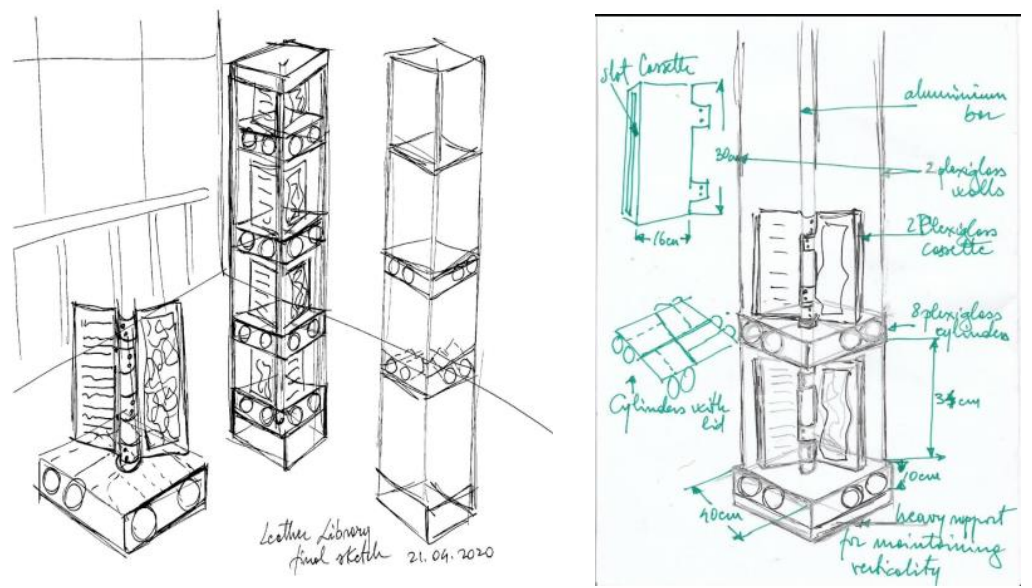

Image 3. Development of the final concept for Leather Library

Designing the technical data of the material library:

Technical data of the Leather Library - 7 modules called "Soldier" with the dimensions of: $200 / 40 \mathrm{~cm}$,

Mass properties of The Soldier:

Mass $=53.61$ kilograms

Volume $=63911.97$ cubic centimeters

Surface area $=21959154.22$ square millimeters

https://doi.org/10.24264/icams-2020.V.10 


\section{Design Thinking in Product Development - Case Study: Leather Library}

Center of mass: (millimeters)

$\mathrm{X}=401.99$

$\mathrm{Y}=-92.53$

$\mathrm{Z}=832.47$

Principal axes of inertia and principal moments of inertia: (kg* square $\mathrm{mm})$ Taken at the center of mass.

$\mathrm{Ix}=(0.00,0.00,1.00) \mathrm{Px}=1329687.86$

Iy $=(-0.35,-0.94,0.00) \mathrm{Py}=23851394.01$

$\mathrm{Iz}=(0.94,-0.35,0.00) \mathrm{Pz}=23870826.61$

Moments of inertia: (kilograms * square millimeters): Taken at the center of mass and aligned with the output coordinate system.

$\mathrm{Lxx}=23868207.11 \mathrm{Lxy}=6184.43 \mathrm{Lxz}=-69548.32$

Lyx $=6184.43$ Lyy $=23853583.82 \mathrm{Lyz}=69622.11$

Lzx $=-69548.32 \mathrm{Lzy}=69622.11 \mathrm{Lzz}=1330117.56$

The space destined for the exhibition of the Leather Library, the ICPI lobby, was designed for two situations: 1. Permanent exhibition; 2. Specialized event, which requires the placement of furniture.

\section{Fourth Stage - Prototyping}

The technical design of "Soldier" (Images 4 and 5) in 2D and 3D was made by the Romanian student, in online collaboration with French and British colleagues, in the difficult situation of state of medical emergency, created by COVID 19.
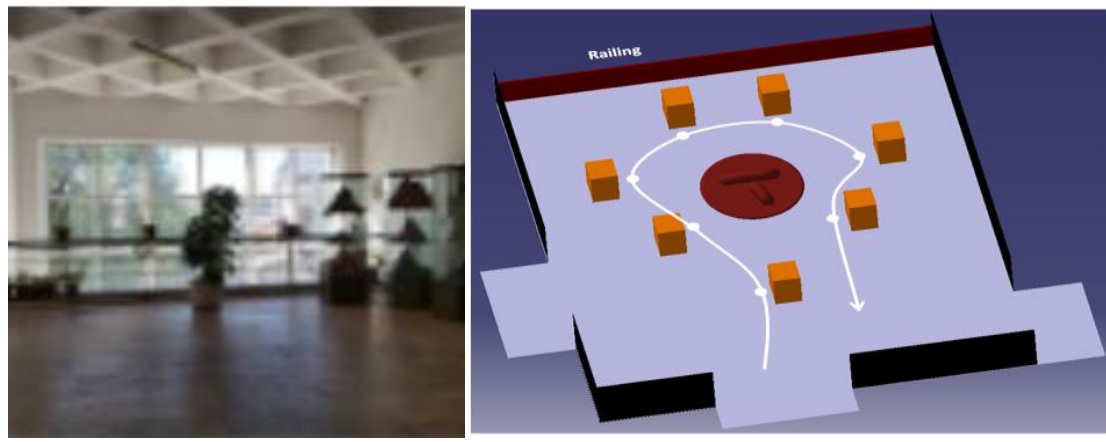

Image 4. The ICPI lobby and 3D design of the Leather Library 

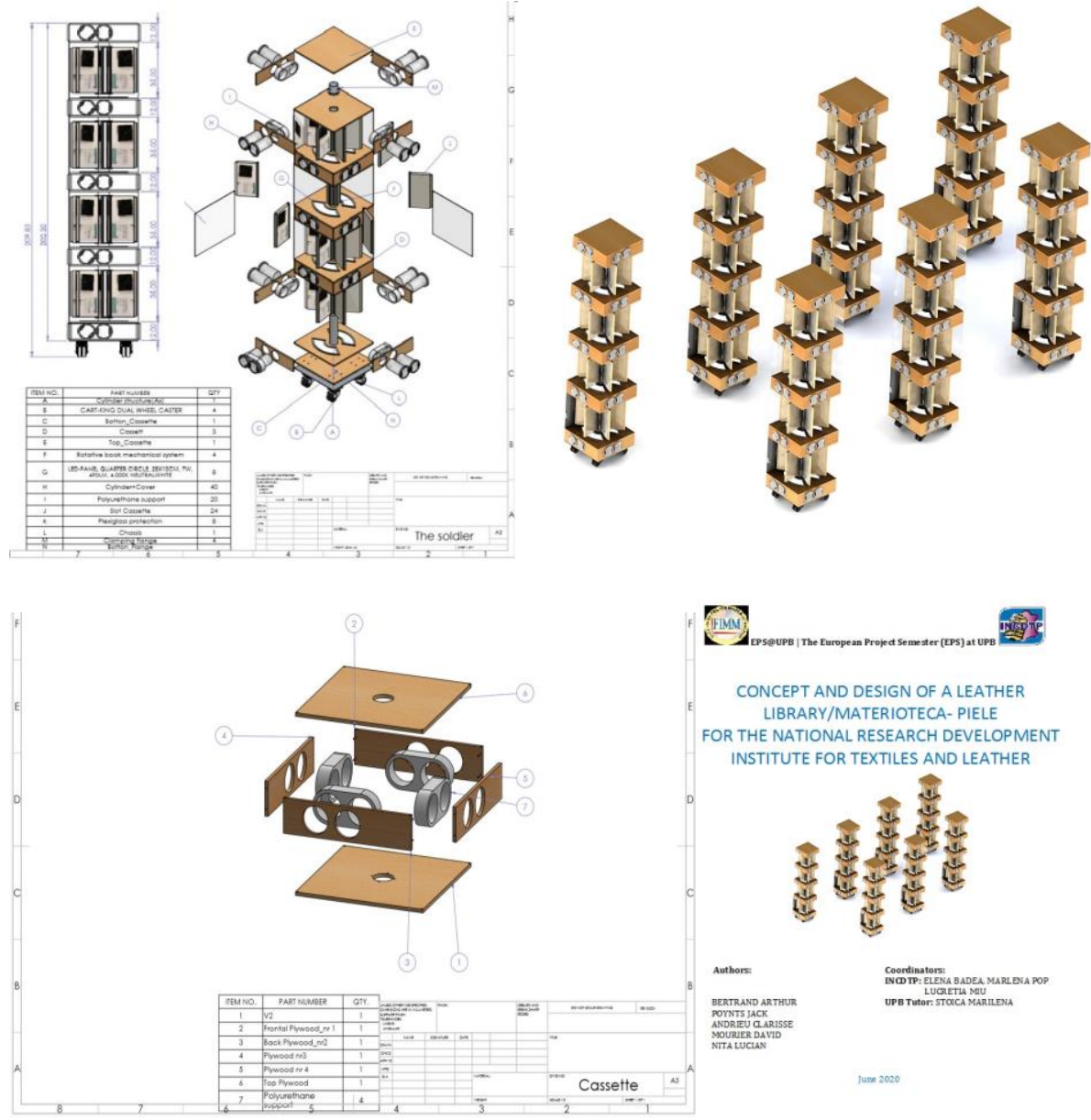

Image 5. Technical design of the Leather Library

\section{Virtual Prototype Testing}

The fifth stage of design thinking, that of testing, through virtual simulation could not be achieved because the universities were closed due to the medical context, and the necessary equipment could not be accessed.

The case study demonstrated that the "design thinking" method solves a much larger number of problems that the design of an object or process raises and leads to the development of the design team through the complex practices learned by students during the project.

\section{CONCLUSIONS}

Through the European Polytechnic Student Project of ICPI in collaboration with UPB, a research / development project in product design was carried out between March and May 2020, called LEATHER LIBRARY.

https://doi.org/10.24264/icams-2020.V.10 
- The Leather Library is an archive of information-structured materials and stored by sampling in cylindrical boxes, and presented using rotating boxes that contain the technical sheet of the leather assortment and its sample. The library consists of 7 mobile modules called "Soldier", each specializing in a specific subfield of advanced research of materials that have collagen in their structure.

- The design method used in product development was Design Thinking, a complex method that starts from the problem and continues with documentary research, ideation, iteration, conceptualization, creative design, environmental design, communication design, technical design and development (layout and prototyping).

- As the EPS students say in the project to support their activity, from the research coordinators "We have learned a lot from a cultural, technical, managerial and human point of view".

\section{REFERENCES}

Anderson, L. and Shattuck, J. (2012), "Design-based research: A decade of progress in education research?", Educational Researcher, 41 16-25, https://doi.org/10.3102/0013189X11428813.

Interaction Design Foundation (2019), "Design Thinking", available at: https://www.interactiondesign.org/literature/topics/design-thinking

Kimbell, L. (2011), "Rethinking design thinking. Part I", Design and culture, 3, 285-306, https://doi.org/10.2752/175470811X13071166525216.

Simon, H.A. (1996), The Sciences of the Artificial, 3rd ed., Cambridge, MA: MIT Press. 


\title{
EFFECTS OF UV, HUMIDITY, AND HIGH TEMPERATURE EXPOSURE ON
} LINEN FIBERS

\author{
IRINA SANDULACHE ${ }^{1}$, MIHAELA-CRISTINA LITE ${ }^{1,2}$, LUCIA-OANA SECAREANU ${ }^{1}$, \\ ELENA-CORNELIA MITRAN ${ }^{1,2}$, OVIDIU IORDACHE ${ }^{1}$, ELENA PERDUM $^{1}$ \\ ${ }^{1}$ The National R\&D Institute for Textiles and Leather Bucharest (INCDTP), 16 Lucretiu \\ Patrascanu, 030508, Bucharest, Romania, office@incdtp.ro \\ ${ }^{2}$ University Politehnica of Bucharest, Faculty of Applied Chemistry and Materials Science, 1-7 \\ Gheorghe Polizu, Bucharest, Romania, secretariat@chimie.upb.ro
}

\begin{abstract}
Generally, the most common damaging factors for linen textile materials are the environmental conditions, their handling, and natural decay. Such environmental factors are ultraviolet (UV) radiation, humidity, and high temperature. Therefore, to investigate the effects these factors may cause, an accelerated weathering test was conducted on linen fabrics, using alternating cycles of UV exposure and humidity, along with relatively high temperatures. The effects of this test were investigated using non-destructive and micro-destructive analysis techniques. Scanning Electron Microscopy (SEM) was used to observe any modifications appearing at the surface of the fibers Energy Dispersive X-Ray Analysis (EDS) was employed in conjunction with SEM for obtaining the spectrum of the chemical elements that were present at the surface of the linen samples. The modifications of functional groups occurring due to the weathering of linen were assessed by Fourier-Transform Infrared Spectroscopy (FT-IR). The color change of the samples was measured with a spectrophotometer. All the acquired information can be used as a starting point for the development of customized environmental parameters for keeping patrimony linen fabrics in museums in optimum conditions, thus preventing further damage. Additionally, the artificially weathered fabrics will be further employed in conservation experiments as substitute for old linen fabrics.
\end{abstract}

Keywords: linen, weathering, textiles

\section{INTRODUCTION}

Textiles have been, for a long time, a significant part of the essential necessities of humankind. The use of textiles commenced with natural fibers of animal and vegetal origin.

There are evidences of the use of linen fibers from more than 30000 years ago and it is still being valued due to its properties. There are many historically significant linen textiles that are currently found in museums nowadays.

Linen is a bast fiber, meaning that it originates from the inner part of the flax plant stem (Linum usitatissimum) (Akin, 2003). The fibers have a diameter of approx. $15-17$ $\mu \mathrm{m}$ and a clockwise rotation around the longitudinal axis (Markova, 2019). The chemical composition of linen fibers is approx. $71 \%$ cellulose, $18.6-20.6$ hemicellulose, $2.2 \%$ lignin, and 1.5\% waxes (Friedrich and Breuer, 2015).

\section{MATERIALS AND METHODS}

For developing the present work, an undyed linen fabric has been used. The fabric has been divided into six $11 \times 9 \mathrm{~cm}$ rectangles. One of the rectangles has been kept as a control sample. The other five fabric rectangles have been submitted to an accelerated weathering process inside an accelerated weathering tester (QUV, Q-Lab). The following weathering method was employed: an $8 \mathrm{~h} \mathrm{UV}$ exposure cycle at $70^{\circ} \mathrm{C}$ using fluorescent UVB-313 lamps, followed by a $4 \mathrm{~h}$ condensation cycle (60\% humidity) at 
$50^{\circ} \mathrm{C}$. One sample has been collected every three days, the final sample being collected after fifteen days of weathering. The total UV and humidity exposure time for each sample is presented in the following table:

Table 1. UV radiation and humidity exposure time of the linen samples

\begin{tabular}{ccc}
\hline Sample & UV time $(\mathrm{h})$ & Humidity time $(\mathrm{h})$ \\
\hline t0 (control) & 0 & 0 \\
t1 & 48 & 24 \\
t2 & 96 & 48 \\
t3 & 144 & 72 \\
t4 & 192 & 96 \\
t5 & 240 & 120 \\
\hline
\end{tabular}

A Nicolet iS50 (ThermoFisher) Fourier-transform infrared spectroscope (FT-IR) with an attenuated total reflection (ATR) accessory was used to investigate the modifications of the functional groups over a spectral range of $4000-400 \mathrm{~cm}^{-1}$.

A FEI Quanta 200 scanning electron microscope (SEM) has been employed is for assessing the morphology of the linen fibers. An Element energy-dispersive X-ray spectroscopy detector (EDS) from EDAX-AMETEK was used in conjunction with the previously mentioned SEM in order to determine the elemental composition of the samples.

The chromatic parameters have been measured with a Datacolor spectrophotometer.

\section{RESULTS AND DISCUSSION}

\section{FT-IR}

The results for all samples are presented in Figure 1:

Linen

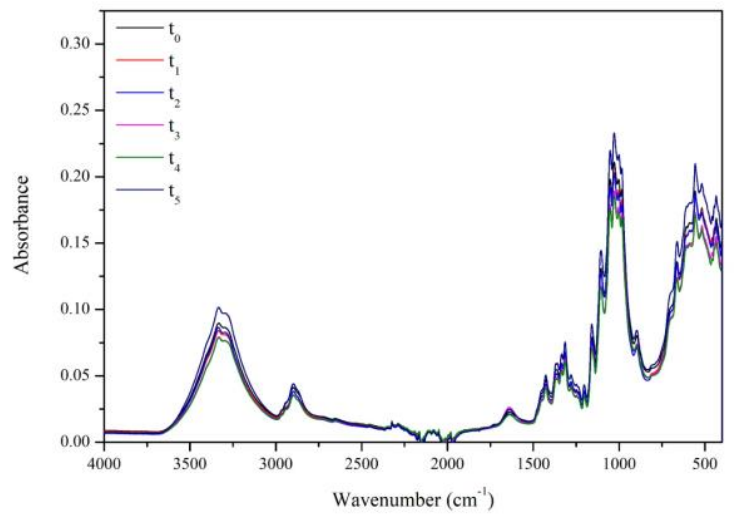

Figure 1. Overlapped FT-IR spectra of the linen samples

The structural differences between the samples were minimal. None of the samples presented new IR absorption bands. For all the samples, the IR spectra corresponded with the literature data (Chung, 2004). 
The specific band positions for the studied materials in this paper, compared to literature data are presented in Table 2.

Table 2. Absorption band positions in IR for the linen samples, compared to literature

\begin{tabular}{ccc}
\hline $\begin{array}{c}\text { Experimental band } \\
\text { position }\left(\mathrm{cm}^{-1}\right)\end{array}$ & $\begin{array}{c}\text { Band position reported } \\
\text { in literature }\left(\mathrm{cm}^{-1}\right)\end{array}$ & Band attribution \\
\hline $3600-3000$ & $3550-3100$ & H-bonded OH stretch \\
2897 & $2980-2800$ & C-H stretching \\
1636 & 1644 & Adsorbed $\mathrm{H}_{2} \mathrm{O}$ \\
1427 & 1429 & $\mathrm{CH}$ wagging (in-plane bending) \\
1361 & 1368 & $\mathrm{CH}$ bending (deformation stretch) \\
1334 & 1337 & OH in-plane bending \\
1314 & 1316 & CH wagging \\
1280 & 1281 & $\mathrm{CH}$ deformation stretch \\
1247 & 1247 & OH in-plane bending \\
1203 & 1203 & OH in-plane bending \\
1159 & 1160 & Asym. Bridge C-O-C \\
1105 & 1108 & Asym. Bridge C-O-C \\
1052 & 1057 & Asym. In-plane ring stretch \\
1028 & 1030 & C-O stretch \\
897 & 900 & Asym. out-of-phase ring stretch: \\
& & C 1 - O-C 4 ; b glucosidic bond \\
\hline
\end{tabular}

\section{SEM}

The modifications appearing at the surface of the linen fibers with the increasing exposure time to the accelerated weathering conditions can be observed in Figure 2.
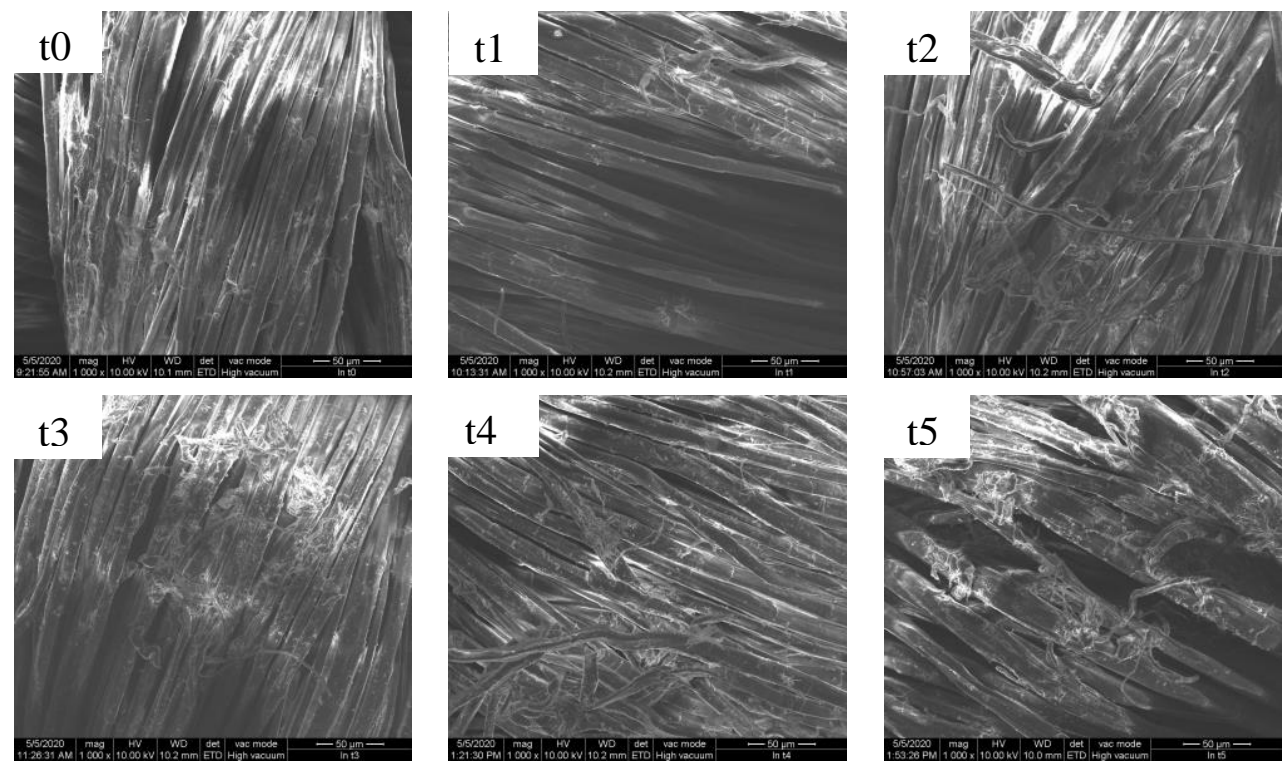

Figure 2. SEM micrographs of the control sample (t0) and the artificially aged samples $(\mathrm{t} 1-\mathrm{t} 5)$ at $1000 \times$ magnification 
With the increasing exposure time to the weathering conditions, the linen fibers have become noticeably damaged. The damage appeared as splits and ruptures, affecting the integrity of the fibers. By the time $\mathrm{t5}$, almost all the fibers were broken. These effects lead to an additional reduction of mechanical properties due to the molecular modifications that resulted from the oxidation at the glycosidic bonds as well as at hydroxyl groups of the cellulose, causing the scission of the macromolecular chains (Kouznetsov et al.,1996).

\section{EDS}

This elemental analysis method was mainly employed to quantify the variation of the oxygen/carbon ratio from the main component of the linen fibers - cellulose.

Table 3. Element quantification

\begin{tabular}{cccc}
\hline Sample & Oxygen $(\%$ weight $)$ & Carbon $(\%$ weight $)$ & O/C ratio \\
\hline t0 & 52.97 & 47.03 & 1.13 \\
t1 & 53.82 & 46.18 & 1.17 \\
t2 & 54.05 & 45.95 & 1.18 \\
t3 & 53.58 & 46.42 & 1.15 \\
t4 & 53.87 & 46.13 & 1.17 \\
t5 & 54.32 & 45.68 & 1.19 \\
\hline
\end{tabular}

A slight increase in the $\mathrm{O} / \mathrm{C}$ ratio can be observed when comparing samples t0 and $\mathrm{t} 5$, that can be attributed to oxidative transformations (i.e., aldehyde groups from the linen fibers into carboxyl groups) (Kleinert, 1972).

\section{Chromatic Parameters}

The color changes are expressed in a qualitative manner by using three variables: shade, resistance, and luminosity (Colour Terms and Definitions, 2008; Ingamells, 1993). The chromatic parameters indicate the visual differences that may appear when a textile material is aged. The parameter $\mathrm{L}^{*}$ represents the brightness of the samples, on a scale from 0 (black) to 100 (white). The $\mathrm{DL}^{*}$ parameter indicates the brightness level of the samples. The saturation $\left(\mathrm{C}^{*}\right)$ represents the intensity of a certain color. The DC* parameter measures the saturation degree of the samples.

Table 4. Chromatic parameters of the linen samples

\begin{tabular}{ccccc}
\hline Sample & $\mathrm{L}^{*}$ & $\mathrm{C}^{*}$ & $\mathrm{DL}^{*}$ & $\mathrm{DC}^{*}$ \\
\hline $\mathrm{t} 0$ & 92.47 & 1.87 & - & - \\
$\mathrm{t} 1$ & 88.94 & 1.97 & -3.52 & 0.10 \\
$\mathrm{t} 2$ & 89.24 & 1.70 & -3.22 & -0.16 \\
$\mathrm{t} 3$ & 89.05 & 2.45 & -3.42 & 0.58 \\
$\mathrm{t} 4$ & 88.98 & 1.39 & -3.49 & -0.48 \\
$\mathrm{t} 5$ & 89.17 & 1.52 & -3.29 & -0.35 \\
\hline
\end{tabular}

All aged samples were darker and less saturated than the control sample. However, sample $\mathrm{t} 3$, which was more saturated than the control, is an exception. The visual aspect of the aged linen samples was a color shift towards a redder shade. This effect might be 
caused by the photooxidation of the linen fabrics during the ultraviolet exposure (Tera et al., 1985).

\section{CONCLUSIONS}

The accelerated weathering experiment that was performed on the linen fabrics revealed, up to a certain point, the effects of ultraviolet radiation and humidity exposure, along with relatively high temperatures. The morphology of the linen fibers was clearly affected by increasing the period of exposure inside the weathering tester, namely, extended fiber ruptures, and splits appeared. These effects have a high negative impact upon the mechanical properties of the linen textiles. At the molecular level though, the modifications imposed by the weathering conditions were not actually significant. However, the visual aspect of the linen samples was affected. The acquired information will be used as a starting point for conservation experiments.

\section{Acknowledgements}

This work was elaborated through Nucleu Program, conducted with MCI support, project no. 4N/2019/PN 191705 01. The publication fee of the paper is funded by the Ministry of Research and Innovation within Program 1 - Development of the national RD system, Subprogram 1.2 - Institutional Performance - RDI excellence funding projects, Contract no. 6PFE/2018.

\section{REFERENCES}

Akin, D.E. (2003), Kirk-Othmer Encyclopedia of Chemical Technology, John Wiley \& Sons, Inc.

Chung, C., Lee, M. and Choe, E.K. (2004), "Characterization of cotton fabric scouring by FT-IR ATR spectroscopy”, Carbohydr. Polym., 58, pp. 417-420, https://doi.org/10.1016/j.carbpol.2004.08.005.

Friedrich, K. and Breuer, U. (2015), Multifunctionality of Polymer Composites: Challenges and New Solutions, William Andrew, pp. 102-143.

Ingamells, W. (1993), Colour for textiles. A user's handbook, Hyperion Books, pp. 4-11.

Kleinert, T.N. (1972), "Ageing of Cellulose. Pt. VI. Natural Ageing of Linen over Long Periods of Time", Holzforschung, 26(2), pp. 46-51, https://doi.org/10.1515/hfsg.1972.26.2.46.

Kouznetsov, D.A., Ivanov, A.A. and Veletksy, P.R. (1996), "Effects of fires and biofractionation of carbon isotopes on results of radiocarbon dating of old textiles: The Shroud of Turin", Journal of Archaeological Science, 23, https://doi.org/10.1006/jasc.1996.0009.

Markova, I. (2019), Textile Fiber Microscopy: A Practical Approach, Wiley, pp. 31-40, https://doi.org/10.1002/9781119320029.

Tera, F.M., Abodu, L.A., Micheal, M.N. et al. (1985), A Journal of Polymer Photochemistry Part II, Vol. 6, p. 475, https://doi.org/10.1016/0144-2880(85)90034-X.

*** (2008), "Colour Terms and Definitions", Journal of the Society of Dyers and Colourists, 89(11), pp. 411422, https://doi.org/10.1111/j.1478-4408.1973.tb03113.x. 
Effects of UV, Humidity, and High Temperature Exposure on Linen Fibers

https://doi.org/10.24264/icams-2020.V.11 


\title{
PROTOCOL FOR IDENTIFICATION AND ASSESSMENT OF NATURAL AND
} SYNTHETIC TEXTILE FIBERS

\author{
LUCIA-OANA SECAREANU ${ }^{1}$, IRINA-MARIANA SANDULACHE ${ }^{1}$, ELENA-CORNELIA \\ MITRAN $^{1,2}$, MIHAELA-CRISTINA LITE ${ }^{1,2}$, ADRIAN ALEXANDRU APOSTOL $^{3}$, OVIDIU \\ IORDACHE $^{1}$, ELENA PERDUM ${ }^{1}$ \\ ${ }^{1}$ The National R\&D Institute for Textiles and Leather, $16^{\text {th }}$ Lucretiu Patrascanu Street, 030508, \\ Bucharest, Romania, office@incdtp.ro \\ ${ }^{2}$ University Politehnica of Bucharest, Faculty of Applied Chemistry and Materials Science, 1-7 \\ Gheorghe Polizu,Bucharest, Romania, secretariat@chimie.upb.ro \\ ${ }^{3}$ University of Bucharest, Faculty of Chemistry, Department of Inorganic Chemistry, Str. \\ Dumbrava Rosie, 23, 020464, Bucharest, Romania, secretariat@chimie.upb.ro
}

\begin{abstract}
Proper identification of textile materials is essential, as people use textiles for clothing and shelter, dental and medical devices, protective firefighting, or even military clothing. There have been several developments regarding fiber identification using instruments such as Fourier transform infrared spectroscopy, Raman spectroscopy, or electron microscopy. However, the traditional methods are prevalent as they are the cheapest alternative. In the present paperwork, an accelerated weathering test was conducted on two different textile materials - cotton (natural fiber) and polypropylene (synthetic fiber). Alternating cycles of UV exposure, along with humidity and relatively high temperatures were employed for the weathering test. In order to evaluate the degradation degree of the two fibers, the results were compared and investigated using nondestructive and micro-destructive analysis techniques such as Scanning Electron Microscopy (SEM), to evaluate the surface modifications of the fibers, and colorimetry, to quantify the color changes. In addition, Fourier-Transform Infrared Spectroscopy (FT-IR) indicated the modifications of functional groups that occurred after the weathering test. A non-destructive technique - X-Ray Diffraction (XRD) was also performed to obtain information about the crystalline structure. The obtained information will be used for cultural heritage studies.
\end{abstract}

Keywords: fibers, accelerated weathering, cultural heritage.

\section{INTRODUCTION}

The history of textile is almost as old as the history of human civilization and as time moves on the history of textile has further enriched itself (Kvavadze et al., 2009). Traditionally, natural fibers have been used in all cultures for making functional products like clothes, bandages, uniform, etc. Clothing and textiles have been important in human history and reflect the materials available to a civilization as well as the technologies that were acquired. The social significance of the finished product reflects their culture (Byko, 2005). It is therefore important to develop new and efficient protocols to proper identify the fibers as this can provide answers that can be further used for cultural heritage studies. Identifying fibers involves observing the physical and chemical properties of the fiber for which there are a wide diversity of instruments available (Robertson, 2010). As fibers behave differently depending on the fibers composition, the light, temperature and humidity they are exposed to (Yang and Ding, 2006) in the present paper two different fibers, cotton (natural fiber) and polypropylene (synthetic fiber), were analyzed after conducting an accelerated weathering test, using non-destructive and micro-destructive techniques such as Scanning Electron Microscopy (SEM), Fourier-Transform Infrared Spectroscopy (FT-IR) and X-Ray Diffraction (XRD). 


\section{MATERIALS AND METHODS}

A QUV instrument from Q-Lab, equipped with fluorescent UVB-313 lamps, was used to simulate an accelerated weathering process on five samples of the two types of fiber. The weathering cycle used consisted of an $8 \mathrm{~h} \mathrm{UV}$ exposure cycle at $70^{\circ} \mathrm{C}$, followed by a $4 \mathrm{~h}$ condensation cycle $\left(60 \%\right.$ humidity) at $50^{\circ} \mathrm{C}$. A sample from each fabric was collected every three days, resulting five samples at different times of exposure.

\section{RESULTS AND DISCUSSION}

\section{SEM}

A FEI Quanta 200 Scanning Electron Microscope was employed for assessing the morphological changes of the samples (Table 1). The magnification used was 500X.

Table 1. SEM micrographs of the control and aged cotton and polypropylene samples

Sampling time $\mathrm{t}_{0}$ (control) Cotton


The accelerated weathering process did not cause a significant modification of the cotton fibers' morphology. However, some fiber breakage was noticed, mostly at the latter periods of exposure. The dry heat caused the desiccation of the cotton fibers, hence their chemical and physical degradation (Timar-Balazsy and Eastop, 1998). Due to the high cellulose content of cotton, the additional UV radiation was absorbed by the fibers, causing more damage.

For the polypropylene samples, the effect of the weathering test was visible via SEM from time $t_{2}$, in terms of fiber breakage. In the last three sampling periods, the fibers were not only broken, but they were also brittle, with cracks appearing transversally on them, confirming the damaging effects of the weathering experiment.

\section{FTIR}

IR spectra were recorded using an FT-IR-ATR instrument from ThermoFisher, over a spectral range of $4000-400 \mathrm{~cm}^{-1}$ (Figures 1 and 2).

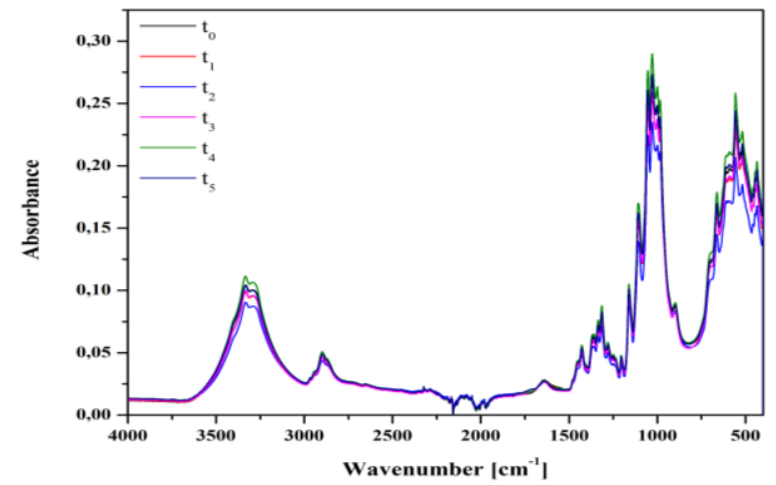

Figure 1. IR absorption spectra of the control and aged cotton samples

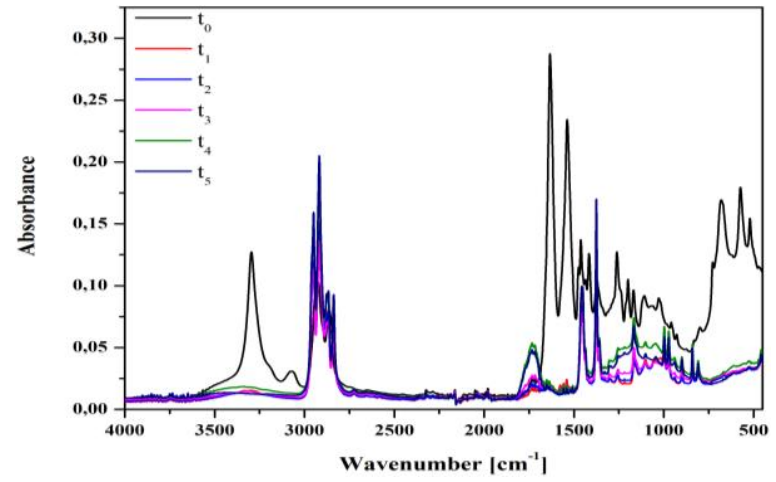

Figure 2. IR absorption spectra of the control and aged polypropylene samples

For cotton fabrics there are no significant changes in the IR absorption spectra of the exposed samples compared to the reference fabric. Only slightly changes in the intensity of the bands can be observed. This indicates that the changes at the structural level are minimal and the cotton fabric is not significantly altered after the exposure to the 
mentioned conditions. Table 2 indicates the IR spectra correspond with the literature data (Chung et al., 2004).

Table 2. IR absorption band positions for cotton compared to literature data

\begin{tabular}{ccc}
\hline $\begin{array}{c}\text { Experimental band } \\
\text { position }\left[\mathrm{cm}^{-1}\right]\end{array}$ & $\begin{array}{c}\text { Band position reported in } \\
\text { literature }\left[\mathrm{cm}^{-1}\right]\end{array}$ & Band attribution \\
\hline $3600-3000$ & $3550-3100$ & H-bonded OH stretch \\
2897 & $2980-2800$ & C-H stretching \\
1636 & 1644 & Adsorbed $\mathrm{H}_{2} \mathrm{O}$ \\
1427 & 1429 & $\mathrm{CH}$ wagging (in-plane bending) \\
1361 & 1368 & $\mathrm{CH}$ bending (deformation stretch) \\
1334 & 1337 & OH in-plane bending \\
1314 & 1316 & CH wagging \\
1280 & 1281 & CH deformation stretch \\
1247 & 1247 & OH in-plane bending \\
1203 & 1203 & OH in-plane bending \\
s1159 & 1160 & Asym. Bridge C-O-C \\
1105 & 1108 & Asym. Bridge C-O-C \\
1052 & 1057 & Asym. In-plane ring stretch \\
1028 & 1030 & C-O stretch \\
897 & 900 & Asym. out-of-phase ring \\
& & stretch: C1-O-C4; b glucosidic bond \\
\hline
\end{tabular}

On the other hand, the overlapped spectra of the polypropylene samples show notable changes, especially in terms of band intensity. The bands at 3296 and $3070 \mathrm{~cm}^{-1}$ in the unexposed fabric spectrum are missing in all the other spectra. This indicates that any trace of supramolecular interaction is destroyed even after short exposure time. The bands at $2950 \mathrm{~cm}^{-1}, 2917 \mathrm{~cm}^{-1}, 2868 \mathrm{~cm}^{-1}, 2837 \mathrm{~cm}^{-1}$ are assigned to the $\mathrm{CH}_{2}$ asymmetrical stretching vibration, $\mathrm{CH}_{3}$ asymmetrical stretching vibration, $\mathrm{CH}_{3}$ symmetrical stretching vibration and symmetrical $\mathrm{CH}_{2}$ stretching vibration, respectively. The bands at $1456 \mathrm{~cm}^{-1}$ and $1375 \mathrm{~cm}^{-1}$ are attributed to the deformation vibration asymmetrical and deformation vibration symmetrical of the $\mathrm{CH}_{3}$ (Souza et al., 2017). These bands suffer an increase of the intensity during the exposure. The significant difference between $t_{0}$ and $t_{1}$ spectra might be due to the presence impurities which seem to be removed in the exposure process. The overlapped spectra of the $t_{1-5}$ samples do not present considerable changes of bands position or intensity.

\section{XRD}

The XRD diffraction measurements were performed on a Proto AXRD diffractometer, using as $\mathrm{X}$-ray the $\mathrm{Cu} \mathrm{K} \alpha$ radiation $(\lambda=1,54 \AA)$, between $2 \theta$ angles 5$35^{\circ}$ (Figures 3 and 4). 


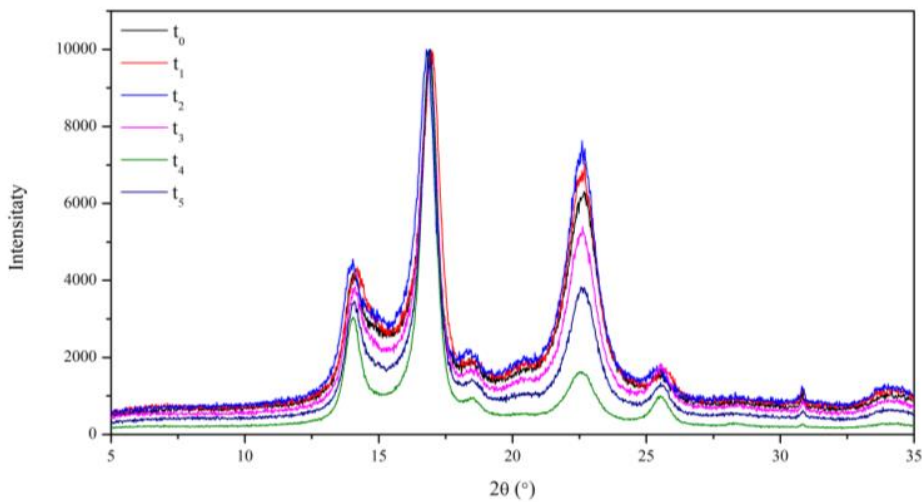

Figure 3. PXRD of the cotton sample, at different exposure times

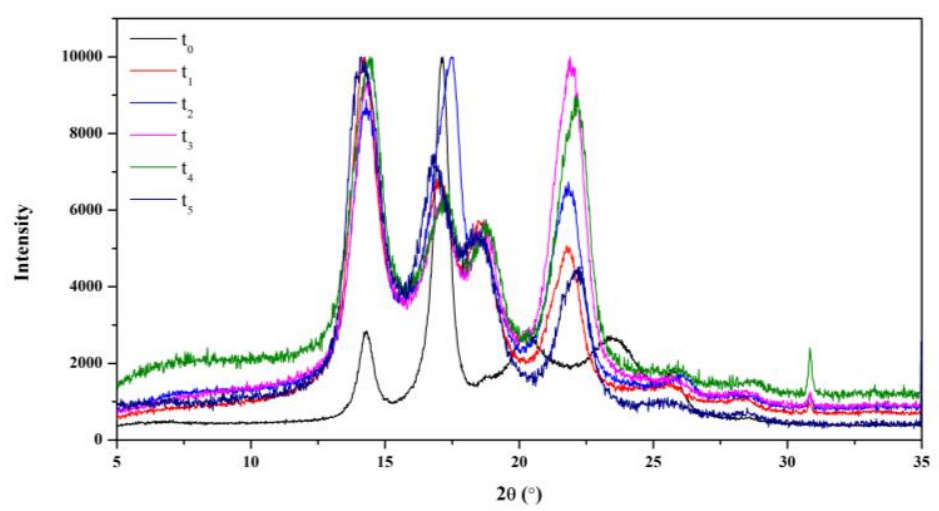

Figure 4. PXRD of the polypropylene sample, at different exposure times

The diffraction lines recorded at different exposure times do not follow a certain trend. In the case of cotton, the crystallinity increases up to 6 days of exposure then decreases. This is observed following the diffraction line at $2 \theta=22,8^{\circ}$, representing the crystallographic plane (002) of the cellulose (Park et al., 2004). A possible explanation is that the degradation destroys first the non-crystalline areas, only later the crystalline ones.

The XRD peaks and corresponding crystallographic plane for polypropylene are listed in table 3 .

Table 3. XRD peaks and corresponding crystallographic plane for polypropylene (Yang and Ding, 2006)

\begin{tabular}{ccc}
\hline $2 \theta\left(^{\circ}\right)$ & Crystallographic plane & Crystalline form \\
\hline 14,0 & $(110)$ & $\alpha$ \\
16,0 & $(300)$ & $\beta$ \\
17,0 & $(040)$ & $\alpha$ \\
18,5 & $(130)$ & $\alpha$ \\
20,5 & $(301)$ & $\beta$ \\
21,5 & $(111)$ & $\alpha$ \\
\hline
\end{tabular}


Polypropylene is present in nature as different polymorphic forms: $\alpha$, the most crystalline, $\beta$, less crystalline and $\gamma$, almost completely amorphous. Table 1 contains the specific diffraction lines for each form (Türkçü, 2014). In this case, the sample contains mostly $\alpha$ polymorphic form. When exposed to the degradation conditions, the amorphous part of the fiber is destroyed, along with the $\beta$ component present in the sample, so the overall crystallinity of the sample is increased. This can be observed by following the increase in intensity of the (110) and (111) lines and the disappearance of the (301) line. It also can be observed a small line forming at $2 \theta=31^{\circ}$, having higher intensities for the most exposed samples.

\section{CONCLUSIONS}

All three performed analyzes show a higher resistance of cotton to polypropylene after the accelerated weathering process was conducted. The changes at the structural level were minimal and the cotton fabric is not notably altered after the exposure to the mentioned conditions. Therefore, we can conclude that even if synthetic fibres are known to be strong and durable, given the exposure conditions of these two fibres, the cotton maintained better. As natural fibres usually have a smaller environmental impact than synthetic fibres due to less chemicals involved in the production process the results are encouraging. Research in this area will continue, in order to improve and help our environment, but also to better maintain our patrimonial objects.

\section{Acknowledgements}

This work was elaborated through Nucleu Program, conducted with MCI support, project no. 4N/2019/PN 191705 01. The publication fee of the paper is funded by the Ministry of Research and Innovation within Program 1 - Development of the national RD system, Subprogram 1.2 - Institutional Performance - RDI excellence funding projects, Contract no. $6 \mathrm{PFE} / 2018$.

\section{REFERENCES}

Byko, M. (2005), "From Electric Corsets to Self-Cleaning Pants: The Materials Science and Engineering of Clothing", JOM, 57(7), 14-18

Chung, C., Lee, M. and Choe, E.K. (2004), "Characterization of cotton fabric scouring by FT-IR ATR spectroscopy", Carbohydr. Polym., 58, 417-420, https://doi.org/10.1016/j.carbpol.2004.08.005.

Kvavadze, E., Bar-Yosef, O., Belfer-Cohen, A., Boaretto, E., Jakeli, N., Matskevich, Z. and Meshveliani, T. (2009), “30,000-Year-Old Wild Flax Fibers”, Science, 325 (5946), 1359, https://doi.org/10.1126/science.1175404

Park, C.H., Kang, Y.K. and Im, S.S. (2004), "Biodegradability of cellulose fabrics", J. Appl. Polym. Sci., 94, 248-253, https://doi.org/10.1002/app.20879.

Robertson, J. (2010), "Identification of textile fibers", Australian Journal of Forensic Sciences, 42(2), 153156, https://doi.org/10.1080/00450611003705802.

Souza, B.R., Benedetto, R.M., Hirayama, D., Raponi, O., Barbosa, L.C.M. and Ancelotti, A.C. (2017), "Manufacturing and Characterization of Jute/PP Thermoplastic Commingled Composite", Mater. Res., 20, 458-465, https://doi.org/10.1590/1980-5373-mr-2017-0104.

Timar-Balazsy, A. and Eastop, D. (1998), Chemical Principles of Textile Conservation, Routledge, p. 16.

Türkçü, H.N. (2014), "Investigation of the Crystallinity and Orientation of Polypropylene with Respect to Temperature Changes Using FT-IR, XRD, and Raman Techniques", Thesis, Department of Chemistry and the Institute of Engineering and Sciences of Bilkent University.

Yang, X. and Ding, X. (2006), "Prediction of outdoor weathering performance of polypropylene filaments by accelerated weathering tests", Geotextiles and Geomembranes, 24(2), 103-109, https://doi.org/10.1016/j.geotexmem.2005.11.002.

https://doi.org/10.24264/icams-2020.V.12 


\title{
MICRO DSC AND NMR MOUSE STUDIES OF COLLAGEN-VEGETABLE TANNIN INTERACTION MECHANISM DURING LEATHER MAKING
}

\author{
CLAUDIU ȘENDREA ${ }^{1}$, MARIA-CRISTINA MICU ${ }^{1}$, EMANUEL HADIMBU ${ }^{1}$, SIMONA \\ MARIA PĂUNESCU ${ }^{1}$, IULIA MARIA CANIOLA ${ }^{1}$, MADALINA IGNAT ${ }^{1}$, LUCRETIA MIU ${ }^{1}$, \\ ELENA BADEA ${ }^{1,2^{*}}$ \\ ${ }^{1}$ INCDTP - Leather and Footwear Research Institute (ICPI) Division, Bucharest, Romania, \\ elena.badea@unito.it \\ ${ }^{2}$ Department of Chemistry, Faculty of Sciences, University of Craiova, Craiova, Romania
}

\begin{abstract}
In this study NMR MOUSE and micro DSC techniques were used to investigate the interaction between collagen and various vegetable tannins during leather making process with the aim of gaining a deeper understanding of different water environment in relation to tannin type. We have previously showed that relaxation times may provide useful information on collagen matrix properties. The vegetable tanned leathers were obtained by patented techniques inspired from ancient recipes at the National R\&D Institute for Textile and Leather, ICPI Division, Bucharest using various vegetable extracts such as myrobalan, gambier and chestnut. Longitudinal and transversal relaxation times $T_{1}$ and $T_{2 \text { eff }}$ were measured using a PM2 portable NMR-MOUSE with 20.05 MHz frequency. Micro DSC measurements were carried out with a high-sensitivity SETARAM Micro-DSC III in the temperature range (5 to 95$){ }^{\circ} \mathrm{C}$ at $0.5 \mathrm{~K} \mathrm{~min}^{-1}$ heating rate. The investigated leathers showed significant differences in the values of spin-spin $\left(T_{2 \mathrm{eff}}\right)$ and spinlattice $\left(T_{1}\right)$ relaxation times depending on tannin type that well corelates with the variation of the calorimetric parameters (denaturation temperature and enthalpy, peak shape). These results highlight the complementarity of the information obtained by the two techniques and open new ways for both designing new leather assortments and analyses of historical and archaeologica leather.
\end{abstract}

Keywords: unilateral NMR, micro DSC, vegetable tanned leather, collagen-tannin interaction

\section{INTRODUCTION}

Since ancient times people have been soaking the skins in natural tannins to dehydrate them and prevent the leather goes stiff and putrescible. The vegetable tannin solutions were made up of organic substance present in trees (such as oak, chestnut or mimosa), or a large number of other types of plants. Starting from the second half of the XIX century chrome tanning became the most common and dominant form of tanning. Chrome tanning brought about a number of innovation in tanning such as a fat liquoring (re-greasing) process and the use of synthetic dyes. Besides, the process was much faster than vegetable tanning. Today, more than $90 \%$ of leather production is based on chrome tanning. However, the leather industry is facing a number of challenges including a continuously tightening regulatory framework for safer and cleaner chemicals and technologies and the ongoing need to differentiate in terms of innovativeness, design, appearance and comfort. So far, vegetable tanning has returned in the leather industry focus, although it requires a high degree of craftmanship.

This paper concerns with the tanning reaction and the origin of hydrothermal stability in vegetable tanned leather. Micro differential scanning calorimetry (micro DSC) was used to quantify the increase in hydrothermal stability upon tanning with various vegetable extracts such as gambier, myrobalan and chestnut. The transverse relaxation time $T_{2}$ measured by Unilateral Nuclear Magnetic Resonance (NMR MOUSE) provided information related to the dynamics of water molecules inside the collagen matrix, while the longitudinal relaxation time $T_{1}$ was related to the collagentannin matrix strength.

https://doi.org/10.24264/icams-2020.V.13 


\section{MATERIALS AND METHODS}

\section{Tannins and Vegetable Tanned Leather}

The vegetable extracts were from Seta S.A., Brazil and Silva Team S.P.A., Italy. Goat hides were tanned using a patented technology inspired from ancient recipes (Miu et al., 2006).

\section{Micro Differential Scanning Calorimetry (Micro DSC) Measurement}

The measurement of hydrothermal stability of pelt and the derived vegetable tanned hides was carried out with a high-sensivity microDSC III Setaram microcalorimeter equipped with Haake DC10 circulator for stabilizing the heating/cooling temperature. Samples were hydrated in $0.5 \mathrm{M}$ acetate buffer solution with $\mathrm{pH} 5.0$ for $1 \mathrm{~h}$, directly in the calorimetric cells, and then heated in the temperature range $(25$ to 95$){ }^{\circ} \mathrm{C}$ at 0.5 $\mathrm{K} \cdot \mathrm{min}^{-1}$ heating rate as previously reported (Carsote and Badea, 2019; Carşote et al., 2016). Three measurements were run using fresh subsamples. Experimental DSC data acquired with the SETARAM SetSoft2000 software were analysed using PeakFit 4.1 (Jandel Scientific) software. Denaturation temperature, $T_{\max }$, was determined as the temperature attained at peak maximum. Temperature span of the transition was reported as peak width at half height, $\Delta T_{1 / 2}$, and specific denaturation enthalpy, $\Delta H$ was calculated as the area under the peak by integrating $C_{p}^{e x}(T)$ curve across the denaturation temperature range.

\section{Unilateral Magnetic Resonance (NMR MOUSE) Measurement}

${ }^{1} \mathrm{H}$ NMR measurements were performed at room temperature using an NMR MOUSE PM2 (Magritek GmbH) controlled by a Kea 2 spectrometer (Magritek GmbH) operating at $27 \mathrm{MHz}{ }^{1} \mathrm{H}$ resonance frequency as described earlier (Badea et al., 2016; Sendrea et al., 2016). This system allows to measure proton relaxation times without any previous preparation of the samples. Effective ${ }^{1} \mathrm{H}$ spin-spin relaxation $T_{2 \mathrm{eff}}$ measurements have been measured using the Carr-Purcell-Meiboom-Gill (CPMG) pulse sequence with an echo-time (TE) of about $25 \mu$ s. The experimental CPMG curves were best analyzed by a combination of double exponential functions. The proton spin-lattice relaxation times $T_{1}$ were measured with a saturation-recovery pulse sequence using a Hahn-echo with an echo time of about $25 \mu$ for detection. The analysis of the saturation recovery data was best performed with the help of a single exponential function.

\section{RESULTS AND DISCUSSION}

\section{Effect on Tannin Type on Leather Hydrothermal Stability}

The micro DSC denaturation curves for the vegetable tanned leathers are shown in Figure 1 together with the DSC curve for pelt (not tanned hide) as comparison. The calorimetric parameters that characterize the collagen matrix denaturation in vegetable tanned leathers, chrome-tanned leather and pelt are listed in Table 1. Hydrolysable (myrobalan and chestnut) tanned leathers show similar denaturation curves and parameters, while the condensed (gambier) tanned leather shows higher $T_{\text {onset }}$ and $T_{\max }$ 
and lower $\Delta T_{1 / 2}$, as expected: thermal stability and structural homogeneity increase as tanning efficiency increases.

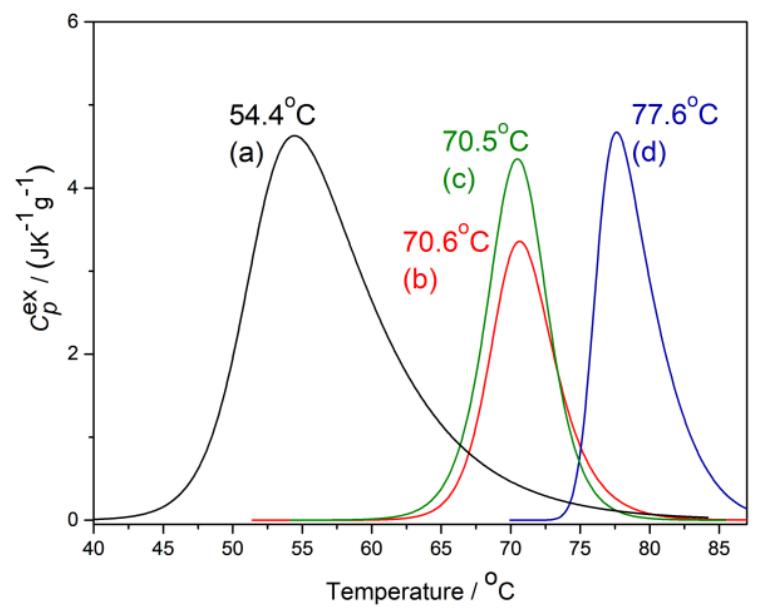

Figure 1. DSC denaturation peaks for vegetable tanned leathers $(b-$ myrobalan, $c-$ chestnut, $d$-gambier) compared to those of pelt (a)

Table 1. DSC parameters of thermal denaturation of vegetable tanned leathers compared to pelt and chrome tanned leather

\begin{tabular}{|c|c|c|c|c|}
\hline Samples & $\Delta H / \mathrm{J} \cdot \mathrm{g}^{-1}$ & $T_{\text {onset }} /{ }^{\circ} \mathrm{C}$ & $T_{\max } /{ }^{\circ} \mathrm{C}$ & $\Delta T_{1 / 2} /{ }^{\circ} \mathrm{C}$ \\
\hline Leather - gambier & 28 & 74.6 & 77.6 & 4.8 \\
\hline Leather - myrobalan & 26 & 65.9 & 70.6 & 5.3 \\
\hline Leather - chestnut & 33 & 65.8 & 70.5 & 5.0 \\
\hline Leather- chrome ${ }^{1}$ & $50-54$ & - & 111.2 & 3.5 \\
\hline Pelt & 56 & 47.3 & 54.5 & 10.4 \\
\hline
\end{tabular}

${ }^{1}$ Values reported by Cucos et al. (2015)

\section{Effect on Tannin Type on Transverse Relaxometric Parameters}

The values of $T_{1}, T_{2 \text { eff_short }}$ and $T_{2 \text { eff_long }}$ are shown in Table 2 . It is noteworthy that $T_{1}$ values increased in the following order: chrome-tanned leather < gambier (catechin) tanned leather < myrobalan (ellagitannin) tanned leather < chestnut (ellagitannin) tanned leather < pelt (not tanned collagen). This behaviour indicates that the collagentannin interaction causes a variation of $T_{1}$ values, and the extent to which $T_{1}$ varies depends on the chemical nature of the tannin. Some of us have already reported that $T_{1}$ values allowed to differentiate between the effects of hydrolysable and condensed tannins on collagen water environment in calf leathers (Badea et al., 2016). By comparing the values of the proton longitudinal relaxation times $T_{1}$ with those of the corresponding temperature of denaturation $T_{\max }$ an inverse correlation is observed: the highest value of $T_{1}$ corresponds to the least thermally stable collagenous material, i.e. pelt (not tanned collagen). 
Table 2. $T_{1}$ and $T_{2 \text { eff }}$ relaxation time values for vegetable tanned leathers compared to chrome tanned leather and parchment (not tanned collagen)

\begin{tabular}{lccc}
\hline Samples & $T_{1} / \mathrm{ms}$ & $T_{\text {2eff_long }} / \mathrm{ms}$ & $T_{\text {2eff_short }} / \mathrm{ms}$ \\
\hline Leather - chestnut & 42.1 & 4.66 & 0.31 \\
Leather - myrobalan & 40.1 & 4.18 & 0.26 \\
Leather - gambier & 36.4 & 2.65 & 0.24 \\
Leather - chrome & 9.6 & 1.29 & 0.13 \\
Parchment & 50.3 & 0.93 & 0.16 \\
\hline
\end{tabular}

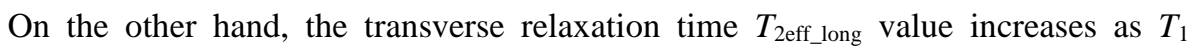
increases for leather while the relaxometric behaviour of parchment is different - it presents the lowest value $T_{2 \text { eff_long }}$ and the highest $T_{1}$ value. We will explain this behaviour by considering that the two $T_{2}$ components correspond to water in various environments. In fact, Rodin et al. (2000) reported that different degree of cross linking in collagen fibres affects the water dynamics. This was directly related to the fibrous collagen microstructure and chain mobilities in the collagen matrix, namely $T_{2 \text { eff_short }}$ relates to the crystalline phase and $T_{2 \text { eff_long }}$ to the amorphous phase (Nishad Fathima $e t$ al., 2010; Sendrea et al., 2017). The increase of chain mobility in vegetable tanned leather by comparison with parchment and chrome-tanned leather could thus be interpreted in terms of a looser packing of collagen fibrils in both the amorphous and crystalline phases. This is in good agreement with the lower hydrothermal stability of vegetable tanned leather by comparison with chrome tanned leather. In case of parchment, the strong dehydration during drying under tension results in a tighter packing of collagen fibrils and restricted chain mobility. It is worthy of note that the second water fraction $T_{\text {2eff_long, }}$ being much more mobile, feels better the influence of the tanning agents. Our results confirm the previous data obtained on sheep leather: $T_{2 \text { eff_long }}$ values discriminate between condensed and hydrolysable tannins (Badea et al., 2016).

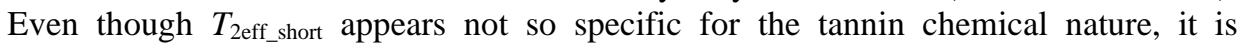
sensitive to the chain mobility constraints, being discriminative for chemically bound and chemically unbound collagen as already reported by some of us (Badea et al., 2016).

\section{CONCLUSIONS}

In the present work unilateral NMR and micro DSC were used to discriminate between the tanning chemistry of hydrolysable and condensed tannins. The effect of collagen-tannin interaction was discusses based on water dynamics in collagenous matrices and their hydrothermal stability. The main conclusions may be summarized as follows:

- $\quad T_{2 \text { eff_long }}$ and $T_{1}$ relaxation values are discriminative for the chemical nature of tannin;

- $\quad T_{2 \text { eff_short }}$ is discriminative between chemically bound (tanned) and unbound (not tanned) collagen;

- $\quad$ an inverse correlation is observed between $T_{\max }$ and $T_{1}$ values;

- $\quad$ the relaxometric parameters confirms a looser packing of collagen fibrils in both the amorphous and crystalline phase in vegetable tanned leather compared to chrome tanned leather. 
Micro DSC and unilateral NMR are complementary techniques that sensitively discriminate for the nature of collagen-tannin interaction and may thus be successfully applied to validate new chrome-free tanning technologies, as well as to characterize historical and archaeological leather.

\section{Acknowledgement}

This work was supported by a grant of the Romanian Ministry of Research and Innovation (now Ministry of Education and Research), CCCDI - UEFISCDI, project number PN-III-P1- 1.2-PCCDI-2017-0878/ no. 55PCCDI/2018, IMPLEMENT.

\section{REFERENCES}

Badea, E., Şendrea, C., Carşote, C., Adams, A., Blümich, B. and Iovu, H. (2016), "Unilateral NMR and thermal microscopy studies of vegetable tanned leather exposed to dehydrothermal treatment and light irradiation", Microchem. J., 129, 158-165, https://doi.org/10.1016/j.microc.2016.06.013.

Carsote, C. and Badea, E. (2019), "Micro differential scanning calorimetry and micro hot table method for quantifying deterioration of historical leather", Herit Sci, 7, 48, https://doi.org/10.1186/s40494-019-0292-8.

Carşote, C., Badea, E., Miu, L. and Della Gatta, G. (2016), "Study of the effect of tannins and animal species on the thermal stability of vegetable leather by differential scanning calorimetry", J. Thermal. Anal. Calorim., 124(3), 1255-1266, https://doi.org/10.1007/s10973-016-5344-7.

Cucos, A., Gaidau, C., Badea, E. and Miu, L. (2015), "Influence of glycerin on denaturation temperature of chrome- and vegetable-tanned leather", Rev. Roum. Chim., 60(11-12), 1093-1096.

Miu, L., Bratulescu, V., Gaidau, C., Bocu, V. and Niculescu O. (2006), Natural leather for bookbinding for heritage and process of making thereof (in Romanian), Romanian patent C14C/2006, available at p.31 at http://www.osim.ro/publicatii/brevete/bopi_2006/bopi0306.pdf.

Nishad Fathima, N., Baias, M., Bluemich, B. and Ramasami, T. (2010), "Structure and dynamics of water in native and tanned collagen fibers: Effect of crosslinking", Int J Biol Macromol, 47, 590-596, https://doi.org/10.1016/j.ijbiomac.2010.08.003.

Rodin, V.V., Sakharov, B.V. and Knight, D.P. (2000), "Hydrated polymer matrices: NMR-relaxation and PFG-NMR in study of molecular mobility", Plast. Massy, 2, 13-24.

Sendrea, C., Badea E. and Adams, A. (2017), "Unilateral NMR and micro DSC study of artificially aged parchments", Rev. Chim., 68(8), 1780-1785, https://doi.org/10.37358/RC.17.8.5764.

Sendrea, C., Carsote, C., Badea, E., Adams, A., Niculescu, M. and Iovu, H. (2016), "Non-invasive characterisation of collagen-based materials by NMR-MOUSE and ATR-FTIR", U.P.B. Sci. Bull., Series Chemistry, 78(3), 27-38. 
Micro DSC and NMR MOUSE Studies of Collagen-Vegetable Tannin Interaction Mechanism During Leather Making

https://doi.org/10.24264/icams-2020.V.13 
VI.

\section{EDUCATION AND DIGITALIZATION}




\title{
FOSTERING ADVANCED TEXTILE CENTERS THROUGH E-LEARNING IN MOROCCO AND JORDAN
}

\author{
RALUCA MARIA AILENI, LAURA CHIRIAC, RAZVAN ION RADULESCU \\ INCDTP, Lucretiu Patrascanu 17, sector 3, Bucharest, Romania, raluca.aileni@incdtp.ro
}

\begin{abstract}
This paper presents some aspects of the need for training and EU best practices and success stories replication to target countries (Morocco and Jordan) in order to establish or upgrade the advanced textile centers. Overall, the proposed teaching and e-learning methods and the aspects of the need for training and EU best practices implementation and success stories replication to target countries (Morocco and Jordan) in order to establish or upgrade the advanced textile centers are essential for higher education organizations involved. In the framework of the FOSTEX Erasmus+ project were identified the best practices across Europe with high potential for being transferrable to the partner countries (Morocco and Jordan) including aspects concerning learning, teaching (courses), dissemination, quality testing, international standardization, equipment, and research projects. We can conclude that the interest of the partners is focused on polymers, protective clothing tests, colour management, product certification, international accreditation, environmental impact (Reach Legislation), colour fastness, washing fastness, and physico-mechanical investigation.
\end{abstract}

Keywords: textile, learning, quantitative, qualitative

\section{INTRODUCTION}

In the framework of the FOSTEX Erasmus+ project, some crucial activities were to identify and analyze EU best practices and successful experiences to be transferred to target countries (Morocco and Jordan) and an electronic database. The database contains best practices identified across Europe with high potential for being transferrable to the partner countries (Morocco and Jordan) and successful EU funded project experiences in capacity building from different sectors. Good practice examples include aspects concerning learning, teaching (courses), dissemination, quality testing, standardization, equipment acquisition, educational, and research projects. The best practices' objectives are to promote research and projects between EU and Moroccan and Jordanian universities in the advanced textiles sector and to create useful research innovation and training network that will continue to generate sustainable results after the end of the project.

However, teaching and learning methods are useful to enhance the educational level of the students. The learning methods to be used (formal or informal type) are necessary for establishing adequate tools and techniques to be used in classes. However, learning in advanced textile materials is the process of acquiring new or modifying existing knowledge, behaviors, skills that can be achieved by examples, theories, and practical examples. Another challenge was to create a training Toolkit for the capacity building consisting of slides, images, and further readings, exercises for each topic covered customized for Jordanian and Moroccan partners' needs.

In order to develop the new advanced textile centers, it is vital to improving the knowledge level through seminars, workshops, e-learning and training, and the infrastructures by the development of new research centers (Jordan), upgrading the existent research centers (Morocco) with new high-quality equipment for material testing, and by promoting the quality in textile materials testing by use of the adequate standards, methods, procedures, and equipment. 
Good practices are lessons already learned about specific aspects concerning teaching, mentoring, dissemination, providing skills, knowledge transfer, coaching for fostering the capacity building in the partner countries (Morocco and Jordan).

In general, the FOSTEX project aims to fill the gap in the area of specialized services for the textile sector, with the establishment of two Textile Centres in Universities of Jordan and the upgrade of two Textile Centres in Morocco through novel and upgraded services such as quality testing, certification of products, human resources training, support of participation to informative seminars, workshops, exhibitions; investigation in the organization of efficient production and enhancing entrepreneurship and integration of refugees into the textile sector; searching for funding opportunities, and improving the textile industry's ability to meet unexpected challenges and to turn into opportunities such as creating protective equipment against COVID-19.

\section{TRAINING TOOLKIT}

In the framework of the FOSTEX Erasmus+ project, have been created a toolkit containing knowledge useful in setting the new textile centers or in upgrading the existing ones and also for applicative and fundamental research investigations.

Among existing testing methods that can be used in textile applicative research or industrial investigation have been selected for the courses, only the methods related to tensile strength testing (Wu and Pan, 2005), testing for protective equipment, washing colour fastness, and colour fastness testing.

The tensile strength testing course module describes the available two methods such as grab (ISO 13934-2:2013, ISO 13935-2:2014) and strip (ISO 13934-1:2013, ISO 13935-1:2014) methods used for tensile strength testing. The strip and grab methods are mainly applicable to woven textile fabrics, fabric containing elastomeric fiber, mechanical or chemical treatment. However, usually, strip and grab methods do not apply to geotextiles, nonwovens, coated fabrics, textile-glass woven fabrics, and fabrics made from carbon fibers or polyolefin tape yarns. Using the strip and grip methods can be investigated the maximum force and elongation at maximum force of test specimens in equilibrium with the standard atmosphere for testing, and test specimens in the wet condition.

The testing for protective textiles and accessories course module highlighted the aspects concerning the technical textiles used to protect against hazards.

The hazard is defined as a situation that can be the cause of harm or damage to the health of the human body. In addition, the hazards types (mechanical, chemical, cold, heat and/or fire, biological agents, radiation) are considered the risks as a combination of the frequency/probability, of occurrence and the consequence of a specified hazard, and the protective textile performance level a number that designates a particular category or range of performance by which the results of testing can be graded.

In the scientific literature are defined several classifications of protective textiles (Raheel, 1994) such as:

- the protection of wearers and/or textiles against insects (Hipler and Elsner, 2006);

- the protection of wearers and/or textiles against heat and fire protection (Horrocks, 2005; Horrocks, 2014; Lawrence, 2014) (firefighters);

- the protection of wearers to water and/or waterproof textiles (water protection) (Türk, 2015);

- the protection of wearers against biological agents (Endrusick et al., 2005);

https://doi.org/10.24264/icams-2020.VI.1 
- the radiance protection of wearers against ultraviolet (UV) rays of the sun (Gies et al., 1998; Ranjan Das, 2010);

- the protection of wearers and/or textiles against electromagnetic-waves (Maity et al., 2013; Koch, 2003) (shielding effect);

- the protection of soldiers and/or textiles for military applications (Wilusz, 2008) against chemical (Anna, 2003), biological, radiological, and nuclear (CBRN) (Magalhães et al., 2017) warfare threats.

Colour fastness-testing represents the resistance testing of the colour of textiles to the different agents (figure 1) to which these materials may be exposed during manufacture and wearing. However, the most used colour fastness tests in the textile industry are color fastness to wash, to rubbing, to perspiration, to light, and hot pressing.

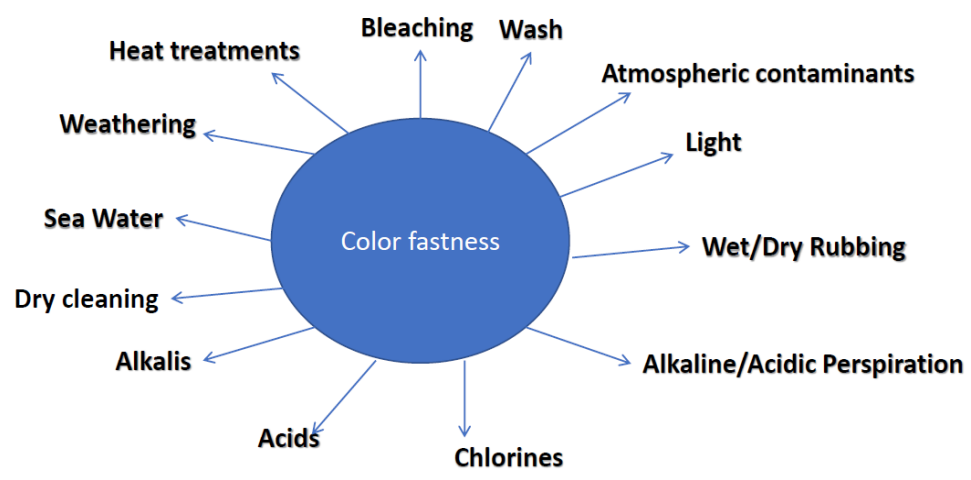

Figure 1. Types of color fastness testing -according ISO 105-A01:2010

In the case of the investigations more appropriate to fundamental research, we can mention the elemental and molecular analysis (Table 1 - Robinson, 2014) used for analysis that can be performed, often with the same instrument and may use light interaction, heat interaction, electric fields or magnetic fields. Each one elemental or molecular analysis uses methods such as:

$\rightarrow$ Qualitative methods that provide information about the identity of atomic/molecular species and functional groups of the sample;

$\rightarrow$ Quantitative methods that provide numerical information about the relative amount of one or more components.

Table 1. Instrumental methods of analysis (Robinson, 2014)

\begin{tabular}{lcccc}
\hline \multicolumn{1}{c}{ Method } & \multicolumn{2}{c}{ Quantitative } & \multicolumn{2}{c}{ Qualitative } \\
& Elemental & Molecular & Elemental & Molecular \\
\hline Atomic absorption spectrometry (AAS) & No & No & Yes & No \\
Atomic emission spectrometry (AES) & Yes & No & Yes & No \\
Capillary electrophoresis (CE) & Yes & Yes & Yes & Yes \\
Gas chromatography (GC) & No & Yes & No & Yes \\
ICP-mass spectrometry (ICP-MS) & Yes & No & Yes & No \\
IR spectroscopy & No & Yes & No & Yes \\
Ion chromatography (IC) & Yes & Yes & Yes & Yes \\
Liquid chromatography (LC) & No & Yes & No & Yes \\
Mass spectroscopy (MS) & Yes & Yes & Yes & Yes \\
Nuclear magnetic resonance (NMR) & No & Yes & No & Yes \\
\hline
\end{tabular}

https://doi.org/10.24264/icams-2020.VI.1 
Fostering Advanced Textile Centers through E-Learning in Morocco and Jordan

\begin{tabular}{lcccc}
\hline \hline \multicolumn{1}{c}{ Method } & \multicolumn{2}{c}{ Quantitative } & \multicolumn{2}{c}{ Qualitative } \\
& Elemental & Molecular & Elemental & Molecular \\
\hline Raman spectroscopy & No & Yes & No & Yes \\
Thermal analysis (TA) & No & Yes & No & Yes \\
UV/VIS spectrophotometry & Yes & Yes & Yes & Yes \\
UV absorption & No & Yes & No & Yes \\
UV fluorescence & No & Yes & No & Yes \\
X-ray absorption (XAS) & Yes & No & Yes & No \\
X-ray diffraction (XRD) & No & Yes & No & Yes \\
X-ray fluorescence (XRF) & Yes & No & Yes & No \\
\hline
\end{tabular}

In the case of the quality testing course module, the adopted approach was to specify the essential terms, definitions, and standards applied in quality (ISO 9000:2015; ISO 9001:2015; ISO 9004:2018), the general terms and definitions relating to conformity assessment to facilitate trade (ISO/IEC 17000:2020), and the general requirements for the competence, impartiality and consistent operation of laboratories (ISO/IEC 17025:2017).

The course module concerning international conformity assessment provided a clear explanation of the certification as a useful tool to add credibility by demonstrating that the product, service, or system meets the expectations of the customers. However, the certification is also known as third party conformity assessment (testing, inspection, and certification) and is essential because, for some industries, certification is a legal or contractual requirement. International Certificate of Conformity $(\mathrm{CoC})$, also named Certificate of Compliance (Amutha, 2017), is a mandatory document necessary for Customs clearance of exports to many countries around the globe. International $\mathrm{CoC}$ is a document certified by a competent authority that the supplied good or service meets the required specifications. Also, the active companies from the textile industry must comply with environmental legislation REACH (Regulation (EC) 1907/2006) designed to ensure a high level of protection of human health and the environment and to manage and control the potential risk to human health and the environment due to the use of chemicals in the European Union.

At the end the all requirements of the textile product (cleaning/washing instructions, size, composition, comfort requirements/specification, physic, chemical, mechanical or electrical requirements/parameters) should be integrated into the technical specification sheet, and some of the information above mentioned should be included in the label, or a hangtag with a barcode.

\section{CONCLUSIONS}

The proposed Training Toolkit is composed of training materials with the necessary technical support relating to the online teaching methods, lesson content, and practical information of the training program. During the online courses was observed an increased interest in lessons related to protective textiles, instrumental analysis, and colour fastness testing. Besides, the participants were very interested in new technologies used for functionalization (plasma) and creating 3D composite materials based textile supports and using polymer matrix and metal microparticles, 3D printing, microwave, and plasma pretreatments. 


\section{REFERENCES}

Amutha, K. (2017), "Sustainable Practices in Textile Industry: Standards and Certificates", in: Sustainability in the Textile Industry, pp. 79-107, Springer, Singapore, https://doi.org/10.1007/978-981-10-2639-3_5.

Anna, D.H. (ed.) (2003), Chemical protective clothing, AIHA, https://doi.org/10.3320/978-1-931504-46-1.

Endrusick, T.L., Gonzalez, J.A. and Gonzalez, R.R. (2005), "Improved comfort of US military chemical and biological protective clothing", Environmental Ergonomics, 3, pp. 369-373, https://doi.org/10.1016/S1572-347X(05)80058-8.

Gies, P.H., Roy, C.R., Toomey, S. and McLennan, A. (1998), "Protection against solar ultraviolet radiation", Mutation Research/Fundamental and Molecular Mechanisms of Mutagenesis, 422(1), pp.15-22, https://doi.org/10.1016/S0027-5107(98)00181-X.

Hipler, U.C. and Elsner, P. (eds.) (2006), Biofunctional textiles and the skin, Vol. 33, Karger Medical and Scientific Publishers, https://doi.org/10.1159/isbn.978-3-318-01349-8.

Horrocks, A.R. (2005), Thermal (heat and fire) protection. Textiles for protection, pp.411-423, Elsevier, https://doi.org/10.1201/9781439823811.ch15.

Horrocks, A.R. (2014), "High performance textiles for heat and fire protection", in: High Performance Textiles and their Applications, pp. 144-175, Woodhead Publishing, https://doi.org/10.1533/9780857099075.144.

ISO 105-A01:2010, Textiles — Tests for colour fastness — Part A01: General principles of testing.

ISO 13934-1:2013, Textiles — Tensile properties of fabrics — Part 1: Determination of maximum force and elongation at maximum force using the strip method

ISO 13934-2:2014, Textiles — Tensile properties of fabrics — Part 2: Determination of maximum force using the grab method.

ISO 13935-1:2014, Textiles — Seam tensile properties of fabrics and made-up textile articles — Part 1 : Determination of maximum force to seam rupture using the strip method.

ISO 13935-2:2014, Textiles — Seam tensile properties of fabrics and made-up textile articles — Part 2: Determination of maximum force to seam rupture using the grab method.

ISO 9000:2015, Quality management systems — Fundamentals and vocabulary.

ISO 9001:2015, Quality management systems — Requirements.

ISO 9004:2018, Quality management — Quality of an organization — Guidance to achieve sustained success

ISO/IEC 17000:2020, Conformity assessment - Vocabulary and general principles.

ISO/IEC 17025:2017, General requirements for the competence of testing and calibration laboratories.

Koch, M., Autoflug GmbH (2003), "Textile base material having an electromagnetic wave shielding", U.S Patent Application 10/448,979.

Lawrence, C. (ed.) (2014), High performance textiles and their applications, Elsevier.

Magalhães, M.J., de Magalhães, S.T., Revett, K. and Jahankhani, H. (2017), "Chemical, Biological, Radiological and Nuclear (CBRN) protective clothing-A review", International Conference on Global Security, Safety, and Sustainability, pp. 331-341, Springer, Cham, https://doi.org/10.1007/978-3-319. 51064-4_26.

Maity, S., Singha, K., Debnath, P. and Singha, M. (2013), “Textiles in electromagnetic radiation protection. Journal of Safety Engineering, 2(2), pp.11-19.

Raheel, M. (ed.) (1994), Protective clothing systems and materials, Vol. 25, CRC Press

Ranjan Das, B. (2010), "UV radiation protective clothing", The Open Textile Journal, 3(1).

Robinson, J.W., Frame, E.S. and Frame II, G.M. (2014), Undergraduate instrumental analysis, CRC press, https://doi.org/10.1201/b15921.

Türk, M., Ehrmann, A. and Mahltig, B. (2015), "Water-, oil-, and soil-repellent treatment of textiles, artificial leather, and leather", The Journal of The Textile Institute, 106(6), pp.611-620, https://doi.org/10.1080/00405000.2014.931108.

Wilusz, E. (ed.) (2008), Military textiles, Elsevier, https://doi.org/10.1201/9781439833247.

Wu, J. and Pan, N. (2005), "Grab and strip tensile strengths for woven fabrics: An experimental verification", Textile research journal, 75(11), pp.789-796, https://doi.org/10.1177/0040517505057525. 
Fostering Advanced Textile Centers through E-Learning in Morocco and Jordan

https://doi.org/10.24264/icams-2020.VI.1 
THE ITERATION METHOD FOR DEVELOPING CREATIVITY IN ECODESIGN

\author{
MARLENA POP ${ }^{1}$, DORINA HORĂTĂU ${ }^{2}$ \\ ${ }^{1}$ INCDTP - Division: Leather and Footwear Research Institute, Bucharest, Romania, \\ pop_marlen@yahoo.ca \\ ${ }^{2}$ UNA, National Art University, Textile Department, Bucharest, Romania, h2dorina@yahoo.com
}

\begin{abstract}
The methods of conceptualization, the theories behind the green-products are issues that this paper wants to address. Spiral Iteration is a method developing creativity in ecodesign for textile and leather industries. It will be demonstrated that exploratory and experimental research in textile design was able to validate the spiral iteration tools and the aesthetic tools allowing the creative to express, through specific visual language, a whole individual universe of Cultural Design, as green identity of cultural sustainable idea of the products.
\end{abstract}

Keywords: ecodesign, cultural design, spiral iteration, green products

\title{
INTRODUCTION
}

Sustainability as an applied science has two distinct directions: an analytical direction and an exploratory one, from the description and analysis of problems, to solving problems by finding solutions defined by evidence-based research (Douglas and Isherwood, 1996). This approach also requires transparency in the methodologies regarding the conceptualization of organic products and the development of new products. The ecodesign standard, ISO $14006 / 2020$, imposes its restrictions to achieve the sustainability of green products. These constraints are in contradiction with the cultural design that facilitates maximum creativity, innovation and culture embedded in the product (Baumgarten and Rautner, 2016).

The typologies of clothing and footwear manufacturing, if we focus our methodological research on this field, refer to "fast fashion", "slow fashion", and arts and crafts or "handmade". These technological systems have different rhythms of product development, determining the different structure of the fashion business. In any anthropized system, Design has the role of showing how objects and processes should be, unlike science, which shows us how phenomena, matter, processes and objects are, being an exploratory and prospective research. That is why design actions are organized in open, creative, consumer-oriented systems, as opposed to systems in scientific research, which are closed and oriented towards solving the technical problem. Research in design has the role of determining and defining through specific methods of scientific research those systems, methods, practical solutions of the design problem in different industrial ecosystems (Norman and Verganti, 2016). The type of research that is perfectly adaptable to the field of design is evidence-based research, in which scientific investigation is humanistic, technical-technological and socio-economic and certifies the evidence necessary for the development of design processes.

\section{Research Methodology in Design}

Ecodesign as a typology of conceptualization and design of the green product is still methodologically unstructured. The green product, both as a technological complexity and as a maker's approach, between slow fashion and fast fashion, there is an arrhythmic industry, neutral in terms of assortments and oriented towards ecodesign and sustainability only in a very small proportion. In this context, the eco-product development methodology, as an experimental methodology, can only be based on a model of discovery and problem 
solving, a model developed by Daniel Clark in 2012 called XProblem (Plano Clark and Ivankova, 2012). This method aims to define the problems and outline a design perspective as close as possible to the realities of the industrial ecosystem. After understanding the environmental issues, a design perspective is discovered or revealed, a design solution is attempted, a test is performed, up to simulation or prototyping, the stage is called Design Iteration. This process is repeated until reaching the optimal design solution that responds to the problem. The methodological steps are the following:

- Immersion in the environment of the problem - implies that design researchers integrate themselves into the environment of the problem to understand the ideas and possible solutions it offers (Desmet and Hekkert, 2016). Thus, in the issue of eco-friendly footwear, immersion in the environment means socio-psychological research in shops, where customer opinions can be taken directly by the designer, sellers' opinions on consumer buying behavior are valuable, the immersion of the designer in the environment of ecofriendly footwear to observe the entire LCA, what are the problems that affect the product design, if the carbon footprint is measured, if the product traceability is respected, the designer's immersion in the distribution process, but also in the product's operating environment, asking customers questions (Pardo and Schweitzer, 2018).

- Convergence - design meetings have the role of converging all issues: those related to product concept materials, technologies, packaging, accessories and advertising and software in a logical and proactive system in the development of the eco-product;

- Divergence - the use of disruptive advantages that lead to innovation. Thus, in iterations made to test the model or a new function of the product, a feature or a new aesthetic direction, reverse decisions are used to create the disruptive advantage. Iterations will lead to creative results, but also to solving the eco-product.

The iterative process in design consists in: identifying the design problem, first of all by defining the needs for that product, choosing those methods and cultural working tools corroborated with technical and economic analysis tools that optimize the specificity of the product range, generating ideas and development of an ideatic prototype (idea sketch, idea modeling, CAD model, sketch folder). Virtual simulation of the product is required to see if it meets all needs (Pop et al., 2018). All mistakes, inadequacies, problems that do not meet the needs are corrected and applied in a new design, along with what was innovative in the previous iteration (Jin and Chusilp, 2006). The iteration process is a spiral process, in continuous development. Fig. 1 shows the CDIO model, a complex spiral of a design iteration.

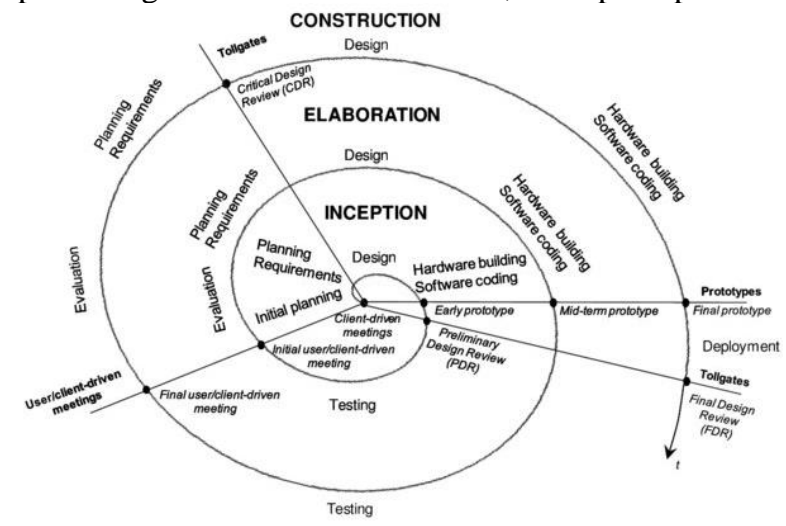

Figure 1. Bermejo, S., 2016 CDIO-Design iteration spiral 
To initiate his/her creative approaches, the designer specialized in ecodesign requires the upstream results, namely the data obtained through individual and company surveys, the limits of the environmental footprints of all partner organizations, carbon footprint measurement and the results of LCAs of all research teams within the project.

\section{PROBLEM SOLVING / HYPOTHESES}

Iteration in design is also called "rapid prototyping" or "spiral prototyping" and is done because simple prototyping is not enough in routine design. Ecodesign requires changing the design in any iteration, knowing from experience so far that ecodesign, due to its technical and economic constraints, is not a creative design.

We assume that the designer specialized in ecodesign needs as many creative iterations as possible to obtain a good design project because he/she goes deeper in the field through this creative tool that reveals the knowledge of the specific field, while a classic, routine design, or a redesign does not require many iterations. It is also assumed that in conceptual design the constraints imposed by the designer to achieve the goal of the final project, in turn, require many iterations. The laboratory experiments performed in the field of footwear fashion and textile design for fashion design aim at validating these hypotheses or not.

\section{EXPERIMENTAL}

\section{Experiment in Footwear Fashion}

The observance of the sustainability requirements disciplines the design of the products and processes, throughout the value chain of the product, which is why designers pay more attention both to the ideation processes and to specific information and research processes. Within the project, a design iteration was made regarding the use of recycled materials, product redesign and development of a design campaign regarding the recycling of materials. The designer Ivona Manea, collaborator of the Il Passo brand, set out to carry out a design campaign, to raise awareness of the need to recycle plastics and a demonstration of a creative design process, using plastic from beach bags. Through a simple iteration, the designer made three variants of a shoe line that will use recycled plastic. Through a simple, creative iteration of the first sketches of ideas, the designer showcased a visual, emotional demonstration of the need for ecological responsibility in moodboards. In images 1 and 2 one can evaluate its entire visual approach, using both the cultural technologies for the development of product concepts and the technical knowledge necessary for footwear design. Cultural technologies in fashion design use content tools as working tools: philosophical imaging, visual archetypes and visual cultural items and also use the cultural tools of expression that are defined by the artistic language, which has its own syntax and stylistics. The transformation of an idea thus obtained into a product concept implies transversal actions, of stylistic declination and commercial (assortment) declination. These are obtained through new creative iterations. Thus, through this process of design thinking, with the help of cultural tools, a greater diversity of cultural objects and creative products arises, transforming the recycled material into a new emotional, cultural and useful product for the informed consumer. 


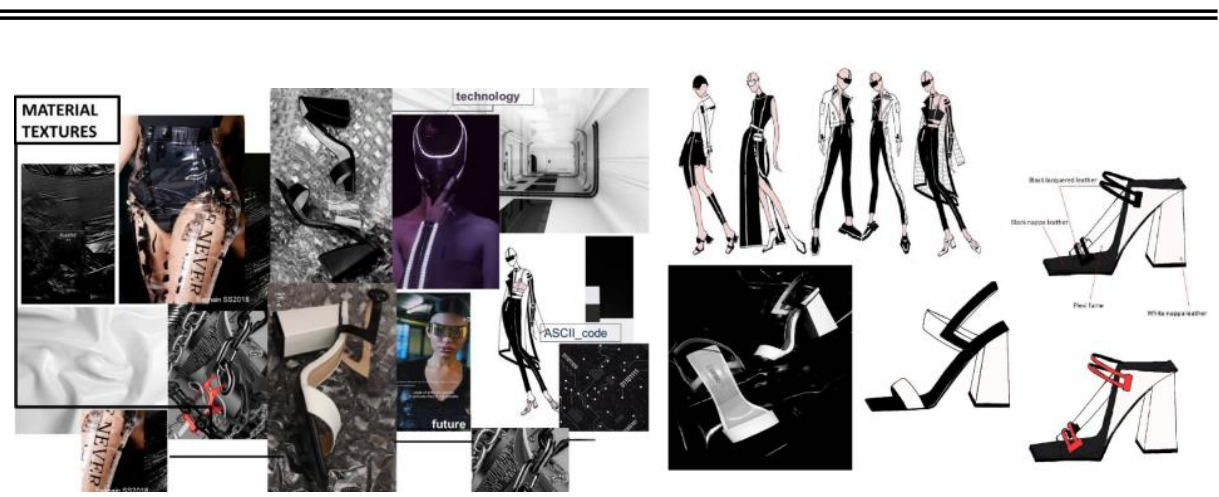

Image 1 and Image 2: Ivona Manea, “ASCII_Code” collection, 2019

\section{Experiment in Textile Design}

An experiment in the field of textile arts and textile design carried out together with students from the National University of Arts (UNA) Bucharest, coordinated by associate professor Dorina Horatau, $\mathrm{PhD}$, aimed to develop the design iteration in three different typologies of textile design: classic design, creative design and conceptual design. The analytical emphasis of the project manager (design research project) was the comparative observation of the development of iteration as a working tool, but also the method of developing individual and group creativity (of the design team). For the student Anamaria Palosanu, the numerous iterations led to projects of high quality through physical prototyping. This experiment in screen printing technique was performed after the student experimented with a mixed technique whose routine iteration did not lead to a satisfactory result in terms of the textile design project for fashion (Image 3).

The routine constraints imposed by the screen-printing technique, through the repetition and inversion of the pattern/color, but also by the "Dropping rain" theme, line composition, forced the student's mind to find a long series of creative iterations.

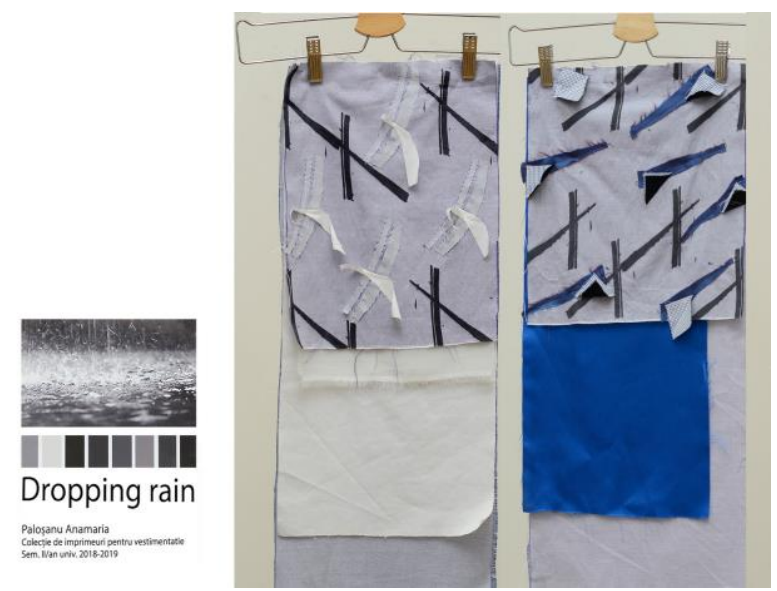

Image 3: Classic iteration in textile design, using a mixed technique - Anamaria Palosanu, $2^{\text {nd }}$ year student in Textile Arts, project coordinator: Assoc. Prof. Dorina Horatau, $\mathrm{PhD}$ 
Not mastering the screen-printing technique and the search for a personal artistic expressiveness were two important impulses in experimenting with creative iterations (Images 4 and 5).
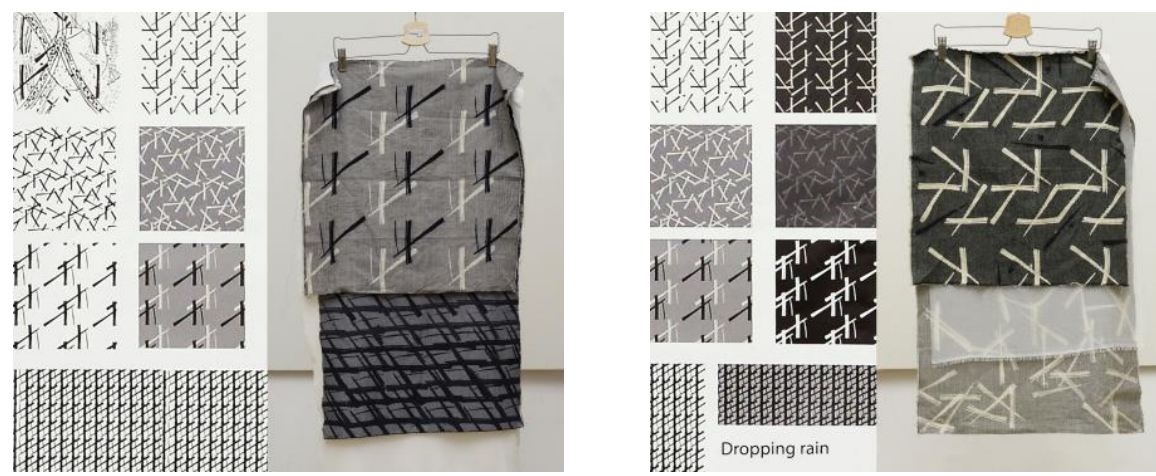

Images 4 and 5: Creative iterations in textile design, through screen printing technique Anamaria Palosanu, "Dropping rain”, 2nd year student, project coordinator: Assoc.

Prof. Dorina Horatau, PhD

It was found that the imposition of constraints in design leads to the development of a larger number of iterations. In conceptual design, when the objective of the design project is to convey the message of the design concept, the refinement of the final solutions can be achieved through creative iterations of the same basic idea, to which are added the stylistic declinations that will allow the development of the iteration spiral. In this student experiment, the concept of the "dropping rain" theme is supported by the graphics of the screen-printing field.

\section{RESULTS AND DISCUSSIONS}

The two types of experiments on the analysis of the practice of creative iterations in fashion design and textile design for eco-product development resulted in over 50 creative iterations expressed through idea sketches and prototypes and two collections of eco-products for footwear and textiles. Creative iterations proved to be useful tools in the development of designers' creativity, both through simple iterations and through iterations based on constraints of the eco-product or of the collection design author.

The basic constraints in ecodesign are, practically, a set of rules that will allow to achieve the objective, namely the green product.

These simple rules refer to:

- observance of innovative design solutions that take into account the entire life cycle of the product, from raw materials to post-consumption;

- observance of rapid prototyping, through successive iterations because only in this way can the optimal solution of designing a viable eco-product be reached,

- a holistic approach, from the first stage of the design process, emphasizing the multifactorial and interdisciplinary synergism, will allow to achieve the objectives, demonstrating that ecodesign is a defining field for green product,

- efficient use of material and energy resources and eliminating all pollutants, all types of destructive messages and symbols; 
- personalized innovation of both the product and green technologies and services towards the responsible customers will change the producer-consumer relationship.

\section{CONCLUSIONS}

The need for creativity of eco-products is the one that imposes on designers several types of iterations, both creative and compelling, in order to obtain a good cultural design project.

The behavior of designers during the iterations is different depending on the type of iteration: if it is a creative, free iteration, the behavior is more relaxed and the iteration is intuitive, and if the iteration arises by constraining the design problem, the iteration sequence is from cognitive to creative.

The basic constraints in ecodesign are, practically, a set of rules that will allow to achieve the objective, namely, the design of the green product. These constraints also require greater attention to all design processes in the product value chain. Creative design needs several iterations to solve the complexity of the green product problem for the clothing and footwear industries.

\section{REFERENCES}

Baumgartner, R.J. and Rauter, R. (2017), "Strategic perspectives of corporate sustainability management to develop a sustainable organization", Journal of Cleaner Production, 140(1), 81-92, https://doi.org/10.1016/j.jclepro.2016.04.146.

Desmet, P. and Hekkert, P. (2007), "Framework of Product Experience”, International Journal of Design, 1(1), ISSN 1991-3761.

Douglas, M. and Isherwood, B.C. (1996), The World of Goods: Towards an Anthropology of Consumption (2nd ed.), London, UK: Routledge.

Jin, Y. and Chusilp, P. (2006), "Study of mental iteration in different design situations", Design Studies, 27(1), 25-55, https://doi.org/10.1016/j.destud.2005.06.003.

Norman, D.A. and Verganti, R. (2014), "Incremental and radical innovation: Design research versus technology and meaning change", Design Issues, 30(1), 78-96, MIT Press, https://doi.org/10.1162/DESI_a_00250.

Pan, Y., Roedl, D., Thomas, J.C. and Blevis, E. (2015), "Fashion thinking: Fashion practices and sustainable interaction design", International Journal of Design, 9(1).

Pardo, R. and Schweitzer, J.P. (2018), A long-term strategy for a European circular economy - setting the course for success, Circular Economy Office, EEB.

Plano Clark, V.L. and Ivankova, N. (2016), Mixed Methods Research: A Guide to the Field, Sage, https://doi.org/10.4135/9781483398341.

Pop, M., Toma, S., Frighi, N. and Pop, M.-A. (2018), "Cultural Work Instruments in Fashion TechnologyPractical Method of Generating Emotional Design”, Journal Textile Science \& Fashion Technology, 1(1), ISSN:2641-192X, https://doi.org/10.33552/JTSFT.2018.01.000505. 


\section{INDEX OF AUTHORS}

A

ABDELBARY, Islam 257

ABIDIN, Mohammad Zainal 169

ACSINTE, Dorel 19

AILENI, Raluca Maria 29, 275

ALBU KAYA, Mădălina

Georgiana 139, 201

ALEKHINA, Victoriy 335

ALEXANDRESCU, Beatrice

Alexandra 373

ALEXANDRESCU, Laurenția 101, 107, 271, 355, 361, 385,

417, 423, 473

ALEXE, Cosmin Andrei 121, 127, 163, 219

ALI, Ahmed 257

ALUVIHARA, Suresh 35

ANICESCU, Maria-Cristina

115

ANUȚA, Valentina 115, 139,

201

APOSTOL, Adrian Alexandru

555

ARÁN AÍS, Francisca 455

AYANOĞLU, Filiz 189, 213

B

BABICH, Antonina Ivanivna 83 BACARDIT, Anna 163, 251, 361

BADEA, Elena 225, 231, 501,

513, 519, 525, 561

BADEA, Ionela 429

BALLUS, Olga 361

BANDARANAYAKE,

P.W.S.K. 35

BARAKAT, Mahmoud 257

BAYOUMI, Engy 263
BECHERITU, Marius 219

BERECHET, Mariana Daniela

121, 127, 225

BONDARYEVA, Anna 43

BOSTACA, Gheorghe 19

BULBOACĂ, Mihaela-Ramona

271

BUNEA, Ana-Maria 139

C

CANIOLA, Iulia-Maria 133, 367, 501, 513, 519, 525, 561

CARBONELL-BLASCO, Pilar 455

CASAS, Concepció 163, 251

CHELARU, Ciprian 49, 127 ,

201

CHIRIAC, Laura 29, 569

CHIRILĂ, Laura 55, 61

CIUPĂGEANU, Dana-

Alexandra 403, 409

COARĂ, Gheorghe 210

CONDE, Mireia 251

CONSTANTIN, Cătălin 447

CONSTANTIN, Daniela Ioana

473

CONSTANTINESCU, Doina

107

CONSTANTINESCU, Rodica

Roxana 49, 55, 121, 133, 151,

157, 367, 513

COSMA, Dragoș-Viorel 61

COSTA, Patrícia 379

CRISTESCU, Ioan 201

CURUȚIU, Carmen 417, 423 
D

DABEES, Ahmed 329

DĂNILĂ, Angela 55

DĂNILĂ, Elena 139, 201

DASHDORJ, Suvda 335

DAVID, Elena 207

DESELNICU, Dana Corina

271, 373, 435, 531

DHANAPAL, Durairaj 67

DINU-PÎRVU, Cristina-Elena

115, 139, 201

DOROGAN, Angela 461

DUMITRIU, Dan 271

DURBACĂ, Ion 71, 277

$\mathbf{E}$

EL ROUBY, Habiba S. 495

ELGAZZAR, Sara 263

ELMESMARY, Hebatallah 311

ENĂSCUȚĂ, Cristina

Emanuela 219

ENE, Alexandra Gabriela 95

EREN, Y1lmaz 145

F

FARCAȘ-FLAMAROPOL,

Dana-Claudia 71, 277

FERDEȘ, Mariana 133, 151, 157

FERREIRA, Maria José 379

FICAI, Anton 101, 107

FICAI, Denisa 101, 107

FOIAȘI, Traian 441, 489, 537

G

GAANBAATAR, Khurelsukh 335

GAIDĂU, Carmen 163, 219, $225,231,237$
GARKAVENKO, Svitlana

Stepanivna 83, 89

GEORGESCU, Mihai 101, 107, $335,361,385,417,423,473$,

481

GHICA, Mihaela Violeta 115, 139, 201

GHICULESCU, Liviu-Daniel

207

GÎDEA, Mihai 219

GIURGINCĂ, Mariana 519

GLIONIK, Anastasia 89

GOHAR, Nermin 283

GONZALEZ-QUIJANO,

Gustavo 19

GRIGORIU, Rodica-Manuela

403, 409

GRIYANITASARI, Gresy 169

GROSU, Cristina 461

GURĂU, Dana 101, 107, 225,

$231,385,417,423,441$

\section{H}

HADDAD, Sandra Samy

George 289, 495

HADÎMBU, Emanuel 501, 513, 561

HAMMAD, Mahmoud A. 495

HANNA, Hany 283, 391

HAROUN, Mai H. 257, 283

HASSANIN, Islam Saleh 297

HORĂTĂU, Dorina 575

HOU, Keyu 303, 317

I

IATAN, Radu 71

IGNAT, Mădălina-Camelia 49, 501, 525, 561

IORDACHE, Ovidiu 77, 177, 549, 555

IOVAN-DRAGOMIR, Alina 323, 507 
IUNGIN, Olga 195

J

JUGĂNARU, Mircea 101, 107

K

KALPAGE, C.S. 35

KARAÇAM, Meryem 183

KASMUDJIASTUTI, Emiliana 169

KAYA, Durmuş Alpaslan 139, 145, 183, 189, 201, 213, 513

KERNESH, Victoria 89

KESHTA, Jilan 311

KHOSBAYAR, Solongo 335

KNEZ, Matjaz 329

KOLEVA, Margarita 243

KUBILIUS, Karolis 399

KULIK, Tetiana 89

$\mathbf{L}$

LĂZĂROIU, Gheorghe 403,

409

LESHCHYSHYN

MYKOLAIVNA, Maryna 83,

89

LI, Jingjing 303, 317

LI, Shunxun 303

LITE, Mihaela-Cristina 77, 177, 549, 555

LUCA, Alexandra 323, 507

M

MA, Jianzhong 237

MAISTRENKO, Lesia 195

MARIN, Maria-Minodora 101,

107, 139, 201

MARINESCU, Maria-Roxana

207

MATEESCU, Alice-Ortansa 61
MATEESCU, Gheorghe 61

MAVİ, Kazim 213

MELESE, Bahiru 67

MELINESCU, Alina 101

MEMECICĂ, Maria 77, 177

MICU, Maria-Cristina 501, 513,

519, 525, 561

MIHĂESCU, Lucian 403

MIHAI, Carmen 95, 347

MITRAN, Elena-Cornelia 77,

177, 549, 555

MITU, Bogdana 447

MIU, Lucreția 225, 231, 501, 513, 519, 525, 561

MLAKER KAČ, Sonja 495

MOGA, Ioana Corina 177

MOKROUSOVA, Olena 43

MONTIEL PARREÑO,

Enrique 441

$\mathbf{N}$

NICULESCU, Mihaela-Doina

219

NICULESCU, Olga 163, 225,

231

NIȚUICĂ, Mihaela 101, 107, $355,361,385,417,423,473$, 481

O

OBRECHT, Matevž 263, 311

OKHMAT, Olena 195

OLARU, Sabina 55, 61, 347,

429

OLLÉ, Lluís 251

OPREA, Ovidiu 101, 107

OPRIȘ, Mircea Constantin 435,

531

ORGILÉS-CALPENA, Elena

455

OTHMAN, Alaa 329

ÖZTÜRK, Şevket 139 
PANTAZI-BĂJENARU, Mirela 441, 489, 537

PĂUN, Gabriela 163

PĂUNESCU, Simona-Maria

501, 513, 519, 525, 561

PERDUM, Elena 77, 177, 549,

555

PINTO, Vera V. 379

POP, Marlena 543, 575

POPA, Lăcrămioara 115, 139,

201

POPESCU, Alina 55

PRISADA, Răzvan Mihai 115

$\mathbf{R}$

RADNAEVA, Vera 335

RĂDULESCU, Ion Răzvan

447, 569

RAHMA, Baher 329

RAJAMANI, Sengoda Gounder 341

RÂPĂ, Maria 121, 127

RENTIZELAS, Athanasios 391

ROȘU, Marcela Corina 61

RUS, Mădălina Alice 95

RUZAFA-SILVESTRE, Carlos 455

$\mathbf{S}$

SĂLIȘTEAN, Adrian 347

SĂNDULACHE, Irina 29, 77 ,

177, 549, 555

SANMUGAM, Kumaravel 67

SÂRBU, Teodor 461

SAVCHUK, Oleksii 195

SCARLAT, Răzvan 95, 447

SECĂREANU, Lucia Oana 77, 177, 549, 555

ŞENDREA, Claudiu 501, 513, $519,525,561$
SHALBUEV, Dmitry 335

SHAWEL, Liya 67

SIMION, Demetra 121, 127,

163, 231, 237

SIMION, Iulia 409

ŠLIBURYTĖ, Auksė 467

SÖNMEZ, Maria 101, 107, 355,

361, 385, 417, 423, 473, 481

SOROLLA, Sílvia 251

SOVETKIN, Nikolay 335

SPOREA, Nicoleta 277

STANCA, Maria 121, 127, 163,

219

S,TEFAN, Adriana 481

STELESCU, Maria Daniela 101, 107, 355, 361, 385, 417, 423, 473, 481

STOLERIU, Ștefania 417, 423

STROE, Cristina Elena 461

SURDU, Elena 71, 77

$\mathbf{T}$

TALIANU, Marina-Theodora

115

ȚIGĂU, Andreea 55

TOMA, Doina 347

TONEA, Stoica 163

TRUȘCĂ, Roxana 107

TUDORAN, Cristian 61

TURCANU, Andreea 121

TÜRKMEN, Musa 213

$\mathbf{U}$

URDA, Alexandra 61

V

VALEIKA, Virgilijus 399, 467

VALEIKIENE, Violeta 399

VASILE, Georgiana 61

VISILEANU, Emilia 447 
W

WANG, Rui 303, 317

WENBO, Zhang 237

$\mathbf{X}$

XIROUCHAKIS, Paul 391

XU, Wei 303

Y

YULIATMO, Ragil 169

$\mathbf{Z}$

ZĂINESCU, Gabriel 133, 367

ZENG, Jie 317

ZHELEVA, Darina 243

ZHOU, Jin 303, 317

ZLOTENKO, Borys 89 


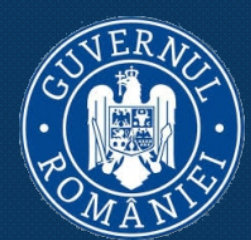

MINISTRY OF EDUCATION AND RESEARCH

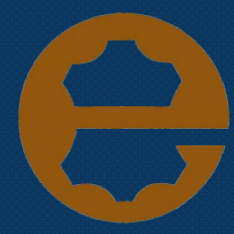

LEATHER ENGINEERING DEPARTMENT EGE UNIVERSITY, TURKEY

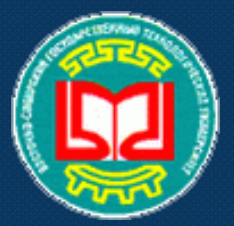

EAST SIBERIA STATE UNIVERSITY OF TECHNOLOGY \& MANAGEMENT, ULAN-UDE, RUSSIA

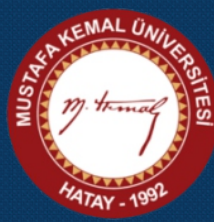

MUSTAFA

KEMAL

UNIVERSITY, TURKEY

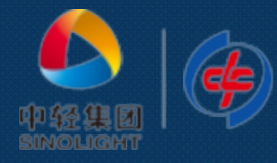

CHINA LEATHER \& FOOTWEAR

RESEARCH INSTITUTE Co. Ltd., CHINA

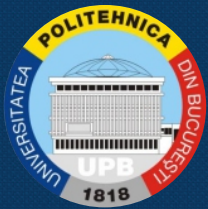

"POLITEHNICA" UNIVERSITY BUCHAREST, ROMANIA

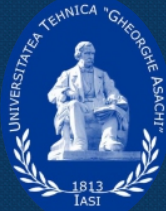

"GH. ASACH" TECHNICAL UNIVERSITY OF IASI, ROMANIA

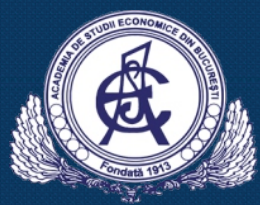

BUCHAREST ACADEMY OF ECONOMIC STUDIES, ROMANIA

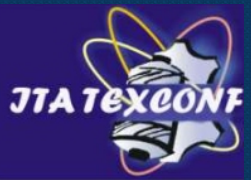

ITA TEXCONF ROMANIAN ENTITY WITHIN INNOVATION \& LEATHER TECHNOLOGICAL TRANSFER

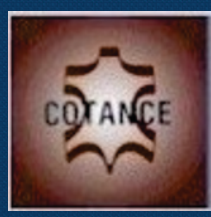

CONFEDERATION OF NATIONAL ASSOCIATIONS OF TANNERS AND DRESSERS OF THE EUROPEAN COMMUNITY

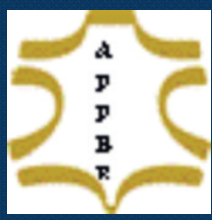

ROMANIAN LEATHER \& FUR PRODUCERS ASSOCIATION

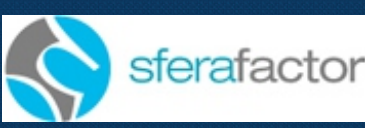

SFERA FACTOR THE ROMANIAN LEATHER

MANUFACTURERS ORGANIZATION

ISSN: 2068-0783 